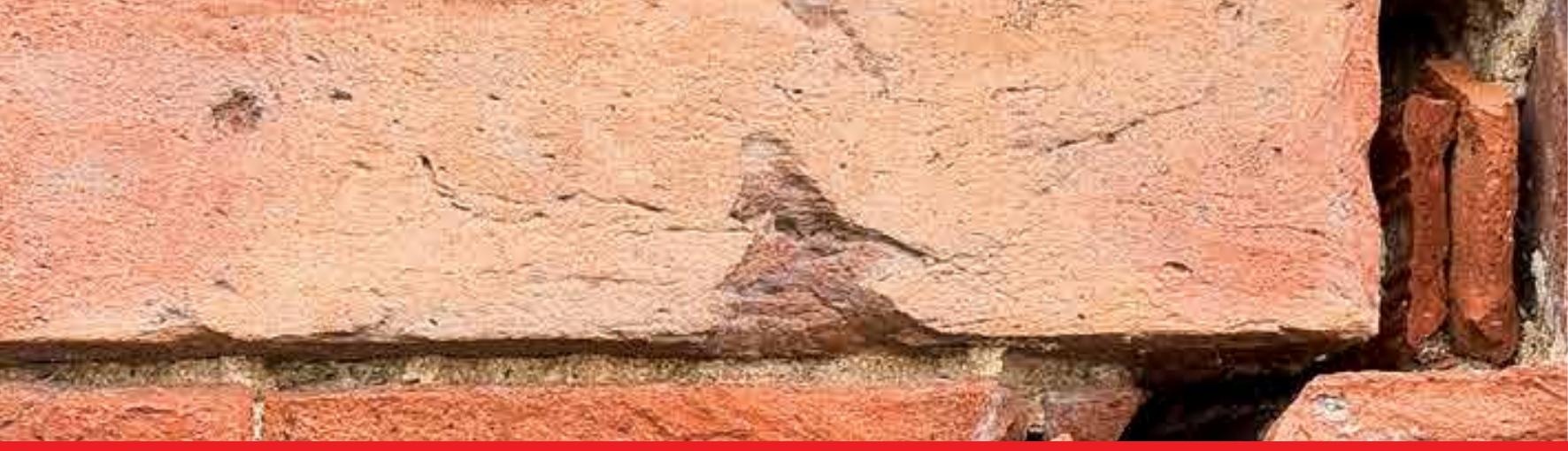

\title{
IntechOpen
}

\section{Earthquake-Resistant Structures}

Design, Assessment and Rehabilitation

Edited by Abbas Moustafa
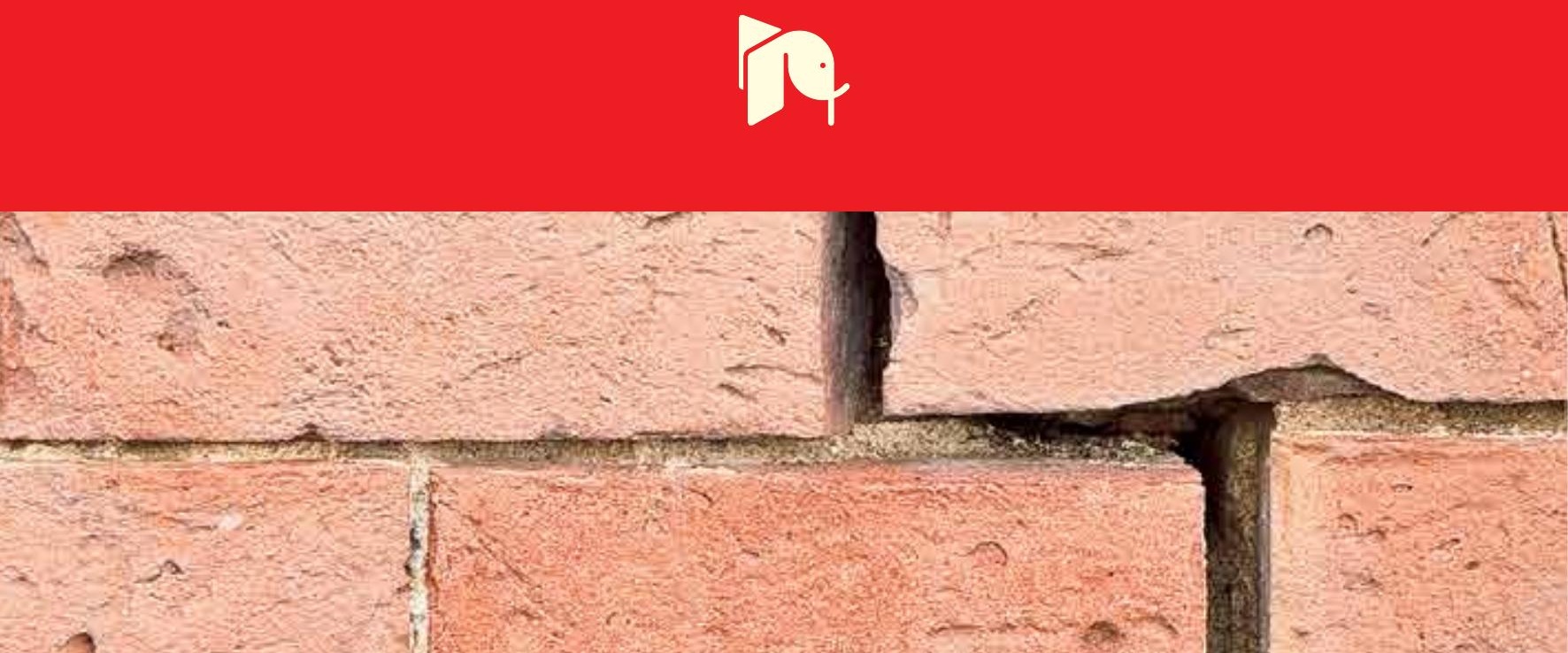



\section{EARTHQUAKE-RESISTANT STRUCTURES - DESIGN, ASSESSMENT AND REHABILITATION}

Edited by Abbas Moustafa 


\section{Contributors}

Triantafyllos Konstantinos Makarios, Wancheng Yuan, Qing-Hai Feng, Chih-Chen Chang, Massimo Bavusi, Romeo Bernini, Vincenzo Lapenna, Antonio Loperte, Francesco Soldovieri, Felice Carlo Ponzo, Antonio Di Cesare, Rocco Ditommaso, Zhu Jian, Halil Sezen, Muhammad Shadab Lodhi, Chong-Shien Tsai, Genda Chen, Ying Huang, Hai Xiao, George Lee, Zach Liang, Xiaosong Ren, Mohammad Reza Tabeshpour, Francesco Castelli, Ernesto Motta, Akira Maekawa, Hasan Kaplan, Salih Yilmaz, Akinola Johnson Olarewaju (Engr.) PhD, Thomas Zimmermann, Alfred Strauss, Rehan Ahmad Khan, Omar A Pineda-Porras, Mario Ordaz, Junichi Abe, Hiroyuki Sugimoto, Tadatomo Watanabe, Mohamed F.M. Fahmy, Zhishen Wu

\section{(c) The Editor(s) and the Author(s) 2012}

The moral rights of the and the author(s) have been asserted.

All rights to the book as a whole are reserved by INTECH. The book as a whole (compilation) cannot be reproduced, distributed or used for commercial or non-commercial purposes without INTECH's written permission. Enquiries concerning the use of the book should be directed to INTECH rights and permissions department (permissions@intechopen.com).

Violations are liable to prosecution under the governing Copyright Law.

\section{(cc) BY}

Individual chapters of this publication are distributed under the terms of the Creative Commons Attribution 3.0 Unported License which permits commercial use, distribution and reproduction of the individual chapters, provided the original author(s) and source publication are appropriately acknowledged. If so indicated, certain images may not be included under the Creative Commons license. In such cases users will need to obtain permission from the license holder to reproduce the material. More details and guidelines concerning content reuse and adaptation can be foundat http://www.intechopen.com/copyright-policy.html.

\section{Notice}

Statements and opinions expressed in the chapters are these of the individual contributors and not necessarily those of the editors or publisher. No responsibility is accepted for the accuracy of information contained in the published chapters. The publisher assumes no responsibility for any damage or injury to persons or property arising out of the use of any materials, instructions, methods or ideas contained in the book.

First published in Croatia, 2012 by INTECH d.o.o.

eBook (PDF) Published by IN TECH d.o.o.

Place and year of publication of eBook (PDF): Rijeka, 2019.

IntechOpen is the global imprint of IN TECH d.o.o.

Printed in Croatia

Legal deposit, Croatia: National and University Library in Zagreb

Additional hard and PDF copies can be obtained from orders@intechopen.com

Earthquake-Resistant Structures - Design, Assessment and Rehabilitation

Edited by Abbas Moustafa

p. cm.

ISBN 978-953-51-0123-9

eBook (PDF) ISBN 978-953-51-6125-7 


\section{We are IntechOpen, \\ the world's leading publisher of Open Access books}

Built by scientists, for scientists

\section{$4,000+$ \\ Open access books available \\ $116,000+$ \\ International authors and editors

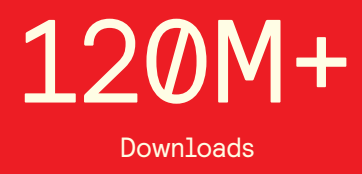

Our authors are among the

151

Countries delivered to

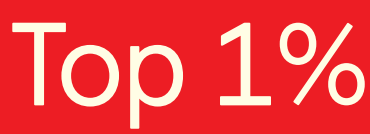

most cited scientists

Contributors from top 500 universities

$12.2 \%$

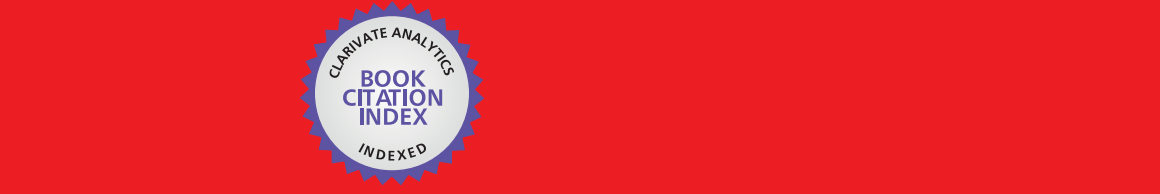

WEB OF SCIENCE ${ }^{\mathrm{M}}$

Selection of our books indexed in the Book Citation Index in Web of Science ${ }^{\mathrm{TM}}$ Core Collection (BKCI)

\section{Interested in publishing with us? \\ Contact book.department@intechopen.com}





\section{Meet the editor}

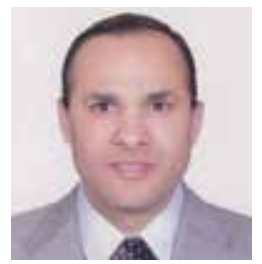

Prof. Moustafa got his doctoral degree in earthquake engineering and structural safety from Indian Institute of Science in 2002. He is currently an associate professor at Department of Civil Engineering, Minia University, Egypt and the chairman of Department of Civil Engineering, High Institute of Engineering and Technology, Giza, Egypt. He is also a consultant engineer and head of structural group at Hamza Associates, Giza, Egypt. Dr. Moustafa was a senior research associate at Vanderbilt University (USA) and a JSPS fellow at Kyoto and Nagasaki Universities (Japan). He has more than 40 research papers published in international journals and conferences. He acts as an editorial board member and a reviewer for several regional and international journals. His research interest includes earthquake engineering, seismic design, nonlinear dynamics, random vibration, structural reliability, structural health monitoring and uncertainty modeling. 



\section{Contents}

\section{Preface XI}

Chapter 1 Seismic Design Forces and Risks 1

Junichi Abe, Hiroyuki Sugimoto and

Tadatomo Watanabe

Chapter 2 Seismic Bearing Capacity of Shallow Foundations

Francesco Castelli and Ernesto Motta

Chapter 3 Design Principles of Seismic Isolation 43

George C. Lee and Zach Liang

Chapter 4 Advanced Base Isolation

Systems for Light Weight Equipments 79

Chong-Shien Tsai

Chapter 5 Seismic Damage Estimation in

Buried Pipelines Due to Future Earthquakes -

The Case of the Mexico City Water System 131

Omar A. Pineda-Porras and Mario Ordaz

Chapter 6 The Equivalent Non-Linear Single Degree of Freedom System of Asymmetric Multi-Storey Buildings in Seismic Static Pushover Analysis 151

Triantafyllos K. Makarios

Chapter 7 Masonry and Earthquakes: Material Properties, Experimental Testing and Design Approaches 177 Thomas Zimmermann and Alfred Strauss

Chapter 8 Seismic Performance of Masonry Building 205 Xiaosong Ren, Pang Li,

Chuang Liu and Bin Zhou

Chapter 9 Seismic Response of Reinforced Concrete Columns 227 Halil Sezen and Muhammad S. Lodhi 
Chapter 10 Seismic Vulnerability Analysis of RC Buildings in Western China 251

Zhu Jian

Chapter 11 Seismic Behavior and Retrofit of Infilled Frames 279

Mohammad Reza Tabeshpour,

Amir Azad and Ali Akbar Golafshani

Chapter 12 Recent Advances in Seismic Response

Analysis of Cylindrical Liquid Storage Tanks 307

Akira Maekawa

Chapter 13 Seismic Reliability Analysis of

Cable Stayed Bridges Against First Passage Failure 337

Rehan Ahmad Khan

Chapter 14 Compound Stochastic Seismic

Vulnerability Analysis and Seismic

Risk Evaluation for Super Large Cable-Stayed Bridge

Feng Qing-Hai, Yuan Wan-Cheng and Chih-Chen Chang

Chapter 15 Assessment and Rehabilitation of Jacket Platforms 381

Mohammad Reza Tabeshpour,

Younes Komachi and Ali Akbar Golafshani

Chapter 16 Seismic Strengthening of

Reinforced Concrete Buildings 407

Hasan Kaplan and Salih Yılmaz

Chapter 17 Bond-Based Earthquake-Proof of RC Bridge Columns Reinforced with Steel Rebars and SFCBs 429

Mohamed F.M. Fahmy and Zhishen Wu

Chapter 18 Electromagnetic Sensing Techniques for Non-Destructive Diagnosis of Civil Engineering Structures 455

Massimo Bavusi, Romeo Bernini, Vincenzo Lapenna,

Antonio Loperte, Francesco Soldovieri, Felice Carlo Ponzo,

Antonio Di Cesare and Rocco Ditommaso

Chapter 19 Steel Building Assessment in Post-Earthquake

Fire Environments with Fiber Optical Sensors 481

Genda Chen, Ying Huang and Hai Xiao

Chapter 20 Response of Underground Pipes to Blast Loads $\mathbf{5 0 7}$

A.J. Olarewaju, R.N.S.V. Kameswara and M.A. Mannan 


\section{Preface}

This book deals with earthquake-resistant structures. It covers the seismic-resistance design of structures to be constructed as well as the safety assessment, strengthening and rehabilitation of existing structures against earthquake loads. The book also includes two chapters on the response of underground pipes to blast loads and post earthquake assessment of steel buildings in fire environment. It contains twenty chapters covering several interesting research topics written by researchers and experts in the field of earthquake engineering. The book provides the state-of-the-art on recent progress on earthquake-resistant structures. It should be useful to graduate students, researchers and practice structural engineers.

Chapter 1 focuses on the seismic design forces and risks. Chapter 2 deals with the estimation of the seismic bearing capacity of shallow foundations. Chapter 3 is devoted to the design principles of seismic base isolation systems for structures. Chapter 4 sheds lights on recent advanced base isolation systems for light weight equipment. The seismic damage estimation in buried pipelines is presented in Chapter 5. Chapter 6 deals with the modelling of equivalent non-linear single-degree-of-freedom system for spatial asymmetric multi-storey buildings. Chapters 7 and 8 tackle the seismic response of masonry structures. Specifically, Chapter 7 deals with material properties, experimental testing and design approaches of masonry structures under earthquake loads. Chapter 8 explores the seismic performance of masonry structures. Chapters 9-11 study the response of reinforced concrete structures to earthquake loads. Chapter 9 handles the seismic response of reinforced concrete columns. Chapter 10 investigates the seismic vulnerability of reinforced concrete buildings. Chapter 11 studies the seismic design and retrofit of infilled frames. Chapter 12 explores the recent advances in seismic design and retrofit of cylindrical storage tanks. Chapter 13 deals with the reliability assessment of cable-stayed bridges against first passage failure. In Chapter 14, the stochastic vulnerability analysis and the risk evaluation of super large cable-stayed bridges are studied. Chapter 15 deals with the assessment and rehabilitation of jacket platforms. The seismic strengthening of reinforced concrete buildings is presented in Chapter 16. Chapter 17 deals with the bondbased proof of reinforced concrete bridges to earthquake loads. The electromagnetic sensing techniques of civil structures are addressed in Chapter 18. Chapter 19 is concerned with the post-earthquake assessment of steel buildings in fire environment. Chapter 20 deals with the response of buried pipelines to blast loads. 
I hope this book benefits graduate students, researchers and engineers working in resistance design of engineering structures to earthquake loads, blast and fire. I thank the authors of the chapters of this book for their cooperation and effort during the review process. Thanks are also due to Mr. Igor Babic and Ms. Ivana Zec for their help during the processing and publishing of the book. I thank also Professor C S Manohar, Indian Institute of Science, Professor Sankaran Mahadevan, Vanderbilt University, and Professor Izuru Takewaki, Kyoto University, for all I have earned from them on earthquake engineering, structural reliability analysis and health assessment of structures.

Prof. Abbas Moustafa

Department of Civil Engineering,

Faculty of Engineering,

Minia University

Egypt 




\title{
Seismic Design Forces and Risks
}

\author{
Junichi Abe, Hiroyuki Sugimoto and Tadatomo Watanabe \\ Hokubu Consultant Corporation, Hokkai Gakuen University \\ Japan
}

\section{Introduction}

In recent years, seismic damages caused by giant earthquakes have occurred in many countries. For example, over 250,000 people were killed by the Haiti Earthquake in January 2010. In addition, over 15,000 people were killed by the Tohoku Japan Earthquake and the coasts of Tohoku Japan were devastated by the massive tidal wave in March 2011.

Meanwhile, The Japanese seismic design criteria for road and railway bridges provide that two levels of earthquake motions - Level 1, which is small in scale but is generated frequently, and Level 2, which is intensive but is not generated frequently - must be used for the verification of seismic performance. For Level 1 earthquake motions, the elastic limit value of a structure is usually adopted as the seismic performance. For Level 2 earthquake motions, on the other hand, the limit value with which a structure does not collapse or is repairable is adopted as the seismic performance depending on the importance of the intended structure.

Level 2 earthquake motions used for verification are based on the records of strong motion seismograms obtained from the Hyogoken-Nanbu and other earthquakes, and seismic waveforms are assigned according to ground type. The earthquake motions are assigned according to classification of the land area of Japan categorized into three types by degree of seismic risk and adjusting the seismic motions using regional correction factors of 1.0, 0.85 and 0.7 depending on the regional classification.

Meanwhile, studies to calculate seismic waveforms unique to the target region of seismic design have been conducted in recent years. Seismic waveforms calculated in these studies were determined by carefully examining past seismic records, ground data, source models and other data of the target region from the viewpoint of earthquake and geotechnical engineering.

In reality, however, earthquakes that generate ground motions stronger than Level 1 but do not exceed Level 2 may occur during the service life of a structure. In current seismic design, direct consideration was not given to changes in performance and risk with seismic motions through time or the importance of applying effective repair and reinforcement methods. These factors cannot be taken fully into account by simply verifying the elastic limit or the limit of reparability or collapse of a structure subject to Level 1 or 2 earthquake motions based on the current seismic design force.

Many seismic risk management studies, which evaluated the loss (seismic risk) caused by the damage or collapse of a structure, have also been conducted in recent years. In these 
papers, seismic risks were calculated using a hazard curve representing the probability of the generation of earthquake motions and a damage curve representing the probability of damage to the structure.

While this damage curve is calculated by statistical procedures using past damage records and analyses, it is necessary to define the damage to a structure with a single index, such as the top horizontal displacement or ductility factor. When damage is defined with an index, it is difficult to precisely associate the index with the repair for the damage. Therefore, these methods are considered difficult to apply them to the examination of seismic risks based on the definition of changes in the damage process and other details due to the difference in design.

To achieve these, it is first necessary to calculate design solutions reflecting the damage and collapse process of a structure under a uniform standard of value for various seismic forces. By calculating seismic risks for respective design solutions and comparing them for different seismic forces, it is possible to find the seismic force with which the total cost including the initial construction cost and seismic risk can be minimized. This is called the "target seismic design force" in this chapter. Although this method involves complex procedures, the necessity for target seismic design forces is expected to be higher for the design of long bridges and other structures that are highly important as lifelines from the viewpoint of the seismic risk management.

This chapter consists of the section presented below.

2. describes a design system with which design solutions are calculated using various seismic forces and the method for calculating the target seismic design forces. 3. explains the method for calculating seismic risks based on the definition of damage.

4. present the results of the analysis of an $\mathrm{RC}$ rigid-frame viaduct as Example of the calculation of the target seismic design forces.

While there is the possibility of loss caused by environmental and other effects besides those of earthquakes during the life cycle of a structure, such additional effects will be studied in the future and this paper limits its focus on the effects of earthquakes.

\section{Target seismic design force}

This section explains the method for calculating the target seismic design forces. In the case of design where the seismic risks of a variety of seismic forces are taken into account, it is assumed that the initial construction cost is low but the seismic risk is high for a structure designed for a low seismic force, while the seismic risk is low but the initial construction cost is high for a structure designed for a high seismic force. By quantifying this seismic risk based on the cost for the repair of damage and other factors to find the seismic risk cost, calculating the total cost by adding this to the initial construction cost and finding its relationship with the seismic force, the target seismic design force and the corresponding design solution can be obtained. Fig. 1 illustrates the flow of finding the target seismic design force and the corresponding design solution. Details of the flow are as described below.

\subsection{Setting of the target structure and region}

The type of the structure to be designed and the region where the structure will be constructed are set. 


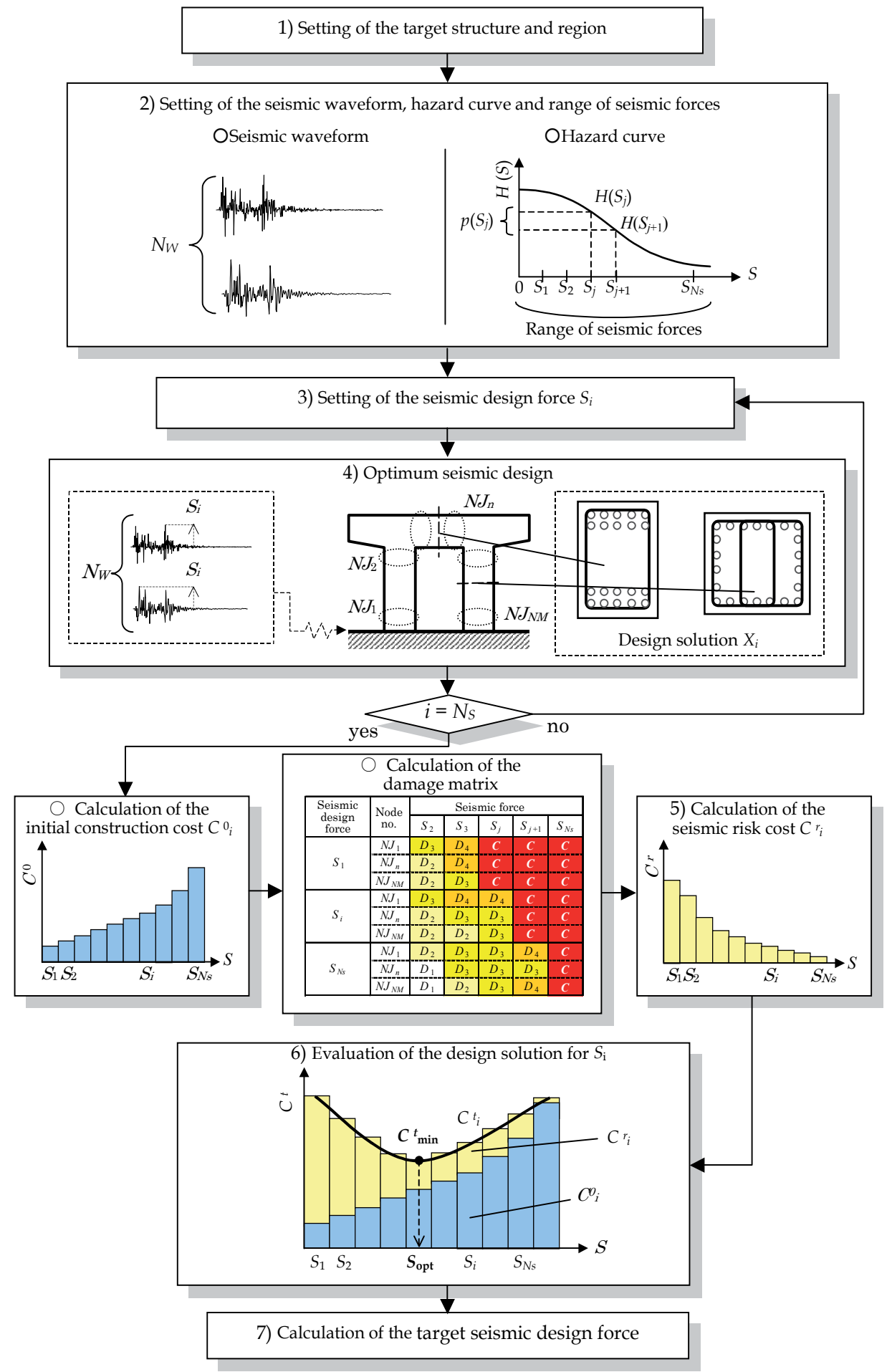

Fig. 1. Flow of calculation of the taqrget seismic design force. 


\subsection{Setting of the seismic waveform, hazard curve and range of seismic forces}

Appropriate seismic waveform and hazard curve are set for the target region. The incremental value $\Delta S$ and division number $N_{S}$ of the seismic forces are also set as shown in Fig. 2.

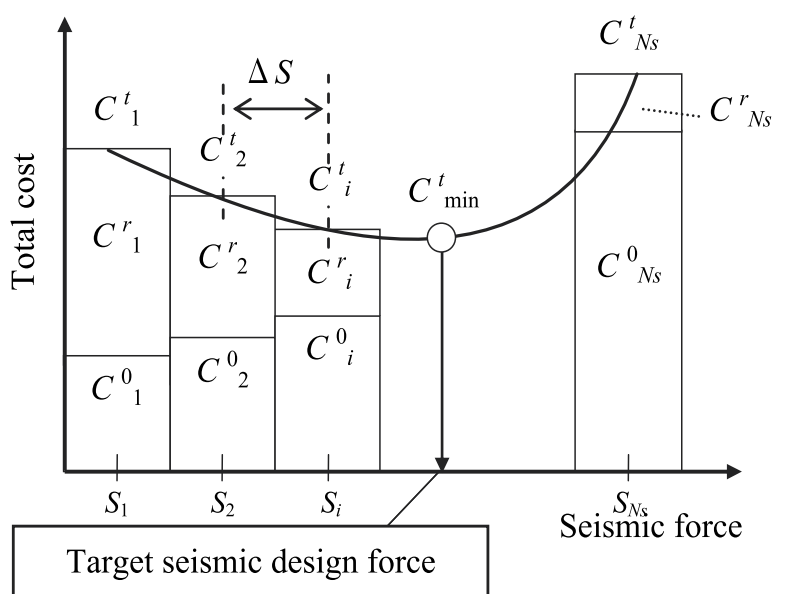

Fig. 2. Relationship between the totalcost and seismic force.

\subsection{Setting of the seismic force}

Based on the range of seismic forces set in 2), the seismic force for optimum seismic design $\mathrm{S}_{\mathrm{i}}\left(\mathrm{i}=1 \sim \mathrm{N}_{\mathrm{S}}\right)$ is set.

\subsection{Optimum seismic design}

Optimization of seismic design is performed for each seismic force $S_{i}\left(i=1 \sim N_{S}\right)$. Details of the formulation of optimum seismic design will be presented later. Time history response analysis is performed by conducting amplitude adjustment to make the maximum amplitude for the seismic waveform set in 2) equal to the seismic force $\mathrm{S}_{\mathrm{i}}$. In this chapter, the optimum solution is calculated through the optimization of the response surface using the RBF network and Genetic Algorithm under the minimized initial construction cost. The initial construction cost of the optimum design solution obtained is presented as $\mathrm{C}_{\mathrm{i}}\left(\mathrm{i}=1 \sim \mathrm{N}_{\mathrm{s}}\right)$.

In this section, the optimum solution is calculated through the optimum seismic design system of the response surface using the RBF network and Genetic Algorithm by the authors.

\subsection{Calculation of the seismic risk cost}

The seismic risk cost $C_{i}\left(i=1 \sim N_{s}\right)$ for each design solution found in 4$)$ is calculated for the range of seismic forces set in 2). It means that analysis and verification are performed $N_{s}$ times for each design solution. The method for calculating the seismic risk cost is as mentioned below.

\subsection{Evaluation of the design solution}

The design solution for a seismic force $S_{i}$ is evaluated by the equation below, as the total cost $\mathrm{C}_{\mathrm{i}}\left(\mathrm{i}=1 \sim \mathrm{N}_{\mathrm{s}}\right)$ found by adding the initial construction cost $\mathrm{C}_{\mathrm{i}}$ of the design solution found in 4 ) to the seismic risk cost $C_{i}$ found in 5),

$$
\mathrm{C}^{\mathrm{t}}{ }_{\mathrm{i}}=\mathrm{C}^{0}{ }_{\mathrm{i}}+\mathrm{C}^{\mathrm{r}}{ }_{\mathrm{i}}
$$




\subsection{Calculation of the target seismic design force}

The above calculation is performed to calculate the total cost $C_{i}$ for each $S_{i}$. Fig. 2 is a conceptual diagram of the relationship between the total cost $C_{i} t_{i}$ and seismic force $S_{i}$. Of these $C_{i}{ }_{i}$ values, the seismic force corresponding to the minimum total cost $C^{t}$ min is the target seismic design force.

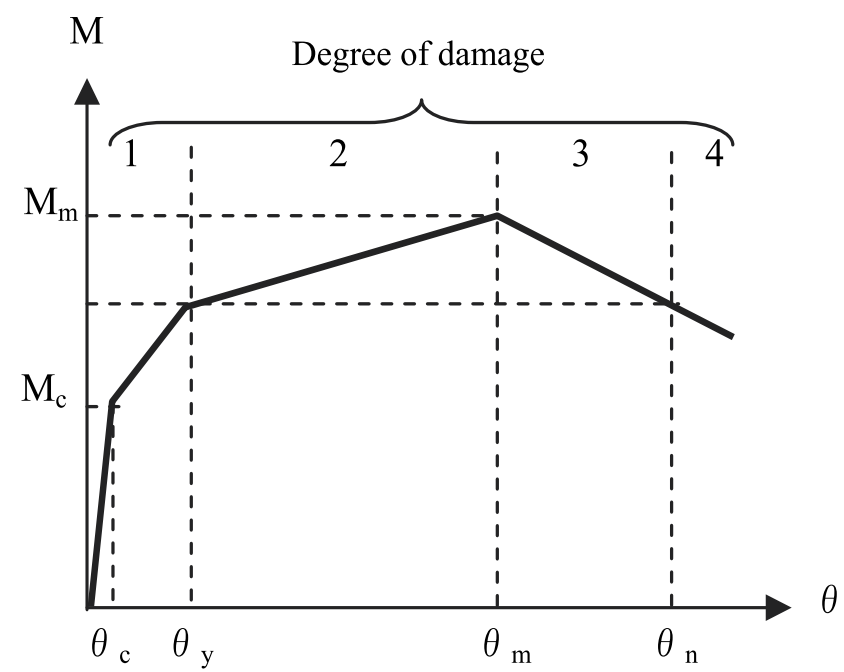

Fig. 3. Skeleton curve and degree of damage.

\section{Seismic risk cost}

As mentioned before, the total cost for each seismic force is calculated by totalling the initial construction and seismic risk costs. The seismic risk cost is usually calculated using damage and hazard curves. However, a damage matrix is constructed by evaluating damage to all elements where nonlinearity is taken into account instead of using a damage curve, and the seismic risk cost is found by calculating repair and other costs.

This section first defines the damage to an RC structure, and then describes the method for calculating seismic risk costs.

\subsection{Definition of damage}

In this chapter, damage is defined for all elements where nonlinearity is taken into account. The $\mathrm{M}-\theta$ relationship of a tetra-linear model, which is represented by the thick black line in Fig. 3, is used as the relationship between the nonlinearity of RC elements and damage , in accordance with the method defined in the Design Code for Railway Structures and instruction manual (seismic design). In the figure, $\mathrm{M}_{\mathrm{c}}$ is the bending moment at the time of cracking, $\mathrm{M}_{\mathrm{y}}$ is the bending moment at the time of yield, $\mathrm{M}_{\mathrm{m}}$ is the maximum bending moment, $\theta_{\mathrm{c}}$ is the angle of rotation at the time of cracking, $\theta_{\mathrm{y}}$ is the angle of rotation at the time of yield, $\theta_{\mathrm{m}}$ is the maximum angle of rotation to maintain $\mathrm{M}_{\mathrm{m}}$, and $\theta_{\mathrm{n}}$ is the maximum angle of rotation to maintain $\mathrm{M}_{\mathrm{y}}$.

Classified degree of damage is defined as degree 1 if the maximum response angle of rotation found from time history response analysis is $\theta_{\mathrm{y}}$ or smaller, degree 2 if it is $\theta_{\mathrm{m}}$ or smaller, degree 3 if it is $\theta_{n}$ or smaller and degree 4 if it exceeds $\theta_{n}$. 
The term "degree 1" represents a condition in which the cracks of concrete member have occurred. The term "degree 2 " represents a condition in which the reinforcing bar in the axial direction has yielded. The term "degree 3 " represents a condition in which the side of compression of concrete member has fractured. The term "degree 4 "represents a condition in which the flexure capacity has decreased by under the yield capacity.

\subsection{Calculation of the damage matrix}

To calculate the seismic risk cost, it is necessary to determine the damage of the structure for a certain seismic force and calculate repair and other costs. As mentioned before, this study uses a damage matrix instead of a damage curve, which is generally used to represent the relationship between the seismic force and damage of the structure.

Fig.4 presents the damage matrix using a single-layer portal rigid-frame structure. In the case of a rigid-frame structure, plastic hinges with the effect of nonlinearity are found at 6 sections in total - the upper and lower ends of each column member and the left and right ends of beam members. The table on fig. 4 shows the node numbers displayed in the rows and seismic forces in the columns. It is a matrix notation of the damage at each node when various seismic forces are input for a certain design solution. In the table, " $\mathrm{C}$ " represents the collapse of the structure. This kind of damage matrix is developed for each of the design solution found for each seismic force.

\subsection{Calculation of seismic risk costs}

In this chapter, the seismic risk cost is calculated using a damage matrix representing the relationship between the seismic force and damage as shown in fig. 4 and a hazard curve representing the relationship between the seismic force and annual probability of excess as shown in Fig. 5. The seismic risk cost is calculated by the equation below,

$$
\mathrm{C}_{\mathrm{i}}^{\mathrm{r}}=\sum_{\mathrm{j}=1}^{\mathrm{Ns}} \mathrm{h}\left(\mathrm{S}_{\mathrm{j}}\right) \cdot \mathrm{c}_{\mathrm{ij}} \cdot \Delta \mathrm{s}\left(\mathrm{i}=1 \sim \mathrm{N}_{\mathrm{S}}\right)
$$

where, $\mathrm{Cr}_{\mathrm{i}}$ is the seismic risk cost of the design solution designed for the $\mathrm{i}$-th seismic force, $\mathrm{h}$ $\left(S_{j}\right)$ is the annual probability of occurrence found from the hazard curve for the $j$-th seismic force $S_{j}, C_{i j}$ is the seismic loss cost for the damage of each element caused by the $j$-th seismic force when the design solution is designed for the i-th seismic force. While the seismic force $S_{j}$ is given as a discrete value in this study, the hazard curve shown in Fig. 5 is a continuous function. In this chapter, the annual probability of occurrence is converted into a discrete value by directly using the difference between the annual probabilities of excess corresponding to the seismic forces $S_{j}$ and $S_{j+1}$. It will be necessary in the future to study the influence on seismic risks in cases where the annual probability of excess is set with consideration to the range of incremental value $\Delta S$.

While there is the possibility of loss caused by repeated sequence earthquakes, such additional effects will be studied in the future and this chapter limits its focus on the effects of a single earthquake.

While there is the possibility of loss caused by repeated sequence earthquakes, such additional effects will be studied in the future and this chapter limits its focus on the effects of a single earthquake. 


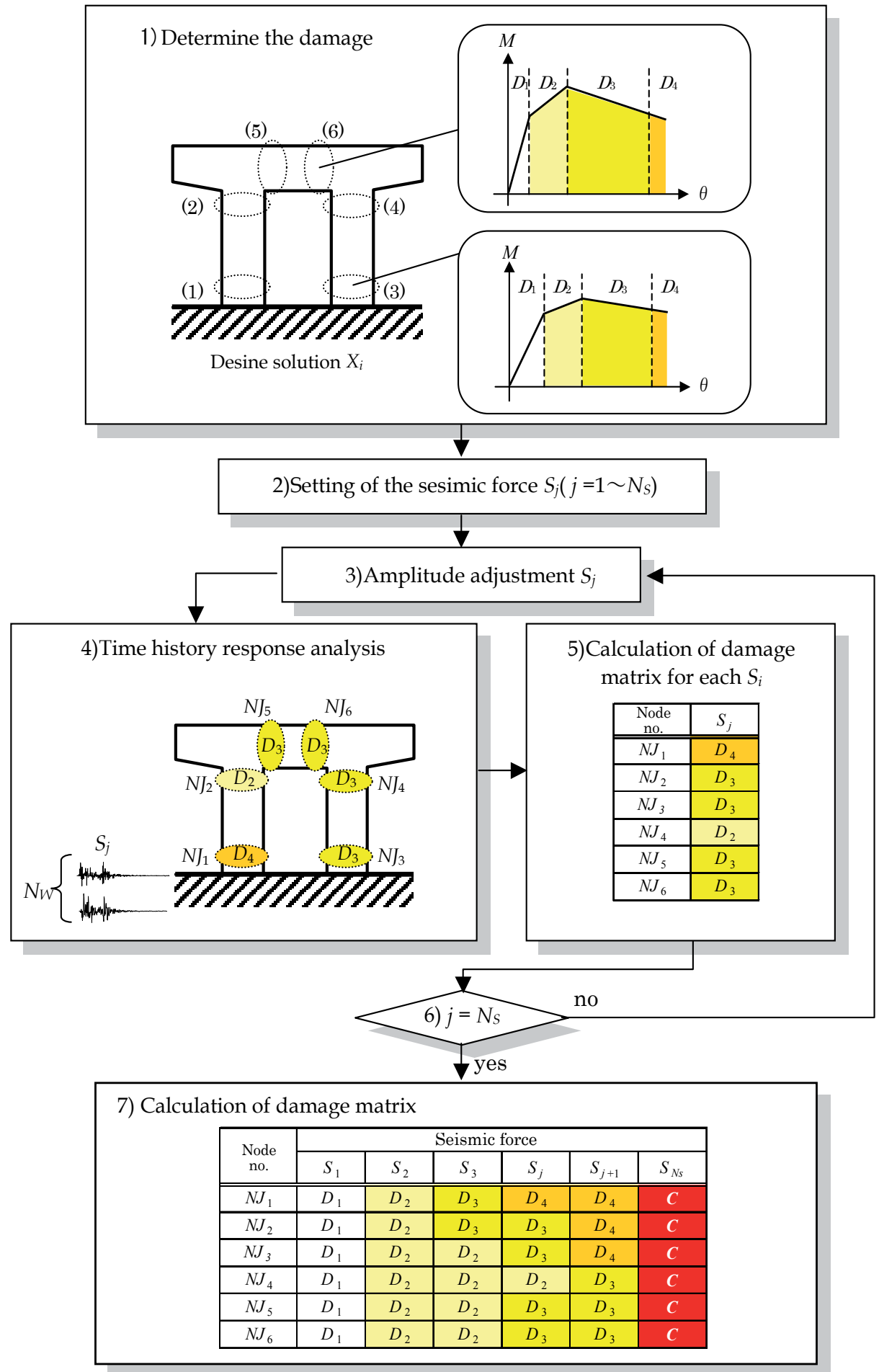

Fig. 4. Flow of the calculation of damage matrix. 


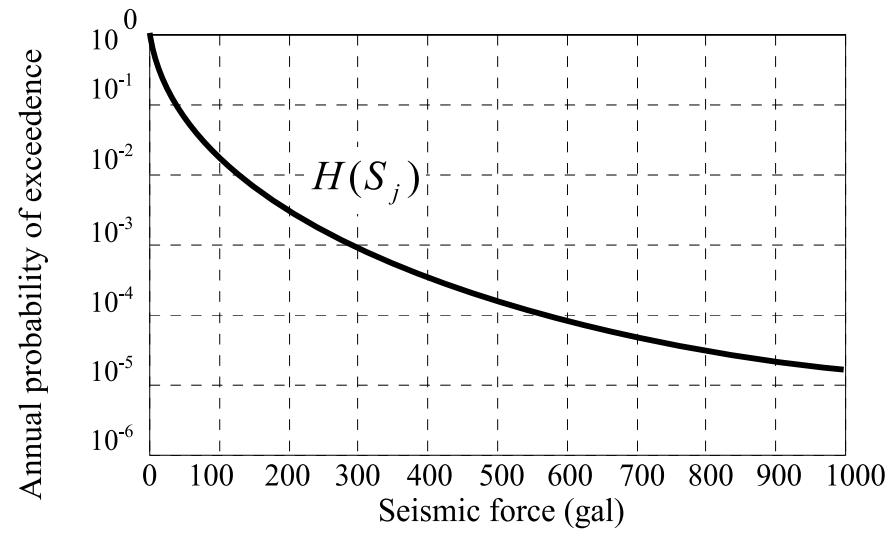

Fig. 5. An example of a hazard curve.

\section{Example of the calculation of the target seismic design force}

In this chapter, the target seismic design force of an RC rigid-frame railway viaduct is calculated. The optimum design problem and examples of numerical calculation will be presented below.

\subsection{Optimum design problem}

A standard single-layer RC rigid-frame railway viaduct with a spread foundation shown in Fig. 6 is used for calculation example. Non-linearity is taken into account for the columns and beam members.

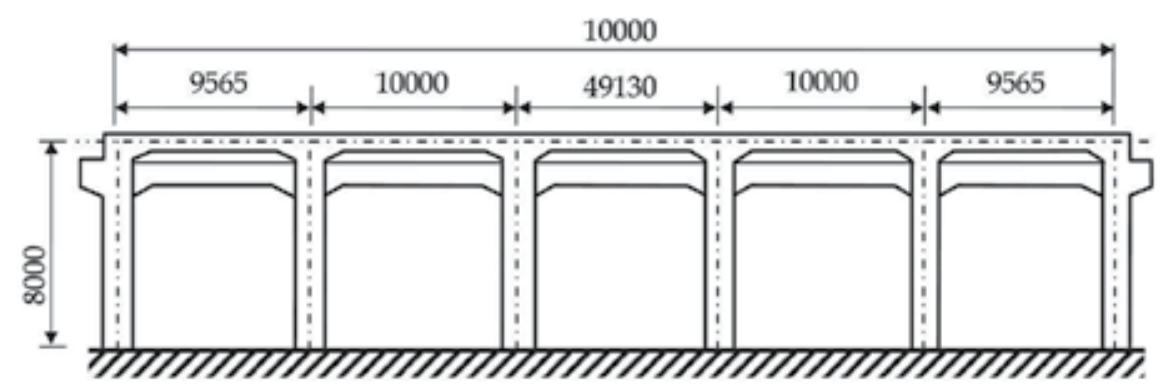

Direction of the bridge axis

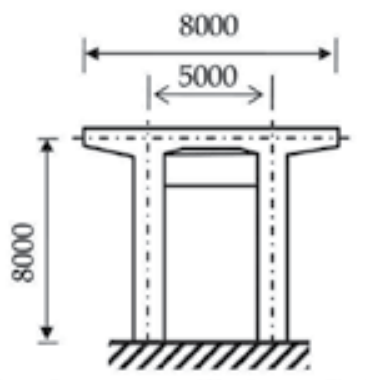

Direction perpendicular to the bridge axis

Fig. 6. Structural model. 
In the optimum design for a certain seismic force $S_{i}$, the initial construction cost, which is the total of the costs related to concrete and reinforcement, is used as the objective function. The objective function is calculated by the equation below

$$
\mathrm{OBJ}=\mathrm{C}_{\mathrm{i}}^{\mathrm{o}}=\mathrm{C}^{\mathrm{c}}+\mathrm{C}^{\mathrm{s}} \rightarrow \min
$$

where, $C^{c}$ is the concrete-related cost (unit) and $C^{s}$ is the reinforcement-related cost (unit). They are calculated by the equations (4) and (5), respectively,

$$
\begin{gathered}
\mathrm{C}^{\mathrm{c}}=\mathrm{a}^{\mathrm{c}} \cdot \mathrm{V}^{\mathrm{c}} \cdot \mathrm{K}^{\mathrm{c}} \\
\mathrm{C}^{\mathrm{s}}=\mathrm{a}^{\mathrm{s}} \cdot \mathrm{V}^{\mathrm{s}} \cdot \mathrm{K}^{\mathrm{s}} \cdot \mathrm{G}^{\mathrm{s}}
\end{gathered}
$$

where, $a_{c}$ is the unit correction factor of concrete, $V_{c}$ is the amount of concrete $\left(\mathrm{m}^{3}\right), \mathrm{K}_{\mathrm{c}}$ is the cost per unit volume of concrete $\left(=65.1 \mathrm{unit} / \mathrm{m}^{3}\right), \mathrm{a}_{\mathrm{s}}$ is the unit correction factor of reinforcement, $V_{s}$ is the amount of reinforcement $\left(\mathrm{m}^{3}\right), K_{s}$ is the cost per unit weight of reinforcement $(=9.1 \mathrm{unit} / \mathrm{kN})$ and $\mathrm{G}_{\mathrm{s}}$ is the unit weight of reinforcement $\left(=77 \mathrm{kN} / \mathrm{m}^{3}\right)$. In this study, $\mathrm{a}_{\mathrm{c}}$ and $\mathrm{a}_{\mathrm{s}}$ are both set as 1.0. The cost per unit volume of concrete and the cost per unit weight of reinforcement are found through conversion from the construction cost, including material cost, cost for scaffolding and personnel cost.

Constraints are found for the verifiability of the angle of rotation and shear force against the seismic force $S_{i}$, and are calculated by the equation below,

$$
\begin{gathered}
\mathrm{g}_{\mathrm{Jk}}^{\mathrm{r}}=\frac{\theta_{\mathrm{Jk}}^{\mathrm{d}}}{\theta_{\mathrm{Jk}}^{\mathrm{m}}}-1 \leq 0\left(\mathrm{~J}=1 \sim \mathrm{N}_{\mathrm{m}}, \mathrm{k}=1 \sim 2\right) \\
\mathrm{g}^{\mathrm{SD}}{ }_{\mathrm{J}}=\frac{\mathrm{V}_{\mathrm{J}}^{\mathrm{d}}}{\mathrm{V}_{\mathrm{J}}^{\mathrm{rd}}}-1 \leq 0 \quad\left(=1 \sim \mathrm{N}_{\mathrm{m}}\right)
\end{gathered}
$$

where, $\mathrm{gr}_{\mathrm{Jk}}$ is the angle of rotation, $\mathrm{gSD}_{\mathrm{J}}$ is the constraint related to shear force, $\theta_{\mathrm{Jk}}$ is the maximum response angle of rotation at the end $\mathrm{k}$ of the member $\mathrm{J}, \theta_{\mathrm{m}}$ is the maximum angle of rotation with which $\mathrm{M}_{\mathrm{m}}$ on the skeleton curve of the end $\mathrm{k}$ of the member $\mathrm{J}$ can be maintained, $\mathrm{Vd}_{\mathrm{J}}$ is the maximum response shear force of the element $\mathrm{J}, \mathrm{Vrd}_{\mathrm{J}}$ is the permissible shear force of the member $\mathrm{J}$ and $\mathrm{N}_{\mathrm{m}}$ is the number of members.

The subjects of design are column and beam members. The cross sections of column members are square and those of beam members are rectangular. There are 7 design variables in total -- the section width $B$, section height $H$, number of reinforcing bars in the axial direction $\mathrm{N}$, number of rows of reinforcing bars in the axial direction $\mathrm{J}_{\mathrm{N}}$, diameter of reinforcing bars in the axial direction $\mathrm{D}$, placing of shear reinforcement $\mathrm{N}_{\mathrm{W}}$ and spacing of shear reinforcement $S_{V}$.

Figs. 7 and 8 display the section specifications and arrangement of shear reinforcement, respectively. The spacing of shear reinforcement in section $2 \mathrm{H}$ of Fig. 8 is $100 \mathrm{~mm}$.

Table 1. lists the potential values of design variables. By setting the minimum spacing of reinforcement as the diameter of reinforcement $\mathrm{D} \times 2.5(\mathrm{~mm})$ and the maximum spacing of reinforcement as $250 \mathrm{~mm}$, the maximum and minimum numbers of reinforcing bars, which are obtained based on the section width and diameter of reinforcing bars, are divided by 8 to find the design variable of the number of reinforcing bars in the axial direction $\mathrm{N}$. 
As materials, concrete with a design standard strength of $24 \mathrm{~N} / \mathrm{mm}^{2}$ and SD345 reinforcement are used.

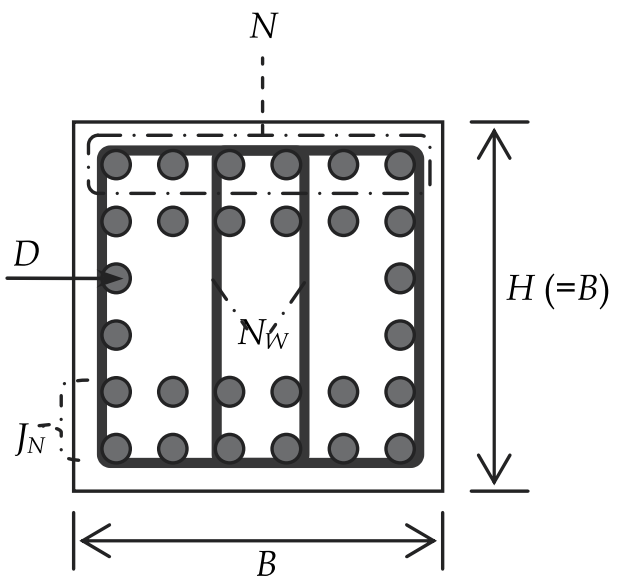

Column member

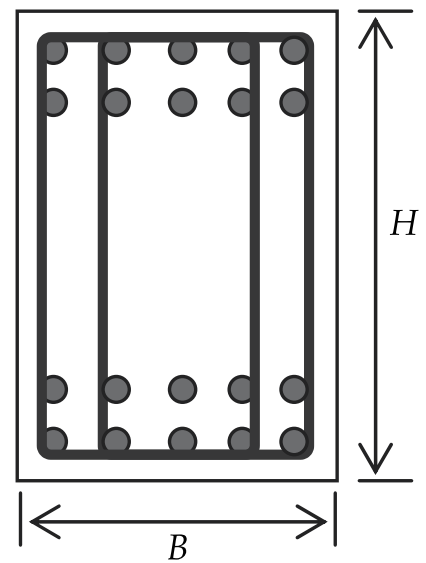

Beam member

Fig. 7. Details of dimensions and reinforcement for $\mathrm{x}$-sections of columns and beams.

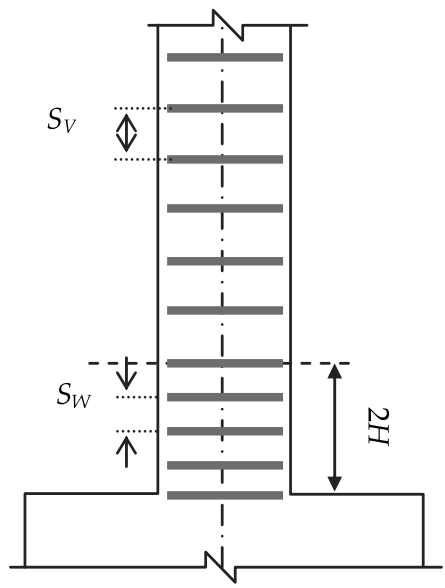

Fig. 8. Details of arrangement of shear reinforcement.

\begin{tabular}{|c|c|}
\hline$B(\mathrm{~mm})$ & $500 \sim 1200(100 \mathrm{~mm}$ intervals $)$ \\
\hline$H(\mathrm{~mm})$ & $B+200 \sim 800(100 \mathrm{~mm}$ intervals $)$ \\
\hline$N$ & 8 types depending on B and H \\
\hline$J_{N}$ & 1 or 2 \\
\hline$D(\mathrm{~mm})$ & 19 or 25 or 29 or 32 \\
\hline$N_{W}$ & $1 \sim 4$ \\
\hline$S_{V}(\mathrm{~mm})$ & 100 or 200 \\
\hline
\end{tabular}

Table 1. Potential values of design variables. 


\subsection{Seismic loss cost}

The repair cost for damage is used as the seismic loss cost. The seismic loss cost is calculated by the equation below,

$$
\mathrm{c}_{\mathrm{ij}}=\sum_{\mathrm{J}=1}^{\mathrm{N}_{\mathrm{m}}} \mathrm{c}_{\mathrm{ijJ}}^{\mathrm{rep}}\left(\mathrm{i}=1 \sim \mathrm{N}_{\mathrm{S}}, \mathrm{j}=1 \sim \mathrm{N}_{\mathrm{S}}\right)
$$

where, $c_{i j}$ is the seismic loss cost for the damage of members caused by the $\mathrm{j}$-th seismic force in a design solution designed for the $\mathrm{i}$-th seismic force, and $\mathrm{c}^{\text {rep }} \mathrm{ijJ}_{\mathrm{j}}$ is the repair cost for the member $\mathrm{J}$ damaged by the $\mathrm{j}$-th seismic force in a design solution designed for the $\mathrm{i}$-th seismic force. The repair cost is determined depending on the repair method applicable to the considered section. In this chapter, different repair methods are adopted for the lower and upper ends of column members and upper beam sections.

Table 2. presents the damage conditions and repair methods corresponding to the damage of different members. Table 3. presents the calculation formulas of repair cost corresponding to the repair methods. Fig. 9 illustrates the calculation model of repair cost.

\begin{tabular}{|c|c|c|c|c|}
\hline \multirow{2}{*}{$\begin{array}{c}\text { Degree } \\
\text { of } \\
\text { damage }\end{array}$} & \multirow{2}{*}{ Damage condition } & \multicolumn{3}{|c|}{ Repair method } \\
\hline & & Culumn (upper end) & Culumn (Iower end) & Upper beam \\
\hline 1 & Slight bending cracking & None & None & None \\
\hline 3 & $\begin{array}{c}\text { Flaking of concrete cover } \\
\text { Buckling of reinforcement in } \\
\text { the axial direction }\end{array}$ & $\begin{array}{c}\text { Scaffolding } \\
\text { Grouting of cracks } \\
\text { Adjustment of reinforcement } \\
\text { Repair of concrete cover }\end{array}$ & $\begin{array}{c}\text { Excavation } \\
\text { Grouting of cracks } \\
\text { Adjustment of reinforcement } \\
\text { Repair of concrete cover }\end{array}$ & $\begin{array}{c}\text { Track removal } \\
\text { Scaffolding } \\
\text { Grouting of cracks } \\
\text { Adjustment of reinforcement } \\
\text { Repair of concrete cover } \\
\text { Bridge-deck waterproofing } \\
\text { Track restoration } \\
\end{array}$ \\
\hline 4 & $\begin{array}{c}\text { Damage of internal concrete } \\
\text { Break of reinforcement in the } \\
\text { axial direction } \\
\text { Break of lateral ties }\end{array}$ & $\begin{array}{c}\text { Temporary support of slab } \\
\text { Scaffolding } \\
\text { Concrete removal } \\
\text { Replacement of reinforcement } \\
\text { Concrete placement }\end{array}$ & $\begin{array}{c}\text { Temporary support of slab } \\
\text { Excavation } \\
\text { Concrete removal } \\
\text { Replacement of reinforcement } \\
\text { Backfill ing }\end{array}$ & $\begin{array}{c}\text { Temporary support of slab } \\
\text { Track removal } \\
\text { Scaffolding } \\
\text { Concrete removal } \\
\text { Replacement of reinforcement } \\
\text { Concrete placement } \\
\text { Bridge-deck waterproofing } \\
\text { Track restoration } \\
\end{array}$ \\
\hline
\end{tabular}

Table 2. Damage conditions and repair methods. 


\begin{tabular}{|c|c|c|c|c|c|}
\hline $\begin{array}{c}\text { Degree } \\
\text { of } \\
\text { damage }\end{array}$ & Repair method & & Unit & Unit price & Calculation formula \\
\hline 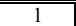 & None & & $\overline{-1}$ & 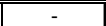 & - $-1-\quad-\quad-$ \\
\hline 2 & $\begin{array}{c}\text { Scaffolding } \\
\text { Grouting of cracks }\end{array}$ & & $\begin{array}{c}\mathrm{m}^{2} \\
\ell\end{array}$ & $\begin{array}{l}2,380 \\
5,500\end{array}$ & $\begin{array}{c}\{(H+0.914 \times 2+0.4 \times 2) \times 2+(H+0.4 \times 2) \times 2\} \times 2 \times H 1 \\
(H \times B \times H) \times 2 \times 10\end{array}$ \\
\hline 3 & $\begin{array}{c}\text { Scaffolding } \\
\text { Grouting of cracks } \\
\text { Repair of concrete cover }\end{array}$ & $\begin{array}{l}1 \\
2\end{array}$ & $\begin{array}{c}\mathrm{m}^{2} \\
\ell \\
\mathrm{m}^{3} \\
\mathrm{~m}^{2}\end{array}$ & $\begin{array}{c}2,380 \\
5,500 \\
22,410 \\
7,090\end{array}$ & $\begin{array}{c}\{(H+0.914 \times 2+0.4 \times 2) \times 2+(H+0.4 \times 2) \times 2\} \times 2 \times H 1 \\
(H \times B \times H \times H) \times 2 \times 10 \\
(H \times B \times H) \times 2 \times 0.35 \\
(H \times H) \times 4 \times 2\end{array}$ \\
\hline 4 & $\begin{array}{l}\text { Temporary support of slab } \\
\text { Scaffolding } \\
\text { Concrete removal } \\
\text { Replacement of reinforcement } \\
\text { Concrete placement }\end{array}$ & $\begin{array}{l}2 \\
1 \\
2\end{array}$ & $\begin{array}{l}\mathrm{m}^{3} \\
\mathrm{~m}^{2} \\
\mathrm{~m}^{3} \\
\mathrm{~kg} \\
\mathrm{~m}^{3} \\
\mathrm{~m}^{2}\end{array}$ & $\begin{array}{c}4,680 \\
2,380 \\
32,000 \\
120 \\
2,700 \\
22,410 \\
7,090\end{array}$ & $\begin{aligned} 10 \times(B 2+H) \times H \\
\{(H+0.914 \times 2+0.4 \times 2) \times 2+(H+0.4 \times 2) \times 2\} \times 2 \times H 1 \\
(H \times B \times H) \times 2 \\
7850 \times A_{S D} \times(H \times 1.5) \times\left((N-1) \times 4+2 \times(N-2) \times\left(J_{N}-1\right)\right)+7850 \times A_{S D} \times N_{W} \times 2 \times \\
\left((1+0.4 \times(N W-1)) \times(B-2 \times 0.04-D)+N_{W} \times(H-2 \times 0.04-D)\right) \\
4 \times(N-1)+2 \times(N-2) \times\left(J_{N}-1\right)+N_{W} \\
(H \times B \times H) \times 2 \\
(H \times H) \times 4 \times 2\end{aligned}$ \\
\hline
\end{tabular}

\begin{tabular}{|c|c|c|c|c|c|}
\hline $\begin{array}{c}\text { Degree } \\
\text { of } \\
\text { damage }\end{array}$ & Repair method & & Unit & Unit price & Calculation formula \\
\hline 1 & None & & - & - & $\begin{array}{lllll} & - & - & - & -\end{array}$ \\
\hline 2 & $\begin{array}{c}\text { Excavation } \\
\text { Grouting of cracks } \\
\text { Backfilling }\end{array}$ & & $\begin{array}{c}\mathrm{m}^{3} \\
\ell \\
\mathrm{m}^{3}\end{array}$ & $\begin{array}{l}6,720 \\
5,500 \\
1,112 \\
\end{array}$ & $\begin{array}{c}\{(H+2) 2-H 2\} \times 0.5 \times 2 \\
(H \times B \times H) \times 2 \times 10 \\
\{(H+2) 2-H 2) \times 0.5 \times 2\end{array}$ \\
\hline 3 & $\begin{array}{c}\text { Excavation } \\
\text { Grouting of cracks } \\
\text { Repair of concrete cover } \\
\text { Backfilling }\end{array}$ & $\begin{array}{l}1 \\
2\end{array}$ & $\begin{array}{l}\mathrm{m}^{3} \\
\ell \\
\mathrm{m}^{3} \\
\mathrm{~m}^{2} \\
\mathrm{~m}^{3}\end{array}$ & $\begin{array}{c}6,720 \\
5,500 \\
22,410 \\
7,090 \\
1,112 \\
\end{array}$ & $\begin{array}{c}\{(H+2) 2-H 2\} \times 0.5 \times 2 \\
(H \times B \times H) \times 2 \times 25 \\
(H \times B \times H) \times 2 \times 0.35 \\
(H \times H) \times 4 \times 2 \\
\{(H+2) 2-H 2) \times 0.5 \times 2\end{array}$ \\
\hline 4 & $\begin{array}{c}\text { Temporary support of slab } \\
\text { Excavation } \\
\text { Concrete removal } \\
\text { Replacement of reinforcement } \\
\text { Repair of concrete cover } \\
\text { Backfilling }\end{array}$ & $\begin{array}{l}2 \\
1 \\
2\end{array}$ & $\begin{array}{l}\mathrm{m}^{3} \\
\mathrm{~m}^{3} \\
\mathrm{~m}^{3} \\
\mathrm{~kg} \\
\mathrm{~m}^{3} \\
\mathrm{~m}^{2} \\
\mathrm{~m}^{3}\end{array}$ & $\begin{array}{c}4,680 \\
6,720 \\
32,000 \\
120 \\
2,700 \\
22,410 \\
7,090 \\
1,112 \\
\end{array}$ & $\begin{array}{c}L 1 \times(B 2+H) \times H \\
\{(H+2) 2-H 2\} \times 0.5 \times 2 \\
(H \times B \times H) \times 2 \\
7850 \times A_{S D} \times(H \times 1.5) \times\left((N-1) \times 4+2 \times(N-2) \times\left(J_{N}-1\right)\right)+7850 \times A_{S D} \times N_{W} \times 2 \times \\
\left((1+0.4 \times(N W-1)) \times(B-2 \times 0.04-D)+N_{W} \times(H-2 \times 0.04-D)\right) \\
4 \times(N-1)+2 \times(N-2) \times\left(J_{N}-1\right)+N_{W} \\
(H \times B \times H) \times 2 \\
(H \times B \times H) \times 4 \times 2 \\
\{(H+2) 2-H 2) \times 0.5 \times 2\end{array}$ \\
\hline
\end{tabular}

\begin{tabular}{|c|c|c|c|c|c|}
\hline \begin{tabular}{|c|}
$\begin{array}{c}\text { Degree } \\
\text { of } \\
\text { damage }\end{array}$ \\
\end{tabular} & Repair method & & Unit & Unit price & Calculation formula \\
\hline 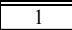 & None & & - & 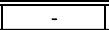 & $\begin{array}{lllll}- & - & - & -\end{array}$ \\
\hline 2 & $\begin{array}{c}\text { Scaffolding } \\
\text { Grouting of cracks }\end{array}$ & & $\begin{array}{c}\mathrm{m}^{2} \\
\ell\end{array}$ & $\begin{array}{l}2,380 \\
5,500\end{array}$ & $\begin{array}{l}(B \times 2+B \times 2) \times H 1 \\
(H \times B \times H) \times 2 \times 10 \\
\end{array}$ \\
\hline 3 & $\begin{array}{c}\text { Track removal } \\
\text { Scaffolding } \\
\text { Grouting of cracks } \\
\text { Repair of concrete cover } \\
\text { Bridge-deck waterproofing } \\
\text { Track restoration }\end{array}$ & $\begin{array}{l}1 \\
2\end{array}$ & $\begin{array}{c}\mathrm{m} \\
\mathrm{m}^{2} \\
\ell \\
\mathrm{m}^{3} \\
\mathrm{~m}^{2} \\
\mathrm{~m}^{2} \\
\mathrm{~m}\end{array}$ & $\begin{array}{c}50,000 \\
2,380 \\
5,500 \\
22,410 \\
7,090 \\
20,000 \\
150,000 \\
\end{array}$ & $\begin{array}{c}L 1 \times 2 \\
(B \times 2+B \times 2) \times H 1 \\
(H \times B \times H) \times 2 \times 25 \\
(H \times B \times H) \times 2 \times 0.35 \\
\{(H-0.3) \times H \times 2+(H \times B)\} \times 2 \\
B 1 \times L 1 \\
L 1 \times 2\end{array}$ \\
\hline 4 & $\begin{array}{c}\text { Temporary support of slab } \\
\text { Track removal } \\
\text { Scaffolding } \\
\text { Concrete removal } \\
\text { Replacement of reinforcement } \\
\text { Concrete placement } \\
\text { Bridge-deck waterproofing } \\
\text { Track restoration } \\
\end{array}$ & $\begin{array}{l}2 \\
1 \\
2\end{array}$ & $\begin{array}{l}\mathrm{m}^{3} \\
\mathrm{~m} \\
\mathrm{~m}^{2} \\
\mathrm{~m}^{3} \\
\mathrm{~kg} \\
\mathrm{~m}^{3} \\
\mathrm{~m}^{2} \\
\mathrm{~m}^{2} \\
\mathrm{~m} \\
\end{array}$ & $\begin{array}{c}4,680 \\
50,000 \\
2,380 \\
32,000 \\
120 \\
2,700 \\
22,410 \\
7,090 \\
20,000 \\
150,000 \\
\end{array}$ & $\begin{array}{c}L 1 \times(\mathrm{B} 2+\mathrm{H}) \times \mathrm{H} \\
L 1 \times 2 \\
(\mathrm{~B} \times 2+\mathrm{B} \times 2) \times \mathrm{H} 1 \\
(\mathrm{~h} 1 \times \mathrm{B} \times \mathrm{h} 1) \times 2 \\
7850 \times A_{S D}{ }^{*} \times(H \times 1.5) \times\left((N-1) \times 4+2 \times(N-2) \times\left(J_{N}-1\right)\right)+7850 \times A_{S D} \times N_{W} \times 2 \times \\
\left((1+0.4 \times(\mathrm{NW}-1)) \times(B-2 \times 0.04-D)+N_{W} \times(H-2 \times 0.04-D)\right) \\
N \times J_{N} \times 2+N_{W} \\
(H \times B \times H) \times 2 \\
\{(H-0.3) \times H \times 2+(H \times B)\} \times 2 \\
B 1 \times L 1 \\
L 1 \times 2 \\
\end{array}$ \\
\hline
\end{tabular}

Table 3. Calculation formulas of repair cost. 


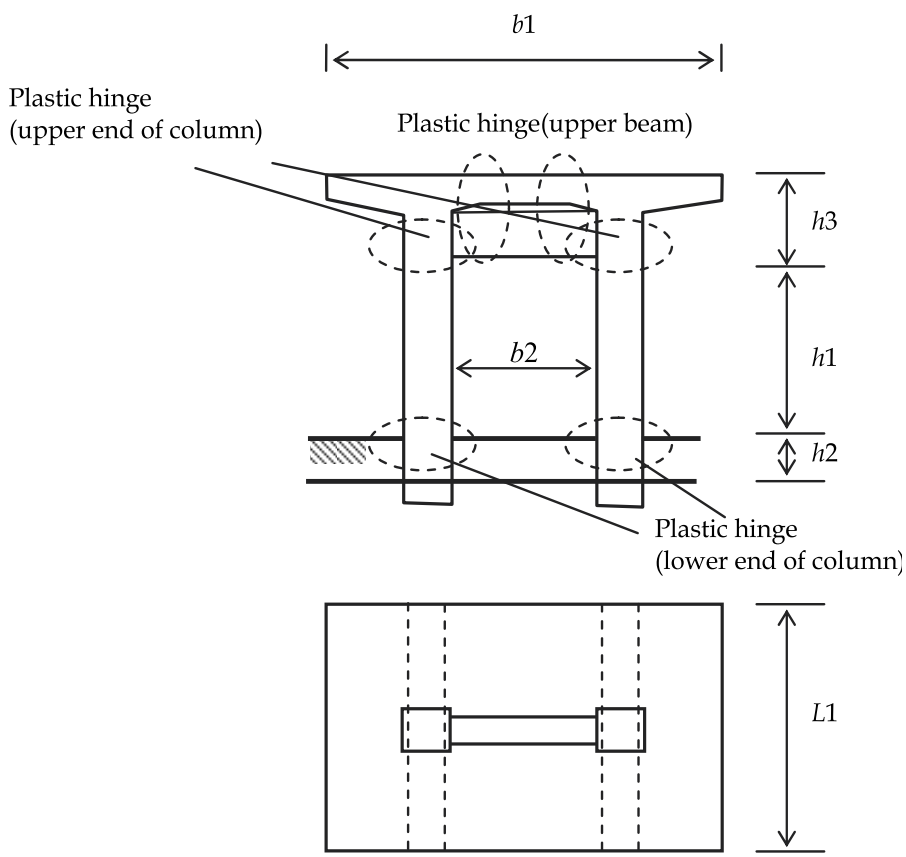

Fig. 9. Calculation model of repair cost.

If the lower ends of all the column members exceed the ultimate angle of rotation, it means that the structure has collapsed and the reconstruction cost replaces the repair cost, which is supposed to be 1.5 times the initial construction cost.

While this definition is based on bending fracture-type collapse, it is also necessary to take the shear fracture-type collapse of structures into account. However, since the seismic force causing bending fracture could be calculated using the damage matrix in this method, it is considered possible to perform analysis based on bending fracture-type collapse by the placement of shear reinforcement, which is not subject to shear fracture caused by the seismic force.

The acceleration waveform of an inland-type earthquake with Level 2 earthquake motion displayed in Fig. 10 is used as the input earthquake motion for time history response analysis and the calculation of the seismic risk cost, and 3 hazard curves $(0.16,0.50$ and 0.84 in fractile) displayed in Fig. 11 are adopted.

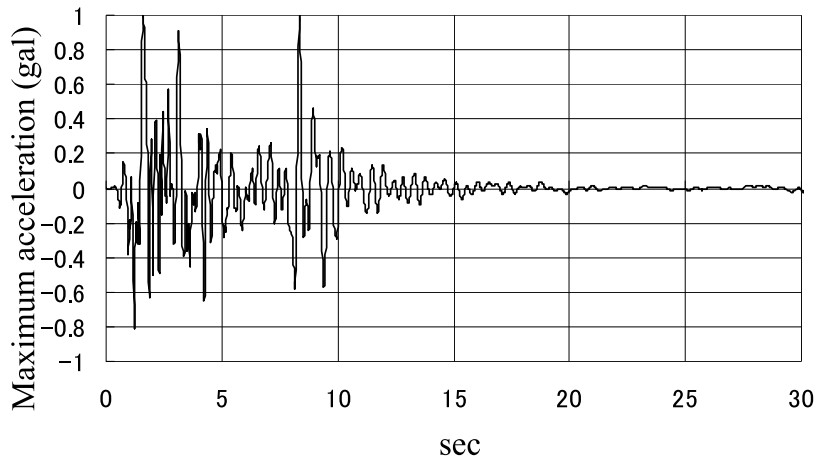

Fig. 10. Acceleration waveform. 


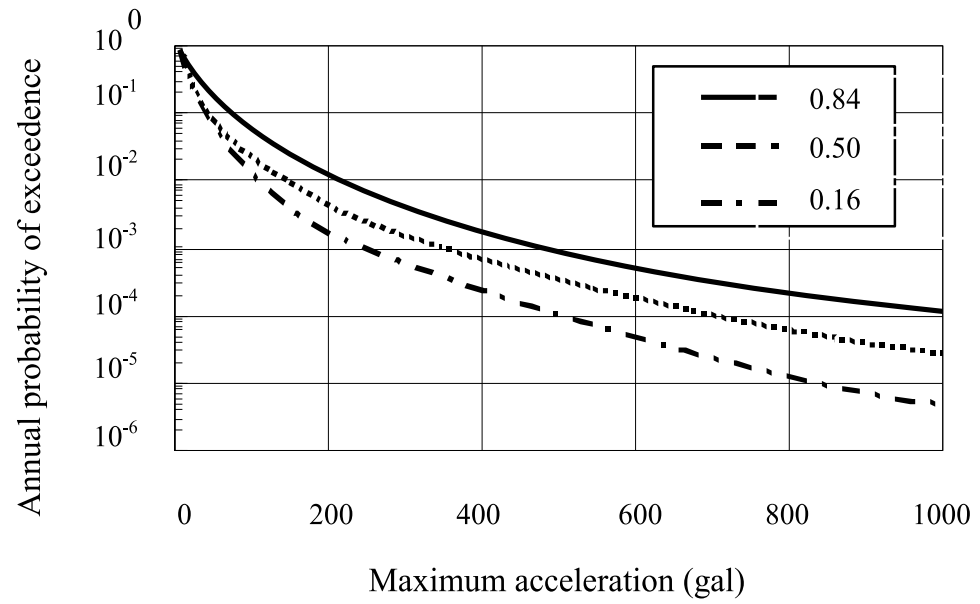

Fig. 11. Hazard curves.

\subsection{Numerical results}

The calculation results for the RC rigid-frame viaduct are presented. The calculation is performed for seismic forces of 50 to 1,000 gal on the assumption that the dividing width $\Delta S$ is $50 \mathrm{gal}$ and the dividing number Ns is 20 for the seismic forces. Since the incremental value of design acceleration must be set taking the influence on design solutions into account, the value in this study is set as 50 gal, which is small enough not to have a significant influence on design solutions. The incremental value of design acceleration can be even smaller if necessary.

Table 4. lists the design solutions found for various seismic forces. In the table, $N_{P}$ and $N_{B}$ represent the numbers of reinforcing bars in the column and beam sections, respectively. Fig. 12 displays the relationship between the seismic force and initial construction cost. In the figure, the symbol represents the initial construction cost.

When the seismic force is within the range of 50 to 400 gal, the initial construction cost is uniform. These are the design solutions with which the objective function becomes minimum by a combination of preset design variables. The initial construction cost tends to increase with increasing seismic force in design solutions of 400 gal or greater. The initial construction cost sharply increases between 750 and 800 gal. As shown in Table 4, this is because the design variables of the two design solutions, $B=900 \mathrm{~mm}$ and $\mathrm{H}=1,200 \mathrm{~mm}$ of $\mathrm{H}$, are necessary when the seismic force is $800 \mathrm{gal}$, while the seismic performance is satisfied with $\mathrm{B}=600 \mathrm{~mm}$ and $\mathrm{H}=800 \mathrm{~mm}$ at 50 gal.

Next, Table 5. presents the damage matrix of design solutions found for various seismic forces (Table 4.). The table shows the seismic forces in rows and input seismic forces for calculation of the damage matrix in columns. The structural model used has nonlinear performance at a total of 28 sections -- 22 in the direction of the bridge axis and 6 in the direction perpendicular to the bridge axis. Although damage is calculated for all members, the maximum values for columns and beams in two directions are presented for each design solution since it is difficult to display all the calculation results. In the table, $\mathrm{P}_{\mathrm{I}}$ is the column member in the direction of the bridge axis, $\mathrm{P}_{\mathrm{O}}$ is the column member in the direction perpendicular to the bridge axis, $\mathrm{B}_{\mathrm{I}}$ is the beam member in the direction of the bridge axis, and $\mathrm{B}_{O}$ is the beam member in the direction perpendicular to the bridge axis. The right side of the thick line represents the cases where the input seismic force exceeds the value used for design. 


\begin{tabular}{|c|c|c|c|c|c|c|c|c|c|}
\hline $\begin{array}{c}\mathrm{Si} \\
\text { (gal) }\end{array}$ & $\begin{array}{c}B \\
(\mathrm{~mm})\end{array}$ & $\begin{array}{c}H \\
(\mathrm{~mm})\end{array}$ & $N_{P}$ & $N_{B}$ & $J_{N}$ & $\begin{array}{c}D \\
(\mathrm{~mm}) \\
\end{array}$ & $N_{W}$ & $\begin{array}{c}S_{V} \\
(\mathrm{~mm})\end{array}$ & $\begin{array}{l}O B J\left(C_{i}^{0}\right) \\
\left.\text { (unit } \times 10^{3}\right) \\
\end{array}$ \\
\hline 50 & 500 & 700 & 3 & 5 & 1 & 22 & 1 & 200 & 6940 \\
\hline 100 & 500 & 700 & 3 & 5 & 1 & 22 & 1 & 200 & 6940 \\
\hline 150 & 500 & 700 & 3 & 5 & 1 & 22 & 1 & 200 & 6940 \\
\hline 200 & 500 & 700 & 3 & 5 & 1 & 22 & 1 & 200 & 6940 \\
\hline 250 & 500 & 700 & 3 & 5 & 1 & 22 & 1 & 200 & 6940 \\
\hline 300 & 500 & 700 & 3 & 5 & 1 & 22 & 1 & 200 & 6940 \\
\hline 350 & 500 & 700 & 3 & 5 & 1 & 25 & 1 & 200 & 6940 \\
\hline 400 & 500 & 700 & 3 & 5 & 1 & 22 & 1 & 200 & 6940 \\
\hline 450 & 500 & 700 & 4 & 6 & 1 & 22 & 1 & 200 & 7219 \\
\hline 500 & 500 & 700 & 3 & 5 & 1 & 22 & 2 & 200 & 7389 \\
\hline 550 & 500 & 700 & 3 & 5 & 1 & 25 & 2 & 200 & 7610 \\
\hline 600 & 500 & 700 & 6 & 10 & 2 & 22 & 2 & 100 & 9646 \\
\hline 650 & 600 & 800 & 4 & 6 & 1 & 22 & 2 & 200 & 10193 \\
\hline 700 & 600 & 800 & 4 & 6 & 1 & 22 & 2 & 200 & 10193 \\
\hline 750 & 600 & 800 & 5 & 7 & 2 & 25 & 2 & 100 & 12000 \\
\hline 800 & 900 & 1200 & 16 & 23 & 1 & 22 & 2 & 200 & 24635 \\
\hline 850 & 1000 & 1200 & 15 & 19 & 1 & 22 & 2 & 200 & 26833 \\
\hline 900 & 1000 & 1200 & 16 & 21 & 1 & 22 & 2 & 200 & 27189 \\
\hline 950 & 1000 & 1200 & 10 & 12 & 1 & 32 & 2 & 200 & 28087 \\
\hline 1000 & 1100 & 1300 & 19 & 23 & 1 & 22 & 2 & 200 & 31852 \\
\hline
\end{tabular}

Table 4. Design solution by seismic force (Si ).

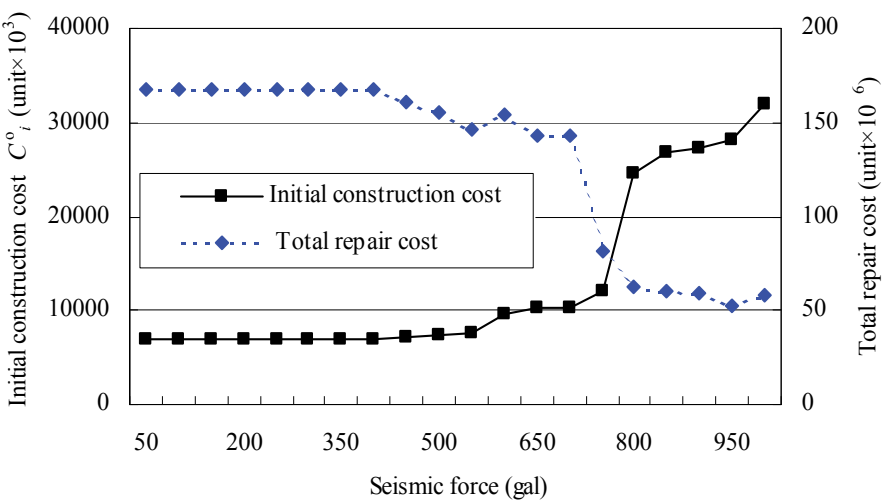

Fig. 12. Initial construction cost and total repair costby seismic force. 


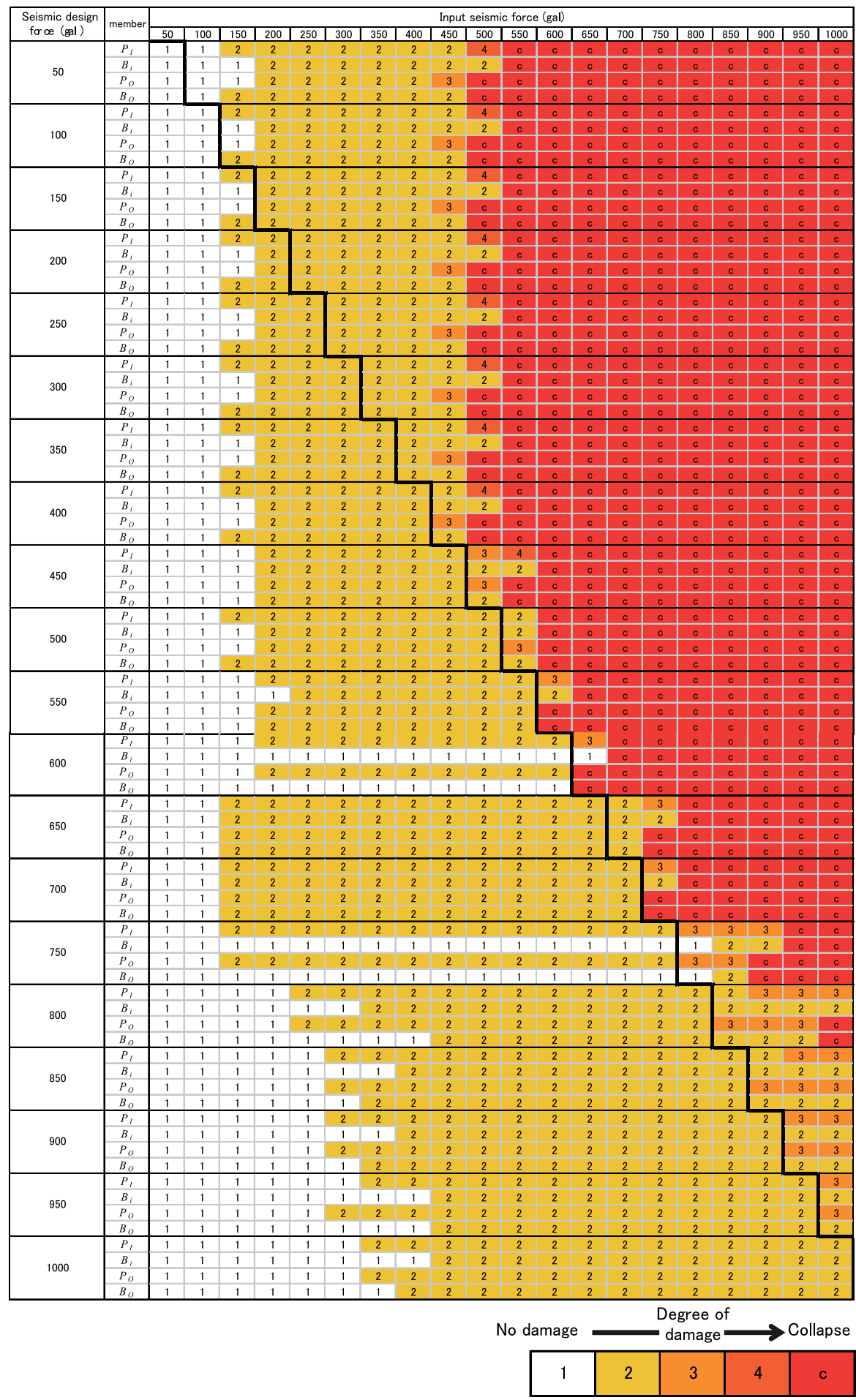

Table 5. Damage matrix. 
Damage is examined for design solutions at 400 gal or more, with which the initial construction cost became the minimum. In design solutions between 400 gal and 800 gal, where the objective function increases sharply, collapse in the direction perpendicular to the bridge axis occurred with a seismic force 50 to 150 gal stronger than the seismic force used for design, while collapse in the direction of the bridge axis occurred with a seismic force 100 to 200 gal stronger. It can thus be seen that the seismic performance in the direction perpendicular to the bridge axis is lower than that in the direction of the bridge axis when the seismic force is stronger than that used for design. In design solutions at 800 gal or more, on the other hand, collapse does not occur even with a seismic force of 1,000 gal.

Next, the symbol $\downarrow$ in Fig. 12 represent the total repair cost for each design solution calculated from the damage matrix in Table 5. The total repair cost is found by totalling the repair costs for all the seismic forces (columns in Table 5.) between 50 and 1,000 gal for each design solution. The total repair cost of each design solution tends to be in inverse proportion to the initial construction cost. The difference in total repair cost is small although the initial construction cost of the design solution at 750 gal is almost double that of the design solution at $800 \mathrm{gal}$. This is because the damage level of the beam member using the design solution at 800 gal is 2 at 350 gal, while the beam member using the design solution at $750 \mathrm{gal}$ is undamaged until the seismic force reached 800 gal. Since the repair of beam members requires scaffolding and other works even if damage is minor, the repair cost is higher compared with that for column members. Also, since collapse would not occur even with a seismic force of 1,000 gal in the case of a design solution for a seismic force of 800 gal or more, the total repair cost is approximately half of that for other design solutions with collapse, except for that at 750 gal.

Figs. 13 to 15 display the relationship between the total cost and seismic force in the case where the repair cost for each design solution, which is calculated using the hazard curve in Fig. 11 and based on the damage matrix in Table 5. , is used as the seismic risk cost. In the figures, the horizontal and vertical axes represent the seismic force and total cost and the white and blue parts indicate the initial construction cost and seismic risk cost, respectively. Each figure presents the results for a $0.16,0.50$ or 0.84 fractile hazard curve. The arrow in each figure indicates the section where the total cost is the lowest, or the target seismic design force.

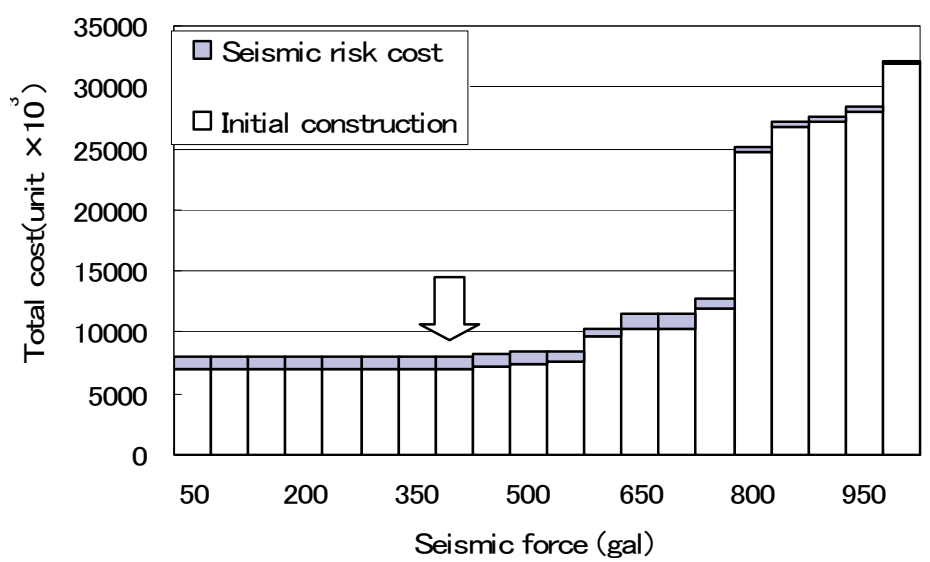

Fig. 13. Relationship between the total cost and seismic force ( 0.16 fractile hazard curve) . 


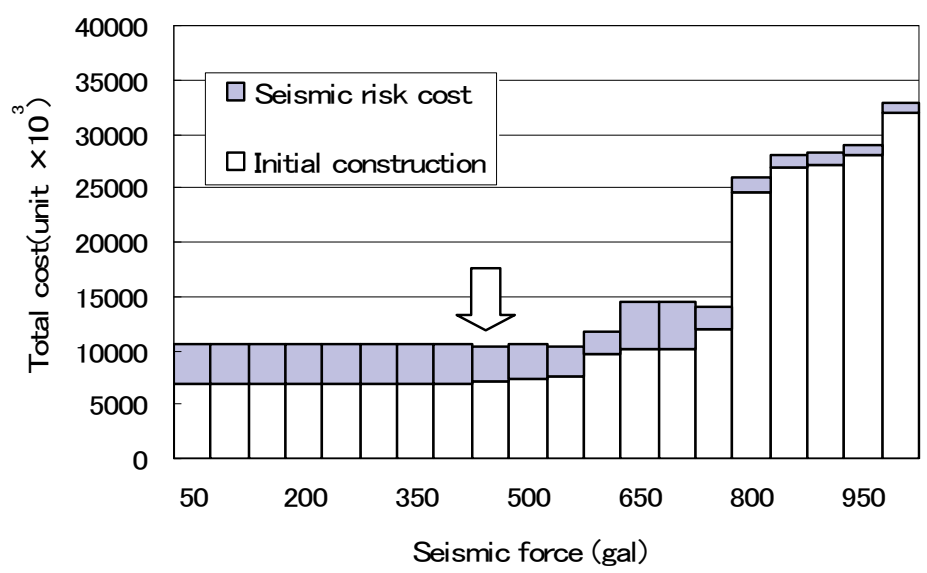

Fig. 14. Relationship between the total cost and seismic force ( 0.50 fractile hazard curve).

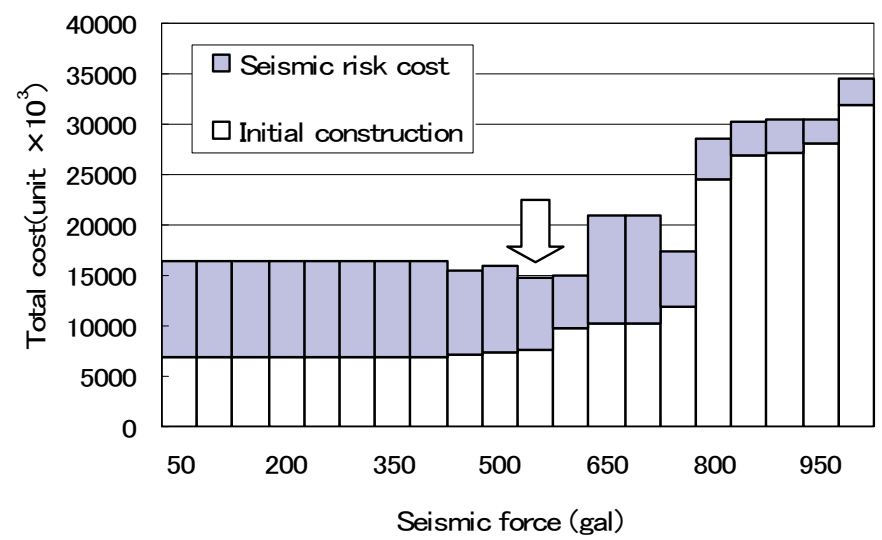

Fig. 15. Relationship between the total cost and seismic force (0.84 fractile hazard curve) .

The target seismic design force is 400, 450 and 550 gal for $0.16,0.50$ and 0.84 fractile hazard curves, respectively. It is confirmed that, even with the same structural model, the target seismic design forces would vary with differences in the occurrence probability of earthquakes. In the relationship between the total cost and seismic force in 0.50 and 0.85 fractile hazard curves, the total cost at 750 gal is locally low. This is because the seismic risks are extremely high at 650 and 700 gal. It can be seen from the damage matrix that damage to beam members started at 150 gal in design solutions designed for 650 and 700 gal. Because the seismic force causing damage is lower compared with other design solutions and the repair cost for beam members is higher, the estimated seismic risk became higher. As a result, the total cost at 750 gal is locally low.

\section{Conclusion}

The current seismic design criteria are based on the verification of seismic performance using Level-1 and -2 seismic forces. However, since earthquake motions that are stronger than Level 1 but do not exceed Level 2 may be generated through time during the service 
life of a structure in reality. Against such a background, this chapter examined target seismic design forces taking seismic risks into account as an attempt to apply seismic risk management to seismic design methods.

The results obtained in this chapter are as listed below.

1. A method for calculating seismic forces with which the total cost can be minimized is presented. The proposed method has the following characteristics:

- The total cost is the total of the initial construction and seismic risk costs. The seismic risk cost includes the costs associated with the damage and collapse of structures.

- The damage of members is calculated by using the nonlinear characteristics related to the damage of members.

- To find the damage and collapse processes of structures, a damage matrix based on the damage conditions of all members with nonlinearity is used to reflect the influence of the repair cost depending on differences in structural type and damage conditions as precisely as possible.

2. The proposed method for calculating target seismic design forces is applied to RC rigidframe railway viaduct. As a result of calculation using three hazard curves with different fractile values, the following knowledge is obtained:

- In calculation example, the target seismic design forces vary with difference in the occurrence probability of earthquakes. When the probability is higher, the target forces also become higher.

A method is presented for the calculation of target seismic design forces, for which the seismic risks of damage and collapse caused by various seismic forces are taken into account. By applying hazard curves unique to this region and seismic waveforms taking regional ground and other properties closely into account to the method presented in this study, the target seismic design force with minimum total cost including seismic risk can be found from the occurrence probability of earthquakes in the target region and damage unique to the target structure. While social consensus based on the accumulation of this kind of study is necessary for the setting of seismic forces to use in seismic design, the authors will be pleased if these studies serve as references for future studies of seismic forces in seismic design.

\section{References}

USGS/EERI Advance Reconnaissance Team (2010). THE MW7.0 HAITI EARTHQUAKE of JANUARY 12,2010 : TEAM REPORT V.1.1

2011 Great East Japan Earthquake-Japan Society of Civil Engineers, Available from http://committees.jsce.or.jp/s_iad/node/9

Railway Technical Research Institute (1999). Design Criteria for Railway Structures and instruction manual - seismic design, ISBN 4-621-04650-0,Maruzen Corporation, Japan.

Japan Road Association(2002). Specifications for Highway Bridges and instruction manual - V seismic design, ISBN 4-88950-248-3, Maruzen Corporation, Japan.

Japan Society of Civil Engineers (2002). Standard Specifications for Concrete Structures - seismic performance verification, ISBN 978-4810601954, Japan Society of Civil Engineers, Japan.

Japan Society of Civil Engineers (2001). Technology for the Seismic Performance Verification of Concrete Structures - Present Status and Future Prospects, Concrete Technology Series 68, ISBN 4-8106-0506-x, Japan Society of Civil Engineers, Japan. 
Special Committee on Seismic Design Methods for Civil Engineering Structures(2000). Third Proposal on Seismic Design Methods for Civil Engineering Structures and instruction manual, Japan.

Earthquake Resistance Standard Subcommittee, Earthquake Engineering Committee Japan Society of Civil Engineers (2003). New Ideas of Level 1 in the Seismic Design of Civil Engineering Structures, Japan.

Sugimoto, H. ; Abe, J. ; Furukawa, K. \& Arakawa, M.( 2004). On Optimum Seismic Design of Steel Piers by RBF Method, The Third China-Japan-Korea Joint Symposium on Optimization of Structural and Mechanical Systems (CJK-OSM 3), pp.463-468, Japan.

Abe, J.; Watanabe, T. \& Sugimoto, H (2006). On Optimum Seismic Design of RC Piers by Constraints Approximation Method Applying RBF Network, JSCE Journal of Structural Mechanics and Earthquake Engineering, Vol. 62, No.2, pp.405-418, Japan.

Abe, J.; Sugimoto, H \& Watanabe, T. (2007). A Study On Calculation of Seismic Design Forces for Structures Considering their Seismic Risks, JSCE Journal of Structural Mechanics and Earthquake Engineering, Vol. 63, No.4, pp.780-794, Japan.

Sugimoto, H.; Lu, B. and Yamamoto, H (1993). A Study on an Improvement of Reliability of GA for the Discrete Structural Optimization, JSCE Journal of Structural Mechanics and Earthquake Engineering, No.471/I-24, pp.67-76,Japan.

Japan Society of Civil Engineers (2007). Standard Specifications for Concrete Structures - designe verification, ISBN 978-4-8106-0414-6, Japan Society of Civil Engineers, Japan.

Railway Technical Research Institute (2004). Design Criteria for Railway Structures and instruction manual - concrete structures, ISBN 978-4621074121,Maruzen Corporation, Japan.

Sugimoto, H.; Watanabe, T. and Saito, H. (2000). A Study of the Optimization of the Earthquake Resistance of an RC Rigid-Frame Viaduct, JSCE Journal of Structural Engineering I, Vol. 46A, pp.385-394,Japan.

Watanabe, T.; Sugimoto; H. \& Asahi, K.(2002). Minimum Cost Design of RC Structures Considering Initial Construction Cost and Repair Cost for Damages Sustained by L2 Earthquake Motions, JSCE Journal of Materials, Concrete Structures and Pavements, No.718/V-57, pp. 81-93, Japan.

Abbas Moustafa.(2011). Damage-based design earthquake loads for SDOF inelastic structures, Journal of Structural Engineering (ASCE).137(3):456-467.

Abbas Moustafa \& Iziri TAKEWAKI (2010). Modeling Critical Ground-Motion Sequencesfor Inelastic Structures,Advances in Structural EngineeringVolume 13 No. 42010 137(3):456-467.

Subcommittee for Studies of Level 2 Earthquake Motions, Earthquake Engineering Committee, Japan Society of Civil Engineers (2000). Report on the Results of Activities of the Subcommittee for Studies of Level 2 Earthquake Motions, Available from http://www.jsce.or.jp/committee/eec2/index.html

Kameda, H.; Ishikawa, H; Okumura, T. \& Nakajima, M.(1997). Probabilistic Scenario Earthquakes -Definition and Engineering Applications-, JSCE Journal of Structural Mechanics and Earthquake Engineering, No.577/I-41, pp.75-87, Japan.

Nagao, T.; Yamada, M; \& Nozu, A.(2005.) Probabilistic Seismic Hazard Analysis With Focus on Fourier Amplitude and Group Delay Time, JSCE Journal of Structural Mechanics and Earthquake Engineering, No.801/I-73, pp. 141-158, Japan.

National Research Institute for Earth Science and Disaster Prevention(2004.) Report of the Review Committee on Engineering Use of Earthquake Hazard Maps, Japan.

Yoshikawa, H.(2008). Seismic design and seismic risk analysis of RC structures. ISBN 978-4621-07955-3, Maruzen Corporation, Japan. 


\title{
Seismic Bearing Capacity of Shallow Foundations
}

\author{
Francesco Castelli ${ }^{1}$ and Ernesto Motta ${ }^{2}$ \\ ${ }^{1}$ Kore University of Enna, Faculty of Engineering and Architecture \\ ${ }^{2}$ University of Catania, Department of Civil and Environmental Engineering
}

Italy

\section{Introduction}

The seismic risk mitigation is one of the greatest challenges of the Civil Engineering and an important contribution toward this challenge can be given by the Geotechnical Earthquake Engineering. Lesson learned by recent destructive earthquakes (January 2010 Port-au-Prince region of Haiti and March 2011 Tohoku Japan), confirms that local soil conditions can play a significant role on earthquake ground motions.

Earthquake-induced damage in Port-au-Prince was devastating and widespread. Yet, there were clearly areas of the city where little to no damage occurred, and areas of the city where an overwhelming majority of the buildings were severely damaged or destroyed.

These types of damage patterns are common in earthquakes, and a wide number of factors need to be considered in order to conclusively piece together the causes.

For a given earthquake, these factors include, but are not limited to: (a) relative distance from the fault rupture plane, $(b)$ construction type and quality, $(c)$ local soil conditions (i.e. strength/stiffness of the soil foundation, depth to bedrock, impedance contrasts, geology), (d) topography (topographic and basin effects), and (e) near fault effects (rupture directivity, fling step, hanging wall effects, polarity effects, etc.). Often several of these factors work together and it can be difficult to identify the primary cause of damage.

Design of foundations in seismic areas needs special considerations compared to the static case. The inadequate performance of structures during recent earthquakes has motivated researchers to revise existing methods and to develop new methods for seismic-resistant design. This includes new design concepts, such as, performance-based design (PBD) (Priestley et al., 2005) and new measures of the structure performance based on energy concepts and damage indexes (Park et al., 1987; Moustafa, 2011).

Similarly, the widespread damage and inadequate performance of code-designed structures during the 1994 Northridge (California) and the 1995 Kobe (Japan) earthquakes have prompted seismologists and engineers of the essential importance of characterizing and modelling near-field ground motions with impulsive nature (Moustafa \& Takewaki, 2010).

For foundations of structures built in seismic areas, the demands to sustain load and deformation during an earthquake will probably be the most severe in their design life.

As stressed by Hudson (1981) the soil-structure interaction is a crucial point for the evaluation of the seismic response of structures. 
Due to seismic loading, foundations may experience a reduction in bearing capacity and increase in settlement. Two sources of loading must be taken into consideration: "inertial" loading caused by the lateral forces imposed on the superstructure, and "kinematic" loading caused by the ground movements developed during the earthquake.

Part 5 of Eurocode 8 (2003) states that foundations shall be designed for the following two loading conditions :

a. inertia forces on the superstructure transmitted on the foundations in the form of axial, horizontal forces and moment ;

b. soil deformations arising from the passage of seismic waves.

In the last years the seismic action has increased in many National Codes according to recent records which show values up to $0.8 \mathrm{~g}$ for very destructive earthquakes. The upgrading of the seismic action requires accurate analyses taking into account all the boundary conditions including the presence of surcharges, sloping ground, depth factors and so on.

With the aim to investigate the influence of these factors on the seismic stability of a shallow foundation, a model based on the limit equilibrium method has been developed.

Many analytical and numerical solutions are today available to evaluate seismic bearing capacity of shallow foundations, and cover area such as the limit equilibrium method, limit analysis, methods of characteristics, finite element analysis and other areas for the computation of the seismic bearing capacity factors required for the design of a foundation. Nevertheless, pseudo-static approaches are more attractive because they are simple, when compared to difficult and more complex dynamic analyses.

Thus, a pseudo-static model to account for reduction in bearing capacity due to earthquake loading is presented. In this model the loading condition consists in normal and tangential forces on the foundation and inertial forces into the soil. An upper bound solution of the limit load of the shallow foundation is found.

Results of the proposed analysis are given in terms of the ratios between seismic and static bearing capacity factors $N_{c}^{*} / N_{c}, N_{q}^{*} / N_{q}$ and $N_{\gamma}^{*} / N_{\gamma}$. Results are also compared with those deduced by other authors using different methods of analysis.

\section{Method of analysis}

The prediction of the bearing capacity of a shallow foundation is a very important problem in Geotechnical Engineering, and in the last decades solutions using limit analysis, slip-line, limit equilibrium and, recently, numerical methods (i.e. finite element and difference finite methods) have been developed.

The problem of static bearing capacity of shallow foundations has been extensively studied in the past by Terzaghi (1943), Meyerhoff (1963), Vesic (1973) and many others. The ultimate load that the foundation soil can sustain is expressed by the linear combination of the three bearing capacity factors $N_{c}, N_{q}$ and $N_{\gamma}$ which depend uniquely on the friction angle of the soil. Further solutions for the bearing capacity were given successively in a more general form, taking into account, by means of corrective factors, of the shape of the foundation, of the load and ground inclination and of the depth and inclination of the bearing surface.

In all these studies, the bearing capacity evaluation is based on the assumption that a failure surface can develop beneath the foundation, according to the well known failure surfaces given by the limit equilibrium method or by the limit analysis. 
Most foundation failures during earthquakes occur due to liquefaction phenomena, even if failures due to reduction in bearing capacity have been observed during Naigata earthquake (1964) Japan and Izmit earthquake (1999) in Turkey (Day, 2002).

Liquefiable soils are categorized by all seismic codes as extreme ground conditions, where, following a positive identification of this hazard, the construction of shallow footings is essentially allowed only after proper soil treatment. More specifically, liquefaction-induced shear strength degradation of the foundation subsoil may result in post-shaking static bearing capacity failure, while excessive seismic settlements may also accumulate. However, the accurate estimation of the degraded bearing capacity and the associated dynamic settlements could potentially ensure a viable performance-based design of shallow footings. Richards et al. (1993) observed seismic settlements of foundations on partially saturated dense or compacted soils. These settlements were not associated with liquefaction or densification and could be easily explained in terms of seismic bearing capacity reduction. In fact, the inertial forces applied on the foundation and in the soil mass reduce the static bearing capacity. Thus, many authors have investigated the seismic bearing capacity giving results in terms of the ratio of the seismic to the static bearing capacity factors $N_{c}^{*} / N_{c}, N_{q}^{*} / N_{q}$ and $N_{\gamma}^{*} / N_{\gamma}$.

The pseudo-static approach is being used to determine bearing capacity of the foundations subjected to seismic loads in non-liquefying soils, considering also the depth effects for an embedded footing and the effect of a sloping ground located at some distance from the footing. Dynamic nature of the load and other factors which affect the dynamic response are not being accounted for.

Ground factors and bearing capacity ratios $N_{c}^{*} / N_{c}, N_{q}^{*} / N_{q}$ and $N_{\gamma}^{*} / N_{\gamma}$ are presented as a function of the friction angle of soil $\phi^{\prime}$, of the ratio $H / B$ between the embedment depth $H$ and the width of the footing $B$, of a slope angle $\beta$ and of the ratio $d / B$ being $d$ the distance from the edge of the slope. The inertial and kinematic effects due to seismic loading have been analyzed in the evaluation of the seismic bearing capacity.

\subsection{Limit equilibrium analysis}

The method of analysis is based on the limit equilibrium technique. The failure mechanism, as shown in Figure 1, is a circular surface which from the foundation propagates until the ground surface is reached (Castelli \& Motta, 2010; 2011).

A similar model was proposed by Castelli \& Motta (2003) for a bearing capacity analysis of a strip footing resting on a soil of limited depth.

The seismic forces are considered as pseudo-static forces acting both on the footing and on the soil below the footing. The ultimate load can be found by a moment equilibrium respect to the centre of the circular surface.

Referring to Figure 1 a moment equilibrium can be written and the mobilizing moment is :

$$
M_{m o b}=\sum_{i=1}^{n_{\text {tot }}} W_{i}\left(1-k_{v}\right) b_{w i}+q_{\lim } \sum_{i=1}^{n_{1}} \Delta x_{i} b_{q l i}+q_{\lim } \sum_{i=1}^{n_{1}} k_{h 1} \Delta x_{i} b_{q l h i}+\sum_{i=1}^{n_{\text {tot }}} k_{h 2} W_{i} b_{\text {whi }}
$$

The resisting moment given by the shear strength $S_{i}$ acting along the base of the slices is :

$$
M_{\text {res }}=R \sum_{i=1}^{n_{\text {tot }}} S_{i}=R \sum_{i=1}^{n_{\text {tot }}} c \Delta x_{i} / \cos \alpha_{i}+R \sum_{i=1}^{n_{\text {tot }}} N_{i} \tan \phi_{i}
$$




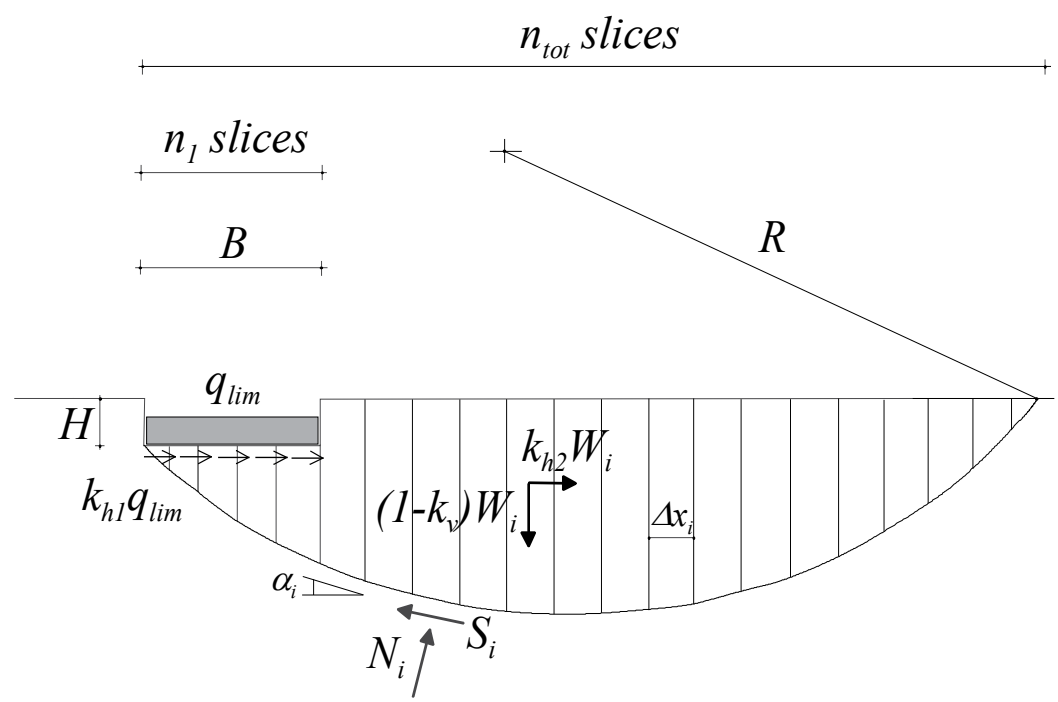

Fig. 1. Failure mechanism and applied forces adopted in the analysis

being :

$$
S_{i}=c \Delta x_{i} / \cos \alpha_{i}+N_{i} \tan \phi_{i}
$$

The force $N_{i}$ resultant of the normal stress distribution acting at the base of the slice can be derived by the Bishop's method of slices (1955) with an equilibrium equation in the vertical direction, so one obtains (see Figure 1), for the slices under the footing where $i=1$ to $n_{1}$ :

$$
N_{i}=\frac{q_{\lim } \Delta x_{i}+W_{i}\left(1-k_{v}\right)-c \Delta x_{i} \tan \alpha_{i}}{\cos \alpha_{i}+\sin \alpha_{i} \tan \phi}
$$

and for the remaining slices $\left(n_{1}+1 \leq i \leq n_{t o t}\right)$ :

$$
N_{i}=\frac{W_{i}\left(1-k_{v}\right)-c \Delta x_{i} \tan \alpha_{i}}{\cos \alpha_{i}+\sin \alpha_{i} \tan \phi}
$$

Thus :

$$
\begin{aligned}
& M_{\text {res }}=R \sum_{i=1}^{\text {ntot }} c \Delta x_{i} / \cos \alpha_{i}+ R \sum_{i=1}^{n_{1}} \frac{q_{\lim } \Delta x_{i}+W_{i}\left(1-k_{v}\right)-c \Delta x_{i} \tan \alpha_{i}}{\cos \alpha_{i}+\sin \alpha_{i} \tan \phi} \cdot \tan \phi+ \\
&+\sum_{i=n 1+1}^{n \text { tot }} \frac{W_{i}\left(1-k_{v}\right)-c \Delta x_{i} \tan \alpha_{i}}{\cos \alpha_{i}+\sin \alpha_{i} \tan \phi} R \tan \phi
\end{aligned}
$$

where :

- $\quad q_{\text {lim }}=$ vertical limit load acting on the footing;

- $c=$ soil cohesion;

- $\Delta x_{i}=$ width of the $i^{\text {th }}$ slice;

- $W_{i}=$ weight of the $\mathrm{i}^{\text {th }}$ slice;

- $R=$ radius of the circular failure surface; 
- $\quad \alpha_{i}=$ angle of the base of the $i^{\text {th }}$ slice;

- $n_{1}=$ number of slices under the footing;

- $n_{\text {tot }}=$ total number of slices;

- $\quad k_{h 1}=$ horizontal seismic coefficient for the limit load;

- $\quad k_{h 2}=$ horizontal seismic coefficient for the soil mass;

- $\quad k_{v}=$ vertical seismic coefficient for the soil mass;

- $\quad b_{w i}=$ distance of the weight $W_{i}$ of the ith slice to the centre of the circular failure surface;

- $\quad b_{w h i}=$ distance of the inertia force $k_{h 2} W_{i}$ of the $i^{\text {th }}$ slice to the centre of the circular failure surface;

- $\quad b_{q l i}=$ distance of the limit load $q_{\text {lim }}$ acting on the $\mathrm{i}^{\text {th }}$ slice to the centre of the circular failure surface;

- $\quad b_{q h l i}=$ distance of the shear limit force $k_{h 1} q_{l i m}$ acting on the $\mathrm{i}^{\text {th }}$ slice to the centre of the circular failure surface.

Substituting the following terms :

$$
\begin{gathered}
a_{1}=R \sum_{i=1}^{n_{\text {tot }}} c \Delta x_{i} / \cos \alpha_{i} \\
a_{2}=R \tan \phi \sum_{i=1}^{n_{1}} \frac{\Delta x_{i}}{\cos \alpha_{i}+\sin \alpha_{i} \tan \phi} \\
a_{3}=R \tan \phi \sum_{i=1}^{n_{1}} \frac{W_{i}\left(1-k_{v}\right)-c \Delta x_{i} \tan \alpha_{i}}{\cos \alpha_{i}+\sin \alpha_{i} \tan \phi} \\
a_{4}=R \tan \phi \sum_{i=n_{1}+1}^{n_{\text {tot }}} \frac{W_{i}\left(1-k_{v}\right)-c \Delta x_{i} \tan \alpha_{i}}{\cos \alpha_{i}+\sin \alpha_{i} \tan \phi} \\
a_{5}=\sum_{i=1}^{n_{\text {tot }}} W_{i}\left(1-k_{v}\right) b_{w i} \\
a_{6}=\sum_{i=1}^{n_{\text {tot }}} k_{h 2} W_{i} b_{w h i} \\
a_{8}=\sum_{i=1}^{n_{1}} k_{h 1} \Delta x_{i} b_{q l h i} \\
a_{7}=\sum_{i=1}^{n_{1}} \Delta x_{i} b_{q l i}
\end{gathered}
$$

and equating $M_{m o b}=M_{\text {res }}$ the limit load is given by :

$$
q_{\lim }=\frac{a_{5}+a_{6}-a_{1}-a_{3}-a_{4}}{a_{2}-a_{7}-a_{8}}
$$


Even if the failure mechanism adopted is quite simple, it allows to investigate a variety of loading and geometric conditions that could have been troublesome using other failure mechanisms and results are in a very good agreement with those obtained by other authors. In fact, referring to the kinematic effect due to the inertia of the soil mass on the seismic bearing capacity, Figure 2 shows a comparison between the results of the present study (for $k_{v}=0$ ), those produced by the method proposed by Paolucci \& Pecker (1997) and those found by Cascone et al. (2004) with the method of characteristics.

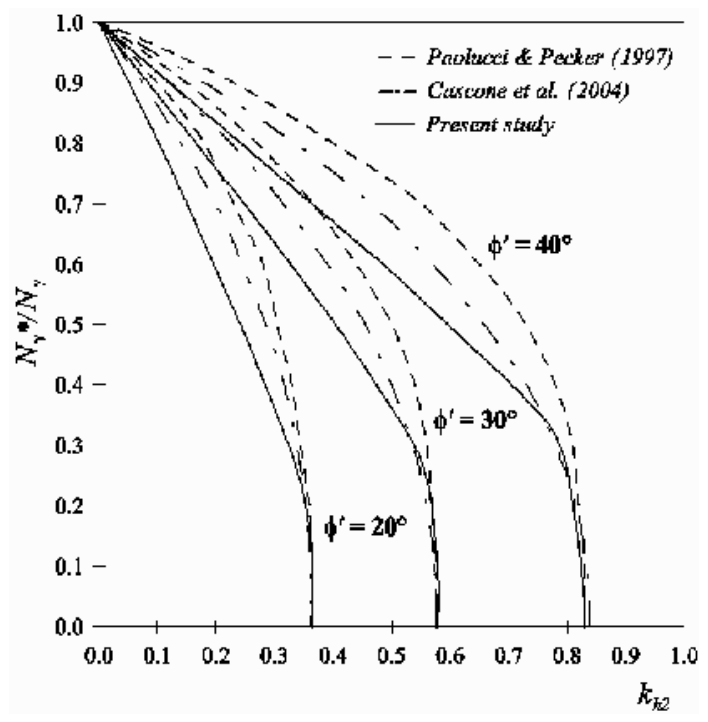

Fig. 2. Seismic ratios as a function of the soil mass inertia

The reduction of the bearing capacity is presented in terms of the ratio $N_{\gamma}^{*} / N_{\gamma}$ as a function of the seismic coefficient $k_{h 2}$ in the soil mass.

Despite of the different methods, the results obtained are in good agreement. However, for low values of $k_{h 2}$, the limit equilibrium approach seems to give the greatest reduction thus it is on the safe side.

\section{Parametric analysis}

To investigate the influence of the depth factor on the seismic stability of a shallow foundation, the model proposed has been applied and an upper bound solution of the limit load is found. Results of the analysis are given in terms of the ratios between seismic and static bearing capacity factors $N_{c}^{*} / N_{c}, N_{q}^{*} / N_{q}$ and $N_{\gamma}^{*} / N_{\gamma}$.

Ground factors and bearing capacity ratios are presented as a function of the friction angle of soil $\phi^{\prime}$ and of the ratio $H / B$ between the embedment depth $H$ and the width of the footing $B$. The inertial and kinematic effects due to seismic loading have been analyzed in the evaluation of the seismic bearing capacity.

For a shallow foundation resting on a cohesionless soil, with horizontal ground surface and in absence of surcharge, the limit load can be expressed by :

$$
q_{\lim }=1 / 2 B \gamma N_{\gamma} i_{\gamma_{i}} i_{\gamma_{k}} d_{\gamma}
$$


where:

- $B$ = width of the footing;

- $\quad \gamma=$ unit weight of soil;

- $\quad N_{\gamma}=$ bearing capacity factor;

- $i_{\gamma}=$ load inclination factor due to the inertia of the structure;

- $i_{\gamma k}=$ reduction factor due to the inertia of the soil mass (kinematic interaction factor);

- $d_{\gamma}=$ depth factor.

The load inclination factor related to the inertia of the structure $\left(i_{j}\right)$ has been discussed by some authors (Pecker \& Salencon, 1991; Budhu \& Al-Karni, 1993; Dormieux \& Pecker, 1995; Paolucci \& Pecker, 1997; Fishmann et al., 2003), while less information are available on the depth factor $\left(d_{\gamma}\right)$ and on the reduction factor due to the inertia of the soil mass $\left(i_{\gamma_{k}}=\right.$ kinematic interaction factor).

Conventionally, the depth factor $\left(d_{\gamma}\right)$ is assumed equal to unit (Brinch Hansen, 1970). Nevertheless, in an analysis in which the effects due to the inertia of the soil mass are taken into consideration, it is also necessary to take into account the inertia of the soil mass corresponding to the embedment depth $H$ of the footing.

\subsection{Depth factor evaluation}

In static conditions the depth factor $d_{\gamma}$ has been evaluated by a parametric analysis, for both drained $\left(\phi^{\prime}=20^{\circ}, 30^{\circ}, 40^{\circ}\right)$ and undrained conditions $(\phi=0)$, varying the ratio $\mathrm{H} / \mathrm{B}$ between the embedment depth $H$ and the width of the footing $B$.

In the present analysis, the depth factor $d_{\gamma}$ for drained conditions is defined as the ratio between the bearing capacity factors $N_{\gamma}{ }^{\prime}$ of a shallow foundation with embedment $H$ and the conventional bearing capacity $N_{\gamma}$ of a shallow foundation with an embedment equal to 0 :

$$
d_{\gamma}=N_{\gamma}{ }^{\prime} / N_{\gamma}
$$

Similarly, for undrained conditions the depth factor $d_{\gamma}{ }^{\circ}$ is defined as the difference between the bearing capacity factors $N_{\gamma}{ }^{\circ}$ of a shallow foundation with an embedment $H$ and the conventional bearing capacity $N_{\gamma}{ }^{\circ}$ of a shallow foundation with an embedment equal to 0 :

$$
d_{\gamma}{ }^{\circ}=\left(N_{\gamma}{ }^{\circ}-N_{\gamma}{ }^{\circ}\right)=N_{\gamma}{ }^{\circ}{ }^{1}
$$

being $N_{\gamma}^{\circ}$ in undrained conditions, as known, equal to 0 .

With reference to equation (10) in Table 1 are reported, as an example, the values of the depth factor $d_{\gamma}{ }^{\circ}$ for the undrained conditions.

\begin{tabular}{c|c}
\hline$H / B$ & $d_{\gamma}^{\circ}($ eq.10) \\
\hline 0 & 0 \\
\hline 0.25 & 0.562 \\
\hline 0.5 & 1.25 \\
\hline 0.75 & 2.062 \\
\hline 1.0 & 3.0 \\
\hline
\end{tabular}

Table 1. Values of the depth factor $d_{\gamma}^{\circ}$ for the undrained conditions 
In Figure 3 are reported the values of the depth factor $d_{\gamma}$ for drained conditions versus $H / B$. For the values of the friction angle of soil $\phi^{\prime}$ taken into consideration, curves shown approximately a linear trend, thus it is possible to express the depth factor $d_{\gamma}$ as a linear function of the ratio $H / B$ according to the equation :

$$
d_{\gamma}=1+\left[(0.85 H / B) \cot g \varphi^{\prime}\right]
$$

For undrained conditions the results obtained (Figure 4) can be conveniently expressed by the following linear equation :

$$
d_{\gamma}^{\circ}=(2 H / B)-0.25
$$

that, obviously, is valid for $H / B>0.125$.

\subsection{Kinematic interaction factor evaluation}

The kinematic interaction factor $i_{\gamma k}$ has been evaluated by a parametric analysis only for drained conditions, varying the friction angle of soil in the range $\phi^{\prime}=20^{\circ}, 30^{\circ}$ and $40^{\circ}$ and the horizontal seismic coefficient for the soil mass $k_{h 2}$ between 0.1 up to 0.3 .

The kinematic interaction factor $i_{\jmath k}$ is defined in this study as the ratio between the bearing capacity factor $N_{\gamma}^{*}$ derived for a given value of the horizontal seismic coefficient $k_{h 2}$, and the conventional bearing capacity factor $N_{\gamma}$ :

$$
i_{\gamma k}=N_{\gamma}^{*} / N_{\gamma}
$$

The numerical analyses have been carried out assuming a vertical seismic coefficient for the soil mass $k_{v}$ equal to $1 / 2 k_{h 2}$. In Figure 5 are reported the values of the kinematic interaction factor $i_{\jmath k}$ obtained for the soil friction angles taken into consideration.

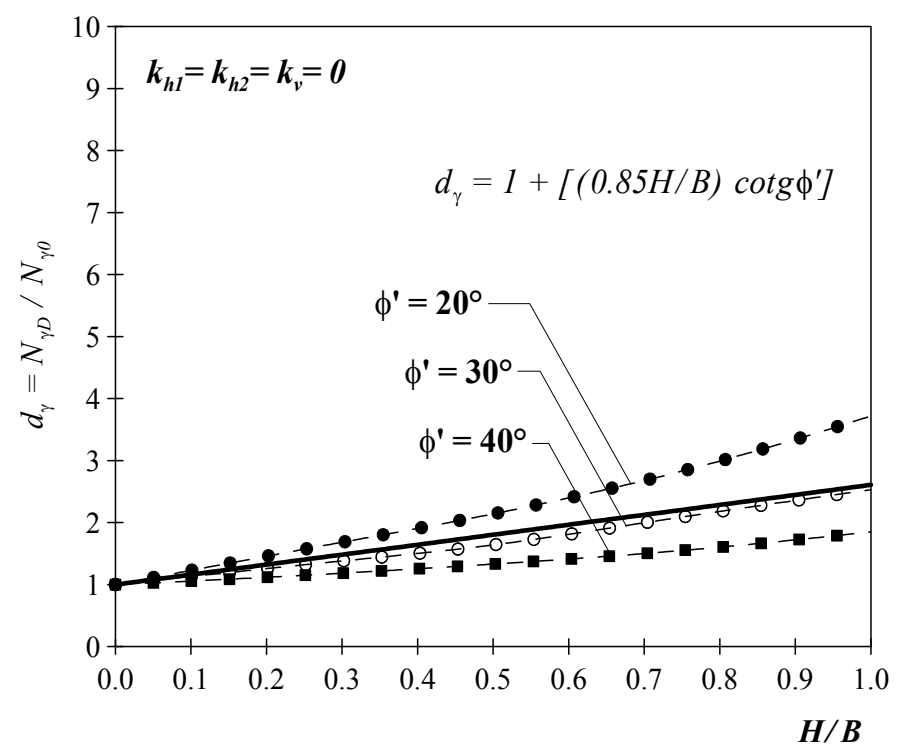

Fig. 3. Depth factor $d_{\gamma}$ for drained conditions 


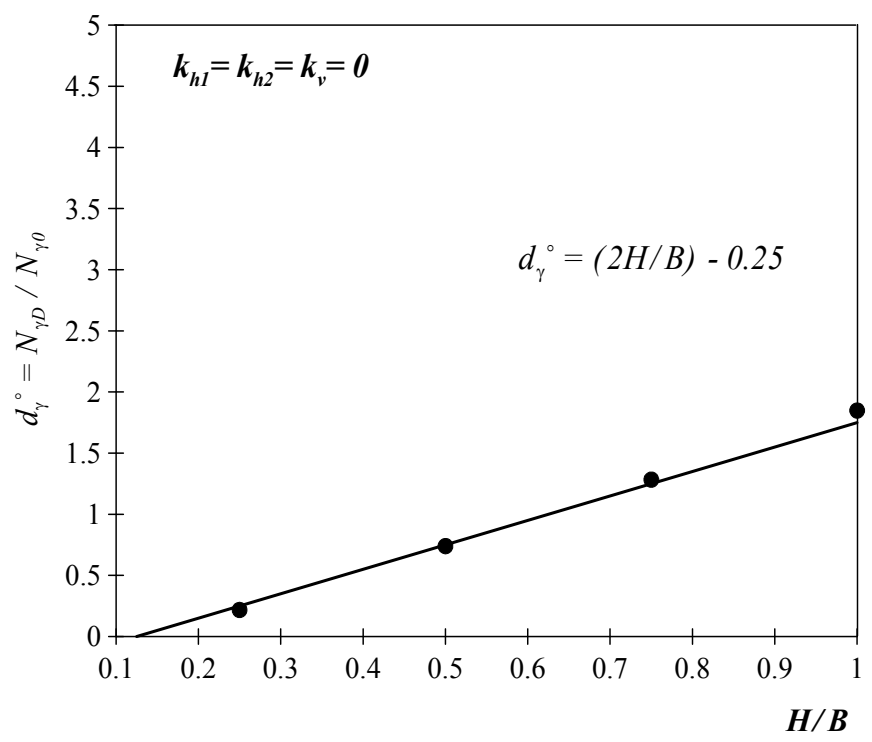

Fig. 4. Depth factor $d_{\gamma}{ }^{\circ}$ for undrained conditions

Curves shown approximately a linear trend, thus it is possible to express the kinematic interaction factor $i_{\jmath k}$ as a linear function of $k_{h 2}$ by the following equation :

$$
i_{\gamma k}=1-k_{h 2} \cot g \phi^{\prime}
$$

It is simple to verify that for $k_{h 2}=\tan \phi{ }^{\prime}$ the kinematic interaction factor $i_{\not k}$ is equal to 0 .

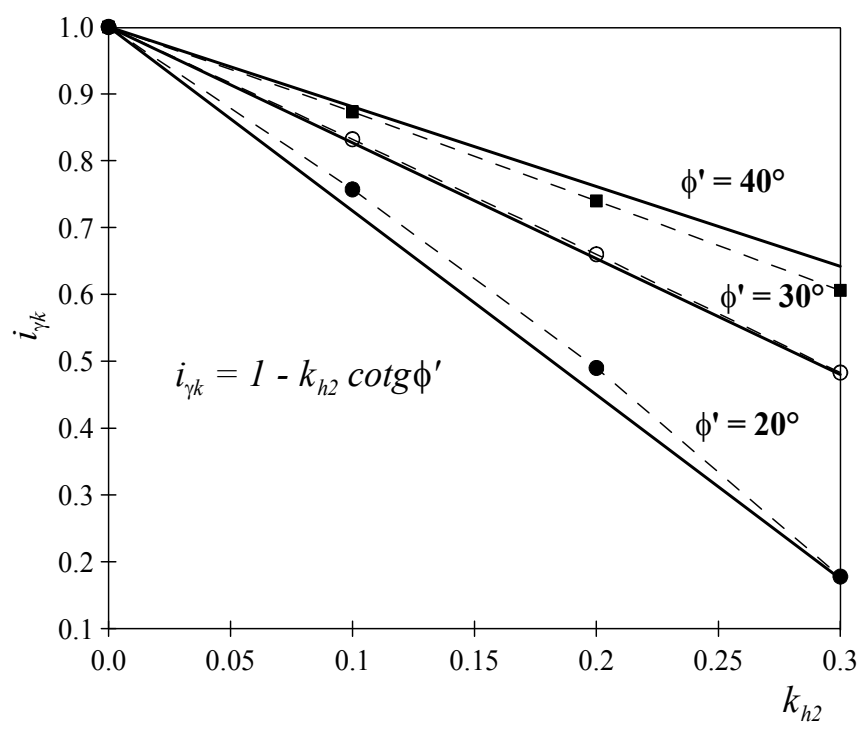

Fig. 5. Values of the kinematic interaction factor $i_{\jmath k}$ 


\section{Bearing capacity of strip footings near slopes}

When a shallow foundation is placed near the edge of a sloping ground the bearing capacity may be reduced both in static (De Buhan \& Gaernier, 1988; Saran et al., 1989; Narita \& Yamaguchi, 1990; Shields et al., 1990; Jao et al., 2001) and seismic conditions (Sawada et al., 1994; Pecker, 1996; Sarma \& Iossifelis, 1990; Sarma \& Chen, 1996; Kumar \& Rao, 2003).

In this case, the failure mechanism is influenced by the distance $d$ of the foundation from the edge of the sloping ground (Figure 6). If the shallow foundation is far enough from the edge, the failure mechanism will be not affected by the slope.

In the last decades extensive studies have been made for two dimensional problems of a strip footing resting on inclined slope surface so that different methods of analysis are available.

In these studies, the bearing capacity evaluation is based on the assumption that a failure surface can develop beneath the foundation, according to a general shear failure surfaces given by the limit equilibrium method or by kinematic mechanisms.

General shear failure is characterized by the existence of a well-defined failure pattern (Terzaghi, 1943), which consists of a continuous slip surface from one edge of the footing to the horizontal or inclined ground surface. Referring to Figure 6, the mobilizing moment is :

$$
\begin{aligned}
& M_{\text {mob }}=\sum_{i=1}^{n_{\text {tot }}} W_{i}\left(1-k_{v}\right) b_{w i}+q_{\lim } \sum_{i=1}^{n_{1}} \Delta x_{i} b_{q l i}+\sum_{i=n_{1}+1}^{n_{\text {tot }}} q_{v}\left(1-k_{v}\right) b_{q v i} \Delta x_{i}+ \\
& +q_{\lim } \sum_{i=1}^{n_{1}} k_{h 1} \Delta x_{i} b_{q l h i}+\sum_{i=1}^{n_{\text {tot }}} k_{h 2} W_{i} b_{w h i}+\sum_{i=n_{1}+1}^{n_{\text {tot }}} k_{h 3} q_{v} b_{q h i}
\end{aligned}
$$

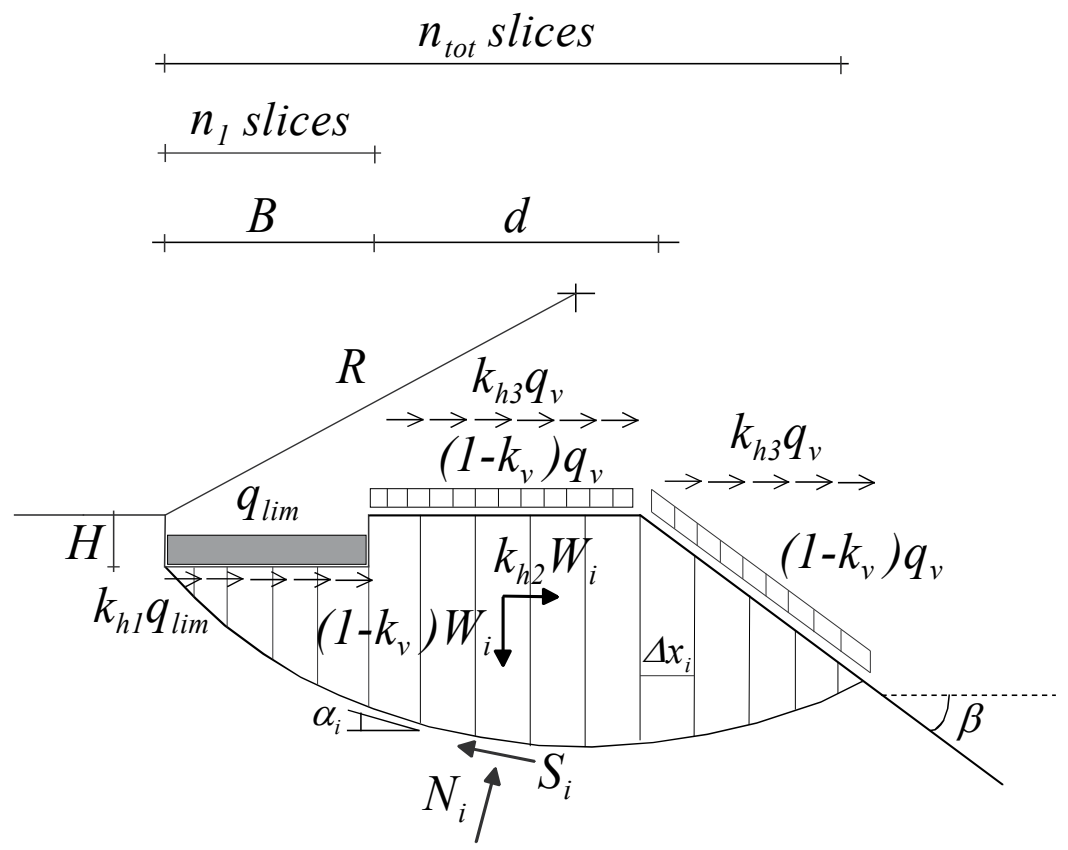

Fig. 6. Strip footings near slopes: failure mechanism and applied forces 
The resisting moment $M_{\text {res }}$ and the shear strength $S_{i}$ acting along the base of the slices are expressed by equation (2) and (3) respectively.

For the slices $i=1$ to $n_{1}$ the force $N_{i}$ resultant of the normal stress distribution acting at the base of the slice, derived by the Bishop's method of slices (1955) with an equilibrium equation in the vertical direction, is given by equation (4), while for the remaining slices $\left(n_{1}+1 \leq i \leq n_{\text {tot }}\right)$ is :

$$
N_{i}=\frac{q_{v}\left(1-k_{v}\right) \Delta x_{i}+W_{i}\left(1-k_{v}\right)-c \Delta x_{i} \tan \alpha_{i}}{\cos \alpha_{i}+\sin \alpha_{i} \tan \phi}
$$

Thus :

$$
\begin{aligned}
& M_{r e s}=R \sum_{i=1}^{n_{\text {tot }}} c \Delta x_{i} / \cos \alpha_{i}+R \sum_{i=1}^{n_{1}} \frac{q_{\text {lim }} \Delta x_{i}+W_{i}\left(1-k_{v}\right)-c \Delta x_{i} \tan \alpha_{i}}{\cos \alpha_{i}+\sin \alpha_{i} \tan \phi} \tan \varphi+ \\
& +\sum_{i=n_{1}+1}^{n_{\text {tot }}} \frac{q_{v}\left(1-k_{v}\right) \Delta x_{i}+W_{i}\left(1-k_{v}\right)-c \Delta x_{i} \tan \alpha_{i}}{\cos \alpha_{i}+\sin \alpha_{i} \tan \phi} R \tan \phi
\end{aligned}
$$

where :

- $\quad q_{\text {lim }}=$ vertical limit load acting on the footing;

- $c=$ soil cohesion;

- $\quad \phi^{\prime}=$ friction angle of soil;

- $\Delta x_{i}=$ width of the $\mathrm{i}^{\text {th }}$ slice;

- $W_{i}=$ weight of the $\mathrm{i}^{\text {th }}$ slice;

- $q_{v}=$ vertical surcharge;

- $\quad R=$ radius of the circular failure surface;

- $\alpha_{i}=$ angle of the base of the $\mathrm{i}^{\text {th }}$ slice;

- $n_{1}=$ number of slices under the footing;

- $n_{\text {tot }}=$ total number of slices;

- $\quad k_{h 1}=$ horizontal seismic coefficient for the limit load;

- $\quad k_{h 2}=$ horizontal seismic coefficient for the soil mass;

- $\quad k_{h 3}=$ horizontal seismic coefficient for the surcharge;

- $\quad k_{v}=$ vertical seismic coefficient for the soil mass and the surcharge;

- $\quad b_{w i}=$ distance of the weight $W_{i}$ of the $\mathrm{i}^{\text {th }}$ slice to the centre of the circular failure surface;

- $\quad b_{w h i}=$ distance of the inertia force $k_{h 2} W_{i}$ of the $i^{\text {th }}$ slice to the centre of the circular failure surface;

- $\quad b_{q v i}=$ distance of the surcharge force $q_{v} \Delta x_{i}$ of the $\mathrm{i}^{\text {th }}$ slice to the centre of the circular failure surface;

- $\quad b_{q h i}=$ distance of the horizontal surcharge force $k_{h 3} q_{v} \Delta x_{i}$ of the $\mathrm{i}^{\text {th }}$ slice to the centre of the circular failure surface;

- $\quad b_{q l i}=$ distance of the limit load $q_{\text {lim }}$ acting on the $\mathrm{i}^{\text {th }}$ slice to the centre of the circular failure surface;

- $\quad b_{q h l i}=$ distance of the shear limit force $k_{h 1} q_{l i m}$ acting on the $\mathrm{i}^{\text {th }}$ slice to the centre of the circular failure surface.

Substituting the following terms :

$$
a_{1}=R \sum_{i=1}^{n_{\text {tot }}} c \Delta x_{i} / \cos \alpha_{i}
$$




$$
\begin{gathered}
a_{2}=R \tan \phi \sum_{i=1}^{n_{1}} \frac{\Delta x_{i}}{\cos \alpha_{i}+\sin \alpha_{i} \tan \phi} \\
a_{3}=R \tan \phi \sum_{i=1}^{n_{1}} \frac{W_{i}\left(1-k_{v}\right)-c \Delta x_{i} \tan \alpha_{i}}{\cos \alpha_{i}+\sin \alpha_{i} \tan \phi} \\
a_{4}=R \tan \phi \sum_{i=n_{1}+1}^{n_{\text {tot }}} \frac{q_{v}\left(1-k_{v}\right) \Delta x_{i}+W_{i}\left(1-k_{v}\right)-c \Delta x_{i} \tan \alpha_{i}}{\cos \alpha_{i}+\sin \alpha_{i} \tan \phi} \\
a_{5}=\sum_{i=1}^{n_{\text {tot }}} W_{i}\left(1-k_{v}\right) b_{w i} \\
a_{6}=\sum_{i=1}^{n_{\text {tot }}} k_{h 2} W_{i} b_{w h i} \\
a_{7}=\sum_{i=n_{1}+1}^{n_{\text {tot }}} q_{v}\left(1-k_{v}\right) \Delta x_{i} b_{q v i} \\
a_{10}=\sum_{i=1}^{n_{1}} k_{h 1} \Delta x_{i} b_{q l h i} \\
a_{8}=\sum_{i=n_{1}+1}^{n_{\text {tot }}} k_{h 3} q_{v} \Delta x_{i} b_{q h i} \\
a_{9}=\sum_{i=1}^{n_{1}} \Delta x_{i} b_{q l i}
\end{gathered}
$$

and equating $M_{m o b}=M_{\text {res }}$ the limit load is given by :

$$
q_{\lim }=\frac{a_{5}+a_{6}+a_{7}+a_{8}-a_{1}-a_{3}-a_{4}}{a_{2}-a_{9}-a_{10}}
$$

For example, referring to the ground slope factor $g_{\gamma}$ taking into account the effect of the sloping ground surface, in Figure 7 the values derived assuming the distance $d$ equal to zero and the slope angle $\beta>0$ (angle that the ground surface makes with the horizontal), have been evaluated for three different friction angles $\left(\phi^{\prime}=20^{\circ}, 30^{\circ}\right.$ and $\left.40^{\circ}\right)$ and compared with those obtained by the well known Brinch Hansen's solution (1970).

The angle $\beta$ is positive when the ground slopes down and away from the footing. According to Brinch Hansen (1970) we have :

$$
g_{\gamma}=(1-0.5 \tan \beta)^{5}
$$

while in the present study we obtain : 


$$
g_{\gamma}=(1-0.5 \tan \beta)^{4.5}
$$

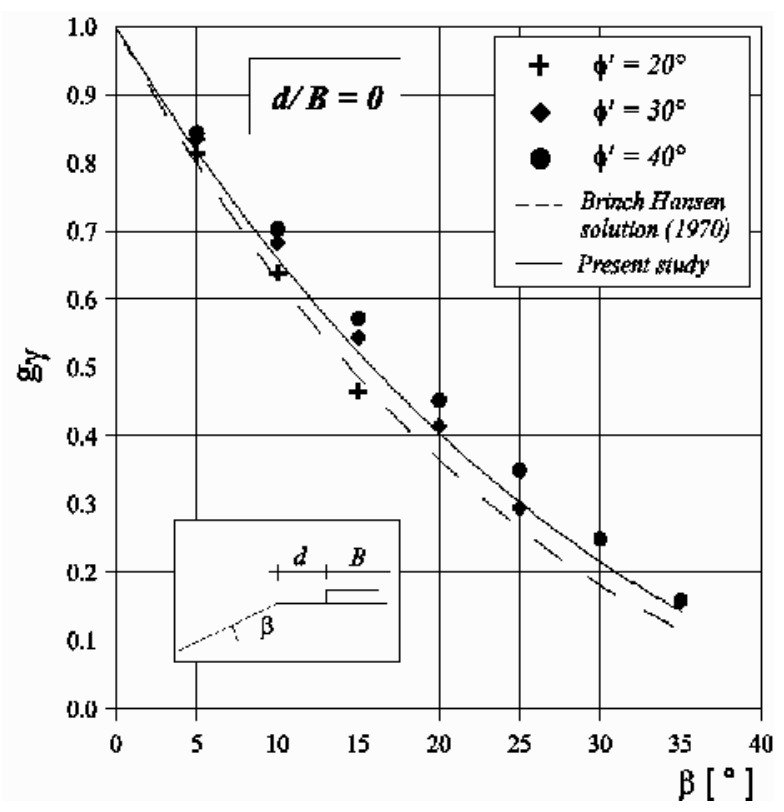

Fig. 7. Values of the ground factors $g_{\gamma}$ and comparison with Brinch Hansen's solution (1970)

\subsection{Seismic analysis}

The recommendations of Eurocode 8 - Part 5 (2003) state that in the calculation of the bearing capacity of a shallow foundation one should include the load inclination and eccentricity arising from the inertia forces of the structure, as well as the possible effects of the inertia in the soil.

Thus, the seismic analysis was carried out considering the following seismic coefficients: $k_{h 1}$ $=0.1$ and 0.2 for the inertia of the structure; $k_{h 2}=0.2$ and 0.4 for the inertia of the soil mass.

The value of $k_{h 1}$ was chosen lower than $k_{h 2}$ because the Eurocode 8 (2003) allows to reduce the seismic action by a behaviour factor associated with the ductility classification of the structures.

This consideration takes to the conclusion that the kinematic effect, and the consequent reduction in bearing capacity due to the soil inertia, cannot be neglected and in some circumstances it's reduction could be more significant than the reduction due to the inertia of the structure (Cascone et al., 2006). In this study the seismic coefficient $k_{h 3}$ of the surcharge was assumed equal to $k_{h 1}$.

The friction angle of soil was chosen in the range $0^{\circ}$ up to $40^{\circ}$, while the angle of the slope near the footing was varied in the range $5^{\circ}$ to $35^{\circ}$. In the seismic analysis the angle of the sloping ground is affected by further limitations, because simple equilibrium considerations, for a cohesionless soil $\left(c^{\prime}=0\right)$, take to the following :

$$
\tan \beta<\frac{\left(1-k_{v}\right) \tan \phi^{\prime}-k_{h, i}}{1-k_{v}+k_{h, i} \tan \phi^{\prime}}(\mathrm{i}=2,3)
$$


or in a simpler form :

$$
\beta<\phi^{\prime}-\theta
$$

where :

$$
\theta=\tan ^{-1}\left[\frac{k_{h, i}}{1-k_{v}}\right](\mathrm{i}=2,3)
$$

In the following, Table 2 shows some limit values of $\beta$, for $k_{v}=0$ and $\phi^{\prime}=20^{\circ}, 30^{\circ}$ and $40^{\circ}$.

\begin{tabular}{c|c|c|c|c}
\hline \multirow{2}{*}{$\phi^{\prime}$} & \multicolumn{4}{|c}{$k_{h 2}, k_{h 3}$} \\
\cline { 2 - 5 } & 0.1 & 0.2 & 0.3 & 0.4 \\
\hline $20^{\circ}$ & $14.29^{\circ}$ & $8.69^{\circ}$ & $3.30^{\circ}$ & - \\
\hline $30^{\circ}$ & $24.29^{\circ}$ & $18.69^{\circ}$ & $13.30^{\circ}$ & $8.19^{\circ}$ \\
\hline $40^{\circ}$ & $34.29^{\circ}$ & $28.69^{\circ}$ & $23.30^{\circ}$ & $18.19^{\circ}$ \\
\hline
\end{tabular}

Table 2. Limit values of $\beta$ for a vertical seismic coefficient $k_{v}=0$

In Figures 8 to 16 the results of the parametric analysis are shown in a synthetic form. The seismic bearing capacity ratios $N_{c}^{*} / N_{c}, N_{q}^{*} / N_{q}, N_{\gamma}^{*} / N_{\gamma}$ are represented as a function of $d / B$ for various slope angles and for different values of the friction angle of soil.

The threshold distance $\left(d_{t}\right)$ at which the sloping ground does not affect anymore the bearing capacity mainly increases with the increasing of the angle of friction and secondarily with the increasing of the seismic coefficient and with the increasing of the slope angle $\beta$.

The embedment depth of the footing does not play a significant role on the threshold distance, however it may produce a considerable increasing of the bearing capacity.

Referring to the $N_{c}^{*} / N_{c}$ ratios, we can observe values of the normalized threshold distances varying between about $d_{t} / B=1$, for an undrained analysis $\left(\phi_{u}=0^{\circ}\right)$, and $d_{t} / B=5$ for $\phi^{\prime}=40^{\circ}$.

For the $N_{q}^{*} / N_{q}$ ratios, we determined values of the normalized threshold distances varying between about $d_{t} / B=2$, for $\phi^{\prime}=20^{\circ}$ and about $d_{t} / B=4$ for $\phi^{\prime}=40^{\circ}$.

Finally for the $N_{\gamma}^{*} / N_{\gamma}$ ratios, we determined values of the normalized threshold distances varying between about $d_{t} / B=1.5$ for $\phi^{\prime}=20^{\circ}$ and about $d_{t} / B=4$ for $\phi^{\prime}=40^{\circ}$.

No significant difference in the threshold distance was found when the inertia of the structure or the inertia of the soil mass is considered.

Furthermore the combined effects of soil and structure inertia can be taken into account by using the superposition of the effects principle.

In this case, at the same way as found by Paolucci \& Pecker (1997) and Cascone et al. (2004), the bearing capacity of the soil self weight under both the seismic loading due to the coefficients $k_{h 1}$ and $k_{h 2}$, can be evaluated through the following equation :

$$
q_{\lim }=\frac{1}{2} B \gamma N_{\gamma_{e}} \approx \frac{1}{2} B \gamma N_{\gamma} e_{\gamma_{i}} e_{\gamma_{k}}
$$

where :

$N_{\gamma e}=$ bearing capacity factor reduced by both acting the coefficients $k_{h 1}$ and $k_{\mathrm{h} 2}$;

$N_{\gamma}=$ static bearing capacity factor;

$e_{\gamma i}=N_{\gamma 1}{ }^{*} / N_{\gamma}$ bearing capacity ratio for structure inertia only $\left(k_{h 1}>0, k_{h 2}=k_{h 3}=0\right)$;

$e_{\gamma k}=N_{\gamma 2}{ }^{*} / N_{\gamma}$ bearing capacity ratio for soil mass inertia only $\left(k_{h 2}>0, k_{h 1}=k_{h 3}=0\right)$. 
Figure 17 shows a comparison between the $N_{\gamma} / N_{\gamma}$ ratio and the product $e_{\gamma} \cdot e_{\gamma k}$ for, as an example, $k_{h 1}=0.1,0.2$ and 0.3 and $k_{h 2}=0.1,0.2$ and 0.3 .

In particular, Figure 17 shows that when the seismic coefficients $k_{h 1}$ and $k_{h 2}$ are small, there is not a significant difference between the $N_{\gamma} / N_{\gamma}$ ratio and the product $e_{\gamma i} \cdot e_{\gamma k}$.

On the contrary, when the seismic coefficients are high enough to produce a great reduction of the limit load, one can find a great difference in using the $N_{\gamma} / N_{\gamma}$ ratio instead of the product $e_{\gamma i} \cdot e_{\gamma k}$.

As example, for $\phi^{\prime}=20^{\circ}, k_{h 1}=0.1$ and $k_{h 2}=0.1$, we have :

$N_{\gamma} / N_{\gamma}=0.565$ and $e_{\gamma i} \cdot e_{\gamma k}=0.587$

while for $\phi^{\prime}=20^{\circ}, k_{h 1}=0.3$ and $k_{h 2}=0.3$, we have :

$N_{\gamma} / N_{\gamma}=0.05$ and $e_{\gamma} \cdot e_{\gamma k}=0.096$.

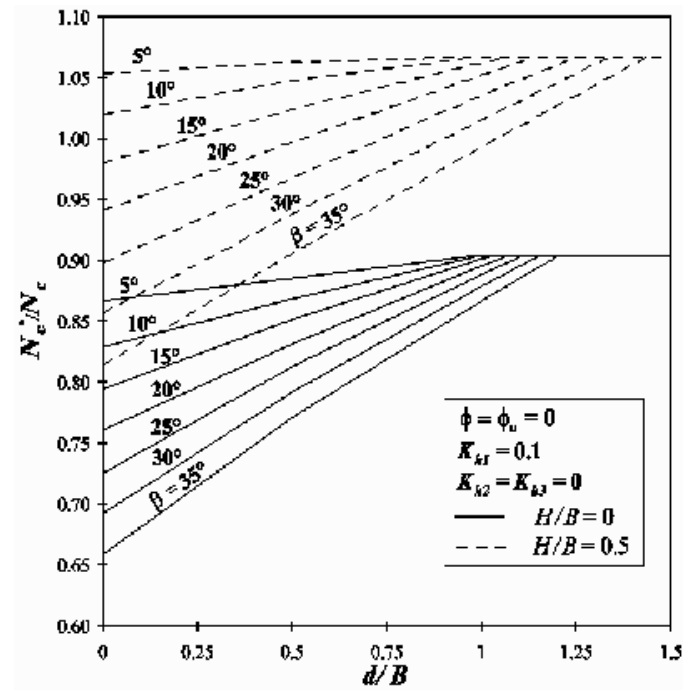

a

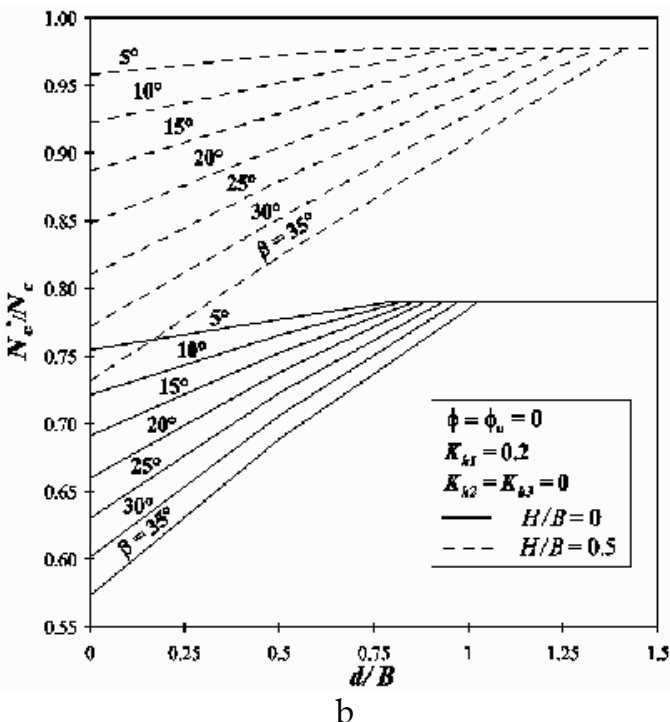

b

Fig. 8. $N_{c}^{*} / N_{c}$ ratios as a function of the normalized $d / B$ slope distance (undrained analysis $\left.\phi=\phi_{u}=0\right)$ for $k_{h 1}=0.1(a)$ and $k_{h 1}=0.2(b)$ 


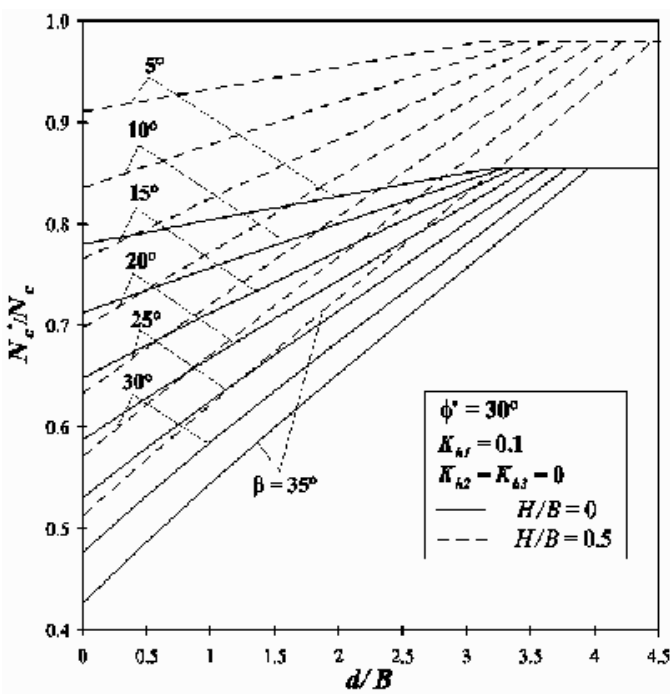

a

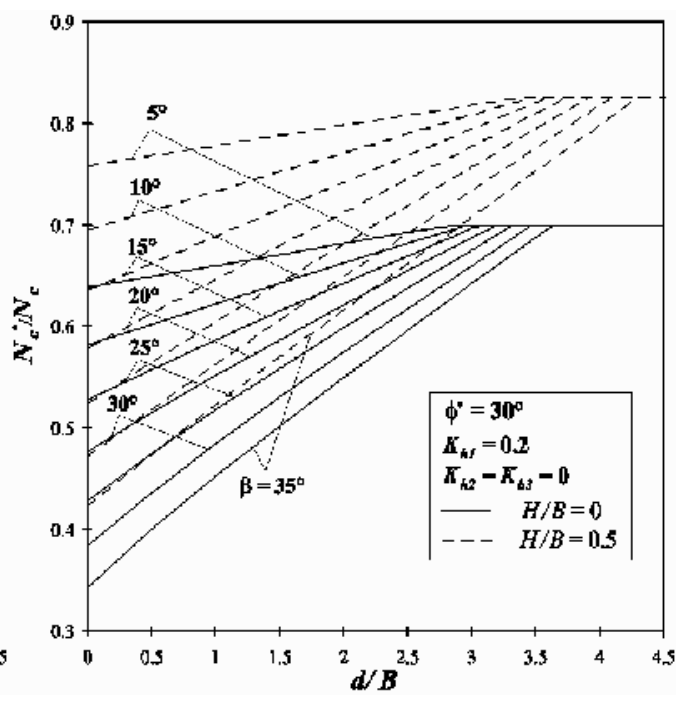

$\mathrm{b}$

Fig. 9. $N_{c}^{*} / N_{c}$ ratios as a function of the normalized $d / B$ slope distance when $\phi^{\prime}=30^{\circ}$ and $k_{h 1}=0.1(a)$ and $k_{h 1}=0.2(b)$

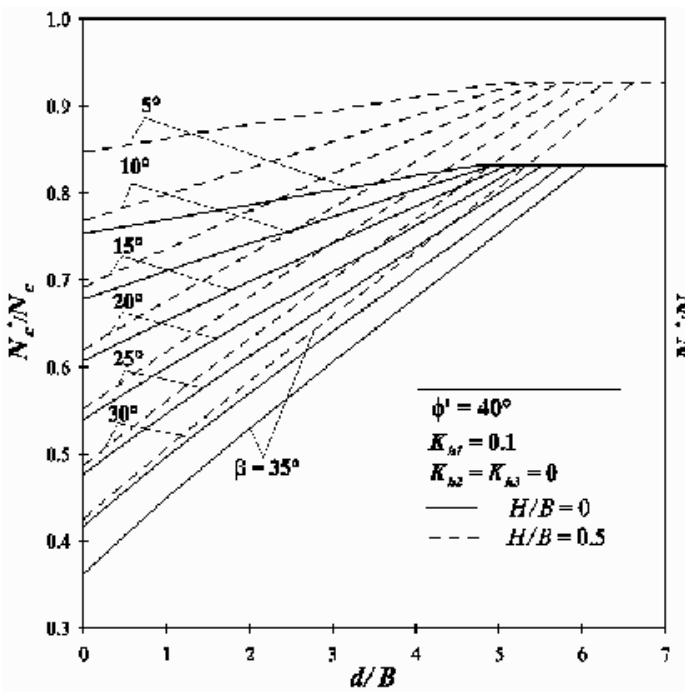

a

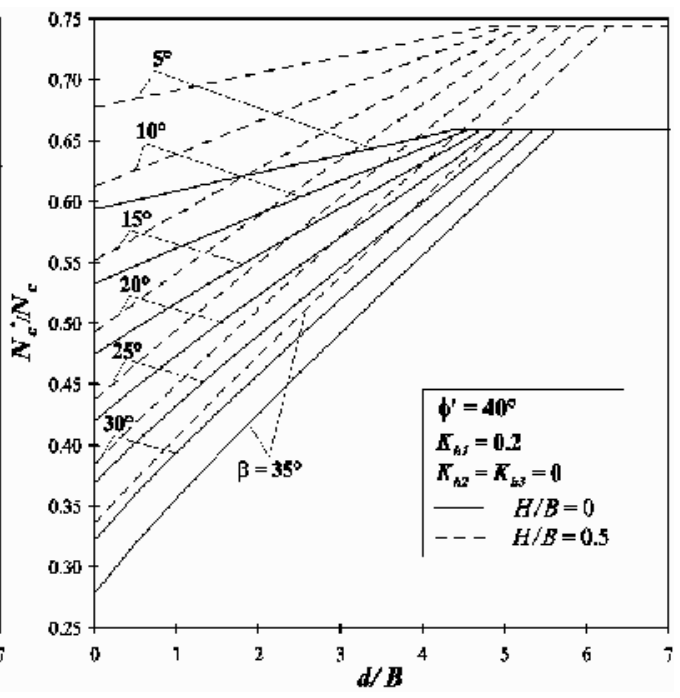

$\mathrm{b}$

Fig. 10. $N_{c}{ }^{*} / N_{c}$ ratios as a function of the normalized $d / B$ slope distance when $\phi^{\prime}=40^{\circ}$ and $k_{h 1}=0.1(a)$ and $k_{h 1}=0.2(b)$ 


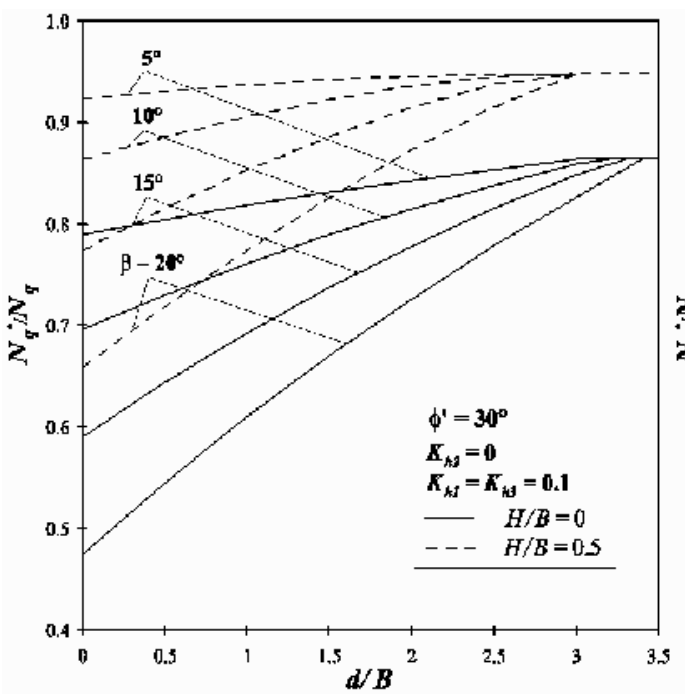

a

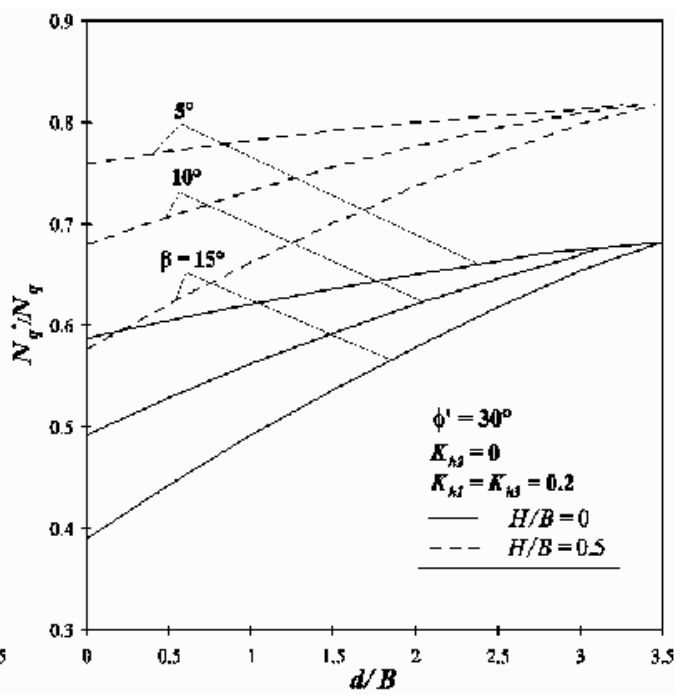

$\mathrm{b}$

Fig. 11. $N_{q}{ }^{*} / N_{q}$ ratios as a function of the normalized $d / B$ slope distance when $\phi^{\prime}=30^{\circ}$ and $k_{h 1}=k_{h 3}=0.1(a)$ and $k_{h 1}=k_{h 3}=0.2(b)$
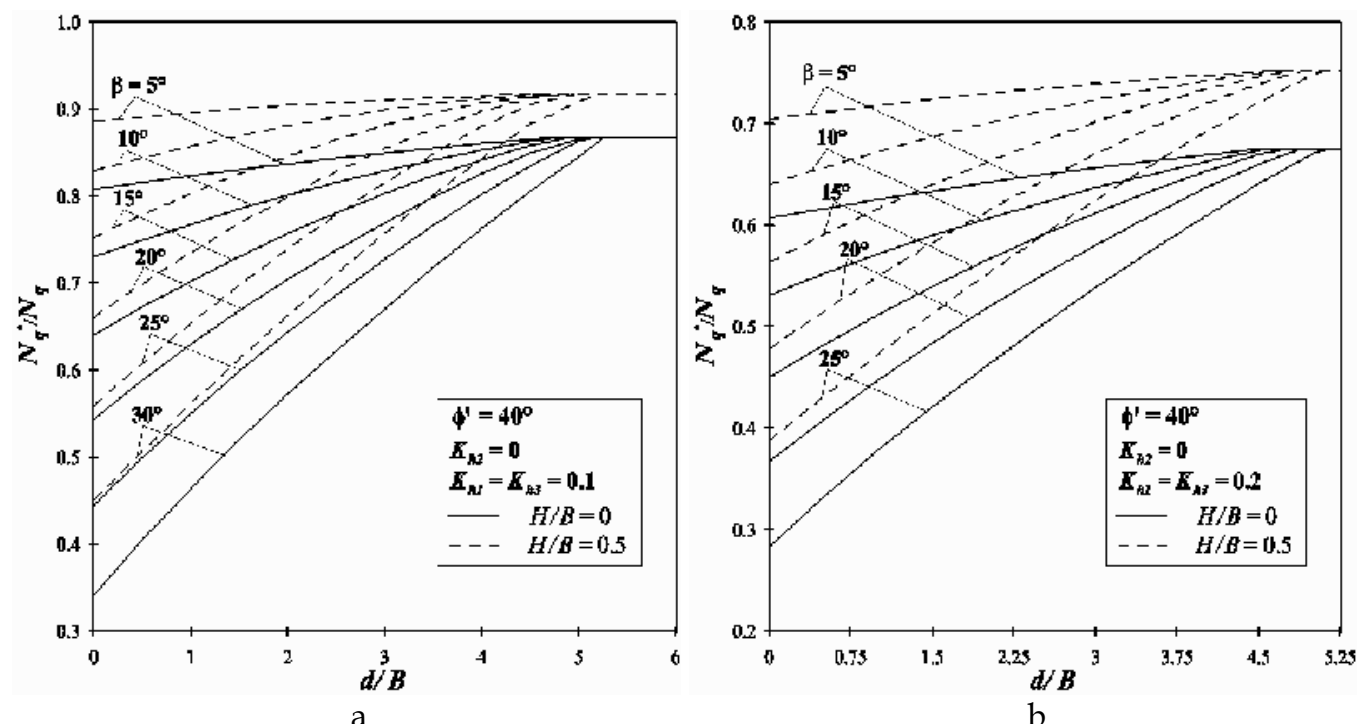

Fig. 12. $N_{q}{ }^{*} / N_{q}$ ratios as a function of the normalized $d / B$ slope distance when $\phi^{\prime}=40^{\circ}$ and $k_{h 1}=k_{h 3}=0.1(a)$ and $k_{h 1}=k_{h 3}=0.2(b)$ 


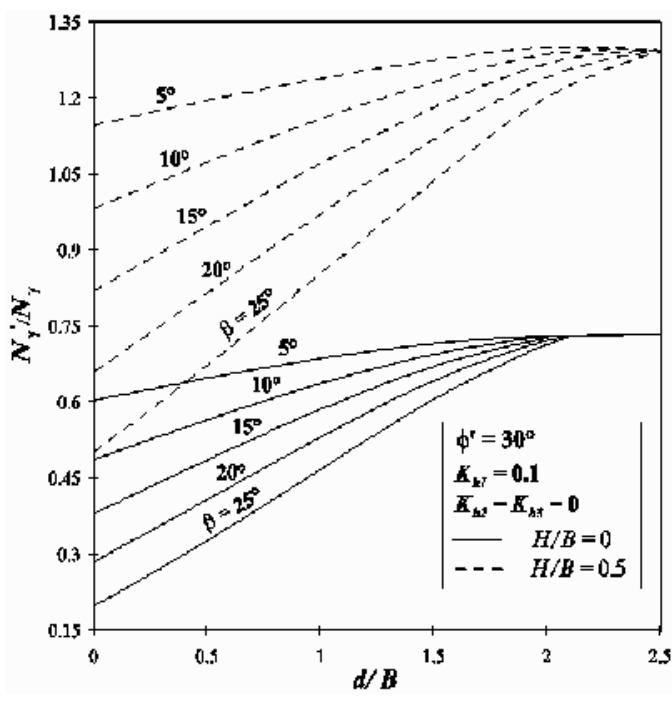

a

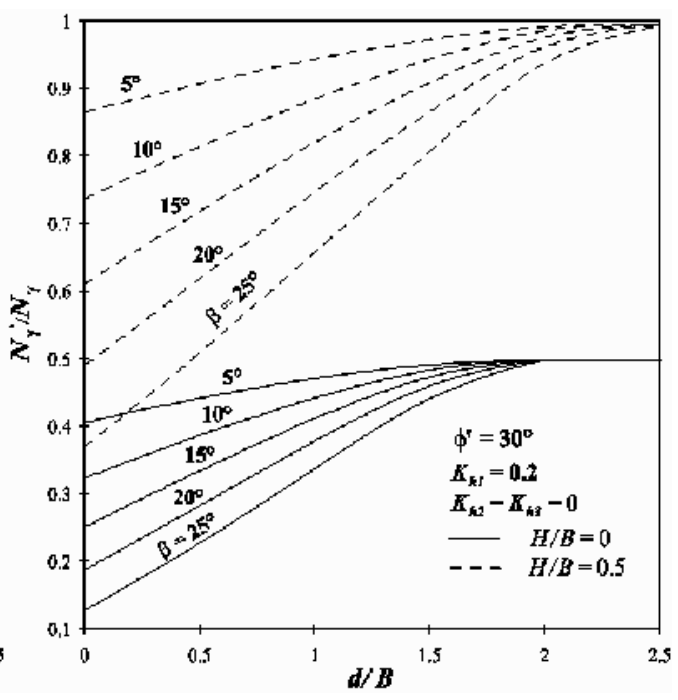

$\mathrm{b}$

Fig. 13. $N_{\gamma}^{*} / N_{\gamma}$ ratios for structural inertia as a function of the normalized $d / B$ slope distance when $\phi^{\prime}=30^{\circ}$ and $k_{h 1}=0.1(a)$ and $k_{h 1}=0.2(b)$

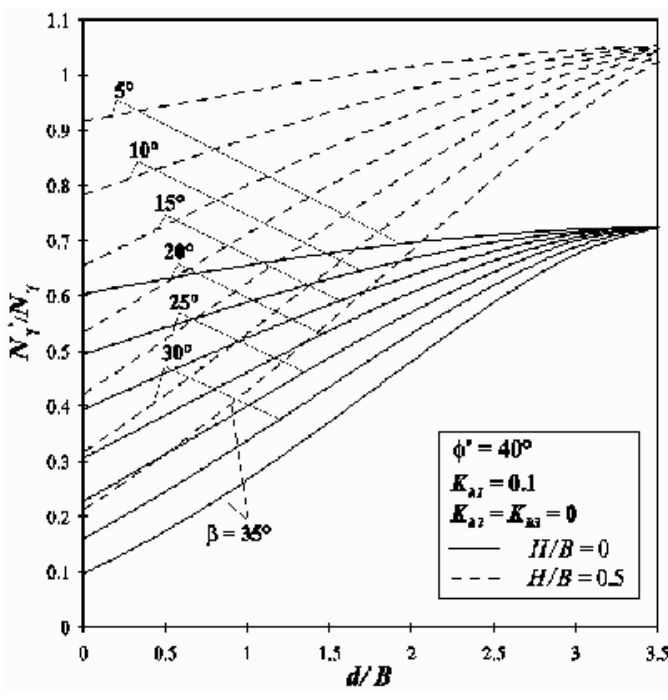

a

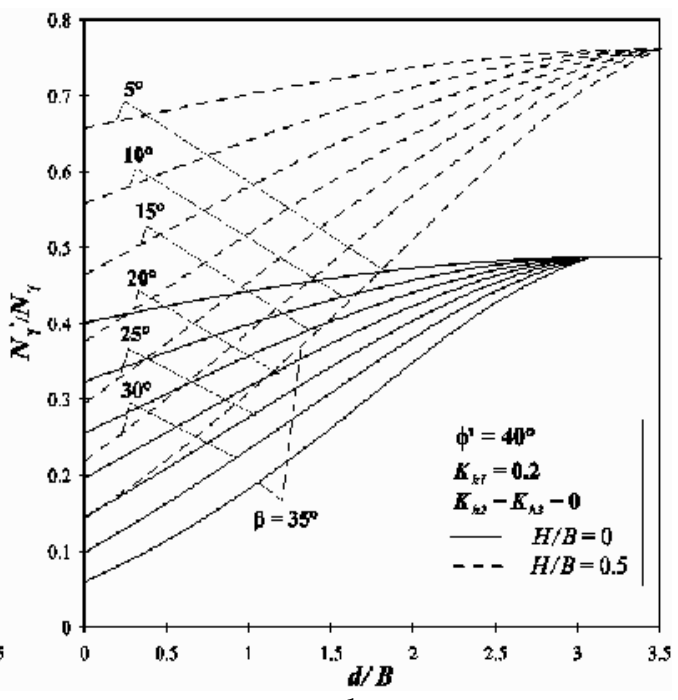

$\mathrm{b}$

Fig. 14. $N_{\gamma}^{*} / N_{\gamma}$ ratios for structural inertia as a function of the normalized $d / B$ slope distance when $\phi^{\prime}=40^{\circ}$ and $k_{h 1}=0.1(a)$ and $k_{h 1}=0.2(b)$ 


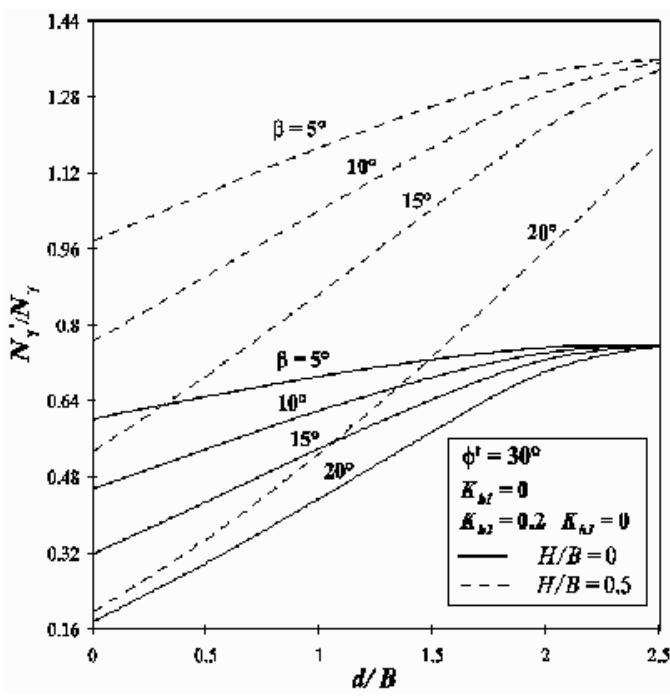

a

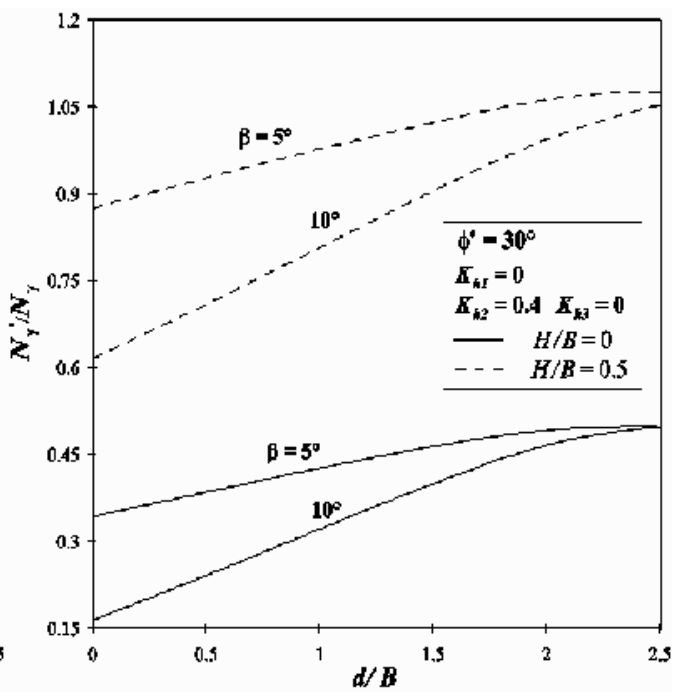

$\mathrm{b}$

Fig. 15. $N_{\gamma}{ }^{*} / N_{\gamma}$ ratios for soil inertia as a function of the normalized $d / B$ slope distance when $\phi^{\prime}=30^{\circ}$ and $k_{h 2}=0.2(a)$ and $k_{h 2}=0.4(b)$

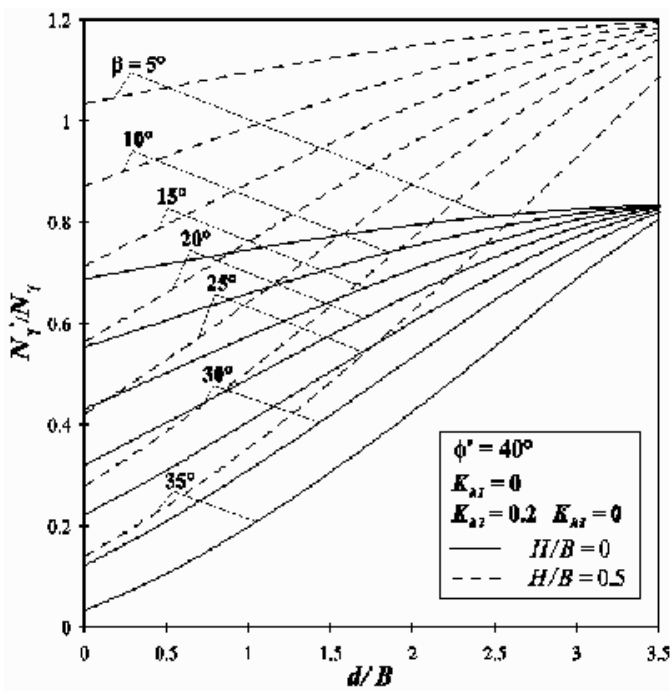

a

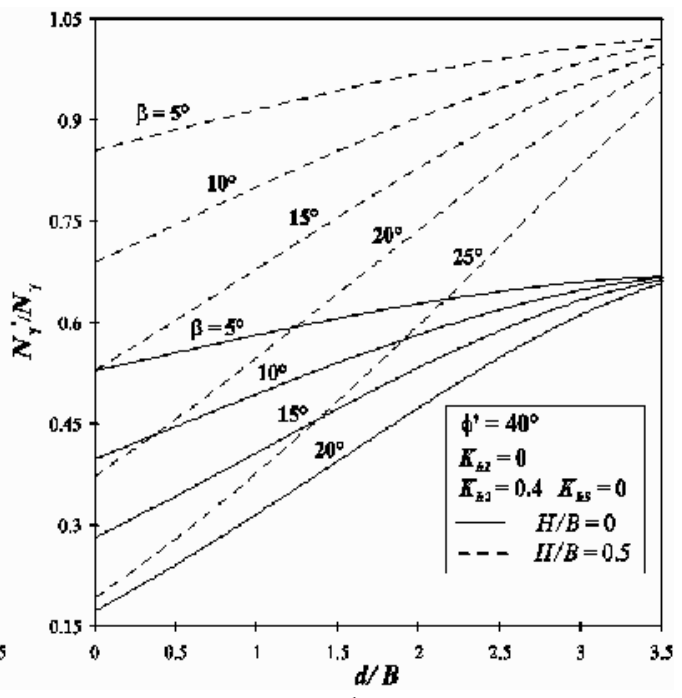

$\mathrm{b}$

Fig. 16. $N_{\gamma}^{*} / N_{\gamma}$ ratios for soil inertia as a function of the normalized $d / B$ slope distance when $\phi^{\prime}=40^{\circ}$ and $k_{h 2}=0.2(a)$ and $k_{h 2}=0.4(b)$ 


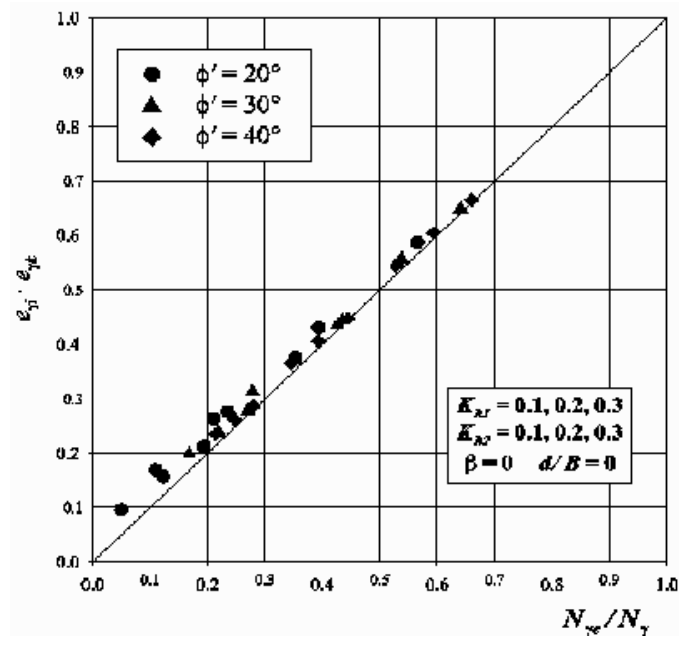

Fig. 17. Comparison between the ratio $N_{\gamma} / N_{\gamma}$ and the product $e_{\gamma 1} \cdot e_{\gamma 2}$

This happens because, when the limit load approaches to zero, the critical surface associated to the simultaneous presence of both the inertial and the kinematic effects is significantly different from that deduced when one considers separately the inertial and the kinematic effects. In this case the superposition of the effects principle may lead to an unconservative design, being the product $e_{\gamma} \cdot e_{\gamma k}$ significantly greater than the $N_{\gamma} / N_{\gamma}$ ratio.

\section{Conclusions}

The design of shallow foundations subject to different static loadings has been an important area of research for geotechnical engineers. The devastating effects of recent earthquakes on shallow foundations has increased the complexity of the problem. Consequently, it is useful to obtain closed-form solutions for the earthquake resistant design of foundations.

Many analytical and numerical solutions are available for the computation of the seismic bearing capacity factors required for the design of shallow foundations.

In the present study the seismic bearing capacity of shallows foundation has been evaluated with the limit equilibrium method.

Numerical analysis shows that, by considering pseudo-static seismic forces, design solutions can be found for the computing of seismic bearing capacity factors for shallow foundations embedded in both horizontal and sloping ground.

Seismic bearing capacity factors with respect to cohesion, surcharge and unit weight components have been computed for a wide range of variation in parameters such as soil friction angle $\left(\phi^{\prime}\right)$, horizontal and vertical seismic coefficients $\left(k_{h}\right.$ and $\left.k_{v}\right)$.

An "upper bound" approach of the limit load was adopted to evaluate the seismic reduction factors to take into account the embedment depth of the footing $\left(i_{\gamma}\right)$ and the inertia of the soil mass $\left(i_{\gamma k}\right)$, as well as, the bearing capacity ratio for structure inertia only $\left(e_{\gamma}\right)$ and the bearing capacity ratio for soil mass inertia only $\left(e_{\gamma k}\right)$.

Some considerations can be formulated:

- In the evaluation of the bearing capacity due to the soil weight it has been observed that the depth of the embedment depth may play a significant role especially for low values of the friction angle. 
- In some cases the seismic reduction in the bearing capacity for the soil inertia (kinematic effect) cannot be ignored, being of about the same amount of that produced by the inertia of the structure.

- $\quad$ The bearing capacity factors decrease appreciably with increases in both $k_{h}$ and $k_{v}$. Bearing capacity decreases as ground inclination $\beta$ increases and as the embedment depth $H$ increases.

- The superposition of the effects principle can be applied to determine the reduced bearing capacity caused by both the seismic actions. However, when the seismic reduction is great, due to high seismic coefficients $k_{h 1}$ and $k_{h 2}$, the superposition of the effects principle may lead to an unconservative design.

- By the simple limit equilibrium method modified bearing capacity factors and simple relations have been proposed which can be used for the practical design of shallow foundations embedded in both horizontal and sloping soil. In many cases the solutions obtained compare well with the previous static results and available results for the seismic conditions.

\section{Acknowledgments}

Funding for this research was provided by "DPC-ReLUIS Research Project 2010 - 2013", Task 2.1 - MT2 "Shallow and Deep Foundations".

\section{References}

Bishop, A.W. (1955). The use of the slip circle in the stability analysis of slopes. Geotechnique, Vol. 5, No.1, pp. 7-17

Brinch Hansen, J. (1970). A revised and extended formula for bearing capacity. Danish Geotechnical Institute - Bulletin no.98, Copenhagen, pp. 5-11

Budhu, M. \& Al-Karni, A. (1993). Seismic bearing capacity of soils. Geotechnique, Vol.43, No.1, pp. 181-187

Cascone, E.; Maugeri, M. \& Motta, E. (2006). Effetto dell'azione sismica sulla valutazione del fattore $N_{\gamma}$. Proceedings V CNRIG, Bari (Italy), 15 September, 2006, pp. 83-97

Cascone, E.; Carfi, G.; Maugeri, M. \& Motta, E. (2004). Effetto dell'inerzia del terreno sul fattore di capacità portante $N_{\gamma}$. Proceedings IARG 2004, Trento (Italy), 7- 9 July, 2004

Castelli, F. \& Motta, E. (2011). Effetto dell'affondamento sul fattore $N_{\gamma}$ per il calcolo del carico limite di una fondazione superficiale in condizioni sismiche, Proceedings ANIDIS 2011, Bari (Italy), 18-22 September 2011

Castelli, F. \& Motta, E. (2010). Bearing capacity of strip footings near slopes. Geotechnical and Geological Engineering Journal, Vol. 28, No.2, pp. 187-198

Castelli, F. \& Motta, E. (2003). Bearing capacity of shallow foundations resting on a soil layer of limited depth. Proceedings International Symposium on Shallow Foundations, FONDSUP2003, Paris, 5-7 November, 2003, 8 p

De Buhan, P. \& Gaernier, D. (1988). Three dimensional bearing capacity analysis of a foundation near a slope. Soils and Foundations, Vol. 38, No.3, pp. 153-163

Dormieux, L. \& Pecker, A. (1995). Seismic bearing capacity of foundation on cohesionless soil. Journal Geotechnical Engineering, ASCE, Vol.121, No.3, pp. 300-303

EN1998 - 1. Eurocode 8 (2003). Design of structures for earthquake resistance Part 1: General Rules, seismic actions and rules for buildings. CENTC250, Brussels, Belgium 
EN1998 - 5. Eurocode 8 (2003). Design of structures for earthquake resistance Part 5: Foundations, retaining structures and geotechnical aspects. CENTC250, Brussels, Belgium

Fishman, K.L.; Richards, R. \& Yao, D. (2003). Inclination factors for seismic bearing capacity. Journal of Geotechnical and Geoenvironmental, ASCE, Vol. 129, No.9, pp. 861-865

Kumar, J. \& Rao, V.B.K. (2003). Seismic bearing capacity of foundations on slopes. Geotechnique, Vol.53, No.3, pp. 347-361

Hudson, D.E. (1981). The role of Geotechnical Engineering in earthquake problems. Proceedings International Conference on Recent Advances in Geotechnical Earthquake Engineering and Soil Dynamics, St. Louis, MO 1981

Jao, M.; Agrawal, V. \& Wang, W.C. (2001). Performance of strip footings on slopes. Proceedings 15 th ICSMFE, Vol.1, pp. 697-699

Meyerhof, G. G. (1963). Some recent research on the bearing capacity of foundations. Canadian Geotechnical Journal, Vol.1, No.1, pp. 16-26

Moustafa, A. (2011). Damage-based design earthquake loads for single-degree-of-freedom inelastic structures. Journal of Structural Engineering, ASCE, Vol.137, No.3, pp. 456-467

Moustafa, A. \& Takewaki, I. (2010). Deterministic and probabilistic representation of nearfield pulse-like ground motion. Soil Dynamics and Earthquake Engineering, Vol.30, pp. $412-422$

Narita, K. \& Yamaguchi, H. (1990). Bearing capacity analysis of foundations on slopes by use of log-spiral sliding surfaces. Soils and Foundations, Vol. 30, No.3, pp. 144-152

Paolucci, R. \& Pecker, A. (1997). Seismic bearing capacity of shallow strip foundations on dry soils. Soils and Foundations, Vol.37, No.3, pp. 95-105

Park, Y. J.; Ang, A. H.-S., \& Wen, Y. K. (1987). Damage-limiting aseismic design of buildings. Earthquake Spectra, Vol.3, No.1, pp. 1-26

Pecker, A. \& Salencon, J. (1991). Seismic Bearing Capacity of Shallow Strip Foundations on Clay Soils. Proceedings of the International Workshop on Seismology and Earthquake Engineering, pp. 287-304

Pecker, A. (1996). Seismic bearing capacity of shallow foundations. Proceedings XI World Conference on Earthquake Engineering, Acapulco, Mexico, 23-28 June 1996, paper No. 2076

Priestley, M.J.N.; Calvi, G.M. \& Kowalsky, M.J. (2005). Displacement-Based Seismic Design of Structures. IUSS Press, Pavia, Italy

Richards, R.; Elms, D.G. \& Budhu, M. (1993). Seismic Bearing Capacity and Settlement of Foundations. Journal of Geotechnical Engineering Division, ASCE, Vol.119, No.4, pp. 662-674

Saran, S.; Sud, V.K. \& Handa, S.C. (1989). Bearing capacity of footings adjacent to slopes. Journal of Geotechnical Engineering, ASCE, Vol.115, GT4, pp. 553-573

Sarma, S.K. \& Chen, Y.C. (1996). Bearing capacity of strip footing near sloping ground during earthquakes. Proceedings XI World Conference on Earthquake Engineering, Acapulco, Mexico, 23-28 June 1996, paper No.2078

Sarma, S.K. \& Iossifelis, I.S. (1990). Seismic bearing capacity factors of shallow strip footings. Geotechnique, Vol. 40, No.2, pp. 265-273

Shields, D.H.; Chandler, N. \& Garnier, J. (1990). Bearing capacity of foundation in slopes. Journal of Geotechnical Engineering, ASCE, Vol.116, GT3, pp. 528-537

Sawada, T.; Nomachi, S. \& Chen, W. (1994). Seismic bearing capacity of a mounted foundation near a down hill slope by pseudo-static analysis. Soils and Foundations, Vol.34, No.1, pp. 11-17

Terzaghi, K. (1943). Bearing capacity. Theoretical soil mechanics, Chapter 8, NY 1943, 118-143

Vesić, A.S. (1973). Analysis of ultimate loads of shallow foundations. Journal of the Soil Mechanics and Foundations Division, ASCE, Vol. 99, SM1, pp. 45-73 


\title{
Design Principles of Seismic Isolation
}

\author{
George C. Lee and Zach Liang \\ Multidisciplinary Center for Earthquake Engineering Research, \\ University at Buffalo, State University of New York \\ USA
}

\section{Introduction}

In earthquake resistance design of structures, two general concepts have been used. The first is to increase the capacity of the structures to resist the earthquake load effects (mostly horizontal forces) or to increase the dynamic stiffness such as the seismic energy dissipation ability by adding damping systems (both devices and/or structural fuses). The second concept includes seismic isolation systems to reduce the input load effects on structures. Obviously, both concepts can be integrated to achieve an optimal design of earthquake resilient structures. This chapter is focused on the principles of seismic isolation.

It should be pointed out that from the perspective of the structural response control community, earthquake protective systems are generally classified as passive, active and semi-active systems. The passive control area consists of many different categories such as energy dissipation systems, toned-mass systems and vibration isolation systems. This chapter addresses only the passive, seismic isolation systems [Soong and Dargush, 1997; Takewaki, 2009; Liang et al, 2011]

Using seismic isolation devices/systems to control earthquake induced vibration of bridges and buildings is considered to be a relatively matured technology and such devices have been installed in many structures world-wide in recent decades. Design guidelines have been established and they are periodically improved as new information based on research and/or field observations become available during the past 20-30 years [ATC 1995; SEAONC 1986; FEMA 1997; IBC 2000; ECS 2000; AASHTO 2010, ASCE 2007, 2010].

Besides the United States, base isolation technologies are also used in Japan, Italy, New Zealand, China, as well as many other countries and regions. [Naiem and Kelly, 1999; Komodromos, 2000; Christopoulos, C. and Filiatrault 2006]

Affiliated with the increased use of seismic isolation systems, there is an increased demand of various isolation devices manufactured by different vendors. This growth of installing seismic isolation devices in earthquake engineering has been following the typical pattern experienced in structural engineering development, which begins from a "statics" platform by gradually modifying the design approach to include the seismic effects based on structural dynamics principles as they develop and new field observations on the responses of real-world structures. The process is typically slow because most studies and laboratory observations have been concentrated on the performances of the devices with scaled-down experiments. Results could not be readily scaled-up for design purposes. At the same time, there were very limited field data on the actual performances of seismically isolated 
structures. In recent years, some limited successful stories were reported in the literature on the seismic performance of base-isolated bridges and buildings during real earthquakes, as well as reports of unsuccessful cases including the failure of isolation bearings and falling spans of bridges and magnification (rather than reduction) of vibration levels of buildings. These structural failures have not been systematically examined for their contributing factors. Some of them include: construction quality, improper choice of the type of isolation bearings, incomplete design principles and methods, unknown ground motion and soil characteristics, etc. In summary, current practice is mainly based on past research and observations on the performance of the isolation devices themselves, with minimum information on the dynamic performance of the structure-device as a system.

The working principle of seismic isolation may be explained in several ways. It is a general understanding that isolation devices/systems are used to reduce the seismic force introduced base shear. Designers often understand the working principles from the viewpoint of design spectrum in that, when the vibration period of a structure is longer than a certain level, continue to increase the period will reduce the magnitude of the spectral value and thus reduce the base shear accordingly. To qualitatively explain the working principle of seismic isolation in this manner is reasonable, but it is insufficient to use it in actual design. Refinements and additional design principles are necessary. Another commonly used explanation of seismic isolation is the "decoupling" of the superstructure vibration from the ground motions excitations to reduce the vibration of structures. This statement again requires quantitative elaborations from the viewpoint of isolation design. In general, an isolation device/system can be viewed as a low-pass mechanical filter of the structure being isolated, to filter out excitations with the undesirable high frequencies to reduce the level of acceleration. In order to establish the cut-off frequency the period of the isolation system must be carefully addressed, and this requires a basic design principle to guide the design.

In order to reduce the base shear, an isolation system must be allowed to deform. This relative displacement cannot be filtered like the absolute acceleration. In general working range, the longer the relative displacement associated with longer periods, the more reduction in base shear can be achieved, except for the fact that the latter will introduce certain negative effects. The most significant issue of large relative displacement is the large P-delta effect and for falling spans in bridges. In this regard, a design principle is needed to achieve the best compromise in seismic isolation design. In reality, the only approach to effectively reduce the relative displacement is to increase damping, which, in turn, will result in higher level of acceleration. This conflicting demand of controlling acceleration and acceptable displacement in essence defines the limiting range of the effectiveness of seismic isolation systems. Quantitatively this issue can be addressed, and this is an important design principle to be conceptually discussed in this chapter.

In Section 2 of this chapter, several important design issues (e.g. P-delta effect, vertical motions, etc.) will be discussed and seismic isolation design principles will be described. In Section 3, the quantitative basis of treating seismically-isolated structures will be briefly reviewed and simplified models will be established for the dynamic analysis and design of the structure-device system. Design methods will be briefly discussed in Section 4, and a newly developed seismic isolation device to address some of the issues facing today's practice is briefly introduced in Section 5. Finally, the key issues and parameters in seismic isolation is summarized and future research needs are briefly noted. 


\section{Some issues and principles of seismic isolation}

In this section, the theories, design and practical considerations of seismic isolation are briefly discussed.

\subsection{State-of-practice on seismic isolation}

The principle of base isolation is typically conceptually explained by using figure 2.1.

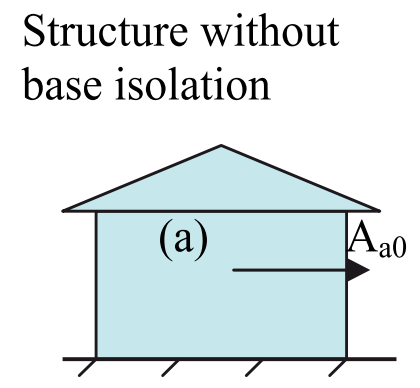

Ground

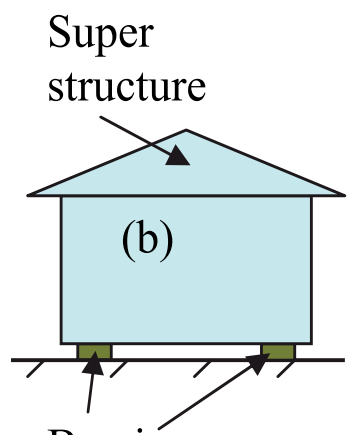

Bearings

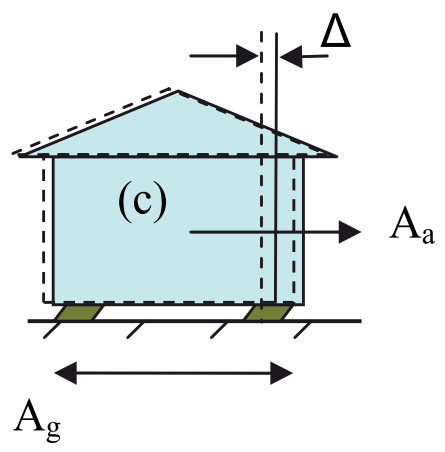

Fig. 2.1. Concept of base isolation

Figure 2.1 (a) and (b) show a structure without and with base isolation, respectively. It is seen that the major difference between (a) and (b) is that in (b), the structure is set on top of isolation several bearings. When the ground moves with acceleration $\mathrm{A}_{\mathrm{g}}$, the superstructure will move accordingly with a displacement $\Delta$, and a reduced level of absolute acceleration, denoted by $A_{a}$, rather than the original level $A_{a 0}$.

This principle has been the basis for many research and development efforts in design guidelines and devices for seismic isolation. These have been many important contributions during the past 25 years led by Kelly and his associates at Berkeley and Constantinou and his associates at Buffalo as well as many others. (See references listed at the end of this chapter.)

\subsection{Basic concept}

The major purpose of using the seismic isolation is to reduce the base-shear of the structure. Physically, large base shear is one of the main reasons of structural damages due to strong horizontal ground accelerations. Thus, to reduce the lateral acceleration is a basic principle.

From the viewpoint of design, many aseismic codes use the base shear as a control parameter. For example, if the base shear of a building is reduced, then the upper story lateral forces floor drifts are also reduced. In the case of a bridge, base shear reduction will minimize damage to the piers.

\subsubsection{Base shear}

Base shear $\mathrm{V}$ can be calculated through various approaches. The following are several examples, first

$$
\mathrm{V}=\mathrm{C}_{\mathrm{s}} \mathrm{W}
$$


where $C_{s}$ is the seismic response factor and $W$ is the total weight of a structure. Base isolation is intended for reducing $\mathrm{C}_{\mathrm{s}}$, second

$$
\mathrm{V}=\sum \mathrm{f}_{\mathrm{Lj}}(\mathrm{kN})
$$

where $f_{L j}$ is the later force of the $j^{\text {th }}$ story of the structure. Base isolation is intended to reduce $\mathrm{f}_{\mathrm{Lj}}$ simultaneously, so that the base shear will be reduced, in addition

$$
\mathrm{V}=\mathrm{K}_{\mathrm{b}} \Delta(\mathrm{kN})
$$

where $K_{b}$ is the lateral stiffness of the bearing system; $\Delta$ is the nominal relative displacement of the bearing. The stiffness $\mathrm{K}_{\mathrm{b}}$ of the bearing system will be much smaller than the structure without the bearing, so that the base shear is reduced.

\subsubsection{Lateral acceleration}

In equation (2.1), $C_{s}$ is in fact the normalized lateral absolute acceleration $A_{a}$, which is in general zero-valued unless earthquake occurs.

$$
\mathrm{C}_{\mathrm{s}}=\mathrm{A}_{\mathrm{a}} / \mathrm{g}
$$

Also note that

$$
\mathrm{W}=\mathrm{Mg}(\mathrm{kN})
$$

where $\mathrm{M}$ is the total mass and $\mathrm{g}=9.8 \mathrm{~m} / \mathrm{s}^{2}$ is the gravity.

In equation (2.2), $\mathrm{f}_{\mathrm{Lj}}$ is also caused by lateral absolute acceleration $\mathrm{a}_{\mathrm{aj}}$ of the $\mathrm{j}^{\text {th }}$ story, that is

$$
\mathrm{f}_{\mathrm{Lj}}=\mathrm{m}_{\mathrm{j}} \mathrm{a}_{\mathrm{aj}}(\mathrm{kN})
$$

where $m_{j}$ is the mass of the $j^{\text {th }}$ floor.

From (2.5) and (2.6), it is seen that, it is difficult to change or reduce the mass $\mathrm{M}$ or $\mathrm{m}_{\mathrm{j}}$ in a design; however, if the acceleration can be reduced, the lateral forces will be reduced. Therefore, we will focus the discussion on the acceleration.

\subsection{Issues of base isolation}

Seismic isolation is considered as a relatively matured technology as evidenced by the many practical applications. These applications have been designed based on codes and provisions that have been established incrementally over time. In the following, seismic isolation principles are examined from a structural dynamics perspective with an objective to suggest additional future research needs.

\subsubsection{Absolute acceleration vs. relative displacement}

Seen in figure 2.1, to achieve the goal of acceleration reduction, in between the ground and the super structure, there will be installed in a group of bearings, which have much soft stiffness so that the period of the total system will be elongated. 
Thus, to achieve acceleration reduction, a major sacrifice is the relative displacement between base and structure must be significantly large. Due to nonlinearities of isolation system, the dynamic displacement can be multiple-centered, which can further notably enlarge the displacement. In addition, permanent residual deformation of bearing may worsen the situation.

Generally speaking, the simplest model of a base isolation system can be expressed as

$$
M \mathrm{a}_{\mathrm{a}}(\mathrm{t})+\mathrm{Cv}(\mathrm{t})+\mathrm{K}_{\mathrm{b}} \mathrm{d}(\mathrm{t})=0
$$

where $\mathrm{C} v(\mathrm{t})$ is the viscous damping force and $\mathrm{C}$ is the damping coefficient; $\mathrm{v}(\mathrm{t})$ is relative velocity.

In most civil engineering structures, the damping force is very small, that is

$$
\mathrm{C} v(\mathrm{t}) \approx 0
$$

Thus, (2.7) can be re-written as

$$
\mathrm{MA}_{\mathrm{a}} \approx \mathrm{K}_{\mathrm{b}} \Delta
$$

where $A_{a}$ and $\Delta$ are amplitudes of the absolute acceleration $\mathrm{a}_{a}(\mathrm{t})$ and relative displacement $\mathrm{d}(\mathrm{t})$. Equation (2.9) describes the relationship between acceleration and displacement of a singledegree-of-freedom (SDOF) system, which can be used to generate the design spectra. Since the damping force is omitted, the generated acceleration is not exactly real, which is referred to as pseudo acceleration, denoted by $A_{s}$. Thus, (2.9) is rewritten as

$$
\mathrm{MA}_{\mathrm{s}}=\mathrm{K}_{\mathrm{b}} \Delta
$$

Furthermore, we have

$$
\mathrm{A}_{\mathrm{s}}=\omega_{\mathrm{b}}^{2} \Delta
$$

where $\omega_{\mathrm{b}}$ is the angular natural frequency of the isolation system

$$
\omega_{\mathrm{b}}=\sqrt{\mathrm{K}_{\mathrm{b}} / \mathrm{M}}(\mathrm{rad} / \mathrm{s})
$$

Since the natural period $\mathrm{T}_{\mathrm{b}}$ is

$$
\mathrm{T}_{\mathrm{b}}=2 \pi / \omega_{\mathrm{b}}
$$

Equation (2.11) can be rewritten as

$$
\mathrm{A}_{\mathrm{s}}=4 \pi^{2} / \mathrm{T}_{\mathrm{b}}^{2} \Delta
$$

From (2.14), the acceleration $\mathrm{A}_{\mathrm{s}}$ and displacement $\Delta$ are proportional, that is

$$
\mathrm{A}_{\mathrm{s}} \propto \Delta
$$

Since $\mathrm{A}_{\mathrm{s}}$ and $\Delta$ are deterministic functions, (2.15) indicates that between $\mathrm{A}_{\mathrm{s}}$ and $\Delta$, only one parameter is needed, usually, the acceleration is considered.

Combine (2.12), (2.13) and (2.14), it is also seen that $A_{s}$ is proportional to the stiffness $K_{b}$, that is 


$$
\mathrm{A}_{\mathrm{s}} \propto \mathrm{K}_{\mathrm{b}}
$$

That is, the weaker the stiffness is chosen, the smaller value of $A_{s}$ can be achieved, which is the basis for current design practice of seismic isolation.

From the above discussion, it seems that, as long as $\mathrm{K}_{\mathrm{b}}$ is smaller than a certain level, the base isolation would be successful.

However, the above mentioned design principle may have several problems if the assumptions and limitations are not examined. First, from (2.10), it is seen that, only if $\Delta$ is fixed, (2.16) holds. On the other hand, if $\mathrm{V}$, that is $\mathrm{A}_{\mathrm{a}}$, is fixed, one can have

$$
\Delta \propto 1 / \mathrm{K}_{\mathrm{b}}
$$

That is, the weaker the stiffness is chosen, the larger value of $\Delta$ can result.

A more accurate model will unveil that, to realize the base isolation, only one parameter, say $\mathrm{A}_{\mathrm{a}}$ or $\Delta$, is not sufficient. This is because $\mathrm{A}_{\mathrm{a}}$ and $\Delta$ are actually independent. In fact, both of them are needed. That is, whenever a claim of the displacement being considered in an isolation design, as long as they are not treated as two independent parameters, the design is questionable. Later, why they must be independent will be explained. Here, let us first use certain group of seismic ground motions as excitations applied on a SDOF system, the ground motion are suggested by Naiem and Kelly (1999) and normalized to have peak ground acceleration (PGA) to be $0.4(\mathrm{~g})$. The responses are mean plus one standard deviation values, plotted in figure 2.2.

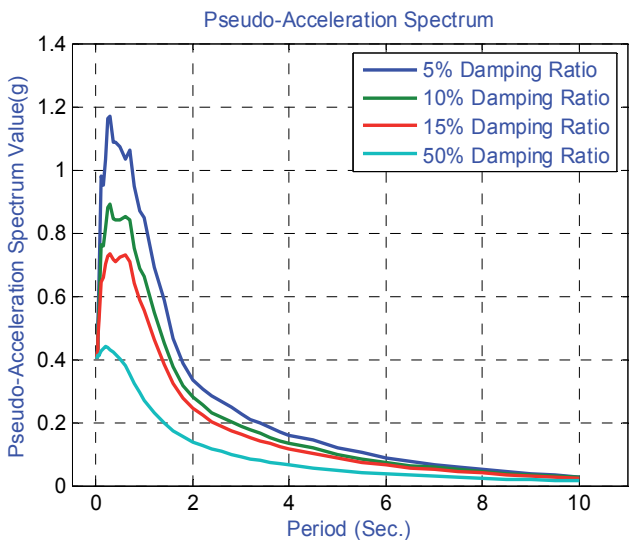

(a)

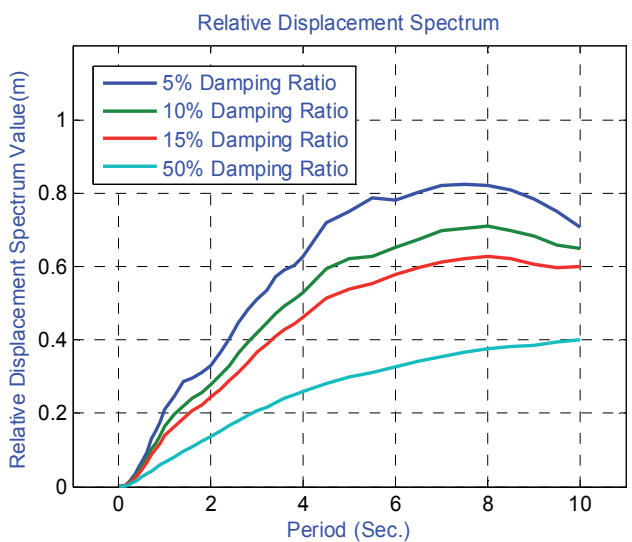

(b)

Fig. 2.2. Statistical seismic responses of SDOF systems

Figure 2.2 (a) shows the accelerations of SDOF systems as functions of period for selected damping ratios. When the period becomes larger, the accelerations do reduce, especially when $\mathrm{T}_{\mathrm{b}}>2(\mathrm{~s})$. The responses are all smaller than $0.4(\mathrm{~g})$ and namely, the acceleration is reduced.

Figure 2.2 (b) shows the displacements. It is seen that, when the periods increase, the displacements can become rather large. When $\mathrm{T}_{\mathrm{b}}>2(\mathrm{~s})$, the responses can be larger than 0.1 (m), especially if the damping ratio is small, say, $5 \%$. 
In figure 2.2 the parameter, damping ratio denoted by $\xi$ is defined by

$$
\xi=\mathrm{C} / 2 \sqrt{\mathrm{MK}_{\mathrm{b}}}
$$

\subsubsection{Displacement and center position}

Another seismic isolation design issue is the self-centering capacity. Because the SDOF system used to generate the response is linear, and many commercially available bearings are nonlinear systems, the displacement time history can be multiple centered. Figure 2.3 shows examples of a bi-linear (nonlinear) system under Northridge earthquake excitations. The plot in Fig. 2.3 (a) is the displacement time history of the bi-linear system with load and unload stiffness ratio $=0.1$ and damping ratio $=0.01$. Here the damping ratio is calculated when the system is linear. The plot in Fig 2.3 (b) is the same system with damping ratio $=0.2$. It is seen that, the biased deformations exist in both cases. This example illustrates that center shifting can enlarge the displacement significantly, even with heavy damping.

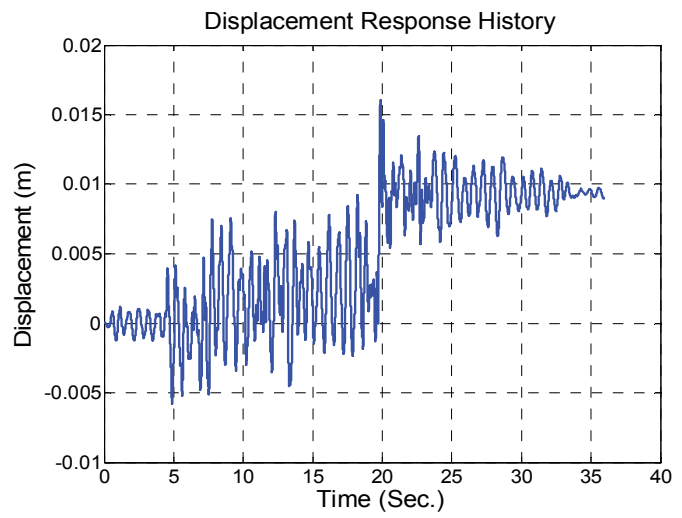

(a) small damping

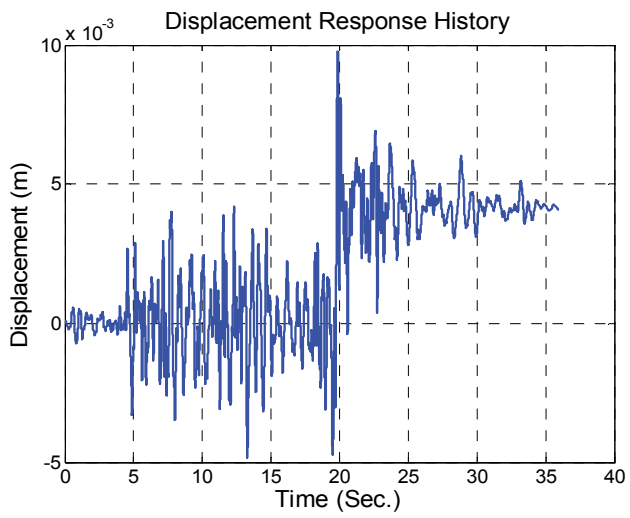

(b) large damping

Fig. 2.3. Multiple centers of displacement responses

Briefly speaking, the current isolation design practice can be based on the spectral analysis, dealing with linear systems with the seismic response coefficient $C_{s}$ given by

$$
\mathrm{C}_{\mathrm{s}}=\mathrm{A}_{\mathrm{s}} / \mathrm{g}=\frac{\mathrm{AS}}{\mathrm{T}_{\mathrm{b}} \mathrm{B}}
$$

and the spectral displacement $d_{D}$ given by

$$
\mathrm{d}_{\mathrm{D}}=\mathrm{D}=\mathrm{C}_{\mathrm{s}} \mathrm{T}_{\mathrm{b}}^{2} / 4 \pi^{2} \mathrm{~g}=\frac{\mathrm{AST}_{\mathrm{b}}}{4 \mathrm{~B}}(\mathrm{~m})
$$

In the above equations, $A$ is the input level of ground acceleration; $S$ is the site factor; $D$, instead of $\Delta$, is used to denote the dynamic amplitude and $\mathrm{B}$ is called the numerical damping coefficient. Approximately

$$
\mathrm{B}=3 \xi+0.9
$$


Equations (2.19) and (2.20) will work for most (but not all) situations but cannot handle the position shifting which is a nonlinear response. Some of the nonlinear modeling issues are discussed in Section 3.

\subsubsection{SDOF and MDOF models}

Many analyses and design of seismic isolations are based on SDOF model, whereas realistic structures are mostly MDOF systems. The acceleration of a higher story of an MDOF system can be much more difficult to reduce.

Equation (2.15) is based on a SDOF system, when the superstructure can be treated as a lumped mass. Namely, the relative deformation among different stories of the structure is negligible. Realistically, such a case is rare; therefore, the acceleration $\mathrm{a}_{\mathrm{aj}}$ at different stories can be different.

A conventional idea is to decouple a MDOF system into several vibration modes. Each mode is treated as a SDOF system. The total response of the MDOF can then obtained through certain method of modal combinations, such as SRSS method. That is, (2.19) can be rewritten as

$$
\mathrm{C}_{\mathrm{si}}=\frac{\mathrm{AS}}{\mathrm{T}_{\mathrm{bi}} \mathrm{B}_{\mathrm{i}}}
$$

where the subscript $i$ stands for the $i^{\text {th }}$ mode. And the $i^{\text {th }}$ spectral displacement $d_{i D},(2.20)$, is rewritten as:

$$
\mathrm{d}_{\mathrm{iD}}=\mathrm{C}_{\mathrm{si}} \mathrm{T}_{\mathrm{bi}}^{2} / 4 \pi^{2} \mathrm{~g}=\frac{\mathrm{AST}_{\mathrm{bi}}}{4 \mathrm{~B}_{\mathrm{i}}}(\mathrm{m})
$$

where

$$
\mathrm{B}_{\mathrm{i}}=3 \xi_{\mathrm{i}}+0.9
$$

Furthermore, for multi-story structures, an additional parameter, mode shape, is needed to distribute the acceleration and displacement at different levels. By denoting the mode shape by $\mathbf{P}_{\mathrm{i}}$, which is a vector with the jth element representing the model displacement $p_{j i}$. The acceleration vector $\mathbf{A}_{\mathrm{si}}$ is given as

$$
\mathbf{A}_{\mathrm{si}}=\Gamma_{\mathrm{i}} \mathrm{C}_{\mathrm{si}} \mathbf{P}_{\mathrm{i}} \mathrm{g}=\left\{\mathrm{a}_{\mathrm{ji}}\right\}\left(\mathrm{m} / \mathrm{s}^{2}\right)
$$

where $a_{\mathrm{ji}}$ is the acceleration of the $\mathrm{i}^{\text {th }}$ mode at the $\mathrm{j}^{\text {th }}$ story and $\Gamma_{\mathrm{i}}$ is the $\mathrm{i}^{\text {th }}$ modal participation factor.

The displacement vector is given by

$$
\mathbf{d}_{\mathrm{si}}=\Gamma_{\mathrm{i}} \mathrm{d}_{\mathrm{iD}} \mathbf{P}_{\mathrm{i}}=\left\{\mathrm{d}_{\mathrm{ji}}\right\}(\mathrm{m})
$$

where $\mathrm{d}_{\mathrm{ji}}$ is the displacement of the $\mathrm{i}^{\text {th }}$ mode at the $\mathrm{j}^{\text {th }}$ story. 


\subsubsection{Acceleration of higher stories}

One of the shortcomings of approximately a MDOF system by a SDOF model is the inability to estimate the responses at the higher levels of a MDOF structure. Typically, through SRSS, the acceleration of the $j^{\text {th }}$ story of MDOF system can be calculated as

$$
\mathrm{a}_{\mathrm{sj}}=\sqrt{\sum_{\mathrm{i}} \mathrm{a}_{\mathrm{ij}}^{2}}\left(\mathrm{~m} / \mathrm{s}^{2}\right)
$$

And the displacement of the $\mathrm{j}^{\text {th }}$ story is

$$
\mathrm{d}_{\mathrm{j}}=\sqrt{\sum_{\mathrm{i}} \mathrm{d}_{\mathrm{ji}}^{2}}(\mathrm{~m})
$$

The reduction of MDOF accelerations is discussed in the literature. The general conclusion is that, (2.25) and (2.27) may not work well so that the acceleration of the higher story $\mathrm{a}_{\mathrm{sj}}$ may not be calculated correctly. In fact, $\mathrm{a}_{\mathrm{sj}}$ can be significantly larger.

The reasons of this inaccuracy mainly come from several factors. The first is the damping effect. The second is the error introduced by using only the first mode for design simplicity (the triangular shape function) as illustrated in figure 2.4
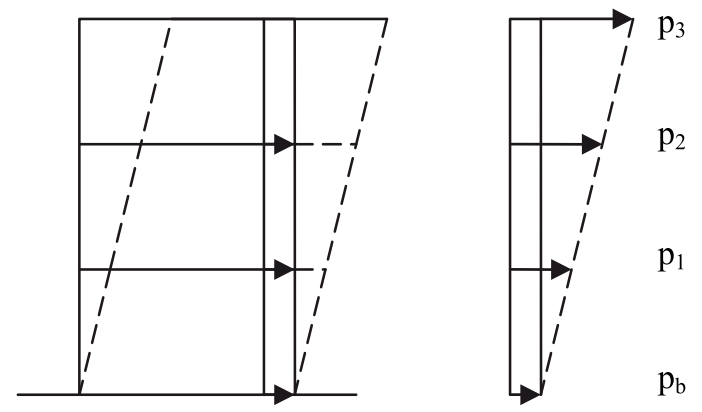

Fig. 2.4. Mode shape function of the $\mathrm{l}^{\text {st }}$ mode

In addition, seen from figure 2.4, even through the acceleration of the base, denoted by $\mathrm{p}_{\mathrm{b}}$ is rather small by using isolators, the top story will have a rather large acceleration.

\subsubsection{Cross effects}

Another shortcoming of using SDOF models is the inability to estimate the cross effect. Typical MDOF structures have "cross effects" in their dynamic responses. Different from a single member of a structure, which has principal axes, a three-dimensional structure often does not have principal axes. This is conceptually shown in figure 2.5 , which is a two story structure. Suppose the first story does have its own principal axes, marked as $\mathrm{x}_{1}-\mathrm{y}_{1}$, and the second story also has its own principal axes, marked as $\mathrm{x}_{2} \mathrm{y}_{2}$. However, from the top view, if $x_{1}$ and $x_{2}$ are not pointing exactly the same direction, say, there exists an angle $\theta$, then the entire structure will not have principal axes in general. In this case, the inputs from any two perpendicular directions will cause mutual responses. The resulted displacement will be further magnified. This the third reason of large displacement. At present, there are no available methods to quantify cross effects associated with seismic responses, although in general this effect in base isolation design may be small for regularly shaped structures. 

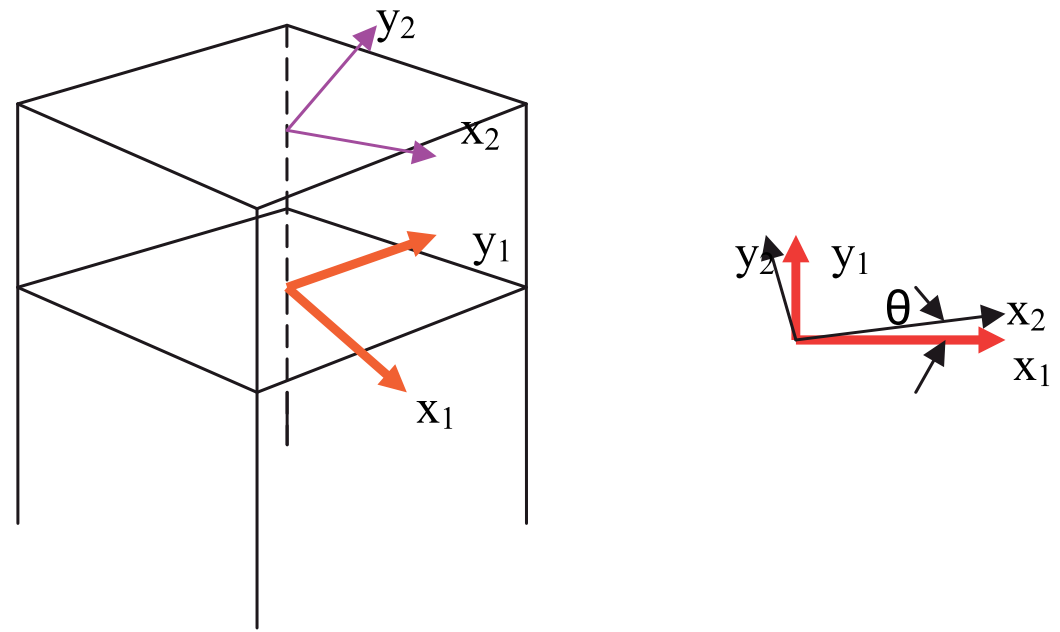

Fig. 2.5. Cross effect

\subsubsection{Overturning moment}

The product of large vertical load and large bearing displacement forms a large P- $\Delta$ effect, conceptually shown in figure 2.6 .

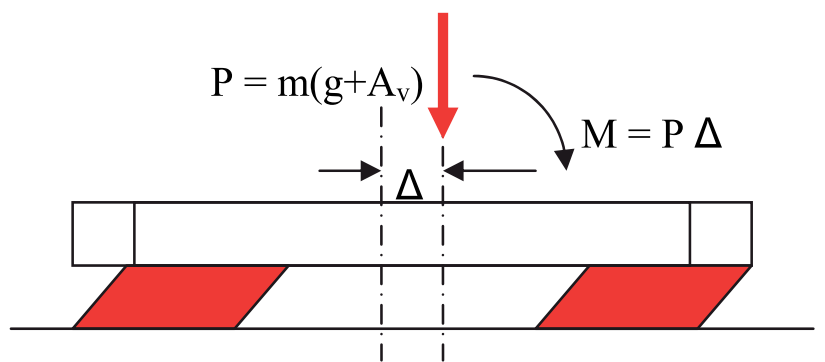

Fig. 2.6. Isolation $P-\Delta$ effect

This large displacement will result in an overturning moment, given by

$$
\mathrm{M}=\mathrm{P} \Delta=\mathrm{m}\left(\mathrm{g}+\mathrm{A}_{\mathrm{v}}\right) \Delta
$$

where, the vertical load $\mathrm{P}$ is a product of the total weight and the vertical acceleration, the vertical acceleration is the sum of gravity $g$ and earthquake induced acceleration $A_{v}$. For example, suppose the total mass is 1000 (ton), the displacement is 0.5 (m), and the additional vertical acceleration is $0.4(\mathrm{~g})$, the total overturning moment will be $6.86(\mathrm{MN}-\mathrm{m})$. This is a large magnitude, which requires special consideration in the design of foundations, structure base as well as bearings.

\subsubsection{Horizontal and vertical vibrations}

As mentioned above, the primary purpose of base isolation is to reduce the horizontal load and/or acceleration. By installing bearings, the lateral stiffness will be significantly reduced so the horizontal vibration can be suppressed. Moreover, by using bearings, the vertical 
stiffness can also be reduced to a certain level and the vertical vibration can be magnified. Since the earthquake induced vertical load is often not significantly large, the vertical vibration is often ignored in design. However, due to the magnification of vertical acceleration as well as the above-mentioned large overturning moment, care must be taken to check the vertical load. In the worst scenario, there can be an uplift force acting on bearings with many of them not manufactured to take the uplift load (e.g. rubber bearings).

\section{Dynamics of seismically isolated MDOF systems}

In this section, base isolation is examined from a different perspective, as a second order mechanical filter of a dynamic system. The working principle of base isolation system is to increase the dynamic stiffness of acceleration without sacrifice too much "dynamic stiffness." Dynamic stiffness is a function of effective period and damping.

\subsection{Models of isolation systems}

\subsubsection{Linear SDOF model}

The linear SDOF model is used here to provide a platform to explain the essence of isolation systems under sinusoidal excitation.

A more detailed base isolation model can be rewritten as

$$
\mathrm{Ma}(\mathrm{t})+\mathrm{Cv}(\mathrm{t})+\mathrm{K}_{\mathrm{b}} \mathrm{d}(\mathrm{t})=-\mathrm{M} \mathrm{a}_{\mathrm{g}}(\mathrm{t})
$$

where $\mathrm{a}(\mathrm{t})$ and $\mathrm{ag}_{\mathrm{g}}(\mathrm{t})$ are respectively the relative and ground accelerations. Note that

$$
\begin{aligned}
& \mathrm{a}_{\mathrm{a}}(\mathrm{t})=\mathrm{a}(\mathrm{t})+\mathrm{a}_{\mathrm{g}}(\mathrm{t}) \\
& \mathrm{a}(\mathrm{t})=\dot{\mathrm{v}}(\mathrm{t})=\ddot{\mathrm{d}}(\mathrm{t})
\end{aligned}
$$

where the overhead dot and double dot stand for the first and the second derivatives with respect to time $t$.

Let the ground displacement $\mathrm{d}_{\mathrm{g}}(\mathrm{t})$ be sinusoidal with driving frequency $\omega_{\mathrm{f}}$,

$$
d_{g}(t)=D \cos \left(\omega_{f} t\right)
$$

The ground acceleration is

$$
a_{g}(t)=A_{g} \cos \left(\omega_{f} t\right)=-D_{g} \omega_{f}^{2} \cos \left(\omega_{f} t\right)
$$

Here, $\mathrm{D}_{\mathrm{g}}$ and $\mathrm{A}_{\mathrm{g}}$ are respectively the amplitudes of the displacement and acceleration. The amplitude of steady state responses of the relative displacement $\mathrm{D}$ can be written as

$$
\mathrm{D}=\frac{\mathrm{D}_{\mathrm{g}} \frac{\omega_{\mathrm{f}}^{2}}{\omega_{\mathrm{b}}^{2}}}{\sqrt{\left(1-\frac{\omega_{\mathrm{f}}^{2}}{\omega_{\mathrm{b}}^{2}}\right)^{2}+\left(2 \xi \frac{\omega_{\mathrm{f}}}{\omega_{\mathrm{b}}}\right)^{2}}}=\frac{\mathrm{r}^{2}}{\sqrt{\left(1-\mathrm{r}^{2}\right)^{2}+(2 \xi \mathrm{r})^{2}}} \mathrm{D}_{\mathrm{g}}=\beta_{\mathrm{d}} \mathrm{D}_{\mathrm{g}}
$$


Here $\mathrm{r}$ is the frequency ratio

$$
r=\frac{\omega_{f}}{\omega_{b}}
$$

and $\beta_{\mathrm{d}}$ is the dynamic magnification factor for the relative displacement

$$
\beta_{\mathrm{d}}=\frac{\mathrm{r}^{2}}{\sqrt{\left(1-\mathrm{r}^{2}\right)^{2}+(2 \xi r)^{2}}}
$$

Note that $\mathrm{r}=0$ means the driving frequency $\omega_{\mathrm{f}}=0$ and the system is excited by static force only. In this case, $\mathrm{D}(\mathrm{r}=0)=0$.

The amplitude of steady state responses of the absolute acceleration $A_{a}$ can be written as

$$
A_{a}=\sqrt{\frac{1+(2 \xi r)^{2}}{\left(1-r^{2}\right)^{2}+(2 \xi r)^{2}}} A_{g}=\beta_{A} A_{g}
$$

and $\beta_{\mathrm{A}}$ is the dynamic magnification factor for the relative displacement

$$
\beta_{\mathrm{A}}=\sqrt{\frac{1+(2 \xi \mathrm{r})^{2}}{\left(1-\mathrm{r}^{2}\right)^{2}+(2 \xi \mathrm{r})^{2}}}
$$

Note that when the driving frequency $\omega_{\mathrm{f}}=0$ and the system is excited by static force only. $\mathrm{A}_{\mathrm{a}}(\mathrm{r}=0)=\mathrm{A}_{\mathrm{g}}$. If there exist a static force $\mathrm{F}_{\mathrm{SA}}$ so that

$$
\mathrm{A}_{\mathrm{g}}=\frac{\mathrm{F}_{\mathrm{SA}}}{\mathrm{M}}
$$

Then, when $r \neq 0$ or $\omega_{\mathrm{f}} \neq 0$, the dynamic response of the acceleration can be seen as

$$
\mathrm{A}_{\mathrm{a}}=\frac{\mathrm{F}_{\mathrm{SA}}}{\mathrm{M}} \beta_{\mathrm{A}}=\frac{\mathrm{F}_{\mathrm{SA}}}{\mathrm{M} / \beta_{\mathrm{A}}}=\frac{\mathrm{F}_{\mathrm{SA}}}{\mathrm{M}_{\beta}}
$$

where $\mathrm{M}_{\beta}$ is called apparent mass or dynamic mass and

$$
\mathrm{M}_{\beta}=\mathrm{M} / \beta_{\mathrm{A}}
$$

That is, the value of the dynamic response $A_{a}$ can be seen as a static force divided by a dynamic mass. From (3.12),

$$
\mathrm{M}_{\beta}=\frac{\mathrm{F}_{\mathrm{SA}}}{\mathrm{A}_{\mathrm{a}}}
$$

The essence described by (3.14) is that the "dynamic mass" equals to a force divided by response. Generally speaking, this term is stiffness. Since the response is dynamic, it can be 
called a dynamic stiffness, $\mathrm{K}_{\mathrm{dA}}$. In this particular case, the dynamic stiffness is the apparent mass.

$$
\mathrm{F}_{\mathrm{SA}}=\mathrm{K}_{\mathrm{dA}} \mathrm{A}_{\mathrm{a}}
$$

Similarly, the relationship between a static force $\mathrm{F}_{\mathrm{Sd}}$ and a dynamic displacement $\mathrm{D}$ can be written as

$$
\mathrm{F}_{\mathrm{Sd}}=\mathrm{K}_{\mathrm{dD}} \mathrm{D}
$$

It can be seen that

$$
\mathrm{K}_{\mathrm{dA}} \propto \frac{1}{\beta_{\mathrm{A}}}
$$

And

$$
\mathrm{K}_{\mathrm{dD}} \propto \frac{1}{\beta_{\mathrm{b}}}
$$

Both the dynamic stiffness and the dynamic factors are functions of frequency ratio $r$. It can be proven that, the proportional coefficients for (3.17) and (3.18) are constant with respect to $r$. That is, we can use the plots of dynamic magnification factors to shown the variations of the dynamic stiffness with respect to r. Figure 3.1(a) and (b) show examples of $\beta_{\mathrm{A}}$ and $\beta_{\mathrm{b}}$. It can be seen from figure 3.1(a), the plot of $\beta_{\mathrm{A}}$, when the frequency ratio varies, the amplitude will vary accordingly. Note that,

$$
r=\frac{\omega_{f}}{\omega_{b}}=\frac{\omega_{f}}{2 \Pi} T_{b}
$$

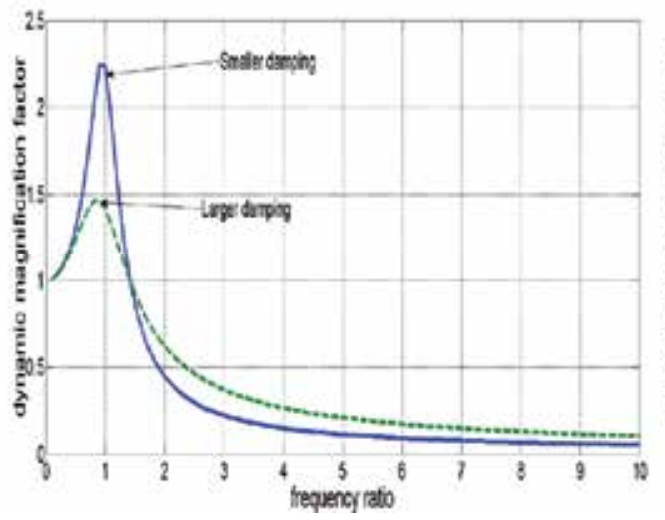

(a) $\beta_{\mathrm{A}}$

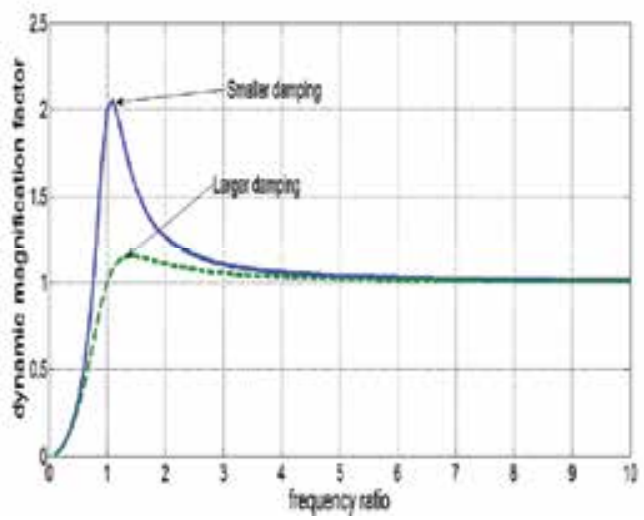

(b) $\beta_{\mathrm{b}}$

Fig. 3.1. Dynamic magnification factors 
That is, if the driving frequency is given, then the frequency ratio $r$ is proportional to the natural period $\mathrm{T}_{\mathrm{b}}$. Therefore, the horizontal plot can be regarded as the varying period. Recall figure 2.2(a) where $\mathrm{x}$-axis is also period. We can realize the similarity of these two plots. First, when the period increases, the amplitudes in both plot increases. After a certain level, these amplitudes start to decrease. It is understandable that, the target of acceleration reduction should be in the region when the accelerations being smaller than a certain level. In figure 3.1 (a), we can clearly realize that this region starts at $r=1.414$, which is called rule 1.4. To let the isolation system start to work, the natural period should be at least 1.4 times larger than the driving period $\mathrm{T}_{\mathrm{f}}=2 \pi / \omega_{\mathrm{f}}$. In the case of earthquake excitation, due to the randomness of ground motions, there is no clear-cut number. However, from figure 2.2 (a), it seems that this number should be even larger than 1.4. In fact, with both numerical simulations and shaking table tests, for linear and nonlinear as well as SDOF and MDOF systems, much higher values of the frequency ratio have been observed by the authors. In general, this limiting ratio should be indeed greater than 1.4.

$$
r>1.4
$$

Additionally, different damping will result in different response level. In figure 3.1(a), it is seen that, when $r>1.4$, larger damping makes the reduction less effective. In figure 2.2 (a), it can be seen that when the period becomes longer, increase damping still further reduce the responses, but the effectiveness is greatly decreased. Note that, this plot is based on statistical results. For many ground excitations, this phenomenon is not always true. That is, increasing damping can indeed reduce the effectiveness of reduction.

Now, consider the displacement. From figure 3.1(b), when the period is near zero, the relative displacement is rather small. Then, as the period increases, the displacement will reach the peak level and then slowly decreases. However, it will never be lower than 1 , which means that, the displacement will never below the level of ground displacement. Increasing the damping, however, will help to reduce the large displacement. This may be seen from Fig. 2.2(b) and it is true for both sinusoidal and earthquake excitations.

\subsubsection{Nonlinear SDOF model}

The above discussion is based on a linear model. Nonlinear system will have certain differences and can be much more complex to analyze. Generally speaking, many nonlinear systems considerably worsen the problem of large displacement. In the working region, increasing the period will decrease the acceleration but the displacement will remain at a high level. Increasing the damping will help to reduce the displacement but does not help the acceleration.

\subsubsection{Effective system}

It is well understood that, in general, a nonlinear dynamic system does not have a fixed period or damping ratio. However, in most engineering applications, the effective period $\mathrm{T}_{\text {eff }}$ and damping ratio $\xi_{\text {eff }}$ are needed, and are calculated from

$$
\mathrm{T}_{\text {eff }}=\frac{2 \pi}{\sqrt{\mathrm{M} / \mathrm{K}_{\mathrm{eff}}}}
$$


And

$$
\xi_{\text {eff }}=\frac{E_{d}}{2 \pi \mathrm{K}_{\text {eff }} \mathrm{D}^{2}}
$$

where $E_{d}$ is the energy dissipation of the nonlinear system during a full vibration cycle.

From (3.21) and (3.22), it is seen that, to have the effective values, the key issue is to establish the effective stiffness $\mathrm{K}_{\text {eff. }}$

In the following, the bi-linear system is used to discuss the effective stiffness, for the sake of simplicity. Although this system is only a portion of the entire nonlinear systems, the basic idea of conservative and dissipative forces seen in the bilinear system can be extended to general nonlinear systems. And, in many cases, nonlinear isolation is indeed modeled as a bi-linear system. Figure 3.2 shows (a) the elastic-perfectly-plastic (EEP) system and (b) the general bi-linear system. Conventionally, the secant 'stiffness" is taken to be the effective stiffness. That is

$$
\mathrm{K}_{\text {eff }}=\mathrm{K}_{\mathrm{sec}}=\frac{\mathrm{f}_{\mathrm{N}}}{\mathrm{D}}
$$

where $D$ is the maximum displacement and $f_{N}$ is the maximum nonlinear force. In the following, since the system is nonlinear and the response is random, we use lower case letters to denote the responses in general situations, unless these responses will indeed reach their peak value.
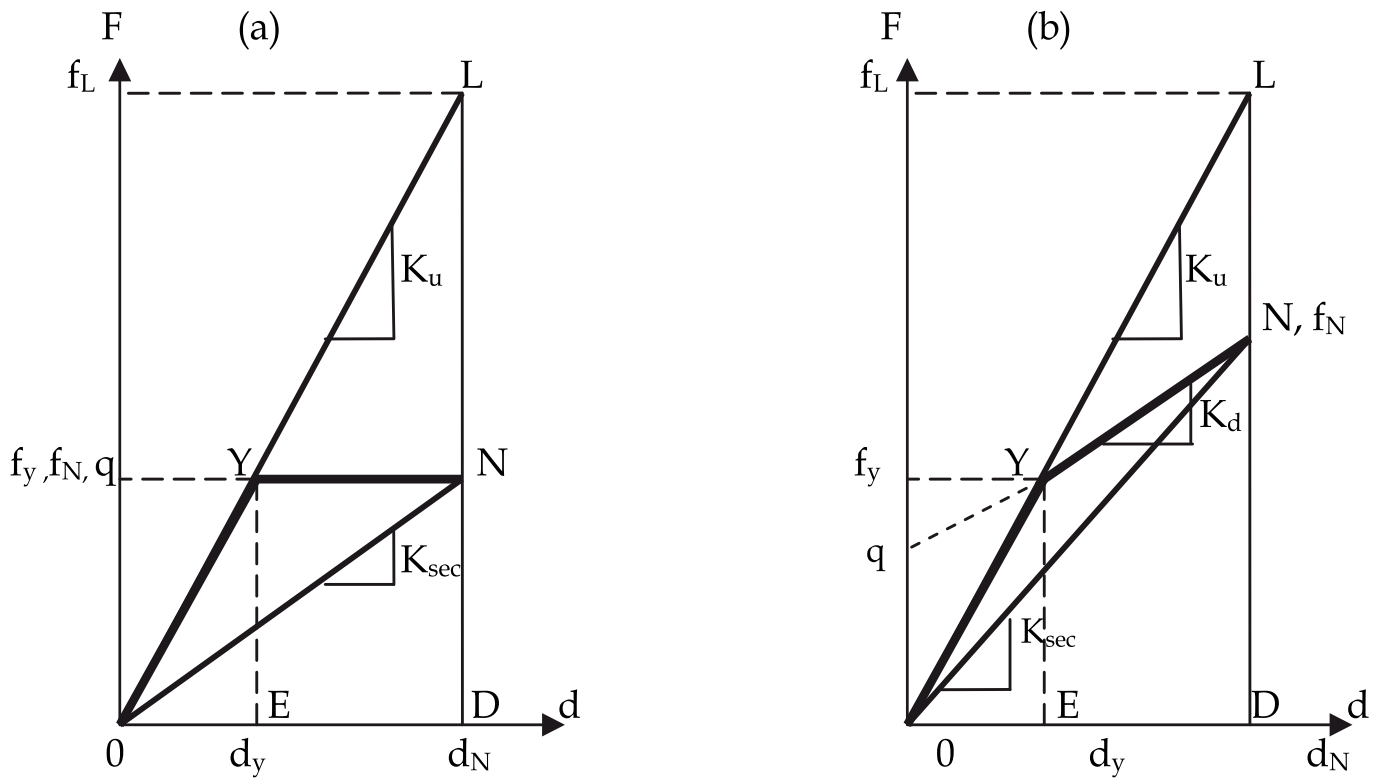

Fig. 3.2. Secant stiffness

In figure 3.2, $\mathrm{d}_{\mathrm{y}}$ and $\mathrm{d}_{\mathrm{N}}$ are the yielding and the maximum displacements. $\mathrm{f}_{\mathrm{y}}$ and $\mathrm{f}_{\mathrm{N}}$ are the yielding and the maximum forces. $K_{d}$ and $K_{u}$ are the loading and unloading stiffness. If the system remains elastic, then we will have a linear system when the displacement reaches the 
maximum value $D$, the force will be $f_{L}$. However, since the system is nonlinear, the maximum force $f_{N}$ will be smaller. Thus the corresponding effective stiffness will be affected.

For seismic isolation, the measurement of the effective stiffness can be considerably overestimated. To see this point, let us consider the definition of stiffness in a linear system. It is well known that the stiffness denotes capability of how a linear system can resist external force. Suppose under a force $\mathrm{f}_{\mathrm{L}}$, the system has a deformation $\mathrm{d}$, and then the rate defines the stiffness. That is

$$
K=\frac{f_{L}}{D}
$$

When the load $f_{L}$ is released, the linear system will return to its original position. Thus, the stiffness also denotes the capacity for how a system can bounce back after the force $f_{L}$ is removed, for at displacement $d$, the linear system will have a potential energy $E_{p}$

$$
E_{p}=\frac{K d^{2}}{2}
$$

Therefore, we can have alternate expression for the stiffness, which is

$$
K=\frac{2 E_{p}}{d^{2}}
$$

Apparently, in a linear system, the above two expressions of the stiffness, described by (3.24) and (3.26), are identical. This is because the potential energy can be written as

$$
E_{p}=\frac{f_{L} d}{2}
$$

However, in a nonlinear system, this equation will no longer hold, because the maximum force $f_{N}$ can contain two components: the dissipative force $f_{d}$ and the conservative force, $f_{c}$,

$$
\mathrm{f}_{\mathrm{N}}=\mathrm{f}_{\mathrm{c}}+\mathrm{f}_{\mathrm{d}}
$$

For example, in Figure 3.2(a), when $\mathrm{f}_{\mathrm{N}}$ is reached,

Thus,

$$
\begin{aligned}
& \mathrm{f}_{\mathrm{c}}=0 \\
& \mathrm{f}_{\mathrm{d}}=\mathrm{q}
\end{aligned}
$$

$$
\mathrm{f}_{\mathrm{N}}=\mathrm{f}_{\mathrm{d}}=\mathrm{q}
$$

Namely, the EPP system does not have a conservative force. Its restoring force is zero. From another example of the general bilinear system shown in figure 3.2 (b),

and

$$
\mathrm{f}_{\mathrm{d}}=\mathrm{q}
$$

$$
\mathrm{f}_{\mathrm{c}}=\mathrm{f}_{\mathrm{N}}-\mathrm{f}_{\mathrm{d}}
$$

Note that, only the conservative force contributes to the potential energy $E_{p}$. That is, 


$$
\mathrm{E}_{\mathrm{p}}=\frac{\mathrm{f}_{\mathrm{c}} \mathrm{d}}{2}<\frac{\mathrm{f}_{N} \mathrm{~d}}{2}
$$

In this case, using (3.24) and (3.26) will contradict each other. In order to choose the right formulae to estimate the effective period and damping, the estimation of effective stiffness must be considered more precisely. Generally speaking, a nonlinear system will have the same problem as the above-mentioned bi-linear system, that is, the restoring force is smaller than the maximum force, as long as the nonlinear system has softening springs.

\subsubsection{Estimation of effective period}

From the above-discussion, when we use an effective linear system to represent a nonlinear system, the effective stiffness should satisfy the following:

$$
\begin{gathered}
K_{\text {eff }}=\frac{2 E_{p}}{d^{2}} \\
K_{\text {eff }}=\frac{f_{c}}{d}
\end{gathered}
$$

By using (3.30) as well as (3.31), the effective stiffness $\mathrm{k}_{\text {eff }}$ will be smaller than the secant stiffness $\mathrm{k}_{\mathrm{sec}}$.

Furthermore, it can be seen that, vibration is caused by the energy exchange between potential and kinetic energies. The natural frequency of a linear system can be obtained by letting the maximum potential energy equal the maximum kinetic energy, that is, through the relation

$$
\frac{k d^{2}}{2}=\frac{M v^{2}}{2}=\frac{M \omega_{b}^{2} d^{2}}{2}
$$

In nonlinear systems, we should modify the above equation as

$$
\frac{k_{\text {eff }} d^{2}}{2}=\frac{m \omega_{\text {eff }}^{2} d^{2}}{2}
$$

Or,

$$
\omega_{\text {eff }}=\sqrt{\frac{k_{\text {eff }}}{M}}=\sqrt{\frac{f_{c} / d}{M}}<\sqrt{\frac{f_{N} / d}{M}}
$$

In other words, considering the dynamic property of a nonlinear system, we should not use the secant stiffness as the effective stiffness. Following this logic, the effective stiffness should be defined in a nonlinear system by considering the restoring potential energy as follows.

In the bi-linear case (see the shaded areas in Figure 3.3), when the system moves from 0 to $d$, the potential energy is 


$$
E_{p}=\frac{1}{2}\left[K_{u} d_{y}^{2}+K_{d}\left(d-d_{y}\right)^{2}\right]
$$

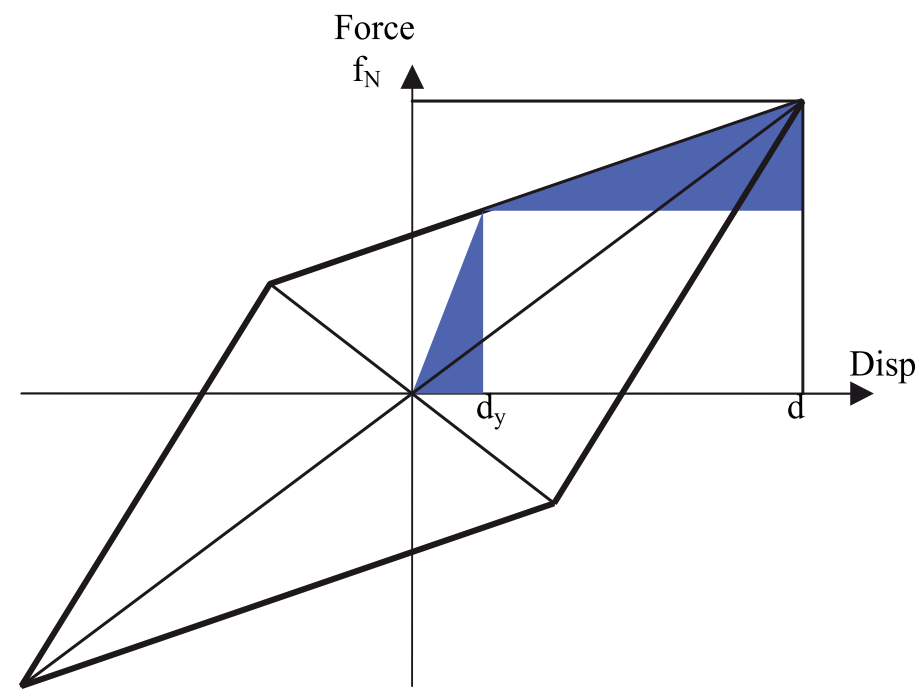

Fig. 3.3. Maximum potential energy of a bi-linear system

Therefore,

$$
K_{\text {eff }}=\frac{2 E_{p}}{d^{2}}=\frac{K_{u} d_{y}^{2}+K_{d}\left(d-d_{y}\right)^{2}}{d^{2}}
$$

By using the displacement ductility $\mu$, we can write

$$
K_{\text {eff }}=\frac{K_{u}+K_{d}(\mu-1)^{2}}{\mu^{2}}
$$

where $\mu$ is the displacement ductility and

$$
\mu=\frac{d}{d_{y}}
$$

The relationship between $K_{d}$ and $K_{u}$ is given as

$$
\mathrm{K}_{\mathrm{d}}=\mathrm{a} \mathrm{K} \mathrm{u}
$$

Using this notation, we have

$$
\mathrm{K}_{\mathrm{eff}}=\frac{1+\mathrm{a}(\mu-1)^{2}}{\mu^{2}} \mathrm{~K}_{\mathrm{u}}
$$

Therefore, the corresponding effective period is 


$$
\mathrm{T}_{\text {eff }}=\sqrt{\frac{\mu^{2}}{1+\mathrm{a}(\mu-1)^{2}}} \mathrm{~T}_{1}=2 \pi \mu \sqrt{\frac{\mathrm{M}}{\left[1+\mathrm{a}(\mu-1)^{2}\right] \mathrm{K}_{\mathrm{u}}}}
$$

From (3.41) and (3.23), the effective stiffness estimated by the secant method can overestimate the period by the factor

$$
\frac{\frac{1+a(\mu-1)}{\mu}}{\frac{1+a(\mu-1)^{2}}{\mu^{2}}}=\frac{1+a(\mu-1)}{1+a(\mu-1)^{2}} \mu
$$

Note that, the term a is smaller than unity and $\mu$ is larger than unity. Therefore, from (3.42), it is seen that the period can be notably underestimated by using the secant stiffness. For example, suppose $\mathrm{a}=0.2$ and $\mu=4$, the factor will be greater than 2 .

Similar to the estimation of effective period, the term $\mathrm{K}_{\text {eff }}$ will also affect the measurement of the effective damping ratio. Using the same logic, the damping ratio can also be underestimated. These double underestimations may cancel each other to a certain degree for acceleration computation, but will certainly underestimate the displacement. For a detailed discussion on structural damping, reference is made to Liang et al (2012).

\subsubsection{MDOF models}

Now, let use consider a linear n-DOF systems along one direction, say the X-direction. The governing equation can be written as

$$
\mathbf{M X}(\mathrm{t})+\mathbf{C X}(\mathrm{t})+\mathbf{K X}(\mathrm{t})=-\mathbf{M} \mathbf{J} a_{g}(\mathrm{t})
$$

Here $\mathbf{M}, \mathbf{C}$ and $\mathbf{K}$ are nxn mass, damping and stiffness matrices, $J=\{1\}_{\mathrm{n} \times 1}$ is the input column vector and

$$
X(t)=\left\{\begin{array}{c}
d_{1}(t) \\
d_{2}(t) \\
\ldots \\
d_{n}(t)
\end{array}\right\}
$$

where $d_{j}(t)$ is the displacement at the $j^{\text {th }}$ location.

If the following Caughey criterion hold

$$
\mathrm{CM}^{-1} \mathbf{K}=\mathrm{K} \mathrm{M}^{-1} \mathbf{C}
$$

The system is proportionally damped, which can be decoupled into n-SDOF systems, which are referred to as n-normal modes, so that the analysis of SDOF system can be used.

In base isolation, to regulate the displacement, large damping must be used. When the damping force is larger, isolation system is often not proportionally damped. That is, (3.45) will no longer hold and the system cannot be decoupled into n SDOF normal modes. In this case, it should be decoupled in $2 \mathrm{n}$ state space by using the state equations. 
Configuration of MDOF structures and non-proportional damping will both affect the magnitude of the accelerations of higher stories. However, the acceleration of the base, as well as the relative displacement between the base and the ground will not be significantly affected by using the normal mode approach. Practically speaking, more accurately estimate the displacement is important then the computation of the acceleration of higher stories.

Furthermore, when the isolation system is non-linear, its first "effective" mode will dominate. Thus, we can use its first "effective" mode only to design the system.

However, care must be taken when the cross effect occurs. It is seen that, if both the motion of both $\mathrm{X}$ and $\mathrm{Y}$ directions, which are perpendicular, are considered, we can have

$$
\left[\begin{array}{ll}
\mathbf{M}_{\mathrm{X}} & \\
& \mathbf{M}_{\mathrm{Y}}
\end{array}\right]\left\{\begin{array}{l}
\ddot{\mathrm{X}} \\
\ddot{\mathrm{Y}}
\end{array}\right\}+\left[\begin{array}{ll}
\mathbf{C}_{\mathrm{XX}} & \mathbf{C}_{\mathrm{XY}} \\
\mathbf{C}_{\mathrm{YX}} & \mathbf{C}_{\mathrm{YY}}
\end{array}\right]\left\{\begin{array}{c}
\dot{\mathrm{X}} \\
\dot{\mathrm{Y}}
\end{array}\right\}+\left[\begin{array}{cc}
\mathbf{K}_{\mathrm{XX}} & \mathbf{K}_{\mathrm{XY}} \\
\mathbf{K}_{\mathrm{YX}} & \mathbf{K}_{\mathrm{YY}}
\end{array}\right]\left\{\begin{array}{c}
\mathrm{X} \\
\mathrm{Y}
\end{array}\right\}=\left[\begin{array}{ll}
\mathbf{M}_{\mathrm{X}} & \\
& \mathbf{M}_{\mathrm{Y}}
\end{array}\right]\left\{\begin{array}{l}
\mathrm{J} \mathrm{a}_{\mathrm{xg}} \\
\mathrm{J} \mathrm{a}_{\mathrm{yg}}
\end{array}\right\}
$$

where $\mathrm{a}_{\mathrm{xg}}$ and $\mathrm{a}_{\mathrm{yg}}$ are ground accelerations along $\mathrm{X}$ and $\mathrm{Y}$ directions respectively, which are time variables and for the sake of simplicity, we omit the symbol ( $t$ ) in equation (3.46). The subscript $X$ and $Y$ denote the directions. The subscript $X X$ means the input is in $x$ direction and the response is also in $X$ direction. The subscript $X Y$ means the input is in $X$ direction and the response is in $\mathrm{Y}$ direction, and so on.

In (3.46), $\mathbf{M}_{X}=\mathbf{M}_{Y}$ are the mass matrices. It is seen that, both $\mathbf{C}_{X Y}=\mathbf{C}_{Y X^{T}}$ and $\mathbf{K}_{X Y}=\mathbf{K}_{Y X^{T}}$ are the cross terms. Generally speaking, if the system is rotated with the help of rotation matrix we can minimize the cross terms $\mathbf{C}_{\mathbf{X Y}}$ and $\mathbf{K}_{X Y}$.

$$
\boldsymbol{\Theta}=\left[\begin{array}{cc}
\cos (\theta) \mathbf{I} & -\sin (\theta) \mathbf{I} \\
\sin (\theta) \mathbf{I} & \cos (\theta) \mathbf{I}
\end{array}\right]_{2 n \times 2 n}
$$

The angle $\theta$ can be chosen from 0 to $90^{\circ}$. However, in this case no matter how the angle $\theta$ is chosen, at least one of $\mathbf{C}_{X Y}$ and $\mathbf{K}_{X Y}$ is not null and hence, the input in $X$ direction will cause the response in $\mathrm{Y}$ direction. That is defined here as the cross effect, which implies energies of ground motions from one direction is transferred to another. There are many reasons to generate the cross effect. In certain cases, the cross effect can considerably magnify the displacement.

\subsection{Bearings and effect of damping 3.2.1 Role of damping}

From the above discussion, it is seen that there are several possible inaccuracies that may exist in estimating the displacement. The results can often be underestimation of displacement in isolation design.

As pointed out earlier, the only way to reduce the displacement in the working range of isolation systems is to increase the damping. For example, from figure 2.2(b), it is seen that, at 3 second period, if the damping ratio is taken to be $50 \%$, the displacement is about $0.2(\mathrm{~m})$ whereas using $5 \%$ only can cause about $0.5(\mathrm{~m})$ displacement, which is about 2.5 times larger.

\subsubsection{Damping and restoring stiffness}

Often base isolators or isolation bearings are designed to provide the required damping. Practically speaking, damping can be generated by several means. 
The first kind of damping is material damping, such as high damping rubber bearings, leadcore rubber bearings as well as metallic dampers. The damping mechanism is generated through material deformations. Note that, high damping rubbers often do not provide sufficient damping.

The second is surface damping. The damping mechanism is generated through surface frictions of two moving parts, such as pendulum bearings, friction dampers. Note that, surface damping often is insufficient and can have a significant variation from time to time.

The third type of damping is viscous damping, which is often provided by hydraulic dampers. This kind of damping is more stable but with higher cost.

Closely related to damping generated by isolator is the restoring stiffness, since both of them are provided by bearings. In fact, the type of method of proving restoring stiffness and damping classifies the type of bearings.

The restoring stiffness can be generated by material deformations, including specific springs, which relies on the deformation to restore potential energy. Releasing of such potential energy can make the bearing return to its center position. Rubber bearings, including high damping rubber bearings, lead core rubbers bearings, steel-rubber-layered bearings fall into this category. Bearings with sliding surface and elastomeric springs also use restoring force generated by material deformation. Bearings with metal ribs which provide both stiffness and damping also fall into this category.

The restoring stiffness can also be generated by geometric shaped. Generally, when horizontal motion occurs, such bearings generate vertical movement which can also restore potential energy. Again, release of potential energy enables the bearing return to its center position. Pendulum sliding bearing falls into this category. Recently, a newly type of roller bearing is developed, which guarantees a low level constant horizontal acceleration, and will be described in Section 5 .

\section{Selected design considerations}

\subsection{Design windows}

As mentioned earlier, the key issue in design of isolation system is an optimal compromise of the acceleration and the displacement, within an acceptable range of the period called "design window" period. The reason to consider the concept of design window is to avoid possible undesired displacement, which in general is independent to acceleration.

\subsubsection{Lower bond of period}

In order to reduce the acceleration, the period of the isolation system cannot be shorter than a given value. Although this value depends upon the site, it can be roughly estimated to be 1.5 to 2 seconds. This value actually establishes the left boundary of the design windows on the design spectrum. This lower limit is well understood. Because of the assumption of negligible damping, acceleration is taking as directly proportional to the displacement. Thus in typical design practice, it is assumed that displacement bound is given once the acceleration limit is established. This is not always true, because acceleration and displacement are in general independent. 


\subsubsection{Upper bond of period}

In order to limit the displacement to a reasonable value, the period of the isolation system cannot be too long. This defines the right side boundary of the design window. In figure 4.1, the design spectrum and the design window for the isolation system is conceptually illustrated.

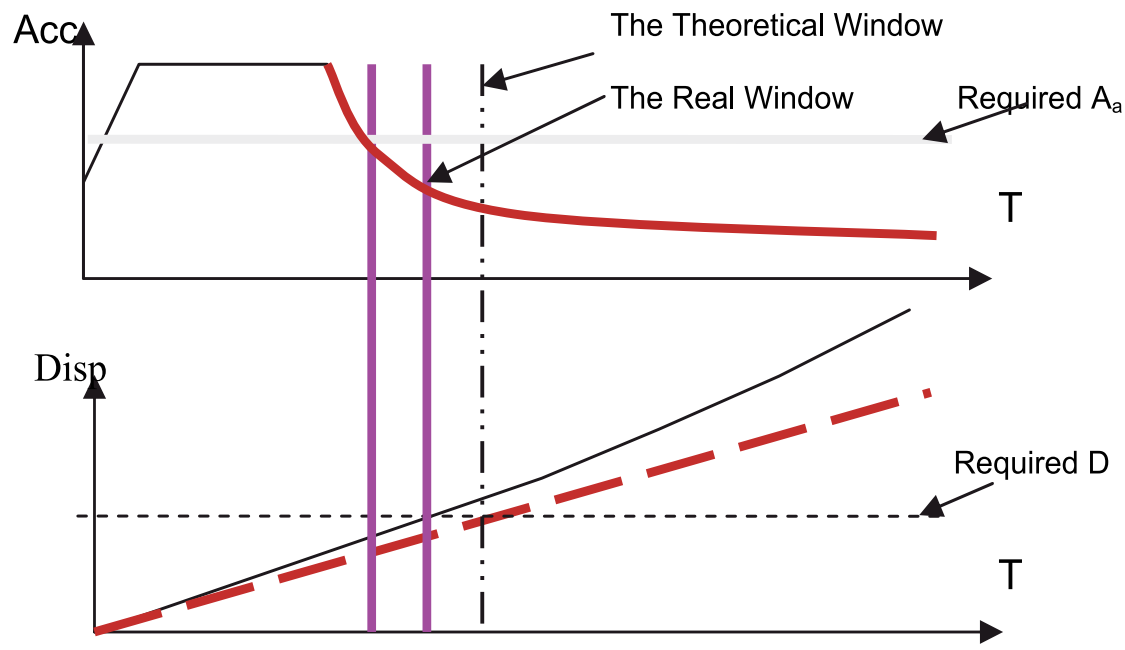

Fig. 4.1. Design Window for Isolation Systems

Figure 4.1 illustrates that if the design has a period that falls outside this "window," it is unacceptable. Within this window, reductions of the acceleration and tolerance displacement must be considered simultaneously. In this sense, a proper isolation system is an optimally compromised design.

It should be noted that, when damping is large, the reduction of acceleration will be reduced. When damping is too low, the displacement will be increased. Thus, the proper damping will have upper and lower period bonds from the viewpoint of isolation design.

\subsection{Left boundary of the design window, acceleration related parameters}

\subsubsection{Design force}

In a typical aseismic design, the base shear $\mathrm{V}$ typically needs to be designed by

$$
\mathrm{s}_{\mathrm{A}} \mathrm{V} \leq[\mathrm{V}]
$$

where [V] is the allowable base shear and $s_{\mathrm{A}}$ is a factor of safety. The base shear $\mathrm{V}$ can be determined by several ways. One is that given by (2.1), which is repeated here.

$$
\mathrm{V}=\mathrm{C}_{\mathrm{s}} \mathrm{W}
$$

To determine the seismic response factor $C_{s}$, the effective stiffness $K_{\text {eff }}$, or $K_{\text {eff }}$ in the case of MDOF system is required. The effective stiffness often should not be determined by using the secant stiffness, as previously explained.

At the same time, the base shear is equal to the product of the lateral stiffness $\mathrm{K}_{\mathrm{b}}$ and the displacement $\mathrm{D}$ of the bearing system plus the damping force $\mathrm{F}_{\mathrm{d}}$ measured at the maximum displacement. 


$$
\mathrm{V}=\mathrm{K}_{\mathrm{b}} \mathrm{D}+\mathrm{F}_{\mathrm{d}}
$$

The specifications related to the damping force for isolators are provided by bearing vendors. For bi-linear damping, the damping force is roughly the dissipative force or the characteristic strength $q$ (see fig. 3.2b). Using the maximum value of $q$, denoted by $Q$,

$$
\mathrm{F}_{\mathrm{d}}=\mathrm{Q}
$$

For friction damping

$$
\mathrm{F}_{\mathrm{d}}=\mu \mathrm{W}
$$

where $\mu$ is the friction coefficient.

For multi-story structures, base on the SRSS method,

$$
V=\sqrt{\sum_{j=1}^{n} f_{L j}^{2}}
$$

where, $\mathrm{f}_{\mathrm{Lj}}$ is the lateral force of the $\mathrm{j}^{\text {th }}$ story and

$$
f_{L j}=m_{j} \sqrt{\sum_{i=1}^{S} a_{j i}{ }^{2}}
$$

in which $m_{j}$ is the mass of the $j^{\text {th }}$ story and $a_{j i}$ is the absolute acceleration of the $i^{\text {th }}$ mode at the $j^{\text {th }}$ story. Note that, for an n-DOF system, there will be n- modal accelerations, and, usually the first few modes will contain sufficient vibration energy of the system. To be on the safe side, the desirable number of modes to be considered is

$$
\mathrm{S}=\mathrm{S}_{\mathrm{r}}+\mathrm{S}_{\mathrm{f}}
$$

where $S_{r}$ is the number of the first few modes that contain 90 95\% vibration energy and

$$
\mathrm{S}_{\mathrm{f}}=1 \sim 3,
$$

as an extra safety margin particularly for irregular structures. This should not add too much computational burden.

To determine $S_{r}$, modal mass ratio $\gamma_{i}$ is often needed,

$$
\gamma_{\mathrm{i}}=\frac{\left(\mathbf{P}_{\mathrm{i}}^{\mathrm{T}} \mathbf{M} \mathrm{J}\right)^{2}}{\mathbf{P}_{\mathrm{i}}^{\mathrm{T}} \mathbf{M} \mathbf{P}_{\mathrm{i}}}
$$

and $S_{r}$ can be determined by

$$
\sum_{\mathrm{i}=1}^{\mathrm{S}_{r}} \gamma_{i}>90 \sim 95 \%
$$

In (4.7) the absolute acceleration $\mathrm{a}_{\mathrm{ji}}$ is the $\mathrm{j}^{\text {th }}$ element of the $\mathrm{i}^{\text {th }}$ acceleration vector $\mathbf{a}_{\mathrm{i}}$, which is determined by 


$$
\mathbf{a}_{\mathrm{i}}=\Gamma_{\mathrm{i}} \mathrm{C}_{\mathrm{si}} \mathbf{P}_{\mathrm{i}}
$$

The term $\mathrm{C}_{\mathrm{si}}$ is the $\mathrm{i}^{\text {th }}$ seismic coefficient, which can be determined through the building or bridge code. The modal participation factor $\Gamma_{\mathrm{i}}$ is given by

$$
\Gamma_{\mathrm{i}}=\frac{\mathbf{P}_{\mathrm{i}}^{\mathrm{T}} \mathbf{M} \mathrm{J}}{\mathbf{P}_{\mathrm{i}}^{\mathrm{T}} \mathbf{M} \mathbf{P}_{\mathrm{i}}}
$$

Equations (4.10), (4.12) and (4.13) contain the mode shape function $\mathbf{P}_{\mathrm{i}}$ which can be a triangular approximation, or more precisely, be given by the following eigen-equation with the normalization by letting the roof modal displacement equal to unity.

$$
\omega_{\mathrm{i}}^{2} \mathbf{P}_{\mathrm{i}}=\left(\mathbf{M}^{-1} \mathbf{K}\right) \mathbf{P}_{\mathrm{i}}
$$

Note that, the base shear calculated from (4.2) only considers the first mode, which may not be sufficiently accurate. Equation (4.3), is theoretically workable but practically speaking, the dissipative force $\mathrm{F}_{\mathrm{d}}$ is difficult to establish.

Note that, in (4.12), the mode shape $\mathbf{P}_{\mathrm{i}}$ is obtained through (4.14), however, $\mathbf{P}_{\mathrm{i}}$ is the displacement mode shape but not necessarily the acceleration mode shape. Therefore, cares must be taken by using (4.12) to calculate the modal acceleration; otherwise a design error can be introduced. For the limited space, the detailed explanation is not discussed in this chapter. Interested reader may see Liang et al 2012.

\subsubsection{Overturning moment}

Because of the potentially large P- $\Delta$ effect, base isolation design must carefully consider the overturning moment to ensure that the uplifting force is not magnified. Furthermore, most bearings cannot take tensions, whereas the overturning moment can generate uplift tension on bearings.

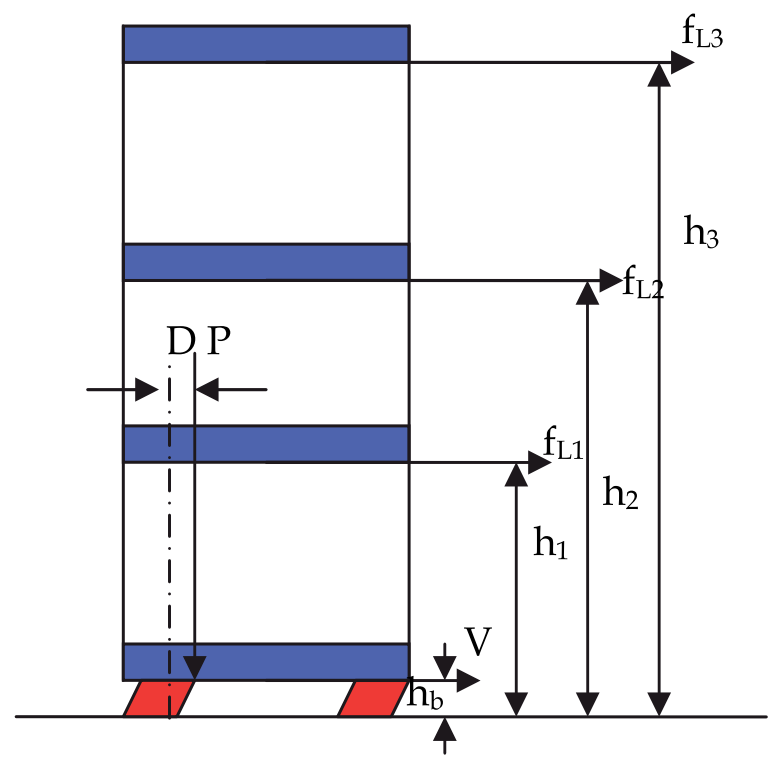

Fig. 4.2. Overturning moment 
From figure 4.2 , it is seen that the total overturning moment is given by

$$
M_{T}=P D+V h_{b}+\sum_{j=1}^{n} f_{L j} h_{j}
$$

If the structure has notable plan irregularity, the additional moment due to the asymmetric distribution of mass must also be considered.

With the help of $\mathrm{M}_{\mathrm{T}}$ and knowing the geometric dimensions of the isolated structure, the uplift force can be calculated, and the corresponding criterion can be set up as

$$
\mathrm{s}_{\mathrm{M}} \mathrm{M}_{\mathrm{T}} \leq\left[\mathrm{M}_{\mathrm{T}}\right]
$$

where $s_{\mathrm{M}}$ is a safety factor and $\left[\mathrm{M}_{\mathrm{T}}\right]$ is allowable moment.

The above two criteria define the left side boundary of the design window (see fig 4.1).

\subsection{Right boundary of design window: Independency of displacement 4.3.1 Independency of displacement}

That the displacement $\mathrm{D}$ and the acceleration $\mathrm{A}_{\mathrm{a}}$ are independent parameters mentioned previously will be briefly explained here. For linear system, from (2.7) we have

$$
\mathrm{d}(\mathrm{t})=-\frac{\mathrm{C}}{\mathrm{K}_{\mathrm{b}}} \mathrm{v}(\mathrm{t})-\frac{\mathrm{M}}{\mathrm{K}_{\mathrm{b}}} \mathrm{a}_{\mathrm{a}}(\mathrm{t})
$$

For large amount of damping, consider the peak values, we have

$$
\mathrm{D} \approx \sqrt{\left(\frac{\mathrm{C}}{\mathrm{K}_{\mathrm{b}}} \mathrm{V}\right)^{2}+\left(\frac{\mathrm{M}}{\mathrm{K}_{\mathrm{b}}} \mathrm{A}_{\mathrm{a}}\right)^{2}}>\frac{\mathrm{M}}{\mathrm{K}_{\mathrm{b}}} \mathrm{A}_{\mathrm{a}}=\omega_{\mathrm{b}}^{2} \mathrm{~A}_{\mathrm{a}}
$$

Additionally, for nonlinear system, softening behavior often occurs, so that the maximum force is not proportional to the maximum displacement. As a result, one cannot simply calculate $\mathrm{D}$ due to the nonlinearity of the most isolation systems, as

$$
\mathrm{D}=\frac{\mathrm{T}_{\mathrm{b}}^{2}}{4 \pi^{2}} \mathrm{C}_{\mathrm{s}} \mathrm{g}
$$

In design, the bearing displacement is as important as the acceleration related parameters. Due to the uncertainties illustrated above, a safety factor should be used before further research results are available.

$$
\mathrm{s}_{\mathrm{D}} \mathrm{D} \leq[\mathrm{D}]
$$

where $s_{\mathrm{D}}$ is a safety factor and [D] is allowable displacement.

\subsubsection{Right boundary due to displacement}

Since the displacement needs to be regulated, the right boundary of the design window is defined. Because of the above-mentioned reasons, by using the safety factor $s_{\mathrm{D}}$, the right boundary will further shift leftwards. 
It is seen that, the resulted window can be rather narrow.

\subsection{Probability-base isolation design}

In recent years, the probability-based design for civil engineering structures against natural and man-made load effects attracts more and more attentions. The basic idea is to treat both the loads and resistance of structures as random variables and to calculate the corresponding failure probability, based on which the load and resistant factors are specified, (see Nowak and Collins, 2000). Probability-based design for base isolation systems should be one of the frontier research areas for earthquake engineering researchers.

\subsubsection{Failure probabilities of base-isolated structures}

In the above discussions of seismic isolation, the design process is a deterministic approach because it is established on deterministic data.

A base-isolated building or bridge is a combination of civil engineering structures and mechanical devices, the bearings. In most cases of mechanical engineering devices, the safety factors are considerably larger than those used by civil engineers. The main reason, among many, is that the civil engineering structures have much larger redundancy. Mechanical devices, on the other hand, do not have such a safety margin. This raises an interesting issue on safety factors for seismically isolated structures.

Take the well known design spectrum, for example. Its spectral value is often generated by the sum of mean value plus one standard deviation. However, the maximum value can be much larger than the spectral value. For example, consider under 99 earthquake statistics of a structure with period $=2$ second and damping ratio $=20 \%$. The mean-plus-one standard deviation value of acceleration is $0.24(\mathrm{~g})$ whereas the maximum value is $0.47(\mathrm{~g})$. And the mean-plus-one standard deviation value of displacement is $0.22(\mathrm{~m})$, whereas the maximum displacement value is $0.45(\mathrm{~m})$.

The question is: Given an isolation design, what is the chance that $0.47(\mathrm{~g})$ acceleration and/or 0.45 (m) displacement could occur? More specifically, if the allowed acceleration [A] and/or the allowed displacement [D] are preset, what is the chance that the acceleration and/or the bearing displacement can exceed the allowed design values? From the viewpoint of probability based design, the above-mentioned chance of exceeding is referred to as the failure probability. Therefore, a new concept of design criteria for seismic isolation may be stated by

$$
\mathrm{p}_{\mathrm{fA}}=\mathrm{P}(\mathrm{A} \geq[\mathrm{A}]) \leq\left[\mathrm{p}_{\mathrm{fA}}\right]
$$

and

$$
\mathrm{p}_{\mathrm{fD}}=\mathrm{P}(\mathrm{D} \geq[\mathrm{D}]) \leq\left[\mathrm{p}_{\mathrm{AD}}\right]
$$

Here, the subscripts $f_{A}$ and $f_{D}$ stand for failure of acceleration and failure of displacement respectively. Symbol $[()$.$] means the allowable value of (.).$

\subsubsection{Computation of failure probabilities}

Figure 4.3 illustrates the probability distributions of a linear isolation system of $\mathrm{T}_{\mathrm{b}}=2$ second and $\xi=20 \%$ under the excitation of 99 earthquake records. Figure 4.3(a) is the absolute acceleration and (b) is the relative displacement. The X-axes are the numerically 
simulated values. The $y$ axes are the probability density function. For example, in figure 4.3(a), this function is denoted as $\mathrm{f}_{\mathrm{A}}(a)$, namely the probability density curve is a function of the level of acceleration $a$.

Suppose the allowed acceleration

The failure probability can be calculated as

$$
[\mathrm{A}]=0.25(\mathrm{~g})
$$

$$
p_{f A}=\int_{0.25}^{\infty} f_{A}(a) d a
$$

The integral is the shadow area. From figure 4.3 (a), we can see that, it is about $16 \%$. This failure probability is not small at all. Note that, in this case, the allowed value being $0.25(\mathrm{~g})$ is satisfied by using the mean-plus-one-standard-deviation value.

As another example, suppose the allowed displacement is

$$
[\mathrm{D}]=0.29(\mathrm{~m})
$$

$$
[\mathrm{D}]=0.29(\mathrm{~m})
$$

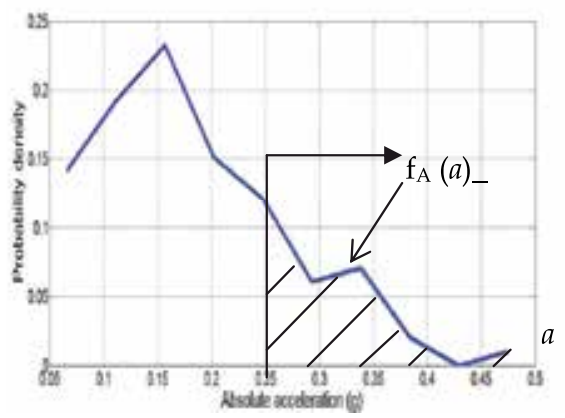

(a)

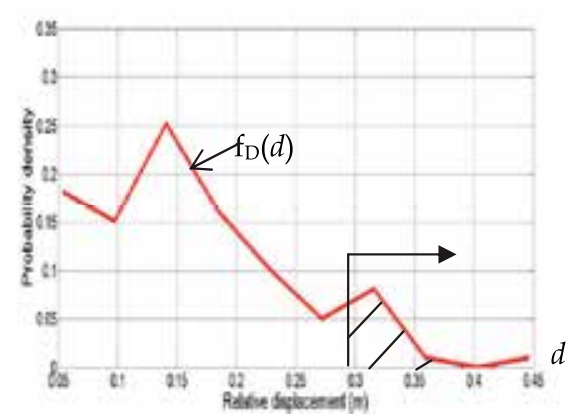

(b)

Fig. 4.3. Probability distributions, $\mathrm{T}_{\mathrm{b}}=2$ (sec), $\xi=20 \%, 99$ records, $\mathrm{PGA}=0.4(\mathrm{~g})$.

The failure probability can be calculated as

$$
\mathrm{p}_{\mathrm{fD}}=\int_{0.29}^{\infty} \mathrm{f}_{\mathrm{D}}(d) \mathrm{d} d
$$

The integral is also the shadow area. From figure 4.3 (b), we can also see that, this failure probability is not small either. Note that, in this case, the allowed value being $0.29(\mathrm{~m})$ is satisfied by using the mean-pulse-one-standard-deviation value.

Generally, the computations can be described as follows

$$
\mathrm{p}_{\mathrm{fA}}=\int_{[\mathrm{A}]}^{\infty} \mathrm{f}_{\mathrm{A}}(a) \mathrm{d} a
$$

for acceleration and

$$
\mathrm{p}_{\mathrm{fD}}=\int_{[\mathrm{D}]}^{\infty} \mathrm{f}_{\mathrm{D}}(d) \mathrm{d} d
$$

for displacement. 
The failure probability implies the chance of not meeting the design requirement. For acceleration, because of the structural redundancy of earthquake resilience, the allowed failure probability can be higher. Anyhow, 15\% chance of failure may not acceptable by the owners of the structures (e.g. seismic isolation systems for nuclear reactors). For displacement, usually a bearing cannot tolerance virtually any exceedance. Thus, the allowed failure probability should also be lower.

This brief discussion above suggests that the design safety considerations for the combined civil engineering structures with mechanical devices may be examined by using a probability-based principle and approach.

\subsection{Seismic isolation bearings}

There are several types of isolation bearings commercially available, such as lead-rubber, high damping rubber as well as sliding bearings. Generally speaking, as long as the bearing system can satisfy the requirement of the above-mentioned design windows in the service life time of the isolation system, any bearing may be employed. Interested readers can consult Komodromos, P.I. (2000). In seismic isolation design, it is suggested that the allowed bearing displacement be used as the primary criterion; the effective period obtained via effective stiffness as the second criterion and providing the proper amount of damping as the third criterion for bearing selections. Because of the difficulty to choose correct type of bearings within the design window, a new type of bearing is introduced as described in Section 5 . .

In the literature, certain non-traditional approaches of using relatively inexpensive bearings are reported. Many inexpensive bearings do not allow large displacements and have very limited service life. Therefore, they should not play an important role in seismic isolation design for bridges. Furthermore, the cost of the isolation bearing is often a smaller fraction of the total cost of bridge construction. In most cases, using inexpensive bearings may not be a good choice.

\section{A new seismic isolation device}

The above discussion on seismic isolation technology suggests that there are many aspects which require further research and development efforts, especially in the area to establish quantitative boundaries of design period in order to achieve optimal designs. In fact, among all commercially available base isolators, none seems to provide a good balance between acceleration reduction and reasonable cost. A recent development, the roller bearing, is briefly explained in this section. It has the promise to utilize damping to achieve desirable compromise in isolative design.

\subsection{Concept of roller bearing}

The core component of this device is the rolling assembly, which generates a desired low and constant acceleration of the superstructure regardless of the magnitude of the ground excitations. To explain the principle of constant acceleration, consider the simplified sketch of a roller traveling on an inclined surface shown in figure 5.1.

In figure 5.1, the inclined surface shown has a constant slope with an angle $\theta$ measured from the horizontal axis. The motion has an absolute acceleration in the horizontal direction, denoted by $a_{h}$. The roller has a relative acceleration $a_{r}$ along the sloping surface, which has a 
vertical component $\mathrm{a}_{\mathrm{v}}$ and a horizontal component $\mathrm{a}_{\mathrm{rh}} . \mathrm{A}_{\mathrm{g}}$ is the ground acceleration which is acting in the horizontal direction without a vertical component: In other words, the roller will have an absolute vertical acceleration, which is $\mathrm{a}_{\mathrm{v}}$.

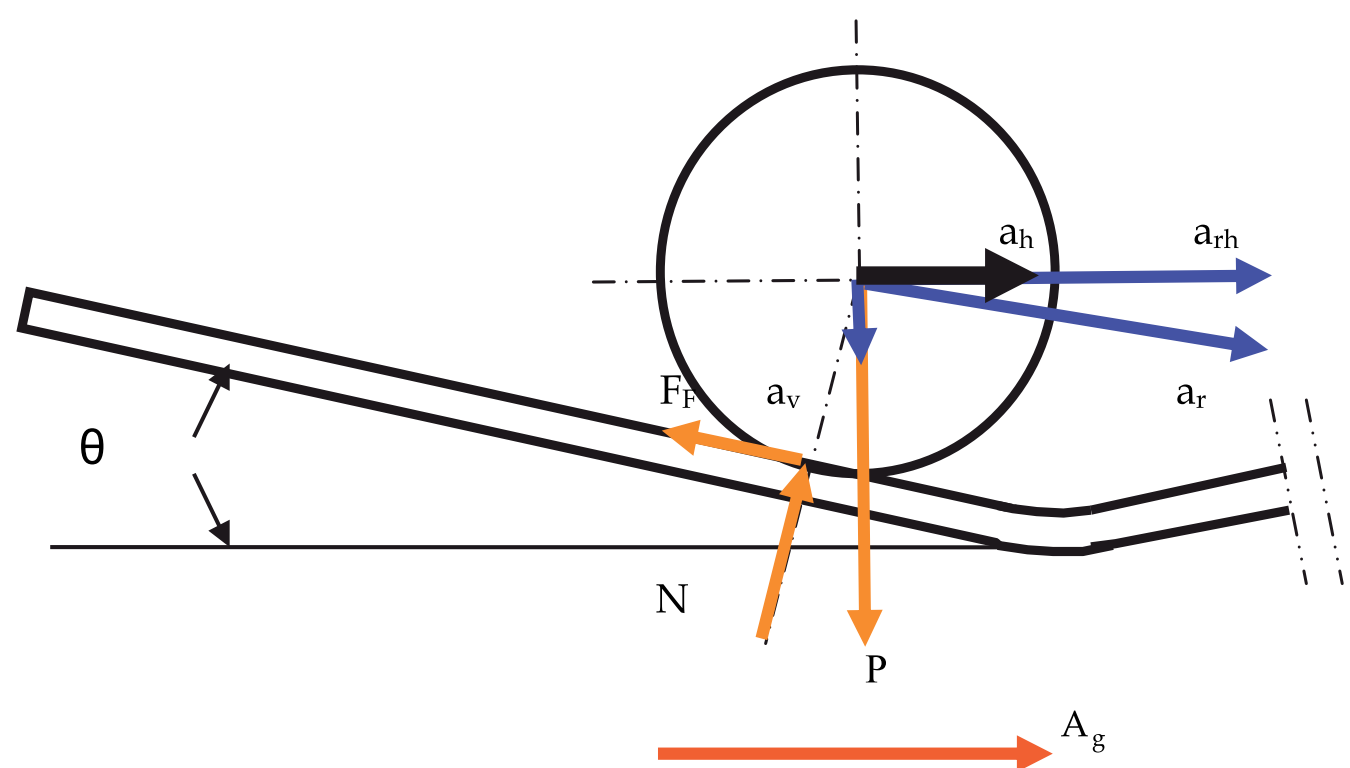

Fig. 5.1. Accelerations and forces of roller bearing

From figure 5.1, it is seen that, the amplitude of the friction force is

$$
\mathrm{F}_{\mathrm{F}}=\mu \mathrm{N}
$$

where $\mu$ is the rolling friction coefficient.

The relative vertical and horizontal accelerations relationships are respectively given by

$$
\mathrm{a}_{\mathrm{v}}=\tan \theta \mathrm{a}_{\mathrm{rh}}
$$

and

$$
a_{h}=a_{r h}+A_{g}
$$

When the angle $\theta$ is sufficiently small, say,

$$
\theta<5^{0}
$$

and let the friction coefficient $\mu$ be less than 0.2 :

$$
\mu<0.2
$$

in this case,

$$
\mathrm{a}_{\mathrm{h}} \approx \frac{\sin \theta-\mu \cos \theta}{\cos \theta+\mu \sin \theta} \mathrm{g}
$$


Here, we assume

$$
\frac{\sin \theta+\mu \cos \theta}{\cos \theta-\mu \sin \theta} \tan \theta \approx 0
$$

Because the quantities $\theta, \mu$ and $g$ are all constant, the horizontal absolute acceleration is a constant. Note that the ground acceleration, $\mathrm{A}_{\mathrm{g}}$, does not appear in equation (5.6) [Lee et al 2007]. In other words, the amplitude of the acceleration of the superstructure will be constant. The first objective of having a constant and low amplitude acceleration of the superstructure thus may be achieved.

Next, if the friction is sufficiently small,

$$
\mu<<\theta
$$

then,

$$
\mathrm{a}_{\mathrm{h}} \approx \theta \mathrm{g}
$$

The rotation friction coefficient of the rolling motion will only be a fraction of one percent. However, with $\theta$ less than $5^{0}$, the angle will be several percent (unit in radians). That is, $\theta$ will be about ten times larger than $\mu$. The condition $\mu<\theta$ is then satisfied.

Next, the vertical acceleration can be given as

$$
\mathrm{a}_{\mathrm{v}} \approx-\mathrm{A}_{\mathrm{g}} \tan \theta
$$

Since $\tan \theta \approx \theta$ is for very small angles, the vertical acceleration due to the horizontal ground excitation $A_{g}$ is very small and can be shown quantitatively that it is negligible [Ou et al 2009].

\subsection{Design parameters}

The major design parameters for the roller isolation bearing are listed as follows:

Maximum vertical load per bearing: $P_{r}[$ kips];

Maximum permanent load: $L_{p m}$ [kips]

Lateral force (use the shear pins controlled the lateral force): $0.12 L_{p m}$ [kips]

Roller diameter $\mathrm{D}_{\mathrm{r}}$ and length $\mathrm{l}_{\mathrm{r}}$ :

$$
1_{\mathrm{r}} \mathrm{D}_{\mathrm{r}} \geq \mathrm{c} \cdot \mathrm{P}_{\mathrm{r}} \frac{\mathrm{E}_{\mathrm{s}}}{8 \sigma_{\mathrm{y}}^{2}}\left[\mathrm{in}^{2}\right]
$$

where

$\sigma_{\mathrm{y}}=$ specified minimum yield strength of the weakest material at the contact surface

$\mathrm{E}_{\mathrm{s}}=$ Young's modulus for material

$\mathrm{P}_{\mathrm{r}}=$ load asymmetrical coefficient

$\mathrm{c}=1$ for single direction rolling bearing

$\mathrm{c}=0.7$ for double direction rolling bearing

Fig. 5.2 shows the configuration of a prototype roller bearing assembly which was manufactured for laboratory studies. Details on the performance and design of roller isolation bearings may be found in publications by the authors and their colleagues that are given in the references. 


\subsection{Roller bearing implementation}

With the support of a Federal Highway Administration grant, the new roller isolation bearing has been installed in an actual bridge to observe the constructability and other related issues in Rhode Island. As of August 2011, the installation is completed and the bridge is open to traffic. Certain monitoring systems are installed so that in time the performance of the roller isolation bearings under ambient live loads can be evaluated. Figure 5.3 contains a number of the New Street Bridge in RI which is the first bridge in the US implemented with the new roller bearings.
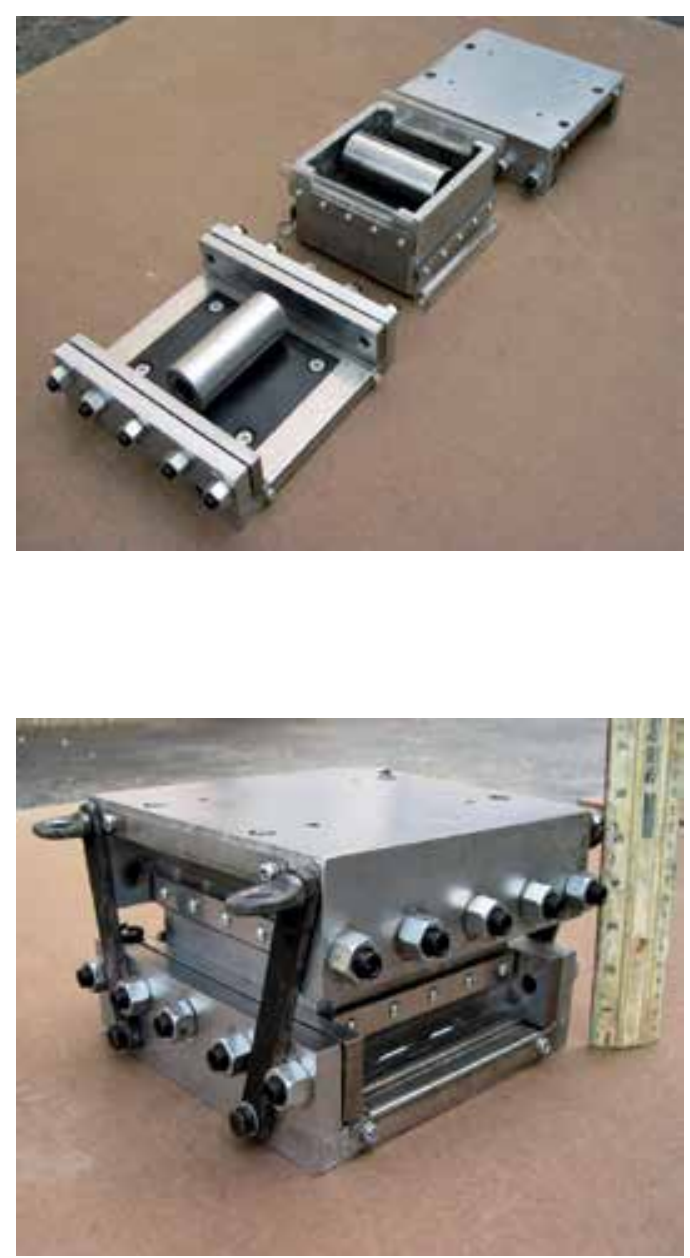

Fig. 5.2. Roller Bearing Assembly 


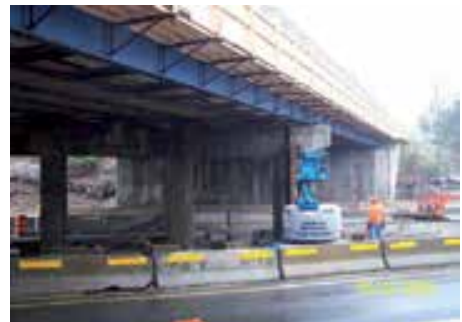

Two Span Bridge Rehabilitation

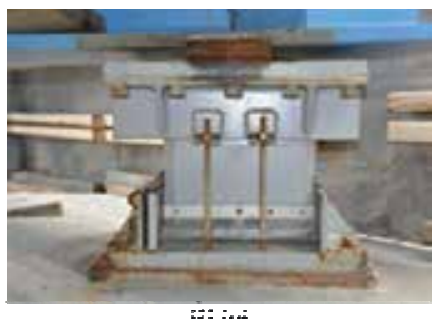

Roller Isolation Bearing

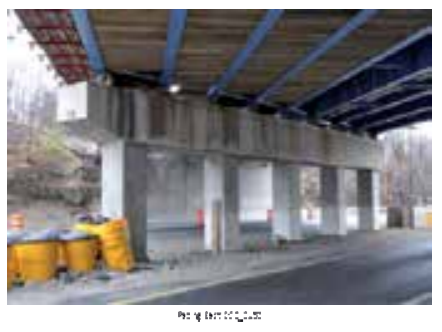

Installation at Completion

Fig. 5.3. Bridge Rehabilitation in Rhode Island

\section{Summary}

Although seismic isolation is considered to be a relatively matured technology, there are several issues need to be further explored. The main concern is that in general, the targeted acceleration reduction and the bearing displacement are independent parameters. That is, giving one does not automatically define the other. While for most available isolation systems today, the design practice has been based on the assumption that they are dependent parameters. For this assumption works for many cases, it does not yield adequate design for special situations that can exist. Therefore, principles and approaches to deal with these two independent parameters are needed.

This chapter first discussed several design issues of seismic isolation followed by an explanation of the cases where current design approaches may introduce large errors. Certain key parameters for isolation design are discussed and some research needs are identified. The references provided are selected representative publications by many contributors. No intention was given in this chapter to provide a careful literature review on the subject of seismic isolation.

The main control parameter of the seismic induced vibration is the natural period or effective period of the isolation system. To reduce the acceleration, longer period is needed. However, the longer the period is the larger displacement will be resulted. Thus, isolation design is viewed from the perspective of compromising between acceleration reduction and displacement tolerance.

The second important parameter is the effective damping ratio, because displacement can only be regulated by damping. The issue becomes complicated because adding damping will affect the acceleration reduction.

A base-isolated structure is typically a combination of civil engineering structure with mechanical engineering devices. The former is often designed with sufficient safety redundancy. However, the isolation devices are "precision" mechanical parts that do not have large tolerance of displacement uncertainty. By using the concept of probability based design, the safety margin for base isolation systems may be established with more confidence.

This chapter emphasizes the importance of a design window to consider the demand of acceleration and the displacement. Since large displacement may induce many engineering problems, such as large artificial P- $\Delta$ effect, etc. the displacement has to be carefully regulated or controlled. This will narrow the available design window. In some cases, no window is available for a reasonable design of base isolation system, suggesting that for such a case it is not effective to use isolation bearings. 
A new type of isolation bearing is briefly explained, which promises to deliver an optimally compromised acceleration reduction and displacement control through addition of damping.

\section{Acknowledgement}

Materials summarized in this chapter are based on studies carried out by the authors in recent years on earthquake protective systems under research grants from the National Science Foundation through the Multidisciplinary Center for Earthquake Engineering Research (ECE 86-07591 and ECE-97-01471) and the Federal Highway Administration (DTFH61-92-C-00106 and DTFH61-98-C-00094).

\section{References}

AASHTO (2010). Guide Specifications for Seismic Isolation Design. Interim 2000. Washington, D.C.

ASCE/SEI standard 41-06 (2007). Seismic Rehabilitation of Existing Buildings, American Society of Civil Engineers.

ASCE/SEI standard 7-10 (2010). Minimum Design Loads of Buildings and Other Structures, American Society of Civil Engineers.

ATC (1995). Structural Response Modification Factors, Report No. ATC-19, Applied Technology Council, Redwood City, California.

Austin, M.A., Lin, W.J. (2004). Energy Balance Assessment of Base-Isolated Structures. J. Engineering Mechanics, 130(3), pp. 347-358

Buckle, I., Constantinou, M., Dicleli, M. and Ghasemi, H. (2006). Seismic Isolation of Highway Bridges, Special Report MCEER-06-SP07, University at buffalo, The State University of New York.

Buckle, I.G. and Mayes, R.L. (1990). Seismic Isolation: History, Application, and Performance - A World View, Earthquake Spectra, 6(2), pp. 161-201.

Christopoulos, C. and Filiatrault, A. (2006). Principles of Supplemental Damping and Seismic Isolation. University Institute for Advanced Study (IUSS) Press, University of Pavia, Italy.

Clark, P. W., Whittaker, A. S., Aiken, L. D. and Egan, J. A. (1993). Performance Considerations for Isolation Systems in Regions of High Seismicity, Proceedings of ATC-17-1 Seminar on Seismic Isolation, Passive Energy Dissipation, and Active Control, San Francisco, CA, March 11-12, pp. 29-40.

Clough, R. and Penzien, J. (2003). Dynamics of Structures. $3^{\text {rd }}$ Ed. Computers and Structures, Inc.

Constantinou, M.C., Caccese, J. and Harris, H.G. (1987). Frictional Characteristics of TeflonSteel Interfaces under Dynamic Conditions, Earthquake Engineering and Structural Dynamics, 15, pp. 751-759.

Constantinou, M.C., Mokha, A.S. and Reinhorn, A.M. (1990). Teflon Bearings in Base Isolation. Part 2: Modeling. Journal of Structural Engineering, ASCE, 116(2), pp. 455474

Constantinou, M.C. (2001). New Developments in the Field of Seismic Isolation, Proceedings of 2001 Structures Congress, ASCE, Washington, DC. 
European Committee for Standardization (2000). Structural Bearings, European Standard EN 1337-1, Brussels.

Fenz, D.M. and Constantinou, M.C. (2006). Behavior of the Double Concave Friction Pendulum Bearing, Earthquake Engineering and Structural Dynamics, Vol. 35, No. 11, pp. 1403-1424.

Fenz, D.M. and Constantinou, M.C. (2008). Spherical Sliding Isolation Bearings with Adaptive Behavior: Theory, Earthquake Engineering and Structural Dynamics, Vol. 37, No. 2, pp. 163-183.

Fenz, D.M. and Constantinou, M.C. (2008). Spherical Sliding Isolation Bearings with Adaptive Behavior: Experimental Verification, Earthquake Engineering and Structural Vibration, Vol. 37, No.2, pp. 185-205.

Hall, J.F., Heaton, T.H., Halling, M.W. and Wald, D.W.J. (1995). Near-Source Ground Motion and Its Effects on Flexible Buildings, Earthquake Spectra, 11(4), pp. 569-605

Hall, J.F. and Ryan, K.L. (2000). Isolated Buildings and the 1997 UBC Near-Source Factors, Earthquake Spectra, 16(2), pp. 393-411

Inaudi, J.A. and Kelly, J.M. (1993). Optimum Damping in Linear Isolation Systems, Earthquake Engineering and Structural Dynamics, 22, pp. 583-598

International Code Council (2000). International Building Code, Falls Church, Virginia.

Kelly, J.M. (1986). Aseismic Base Isolation: A Review and Bibliography, Soil Dynamics and Earthquake Engineering, 5, 202-216

Kelly, J.M. (1993). State-of-the-Art and State-of-the-Practice in Base Isolation, Proceedings of ATC-17-1 Seminar on Seismic Isolation, Passive Energy Dissipation, and Active Control, San Francisco, California, pp.9-28

Kelly, J.M. (1997). Earthquake Design with Rubber, Springer-Verlag, Inc.

Kelly, J.M. (1999). The Role of Damping in Seismic Isolation, Earthquake Engineering and Structural Dynamics, 28, pp. 3-20.

Kelly, J.M. (1999). The Current State of Base Isolation in United States, Proceedings of the 2nd World Conference on Structural Control, Kyoto, Japan, 1, 1043-1052

Kelly, J.M. (2004), Chapter 11, Seismic Isolation, in Earthquake Engineering, Borzognia \& Bertero (eds), CRC Press LLC.

Komodromos, P.I. (2000). Seismic Isolation for Earthquake-Resistant Structures, Southampton, UK : WIT Press

Lee, G.C., Ou Y.-C, Liang Z., Niu T., Song J. (2007). Principles and Performance of Roller Seismic Isolation Bearings for Highway Bridges, MCEER Technical Report MCEER-07-0019, December 2007.

Lee, G.C., Ou, Y.-C., Niu, T., Song, J., and Liang, Z. (2010). Characterization of a Roller Seismic Isolation Bearing with Supplemental Energy Dissipation for Highway Bridges, Journal of Structural Engineering, ASCE, 136(5), 502-510, May 2010.

Liang, Z. and Lee, G. C. (1991). Damping of structures, Part 1, Theory of complex Damping. Technical Report NCEER-91-0004, State University of New York at Buffalo.

Liang, Z., Lee, G. C., Dargush, G. and Song, J. W. (2012). Structural Damping: Applications in Seismic Response Modification. CRC Press, 2012. 
Lin, T.W., Hone, C.C. (1993). Base Isolation by Free Rolling Rods under Basement. Earthquake Engineering and Structural Dynamics, 22, pp. 261-273.

Mosqueda, G., Whittaker, A.S. and Fenves, G.L. (2004). Characterization and Modeling of Friction Pendulum Bearings Subjected to Multiple Components of Excitation. Journal of Structural Engineering, 130(3), pp. 433-442.

Mostaghel, N. and Khodaverdian, M. (1987). Dynamics of Resilient-Friction Base Isolator (RFBI), Earthquake Engineering and Structural Dynamics, 11, pp. 729-748.

Naeim, F. and Kelly, J.M. (1999). Design of Seismic Isolation Structures from Theory to Practice, John Wiley \& Sons Inc.

Nowak, A. S. and Collins, K. R. (2000) Reliability of Structures McGraw Hill

Ou, Y.-C., Song, J., and Lee, G.C. (2010). A parametric study of seismic behavior of roller seismic isolation bearings for highway bridges. Earthquake Engineering and Structural Dynamics, 39(5), 541-559,April 2010.

Robinson, W.H. (1982). Lead-Rubber Hysteretic Bearings Suitable for Protecting Structures During Earthquakes, Earthquake Engineering and Structural Dynamics, 10, pp. 593-604.

Roussis, P.C. and Constantinou, M.C. (2006). Uplift-Restraining Friction Pendulum Seismic Isolation System, Earthquake Engineering and Structural Dynamics, Vol. 35, No. 5, April 2006, pp. 577-593.

Skinner, R.I., Robinson, W.H. and McVerry, G.H. (1993). An Introduction to Seismic Isolation, John Wiley \& Sons Ltd.

Soong, T.T. and Dargush, G. F. (1997) Passive Dissipation Systems in Structural Engineering John Wiley \& Sons, New York,

Structural Engineers Association of Northern California (SEAONC) (1986). Tentative Seismic Isolation Design Requirements, Yellow Book.

Takewaki, I. (2009) Building Control with passive Dampers, Optimal Performance-based Design for Earthquakes, ohn Wiley \&

Thompson, A.C.T., Whittaker, A.S., Fenves, G.L. and Mahin, S.A. (2000). Property Modification Factors for Elastomeric Seismic Isolation Bearings, Proc. 12th World Conference on Earthquake Engineering, New Zealand.

Tsopelas, P. and Constantinou, M.C. (1997). Study of Eiastoplastic Bridge Seismic Isolation System, Journal of Structural Engineering, Vol. 123, No.4, pp. 489-498.

Tsopelas, P., Constantinou, M.C., Kim, Y-S., and Okamoto, S. (1997). Experimental Study of FPS System in Bridge Seismic Isolation. Structural Engineering and Structural Dynamics, Vol. 25, No. 1, pp. 65-78.

Wang, J. (2005). Seismic Isolation Analysis of a Roller Bearing Isolation System, Ph.D. Dissertation, University at Buffalo, State University of New York, Buffalo, NY, July 2005.

Whittaker, A.S., Constantinou, M.C., and Tsopelas, P. (1998). Displacement Estimates For Performance-Based Seismic Design, Journal of Structural Engineering, Vol. 124, No.8, pp. 905-912.

Zayas, V.A., Low, S.S. and Mahin, S.A. (1990). A Simple Pendulum Technique for Achieving Seismic Isolation, Earthquake Spectra, 6, pp. 317-334 
Zhou, Q., Lu, X.L., Wang, Q.M., Feng, D.G., Yao, Q.F. (1998). Dynamic Analysis on Structures Base-isolated by a Ball System with Restoring Property. Earthquake Engineering and Structural Dynamics, 27, pp. 773-791. 


\title{
Advanced Base Isolation Systems for Light Weight Equipments
}

\author{
Chong-Shien Tsai \\ Department of Civil Engineering, Feng Chia University, Taichung, \\ Taiwan
}

\section{Introduction}

This chapter is intended to introduce the earthquake proof technology particularly in the area of base isolation systems that have been used to protect light weight structures, such as motion sensitive equipment, historic treasures, and medical instruments, etc., from earthquake damage. This chapter presents theoretical background, experimental studies, numerical analyses, and the applications of the advanced isolation systems consisting of rolling- and sliding-type isolation systems for light weight structures. The efficiency of these isolators in reducing the seismic responses of light weight equipment was also investigated in this study. In addition, the results from theoretical and experimental studies for these isolators are compared and discussed.

\subsection{General background}

One of the greatest casualties in recorded history is the Huaxian earthquake that occurred in China in 1556, causing over 830,000 deaths (Kanamori, 1978). The Tangshan great earthquake that struck the northeastern part of China in 1976 killed 242,769 people, according to official sources, although some estimates of the death toll are as high as 650,000 (Kanamori, 1978). The Mw 7.0 earthquake (Eberhard et al., 2010) that struck the Republic of Haiti on January 12, 2010, resulted in a death toll, as reported by the Government of Haiti, that exceeds 217,000, with an additional 300,000 people injured. The earthquake damaged nearly 190,000 houses, of which 105,000 were completely destroyed, and left long term suffering for the residents of the country. The moment magnitude 9.0 Tohoku earthquake (Takewakin et al., 2011) that struck eastern Japan on March 11, 2011, is one of the most five powerful earthquakes in the world since modern measurements began in 1900, killing more than 20,000 people and causing huge damage and economic loss that cannot be ignored.

Traditionally designed structures have used the strength and ductility of their structural members to resist the seismic forces or dissipate earthquake induced energy. However, many past earthquakes have proven that structures collapse or lose their functionality when the ductility capacity of the structures is consumed during the earthquake. Even if the structures survive earthquakes through excellent designs to provide more strength or ductility to the structures, the vibration sensitive equipment located in the structures may still lose its functionality due to floor accelerations. 
Several techniques exist to minimize earthquake effects on structures, such as light-weight structure design, improving the ductility capacities of structures, and structural control (earthquake proof technology), etc. Structural control technology has been recognized as an effective tool in seismic mitigation, and can be classified as active, passive, hybrid and semiactive controls, which can be clarified by the following equation:

$$
M \ddot{u}+C \dot{u}+K u=-M B \ddot{u} g+F(\ddot{u}, \dot{u}, u, t)
$$

where $\mathrm{M}, \mathrm{C}$, and $\mathrm{K}$ are the mass, the damping, and stiffness matrices, respectively, of a structure, which are the natural characteristics of a structure; $\ddot{u}, \dot{u}$ and $u$ denote the vectors of the relative acceleration, velocity, and displacement with respect to supports, respectively, which are structural responses during earthquakes; $\ddot{u}_{g}$ is the ground acceleration; $\mathrm{B}$ is the displacement transformation matrix; and $F(\ddot{u}, \dot{u}, u, t)$ depicts the control force that is an external force provided by various types of power and control systems.

Active control technology has a control force used to activate the control system. The control force is generated through the control signal which is based on the results calculated from the measured responses of the structure and the specified control algorithm. The structural responses can be lessened by changing its characteristic through the control force in the second term on the right hand side of Eq. (1) which could be proportional to the measured displacement, velocity and acceleration of the structure during earthquakes. From a mathematical point of view, we can then move the control force from the right hand side to the left hand side of Eq. (1) to combine with the corresponding terms depending on the values of the control fore proportional to. As a result, the mass, damping, and stiffness matrices of a structure are modified by the control force, but not by an actual device.

In the passive control, there is no external control force, $F(\ddot{u}, \dot{u}, u, t)$, in the system, which means that there is no second term on the right hand side of Eq. (1). The mass, damping, or stiffness which are the first three terms on the left hand side are modified by adding actual devices to the structure (Soong and Dragush, 1997; Takewakin, 2009). The device used to modify the mass matrix is named the tuned mass damper. Any actual devices used to modify the second and third terms on the left hand side of Eq. (1) are called energy absorbing systems (or dampers). A device such as a fluid damper producing an internal force that is strongly dependent on the relative velocity between the two ends of the device is called a velocity dependent device (damper). On the other hand, a device such as a friction and yielding dampers producing an internal force that is strongly dependent on the relative displacement between the two ends of the device is called a displacement dependent device (damper). Usually, the velocity dependent device produces minimum internal forces at the moments of maximum displacement due to zero velocity, which means that this type device provides no damping effect to the structure while the structure deforms at critical moments of earthquakes. On the other hand, the displacement dependent device produces maximum internal forces at maximum displacements. This means that this type device can provide maximum damping effect at the moments of maximum displacement and immediately reduce the structural responses at the most critical time of structural responses. The discussions of the advantages and disadvantages of these two types of devices are out of the scope of this chapter. 
A system called the base (seismic) isolation system inserts a soft layer or device (base isolator) between the structure and its foundation to isolate earthquake-induced energy trying to penetrate into the structure, thereby protecting the structure from earthquake damage (Skinner et al., 1993; Naeim and Kelly, 1999). A base isolation system is used to minimize the seismic force which is the first term on the right hand side of Eq. (1) in two ways: (i) by reflecting the seismic energy by lengthening the natural period of the entire system including the structure and the base isolator and (ii) by absorbing the seismic energy through the hysteretic loop of the isolator displacement and the force induced in the isolator. The combination of the active and passive control is called hybrid control, which also needs a large control force for controlling structural responses. By contrast, semi-active control uses substantially smaller control force in the manner of an on and off switch to improve the efficiency of the passive control system through an active control algorithm, but not massive control force. In conclusion, structural control technology protects structures through mechanisms that are used to prohibit the seismic energy from transmitting into major members such as the beams, columns and walls of a structure, which are used for supporting structural weight.

In the past, there have been a lot of papers and reports concerning the use of vibration isolation technology to increase the precision of machines by isolating vibration sources resulting from the environment, such as moving vehicles, or for improving human comfort by isolating vibration sources that result from machines and moving vehicles (Rivin, 2003). Recently, the isolation technology has been acknowledged as an effective technique to promote the earthquake resistibility of the structures by controlling structural responses during earthquakes on the basis of theoretical and experimental results and earthquake events (Naeim and Kelly, 1999). Several theoretical studies have been made on the applications of the base isolation technology to critical equipment in seismic mitigation (Alhan and Gavin, 2005; Chung et al., 2008). However, little attention has been given to the experimental study of the efficiency of base isolation on the protection of vibration sensitive equipment in the events of earthquakes, especially for experimental investigations under tridirectional seismic loadings (Tsai et al., 2005b, 2007, 2008a; Fan et al., 2008).

This chapter is aimed at the seismic isolation, especially for the equipments in hospitals and facilities of emergency departments used for saving peoples' lives. These are of extreme importance and should be kept functional during and after earthquakes.

\subsection{Background of rolling types of bearings}

To the best of the author's knowledge, the isolation system with doubled spherical concave surfaces and a rolling ball located between these two concave surfaces was first patented by Touaillon in 1870, as shown in Fig. 1(a). Several similar isolation systems with a ball located between two spherical concave surfaces were also proposed (Schär, 1910; Cummings, 1930; Bakker, 1935; Wu, 1989), as shown in Figs. 1(b)-1(e). In 1997, Kemeny propounded a ball-incone seismic isolation bearing that includes two conical concave surfaces and a ball seated between the conical surfaces, as shown in Fig. 1(f). The dynamic behavior of the ball-in-cone isolation system has been investigated (Kasalanati et al., 1997). In addition, Cummings (1930) also proposed a seismic isolation system with a rolling rod of a cylinder sandwiched between two concave surfaces, as shown in Fig. 1(c). Lin and Hone (1993), Tsai et al. (2006b) and M. H. Tsai et al. (2007) conducted research on the effectiveness of this type of base isolation system in seismic mitigation, as shown in Figs. 2(a) and 2(b). Kim (2004) proposed 
a seismic isolation system that has rollers of a bowling shape to roll in the friction channel, as shown in Fig. 2(c). Tsai (2008a, b) revealed seismic isolation systems each consisting of shafts rolling in the concave slot channels, as shown in Figs. 2(a) and 2(d). These devices are capable of resisting the uplift while the vertical force in the isolator becomes negative under severe earthquakes.

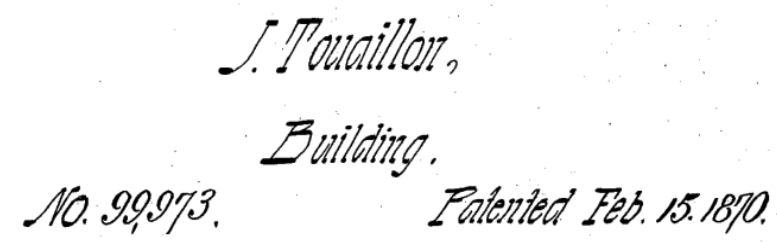

\section{Fig. 1}

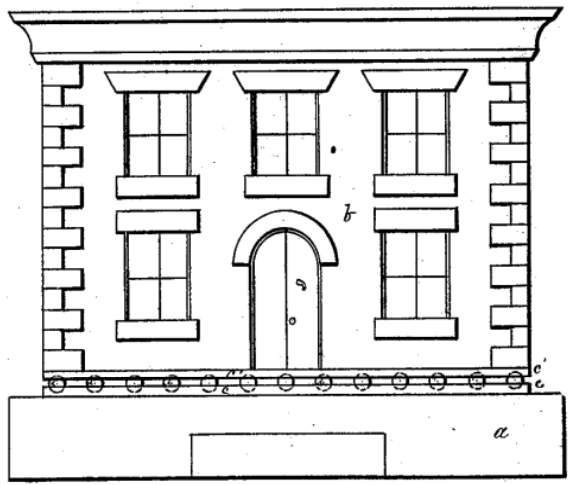

Fig 2

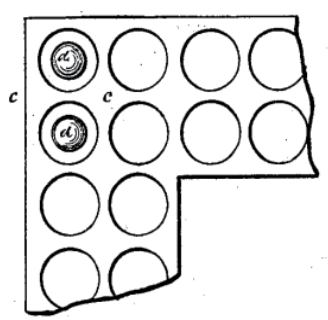

rig. 3

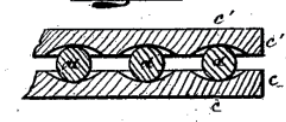

Fio. 4

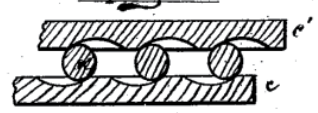

Wilnesses
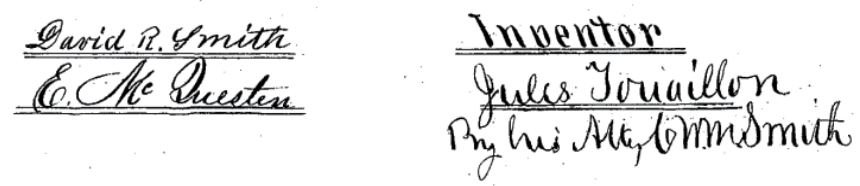

Fig. 1(a). Touaillon's original patent (1870) 
F. SCHÄR,

FOUNDATION FOR BUILDINGS.

951,028 .

APPLIOATION FILED JULY 27, 1909.

Fig. 1

Patented Mar. 1, 1910.
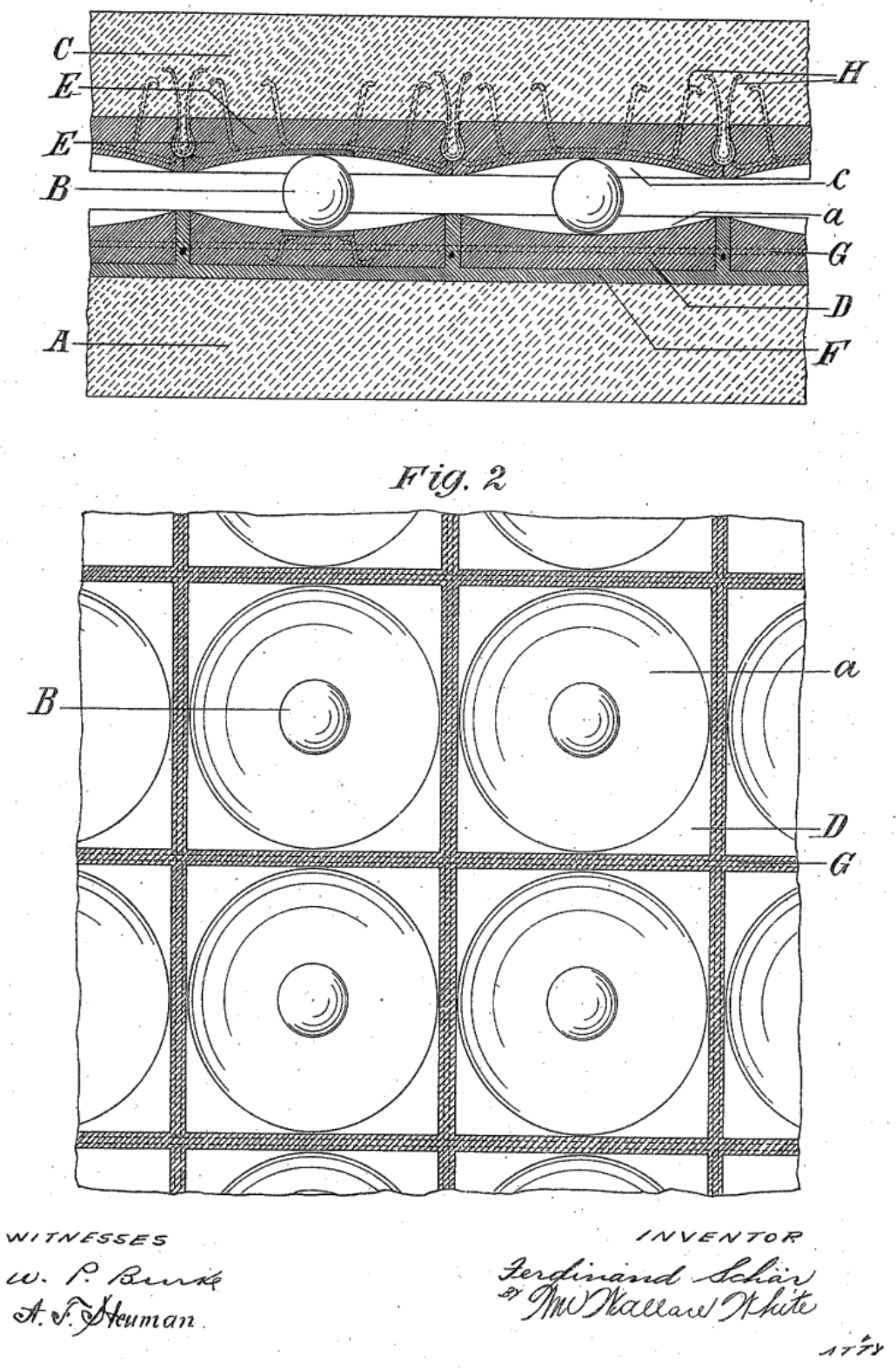

Fig. 1(b). Schär's original patent (1910) 
June 3, 1930.

F. D. CUMMINGS

$1,761,659$

BUILDING CONSTRUCTION (QUAKEPROOF BUILDING)

Filed Jan. 18, 1928

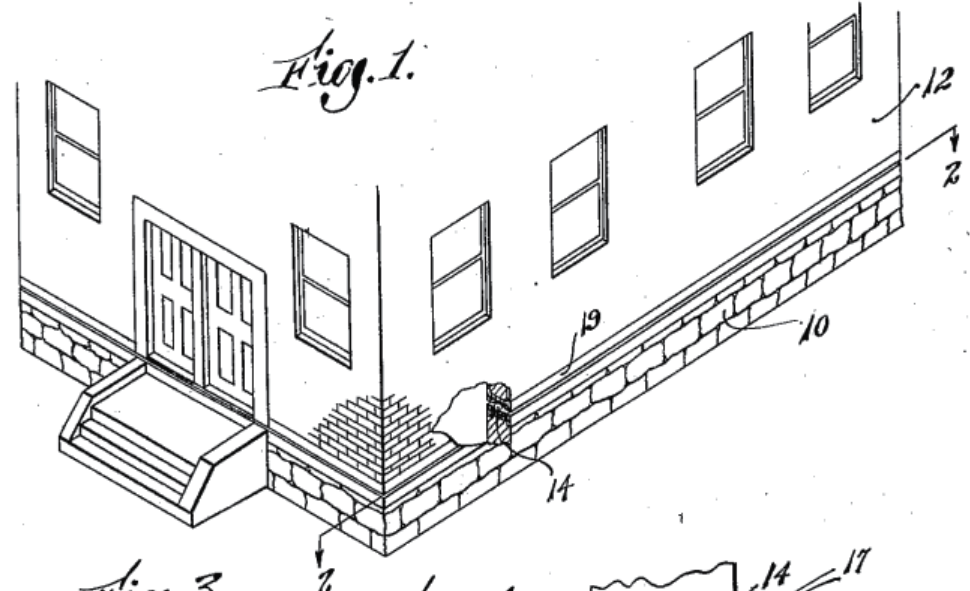

Higl.3.
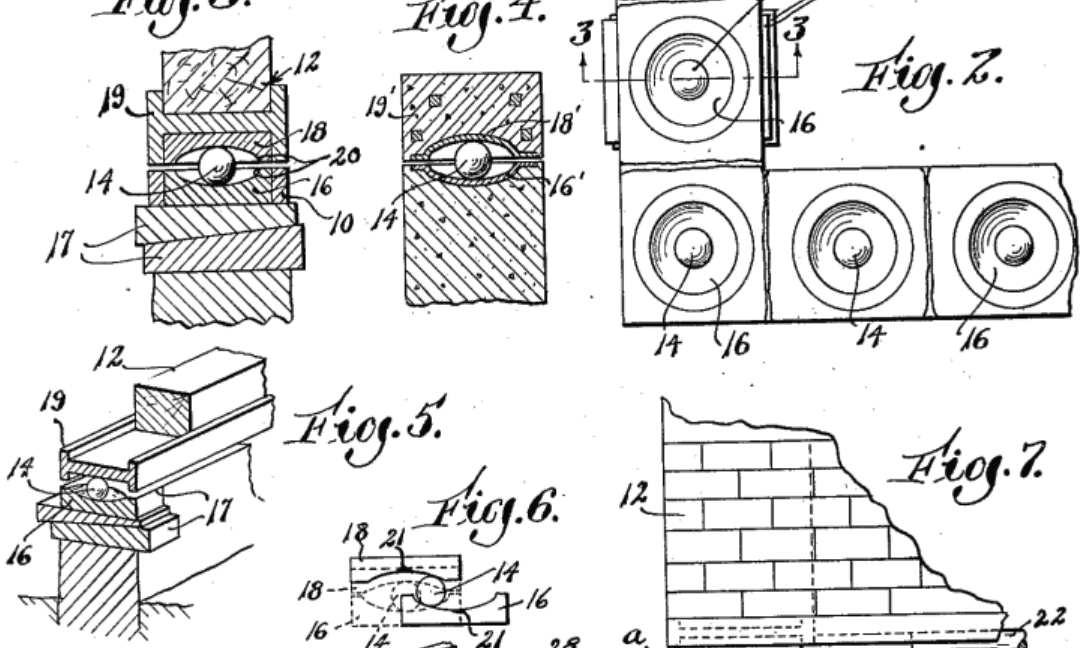

Hiog.5.
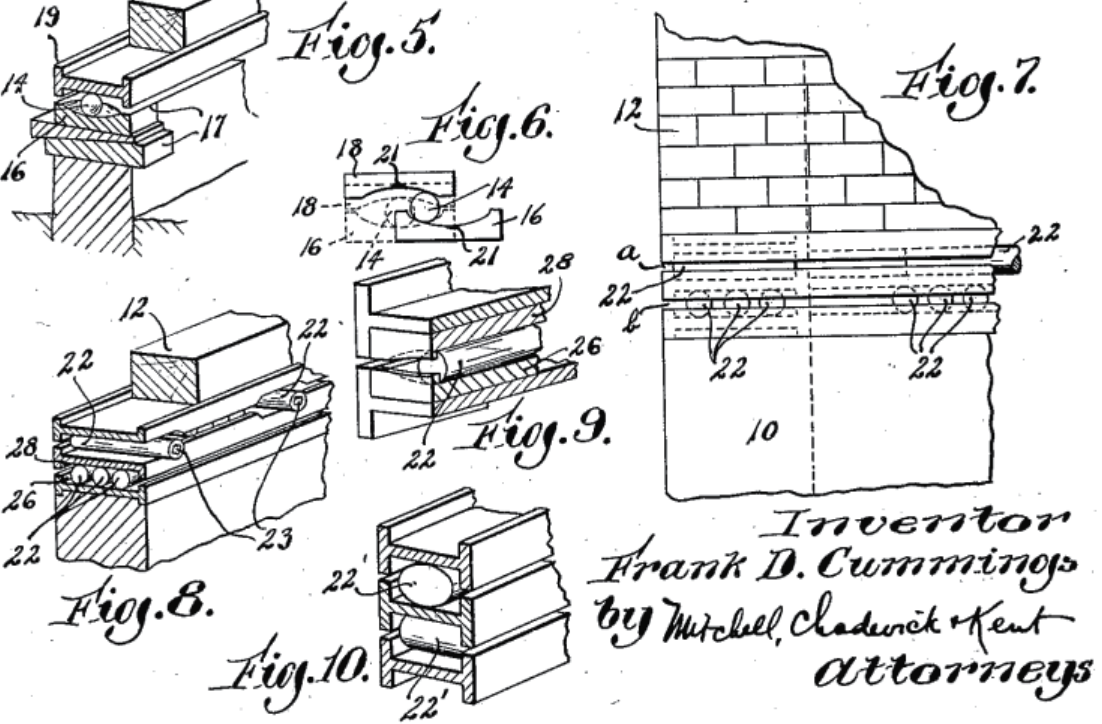

Fig. 1(c). Cummings' original patent (1930) 


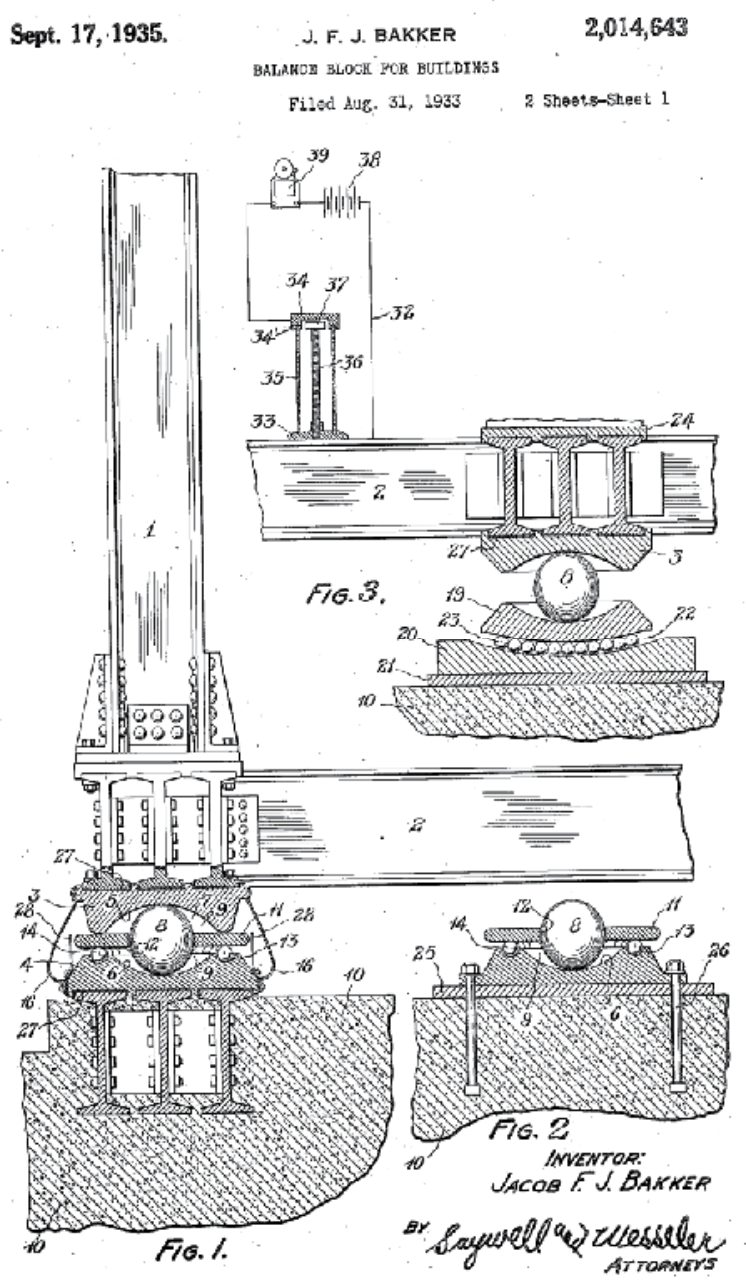

Fig. 1(d). Bakker's original patent (1935)

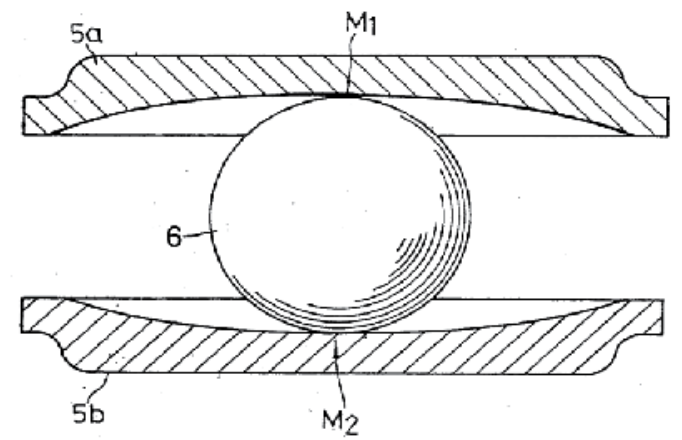

Fig. 1(e). Wu's original patent (1989) 


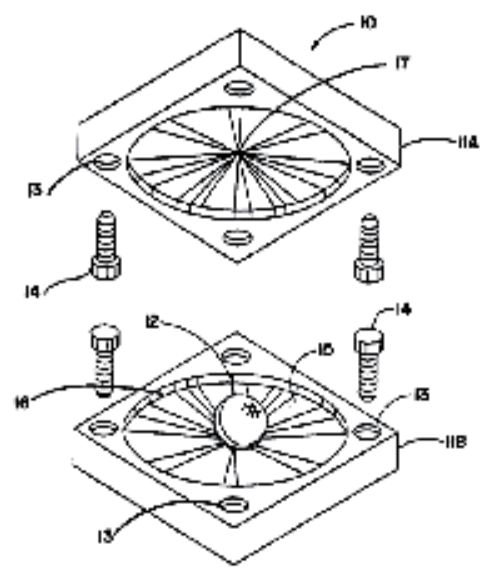

Fig. 1(f). Kemeny's original patent (1997)

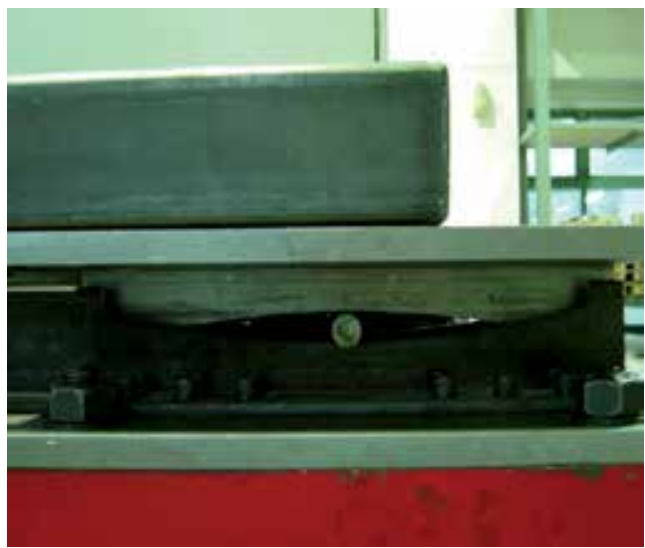

Fig. 2(a). Rolling rod isolation system

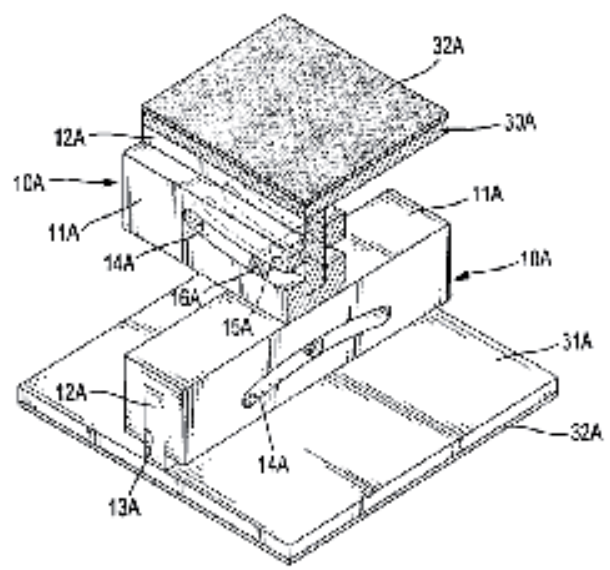

Fig. 2(b). Tsai's original patent (2008a) 


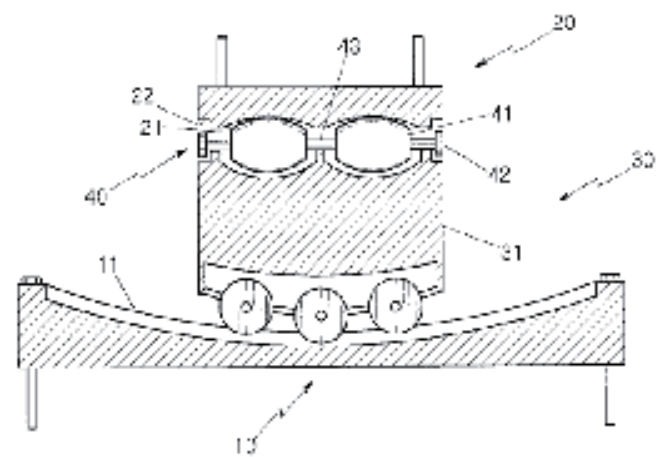

Fig. 2(c). Kim's original patent (2004)

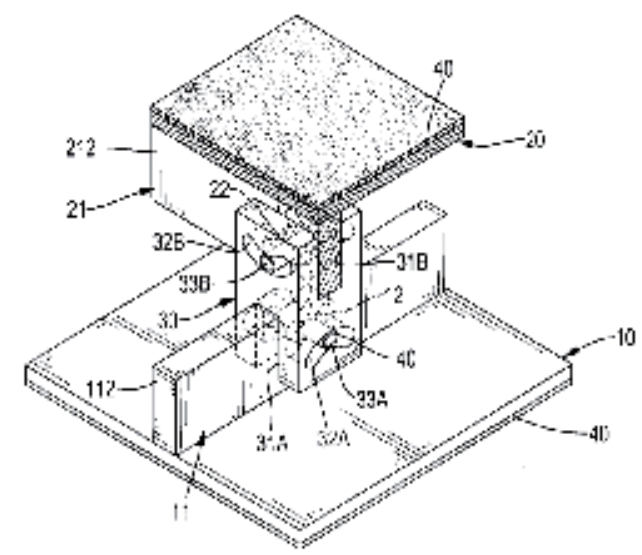

Fig. 2(d). Tsai's original patent (2008b)

The isolation system with two concave surfaces and a rolling ball (Touaillon, 1870; Wu, 1989; Kemeny, 1997) possesses some shortcomings even under small loadings like equipment and medical instruments, such as negligible damping provided by the system, a highly concentrated stress resulted from the weight of the equipment on the rolling ball and the concave surfaces due to the small contact area, and scratches and damage to the concave surfaces caused by the ball rolling motions during earthquakes. The rolling ball has a tendency to move even under environmental loadings such as human activities during regular services. In addition, the bearing size is large because of the large bearing displacements under seismic loadings due to insufficient damping provided by the rolling motion of the ball on the concave surfaces in the system.

To supply more damping to the isolation system and simultaneously reduce the bearing size as a consequence of smaller bearing displacements during earthquakes, Tsai et al. (2006a) proposed a ball pendulum system (BPS). As shown in Figs. 3(a) and 3(b), this system comprises two spherical concave surfaces and a steel rolling ball covered with a special damping material to provide horizontal and vertical damping to tackle the problems mentioned above. A series of shaking table tests conducted by Tsai et al. (2006a) have proven that the BPS isolator can enhance the seismic resistibility of vibration sensitive equipment under severe earthquakes with smaller displacements compared to an isolation 
system with negligible damping. However, the special material covering the steel ball that supports the weight of the vibration sensitive equipment for a long period of time in its service life span might result in permanent deformation due to plastic deformation in the damping material. It may damage or flat the contacting surface of the special damping material after sustaining a certain period of service loadings and affect the isolation efficiency.

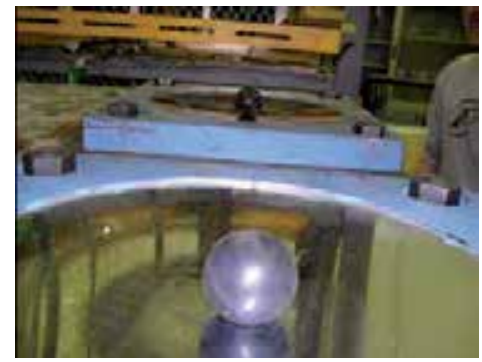

Fig. 3(a). Open-up view of ball pendulum isolation system

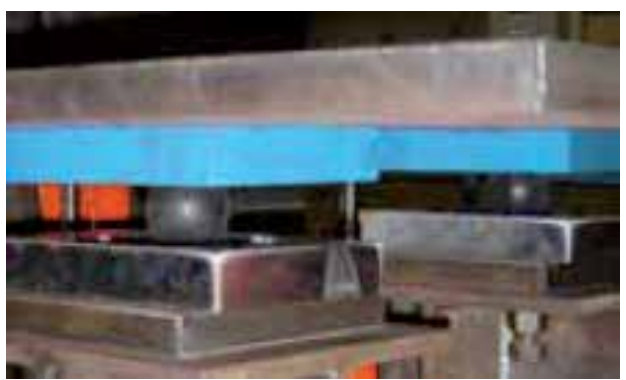

Fig. 3(b). Test set-up for ball pendulum isolation system

An alternative approach for increasing damping and lessening the isolator displacement is to add a damping device to the isolation system (Fan et al., 2008). Fathali and Filiatrault (2007) presented a spring isolation system with restraint which is a rubber snubber to play a role of displacement restrainer to limit the isolator displacement. In general, the displacement restrainer will involve impact mechanisms as a result of contact made with isolated equipment, which lead to amplified acceleration responses and large dynamic forces.

To increase damping for a rolling bearing and to prolong the service life of a bearing, an isolation system called the static dynamics interchangeable-ball pendulum system (SDIBPS) shown as Figures 4(a) and 4(b) was proposed by Tsai et al. (2008a). The SDI-BPS system consists of not only two spherical concave surfaces and a steel rolling ball covered with a special damping material to provide supplemental damping and prevent any damage and scratches to the concave surfaces during the dynamic motions induced by earthquakes but also several small steel balls that are used to support the static weight to prevent any plastic deformation or damage to the damping material surrounding the steel rolling ball during the long term of service loadings. Because the concave surfaces are protected by the damping material covering the steel ball from damage and scratches, they may be designed as any desired shapes in geometry, which can be spherical, conical or 
concave surfaces with variable radii of curvature. The natural period of the SDI-BPS isolator depends only on the radii of curvature of the upper and lower concave surfaces, but not a function of the vertical loading (static weight). It can be designed as a function of the isolator displacement, and predictable and controllable for various purposes of engineering practice.

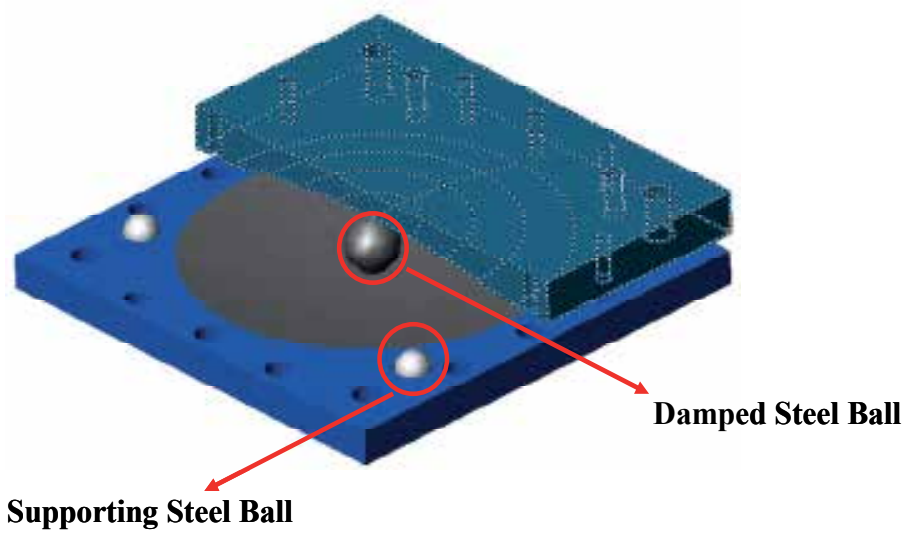

Fig. 4(a). Exploded perspective view of static dynamics interchangeable-ball pendulum system (SDI-BPS)

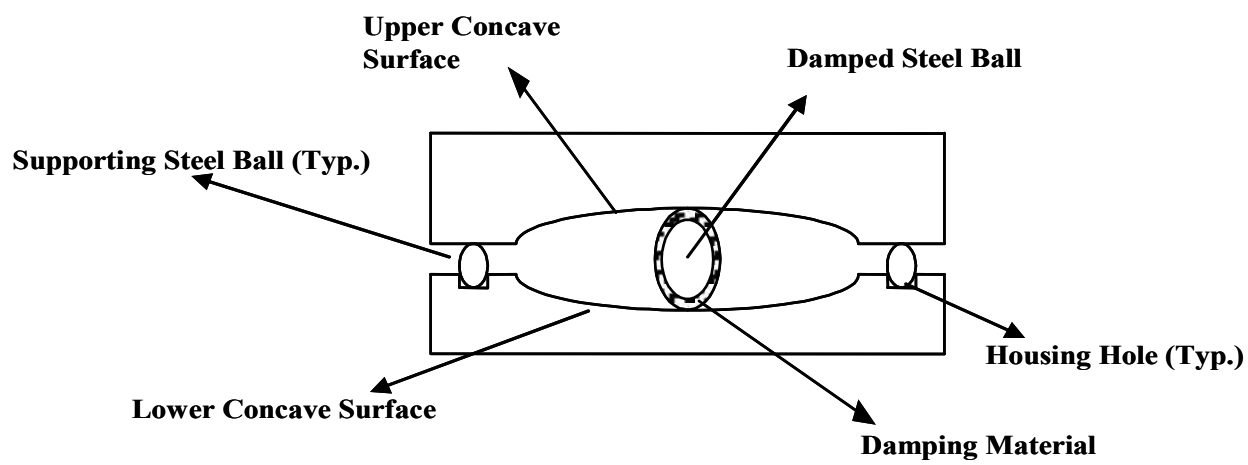

Fig. 4(b). Cross-sectional view of SDI-BPS

\subsection{Background of sliding types of bearings}

Most sliding types of isolation systems are suitable not only for light weight structures such as equipment and medical instruments but also for very heavy structures such as buildings and bridges. These types of bearings provide damping through frictional mechanism between sliding surfaces. As shown in Fig. 5(a), Penkuhn (1967) proposed a sliding isolation system including a concave sliding surface and a universal joint to accommodate the rotation resulted from the superstructure and the sliding motion of the universal joint, and suggested that the superstructure be supported by a rigid supporting base which was in turn supported by three proposed isolation bearings. Zayas (1987) and Zayas et al. (1987) 
proposed a friction pendulum system (FPS) with a concave sliding surface and an articulated slider, as shown in Figs 5(b) and 5(c). Through extensive experimental and numerical studies, the FPS isolator has proven to be an efficient device for reducing the seismic responses of structures (Zayas et al., 1987; Al-Hussaini et al., 1994).

Oct. 17, 1967

A. L. K. PENKUHN

$3,347,002$

THREE FOIKT FOUNDATION YOR EUILDIKG STMUGTURES

Original F1led Fob. 12, 1964
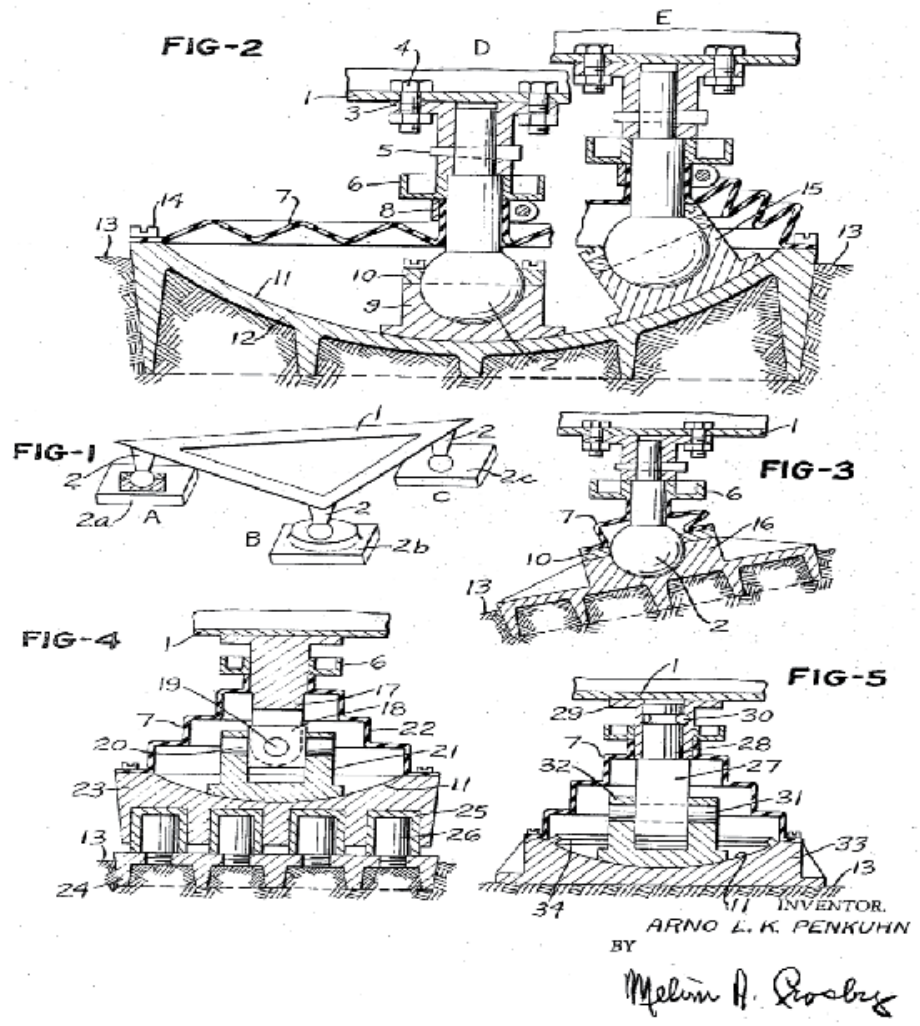

Fig. 5(a). Penkuhn's original patent (1967)

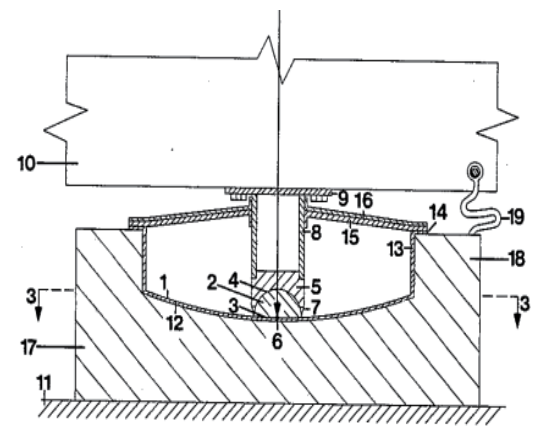

Fig. 5(b). Cross-sectional view of the friction pendulum system 


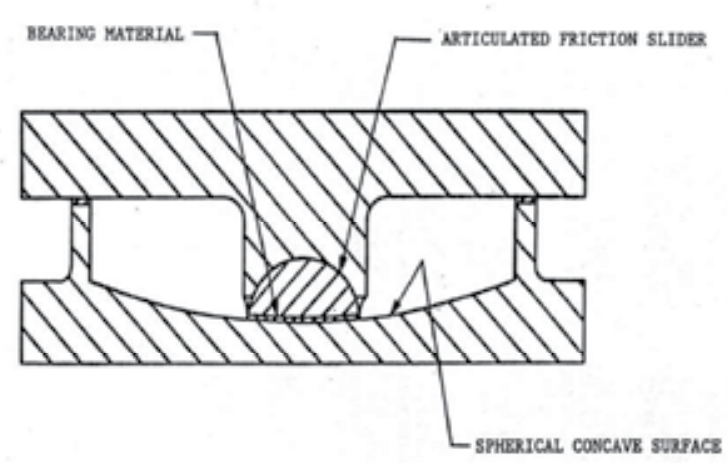

Fig. 5(c). Zayas's original patent (1987)

To avoid the possibility of resonance of the isolator with long predominant periods of ground motions, Tsai et al. (2003a) presented an analytical study for a variable curvature FPS (VCFPS). In order to enhance the quakeproof efficiency and reduce the size of the FPS isolator, Tsai $(2004 a, b)$ and Tsai et al. (2003b, 2005a,b, 2006c) proposed a sliding system called the multiple friction pendulum system (MFPS) with double concave sliding surfaces and an articulated slider located between the concave sliding surfaces, as shown in Figs. 6(a)-6(f). Based on this special design, the displacement capacity of the MFPS isolator is double of the FPS isolator that only has a single concave sliding surface, and the bending moment induced by the sliding displacement for the MFPS isolator is an half of that for the FPS isolator. Moreover, the fundamental frequency is lower than that of the FPS as a result of the series connection of the doubled sliding surfaces in the MFPS isolation system, and the bearing is a completely passive apparatus, yet exhibits adaptive stiffness and adaptive damping by using different coefficients of friction and radii of curvature on the concave sliding surfaces to change the stiffness and damping to predictable values at specifiable and controllable displacement amplitudes. Hence, the MFPS device can be given as a more effective tool to reduce the seismic responses of structures even subjected to earthquakes with long predominant periods, and be more flexible in design for engineering practice. In addition, Fenz and Constantinou (2006) conducted research and published their results on this type of base isolation system with double sliding surfaces. Kim and Yun (2007) reported the seismic response characteristics of bridges using an MFPS with double concave sliding interfaces.

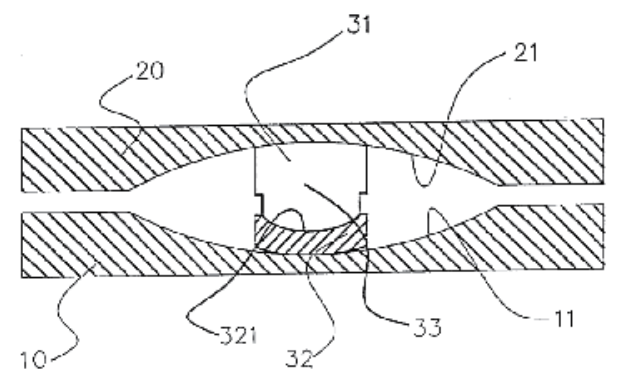

Fig. 6(a). Tsai's original patent (2004a) 


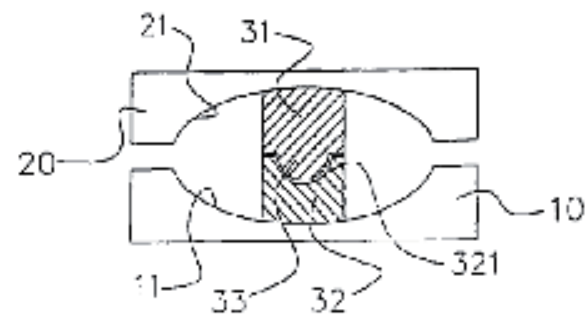

Fig. 6(b). Tsai's original patent (2004a)

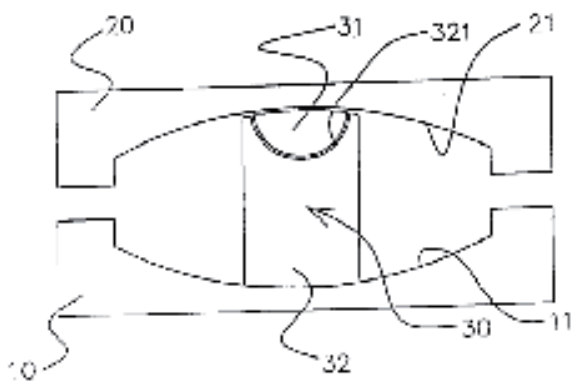

Fig. 6(c). Tsai's original patent (2004a)

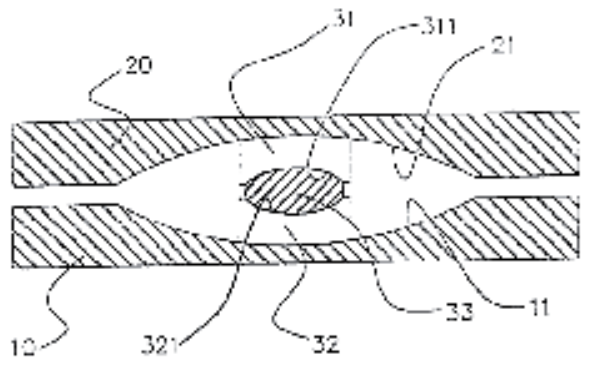

Fig. 6(d). Tsai's original patent (2004b)

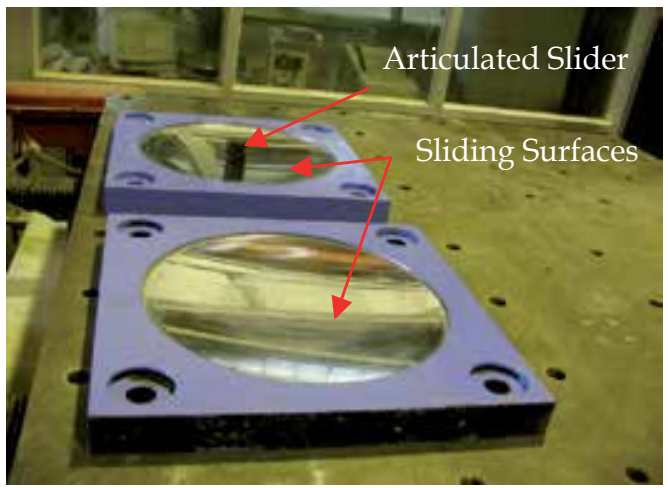

Fig. 6(e). Open-up view of MFPS isolator 


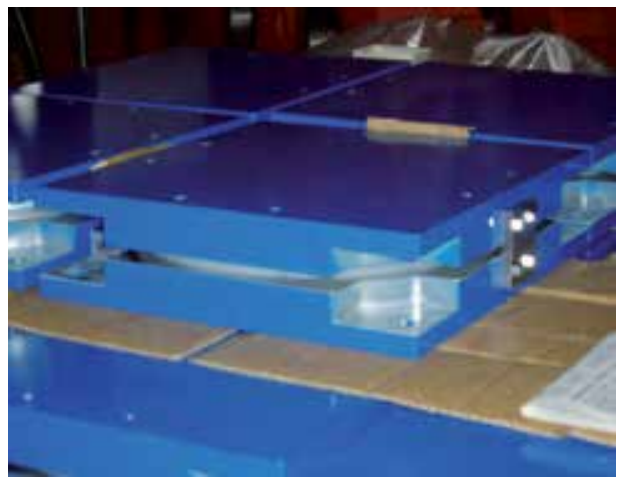

Fig. 6(f). Assembled MFPS isolator

Furthermore, Tsai (2003) proposed several other types of MFPS isolators, as shown in Figs. 7(a)-7(f), each with multiple sliding interfaces, which essentially represent that each FP isolation system above and below the slider has multiple sliding interfaces connected in series (Tsai et al., 2008b, 2010a). Fenz and Constantinou (2008a, b) published their research on the characteristics of an MFPS isolator with four sliding interfaces under unidirectional loadings. Morgan and Mahin $(2008,2010)$ investigated the efficiency of an MFPS isolator with four concave sliding interfaces on seismic mitigation of buildings. As shown in Figs. 7(g) and 7(h), Tsai et al. (2010a, b) proposed an MFPS isolator with numerous sliding interfaces (any number of sliding interfaces). As explained earlier, these types of bearings, each having $N$ number of sliding interfaces, possess adaptive features of stiffness and damping by adopting different coefficients of friction and radii of curvature on the concave sliding surfaces to result in changeable stiffness and damping at specified displacement amplitudes. Tsai et al. (2011a) published experimental investigations on the earthquake performance of these types of friction pendulum systems. The efficiency of the MFPS isolator with multiple sliding interfaces in mitigating structural responses during earthquakes has been proven through a series of shaking table tests on a full scale steel structure.

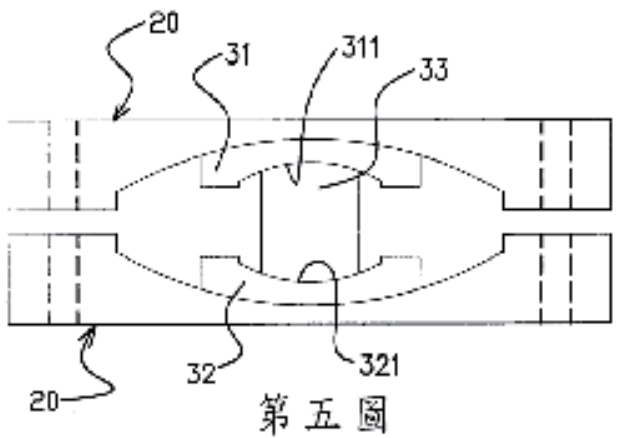

Fig. 7(a). Tsai's original patent (2003) 


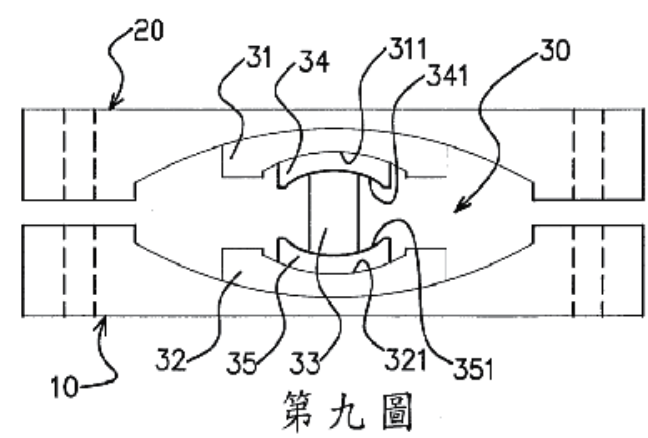

Fig. 7(b). Tsai's original patent (2003)

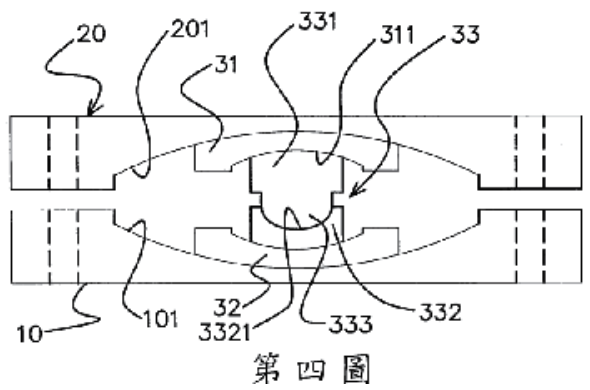

Fig. 7(c). Tsai's original patent (2003)

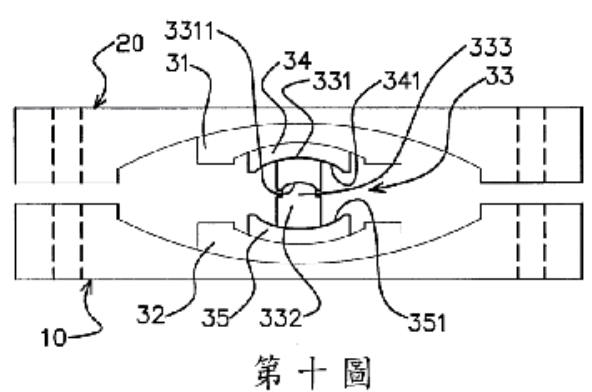

Fig. 7(d). Tsai's original patent (2003)

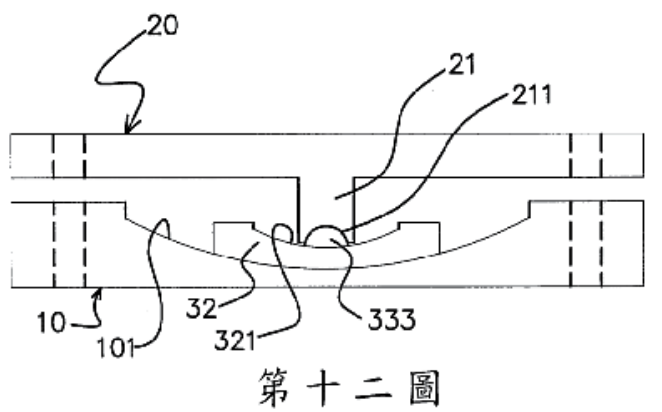

Fig. 7(e). Tsai's original patent (2003) 


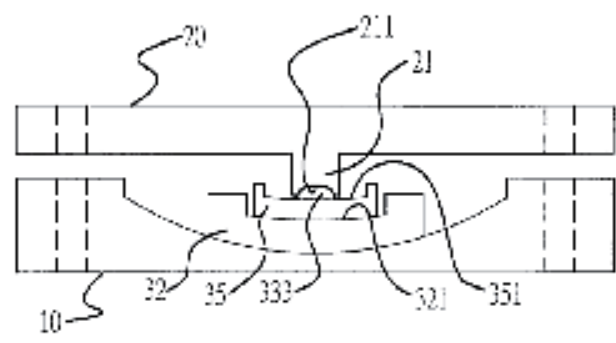

$$
\text { 第二 - 或 }
$$

Fig. 7(f). Tsai's original patent (2003)

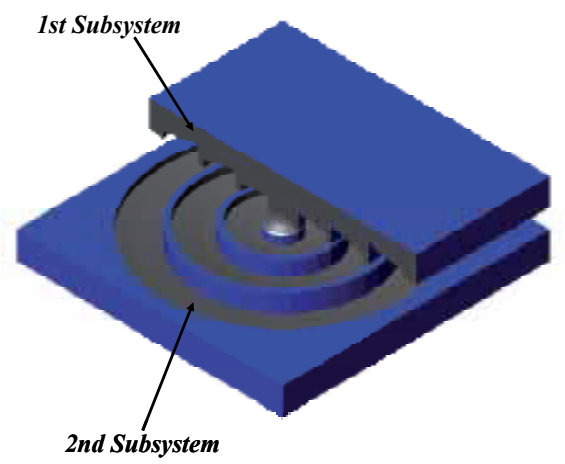

Fig. 7(g). Exploded view of MFPS isolator with six sliding surfaces

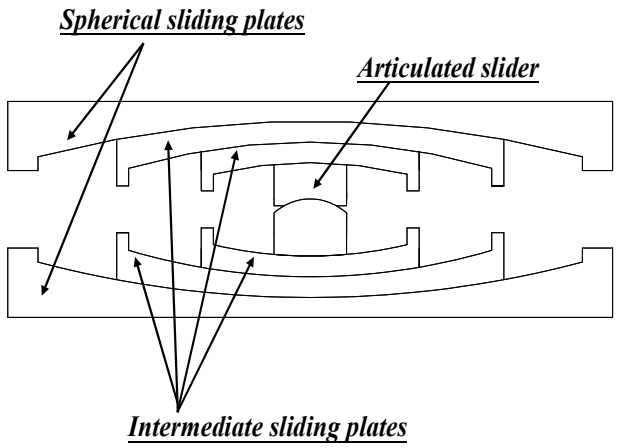

Fig. 7(h). Cross-sectional view of MFPS isolator with six sliding surfaces

An isolation system, as shown in Fig. 8(a) and called the XY-FP isolator, consisting of two orthogonal concave beams interconnected through a sliding mechanism has been published by Roussis and Constantinou (2005). The FP isolator (XY-FP) possesses the uplift-restraint property by allowing continuous transition of the bearing axial force between compression and tension, and has different frictional interface properties under compressive and tensile normal force in the isolator. A device, as shown Fig. 8(b), similar to the design concept but without the uplift-restraint property was also proposed by Tsai (2007). This device has an 
articulated slider seated between the FP bearings in the $\mathrm{X}$ and $\mathrm{Y}$ directions to accommodate the rotation as a result of sliding motion of the articulated slider and to maintain the isolated structure standing vertically during earthquakes.

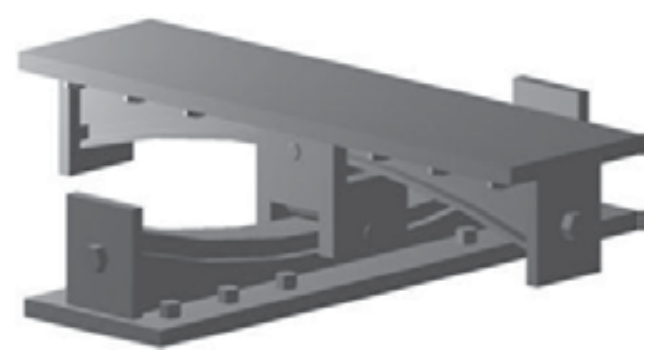

Fig. 8(a). X-Y (Adatped from Roussis and Constantinou 2005)

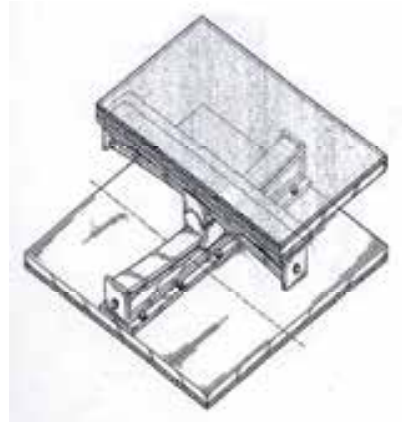

Fig. 8(b). Tsai's original patent (2007)

Tsai et al. (2010c) proposed a trench friction pendulum system (TFPS), as shown in Figs. 9(a) and $9(\mathrm{~b})$, that consists of one trench concave surface in each of two orthogonal directions, and an articulated slider situated between the trench concave surfaces to accommodate the rotation induced by the sliding motion of the slider. The TFPS possesses independent characteristics such as the natural period and damping effect in two orthogonal directions, which can be applied to a bridge, equipment or a structure with considerably different natural periods in two orthogonal directions.

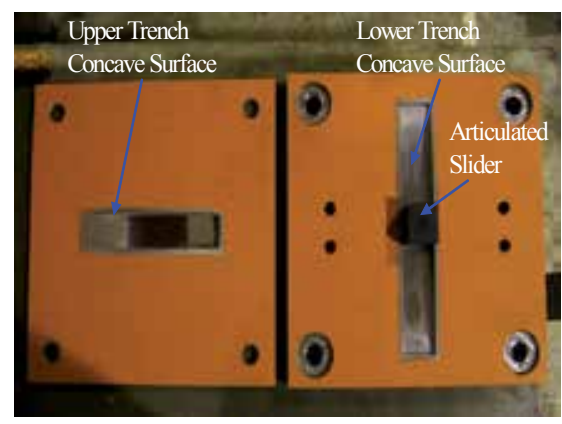

Fig. 9(a). Open-up view of trench friction pendulum system 


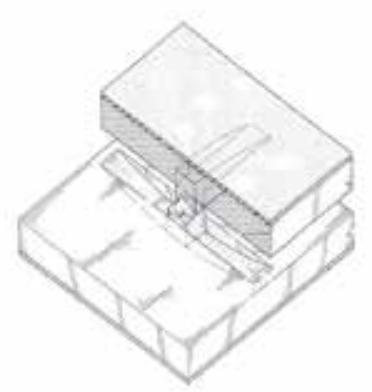

Fig. 9(b). A perspective view of trench friction pendulum system

In order to further enhance the functionality of the TFPS isolator (Tsai et al., 2010c), a base isolation system named the multiple trench friction pendulum system (MTFPS) with numerous intermediate sliding plates was proposed by Tsai et al. (2010d). As shown in Figures 10(a) and 10(b), the MTFPS isolator has multiple concave sliding interfaces that are composed of several sliding surfaces in each of two orthogonal directions, and an articulated slider located among trench concave sliding surfaces. The MTFPS represents more than one trench friction pendulum system connected in series in each direction. The friction coefficient, displacement capacity, and radius of curvature of each trench concave sliding surfaces in each direction can be different. The natural period and damping effect for a MTFPS isolator with several sliding surfaces can change continually during earthquakes. Therefore, a large number of possibilities of combinations are available for engineering designs. Such options are dependent on the needs of engineering practicing.

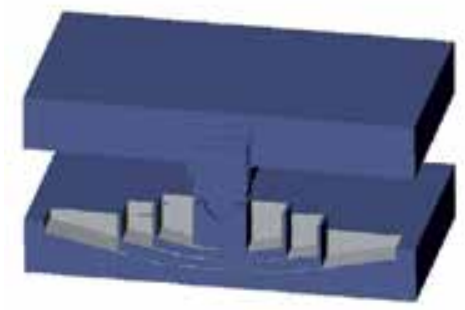

Fig. 10(a). Cross-sectional view of multiple trench friction pendulum system

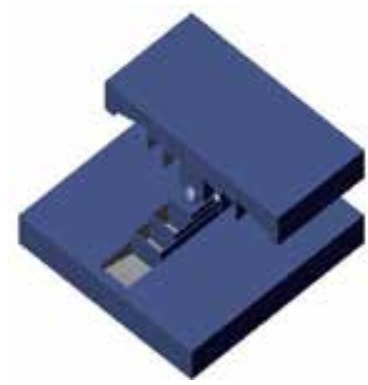

Fig. 10(b). Perspective view of multiple trench friction pendulum system 
As shown in Figs. 11(a) and 11(b), Tsai et al. (2007, 2008b) developed a direction-optimized friction pendulum system (DO-FPS) which consists of a spherical concave surface, a trench concave surface, as shown in Fig. 11(a), or a trajectory concave surface, as shown in Fig. 11(b), and an articulated slider. The DO-FPS isolator possesses important characteristics such as the natural period, displacement capacity and damping effect, which are functions of the directional angle of the sliding motion of the articulated slider during earthquakes. In order to improve the contact between the spherical and trench surfaces (or the trajectory concave surface), the slider consists of circular and square contact surfaces to match the spherical and trench surfaces, respectively. To further enhance the contact, it possesses a special articulation mechanism to accommodate any rotation in the isolator and maintain the stability of the isolated structures during earthquakes. In addition, the DO-FPS isolator can continually change the natural period and adjust the capacity of the bearing displacement and damping effect as a result of the change of the angle between the articulated slider and trench concave surface during earthquakes. This isolation system exhibits adaptive stiffness and adaptive damping by using different coefficients of friction and radii of curvature on the spherical and trench (or the trajectory) concave sliding surfaces to change the stiffness and damping to predictable values at specified and controllable angle of the sliding motion of the slider in the isolator although it is a completely passive device.

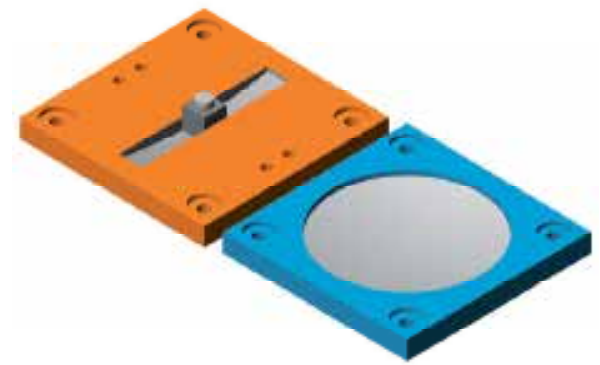

Fig. 11(a). Open-up view of first type direction optimized-friction pendulum system

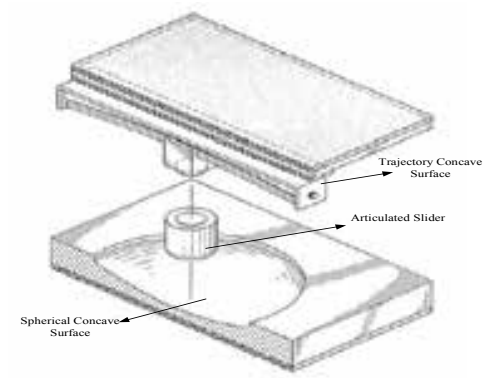

Fig. 11(b). Open-up view of second type direction optimized-friction pendulum system

As shown in Figures 12(a) and 12(b), Tsai et al. (2010e, 2011b) proposed and studied in theory and experiment on a base isolator that features variable natural period, damping effect and displacement capacity, named the multiple direction optimized-friction pendulum system (MDO-FPS). This device is mainly composed of several spherical concave sliding surfaces, several trench sliding concave surfaces and an articulated slider located 
among these spherical and trench concave sliding surfaces to make the isolation period changeable with the sliding direction from only the multiple trench sliding interfaces to the combinations of the multiple trench sliding interfaces and the multiple spherical sliding interfaces.

In addition, this bearing may have $N$ number of sliding interfaces in the trench and spherical surfaces to possess adaptive features of stiffness and damping by using different coefficients of friction and radii of curvature on the trench and spherical concave surfaces leading to changeable stiffness and damping at specified displacement amplitudes. Therefore, the MDO-FPS isolator possesses important characteristics in natural period, damping effect and displacement capacity, which are functions of the direction of the sliding motion, coefficients of friction and radii of curvature on sliding interfaces, and sliding displacements.

The advantage of the isolator is able to change its natural period, damping effect and displacement capacity continually during earthquakes to avoid possibility of resonance induced by ground motions. This base isolator has more important features and flexibility than other types of base isolation devices for engineering practice. Practicing engineers will be able to optimize the isolator at various levels of earthquakes by adopting suitable parameters of friction coefficients and radii of curvature of the sliding interfaces.

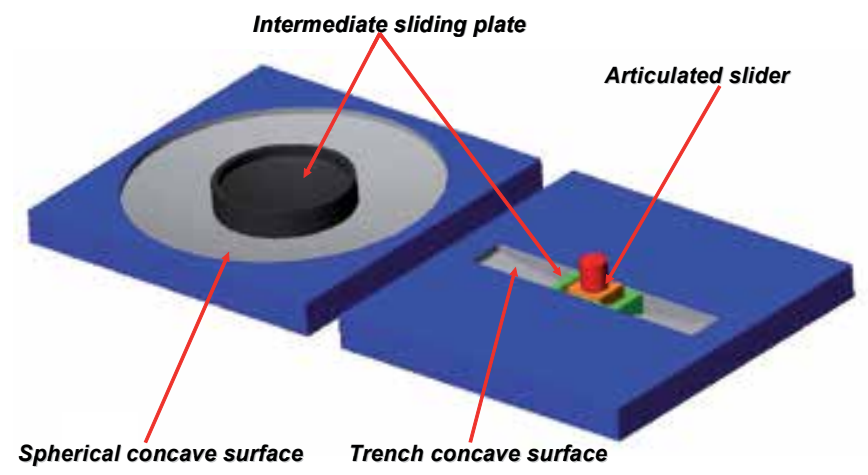

Fig. 12(a). Open-up view of MDO-FPS

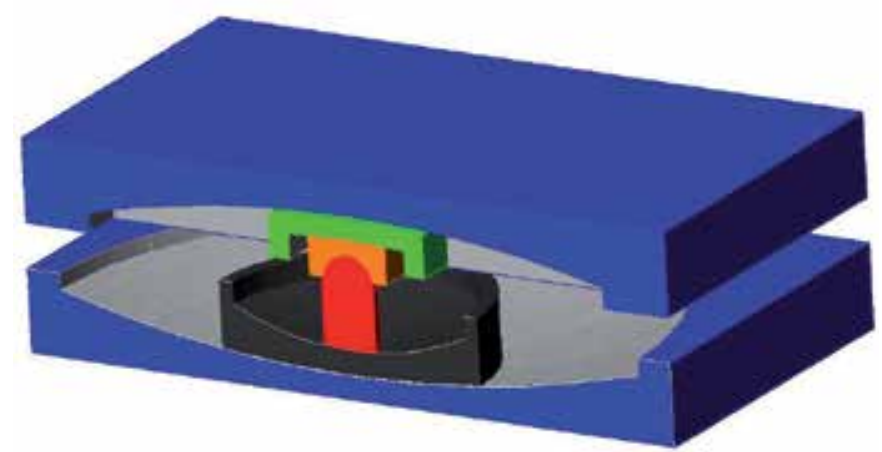

Fig. 12(b). Cross-sectional view of multiple direction optimized-friction pendulum system (MDO-FPS) 


\section{The static dynamics interchangeable - Ball pendulum system}

The dynamics interchangeable-ball pendulum system (SDI-BPS) is schematized in Figs. 4(a) and $4(\mathrm{~b})$ consisting of one upper concave surface (not necessary a spherical shape), one lower concave surface, several supporting steel balls to provide supports for long terms of service loadings and the frictional damping effect to the isolator at small displacements (see Case 1 of Fig. 13), several housing holes to lodge the supporting steel balls and one damped steel ball covered by damping materials to uphold the vertical loads resulting from the static and seismic loadings at large displacements (see Case 3 of Fig. 13) and supply additional damping to the bearing by deforming the damping material that could be a rubber material during earthquakes.

As shown in Case 1 of Fig. 13, almost all static loadings as a result of the weight of the equipment are sustained by the supporting steel balls and negligible loadings are taken by the damped steel ball while the system is under long terms of service loadings.

In the event of an earthquake, the static loadings and the dynamic loadings induced by the ground or floor accelerations are still supported by the supporting steel balls while the horizontally mobilized force is less than the total frictional force from the supporting steel balls, and the damped steel ball remains inactivated, similar to Case 1 of Fig. 13. The frictional force depends on the contact area and the coefficient of friction among the upper concave surface, the supporting steel balls and the housing holes located on the lower concave surface. This contact area and friction coefficient can be properly designed for the purpose of adjusting the frictional force and damping.

When the horizontal force exceeds the frictional force, the damped steel ball is activated and starts rolling on the concave surfaces. The vertical force resulting from the static and dynamic loadings is shared by the damped steel ball and the supporting steel balls. Simultaneously, the damping effect is provided by the supporting steel balls due to the frictional force and the damped steel ball as a result of the deformation of the damping material enveloping the damped steel ball under the condition of small isolator displacement, as shown in Case 2 of Fig. 13. The natural period of the isolated system is then dominated by the radii of curvature of the concave surfaces, which is equal to $2 \pi \sqrt{\frac{R_{1}+R_{2}}{g}}$.

Where $R_{1}$ and $R_{2}$ are the radii of curvature of the upper and lower spherical concave surfaces, respectively; and $g$ is the gravity constant.

If the system is subjected to a large isolator displacement during an earthquake, the supporting steel balls will be detached from the upper concave surface, and the total vertical and horizontal loads will be supported by the damped steel ball only to result in more damping effect due to the larger deformation of the damping material, and no damping effect results from the frictional force caused by the supporting steel balls, as depicted in Case 3 of Fig. 13. Furthermore, the natural period of the isolated equipment is governed by the radii of curvature of the concave surfaces in this stage. The damping effect for the isolator is only provided by the deformation of the damping material covering the damped steel ball in the course of motions to reduce the size of the isolator as a result of smaller isolator displacements caused by earthquakes in comparison to a rolling isolation system with negligible damping.

As shown in Case 4 of Fig. 13, because the component of the gravity force from the equipment weight tangential to the concave surface provides the restoring force, the 
isolator will be rolling back to the original position without a significant residual displacement after earthquakes. Therefore, the damped steel ball is subjected to temporary loadings induced by earthquakes only, and the static loadings in the life span of service won't cause any permanent deformation to the damping material enveloping the damped steel ball.

In general, in the case of a service loading or a small earthquake, the static load is supported by the mechanism composed of the upper and lower concave surfaces and supporting steel balls with negligible supporting effect from the damped steel ball. On the other hand, in the events of medium and large earthquakes, the entire loads including static and dynamic loads are supported by the mechanism offered by the upper and lower concave surfaces and the damped steel ball while the isolation system is activated. These two mechanisms are interchangeable between the cases of static loading from the weight of equipment and seismic loading from the ground or floor acceleration.

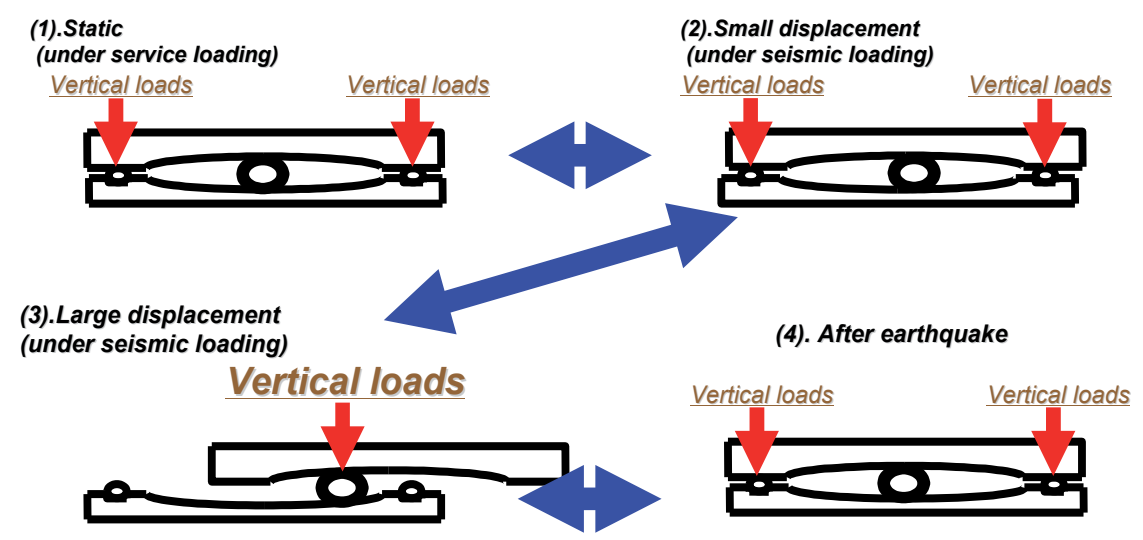

Fig. 13. Movements of a SDI-BPS isolator under service and seismic loadings 


\subsection{Characteristic of the SDI-BPS isolator}

The test setup of the SDI-BPS isolator is depicted in Figures 14(a) and 14(b). In this test, the damped steel ball consisted of a steel rolling ball of $44.55 \mathrm{~mm}$ in diameter covered with a thickness of $6.75 \mathrm{~mm}$ damping material which was made of natural rubber material with hardness of 60 degrees in the IRHD standard (International Rubber Hardness Degree). The main purpose of this test was to investigate the mechanical behavior of the damped steel ball, therefore, supporting balls were removed during the component tests and all damping effect resulting from the system was provided by the damped steel ball. Figure 15 shows the relationship of the horizontal force to the horizontal displacement while the system was subjected to a vertical load of $4.56 \mathrm{KN}$ and a harmonic displacement of $50 \mathrm{~mm}$ with a frequency of $0.3 \mathrm{~Hz}$. The enclosed area shown in the Fig. 15 provides a damping effect into the isolation system. The test result demonstrates that the deformations of the rubber material leaded to significant damping effects in the system with negligible deformation occurring in the steel material.

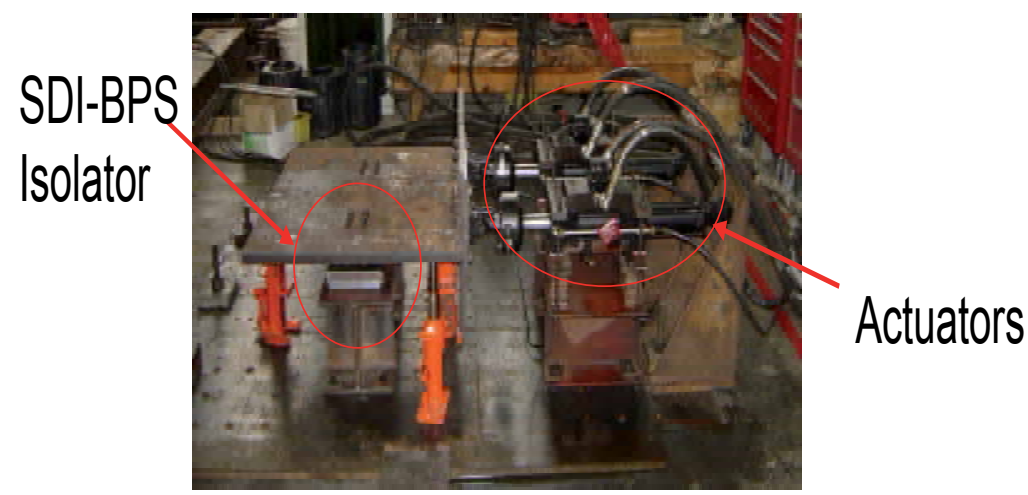

Fig. 14(a). Setup for component tests of the SDI-BPS isolator

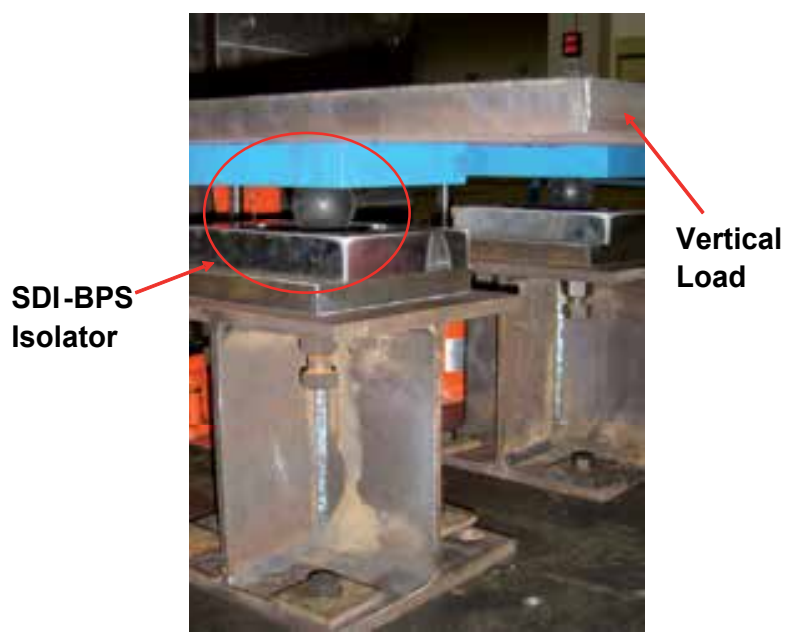

Fig. 14(b). Close view of component test of SDI-BPS isolator 


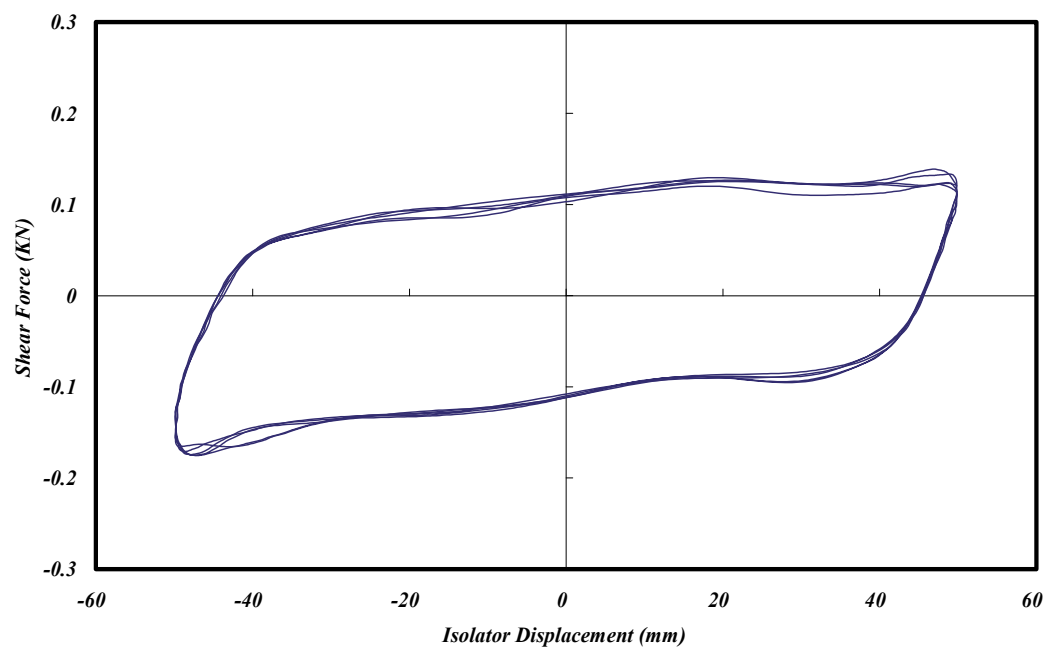

Fig. 15. Hysteresis loop of SDI-BPS isolator without supporting balls

\subsection{Shaking table tests of motion sensitive equipment with SDI-BPS isolators}

This section will investigate the performance of the SDI-BPS isolator installed in the motion sensitive equipment on seismic mitigation under tri-directional earthquakes through a series of shaking table tests of the vibration sensitive equipment isolated with the SDI-BPS isolator under tri-directional earthquakes. As shown in Figure 16, the tested vibration sensitive equipment with six inner layers was used to house the network server. The lengths in the two horizontal directions were $0.8 \mathrm{~m}$ and $0.6 \mathrm{~m}$, respectively, and $1.98 \mathrm{~m}$ in height. The mass of the empty equipment was $110 \mathrm{~kg}$. A mass of $108 \mathrm{~kg}$ at each layer from the first to the third layer and $54 \mathrm{~kg}$ each at the rest of the layers was added. In the case of the fixed base equipment, the natural frequency was $5.66 \mathrm{~Hz}$. In the case of the isolated system, four SDIBPS isolators with a radius of curvature of $1.0 \mathrm{~m}$ representing 2.84 seconds in natural period for the isolated equipment were installed beneath the equipment.

The input ground motions in these tests included the 1995 Kobe earthquake (Japan) and the 1999 Chi-Chi earthquake (recorded at TCU084 station, Taiwan). Figures 17(a) and 17(b) show the comparisons of the acceleration responses at the top layer of the equipment between the fixed base and SDI-BPS-isolated systems under tri-directional earthquakes. It is observed from these figures that the SDI-BPS isolator can effectively isolate the seismic energy trying to transmit into the vibration sensitive equipment during earthquakes. Figures 18(a) and 18(b) show the hysteresis loops of the SDI-BPS isolator under the various tridirectional earthquakes. These figures illustrate that the SDI-BPS isolator can provide damping to limit the bearing displacement, and accordingly, the bearing size was decreased. It also infers from these figures that a frictional type of damping was provided by the isolation system in small displacements and other type of damping was provided by the damped steel ball of the isolation system for large displacements, referring to the hysteresis loop in Figure 15, because the upper concave surface was lifted and away from the supporting steel balls without any contact. The isolator displacement history depicted in Figure 19 demonstrates that the response of equipment had been reduced by the isolation system with acceptable displacements in the isolators and that the isolator displacement approached zero in the end of the earthquake with negligible residual displacement. 


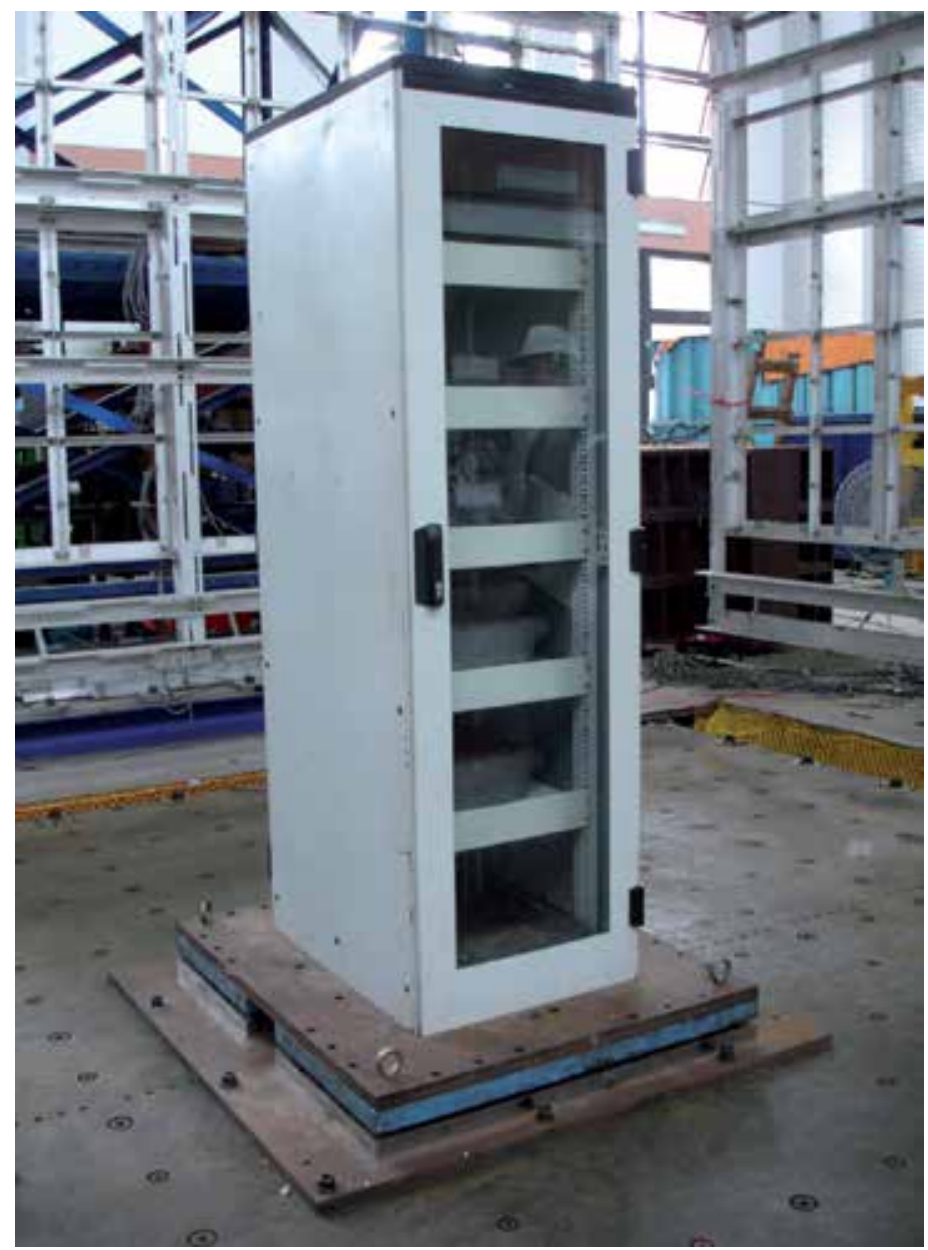

Fig. 16. Vibration sensitive equipment isolated with SDI-BPS isolators 


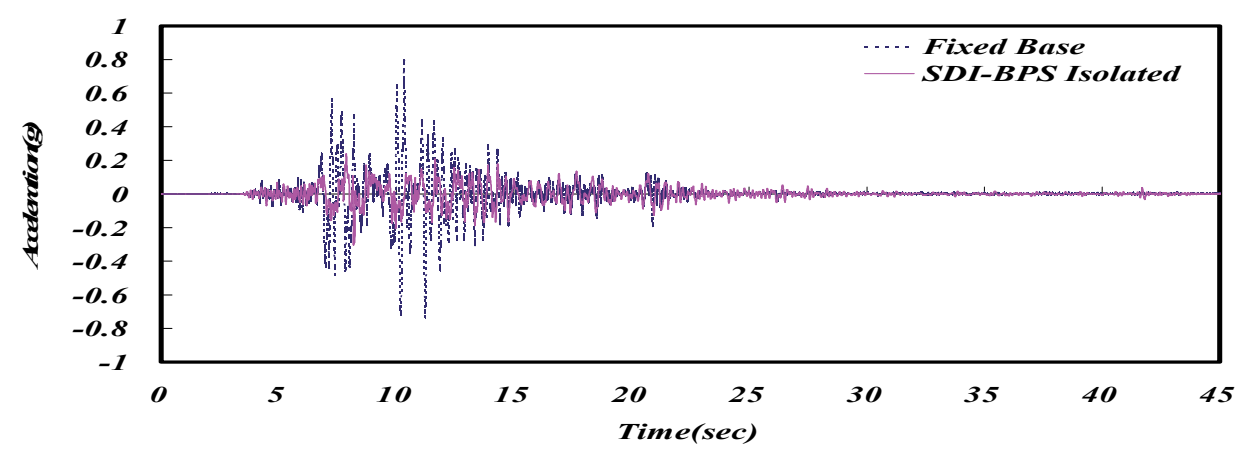

Fig. 17(a). Comparison of X-directional acceleration response at top layer between fixed-base and SDI-BPS-isolated systems under tri-directional Kobe earthquake (PGA $=$ X0.388g + $\mathrm{Y} 0.265 \mathrm{~g}+\mathrm{Z} 0.116 \mathrm{~g})$

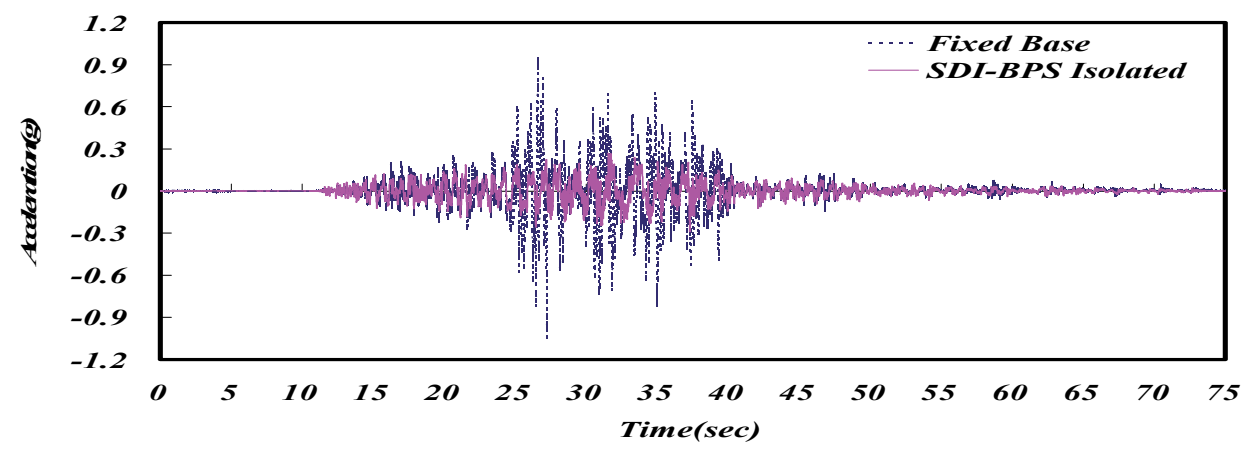

Fig. 17(b). Comparison of acceleration response in X-direction at top layer between fixedbase and SDI-BPS-isolated systems under tri-directional Chi-Chi (TCU084 Station) earthquake $(\mathrm{PGA}=\mathrm{X} 0.673 \mathrm{~g}+\mathrm{Y} 0.271 \mathrm{~g}+\mathrm{Z} 0.159 \mathrm{~g})$ 


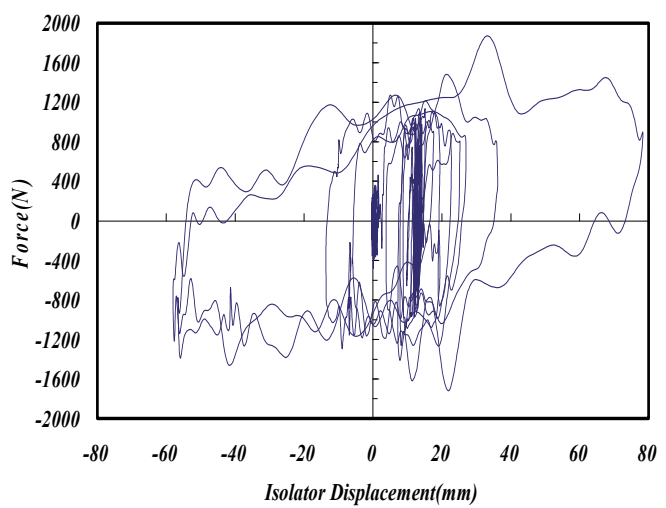

Fig. 18(a). Hysteresis loops of SDI-BPS isolator in $X$ direction under tri-directional Kobe earthquake (PGA $=$ X0.388g $+Y 0.265 g+Z 0.116 g)$

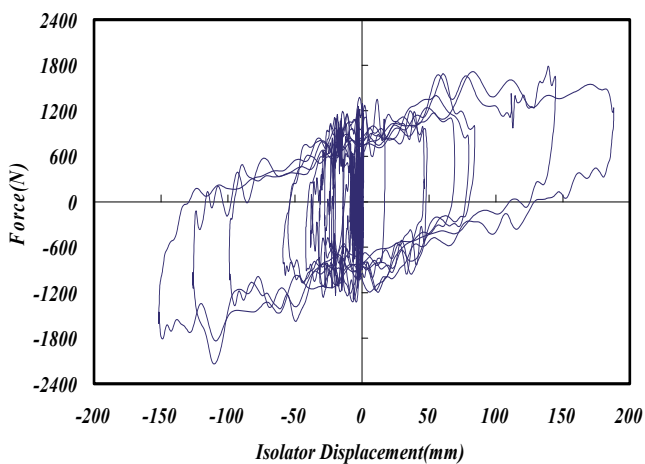

Fig. 18(b). Hysteresis loops of SDI-BPS isolator in $\mathrm{X}$ direction under tri-directional Chi-Chi (TCU084 Station) earthquake (PGA $=$ X0.673g + Y0.271g + Z0.159g)

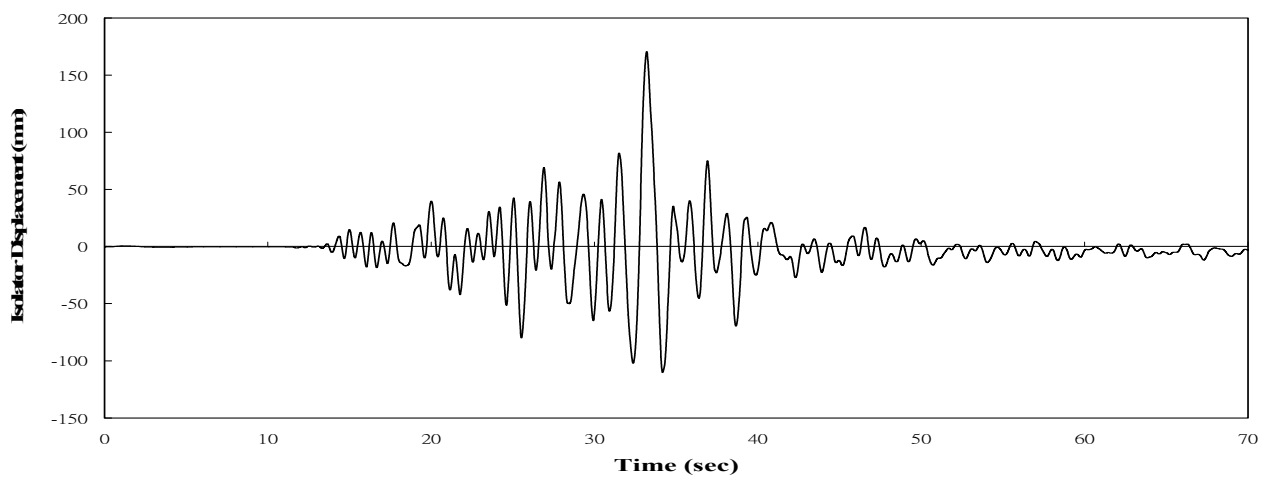

Fig. 19. Isolator displacement history in $\mathrm{X}$-direction while isolated system subjected to tridirectional Chi-Chi (TCU084 Station) earthquake (PGA $=$ X0.513g + Y0.170g +Z0.151g) 


\section{The direction-optimized friction pendulum system}

A seismic isolation system, called the direction-optimized friction pendulum system (DOFPS), consists of a concave trench, a plate with a spherically concave surface, and an articulated slider located in between, as shown in Figs. 20(a) and 20(b). The trench and the plate can usually possess different curvature radii, friction coefficients, and displacement capacities. As shown in Fig. 20(b), the concave trench is connected in series with the spherically concave plate in the X-direction, and this provides both the maximum natural period and the maximum displacement capacity. By contrast, in the Y-direction the isolator consists of just the spherically concave plate, and this provides both the minimum natural period and the minimum displacement capacity. The natural period and the displacement capacity vary with direction and are functions of the sliding angle between the articulated slider and the concave trench. In engineering practice, the DO-FPS isolator can be designed for differences in natural period and displacement capacity for the purpose of costeffectiveness. During an earthquake, the DO-FPS isolator will adjust the natural period automatically to avoid the possibility of resonance. Following the earthquake, the concave trench and the spherically concave plate can offer a centering mechanism to return the isolated structure to its original position without significant residual displacements.

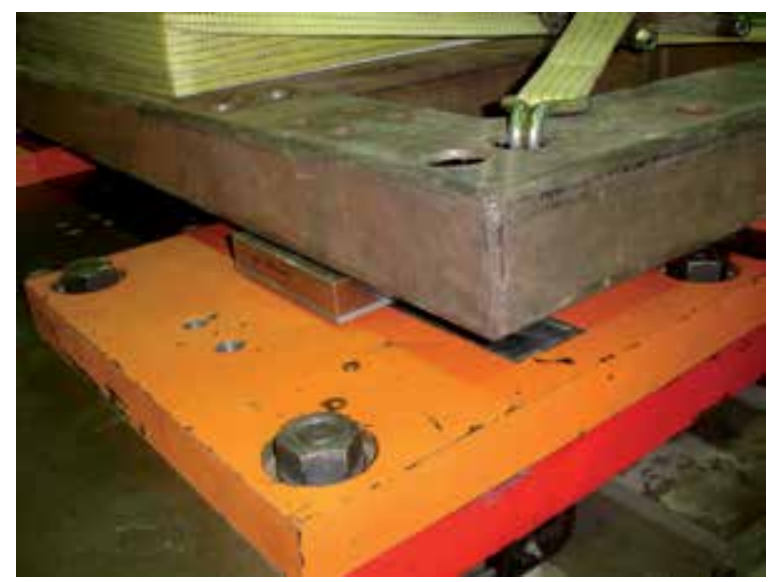

Fig. 20(a). Assembly of direction optimized friction pendulum system

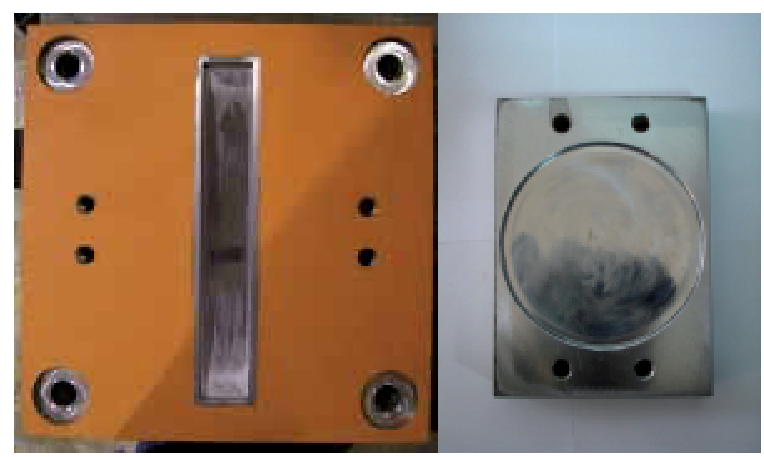

Fig. 20(b). An open-up view of the DO-FPS isolator with trench and concave surface 


\subsection{Properties of the direction-optimized friction pendulum system}

As shown in Figs. 21(a) and 21(b), an articulated slider is located between the concave trench and the spherically concave plate. The articulated slider includes two parts: the lower part at the trench side can have a rectangular cross section, while the upper part at the plate side can have a circular cross section. These two parts are joined with a hemispherical joint to produce an articulation mechanism in the middle of the slider. Hence, the articulated slider incorporates a rotational function to accommodate the relative rotation during its movement. As shown in Fig. 21(a), the slider is coated with Teflon composite on top, bottom, and two sides to reduce the frictional forces at its interfaces with not only the concave surfaces of the trench and plate but also the side walls of the trench.

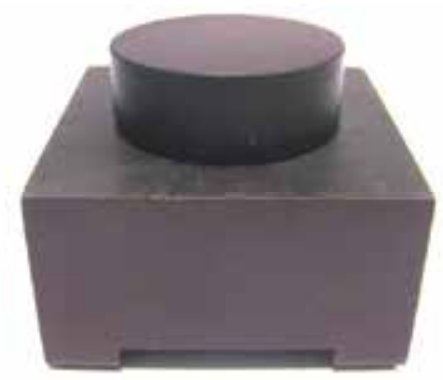

Fig. 21(a). Assembly of articulated slider coated with Teflon composite

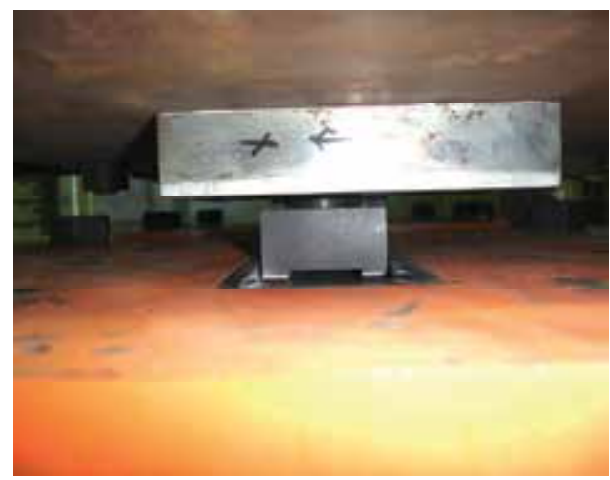

Fig. 21(b). Slider located between the concave trench and the spherical concave surface.

As shown in Fig. 21(b), the width of the articulated slider almost equals that of the trench; therefore, the sides of the slider and the walls of the trench almost remain in contact. These two sides of the articulated slider can thus provide additional damping in the direction parallel to the concave trench to help dissipate seismic energy.

\subsection{Component tests of sliding interfaces}

Figures 22(a) and 22(b) respectively show the schematic design and the test setup for the Teflon composite coated on the sliding surfaces. To examine the sliding characteristic of the sliding surface, the steel plates were coated with the Teflon composite and the high density chrome, respectively, to rub against each other. During the tests, the vertical pressures imposing at the interface are variable values. 


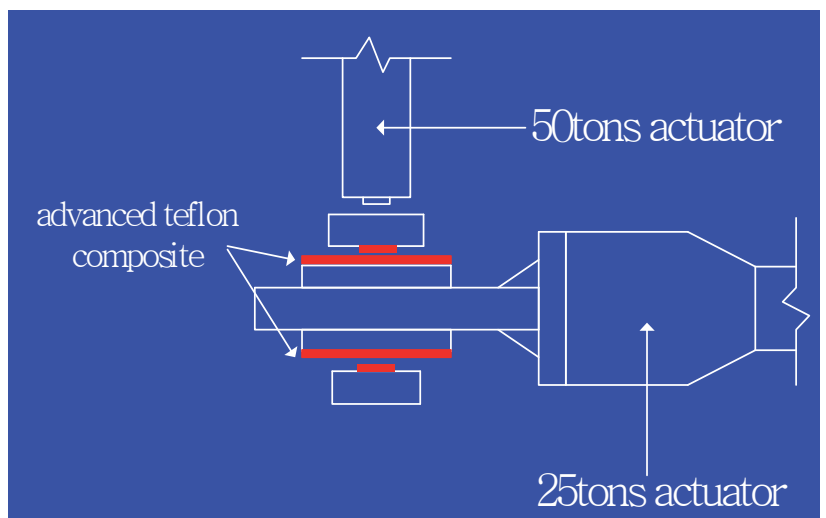

Fig. 22(a). The schematic design for the test of the Teflon composite

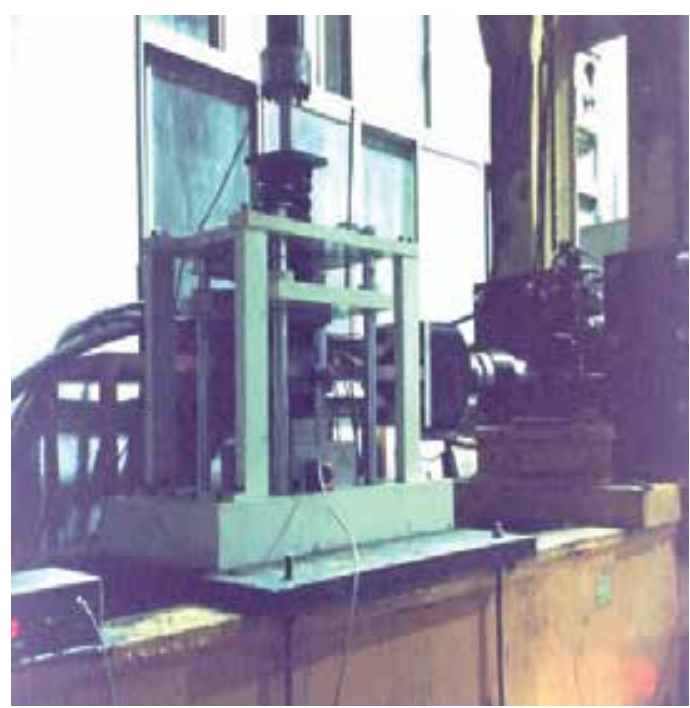

Fig. 22(b). Test setup for the Teflon composite

Figure 23 depicts a typically hysteretic loop when the vertical pressure was 98Mpa. Figure 24 demonstrate that the coefficients of friction are functions of the vertical pressure on the contact surface and the velocity of the sliding motion. In accordance with the analytical model proposed by Tsai (2005a), the total friction force acting on the sliding interface can be expressed as:

$$
\mu\left(\dot{u}_{b}\right)=\frac{A}{\lambda_{1} A+\lambda_{2} P} \cdot\left\{1+\alpha\left[1-\exp \left(-\beta\left|\dot{u}_{b}\right|\right)\right]\right\} \times \text { Coef }
$$

where $A$ represents the contact area at the sliding interface; $\lambda_{1}$ and $\lambda_{2}$ denote the parameters associated with the quasi-static friction force at the zero velocity; $P$ is the contact force normal to the sliding surface; and $\alpha$ is the amplification factor used to describe the increase of friction force with increasing the sliding velocity; and $\beta$ is the parameter which controls the variation of the friction coefficient with sliding velocity; and $\dot{u}_{b}$ is the siding velocity of the base isolator. 
The term $A / \lambda_{1} A+\lambda_{2} P$ is used to describe the friction coefficient at zero velocity. According to the experimental observations, the friction coefficient depends on the sliding velocity. Therefore, the term $1+\alpha\left[1-\exp \left(-\beta\left|\dot{u}_{b}\right|\right)\right]$ is used to describe the amplification factor of the friction coefficient relative to that at zero velocity. The coefficient, Coef, is a decay function representing the phenomenon of degradation of the friction force with the increase of the number of cyclic reversals. The coefficient of Coef can be shown as:

$$
\text { Coef }=\left(1-\gamma_{1}\right)+\gamma_{1} \cdot \exp \left(-\gamma_{2} \cdot \int_{0}^{t} \frac{F_{t}-F_{t}^{0}}{F_{t}^{0}} \cdot d u_{b}\right)
$$

where $\gamma_{1}$ and $\gamma_{2}$ are parameters to describe the decay behavior of the friction coefficient at the Teflon interface associated with the energy accumulation in the history of the sliding motion; $F_{t}^{0}$ is the friction force when the sliding velocity is equal to zero; $F_{t}$ is the friction force at current time $t$; and $d u_{b}$ is the displacement increment of the base isolator. The effect of the coefficient, Coef, may be neglected for the purpose of engineering practice.

$$
\begin{gathered}
\lambda_{1}=21.120, \quad \lambda_{2}=1.221 \times 10^{-7}(1 / \mathrm{Pa}) \\
\alpha=1.903, \beta=100.000(\mathrm{sec} / \mathrm{m}) \\
\gamma_{1}=0.1390, \quad \gamma_{2}=7.1537(1 / \mathrm{m})
\end{gathered}
$$

According to the model proposed by Al-Hussaini et al. (1994), the friction coefficient can be represented in the following form:

$$
\mu\left(\dot{u}_{b}\right)=\mu_{\max }-\left(\mu_{\max }-\mu_{\min }\right) \exp \left(-\beta\left|\dot{u}_{b}\right|\right)
$$

where $\mu_{\min }$ and $\mu_{\max }$ are the friction coefficients at zero sliding velocity and high sliding velocity, respectively.

Figure 25 shows the comparison between the theoretical and experimental results. It can be concluded that the numerical result obtained from the mathematical model has good agreement with the experimental results.

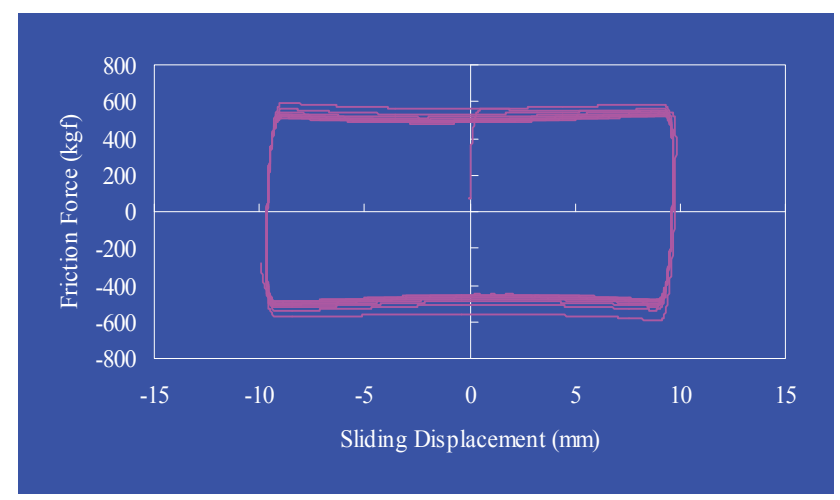

Fig. 23. Typical hysteretic loop for the Teflon composite under a vertical pressure of $98 \mathrm{Mpa}$ and a Frequency of $1 \mathrm{~Hz}$ 


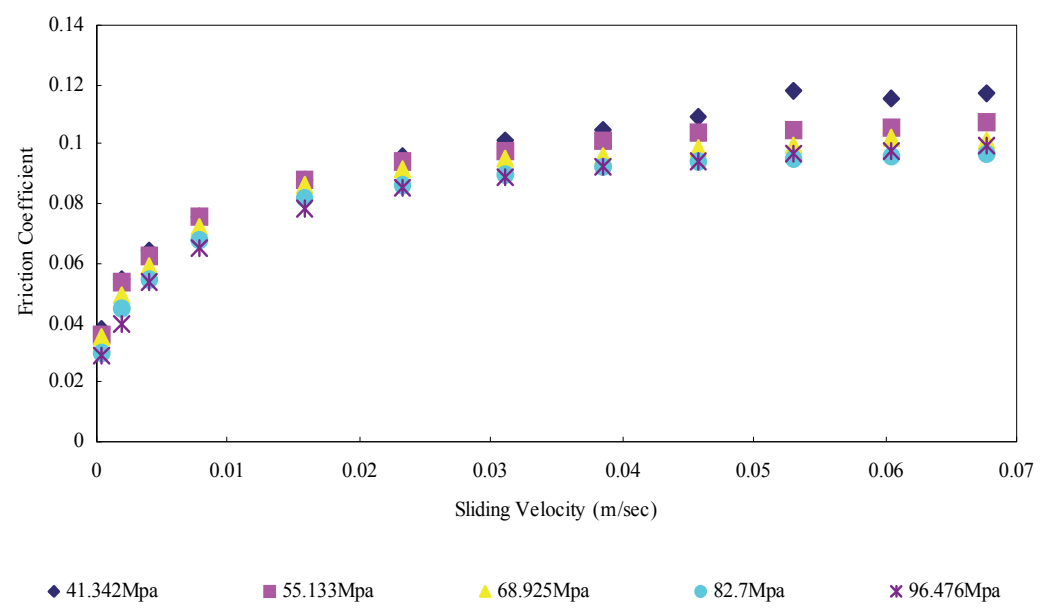

Fig. 24. Friction coefficients under various vertical pressures

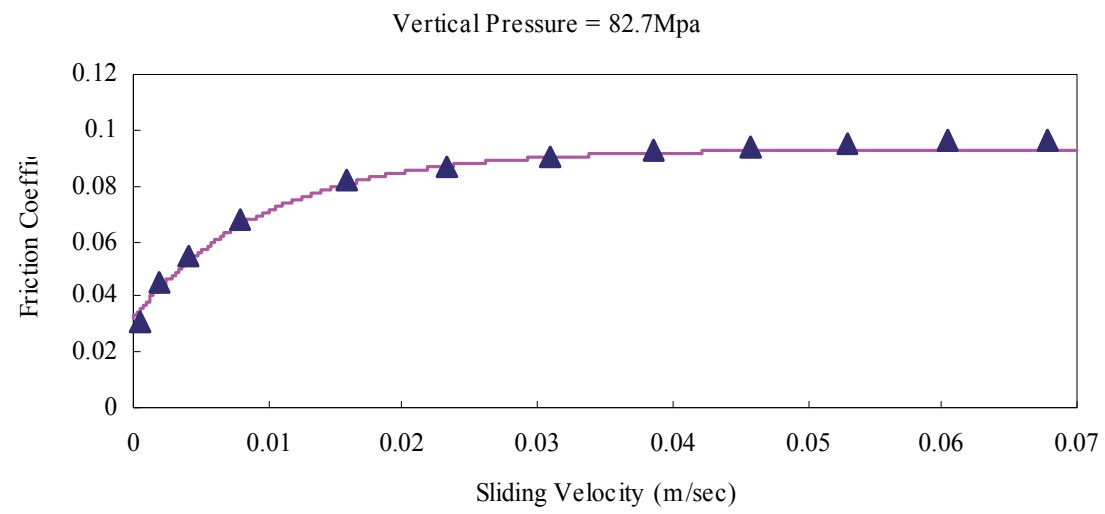

- Experimental Results

Numerical Results

Fig. 25. Friction coefficients under various sliding velocities

\subsection{Equation of motion for a rigid mass isolated with DO-FPS isolators}

As shown in Fig. 26, the time history of ground accelerations can be represented by the method of interpolation of excitation by assuming that the ground motion is a linear variation between time $t_{i-1}$ and $t_{i}$. If a rigid mass is isolated with DO-FPS isolators, as shown in Fig. 27, the equation of motion can be expressed as:

$$
m \ddot{u}_{b}+c_{b} \dot{u}_{b}+k_{b} u_{b}=-\mu\left(\dot{u}_{b}\right) m g \operatorname{sgn}\left(\dot{u}_{b}\right)-m \ddot{u}_{g}^{i-1}-\frac{m \ddot{u}_{g}^{i}-m \ddot{u}_{g}^{i-1}}{\Delta t} \tau
$$

where $m, c_{b}$ and $k_{b}$ are the mass, damping coefficient and horizontal stiffness of the isolated mass, respectively; $u_{b}$ is the displacement of the base isolator relative to the ground; $\mu\left(\dot{u}_{b}\right)$ is the friction coefficient of the sliding surface, which is a function of sliding velocity; and $\ddot{u}_{g}^{i}$ is the ground acceleration at time $t_{i}$. 


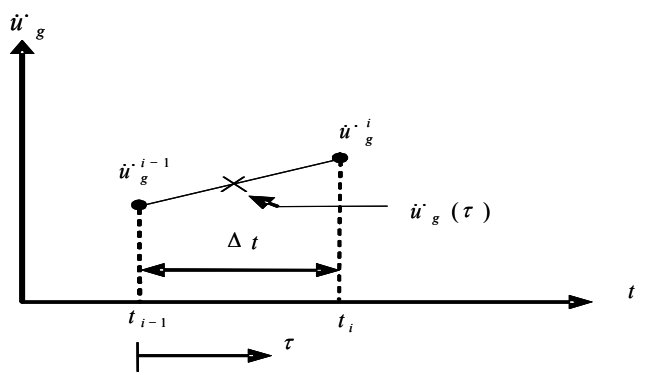

Fig. 26. Linear Interpolation for Ground Motions

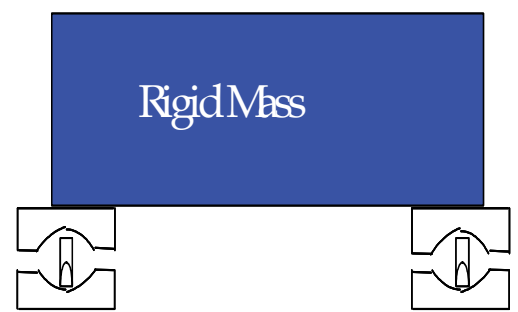

Fig. 27. A rigid mass isolated with DO-FPS isolator

The transient response of Eq. (5) can be given by:

$$
u_{c}(\tau)=\left(A \cos \omega_{d} \tau+B \sin \omega_{d} \tau\right) \exp \left(-\xi \omega_{n} \tau\right)
$$

where $\omega_{n}$ is the natural frequency; $\omega_{d}=\omega_{n} \sqrt{1-\xi^{2}}$ is the damped frequency; $\xi$ is the viscous damping ratio.

The particular solution of Eq. (5) between time $t_{i-1}$ and $t_{i}$ can be given by:

$$
u_{p}(\tau)=\frac{1}{\omega_{n}^{2}}\left[-\mu\left(\dot{u}_{b}\right) g \operatorname{sgn}\left(\dot{u}_{b}\right)-\ddot{u}_{g}^{i-1}+\frac{2 \xi}{\omega_{n} \Delta t}\left(\ddot{u}_{g}^{i}-\ddot{u}_{g}^{i-1}\right)\right]-\frac{\ddot{u}_{g}^{i}-\ddot{u}_{g}^{i-1}}{\omega_{n}^{2} \Delta t} \tau
$$

The sliding displacement of the base isolator between time $t_{i-1}$ and $t_{i}$ can be obtained from Eqs. (6) and (7) as:

$$
\begin{aligned}
& u_{b}(\tau)=\left(A \cos \omega_{d} \tau+B \sin \omega_{d} \tau\right) \exp \left(-\xi \omega_{n} \tau\right) \\
& +\frac{1}{\omega_{n}^{2}}\left[-\mu\left(\dot{u}_{b}\right) g \operatorname{sgn}\left(\dot{u}_{b}\right)-\ddot{u}_{g}^{i-1}+\frac{2 \xi}{\omega_{n} \Delta t}\left(\ddot{u}_{g}^{i}-\ddot{u}_{g}^{i-1}\right)\right]-\frac{\ddot{u}_{g}^{i}-\ddot{u}_{g}^{i-1}}{\omega_{n}^{2} \Delta t} \tau
\end{aligned}
$$

At the beginning of each time step, the sliding displacement is equal to that at the end of the previous time step, i.e. $u_{b}(0)=u_{b}^{i-1}$.

The coefficient $A$ of Equation (6) can be obtained as:

$$
A=u_{b}^{i-1}-\frac{1}{\omega_{n}^{2}}\left[-\mu\left(\dot{u}_{b}\right) g \operatorname{sgn}\left(\dot{u}_{b}\right)-\ddot{u}_{g}^{i-1}+\frac{2 \xi}{\omega_{n} \Delta t}\left(\ddot{u}_{g}^{i}-\ddot{u}_{g}^{i-1}\right)\right]
$$


The derivative of Eq. (8) respect to $\tau$ leads to:

$$
\begin{aligned}
& \dot{u}_{b}(\tau)=\left(-\omega_{d} A \sin \omega_{d} \tau+\omega_{d} B \cos \omega_{d} \tau\right) \exp \left(-\xi \omega_{n} \tau\right) \\
& -\xi \omega_{n}\left(A \cos \omega_{d} \tau+B \sin \omega_{d} \tau\right) \exp \left(-\xi \omega_{n} \tau\right)-\frac{\ddot{u}_{g}^{i}-\ddot{u}_{g}^{i-1}}{\omega_{n}^{2} \Delta t}
\end{aligned}
$$

Backsubstitution of $\dot{u}_{b}(0)=\dot{u}_{b}^{i-1}$ into Eq. (10) results in:

$$
B=\frac{1}{\omega_{d}}\left(\dot{u}_{b}^{i-1}+\xi \omega_{n} A+\frac{\ddot{u}_{g}^{i}-\ddot{u}_{g}^{i-1}}{\omega_{n}^{2} \Delta t}\right)
$$

The sliding acceleration of the base isolator relative to the ground can be given by:

$$
\begin{aligned}
& \ddot{u}_{b}(\tau)=\left(-\omega_{d}^{2} A \cos \omega_{d} \tau-\omega_{d}^{2} B \sin \omega_{d} \tau\right) \exp \left(-\xi \omega_{n} \tau\right) \\
& -2 \xi \omega_{n}\left(-\omega_{d} A \sin \omega_{d} \tau+\omega_{d} B \cos \omega_{d} \tau\right) \exp \left(-\xi \omega_{n} \tau\right) \\
& +\xi^{2} \omega_{n}^{2}\left(A \cos \omega_{d} \tau+B \sin \omega_{d} \tau\right) \exp \left(-\xi \omega_{n} \tau\right)
\end{aligned}
$$

\subsubsection{Condition for non-sliding phase}

The kinetic friction coefficient has been considered as the same as the static friction coefficient. Therefore, as the summation of the inertia and restoring forces imposing at the base raft is lower than the quasi-static friction force, i. e.:

$$
\left|m\left(\ddot{u}_{b}+\ddot{u}_{g}\right)+c_{b} \dot{u}_{b}+k_{b} u_{b}\right|<\mu_{\min } m g
$$

Then the structure will behave as a conventional fixed base structure, and the sliding displacement, sliding velocity and relative acceleration are:

$$
u_{b}=\text { constant }, \dot{u}_{b}=\ddot{u}_{b}=0
$$

\subsubsection{Initiation of sliding phase}

The base isolated rigid mass will behave as a fixed base structure unless the static friction force can be overcome. During the sliding phase, the equation given in the following should be satisfied:

$$
\left|m\left(\ddot{u}_{b}+\ddot{u}_{g}\right)+c_{b} \dot{u}_{b}+k_{b} u_{b}\right| \geq \mu_{\text {min }} m g
$$

Because the time increment adopted in the time history analysis (e.g. $\Delta t=0.0005 \mathrm{sec}$ ) is quite smaller than that of the sampling time of the earthquake history, it is reasonable to assume that the direction of sliding at the current time step is the same as the previous time step. It should be noted that the direction of sliding remains unchanged during a particular sliding phase. At the end of each time step, the validity of inequality Eq. (15) should be checked. If the inequality is not satisfied at a particular time step, then the structure enter a non-sliding phase and behaves as a fixed base structure. 


\subsubsection{Simplified mathematical model for DO-FPS isolator}

The simplified model based on the equilibrium at the slider of the DO-FPS isolator can be shown in the following. As shown in Fig. 28, horizontal forces $F_{1}$ and $F_{2}$ imposing at the concave trench and spherical sliding surfaces, respectively, can be expressed as:

$$
F_{1}=\frac{W}{R_{1}} u_{1}+\mu W \operatorname{sgn}\left(\dot{u}_{1}\right)
$$

and

$$
F_{2}=\frac{W}{R_{2}} u_{2}+\mu W \operatorname{sgn}\left(\dot{u}_{2}\right)
$$

where $W$ is the vertical load resulting from the superstructure; $R_{1}$ and $R_{2}$ represent the radii of curvature of the concave trench and spherical sliding surfaces, respectively; $u_{1}$ and $u_{2}$ depict the horizontal sliding displacements of the slider relative to the centers of the concave trench and spherical sliding surfaces, respectively; $\mu$ represents the friction coefficient for the Teflon composite interface which depends on the sliding velocity; and $\dot{u}_{1}$ and $\dot{u}_{2}$ are the sliding velocities of the articulated slider.

Rearrangement of Eqs. (16) and (17) leads to:

$$
u_{1}=\frac{F_{1}-\mu W \operatorname{sgn}\left(\dot{u}_{1}\right)}{W / R_{1}}
$$

and

$$
u_{2}=\frac{F_{2}-\mu W \operatorname{sgn}\left(\dot{u}_{2}\right)}{W / R_{2}}
$$

With the aid of equilibrium at the articulated slider $\left(F=F_{1}=F_{2}\right)$, the total relative displacement between the centers of the concave trench and spherical sliding surfaces can be obtained as:

$$
u_{b}=u_{1}+u_{2}=\frac{F-\mu W \operatorname{sgn}\left(\dot{u}_{1}\right)}{W / R_{1}}+\frac{F-\mu W \operatorname{sgn}\left(\dot{u}_{2}\right)}{W / R_{2}}
$$

Rearrangement of Eq. (20) results in the base shear force:

$$
F=\frac{W}{R_{1}+R_{2}} u_{b}+\frac{R_{1} \mu W \operatorname{sgn}\left(\dot{u}_{1}\right)+R_{2} \mu W \operatorname{sgn}\left(\dot{u}_{2}\right)}{R_{1}+R_{2}}=K_{b} u_{b}+F_{f}
$$

where $K_{b}$ represents the horizontal stiffness of the DO-FPS isolator and can be expressed as:

$$
K_{b}=\frac{W}{R_{1}+R_{2}}
$$

Hence, the isolation period of the DO-FPS isolated structure in the direction of the concave trench surface is as follows: 


$$
T_{b}=2 \pi \sqrt{\frac{m}{k_{b}}}=2 \pi \sqrt{\frac{R_{1}+R_{2}}{g}}
$$

Based on the exact solution, the relationship between the base shear force and sliding displacement can be obtained by using Eqs. (21) and (8).

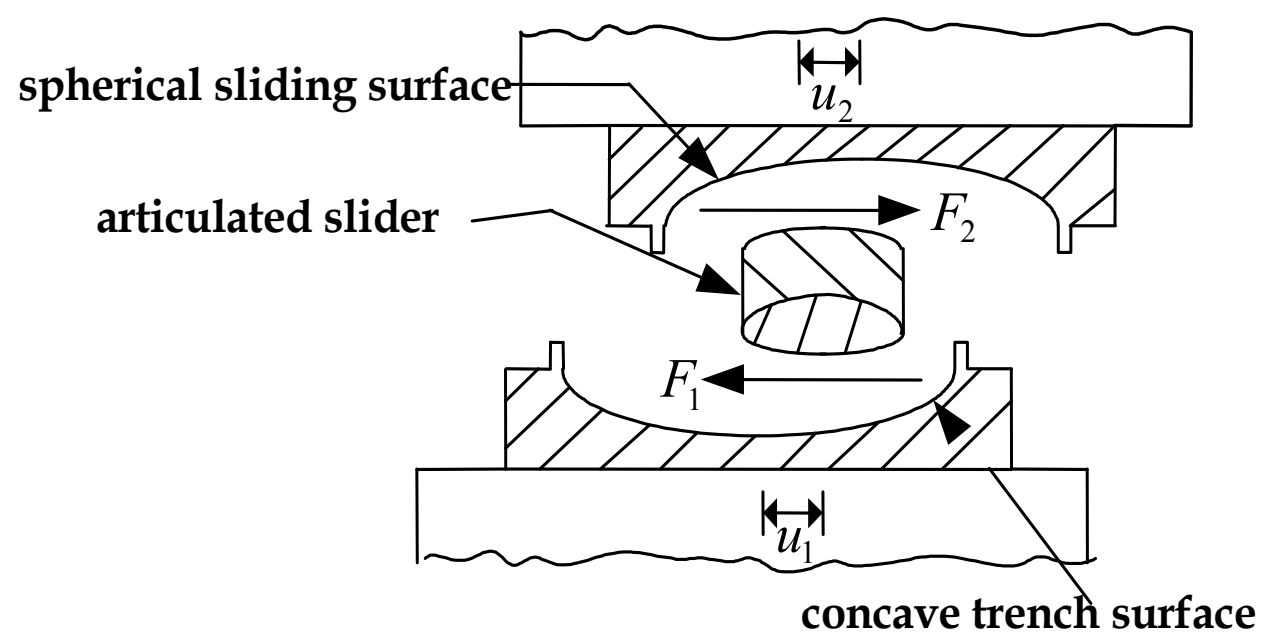

Fig. 28. The equilibrium of the forces on the spherical sliding and concave trench surfaces

\subsubsection{Comparison of experimental and analytical results}

In order to verify the accuracy of the exact solution derived in the previous sections, the shaking table tests of a rigid mass isolated with DO-FPS isolators were performed in Taiwan. The strong ground motions of the 1999 Chi-Chi (TCU084) have been given as earthquake loads during the shaking table tests. The DO-FPS isolator adopted in the shaking table tests has a concave trench and spherical sliding surfaces of $2.236 \mathrm{~m}$ in radius of curvature. The fundamental period of the base isolated structure in the trench direction is $4.242 \mathrm{sec}$.

The comparisons of the bearing displacement and velocity histories between the experimental and analytical results under the 1999 Chi-Chi earthquake (TCU084, EW component) of 1.211g in PGA are shown in Figs. 29(a) and 29(b), respectively. These figures tell us that the proposed solution can well simulate the sliding displacement and velocity of the nonlinear behavior of the device under strong ground motions. Figures 30 (a) and 30(b) display the comparison between the recorded force-displacement loop and the calculated results for the DO-FPS base isolator. The results obtained from the numerical analysis are quite consistent with those from the experiments. The proposed algorithm can be given as a good tool for engineering professions to preliminarily design the displacement capacity and isolation period, etc. It should be noted that the method for the numerical analysis proposed in the previous section is only suitable for unidirectional loadings. 


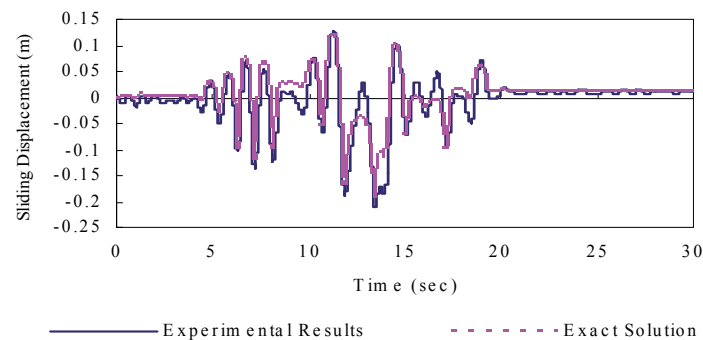

Fig. 29(a). Comparison of sliding displacement of DO-FPS isolator during 1999 Chi-Chi earthquake (TCU084, EW Component) of 1.211g in PGA

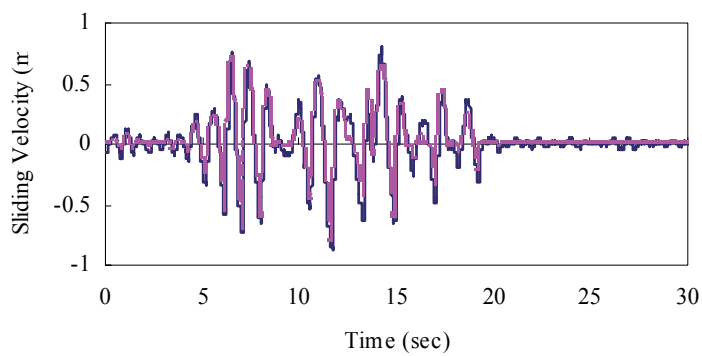

Experimental Results _ - - - - Exact Solution

Fig. 29(b). Comparison of bearing sliding velocity between experimental and numerical results under 1999 Chi-Chi earthquake (TCU084, EW Component) of 1.211g in PGA

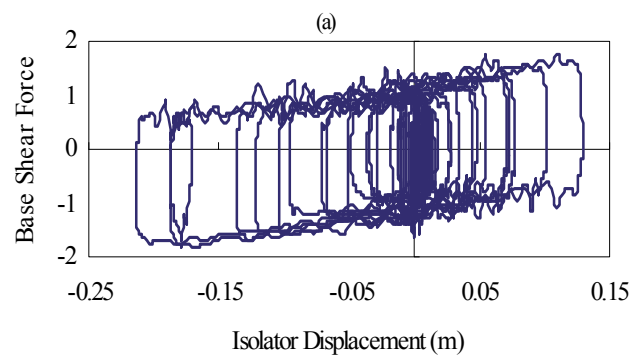

Fig. 30(a). Hysteresis loop of DO-FPS isolator under 1999 Chi-Chi earthquake (TCU084, EW Component) of 1.211g in PGA: (a) Experimental Results

(b)

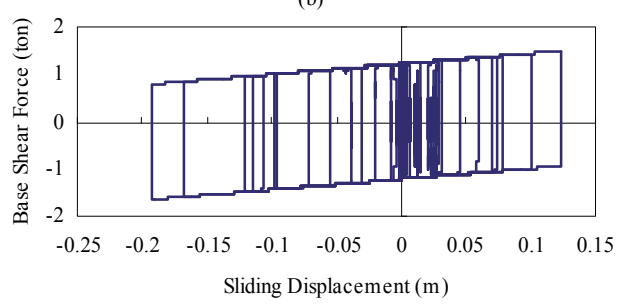

Fig. 30(b). Hysteresis loop of DO-FPS isolator under 1999 Chi-Chi earthquake (TCU084, EW Component) of 1.211g in PGA: (b) Exact Solution 


\subsection{Shaking table tests of equipment isolated with DO-FPS isolators}

In order to examine the seismic behavior of motion sensitive equipment isolated with a direction-optimized friction pendulum system, a series of shaking table tests were carried out in the Department of Civil Engineering at Feng Chia University, as shown Fig. 31. In this full-scale experiment, a modem rack was adopted to simulate high-technology equipment such as server computers and workstations. The dimensions of the critical equipment were $0.8 \mathrm{~m} \times 0.6 \mathrm{~m} \times 1.98 \mathrm{~m}$ (length $\times$ width $\times$ height). Within the critical equipment were six levels, and lumped masses in the range from $50 \mathrm{~kg}$ to $100 \mathrm{~kg}$ were placed on these in $10 \mathrm{~kg}$ increments from top to bottom. The fundamental period of the critical equipment without isolators was measured in the shaking table test as $0.18 \mathrm{~s}$.

In order to prove the benefit provided by the DO-FPS isolator, we used two extreme conditions with angles of $0^{\circ}$ and $90^{\circ}$, respectively. The input ground motions included those of the earthquakes at El Centro (USA, 1940), Kobe (Japan, 1995), and Chi-Chi (station TCU084, Taiwan, 1999). Accelerometers and LVDTs were installed to measure the accelerations and displacements of the critical equipment plus DO-FPS isolators when subjected to the various ground motions.

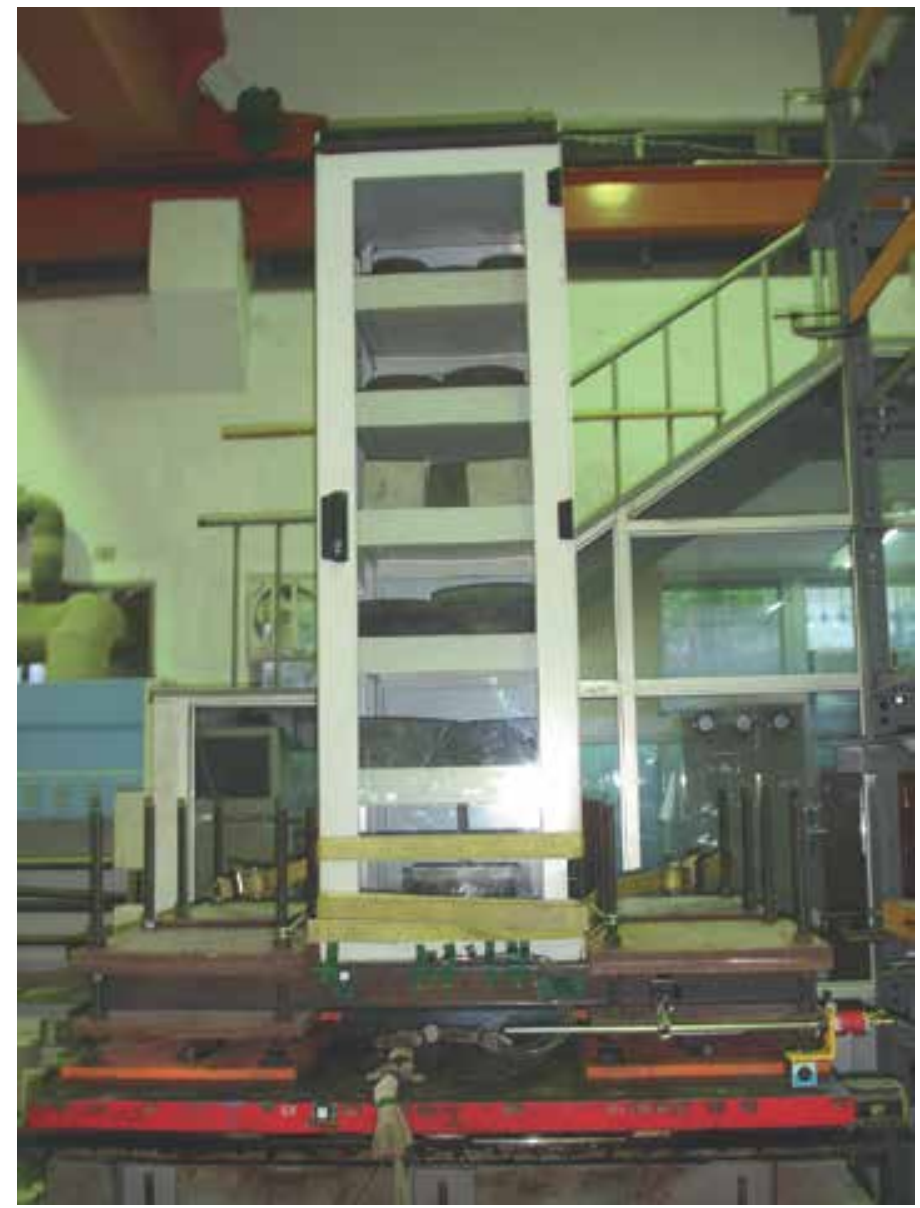

Fig. 31. Critical equipment isolated with DO-FPS isolators 
Figures 32(a) and 32(b) show comparisons of the roof acceleration responses of the critical equipment with and without DO-FPS isolators for angles of $0^{\circ}$ and $90^{\circ}$, respectively, under the conditions of the El Centro earthquake (PGA of 0.4g). Figures 33(a) and 33(b) show analogous comparisons for the Kobe earthquake (PGA of $0.35 \mathrm{~g}$ ), while Figures 34(a) and 34(b) show those for the Chi-Chi earthquake (PGA of $0.3 \mathrm{~g})$. These results illustrate that the direction-optimized friction pendulum system effectively reduced the responses of the critical equipment by lengthening its fundamental period.

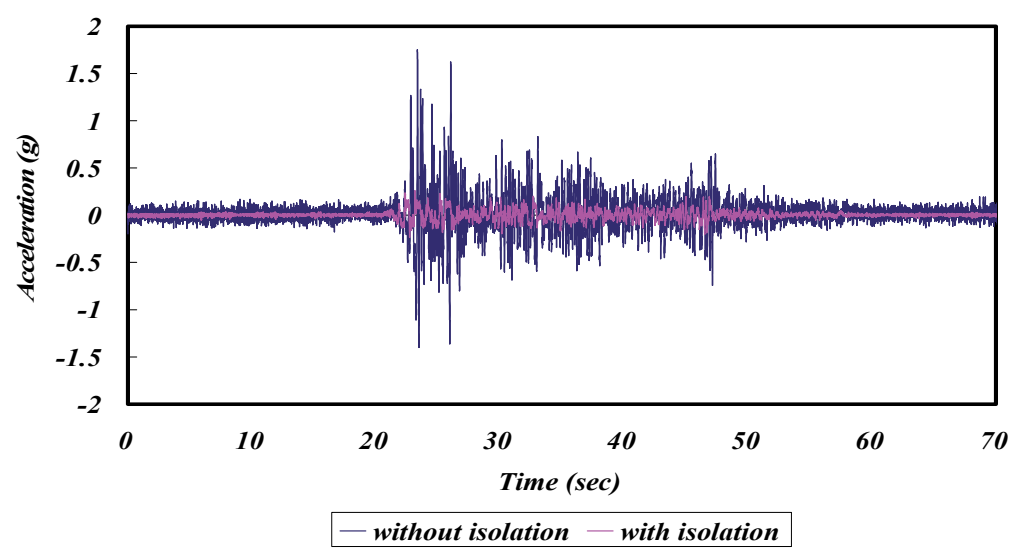

Fig. 32(a). Roof acceleration of equipment with and without DO-FPS isolators at angle of $90^{\circ}$ under El Centro earthquake of 0.4g PGA

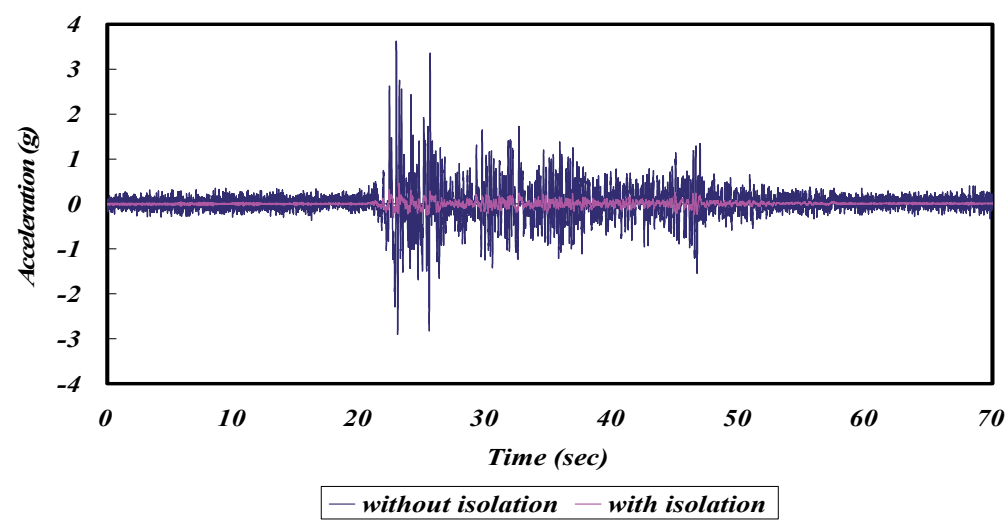

Fig. 32(b). Roof acceleration of equipment with and without DO-FPS isolators at angle of $0^{\circ}$ under El Centro earthquake of $0.4 \mathrm{~g}$ PGA 


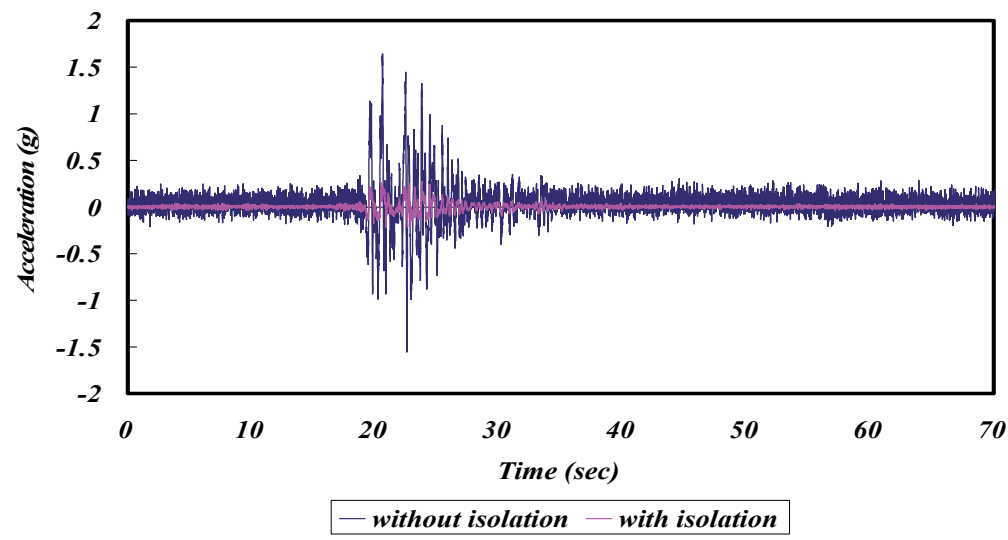

Fig. 33(a). Roof acceleration of equipment with and without DO-FPS isolators under at angle of $90^{\circ}$ Kobe earthquake of $0.35 \mathrm{~g}$ PGA

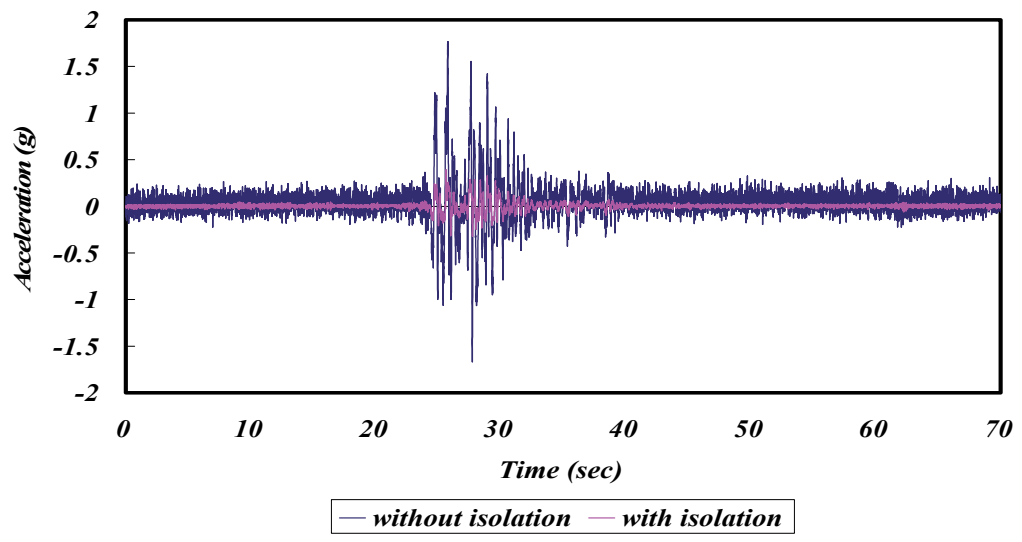

Fig. 33(b). Roof acceleration of equipment with and without DO-FPS isolators at angle of $0^{\circ}$ under Kobe earthquake of $0.35 \mathrm{~g}$ PGA 


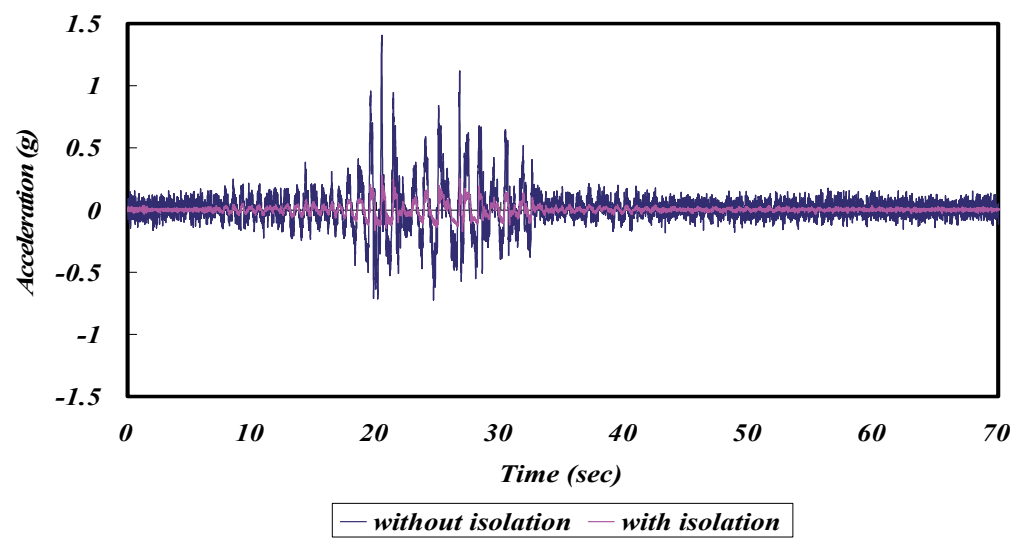

Fig. 34(a). Roof acceleration of equipment with and without DO-FPS isolators under at angle of $90^{\circ}$ Chi-Chi earthquake of $0.3 \mathrm{~g}$ PGA

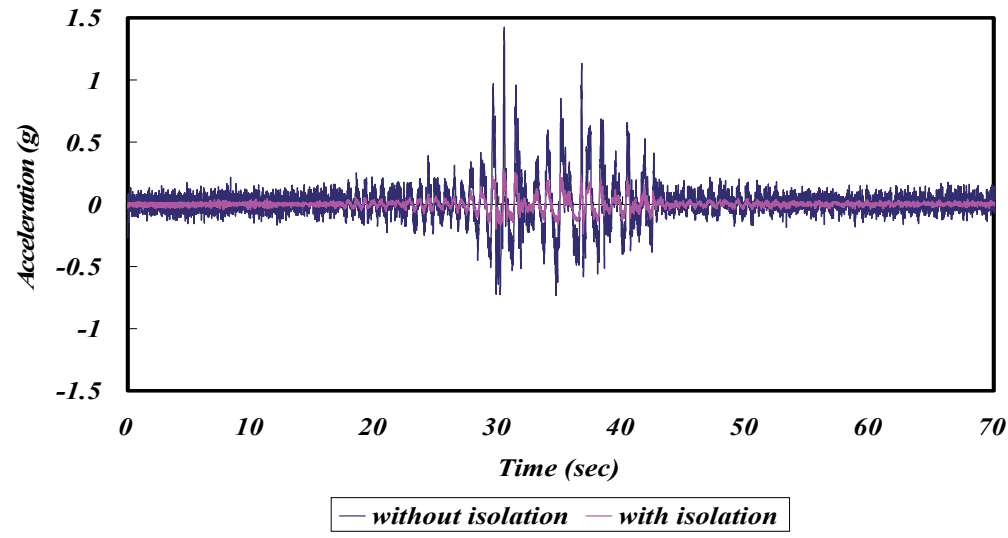

Fig. 34(b). Roof acceleration of equipment with and without DO-FPS isolators at angle of $0^{\circ}$ under Chi-Chi earthquake of 0.3g PGA 


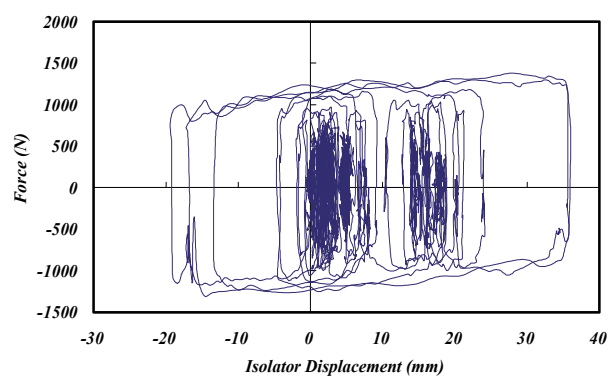

Fig. 35(a). Hysteresis loop of DO-FPS isolator at angle of $90^{\circ}$ during $0.4 \mathrm{~g}$ El Centro ground motion

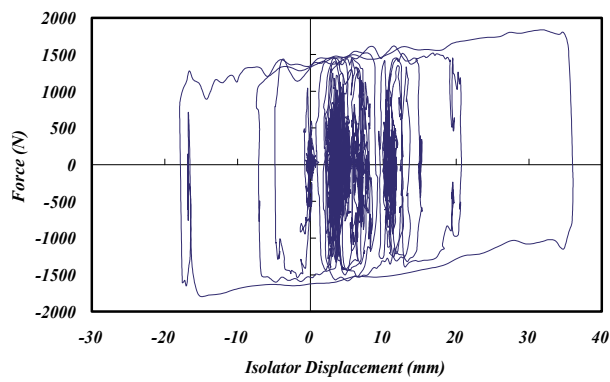

Fig. 35(b). Hysteresis loop of DO-FPS isolator at angle of $0^{\circ}$ during $0.4 \mathrm{~g}$ El Centro ground motion

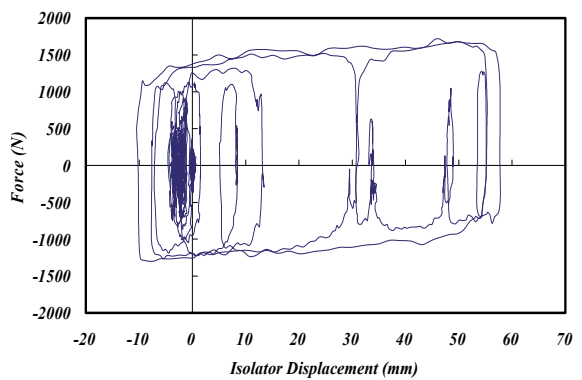

Fig. 36(a). Hysteresisloop of DOFPS isolator at angle of $90^{\circ}$ during $0.35 \mathrm{~g}$ Kobe ground motion

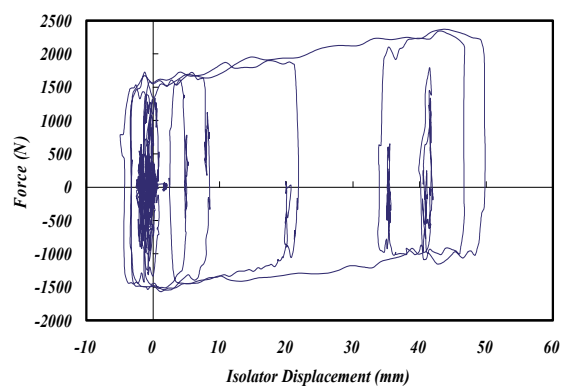

Fig. 36(b). Hysteresis loop of DO-FPS isolator at angle of $0^{\circ}$ during $0.35 \mathrm{~g}$ Kobe ground motion 


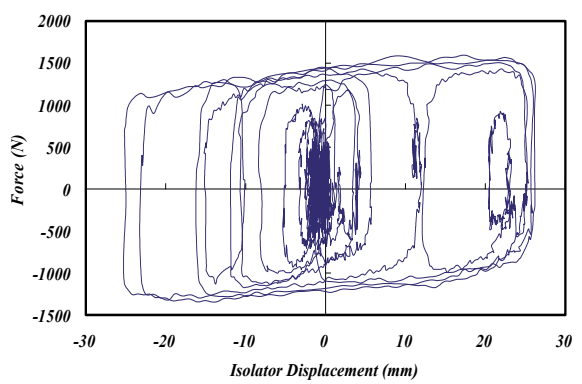

Fig. 37(a). Hysteresis loop of DOFPS isolator at angle of $90^{\circ}$ during $0.3 \mathrm{~g}$ Chi-Chi ground motion

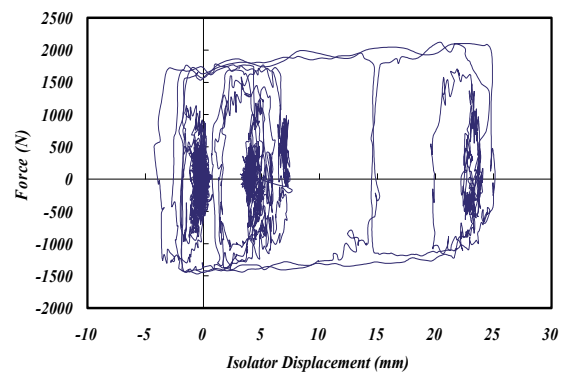

Fig. 37(b). Hysteresis loop of DOFPS isolator at angle of $0^{\circ}$ during $0.3 \mathrm{~g}$ Chi-Chi ground motion

Figures 35(a) to 37(b) show the hysteresis loops of the DO-FPS isolator under the various earthquake conditions. The enclosed areas in these figures demonstrate that the DO-FPS isolator provided excellent damping of the entire system during the simulated earthquakes. Figures 38(a) to 40(b) show the roof displacement responses of the critical equipment. These figures illustrate that the DO-FPS isolator has a good mechanism for bringing the isolator back to its original position without significant displacements. 


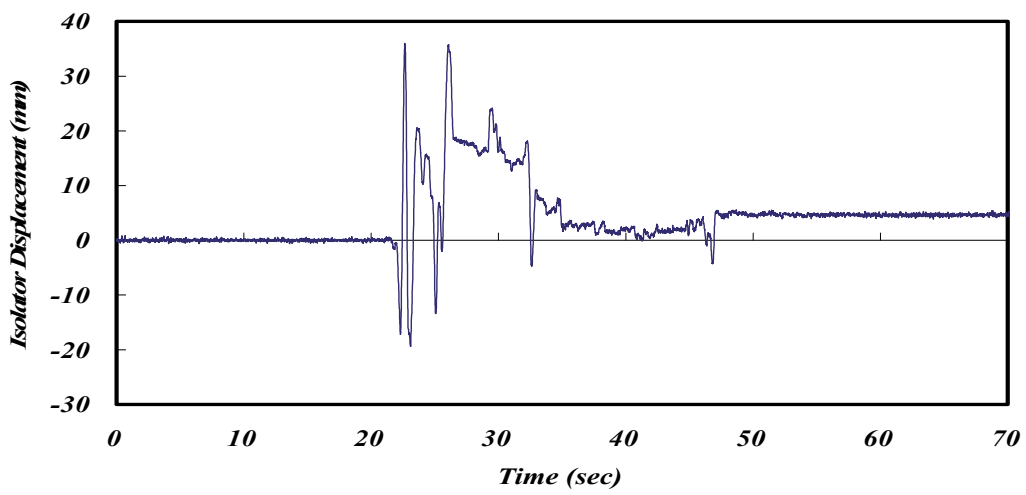

Fig. 38(a). Base isolator displacement of DOFPS isolator at angle of $90^{\circ}$ during $0.4 \mathrm{~g} \mathrm{El}$ Centro ground motion

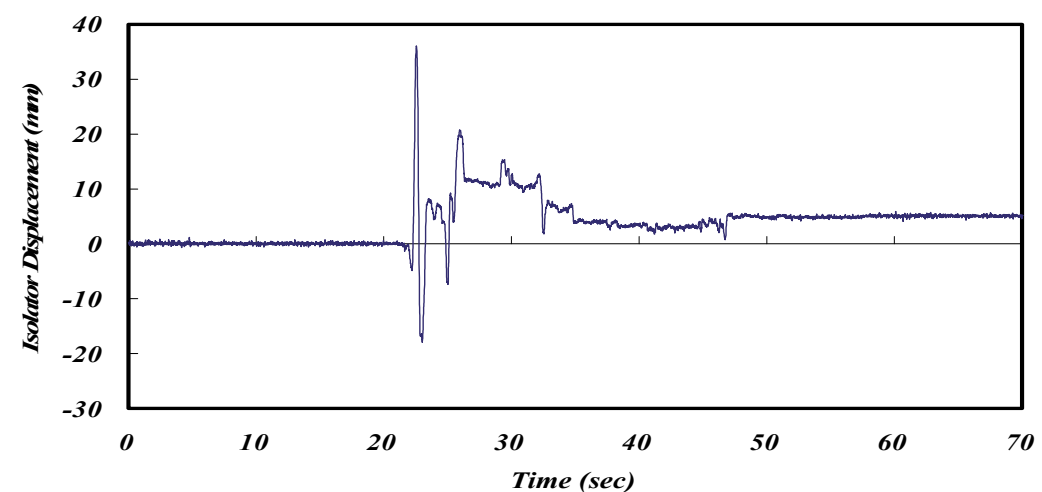

Fig. 38(b). Base isolator displacement of DO-FPS isolator at angle of $0^{\circ}$ during $0.4 \mathrm{~g}$ El Centro ground motion

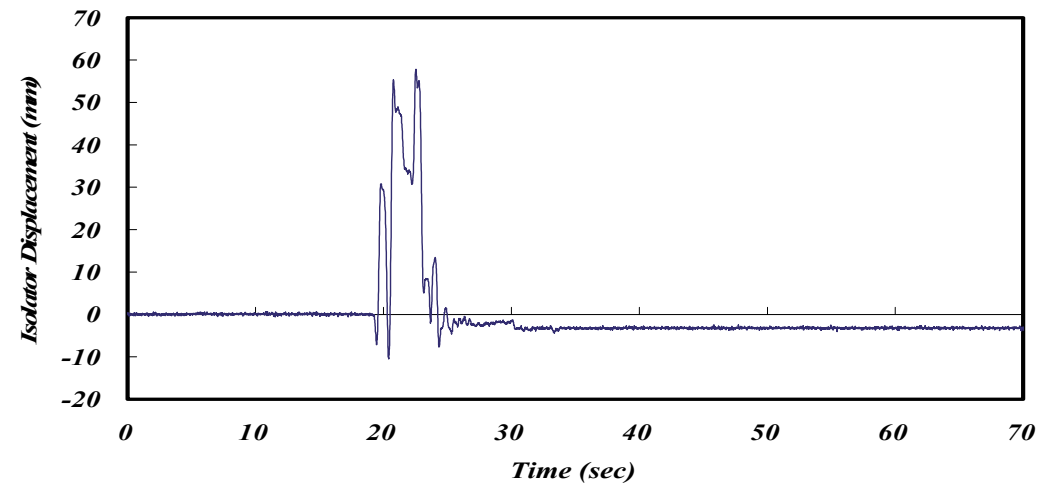

Fig. 39. Base isolator displacement of DO-FPS isolator at angle of $90^{\circ}$ during $0.35 \mathrm{~g}$ Kobe ground motion 


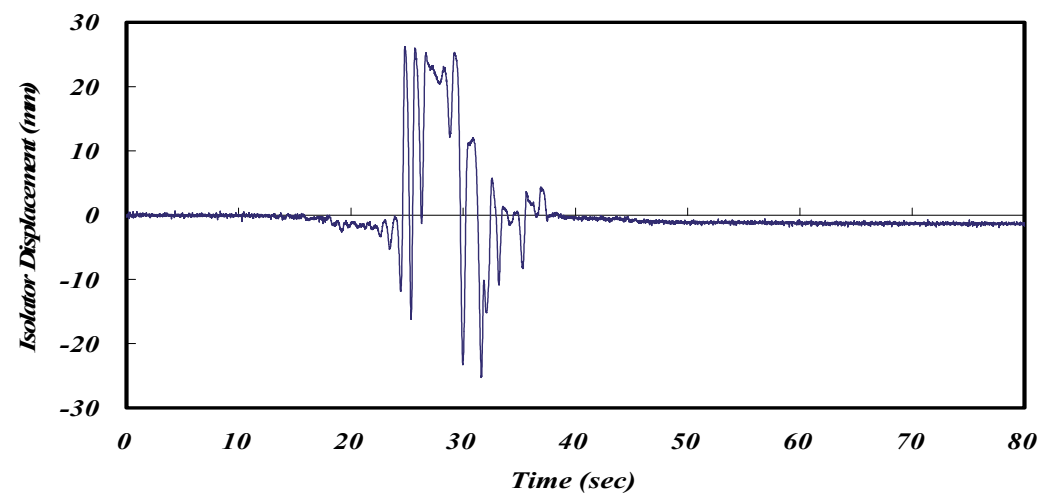

Fig. 40(a). Base isolator displacement of DO-FPS isolator at angle of $90^{\circ}$ during $0.3 \mathrm{~g}$ Chi-Chi ground motion

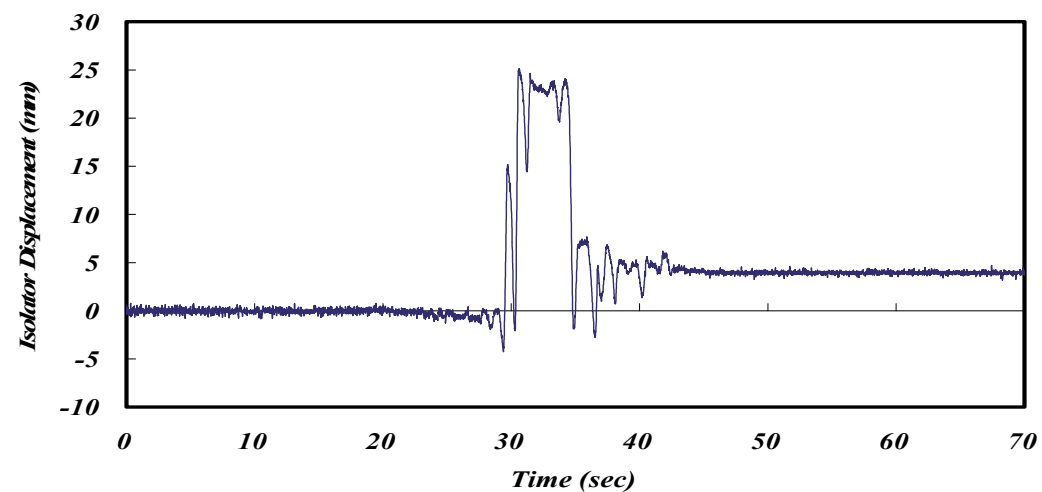

Fig. 40(b). Base isolator displacement of DO-FPS isolator at angle of $0^{\circ}$ during $0.3 \mathrm{~g}$ Chi-Chi ground motion

As shown in Tables 1 and 2, the maximum roof accelerations of critical equipment have been reduced remarkably under various types of earthquakes. Therefore, the DO-FPS isolator can be recognized as an effective tool for upgrading the seismic resistance of high-technology facilities by isolating earthquake induced energy trying to impart into the equipment. This device also supplies significant damping for the isolation system through the frictional force resulting from the sliding motion of the slider on the sliding surface to reduce the isolator displacement and the bearing size.

\section{Discussions}

Earthquake proof technologies such as energy absorbing systems and base isolation systems have been accepted as powerful tools to protect structures and equipments from earthquake damage. At the same time, what we should bear in mind is that the design earthquakes utilized for estimating the performance of such technologies in safeguarding structures and equipments still leave room for future research. More recently, a new methodology based on 
damages indices to obtain design earthquake loads for seismic-resistant design of traditional building structures was proposed by Moustafa (2011). The prerequisite for examining the efficiencies of the earthquake proof technologies in protecting building, bridge, and lifeline structures and equipments is having rational design earthquake loads associated with the occurrence and their characteristics (e.g. time, location, magnitude, frequency content and duration, etc.). More research efforts in this subject are needed to make this possible.

\begin{tabular}{|c|c|c|c|c||}
\hline \multirow{2}{*}{ Max. Response } & \multicolumn{4}{|c||}{ Roof Acceleration(g) } \\
\hline \multirow{2}{*}{ Earthquake } & $\begin{array}{c}\text { Amplitude } \\
(\text { PGA) }\end{array}$ & $\begin{array}{c}\text { Fixed-Base } \\
\text { Structure }\end{array}$ & $\begin{array}{c}\text { Isolated } \\
\text { Structure }\end{array}$ & $\begin{array}{c}\text { Response } \\
\text { Reduction }\end{array}$ \\
\hline \multirow{3}{*}{ El Centro } & $0.2 \mathrm{~g}$ & 0.846 & 0.200 & $76.35 \%$ \\
\cline { 2 - 5 } & $0.3 \mathrm{~g}$ & 1.492 & 0.239 & $83.96 \%$ \\
\cline { 2 - 5 } & $0.4 \mathrm{~g}$ & 1.798 & 0.259 & $85.61 \%$ \\
\hline \multirow{3}{*}{ Kobe } & $0.2 \mathrm{~g}$ & 0.589 & 0.187 & $68.26 \%$ \\
\cline { 2 - 5 } & $0.3 \mathrm{~g}$ & 1.330 & 0.248 & $81.35 \%$ \\
\cline { 2 - 5 } & $0.35 \mathrm{~g}$ & 1.687 & 0.274 & $83.75 \%$ \\
\hline \multirow{2}{*}{ Chi-Chi } & $0.2 \mathrm{~g}$ & 0.605 & 0.177 & $70.79 \%$ \\
\cline { 2 - 5 } & $0.3 \mathrm{~g}$ & 1.431 & 0.275 & $80.80 \%$ \\
\hline
\end{tabular}

Table 1. Maximum roof accelerations and earthquake efficiency for the DO-FPS isolated critical equipment at angle of $90^{\circ}$

\begin{tabular}{|c|c|c|c|c||}
\hline \multirow{2}{*}{ Max. Response } & \multicolumn{4}{|c||}{ Roof Acceleration(g) } \\
\hline \multirow{2}{*}{ Earthquake } & $\begin{array}{c}\text { Amplitude } \\
\text { (PGA) }\end{array}$ & $\begin{array}{c}\text { Fixed-Base } \\
\text { Structure }\end{array}$ & $\begin{array}{c}\text { Isolated } \\
\text { Structure }\end{array}$ & $\begin{array}{c}\text { Response } \\
\text { Reduction }\end{array}$ \\
\hline \multirow{3}{*}{ El Centro } & $0.2 \mathrm{~g}$ & 0.827 & 0.285 & $65.53 \%$ \\
\cline { 2 - 5 } & $0.3 \mathrm{~g}$ & 2.204 & 0.370 & $83.21 \%$ \\
\cline { 2 - 5 } & $0.4 \mathrm{~g}$ & 3.723 & 0.472 & $87.31 \%$ \\
\hline \multirow{3}{*}{ Kobe } & $0.2 \mathrm{~g}$ & 0.599 & 0.203 & $66.19 \%$ \\
\cline { 2 - 5 } & $0.3 \mathrm{~g}$ & 1.345 & 0.293 & $70.39 \%$ \\
\cline { 2 - 5 } & $0.35 \mathrm{~g}$ & 1.813 & 0.398 & $78.03 \%$ \\
\hline \multirow{2}{*}{ Chi-Chi } & $0.2 \mathrm{~g}$ & 0.618 & 0.228 & $63.08 \%$ \\
\cline { 2 - 5 } & $0.3 \mathrm{~g}$ & 1.451 & 0.282 & $80.56 \%$ \\
\hline
\end{tabular}

Table 2. Maximum roof accelerations and earthquake efficiency for the DO-FPS isolated critical equipment at angle of $0^{\circ}$ 


\section{Conclusions}

The isolators presented in this chapter, which provide damping as a result of the deformed material or the frictional force between the sliding interfaces, have rectified the drawbacks of the rolling ball isolation system, such as little damping provided by the system, highly concentrated stress produced by the rolling ball or cylindrical rod due to the small contact area between the rolling ball (or cylindrical rod) and the concave surfaces, and scratches and damage to the concave surfaces caused by the ball or cylindrical rod motions during earthquakes. The presented isolators not only effectively lengthen the natural period of the vibration sensitive equipment but also provide significant damping to reduce the bearing displacement and size and the protection to the contact area between the damped steel ball (or articulated slider) and the concave surface to prevent any damage or scratch on the concave surfaces. Further, the advanced isolators possess a stable mechanical behavior during the life span of service. In addition, the isolators can isolate energy induced by earthquakes to ensure the safety and functionality of the vibration sensitive equipment located in a building. It can be concluded from these studies that the presented isolators in this chapter, including the rolling and sliding types of isolators, exhibit excellent features for preventing vibration sensitive equipment from earthquake damage.

\section{References}

Alhan, C. and Gavin, H. P. (2005). Reliability of Base Isolation for the Protection of Critical Equipment from Earthquake Hazards. Engineering Structures, Vol. 27, 1435-1449.

Al-Hussaini, T. M.; Zayas, V. A. and Constantinou, M. C. (1994). Seismic Isolation of Multistory Frame Structures Using Spherical Sliding Isolation Systems. NCEER Technical Report, NCEER-94-0007, National Center for Earthquake Engineering Research, State University of New York at Buffalo, NY.

Bakker, J. F. J. (1935). Balance Block for Buildings. US Patent No. 2014643.

Chung, L. L.; Yang, C. Y.; Chen, H. M. and Lu, L. Y. (2008). Dynamic Behavior of Nonlinear Rolling Isolation System. Structural Control and Health Monitoring, DOI: 10.1002/stc.305.

Cummings, F. D. (1930). Building Construction (Quakeproof Building). US Patent No. 1761659.

Eberhard, M. O.; Baldridge, S.; Marshall, J.; Mooney, W. and Rix, G. J. (2010). The MW 7.0 Haiti Earthquake of January 12, 2010: USGS/EERI Advance Reconnaissance Team Report. Report 2010-1048, U.S. Department of the Interior, U.S. Geological Survey.

Fan, Y. C.; Loh, C. S.; Yang, J. N. and Lin, P. Y. (2008). Experimental Performance Evaluation of an Equipment Isolation Using MR Dampers. Earthquake Engineering and Structural Dynamics, DOI: $10.1002 /$ eqe.844.

Fathali, S. and Filiatrault, A. (2007). Experimental Seismic Performance Evaluation of Isolation/Restraint Systems for Mechanical Equipment, Part 2: Light Equipment Study. Technical Report MCEER-07-0022, University at Buffalo, The State University of New York.

Fenz, D. M. and Constantinou, M. C. (2006). Behavior of the Double Concave Friction Pendulum Bearing. Earthquake Engineering and Structural Dynamics, Vol. 35, No. 11, 1403-1424. 
Fenz, D. M. and Constantinou, M. C. (2008a). Spherical Sliding Isolation Bearings with Adaptive Behavior-Theory. Earthquake Engineering and Structural Dynamics, Vol. 37, No. 2, 168-183.

Fenz, D. M. and Constantinou, M. C. (2008b). Mechanical Behavior of Multi-spherical Sliding Bearings. Technical Report MCEER-08-0007, Multidisciplinary Center for Earthquake Engineering Research, State University of New York at Buffalo, NY.

Kanamori, H. (1978). Quantification of Earthquakes. Nature, Vol. 271, No. 5644, 411-414.

Kasalanati, A.; Reinhorn, A. M.; Constantinou, M. C. and Sanders, D. (1997). Experimental Study of Ball-in-cone Isolation System. Proceedings of the structures congress $X V$, ASCE, Vol. 2. Portland, Oregon, 1191-1195.

Kemeny, Z. A. (1997). Ball-in-cone Seismic Isolation Bearing. US Patent No. 5599106.

Kim, J. K. (2004). Directional Rolling Pendulum Seismic Isolation Systems and Roller Assembly Therefore. US patent No. 6725612, Filing date: March 11, 2002.

Kim, Y. S. and Yun, C. B. (2007). Seismic Response Characteristics of Bridges Using Double Concave Friction Pendulum Bearings with Tri-linear Behavior. Engineering Structures, Vol. 29, No. 11, 3082-3093.

Lin, T. W. and Hone, C. C. (1993). Base Isolation by Free Rolling Rods under Basement. Earthquake Engineering and Structural Dynamics, Vol. 22, 261-73.

Morgan, T. A. and Mahin, S. A. (2008). The Optimization of Multi-stage Friction Pendulum Isolators for Loss Mitigation Considering a Range of Seismic Hazard. In the 14th World Conference on Earthquake Engineering, Beijing, China, Paper No. 11-0070.

Morgan, T. A. and Mahin, S. A. (2010). Achieving Reliable Seismic Performance Enhancement Using Multi-stage Friction Pendulum Isolators. Earthquake Engineering and Structural Dynamics, Vol. 39, 1443-1461.

Moustafa, A. (2011). Damage-Based Design Earthquake Loads for Single-Degree-OfFreedom Inelastic Structures. Journal of Structural Engineering, ASCE, Vol. 137, No. 3, March, 2011, 456-467.

Naeim, F. and Kelly, J. M. (1999). Design of Seismic Isolated Structures: from Theory to Practice. John Wiley \& Sons, ISBN: 0-471-14921-7, New York.

Penkuhn, A. L. K. (1967). Three Point Foundation for Building Structures. US Patent No. 3347002.

Rivin, E. I. (2003). Passive Vibration Isolation. The American Society of Mechanical Engineers, ISBN: 0-7918-0187-X.

Roussis, P. C. and Constantinou, M. C. (2005). Experimental and Analytical Studies of Structures Seismically Isolated with an Uplift-restraint Isolation System. Technical Report MCEER005-0001, Buffalo, NY.

Schär, F. (1910). Foundation for Buildings. US Patent No. 951028.

Skinner, R. I.; Robinson, W. H. and McVerry, G. H. (1993). An Introduction to Seismic Isolation. John Wiley \& Sons, ISBN: 0-471-93433-X, New York.

Soong, T. T. and Dargush, G. F. (1997). Passive Energy Dissipation Systems in Structural Engineering. John Wiley \& Sons, ISBN: 0-471-96821-8, New York.

Takewakin, I. (2009). Building Control with Passive Dampers. John Wiley \& Sons, ISBN: 978-0470-82491-7, Singapore. 
Takewakin, I.; Murakami, S.; Fujita, K.; Yoshitomi, S. and Tsuji, M. (2011). The 2011 off the Pacific Coast of Tohoku Earthquake and Response of High-rise Buildings under Long-period Ground Motions. Soil Dynamics and Earthquake Engineering, doi:10.1016/j.soildyn. 2011.06.001.

Tsai, C. S. (2003). Improved Structures of Base Isolation Systems. Taiwan patent No. 207126, Publication Number: 00542278, Publication date: July 11, 2003, Application number: 091210175, Filing date: July 4, 2002 [in Chinese].

Tsai, C. S. (2004a). Structure of an Anti-shock Device. US Patent No. 6688051, Filing date: March 7, 2002.

Tsai, C. S. (2004b). Structure of an Anti-shock Device. US Patent No. 6820380, Filing date: Sept. $10,2003$.

Tsai, C. S. (2007). Foundation Shock Eliminator. US Patent No. 7237364, Filing Date: July 2, 2004.

Tsai, C. S. (2008a). Foundation Shock Suppressor. US Patent No. 7338035, Filing date: Dec. 9, 2004.

Tsai, C. S. (2008b). Anti Shock Device. US Patent No. 7409799, Filing date: Dec. 13, 2005.

Tsai, C. S.; Chiang, T. C. and Chen, B. J. (2003a). Finite Element Formulations and Theoretical Study for Variable Curvature Friction Pendulum System. Engineering Structures, Vol. 25, No. 14, 1719-1730.

Tsai, C. S.; Chiang, T. C. and Chen, B. J. (2003b). Seismic Behavior of MFPS Isolated Structure under Near-fault Earthquakes and Strong Ground Motions with Long Predominant Periods. In the 2003 ASME Pressure Vessels and Piping Conference, Seismic Engineering, Cleveland, Ohio, U. S. A., J. C. Chen, Editor, Vol. 466, 7379.

Tsai, C. S.; Chiang, T. C. and Chen, B. J. (2005a). Experimental Evaluation Piecewise Exact Solution for Predicting Seismic Responses of Spherical Sliding Type Isolated Structures. Earthquake Engineering and Structural Dynamics, Vol. 34, No. 9, 10271046.

Tsai, C. S.; Cheng, C. K.; Chen, M. J. and Yu, S. H. (2005b). Experimental Study of MFPSisolated Sensitive Equipment. in the 2005 ASME Pressure Vessels and Piping Conference, Seismic Engineering, C. S. Tsai, editor, Denver, Colorado, U.S.A., 8:1118.

Tsai, C. S.; Lin, Y. C.; Chen, W. S.; Chen, M. J. and Tsou, C. P. (2006a). The Material Behavior and Isolation Benefits of Ball Pendulum System. in the 2006 ASME Pressure Vessels and Piping Conference, Seismic Engineering, James F. Cory, editor, Vancouver, BC, Canada, Paper No. PVP2006-ICPVT11-93252.

Tsai, C. S.; Chen, W. S.; Yu, S. H. and Yang, C. T. (2006b). Shaking Table Tests of Critical Equipment with Simple Isolators. in the 2006 ASME Pressure Vessels and Piping Conference, Seismic Engineering, James F. Cory, Editor, Vancouver, BC, Canada., July 23-27, No. PVP2006-ICPVT11-93250.

Tsai, C. S.; Chen, W. S.; Chiang, T. C. and Chen, B. J. (2006c). Component and Shaking Table Tests for Full-scale Multiple Friction Pendulum System. Earthquake Engineering and Structural Dynamics, Vol. 35, No. 11, 1653-1675. 
Tsai, C. S.; Chen, W. S.; Chiang, T. C. and Lin, Y. C. (2007). Application of Direction Optimized-Friction Pendulum System to Seismic Mitigation of Sensitive Equipment. in the 2007 ASME Pressure Vessels and Piping Conference, Seismic Engineering, Artin Dermenjian, editor, San Antonio, Texas, U.S.A., Paper No. PVP2007-26552.

Tsai, C. S.; Chen, W. S.; Lin, Y. C.; Tsou, C. P.; Chen, C. C. and Lin, C. L. (2008a). Shaking Table Tests of Motion Sensitive Equipment Isolated with Static Dynamics Interchangeable-Ball Pendulum System. In: the 14th World Conference on Earthquake Engineering, Beijing, China, Paper No. 11-0010.

Tsai, C. S.; Lu, P. C.; Chen, W. S.; Chiang, T. C.; Yang, C. T. and Lin, Y. C. (2008b). Finite Element Formulation and Shaking Table Tests of Direction-Optimized Friction Pendulum System. Engineering Structures, Vol. 30, No. 9, 2321-2329.

Tsai, C. S.; Lin, Y. C. and Su, H. C. (2010a). Characterization and Modeling of Multiple Friction Pendulum Isolation System with Numerous Sliding Interfaces. Earthquake Engineering and Structural Dynamics, Vol. 39, No. 13, 1463-1491.

Tsai, C. S.; Lin, Y. C. and Su, H. C. (2010b). Seismic Responses of a Building Isolated with Multiple Friction Pendulum System Subjected to Multi-directional Excitations. In the 2010 ASME Pressure Vessels and Piping Conference, Seismic Engineering, O'Brien, Cheryl C. (ed.), Bellevue, Washington, U. S. A., Paper No. PVP2010-25587.

Tsai, C. S.; Chen, W. S. and Chiang, T. C. (2010c). Experimental and Numerical Studies of Trench Friction Pendulum System. Structural Engineering and Mechanics, An International Journal, Vol. 34, No. 2.

Tsai, C. S. and Lin, Y. C. (2010d). Mechanical Characteristics and Modeling of Multiple Trench Friction Pendulum System with Multi-intermediate Sliding Plates. International Journal of Aerospace and Mechanical Engineering, Vol. 4, No. 1.

Tsai, C. S.; Lin, Y. C. and Su, H. C. (2010e). Characteristic and Modeling of multiple Direction Optimized-Friction Pendulum System with Numerous Sliding Interfaces Subjected to Multi-directional Excitations. In the 2010 ASME Pressure Vessels and Piping Conference, Seismic Engineering, O'Brien, Cheryl C. (ed.), Bellevue, Washington, U. S. A., Paper No. PVP2010-25598.

Tsai, C. S.; Wang, Y. M. and Su, H. C. (2011a). Experimental Study of a Full Scale Building Isolated with Multiple Friction Pendulum System with Multiple Sliding Interfaces. In the 2011 ASME Pressure Vessels and Piping Conference, Seismic Engineering, O'Brien, Cheryl C. (ed.), Baltimore, Maryland, U. S. A., Paper No. PVP2011-57352.

Tsai, C. S.; Hsueh, C. I. and Su, H. C. (2011b). Experimental Investigation on Performance of Multiple Direction-Optimized Friction Pendulum System with Multiple Sliding Interfaces in Mitigating Structural Responses during Earthquakes. In the 2011 ASME Pressure Vessels and Piping Conference, Seismic Engineering, O'Brien, Cheryl C. (ed.), Baltimore, Maryland, U. S. A., Paper No. PVP2011-57598.

Tsai, M. H.; Wu, S. Y.; Chang, K. C. and Lee, G. C. (2007). Shaking Table Tests of a Scaled Bridge Model with Rolling-type Seismic Isolation Bearings. Engineering Structures, Vol. 29, 694-702. 
Touaillon, J. (1870). Improvement in Buildings. US Letters Patent No. 99973.

Wu, C. J. (1989). Anti-earthquake Structure Insulating the Kinetic Energy of Earthquake from Buildings. US Patent No. 4881350.

Zayas, V. A. (1987). Earthquake Protective Column Support. US Patent No. 4644714.

Zayas, V. A.; Low, S. S. and Mahin, S. A. (1987). The FPS Earthquake Resisting System Report. EERC Technical Report, UBC/EERC-87/01, University of California, Berkeley, CA. 


\title{
Seismic Damage Estimation in Buried Pipelines Due to Future Earthquakes - The Case of the Mexico City Water System
}

\author{
Omar A. Pineda-Porras ${ }^{1}$ and Mario Ordaz ${ }^{2}$ \\ ${ }^{1}$ Energy and Infrastructure Analysis (D-4), Los Alamos National Laboratory, Los Alamos, \\ 2Instituto de Ingeniería, Ciudad Universitaria, Universidad Nacional \\ Autónoma de México (UNAM) \\ ${ }^{1}$ USA \\ ${ }^{2}$ Mexico
}

\section{Introduction}

Since the mid-70s, there have been advances in the development of models to better understand how earthquakes affect buried pipelines. These natural events can cause damage due to two phenomena: seismic wave propagation and permanent ground deformation. The combined effect of both phenomena in pipeline damage estimation is a subject still complex to address, especially if the objective is to estimate damage due to future earthquakes. In this chapter, the damage assessment methods only consider the impact of seismic wave propagation. The effects of permanent ground deformation phenomena, like ground subsidence, landslides, and ground rupture, are omitted.

The exceptional damage caused by the 1985 Michoacan earthquake in Mexico City has encouraged researchers to develop sophisticated tools to estimate ground motion in the Valley of Mexico from Pacific coastal earthquakes, including the important site effects largely observed in the city. These tools have helped to better understand how earthquakes affect buildings and other structures like pipeline systems. The most remarkably case of pipeline damage caused by the 1985 seismic event is the extensive damage suffered by the Mexico City Water System (MCWS) that left almost 3.5 million people without water, and caused water service disruptions over a period of two months. The 1985 MCWS damage scenario has been extensively analyzed for developing models to better understand how seismic wave propagation affects buried pipelines; some of those models are employed in the future damage prediction methods described in this manuscript.

Fragility functions are typically the tools most used to assess seismic damage in buried pipelines. These functions relate pipeline damage with seismic intensity. Pipeline damage is generally expressed as a linear pipe repair density. Seismic intensity is usually quantified through a seismic parameter. There are many seismic parameters used as arguments of fragility functions; the most important of these are described in Section 2. Section 3 describes the most important fragility functions proposed until now, including the two employed in the seismic damage estimation for the MCWS presented in Section 4. Finally, Section 5 contains a summary of the most important conclusions of this work. 


\section{Seismic parameters related to damage in buried pipelines}

An historical revision of all the seismic parameters employed to represent seismic intensity in fragility functions is summarized in this section. The seismic parameters described in detail are Mercalli modified intensity (MMI), peak ground acceleration (PGA), peak ground velocity (PGV), maximum ground strain, and a recently proposed composite parameter (Section 2.5). Other parameters used as fragility function arguments are not included here because there is not enough evidence of their relationship with pipeline damage; among them are permanent ground displacement, Arias intensity, spectral acceleration, and spectral intensity.

\subsection{Mercalli modified intensity}

Though it is a parameter of subjective nature, MMI was used as damage indicator for pipelines in the 80s and 90s (Eguchi 1983 and 1991; Ballantyne et al. 1990; and, O'Rourke T. et al. 1998). A likely reason for the development of MMI-based fragility relations in the past was the extended use of that parameter to describe damage to aboveground structures. Lately, the installation of seismic stations and the availability of seismic records have made it easier to estimate parameters like PGA and PGV, which are better related to buried pipeline damage.

\subsection{Peak Ground Acceleration (PGA)}

PGA was largely employed as a damage indicator for pipelines during 25 years, from the study of Katayama et al. (1975), to the PGA-based fragility function of Isoyama et al. (2000). Though it has been largely demonstrated that PGV is related more closely to pipeline damage than PGA, as it is further explained in the following paragraphs, there are several reasons to explain why PGA, instead of PGV, was used to create some fragility functions before 2000. Most seismic stations record time histories of acceleration instead of velocity; then, PGA can be directly obtained from seismic records without involving the integration process needed for computing PGV. Most attenuations laws provide estimates of PGA (before 2000, PGV attenuation laws were limited); thus, for practical purposes, PGA was the ideal parameter for analyzing pipeline damage, and therefore, creating pipeline fragility relations.

\subsection{Peak Ground Velocity (PGV)}

PGV is by far the most widely used seismic parameter for pipeline seismic fragility functions. Generally, PGV shows good correlation with pipeline damage, although some studies (Sections 2.4 and 2.5) have shown that, for pipeline located in soft soils, there are some complications, mainly due to the assumptions related to PGV's use as a damage indicator.

PGV is better related to pipeline damage than PGA mainly due to two reasons: 1) PGV is related to ground strain -the main cause of pipeline damage due to seismic wave propagation (Section 2.3.2)-; and, 2) PGA is more related to inertia forces -forces that do not affect buried structures like pipelines-. Many studies have empirically demonstrated that PGV is better pipeline damage predictor than PGA (O'Rourke T. et al. 1998; Isoyama et al. 2000; and, Pineda 2002). 
PGV has been extensively used as damage indicator for pipelines considering two assumptions: 1) PGV is directly related with maximum ground strain $\left(\varepsilon_{\mathrm{g}}\right)$; and 2) transient ground strain is the main cause of pipeline damage due to seismic wave propagation. The relationship between PGV and $\varepsilon_{\mathrm{g}}$ can be analyzed in Equation 1 (Newmark 1967), where C is seismic wave velocity. From Equation 1, PGV is directly related to $\varepsilon_{\mathrm{g}}$ only if $\mathrm{C}$ is constant. Since $\varepsilon_{\mathrm{g}}$ is non-dimensional, PGV and C must be expressed with the same velocity units.

$$
\varepsilon_{g}=\frac{P G V}{C}
$$

\subsection{Maximum ground strain $\left(\varepsilon_{g}\right)$}

Because transient ground strain is the assumed main cause of pipeline damage due to seismic wave propagation, $\varepsilon_{\mathrm{g}}$ is straightforwardly the optimum parameter for analyzing the relationship between pipeline damage and seismic intensity. Rigorously, $\varepsilon_{\mathrm{g}}$ can be estimated from displacement time histories $\mathrm{D}(\mathrm{t})$ (Equation 2). In Equation 2, $\mathrm{x}$ is a space variable, $\varepsilon(\mathrm{t})$ is ground strain time history, and max represents the maximum of the expression between absolute value brackets | $\mid$.

$$
\varepsilon_{g}=\max |\varepsilon(t)|=\boldsymbol{m a x}\left|\frac{\partial D(t)}{\partial x}\right|
$$

There are three major problems for estimating $\varepsilon_{\mathrm{g}}$ through Equation 2. First, $\varepsilon_{\mathrm{g}}$ is generally obtained through the double integration of acceleration time histories; this process causes loss of information due to the involved mathematical operations. Procedures like correction of base line, filtering and tapering could generate ambiguous results if the parameters used in those operations are modified. Second, the derivation process of $\varepsilon_{\mathrm{g}}$ with respect to a space variable (x) implies that the seismic records used in the analysis need to be referenced to an absolute time scale; this is a very significant limitation because only ground motion information from seismic arrays using the same time reference, and preferably located in the place of interest (e.g., the zone covered by a pipeline system), would be useful. The third and probably the most important problem is the high cost involved in installing and operating seismic arrays covering large extensions (e.g., area covered by a pipeline network).

In order to avoid the above-mentioned problems of Equation 2, Equation 1 has been used to obtain conservative estimates of $\varepsilon_{\mathrm{g}}$. PGV can be easily obtained from seismic records or other sources (e.g., attenuation laws); on the contrary, C is far from being easy to obtain, which complicates the estimation of $\varepsilon_{\mathrm{g}}$. We include two examples to show the complexity of estimating $C$ for the purpose of estimating $\varepsilon_{\mathrm{g}}$ with Equation 1.

In the first example, the $\varepsilon_{\mathrm{g}}$-based fragility relation proposed by $\mathrm{O}^{\prime}$ Rourke and Deyoe (2004) was computed by assuming C values of $500 \mathrm{~m} / \mathrm{sec}$ for Rayleigh waves (surface waves), and 3,000 m/sec for S-waves (body waves). Later, the study of Paolucci and Smerzini (2008) suggests that the apparent propagation velocity of S-waves is closer to $1,000 \mathrm{~m} / \mathrm{sec}$. O'Rourke M. (2009) employed the new suggested $C$ value for $S$-waves and proposed a new version of the 2004 fragility relation. Changing $C$ from $3,000 \mathrm{~m} / \mathrm{sec}$ to $1,000 \mathrm{~m} / \mathrm{sec}$ in Equation 1 implies that $\varepsilon_{\mathrm{g}}$ increases with a factor of three.

The second example deals with the estimation of $\varepsilon_{g}$ in soft soil zones. Singh et al. (1997) analyzed ground strains at the Roma micro-array in Mexico City for four earthquakes. They 
concluded that Equation 1 could be used to estimate $\varepsilon_{\mathrm{g}}$ by using a phase velocity (Rayleigh waves) of $600 \mathrm{~m} / \mathrm{sec}$ instead of the value of $\mathrm{C}$ at the natural period of lakebed sites (estimated as 1,500 m/sec). Singh et al. (1997) indicate that the discrepancy in the value of C could be due to local heterogeneities within the array. .

PGV is a more convenient parameter than $\varepsilon_{\mathrm{g}}$ for analyzing pipeline damage due to seismic wave propagation for three reasons. First, PGV is easier to estimate than $\varepsilon_{\mathrm{g}}$. Second, many studies have proved that PGV is well correlated with pipeline damage. Third, theoretically, there is a direct relationship between PGV and pipeline damage considering two assumptions already mentioned in this section. Notwithstanding these three points, there is evidence of a case in which PGV is not the best parameter for relating pipeline damage with seismic intensity: the particular case of Mexico City.

\subsection{The novel composite parameter PGV²/PGA}

As it will be described in Section 3.4, Pineda and Ordaz (2007 and 2009) demonstrated that $\mathrm{PGV}^{2}$ /PGA is better correlated to pipeline damage than PGV alone for soft soils; a plausible explanation for this is the strong relationship of $\mathrm{PGV}^{2} / \mathrm{PGA}$ to permanent ground displacement (PGD), which is a ground motion parameter related to very-low frequency contents. Though in the past O'Rourke (1998) demonstrated that PGV is better pipeline damage predictor than PGD, studies that focused exclusively on the relationship between pipeline damage and PGD (or PGV 2 PGA) for soft soil sites have not been done yet. Finally, two things must be noted: 1) Pineda and Ordaz (2007 and 2009) employed PGV $2 /$ PGA instead of PGD due to the rigorous theoretical relationship between both parameters; the availability of detailed PGA and PGV maps for the 1985 Michoacan event (see Pineda 2006 for details about those maps); and the lack of information on ground motion to produce reliable PGD maps for the 1985 earthquake (Pineda 2006). Second, Pineda and Ordaz (2007 and 2009) define soft soils as those soils with natural periods equal to or higher than $1.0 \mathrm{sec}$.

\section{Seismic fragility functions for buried pipelines}

Buried pipeline seismic fragility functions relate pipeline damage rates with different levels of seismic intensity. Damage rates are usually defined as the number of pipe repairs per unit length of pipeline (e.g., number of repair per kilometer, rep/ km). Seismic intensity can be quantified through a diverse group of ground motion parameters computed from seismic records (Section 2). Though there are many studies focused on computing analytical pipeline fragilities (Hindy and Novak 1979; O'Rourke M. and El Hmadi 1988; and Mavridis and Pitilakis 1996). This study only includes empirical pipeline fragility functions computed from pipeline damage documented after earthquakes. For the sake of brevity, equations on the fragility relations are not included here; but can be obtain from those studies in the reference section of this chapter.

\subsection{Early fragility functions for buried pipelines}

As noted previously, empirical correlation between buried pipeline damage and ground motion intensity parameters has been studied since the mid-70s. Katayama et al. (1975) employed pipeline damage scenarios from six earthquakes; four in Japan (Kanto, 9/01/1923; Fukui, 6/28/1948; Niigata, 6/16/1964; and, Tokachi-oki, 5/16/1968), one in Nicaragua (Managua, 12/23/1972), and one in the United States (San Fernando, 2/09/1971) 
to compute fragility functions for segmented cast iron (CI) and asbestos cement (AC) pipelines in terms of PGA. Katayama et al. (1975) included fragility functions for poor, average, and good soil conditions.

Early in the 80s, Isoyama and Katayama (1982) employed the 1971 San Fernando earthquake damage scenario to compute a PGA-based fragility function. The same damage data and information on other three pipeline damage scenarios (Santa Rosa, 10/01/1969; Nicaragua, 12/23/1972; and Imperial Valley, 10/15/1979) was used by Eguchi (1983 and 1991) to compute a set of fragility functions in terms of MMI for the following pipeline types: welded steel gas welded joints (WSGWJ), AC, concrete (C), PVC, CI, welded steel caulked joints (WSCJ), welded steel arc-welded joints-Grades A \& B steel (WSAWJ A\&B), polyethylene (PE), ductile iron (DI), and welded steel arc-welded joints-Grade X steel-(WSAWJ X). Eguchi (1983 and 1991) concluded that AC and concrete pipes are more vulnerable than PVC pipes; and PVC pipes are more vulnerable than CI pipes and welded steel pipes with caulked joints. DI pipes experienced on average about 10 times fewer repairs per unit length than the worst performing pipes; and finally, the repair rate of $X$-grade steel pipes with arcwelded joints was approximately ten times smaller than that of DI pipes.

In the late 80s, Barenberg (1988) proposed the first documented PGV-based fragility function for buried CI pipelines employing damage data from three U.S. earthquakes (Puget Sound, 4/29/1965; Santa Rosa, 10/01/1969; and, San Fernando, 2/09/1971). The fragility function of Barenberg (1988) suggests that a doubling of PGV will lead to an increase in the pipeline damage rate by a factor of about 4.5 .

Early in the 90s, Ballantyne et al. (1990) expanded the pipeline damage data of Barenberg (1988) with damage information from other three U.S. earthquakes (Puget Sound, 4/29/1949; Coalinga, 5/02/1983; and Whittier Narrows, 10/01/1987) and proposed new fragility functions by using MMI as a measure of seismic intensity.

Three PGA-based fragility functions were also published in the early 90s. The Technical Council on Lifeline Earthquake Engineering (TCLEE) of the American Society of Civil Engineers (ASCE) published a comprehensive study on seismic loss estimation for water systems (ASCE-TCLEE 1991) in which PGA-based fragility relations were computed from a reanalysis of the damage data of Katayama et al. (1975) and the 1983 Coalinga pipeline damage scenario. Hamada (1991) proposed another PGA-based fragility function by analyzing the damage scenarios of earthquakes from United States (San Fernando, 2/09/1971) and Japan (Miyagiken-oki, 6/12/1978; and Nihonkai-chubu, 5/26/1983). O'Rourke T. et al. (1991) related pipeline damage with PGA employing damage scenarios from seven earthquakes: six from U.S. (San Francisco, 4/18/1906; Puget Sound, 4/29/1965; Santa Rosa, 10/01/1969; San Fernando, 2/09/1971; Imperial Valley, 10/15/1979; Coalinga, 5/02/1983; and Loma Prieta, 10/18/1989), and one from Japan (Miyagiken-oki, 6/12/1978).

\subsection{Fragility functions in terms of PGV}

A notable change in the literature on seismic fragility functions for pipelines is observed from 1993, when PGV began to be the preferred seismic parameter for pipeline fragility relations, and PGA and MMI were no longer used for developing new fragility functions (with some exemptions described later in this section).

O'Rourke and Ayala (1993) proposed a new pipeline fragility function in terms of PGV by using the damage data points of Barenberg (1988) and damage information from three earthquakes, one from the United States (Coalinga, 5/02/1983), and two from Mexico 
(Michoacan, 9/19/1985; and, Tlahuac, 4/25/1989). The damage data employed for computing the fragility function are related to pipelines made of $\mathrm{AC}, \mathrm{CI}$, concrete, and prestressed concrete cylinder pipes. The fragility relation of O'Rourke and Ayala (1993) was later incorporated into the U.S. Federal Emergency Management Agency's loss assessment methodology HAZUS-MH (FEMA 1999). This fragility function can be used for damage prediction of brittle pipelines. For ductile pipelines, the fragility relation must be multiplied by a suggested factor of 0.3 (FEMA 1999).

Eidinger et al. (1995) and Eidinger (1998) reanalyzed the pipeline damage data of O'Rourke and Ayala (1993), along with information from the 1989 Loma Prieta pipeline damage scenario, in order to propose a set of fragility functions in terms of PGV considering pipe material, joint type, and soil corrosiveness. Edinger's fragility functions estimated damage for CI, welded steel (WS), AC, concrete, PVC, and DI pipes.

Hwang and Lin (1997) computed a pipeline fragility function in terms of PGA by analyzing pipeline damage data obtained from six previous studies (Katayama et al. 1975; Eguchi 1991; ASCE-TCLEE 1991; O’Rourke T. et al. 1991; Hamada 1991; and Kitaura and Miyajima 1996).

O'Rourke T. et al. (1998) employed a GIS-based methodology to investigate factors affecting the water supply service of the Los Angeles Department of Water and Power and the Metropolitan Water District, after the 1994 Northridge earthquake. Analyses of the relationship between damage rate and seismic intensity employed seven seismic parameters: MMI, PGA, PGV, PGD, Arias Intensity, Spectral Acceleration, and Spectral Intensity (SI). Pipeline fragility relations in terms of MMI, SI, PGA, and PGV, are also included in the study. O'Rourke T. et al. (1998) concluded that PGV relates to the pipeline damage better than any other parameter and proposed PGV-based fragilities for steel, CI, DI, and AC pipelines. Later, O'Rourke T. and Jeon (1999) developed a fragility relationship (for CI pipes) for scaled velocity, a parameter based on PGV but normalized for the effects of pipe diameter.

Isoyama et al. (2000) computed fragility functions in terms of PGA and PGV by analyzing the pipeline damage scenario left by the 1995 Hyogoken-nanbu earthquake. A multivariate analysis computed empirical correction factors to account for pipe material, pipe diameter, ground topography, and liquefaction, in the fragility relation.

The American Lifeline Alliance (ALA), a public-private partnership between the FEMA and the ASCE, published a set of algorithms to compute the probability of damage from earthquake effects to several components of water supply systems (ALA 2001). For buried pipelines, the PGV-based fragility function published by the ALA was computed from a set of 81 damage rate-PGV data points from 12 seismic damage scenarios. Similar to the fragility functions of Eidinger et al. (1995) and Eidinger (1998), the ALA's fragility relation provides factor to account for pipe material, joint type, and soil corrosiveness.

Jeon and O'Rourke T. (2005) reanalyzed the pipeline damage data from a previous study (O'Rourke T. et al. 1998). They compared the correlation between CI pipeline damage rates from the 1994 Northridge earthquake and PGV computed in different ways (geometric mean PGV, maximum PGV, and maximum vector magnitude of PGV). Their results show that maximum PGV, computed as the peak recorded value, is better correlated with pipeline damage. Jeon and O'Rourke T. (2005) also provide fragility functions for WSJ Steel, CI, DI, and $\mathrm{AC}$, pipelines. 


\subsection{Fragility functions in terms of maximum ground strain}

O'Rourke M. and Deyoe (2004) analyzed the differences of the fragility relations published by O'Rourke M. and Ayala (1993) and O'Rourke T. and Jeon (1999). The analysis identified some reasons for the differences, including the wave type that dominated each seismic scenario, the presence of corrosion in some pipes, and the low statistical reliability of some data points. By removing doubtful data points and classifying the remaining data points according to the presumably dominating wave type, O'Rourke M. and Deyoe computed PGV-based pipeline fragility functions for surface waves (Rayleigh) by assuming phase velocity of $500 \mathrm{~m} / \mathrm{sec}$, and body waves (S-waves) by assuming apparent velocity of $3,000 \mathrm{~m} / \mathrm{sec}$. They also proposed a fragility function in terms of $\varepsilon_{\mathrm{g}}$. The new $\varepsilon_{\mathrm{g}}$-based fragility function also considers the effect of permanent ground deformation because O'Rourke and Deyoe (2004) included repair rate- $\varepsilon_{\mathrm{g}}$ data points from the 1994 Northridge earthquake (Sano et al. 1999) and from Japan (Hamada and Akioka 1997). A recent modification to the $\varepsilon_{\mathrm{g}}$-based fragility relation (O'Rourke M. 2009) uses an apparent velocity of $1000 \mathrm{~m} / \mathrm{sec}$ for S-waves; they base that assumption on a study by Paolucci and Smerzini (2008).

\subsection{Seismic fragility functions based on the 1985 MCWS damage scenario}

In the literature, there are two observed tendencies for developing fragility functions: the use of damage scenarios for several pipelines systems and earthquakes; and the use of damage scenarios for only a specific pipeline system and earthquake. On the one hand, while the first tendency provides general pipeline fragilities, characterized by its wide applicability due to the typical mixture of pipeline types and other factors (ALA, 2001), it is also usually related to high uncertainty levels. On the other hand, using information about a specific pipeline system and well-studied damage scenarios, the uncertainty could be controlled because the number of unknown variables related to pipeline damage (e.g., variables related to earthquake environment, soil properties, and pipeline conditions), for that particular study case, is lower than if several systems and events are included in the analysis. In order to reduce the uncertainty in the damage estimation for the MCWS, in this study only two fragility functions (Pineda and Ordaz, 2003 and 2007) are used to estimate the damage in the pipeline system.

Pineda and Ordaz (2003) proposed a fragility formulation for buried pipelines employing the 1985 damage scenario published by Ayala and O'Rourke M. (1989), and the detailed maps for Mexico City proposed by Pineda and Ordaz (2004). In order to analyze the variability of the relationship between damage rates $(\mathrm{rep} / \mathrm{km})$ and PGV for several ranges of seismic intensity, nine scenarios were used to generate a PGV-based fragility function. In this study, the functional form chosen for the fragility relation is the cumulative normal function because it better fit the cloud of damage rate-PGV data points when compared with the resulting fit employing other function types (linear, power, etc.). This fragility function is employed in the damage assessment of Section 4.2.

In a subsequent study, Pineda and Ordaz (2007) proposed PGV2/PGA as a new seismic parameter for buried pipelines. From a theoretical development, they found that $\lambda_{\mathrm{pr}}$, defined by Equation 3, interrelate the peak ground response parameters PGA, PGV, and PGD. $\lambda_{\text {pr }}$ has three relevant characteristics: it is non-dimensional; it is always greater than or equal to 1.0; and it can be a measure of spectra bandwidth. 


$$
\lambda_{p r}=\frac{P G A \cdot P G D}{P G V^{2}}
$$

By isolating PGD in Equation 3, it is found that PGD and PGV $2 /$ PGA have a direct relationship through $\lambda_{\text {pr }}$ (Equation 4). Based on the assumption that ground strain, the main cause for the pipeline damage, is related to PGD; pipeline damage is then also related to $\mathrm{PGV}^{2} / \mathrm{PGA}$ and $\lambda_{\text {pr }}$. Because $\lambda_{\text {pr }}$ varies in a delimited range for the places where the MCWS is located in Mexico City, it was implicitly included in the fragility formulation. This $\mathrm{PGV}^{2}$ /PGA-based fragility function is used in the damage assessment discussed in Section 4.3 .

$$
P G D=\lambda_{p r}\left(P G V^{2} / P G A\right)
$$

Recently, Pineda and Ordaz (2009) computed fragility relations for 48-inch segmented pipelines considering the effects of ground subsidence, a phenomenon largely observed in the Valley of Mexico. They analyzed the relationship between pipeline damage and seismic intensity (measured in terms of $\mathrm{PGV}^{2} / \mathrm{PGA}$ ) for two levels of differential ground subsidence (DGS). The proposed fragility functions fall above and below a previous fragility relation for 48-inch pipelines that does not explicitly consider the effects of DGS in the damage (Pineda 2006).

\section{Seismic damage estimation in the MCWS due to future earthquakes}

The main objective of this section is to describe a new approach for estimating pipeline damage at the MCWS due to future earthquakes nucleated at the subduction zones of the Pacific Mexican coast. This new study is based on a fragility function in terms of PGV $2 /$ PGA (a seismic parameter described in Section 2.5) that has shown better correlation with buried pipeline damage (Pineda and Ordaz, 2007) than PGV alone. Pineda and Ordaz (2003) previously employed this parameter to predict future damage at the MCWS.

\subsection{Description of the MCWS and its 1985 damage scenario}

The MCWS pipeline network is formed by more than $600 \mathrm{~km}$ of pipes, with diameters ranging from 20- to 72-inches, and made of several pipe materials: asbestos-cement, concrete, cast iron, and steel. This pipeline system is particularly vulnerable to earthquakes because it is mainly located in the lake zone of the Valley of Mexico where deep clay deposits cause great dynamic amplification of seismic waves.

The MCWS was severely affected by the 1985 earthquake, causing extensive damage that left around $40 \%$ of the 8.5 million people living in the Federal District without water service. A comprehensive report on the damage was published by Ayala and O'Rourke M. (1989). They observed that two-thirds of the damage was located at pipe joints; and that the observed failure types include lateral crushing of pipes, and crushing and unplugging of joints. The authors state that the main apparent cause for the extensive damage in the pipeline network was the propagation of surface waves (Rayleigh waves). These greatly amplified waves caused that most of the damage were localized in sites with natural period higher than $2 \mathrm{~s}$, and with clay deposits between 30-m and 70-m deep. The authors also indicate that the second most important factor that likely influenced the great damage in the pipeline system was the largely observed ground settlement typical of the lake zone, a phenomenon that could have reduced the pipeline capacity to withstand wave propagation. 


\subsection{General aspects of the estimation of future damage in the MCWS}

The MCWS 1985 damage scenario has been used to compute fragility functions to better understand the relationship between pipeline damage and seismic intensity (Section 3.4). Two of those fragility functions, the one in terms of PGV (Pineda and Ordaz, 2003), and another in terms of $\mathrm{PGV}^{2} / \mathrm{PGA}$ (Pineda and Ordaz, 2007) are used in the future damage estimation described in Sections 4.3 and 4.4, respectively.

In general, the procedure to estimate the expected number of pipe repairs for a set of earthquake scenarios is the same, independently of the seismic parameter of interest (PGV or $\mathrm{PGV}^{2}$ /PGA). The main steps are 1) A set of postulated earthquake scenarios is calculated with the Program Z (Ordaz et al. 1996) for magnitudes (m) between 6.6 and 8.4, and for focal distances (R) between 250 and $450 \mathrm{~km}$. 2) The MCWS network is divided in segments of $100 \mathrm{~m}$ or shorter, and the seismic parameter value (PGV or PGV2/PGA) is calculated at each segment mid-point for each earthquake scenario. 3) The expected number of pipe repairs for each earthquake scenario is then computed by adding all the damage rate-length products for the whole pipeline network. The summary of these calculations is shown in Sections 4.3 and 4.4.

\subsection{Future damage estimation using PGV}

Based on the 1985 MCWS pipeline damage scenario, Pineda and Ordaz (2003) proposed a fragility function to estimate damage to buried pipelines. In Figure 1 the fragility function is showed along with the data points employed in its calculation. The 1985 damage scenario was divided in zones depending on the PGV values; a total of nine different PGV intervals (I1 to I9 in Figure 1) were used to generate the data points for the relationship damage ratePGV. More details about the calculation of this fragility curve are found at the 2003 paper. In Equation 5, RR is the expected number of pipe repairs per kilometer of pipeline, $\Phi$ represents the cumulative normal function defined by Equation 6, where $\mu$ and $\sigma$ are parameters related to the mean and standard deviation of the damage rate-PGV relationship. In Equations 5 and 6, PGV is in $\mathrm{cm} / \mathrm{sec}$.

$$
\begin{gathered}
R R=\left\{\begin{array}{ccc}
0 & \text { if } & 0<P G V<5.35 \mathrm{~cm} / \mathrm{sec} \\
0.1172+0.7281 \cdot \Phi(P G V ; 51.8964,19.7811) & \text { if } & 5.35 \leq P G V<95 \mathrm{~cm} / \mathrm{sec} \\
0.00137 \cdot P G V+0.70458 & \text { if } & P G V \geq 95 \mathrm{~cm} / \mathrm{sec}
\end{array}\right. \\
\Phi(P G V ; \mu, \sigma)=\int_{-\infty}^{P G V} \frac{1}{\sqrt{2 \pi} \sigma} e^{-(1 / 2)[(v-\mu) / \sigma]^{2}} d v
\end{gathered}
$$

A total of 75 earthquakes scenarios were employed to estimate the MCWS damage due to future seismic events nucleated at the subduction zones of the Pacific Mexican coast. These scenarios represent events with magnitudes between 6.6 and 8.4, and focal distances between 250 and $450 \mathrm{~km}$. The number of pipe repairs for each scenario was rounded to the nearest integer (Table 1). These results can also be observed in Figure 2. It is observed that for magnitudes higher than 7.6, there is an exponential tendency in the variation of the number of repairs (NR), and magnitude. In fact, after fitting a double exponential function in terms of $m$ and $R$, an equation to simplify the estimation of NR was obtained (Equation 7). NR, then, can be estimated with Equation 7 (where e is the exponential parameter) for earthquake scenarios with magnitudes equal or higher than 7.6, and focal distances between 
$250 \mathrm{~km}$ and $450 \mathrm{~km}$. The fitting of Equation 7 is a good representation of the damage estimates from Table 1 because the R-square parameter was 0.996 . The results for magnitudes lower than 7.6 did not have any clear tendency on the $\mathrm{m}$ and $\mathrm{R}$ domains, so it was no possible to find a similar function to the one shown in Equation 7.

$$
N R=0.00075 \cdot e^{1.6295 m} \cdot e^{-0.00344 R}
$$

Equation 7 shows how NR is related to $\mathrm{m}$ and R; however, for a better damage prediction, the figures from Table 1 must have preference over calculations with the equation due to the added error caused by the fitting.

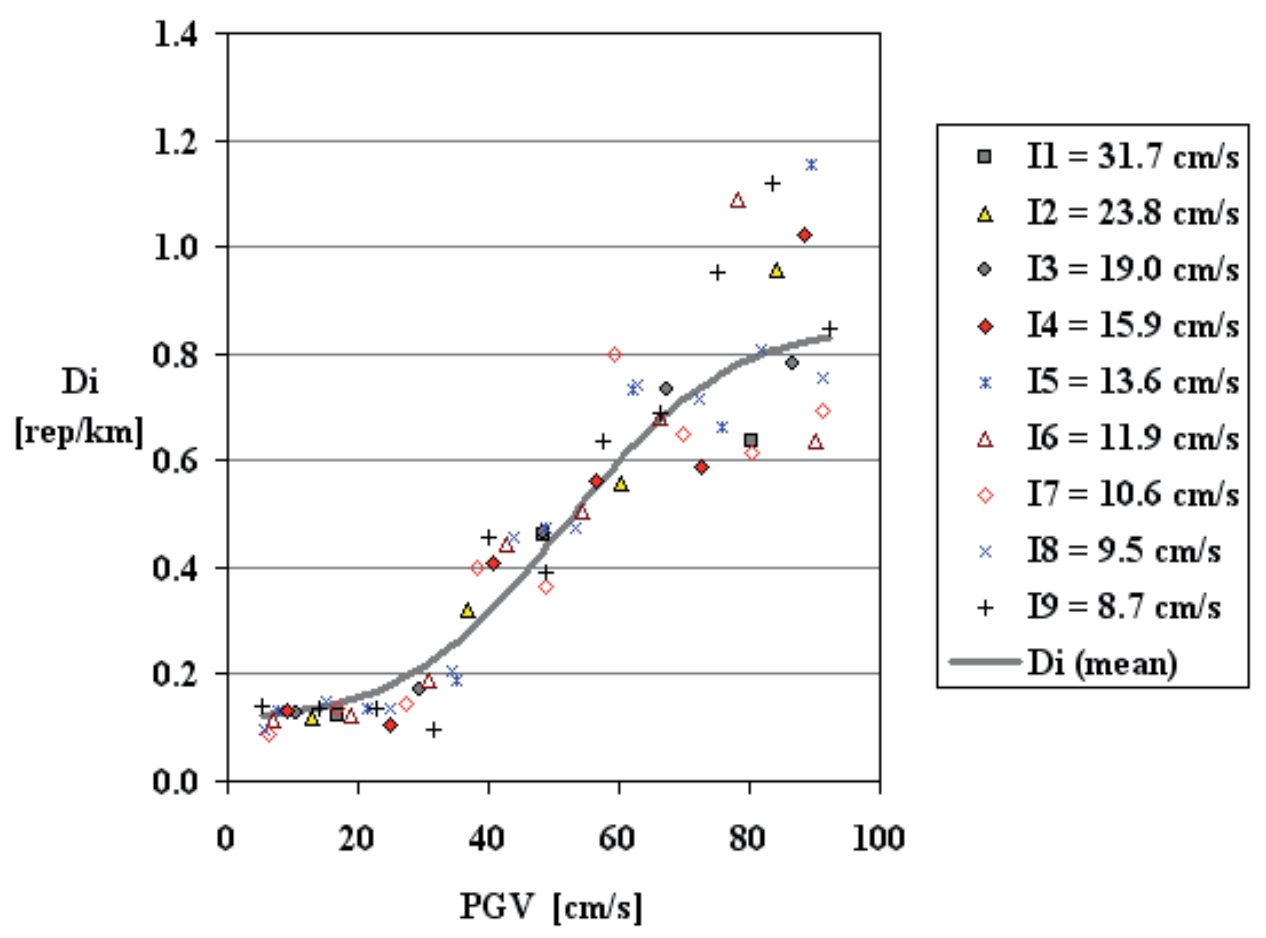

Fig. 1. Fragility Function for the MCWS in terms of PGV (Pineda and Ordaz, 2003). Di is damage rate in number of pipe repairs per kilometer of pipeline (it is also called RR in this manuscript) 
Seismic Damage Estimation in Buried Pipelines Due to

\begin{tabular}{|c|c|c|c|c|c|}
\cline { 2 - 7 } \multicolumn{1}{c|}{} & \multicolumn{5}{c|}{ FOCAL DISTANCE } \\
\hline MAGNITUDE & $\mathbf{R}=\mathbf{2 5 0} \mathbf{~ k m}$ & $\mathbf{R}=\mathbf{3 0 0} \mathbf{~ k m}$ & $\mathbf{R}=\mathbf{3 5 0} \mathbf{~ k m}$ & $\mathbf{R}=\mathbf{4 0 0} \mathbf{~ k m}$ & $\mathbf{R}=\mathbf{4 5 0} \mathbf{~ k m}$ \\
\hline 6.6 & 1 & 0 & 0 & 0 & 0 \\
\hline 6.7 & 4 & 1 & 0 & 0 & 0 \\
\hline 6.8 & 10 & 3 & 1 & 0 & 0 \\
\hline 6.9 & 18 & 7 & 2 & 1 & 0 \\
\hline 7.0 & 27 & 15 & 7 & 2 & 1 \\
\hline 7.1 & 38 & 24 & 13 & 7 & 3 \\
\hline 7.2 & 47 & 35 & 23 & 14 & 7 \\
\hline 7.3 & 54 & 42 & 33 & 23 & 14 \\
\hline 7.4 & 59 & 50 & 40 & 33 & 23 \\
\hline 7.5 & 66 & 57 & 47 & 39 & 32 \\
\hline 7.6 & 76 & 63 & 54 & 45 & 38 \\
\hline 7.8 & 102 & 89 & 70 & 60 & 51 \\
\hline 8.0 & 141 & 115 & 98 & 87 & 68 \\
\hline 8.2 & 208 & 165 & 134 & 113 & 99 \\
\hline 8.4 & 289 & 240 & 198 & 163 & 136 \\
\hline
\end{tabular}

Table 1. Expected number of pipe repairs in the MCWS due to postulated earthquake scenarios (PGV-based model; Pineda and Ordaz, 2003)

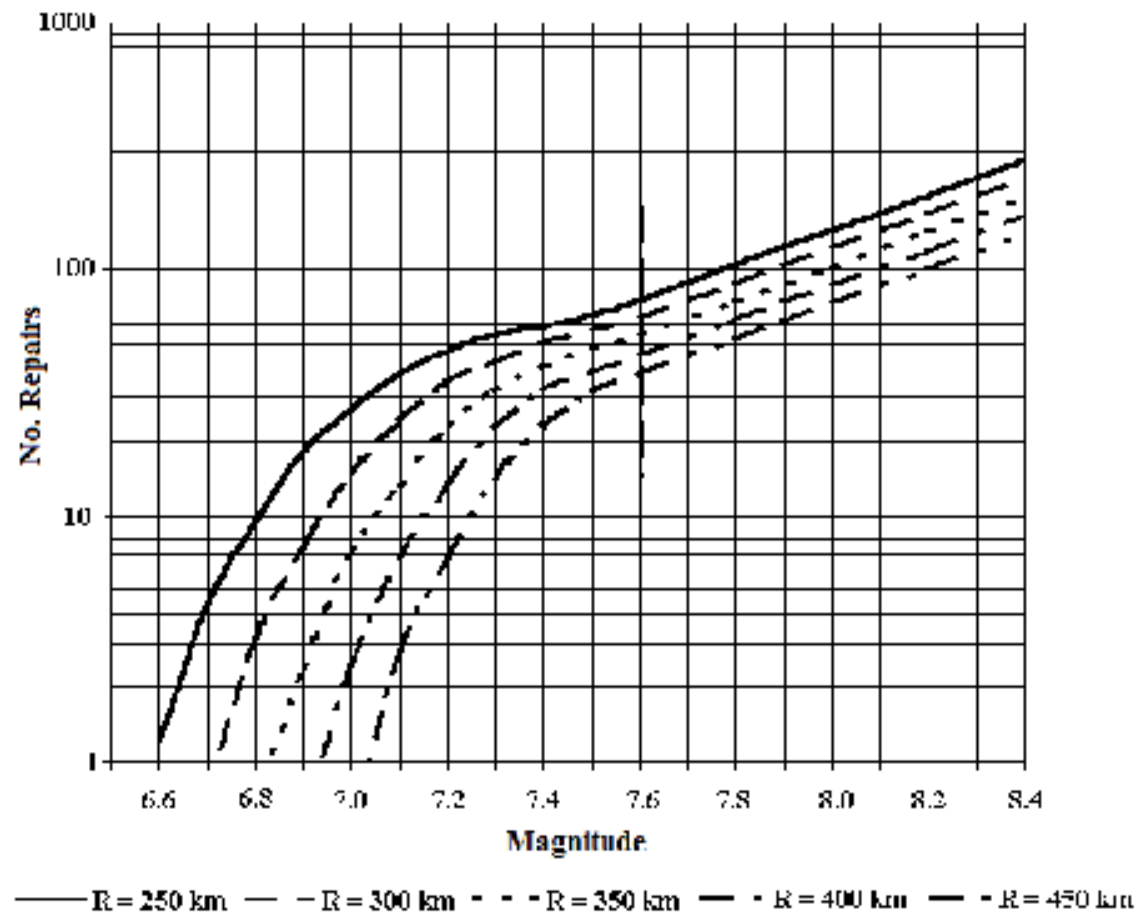

Fig. 2. Seismic damage prediction model for the MCWS based on a PGV-based fragility function (Pineda and Ordaz, 2003) 


\subsection{Future damage estimation using the composite parameter PGV²/PGA}

The PGV $/$ PGA-based fragility function proposed by Pineda and Ordaz (2007) for the MCWS (Figure 3) has three parts where the damage rate can be - zero, constant, or linearly dependent of $\mathrm{PGV}^{2}$ /PGA (Equation 8). The no-damage zone is defined for seismic intensity levels not associated to pipeline damage in the 1985 damage scenario. A likely explanation of the constant-damage zone is the presumably about-to-fail precondition of some pipe segments previously to the 1985 event. The PGV²/PGA map employed by Pineda and Ordaz (2007) to generate the fragility function is shown in Figure 3.

$$
R R=\left\{\begin{array}{ccc}
0 & \text { if } & P G V^{2} / P G A<1.8 \mathrm{~cm} \\
0.122 & \text { if } & 1.8 \leq P G V^{2} / P G A<8.72 \mathrm{~cm} \\
0.032\left(P G V^{2} / P G A\right)-0.157 & \text { if } & P G V^{2} / P G A \geq 8.72 \mathrm{~cm}
\end{array}\right.
$$

In Figure 4, the PGV 2 PGA-based fragility function is showed along with the data points employed in its calculation. In a similar way to the employed to calculate the PGV-based fragility function (Section 4.3), the 1985 damage scenario was divided in zones depending on the PGV 2 / PGA values; a total of nine different PGV $2 /$ PGA intervals (I1 to I9 in Figure 3) were used to generate the data points for the relationship damage rate- PGV ${ }^{2} / \mathrm{PGA}$. The 2007 paper contains more details about the calculation of this fragility curve.

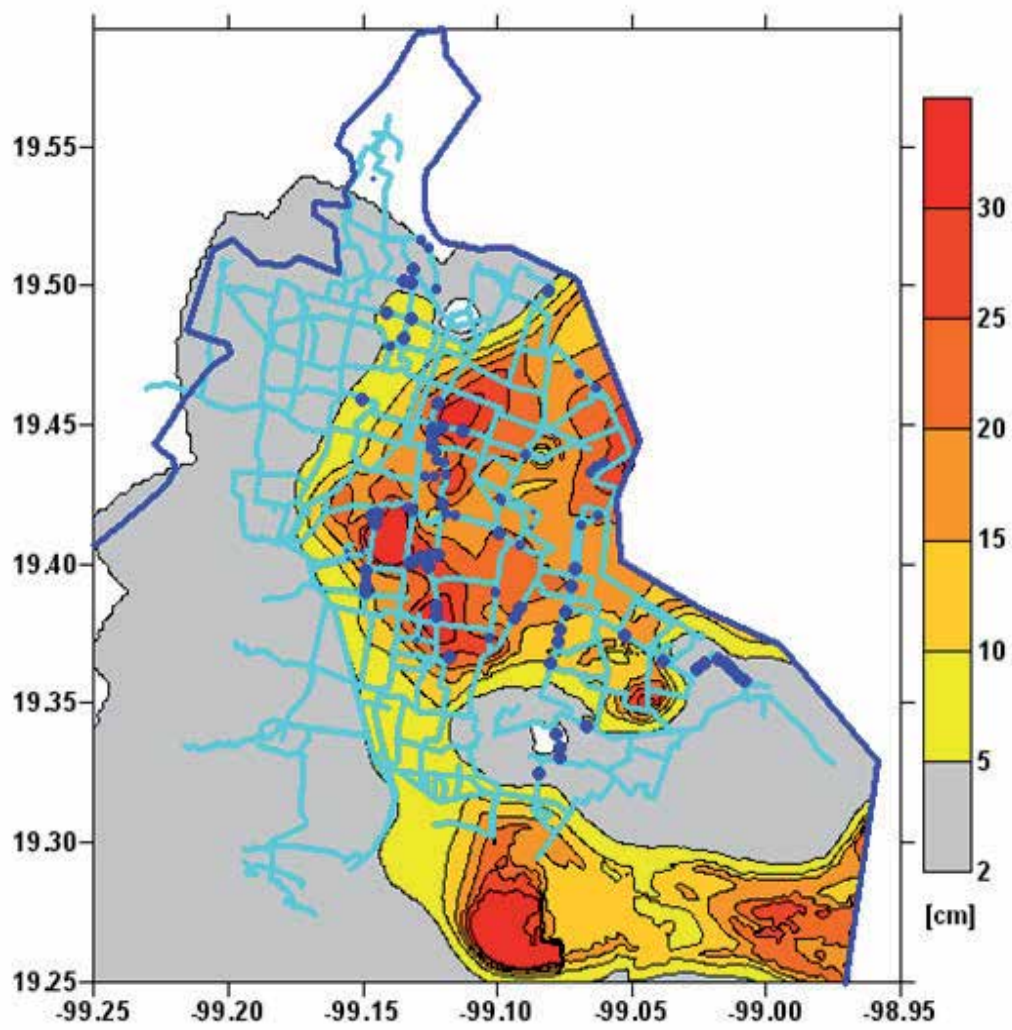

Fig. 3. Mexico City water system, PGV²/PGA map, and damage sites after the 1985 Michoacan earthquake 


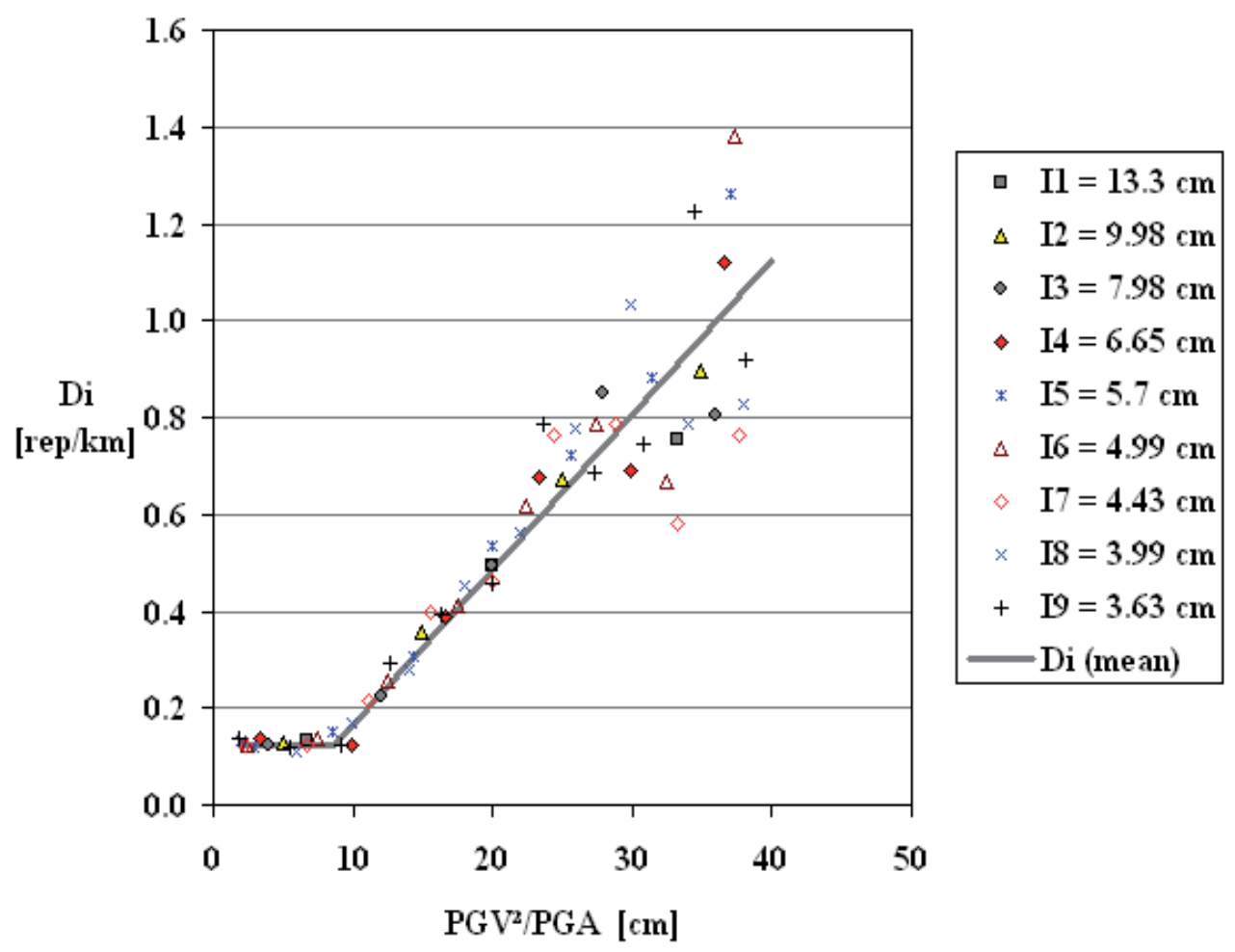

Fig. 4. Fragility Function for the MCWS in terms of PGV²/PGA (Pineda and Ordaz, 2007). Di is damage rate (rep/ $\mathrm{km})$; it is also called RR through this manuscript

The damage estimation for the MCWS employing the fragility function in terms of $\mathrm{PGV}^{2}$ /PGA was done with 80 earthquake scenarios calculated with the Program Z (Ordaz et al. 1996) for seismic events nucleated at the Mexican Pacific subduction zones with magnitudes between 6.6 and 8.4, and focal distances between 250 and $450 \mathrm{~km}$, as it was done with the model described in Section 4.3. Figure 5 shows an example of postulated seismic scenario for a magnitude of 8.4 and focal distance of $250 \mathrm{~km}$. This event is much stronger than the 1985 Michoacan event $(m=8.1 ; R=295 \mathrm{~km})$ because it has a larger magnitude and a shorter focal distance.

The expected number of pipe repairs for the 80 earthquake scenarios are shown in Table 2 and plotted in Figure 6. In this prediction model, an exponential relationship between the number of pipe repairs and magnitude is observed for magnitudes higher than 7.8 for all focal distances; this relationship can be represented by the exponential function shown in Equation 9, where $a$ and $b$ are fitting parameters that vary depending on the focal distance values (Table 3). The fit of Equation 9 for all five focal distances is good because the Rsquare parameters have values close to one. Figure 7 shows the fitted curves with Equation 9 using the parameters from Table 3. These curves can be compared with the original data points from Table 2. Because there is no a clear tendency in the variation of NR with respect to $\mathrm{m}$ and $\mathrm{R}$ for magnitudes lower than 7.8 , a fitted curve could not be found.

$$
N R=a \cdot e^{b \cdot m}
$$




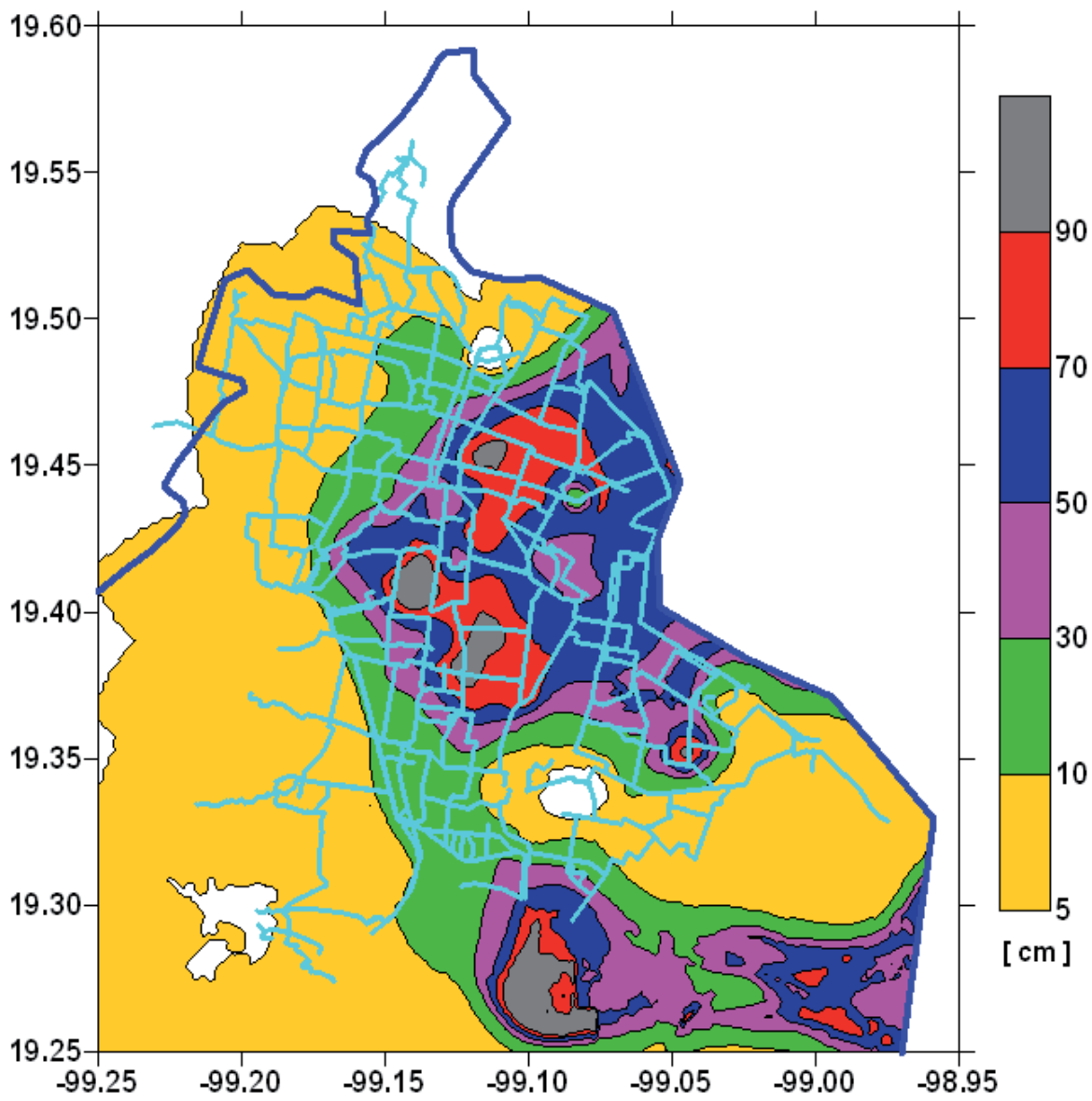

Fig. 5. Earthquake scenario map for a postulated event $(\mathrm{m}=8.4 ; \mathrm{R}=250 \mathrm{~km})$ and the MCWS

\subsection{Comparison of results between the PGV and PGV'/PGA models}

A comparison between both prediction models reveals that the PGV $2 /$ PGA-based model (Section 4.4) predicts a lower number of pipe repairs than the PGV-based model (Section 4.3), for magnitudes around 8.0 and lower; and for higher magnitudes, the PGV $2 / P G V-$ based model predicts a higher number of pipe repairs (Figure 8). These results mean that the 2003 model overestimates the damage in the MCWS for earthquakes with magnitudes up to 8.0, and underestimates damage figures for stronger earthquakes. This conclusion is based on the fact that the proposed model uses a parameter better related to buried pipeline damage.

One important advantage of the PGV²/PGA model is the linear relationship between RR and $\mathrm{PGV}^{2} / \mathrm{PGA}$ in the fragility function. This simple functional form makes it easier to assess the damage for very strong earthquakes (events stronger than the 1985 quake). On the contrary, the PGV model could be unreliable for earthquakes stronger than the 1985 quake because the PGV-based fragility function assumes a linear relationship RR-PGV for PGV values higher than $95 \mathrm{~cm} / \mathrm{sec}$, something that could not be demonstrated in the 2003 study. 
Seismic Damage Estimation in Buried Pipelines Due to

\begin{tabular}{|c|c|c|c|c|c|}
\cline { 2 - 6 } \multicolumn{1}{c|}{} & \multicolumn{5}{c|}{ FOCAL DISTANCE } \\
\hline MAGNITUDE & $\mathbf{R}=\mathbf{2 5 0} \mathbf{~ k m}$ & $\mathbf{R}=\mathbf{3 0 0} \mathbf{~ k m}$ & $\mathbf{R}=\mathbf{3 5 0} \mathbf{~ k m}$ & $\mathbf{R}=\mathbf{4 0 0} \mathbf{~ k m}$ & $\mathbf{R}=\mathbf{4 5 0} \mathbf{~ k m}$ \\
\hline 6.6 & 0 & 0 & 0 & 0 & 0 \\
\hline 6.7 & 0 & 0 & 0 & 0 & 0 \\
\hline 6.8 & 2 & 0 & 0 & 0 & 0 \\
\hline 6.9 & 5 & 1 & 0 & 0 & 0 \\
\hline 7.0 & 9 & 4 & 1 & 0 & 0 \\
\hline 7.1 & 14 & 8 & 4 & 1 & 0 \\
\hline 7.2 & 19 & 13 & 8 & 4 & 1 \\
\hline 7.3 & 25 & 18 & 13 & 8 & 4 \\
\hline 7.4 & 32 & 24 & 18 & 13 & 8 \\
\hline 7.5 & 39 & 35 & 29 & 23 & 18 \\
\hline 7.6 & 46 & 39 & 35 & 32 & 28 \\
\hline 7.7 & 56 & 47 & 39 & 36 & 32 \\
\hline 7.8 & 74 & 58 & 48 & 40 & 36 \\
\hline 8.0 & 147 & 124 & 98 & 73 & 59 \\
\hline 8.2 & 261 & 216 & 183 & 158 & 138 \\
\hline 8.4 & 474 & 390 & 328 & 279 & 241 \\
\hline
\end{tabular}

Table 2. Expected number of pipe repairs in the MCWS due to postulated earthquake scenarios (PGV 2 /PGA-based model)

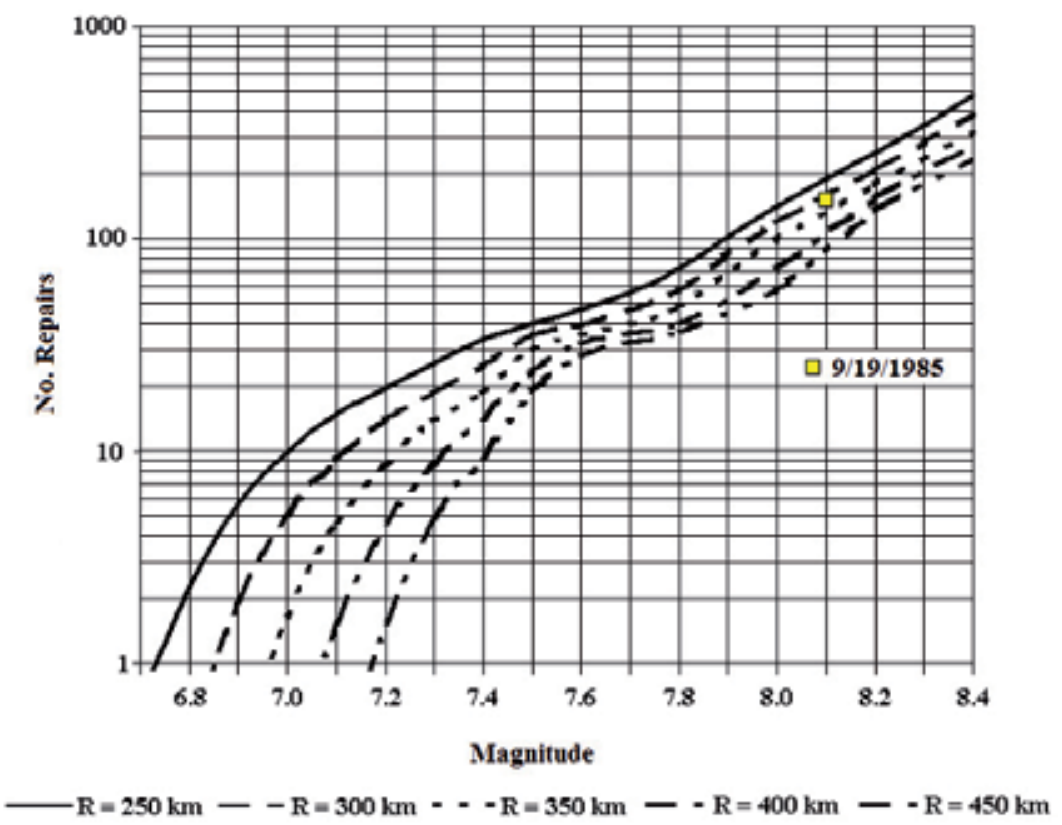

Fig. 6. Seismic damage prediction model for the MCWS based on a PGV²/PGA-based fragility function. The square marker corresponds to the 1985 Michoacan earthquake case $(\mathrm{m}=8.1 ; \mathrm{R}=295 \mathrm{~km})$ 


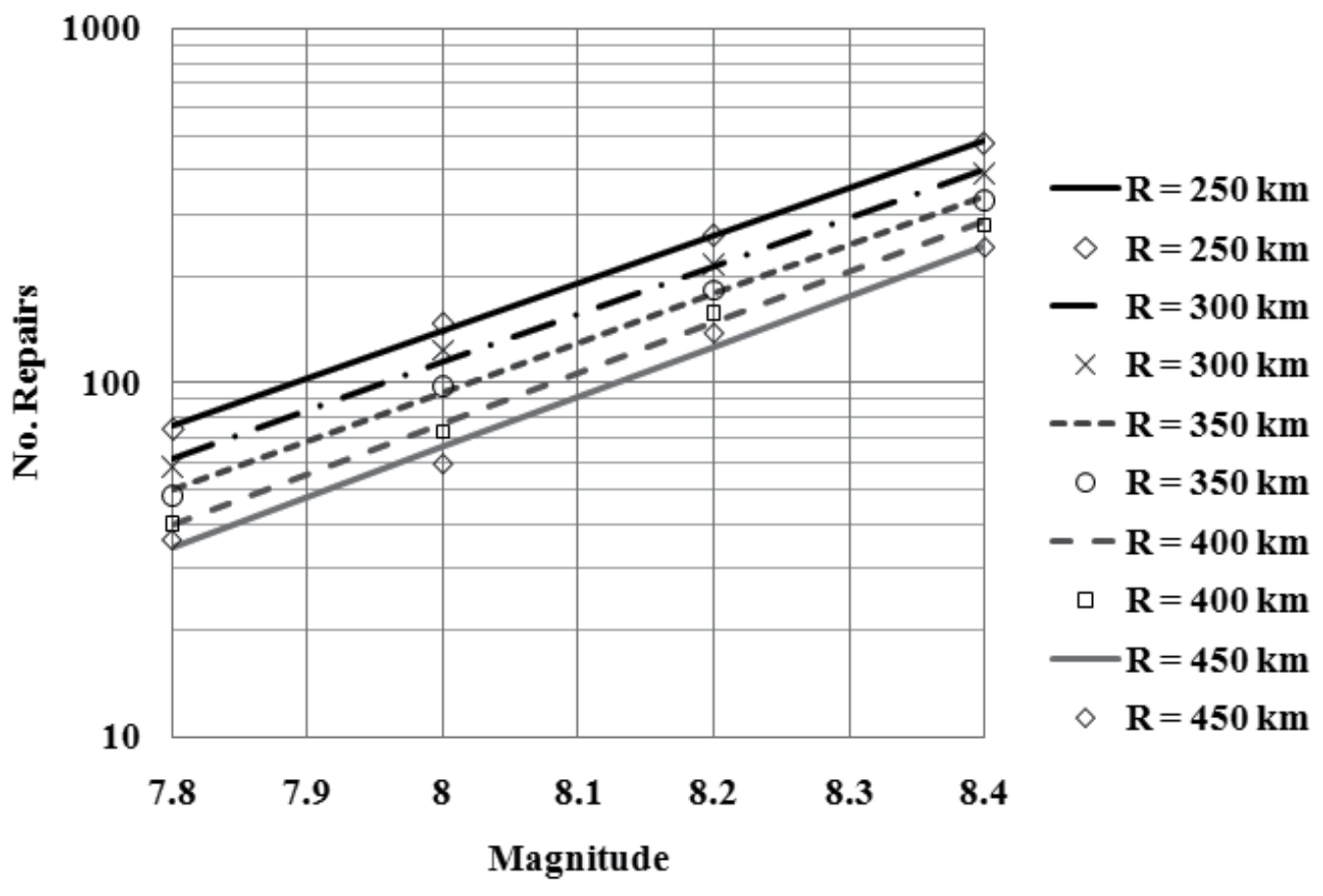

Fig. 7. Fitted curves for Equation 9

\begin{tabular}{|c|c|c|c|c|c|}
\cline { 2 - 6 } \multicolumn{1}{c|}{} & \multicolumn{5}{c|}{ FOCAL DISTANCE } \\
\cline { 2 - 6 } \multicolumn{1}{c|}{} & $\mathbf{R}=\mathbf{2 5 0} \mathbf{~ k m}$ & $\mathbf{R}=\mathbf{3 0 0} \mathbf{~ k m}$ & $\mathbf{R}=\mathbf{3 5 0} \mathbf{~ k m}$ & $\mathbf{R}=\mathbf{4 0 0} \mathbf{~} \mathbf{m}$ & $\mathbf{R}=\mathbf{4 5 0} \mathbf{~ k m}$ \\
\hline $\mathrm{a}$ & $2.972 \mathrm{E}-09$ & $1.448 \mathrm{E}-09$ & $8.453 \mathrm{E}-10$ & $2.916 \mathrm{E}-10$ & $2.927 \mathrm{E}-10$ \\
\hline $\mathrm{b}$ & 3.073 & 3.1369 & 3.18 & 3.287 & 3.268 \\
\hline $\mathrm{R}^{2}$ & 0.998 & 0.995 & 0.998 & 0.997 & 0.99 \\
\hline
\end{tabular}

Table 3. Parameters a and b for Equation 9, and R-square parameters 


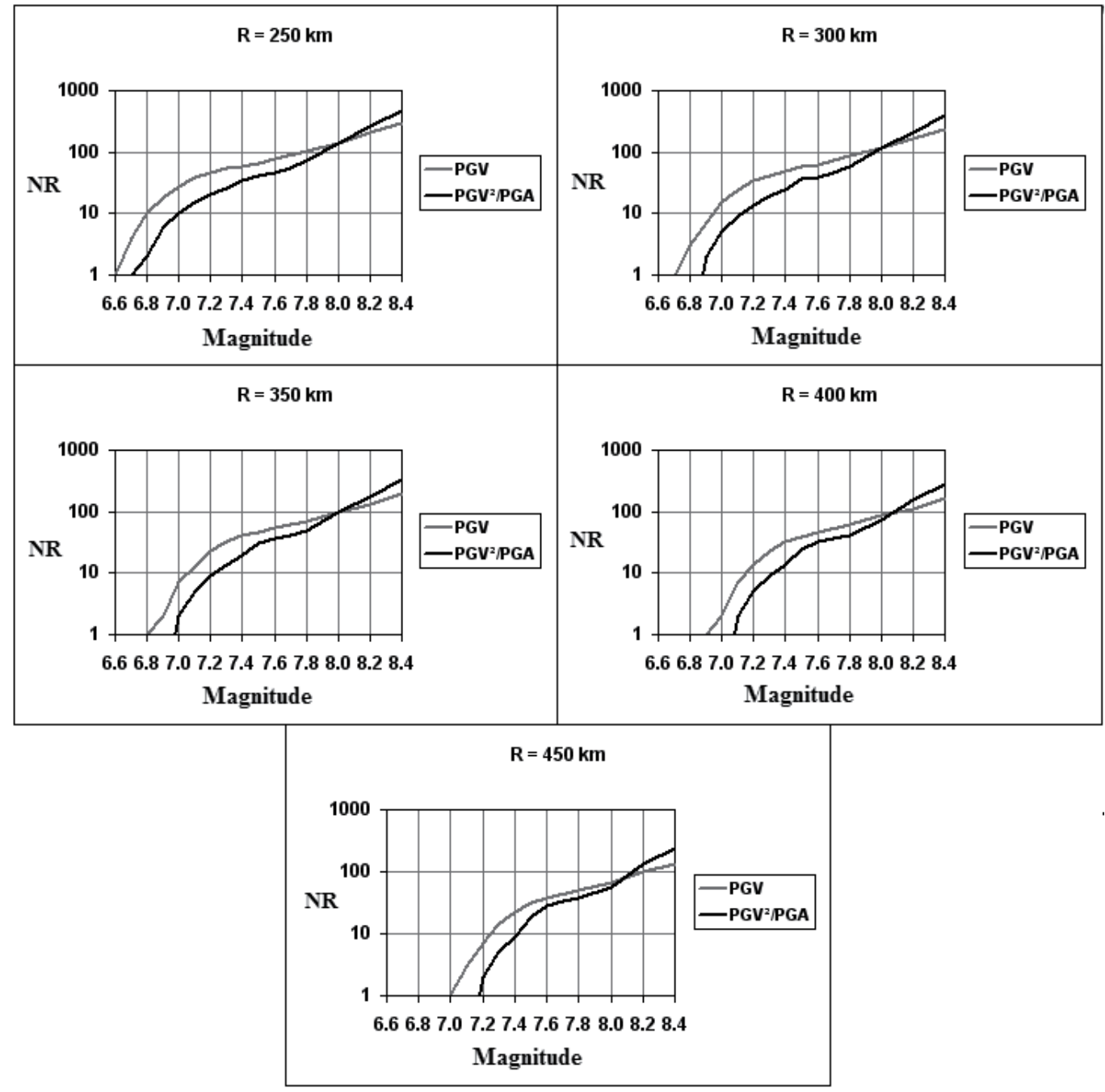

Fig. 8. Comparison of results between the PGV and PGV²/PGA models

\section{Conclusions}

One important challenge in the field of earthquake engineering for pipelines is the damage estimation due to future events. In this chapter, the reader will find a comprehensive stateof-the-art revision of the seismic parameters that have been employed as damage indicators for pipelines, and the most important seismic fragility functions proposed until now. In order to show a case of damage estimation due to future earthquake, we describe two prediction models for the Mexico City Water System (MCWS).

The results of this research reveal that a previous damage estimation study for the MCWS, based on a PGV fragility function, overestimated the expected number of pipe repairs caused by earthquakes with magnitudes around 8.0 to 8.1 and lower, and underestimated the damage for stronger earthquakes. This new study employs a recently proposed fragility 
function for the MCWS in terms of $\mathrm{PGV}^{2}$ /PGA: A composite parameter in terms of PGA, and PGV. Because a previous study (Pineda and Ordaz, 2007) demonstrates that PGV $2 /$ PGA is better related to pipeline damage in Mexico City than PGV alone, the results of the future damage estimation for the MCWS showen here are believed to be more reliable than those obtained with the PGV-based fragility function (Pineda and Ordaz, 2003).

\section{Acknowledgements}

This research has been partially funded by the Energy and Infrastructure Analysis (D-4) group at Los Alamos National Laboratory and the Institute of Engineering of the National Autonomous University of Mexico (UNAM).

\section{References}

American Lifelines Alliance, ALA (2001). Seismic Fragility Formulations for Water Systems, American Society of Civil Engineers (ASCE) and Federal Emergency Management Agency (FEMA). www.americanlifelinesalliance.org

ASCE-TCLEE (1991). Seismic Loss Estimation for a Hypothetical Water System. Technical Council on Lifeline Earthquake Engineering (TCLEE) of the American Society of Civil Engineers (ASCE), Monograph No.2, C.E. Taylor (eds).

Ayala, G. \& O'Rourke, M. (1989). Effects of the 1985 Michoacan Earthquake on Water Systems and other Buried Lifelines in Mexico, Multidisciplinary Center for Earthquake Engineering Research, Technical Report NCEER-89-0009, New York.

Ballantyne, D. B.; Berg, E.; Kennedy, J.; Reneau, R. \& Wu, D. (1990). Earthquake Loss Estimation Modeling for the Seattle Water System, Report to U.S. Geological Survey under Grant 14-08-0001-G1526, Technical Report, Kennedy/Jenks/Chilton, Federal Way, WA.

Barenberg, M.E. (1988). Correlation of Pipeline Damage with Ground Motions. Journal of Geotechnical Engineering, ASCE, June, 114 (6), 706-711.

Eidinger, J. (1998). Water Distribution System - The Loma Prieta, Californa, Earthquake of October 17, 1989 - Lifelines, USGS, Professional Paper 1552-A, Anshel J. Schiff (ed.), U.S. Government Printing Office, Washington, A63-A78.

Eidinger, J.; Maison, B.; Lee, D. \& Lau, B. (1995). East Bay Municipal District Water Distribution Damage in 1989 Loma Prieta Earthquake. Proceedings of the Fourth U.S. Conference on Lifeline Earthquake Engineering, ASCE-TCLEE, Monograph No. 6, 240247.

Eguchi, R. T. (1983). Seismic Vulnerability Models for Underground Pipes. Proceedings of Earthquake Behavior and Safety of Oil and Gas Storage Facilities, Buried Pipelines and Equipment, ASME, PVP-77, New York, June, 368-373.

Eguchi, R. T. (1991). Seismic Hazard Input for Lifeline Systems. Structural Safety, 10, 193-198. FEMA (1999). Earthquake Loss Estimation Methodology HAZUS-MH - Technical Manual. FEMA, Washington DC, http://www.fema.gov/hazus.

Hamada, M. (1991). Estimation of Earthquake Damage to Lifeline Systems in Japan. Proceedings of the Third Japan-U.S. Workshop on Earthquake Resistant Design of Lifeline Facilities and Countermeasures for Soil Liquefaction, San Francisco, CA, December 1719, 1990; Technical Report NCEER-91-0001, NCEER, State University of New York at Buffalo, Buffalo, NY, 5-22. 
Hamada, M. \& Akioka, Y. (1997). Liquefaction Induced Ground Strain and Damage to Buried Pipes. Proceedings of Japan Society of Civil Engineers Earthquake Engineering Symposium, Vol. 1, pp. 1353-1356 (in Japanese).

Hindy, A. \& Novak, M. (1979). Earthquake Response of Underground Pipelines. Earthquake Engineering and Structural Dynamics, 106, 451-476.

Hwang, H. \& Lin, H. (1997). GIS-based Evaluation of Seismic Performance of Water Delivery Systems. Technical Report, CERI, University of Memphis, Memphis, TN.

Isoyama, R.; Ishida, E.; Yune, K. \& Shirozu, T. (2000). Seismic Damage Estimation Procedure for Water Supply Pipelines. Proceedings of the Twelfth World Conference on Earthquake Engineering, CD-ROM Paper No. 1762, 8pp.

Isoyama, R. \& Katayama, T. (1982). Reliability Evaluation of Water Supply Systems during Earthquakes. Report of the Institute of Industrial Science, University of Tokyo, 30 (1) (Serial No. 194).

Jeon, S. S. \& O'Rourke, T. D. (2005). Northridge Earthquake Effects on Pipelines and Residential Buildings. Bulletin of the Seismological Society of America, 95 (1), 294-318.

Katayama, T.; Kubo, K. \& Sato, N. (1975). Earthquake Damage to Water and Gas Distribution Systems. Proceedings of the U.S. National Conference on Earthquake Engineering, EERI, Oakland, CA, 396-405.

Kitaura, M. \& Miyajima, M. (1996). Damage to Water Supply Pipelines. Special Issue of Soils and Foundations. Japanese Geotechnical Society, Japan, January, 325-333.

Mavridis, G. \& Pitilakis, K. (1996). Axial and Transverse Seismic Analysis of Buried Pipelines. Proceedings of the Eleventh World Conference on Earthquake Engineering, Acapulco, Mexico, 81-88.

Newmark, N. M. (1967). Problems in Wave Propagation in Soil and Rocks. Proceedings of the International Symposium on Wave Propagation and Dynamic Properties of Earth Materials, University of New Mexico Press.

Ordaz, M.; Perez Rocha, L.E.; Reinoso, E.; Montoya, C. \& Arboleda, J. (1996-2002) Program Z, Instituto de Ingeniería, National Autonomous University of Mexico (UNAM).

O'Rourke, M. J. (2009). Analytical Fragility Relation for Buried Segmented Pipe. Proceedings of the TCLEE 2009: Lifeline Earthquake Engineering in a Multihazard Environment. Oakland, CA, 771-780.

O'Rourke, M. J. \& Ayala, G. (1993). Pipeline Damage due to Wave Propagation. Journal of Geotechnical Engineering, ASCE, 119 (9), 1490-1498.

O'Rourke, M. J. \& Deyoe, E. (2004). Seismic Damage to Segmented Buried Pipe. Earthquake Spectra, (20) 4, 1167-1183.

O'Rourke, M. J. \& El Hmadi, K. (1988). Analysis of Continuous Buried Pipelines for Seismic Wave Effects. Earthquake Engineering and Structural Dynamics, 16, 917-929.

O'Rourke, T. D. \& Jeon, S. S. (1999). Factors Affecting the Earthquake Damage of Water Distribution Systems. Proceedings of the Fifth U.S. Conference on Lifeline Earthquake Engineering, Seattle, WA, ASCE, Reston, VA, 379-388.

O’Rourke, T. D.; Stewart, H. E.; Gowdy, T. E. \& Pease, J. W. (1991). Lifeline and Geotechnical Aspects of the 1989 Loma Prieta Earthquake. Proceedings of the Second International Conference on Recent Advances in Geotechnical Earthquake Engineering and Soil Dynamics, St. Louis, MO, 1601-1612. 
O'Rourke, T. D.; Toprak, S. \& Sano, Y. (1998). Factors Affecting Water Supply Damage Caused by the Northridge Earthquake. Proceedings of the Sixth U.S. National Conference on Earthquake Engineering.

Paolucci, R. \& Smerzini, C. (2008). Earthquake-induced Transient Ground Strains from Dense Seismic Networks. Earthquake Spectra, 24 (2), 453-470.

Pineda, O. (2002). Estimación de Daño Sísmico en la Red Primaria de Distribución de Agua Potable del Distrito Federal. Master of Engineering Thesis, Institute of Engineering, National Autonomous University of Mexico (UNAM), Mexico City. (In Spanish)

Pineda, O. \& Ordaz, M. (2003). Seismic Vulnerability Function for High-diameter Buried Pipelines: Mexico City's Primary Water System Case. 2003 ASCE International Conference on Pipeline Engineering and Construction, American Society of Civil Engineers, Baltimore, USA.

Pineda, O. \& Ordaz, M. (2004). Mapas de Velocidad Máxima del Suelo para la Ciudad de México. Revista de Ingeniería Sísmica, Mexican Society of Earthquake Engineering; (71) 37-62. (In Spanish)

Pineda, O. (2006). Estimación de Daño Sísmico en Tuberías Enterradas. Doctorate of Engineering Thesis, Institute of Engineering, National Autonomous University of Mexico (UNAM), Mexico City. (In Spanish)

Pineda, O. \& Ordaz, M. (2007). A New Seismic Intensity Parameter to Estimate Damage in Buried Pipelines due to Seismic Wave Propagation. Journal of Earthquake Engineering, (11) 773-786.

Pineda, O. \& Ordaz, M. (2010). Seismic Fragility Formulation for Segmented Buried Pipeline Systems Including the Impact of Differential Ground Subsidence. Journal of Pipeline Systems Engineering and Practice, ASCE, Vol. 1, No. 4, pp. 141-146.

Sano, Y.; O'Rourke, T. \& Hamada, M. (1999). GIS Evaluation of Northridge Earthquake Ground Deformation and Water Supply Damage. Proceedings of Fifth U.S. Conference on Lifeline Earthquake Engineering, TCLEE Monograph No.16, ASCE, pp. 832-839.

Singh, S. K.; Santoyo, M.; Bodin, P. \& Gomberg, J. (1997). Dynamic Deformations of Shallow Sediments in the Valley of Mexico. Part II: Single-station Estimates. Bulletin of the Seismological Society of America, 87, 540-550. 


\title{
The Equivalent Non-Linear Single Degree of Freedom System of Asymmetric Multi-Storey Buildings in Seismic Static Pushover Analysis
}

\author{
Triantafyllos K. Makarios \\ Hellenic Institute of Engineering Seismology E Earthquake Engineering, \\ Greece
}

\section{Introduction}

In order to estimate seismic demands at low seismic performance levels (such as life safety or collapse prevention), the application of Non-Linear Response History Analysis (NLRHA) is recommended for reasons related to its accuracy. However, for the purposes of simplification, the application of Pushover Analysis is also often recommended. Here, in order to obtain the seismic demands of asymmetric multi-storey reinforced concrete $(\mathrm{r} / \mathrm{c})$ buildings, a new seismic non-linear static (pushover) procedure that uses inelastic response acceleration spectra, is presented. The latter makes use of the optimum equivalent NonLinear Single Degree of Freedom (NLSDF) system, which is used to represent a randomlyselected, asymmetric multi-storey $\mathrm{r} / \mathrm{c}$ building. As is proven below, for each asymmetric multi-storey building, a total of twelve suitable non-linear static analyses are required according to this procedure, while at least two hundred and twenty-four suitable non-linear dynamic analyses are needed in the case of NLRHAnalysis, respectively, while if accidental ecentricity is ignored (or external floor moments loads around the vertical axis are used) then a total number of fifty six NLRH Analyses are required. The seismic non-linear static procedure that is presented here is a natural extension of the documented seismic equivalent static linear (simplified spectral) method that is recommended by the established contemporary Seismic Codes, with reference to torsional provisions. From the numerical parametric documentation of this proposed seismic non-linear static procedure, we can reliably evaluate the extreme values of floor inelastic displacements, with reference to results provided by the Non-Linear Response History Analysis.

More specifically, it is well-known from past research that, as regards the pushover procedure (i.e. pushover analyses of buildings with inelastic response acceleration spectra), its suitability for use on asymmetric multi-storey $\mathrm{r} / \mathrm{c}$ buildings is frequently questioned because of the following five points:

1. For a known earthquake, an ideal equivalent NLSDF system, which represents the real asymmetric multi-storey $\mathrm{r} / \mathrm{c}$ building, must first be defined, in order to calculate the seismic target-displacement. In other words, the above-mentioned $r$ /c NLSDF system has an equivalent viscous damping ratio $\xi=0.05$ and is combined with "inelastic acceleration spectra with an equivalent viscous damping ratio $\xi=0.05$ "; thus its demand seismic target-displacement is obtained for each earthquake level. The latter 
displacement is transformed into a demand extreme seismic displacement of the monitoring point of the real Multi-Degree of Freedom (MDoF) system, namely the real multi-storey building. After that, the remaining demand seismic displacements (and seismic stress on the members) at the other points of the building are easily calculated using the pushover analysis image. Therefore, successfully defining the ideal equivalent NLSDF system is always the most important part of this procedure, and for this reason, it also constitutes a major concern. Many attempts have been made in the past to resolve this problem in the planar frames (Saiidi \& Sozen, 1981; Fajfar \& Fischinger, 1987a,b; Uang \& Bertero, 1990; Qi \& Moehle, 1991; Rodriquez, 1994; Fajfar \& Gaspersic, 1996; Hart \& Wong, 1999; Makarios, 2005;). On the other hand, in the case of asymmetric multi-storey buildings, a mathematically documented optimum equivalent Non-Linear Single Degree of Freedom System can be defined (Makarios, 2009). According to the latter proposal, three coupled degrees of freedom (two horizontal displacements and a rotation around a vertical axis that passes through the floor mass centre) are taken into account for each floor mass centre. The above-mentioned definition of the optimum equivalent NLSDF system was mathematically derived by observing suitable dynamic loadings on the floor mass centres of the multi-storey building, using simplified assumptions. The use of this optimum equivalent NLSDF system, in combination with the inelastic design spectra, provides an acceptable evaluation of the extreme (maximum/minimum) seismic floor displacements required for a known design earthquake, and for this reason constitutes the core issue of the present chapter.

2. It is known that in the case of Response History Analysis, three uncorrelated accelerograms (Penzien \& Watabe, 1975;) are used simultaneously along the three axes $x$, $y \& z$. For this reason, in the case of Response Spectrum Analysis (RSA) or Simplified Spectral Method (SSM) or the equivalent static method, the three response acceleration spectra are "statistically independent" (sect.3.2.2.1(3)P of Eurocode EN-1998.01). This practically means that the response spectra (in the case of RSA), or the lateral floor static forces (in the case of SSM), must be inserted separately for each main direction of the building, and then a suitable superposition (i.e. rule of Square Root of Sum of Squares SRSS) must be applied to the results of each independent analysis, always in the linear area. However, in the non-linear area, as in the example of static pushover analysis, where superposition is generally forbidden due to its non-linearity, a basic question arises; which is the most suitable way of taking the "spatial seismic action" of the three seismic components into account? In order to answer this question, the rule of Eq.(1) that has resulted from a parametric numerical analysis, provides an approximate evaluation of the spatial seismic action during the static pushover procedure (Makarios, 2011):

$$
E_{\text {ex }}= \pm\left(E_{\text {, }}^{\kappa}+E_{\text {,II }}^{\kappa}\right)^{1 / \kappa}
$$

where $\kappa=0.75$ is a mean value in the case of displacements/deformations, and $E_{\text {, }}$ is the extreme seismic demand inelastic displacement/deformation due to static pushover analysis using the first seismic component only, along the horizontal real (or fictitious) principal $I$-axis of the building. Similarly, $E_{\text {,II }}$ is the extreme seismic demand inelastic displacement/deformation due to pushover analysis using the second seismic component only, the other horizontal real (or fictitious) principal II-axis of the building. 
On the contrary, when $E_{\text {, }}$ and $E_{\text {,II }}$ represent stress, then $\kappa=2.00$, as is the case in the linear area. In Eq.(1) above, we consider that the vertical seismic component is often ignored (for example, when the vertical ground acceleration is less than $0.25 g$, according to sect.4.3.3.5.2(1)/EN-1998.01; $g$ is the acceleration of gravity).

3. What is the most suitable "monitoring point" and its suitable characteristic degree of freedom (control displacement) that is related to the degree of freedom of the equivalent NLSDF system, in the case of asymmetric buildings? As has been proven by the mathematical analysis provided below, concerning the definition of the optimum NLSDF system, the centre of mass of the last floor at the top of the building can play the role of the "monitoring point", whilst its "control displacement" must be parallel to the lateral floor static forces.

4. What is the most suitable distribution of lateral floor static forces in elevation? There are various opinions on this matter, since the distribution can be "triangular", "uniform" or in accordance with the pure translational "fundamental mode-shape". Moreover, the distribution can be adapted to the fundamental mode-shape of the building in each step of the pushover analysis, an issue related to the action of higher order mode-shapes in tall buildings. According to sect.4.3.3.4.2.2(1)/EN-1998.01, at least two different distributions ("uniform" and "first mode-shape") in elevation should always be taken into account. On the other hand, fundamental mode-shape distribution is often applied to common asymmetric buildings, while in the case of very tall buildings, Non-Linear Response History Analysis is mainly reccommended.

5. At which point in the plan should the lateral floor static forces be applied during the pushover analysis of asymmetric buildings? According to sect.4.3.3.4.2.2(2)P/EN1998.01, the lateral static loads must be applied at the location of the mass centres, while simultaneously, accidental eccentricities should also be taken into account. Moreover, it is known that in the case of dynamic methods, such as "Response Spectrum Analysis" or "Response History Analysis", accidental eccentricities are also considered, with a suitable movement of the floor mass centres by $e_{\mathrm{a} i}= \pm 0.05 L_{i}$ or $\pm 0.10 L_{i}$, where $L_{i}$ is the dimension of the building's floor that is perpendicular to the direction of the seismic component (sect.4.3.2(1)P/EN-1998.01). However, in the case of static seismic methods, such as the "equivalent static method", "simplified spectrum method", "lateral force method" and "static pushover analysis", the accidental eccentricities are examined with a suitable movement (by $e_{\mathrm{a} i}= \pm 0.05 L_{i}$ or $\pm 0.10 L_{i}$ ) of the point at which the lateral floor static forces are applied. In the latter case, and for one seismic component, the final two design eccentricities $\left(\max e_{\mathrm{II}, i} \& \min e_{\mathrm{II}, i}\right.$ for $i$ floor) consist of the following two parts: In order to take into account the dynamic amplification of the torsional effects of the building, two dynamic eccentricities $e_{\mathrm{f}, i} \& e_{\mathrm{r}, i}$ are examined (first part). According to several Seismic Codes (NBCC-95, EAK/2003), dynamic eccentricities are defined as $e_{\mathrm{f}, i}=1.50 \cdot e_{\mathrm{o}} \& e_{\mathrm{r}, i}=0.50 \cdot e_{\mathrm{o}}$, where $e_{\mathrm{o}}$ is the static eccentricity of the building along the examined horizontal principal axis of the building, perpendicular to the direction of the seismic component. Dynamic eccentricities are measured from the real/fictitious stiffness centre of the building to its mass centre (CM); more accurate closed mathematical relationships of the former have been developed by Anastassiadis et al (1998). As we can observe, a calculation of (a) the location of the real/fictitious stiffness 
centre of the asymmetric building in the plan, (b) the orientation of its horizontal principal directions $I \& I I$ and (c) the magnitude of its torsional-stiffness radii, must be carried out before the seismic static pushover analysis begins. For this triple purpose, the fictitious elastic Reference Cartesian System $\mathrm{P}_{\mathrm{o}}(I, I I, I I I)$ of the asymmetric multistorey building is the most rational, documented method for use (Makarios \& Anastassiadis 1998a,b; Makarios, et al 2006; Makarios, 2008;). On the contrary, it is worth noting that, various other floor centres, such as centres of rigidity/twist/shear, are not suitable for seismic design purposes, because they are dependent on external floor lateral static loads (Cheung \& Tso, 1986; Hejal \& Chopra, 1987;). At a next stage, the static eccentricities $e_{\mathrm{O}, \mathrm{I}}$ and $e_{\mathrm{O}, \mathrm{II}}$ of an asymmetric multi-storey building are defined as the distance between the floor mass centre $(\mathrm{CM})$ and the real/fictitious stiffness centre $\mathrm{P}_{\mathrm{o}}$ of the building, along the two horizontal principal I \& II -axes.

In relation to my previous point, and in order to account for uncertainties as regards the real location of floor mass centres, as well as the spatial variation of the seismic motion, accidental eccentricities are also examined (second part). However, it is well-known that according to documented Seismic Codes with reference to torsional provisions, the critical horizontal directions for static lateral loading (in linear equivalent static seismic methods) are the real/fictitious principal I \& II-axes of a building. Therefore, the documented static pushover procedure must define the same point; the lateral floor static loads that will be used in the static pushover analysis must be oriented along the real/fictitious principal I \& II-axes of the building. In this case, and since the final design eccentricities (maxe \& mine along the two principal I \& II-axes) have also been used, we can observe that this non-linear static pushover analysis is, undoubtedly, a natural extension of the linear equivalent static seismic method.

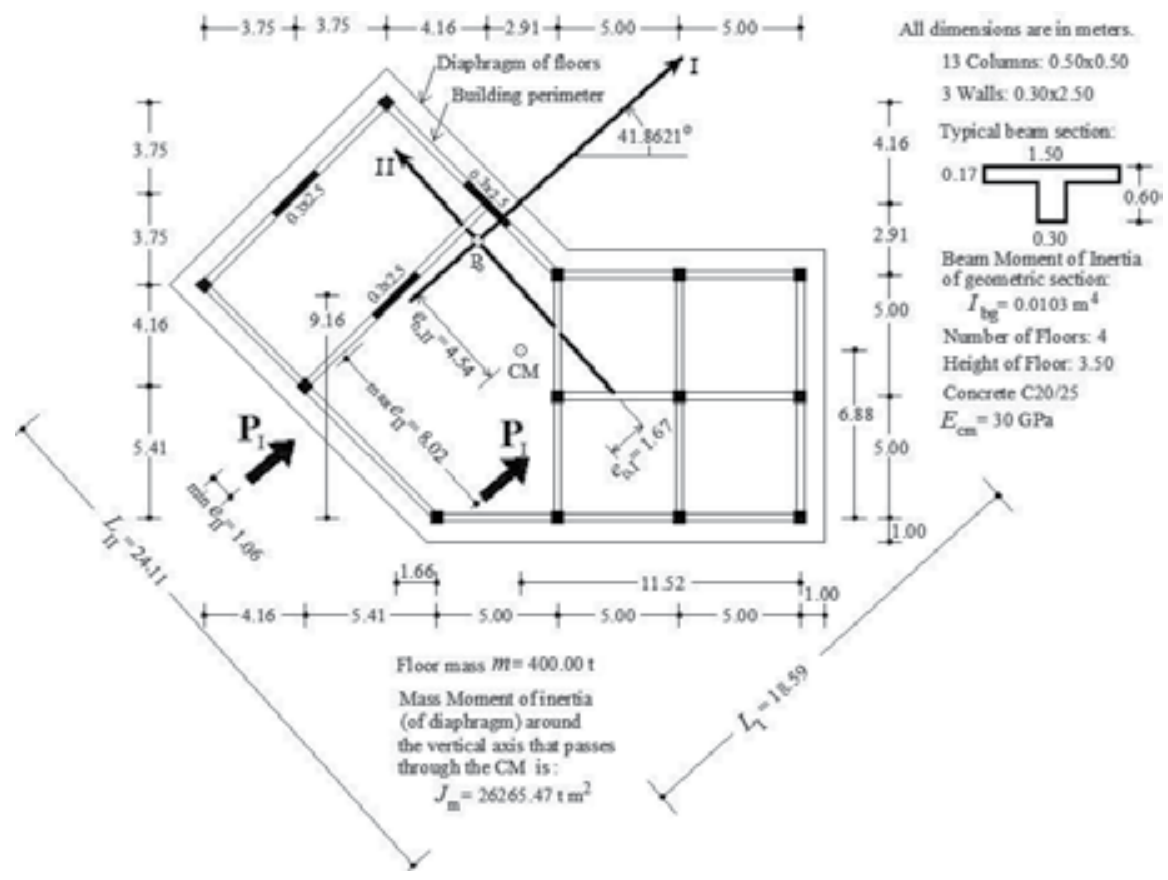

Fig. 1. Typical plan of an asymmetric four-storey $\mathrm{r} / \mathrm{c}$ building. 
For illustration purposes, we can see the numerical example of an asymmetric four-storey $\mathrm{r} / \mathrm{c}$ building with a typical plan, whose real/fictitious principal axes are initially unknown (Fig.1). The location in the plan and the orientation of the $\mathrm{P}_{\mathrm{o}}(I, I I, I I I)$ system have been calculated according to the above-mentioned relative references, using a 50\% reduction of the stiffness properties of all structural elements, due to cracks (sect.4.3.1(7)/EN-1998.01). The position of all floor mass centres (CM) coincides with the geometric centre of the floor rigid diaphragms. Next, the static eccentricities are calculated as $e_{0, \mathrm{I}}=1.67$ \& $e_{\mathrm{O}, \mathrm{II}}=4.54$ metres, along the fictitious principal $I \& I I$-axes, respectively. The two torsionalstiffness radii $r_{\text {I }} \& r_{\text {II }}$ are calculated as $r_{\text {I }}=9.35 \& r_{\text {II }}=7.90$ metres on level $0.8 H$ of the building (namely, on the $3^{\text {rd }}$ floor; $H$ is the total height of the building) according to the theory of Dynamics of Structures (Makarios, 2008;), and along I \& II-axes, respectively. Note that, in the case of a single-storey building, the torsional-stiffness radius $r_{\mathrm{I}}$ is for example calculated by the square root of the ratio of the torsional stiffness $k_{\mathrm{III}}$ of the building around its Stiffness Centre to the lateral stiffness $k_{\mathrm{II}}$ along principal II-axis (i.e. $r_{\mathrm{I}}=\sqrt{k_{\mathrm{III}} / k_{\mathrm{II}}}$, $\left.r_{\mathrm{II}}=\sqrt{k_{\mathrm{III}} / k_{\mathrm{I}}}\right)$. Also, the radius of gyration of a typical floor rigid diaphragm is calculated as $l_{\mathrm{s}}=\sqrt{J_{\mathrm{m}} / m}=\sqrt{26265.47 / 400}=8.10$ metres (see data in Fig.1). In order to calculate the final design eccentricities for loading $\mathrm{P}_{\mathrm{I}}$ along the principal $I$-axis of the building, we have:

$$
\begin{aligned}
& \max _{\mathrm{II}}=e_{\mathrm{f}, i}+e_{\mathrm{a} i}=1.50 \cdot e_{\mathrm{o}, \mathrm{II}}+0.05 \cdot L_{\mathrm{II}}=1.50 \cdot 4.54+0.05 \cdot 24.11=8.02, \\
& \min e_{\mathrm{II}}=e_{\mathrm{r}, i}+e_{\mathrm{a} i}=0.50 \cdot e_{\mathrm{o}, \mathrm{II}}-0.05 \cdot L_{\mathrm{II}}=0.50 \cdot 4.54-0.05 \cdot 24.11=1.06
\end{aligned}
$$

The envelope of the two individual static loading states, using the above final design eccentricities, produces the results $E_{\text {, }}$ of the first seismic component (along principal $I$ axis). The results $E_{\text {,II }}$ of the second seismic component (along principal II-axis) are obtained in a similar way.

Please note the following important point: According to sect.4.2.3.2(6)/EN-1998.01, the building does not satisfy the criterion of regularity in plan, because $e_{\mathrm{o}, \mathrm{II}}=4.54$ is not lower than $0.3 \cdot r_{\mathrm{II}}=0.3 \cdot 7.90=2.37$. Furthermore, $r_{\mathrm{II}}=7.90$ is lower than $l_{\mathrm{s}}=8.10$ and, according to sect.4.3.3.1(8)d/EN-1998.01, the condition $r_{\mathrm{II}}^{2}>l_{\mathrm{s}}^{2}+e_{\mathrm{o}, \mathrm{II}}^{2}$ is not true. Consequently, the present building has to be simulated as a fully spatial model, according to both the elastic analysis (sect.4.3.3.1(7)/EN-1998.01) and the non-linear static (pushover) analysis (sect.4.3.3.4.2.1(2)P/EN-1998.01).

To conclude, the real/fictitious principal elastic Cartesian system $P_{\mathrm{o}}(I, I I, I I I)$ of the asymmetric multi-storey building is initially calculated. In order to calculate the real/fictitious principal elastic Cartesian system $P_{\mathrm{o}}(I, I I, I I I)$ of the asymmetric multi-storey $\mathrm{r} / \mathrm{c}$ building, all structural elements (columns, walls, beams, cores) of the building model must have effective flexural and shear stiffness, constant along their whole length, 
corresponding to $50 \%$ of their geometric section values. This effective stiffness is more rational, since it leads to a realistic "total lateral stiffness" of the building in the post-elastic, non-linear area. However, if we use much lower values of effective stiffness (i.e. equal to $0.20 E I$ or less), then an artificial increase of the eigen-periods occurs. In such a case, one possible result is that the building model is inadequately seismically loaded, because the state of co-ordination between the fundamental eigen-period of the building and the fundamental period of the actual seismic ground strong motion is removed. Existing views about the use of very small effective flexural stiffness values stem from the following assumption, that "...plastic hinges appeared simultaneously at both ends of each structural element of the multi-storey building." However, this is rather incorrect, because many structural elements do not yield, even in the "near collapse state" of the building, as we can observe from the seismic NLRHA. On the other hand, cracks appeared on the plastic hinges of the $\mathrm{r} / \mathrm{c}$ elements with yielding steel bars, while at the next moment, when the bending of the elements has the opposite sign, some cracks on the plastic hinges cannot fully close, because the yielded steel bars obstruct their closure. Additionally, in the frame of the static pushover analysis of irregular in the plan, asymmetric multi-storey buildings, a Cartesian system $\mathrm{CM}(x y z)$ that has the same orientation with the known fictitious elastic system $P_{\mathrm{o}}(I, I I, I I I)$ of the building must be adopted as a global reference system. Thus, the lateral floor static loads, according to the static pushover analyses, must be oriented along the two horizontal principal I \& II-axes of the building. Otherwise, it is well-known from the linear area that it is not possible to calculate the envelope of the floor displacements/stress of an asymmetric building. In other words, the loading along the principal axes of a building creates the most unfavourable state for static methods. Moreover, as a general conclusion, final design eccentricities $\left(\max e_{\mathrm{I}}, \min e_{\mathrm{I}}, \max e_{\mathrm{II}} \& \min e_{\mathrm{II}}\right)$ must always be taken into account in pushover analysis, and thus the present non-linear static pushover procedure is a natural extension of the equivalent linear static method, with mathematical consistency.

With reference to the simulation for the non-linear analysis, we consider that each structural column/beam consists of two equivalent sub-cantilevers (Fig.2a,b). Each sub-cantilever is represented by an elastic beam element with a non-linear spring at its base. This inelastic spring represents the inelastic deformations that are lumped at the end of the element. In order to identify the characteristics of a plastic hinge, it is assumed that each member (beam/column) deforms in antisymmetric bending. The point of contraflexure is approximately located at the middle of the element and for this reason, two sub-cantilevers appear for each member. Therefore, each sub-cantilever has a "shear length" $L_{\mathrm{s}, i}$, where its chord slope rotation $\theta$ characterizes the Moment-Chord Slope Rotation $(M-\theta)$ diagram of the non-linear spring in the end section. In practice, the two critical end-sections of the clear length of the beams and columns, as well as the critical section at the base of the walls/cores of the building model, must be provided with suitable non-linear springs. In order to calculate the $M-\theta$ diagram of a plastic hinge, two methods are frequently implemented: firstly, the most rational method involves the use of suitable semi-empirical, non-linear diagrams of Moment-Chord Slope Rotation $(M-\theta)$ of the "shear length" of each sub-cantilever, taken from a large database of experimental results, despite their considerable scattering (Panagiotakos \& Fardis 2001). Such relations are proposed by Eurocode EN-1998.03 (Annex A: sections A.3.2.2 to A.3.2.4). Secondly, the calculation of $M-\theta$ diagrams can alternatively be achieved via Moment-Curvature 
$(M-\varphi)$ diagrams, which can be calculated using the "fiber elements" (i.e. XTRACT, 2007;). Next, using a suitable experimental lengh $L_{\mathrm{p}}$ for each plastic hinge, $M-\theta$ diagrams are calculated (Fig.2c) by the integral of curvatures in length $L_{\mathrm{p}}$. More specifically, the yielding rotation can be calculated by $\theta_{\mathrm{y}}=\varphi_{\mathrm{y}} \cdot L_{\mathrm{s}} / 3$ and the plastic rotation by $\theta_{\mathrm{p}} \approx\left(\varphi_{\mathrm{u}}-\varphi_{\mathrm{y}}\right) \cdot L_{\mathrm{p}}$, where $\varphi_{\mathrm{y}}$ is the yielding curvature of the end section and $\varphi_{\mathrm{u}}$ is the ultimate one by "fiber element" method, whilst the ultimate rotation $\theta_{\mathrm{u}}$ is always calculated from the sum of both the yielding rotation $\theta_{\mathrm{y}}$ and the plastic rotation $\theta_{\mathrm{p}}$. However, using the second method, only flexural deformations are taken into account, while other sources of inelastic properties are ignored. For example, shear \& axial deformations, slippage and extraction of the main longitudinal reinforcement and opened cracks with yielding steel bars, should also be taken into account. In order to avoid all these issues, the initial method of semi-empirical Moment-Chord Slope Rotation $(M-\theta)$ diagrams, either a combination of the two methods, is often selected. Moreover, the maximum available ultimate rotation of a plastic hinge in Fig.(2a) very closely approximates (in practice) the ultimate chord slope rotation $\theta$ of the equivalent sub-cantilever in Fig.(2b). On the other hand, other simulations of plastic hinges, such as the one based on the "dual component model", where each member is replaced by an elastic element (central length of the element) and an elasto-plastic element (plastic hinge) in parallel, are not suitable for analyzing reinforced concrete members, because then the "stiffness degradation" for repeated dynamic seismic loading cycles is ignored. Also, in many cases, and especially for existing $\mathrm{r} / \mathrm{c}$ structures, a "shear failure" on structural members can occur because the mechanism of the shear forces in the plastic hinges is destroyed, and therefore doubts arise concerning the validity of the well-known modified "Morsch's truss-model" after the yielding. Therefore, the available plastic rotation $\theta_{\mathrm{p}}$ of a plastic hinge is reduced (by up to $50 \%$ in several cases); (see Eq.(A.12) of EN-1998.03). Also, in the case where plastic hinges appeared simultaneously at both ends of an structural element, the flexural effective stiffness of the section is calculated by $E I_{\text {eff }}=M_{\mathrm{y}} \cdot L_{\mathrm{s}} / 3 \theta_{\mathrm{y}}$, (arithmetic mean value of the two antisymmetric bending states of the element).

Last but not least, in order to calculate the non-linear Moment-Chord Slope Rotation $(M-\theta)$ diagrams, in contrast to the initial design state, the mean values of the strength of the materials must be used (namely, $f_{\mathrm{cm}}=f_{\mathrm{ck}}+8$ in MPa for concrete and $f_{\mathrm{sm}}=1.10 f_{\mathrm{sk}}$ for steel, where $f_{\mathrm{ck}}$ and $f_{\mathrm{sk}}$ are the characteristic values). If some structural elements have a low available ductility $(\mu<2)$, such as low-walls with a shear span ratio $a_{\mathrm{s}}<1.50$ at their base, columns/beams with inadequate strength in shear stress, brittle members such as shortcolumns with $a_{\mathrm{s}}<2.50$ etc, then these must be checked in relation to stress only. With reference to the "shear length $L_{\mathrm{s}}$ " of a sub-cantilever, we can note that it is calculated by $L_{\mathrm{s}}=M / V$, where $M$ is the flexural yielding moment and $V$ is the respective shear force on a plastic hinge. In the case of tall-walls, the "shear length" $L_{\mathrm{s}}$ can be calculated by the distance of the zero-moment point to the base in elevation, from a temporary lateral static floor force. 
The "shear span ratio" is calculated by $a_{\mathrm{s}}=M / V h=L_{\mathrm{s}} / h$, where $h$ is the dimension of the section that is perpendicular to the axis of the flexural moment $M$. Moreover, the yielding moment $M$ of the diagram $M-\theta$ cannot be greater than the value $\max M=V_{R} L_{\mathrm{s}}$, where $V_{R}$ is calculated by Eq.(A.12) of Eurocode EN/1998.03, with the axial force $N$ of the column at zero (i.e. due to the action of the vertical seismic building vibration of vertical and horizontal seismic components) and the plastic ductility of the element is equal to $\mu_{\Delta}^{\mathrm{pl}}=\theta_{\mathrm{p}} / \theta_{\mathrm{y}}=5$ (conservative value), because then the "brittle shear failure" appears prematurely, due to the doubts of the validity of the modified "Morsch's truss-model" after the yielding.

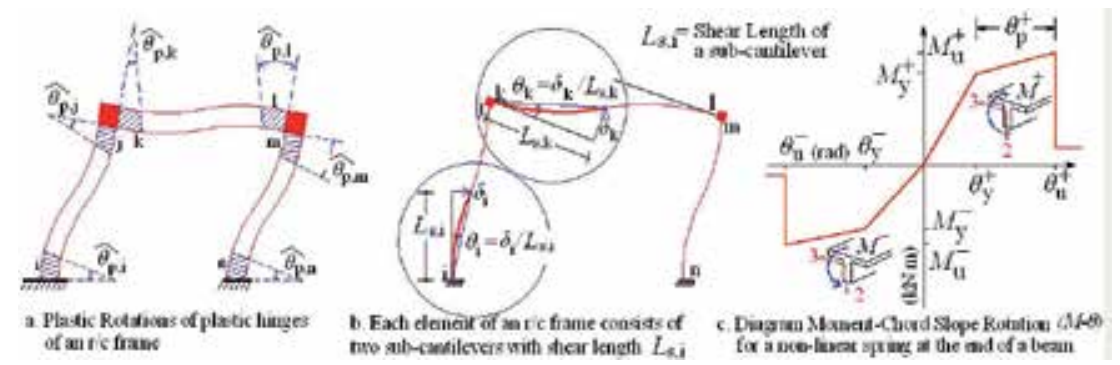

Fig. 2. a. Plastic rotations of plastic hinges; b. Two equivalent sub-cantilevers of each structural column/beam; c. Diagram $M-\theta$ of inelastic springs at the ends of the elements (where $\theta$ is the chord slope rotation).

On the other hand, in the case of seismic Non-Linear Response History Analysis (NLRHA), we have to use suitable pairs (or triads) of uncorrelated accelerograms as seismic action. However, the seismic demands of asymmetric multi-storey buildings are often unreliable for the following reasons:

i. It is possible for the accelerograms to be deemed unsuitable (they often do not have the necessary frequency content or are inadequate as regards the number of strong cycles of the dynamic loading or the strong motion duration). In order to cope with this problem, according to contemporary seismic codes, at least seven triads (or pairs, in the case where the vertical seismic component is ignored) of accelerograms must be taken into consideration and then, the average of the response quantities from all these analyses should be used as the "final design values" of the seismic action effect. Note that, for each pair (or triad) of accelerograms, the latter must act simultaneously (sect.3.2.3.1.1(2)P/EN1998.01) and be "statistically independent". This means that the "correlation factor" among these accelerograms must be zero (uncorrelated accelerograms). However, each accelerogram of each pair must be represented by the same response acceleration spectrum (sect.3.2.2.1(3)P/EN-1998.01). The acceleration response spectra (with 0.05 equivalent viscous damping ratio) of artificial (or recorded) accelerograms should match the elastic response spectra for 0.05 equivalent viscous damping ratio. This should occur over a wide range of periods (or at least between $0.2 \mathrm{~T}$ and $2 \mathrm{~T}$, where $T$ is the fundamental period of the structure), as is defined by sect.3.2.3.1.2(4c)/EN-1998.01. Also, no value of the "mean elastic spectrum", which is calculated based on all the used accelerograms, should be less than $90 \%$ (or greater than $110 \%$ ) of the corresponding value of the tagret elastic response spectrum. The peak ground acceleration of each accelerogram must always be equal to $a_{\mathrm{g}} \cdot S$, where $a_{\mathrm{g}}$ is the design ground acceleration of the local area and 
$S$ is the soil factor according to EN-1998.01. Simultaneously, for strong earthquakes, the minimum duration of the strong motion of each accelerogram must be sufficient (about $15 \mathrm{~s}$ in common cases), whilst, from personal observations, I consider that there should be a minimum number of approximately ten "large" and thirty-five "significant" loading loops, due to the dynamic seismic cyclic loading (i.e. a "large" cycle has an extreme ground acceleration of over 0.75PGA and a "significant" cycle has an extreme ground acceleration of between 0.30PGA and 0.75PGA). However, the subject of the exact definition of the Design Basis Earthquake and the Maximum Capable Earthquake of a seismic hazard area is open to question, while recently, a new framework for the simulation and definition of the seismic action of Design Earthquake Basis for the inelastic single degree of freedom system, using the Park \& Ang damage index on the structures, has been developed (Moustafa, 2011;).

ii. It is possible for the numerical models from the building simulation to be inadequate, because many false assumptions about the non-linear dynamic properties of plastic hinges can be inserted into the building model. For example, each non-linear spring must possess a suitable dynamic model $M-\theta$ for cyclic dynamic seismic loading (Dowell et al, 1998; Takeda et al, 1970; Otani, 1981; Akiyama, 1985;), where after each loading cycle, a suitable "stiffness degradation" must be taken into account (Fig.3).

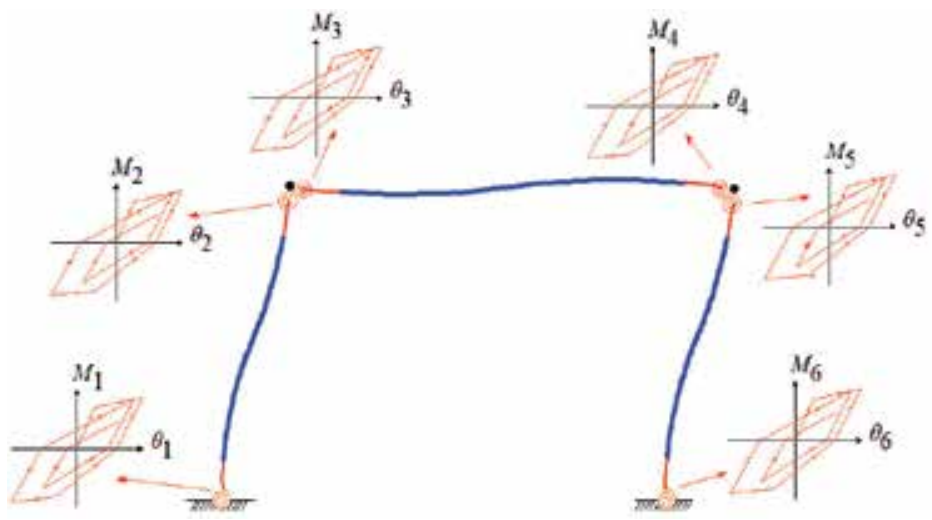

Fig. 3. Non-linear model of a planar frame, where each spring possesses a suitable diagram for cyclic dynamic seismic loading.

iii. It is possible for the numerical integration method to be inadequate, as regards accuracy \& stability.

iv. The critical dynamic loading orientation of the pair of horizontal seismic components is unknown or does not exist and leads to the examination of various other orientations (at least one more orientation with a rotation of 45 degrees relative to the initial principal horizontal orientation must be examined). Alternative, a special methodology about the examination of various orientations usimg pairs of accelerograms, gives the envelope of the most unfavourable results (in the linear area only) was presented by Athanatopoulou (2005). Note that, for each pair of accelerograms, all possible sign combinations of seismic components must be taken into account, resulting in four combinations for each pair. Moreover, seven (7) pairs must be taken into account. In addition, due to the accidental eccentricities, four different positions of the floor mass centres must be considered. Therefore, ( 2 orientations $) \times(4$ combinations of signs per pair $) \times(7$ pairs $) \times(4$ positions of the 
mass centres)=224 NLRH Analyses. Alternatively, in order to reduce the number of said analyses, the action of accidental eccentricities can be taken into account using equivalent external floor moments $M_{\mathrm{m}, \mathrm{i}}$ around the vertical axis, which are calculated by the floor lateral static forces, $M_{r_{\mathrm{i}}}= \pm F_{r_{\mathrm{i}}} \times e_{\mathrm{ai}, \mathrm{m}}$, where $F_{r_{\mathrm{i}}}$ is the lateral floor static force and $e_{\mathrm{ai}, \mathrm{m}}$ is a mean accidental eccentricity $\left(e_{\mathrm{ai}, \mathrm{m}}=\sqrt{e_{\mathrm{ai}, \mathrm{I}}^{2}+e_{\mathrm{ai}, \mathrm{II}}^{2}}\right)$ for the case where the base shear is same for both principal directions of the building. Otherwise, the external moments can derive by $M_{\mathrm{m}, \mathrm{i}}= \pm \sqrt{M_{\mathrm{I}, \mathrm{i}}^{2}+M_{\mathrm{II}, \mathrm{i}}^{2}}=\sqrt{\left(F_{\mathrm{I}, \mathrm{i}} \times e_{\mathrm{ai}, \mathrm{II}}\right)^{2}+\left(F_{, \mathrm{II}, \mathrm{i}} \times e_{\mathrm{ai}, \mathrm{I}}\right)^{2}}$. In this case, we arrive at a total number of fifty six (56) NLRH Analyses.

Consequently, the most important issues that we come across during the static pushover analysis are the following: (1) Why is the use of a single degree of freedom system that represents the real structure required? (2) How can the spatial action of the two horizontal seismic components during the static pushover analysis be taken into account? (3) What is the most suitable monitoring point in the case of asymmetric buildings? (4) Which distribution of lateral floor static forces is most suitable? and (5) At which point, in the plan of an asymmetric building, should the lateral floor static forces be applied? The design dynamic eccentricities and accidental eccentricities must also be taken into account, which means that the real or fictitious elastic axis of the building with the real or fictitious horizontal main elastic axes, where the lateral static forces must be oriented during the static pushover analysis, must be calculated. On the other hand, the Non-Linear Response History Procedure has a high computational cost and, simultaneously, many of its results present a high sensitivity. For these reasons, in practice, the mathematically documented static non-linear (pushover) analysis, presented below in this chapter, is a reliable alternative approximate method, which can envelope the accurate seismic demand floor inelastic displacements by the above NonLinear Response History Procedure, in a rational way. However, it is known that the remaining seismic demands, such as the inter-storey drift ratios, real distribution of the plastic hinges on the building, yielding/failure mechanism of the building, etc, are only predicted correctly by using Non-Linear Response History Analysis.

\section{The equivalent non-linear SDF system of asymmetric multi-storey buildings in non-linear static pushover analysis}

Let us consider an asymmetric multi-storey building consisting of a rigid deck (diaphragm) on each floor, where the total mass $m_{r_{i}}$ of each floor is concentrated at the floor geometric centre $\mathrm{CM}_{\text {, }}$ of the diaphragm, and the multi-storey building has a vertical mass axis, where all floor concentrated masses are located. Each centre $\mathrm{CM}_{r_{\mathrm{i}}}$ therefore has the translational mass $m_{r_{i}}$ for any horizontal direction, while it has the floor mass inertia moment $J_{\mathrm{m}, i}=m_{, i} l_{\mathrm{s}, i}^{2}$ due to its diaphragm (where $l_{\mathrm{s}, i}$ is the floor inertia radius). Every diaphragm is supported on massless, axially loaded columns and structural walls, in reference to the global Cartesian mass system $\operatorname{CM}(x, y, z)$ that is parallel to the known fictitious principal Cartesian reference system $\mathrm{P}_{\mathrm{o}}(I, I I, I I I)$, as presented in the previous paragraph. Next, the original point CM has three degrees of freedom for each floor; two horizontal displacements $u_{\mathrm{x}, \mathrm{i}}$ and $u_{\mathrm{y}, \mathrm{i}}$ relative to the ground, along axes $x$ and $y$ respectively, and a rotation $\theta_{\mathrm{z}, \mathrm{i}}$ around the vertical $z$-axis (Fig.4). Moreover, for the needs of this mathematical analysis, we consider that this $\mathrm{N}$-storey building is loaded with the $3 \mathrm{~N}$-dimensional loading vector $\mathbf{P}$ of external lateral floor static loading, 
where the floor forces are oriented in parallel to one fictitious principal axis (i.e. along the IIaxis) of the building. The loading vector $\mathbf{P}$ relating to the $3 \mathrm{~N}$-dimensional vector $\mathbf{u}$ of the degrees of freedom of the floor masses of the asymmetric multi-storey building is formed according to the pure translational (along the principal II-axis) mode-shape's distribution of lateral floor static forces in elevation (Eqs.2a,b). For the needs of this analysis, the lateral floor static forces of the loading vector $\mathbf{P}$ in the plan, are located at max $e_{\mathrm{I}}$ distance from the fictitious stiffness centre $P_{o}$ to the mass centre of the building (Fig.4a). Next, a primary static pushover analysis of the asymmetric $\mathrm{N}$-storey building is performed, using this increased static loading vector $\mathbf{P}$, until the collapse of the building model.

$$
\mathbf{u}=\left\{\begin{array}{c}
\mathbf{u},{ }_{1} \\
\mathbf{u},,_{2} \\
\ldots \\
\mathbf{u},,_{i} \\
\ldots \\
\mathbf{u},,_{\mathrm{N}}
\end{array}\right\}, \mathbf{P}=\left\{\begin{array}{c}
\mathbf{P}_{,_{1}} \\
\mathbf{P}_{,_{2}} \\
\ldots \\
\mathbf{P}_{,_{i}} \\
\ldots \\
\mathbf{P}_{, \mathrm{N}}
\end{array}\right\}
$$

where $\mathbf{u}_{i}=\left\{\begin{array}{c}u_{\mathrm{x}, i} \\ u_{\mathrm{y}, i} \\ \theta_{\mathrm{z}, i}\end{array}\right\}$ and $\mathbf{P}_{,_{i}}=\left\{\begin{array}{c}P_{\mathrm{x}, i} \\ P_{\mathrm{y}, i} \\ M_{\mathrm{z}, i}\end{array}\right\}=\left\{\begin{array}{c}0 \\ P_{,_{i}} \\ P_{s_{i}} \cdot\left(\max _{\mathrm{I}, i}-e_{\mathrm{o}, \mathrm{I}}\right)\end{array}\right\}$ for each floor $i$ of the building.

Next, let us consider an intermediate step of this pushover analysis near the middle of the inelastic area. On each floor $i$, the vector of inelastic displacements of the mass centre of level $i$ of the building is $\mathbf{u}_{, i, \mathrm{o}}=\left\{\begin{array}{lll}u_{\mathrm{x}, i, \mathrm{o}} & u_{\mathrm{y}, i, \mathrm{o}} & \theta_{z, i, \mathrm{o}}\end{array}\right\}^{\mathrm{T}}$, while the global vector of inelastic displacements of all floor mass centres constitutes the $3 \mathrm{~N}$-dimensional vector $\mathbf{u}_{0}$, Eq.(3). It follows that this vector constitutes the initial state (index " $\mathrm{o}$ ") of displacements in the following mathematical analysis:

$$
\mathbf{u}_{\mathrm{o}}=\left\{\begin{array}{c}
u_{x, 1, \mathrm{o}} \\
u_{y, 1, \mathrm{o}} \\
\theta_{z, 1, \mathrm{o}} \\
u_{x, 2, \mathrm{o}} \\
u_{y, 2, \mathrm{o}} \\
\theta_{z, 2, \mathrm{o}} \\
\ldots \\
\ldots \\
u_{x, \mathrm{~N}, \mathrm{o}} \\
u_{y, \mathrm{~N}, \mathrm{o}} \\
\theta_{z, \mathrm{~N}, \mathrm{o}}
\end{array}\right\}=\left\{\begin{array}{c}
u_{x, 1, \mathrm{o}} / u_{y, \mathrm{~N}, \mathrm{o}} \\
u_{y, 1, \mathrm{o}} / u_{y, \mathrm{~N}, \mathrm{o}} \\
\theta_{z, 1, \mathrm{o}} / u_{y, \mathrm{~N}, \mathrm{o}} \\
u_{x, 2, \mathrm{o}} / u_{y, \mathrm{~N}, \mathrm{o}} \\
u_{y, 2, \mathrm{o}} / u_{y, \mathrm{~N}, \mathrm{o}} \\
\theta_{z, 2, \mathrm{o}} / u_{y, \mathrm{~N}, \mathrm{o}} \\
\cdots \\
\cdots \\
u_{x, \mathrm{~N}, \mathrm{o}} / u_{y, \mathrm{~N}, \mathrm{o}} \\
1.00 \\
\theta_{z, \mathrm{~N}, \mathrm{o}} / u_{y, \mathrm{~N}, \mathrm{o}}
\end{array}\right\} u_{y, \mathrm{~N}, \mathrm{o}}=\left\{\begin{array}{c}
\psi_{x, 1, y, \mathrm{~N}} \\
\psi_{y, 1, y, \mathrm{~N}} \\
\psi_{z, 1, y, \mathrm{~N}} \\
\psi_{x, 2, y, \mathrm{~N}} \\
\psi_{y, 2, y, \mathrm{~N}} \\
\psi_{z, 2, y, \mathrm{~N}} \\
\cdots \\
\cdots \\
\psi_{x, \mathrm{~N}, y, \mathrm{~N}} \\
1.00 \\
\psi_{z, \mathrm{~N}, y, \mathrm{~N}}
\end{array}\right\} u_{y, \mathrm{~N}, \mathrm{o}}=\boldsymbol{\psi}_{y, \mathrm{~N}, \mathrm{o}} \cdot u_{y, \mathrm{~N}, \mathrm{o}}
$$




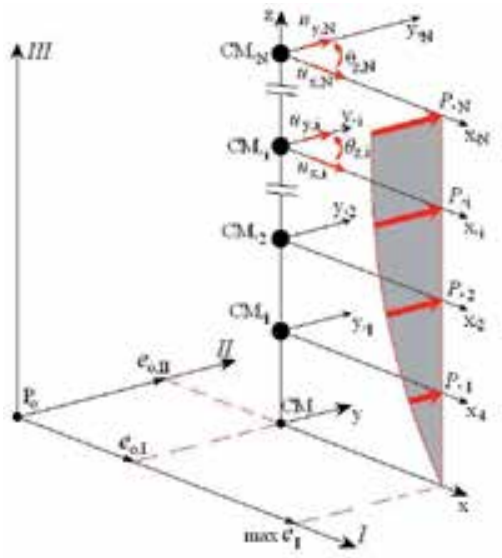

a. Primary static posbover analysis

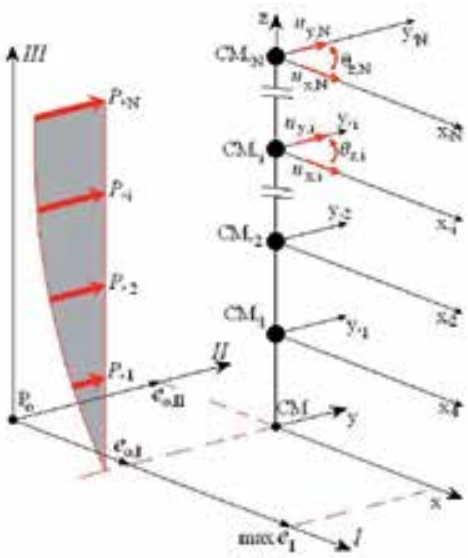

b. Secondary static pushover analysis

Fig. 4. Spatial Asymmetric Multi-storey System. Primary and Secondary pushover analysis.

Next, consider the known pure translational mode shape distribution $\mathbf{Y}$ of the lateral floor forces in elevation (from the linear modal analysis), where, Eq.(4);

$$
\mathbf{Y}=\left\{\begin{array}{llllll}
Y_{1} & Y_{2} & \ldots & Y_{i} & \ldots & Y_{\mathrm{N}}
\end{array}\right\}^{\mathrm{T}}
$$

with $Y_{\mathrm{N}}=1.00$.

In order to define the optimum equivalent Non-Linear SDF system, the following two assumptions are set (Makarios, 2009):

$1^{\text {st }}$ Assumption: Vector $\boldsymbol{\psi}_{y, \mathrm{~N}, \mathrm{o}}$ of the inelastic displacement distribution of the floor mass centres of the asymmetric multi-storey building is a 'notional mode-shape'.

$2^{\text {nd }}$ Assumption: The lateral force $\mathbf{P}$ causes translational and torsional accelerations on the concentrated mass of the asymmetric multi-storey building. Therefore, if we consider that the vector loading $\mathbf{P}$ of the external loads is a function of time $t$, i.e. $\mathbf{P}(t)$ is a dynamic loading, this function is written as follows in Eq.(5):

$$
\mathbf{P}=\left\{\begin{array}{c}
P_{x, 1} \\
P_{y, 1} \\
M_{z, 1} \\
P_{x, 2} \\
P_{y, 2} \\
M_{z, 2} \\
\cdots \\
\cdots \\
P_{x, \mathrm{~N}} \\
P_{y, N} \\
M_{z, \mathrm{~N}}
\end{array}\right\}=\left\{\begin{array}{c}
0.00 \\
P_{, 1} \\
\left(\max _{\mathrm{I}, 1}-e_{\mathrm{o}, \mathrm{I}}\right) \cdot P_{, 1} \\
0.00 \\
P_{, 2} \\
\left(\max _{\mathrm{I}, 2}-e_{\mathrm{o}, \mathrm{I}}\right) \cdot P_{, 2} \\
\cdots \\
\cdots \\
0.00 \\
P_{\mathrm{N}} \\
\left(\max _{\mathrm{I}, \mathrm{N}}-e_{\mathrm{o}, \mathrm{I}}\right) \cdot P_{, \mathrm{N}}
\end{array}\right\} \mathrm{f}(t)=\left\{\begin{array}{c}
0.00 \\
Y_{1} \\
\left(\max _{\mathrm{I}, 1}-e_{\mathrm{o}, \mathrm{I}}\right) \cdot Y_{1} \\
0.00 \\
Y_{2} \\
\left(\max _{\mathrm{I}, 2}-e_{\mathrm{o}, \mathrm{I}}\right) \cdot Y_{2} \\
\cdots \\
\cdots \\
0.00 \\
1.00 \\
\left(\max _{\mathrm{I}, \mathrm{N}}-e_{\mathrm{o}, \mathrm{I}}\right)
\end{array}\right\} P_{\mathrm{N}} \cdot \mathrm{f}(t)=\mathbf{i} P_{\mathrm{N}} \cdot \mathrm{f}(t),
$$


where $\mathrm{f}(t)$ is a known, suitable, increasing, linear, monotonic time function (i.e. $\mathrm{f}(t)=\mathrm{a} \cdot t+\mathrm{b}$, where $\mathrm{a} \& \mathrm{~b}$ are coefficients) and $P_{,_{i}}$ is the value of the floor lateral force.

Therefore, the equation of the motion of the masses of the multi-storey building, which are loaded with the dynamic loading $\mathbf{P}(t)$, is described by the mathematical system of linear second order differential N-equations, which can be written in matrix form, Eq.(6):

$$
\mathbf{M} \ddot{\mathbf{u}}_{\mathrm{o}}(t)+\mathbf{C} \dot{\mathbf{u}}_{\mathrm{o}}(t)+\mathbf{K} \mathbf{u}_{\mathrm{o}}(t)=\mathbf{P}(t)
$$

where $\mathbf{u}_{\mathbf{o}}(t)$ is the $3 \mathrm{~N}$-dimensional vector of displacements from Eq.(3), $\mathbf{K}$ and $\mathbf{M}$ are the $3 \mathrm{~N} x 3 \mathrm{~N}$-dimensional square symmetric matrices (Eqs.7a,b) of lateral elastic stiffness and masses respectively, while $\mathrm{C}$ is the damping $3 \mathrm{~N} \times 3 \mathrm{~N}$ matrix.

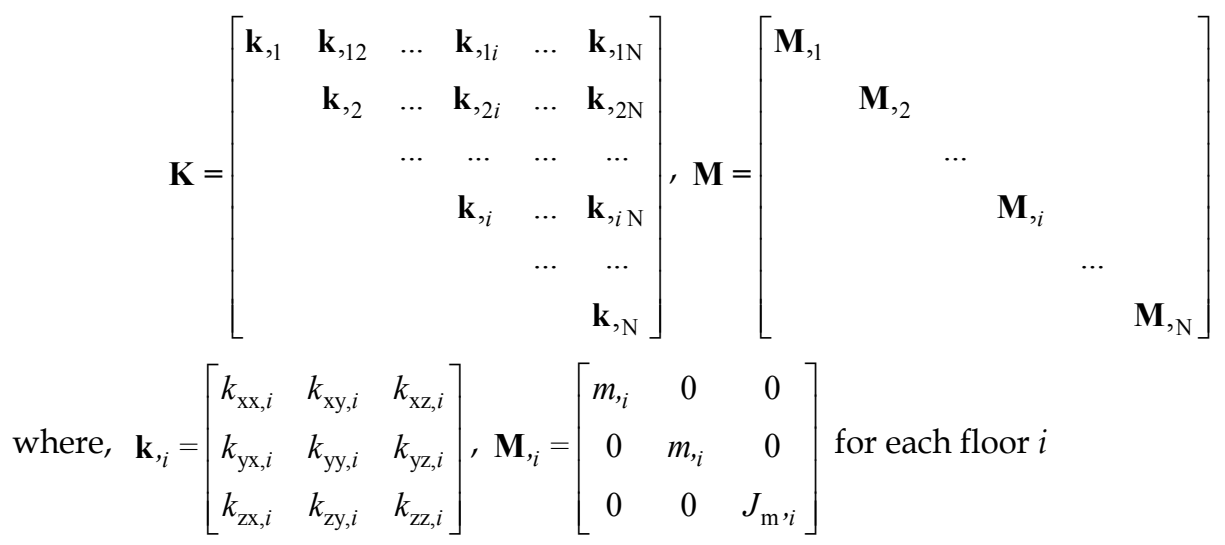

and where $J_{\mathrm{m}, i}=m_{, i} l_{\mathrm{s}, i}^{2}$ is the mass inertia moment of the diaphragm (floor) around the vertical axis passing through the mass centre, and $l_{\mathrm{s}, i}$ is the floor inertia radius. The floor lateral stiffness sub-matrix $\mathbf{k}_{i_{i}}$, as well as the global lateral stiffness matrix $\mathbf{K}$ of the building, are calculated with reference to the global Cartesian mass system $\mathrm{CM}(x, y, z)$ using a suitable numerical technique pertaining to the "stiffness condensation" of the total stiffness matrix. Alternatively, we enforce a displacement (that is equal to one) of a floor mass centre along one degree of freedom and simultaneously all the remaining degrees of freedom of the building are fixed. The generated reactions on the degrees of freedom provide the coefficients of a column of the global lateral stiffness matrix $\mathbf{K}$, and this procedure is repeated for all other mass degrees of freedom of the building.

Using Eqs.(2) , (3), (5) \& (7), Eq.(6) is written as follows:

$$
\mathbf{M} \boldsymbol{\psi}_{\mathrm{y}, \mathrm{N}, \mathrm{o}} \cdot \ddot{\mathbf{u}}_{\mathrm{y}, \mathrm{N}, \mathrm{o}}(t)+\mathbf{C} \boldsymbol{\psi}_{\mathrm{y}, \mathrm{N}, \mathrm{o}} \cdot \dot{\mathbf{u}}_{\mathrm{y}, \mathrm{N}, \mathrm{o}}(t)+\mathbf{K} \boldsymbol{\psi}_{\mathrm{y}, \mathrm{N}, \mathrm{o}} \cdot \mathbf{u}_{\mathrm{y}, \mathrm{N}, \mathrm{o}}(t)=\mathbf{i} \mathrm{P}_{\mathrm{N}} \cdot \mathrm{f}(t)
$$

Next, we pre-multiply Eq.(8) by the $\boldsymbol{\psi}_{\mathrm{y}, \mathrm{N}, \mathrm{o}}^{\mathrm{T}}$ vector:

$$
\begin{aligned}
& \boldsymbol{\psi}_{\mathrm{y}, \mathrm{N}, \mathrm{o}}^{\mathrm{T}} \mathbf{M} \boldsymbol{\psi}_{\mathrm{y}, \mathrm{N}, \mathrm{o}} \cdot \ddot{\mathbf{u}}_{\mathrm{y}, \mathrm{N}, \mathrm{o}}(t)+\boldsymbol{\psi}_{\mathrm{y}, \mathrm{N}, \mathrm{o}}^{\mathrm{T}} \mathbf{C} \boldsymbol{\psi}_{\mathrm{y}, \mathrm{N}, \mathrm{o}} \cdot \dot{\mathbf{u}}_{\mathrm{y}, \mathrm{N}, \mathrm{o}}(t)+\boldsymbol{\psi}_{\mathrm{y}, \mathrm{N}, \mathrm{o}}^{\mathrm{T}} \mathbf{K} \boldsymbol{\psi}_{\mathrm{y}, \mathrm{N}, \mathrm{o}} \cdot \mathbf{u}_{\mathrm{y}, \mathrm{N}, \mathrm{o}}(t)= \\
& =\boldsymbol{\psi}_{\mathrm{y}, \mathrm{N}, \mathrm{o}}^{\mathrm{T}} \mathbf{i} \cdot \mathrm{P}_{\mathrm{N}} \cdot \mathrm{f}(t)
\end{aligned}
$$


where $\boldsymbol{\psi}_{\mathrm{y}, \mathrm{N}, \mathrm{O}}^{\mathrm{T}} \mathbf{i}$ is a factor.

In this case, it is clear that the base shear $V_{\mathrm{o} y}(t)$ of the building, for every time $t$, is given as follows:

$$
V_{\mathrm{o} y}(t)=P_{\mathrm{N}} \cdot \mathrm{f}(t) \cdot \sum_{i=1}^{\mathrm{N}} Y_{i}
$$

Therefore, the lateral force $P_{\mathrm{N}} \cdot \mathrm{f}(t)$ at the top of the frame is given by:

$$
P_{\mathrm{N}} \cdot \mathrm{f}(t)=\frac{V_{\mathrm{o} y}(t)}{\sum_{i=1}^{\mathrm{N}} Y_{i}}
$$

Eq.(9) is divided by the number $\boldsymbol{\psi}_{\mathrm{y}, \mathrm{N}, \mathrm{o}}^{\mathrm{T}} \mathbf{i}$ and by inserting Eq.(3), we arrive at:

$$
\begin{aligned}
& \frac{\boldsymbol{\psi}_{\mathrm{y}, \mathrm{N}, \mathrm{o}}^{\mathrm{T}} \boldsymbol{M} \boldsymbol{\psi}_{\mathrm{y}, \mathrm{N}, \mathrm{o}} \cdot \sum_{i=1}^{\mathrm{N}} Y_{i}}{\boldsymbol{\psi}_{\mathrm{y}, \mathrm{N}, \mathrm{o}}^{\mathrm{T}} \mathbf{i}} \cdot \ddot{u}_{y, \mathrm{~N}, \mathrm{o}}(t)+\frac{\boldsymbol{\psi}_{\mathrm{y}, \mathrm{N}, \mathrm{o}}^{\mathrm{T}} \mathbf{C} \boldsymbol{\psi}_{\mathrm{y}, \mathrm{N}, \mathrm{o}} \cdot \sum_{i=1}^{\mathrm{N}} Y_{i}}{\boldsymbol{\psi}_{\mathrm{y}, \mathrm{N}, \mathrm{o}}^{\mathrm{T}} \mathbf{i}} \cdot \dot{u}_{y, \mathrm{~N}, \mathrm{o}}(t)+ \\
& +\frac{\boldsymbol{\psi}_{\mathrm{y}, \mathrm{N}, \mathrm{o}}^{\mathrm{T}} \mathbf{K} \boldsymbol{\psi}_{\mathrm{y}, \mathrm{N}, \mathrm{o}} \cdot \sum_{i=1}^{\mathrm{N}} Y_{i}}{\boldsymbol{\psi}_{\mathrm{y}, \mathrm{N}, \mathrm{o}}^{\mathrm{T}} \mathbf{i}} \cdot u_{y, \mathrm{~N}, \mathrm{o}}(t)=V_{\mathrm{o} y}(t)
\end{aligned}
$$

Eq.(12) presents an equation of the motion of a single-degree-of-freedom (SDF) system. This equation must be transformed, so that the "optimum equivalent Non-linear SDF system" represents the initial asymmetric multi-storey building. As we can see in Eq.(12), the degree of freedom is the displacement $u_{y}, \mathrm{~N}$ of the mass centre CM, while loaded with the base shear $V_{\text {oy }}$. Thus, the "effective lateral stiffness" $k^{*}$ of this SDF system must be obtained through an additional, special, secondary, non-linear (pushover) analysis of the initial, asymmetric multi-storey building, where the lateral static force is applied to the mass centre $\mathrm{CM}$ along the $y$-axis (Fig.4b). Hence, the lateral stiffness $k^{*}$ of the first branch and the lateral stiffness $\alpha \cdot k^{*}$ of the second branch of the bilinear diagram $V_{\mathrm{oy}}-u_{y, \mathrm{~N}}$ are the result of this special, secondary pushover analysis (Fig.5a). Therefore, we can consider that the slopes $k^{*}$ and $\alpha \cdot k^{*}$ are already known from the secondary pushover analysis. Thus, for reasons of convergence between the known analytic SDF system of Eq.(12) and the (unknown at present) required optimum equivalent NLSDF system of the asymmetric multi-storey building, Eq.(12) is multiplied with the coefficient $L=k^{*} / k_{o}$, hence:

$$
m^{*} \cdot \ddot{u}_{y, \mathrm{~N}, \mathrm{o}}(t)+c^{*} \cdot \dot{u}_{y, \mathrm{~N}, \mathrm{o}}(t)+k^{*} \cdot u_{y, \mathrm{~N}, \mathrm{o}}(t)=L \cdot V_{\mathrm{o} y}(t)
$$


where,

$$
\begin{gathered}
m^{*}=\frac{k^{*}}{k_{\mathrm{o}}} \cdot \frac{\boldsymbol{\psi}_{\mathrm{y}, \mathrm{N}, \mathrm{o}}^{\mathrm{T}} \mathbf{M} \boldsymbol{\psi}_{\mathrm{y}, \mathrm{N}, \mathrm{o}} \cdot \sum_{i=1}^{\mathrm{N}} Y_{i}}{\boldsymbol{\psi}_{\mathrm{y}, \mathrm{N}, \mathrm{o}}^{\mathrm{T}} \mathbf{i}} \\
k_{o}=\frac{\boldsymbol{\psi}_{\mathrm{y}, \mathrm{N}, \mathrm{o}}^{\mathrm{T}} \mathbf{K} \boldsymbol{\psi}_{\mathrm{y}, \mathrm{N}, \mathrm{o}} \cdot \sum_{i=1}^{\mathrm{N}} Y_{i}}{\boldsymbol{\psi}_{\mathrm{y}, \mathrm{N}, \mathrm{o}}^{\mathrm{T}} \mathbf{i}}
\end{gathered}
$$

$\boldsymbol{\psi}_{\mathrm{y}, \mathrm{N}, \mathrm{o}}^{\mathrm{T}} \mathbf{M} \boldsymbol{\psi}_{\mathrm{y}, \mathrm{N}, \mathrm{o}}=$ Coefficient, $\boldsymbol{\psi}_{\mathrm{y}, \mathrm{N}, \mathrm{o}}^{\mathrm{T}} \mathbf{i}=$ Coefficient,

$\boldsymbol{\psi}_{\mathrm{y}, \mathrm{N}, \mathrm{o}}^{\mathrm{T}} \mathbf{K} \boldsymbol{\psi}_{\mathrm{y}, \mathrm{N}, \mathrm{o}}=$ Coefficient, $\sum_{i=1}^{\mathrm{N}} Y_{i}=$ Coefficient,

Therefore, the optimum equivalent NLlinear SDF system is represented by Eq.(13) and is presented in Fig.(6a). In addition, we can assume that the NLSDF system possesses the equivalent viscous damping $c^{*}$, hence:

$$
\boldsymbol{c}^{*}=\frac{k^{*}}{k_{\mathrm{o}}} \cdot \frac{\boldsymbol{\psi}_{\mathrm{y}, \mathrm{N}, \mathrm{o}}^{\mathrm{T}} \mathbf{C} \boldsymbol{\psi}_{\mathrm{y}, \mathrm{N}, \mathrm{o}} \cdot \sum_{i=1}^{\mathrm{N}} Y_{i}}{\boldsymbol{\psi}_{\mathrm{y}, \mathrm{N}, \mathrm{o}}^{\mathrm{T}} \mathbf{i}}=2 \cdot m^{*} \cdot \omega^{*} \cdot \xi
$$

where, $\omega^{*}$ is the circular frequency (in rad/s) of the equivalent vibrating SDF system in the linear range:

$$
\omega^{*}=\sqrt{k^{*} / m^{*}}
$$

and $\xi$ is the equivalent viscous damping ratio that corresponds to the critical damping of the SDF system (about $5 \%$ for reinforced concrete).

In order to transform the diagram $V_{\mathrm{o} y}-u_{y, \mathrm{~N}}$ of Fig. $(5 \mathrm{a}, \mathrm{b})$ into the capacity curve $P^{*}-\delta^{*}$ of Fig.(5b) of the equivalent NLSDF system, factor $\Gamma$ is directly provided by:

$$
\Gamma=m_{\text {tot }} / m^{*}
$$

where,

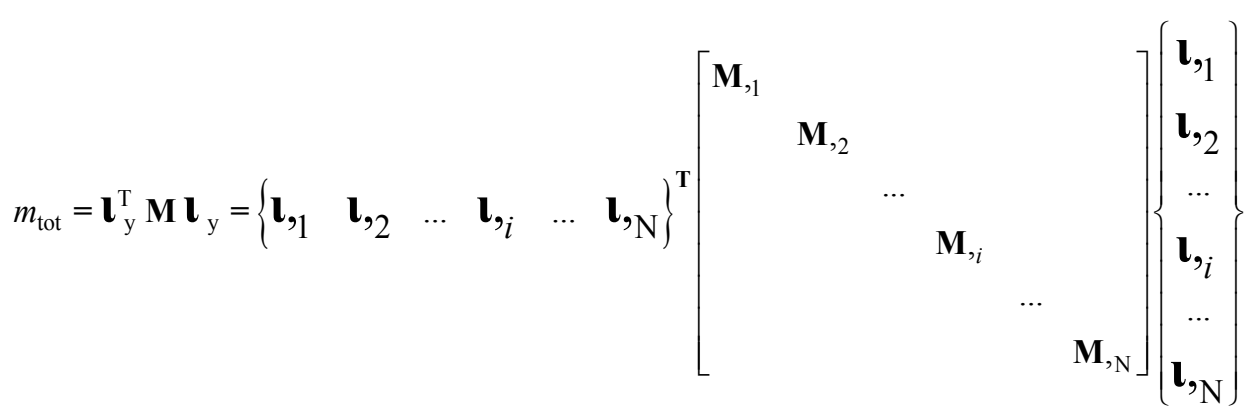


with $\quad \mathbf{l}_{i}^{\mathrm{T}}=\left\{\begin{array}{lll}0 & 1 & 0\end{array}\right\}^{\mathrm{T}}$

Moreover, $\mathbf{l}_{\mathrm{y}}$ is the global 'influence vector', that represents the displacements of the masses resulting from the static application of a unit horizontal ground displacement of the building along the seismic loading (i.e. $y$-direction). Hence, the optimum equivalent NLSDF system is characterized by Eq.(13) and has a bilinear capacity curve $\left(P^{*}-\delta^{*}\right)$ according to Fig.(5b).

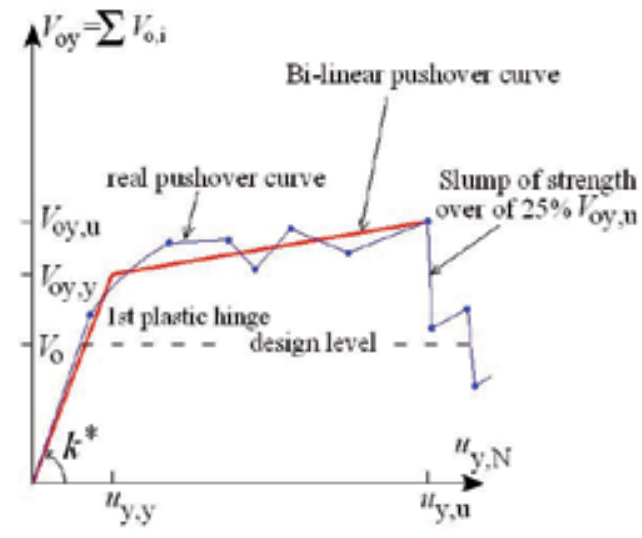

a. Pushover Curve of MDoF system by the Secondary Analysis

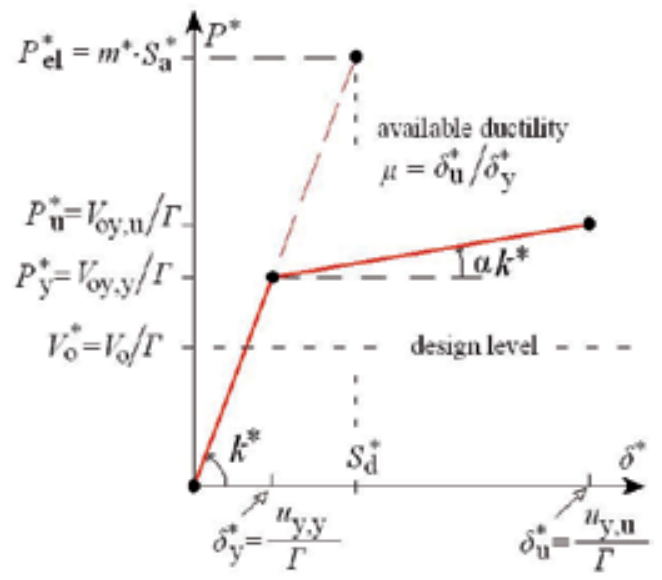

b. Capacity Curve of equivalent NLSDF syztem

Fig. 5. Transformation of the Pushover Curve of the Multi Degree of Freedom system into the Capacity Curve of the optimum equivalent NLSDF system.

The maximum elastic base shear $P_{\mathrm{el}}^{*}$ of the infinitely elastic SDF system, the yielding base shear $P_{\mathrm{y}}^{*}$ of the respective equivalent non-linear SDF system, its ultimate base shear $P_{\mathrm{u}}^{*}$, its yielding displacement $\delta_{\mathrm{y}}^{*}$ and its ultimate displacement $\delta_{\mathrm{u}}^{*}$, are given (Fig.5b):

$$
\begin{gathered}
P_{\mathrm{el}}^{*}=m^{*} \cdot S_{\mathrm{a}}^{*}, P_{\mathrm{y}}^{*}=\frac{V_{\mathrm{o} y, \mathrm{y}}}{\Gamma}, P_{\mathrm{u}}^{*}=\frac{V_{\mathrm{o} y, \mathrm{u}}}{\Gamma} \\
\delta_{\mathrm{y}}^{*}=\frac{u_{y, \mathrm{y}}}{\Gamma}, \delta_{\mathrm{u}}^{*}=\frac{u_{y, \mathrm{u}}}{\Gamma}
\end{gathered}
$$

The "effective period" $T^{*}$ of the optimum equivalent NLSDF system is given by Eq.(21), Fig.(6a):

$$
T^{*}=2 \pi \sqrt{\frac{m^{*}}{k^{*}}}=2 \pi \sqrt{\frac{m^{*} \cdot \delta_{\mathrm{y}}^{*}}{P_{\mathrm{y}}^{*}}}
$$


Next, in order to evaluate the demand seismic displacement of the asymmetric multi-storey $\mathrm{r} / \mathrm{c}$ building, we must first calculate the seismic target-displacement $\delta_{t \text {,inel }}^{*}$ of the optimum equivalent NLSDF system using the known Inelastic Response Spectra, since this is the only credible option; otherwise, a Non-Linear Response History procedure must be performed. Therefore, the inelastic seismic target-displacement $\delta_{\mathrm{t} \text {,inel }}^{*}$ is calculated directly using Eq.(22), Fig.(6b). Secondly, we must transform the inelastic seismic target-displacement $\delta_{t, \text { inel }}^{*}$ into the target-displacement of the monitoring point (namely, the centre mass at the top level of the real initial asymmetric multi-storey building, along the examined direction), multiplying it with factor $\Gamma$, Eq.(28). Finally, the maximum required seismic displacements of the other positions of the multi-storey building can be obtained from that step in the known primary pushover analysis, where the seismic target-displacement of Eq.(28) of the monitoring point appears.

\section{The equivalent NLSDF system}

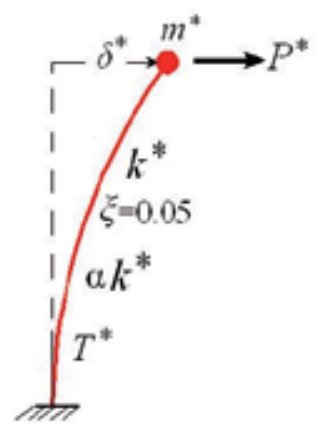

a.

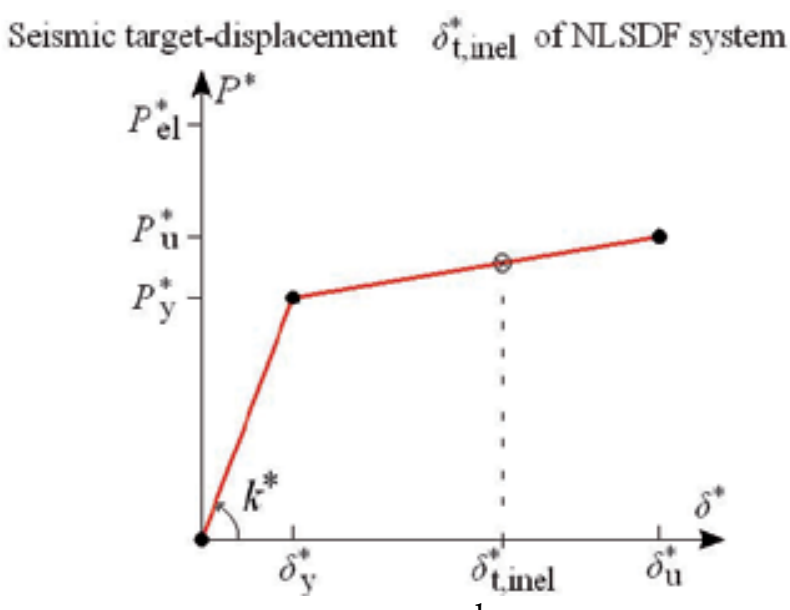

b.

Fig. 6. a. The optimum equivalent NLSDF system, b. The seismic target-displacement of the equivalent NLSDF system.

More specifically, with reference to the optimum equivalent NLSDF system, its elastic spectral acceleration $S_{\mathrm{a}}^{*}$ is calculated by the elastic response acceleration spectrum, for the known period $T^{*}$. Consequently, the maximum required inelastic seismic displacement (target-displacement) $\delta_{\mathrm{t}}$ of the new equivalent NLSDF system arises from the inelastic spectrum of a known earthquake, according to the following equations:

If $\frac{P_{\mathrm{y}}^{*}}{m^{*}}<S_{\mathrm{a}}^{*}$, then the response is non-linear and post-elastic and thus the targetdisplacement is inelastic, because this means that the yielding base shear $P_{\mathrm{y}}^{*}$ of the NLSDF system is less than its elastic seismic base shear; therefore this system yields:

$$
\delta_{\mathrm{t}, \text { inel }}^{*}=L \cdot \frac{\mu_{\mathrm{d}}}{R_{\mathrm{y}}} \cdot \frac{S_{\mathrm{a}}^{*}}{\left(\omega^{*}\right)^{2}}=L \cdot \frac{\mu_{\mathrm{d}}}{R_{\mathrm{y}}} \cdot \frac{S_{\mathrm{a}}^{*}}{4 \pi^{2}} \cdot\left(T^{*}\right)^{2}=L \cdot \frac{\mu_{\mathrm{d}}}{R_{\mathrm{y}}} \cdot S_{\mathrm{d}}^{*}
$$


where,

$S_{\mathrm{a}}^{*}=S_{\mathrm{a}}\left(T^{*}\right)$ and $S_{\mathrm{d}}^{*}=S_{\mathrm{d}}\left(T^{*}\right)$ are the "elastic spectral acceleration" and the "elastic spectral displacement" from the elastic response acceleration and displacement spectrum respectively, for equivalent viscous damping ratio $\xi=0.05$ for $\mathrm{r} / \mathrm{c}$ structures,

$R_{\mathrm{y}}=P_{\mathrm{el}}^{*} / P_{\mathrm{y}}^{*}$ is the reduction factor of the system (Fig.5b)

$L=k^{*} / k_{o}$ the "convergence factor" between the initial Multi Degree of Freedom system and the NLSDF

$\mu_{\mathrm{d}}$ is the demand ductility of this non-linear SDF system (Eq.23):

$$
\mu_{\mathrm{d}}=1+\frac{\left(R_{\mathrm{y}}^{c}-1\right)}{c}
$$

$c$ is a coefficient due to the second branch slope from Eqs. $(24,25,26)$, using a suitable slope ratio $\alpha=0 \%, 2 \%, 10 \%$ of the equivalent NLSDF system (Fig.5b), according to Krawinkler \& Nassar (1992):

$$
\begin{gathered}
c=\frac{T^{*}}{1+T^{*}}+\frac{0.42}{T^{*}} \text { for the second branch slope } \alpha=0 \% \\
c=\frac{T^{*}}{1+T^{*}}+\frac{0.37}{T^{*}} \text { for the second branch slope } \alpha=2 \% \\
c=\frac{\left(T^{*}\right)^{0.8}}{1+\left(T^{*}\right)^{0.8}}+\frac{0.29}{T^{*}} \text { for the second branch slope } \alpha=10 \%
\end{gathered}
$$

Note that the demand ductility $\mu_{\mathrm{d}}$ by Eq.(23) simultaneously satisfies both the rule of "equal energies" and the rule of "equal displacements" according to Veletsos \& Newmark (1960) and Newmark \& Hall (1982).

If $\frac{P_{\mathrm{y}}^{*}}{m^{*}} \geq S_{\mathrm{a}}^{*}$, then the response is linear elastic and thus the target-displacement $\delta_{\mathrm{t}, \mathrm{el}}^{*}$ is elastic, because this means that the yielding base shear $P_{\mathrm{y}}^{*}$ of the NLSDF system is greater than the elastic seismic base shear and therefore this system remains in the linear elastic area,

$$
\delta_{\mathrm{t}, \mathrm{el}}^{*}=L \cdot \frac{S_{\mathrm{a}}^{*}}{\left(\omega^{*}\right)^{2}}=L \cdot \frac{S_{\mathrm{a}}^{*} \cdot\left(T^{*}\right)^{2}}{4 \pi^{2}}
$$

It is worth noting that, in order to achieve adequate seismic stability of the building, the demand ductility $\mu_{\mathrm{d}}$ must be less than the available ductility $\mu=\delta_{\mathrm{u}}^{*} / \delta_{\mathrm{y}}^{*}$. Next, in the first 
approach (index "1"), the maximum required seismic displacement $u_{y, \mathrm{~N}, 1}$ of the mass at the top level of the asymmetric multi-storey building, along $\mathrm{y}$-direction, is directly given by:

$$
u_{y, \mathrm{~N}, 1}=\Gamma \cdot \delta_{\mathrm{t}}^{*}
$$

Finally, as mentioned above, the other maximum required seismic displacements $u_{x, i}$ and $\theta_{z, i}$ of the mass of the multi-storey building are obtained from that step in the known primary pushover analysis, where the displacement $u_{y, \mathrm{~N}, 1}$ appears. If an optimum approach is required, then we can repeat the calculation of Eqs.(3-28), using the new displacements $u_{x, i}, u_{y, i}, \theta_{z, i}$ instead of $u_{x, i, \mathrm{o}} \quad u_{y, i, \mathrm{o}} \quad \theta_{z, i, \mathrm{o}}$; the third approach is not usually needed. Finally, for each lateral loading, we follow the same methodology with a suitable index alternation (i.e. if the lateral floor forces are parallel to $x$-axis, then $\mathbf{u}_{\mathrm{o}}=\boldsymbol{\psi}_{x, \mathrm{~N}, \mathrm{o}} \cdot u_{x, \mathrm{~N}, \mathrm{o}}$ ， $\mathbf{P}_{, i}=\left\{\begin{array}{lll}P_{x, i} & 0 & M_{z, i}\end{array}\right\}^{\mathrm{T}}, \quad \mathbf{l}_{i}^{\mathrm{T}}=\left\{\begin{array}{lll}1 & 0 & 0\end{array}\right\}^{\mathrm{T}}$, etc $)$.

To sum up, the above-mentioned optimum equivalent Non-Linear Single Degree of Freedom System allows us to provide the definition of the behavior factor of the multi-storey building. Indeed, it is well known that the global behavior factor $q$ of a system is mathematically defined by the single degree of freedom system only. Therefore, in order to define the behavior factor of an asymmetric multi-storey building, an equivalent SDF system (such as the above optimum equivalent NLSDF system) must be estimated at first, and then its behavior factor, which refers to the asymmetric multi-storey building, is easy to determine. According to recent research (Makarios 2010), the "available behavior factor" $q_{\mathrm{av}}$ of the optimum equivalent NLSDF system indirectly represents the "global available behavior factor" of the asymmetric multi-storey building, while $q_{\text {av }}$ is given by Eq.(29). Note that, in order for a building to cope with seismic action, the total "available behavior factor" $q_{\mathrm{av}}$ has to be higher than the "design behavior factor" $q$, that was used for the initial design of the building.

$$
q_{\mathrm{av}}=q_{\mathrm{o}} \cdot q_{\mathrm{m}} \cdot q_{\mathrm{p}}
$$

where $q_{\mathrm{o}}$ is the "overstrength partial behavior factor" that is part of the $q_{\mathrm{av}}$ and is related to the "extent of static-indefiniteness / superstaticity" of the asymmetric multi-storey building, as well as the constructional provisions of the Reinforced Concrete Code used, and can be calculated by Eq.(30), where $V_{\mathrm{o}}^{*}$ is the design base shear of the NLSDF system $\left(V_{\mathrm{o}}^{*}=m^{*} \cdot S_{\mathrm{a}}\right)$ :

$$
q_{\mathrm{o}}=P_{\mathrm{y}}^{*} / V_{\mathrm{o}}^{*}
$$

Furthermore, $q_{\mathrm{m}}$ is the "ductility partial behavior factor" that is another part of $q_{\mathrm{av}}$ that is related to the ductility of the equivalent elasto-plastic SDF system, and is calculated by Eq.(31), which is an inversion of Eq.(23):

$$
q_{\mathrm{m}}=[c(\mu-1)+1]^{1 / c}
$$


where $\mu=\delta_{\mathrm{u}}^{*} / \delta_{\mathrm{y}}^{*}$ is now the "available ductility" of the NLSDF system.

Next, $q_{\mathrm{p}}$ is the "post-elastic slope partial behavior factor" that is the last part of the $q_{\mathrm{av}}$ and is related to the slope of the post-elastic (second) branch of the bilinear capacity curve of the equivalent NLSDF system and is calculated by Eq.(32):

$$
q_{\mathrm{p}}=P_{\mathrm{u}}^{*} / P_{\mathrm{y}}^{*}
$$

Note that, if the second (post-elastic) branch has a negative slope (i.e. intense action of second order phenomena), then the "post-elastic slope partial behavior factor" $q_{\mathrm{p}}$ is less than one.

Next, with reference to the spatial seismic action (signs of seismic components, simultaneous action of the two horizontal seismic components) in the presented non-linear static (pushover) procedure, we can observe the following issues:

Being aware, from the above mathematical analysis, that two static pushover analyses are needed for one location of lateral floor static forces, we can conclude that, if we use the final four design eccentricities, then twelve (12) individual static pushover analyses are required, as following:

- For the first seismic horizontal component along principal I-axis, six load cases are needed, Fig.(7). Note that from each primary analysis (where one "final design eccentricity" out of the four is used), the demand seismic displacements of the asymmetric multi-storey building are obtained, whilst from the secondary analysis (where the lateral static forces are located on the mass centres) the effective lateral stiffness $k^{*}$ of its NLSDF is defined. Then, the twelve individual pushover analyses are analytically provided:

1st case: Lateral static loads along positive principal $(+) I$-axis, with final design eccentricity max $e_{\text {II }}$ (primary pushover analysis, $E_{1}$ ).

$2^{\text {nd }}$ case: Lateral static loads along negative principal (-)I-axis, with final design eccentricity max $e_{\mathrm{II}}$ (primary pushover analysis, $E_{2}$ ).

$3^{\text {rd }}$ case: Lateral static loads along positive principal $(+) I$-axis, with final design eccentricity $\min e_{\mathrm{II}}$ (primary pushover analysis, $E_{3}$ ).

$4^{\text {th }}$ case: Lateral static loads along negative principal (-)I-axis, with final design eccentricity min $e_{\mathrm{II}}$ (primary pushover analysis, $E_{4}$ ).

$5^{\text {th }}$ case: Lateral static loads that are located on the floor mass centres along positive principal (+) $I$-axis (secondary pushover analysis, useful for the $1^{\text {st }}$ and $3^{\text {rd }}$ cases).

$6^{\text {th }}$ case: Lateral static loads that are located on the floor mass centres along negative principal (-)I-axis (secondary pushover analysis, useful for the $2^{\text {nd }}$ and $4^{\text {th }}$ cases).

The envelope of the results from the previous primary pushover analyses $\left(1^{\text {st }}, 2^{\text {nd }}, 3^{\text {rd }} \&\right.$ $4^{\text {th }}$ ) is symbolized as $E_{\text {I }}$; it represents the demands of the first seismic component along principal I-axis:

$$
E_{,_{\mathrm{I}}}=\max \left(\left|E_{1}\right|,\left|E_{2}\right|,\left|E_{3}\right|,\left|E_{4}\right|\right)
$$


- $\quad$ For the second seismic horizontal component along principal II-axis, six more load cases are needed, Fig.(8):

7th case: Lateral static loads along positive principal $(+) I I$-axis, with final design eccentricity $\max e_{\mathrm{I}}$ (primary pushover analysis, $E_{7}$ ).

$8^{\text {th }}$ case: Lateral static loads along negative principal (-)II-axis, with final design eccentricity $\max e_{\mathrm{I}}$ (primary pushover analysis, $E_{8}$ ).

9th case: Lateral static loads along positive principal (+)II-axis, with final design eccentricity $\min e_{\mathrm{I}}$ (primary pushover analysis, $E_{9}$ ).

$10^{\text {th }}$ case: Lateral static loads along negative principal (-)II-axis, with final design eccentricity $\min e_{\mathrm{I}}$ (primary pushover analysis, $E_{10}$ ).

11th case: Lateral static loads that are located on the floor mass centres along positive principal (+)II-axis (secondary pushover analysis, useful for the $7^{\text {th }} \& 9^{\text {th }}$ cases).

$12^{\text {th }}$ case: Lateral static loads that are located on the floor mass centres along negative principal (-)II-axis (secondary pushover analysis, useful for the $8^{\text {th }} \& 10^{\text {th }}$ cases).
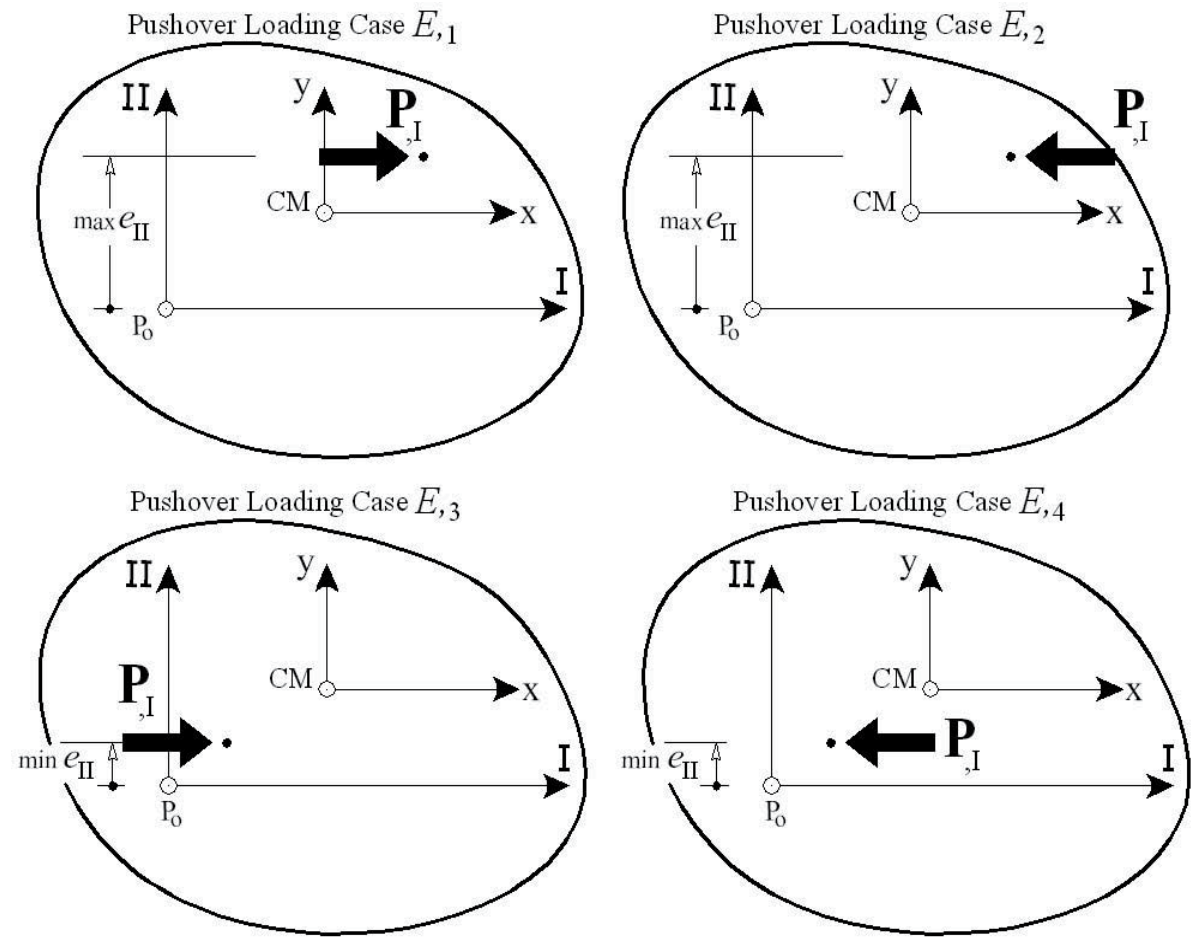

Fig. 7. The envelope of these four pushover analyses represents the results of the first seismic component $E_{\text {,I }}$. 

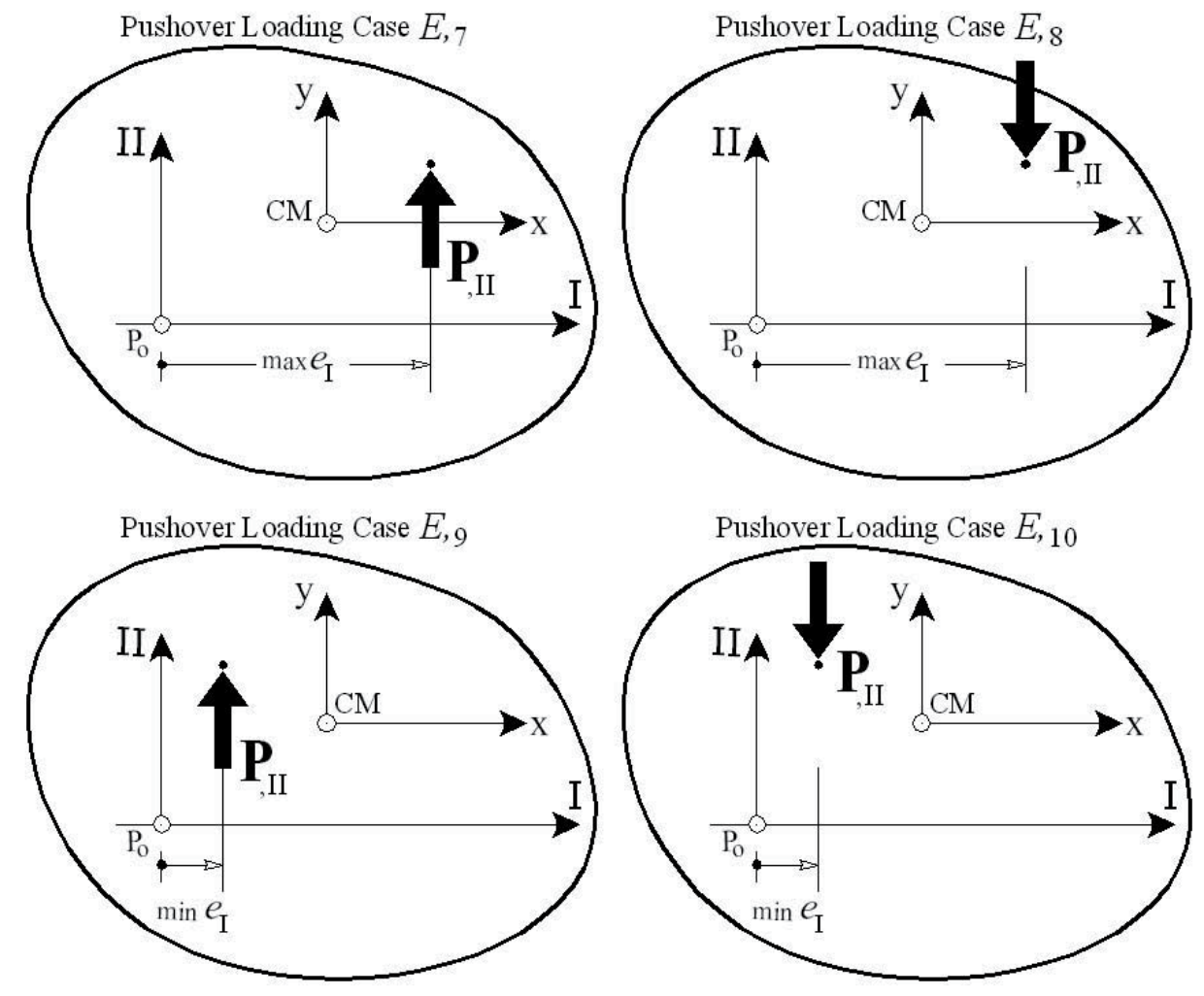

Fig. 8. The envelope of these four pushover analyses represents the results of the second seismic component $E_{\text {,II }}$. 
The envelope of the results from the pushover analyses $\left(7^{\text {th }}, 8^{\text {th }}, 9^{\text {th }} \& 10^{\text {th }}\right)$ is symbolized as $E_{\text {,II }}$ and represents the demands of the second seismic component along principal II-axis:

$$
E_{\text {III }}=\max \left(\left|E_{7}\right|,\left|E_{8}\right|,\left|E_{9}\right|,\left|E_{10}\right|\right)
$$

Note that, the use of the two signs of seismic components is necessary, because superpositions are generally forbidden in the non-linear area (sect.4.3.3.4.1(7)P/EN-1998.01). With reference to the simultaneous action of the two horizontal seismic components, Eq.(1) must be applied in order to obtain the final result of the extreme seismic demands by the spatial seismic action (where $E_{\text {, I }} \& E_{\text {,II }}$ are calculated by Eqs.(33-34). Note that suitable numerical examples, which demostrate the applicability of the proposed method, have been provided in other papers (Makarios, 2009 \& 2011), where the correctness of these final results has been verified by the Non-Linear Response History Analysis.

In addition, contemporary Sesmic Codes, such as Eurocode EN-1998, do not give details and quidance regarding the static seismic pushover analyis of irregular in plan, asymmetric, multistorey r/c buildings. In reality, Annex B of EN-1998.01 refers to the calculation of the seismic target-displacement of planar frames only, whilst for spatial irregular in plan multistorey buildings there are no relevant provisions. The present work intends to precisely cover this gap. The present non-linear static (pushover) procedure is a natural extension of the established equivalent linear (simplified spectral) static method, recommended by the Seismic Codes (EAK/2003, NBCC/1995), which are adequately documented as regards torsional provisions. The twelve static pushover analyses of the irregular asymmetric building involve an important computational cost, but on the other hand, this is much lower compared to the two hundred and twenty-four (224) non-linear dynamic analyses that the Non-Linear Response History Procedure requires. Thus, the presented non-linear static (pushover) procedure is a very attractive option, despite its twelve non-linear static analyses. In addition, within the framework of the proposed procedure, there is also natural supervision, which constitutes a very important issue.

\section{Conclusions}

Consequently, the most important issues that we come across during the static pushover analysis, in order to avoid the exact non-linear response history analysis, have been noted. More specifically, we have explained (1) why it is necessary to use a single degree of freedom system that represents the real structure; (2) how the spatial action of the two horizontal seismic components during the static pushover analysis can be taken into account; (3) what is the most suitable monitoring point in the case of asymmetric buildings; (4) which distribution of the lateral floor static forces is suitable; and (5) at which point in the plan of an asymmetric building, lateral floor static forces should be applied. Design dynamic eccentricities plus accidental eccentricities must be taken into account. This point leads to the calculation of the real or fictitious elastic axis of the building and the real or fictitious horizontal main elastic axes, where the lateral static forces must be oriented during the static pushover analysis (Fig.1). Extensive guidance has been given about the simulation of plastic hinges using Eurocode EN/1998.03. Moreover, various important issues have been discussed concerning seismic non-linear response history analysis. Next, a documented proposal of the optimum 
equivalent NLSDF system that represents asymmetric multi-storey buildings is presented. The definition of the NLSDF system is mathematically derived, by studying suitable dynamic loadings on the masses of each $\mathrm{r} / \mathrm{c}$ system and using simplified assumptions. The coupling of the translational and the torsional degrees-of-freedom of the asymmetric building has also been taken into consideration, without dividing the asymmetric building into various, individual (two planar) subsystems. This NLSDF system is used in combination with the inelastic design spectra in order to calculate the seismic demands. The natural meaning of the characteristics of the asymmetric building, such as the fundamental eigenperiod, is not distorted by the optimum equivalent NLSDF system, since both the fundamental period of the multi-storey building and the period of the NLSDF system are very close, due to the fact where the total methodology is derived in a mathematical way. Extended numerical comparisons have verified the correctness of the use of the equivalent NLSDF system (Makarios, 2009 \& 2011;). However, on the other hand, it is a known fact that, the sequence of plastic hinges on a building and the yielding/failure mechanism of multi-storey buildings are not correctly calculated by the various pushover analyses, which can only provide approximate results; nevertheless, this question is beyond the scope of the present chapter.

\section{References}

Akiyama, H. 1985. Earthquake-resistant limit-state design for buildings, University of Tokyo, Press, Tokyo.

Anastassiadis K, Athanatopoulou A, Makarios T. 1998. Equivalent Static Eccentricities In the Simplified Methods of Seismic Analysis of Buildings. Earthquake Spectra the Profes. Jour. of the Earth. Engin. Research Inst.; 1998; vol. 14, Number 1, 1-34.

Athanatopoulou AM. 2005. Critical orientation of three correlated seismic components. Journal Engineering Structures, 27, 2, January, pp.301-312.

Cheung V WT, Tso WK. 1986. Eccentricity in Irregular Multistorey Buildings. Can. Journ. Civ. Eng. 13, 46-52.

Dowell R, Seible F, Wilson E L. 1998. Pivot Hysterisis Model for Reinforced Concrete Members. ACI Structural Journal, Technical Paper, Title no. 95-S55, SeptemberOctober, pp 607-617.

EAK/2003: Hellenic Seismic Code/2003; FEK184B /20.12.1999, FEK 781/18.07.2003, FEK 1154/12.08.2003 (in Greek).

EN-1998.01. 2004. Eurocode 8: Design of structures for earthquake resistance - Part 1: General rules, seismic actions and rules for buildings. European Commitetee for Standardization, Brussels.

EN-1998.03. 2005. Eurocode 8: Design of structures for earthquake resistance - Part 3: Assessment and retrofitting of building. European Commitetee for Standardization, Brussels.

Fajfar P, Fischinger M. 1987a. Non-linear Seismic Analysis of RC Buildings: Implications of a Case Study. European Earthquake Engineering;1:31-43.

Fajfar P, Fischinger M. 1987b. N2 - a method for non-linear seismic analysis of regular buildings. Proc. 9th World conf. Earthquake Eengin., Tokyo, Kyoto; vol. 5:111-116.

Fajfar P, Gaspersic P. 1996. The N2 Method for the Seismic Damage Analysis of RC Buildings. Earthq. Engin.E Struct. Dynamics. vol.25: 31-46.

Hart G, Wong K. 1999. Structural Dynamics for Structural Engineers. Willey, New York, USA. 
Hejal R, Chopra AK. 1987. Earthquake Response of Torsionally-Coupled Building. Report No U.S.B./e.e.r.c.-87/20, Berkeley.

Krawinkler H, Nassar AA. 1992. Seismic design based on ductility and cumulative damage demands and capacities. Nonlinear Seismic Analysis and Design of Reinforced Concrete Buildings, eds P.Fajfar and H. Krawinkler. New York: Elsevier Applied Science, 2339.

Makarios T, Anastassiadis K. 1998a. Real and Fictitious Elastic Axis of multi-storey Buildings: Theory. The Structural Design of Tall Buildings Journal, 7,1, 33-55.

Makarios T, Anastassiadis K. 1998b. Real and Fictitious Elastic Axis of multi-storey Buildings: Application. The Structural Design of Tall Buildings Journal, 7, 1,57-71.

Makarios T. 2005. Optimum definition of equivalent non-linear SDF system in pushover procedure of multistory r/c frames. Engineering Structures Journal v.27, 5, April, pp.814-825.

Makarios T, Athanatopoulou A, Xenidis H. 2006. Numerical verification of properties of the fictitious elastic axis in asymmetric multistorey buildings. The Structural Design of Tall and Special Buildings Journal, 15, 3, 249-276.

Makarios T. 2008. Practical calculation of the torsional stiffness radius of multistorey tall buildings. Journal of the Structural Design of Tall \& Special Buildings. 2008; 17, 1, 39-65.

Makarios T. 2009. Equivalent non-linear single degree of freedom system of spatial asymmetric multi-storey buildings in pushover procedure. Theory \& applications. Journal of the Structural Design of Tall \& Special Buildings, 18,7, pp.729-763.

Makarios T. 2011. Seismic non-linear static new method of spatial asymmetric multi-storey r/c buildings. Journal of the Structural Design of Tall $\mathcal{E}$ Special Buildings, DOI: 10.1002/tal.640, (in press).

Moustafa A. 2011. Damage-based design earthquake loads for single-degree-of-freedom inelastic structures. Journal of Structural Engineering, ASCE, 137(3), 456-467.

National Building Code of Canada (NBCC/95): Associate Committee on the National Building Code. National Research Council of Canada, 1995.

Newmark NM, Hall WJ. 1982. Earthquake Spectra and Design. Earthquake Engineering Research Institute, Berkeley, CA.

Panagiotakos T, Fardis M. 2001. Deformations of reinforced concrete members at yielding and ultimate. ACI Structural Journal, March-April; v.98, No.2. pp 135-148.

Penzien J, Watabe M. 1975. Characteristics of 3-Dimensional Earthquake Ground Motions. Earthquake Engin. \& Struct. Dynamics. vol. 3, pp365-373.

Qi X, Moehle JP. 1991.Displacement design approach for reinforced concrete structures subjected to earthquakes. Report No. UCB/EERC-91/02, Univercity of California, Berkeley.

Otani, S. 1981. Hysteretic models of reinforced concrete for earthquake response analysis. J. Fac. Eng., University of Tokyo, 36(2), 407-441.

Rodriguez M.1994. A measure of the capacity of earthquake ground motions to damage structures. Earthquake Engin. Struct. Dynam.; 23:627-643.

Saiidi M, Sozen MA. 1981. Simple Nonlinear Seismic Analysis of r/c Structures. Journal of the Structural Division, ASCE 1981; 107:937-952.

Takeda T, Sozen M.A, Nielsen N.N . 1970. Reinforced Concrete Response to Simulated Earthquakes. Journal Structures Engin. Div, ASCE, v.96, No 12, pp 2557-2573 
Uang C, Bertero VV.1990. Evaluation of Seismic Energy in Structures. Earthq. Eng. \& Struct. Dynam. vol.19:77-90.

Veletsos AS, Newmark NM. 1960. Effects of inelastic behavior on the response of simple system to earthquake motions. Proccedings of the $2^{\text {nd }}$ World Conference on Earthquake Engineering. Japan; 2: pp895-912.

XTRACT. v.3.0.8. 2007. Cross-sectional X sTRuctural Analysis of ComponenTs. Imbsen Software System. 9912 Business Park Drive, Suite 130, Sacramento CA 95827. 


\title{
Masonry and Earthquakes: Material Properties, Experimental Testing and Design Approaches
}

\author{
Thomas Zimmermann and Alfred Strauss \\ University of Natural Resources and Life Sciences Vienna \\ Institute for Structural Engineering \\ Austria
}

\section{Introduction}

Earthquakes are natural disasters which can occur without warning and they can affect large areas. Earthquakes are often accompanied by aftershocks which may cause additional damage to an already damaged structure or lead to failure. Consequences of earthquakes, such as rock falls, fires, explosions etc., can be very large in the affected areas. An example is the 1906 earthquake in San Francisco. Thereby many lives were killed by the ensuing fire. But not only past events have had fatal consequences, also events of recent years caused countless deaths and consequential damages e.g. the 2010 earthquake in Haiti with a death toll of more than 250,000 people (Eberhard et al., 2011) or the 2011 earthquake in Fukushima, Japan with high consequential damages e.g. to the nuclear power plant (Takewaki et al., 2011) or both earthquakes in Christchurch, New Zealand with high damage on cultural heritage (Ingham \& Griffith, 2011; Ingham et al., 2011).

\section{Masonry}

\subsection{Historical overview in masonry construction}

Masonry is one of the oldest types of construction. Taking account structural-physical properties and the quite easy construction process, this construction system is used until today. The building material brick was easy to produce. In ancient times, clay was put into a model, the surface was smoothed and then the brick was exposed to air for drying. In later times the raw material was extruded and baked in a kiln. Masonry construction methods were already well known in ancient times, about 5000 BC bricks were used in Mesopotamia. A wellknown example for the usage of masonry in these times is the tower of Babel, which was built around $600 \mathrm{BC}$. Clay bricks were used as building material and bitumen as mortar. Even then masonry offered a faster and cheaper alternative to natural stones in the construction process. In contrast to natural stone construction methods, manufacturing of regular building stones was a revolution and enables systematic construction methods. Monumental structures, for instance the Pantheon in Rome were built by using masonry. Masonry construction methods do not offer the possibility of build plane top panels and lintels, therefore the construction of arches was developed and enhanced during that period (Maier, 2002).

Masonry always has to be constructed in bond to guarantee an adequate bearing capacity. Depending on the time period, different bond types were used, additionally it has to be 
distinguished between pure and mixed masonry. Especially in the Early Middle Ages, mixed masonry was constructed. The facings of the wall were built with bricks but the core was filled up with quarry stones and lower quality bricks. Later on, masonry was used through the whole thickness of a structure, with continuous horizontal joints in each row, independent from the thickness of the wall.

By placing bricks in an adequate way, a cross bonding through the whole thickness of the wall was achieved. As a result of this the decisive bond of the bricks depends on the thickness of the wall. Walls with a thickness of the width of a brick usually were built in a stretching bond and walls with a thickness of the length of a brick in a heading bond. Further masonry was built with different bond types e.g. Markish or Wendish, Gothic or Monastic bond. Thereby in every brick layer one header brick is followed by a few stretcher bricks.

In the $16^{\text {th }}$ century, structured bonds like cross bond and block bond were used, in which a stretcher course is followed by a header course. In the 17th century, the Dutch or Flemish bond has been used. Thereby a header course is followed by a mixed layer of headers and stretchers. The common bond methods are depictured in Fig. 1.

The bearing capacity of a masonry bond is reached by avoiding vertical joints which go through multiple layers of construction stones. An additional capacity can be reached by using anchorages, which set up a force-fit connection of opposite walls and especially of masonry and wooden floor slabs. In the rebuilding period after World War II masonry construction became important again.

Developing of reinforced concrete and enhancing of the corresponding construction methods showed the limits of masonry construction methods. Nevertheless masonry is still used in restoration of historic buildings and in housing construction. It becomes more important again during the last years, because of the increasing requirements to thermal insulation, almost special large-sized honeycomb bricks are used.

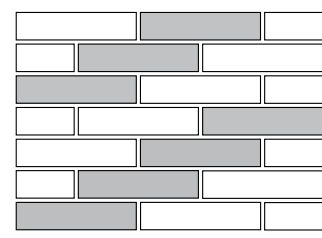

(a)

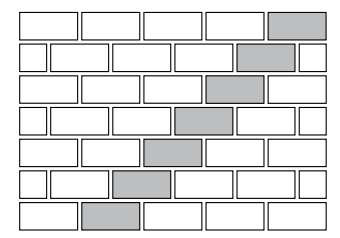

(b)

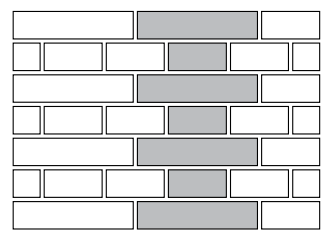

(c)

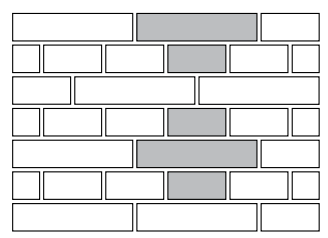

(d)

Fig. 1. Various bonds of masonry (a) stretching bond, (b) heading bond, (c) block bond, (d) cross bond; (Bargmann, 1993)

\subsubsection{Bricks}

In contrast to natural stones, which have to be cut to the appropriate form and size, artificial construction stones as bricks are shaped by the models they are made with. The requirements to construction stones and mortar are defined in various codes. These codes define basic materials, definitions, dimensions, classes of raw density and strength and the assurance of quality.

Nowadays research objectives in brick design should offer the following properties: (a) increasing of efficiency in production process of construction stones; (b) increasing of the bricklayer's capacity; (c) increasing of the bearing capacity; (d) increasing of the building physics. 
An important change in the production process was made with the industrial revolution, when the process was automatized. The change from the manual handling to automatically extruding machines yields in constant shapes. Moreover it ensures an alignment of the clay minerals due to the high pressure. This orientation of the clay minerals is the texture. It causes the anisotropic material behaviour depending on the direction of the brick. During the time various shapes and dimensions of bricks for different purposes occurred.

\subsubsection{Mortar}

Mortar is used to build up compensating layers to connect not accurately fitting bricks to an accurate and usable masonry. The most important characteristics of masonry mortar are its processability, its plasticity, water retention capacity, compressive strength and the bond strength between bricks and mortar. The components of mortar are binding agent, additives and water. Throughout additives and admixtures certain properties can be achieved.

The classification of mortars is based on the type of the binding agent, the production process and the type. Binding agents can be distinguished in non-hydraulic and hydraulic binders.

Non-hydraulic binders become hardened only on air and must not be under permanent moisture and water influence. Non-hydraulic binders are loam, common lime, gypsum, anhydrite, magnesia and fireclay.

Hydraulic binders become hardened both on air and under water and are resistant against permanent moisture. Hydraulic binders are cement, special types of lime and mixed binders. Mortars can be produced on construction side or in plant.

Lime was used as binding agent for masonry verifiable in 1000 BC (Hilsdorf, 1965) and it is up to now the determining binding agent for masonry mortar. Already in ancient times it was well known that admixtures like volcanic ashes or brick powder have a positive influence on strength and moisture resistance of the mortar (Grimm, 1989). Organic components enhance or modify manufacturing and hardening process, especially casein has an improving influence on water demand and water retention capacity (Conrad, 1990).

Extensive analyses of historic mortars have shown information about their composition, mixture and characteristics, which are quite different to modern mortars (Schäfer \& Hilsdorf, 1990; Wisser \& Knöfel, 1987). Additional components like tuff and puzzolan can be added to lime mortar to make the mortar hardening under water.

Table 1 gives an overview of different mortar types. Nowadays the mortar layer becomes thinner and thinner because the surface of modern bricks is very flat ensured by the production process. Further the thermal properties of mortar are improved continuously.

\subsection{Material properties: Bricks, mortar, masonry}

\subsubsection{Bricks}

Older structures often built with bricks, which are not conforming to today's ones. A comparison of old bricks in terms of material properties and characteristics can be found in (Egermann \& Mayer, 1987). The following types were tested: (a) usual common brick (MZ); (b) extruded brick (SM); (c) hand-smoothed brick (HM); (d) historical brick 1796 (QU); (e) historical brick $1884(\mathrm{BE})$.

The bricks SM and HM are made from the same raw material as the usual common brick $\mathrm{MZ}$, but they were baked with $800^{\circ} \mathrm{C}$ instead of $1000^{\circ} \mathrm{C}$. The historical brick QU from 1796 has been made manually, and the historical brick BE was formed by a screw extruder. 


\begin{tabular}{ll}
\hline Loam-mortar & $\begin{array}{l}\text { made out of moistured loam with added chaffed components, } \\
\text { hardening through drying; application: indoor rooms, well } \\
\text { protected outdoor walls, clay floor in agricultures }\end{array}$ \\
\hline Gypsum-mortar & $\begin{array}{l}\text { made out of gypsum with sand and lime; application: gypsum, } \\
\text { sand, lime as wall and ceiling plaster }\end{array}$ \\
\hline Lime-mortar & $\begin{array}{l}\text { lime-sand mortar is the usual mortar in structural engineering, } \\
\text { made out of slaked-lime, sand and water }\end{array}$ \\
\hline $\begin{array}{l}\text { Lime-cement- } \\
\text { mortar }\end{array}$ & $\begin{array}{l}\text { extended cement-mortar is made by add cement to lime-mortar; } \\
\text { application: if lime-mortar cannot be used as a result of the type of } \\
\text { the bricks, the strength of the mortar and the expected moisture } \\
\text { (lime-sand bricks, floating bricks, loaded piles and arches, weather } \\
\text { sides and outside wall plaster }\end{array}$ \\
\hline Cement-mortar & $\begin{array}{l}\text { made out of cement and additional sand; application: for heavily } \\
\text { used constructions and for structural members (piles, arches) } \\
\text { which are exposed to moisture (foundations) }\end{array}$ \\
\hline $\begin{array}{l}\text { Raw-cement- } \\
\text { mortar }\end{array}$ & cement, sand and additional components: fly-ash, gravel, stones \\
\hline Floor-mortar & terrazzo-mortar, magnesia-mortar, xylolite, pavement \\
\hline
\end{tabular}

Table 1. Mortar types (Bargmann, 1993)

\begin{tabular}{lcccccccccc}
\hline Specimen & Density & \multicolumn{2}{c}{$\begin{array}{c}\text { Compressive } \\
\text { strength in } \\
\text { load direction } \\
{[\mathrm{MPa}]}\end{array}$} & $\begin{array}{c}\text { Splitting } \\
\text { tensile } \\
\text { strength } \\
{[\mathrm{MPa}]}\end{array}$ & $\begin{array}{c}\text { Elastic } \\
\text { modulus in } \\
\text { load direction } \\
{[\mathrm{MPa}]}\end{array}$ & $\begin{array}{c}\text { Poisson's } \\
\text { ratio in load } \\
\text { direction } \\
{[-]}\end{array}$ \\
\hline MZ & 1.83 & 1.6 & 43.0 & 18.1 & 3.94 & 32.4 & 22669 & 5.5 & 0.19 & 6.4 \\
$\mathrm{SM}$ & 1.90 & 1.2 & 31.3 & 16.3 & 3.76 & 18.0 & 11867 & 18.7 & 0.13 & 12.5 \\
$\mathrm{HM}$ & 1.82 & 1.4 & 15.6 & 22.2 & 1.82 & 21.7 & 5716 & 21.2 & 0.10 & 33.5 \\
QU & 1.65 & 4.3 & 9.5 & 56.2 & 0.52 & 37.4 & 2726 & 11.7 & 0.14 & 19.1 \\
BE & 1.49 & 72.3 & 13.9 & 38.5 & 2.42 & 40.1 & 8379 & 35.2 & 0.21 & 21.9 \\
\hline
\end{tabular}

Table 2. Properties of historical bricks (Egermann \& Mayer 1987)

As it can be seen in Table 2, the strength properties of hand-smoothed (HM) and historical bricks (QU, BE) are by trend lower than from machine-made bricks (MZ, SM). The scattering increases due to the manual production process. The consequent enhancement of the oxidation technique mainly led to a reduction of the spreading in the mechanic parameters. On the other hand the shaping methods influence other important parameters which are decisive for research of the structures. As discussed above, the orientation of clay minerals have a significant importance on the compressive strength and can be influenced by the production process. Taking account the compressive strengths of different brick types the results of the experimental study shows that in reference to common bricks (MZ) compressive strength of the extruded bricks (SM) is $73 \%$ and the hand-smoothed (HM) is just $37 \%$. The compressive strengths of historical bricks (QU, BE) is below the strength of the common bricks. 
The bearing capacity of masonry is essentially influenced by the splitting tensile strength of bricks. Common (MZ) and extruded bricks (SM) have approximately the same values for splitting tensile strength, whereas the value of the hand-smoothed bricks is just at $50 \%$.

If a masonry element is loaded by a centric compressive load, the failure results from cracks caused by tensile stresses. This tensile stress state develops due to different lateral Poisson ratios of bricks and mortar. Additionally, the production process has an important influence on stiffness. In reference to common bricks (MZ) the elastic modulus of the extruded bricks $(\mathrm{SM})$ is $50 \%$ and the hand-smoothed bricks (HM) have a value of about $25 \%$. As written above, the orientation of the clay minerals causes a relationship of the strength and deformation parameters to the considered direction of loading. Particularly common bricks (MZ) and extruded bricks (SM) show this behaviour. Hand-smoothed and historical bricks (HM, QU, BE) do not show this behaviour according to the considered direction. Therefore it can be concluded, that shaping under high pressure induces an anisotropic material behaviour. In addition to the strength values of construction stones given in Table 3, research results on historical Viennese bricks from the $19^{\text {th }}$ century are given in (Furthmüller \& Adam, 2009; Pech, 2010; Zimmermann \& Strauss, 2010a). Properties of contemporary brick types, mortar and masonry can be found in (Schubert \& Brameshuber, 2011).

\begin{tabular}{lcccccccccc}
\hline Reference & $\begin{array}{c}\text { Compressive } \\
\text { strength } \\
{[\mathrm{MPa}]}\end{array}$ & $\begin{array}{c}\text { Tensile } \\
\text { strength } \\
{[\mathrm{MPa}]}\end{array}$ & $\begin{array}{c}\text { Elastic } \\
\text { modulus } \\
{[\mathrm{MPa}]}\end{array}$ & $\begin{array}{c}\text { Fracture } \\
\text { energy } \\
{\left[\mathrm{Nmm} / \mathrm{mm}^{2}\right]}\end{array}$ & \multicolumn{2}{c}{$\begin{array}{c}\text { Density } \\
\mathrm{kg} / \mathrm{m}^{3}\end{array}$} \\
\hline & mean & cov & mean & cov & mean & cov & mean & cov & mean & $\mathrm{cov}$ \\
\hline (a) & 29.5 & 34.6 & 2.1 & 33.3 & 12055 & 25.6 & 0.056 & 3.6 & 1510 & 4.0 \\
(b) & 22.5 & 26.6 & - & - & - & - & - & - & - & - \\
(c) & 19.3 & 39.7 & - & - & 13489 & 52.6 & - & - & 1467 & 6.6 \\
\hline
\end{tabular}

Table 3. Material parameters from historical Viennese bricks from the 19th century (a): (Furthmüller \& Adam, 2009), (b): (Pech, 2010), (c): (Zimmermann \& Strauss, 2010a)

\subsubsection{Mortar}

The percentage of binding agent in the hardened state in comparison to the additives is 1:2 up to $1: 3$ in reference to weight. The whole hydraulic part of the binding agent, including puzzolanic additives is $10-25 \%$. The configuration of the mortar has a high influence on the material characteristics. For compressive bearing capacity of masonry the influence factors are the compressive and tensile strength of the mortar and additionally its deformation behaviour. For shear and flexural capacity, initial shear strength and tensile bond strength are the decisive parameters. It is quite difficult to get adequate test specimens from masonry. Therefore these characteristics mostly have to be estimated (Schubert \& Brameshuber, 2011).

If masonry is under compressive load, (see Sec. 2.2.3), tensile strength of bricks is decisive for the capacity. The modulus of lateral elongation of the mortar is crucial for the tensile stress state inside the bricks. Compressive strength of mortar is influenced especially by binding agent, the percentage of the components and porosity. Pure lime-mortars have compressive strength in a range from 1.0 to $2.0 \mathrm{MPa}$. If the hydraulic fraction increases, then also the compressive strength increases, which can be up to $5.0 \mathrm{MPa}$ and more for lime-cement-mortar. If the hydraulic fraction increases, the elastic modulus increases and ductility decreases. Bonding characteristics between mortar and bricks can be specified 
with the following parameters: (a) shear strength $f_{v k}$; (b) tensile bond strength $f_{h z ;}$ (c) coefficient of friction $\mu$. Bonding characteristics are mainly influenced by the mortar type and its components. The type of bricks and further the moisture characteristics has an influence.

\subsubsection{Masonry}

Masonry is defined as a composite material. The bearing behaviour under compressive, tensile, flexural and shear load is different to homogenous materials like concrete or steel. The composite material itself consists of the singular bricks, horizontal and vertical joints. Depending on the scale, masonry can be seen as (a) inhomogenous or (b) homogenous construction material. In case (a) the characterisation has to be made separately for bricks, for mortar and their interaction, in case (b) the characterisation can be done with global parameters on the smeared masonry element; see Fig. 2.

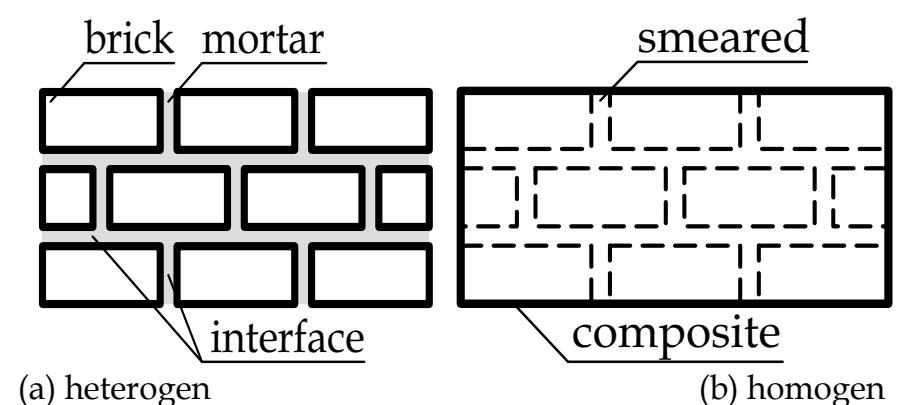

Fig. 2. Characterisation of masonry depending on the scale

\subsubsection{Compressive strength}

Compressive strength of masonry is much higher than its tensile or flexural strength. As a result masonry is mainly used for structures under compressive load and the bearing capacity of masonry is described generally by the parameter compressive strength $f_{k}$. Taking into account the different components of masonry, the strengths are different. The compressive strength of the bricks $f_{b}$ is much higher than the compressive strength of the mortar $f_{m}$, therefore the failure mechanism of masonry under compressive load can be characterised.

Compressive strength of masonry can be seen as a function of the strength of the bricks and the strength of the mortar. The formula was detected empirically with the influences of the singular strengths on the overall strength of masonry and shows a nonlinear behaviour between the strengths of bricks and mortar. According to EC 6 the characteristic compressive strength of masonry $f_{k}$, which is defined as the $5 \%$-fractile value, can be determined from the mean values of the compressive strength of bricks and mortar. These mean values can be determined by execute tests according to EN 1052-1 or from the following equation:

$$
f_{k}=K \cdot f_{b}^{\alpha} \cdot f_{m}^{\beta}
$$

The factor $K$ and the exponents $\alpha$ and $\beta$ have to be taken from EC 6, e.g. for old bricks $K=$ 0.60 the exponents $\alpha=0.65$ and $\beta=0.25$. The relation of eq (1) is just valid for compressive 
loads perpendicular to the horizontal joints. If the compressive load is applied in the direction of horizontal joints, the strength has to be reduced as a result of the influence of the vertical joints. Researches from (Glitzka, 1988) quantify this reduction as follows:

$$
f_{k||}=0.75 f_{k}
$$

If masonry is under compressive loads, deformations occur in parallel as well as in perpendicular direction in accordance to the load direction. As a result from the differences in material behaviour of bricks and mortar, horizontal stress occurs and causes failure of masonry. There are also differences in the lateral deformations of the masonry components, which can be observed, if masonry is loaded until the uniaxial compressive strength of mortar is almost reached. In this stress state the lateral elongation of mortar is much larger than those of the bricks, but the lateral deformations of the mortar are restricted by the bricks. Hence in the mortar occurs a spatial compressive stress state, whereas the brick is loaded by compression and tension.

The compressive strength of masonry under compressive loads is mostly determined by lateral tensile strength of bricks and the elastic modulus of mortar. However the elastic modulus can be identified in the tests quite difficulty. Therefore, in most applications the compressive strength of the bricks $f_{b}$ is taken as the initial parameter for defining the overall compressive strength of masonry. Some correlation factors between lateral tensile strength $f_{b t}$ and compressive strength of bricks are listed in the literature (Schubert \& Brameshuber, 2011). For common bricks, this correlation is:

$$
f_{b t}=0.026 f_{b}
$$

Tensile stresses inside of bricks cause cracks and fracture of bricks. To follow the progression of the cracks up to failure, the interaction phases between bricks and mortar have to be considered.

If masonry is put under compressive load and vertical cracks appear, the limit of lateral tensile strength has been exceeded, and on the appearing cracks, lateral tensile stress is reduced. If the load increases, the proximate cross-sections carry the lateral tensile stress up to an exceeding of the next maximum possible tensile stress state. After a few formations of cracks, the structure fails. The function of the critical stress state is depicted as the enveloping line of the fracture and shows the local reachable limit state. If masonry is under a marginal vertical compressive loading, the tensile stress state inside the bricks is below the enveloping curve of fracture. Therefore vertical stress can be increased, until the brick fails in tension or the mortar fails in compression.

The material performance of masonry under compressive load is defined in EC 6 by means of the parable-rectangle-diagram, see Fig. 3 . The limit strain is defined with $\varepsilon_{\mathrm{m} 1}$ and $\varepsilon_{\mathrm{mu}}$.

Another important parameter for defining the material behaviour of masonry is the elastic modulus. According to EC 6 the short time elastic modulus can be determined as the secant modulus from tests according to EN 1052-1 or calculated directly from compressive strength:

$$
E=1000 f_{k}
$$

From experimental tests, see also Sec. 2.3, for masonry made of solid bricks with the oldAustrian shape type (height $6.5-7.5 \mathrm{~cm}$ ) independent from the mortar type, a deviation from the recommended values for the elastic modulus was found out. 


$$
E=300 f_{k}
$$

The reason for the lower elastic modulus for masonry made from solid bricks can be seen in the higher percentage of horizontal joints per altitude compared to masonry made out of new honeycomb bricks. Commonly used honeycomb bricks have a height of $25 \mathrm{~cm}$ and therefore just 4 horizontal joints per meter altitude difference in relation to 13 joints (masonry made of solid bricks), which is a multiplying factor of 3.3 for the joints. The same ratio can be found in the correlation factors for elastic modulus and compressive strength.

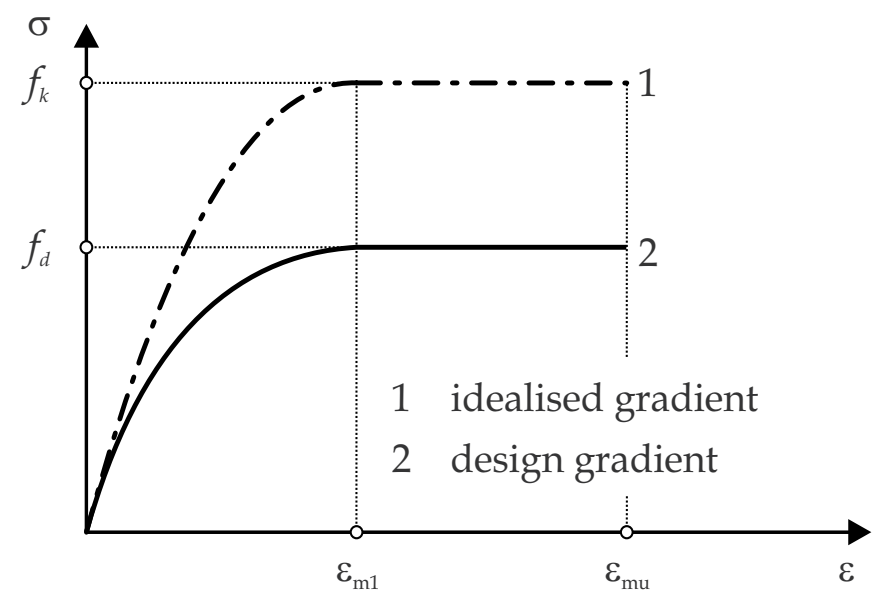

Fig. 3. Stress-Strain-Curve of masonry under compressive load

\subsubsection{Tensile strength}

Generally in masonry walls there is no constant tensile load over the whole cross section perpendicular to the horizontal joint. Besides the dead load, in most cases, masonry has to bear vertical loads. Anyway if there occur a tensile load, two mechanisms of failure can be distinguished: (a) failure of bond between mortar and brick, which is influenced mainly by tensile bond strength $f_{h z}$ between these components, and (b) failure of bricks, if tensile bond strength between mortar and bricks is larger than the tensile strength of bricks.

According to the codes of masonry construction, a planned tensile load perpendicular to the horizontal joints has to be avoided, because the resistance and capacity perpendicular to the horizontal joints has a large scatter.

EC 6 defines boundary conditions, when tensile strength perpendicular to the horizontal joints may be considered. In these cases, the failure of the structural member must not cause a failure of the whole structure. Tensile load in the direction of horizontal joints results from a load in the direction of the wall. To bear these tensile stresses, the walls have to be built in bond, whereat it is convenient to overpressure the occurring tensile stresses with perpendicular compressive stresses.

Tension bearing capacity of masonry in the parallel direction to the horizontal joints is defined mainly by the characteristics of the mortar. To define the deformation behaviour of masonry walls parallel and perpendicular to the horizontal joints extensive research is documented in (Bakes, 1983). However in this analysis the friction between the mortar of the vertical joint and the brick was neglected. Results show until fracture an almost linear material behaviour. Fig. 4 shows different failure modes caused by tension. 

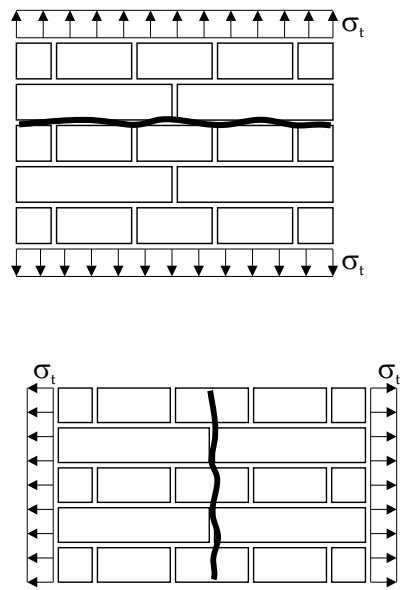

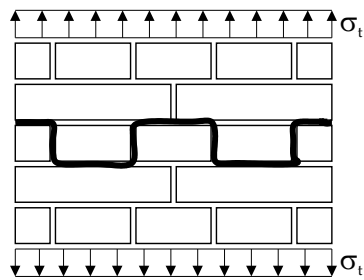

(a)

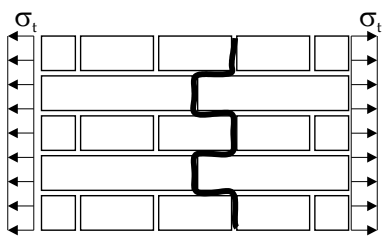

(b)
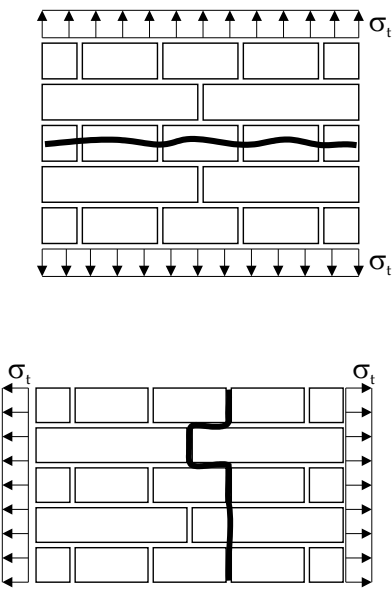

Fig. 4. Masonry under tension load, (a) tension perpendicular to horizontal interface (b) tension parallel to horizontal interface

\subsubsection{Flexural behaviour}

In contrast to the pure tensile load perpendicular to the horizontal joints, which can be excluded in nearly all load cases, flexural loading is a load case which is quite common. If a wall is loaded by wind or earth pressure perpendicular to its surface, then flexural stresses in the perpendicular and the parallel direction of the horizontal joints occur (Fig. 5). Designing principles assume no tensile or flexural stresses perpendicular to the horizontal joints. According to EC 6, the gap in the horizontal joints is just acceptable until to the half of the cross section.

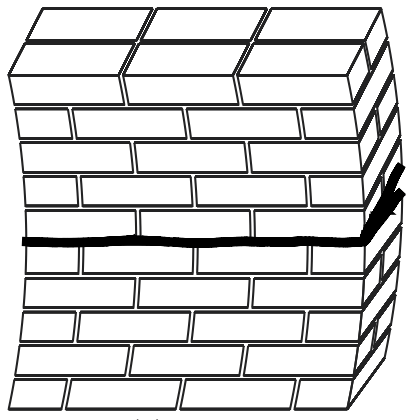

(a)

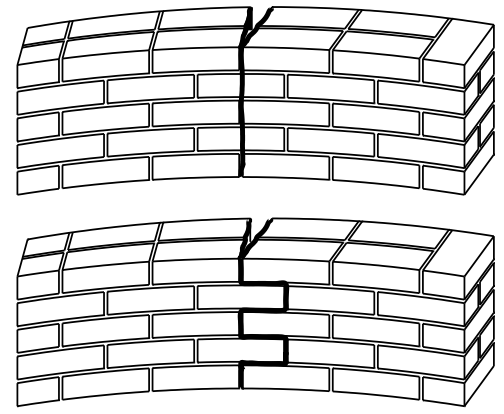

(b)

Fig. 5. Flexural load (a) parallel to the horizontal joints $f_{x k 1}$ and (b) perpendicular to the horizontal joints $f_{x k 2}$

\subsubsection{Shear strength}

The global bearing behaviour of structures made of masonry is influenced by loads acting in horizontal direction like wind and earthquake loads. For the load transfer vertical shear walls are required, which are loaded with shear forces in the wall direction. Therefore the behaviour of the shear wall is decisive for the bearing capacity of the whole structure. If an element is cut from the shear wall normal stresses are acting in vertical direction and shear 
stresses are acting at all four edges. This theory assumes that the vertical joints between the bricks nearby the fracture state do not transfer shear stresses, and consequently the vertical joints are neglected because of the low value of the stresses. Additionally the shrinking process in the mortar reduces the bond between mortar interface and brick. Further due to the low compression state in vertical direction no significant friction forces can be developed in the vertical joints.

Through the combination of normal and shear forces in the direction of the wall, a two-axial loading is induced. A plane stress state develops in the direction of the shear wall. The theory of Mann \& Müller is defined for a stretcher bond with an overlapping of the stretcher of a half length of the bricks and a ratio of width and length of the bricks of 1:2. The shear stresses inside the horizontal joints induce a torque, which is compensated by the equilibrium on every single brick by a pair of forces. Assuming a linear distribution of stresses over the half length of the brick the stress state can be calculated as follows:

$$
\sigma_{x 1,2}=\sigma_{x} \pm \tau \cdot \frac{Q_{x}}{\Delta y} \text { with } Q_{x}=2 \cdot \tau \cdot \Delta x
$$

The fracture depends on the ratio of the different loads and the material parameters and can be distinguished into four different failure modes (Mann \& Müller, 1978): (a) failure of masonry due to compression; (b) rocking (gap in the horizontal joints at the bottom part of the wall); (c) friction failure of the horizontal joints; (d) tension failure of the bricks.

Failure of masonry due to compression (line $a$ in Fig. 6) appears, if the maximal compressive stress $\sigma_{x 1}$ becomes higher than the compressive strength $f_{k}$ of masonry.

$$
\tau=\left(f_{k}-\sigma_{x}\right) \cdot \frac{\Delta y}{2 \Delta x}
$$

Rocking (b) appears, if the minimal compressive stress $\sigma_{\times 2}$ becomes zero. It is assumed that the horizontal joints cannot bear tensile stresses (eq. (8), $b_{1}$ Fig. 6). If a tensile strength is considered $\left(f_{h z}=\right.$ tensile bond strength), the fracture condition can be formulated as stated in eq. (9) (b Fig. 6).

$$
\begin{gathered}
\tau=\sigma_{x} \cdot \frac{\Delta y}{2 \Delta x} \\
\tau=\left(\sigma_{x}+f_{h z}\right) \cdot \frac{\Delta y}{2 \Delta x}
\end{gathered}
$$

Friction failure (line $c$ in Fig. 6) appears, if in the area of the horizontal joints with minimal compressive stress the friction resistance is exceeded. The fracture condition can be defined with Mohr-Coulomb's law.

$$
\tau=f_{v k o}+\mu \cdot \sigma_{x}
$$

The compressive stresses $\sigma_{x 1,2}$ and the shear loads as shown in eq. (6) induce a principal stress state inside the bricks, which results in the fourth failure mode, tensile failure of bricks (d Fig. 6). The principal stress state induces the fracture of the bricks, and therefore the tensile strength of bricks $f_{b t}$ becomes decisive. 


$$
\tau=\frac{f_{b t}}{2.3} \cdot \sqrt{1+\frac{\sigma_{x}}{f_{b t}}}
$$

The discussed failure modes can be pictured as one curve in the $\sigma \tau$-diagram (Fig. 6). The curve encloses the area, in which no fracture and failure occurs. Stress states which are outside of the curve, lead to one of the four failure modes due to the discussed criteria.

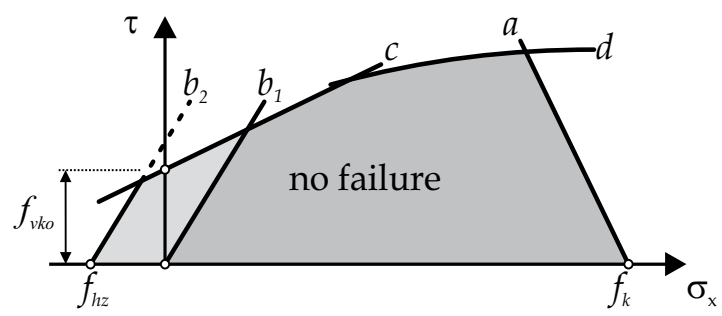

Fig. 6. Curve of the failure mode in the $\sigma \tau$-diagram

\subsection{Experimental testing}

From the 1950ies up to now, extensive research has been done with respect to the correlation of the strengths of used materials and the bearing capacity of structures, whereas most of interest was spent on uniaxial compressive strength. The research led to different calculation models and during the last centuries to a harmonisation of the European codes and standardised design concepts.

For definition of the design parameters of bricks and mortar, testing values are required. Therefore it was a need to standardise the testing methods and applications. On the other hand, efforts were put on resistance against horizontal loads, i.e. shear or dynamic loads and mostly also in combination with bending. As a result of a higher sensitivity in respect to earthquake it was necessary to adapt the codes. This adaption causes quite complex calculation methods and the need of additional material parameters. Various testing methods were discussed for determining the needed material parameters. A simple approach, which has been included into the European codes, is due to basic parameters, i.e. the compressive strengths of bricks and mortar. For defining the horizontal resistance, this approach needs more information, which can be given by the coefficient of friction $\mu$, the initial shear strength $f_{v k o}$ and the tensile strength of the bricks $f_{b t}$. Therefore standardised values are available, which can be taken for the calculation and design or derived from the basic parameters, correlation factors are listed in (Schubert \& Brameshuber, 2011).

For existing structures, it is required to get information about the basic material parameters in a non-destructive way as possible. There are a few non-destructive testing methods available, but they do not offer a direct conclusion on the existing strengths. One possibility is the sampling of wall-like specimens. This gives a deep insight into the strength of the tested component. However, by sampling test specimens of the building the structural integrity may get disturbed and in the extreme case the stability against collapse decreases.

Moreover, there are remarkable costs and therefore in most of the cases the number of test samples is not sufficient enough for a detailed static assessment of the existing structure. Especially in older structures the scatter of material parameters are considerably high and then a large number of samples is required to make serious assessments. 


\subsubsection{Testing methods of static parameters}

In-situ testing methods of mortar and bricks have been enhanced to avoid disturbing existing structures. Additionally, the results were calibrated on wall specimens and on model structures and there are lots of standardised testing methods for determining parameters like brick size, shape, density etc.

- $\quad$ testing of masonry compressive strength by direct methods:

- testing in laboratory of adequate, new constructed masonry specimens according to EN 1052-1 - Determination of compressive strength.

- $\quad$ in-situ testing on structures, e.g. Flat-Jack-Test (Pech \& Zach, 2009).

- sampling of test specimens and testing in laboratory, according to EN 1052-1 Determination of compressive strength.

- $\quad$ sampling of representative test specimens for laboratory testing

- testing of shear strength of masonry:

- $\quad$ adequate testings on masonry specimens, no standardised methods available

- definitions and specifications of storage, size of test specimens, load application, boundary conditions can be found in the Mauerwerk Kalender

- $\quad$ testing of flexural strength of masonry:

- general:

- different test setups in literature, no valid results, many influencing parameters cannot be evaluated yet

- $\quad$ values are not necessarily required for the assessment of existing structures

- test method according to EN 1052-2 - Determination of flexural strength:

- flexural in the plane of the wall-panel

- test setup for determining the flexural strength parallel and perpendicular to the horizontal joints

- test method according to EN 1052-5 - Determination of bond strength by the bondwrench method:

- for torque application e.g. at the top of the wall, pure torque load

- there cannot be estimated a common relationship between the test results of tensile bond strength and shear strength

- testing of tension strength of masonry:

- There is no standardised test method available. In the ESECMaSE-Projekt the "Direct tension Test" was proposed as test method.

- testing of compressive strength of the components (indirect testing of masonry) and calculation of the overall compressive strength of masonry:

- testing of strength of the bricks according to EN 772-1 - Determination of compressive strength. Compressive strength of the bricks is determined perpendicular to the horizontal joints, which is sufficient precise. The possibility of a reduced bearing capacity due to diagonal principal compressive stresses is neglected.

- testing of strength on small sized test specimens

- $\quad$ testing of strength by rebound hammer (Pech \& Zach, 2009).

- testing of mortar strength on a standardised prism, according to EN 1015-11 Determination of flexural and compressive strength of hardened mortar

- $\quad$ testing of mortar strength by stamp compression test (Pech \& Zach, 2009). 
- testing of mortar strength by determination of penetration resistance and the related deformation

- $\quad$ testing of splitting tensile strength of bricks:

- There is no standardised test method available. Testing can be performed analogous to concrete, where load application is done with stripes of a width of $10 \mathrm{~mm}$. The test method can also be applied on drill cores.

- testing of flexural strength of bricks:

- testing method according to RILEM-Recommendations TC 76 - flexural strength of units (de Vekey, 1988)

- $\quad$ testing of centric brick tensile strength:

- There is no standardised test method available.

- testing of coefficient of friction and initial shear strength between brick and mortar:

- method according to EN 1052-3 - Determination of initial shear strength.

- additional physical analysis on bricks:

- $\quad$ EN 772-13 - Determination of net and gross dry density of masonry units.

- EN 772-16 - Determination of dimensions.

For estimating the strength of the bricks and as basic value for the calculative determination of the masonry compressive strength from the values from the components (e.g. according to national part of EC 6) the bricks have to be tested according to EN 772. As an alternative for existing structures, the compressive strength can be determined in a non-destructive way by rebound and penetration methods.

Estimating of compressive strength of the bricks on existing structures has to follow EN 1998-3 for the required minimum number of testing samples. Existing structures are defined as those objects, which were built before the actual standards for masonry were valid, therefore these objects cannot ensure the required quality. The required amount of tests for a sufficient result of an existing structure with homogenous material and a knowledge class KL3 it is necessary to perform one test series per $1000 \mathrm{~m}^{2}$ total floor area or two test serials per structure. For a knowledge class KL2 $50 \%$ of the required tests for KL3 have to be done. The definition of the knowledge classes is standardised in EN 19983 and depends on the geometry, constructional details and the materials. According to EN 1998-3 a test series is defined by the following parameters: (a) at least three test specimens (masonry) or (b) at least three test locations for testing strength of the components by taking specimens of the bricks and mortar for compressive strength tests or (c) at least six test locations for testing strength of the components by rebound and penetration methods.

After determining the test locations, the bricks usually are taken from masonry by means of a masonry saw or they are chiselled out. The test locations have to be documented. In order to minimize the size of the disturbed masonry, instead of the five to six full bricks according to EN 772-1, four to six half bricks can be taken instead. Although the size of the test specimens generally should not be decisive for compressive strength, especially the inhomogeneities in historical bricks can be the reason for high scatter and unsafe test results. For average determination of a test location there have to be taken five values, divergent from EN 772-1. In case of non-destructive testing with the rebound hammer there have to be taken 10 individual test results for each test location for determining the compressive strength. 
In addition to performing the tests, they should be documented. The documentation should include the following parts: (a) object/structure; (b) date of testing; (c) situation of the test locations (identification on building plan); (d) testing method and standardisation to normative values; (e) characteristic masonry strength for each test location, test serial, type of masonry; (f) compressive strengths of bricks and mortar for each test location, test serial, type of masonry if the components were tested individually; (g) type of construction stones according to EN 771-1.

\subsubsection{Testing methods of dynamic parameters}

By investigation of the behaviour of masonry walls under cyclic load, essential information of the load-displacement-curve can be determined. The load-displacement-behaviour depends on the decisive failure mode. By means of pseudo-dynamic experiments, the dynamic behaviour of structures can be determined depending on time.

The experimental research of the cyclic behaviour of masonry walls has been investigated during the last years in lots of research projects, e.g. ESECMaSE and SEISMID. Usually, shear wall tests were performed, in which under constant vertical loads a cyclic horizontal load is applied in a quasi-static way. The defined boundary condition on the top of the wall is either a fixed support or a cantilever arm, which has the possibility of free rotation. This experimental setup allows the determination of special dynamic parameters, e.g. energy dissipation and hysteretic damping. The obtained test data can be used for further relevant parameters for seismic design concepts, like behaviour factor, stiffness and stiffness degradation. This topic is discussed in (Knox \& Ingham, 2011; Tomazevic et al., 1996a; Zimmermann et al., 2010a; Zimmermann et al., 2010b). A further possibility for experimental testing is the performance of shake table tests on a vibrating table. In contrast to the shear walls discussed above, precise acceleration spectra can be taken for loading. The direct analysis of whole structures (walls, slabs, openings and floors) allows a better consideration of the load bearing behaviour. Experimental work is reported e.g. in (Benedetti et al., 1998; Tomazevic et al., 1996b; Tomazevic, 2007).

\section{Seismic loads}

Seismic loads are considered in EC 8. Part 1 specifies the basics, the loads from seismic impacts and structural design concepts in seismic influenced regions. The code specifications cover the design concepts by requirements on geometry, design by verification of the load bearing capacity and considerations for construction details. The other parts of EC 8 include specifications for bridges (2), existing structures (3), silos, tanks and pipelines (4), foundations and retaining walls (5) and towers, pylons and chimneys (6). Each structure has to be designed considering the unfavourable limit states, including and not including seismic loads. Therefore, depending on the used material, the corresponding $\mathrm{EC}$ is the basis for design, considering the specifications of EC 8.

\subsection{Seismic zones}

The exposure to seismic loads is specified by one single parameter, which is the referencetop level ground acceleration $a_{g R}$ for foundation class A. In Austria, the value of this ground acceleration is from $0.18 \mathrm{up}$ to $1.34 \mathrm{~m} / \mathrm{s}^{2}$. This reference-top level ground acceleration has to be multiplied with the coefficient of importance $\gamma_{\mathrm{I}}$ to obtain the ground acceleration $a_{g}$. 


$$
a_{g}=\gamma_{I} \cdot a_{g R}
$$

The reference-top level ground acceleration at a coefficient of importance of $\gamma_{\mathrm{I}}=1.0$ corresponds to a reference exceeding probability of $P_{N C R}=10 \%$ within 50 years or a reference recurrence period of $T_{N C R}=475$ years.

\subsection{Categories of importance}

Failure of a structure, its impacts on human life, public safety and social an economic effects are defined by means of the categories of importance and the related coefficients of importance $\gamma_{\mathrm{I}}$. In case of structural design, structures with a low importance for public safety are category I (e.g. structures with an agricultural using, $\gamma_{I}=0.8$ ), common structures are category II, structures where gatherings have to be considered (e.g. school buildings) are category III and the most important structures are classified in category IV (e.g. hospitals) both categories have a range of $\gamma_{\mathrm{I}}=1.0$ to 1.4 .

\subsection{Foundation classes}

Foundation has an applicable influence on the exposure of a structure to seismic loads. Foundation can be divided in classes $\mathrm{A}-\mathrm{E}, \mathrm{S}_{1}$ and $\mathrm{S}_{2}$, according to EC 8. The classification of the local foundation should be done considering the shear wave velocity $v_{s 30}$, if the value is known; otherwise the classification should be done with the number of blows of the Standard-Penetration-Test, $N_{S P T}$. Additional investigations to the required static analysis are necessary, if the local foundation is classified to $S_{1}$ or $S_{2}$, or if the structure has the category of importance III or IV.

\subsection{Response spectrum}

The dynamic impact of an earthquake on a structure is generally characterised by a horizontal response spectrum. Thereby on the abscissa it is plotted the natural oscillation time $T$ and on the ordinate the maximal amplitude of the response acceleration of a planar single degree of freedom, which has a constant natural oscillation time over the duration of the seismic impacts. The response spectrum is timely independent and depicts the smoothing and enclosing distribution of many earthquakes.

\subsubsection{Horizontal-elastic response spectrum}

The horizontal seismic impact can be described by means of two orthogonal components, which are independent from each other and can be characterised by the same response spectrum. The horizontal component of the elastic response spectrum $S_{a}(T)$ is defined at $5 \%$ viscous damping by four groups. In general there are two modes of spectra, type 1 and type 2 . Type 1 is assumed for larger magnitudes of surface waves, $M_{S}>5.5$ and type 2 for smaller magnitudes, $M_{S} \leq 5.5$. The parameters of the elastic response spectrum are listed in EC 8. In Fig. 7 the characteristics of the response spectrum are depicted for the different foundation classes.

The elastic acceleration spectrum $S_{a}(T)$, depending on the settling time $T$, can directly be transformed into an elastic displacement response spectrum $S_{D e}(T)$ by following condition:

$$
S_{D e}(T)=S_{a}(T) \cdot\left[\frac{T}{2 \pi}\right]^{2}
$$




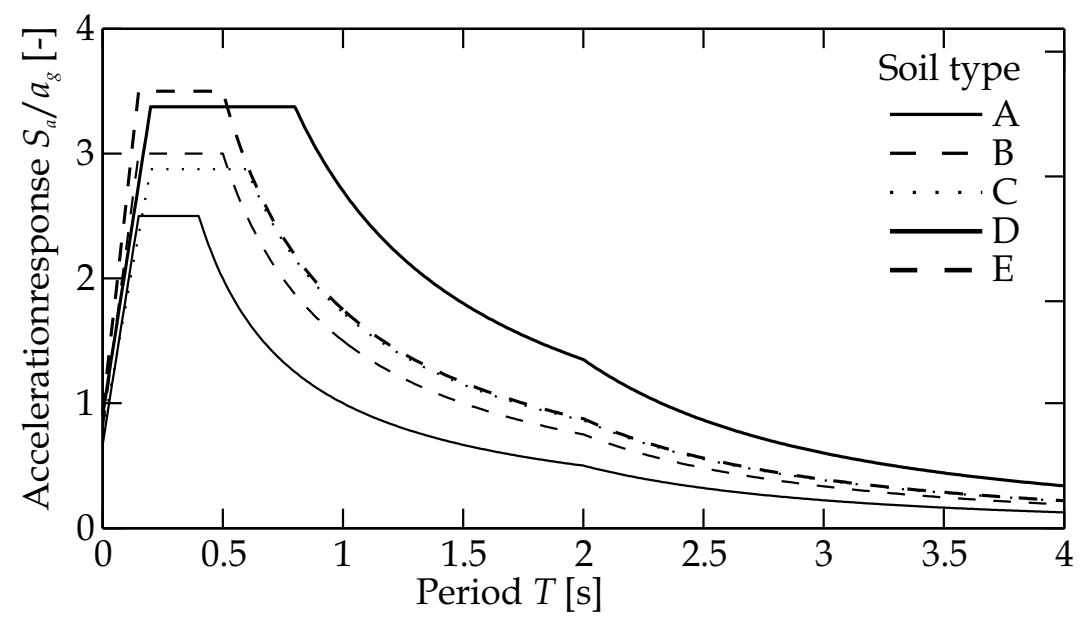

Fig. 7. Elastic response spectra of type 1 for foundation classes A - E with $5 \%$ viscous damping

The correlation of spectral acceleration and displacement for a single degree of freedom with a predefined natural period is described by the ADR-spectrum (Acceleration Displacement Response), whereby the abscissa shows the time of displacement response $S_{D e}(T)$ and the ordinate the acceleration response $S_{a}(T)$. For periods exceeding $4.0 \mathrm{~s}$, the elastic acceleration response spectrum of type 1 can be obtained from the elastic displacement response spectrum by inverting eq. (13), according to EC 8, Annex A.

\subsubsection{Vertical-elastic response spectrum}

The vertical component of seismic impact can be neglected in Austria and is therefore listed only for ensuring completeness. It can be described by the vertical elastic response spectrum $S_{v e}(T)$. Just as the horizontal spectrum, the vertical spectrum can be divided in four groups, whereby the maximal values are considerably smaller. In general the dominant impact is the horizontal response spectrum.

\subsubsection{Design value of ground displacement}

The design value of ground displacement $d_{g}$ can be estimated in accordance with the design value of the ground acceleration as follows:

$$
d_{g}=0.025 \cdot a_{g} \cdot S \cdot T_{C} \cdot T_{D}
$$

\subsubsection{Design spectrum}

Observations of earthquake impacts have shown that structures can reduce seismic impacts by nonlinear reactions. To use the advantages of a linear calculation, the energy dissipation is considered by a reduced response spectrum (= design spectrum). The ratio of the estimated maximal exposure to the real appearing lower exposure is defined by the behaviour factor $q$. This coefficient is an approximation of the ratio of the seismic forces which would act on the structure if the response at $5 \%$ viscous damping would be completely elastic and of those forces which can be used for a barely satisfactory linear design. Table 4 gives an overview of the behaviour factor $q$. 


\begin{tabular}{lcc}
\hline Material & Range & Relevant code \\
\hline concrete & $1.50-5.85$ & EC 8 Table 5.1 \\
\hline steel & $\leq 1.50-6.50$ & EC 8 Table 6.1 resp. Table 6.2 \\
\hline bond between concrete and steel & $\leq 1.50-6.50$ & EC 8 Table 6.2 resp. Table 7.2 \\
\hline wood & $1.50-5.00$ & EC 8 Table 8.1 \\
\hline masonry & $1.50-3.00$ & EC 8 Table 9.1 \\
\hline
\end{tabular}

Table 4. Behaviour factor $q$ according to EC 8

The four groups of the horizontal design spectrum can be defined as follows:

$$
\begin{aligned}
& 0 \leq T \leq T_{B}: S_{d}(T)=a_{g} \cdot S \cdot\left[\frac{2}{3}+\frac{T}{T_{B}} \cdot\left(\frac{2.5}{q}-\frac{2}{3}\right)\right] \\
& T_{B} \leq T \leq T_{C}: S_{d}(T)=a_{g} \cdot S \cdot \frac{2.5}{q} \\
& T_{C} \leq T \leq T_{D}: S_{d}(T)\left\{\begin{array}{l}
=a_{g} \cdot S \cdot \frac{2.5}{q} \cdot\left[\frac{T_{C}}{T}\right] \\
\geq \beta \cdot a_{g} \text { with } \beta=0.2
\end{array}\right. \\
& T_{D} \leq T \leq 4 s: S_{d}(T)\left\{\begin{array}{l}
=a_{g} \cdot S \cdot \frac{2.5}{q} \cdot\left[\frac{T_{C} T_{D}}{T^{2}}\right] \\
\geq \beta \cdot a_{g} \text { with } \beta=0.2
\end{array}\right.
\end{aligned}
$$

in which $S_{d}(T)=$ elastic design response spectrum $a_{g}=$ design ground acceleration according eq. (12), $T=$ settling time, $S=$ soil parameter, $T_{B}, T_{C}, T_{D}=$ period, $q=$ behaviour factor and $\beta$ $=$ lower bound of spectrum, recommended value is 0.2 .

If in eq. (15) the vertical component $a_{v g}$ is used instead of the design ground acceleration $a_{g}$ and $S=1$, all four groups of the vertical design spectrum can be defined. The coefficient of behaviour should not exceed 1.5 for masonry.

\subsubsection{Alternative Interpretation of seismic impacts}

Alternatively, seismic impacts can be considered by means of natural or simulated time dependent distributions of acceleration. In spatial models of a structure there have to be considered three simultaneous time dependent distributions of acceleration, whereby the same distribution may not be used for both horizontal directions. Simulated distributions of acceleration have to be generated in a way, that the response spectra describe the elastic response spectra of Sec. 3.4.1 and Sec. 3.4.2 for $5 \%$ viscous damping. The duration of the distributions of acceleration has to be consistent to the characteristics of the earthquake, which refers to $a_{g}$ and to the earthquake's magnitude. If there is no further information available, the minimum duration of the stationary part of the distribution of acceleration should be considered with $10 \mathrm{~s}$. Moreover it should be used a minimum of three distributions of acceleration, whose average for the zero period yields at least to a value of $a_{g} \cdot S$ for the considered location. No ordinal value in the range of $0.2 T_{1}$ up to $2.0 T_{1}$ shall be smaller than $90 \%$ of the corresponding value of the design spectrum, whereby $T_{1}$ is the natural period of the structure in the related direction. 


\subsection{Seismic design}

Seismic loads are inertial loadings, which act on the mass points and they are the result of multiplying mass with acceleration. Hence the loading is not applied external on the structure, but is produced by ground movements and deformations in the structure itself. Therefore seismic loading depends on both the place of location and on the structure itself. In earthquakes prone areas, the seismic aspects have to be considered already in the conceptual state of designing structures, therewith both the requirements on structural safety and minimizing failure effects can be fulfilled with bearable costs, compare with EC 8 Part 2.1, (Bachmann, 2002a, 2002b).

\subsubsection{General principles}

Generally, structures have to be designed from the constructive aspect as easy as possible for ensuring a definite and direct load transfer, avoiding uncertainties in modelling and increasing the safety of the structure. In buildings, the floor slabs have a decisive significance. In the plane of the slab the inertial forces are bounded and transferred to the vertical members. Therefore the slabs should have a sufficient stiffness in their plane and the behaviour of horizontal panels. Particularly in case of mixed structures, large openings and changes of the stiffness, the behaviour of the panel and the interaction between horizontal and vertical structural members should be ensured.

For avoiding non-uniform torsional loading on bearing structural members it has to be ensured that stiffness is distributed preferably constant around the structure and a sufficient torsional stiffness is warranted. In addition to constructive aspects, an adequate foundation and connective elements to the superstructure are required for ensuring a homogenous seismic loading of the structure and the load transfer to the ground. Structures with loadbearing walls with diverging values of length and stiffness should have a box-shaped or cellular foundation; separate parts of the foundation should be connected with a ground slab or a flexible foundation beam.

\subsubsection{Criteria of regularity}

For purposes of seismic design of construction it has to be distinguished between regular and non-regular structures. This differentiation influences the calculation model, the calculation method and the behaviour factor, compare Table 5.

\begin{tabular}{ccccc}
\hline \multicolumn{2}{c}{ Regularity } & \multicolumn{2}{c}{ Acceptable simplifications } & Behaviour factor \\
\hline ground plan & vertical section & model & $\begin{array}{c}\text { linear-elastic } \\
\text { calculation }\end{array}$ & \\
\hline Yes & Yes & plane & simplified & reference value \\
\hline Yes & No & plane & modal & reduced value \\
\hline No & Yes & spatial & simplified & reference value \\
\hline No & No & spatial & modal & reduced value \\
\hline
\end{tabular}

* according EC 8 Part 4.3.3.1(8) under special circumstances an planar model can be used in each of the both directions

Table 5. Seismic design according to constructive regularity

A structure has to fulfil the following requirements that it can be classified as regularly in respect to the ground plan: The distribution of the horizontal stiffness and mass in 
accordance to two perpendicular axes should be symmetric. The shape of the ground plan has to be compact and should be enhanced by a polygon line, offsets and niches must not contain more than $5 \%$ of the floor area. Comparing to the stiffness of the horizontal members, the slabs have to assure a sufficient stiffness in their plane to ensure the load transfer. Slenderness ratio $\lambda$ has to fulfil $L_{\max } / L_{\min } \leq 4$, in which $L_{\max }$ and $L_{\min }$ are the maximum and minimum perpendicular dimensions of the structure.

For each floor and in each direction of calculation the effective excentricity has to be in $x$ direction $e_{0 x} \leq 0.30 r_{x}$ and $r_{x} \geq l_{s}$ and in $y$-direction $e_{0 y} \leq 0.30 r_{y}$ and $r_{y} \geq l_{s}$ respectively, in which $e_{0}$ is the distance between centre of stiffness and centre of mass, $r$ is the radius of torsion and $l_{s}$ the radius of inertia of the floor mass. In case of one floor the radius of torsion is defined as the square root from the torsion stiffness in reference to the horizontal stiffness. The radius of inertia is defined as the square root of the polar moment of inertia in reference to the centre of mass. In case of several floors, the centre of stiffness and the radius of torsion can be determined just approximately. Simplifying it can be regarded as regular, if load-bearing structural members range from the foundation up to the top edge of the structure and if the bending lines of the stiffening systems under horizontal loading are not different. An approximate approach for the calculation is defined in the national part of EC 8, Annex B.

That a structure can be determined as regularly in respect to the vertical section, it has to conform to the requirements below. All horizontally operating stiffening systems have to range from the foundation up to the top edge of the structure, respectively up to the adequate height of structural members. Horizontal stiffness and the mass of the respective floors have to be constant over their height or decrease steadily from the bottom to the top. In case of frame structures, the ratio of the real strength of proximate floors to the required strength from the calculation should not diverge too much. If there are offsets, the conditions from Fig. 8 must be considered.

The offsets have to be designed symmetrically and may not exceed more than $20 \%$ of the previous dimensions. In case of a single offset within the lower $15 \%$ of the total height, the offset may not exceed $50 \%$ of the ground plan dimension. Then the continuous part below should be able to bear at least $75 \%$ of the total horizontal shear load. In the event of asymmetric offsets, in each vertical section the sum of offsets may not exceed $30 \%$ of the dimensions of the ground plan and each offset may not be larger than $10 \%$ of the previous dimension, see Fig. 8 . In general, the requirements of EC 8 with regard to regularity in ground plan and vertical section can be summarized, that a compact construction type with symmetric distributed mass and stiffness has a positive influence on the seismic loadbearing capacity, compare Fig. 9.

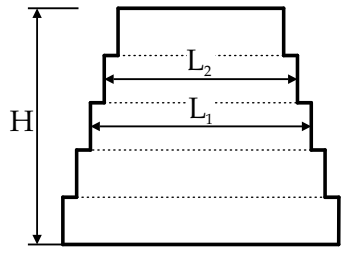

$\left(L_{1}-L_{2}\right) / L_{1} \leqslant 0.20$

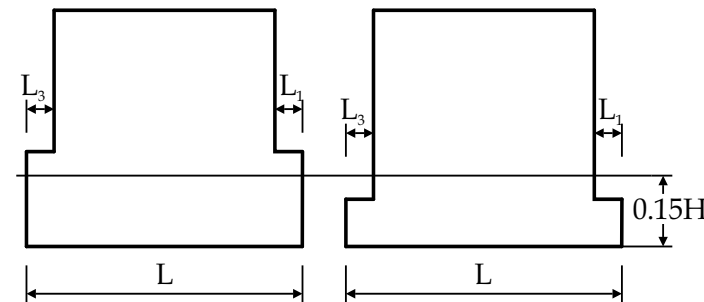

$\left(L_{3}+L_{1}\right) / L \leqslant 0.50$

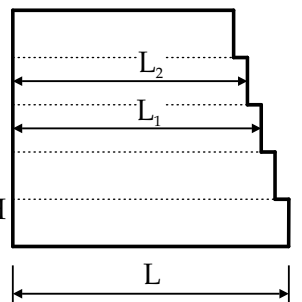

$\left(L-L_{2}\right) / L \leqslant 0.30$

$\left(L_{1}-L_{2}\right) / L_{1} \leqslant 0.10$

Fig. 8. Criteria of regularity of structures in reference to the vertical section, according to EC 8 


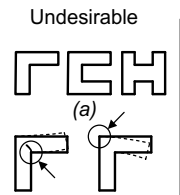

(b)
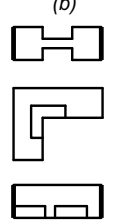

(d)

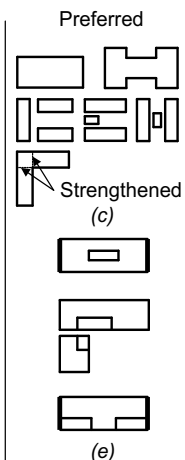

(e)
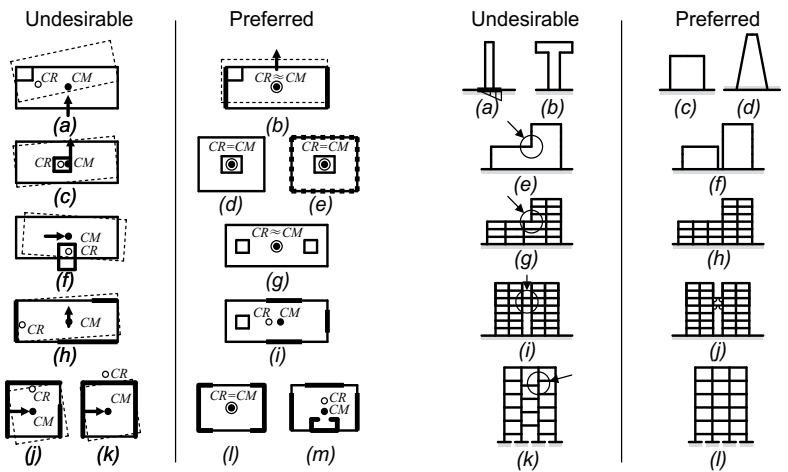

$\mathrm{CM}=$ centre of mass, $\mathrm{CR}=$ centre of stiffness

(a) horizontal setup

(b) distribution of mass and horizontal stiffness,

(c) vertical setup

Fig. 9. Comparison of disadvantageous and favourable structural characteristics, from (Paulay \& Priestley, 1996)

\subsubsection{Coefficients of combination}

In the seismic load case, variable loads have to be considered in a reduced way. Their value is defined by the factors of combination $\psi_{\mathrm{E}, \mathrm{l}}=\varphi \cdot \psi_{2,1}$. Thereby $\psi_{2, \mathrm{i}}$ is the value, which takes account of the quasi constant part, as recommended in Annex A1, EC 0. The factor $\varphi$ defines the probability of a simultaneous occurrence of the variable loads in each floor. In Austria it is recommended to use $\varphi=1.0$, or the values from Table 6 .

The masses, which are required for calculating the inertial forces, which have to be applied in case of seismic loads, result from the dead loads from the permanent loads and the variable loads, which are reduced by the factor $\psi_{\mathrm{E}, \mathrm{i}}$.

$$
\sum G_{k, i}+\sum \psi_{E, i} \cdot Q_{k, i}
$$

\begin{tabular}{lll}
\hline \multicolumn{1}{c}{ Type of loading } & \multicolumn{1}{c}{ Floor } & $\varphi$ \\
\hline \multirow{2}{*}{$\begin{array}{l}\text { Category A - C } \\
\text { according EC 1 }\end{array}$} & housetop & 1.0 \\
\cline { 2 - 3 } & floors with an related usage & 0.8 \\
\cline { 2 - 3 } & independent usage of floors & 0.5 \\
\hline $\begin{array}{l}\text { Category D - F } \\
\begin{array}{l}\text { according EC 1 und } \\
\text { archives }\end{array}\end{array}$ & - & 1.0 \\
\hline
\end{tabular}

Table 6. Values for the calculation of $\psi_{\mathrm{E}, \mathrm{i}}$

\subsubsection{Computation of structures}

Modelling has to ensure, that the distribution of masses and stiffness is described correctly, in case of nonlinear analysis the distribution of strength, too. The structural model should also take account of the connecting zones to the deformation of the structure, e.g. ending zones of beams or columns. In case of structures made of concrete, reinforced concrete, 
composite constructions or masonry, the stiffness of the load-bearing structural members should be determined by considering the formation of cracks. If no calculative analysis of the cracked members is available, approximately the half of the stiffness of the uncracked members can be assumed.

Bracing elements in masonry, which have an essential impact on the horizontal stiffness, should be considered. The ductility of the foundation has to be regarded, if it influences the model in a negative way and can optionally be regarded if there is a positive impact. The definitions of EC 8 recommend that the stiffness and the masses of the structure should be summarized to a substitute beam. Then the seismic loads are calculated for each floor and then redivided to the load-bearing structural members. This procedure presumes that the load distribution takes place by floor slabs with an adequate stiffness.

Alternatively, the seismic loading can also be calculated directly at a spatial model, if the recommendations from EC 8 are used analogously. This model even allows the calculation of structures, which do not have a sufficient panel effect of the floor slabs. In Table 7 the calculation methods for the load case seismic loads are summarized.

\begin{tabular}{|c|c|c|c|c|}
\hline & Force method & $\begin{array}{l}\text { Response } \\
\text { spectrum }\end{array}$ & Pushover & Timehistory \\
\hline Method & static & static & static & dynamic \\
\hline \multirow{2}{*}{ Model } & linear & linear & nonlinear & nonlinear \\
\hline & plane & plane, spatial & plane, spatial & plane, spatial \\
\hline Torsion & $\begin{array}{l}\text { simplified } \\
\text { approach }\end{array}$ & $\begin{array}{c}\text { plane: simplified } \\
\text { approach, spatial: in } \\
\text { model }\end{array}$ & $\begin{array}{c}\text { plane: simplified } \\
\text { approach, spatial: in } \\
\text { model }\end{array}$ & $\begin{array}{c}\text { plane: simplified } \\
\text { approach, } \\
\text { spatial: in model }\end{array}$ \\
\hline $\begin{array}{l}\text { Considering } \\
\text { nonlinearity }\end{array}$ & $\begin{array}{c}\text { global by coefficient } \\
\text { of behaviour }\end{array}$ & $\begin{array}{c}\text { global by coefficient } \\
\text { of behaviour }\end{array}$ & in model & in model \\
\hline Load & response spectrum & response spectrum & response spectrum & time response \\
\hline Calculation & $\begin{array}{l}\text { analysis with static } \\
\text { resultant forces }\end{array}$ & $\begin{array}{c}\text { modal analysis with } \\
\text { quadratic } \\
\text { superposition of } \\
\text { state variables }\end{array}$ & $\begin{array}{c}\text { Pushover- } \\
\text { calculation, } \\
\text { continuous } \\
\text { increasing external } \\
\text { forces }\end{array}$ & $\begin{array}{l}\text { at least three } \\
\text { time response } \\
\text { calculations with } \\
\text { static analysis }\end{array}$ \\
\hline Uncertainties & $\begin{array}{c}\text { modelling, dynamic } \\
\text { of the structure, } \\
\text { material behaviour }\end{array}$ & $\begin{array}{c}\text { modelling, dynamic } \\
\text { of the structure, } \\
\text { material behaviour }\end{array}$ & $\begin{array}{c}\text { modelling, dynamic } \\
\text { of the structure }\end{array}$ & modelling \\
\hline Regularity & very high & $\begin{array}{l}\text { plane: high } \\
\text { spatial: none }\end{array}$ & $\begin{array}{l}\text { plane: high } \\
\text { spatial: none }\end{array}$ & $\begin{array}{l}\text { plane: high } \\
\text { spatial: none }\end{array}$ \\
\hline Traceability & very easy & easy & easy & difficult \\
\hline $\begin{array}{l}\text { Utilisation of } \\
\text { bearing } \\
\text { reserves }\end{array}$ & low & low & good & very good \\
\hline $\begin{array}{l}\text { Computational } \\
\text { effort }\end{array}$ & low & middle & high & very high \\
\hline
\end{tabular}

Table 7. Overview about the calculation methods for seismic loads (Zimmermann \& Strauss, 2010b) 


\subsubsection{Resultant force method}

The resultant force method or the simplified response spectrum method can be applied, if the structure complies with the requirements of regularity in vertical section from Sec. 3.5.2 and if the natural period $T_{1}$ in each main direction is lower than $4 T_{C}$ and $2 \mathrm{~s}$ respectively. Then it can be assumed, that the higher mode shapes have no influence on the total seismic load and that they can be neglected. The total seismic force $F_{b}$ for each main direction is calculated by:

$$
F_{b}=S_{d}\left(T_{1}\right) \cdot m \cdot \lambda
$$

in which $S_{d}\left(T_{1}\right)=$ ordinate of the design spectrum, $m=$ total mass of the structure and $\lambda=$ correction coefficient for the participation of the mass. For more than two floors and $T_{1} \leq 2 T_{C}$ it is recommended $\lambda=0.85$, otherwise $\lambda=1.0$. The natural period $T_{1}$ can be determined by an approximation procedure, e.g. the energy method (Flesch, 1993). For buildings up to a height of $40 \mathrm{~m}$, the following approach can be used:

$$
T_{1}=C_{t} \cdot H^{3 / 4}
$$

in which $C_{t}=0.085$ for flexural resistant steel frameworks, $C_{t}=0.075$ for frameworks made of reinforced concrete and $C_{t}=0.050$ for all other structures; $H=$ height of the structure.

Alternatively, in case of structures with shear walls made of concrete or masonry, the value for $C_{t}$ can be defined as follows:

$$
C_{t}=0.075 /\left(\sqrt{\sum\left[A_{i} \cdot\left(0.2+\left(l_{w, i} / H\right)^{2}\right)\right]}\right)
$$

in which $A_{i}=$ effective cross section of the shear wall $i$ and $l_{w, i}=$ length of the shear wall $i$, under the condition of $l_{w, i} / H \leq 0.9$.

The distribution of the horizontal seismic loads is based on the mode shapes or can be assumed as a triangular distributed load over the height, if the horizontal displacement of the eigenmode is approximated to be linear over the height.

$$
F_{i}=F_{b} \cdot \frac{s_{i} \cdot m_{i}}{\sum s_{j} \cdot m_{j}} \quad F_{i}=F_{b} \cdot \frac{z_{i} \cdot m_{i}}{\sum z_{j} \cdot m_{j}}
$$

Thereby $F_{i}=$ the applying horizontal load on floor $i, s_{i}, s_{j}=$ displacement of the masses $m_{i}, m_{j}$ and $z_{i}, z_{j}=$ height of the masses. If the horizontal seismic loads are calculated as loads of the floors, the assumption of load transfer by rigid floor panels has to be fulfilled. In case of the separate structural members, the additional loading resulting from accidentally torsion load has to be considered with a coefficient $\delta=1+0.6\left(x / L_{e}\right)$, which is a multiplying factor for the seismic load. Thereby $x=$ distance from the structural member from centre of mass and $L_{e}=$ distance between the outside structural members perpendicular to the considered direction of seismic impact.

\subsubsection{Multimodal response spectrum}

If the criteria of regularity in respect of vertical section are not fulfilled and other modal shapes than the natural eigenmode are decisive, the multimodal response spectrum method has to be applied instead of the simplified response spectrum method, see Sec. 3.5.2. This 
method can be used for each type of structure. In this dynamic calculation method the whole structure is divided into individual single degree of freedoms and the reaction under applied dynamic load is identified for each single degree of freedom $i$ with a natural period $T_{i}$. This reaction can be determined from the design spectrum, the shear force $F_{b, i}$ is then:

$$
F_{b, i}=S_{d}\left(T_{i}\right) \cdot m_{i, e f f}
$$

in which $S_{d}\left(T_{i}\right)=$ ordinate of the design spectrum for the natural period $T_{i}$ and $m_{i, e f f}=$ effective modal mass of the $i$-th eigenmode.

All decisive mode shapes have to be considered, which have a significant impact on the structural response. An eigenmode is decisive, if the sum of the effective modal mass is at least $90 \%$ of the total mass. In addition, no eigenmode may be neglected, which modal mass has more than $5 \%$ of the total mass. If these requirements cannot be fulfilled, the number $k$ of the modal inputs which have to be taken into account should be at least $k \geq 3 n^{0.5}$; thereby $n=$ number of floors.

The period of the last eigenmode $T_{k}$ which should be considered may not exceed $0.2 \mathrm{~s}$. For each eigenmode $i$ the maximal loading values can be determined. As a result, the singular modal parts can be added to the reaction of the whole structure. If all decisive mode shapes can be assumed as independent from each other, the maximal value of the seismic loading follows with the SRSS-formula (Square Root of Sum of Squares):

$$
E_{E}=\sqrt{\sum E_{E, i}}
$$

in which $E_{E}=$ seismic load value and $E_{E, i}=$ seismic load value of the $i$-th eigenmode. Mode shapes are oscillating independently from each other, if the difference between the singular eigenfrequencies is large enough. This is fulfilled, if for two consecutive periods $i$ and $j T_{j} \leq$ $0.9 T_{i}$. If this condition is not reached, other methods for combination have to be applied, e.g. the CQC-method (Complete Quadratic Combination). Thereby the load value is:

$$
E_{E}=\sqrt{\sum \sum E_{E, i} \cdot \rho_{i j} \cdot E_{E, j}}
$$

in which $\rho_{\mathrm{ij}}=$ factor of interaction. The factor of interaction takes account of the modal damping in reference of the mode shapes $i$ and $j$ and the ratio of the circular eigenfrequencies $\omega_{\mathrm{i}}$ and $\omega_{\mathrm{j}}$. This method is documented e.g. in (Clough \& Penzien, 1995; Flesch, 1993). Torsion loadings at spatial models can be incorporated by additional torsional moments $M_{a, i}$ around the vertical axis of each floor $i$ :

$$
M_{a, i}=e_{a, i} \cdot F_{i} \quad \text { with } e_{a, i}= \pm 0.05 \cdot L_{i}
$$

in which $F_{i}=$ horizontal force in floor $i, e_{a, i}=$ accidental excentricity of floor mass $i$ and $L_{i}=$ floor dimension perpendicular to the direction of seismic load.

\subsubsection{Nonlinear static method (Pushover)}

Alternatively to response spectrum methods, nonlinear methods, e.g. the nonlinear static pushover method can be applied (Chopra \& Goel, 1995). The characterisation of the material behaviour has to be done with a bi-linear force-deformation-relation. For concrete and masonry, the linear-elastic stiffness of the bi-linear relationship should coincide with that from 
cracked cross sections. For ductile members the secant stiffness to the yield point should be taken for the bi-linear relation. After the yield point, the tangential stiffness can be appropriated. In case of brittle materials, the tangential stiffness of the force-deformationrelation should be considered. If there are no further specifications, the material characteristics should be based on average values. For new structures, the material parameters can be taken from the codes EC 2 - EC 6 or from other appropriate European standards.

At nonlinear static calculation, the horizontal loads are increased monotonous under constant dead loads and the gained load-deformation-curves of the singular load-bearing structural members are superposed. As a result the capacity curve of the structure is achieved. This calculation method can be used for both determining the bearing capacity of existing and of new structures (Chopra, 2002; Clough \& Penzien, 1995). Instead of the behaviour factor $q$, which incorporates the energy dissipation and the nonlinear effects in a global way, the real nonlinear material behaviour is considered. Depending on the criteria of regularity, in the calculation either two plane models for each of the both horizontal main directions are set up or a spatial model is used. In case of low masonry structures (s 3 floors), whose load bearing walls are loaded mainly by shear loads, each floor can be considered singularly. For distributing the horizontal loads two approaches should be applied, on the one hand a modal, and on the other hand a mass-proportional distribution of the horizontal loads, referring to eq (20).

The horizontal loads have to be applied in in the centres of mass, whereby accidental excentricities according eq (24) have to be considered. From the nonlinear static calculation, the curve of capacity of the structure has to be defined in a range of $0-150 \%$ of the aimed displacement, whereby the control displacement of the capacity curve can be assumed in the centre of mass of the top of the structure. The aimed displacement is determined by the displacement of an equivalent single degree of freedom. The method for determining the aimed displacement is regulated in EC 8, Annex B.

\subsubsection{Nonlinear dynamic calculation (Timehistory)}

Seismic loads can also be determined by means of simulated or measured time responses of the ground acceleration. Solving eq. (25) yields to the variation in time of the responded oscillations of the system in the considered degrees of freedom (Chopra, 2002; Clough \& Penzien, 1995).

$$
[M]\{\ddot{x}\}+[C]\{\dot{x}\}+[K]\{x\}=\{f(t)\}
$$

Thereby $\ddot{x}=$ acceleration, $\dot{x}=$ velocity and $x=$ displacement vector, $f(t)=$ load vector, $\mathbf{M}=$ mass matrix, $\mathbf{C}=$ damping matrix and $\mathbf{K}=$ stiffness matrix. The time responses of all decisive parameters have to be quantified separately, because the maximum values of the displacement parameters $x_{j}(t)$ do not appear at the same time. The structural response oscillation depends on the characteristics of the applied variation of time and therefore at least three time responses have to be considered. For solving the differential equation system in eq. (25), (a) the modal method for linear systems, or (b) the direct integration method for linear and nonlinear systems can be used.

By means of the modal method the linked differential equation system is decoupled by transformation of the variables. As a result for linear elastic systems the displacements can be described as a linear combination of the mode shapes. Solving the decoupled differential equation yields to the time response of the $i$-th modal response oscillation. An advantage in 
contrast to the response spectrum method is that the maximal response can be determined more accurately and in terms of the direct integration the calculation effort decreases. The main disadvantage is that only linear material behaviour can be incorporated.

The method of the direct integration solves eq. (25) directly with the aid of a numeric integration. As a result of this, variable constitutive equations and damping mechanisms (apart from Rayleigh-damping) can be regarded. The main disadvantage of this method is the huge calculation effort, and the need of adequate numerical models for the description of the nonlinear material behaviour under cyclic loading.

The scope of the two described time-history methods is primarily in the assessment of existing structures. Further information is given in (Bachmann, 2002b; Chopra, 2002; Clough \& Penzien, 1995; Flesch, 1993).

\section{Damage quantification}

The quantification of damage is an important task for evaluating the condition of the structure and the degradation over time caused by loads and/or environmental impacts. Damage indexes can be used for structural assessment and further such indexes can be used for decision making of repair and of demolition respectively. Additionally different cost factors can be considered for the decision process and life cycle assessment of structures (Frangopol et al., 2009; Strauss et al. 2010; Strauss et al., 2008).

Damage indexes are mathematical models for a quantitative assessment and they are of substantial importance to estimate critical condition states of structures. The calculation of damage indexes and different studies can be found in the literature, e.g. (Fajfar, 1992; Cosenza et al., 1993; Moustafa, 2011).

Damage indexes can also be correlated with experimental test results. If a structure is subjected to repeated pseudo-dynamic load reversals the increasing degree of damage can be described by damage indexes (Tomazevic, 1998).

\section{References}

Bachmann, H. (2002a). Erdbebengerechter Entwurf von Hochbauten - Grundsätze für Ingenieure, Architekten, Bauherren und Behörden, Richtlinien des BWG, Bern.

Bachmann, H. (2002b). Erdbebensicherung von Bauwerken. Birkhäuserverlag, Basel-BostonBerlin, ISBN: 3-7643-6941-8

Bakes, H.-P. (1983). Zugfestigkeit von Mauerwerk und Verformungsverhalten unter Zugbeanspruchung, TU Aachen

Bargmann, H. (1993). Historische Bautabellen, Werner Verlag

Benedetti, D.; Carydis, P. \& Pezzoli, P. (1998). Shaking table test on 24 simple masonry buildings, Earthquake Engineering and Structural Dynamics, Vol. 27, pp. 67-90

Conrad, D. (1990). Kirchenbau im Mittelalter - Bauplanung und Bauausführung. 1. Auflage Leipzig

Cosenza, C.; Manfredi, G. \& Ramasco, R. (1993). The use of damage functionals in earthquake engineering: comparison between different methods, Earthquake Engineering \& Structural Dynamics, Vol. 22, pp. 855-868

Chopra, A. (2002). Dynamics of structures: Theory and Application to Earthquake, Prentic-Hall Inc. A Simon \& Schuster Company, Englewood Cliffs, NJ, USA, 3rd Edition. 
Chopra, A. \& Goel, R.K. (1995). Capacity-Demand-Diagram Methods for Estimating Seismic Deformations of Inelastic Structures: SDF Systems, Technical Report, Pacific Earthquake Engineering Research Centre, UCA Berkeley, CA, USA.

Clough, R.W. \& Penzien, J. (1995). Dynamics of structures, Computer \& Structures Inc. Berkeley, CA, USA, $3^{\text {rd }}$ Edition.

de Vekey, R. (1988). General recommendations for methods of testing load-bearing unit masonry, Materials and Structures, Vol. 21, No. 3, pp. 229-231

Eberhard, M.O.; Baldridge, S.; Marshall, J.; Mooney, W. \& Rix, G.J. (2010). The MW 7.0 Haiti earthquake of January 12, 2010, USGS/EERI Advance Reconnaissance Team report: U.S. Geological Survey Open-File Report 2010-1048, available online: http://pubs.usgs.gov/of/2010/1048/

Egermann, R. \& Mayer, K. (1987). Die Entwicklung der Ziegelherstellung und ihr Einfluss auf die mechanischen Eigenschaften von Mauerziegeln, In: Erhalten historischer Bauwerke, Sonderforschungsauftrag 315, Universität Karlsruhe, Ernst \& Sohn, Berlin 1987, pp. $107-130$

ESECMaSE - Forschungsprojekt: Enhanced Safety and Efficient Construction of Masonry Structures in Europe, $h t t p: / / w w w . e s e c m a s e . o r g$

Fajfar, P. (1992). Equivalent ductility factors, taking into account low cyclic fatigue, Earthquake Engineering \& Structural Dynamics, Vol. 21, pp. 837-848

Flesch, R. (1993). Baudynamik: praxisgerecht, Bauverlag, Wiesbaden - Berlin, ISBN: 3-76253010-6

Frangopol, D.M.; Strauss, A. \& Bergmeister, K. (2009). Lifetime cost optimization of structures by a combined condition-reliability approach, Engineering Structures, Vol. 31, No. 7, pp. $1572-1580$

Furtmüller, T. \& Adam, C. (2009). Numerische Simulation des seismischen Verhaltens von Mauerwerk in Gründerzeithäusern, SEISMID: Report No. 06/2319-23, TU Universität Innsbruck

Glitzka, H. (1988). Druckbeanspruchung parallel zur Lagerfuge, In: Mauerwerk Kalender 1988, Ernst \& Sohn

Grimm, H. D. (1989). Historische Mörtel - Auswertung von Untersuchungsergebnissen hinsichtlich historischer und geographischer Herkunft, Technische Universität Braunschweig.

Hilsdorf, H. K. (1965). Untersuchungen über die Grundlagen der Mauerwerksfestigkeit, Materialprüfamt für das Bauwesen der TU München, Report No. 40

Ingham, J. \& Griffith, M. (2011). Performance of unreinforced masonry buildings during the 2010 darfield (Christchurch, NZ) earthquake, Australian Journal of Structural Engineering, Vol. 11, No. 3, pp. 207-224

Ingham, J.; Biggs, D.T. \& Moon, L.M. (2011). How did unreinforced masonry buildings perform in the February 2011 Christchurch earthquake?, Structural Engineer, Vol. 89, No. 6, pp. 14-18.

Knox, M. \& Ingham, J. (2011). Experimental Testing to Determine Failure Patterns in URM Pier/Spandrel Sub-Structures, In: Proceedings of 9th Australasian Masonry Conference, Queenstown, New Zealand, February 2011

Mann, W. \& Müller, H. (1978). Mauerwerk Kalender 1978, Ernst \& Sohn

Maier, J. (2002). Handbuch historisches Mauerwerk, Birkhäuser 
Moustafa, A. (2011). Damage-Based Design Earthquake Loads for Single-Degree-Of-Freedom Inelastic Structures, Journal of Structural Engineering, Vol. 137, No. 3, pp. 456-467

Müller, F. P. \& Keintzel, E.: (1984). Erdbebensicherung von Hochbauten, Ernst \& Sohn, Berlin.

Paulay, T. \& Priestley, M.J.N. (1996). Seismic Design of Reinforced Concrete and Masonry Buildings, John Wiley \& Sons Inc.

Pech, A. (2010). Forschungsprogramm zur Verifizierung der konstruktiven Kennwerte von altem Vollziegelmauerwerk nach EC 6, Testreport MA39 - VFA 2009-1396.01

Pech, A. \& Zach, F. (2009). Mauerwerksdruckfestigkeit - Bestimmung bei Bestandsobjekten, Mauerwerk Vol. 13, No. 9, pp. 135-139, Ernst \& Sohn

Schäfer, J. \& Hilsdorf, H. K. (1990). Historische Mörtel in historischem Mauerwerk, In: Erhalten historischer bedeutsamer Bauwerke, Ernst \& Sohn, Berlin

Schubert, P. \& Brameshuber, W. (2011). Eigenschaften von Mauersteinen, Mauermörtel, Mauerwerk und Putzen, In: Mauerwerk Kalender 2011, Ernst \& Sohn, Berlin

Strauss, A.; Frangopol, D.M. \& Kim, S. (2008). Use of monitoring extreme data for the performance prediction of structures: Bayesian updating, Engineering Structures, Vol. 30, No. 12, pp. 3654-3666

Strauss, A.; Frangopol, D. \& Bergmeister, K. (2010). Assessment of Existing Structures Based on Identification, Journal of Structural Engineering, Vol. 136, No. 1, pp. 86-97

SEISMID - Forschungsprojekt: Seismic System Identification, $h t t p: / / w w w . s e i s m i d . c o m$

Takewaki, I.; Murakami, S.; Fujita, K.; Yoshitomi, S. \& Tsuji, M. (2011). The 2011 off the Pacific coast of Tohoku earthquake and response of high-rise buildings under long-period ground motions, Soil Dynamics and Earthquake Engineering, In Press

Tomazevic, M.; Lutman, M. \& Weiss, P. (1996a). Seismic Upgrading of Old Brick-Masonry Urban Houses: Tying of Walls with Steel Ties, Earthquake Spectra, Vol. 12, No. 3, pp. 599-622

Tomazevic, M.; Lutman, M. \& Petkovic, L. (1996b). Seismic Behaviour of Masonry Walls: Experimental Simulation, Journal of Structural Engineering Vol. 122, pp. 1040-4047

Tomazevic, M. (1998). Correlation between damage and seismic resistance of masonry walls and buildings, Bernardini, Alberto (ed.): Seismic damage to masonry buildings: Proceedings of the International workshop on measures of seismic damage to masonry buildings, Monselice, Padova, Italy, June 1998, Rotterdam; Brookfield: A. A. Balkema, pp. 161167,1999

Tomazevic, M. (2007). Damage as measure for earthquake-resistant design of masonry structures: Slovenian experience, Canadian Journal of Civil Engineering Vol. 34, pp. 1403-1412

Wisser, S. \& Knöfel, D. (1987). Untersuchungen an historischen Putz- und Mauermörteln. T.1 Analysengang, In: Bautenschutz + Bausanierung Vol. 10, No. 3, pp. 124-126

Zimmermann, T. \& Strauss, A. (2010a). Leistungsfähigkeit von alten Ziegelmauerwerk in Bezug auf zyklische Belastung, Bauingenieur Vol. 85, pp. S2-S9

Zimmermann, T. \& Strauss, A. (2010b). Gründerzeit Mauerwerk unter Erdbebenbelastung Vergleich zwischen normativen Ansätzen und messtechnischen Ergebnissen, Bautechnik Vol. 87, No. 9 pp. 532-540

Zimmermann, T.; Strauss, A. \& Bergmeister, K. (2010a). Numerical investigations of historic masonry walls under normal and shear load, Construction and Building Materials Vol. 24, No. 8, pp. 1385-1391 
Zimmermann, T.; Strauss, A.; Lutman, M. \& Bergmeister, K. (2010b). Stiffness Identification and Degradation of Masonry under Seismic Loads, In: Proceedings of $8^{\text {th }}$ IMC, Dresden, Germany, July 2011

Standards (all standards are published by Austrian Standards, Austria and available online via: www.as-search.at)

EN 771-1 (2011). Specifications for masonry units - Part 1: Clay masonry units

EN 772-1 (2011). Methods of test for masonry units - Part 1: Determination of compressive strength

EN 772-13 (2000). Methods of test for masonry units - Part 13: Determination of net and gross dry density of masonry units

EN 772-16 (2005). Methods of test for masonry units - Part 16: Determination of dimensions

EN 1015-11 (2007). Methods of test for mortar for masonry - Part 1: Determination of flexural and compressive strength of hardened mortar

EN 1052-1 (1999). Methods of test for masonry - Part 1: Determination of compressive strength

EN 1052-2 (1999). Methods of test for masonry - Part 2: Determination of flexural strength

EN 1052-3 (2007). Methods of test for masonry - Part 3: Determination of initial shear strength

EN 1052-5 (2005). Methods of test for masonry - Part 5: Determination of bond strength

EN 1990 (2006). Eurocode 0 - Basis of structural design

EN 1991-1-1 (2011). Eurocode 1 - Actions on structures - Part 1-1: General actions - Densities, self-weight, imposed loads for buildings

EN 1996-1-1 (2006). Eurocode 6 - Design of masonry structures - Part 1-1: General rules for reinforced and unreinforced masonry structures

EN 1998-1 (2011). Eurocode 8 - Design of structures for earthquake resistance - Part 1: General rules, seismic actions and rules for buildings

EN 1998-3 (2005). Eurocode 8 - Design of structures for earthquake resistance - Part 3: Assessment and retrofitting of buildings 


\title{
Seismic Performance of Masonry Building
}

\author{
Xiaosong Ren, Pang Li, Chuang Liu and Bin Zhou \\ Institute of Structural Engineer and Disaster Reduction, Tongji University,
}

China

\section{Introduction}

The frequent occurrence of huge earthquake in the recent years results catastrophic losses to the people's life and property, which is mainly caused by the devastation of many buildings. The Tangshan Earthquake (Ms 7.8,1976) and Wenchuan Earthquake (Ms 8.0,2008) should be mentioned here for the destructive influence on the development of China in the recent ages(Housner \& Xie, 2002; Liu \& Zhang,2008; Wang,2008). After the trial version of seismic design code as TJ 11-74, the Chinese seismic design code was published first in 1978 (as TJ 11-78), revised in 1989 (as GBJ 11-89) , 2000 (as GB 50011-2001) and 2010 (as GB 50011-2010). The minor earthquake, moderate earthquake and major earthquake are concerned in the seismic fortification. The moderate earthquake is defined to be of local fortification intensity. The minor earthquake means the frequent earthquake, whose intensity is about 1.5 degree lower, while the major earthquake means the rare earthquake, whose intensity is about 1 degree higher. The exceeding probability during 50 years as $P_{f}$, the maximum acceleration of ground $a_{\max }$ and the maximum coefficient of horizontal earthquake action as $\alpha_{\max }$ for local fortification intensity 7 are summarized in Table 1 . It is seen that the relative major earthquake action, which is defined as the ratio of major earthquake action and minor earthquake action is 6.3 for local fortification intensity 7 .

\begin{tabular}{|c|c|c|c|}
\hline Condition & $P_{f}$ & $a_{\max }\left(\mathrm{cm} / \mathrm{s}^{2}\right)$ & $\alpha_{\max }$ \\
\hline Minor earthquake & $63.2 \%$ & 35 & 0.08 \\
\hline Moderate earthquake & $10 \%$ & 100 & 0.23 \\
\hline Major earthquake & $2-3 \%$ & 220 & 0.50 \\
\hline
\end{tabular}

Table 1. Main parameters for local fortification intensity 7 according to the Chinese code.

In order to realize the seismic objective in China, which is defined as no failure under minor earthquake, repairable damage under moderate earthquake and no collapse under major earthquake, the seismic design procedure should be finished in two steps. The strength and lateral deformation in the elastic range must be checked under minor earthquake action, while for some specified structures, the elasto-plastic deformation analysis should also be done to verify the collapse-resistant capacity of structures under major earthquake action.

Because of the relative low construction cost, masonry building is the widely used structural type in China. For this reason, the seismic damage of masonry buildings was specially investigated by the first author just after the 5.12 Wenchuan Earthquake of 2008. Based on 
the seismic damage collected in the disaster area, the seismic performance of masonry building is discussed. In order to ensure the collapse-resistant capacity, the ductility of structures should be involved. It is necessary to set more margin of shear strength in the design. The method of parcelling masonry structure with reinforced concrete members is suggested to retrofit the existing masonry buildings.

\section{Seismic damage caused by 5.12 Wenchuan earthquake}

The severe damage of buildings caused by 5.12 Wenchuan Earthquake of 2008 is really a good lesson for engineering community to understand more about the seismic performance of structures. The first author took part in the work of site urgent structural assessment in a small mountainous county, Qingchuan County. Among the concerned 133 buildings, there are 6 buildings of reinforced concrete structure and 127 masonry buildings. Till now, there are 44 big after-shocks of magnitudes larger than Ms 5.0, while 9 big after-shocks took place in Qingchuan County, including the largest one (Ms 6.4,16:21, May 25th, 2008). Qingchuan County belongs to the extremely heavy disaster area. The actual seismic intensity of Qingchuan County is 9, which exceeds the level of major earthquake (about intensity 8.5) of the previous fortification intensity 7 in this region.

No steel structure was found in Qingchuan County. The amount of the reinforced concrete structure is about $10 \%$ and the others are masonry buildings. The masonry buildings were constructed mainly after 1980 and seismic proof was generally considered by the previous design code. About $50 \%$ of masonry buildings collapsed or nearly collapsed while the ratio of reinforced concrete structure is about $20 \%$. The seismic damage of masonry buildings investigated in this area is mainly stated (Lu and Ren, 2008).

\subsection{Through diagonal cracks or through X-shape cracks on the wall}

Through diagonal cracks or through $\mathrm{X}$-shape cracks are the common earthquake induced damages on the walls of the masonry buildings, as shown in Fig.1.This kind of earthquake damage belongs to shear failure, which is caused by the principal tensile stress exceeding shear strength of masonry. The through $\mathrm{X}$-shape cracks are very popular on the longitudinal walls, especially between the door or window openings of nearly every floor. The diagonal cracks usually appear mostly on the bearing transverse walls. These cracks may lead to the obvious decrease of structural capacity and even collapse of the buildings.
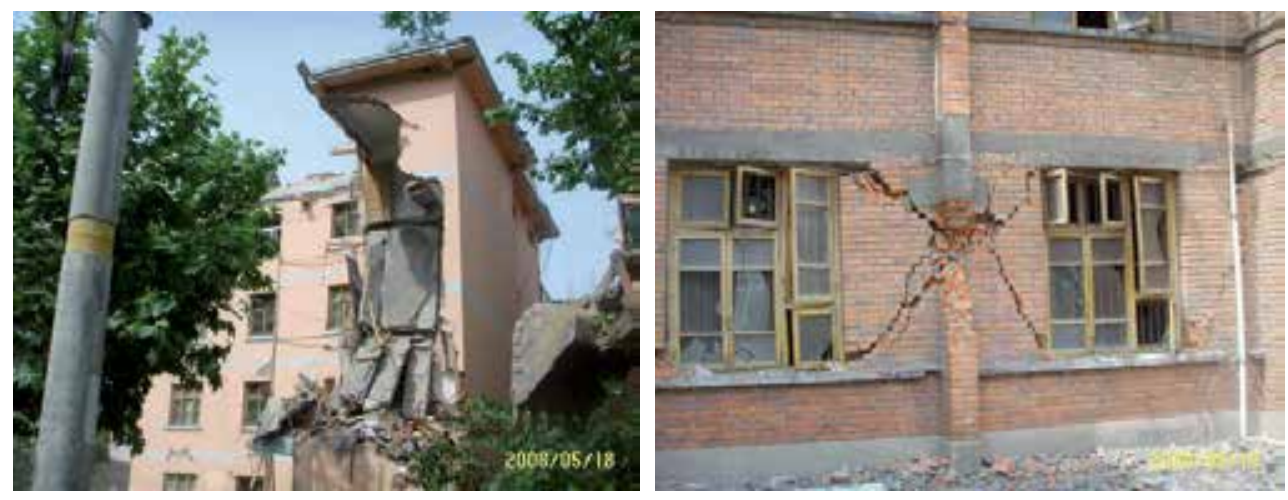

Fig. 1. Through diagonal or X-shape cracks on the wall 


\subsection{Horizontal crack on the wall}

Another main seismic damage is the horizontal crack on the wall. Horizontal cracks usually appear at the wall near the elevation of floor or roof, which enlarges the damage and results in collapse of pre-cast hollow slab. Meanwhile, horizontal cracks also appear on the end of some bearing brick columns, which lead to the decrease and even loss of the structural capacity. This kind of cracks means horizontal shear failure of walls. It is deduced that the large vertical ground motion may lead to this kind of earthquake damage. Typical phenomenon of horizontal cracks on the wall is shown in Fig. 2.
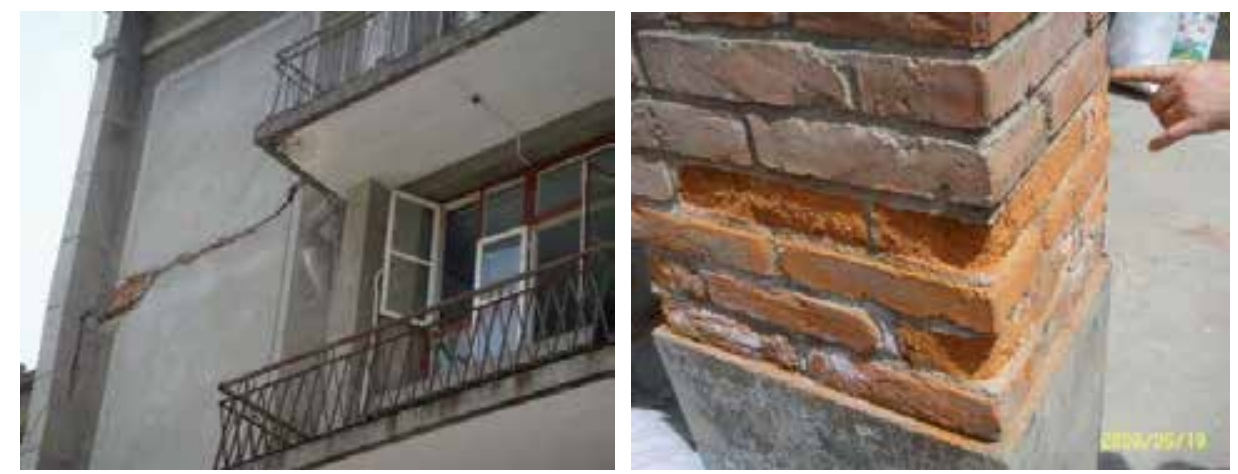

Fig. 2. Horizontal cracks on the wall or the bearing brick column

\subsection{Damage of the stair part}

Comparing with the other parts, the damage of stair part is relative severe because of the relative large stiffness of the slope structural members. Fig. 3 shows the severe damage of the stair part in the building with irregular plan. From the layout of this building, it is seen that the stair part is the convex part of the T-shaped plan.
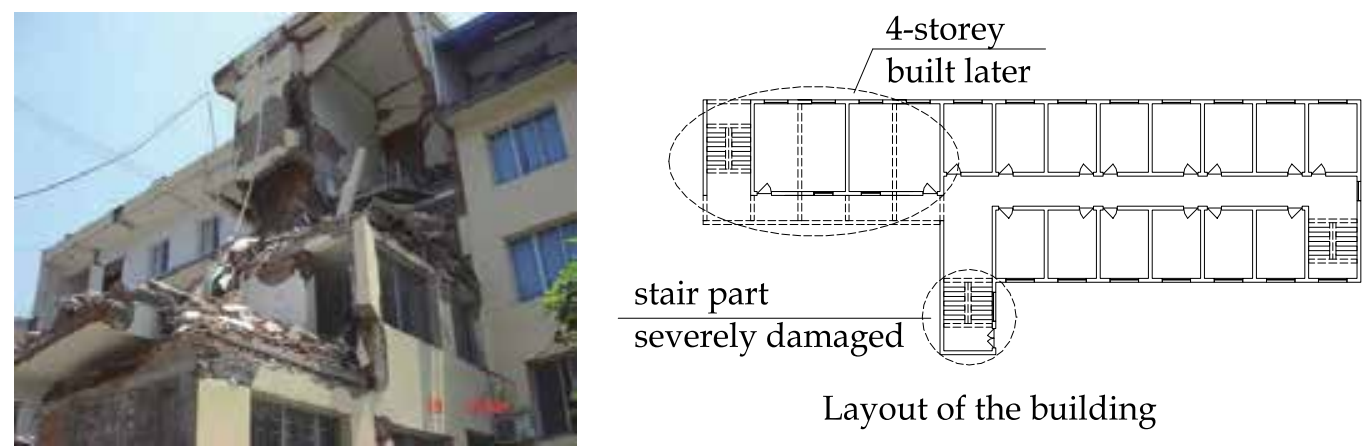

Layout of the building

Fig. 3. Partial collapse of stair part

\subsection{Damages of nonstructural components}

Severe damages on nonstructural components, such as horizontal crack, diagonal crack, even partial collapse can be easily found due to no reliable connections with the main structures. Fig.4 shows the partial collapse of the parapet, which even leads to the damage of the roof slab. Fig. 5 shows the falling of the corridor fence, severe damage of the 
protruding member in the roof. These are typical phenomena of the seismic induced damage of the nonstructural components.
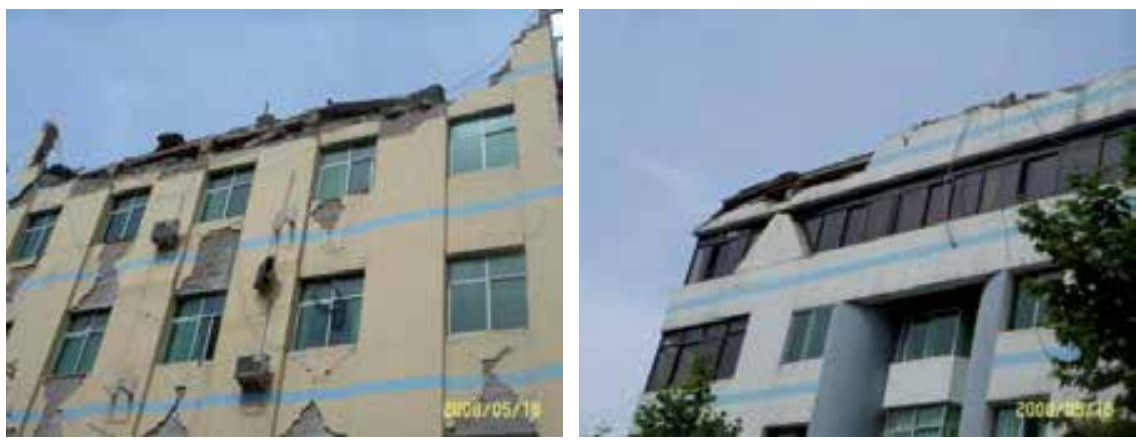

Fig. 4. Failure of the parapet wall
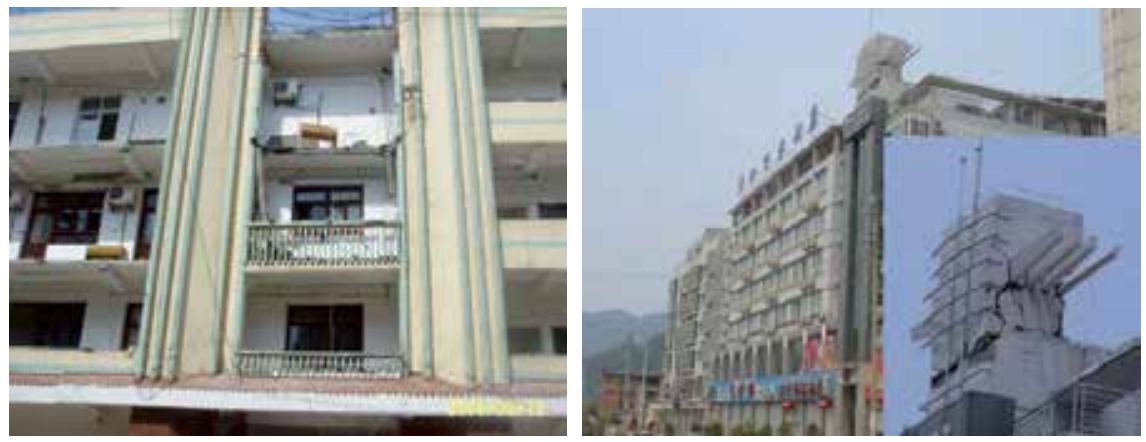

Fig. 5. Falling of the corridor fence and horizontal crack of the protruding member

\subsection{Damage caused or aggravated by structural irregularity}

Two examples are given here to show the harmful influence of structural irregularity on the building damage. Fig. 6 shows the severe damage of the L-shaped building with unequal height. Fig.7 shows the severe damage in the part of staggered elevation.
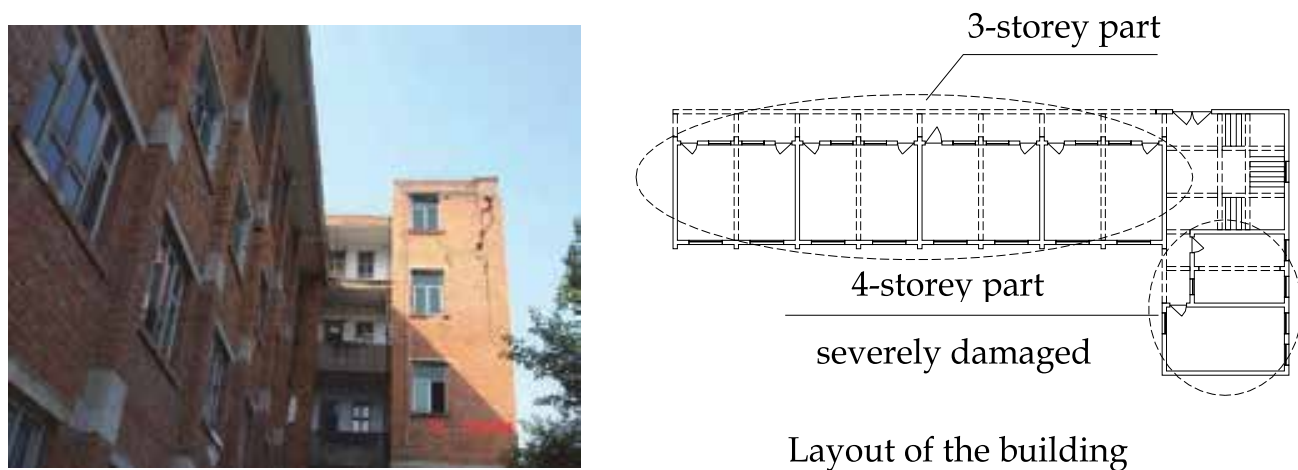

Layout of the building

Fig. 6. Severe damage of masonry buildings with plan irregularity 

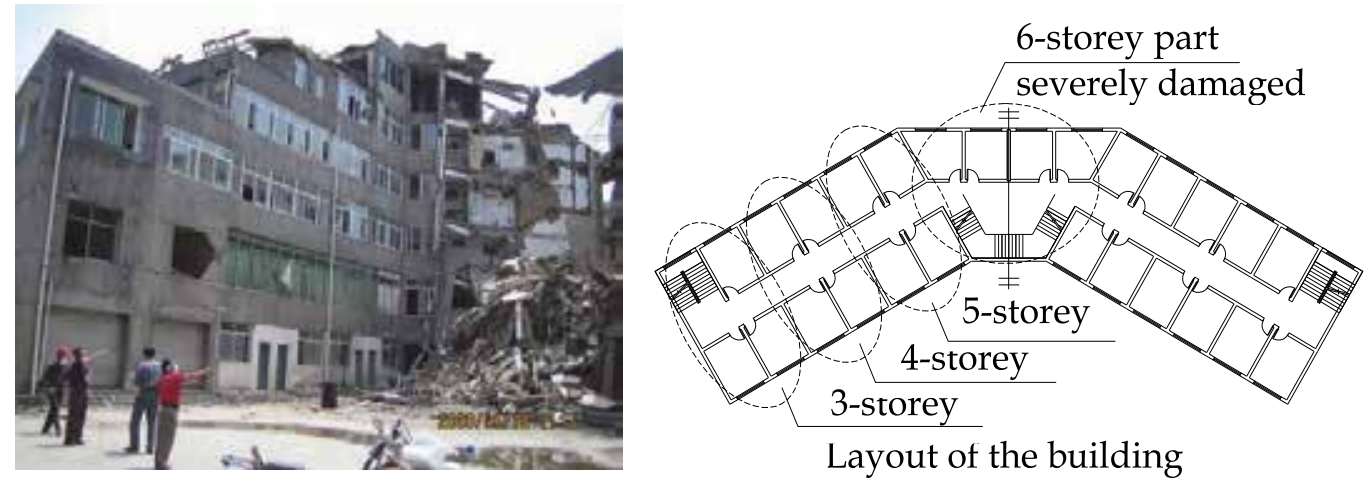

Fig. 7. Severe damage of masonry buildings with elevation irregularity

\section{Discussion on the current seismic design method}

\subsection{Conceptual design}

In order to get better seismic performance, the conceptual design is always very important to achieve besides the seismic analysis, especially in the early stage of architectural scheme. The current seismic code GB50011-2010 stipulates regulations for seismic conceptual design in detail. The consensus is reached by the engineering community that strictly following the seismic conceptual requirement should perform better seismic performance and at least minimize the possibility of the structural collapse (Wang, 2008; Zhang et al., 2008; Ren et al., 2008). The main key points for seismic conceptual design are emphasized here.

\subsubsection{Structural regularity}

Reasonable architecture arrangement may play an important role in the seismic conceptual design, with emphasis on the simplicity and symmetry in plan and elevation for uniform stiffness distribution of structures. Detailed description for regularity is given in the code.

More attention to enhance the structural ductility should be paid to the irregular structures if the seismic joint is not feasible to set, although separating the irregular structure into regular parts by seismic joint is a simple and good way in usual condition. Try to avoid the structural system of one bay transverse bearing wall with outside corridor supported by the cantilever beam.

\subsubsection{Structural integrity}

In order to get the largest possible number of redundancies subjected to earthquake action, structures should be fully integrated by structural members. Good structural integrity will guarantee the good seismic performance of structures.

The measure for structural integrity of masonry buildings should be the reinforced concrete members, such as tie beam, column and cast-in-site slab. The enhancement of the small size masonry wall segments for seismic protection is very important, as these segments are proved to be the weak parts subjected to earthquake in practice. The valid connection between the nonstructural components and the main structure should also be set properly. 


\subsection{Shear strength check}

In the current seismic design code, only the shear strength check under minor earthquake is stipulated for seismic design analysis of masonry buildings.

The seismic shear capacity is contributed not only by the masonry wall segment but also by reinforced concrete member. It is checked by

$$
V \leq R=\frac{1}{\gamma_{R E}}\left[\eta_{c} f_{V E} A+\xi f_{t} A_{c}+0.08 f_{y} A_{s}\right]
$$

In Equation (1), $V$ is the shear force on the wall, $R$ is the structural resistance, $\gamma_{R E}$ is the seismic adjusting factor which is taken as 1 for bearing wall and 0.75 for self-bearing wall, $\eta_{c}$ is the confined factor of wall which is usually taken as $1, f_{V E}$ is the design value for seismic shear strength of wall, $A$ is the net cross area of the wall, $\xi$ is the participation factor of reinforced concrete tie column in the middle which is taken as 0.4 or 0.5 by the number of tie column, $f_{t}$ and $A_{c}$ is the design tensile strength of concrete and the cross area of the tie column in middle, $f_{y}$ and $A_{s}$ is the design tensile strength and the total area of reinforcements of tie column in middle.

It should be mentioned here that the design value of the shear strength $f_{V E}$ is got by the primary design value of shear strength $f_{V}$ and the normal stress influence factor $\xi_{N}$, i.e.

$$
f_{V E}=\xi_{N} f_{V}
$$

The normal stress influence factor is determined by the pressure of the cross section corresponding to the gravity load. It is in the range of 1.0 to 4.8 . The beneficial influence of normal stress on the shear strength is caused by the friction in the wall.

For convenience, the strength check parameter, which is defined as the structural resistance divided by the shear force, is set for shear strength check for the wall segment. Satisfactory result means the strength check parameter is no less than 1 as

$$
S I=\frac{R}{V} \geq 1
$$

\subsection{Introspection on the design analysis}

The engineering practice showed that strength and deformation are two import factors to evaluate the structural performance. The deformation check is valuable to proceed. The elastic deformation analysis is quite helpful to find some seismic weak parts, such as the torsional irregularity, discontinuous displacement. And the elasto-plastic deformation check can directly verify the structural performance under major earthquake action.

The seismic design of masonry structure is dominated by the shear strength check under minor earthquake. As neither elastic nor elasto-plastic deformation check is involved, it is somewhat questionable to guarantee the collapse resistant capacity under major earthquake (Ren, Weng \& Lu,2008).

1. The investigated seismic damage shows the two way relationship between shear strength and axial strength of the wall. It is different from the theoretical assumption in the current design code that only the shear strength is affected by the axial strength. Once the shear failure happened, the mortar will break and have crack on it, which means that the axial strength will decrease. For the difference of masonry block or mortar and the influence of 
construction quality, it is difficult to get the unified model for elasto-plastic deformation analysis. As no convincing progress in the elasto-plastic analysis of masonry structure, the current strength check method should be improved.

2. In practice, the structural ductility is proved to be the key for the collapse-resistant capacity of masonry buildings under the major earthquake. As the ductility of masonry structure is about 1-3, which is less than the normal range as 3-5 and 5-10 for the ductility of reinforced concrete structure and steel structure, the masonry structure is more like the brittle structure. In usually condition, the major earthquake action is about 4.5 to 6.3 times the minor earthquake action. If the strength check parameter is close to 1 , which means not many margins for strength, severe damage and even collapse will happen on the structure. It is conflicted with the demand of no collapse under the major earthquake and necessary for more margins of shear strength in the seismic design.

3. The seismic design code emphasizes on the design details such as tie columns and beams for better structural regularity and integrity. Although the shear strength check method in current code can take these factors into consideration, no quantitative index can be got to evaluate the collapse resistant capacity under major earthquake action. To process the shear strength with the demand of deformation capacity may be the feasible way to evaluate the collapse resistant capacity of masonry structure.

4. The earthquake action on the structure is in any arbitrary direction. The earthquake damage shows that once the failure or partial failure of the wall happens in one direction, the wall will easily be broken in another direction due to the out-of-plan stability problem. Hence it is important to keep uniform seismic capacity in two directions. In usual conditions, the door and window will bring very different seismic capacity in two directions. These wall segments near the door and windows are usually the weak parts of the structure. The ratio of seismic capacity in two directions should be limited to a certain value.

\section{Analysis of a severely damaged building}

A typical severely damaged 3-storey masonry school building is found in Qingchuan County (Ren \& Tao, 2011). Typical damaged longitudinal and transverse walls in first storey are shown in Fig. 8. The detailed position of damaged walls is marked in the layout (Fig. 9). As the through cracks are on the load-bearing walls, the structural capacity of this building decreases remarkably. Specially mentioned here, the damaged wall segment in longitudinal direction is in a quite dangerous state as it may collapse or partially collapse in the strong after-shock.
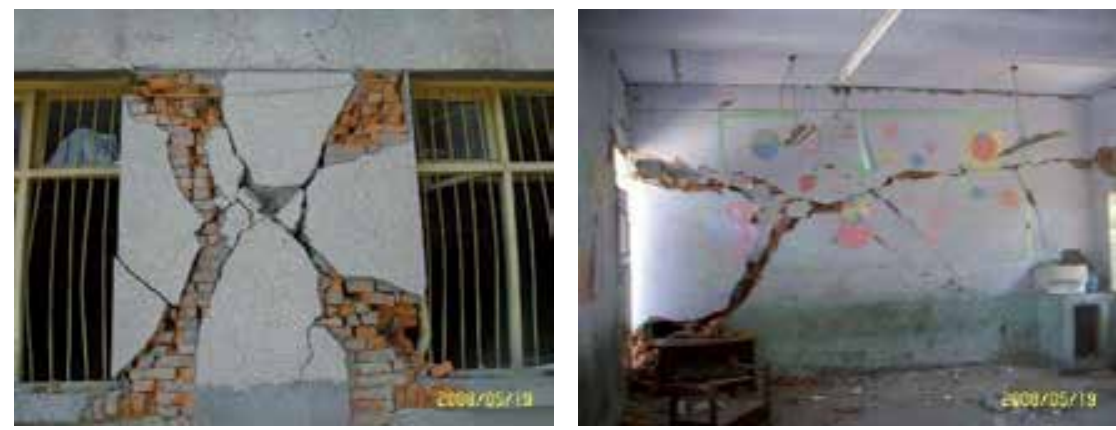

Fig. 8. Through cracks on the longitudinal and transverse load-bearing wall 
damaged wall segment (shown in Fig. 8) damaged wall segment (shown in Fig. 8)

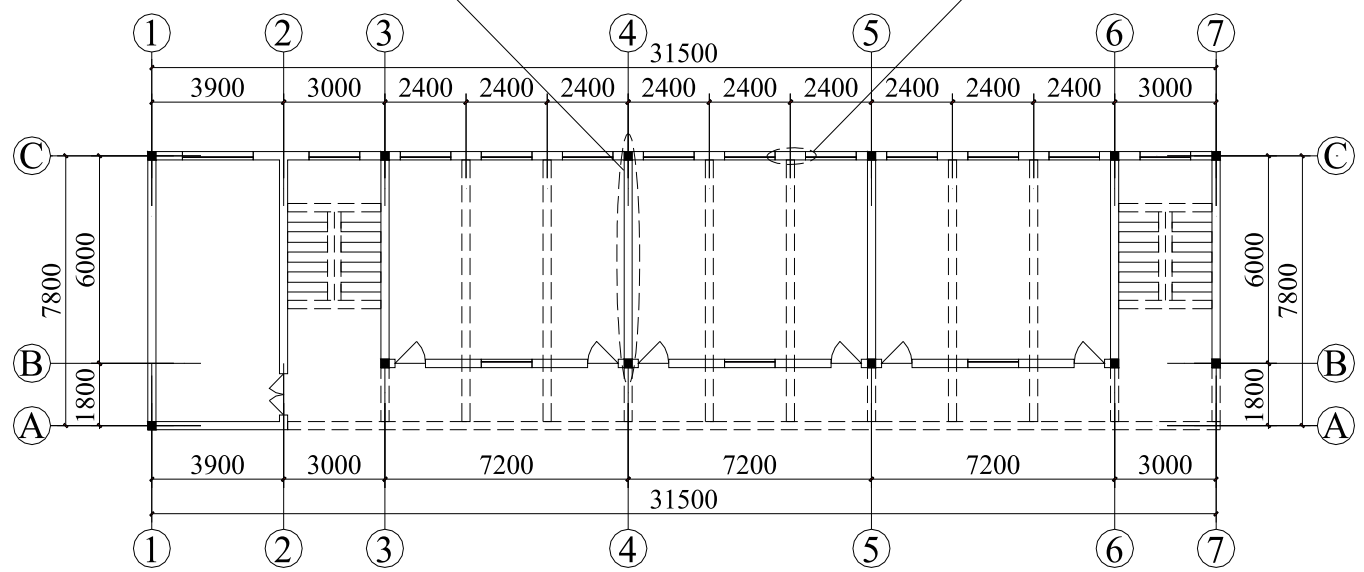

Fig. 9. Structural layout and the position of the severely damaged wall segments

\subsection{Shear strength check under minor earthquake}

An authorized design and analysis software PMCAD/PKPM is used for shear strength check of masonry structure (http://www.pkpm.com.cn/). In site, the mortar, the brick and the concrete are deduced to be M5, MU7.5 and C20. The wall thickness is $240 \mathrm{~mm}$. The storey height is $3.2 \mathrm{~m}$. The live and dead load on the floor is 2.0 and $4.0 \mathrm{kN}$ per square meter respectively.

Qualified result under minor earthquake is shown in Fig.10, as the strength check parameter is larger than 1. The damaged wall segments as shown in Fig. 9 are verified to be the most dangerous segment in transverse and longitudinal direction because of the smallest value of shear strength check parameter, which are 1.26 and 2.19 respectively.

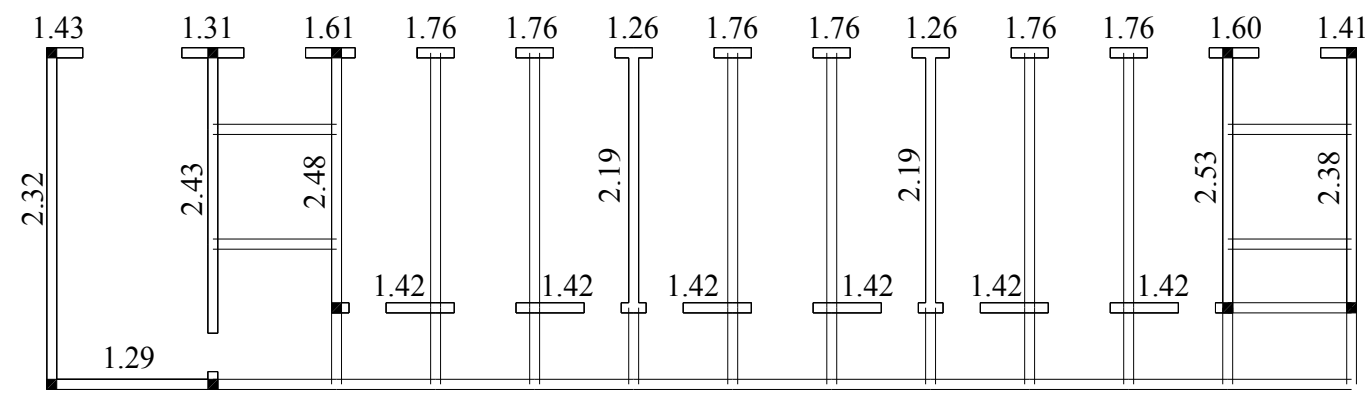

Fig. 10. Shear strength check parameter of the bottom storey

\subsection{Further analysis}

Although satisfactory check for intensity 7 is found according to the current design code, why so severe damage happened on the wall segments? The poor ductility of the masonry structure is the main reason. Here give a simple explanation for it.

Assuming the masonry structures has an idealized curve for shear force $\mathrm{V}$ and storey drift $\Delta$ as shown in Fig. 11, line 1-2-3 means the elasto-plastic behaviour while line 1-2-4 represents 
the elastic model. Here point 1, 2 indicate the design state and the yield state of the structure. Point 4 can be determined by point 1 multiplying the relative real earthquake, which is the ratio of the real earthquake action vs. the design earthquake action (or the minor earthquake action). The failure point 3 can be determined by the equal area of two shadowed region in Fig. 11. The deformation ratio of point 3 vs. point 2 is defined as the ductility.

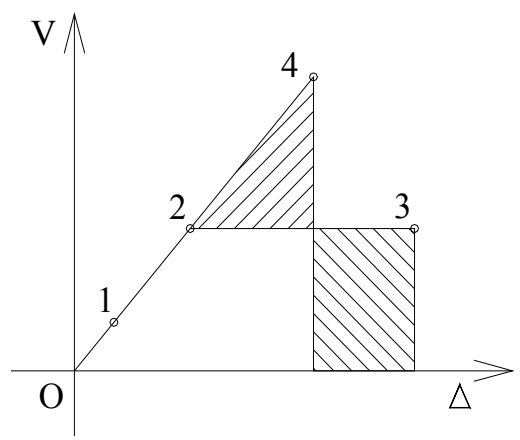

Point 1:design state

(subjected to minor earthquake)

Point 2:yield state

Point 3:ultimate state for elasto-plastic model

(subjected to actual earthquake)

$\Delta_{3} / \Delta_{2}$ is the ductility of structure

Point 4:ultimate state for elastic model

(subjected to actual earthquake)

Fig. 11. Idealized curve of storey shear force vs. storey drift

When subjected to major earthquake, point 3 can be determined by the equal area of two shadowed regions in Fig. 11. The ratio of ultimate strength vs. design strength $\left(V_{3} / V_{1}\right.$ or $V_{2} / V_{1}$ ) is usually about 2.5 to 3 , an average value 2.7 is used here. For different fortification intensity, the minimum ductility can be got. From Table 2, it is found that the objective of no collapse under major earthquake may not be easily realized by the shear strength check under minor earthquake, as the ductility of masonry buildings is usually about 1-3.

\begin{tabular}{|c|c|c|c|}
\hline Local fortification intensity & 7 & 8 & 9 \\
\hline Relative major earthquake action & 6.3 & 5.6 & 4.4 \\
\hline Minimum ductility required & 3.22 & 2.65 & 1.83 \\
\hline
\end{tabular}

Table 2. Minimum ductility required for different local fortification intensity

In usual condition, the ductility for this kind of masonry buildings with not many reinforced concrete members is no large than 2 , the severe damage will happen in the condition of an earthquake action at the level of 5 to 6 times the minor earthquake action, which is smaller than the major earthquake action. This means the possibility of losing structural capacity under major earthquake action, which is about 6.3 times the design earthquake action (minor earthquake action) for fortification intensity 7. So it is not difficult to understand the severe damage on the load bearing walls, as shown in Fig. 8.

\section{Suggestion for more margin}

\subsection{Improved shear strength check under minor earthquake}

As the difficulty to make the elasto-plastic deformation check of masonry buildings, the feasible way for better seismic performance is to set more margin of shear strength under minor earthquake action. Enough shear resistant capacity may be got under the major earthquake action to prevent the loss on axial strength due to the horizontal crack. 
For the improvement on Equation (3), the shear strength check under minor earthquake is suggested to satisfy

$$
S I=\frac{R}{V} \geq \psi
$$

Where, $\psi$ is the modified limitation with consideration of the structural ductility. Equation (4) means higher requirement of structural capacity comparing with Equation (3). By the simplified model, the suggested parameter $\psi$ can be got by the equal area of two shadowed regions in Fig. 11. It is seen that larger modified limitation should be set for the structure with smaller ductility.

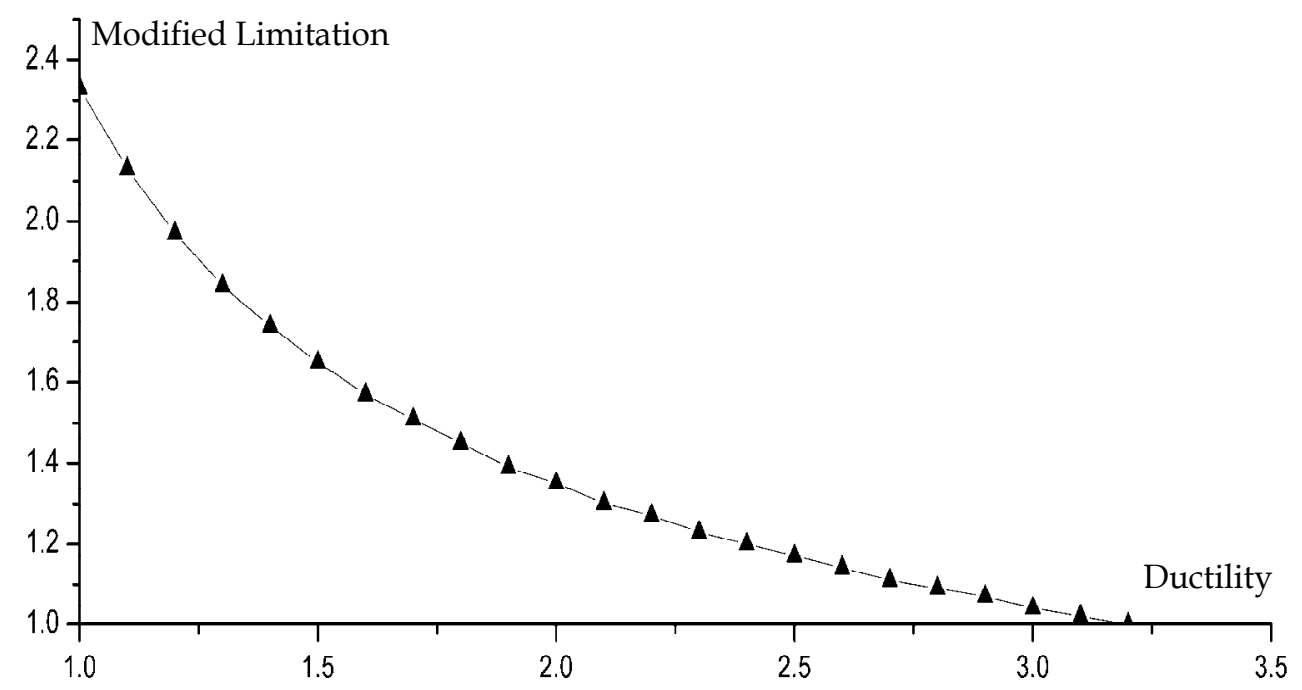

Fig. 12. Modified limitation of shear strength check for different ductility

In the meanwhile, to keep uniform seismic capacity in two directions is also very import for better seismic performance. The strength check parameter in two directions should be close to each other. Referring to regulations concerning the stiffness regularity in the current seismic design code, the ratio of the strength check parameter in two directions should be no less than 0.8 . Considering the openings on the wall in the actual condition, some reinforced concrete member s should be used to replace the small masonry wall segment. The structure is transformed to the composite structure of masonry and reinforced concrete, which is quite different from the ordinary masonry structure. Due to the large number and section size of the reinforced concrete member, the structure has more strength along with more ductility. The collapse resistant capacity of masonry buildings should be greatly improved.

\subsection{Illustrative analysis}

The damaged school building is illustrated here as an example here to demonstrate the authors' suggestion. As less tie columns are set, it is suggested to strengthen the structure in the longitudinal direction. As shown in Fig. 13, the small wall segments in axis B and C 
should be substituted by reinforced concrete member with section $900 \mathrm{~mm} X 240 \mathrm{~mm}$, while the reinforced concrete tie-columns should be set in axis A at the outside corridor part.

Similar structural analysis by PKPM software is done for strength check under minor earthquake. The main results are shown in Fig, 14 and Table 3. By comparison with Fig.10, it can be seen that the shear strength check ratio along transverse direction is raised from 2.19-2.53 to 2.68-2.80 and the parameter along longitudinal direction is raised from 1.26-1.76 to 1.74-3.07. The strengthened scheme can greatly enhance the seismic capacity.

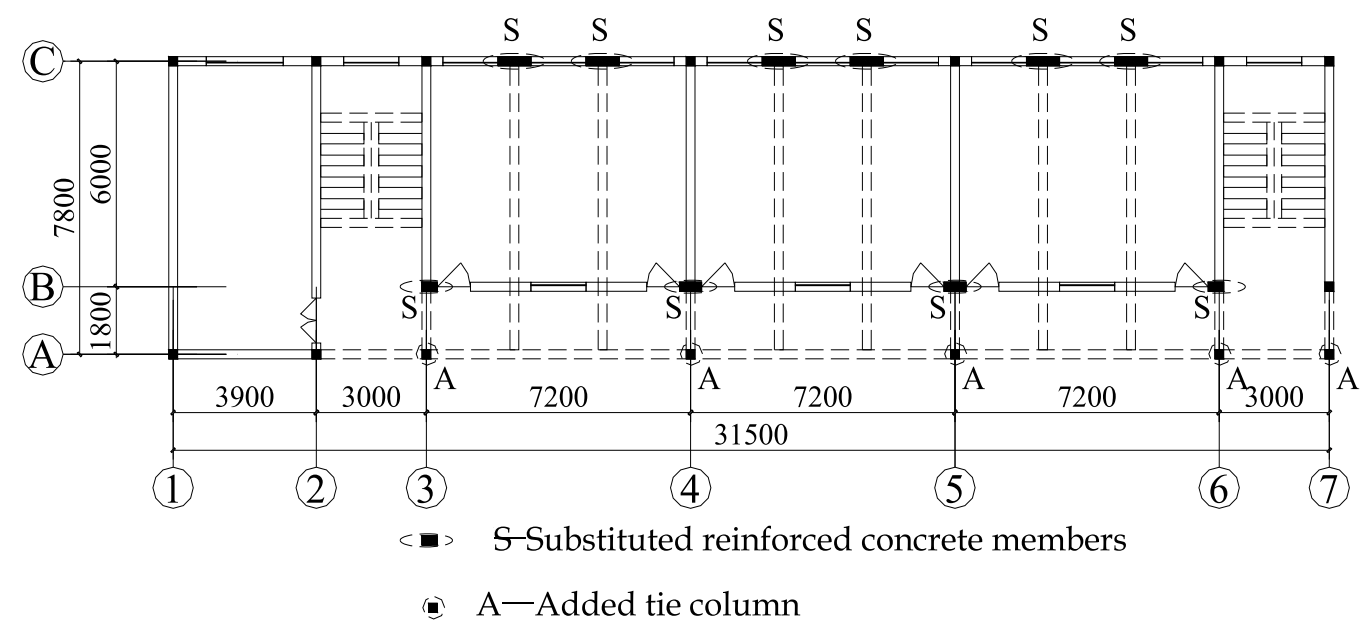

Fig. 13. Strengthened structural layout

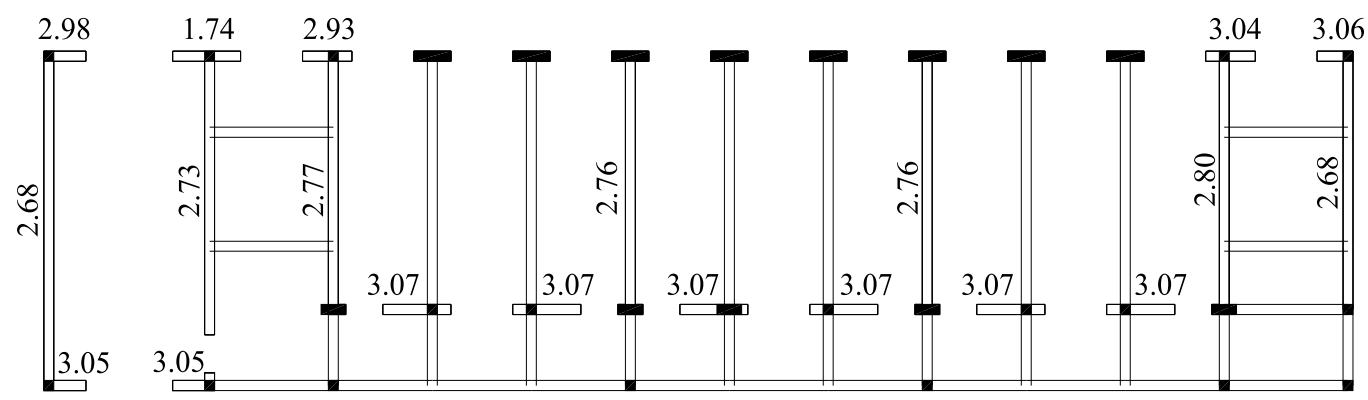

Fig. 14. The shear strength check parameter of the bottom storey of strengthened scheme

\begin{tabular}{|l|c|c|}
\hline \multicolumn{1}{|c|}{ Item } & Original & Strengthened \\
\hline Average strength check parameter in transverse direction & 2.37 & 2.74 \\
\hline Average strength check parameter in longitudinal direction & 1.65 & 3.05 \\
\hline Ratio of average strength check parameter in two directions & $0.70<0.8$ & $0.89>0.80$ \\
\hline
\end{tabular}

Table 3. Average shear strength check parameter for the original and strengthened structure 


\subsection{Ductility evaluation}

A simplified finite element plane model with two reinforced tie columns in the edge is used for ductility evaluation of the original and strengthened structure. It is used to simulate the longitudinal wall. The width of the wall is determined as $2.2 \mathrm{~m}$. The width of the reinforced concrete member is determined as $60 \mathrm{~mm}$ and $300 \mathrm{~mm}$ for. The area of the steel bar in the tie column is also determined as the average area of steel bar for the wall segment.

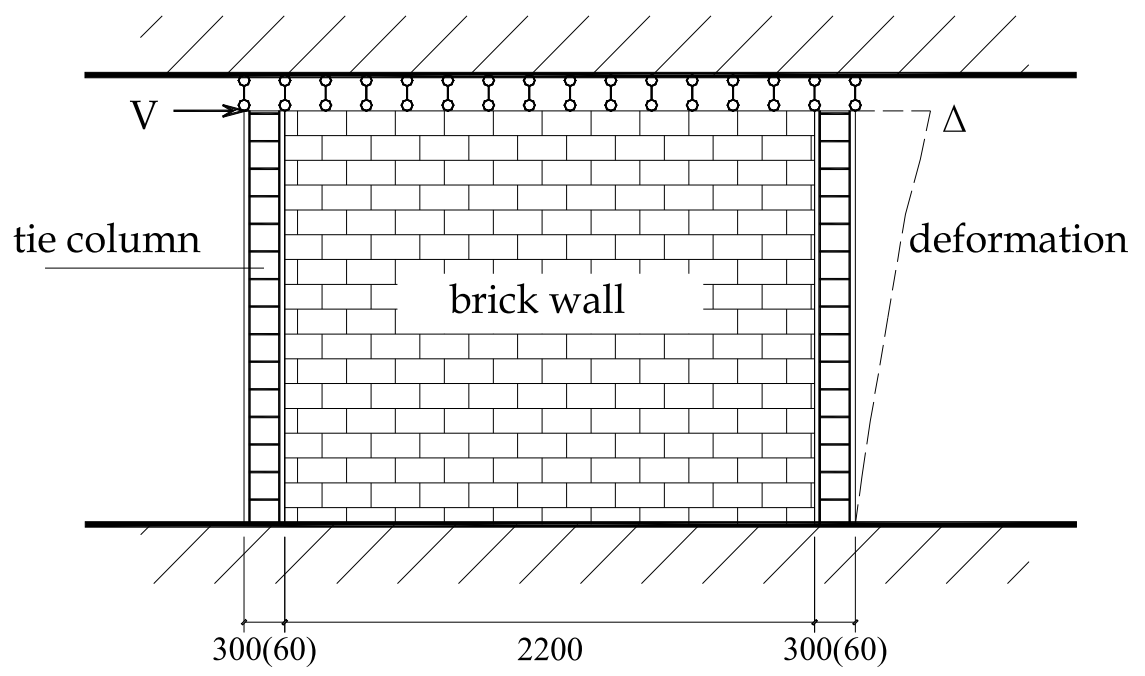

Fig. 15. Finite element model for ductility evaluation

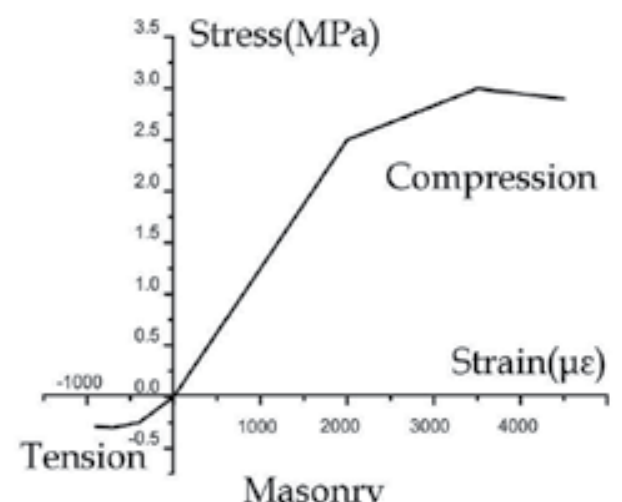

Masonry

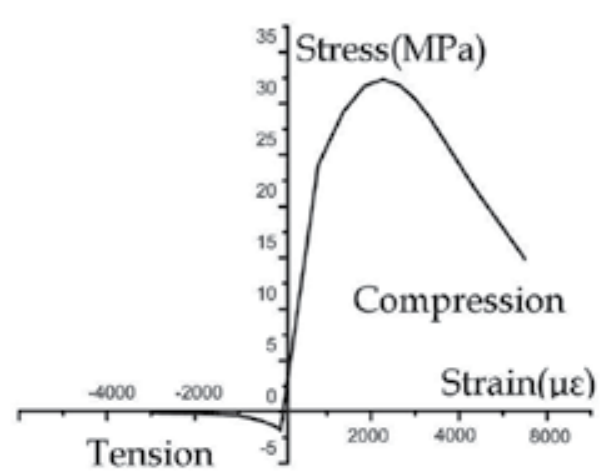

Concrete

Fig. 16. Constitutive model for masonry and concrete

From the skeleton curve shown in Fig. 17, it is seen that the yield load and ultimate load of the strengthened structure is about twice the value of the original structure. Moreover, the ductility of the structure should be deduced from the hysteretic curve by the principle of no obvious decrease of the primary strength. The ductility is raised from 1.65 to 2.50 . From Fig.12, the corresponding modified limitation is determined as 1.55 and 1.17 respectively. The original and the strengthened structure can not and can meet the authors' suggestion. 

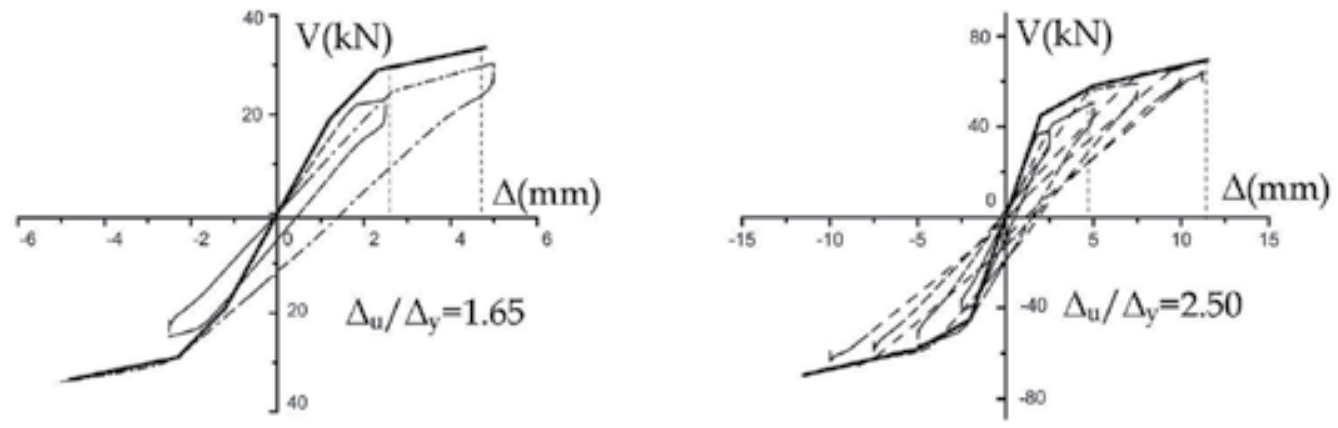

Fig. 17. Hysteretic curve and deduced skeleton curve for the different condition

\begin{tabular}{|c|c|c|}
\hline Item & Original condition & Strengthened condition \\
\hline Elastic stiffness $(\mathrm{N} / \mathrm{mm})$ & $1.58 \times 10^{5}$ & $2.25 \times 10^{5}$ \\
\hline Yield displacement $(\mathrm{mm})$ & 1.20 & 2.00 \\
\hline Crack load(N) & $1.90 \times 10^{5}$ & $4.5 \times 10^{5}$ \\
\hline Ultimate load(N) & $3.10 \times 10^{5}$ & $6.95 \times 10^{5}$ \\
\hline
\end{tabular}

Table 4. Main results for finite element analysis

\section{Seismic retrofit by parcelled reinforced members}

In 2001, a six storey masonry building in Shanghai was chosen as the first engineering case for the comprehensive transformation of residence. Duplex apartments with slope roof were added in the seventh floor. The ground floor residents moved to the corresponding apartment in the seventh floor. And the ground floor was used to be the space for public community. Elevators were also set. It is an active attempt to aim at the improvement on the residential function along with raising the level of seismic protection (Ren \& Liu,2010).

\subsection{Engineering background and strength check}

The original structure was built in 1986. The building was found in a good condition by site test. The grade of masonry and mortar could meet the design demands of MU10 and M10, while the strength of concrete is deduced to be of grade C25.

The structure is a supported-on-transverse-wall system while the stair part is a supportedon-longitudinal-wall subsystem. Reinforced concrete ring beams are set in every floor, but no tie column is set. And prefabricated slab is used for floor. It is found that the potential capacity in vertical direction is exerted for the static strength demand of adding storey while the capacity of seismic resistance is insufficient, especially in longitudinal direction. For the difficulty to retrofit the structure by direct method, the strengthening strategy of load transferring is applied here for the improvement of poor seismic capacity. In Fig.18, the dashed lines represent the demolished walls while the black lines represent the parcelled reinforced concrete members. 


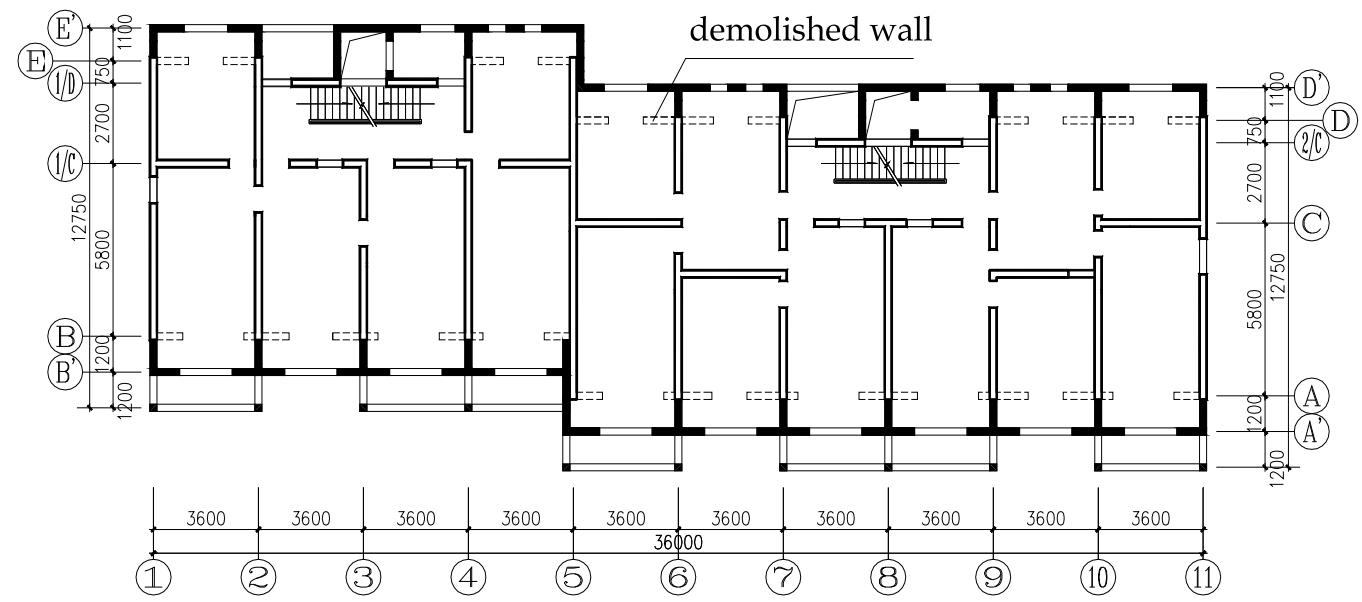

Fig. 18. Structural layout after comprehensive transformation

An eight storey model is established by PKPM software. The overhead floor is treated as the first storey in the model, and the added floor including the duplex part is treated as the eighth storey in the model with the load of one and half storey on it. Detailed results of shear strength check are got. The weakest structural member is located in the 2nd floor, which is shown in Fig. 19. The seismic performance is effectively enhanced.

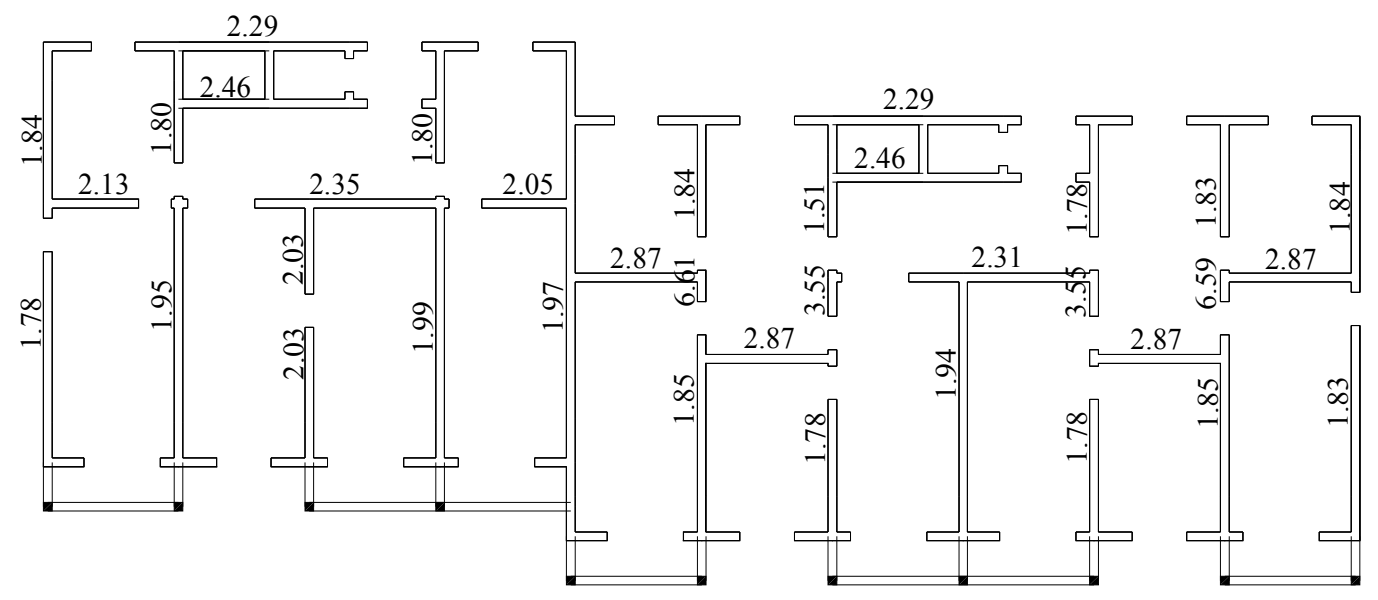

Fig. 19. The shear strength check parameter of the second storey 
Moreover, the average parameter for the shear strength check under minor earthquake can be got. From Table 5, the satisfactory shear strength check can be found according to the authors' suggestion.

\begin{tabular}{|c|c|c|c|}
\hline Model storey & Transverse direction & Longitudinal driection & Ratio of two directions \\
\hline 1 & 2.70 & 3.82 & 0.71 \\
\hline 2 & 2.72 & 2.67 & 0.98 \\
\hline 3 & 2.70 & 2.65 & 0.98 \\
\hline 4 & 2.70 & 2.73 & 0.99 \\
\hline 5 & 2.92 & 2.89 & 0.99 \\
\hline 6 & 3.30 & 3.32 & 0.99 \\
\hline 7 & 4.07 & 4.18 & 0.97 \\
\hline 8 & 7.80 & 8.41 & 0.93 \\
\hline
\end{tabular}

Table 5. The average shear strength check parameter under minor earthquake

Using the SATWE/PKPM program for further analysis under minor earthquake, the lateral storey stiffness can be got.(http://www.pkpm.com.cn). For comparison, another eight storey masonry model without the outside reinforced concrete walls is also established. The longitudinal analytical results are summarized in Table 6. Making comparison between the storey stiffness with and without reinforced concrete walls, the proportion of RC (reinforced concrete) part stiffness is got. It is seen that the earthquake action on the masonry part is greatly reduced. The first 3 natural modes are longitudinal, horizontal and torsional modes, and the corresponding periods are $0.46 \mathrm{~s}, 0.35 \mathrm{~s}, 0.30 \mathrm{~s}$. For the little influence of the mode of torsion, the analysis of longitudinal and transverse directions could be made separately. The largest storey drift is $1 / 2691$, which is in fourth model storey or the third storey of actual structure.

\begin{tabular}{|c|c|c|c|c|}
\hline $\begin{array}{c}\text { Model } \\
\text { storey }\end{array}$ & $\begin{array}{c}\text { Masonry stiffness } \\
(\mathrm{kN} / \mathrm{m})\end{array}$ & $\begin{array}{c}\text { Storey stiffness } \\
(\mathrm{kN} / \mathrm{m})\end{array}$ & $\begin{array}{c}\text { Proportion of } \\
\text { RC part } \\
\text { stiffness }\end{array}$ & Storey drift \\
\hline 1 & $1.56 \times 10^{7}$ & $1.39 \times 10^{8}$ & $88.7 \%$ & $<1 / 9999$ \\
\hline 2 & $1.19 \times 10^{6}$ & $5.09 \times 10^{6}$ & $76.6 \%$ & $1 / 4474$ \\
\hline 3 & $8.61 \times 10^{5}$ & $3.45 \times 10^{6}$ & $75.0 \%$ & $1 / 2946$ \\
\hline 4 & $7.54 \times 10^{5}$ & $3.00 \times 10^{6}$ & $74.8 \%$ & $1 / 2691$ \\
\hline 5 & $6.93 \times 10^{5}$ & $2.76 \times 10^{6}$ & $74.9 \%$ & $1 / 2780$ \\
\hline 6 & $6.47 \times 10^{5}$ & $2.61 \times 10^{6}$ & $75.3 \%$ & $1 / 3174$ \\
\hline 7 & $6.06 \times 10^{5}$ & $2.52 \times 10^{6}$ & $75.9 \%$ & $1 / 4126$ \\
\hline 8 & $3.64 \times 10^{5}$ & $2.04 \times 10^{6}$ & $82.2 \%$ & $1 / 10500$ \\
\hline
\end{tabular}

Table 6. Analytical results along the longitudinal direction

\subsection{Lumped storey model for elasto-plastic analysis}

For the status of more margins along transverse direction, the elasto-plastic analysis in longitudinal direction is done to verify the collapse resistant capacity under major earthquake.

The masonry part and RC (reinforced concrete) part of every storey are represented by two lumped joints connected by a rigid rod. The lumped storey model is shown in Fig. 20. As 
stated before, the stress-strain relationship for masonry part is a multi-line curve while it is a curve for reinforced concrete part. Here the proposed skeleton curves for the masonry and reinforced concrete part are demonstrated in Fig. 20.
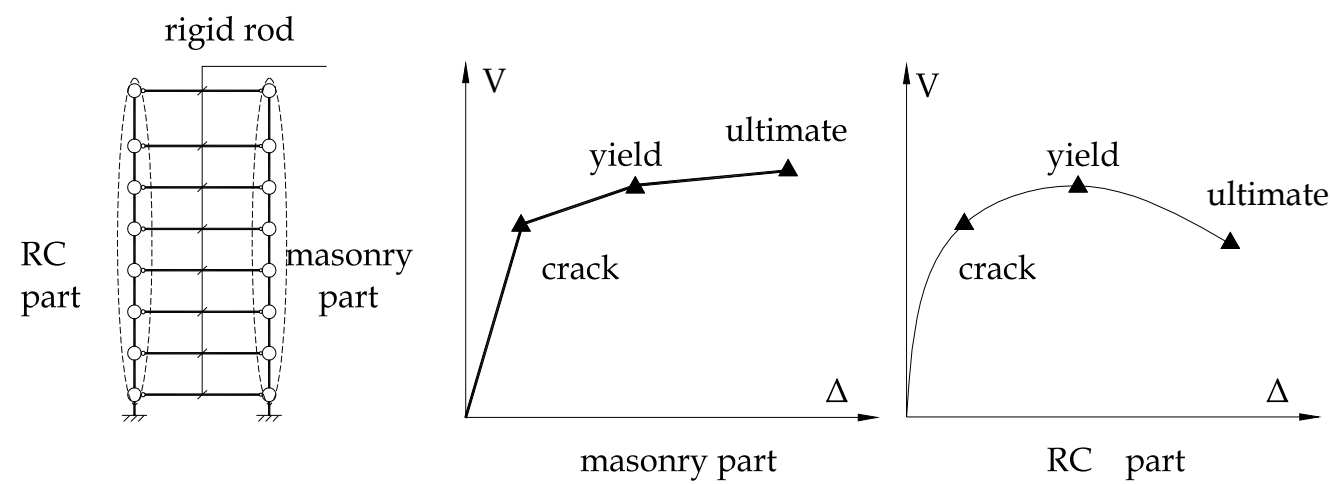

Fig. 20. Lumped storey model and proposed skeleton curve for two parts

From the past work carried out mainly by shaking table test and static push test (Zhu et al. 1980,1983; Xia, et al.,1989; Tomazevic, et al. 1996,1997; Benedett, et al. 1998,2009;Weng et al. 2002; Hori, et al. 2006;), three key points in the curve are denoted as primary crack, yield and ultimate. Although reaching the state of ultimate usually does not mean the complete failure of the structure, the descending stage from the ultimate state to the complete failure state is remarkably different from each other and usually neglected in the analysis. The values of storey drift of three key points are 1/1200,1/500,1/250, while the three key points' shear forces are $50 \%, 90 \%, 100 \%$ of the ultimate shear force. Detailed parameters for the skeleton cure will be gotten by the elastic storey stiffness resulted in the SATWE/PKPM analysis.

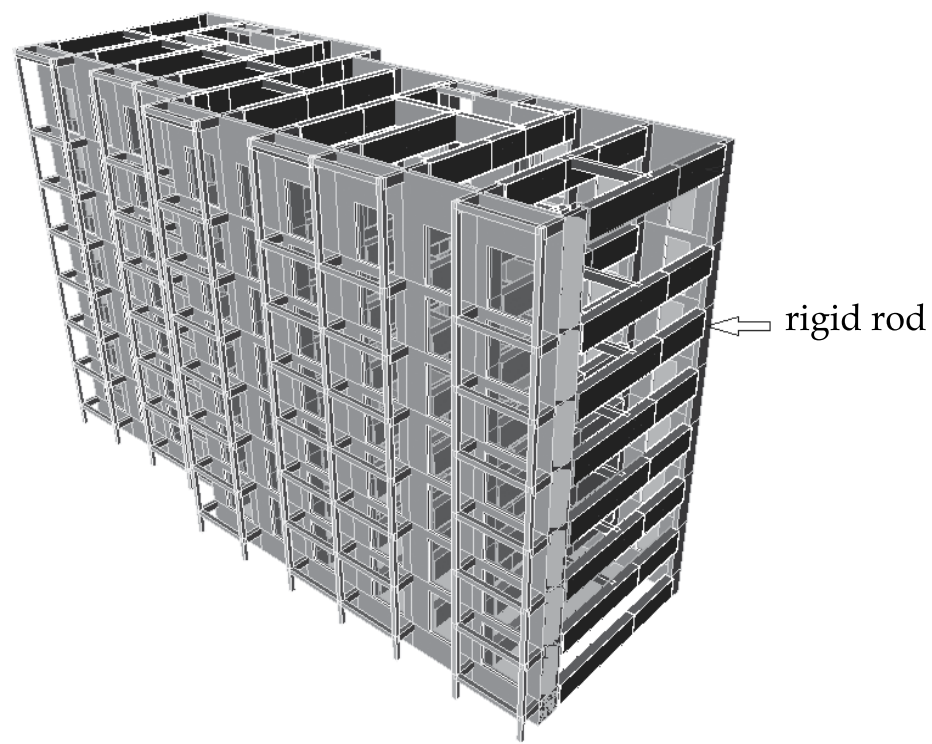

Fig. 21. Equivalent structure model for the analysis of skeleton curve of the RC part 
An equivalent the PMCAD/PKPM model is established for the deduced skeleton curve of reinforced concrete part by the EPDA/PKPM software (http://ww.pkpm.com.cn). The masonry members are treated as the rigid rods with lumped mass on the model, which is marked as dark beam in Fig.21. The dynamic characteristic of this equivalent model is proved to be nearly the same as the actual structural model. In order to fit the actual case, the skeleton curve will be described as symmetric curve artificially. The three key points as crack point, yield point and ultimate point can be determined by the characteristics as obvious decrease in stiffness, stiffness degrading and the maximum deformation. The results are summarized in Table 7.

\begin{tabular}{|c|c|c|c|c|c|c|}
\hline $\begin{array}{c}\text { Model } \\
\text { storey }\end{array}$ & $\begin{array}{c}\text { Crack } \\
\text { deformation } \\
(\mathrm{mm})\end{array}$ & $\begin{array}{c}\text { Crack } \\
\text { load } \\
(\mathrm{kN})\end{array}$ & $\begin{array}{c}\text { Yield } \\
\text { deformation } \\
(\mathrm{mm})\end{array}$ & $\begin{array}{c}\text { Yield } \\
\text { load } \\
(\mathrm{kN})\end{array}$ & $\begin{array}{c}\text { Ultimate } \\
\text { deformaton } \\
(\mathrm{mm})\end{array}$ & $\begin{array}{c}\text { Ultimate } \\
\text { load } \\
(\mathrm{kN})\end{array}$ \\
\hline 1 & 0.14 & $1.4 \times 10^{4}$ & 0.5 & $2.2 \times 10^{4}$ & 0.7 & $2.4 \times 10^{4}$ \\
\hline 2 & 2.0 & $1.5 \times 10^{4}$ & 11.0 & $2.2 \times 10^{4}$ & 18.0 & $2.4 \times 10^{4}$ \\
\hline 3 & 2.8 & $1.5 \times 10^{4}$ & 7.5 & $2.0 \times 10^{4}$ & 11.0 & $1.6 \times 10^{4}$ \\
\hline 4 & 3.2 & $1.4 \times 10^{4}$ & 7.5 & $1.8 \times 10^{4}$ & 10.0 & $1.4 \times 10^{4}$ \\
\hline 5 & 3.0 & $1.2 \times 10^{4}$ & 8.0 & $1.5 \times 10^{4}$ & 10.0 & $1.3 \times 10^{4}$ \\
\hline 6 & 3.2 & $1.5 \times 10^{4}$ & 7.0 & $1.3 \times 10^{4}$ & 11.0 & $1.0 \times 10^{4}$ \\
\hline 7 & 2.2 & $8.0 \times 10^{3}$ & 7.0 & $9.0 \times 10^{3}$ & 10.0 & $7.0 \times 10^{3}$ \\
\hline 8 & 2.0 & $4.4 \times 10^{3}$ & 5.5 & $5.5 \times 10^{3}$ & 10.0 & $4.2 \times 10^{3}$ \\
\hline
\end{tabular}

Table 7. Key parameters for skeleton curve of reinforced concrete part

The lumped storey model has similar structural characteristics of the space model by PKPM software. The first vibration mode is of the first grade transversal vibration mode with natural period $0.44 \mathrm{~s}$, while the result of space structural model is $0.45 \mathrm{~s}$. And the story drift by the method of earthquake spectrum for Shanghai region (maximum horizontal influence coefficient 0.08 ) is quite close to the results by PKPM software, which is shown in column "response spectrum" of Table 8.

\subsection{Elasto-plastic analysis under major earthquake}

Two artificial records and a natural record are used as the ground acceleration input for the lumped storey model. The peak acceleration is $220 \mathrm{~cm} / \mathrm{s}^{2}$. The response spectrum for the input seismic record and the design spectrum for Shanghai code are also shown in Fig.22. The symbol A-1,A-2 and N-1 in the following paragraphs represent the condition of two artificial seismic records and the natural seismic record.

The storey drift in different conditions is summarized in Table 8. The data in column "A-1", "A-2" and "N-1" is the elasto-plastic storey drift under three input ground acceleration. The data in column "average" is the average storey drift under three records. The data in column "response spectrum" is the elastic storey drift under minor earthquake action. Using the ratio of average storey drift divided by the elastic storey drift under major earthquake action, which the data in column "response spectrum" multiply 6.3, the amplifying factor for storey drift is listed in the last column of Table 8. 

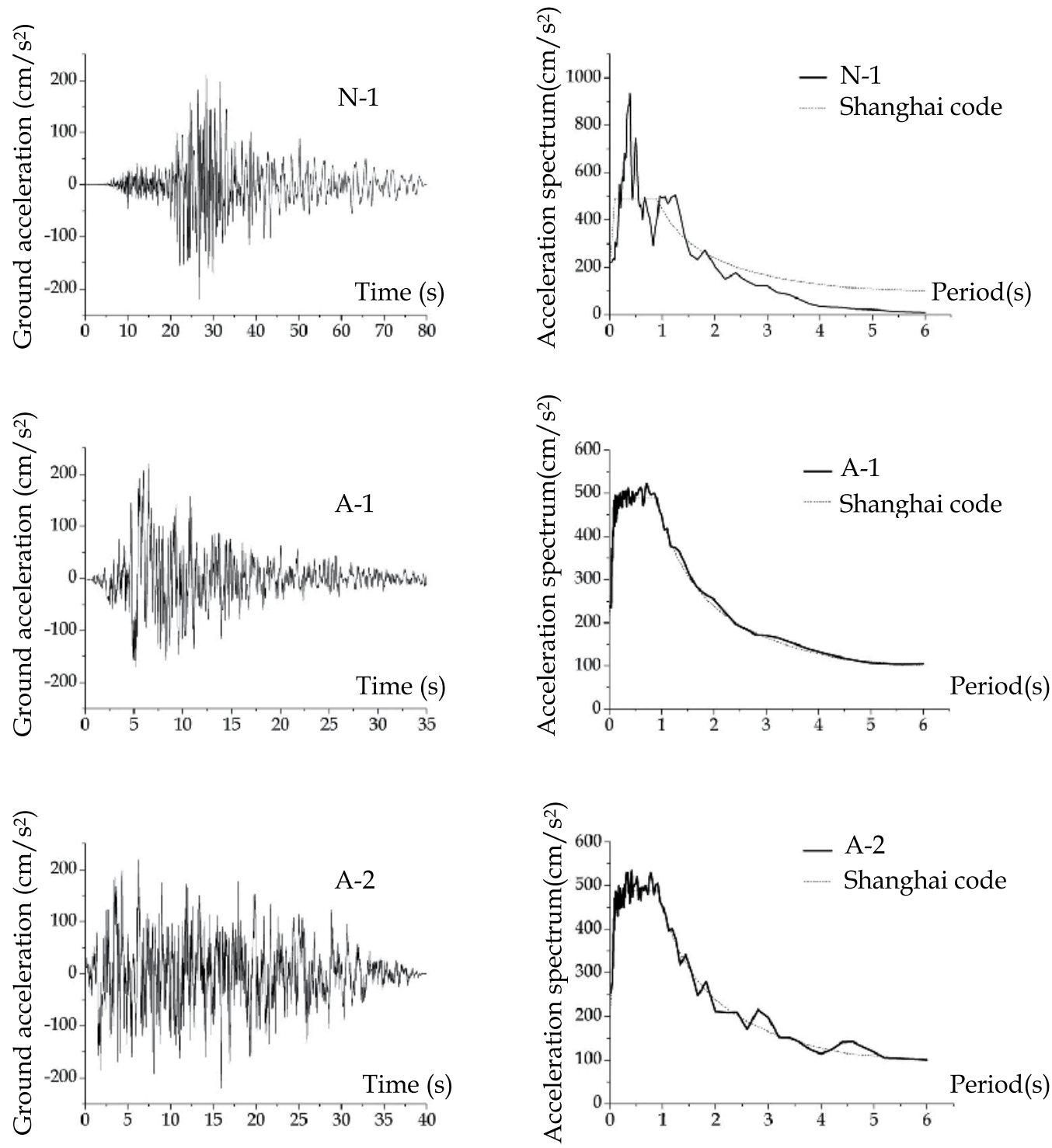

Fig. 22. Input ground acceleration and the response spectrum 


\begin{tabular}{|c|c|c|c|c|c|c|}
\hline $\begin{array}{c}\text { Model } \\
\text { storey }\end{array}$ & A-1 & A-2 & N-1 & Average & $\begin{array}{c}\text { Response } \\
\text { spectrum }\end{array}$ & $\begin{array}{c}\text { Amplifying } \\
\text { factor for storey } \\
\text { drift }\end{array}$ \\
\hline 1 & $1 / 6292$ & $1 / 5450$ & $1 / 4846$ & $1 / 5467$ & $1 / 51395$ & 1.12 \\
\hline 2 & $1 / 572$ & $1 / 418$ & $1 / 322$ & $1 / 414$ & $1 / 4071$ & 1.20 \\
\hline 3 & $1 / 511$ & $1 / 433$ & $1 / 311$ & $1 / 401$ & $1 / 3141$ & 1.11 \\
\hline 4 & $1 / 453$ & $1 / 399$ & $1 / 304$ & $1 / 375$ & $1 / 2812$ & 1.23 \\
\hline 5 & $1 / 427$ & $1 / 372$ & $1 / 284$ & $1 / 351$ & $1 / 2919$ & 1.34 \\
\hline 6 & $1 / 491$ & $1 / 449$ & $1 / 407$ & $1 / 446$ & $1 / 2979$ & 1.06 \\
\hline 7 & $1 / 660$ & $1 / 633$ & $1 / 478$ & $1 / 578$ & $1 / 4000$ & 1.10 \\
\hline 8 & $1 / 579$ & $1 / 676$ & $1 / 509$ & $1 / 580$ & $1 / 3784$ & 1.03 \\
\hline
\end{tabular}

Table 8. Elasto-plastic storey drift, elastic storey drift and the amplifying factor

From Table 8 , it is seen that the strengthened structure can satisfy the demand of no collapse under major earthquake as the maximum storey drift is less than $1 / 250$, which is the drift of ultimate point of masonry part. It is shown that the fourth and fifth storey have more plastic deformation than other storeys for the relative large values of amplifying factor for storey drift. The whole degree of structural plasticity is not very large. Moreover, the structural response under natural record is large than the reponse in aritificial records due to the resonant period near the structual period, which can be found on its curve of response spectrum in Fig. 22.

The distribution of storey shear can also be obtained. Table 9 is the result of the proportion of storey shear on the reinforced concrete walls. The column "A-1", "A-2", and "N-1" is the proportion under three input ground acceleration, while the last column represents the average data of storey shear proportion. It is seen that the values are about $84 \% \sim 90 \%$ and larger than the the corresponding values in elastic range. This means that parcelled reinforced concrete walls contribute much more strength in plastic stage than in elastic stage. And the small changes in the condition of A-1, A-2 and N-1 demonstrate the redistribution of storey shear in the plastic range.

\begin{tabular}{|c|c|c|c|c|}
\hline Model storey & A-1 & A-2 & N-1 & Average \\
\hline 1 & $86.2 \%$ & $89.7 \%$ & $86.9 \%$ & $87.6 \%$ \\
\hline 2 & $86.0 \%$ & $86.0 \%$ & $86.1 \%$ & $86.0 \%$ \\
\hline 3 & $86.2 \%$ & $86.2 \%$ & $86.1 \%$ & $86.2 \%$ \\
\hline 4 & $85.7 \%$ & $85.1 \%$ & $85.4 \%$ & $85.4 \%$ \\
\hline 5 & $85.3 \%$ & $85.3 \%$ & $85.2 \%$ & $85.3 \%$ \\
\hline 6 & $84.1 \%$ & $84.3 \%$ & $84.3 \%$ & $84.2 \%$ \\
\hline 7 & $85.7 \%$ & $85.7 \%$ & $85.7 \%$ & $85.7 \%$ \\
\hline 8 & $85.4 \%$ & $85.1 \%$ & $86.2 \%$ & $85.6 \%$ \\
\hline
\end{tabular}

Table 9. Proportion of storey shear distribution for reinforced concrete part 
Although the earthquake action on the masonry part is limited after the adding of reinforced concrete walls, the masonry part is still the key for satisfying the demand of no collapse under major earthquake.

\section{Conclusion}

The discussion of the seismic performance of masonry building is presented here. The collapse-resistant capacity under major earthquake action is somewhat questionable according to the current design code. It is of great importance for better structure performance subjected to major earthquake. For the purpose of "no failure under minor earthquake, repairable damage under moderate earthquake and no collapse under major earthquake", the structural ductility should be improved. More shear strength margin along with structural regularity and integrity are suggested in order to get better seismic performance. The suggestion can be easily realized in the current design analysis procedure. Besides a typical damaged masonry building illustrated, a success engineering case of retrofitting existing masonry building by parcelled reinforced concrete members is also presented to proceed seismic design by the authors' suggestion. The seismic retrofit by parcelled reinforced concrete member is quite effective for the increment of the strength and the enhancement of the ductility. As a lot of masonry buildings exist in China and will not be demolished in a short time, parcelling reinforced members is the feasible and practical way for better seismic performance of existing masonry buildings.

\section{Acknowledgment}

Financial support from Shanghai Natural Science Foundation (No. 09ZR1433400) is gratefully acknowledged.

\section{References}

Benedett, D. Carydis, P. and Pezzoli, P. (1998), Shaking Table Test on 24 Simple Masonry Buildings, Earthquake Engineering and Structural Dynamics, Vol.27,No.1,pp. 67-90

Benedett, D. Carydis, P. and Pezzoli, P. (2009), Cyclic Behavior of Combined and Confined Masonry Walls, Engineering Structures, Vol.38,No.1,pp. 240-259

Bruneau, M. (1994) State-of-the-art Report on Seismic Performance of Unreinforced Masonry Building. Journal of Structural Engineering, ASCE,Vol. 120,No.1,pp.230251.

Hori, N. Inoue, N. Purushotam, D. and Kobayashi, T. (2006), Experimental and Analytical Studies on Earthquake Resistant Behavior of Confined Concrete Block Masonry Structures, Earthquake Engineering and Structural Dynamics, Vol.35,No.1, pp.16991719

Housner, G. W. and Xie, L. L. ed. (2002), The Great Tangshan Earthquake of 1976, Technical Report EERL 2002.001. California Institute of Technology, Pasadena, California.

http://www.pkpm.com.cn/ 
Liu, X. H., Zhang H.X.(1981) A Study of Aseismic Characteristics of Masonry Buildings with Reinforced Concrete Tie-columns, Journal of Building Structures, Vol. 2,No.6,pp.4755. (in Chinese)

Lu, X.L. and Ren, X.S.(2008), Site Urgent Structural Assessment of Buildings in Earthquakehit Area of Sichuan and Primary Analysis on Earthquake Damages, Proceedings of 14th World Conference on Earthquake Engineering, No. S31-034, Bejing, China,Oct. 2008.

National Standards of the People's Republic of China (2001). Code for Design of Masonry Structures (GB 50003-2001) (English version), Architecture \& Building Press. Beijing, China

National Standards of the People's Republic of China (2001). Code for Design of Concrete Structures (GB 50010-2001) (English version), China Architecture \& Building Press. Beijing, China

National Standards of the People's Republic of China (2001). Code for Seismic Design of Buildings (GB 50011-2001) (English version), China Architecture \& Building Press. Beijing, China

Ren X. S., Liu C. (2010). Seismic Analysis of Multi-story Masonry Structure Parceled with Reinforced Concrete Wall [J], Journal of Building Structures,2010,31 (Supplementary Issue No.2): 334-339. (in Chinese)

Ren X. S., Weng D.G., Lu X.L(2008). Earthquake Damage of Masonry Buildings in Sichuan Province and Discussion on Seismic Design of Pimary and Middle School Buildings, Earthquake Engineering and Retrofitting, Vol.30,No.4,pp.71-76. (in Chinese)

Ren X. S., Tao Y.F.(2011),Discussion on The Seismic Design Analysis Method of masonry Building,Proceedings of International Conference on Structure and Building Material, pp.3952-3957, Guangzhou,China, Jan. 2011

Tomazevic, M. Lutman, M. and Petkovic, L. (1996), Seismic Behavior of Masonry Walls: Experimental Simulation, Journal of Structural Engineering, ASCE, Vol.122,No.9, pp.1040-1047.

Tomazevic, M. and Klemenec, I. (1997), Seismic Behavior of Masonry Walls: Experimental Simulation, Earthquake Engineering and Structural Dynamics, Vol.26,No.1, pp.10591071

Wang Y.Y. (2008). Lessons Learned from the "5.12" Wenchuan Earthquake: Evaluation of Earthquake Performance Objectives and the Importance of Seismic Conceptual Design Principles, Earthquake Engineering and Engineering Vibration, Vol.7,No.3,pp. 255-262.

Weng D.G., Lu X.L., Ren X.S. et al. (2002), Experimental Study on Seismic Resistant Capacity of Masonry Walls, Proceedings of 4th Multi-lateral Workshop on Development of Earthquake Technologies and their Integration for the Asia-Pacific Region(EQTAP),Tokyo, Japan,Sept. ,2002

Xia J. Q., Huang Q. S.(1989), Model Test of Brick Masonry Buildings Strengthened with Reinforced Concrete Tie Columns, Earthquake Engineering and Engineering Vibration, Vol. 9, No.2,pp. 83-96. (in Chinese) 
Zhu B.L., Wu M.S., Jiang Z.X. (1980), The Test Study on the Behavior of Masonry under the Reversed Load, Journal of Tongji University, No.2,pp.1-14. (in Chinese)

Zhu B.L., Jiang Z.X., Wu M.S. (1983). Study on the Seismic Behavior of Masonry Buildings with Reinforced Concrete Tie-columns, Journal of Tongji University, No.1,pp.21-43. (in Chinese) 


\title{
Seismic Response of Reinforced Concrete Columns
}

\author{
Halil Sezen and Muhammad S. Lodhi \\ Department of Civil and Environmental Engineering and Geodetic Science, \\ The Ohio State University, Columbus, Ohio, \\ USA
}

\section{Introduction}

There are a large number of reinforced concrete buildings in seismically active areas of the world that are not built in accordance with modern seismic design provisions such as those published by American Concrete Institution ([ACI] Committee 318, 2008). In the United States and other parts of the developed world, these buildings were constructed between 1930s to mid 1970s according to the building code requirements of that time. Even today, in low to moderate seismic regions and in some developing countries that are in process of developing and implementing their seismic codes, reinforced concrete structures are being designed and built without essential seismic details deemed vital to withstand large lateral loads. These buildings often have low lateral displacement capacities and undergo rapid degradation of shear strength and axial load carrying capacity during strong ground motions and hence are extremely vulnerable to excessive structural damage or collapse during future earthquakes.

In the past, the earthquakes have caused wide spread damage to the reinforced concrete structures with inadequate seismic design and construction practices. For example, during Kashmir (Pakistan) earthquake of 2005 and Haiti earthquake of 2010, extensive structural damage to residential, commercial and government buildings was observed (Earthquake Engineering Research Institute [EERI], 2005; Mid-America Earthquake [MAE] Center, 2005; U.S. Geological Survey [USGS]/EERI, 2010). The damage was attributed largely to lack of earthquake-resistant design, poor standard of construction and inferior quality of building materials. In majority of the collapsed or damaged structures, structural types, member dimensions and detailing practices (insufficient lap length, improper lap location and lack of confinement in columns etc) were found inadequate to resist forces imposed by these earthquakes. The 2011 off The Pacific Coast of Tohoku (Japan) earthquake is a modern day example of large scale devastation to a highly industrial nation in which building and infrastructure is well designed and constructed. Although, majority of causalities and large scale destruction of infrastructure was caused by ensuing tsunami, limited damage to the buildings due to ground shaking was reported (Pacific Earthquake Engineering Research Center [PEER]/EERI/Geotechnical Extreme-Event Reconnaissance [GEER]/Tsunami Field Investigation Team, 2011; Takewaki et al. 2011). However, extensive and severe structural damage was observed in older residential and commercial buildings that were constructed prior to 1978 code revision of Japan, whereas modern structures built to withstand seismic demands did not sustain any substantial and widespread damage (Aydan \& Tano, 2011). 
The existing reinforced concrete buildings with deficient seismic design can, however, be retrofitted to enhance their performance during future earthquakes. The need to assess their vulnerability to earthquake damage, and suggest desired level of retrofit, requires evaluation of their expected behavior in terms of strength and deformation capacity. In addition to the retrofitting requirements, there may be many situations where structures are required to be analyzed accurately to evaluate their structural responses. For example, in performance- and displacement-based design philosophy (Priestley et al, 2007, Structural Engineers Association of California [SEAOC], 2002), important existing buildings and planned future structures may need to be evaluated to determine their maximum load carrying capacity, ultimate deformation capacity, progression of the damage, and collapse mechanism. However, a realistic seismic damage analysis, in pre- or post-earthquake scenario, requires development of analytical models to accurately predict non-linear structural behavior during the seismic event (Mergos \& Kappos, 2010).

The expected behavior of a structure can be evaluated by determining load-deformation responses of the concrete elements, such as beams, columns and shear walls, considering all potential failure mechanisms associated with axial, flexure and shear behavior. The pattern of damage observed during past earthquakes suggests that columns are the most critical elements that sustain damage and lead the potential building failure. Hence, understanding of their response to applied seismic loads is vital for overall assessment of the structural performance. This paper presents a procedure for response estimation of reinforced concrete columns subjected to lateral loads, with focus on modeling columns commonly found in the older existing buildings. Traditionally, these columns have insufficient and widely spaced transverse reinforcement and non-seismic details such as 90-degree end hooks and splicing of the longitudinal bars in the regions experiencing largest inelastic deformations near column ends. Due to such deficiencies, columns may not have sufficient shear strength to develop plastic hinges at the ends. Also, wide spacing of column ties does not provide good confinement to the core concrete resulting in non-ductile behavior and sudden brittle failure. Although, this study focuses on modeling columns with poor seismic details, the proposed analytical procedure is equally applicable to predict structural response of the columns designed to meet the requirements of modern seismic codes.

\section{Research background and overview}

When a typical fixed ended reinforced concrete column is subjected to the lateral loads at its ends, it undergoes total lateral deformation that is mainly comprised of three components due to flexure, reinforcement slip and shear mechanisms (Setzler \& Sezen, 2008; Sezen \& Moehle, 2004) as shown in Figure 1.
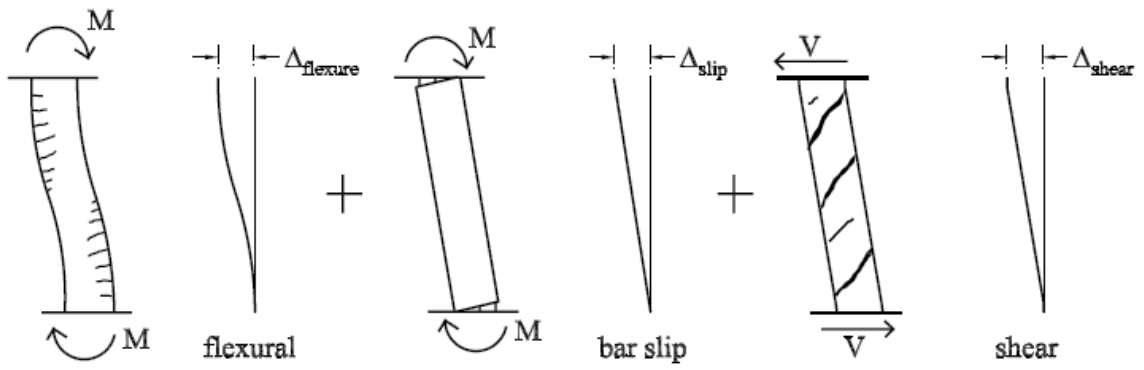

Fig. 1. Components of the total lateral deformation in a fixed ended column 
For a column whose behavior is dominated by flexure, issues regarding its performance evaluation have been studied well and current design procedures for strength estimation are generally considered well established. Among available approaches, most of which are either based on lumped plasticity models or distributed nonlinearity models, fiber models are considered advanced analytical procedures that can conveniently be employed for evaluating structural response. It must however be noted that fiber models are appropriate tools for analyzing flexural performance only and behavior of the columns dominated by shear related mechanisms can not be simulated.

For evaluating shear response of structural elements, such as beams and columns, many analytical models and theories have been presented in the past. Some of the most commonly used approaches are strut and tie models (Mörsh, 1902; Ritter, 1899; Schlaich et al., 1987) and empirical formulations/rational theories based on experimental observations such as Arakawa equation (Arakawa, 1970), Modified Compression Field Theory (MCFT) (Vecchio \& Collins, 1986) and Disturbed Stress Field Model (DSFM) (Vecchio, 2000). These approaches are fundamentally different in their theoretical modeling and conceptual development. Their applicability to structural members, computational demand and accuracy also vary in wide range from one approach to the other. Hence, accurate modeling of the shear behavior in beams and columns still remains elusive. MCFT is a powerful tool to model the response of reinforced concrete elements subjected to in-plane shear and normal stresses. However, in order to evaluate flexure-shear response of the reinforced concrete columns by MCFT, the member needs to be discretized into large number of biaxially stressed elements and analyzed using nonlinear finite element procedure (Vecchio, 1989). Vecchio and Collins (Vecchio \& Collins, 1988) extended concept of MCFT to fiber model approach for response estimation of reinforced concrete beams loaded in combined axial, shear and flexural forces. In this approach, concrete fibers are treated as biaxially stressed elements in the cross section and analyzed for in-plane stress field based on MCFT. Later, this approach was improved for accurate determination of shear stress distribution on the cross section and advanced formulations were implemented successfully into a non-linear section analysis computer program called Response-2000 (Bentz, 2000). The application of the MCFT in finite element approach or sectional analysis approach yields reliable flexureshear response, but results in fastidious computations which are not simple for practical applications.

Total lateral deformation of a concrete column is mainly comprised of the flexure and shear components. These mechanisms interact with each other and corresponding deformations do not occur independently. For example, in the web of a reinforced concrete column, axial strain due to flexural mechanism will increase principal tensile strain and width of the shear crack resulting in lower shear capacity of the element. On the other hand, it has been established by experimental evidence that principal compressive stress in the concrete is function of principal compressive strain as well as of principal tensile strain (Vecchio \& Collins, 1986). Compressive strength and stiffness of concrete decrease as tensile strains increase. The concrete in the web of laterally loaded element is subjected to shear stresses in addition to the normal stresses due to axial load and flexure. As the shear stresses increase, principal tensile strains increase which will decrease compressive strength of the concrete resulting in lower flexural strength of the element. 
Therefore, any numerical procedure that aims to model overall lateral load-displacement relationship must take the interaction of flexural and shear mechanisms into account. Recently, Mostafaei and Kabeyasawa (Mostafaei \& Kabeyasawa, 2007) presented AxialShear-Flexural Interaction (ASFI) approach for the displacement-based analysis of reinforced concrete elements such as beams, columns and shear walls by considering interaction between axial, shear and flexural mechanisms. This macro-model based approach consists of two models evaluating axial-flexural and axial-shear responses simultaneously to obtain total response of elements subjected to axial, flexural and shear loads. In this approach, axial-flexural behavior is simulated by employing conventional section analysis or fiber model whereas axial-shear response is determined through MCFT by considering one integration point in the in-plane stress conditions. The axial-flexural and axial-shear mechanisms are coupled in average stress-strain field considering axial deformation interaction and softening of concrete compression strength while satisfying compatibility and equilibrium conditions. Although, ASFI approach reduces computational demand considerably as compared to other models implementing MCFT into finite element analysis approach or sectional analysis approach, computational process is still intense and complicated due to coupling of the axial-flexure and axial-shear mechanisms and requires a deliberate iterative scheme at each loading step. However, few concepts from ASFI approach are utilized in the model proposed in this study.

Few studies in the recent past have also addressed the issues of stiffness degradation and strength deterioration in the reinforced concrete elements dominated by shear or shearflexure behaviors. These studies represent advanced formulations for fiber-based element (Ceresa et al., 2007, 2009; Chao \& Loh, 2007; Mullapudi \& Ayoub, 2008, 2010; Xu \& Zhang, 2011; Zhang \& Xu, 2010) and Macro-element model (Mergos \& Kappos, 2008, 2010) and consider interaction between inelastic shear and nonlinear flexural behaviors with different conceptual backgrounds, solution strategies and implementation complexities. A state-of-art review is presented on fiber elements with focus on concentrated plastic-hinge type model that can be implemented in displacement-based finite element programs (Ceresa et al., 2007).

Currently available studies for response estimation of non-ductile reinforced concrete columns show that the approaches that can predict structural behavior with good accuracy employ complicated and computation-intensive procedures that may not be amenable and are difficult to implement. As a result, many approaches try to simplify the process by making simplifying assumptions but in most cases this is done at the cost of accuracy. A suitable procedure is proposed here to address critical modeling issues while predicting the response accurately and keeping overall computational process simple with easy implementation. The authors believe that the proposed model can effectively be employed to predict the strength and total lateral displacement capacity, considering the deformation components due to flexure, shear and reinforcement slip. Implementation of the proposed procedure results in satisfactory response envelope curves which can be used for development of cyclic response models.

\section{The proposed analytical procedure for response estimation of columns}

Flexural and shear deformations in the proposed model are calculated independently while considering the interaction between these mechanisms and then combined together depending upon dominant failure mode. The flexural deformations are determined through 
fiber section model considering shear effects by employing compressive constitutive law for cracked concrete. Shear deformations are calculated by combination of MCFT (Vecchio \& Collins, 1986) and shear response envelope by Sezen (Sezen, 2008), while considering effect of axial strains due to flexure on shear mechanism. Lateral deformation component due to reinforcement slip in beam-column joint regions is determined separately and added to the flexural and shear deformation components to obtain total response. The interaction between flexure and shear mechanisms allows for accurate response estimation while decoupled flexural analysis minimizes complexity of calculations and makes the analysis process relatively simple and easy. In addition, buckling of compression bars under large compressive strains is also incorporated in the analysis by employing separate stress-strain relationships for reinforcing steel in compression. The effects of concrete tension strength and softening of cracked concrete in compression are also considered. The details of the components deformation models and total deformation model are presented in the following sub-sections.

\subsection{Flexural analysis and deformations}

Flexural deformations in the proposed procedure are determined from fiber section analysis in one-dimensional stress field. For the reinforced concrete elements subjected to bending moment and axial load, such as beams or columns, fiber model approach is usually handy and accurate approach if actual stress distribution across the depth of the cross-section is considered. The reliability of the analysis is also directly related to the ability of the constitutive material models to accurately simulate material behavior and level of simplifying assumptions during the analysis. In this approach, a reinforced concrete crosssection is discretized into finite number of concrete and steel fibers. Each of the fibers is idealized as a uniaxial element with its unique stress-strain relationship. Bernoulli's principle, that plane section before bending remains plane after bending, is the main hypothesis in the analysis and implies that the longitudinal strain in concrete and steel at any point in the cross section is proportional to its distance from neutral axis resulting in linear strain distribution. Based upon the resulting strain profile, stress distribution for concrete and reinforcing steel can be determined in accordance with their respective stressstrain relationships. By satisfying equilibrium equations at the cross section, the moment capacity of the section is determined. The process is repeated number of times by incrementing longitudinal stain until either the concrete or steel fails as per defined failure criterion.

In conventional flexural section analysis, the concrete behavior is simulated by its response usually derived from standard cylinder test where it is subjected to uniaxial compression. The strain conditions for the concrete in the web of a laterally loaded reinforced concrete beam or column are significantly different from those in a cylinder test. The concrete in a cylinder test is subjected to only small tensile strains primarily due to Poisson's effect, whereas, the concrete in the web experience shear stresses in addition to the normal stresses due to axial load and flexure. Due to applied shear stresses, concrete in the web cracks diagonally in the direction normal to principal tensile strain. As mentioned earlier, experimental evidence has shown that that principal compressive stress in the concrete is not only the function of principal compressive strain but is also affected by the coexisting principal tensile strain in a way that compressive strength and stiffness of the concrete 
decrease as tensile strains increase (Vecchio \& Collins, 1986). This implies that the concrete subjected to combined normal compressive and shear stresses is weaker in compression than the concrete subjected to normal compressive stresses only. Hence, the concrete in the web of a laterally loaded column must exhibit weaker and softer response as compared to the concrete subjected to uniaxial compression in cylinder test. The behavior of the cracked concrete in the manner explained above is called compression-softening and is illustrated in Figure 2. In this figure, $f_{c}^{\prime}$ is compressive strength of the concrete, $f_{c 2}$ is principal compressive stress in the concrete and $\varepsilon_{c 2}$ is principal compressive strain in the concrete.

The effect of shear stress on degrading compressive strength of the concrete can be taken into account by considering compressive stress-strain relationships of diagonally cracked concrete in flexural section analysis instead of employing conventional constitutive relationship for uniaxially compressed concrete. This can be done by softening the response of concrete in uniaxial compression by a factor which decreases as shear deformations increase. This factor, known as compression softening factor $\beta$, is function of principal tensile strain in the concrete and is defined as following (Vecchio \& Collins, 1986).

$$
\beta=\frac{1}{0.8-0.34 \frac{\varepsilon_{c 1}}{\varepsilon_{c o}}} \leq 1.0
$$

where $\varepsilon_{c o}$ is concrete strain corresponding maximum concrete cylinder strength and $\varepsilon_{c 1}$ is principal tensile strain in the concrete which can be determined through in-plane shear analysis of the flexural element. The procedure for determining principal tensile strain in the concrete and compression softening factor is explained in the subsequent section.

In addition to considering cracked concrete behavior in the fiber model, enhancement in the strength and ductility of the concrete due to confinement and contribution of the concrete tensile properties to section moment capacity must also be considered in the analysis. For determining realistic moment capacity and analyzing the buckling of the longitudinal bars under excessive compressive strains, confined core concrete and unconfined cover concrete are modeled separately with their respective stress-strain relationships.

Fiber model analysis results in a moment-curvature relationship for given geometric and material properties, reinforcement details and applied axial load for the cross-section being analyzed. From here, lateral load $V$ corresponding to respective moment capacity, resulting average shear stress from flexural analysis $\tau_{f}$ and maximum lateral force sustainable by the column can be calculated with the help of following equations.

$$
V=\frac{M}{a} \quad \tau_{f}=\frac{V}{b d} \quad V_{p}=\frac{M_{p}}{a}
$$

where, $M$ is the flexural section moment capacity at any load level, $b$ is width of the section, $d$ is the effective depth of the section, $a$ is the shear span equal to cantilever column length and one half of the length a fixed ended column, and $M_{p}$ is maximum moment capacity from flexural section analysis.

Flexure deformations are calculated with the help of plastic hinge model in which elastic and inelastic curvatures are idealized separately. In this model, a linear curvature 
distribution is assumed in the elastic range over the length of the column, and the inelastic curvatures are lumped at the column end over the plastic hinge length. The conceptual illustration of the plastic hinge model for a cantilever column is presented in Figure 3. Hence, lateral displacement due to flexure $\Delta_{f}$ can be calculated by integrating curvature over the length of the column as per Equation 3.

$$
\Delta_{f}=\int_{0}^{a} \phi(x) x d x
$$

where $\phi(x)$ is section curvature at distance $x$ measured along column axis, and $\phi_{y}$ is curvature at yield point. The plastic hinge length $L_{p}$ is taken as one-half of the section depth $h$.

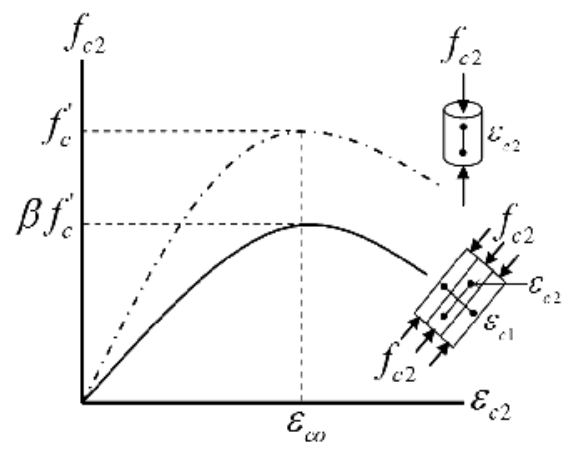

Fig. 2. Behavior of the cracked concrete in compression

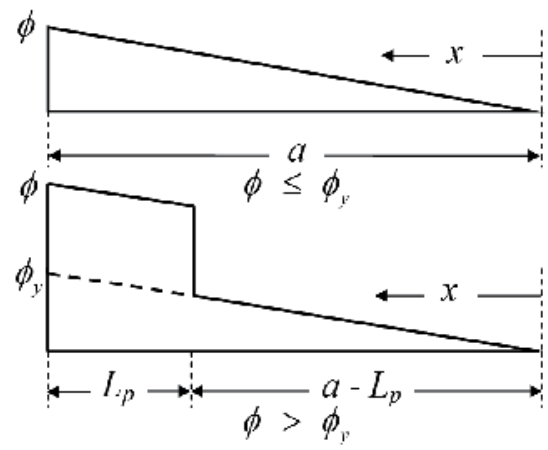

Fig. 3. Plastic hinge model for calculating flexural displacements

\subsection{Reinforcement slip deformations}

When a reinforced concrete column is subjected to bending moment, strain accumulates in the embedded length of the tensile reinforcing bars. This causes the bars to extend or slip relative to the anchoring concrete at column fixed end(s). The extension is commonly known as reinforcement slip and leads to rigid-body rotation of the column, as shown in Figur 1. This results in an additional lateral displacement component that can be as large as 25 to 40 
$\%$ of the total lateral displacement (Sezen, 2002). Flexural deformations determined from conventional fiber section analysis (moment-curvature analysis) do not account for lateral deformations caused by reinforcement slip at column ends. Therefore, these deformations must be calculated separately and added to the other deformation components due to flexure and shear to calculate the total lateral displacement.

In this study, lateral displacement due to reinforcement slip is calculated using the model illustrated in Figure 4 (Sezen \& Setzler, 2008).
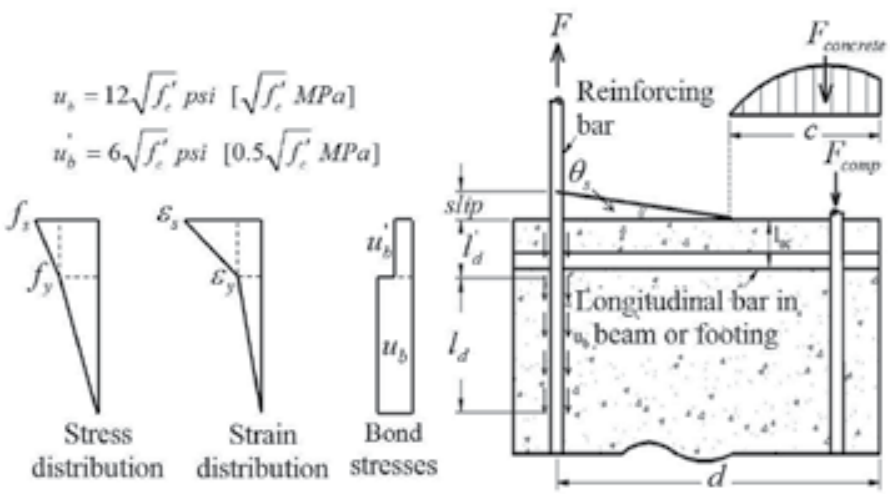

Fig. 4. Reinforcement slip model (Sezen \& Setzler, 2008)

The model approximates the bond stress as bi-uniform function with different values for elastic and inelastic steel behaviors. The bond stress in the elastic and inelastic range is taken as $u_{b}=12 \sqrt{f_{c}^{\prime}} p s i\left(1 \sqrt{f_{c}^{\prime}} M P a\right)$ and $u_{b}^{\prime}=6 \sqrt{f_{c}^{\prime}} p s i\left(0.5 \sqrt{f_{c}^{\prime}} M P a\right)$, respectively, where $f_{c}^{\prime}$ is concrete compressive strength. Slip $s$ at the loaded end of the reinforcing bar can be calculated by integrating bi-linear strain distribution $\varepsilon_{s}(x)$ over the development length as follows.

$$
S=\int_{0}^{l_{d}+l_{d}^{\prime}} \varepsilon_{s}(x) d x
$$

where $l_{d}=\frac{f_{s} d_{b}}{4 u_{b}}$ and $l_{d}^{\prime}=\frac{\left(f_{s}-f_{y}\right) d_{b}}{4 u_{b}^{\prime}}$ are development lengths for the elastic and inelastic portion of the bar, respectively. Hence, integrating Equation 4, extension or slip of the reinforcing bars is

$$
s= \begin{cases}\frac{\varepsilon_{s} l_{d}}{2}=\frac{\varepsilon_{s} f_{s} d_{b}}{8 u_{b}} & \text { for } \varepsilon_{s} \leq \varepsilon_{y} \\ \frac{\varepsilon_{y} l_{d}}{2}+\frac{\left(\varepsilon_{s}+\varepsilon_{y}\right) l_{d}^{\prime}}{2}=\frac{\varepsilon_{y} f_{y} d_{b}}{8 u_{b}}+\frac{\left(\varepsilon_{s}+\varepsilon_{y}\right)\left(f_{s}-f_{y}\right) d_{b}}{8 u_{b}^{\prime}} & \text { for } \varepsilon_{s}>\varepsilon_{y}\end{cases}
$$

where $\varepsilon_{s}$ is the strain at loaded end of the bar, $\varepsilon_{y}$ is steel yield strain, $f_{s}$ is stress at loaded end of the bar, $f_{y}$ is steel yield stress, and $d_{b}$ is diameter of the longitudinal bar. The 
reinforcement slip is assumed to occur in tension bars only and cause the rotation about the neutral axis as shown in Figure 4. Rotation caused due to reinforcement slip can be calculated as,

$$
\theta_{s}=\frac{s}{d-c}
$$

where $d$ and $c$ are the distances from the extreme compression fiber to the centroid of the tension steel and neutral axis, respectively. The lateral displacement due to slip at free end of a cantilever column can be calculated as the product of slip rotation $\theta_{s}$ and length of the column $L$ as,

$$
\Delta_{s}=\theta_{s} L
$$

\subsection{Shear deformations}

Shear deformations in reinforced concrete members have traditionally been ignored in design and research due to lack of their complete understanding and being difficult to measure, independent of other deformation components, in an experimental set up or a real structure. For a well designed reinforced concrete column, shear deformations are small as compared to the flexural deformations and are often less than 10 percent of total deformations. Contrary, for a reinforced concrete column not designed according to stricter seismic design provisions, shear behavior could be the governing failure criterion. Shear deformations in such shear critical reinforced concrete column could contribute large percentage towards total deformations and hence can not be ignored if an accurate analysis of deformation capacity is required.

Shear deformations in the proposed model are calculated using a combination of MCFT (Vecchio \& Collins, 1986) and post-peak shear response envelope (Patwardhan, 2005; Sezen, 2008). In this model, pre-peak non-linear shear force-shear deformation response is obtained from in-plane analysis of the shear element based on MCFT while considering the interaction of the axial strain (Mostafaei \& Kabeyasawa, 2007). Axial strain obtained from flexural section analysis is incorporated into the total axial strain of the shear element to include the effect of flexural behavior on shear response. After the peak strength has reached, shear strength is first assumed to remain constant at its peak value until the onset of the shear strength degradation and then declines linearly with increasing shear deformations to the point of axial load failure (Figure 5). At the point of axial load failure, lateral strength is assumed zero. The peak strength $V_{\text {peak }}$ in the proposed shear response model refers to the point where response estimation by MCFT terminates either due to shear failure or reaching the load step corresponding to the peak flexural strength prior to experiencing shear failure. Hence, the peak strength $V_{\text {peak }}$ is the minimum of the shear strength of the column $V_{n}$ and shear force corresponding to the maximum moment that can be carried by the section $V_{p}$.

Shear displacements at the onset of shear degradation $\Delta_{v, u}$ can be calculated as follows (Patwardhan, 2005; Sezen, 2008).

$$
\Delta_{v, u}=\left(4-12 \frac{v_{n}}{f_{c}^{\prime}}\right) \Delta_{v, n}
$$




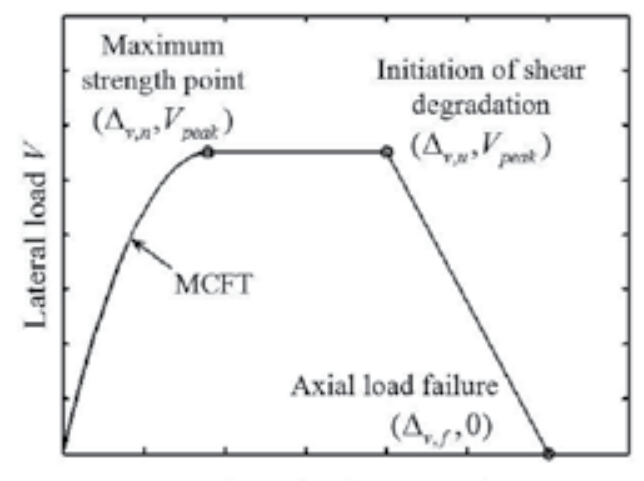

Shear displacement $\Delta_{v}$

Fig. 5. Shear response model

where $v_{n}=\frac{V_{\text {peak }}}{b d}$ is the shear stress at peak strength, $f_{c}^{\prime}$ is the concrete compressive strength, and $\Delta_{v, n}$ is the shear displacement corresponding to the peak strength as determined from MCFT analysis. The shear displacement at axial load failure $\Delta_{v, f}$ is calculated as,

$$
\Delta_{v, f}=\Delta_{A L F}-\Delta_{f, f}-\Delta_{s, f} \geq \Delta_{v, u}
$$

where $\Delta_{A L F}$ is the total displacement at axial load failure, $\Delta_{f, f}$ and $\Delta_{s, f}$ are the flexural and slip displacement at the point of axial load failure, respectively. The total displacement at axial load failure is determined by the expression based on a shear friction model and an idealized shear failure plane (Elwood \& Moehle, 2005).

$$
\Delta_{A L F}=\frac{0.04 L\left(1+\tan ^{2} \theta\right)}{\left(\tan \theta+P\left(\frac{s_{h}}{A_{s v} f_{y v} d_{c} \tan \theta}\right)\right)}
$$

where $\theta$ is the angle of the shear crack, $P$ is the axial load, $A_{s v}$ is the area of transverse steel with yield strength $f_{y v}$ at spacing $s_{h}$, and $d_{c}$ is the depth of the core concrete measured to the centerlines of the transverse reinforcement. In the derivation, $\theta$ is assumed to be 65 degrees. The values of $\Delta_{f, f}$ and $\Delta_{s, f}$ in Equation 9 are determined according to the expected failure mode and classification of the column into categories as explained in subsequent subsection.

\subsection{Total lateral response}

In order to model the response of a column subjected to lateral loading, three deformation components should be combined together considering their interconnectedness. In this study, total column lateral response is modeled as a set of three springs in series; each spring representing lateral displacement component due to flexure, bar slip and shear. Each 
spring is subjected to same force and total displacement is sum of responses of each spring. The pre-peak total response is obtained by simply adding deformation components due to flexure, bar slip and shear mechanism as described above. After reaching the peak, the mechanism limiting the peak strength (flexure or shear) will dominate the behavior. The procedure for combining deformation components for post peak is explained below and the model is illustrated in Figure 6.

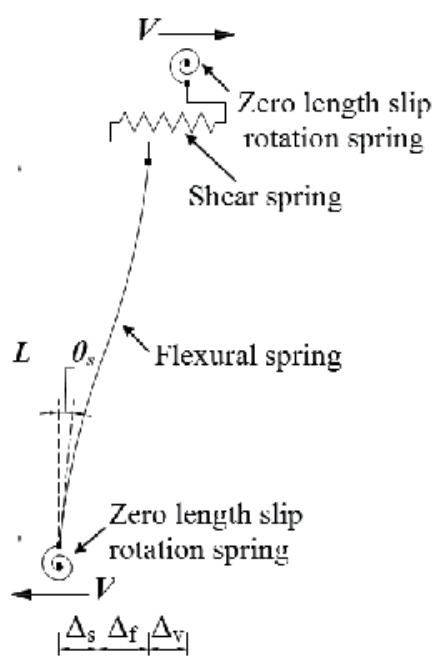

Fig. 6. Spring representation of total response model

For post-peak behavior, the column is classified into one of the five categories based on a comparison of its shear, yield and flexural strength and rules are specified for the combination of the deformation components for each category (Setzler \& Sezen, 2008). The yield strength $V_{y}$ is defined as the lateral load corresponding to the first yielding of the tension bars in the column and flexural strength $V_{p}$ is the lateral load corresponding to the peak moment calculated from flexural analysis. Both of these loads are calculated from moment-curvature analysis of the fiber model as explained above. The shear strength $V_{n}$ for the columns failing in shear prior to the reaching flexural strength or failing close to flexural strength is determined from the proposed shear model, where $V_{n}=V_{\text {peak }}$ as discussed above. For other columns where peak strength by the proposed shear model is close to the flexural strength, shear strength is calculated as a function of displacement ductility (Sezen \& Moehle, 2004).

$$
V_{n}=k\left(V_{c}+V_{s}\right)=k\left[\left(\frac{6 \sqrt{f_{c}^{\prime}}}{a / d} \sqrt{1+\frac{P}{6 \sqrt{f_{c}^{\prime}} A_{g}}}\right) 0.80 A_{g}+\frac{A_{s v} f_{y v} d}{s}\right]
$$

where $V_{c}$ is the concrete contribution to shear strength, $V_{s}$ is the steel contribution to shear strength, $A_{g}$ is gross cross-sectional area, $a / d$ is the aspect ratio and $k$ is a factor related to the displacement ductility which is the ratio of the maximum displacement to the yield displacement. The value for $k$ varies from 0.7 to 1.0 for displacement ductilites from less than 2 to grater than 6 respectively. In this study, the value for $\mathrm{k}$ is taken as 1.0 as classification of the columns is based on initial or low-ductility shear and flexural strengths. 
The classification system and rules governing the post peak response in each category are described below and are illustrated in Figure 7. Peak response of the column is limited by the smaller of the shear strength and flexural strength, however post-peak response is assumed to be governed by the limiting mechanism (i.e., flexure or shear).

\subsubsection{Category - I $\left(V_{n}<V_{y}\right)$}

In this category of the columns, shear strength is less than the yield strength and column fails in shear while the flexural behavior remains elastic. The deformation at peak strength (i.e., shear strength) is the sum of deformations in each spring at the peak strength. After the peak strength is reached, the shear behavior dominates the response. As the shear strength degrades, the flexure and slip springs unload along their initial responses. The post-peak deformation at any lateral load level is the sum of the post-peak shear deformation and the pre-peak flexural and slip deformations corresponding to that load.

\subsubsection{Category - II $\left(V_{y} \leq V_{n}<0.95 V_{p}\right)$}

The shear strength is less than flexural strength and column fails in shear, however inelastic flexural deformation occurring prior to shear failure affects the post-peak behavior. The deformation at peak strength is the sum of the deformations in each spring at the peak strength. Shear deformations continue to increase after the peak shear strength is reached, but the flexure and shear springs are locked at their peak strength values. Hence, post-peak deformations at any lateral load level is the sum of flexural and slip deformations at the peak strength and post-peak shear deformation at that load.

\subsubsection{Category - III $\left(0.95 V_{p} \leq V_{n} \leq 1.05 V_{p}\right)$}

The shear and flexural strengths are nearly identical. Shear and flexural failure are assumed to occur "simultaneously," and both mechanisms contribute to the post-peak behavior. The post-peak deformation at any lateral load level is the sum of the post-peak flexure, slip, and shear deformations corresponding to that load.

\subsubsection{Category - IV $\left(1.05 V_{p}<V_{n} \leq 1.4 V_{p}\right)$}

The shear strength is greater than the flexural strength and the column may potentially fail in the flexure, however large shear deformations affect the post-peak behavior and shear failure may occur as the displacements increase. The deformation at peak strength is the sum of the deformations in each spring at the peak strength. After the peak strength is reached, flexural and slip deformations continue to increase according to their models, but the shear spring is locked at its value at peak strength. The post-peak deformation at any lateral load level is the sum of the post peak flexural and slip deformations corresponding to that load and the shear deformation at peak strength.

\subsubsection{Category - V $\left(V_{n}>1.4 V_{p}\right)$}

The shear strength is much greater than the flexural strength and column fails in flexure while shear behavior remains elastic. The peak strength of the column is the flexural strength calculated from the flexure model. If the column strength degrades, flexural and 
slip deformations continue to increase according to their models, while the shear spring unloads with an unloading stiffness equal to its initial stiffness. The post-peak deformation at any lateral load level is the sum of the post-peak flexural and slip deformations and the pre-peak shear deformation corresponding to that load.

For category-I columns, $\Delta_{f, f}$ and $\Delta_{s, f}$ values to be used in Equation 9 are assumed zero.

For the category-II columns, shear strength is lesser than flexural strength and these values are taken as the flexural and slip deformations at the load equal to the shear strength of the columns. For categories III, IV, and V specimens, $\Delta_{f, f}$ and $\Delta_{s, f}$ are the maximum calculated flexural and slip deformations.

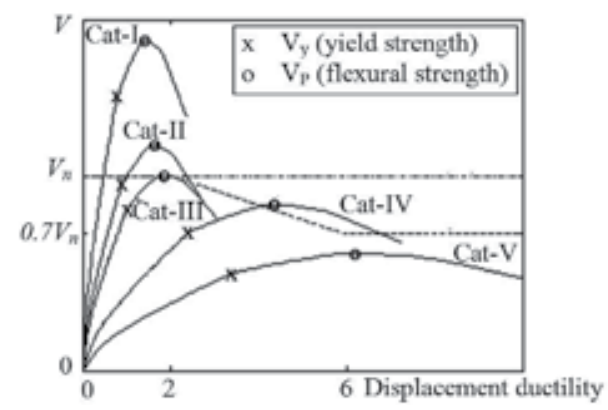

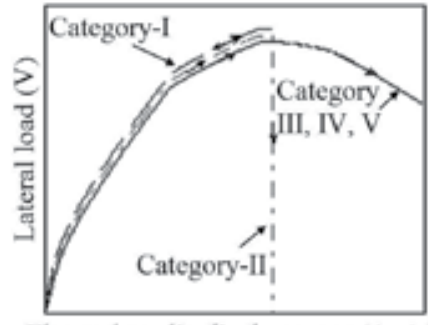

Flexural or slip displacement $\left(\Delta_{6}, \Delta_{s}\right)$

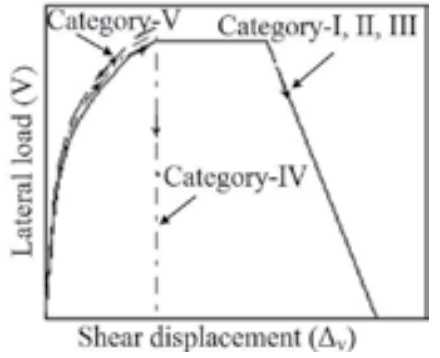

Shear displacement $\left(\Delta_{v}\right)$

Fig. 7. Classification of columns into categories and rules governing combination of the deformation components (Setzler \& Sezen, 2008)

\section{Interaction between flexure and shear mechanisms}

When a fixed-ended reinforced concrete column is subjected to lateral loading, such as during an earthquake, flexural and shear mechanisms interact with each other and affect overall response of the column. The interaction between flexural and shear deformations in the proposed analytical procedure is based on the ASFI approach (Mostafaei \& Kabeyasawa, 2007). Interaction methodology in ASFI approach couples axial-flexure and axial-shear models with each other. Both mechanisms have to be evaluated simultaneously which makes ASFI approach relatively complicated and computationally intensive. The computational effort can be reduced significantly, if the analyses for flexural and shear behavior can be performed independently. Therefore, in the proposed procedure, the interaction of the shear deformations on flexural performance, and vice versa, are considered in a simplified manner that allows easy implementation and decoupled flexural and shear response evaluations. 


\subsection{Interaction of concrete compression softening}

Cracked concrete behavior is considered in flexural section analysis to represent degradation in compressive strength of the concrete due to applied shear stresses. This requires determination of the compression softening factor $\beta$ to lower concrete stresses in uniaxial compression (Figure 2). The procedure for determining compression softening factor is adopted from the recently developed model called Uniaxial-Shear-Flexure Model (USFM) (Mostafaei \& Vecchio, 2008). This is an approximate approach for response estimation of reinforced concrete elements which is derived after simplifying ASFI approach. USFM employs few fundamental equations of the MCFT and two assumptions on average principal compressive strain and average centroidal axial strain of the element to determine average principal tensile strain. The details of formulation, implementation and verification of USFM approach can be found in (Mostafaei \& Vecchio, 2008).

Compression softening factor $\beta$, as defined in Equation 1, is a function of concrete principal tensile strain $\varepsilon_{c 1}$ of the element being analyzed. The procedure to approximately determine principal tensile strain and subsequently compression softening factor for a fixed ended column subjected to in-plane lateral load is illustrated in Figure 8. For an element considered between inflection point and one of the end sections of the column, $\varepsilon_{c 1}$ can be determined from the following MCFT equation.

$$
\varepsilon_{c 1}=\varepsilon_{x}+\varepsilon_{y v}-\varepsilon_{c 2}
$$

where, $\varepsilon_{x}$ is average axial strain at the centroid for the element and is obtained by averaging the values of centroidal axial stain at one of the end section $\varepsilon_{0}$ and axial strain of the inflection point $\varepsilon_{x a}$. Likewise $\varepsilon_{c 2}$ is average concrete principal compressive strain for the element. Its value, as per USFM assumption (Mostafaei \& Vecchio, 2008), can be taken as the average of the uniaxial concrete compressive strain corresponding to resultant compressive force of the stress block at end section $\varepsilon_{c}$ and axial strain at the inflection point $\varepsilon_{x a}$. Hence,

$$
\varepsilon_{x}=\frac{\left(\varepsilon_{0}+\varepsilon_{x a}\right)}{2} \quad \varepsilon_{c 2}=\frac{\left(\varepsilon_{c}+\varepsilon_{x a}\right)}{2}
$$

The other unknown quantity in Equation 12, strain of the transverse reinforcement $\varepsilon_{y v}$, can be determined from the following MCFT based relationship.

$$
\varepsilon_{y v}=\sqrt{b^{2}+c}-b
$$

where,

$$
b=\frac{f_{c 1}}{2 \rho_{y} E_{s y}}-\frac{\varepsilon_{c 2}}{2}, c=\frac{\left(\varepsilon_{x}-\varepsilon_{c 2}\right)\left(f_{c 1}-f_{c x}\right)+f_{c 1} \varepsilon_{c 2}}{\rho_{y} E_{s y}}, f_{c x}=f_{x}-\rho_{x} f_{s x}
$$

where $\rho_{y}$ is transverse reinforcement ratio, $E_{s y}$ is modulus of elasticity of transverse reinforcement, $f_{c x}$ is concrete stress in longitudinal axis of the column, $f_{x}$ is applied axial stress, $\rho_{x}$ is longitudinal reinforcement ratio, $f_{s x}$ is longitudinal steel stress obtained from 
section analysis based on average centroidal strain, $f_{c 1}=0.145 \sqrt{f_{c}^{\prime}}$ is concrete principal tensile stress (in $\mathrm{MPa}$ ), $\varepsilon_{x}$ is normal strain at the centroid and $\varepsilon_{c 2}$ is average concrete principal compressive strains, both determined from Equation 13.

After calculating concrete principal tensile strain $\varepsilon_{c 1}$ from Equation 12, compression softening factor $\beta$ is determined from Equation 1 for a given curvature. This is the estimated value of $\beta$ which is employed in fiber model analysis to lower concrete stresses. In the proposed procedure, compression softening factor determined with the help of above mentioned procedure is employed till peak flexural strength and then a constant value equal to the last lowest is used for post-peak flexural analysis.

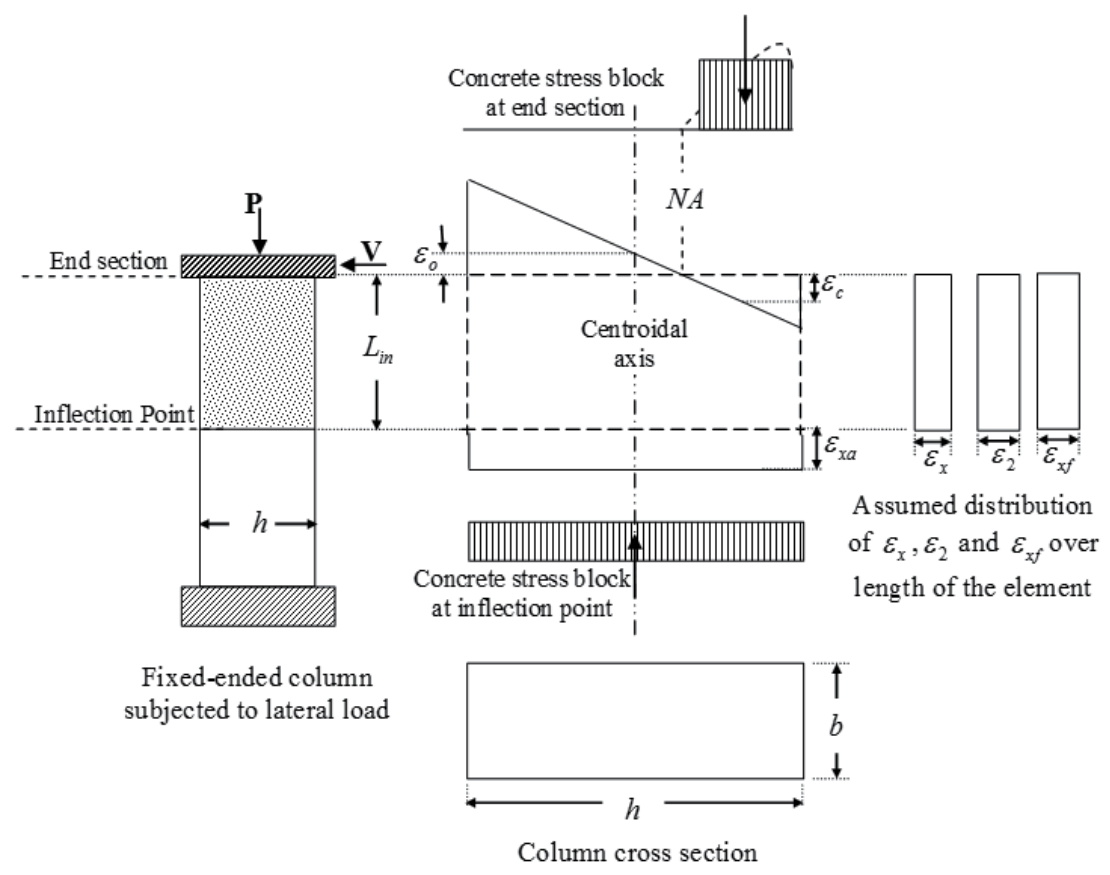

Fig. 8. Interaction of compression softening and axial strains (Mostafaei \& Kabeyasawa, 2007; Mostafaei \& Vecchio, 2008).

\subsection{Interaction of axial strains}

The effect of flexural deformations on shear behavior is considered by incorporating axial strain and shear stress due to flexure into in-plane analysis of the shear element based on axial strain interaction methodology of ASFI approach (Mostafaei \& Kabeyasawa, 2007) and equilibrium of shear stresses in flexural and shear mechanisms. In this procedure, interaction of axial strain is taken into account by adding flexibility component of axial deformation due to flexure to the corresponding flexibility component of axial-shear model. By employing flexural shear stress to in-plane stress-strain relationship of the shear element, shear deformations are determined. The procedure for axial deformation interaction and determination of shear strain is described here for a fixed ended column subjected to lateral load. 
The length of the column between inflection point and one of the end sections is considered as a shear element subjected to constant normal stress due to applied axial load and average shear stresses due to applied lateral load. Performing flexural analysis on fiber model of the end section and inflection point, average centroidal axial strain due to flexure $\varepsilon_{x f}$ (Figure 8) and corresponding flexibility component $f_{x f}$ can be determined with the help of following ASFI equations (Mostafaei \& Kabeyasawa, 2007).

$$
\varepsilon_{x f}=\frac{\left(\varepsilon_{o}-\varepsilon_{x a}\right)}{2}, f_{x f}=\frac{\varepsilon_{x f}}{\sigma_{x}}, \quad \sigma_{x}=\frac{P}{b d}
$$

where, $\sigma_{x}$ is applied axial stress in longitudinal direction of the column and can be determined by dividing the applied axial load $P$ by the effective area of the cross section. A stress-strain relationship in terms of flexibility matrix for an in plane shear element (axialshear model) can be defined as,

$$
\left(\begin{array}{lll}
f_{11} & f_{12} & f_{13} \\
f_{21} & f_{22} & f_{23} \\
f_{31} & f_{32} & f_{33}
\end{array}\right)\left\{\begin{array}{l}
\sigma_{x} \\
\sigma_{y} \\
\tau_{x y}
\end{array}\right\}=\left\{\begin{array}{l}
\varepsilon_{x} \\
\varepsilon_{y} \\
\gamma_{x y}
\end{array}\right\}
$$

where $f_{i j}(i, j=1,2,3)$ are flexibility components of in plane shear model, $\sigma_{x}$ is normal applied stresses in longitudinal direction, $\sigma_{y}$ is normal stress in transverse direction, $\tau_{x y}$ is shear stress, $\varepsilon_{x}$ is normal strain in axial direction, $\varepsilon_{y}$ is normal strain in transverse direction, and $\gamma_{x y}$ is shear strain.

Axial strain due to flexure $\varepsilon_{x f}$ can be taken into account in the axial-shear model by adding flexibility component obtained from Equation 16 into Equation 17.

$$
\left(\begin{array}{ccc}
\left(f_{11}+f_{x f}\right) & f_{12} & f_{13} \\
f_{21} & f_{22} & f_{23} \\
f_{31} & f_{32} & f_{33}
\end{array}\right)\left\{\begin{array}{l}
\sigma_{x} \\
\sigma_{y} \\
\tau_{x y}
\end{array}\right\}=\left\{\begin{array}{c}
\varepsilon_{x}+\varepsilon_{x f} \\
\varepsilon_{y} \\
\gamma_{x y}
\end{array}\right\}
$$

In Equation 18, stresses in transverse direction (clamping stresses) are zero due to inexistence of lateral external force along the column, i.e., $\sigma_{y}=0$. In addition, the applied shear stress $\tau_{x y}$ of the element is taken from flexural section analysis (Equation 2) as $\tau_{x y}=\tau_{f}$. In Equation 18, knowing the applied stresses, corresponding strains can be calculated. The flexibility matrix is obtained by inverting material stiffness matrix of the shear element formulated using secant stiffness methodology of the MCFT approach (Vecchio \& Collins, 1986).

\section{Buckling of compression bars}

Longitudinal reinforcing bars in columns may experience inelastic axial compression under severe loading and exhibit large lateral deformation known as buckling. The behavior in the compressive face of a concrete member at overload depends on a variety of factors such as, size and shape of the cross-section, the amount of longitudinal compression steel, the amount of transverse reinforcement providing confinement to the section, thickness of the 
cover concrete, and stress-strain properties for the steel and concrete (Potger et al., 2001). The tendency for the compressively loaded steel bars to buckle and deflect outwards is initially resisted by the lateral restraint provided by the surrounding cover concrete as well as the transverse steel ties. As the compressive loads increase and approach the section capacity, the concrete surrounding the compressive bars carries large longitudinal compressive stress, and eventually becomes prone to longitudinal cracking, and spalling. After the cover concrete spalls off, ties restrain lateral movement and buckling.

In this study, compression steel stresses were reduced to account for buckling using the model shown in Figure 9. According to this model, compression stresses in longitudinal bars start to decrease when unconfined cover concrete starts to spall. When this happens, corresponding strain in the relevant steel layer can be calculated from flexural strain distribution across the cross section depth. This strain is $\varepsilon_{s p}$ as shown in the figure. This point can fall anywhere on typical stress-strain relationship for steel depending upon the level of flexural strain. Steel stresses follow their usual constitutive stress-strain relationship until strain reaches this limit. Then compression stresses in reinforcement follow new path defined by a line joining peak stress point to residual strength point having a slope $m$, which is calculated from following relationship (Inoue \& Shimizu, 1988).

$$
m=100 \varepsilon_{y x}\left(\frac{1}{\sqrt{1+500 \lambda^{2}}}-1\right) E_{s x} ; \lambda=\frac{\alpha s_{h}}{i_{r}}
$$

where, $\varepsilon_{y x}$ is yield strain of longitudinal bars, $E_{s x}$ is modulus of elasticity for longitudinal steel, $\alpha$ is 1.0 for corner bars and 0.5 for intermediate bars, $s_{h}$ is stirrup or tie spacing, and $i_{r}$ is radius of gyration of longitudinal bar.

Diameter of the longitudinal bar and spacing of the transverse reinforcement is important parameters that affect the buckling of the compression bars (Monti \& Nuti, 1992). Smaller diameter bars restrained by widely spaced ties are most likely to undergo lateral deformations and buckling much earlier during loading history than larger diameter bars confined by closely spaced transverse reinforcement. Therefore, in the proposed model, for tie spacing to bar diameter ratio $s_{h} / d_{b}$ of less than 5.00 , no buckling is considered and compressive behavior of the reinforcement is similar to its tensile behavior. For $s_{h} / d_{b}$ ratio above 11.00, the bars are considered to buckle as soon as reinforcement yields. For $s_{h} / d_{b}$ ratios between 5.00 and 11.00, post-buckling softening is considered soon after spalling of the cover concrete with the proposed model.

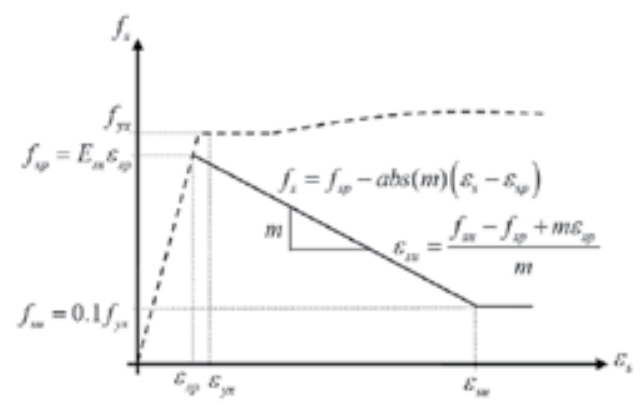

Fig. 9. Proposed compression bar buckling model 


\section{Analytical steps for implementation of the proposed procedure}

Total lateral displacement of a concrete column can be calculated under lateral loads. Note that the procedure can be used to model the response prior to peak strength (under increasing loads) and also beyond peak response (possible decreasing lateral loads due to strength and stiffness degradation). The major steps of the proposed procedure are to:

- Define uniaxial material properties for unconfined and confined concrete, and reinforcing steel. Include the effect of compression softening of concrete (Equation 1 and Figure 2). Consider the effect of compression bar buckling under large axial deformations (Equation 19 and Figure 9).

- Define fiber cross section for flexural analysis and perform moment-curvature analysis of the cross section.

- Calculate lateral load versus flexural displacement by integrating curvatures over the height of column (Equations 2 and 3).

- Calculate lateral load versus reinforcement slip displacement (Equations 5 through 7).

- Perform MCFT analysis to lateral load versus shear displacement up to maximum shear strength (Figure 5) while considering the interaction of axial strains due to flexure (Mostafaei \& Kabeyasawa, 2007). Alternatively, an approximate method (Sezen, 2008) can be used and this step can be skipped. Obtain lateral load versus shear displacement envelope (Figure 5).

- Classify the column and combine flexure, slip, and shear responses (Section 3.4, and Figure 7). During calculation of combined or total displacement, at each step, consider the interaction between axial-shear and flexure mechanisms (Section 4 and Figure 8).

\section{Model verification}

The proposed procedure is implemented to evaluate response of previously tested reinforced concrete columns (Sezen, 2002) and predicted responses are compared with experimental test data. The columns tested by (Sezen, 2002) are very useful as these provide experimental force-displacement data for each of the flexure, slip, and shear components individually in addition to the overall response. Hence, the experimental data from these columns are used to validate the component and total deformation models proposed in this study (Lodhi, 2010; Sezen \& Moehle 2006).

These columns were lightly reinforced and had shear and flexural design strengths very close to each other. These are $18 \mathrm{in} .(457 \mathrm{~mm})$ square columns with fixed ends at top and bottom having height of 116 in. (2946 mm). The columns had eight No.9 bars and No. 3 column ties with 90-degree end hooks spaced at 12 in. (305 mm). Specimens- 1 and -4 were tested with a constant axial load of $150 \mathrm{kip}(667 \mathrm{KN})$, whereas, Specimen-2 was tested under a constant axial load of $600 \mathrm{kip}(2670 \mathrm{KN})$. The columns were tested under unidirectional cyclic lateral loading, except for Specimen-4, which was tested under monotonically increasing load after few initial cycles of elastic loading. All of the test specimens are modeled with average concrete compressive strength of 3.08 psi (21.2 MP). The yield strength of longitudinal and transverse reinforcement are taken to be $63 \mathrm{ksi}(434 \mathrm{MP})$ and 69 ksi $(476 \mathrm{MP})$, respectively. Other details of test specimens, material properties used for the development of reinforcing steel and unconfined and confined concrete models can be found in (Setzler \& Sezen, 2008; Sezen, 2002, 2008). 
Lateral load-flexural displacement relationships for Specimen-1 and 4 are presented in Figure 10 (a) and 10(b), respectively. In this comparison, test specimens are also analyzed using another displacement component model (Setzler \& Sezen, 2008). This model also treats deformations due to flexure, bar slip and shear individually, however, does not consider softening of concrete compression strength, concrete tensile behavior and buckling of compression bars in flexural analysis. It can be seen that both approaches predict identical pre-peak responses, which matches very well with the experimental data. Peak load and deformation at peak load is also estimated very well by both approaches. For post peak behavior, however, predicted responses are quite different. After reaching the strains corresponding to the start of compression bar buckling, response predicted by the proposed procedure gradually drops and generally follows stiffness of the measured response. The diverging near-peak and post-peak predicted responses by both approaches highlight the need to consider concrete softening and bar buckling effect in the analysis. Figure 10 (c) and 10(d) presents load-displacement relationships due to reinforcement slip for Specimen-1 and 2 , respectively. The predicted responses by displacement component model and proposed method produce almost identical response up until peak load and then diverge in the post peak range. Again, this highlights the need for considering buckling of compression bars in the flexural analysis.

Lateral load-shear displacement relationships for Specimen-1 and 2 are presented in Figure 10(e) and 10(f), respectively. For comparison of the predicted shear responses, the columns are also analyzed with ASFI approach (Mostafaei \& Kabeyasawa, 2007). The predicted responses by ASFI approach and proposed procedure are identical until observed peak and follows experimental data generally well. Peak shear strength is generally captured well by both approaches. For post-peak behavior, proposed model shows strength degradation as deformations increase. In ASFI approach, after reaching peak load, shear deformations are calculated from secant stiffness at peak strength, which is kept constant for post peak behavior. As a result, post-peak predicted shear response in ASFI approach does not show shear strength degradation.

Figure 11 shows the comparison of predicted and experimental lateral load-total displacement relationships for Specimen-1, 2 and 4. Shear strength of Specimen-1 (Figure 11(a)) is calculated as 69.0 kips and flexural strength from moment-curvature analysis is 70.0 kips. Hence, this specimen is classified as category-III column, for which total displacement at any point in the response is sum of flexural, slip and shear displacement at that load step. With the proposed procedure, initial response is predicted very well up to the peak strength. Peak strength and deformation at peak load and the post peak response are captured well. The Specimen-2 (Figure 11(b)) has shear and flexural strengths of 92.0 and 72.0 kips, respectively, and is classified as category-IV column. For this column, shear deformation is frozen at its value at peak strength (flexural strength, $72.0 \mathrm{kips}$ ) and added to flexural and slip displacements for post-peak response. Predicted response by the proposed approach slightly overestimates the pre-peak stiffness and peak load in the positive direction and follows post peak experimental response fairly well in both directions. Specimen-4 (Figure 11(c)) is identical to Specimen-1 except that it was tested under monotonically increasing lateral load after few initial elastic cycles. Comparison of shear and flexural strength classifies this column into category-III column. The predicted response by the proposed procedure follows the trend in experimental data but slightly overestimates the initial stiffness and peak strength. 


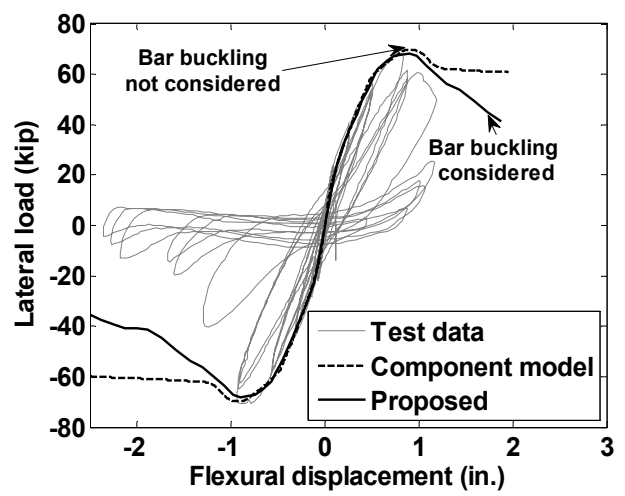

(a)

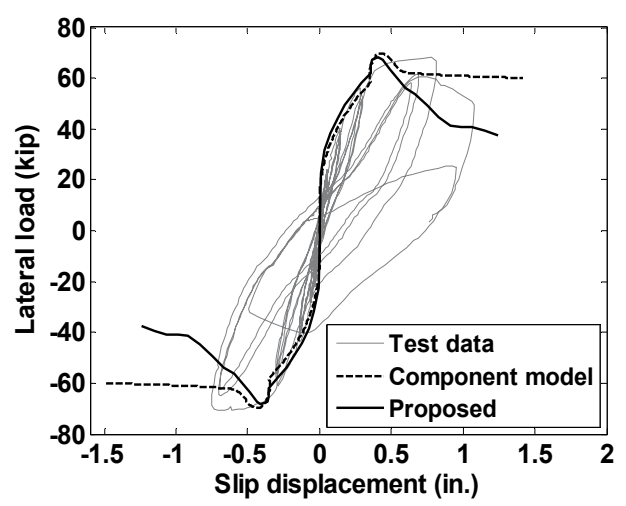

(c)

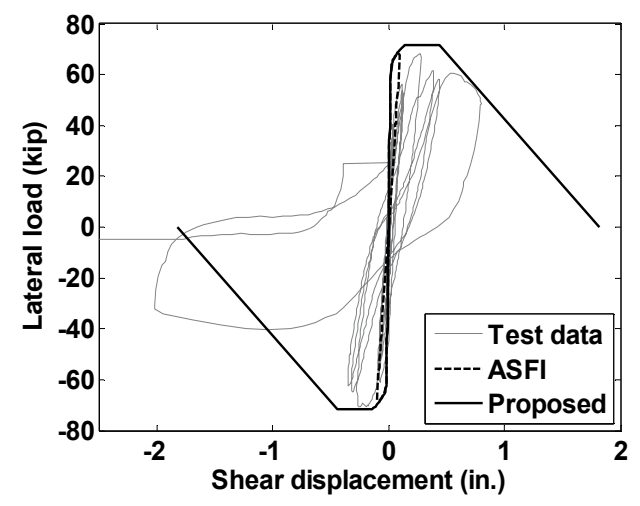

(e)

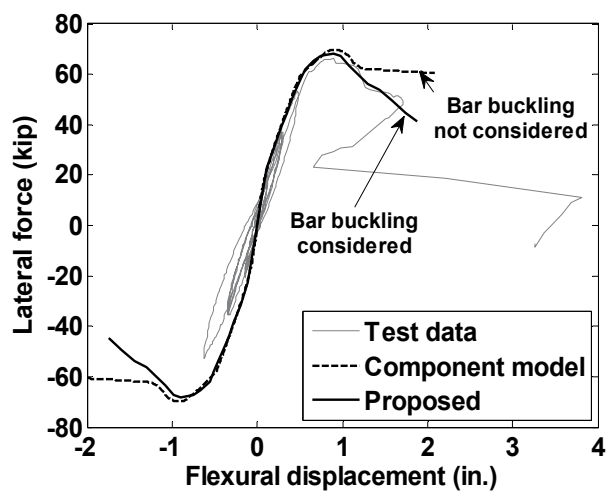

(b)

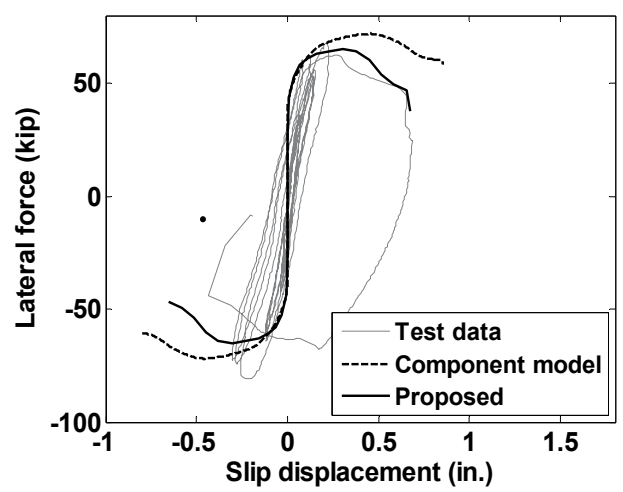

(d)

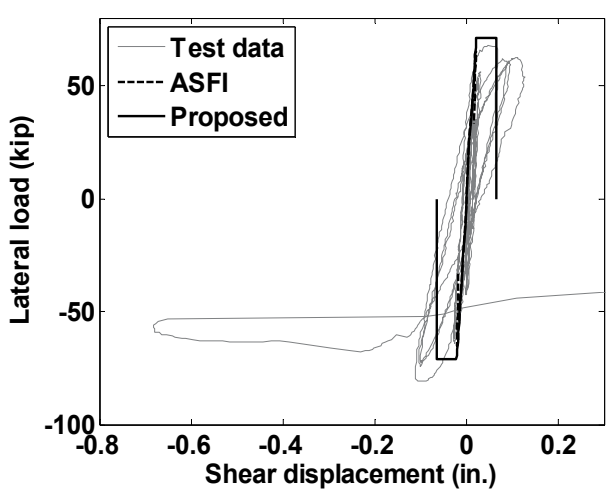

(f)

Fig. 10. Experimental and analytical results for: (a) flexural response of Specimen 1; (b) flexural response of Specimen 4; (c) reinforcement slip response of Specimen 1; (d) reinforcement slip response of Specimen 2; (e) shear response of Specimen 1; and (f) shear response of Specimen 2 


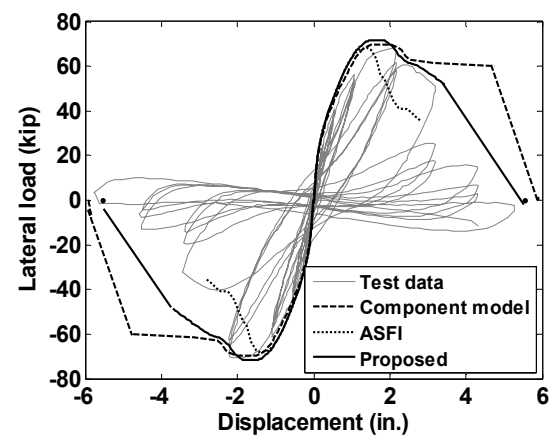

(a)

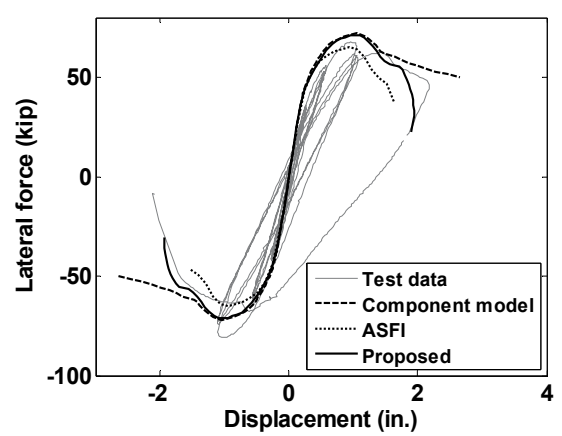

(b)

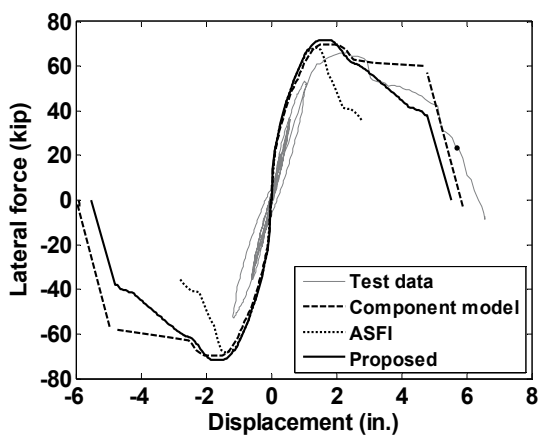

(c)

Fig. 11. Lateral load - displacement relationships : (a) Specimen 1; (b) Specimen 2; and (c) Specimen 4

\section{Conclusions}

A procedure is proposed for response estimation of reinforced concrete columns subjected to lateral loads. The procedure determines flexure, bar slip and shear deformations individually considering interaction between these mechanisms. The axial-flexure mechanism is decoupled from axial-shear model, allowing a relatively simpler analytical procedure. The flexure model in the proposed procedure incorporates concrete tensile behavior, interaction of compression softening and buckling of compression bars into the flexural analysis. The shear model includes the effect of flexural deformation on shear behavior. The pre-peak response is evaluated by employing MCFT, which cannot be used when the strength starts to degrade beyond peak strength. A post peak shear response envelope from displacement component model is adopted for predicting post peak shear behavior. All deformation components, i.e., flexural, bar slip and shear, are added together to get total response of the column. The total/combined peak response is limited by the smaller of the shear and flexural strength of the column and limiting mechanism governs the post peak response. The proposed procedure employs relatively simple calculations for the overall response estimation. The comparison of the predicted and observed responses indicates that the proposed procedure is a suitable displacement-based evaluation process that performs well in predicting the individual displacement components and total response. 


\section{References}

ACI Committee 318. (2008). Building Code Requirements for Structural Concrete (ACI 318-08) and Commentary (ACI 318R-08), American Concrete Institution, ISBN 0-87031-264-2, Farmington Hills, Michigan, USA

Arakawa, T. (1970). Allowable Unit Shearing Stress and Design Method of Shear Reinforcement for Reinforced Concrete Beams. Concrete Journal, Vol.8, No.7, pp. 1120 (in Japanese)

Aydan, O. \& Tano, H. (2011). Shaking-Induced Damage to Buildings by M 9.0 East Japan Mega Earthquake on March 11, 2011, In: Tohoku Pacific Ocean Earthquake and Tsunami - Quick observations from the PEER/EERI/GEER/Tsunami Field Investigation Team, 18.08.2011, Available from: http://www.jsce.or.jp/committee/eec2/eq_repo rt/201103tohoku/Aydan3.pdf

Bentz, E.C. (2000). Sectional Analysis of Reinforced Concrete Members. PhD Thesis, Department of Civil Engineering, University of Toronto, Toronto, Canada, 2000

Ceresa, P.; Petrini, L. \& Pinho, R. (2007). Flexure-shear fiber beam-column elements for modeling frame structures under seismic loading-state of the art. Journal of Earthquake Engineering, Vol. 11, pp. 46-88, ISSN 1363-2469

Ceresa, P.; Petrini, L.; Pinho, R. \& Sousa, R. (2009). A fiber flexure-shear model for seismic analysis of RC-framed structures. Earthquake Engineering and Structural Dynamics, Vol. 38, No. 5, pp. 565-586

Chao, S.H. \& Loh, C.H. (2007). Inelastic response analysis of reinforced concrete structures using modified force analogy method. Earthquake Engineering and Structural Dynamics, Vol. 36, pp. 1659-1683

Earthquake Engineering Research Institute. (December 2005). First Report on the Kashmir Earthquake of October 8, 2005, EERI Special Earthquake Report-December 2005/EERI's Learning from Earthquakes Program, 18.08.2011, Available from: http:/ /www.eeri.org /lfe/pdf/kashmir_eeri_1st_report.pdf

Inoue, K. \& Shimizu, N. (1988). Plastic Collapse Load of Steel Braced Frames Subjected to Horizontal Force. Journal of Structural and Construction Engineering, Vol. 388, pp. 5969 (in Japanese)

Lodhi M.S. (2010). Response estimation of reinforced concrete columns subjected to lateral loads. M.S. Thesis, The Ohio State University, Columbus, Ohio, 2010.

Mid-America Earthquake Center, University of Illinois Urbana-Champaign. (2005). The Kashmir Earthquake of October 8, 2005 - A Quick Look Report, MAE Center Report No. 05-04, 18.08.2011, Available from: http://mae.cee.uiuc.edu/documents/cd_ro m_series/05-04/Report05-04.pdf

Mergos, P.E. \& Kappos, A.J. (2008). A distributed shear and flexural flexibility model with shear-flexure interaction for R/C members subjected to seismic loading. Earthquake Engineering and Structural Dynamics, Vol. 37, PP. 1349-1370

Mergos, P.E. \& Kappos, A.J. (2010). Seismic damage analysis including inelastic shearflexure interaction. Bulletin of Earthquake Engineering, Vol. 8, pp. 27-46

Monti, G. \& Nuti, C. (1992). Non-linear Cyclic Behavior of Reinforcing Bars Including Buckling. Journal of Structural Engineering, Vol. 118, No. 12, pp. 3269-3284

Morsch, E. (1922). Der Eisenbetonbau-Seine Theorie und Anwendung, (5th Edition, Vol. 1, Part 1), Wittwer, Stuttgart, Germany 
Mostafaei, H. \& Kabeyasawa, T. (2007). Axial-Shear-Flexure Interaction Approach for Reinforced Concrete Columns. ACI Structural Journal 2007, Vol. 104, No. 2, pp. 218226

Mostafaei, H. \& Vecchio, F.J. (2008). Uniaxial Shear-Flexure Model for Reinforced Concrete Elements. Journal of Structural Engineering, Vol 134, No. 9, pp. 1538-1547

Mullapudi, T.R. \& Ayoub, A.S. Modeling of the seismic behavior of shear-critical reinforced concrete columns. Engineering Structures 2010; 32:3601-3615.

Mullapudi, T.R.; Ayoub, A.S. \& Belarbi, A. (2008). A fiber beam element with axial, bending and shear interaction for seismic analysis of RC structures, Proceedings of the 14th World Conference on Earthquake Engineering, Beijing, China, 2008

Pacific Earthquake Engineering Research Center/Earthquake Engineering Research Institute/Geotechnical Extreme Event Reconnaissance/Tsunami Field Investigation Team. (2011). Tohoku Pacific Ocean Earthquake and Tsunami - Quick observations from the PEER/EERI/GEER/Tsunami Field Investigation Team, 18.08.2011, Available from: http://peer.berkeley.edu/news/wp-content/uploads/2011/04/T ohoku-short-interim-report.pdf

Patwardhan, C. (2005). Strength and Deformation Modeling of Reinforced Concrete Columns. M.S.Thesis, The Ohio State University, Columbus, Ohio, 2005

Potger, G.M.; Kawano, A.; Griffith, M.C. \& Warner, R.F. (2001). Dynamic Analysis of RC Frames Including Buckling of Longitudinal Steel Reinforcement, Proceedings of the NZSEE Conference, 2001, Paper No. 4.12.01

Priestley, M.J.N.; Calvi, G.M. \& Kowalsky, M.J. (2007). Displacement-Based Seismic Design of Structures, IUSS Press, Pavia, Italy

Ritter, W. (1899). Die Bauweise Hennebique. Schweizerische Bbauzeitung, Vol. 33, No. 7, pp 59-61

Schlaich, J.; Schafer, I. \& Jennewein, M. (1987). Towards a Consistent Design of Structural Concrete. Journal of the Prestressed Concrete Institute, Vol. 32, No. 3, pp. 74-150

Setzler, E.J. \& Sezen, H. (2008). Model for the lateral behavior of reinforced concrete columns including shear deformations. Earthquake Spectra, Vol. 24, No. 2, pp. 493-511

Sezen H., and Moehle J.P. (2006). Seismic tests of concrete columns with light transverse reinforcement. ACI Structural Journal, Vol. 103, No. 6, pp. 842-849

Sezen, H. (2002). Seismic Behavior and Modeling of Reinforced Concrete Building Columns. Ph.D Thesis, University of California, Berkeley, 2002

Sezen, H. (2008). Shear deformation model for reinforced concrete columns. Structural Engineering and Mechanics, Vol. 28, No. 1, pp. 39-52

Sezen, H., \& Moehle, J.P. (2004). Strength and deformation capacity of reinforced concrete columns with limited ductility, Proceedings of the 13th World Conference on Earthquake Engineering, Vancouver, Canada, 2004

Sezen, H. \& Moehle, J.P. (2004). Shear Strength Model for Lightly Reinforced Concrete Columns. Journal of Structural Engineering, Vol. 130, No. 11, pp. 1692-1703

Sezen, H. \& Setzler, E.J. (2008). Reinforcement Slip in Reinforced Concrete Columns. ACI Structural Journal, Vol. 105, No. 3, pp. 280-289

Structural Engineers Association of California (SEAOC). (2002). Performance based seismic design engineering of buildings. Vision 2000 Report, Sacramento, CA, USA

Takewaki, I.; Murakami, S.; Fujita, K.; Yoshitomi, S. \& Tsuji, M. (2011). The 2011 off the Pacific coast of Tohoku earthquake and response of high-rise buildings under long- 
period ground motions. Soil Dynamics and Earthquake Engineering, doi:10.1016/j.soil dyn.2011.06.001 (In press)

U.S Geological Survey/Earthquake Engineering Research Institute Advance Reconnaissance Team. (February 23, 2010). The $\mathrm{M}_{\mathrm{w}} 7.0$ Haiti Earthquake of January 12, 2010, USGS/EERI Team Report V.1.1, 18.08.2011, Available from: http://www.eqclearingh ouse.org/20100112-haiti/wp-content/uploads/2010/02/USGS_EERI_HAITI_V1.1. pdf

Vecchio, F.J. (1989). Nonlinear finite element analysis of reinforced concrete membranes. ACI Structural Journal, Vol. 86, No. 1, pp. 26-35

Vecchio, F.J. (2000). Disturbed stress field model for reinforced concrete: formulation. Journal of Structural Engineering, Vol. 126, No. 9, pp. 1070-1077

Vecchio, F.J. \& Collins, M.P. (1986). The modified compression field theory for reinforced concrete elements subjected to shear. ACI Journal Proceedings, Vol. 83, No. 2, pp. 219-231

Vecchio, F.J. \& Collins, M.P. (1988). Predicting the Response of Reinforced Concrete Beams Subjected to Shear Using Modified Compression Field Theory. ACI Structural Journal, Vol. 85, No. 3, pp. 258-268

Williams, M.S. \& Sexsmith, R.G. (1995). Seismic Damage Indices for Concrete Structures: A State-of-the-Art Review. Earthquake Spectra, Vol. 11, No. 2, pp. 319-349

Xu, S.Y. \& Zhang, J. (2011). Hysteretic shear-flexure interaction model of reinforced concrete columns for seismic response assessment of bridges. Earthquake Engineering and Structural Dynamics, Vol. 40, pp. 315-337

Zhang, J.; Xu, S.Y. \& Tang, Y. (2010). Inelastic displacement demand of bridge columns considering shear-flexure interaction. Earthquake Engineering and Structural Dynamics, DOI 10.1002/eqe.1056 


\title{
Seismic Vulnerability Analysis of RC Buildings in Western China
}

\author{
Zhu Jian \\ Ning Xia University \\ China
}

\section{Introduction}

Kobe earthquake in Japan (Ms6.9) happened in 1995 Jan 17 at five o'clock in the morning, depth of the seismic focus was $20 \mathrm{~km}$, the seismic characteristic was shallow vertical focus earthquake, peak acceleration was 813 gal, predominant period was $0.3 \sim 0.5 \mathrm{~s}$, The main sediments of the region under 20 30 meters were sandy silt, and Generally to moderately weathered rock. 18 thousand buildings were been damaged and 1.2 thousand buildings were collapsed, half timber houses which built before 1980'were destroyed seriously, at the same time middle-storey reinforced-concrete (RC) buildings which were $7 \sim 8$ layer buildings damaged also seriously, the mainly damage showing was integral overthrow of buildings, in addition columns between third to forth layer of RC buildings were squashed in the earthquake, and were collapsed in the middle of structures.

Tohoku Earthquake (Ms9.0) in Japan occurred on March,11 2011, it is regarded as the most devastating killer earthquake after the 1923 great kanto earthquake in Japan, in which almost 30000 people were killed or missed in the earthquake and the subsequent monster tsunami. The maximum height of the tsunami is reported to have been almost $40 \mathrm{~m}$. The recorded maximum peak ground acceleration was 2933 gal and large long-period wave components were recorded in Tokyo during the 2011 off the Pacific coast of Tohoku Earthquake. It is remarkable in this earthquake that the number of collapsed or damaged buildings and houses remains unclear because most of the damage resulted from the tsunami (Takewaki,2011).

Northridge earthquake(Ms 6.7) happened in 1994 Jan 17 at five o'clock in the morning, this is terrible earthquake with tremendous horizontal and vertical acceleration evenly to reach $1.0 \mathrm{~g}, 2500$ houses were been collapsed and 4000 houses were been damaged seriously by powerful natural energy, the main characteristic of buildings' damage was shear failure of columns in old second to third layer timberwork apartment blocks, and RC frames were damaged very small include several 40 layer high-rise RC buildings.

Taiwan Chichi earthquake (Ms7.6) happened in 1999 Sep 21 at one o'clock in the morning, depth of the seismic focus was $8 \mathrm{~km}$, and the earthquake was powerful shallow focus earthquake, damaged data of 8733 buildings were collected post earthquake, one to three layer RC buildings were damaged seriously, or about 52.5 percent, among which old RC buildings built before 1982 based insufficient seismic design and outdated seismic code were destroyed deeply, and occupied about 59.4 percent. Small high-rise shear-wall RC buildings were damaged tiny, and which occupied 6.4 percent. 
China Wenchuan earthquake(Ms8.0) happened in 2008 May 12 at two o'clock in the afternoon, in the seismic region are three main geological fault zones, and the Wenchuan earthquake occurred in Longmenshan (LMS) geological fault zone, total length of LMS is 530 kilometer $(\mathrm{km})$, width of which is $40 \sim 50 \mathrm{~km}$, presenting northeast-southwest running, and leaning to northwest about 30 70 angles, LMS divided into two parts by Jiangyou(JY) city in Sichuan province, northeast part of which is geological fault zone during Early Pleistocene-middle Middle Pleistocene, southwest part of which is fault zone during Holocene, the earthquake was happened in southwest part, depth of the seismic focus was $14 \mathrm{~km}$, and fracture length was $240 \mathrm{~km}$, the process of fracture consisted of several continuous events, every event was a earthquake which magnitude was Ms7.2 to Ms7.6, all type of buildings were damaged overlying in the continuous vibration, the peak acceleration recorded was 957.8gal, waves diffused all directions in three-dimensional, at the same time vibration along northeast of fracture zone was more powerfully, and continued for about 100 seconds, spreading to 16 provinces of China, specialists from State Seismological Bureau of China surveyed 500 thousand square kilometer, and in where 2419 square kilometer earthquake intensity reached 11 scale, far surpass design intensity in the region where is 7 scale.

In the Wenchuan earthquake, 5.46 million buildings were collapsed, number of serous damaged buildings were 5.93 million, amount to total damaged buildings exceeded 15 million, old brick masonry structures built 70s 80s damaged most seriously, in the next place low-rise RC buildings destroyed also severely, which were integral overthrow of base layer columns yielded because of wrong site, low material strength and fault layout. With Indian plate moving to north continuously and squeezing Asian plate, in the near future there is still high risk of major earthquake happen again in western China region, so how to evaluate reliability and vulnerability of the lifeline systems for future earthquake in the area and search reasonable design practice of seismic strengthening of these buildings is urgent mission.

\section{Summarize methods of structural seismic vulnerability analysis}

Damage from earthquake is comprehensive, there are different ways for various damage of engineering, seismic technician classify the vulnerability assessment as four sorts: empirical, judgmental, experimental and analytical according to whether the damage data used in their generation derives mainly from observed post-earthquake surveys, expert opinion, analytical simulations or combinations of these respectively.

As described below, the seismic vulnerability assessment of buildings at large geographical scales has been first carried out in the early 70's, through the employment of empirical methods initially developed and calibrated as a function of macro-seismic intensities. This came as a result of the fact that, at the time, hazard maps were, in their vast majority, defined in terms of these discrete damage scales (earlier attempts to correlate intensity to physical quantities, such as PGA, led to unacceptably large scatter). Therefore these empirical approaches constituted the only reasonable and possible approaches that could be initially employed in seismic risk analyses at a large scale.

(Whitman, 1973) first proposed the use of damage probability matrices for the probabilistic prediction of damage to buildings from earthquakes. The concept of a DPM is that a given 
structural typology will have the same probability of being in a given damage state for a given earthquake intensity. The format of the DPM was suggested by (Whitman, 1973), where example proportions of buildings with a given level of structural and non-structural damage are provided as a function of intensity (note that the damage ratio represents the ratio of cost of repair to cost of replacement). (Whitman, 1973) compiled DPMs for various structural typologies according to the damaged sustained in over 1600 buildings after the 1971 San Fernando earthquake.

One of the first European versions of a damage probability matrix was produced by (Braga et al., 1982), which was based on the damage data of Italian buildings after the 1980 Irpinia earthquake, and this introduced the binomial distribution to describe the damage distributions of any class for different seismic intensities. The binomial distribution has the advantage of needing one parameter only which ranges between 0 and 1. On the other hand it has the disadvantage of having both mean and standard deviation depending on this unique parameter. The buildings were separated into three vulnerability classes (A, B and C) and a DPM based on the MSK scale was evaluated for each class. This type of method has also been termed 'direct' (Corsanego \& Petrini, 1990) because there is a direct relationship between the building typology and observed damage. The use of DPMs is still popular in Italy and proposals have recently been made to update the original DPMs of Braga. (Di Pasquale, 2005) have changed the DPMs from the MSK scale to the MCS (Mercalli-Cancani-Sieberg) scale because the Italian seismic catalogue is mainly based on this intensity, and the number of buildings has been replaced by the number of dwellings so that the matrices could be used in conjunction with the 1991 Italian National Statistical Office (ISTAT) data. (Dolce,2003) have also adapted the original matrices as part of the ENSeRVES (European Network on Seismic Risk, Vulnerability and Earthquake Scenarios) project for the town of Potenza, Italy. An additional vulnerability class D has been included, (Grüntal, 1998) using the EMS98 scale to account for the buildings that have been constructed since 1980. These buildings should have a lower vulnerability as they have either been retrofitted or designed to comply with recent seismic codes.

Judgmental method was based structural damage data can be considered the greatest cause of life and monetary loss in the majority of seismic events, vulnerability curves were been received under-predict the damage observed in buildings after earthquake, for example: Miyakoshi et al. [6] used damage data observed in RC buildings after the Kobe earthquake (Japan, 1995) in constructing damage parameter equation, (Yamazaki \& Murao, 2000) also made up of empirical vulnerability curve though analyzing damage data observed in buildings after the Kobe earthquake, (Orsini et al.,1999) used the Parameterless Scale of Intensity (PSI) ground-motion parameter to derive vulnerability curves for apartment units in Italy. Both studies subsequently converted the PSI to PGA using empirical correlation functions, such that the input and the response were not defined using the same parameter.

The use of observed damage data to predict the future effects of earthquakes also has the advantage that when the damage probability matrices are applied to regions with similar characteristics, a realistic indication of the expected damage should result and many uncertainties are inherently accounted for. However, there are various disadvantages associated with the continued use of empirical methods: 
1. A macro-seismic intensity scale is defined by considering the observed damage of the building stock and thus in a loss model both the ground motion input and the vulnerability are based on the observed damage due to earthquakes.

2. The derivation of empirical vulnerability functions requires the collection of postearthquake building damage statistics at sites with similar ground conditions for a wide range of ground motions: this will often mean that the statistics from multiple earthquake events need to be combined. In addition, large magnitude earthquakes occur relatively infrequently near densely populated areas and so the data available tends to be clustered around the low damage/ground motion end of the matrix thus limiting the statistical validity of the high damage/ground motion end of the matrix.

3. The use of empirical vulnerability definitions in evaluating retrofit options or in accounting for construction changes (that take place after the earthquakes on which those are based) cannot be explicitly modeled; however simplifications are possible, such as upgrading the building stock to a lower vulnerability class.

4. Seismic hazard maps are now defined in terms of PGA (or spectral ordinates) and thus PGA needs to be related to intensity; however, the uncertainty in this equation is frequently ignored. When the vulnerability is to be defined directly in terms of PGA, where recordings of the level of the ground shaking at the site of damage are not available, it might be necessary to predict the ground shaking at the site using a ground motion prediction equation; however, again the uncertainty in this equation needs to be accounted for in some way, especially the component related to spatial variability.

5. When PGA is used in the derivation of empirically-defined vulnerability, the relationship between the frequency content of the ground motions and the period of vibration of the buildings is not taken into account.

Laboratory testing represents the third alternative tool for vulnerability assessment. The main advantage of this method is the freedom in selecting model to suite the application. However, this method is hampered by the limitations of scale, laboratory and equipments capacities. Another factor affecting the reliability of this tool is the effect of loading type and routine on the response. Furthermore, the deficiency in modeling soil-structure interactions represents one of the main disadvantages of using the laboratory testing as a seismic vulnerability assessment tool. Pseudo dynamic testing has been used in several studies. However, the slow rate of testing represents a main obstacle in using this type of testing for vulnerability assessment where large sets of data points are required to make a comprehensive assessment. Recently sub-structuring and distributed testing have been used in different studies.

The last tool is analysis vulnerability method. The method has received much attention from researchers in recent years. Many researchers of countries such as (M.A.Erberik, 2004), (Rossetto. T, 2005), (S. Kircil,2006), (Jun Ji,2007) and (Barbara Borzi,2008) have studied seismic characteristic and vulnerability of many kinds of structures like reinforced concrete buildings, masonry buildings, bridges, museums and dams. Analysis offers two main advantages: the ease of controlling the level of refinement and feasibility of parametric studies. However, results of recent studies emphasized several issues that need to be resolved in order to enhance the use of analysis in realistic vulnerability assessment, including influence level between different models, ground vibration importing selecting, comprehensive structures analysis and computing consume. So analysis method is selected based all four tools characteristic, and the analysis tool will be used in classic RC buildings of western region in China in connecting fielding damage data observed after Wenchuan earthquake (2008) in China. 


\section{Response spectrum fitting stochastic artificial waves}

Probability response spectrum is an elastic response spectrum, which is enough as external load for most sample and general layout buildings of seismic design, but which is not suitable for comprehensive and lifeline structures, so nonlinear dynamic analysis is very necessary and importable to the lifeline structures. In fact, actual earthquake recorded waves are not easy for attainable in engineering research, and why is the reason how to use spectrum density and design response spectrum for fitting artificial stochastic seismic waves.

\subsection{Analysis procedure}

Artificial seismic waves analysis methods could be divided into engineering simulation method and seismology simulation method. Earthquake focus also be divided into many element focus in seismology simulation method, and which element focus excite site vibration in using theoretical or experimental ways, that in the end gathering site vibration by total element focus and to move forward to construct site seismic function. Engineering simulation method is made up appropriate seismic function in accordance with seismic Fourier frequency spectrum, energy spectrum density and response spectrum, engineering method has been accepted by engineering technician because of well academic foundation. So engineering simulation method is been selected in Matlab7.0 programming and simulate and fit artificial seismic stochastic waves according with design response spectrum. Five steps are following:

1. initial setting artificial seismic waves;

2. compute response spectrum of artificial seismic waves;

3. compare initial response spectrum with target response spectrum and compute both ratio;

4. regulate artificial seismic waves on the base of response ratio;

5. repeat step 2 and degree of fitting satisfy demand.

\subsection{Artificial seismic waves simulation}

There are three methods for artificial waves: tri-angle series method, stochastic impulse method and natural regression method, the third ways raised in 1970s, but the first method is accepted widely.

We suppose that an zero mean value $(E[x(t)=0])$ steady state diffuse scattering wave shape time history is $x(t)$, which may be showed by Fourier analysis, as shown in Eq.(1):

$$
x(t)=\sum_{k} A_{k} \exp \left[i\left(\omega_{k} t+\phi_{k}\right)\right]
$$

Where $\omega_{k}=k \Delta \omega, \Delta \omega=2 \pi / T_{d}, T_{d}$ is seismic time history, $A_{k}=A\left(\omega_{k}\right)$ is Fourier amplitude spectrum value, $\phi_{k}$ is phase angle, so we can decided $A_{k}, \phi_{k}$ according with vibration characteristic value, in other word, we can create steady vibration time history based above function. In which $\phi_{k}$ is created by random phase way and random range from 0 to $2 \pi$, certainly it my also take from real earthquake recorded waves. Frequency of vibration could shown by spectrum density function $S_{\ddot{X} g}(\omega)$, and $S(\omega)$ has relation with $A\left(\omega_{k}\right)$, $E\left[x(t)^{2}\right]=\int_{-\infty}^{\infty} S(\omega) d \omega=\sum_{k} A_{k}{ }^{2}$, mean $S\left(\omega_{k}\right) \Delta \omega=\frac{A_{k}{ }^{2}}{2}$, mean also $A_{k}=\left[2 S\left(\omega_{k}\right) \Delta \omega\right]^{\frac{1}{2}}$. 
In the mean time (Kaul, 1978) computed relation energy spectrum density function with response spectrum on the base of diffuse scattering theory shown as Eq. (2):

$$
S\left(\omega_{k}\right)=\frac{\left[\zeta / \pi \omega_{k}\right] S_{a}^{2}\left(\omega_{k}, \zeta\right)}{\operatorname{In}\left[\omega_{k} T_{d} /-\pi \operatorname{In}(1-p)\right]}
$$

Where $S_{a}$ is accelerate response spectrum, $\zeta$ is system damping ratio and initial setting as $5 \%, p$ is surpass probability and setting as $10 \%, T_{d}$ is time history 30 seconds, initial steady artificial seismic time history are shown by programmed with Matlab 7.0 in Fig.1:

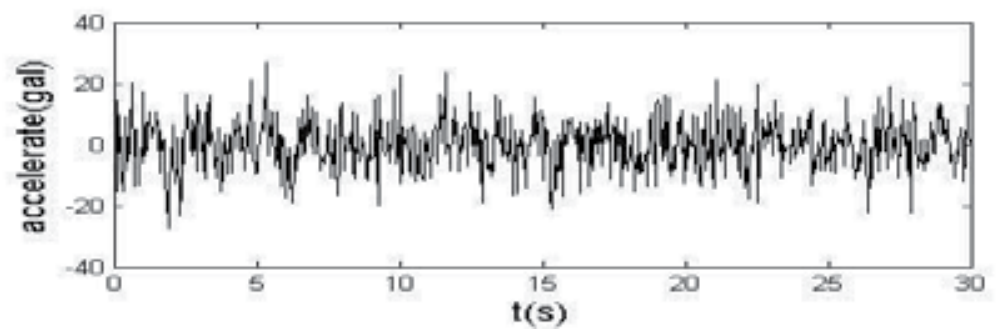

Fig. 1. Initial steady artificial vibration time history

Real earthquake wave is not an sample steady vibration, in consideration of initial vibration phase, main vibration phase and decline phase, so artificial waves only shown more accurately as stationary time history, in present, common way is steady state $x(t)$ multiply by decided time varying envelop function $g(t)$, that is to say, change stationary state into steady form.

Seismic acceleration could be shown as:

$$
A_{g}(t)=g(t) \ddot{X}_{g}(t)
$$

Where $\ddot{X}_{g}(t)$ is $x(t), g(t)$ is envelop curve, so structure of spectrum frequency is not vary with time but variance is changing with time.

Generally envelop function is decided in according with seismic total duration and strong vibration duration. Seismic total duration means earthquake time history from begin time to end time, we can know that seismic duration change from several seconds to dozens of seconds and at most one to two minutes, (Murphy and O'Brien,1977) from American analysis about 400 recorded earthquake waves and found the vast majority of recorded waves duration within $25 \sim 40$ s when the total duration within 2 100s, so 30s was decided as standard artificial wave duration. After engineering technician analyze vast actual seismic recorded acceleration $a(t)$, they found an common characteristic: stationary of intensity and frequency spectrum, vibration intensity divided as ascent stage, strong vibration sustain stage and weakening stage, meanwhile stationary in every stage. In engineering we often pay attention to strong vibration stage called strong vibration duration $T_{\alpha}$, because importance of strong vibration duration has been realized by engineering technician, but definition of which is not definite, so in the article relative duration based 
energy was been considered, and (Husid,1974), (Trifunac \& Bardy,1975) studied that they thought using $\int_{0}^{t} a^{2}(t) d t$ as time-vary characteristic of earthquake vibration energy, and shown as Eq.(4):

$$
I(t)=\frac{\int_{0}^{t} a^{2}(t) d t}{\int_{0}^{T} a^{2}(t) d t}
$$

Where $\mathrm{T}$ is total duration of seismic wave, $I(t)$ is function within $0 \sim 1$. Definition of strong vibration duration is :

$$
T_{\alpha}=t_{2}-t_{1}
$$

The relation of $t_{2}$ and $t_{1}$ is:

$$
I\left(t_{2}\right)-I\left(t_{1}\right)=\alpha \%
$$

Japanese and American researchers (Takjzawa,1980), (Jennings \& Housner,1968) compared $90 \%, 80 \%, 70 \%, 60 \%$ and $50 \%$ of energy duration for destructive effect of buildings and decided $70 \%$ of energy duration was more fitting to actual situation, the article author also analysed and computed representative seismic record in Tab.(1), in the same time considered standard deviation $\pm 5 \%$, and energy in ascent stage occupy $10 \%$ of total energy , moreover $I\left(t_{1}\right)$ is $10 \%, I\left(t_{2}\right)$ is $80 \%$.

\begin{tabular}{|c|c|c|c||c|c|c|c|}
\hline $\begin{array}{c}\text { Earthquake } \\
(\mathrm{T})\end{array}$ & $\begin{array}{c}10 \% \\
t_{1}\end{array}$ & $\begin{array}{c}80 \% \\
t_{2}\end{array}$ & $\begin{array}{c}\text { Strong } \\
\text { duration } \\
T_{\alpha}\end{array}$ & $\begin{array}{c}\text { Earthquake } \\
(\mathrm{T})\end{array}$ & $\begin{array}{c}10 \% \\
t_{1}\end{array}$ & $\begin{array}{c}80 \% \\
t_{2}\end{array}$ & $\begin{array}{c}\text { Strong } \\
\text { duration } \\
T_{\alpha}\end{array}$ \\
\hline $\begin{array}{c}\text { EI-centro } \\
(37.03 \mathrm{~s})\end{array}$ & $6.12 \mathrm{~s}$ & $10.18 \mathrm{~s}$ & $4.06 \mathrm{~s}$ & $\begin{array}{c}\text { Northridge } \\
(15.01 \mathrm{~s})\end{array}$ & $1.90 \mathrm{~s}$ & $3.80 \mathrm{~s}$ & $1.90 \mathrm{~s}$ \\
\hline $\begin{array}{c}\text { Taftew } \\
(27.11 \mathrm{~s})\end{array}$ & $1.87 \mathrm{~s}$ & $7.73 \mathrm{~s}$ & $5.86 \mathrm{~s}$ & $\begin{array}{c}\text { Chichi long } \\
(39.98 \mathrm{~s})\end{array}$ & $10.26 \mathrm{~s}$ & $15.00 \mathrm{~s}$ & $4.74 \mathrm{~s}$ \\
\hline $\begin{array}{c}\text { Kobe } \\
(46.38 \mathrm{~s})\end{array}$ & $4.00 \mathrm{~s}$ & $8.50 \mathrm{~s}$ & $4.50 \mathrm{~s}$ & $\begin{array}{c}\text { Nanjin } \\
(16.10 \mathrm{~s})\end{array}$ & $3.00 \mathrm{~s}$ & $8.90 \mathrm{~s}$ & $5.90 \mathrm{~s}$ \\
\hline $\begin{array}{c}\text { Tianjinew } \\
(19.08 \mathrm{~s})\end{array}$ & $7.26 \mathrm{~s}$ & $10.01 \mathrm{~s}$ & $2.75 \mathrm{~s}$ & $\begin{array}{c}\text { Qiananns } \\
(22.10 \mathrm{~s})\end{array}$ & $1.79 \mathrm{~s}$ & $4.74 \mathrm{~s}$ & $2.95 \mathrm{~s}$ \\
\hline $\begin{array}{c}\text { Tangshanew } \\
(49.20 \mathrm{~s})\end{array}$ & $20.9 \mathrm{~s}$ & $39.4 \mathrm{~s}$ & $18.5 \mathrm{~s}$ & $\begin{array}{c}\text { Wenchuan } \\
(500 \mathrm{~s})\end{array}$ & $11.92 \mathrm{~s}$ & $91.7 \mathrm{~s}$ & $79.78 \mathrm{~s}$ \\
\hline
\end{tabular}

Table 1. Statistics and analysis of strong vibration duration in earthquake record

Tri-stage curve and exponent curve have been accepted widely now by engineering because of simple, visualized and physical significance (Ohsaki,1978;Kaul,1978;Amin \& Ang, 1968 ) , tri-stage curve could be shown as: 


$$
g(t)=\left\{\begin{array}{lc}
\left(t / t_{1}\right)^{2} & t \leq t_{1} \\
1 & t_{1}<t \leq t_{2} \\
\exp \left[-c\left(t-t_{2}\right)\right] & t>t_{2}
\end{array}\right.
$$

Where $t_{1}, t_{2}$ and $c$ are model parameters, $t_{1}$ and $t_{2}$ are begin moment and end moment, $c$ present weakening velocity in descent stage, $T_{s}$ is vibration duration in strong vibration duration for 70\% energy of total seismic energy, in which $t_{1}=0.5 T_{s}, t_{2}=1.2 T_{s}, c=2.5 / T_{s}$. Exponent curve is shown as $g(t)=\beta t e^{-\alpha t}$,in which $\alpha$ and $\beta$ is model parameter, $\alpha=0.01$, $\beta=0.028$.

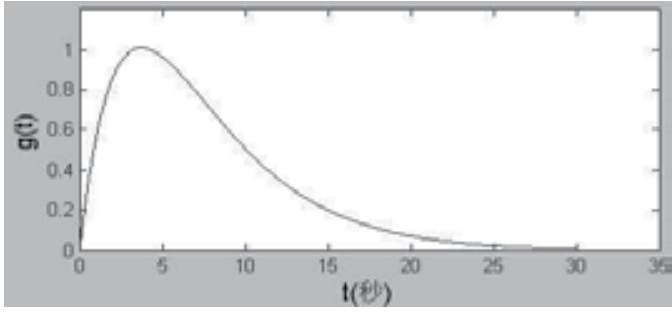

a

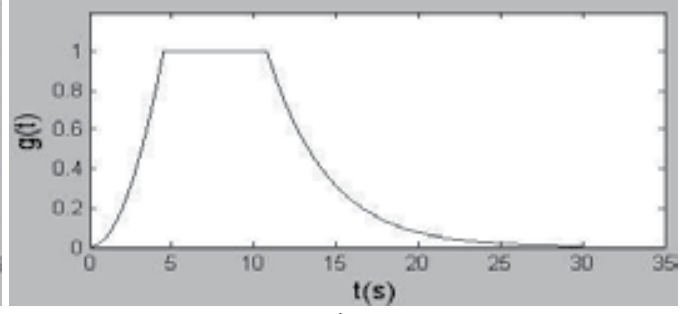

$\mathrm{b}$

Fig. 2. Envelop curve (a: exponent form, b: tri-stage form)

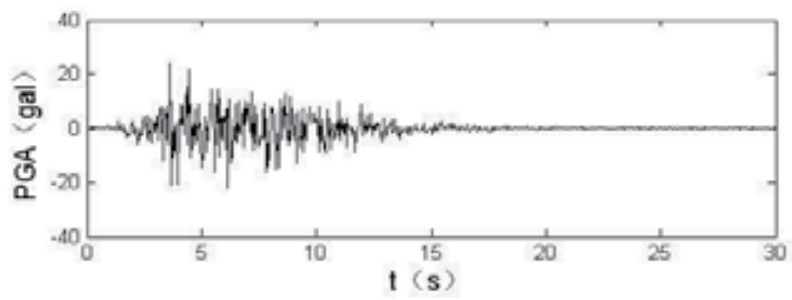

Fig. 3. Initial stationary artificial stochastic acceleration time history

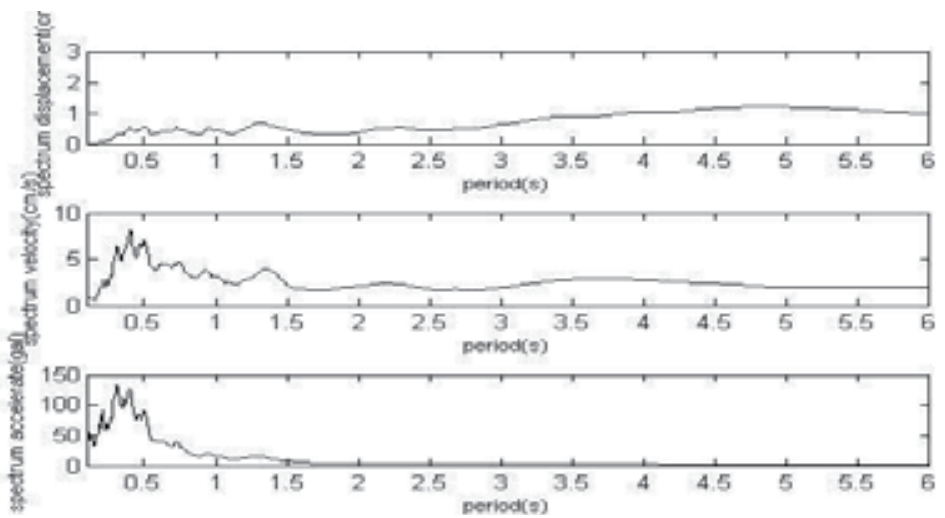

Fig. 4. Initial artificial stochastic response spectrum(from top to bottom: spectrum displacement, spectrum velocity, spectrum acceleration) 


\subsection{Artificial seismic record update}

If initial artificial seismic response spectrum from record $a_{0}(t)$ isn't meet degree of fitting with specify earthquake response spectrum or code response spectrum, there has need to update for more precision. Generally two ways now have been used for updating. The article used the second method.

First method :

$$
A_{i}^{\prime}(t)=\frac{S_{V}}{S_{V}^{\prime}} A_{i}(t)
$$

Where $A_{i}^{\prime}(t)$ is updated Fourier spectrum, $A_{i}(t)$ is Fourier spectrum of initial artificial record, $S_{V}$ is target response spectrum velocity, $S_{V}{ }^{\prime}$ is spectrum velocity of initial record. Second method :

$$
S_{g}{ }^{\prime}(\omega)=S_{g}(\omega)\left[\frac{\operatorname{TRSA}(\omega)}{\operatorname{SRSA}(\omega)}\right]^{2}
$$

Where $S_{g}{ }^{\prime}(\omega)$ is updated power spectrum density, $S_{g}(\omega)$ is initial power spectrum density, $\operatorname{TRSA}(\omega)$ is intend to compared target response spectrum, $S R S A(\omega)$ is computed stationary artificial seismic response spectrum, next step compute new steady artificial wave and multiply seismic envelop curve for updating new stationary artificial seismic wave, the updating could be repeated several times until degree of fitting is satisfy with accuracy. It is aware of that we need to control frequency in updating process, at first spectrum acceleration value of control points need be computed out, secondly comparing with control points of frequency, adjust ratio between two points need linear interpolation method to compute. As shown in Tab. 2.

\begin{tabular}{|c|c|c|}
\hline \hline The number of control points & \multicolumn{2}{|c|}{ Frequency Range (Hz) } \\
\hline \hline 5 & 0.10 & 0.18 \\
\hline 29 & 0.20 & 3.00 \\
\hline 4 & 3.15 & 3.60 \\
\hline 7 & 3.80 & 5.00 \\
\hline 11 & 5.25 & 8.00 \\
\hline 14 & 8.50 & 15.00 \\
\hline 4 & 16.00 & 30.00 \\
\hline
\end{tabular}

Table 2. Frequency adjust control points for artificial wave

Fig. 5-6 are updated samples for intensity 9 from (Chinese Seismic Code, 2001). The program iterative computed 5 8 times and result meet degree of fitting with Chinese code response spectrum, now artificial wave after updated was fitting to code response spectrum, 120 seismic record fitting to major earthquake and code response spectrum were created in this way and become applied load for next computation. 

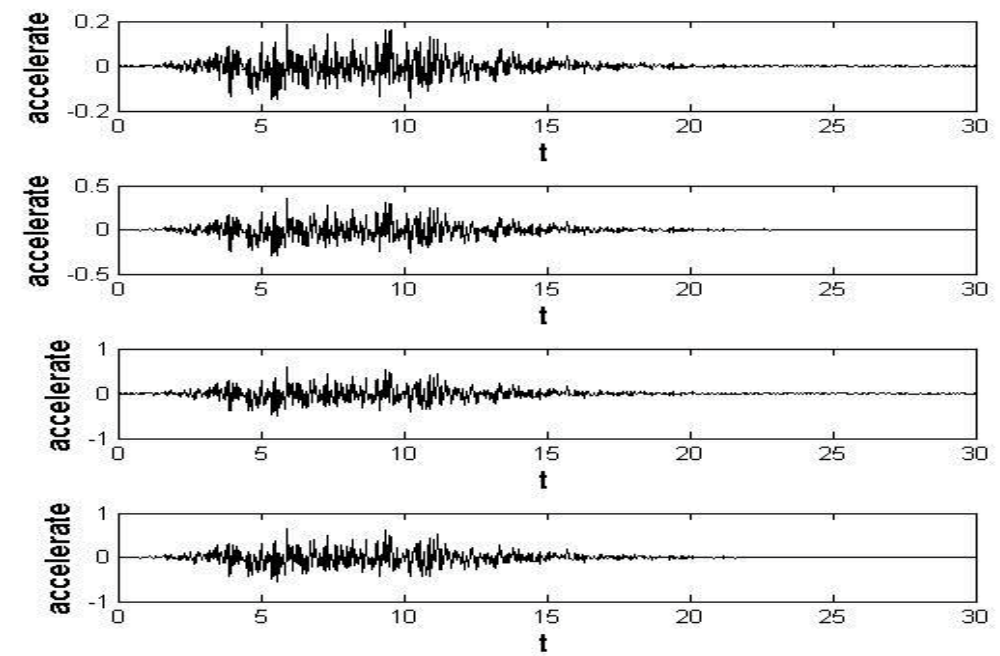

Fig. 5. Updating process of artificial seismic acceleration

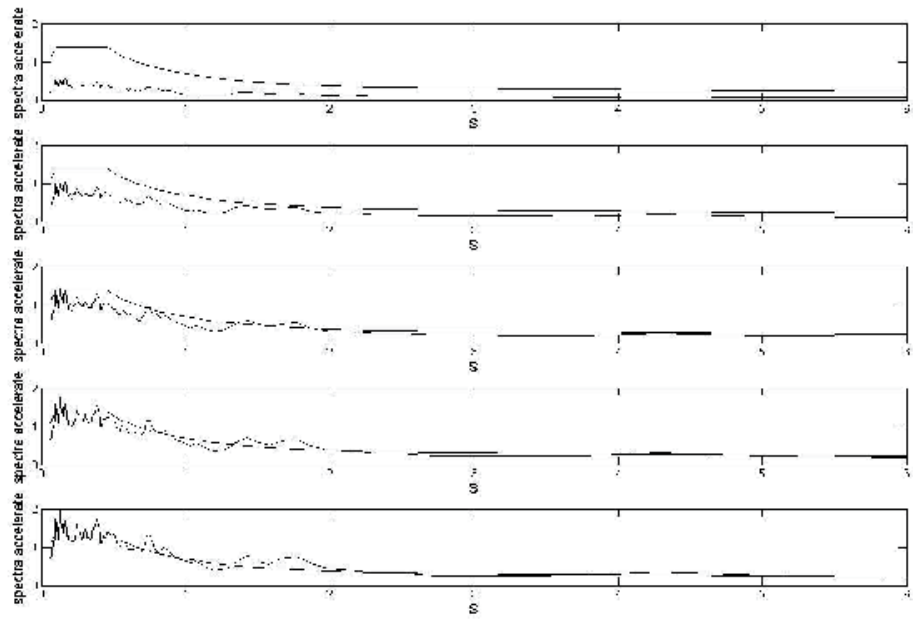

Fig. 6. Updating process of artificial response spectrum

\section{Proposed damage index of frame buildings}

At the moment seismic theory of China is more emphasis in prevent buildings collapse instead of prevent structural damage, first aim of buildings design is prevent collapse in major earthquake but obvious plastic deformation and damage is evitable, and key point is how to assure structural damage within acceptable degree based moment code, especially many lifeline constructions for example: nuclear energy station, bridge, tunnel, gymnasium and important factory buildings, so design based performance now have been approved widely, and content of structural vulnerability index is significant part of design based performance. Damage index in early research mainly emphasis in member ductility, ductility ratio could be define as curvature $\mu_{\phi}$, rotation $\mu_{\theta}$ and deformation $\mu_{\delta}$, 


$$
\mu_{\phi}=\phi_{m} / \phi_{y} \quad \mu_{\theta}=\theta_{m} / \theta_{y} \quad \mu_{\delta}=\delta_{m} / \delta_{y}
$$

Where $\phi_{m}, \theta_{m}$ and $\delta_{m}$ are respectively maximum curvature, maximum rotation and maximum deformation of member end, $\phi_{y}, \theta_{y}$ and $\delta_{y}$ are respectively yielding curvature, yielding rotation and yielding deformation.

(Banon,1982) also proposed a correctional ductility parameter that considering structural rigid and strength degeneration in same time,

$$
F D R=K_{0} / K_{m}
$$

Where $K_{o}$ is initial tangent stiffness, $K_{m}$ is equivalent tangent stiffness after maximum reaction. But in fact whether ductility ratio or correctional ductility ratio all could not give accurate judgment for structural damage and invalid.

Another damage index is relative storey displacement or relative storey rotation, in which relative storey rotation is widely accepted as estimate damage index at present in fragility research, in many research articles the index have taken as fragility standard in reinforced concrete buildings, and that relate to compatibility and effective in judging structural damage, that is to say that the index has characteristic in revealing both whole damage and local damage, and more better express structural whole damage compare with building bearing capacity, HAZUS99 series damage evaluation manual of US also adopted relative storey rotation as seismic vulnerability index.

Above both index could not reflect material accumulative damage effect, so other scholars lately proposed some index with accumulative damage effect, in which Park-Ang damage index is widely influence, the index is consist of two parts as shown in Eq. (12), and one is transformation effect and second is absorbing energy effect.

$$
D=\frac{\delta_{m}}{\delta_{u}}+\beta_{e} \frac{\int d E}{F_{y} \delta_{u}}
$$

Where $D$ damage parameter, $\delta_{m}$ is maximum seismic transformation, $\int d E$ is accumulative absorbing hysteresis energy, $\delta_{u}$ is ultimate deformation under monotonic loading, $F_{y}$ is yielding strength with longitudinal steel bars, $\beta_{e}$ is constant damage parameter under considering hysteresis loading, which is connect with ratio of shear span to effective depth of section, axial-load ratio, ratio of longitudinal reinforcement and stirrup ratio.

Damage parameter $D$ is function with structural maximum deformation $\delta_{m}$ and whole hysteresis energy $\int d E$, which is connect to loading time history, while the quantities $\beta_{e}$, $\delta_{u}$ and $F_{y}$ are independent of the loading history and are determined from experimental tests (Moustafa,2011), $D \geq 1$ means that buildings damage completely and could not bear loading.

$$
\beta_{e}=\left[0.37 n_{0}+0.36\left(k_{p}-0.2\right)^{2}\right] 0.9^{100 \rho_{c}}
$$


Where $n_{0}=N /\left(b d f_{c}^{\prime}\right)$ is standard axial loading, which is 0.05 when it less than $0.05, \rho_{c}$ is stirrup ratio, which is stirrup volume compare to core concrete volume, and is 0.004 when less than $0.004, k_{p}=\rho_{p} f_{y p} /\left(0.85 f_{c}^{\prime}\right)$ is standard reinforcement ratio.

$$
\delta_{u}=0.0052\left(\frac{l_{s}}{d}\right)^{0.93}\left(0.85 k_{p}\right)^{-0.27}\left(\frac{100 \rho_{c}}{n_{0}}\right)^{0.48}\left(\frac{f_{c}^{\prime}}{0.6895}\right)^{-0.15} l_{c}
$$

Where $l_{s}$ is span length with shearing, $l_{c}$ is respective member length or height, $f_{c}^{\prime}$ is uniaxial concrete compression strength, unit is $\mathrm{kN} / \mathrm{cm}^{2}, d$ is effective height, units is $\mathrm{cm}$.

In 1985, Park suggested that set $D=0.4$ as limit line between repairable and irreparable, in 1987, he proposed more detailed qualitative classified chart of concrete buildings' damage, as shown in Tab. 3.

\begin{tabular}{|c|c|}
\hline$D<0.1$ & Undamaged or localized trivial crack \\
\hline $0.1 \leq D<0.25$ & Light damage - trivial crack throughout \\
\hline $0.25 \leq D<0.4$ & Moderate damage-severe crack and localized buckling \\
\hline $0.4 \leq D<1$ & Severe damage-concrete crashing and reinforcing bars exposure \\
\hline$D \geq 1$ & collapse \\
\hline
\end{tabular}

Table 3. Qualitative damage description for concrete buildings (Park \& Ang, 1985)

Strength and stiffness degeneration is main characteristic of material and structure damage, because of high variability of stiffness degeneration in cylinder-load lead to difficult in actual application, in 2005 Colombo-Negro proposed modified Park-Ang damage parameter model corresponding to strength degeneration, and which is defined:

$$
\begin{aligned}
D & =1-\frac{M_{a c}}{M_{y o}} \\
& =1-\left(\left(1-\frac{\mu_{\max }}{\mu_{u}}\right)^{1 / \beta_{1}} \cdot 0.5\left(1-\tanh \left(\beta_{2} \frac{\int d E}{E_{u}^{*}}-\pi\right)\right) \cdot \exp \left(-\beta_{3} \frac{\int d E}{E_{u}^{*}}\right)\right)
\end{aligned}
$$

Where $M_{a c}$ is yield force or moment actual degradation value, $M_{y o}$ is force or moment of theoretical yielding point on skeleton envelop curve, $\mu_{\max }$ is attainable deformation ductility, $\mu_{u}$ is ultimate ductility, $E_{u}^{*}$ is ultimate cylinder energy, $\beta_{1}$ is coefficient of harden slope or soften slope in stress-strain curve, $\beta_{2}$ is consuming energy point in structure damage model when resistance force sloping, $\beta_{3}$ is strength dropping ratio in fragility damage model, as shown in Tab. 4. 


\begin{tabular}{|c|c|c|c|c|c|}
\hline coefficient & $(\mathrm{a})$ & $(\mathrm{b})$ & $(\mathrm{c})$ & $(\mathrm{d})$ & $(\mathrm{e})$ \\
\hline $\begin{array}{c}\text { Ductility-based strength decay } \\
\text { parameter } \beta_{1}\end{array}$ & 0.10 & 0.10 & 0.10 & 0.15 & 0.15 \\
\hline $\begin{array}{c}\text { Energy-based ductility strength decay } \\
\text { parameter } \beta_{2}\end{array}$ & 2.40 & 3.20 & 7.00 & 0.10 & 0.10 \\
\hline $\begin{array}{c}\text { Energy-based brittle strength decay } \\
\text { parameter } \beta_{3}\end{array}$ & 0.10 & 0.10 & 0.10 & 9.00 & 2.20 \\
\hline
\end{tabular}

(a) Damage behavior of well confined reinforced concrete columns. (b) Damage behavior of concrete-filled steel rectangular columns. (c) Damage behavior of welded steel joints. (d) Damage behavior of poorly confined reinforced concrete columns. (e) Damage behavior of shear-deficient reinforced concrete walls.

Table 4. Damage model coefficient suggested value (Colombo \& Negro,2005)

Where assuming system is double linear hysteresis model, post-yield stiffness is equal $3 \%$ of elastic yielding stiffness, so when ductility $=\mu$, hysteresis consumed energy is shown as Eq.(16).

$$
\int d E=\frac{1}{2} \mu_{y} f_{e}-\mu_{y} f_{y}\left(\mu-\frac{1}{2}\right)
$$

Where $\mu_{y}$ is yielding deformation, $f_{e}$ is yielding strength of equivalent linear system, $\frac{1}{2} \mu_{y} f$ is energy of equivalent linear system, $\mu_{y} f_{y}\left(\mu-\frac{1}{2}\right)$ is energy of plastic system, ultimate hysteresis energy is

$$
E^{*}=\mu_{y} f_{e}\left(\frac{1}{2}-\mu_{u} \bar{f}_{y u}+\frac{1}{2} \bar{f}_{y u}\right)
$$

Where $\bar{f}_{y u}$ ultimate standard yielding strength, and assumed as 0.8 , in according with above equations that could compute Colombo-Negro adjusted damage coefficient of specific structure

\begin{tabular}{|c|c|}
\hline Damage Scale & Value Range \\
\hline Light damage & $0.01-0.10$ \\
\hline Moderate damage & $0.10-0.40$ \\
\hline Severe damage & $0.40-0.70$ \\
\hline collapse & $>0.70$ \\
\hline
\end{tabular}

Table 5. Colombo-Negro suggested revised damage index for different limit states

One of primary object for calculate damage parameters is decided level of limit-value of different damage coefficient, and provide vulnerability scale for later seismic vulnerability analysis.

\section{Finite element and frame modeling}

In order to assess the adequacy of results obtained from Finite Element (FE) analyses have been carried out. The reinforced concrete models have been conducted with SeismoStruct 
V5.0, a fibre-element based professional 3D program for seismic analysis of framed structures, which can be freely downloaded from the Internet. The program is capable of predicting the large displacement behavior and the collapse load of framed structures under static or dynamic loading, duly accounting for geometric nonlinearities and material inelasticity. Section with fibre-element of column or beam is shown as Fig.7, and 200 fibre elements fulfill calculated requirement.

Distributed fibre inelasticity elements are becoming widely employed in earthquake engineering applications, Whilst their advantages in relation to nonlinear constant-confined concrete model theory proposed by (Madas,1992), (Martinez-Rueda J.E. \& Elnashai A.S.,1997) in 1990s, which uniaxial stain-stress relationship shown as Fig.8a, skeleton envelop curve could reflect confinement effect and hysteresis-stiffness degenerated characteristic. Steel stress-strain relationship proposed by (Menegotto and Pinto,1973), coupled with the isotropic hardening rules proposed by (Filippou,1983). The current implementation follows that carried out by (Monti \& Nuti, 1992). Its employment should be confined to the modelling of reinforced concrete structures, particularly those subjected to complex loading histories, where significant load reversals might occur. As discussed by (Prota et al.,2009), with the correct calibration, this model, initially developed with ribbed reinforcement bars in mind, can also be employed for the modelling of smooth rebars, often found in existing structures, as shown in Fig.8b.

In the article frame models proposed by (Ghobarah et al.,1999), who had been calculated on the based above fibre element, results coincide with results from general finite element program Drain-2D, in the same time, its accuracy in predicting the seismic response of reinforced concrete structures has been demonstrated through comparisons with experimental results derived from pseudo dynamic tests carried out on large-scale models (Casarotti et al.,2005).

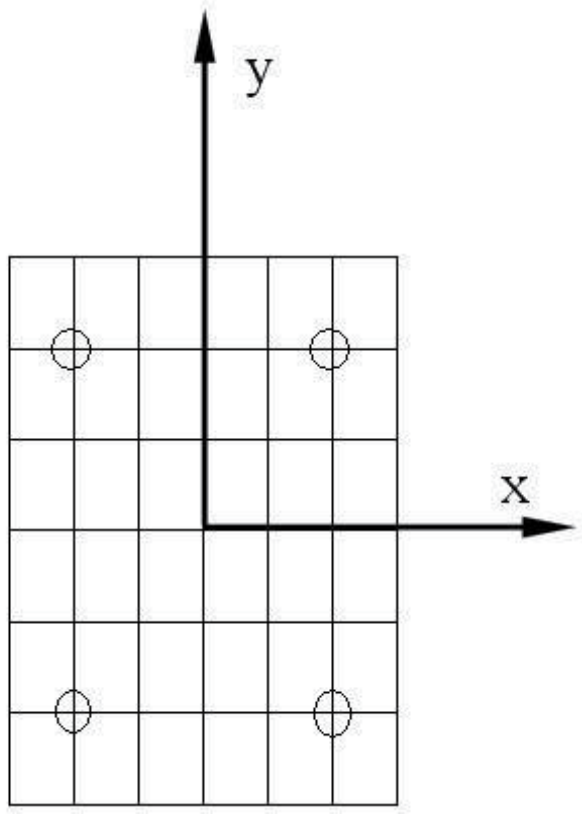

Fig. 7. Fibre in Section 


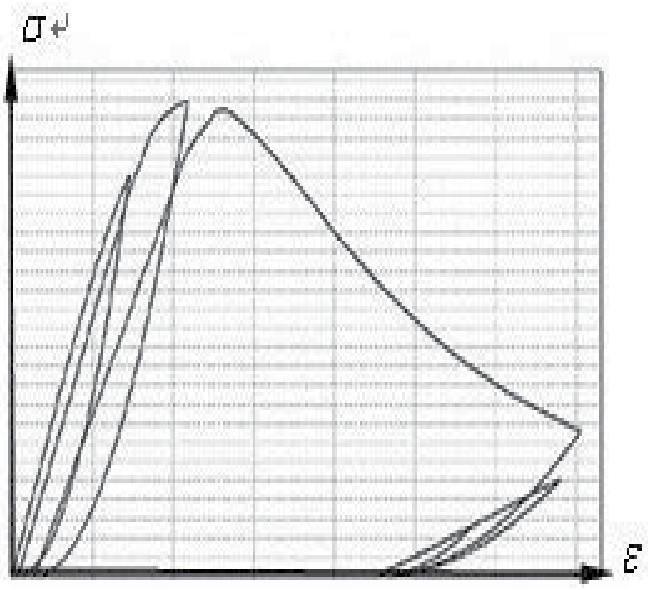

a

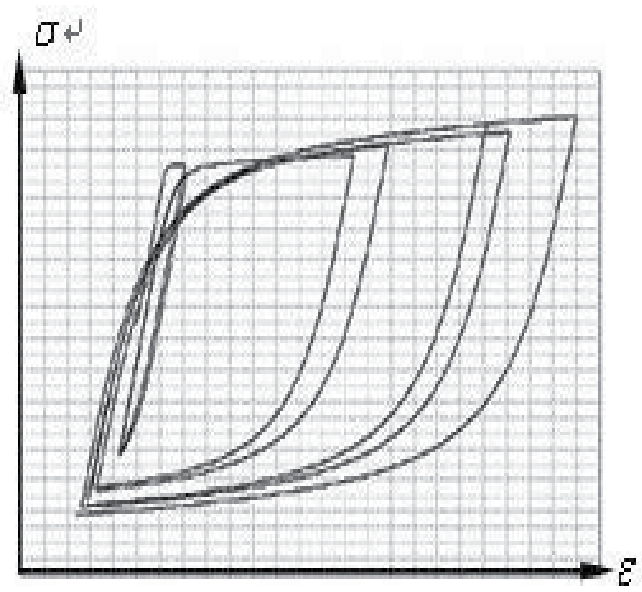

$\mathrm{b}$

Fig. 8. Material stress-strain model (a: concrete b: steel bar)

\section{Seismic vulnerability analysis of RC shear wall frame buildings}

At present, there are still many RC buildings in Western China regions. Most of them are small high-rise (12 16 storey) RC frames in recent years. The sample buildings were designed according to the prescriptions for loading, material, member dimensioning and detailing of the seismic design and gravity load design codes in place in China in 2002. The full design of the sample RC frame in Western China is presented in Fig.9

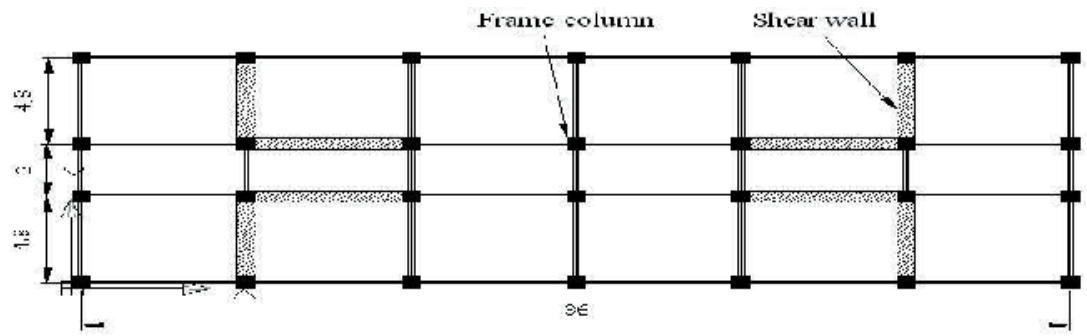

Fig. 9. Plan of typical frame-shear wall structure of 12-story

The structure consists of seven frames with bay width of $4.8 \mathrm{~m}, 3 \mathrm{~m}, 4.8 \mathrm{~m}$ respectively and frame spacing of $6 \mathrm{~m}$. It is symmetrical in plan and elevation, and RC beam section is $0.60 \mathrm{~m} \times 0.30 \mathrm{~m}$, at the same time RC column is $0.60 \mathrm{~m} \times 0.60 \mathrm{~m}$, which connected beams around the building at the corner and intersect with inner columns of building. Thickness of RC shear-wall is $0.3 \mathrm{~m}$ and floors consist of cast-in place reinforced concrete slabs is $120 \mathrm{~mm}$ thick.

Diameter longitudinal reinforcements of columns and beams' section are revealed in Fig.10, 8mm diameter stirrups must be spaced $100 \mathrm{~mm}$ apart at the extremes and $200 \mathrm{~mm}$ at the centre of the elements. The stress method used for the design is according to China seismic code.

The gravity load scenario consists of dead load and live load. When calculating the dead load, the weight of the structural members and the infill walls was included. The live load 
used was $2.5 \mathrm{kN} / \mathrm{m}^{2}$, which is typical for school building. Other types of loading, such as wind and snow, were not considered.
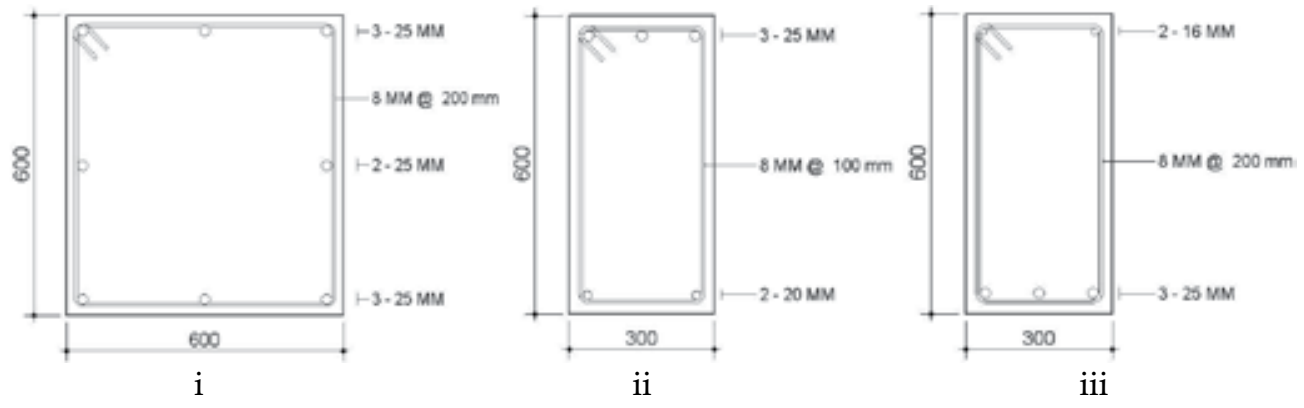

Fig. 10. Reinforced bars of section (i: column ii: side-beam iii:mid-beam )

Because of complicated climate in China all of year, the concrete strength must at least C30 according to China seismic code, reinforced bar (HPB235 HRB335) strength is 235 and 335 Mpa respectively, concrete strength is $30 \mathrm{Mpa}$, the stress-stain relationship are illustrated in Fig.8.

Ground motion characteristics have a significant effect on the vulnerability curves and special attention is required during the record selection phase in Fig.11.
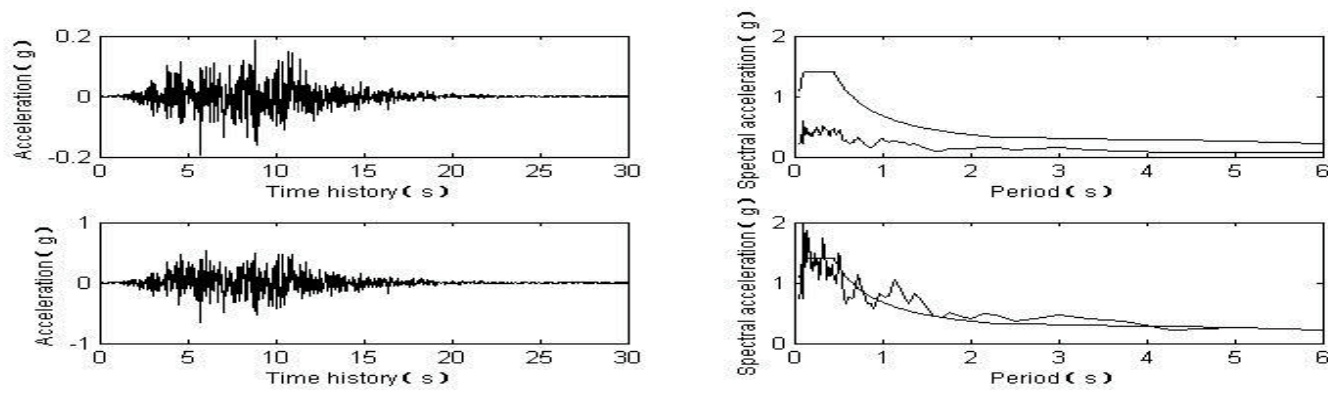

Fig. 11. Stochastic record compatible with the target spectrum of China code

\subsection{Determination of limit states}

The aim of this analysis is to evaluate the building's potential seismic performance. Four limit state conditions have been taken into account: light damage, moderate damage, extensive damage and complete damage. So if a building deformation beyond the extensive damage limit state it might not be economically advantageous to repair the building because many of the school buildings of Sichuan region were being set up without carefully thought of structural seismic codes of China.

\begin{tabular}{|c|c|c|c|c|c|}
\hline $\begin{array}{c}\text { Limit } \\
\text { state }\end{array}$ & $\begin{array}{c}\text { Inter-storey } \\
\text { drift }(\mathrm{mm})\end{array}$ & $\begin{array}{c}\text { Inter-storey } \\
\text { drift ratio(\%) }\end{array}$ & $\begin{array}{c}\text { Limit } \\
\text { state }\end{array}$ & $\begin{array}{c}\text { Inter-storey } \\
\text { drift }(\mathrm{mm})\end{array}$ & $\begin{array}{c}\text { Inter-storey } \\
\text { drift ratio(\%) }\end{array}$ \\
\hline $\begin{array}{c}\text { Light } \\
\text { Moderate }\end{array}$ & 6.6 & 0.20 & Extensive & 33.0 & 1.00 \\
Complete & 82.5 & 2.00 \\
\hline
\end{tabular}

Table 6. Limit states and corresponding inter-storey drifts ratios(ISD \%) 


\subsection{Vulnerability curve}

Dynamic time-history analysis is used to evaluate the seismic response and to derive the vulnerability curve. This approach is the most tedious but it is also the more accurate way to assess the vulnerability of RC buildings in China. The selected frame was subject to each group of the stochastic artificial records.

The stochastic damage scatter diagram and damage versus hazard relationship of the typical $\mathrm{RC}$ frame is illustrated in Fig.12. The damage axis (y-axis) described as the hazard axis ( $\mathrm{x}-$ axis) is described as spectral acceleration pga. Each vertical line of scattered data corresponds to an intensity level. The horizontal lines in the figure represent the limit states used in this study and described in terms of ISD\%.

A statistical distribution is fitted to the data for each intensity level on each vertical line. The normal parameters, the mean $M_{d s}$ and standard deviation $\beta_{d s}$ of the damage state are calculated for each of these $S_{k}$ intensity levels. At each intensity level, the probability of exceeding each limit state is calculated. LS1, LS2, LS3 and LS4 represent the limit states for light, moderate, extensive and complete damage, respectively, as mentioned above. The mean and standard deviation values of the response data are also given in the Fig.13.

The probability of exceedance of a certain limit state is obtained by calculating the area of the standard normal distribution over the horizontal line of that limit state.
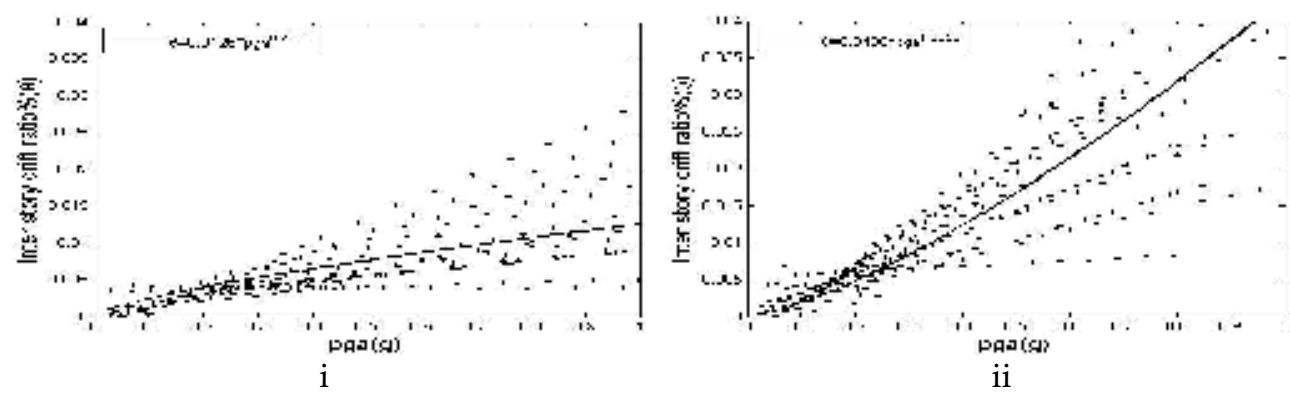

Fig. 12. Damage scatter dots relationship with PGA-ISD $\%_{\max }$ (i:X axis; ii:Y axis)
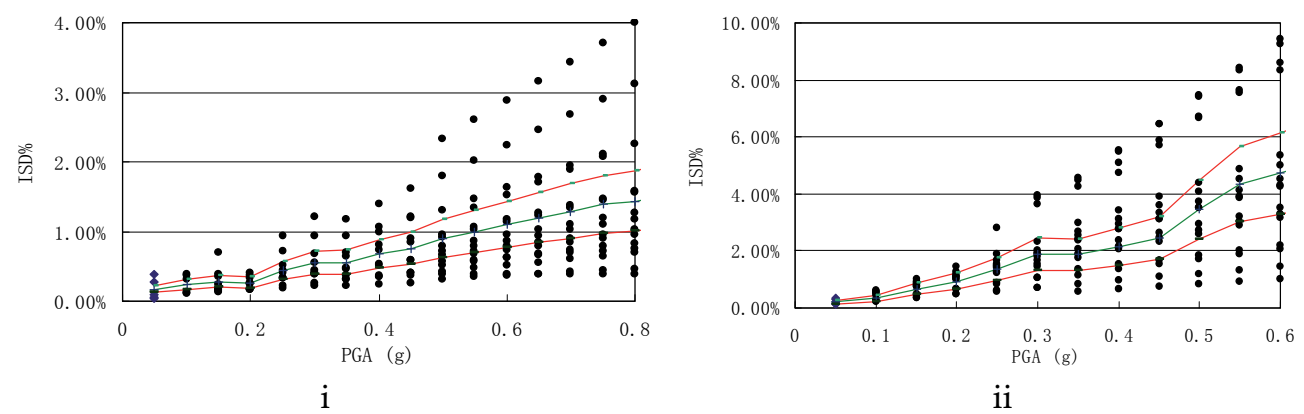

Fig. 13. Damage column dots plot with PGA-ISD\% ${ }_{\max }$ (i:X axis; ii:Y axis)

After calculating the probability of exceedance of the limit state for each intensity level, the vulnerability curve can be constructed by plotting the calculated data versus spectral acceleration. In this study, a standard deviation fit is assumed as 0.3. 
Figure.14 represents the vulnerability curves of typical frame-shear wall buildings in China with different spectral characteristic parameter. The curves become flatter as the nature of the statistical distribution of the response data. Vertical curves would represent deterministic response.
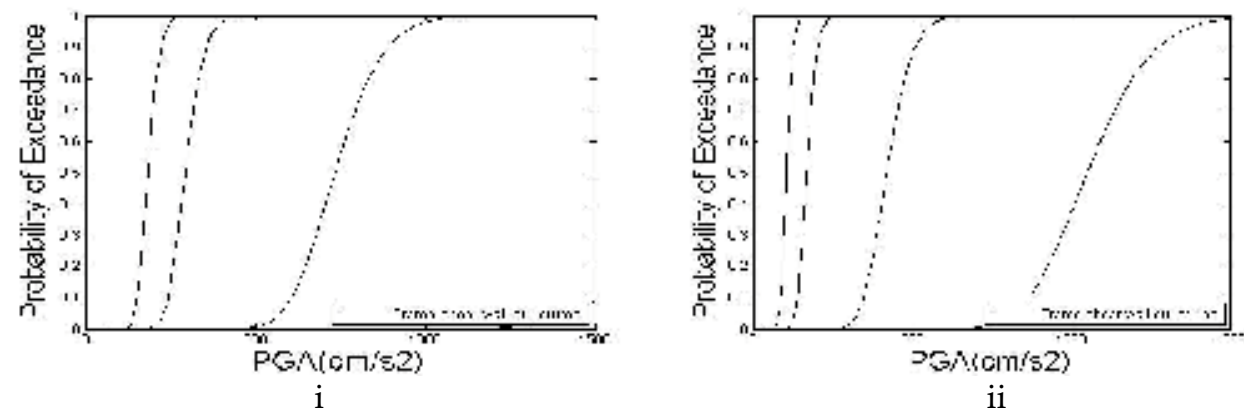

Fig. 14. Vulnerability curves with respected PGA (i : X axis; ii : Y axis)

\section{Seismic vulnerability analysis of structures with mid-story seismic isolation and reduction (MIRS)}

At present, there are many new built RC buildings in China large cities because of rapid developing economy. The buildings are designed according to the prescriptions for loading, material, member dimensioning and detailing of the seismic design and gravity load design codes in place in China in 2002.

The full design of the typical 12 stories MIRS in China is presented in Fig.15.

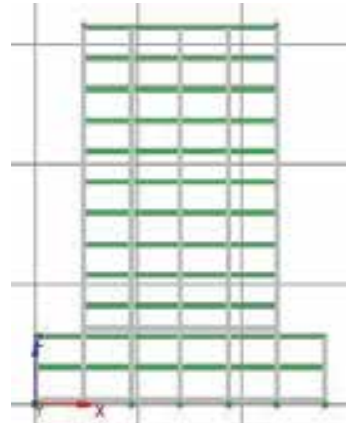

i. front façade

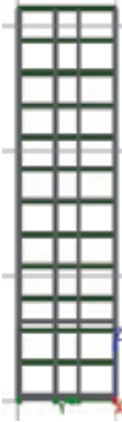

ii. left

facade view

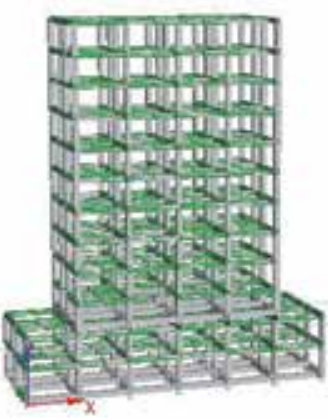

iii. 3D globe view

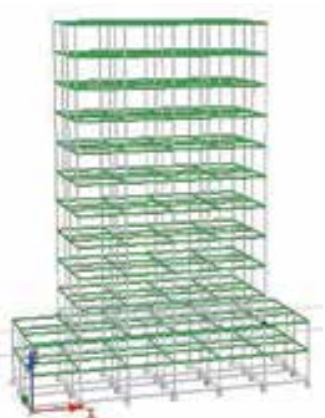

iv. 3D finite model with inelastic bars

Fig. 15. The full design of the typical MIRS in China

The structure consists of seven frames with bay width of $4.8 \mathrm{~m}, 3 \mathrm{~m}, 4.8 \mathrm{~m}$ respectively and frame spacing of $6 \mathrm{~m}$. It is symmetrical in plan and elevation, and RC beam $\left(0.6 \times 0.35 \mathrm{~m}^{2}\right)$ around the exterior perimeter and along the top of interior longitudinal and transverse columns in all the floors of the building including ground base level according China code. At the same time RC column $\left(0.6 \times 0.6 \mathrm{~m}^{2}\right)$ which connected beams around the building at the corner and intersect with inner columns of building. Story height is $3.3 \mathrm{~m}$, floors consist of 
cast-in place reinforced concrete slabs is $120 \mathrm{~mm}$ thick. Reinforcement of section of MIRS is shown in Fig.16.
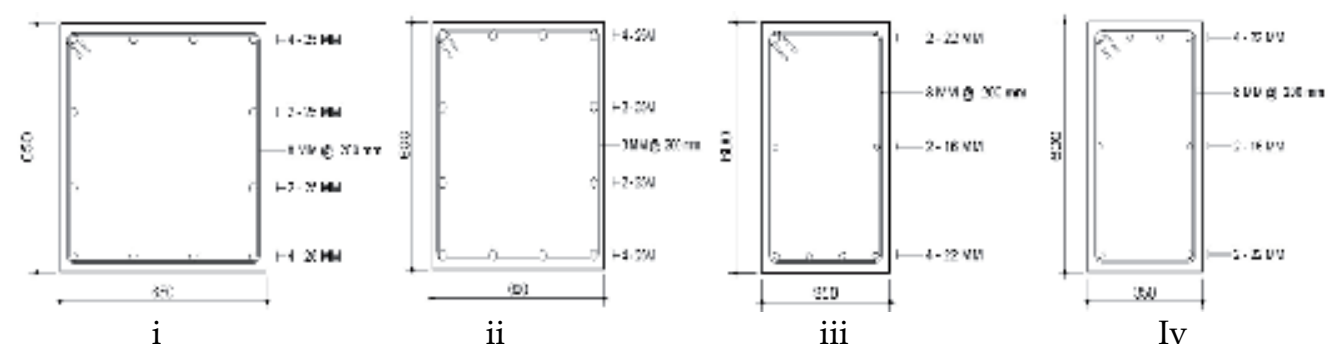

Fig. 16. Reinforcement of section of MIRS (i: column section of subway platform ii:column section on isolation layer iii: mid-beam section iv: side-beam section)

Columns have twelve $20 \mathrm{~mm}$ diameter longitudinal reinforcements, $8 \mathrm{~mm}$ diameter stirrups must be spaced $100 \mathrm{~mm}$ apart at the extremes and $200 \mathrm{~mm}$ at the centre of the elements, beams have four tension bars and two compressive bars. The stress method used for the design is according to China seismic code.

The gravity load scenario consists of dead load and live load. When calculating the dead load, the weight of the structural members and the infill walls was included. The live load used was $2.5 \mathrm{kN} / \mathrm{m}^{2}$, which is typical for city tall building. Other types of loading, such as wind and snow were not considered.

\subsection{Material and member property}

Because of moist climate in Sichuan district all of year, the concrete strength must at least C30 according to China seismic code, reinforced bar strength is 235 and 335 Mpa respectively, concrete strength is $30 \mathrm{Mpa}$.

Design of laminated rubber bearing is chosen LRB-G4-850-180 based China isolation design code(CECS126,2001), the key parameters of bearing are: equivalent damping ratio is 0.27 , secant stiffness ratio is 0.128 ,initial stiffness $k_{0}$ is $18100 \mathrm{KN} / \mathrm{m}$, yielding force $F_{y}$ is $203 \mathrm{KN}$ and post yielding ratio is 0.1 , laminated rubber bearing stress-strain relationship is shown in Fig.17.

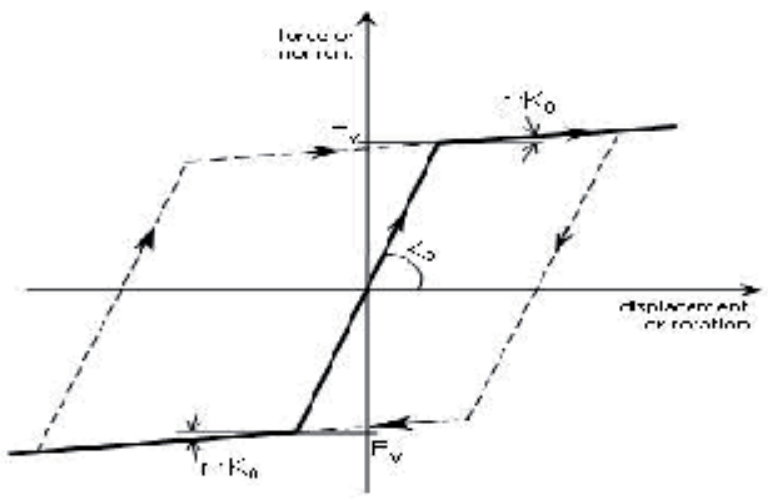

Fig. 17. Rubber bearing stress-strain model 


\subsection{Analytical model}

In order to evaluating seismic vulnerability of MIRS accurately, the building is modeled as a 3-D pole frame with lumped masses the study when assessing seismic response. Inelastic frame elements means 3D beam-column elements capable of modeling members of space frames with geometric and material nonlinearities with $5 \%$ global damp coefficient.

Ground motion characteristics have a significant effect on the fragility curves and special attention is required during the record selection phase. Considering this fact, 30 corrected stochastic artificial ground motions have been used in this study from different PGA covering a wide range of characteristics with a magnitude range between 5.1 and 7.8 . The motions are generally recorded on soft to medium sites according to Sichuan region's circumstance where basis prominent period range from $0.35 \mathrm{~s}$ to $0.45 \mathrm{~s}$ according China seismic code and crossed over the foundation in random orientation within $\mathrm{x}$-direction or $\mathrm{y}$ direction or both direction, as shown in Fig.11.

\subsection{Damage level definition}

The aim of this analysis is to evaluate the building's potential seismic performance, establishing a relation between the intensity of the seismic action and different damage states up to collapse. so damage level is defined according to the cracking, yielding or collapse of a set of elements or connections in the structure, as presented following Table.7 .

In view of being prone to brittle shear injury of inner brick masonry wall under strong motion, four limit state conditions have been taken into account: light damage, moderate damage, extensive damage and complete damage. So if a building deforms beyond the extensive damage limit state it might not be economically advantageous to repair the building because many of the school buildings of Sichuan region were being set up without carefully thought of structural seismic codes of China.

\begin{tabular}{|c|c|c|}
\hline Limit state & Inter-story drift $(\mathrm{mm})$ & ISD ratio(\%) \\
\hline Light damage & 6.6 & 0.20 \\
Moderate damage & 16.5 & 0.50 \\
Extensive damage & 33.0 & 1.00 \\
Collapse & 99.0 & 3.00 \\
\hline
\end{tabular}

Table 7. Limit States and corresponding inter-story drifts ratios(ISD\%)

\subsection{Fragility curve}

Dynamic time-history analysis is used to evaluate the seismic response and to derive the fragility curve. This approach is the most tedious but it is also the more accurate way to assess the vulnerability of MIRS in China. The selected frame was subject to each group of the stochastic artificial records. The stochastic damage scatter diagram of the typical MIRS is illustrated in Fig.18.

A statistical distribution is fitted to the data for each intensity level on each vertical line. The normal parameters, the mean $M_{d s}$ and standard deviation $\beta_{d s}$ of the damage state are calculated for each of these Sa intensity levels. At each intensity level, the probability of exceeding each limit state is calculated. LS1, LS2, LS3 and LS4 represent the limit states for light, moderate, extensive and complete damage, respectively, as mentioned above. The mean and standard deviation values of the response data are also given lately. 

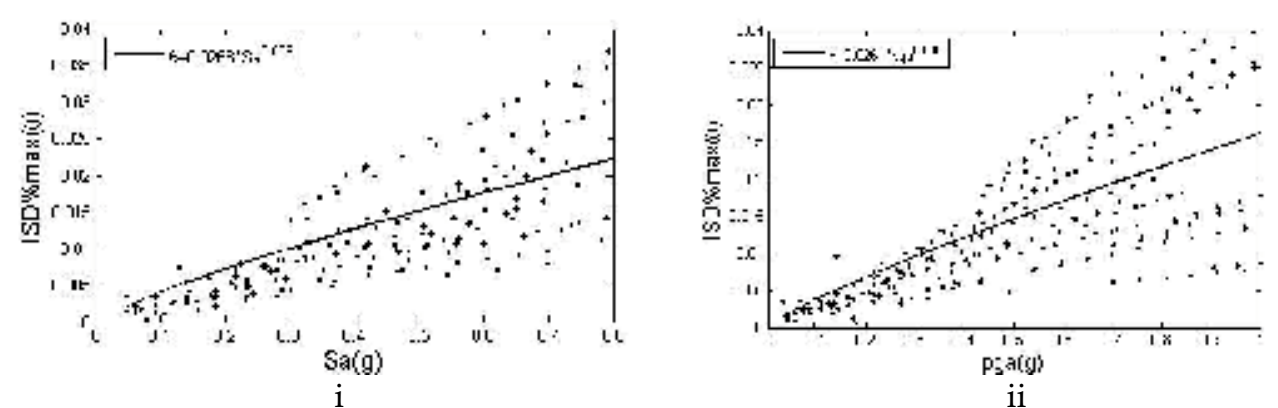

Fig. 18. Stochastic damage scatter diagram for MIRS(i : Sa; ii: PGA)

The probability of exceedance of a certain limit state is obtained by calculating the area of the standard normal distribution over the horizontal line of that limit state.

After calculating the probability of exceedance of the limit state for each intensity level, the vulnerability curve can be constructed by plotting the calculated data versus spectral acceleration. In this study, a standard deviation fit is assumed as 0.3.

Fig.19 represents the fragility curves of typical MIRS in China with different spectral characteristic parameter. The curves become flatter as the nature of the statistical distribution of the response data. Vertical curves would represent deterministic response.
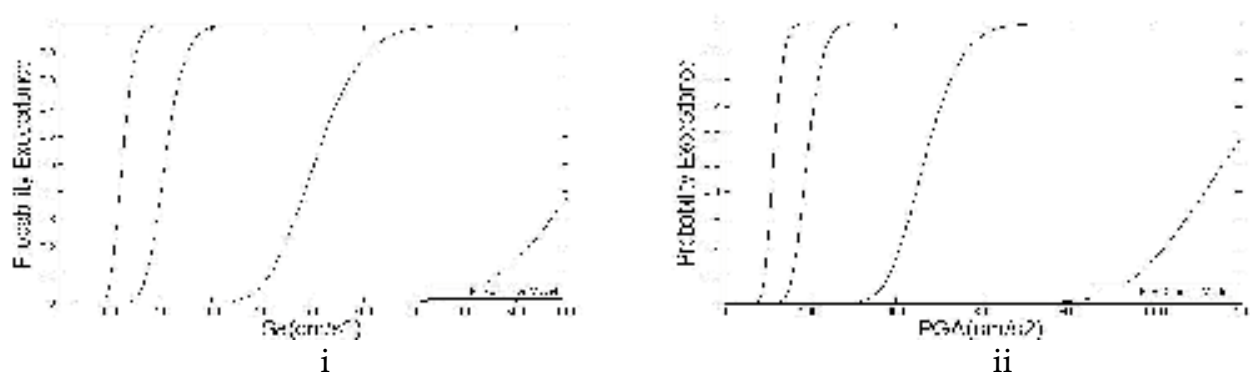

Fig. 19. Vulnerability curve for typical MIRS of China (i : Spectral accelerate; ii: PGA)

\section{Seismic vulnerability analysis of RC industrial buildings}

At present, there are many new built and old RC industrial buildings in Western China because of rapid developing economy. The buildings are designed according to the prescriptions for loading, material, member dimensioning and detailing of the seismic design and gravity load design codes of China.

The full design of the typical 12 stories MIRS in China is presented in Fig.20.

The structure consists of two frames with general configuration of bent widths and bay widths of $6 \mathrm{~m}$ and $24 \mathrm{~m}$ respectively, so the whole building have 66 meters long with 12 columns and $24 \mathrm{~m}$ width, It is symmetrical in plan and elevation, and rectangular reinforced concrete ring beam $\left(\begin{array}{lll}0.30 & 0.40 \mathrm{~m} 2\end{array}\right)$ on the bracket of two side longitudinal columns of the building. At the same time RC column $\left(\begin{array}{lll}0.40 & 0.60 \mathrm{~m} 2\end{array}\right)$ which connected ring beam as confined frame element array along the exterior side of the building at the intersect with inner confined brick wall between columns. The roof of building which 
height selected is $9.6 \mathrm{~m}$ consist in steel fibres truss, the truss depth is changed from $2.4 \mathrm{~m}$ in centre to $1.5 \mathrm{~m}$ of two sides, there are four kind of circular hollow rod being defined to using with diameter from $0.03 \mathrm{~m}$ to $0.05 \mathrm{~m}$, and thickness of bar' section is also verify from 2 to $3 \mathrm{~mm}$.

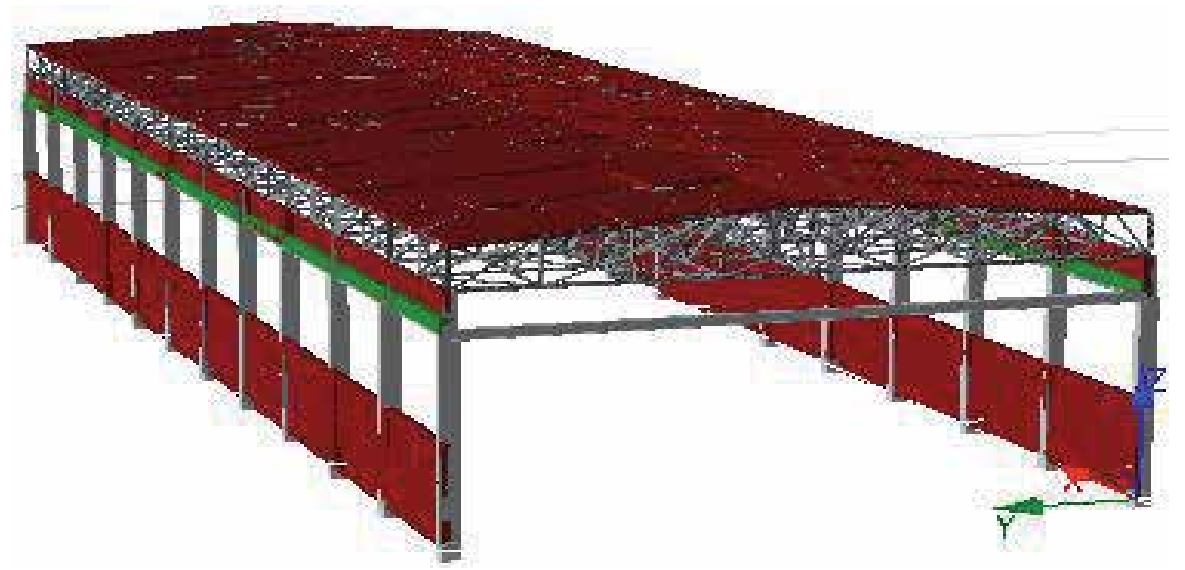

Fig. 20. The full design of the typical industrial buildings in China

The building's wall between columns generally consist of load-bearing infill masonry walls commonly made of clay bricks in Sichuan district of western China, confined by reinforced concrete vertical columns and width and thickness of wall are changed from $0.24 \mathrm{~m}$ to $0.37 \mathrm{~m}$ according China masonry code. Columns must have four $24 \mathrm{~mm}$ and $28 \mathrm{~mm}$ diameter longitudinal reinforcements, $8 \mathrm{~mm}$ diameter stirrups must be spaced $100 \mathrm{~mm}$ apart at the extremes and $200 \mathrm{~mm}$ at the centre of the elements. The stress method used for the design is according to China code.
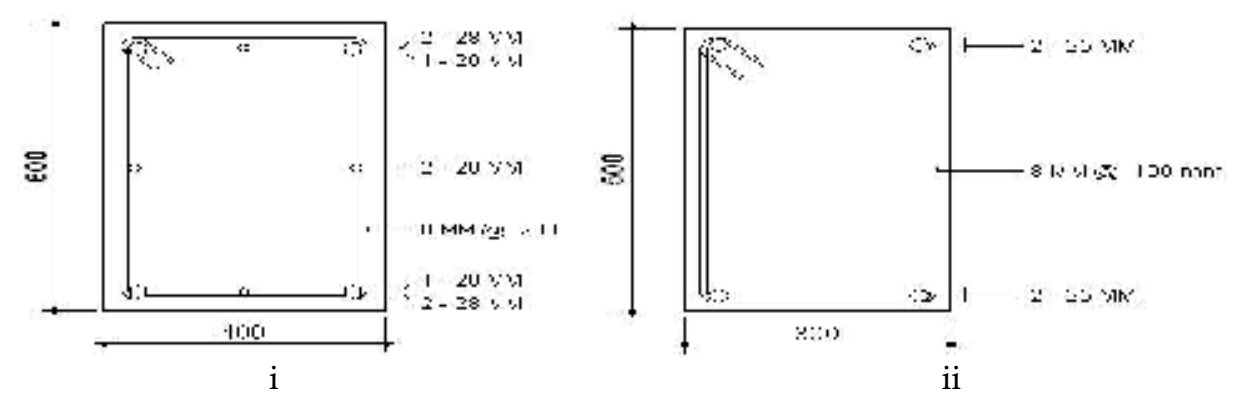

Fig. 21. RC industrial buildings' section (i: column ii: beam)

The industrial building was designed according to the China code for design of structure load for both gravity and seismic loads. The gravity load scenario consists of dead load and live load. When calculating the dead load, the weight of the structural members and the masonry infill walls was included. The roof live load used was $0.5 \mathrm{kN} / \mathrm{m}^{2}$, which is typical for an industrial building. Other types of loading, such as wind and snow, were not considered. At the same time lifting capacity of crane is considered randomly from 22 to 66 ton base on crane span and working condition. 


\subsection{Material and member property}

Because of moist climate in Sichuan district all of year, the clay brick strength must at least MU15 and the mortar strength must at least M10 according to China masonry code, so typical masonry shear strength is 0.27-1 Mpa. Bilinear stress-strain relationships with strain hardening were used for reinforced members which yield strength is $200 \mathrm{Mpa}$ and $300 \mathrm{Mpa}$, concrete strength is $30 \mathrm{Mpa}$ in considering of that many industrial buildings in Sichuan district have been built two decades ago, some respective buildings among those built even without any consideration of horizontal seismic loads. And coefficient of variation of $30 \%$ have been considered for steel and concrete respectively. Uniaxial nonlinear constant confinement concrete model that constant confining pressure is assumed throughout the entire stress-strain range is proposed by (Madas,1992) to apply to element of concrete.

\subsection{Generation of ground stochastic motion input}

Ground motion characteristics have a significant effect on the fragility curves and special attention is required during the record selection phase. Considering this fact, 90 corrected stochastic artificial ground motions have been used in this study from different PGA covering a wide range of characteristics with a magnitude range between 5.1 and 7.8 . The motions are generally recorded on soft to medium sites according to Sichuan region's circumstance where basis prominent period range from $0.35 \mathrm{~s}$ to $0.45 \mathrm{~s}$ according China seismic code and crossed over the foundation in random orientation within $\mathrm{x}$-direction or $\mathrm{y}$ direction or both direction. as shown in Fig.11.

\subsection{Damage level definition}

The aim of this analysis is to evaluate the building's potential seismic performance, establishing a relation between the intensity of the seismic action and different damage states up to collapse. so damage level is defined according to the cracking, yielding or collapse of a set of elements or connections in the structure, as presented following Table.8 .

Four limit state conditions have been taken into account: light damage, moderate damage, extensive damage and complete damage as table1 So if a building deforms beyond the extensive damage limit state it might not be economically advantageous to repair the building because many of the industrial buildings of Sichuan region were be set up without carefully thought of structural seismic codes of China.

\begin{tabular}{|c|c|c|}
\hline Limit state & Inter-story drift $(\mathrm{mm})$ & ISD ratio(\%) \\
\hline Light damage & 24 & 0.25 \\
Moderate damage & 38.5 & 0.40 \\
Extensive damage & 96.0 & 1.00 \\
Collapse & 240 & 2.50 \\
\hline
\end{tabular}

Table 8. Limit States and corresponding inter-story drifts ratios (ISD\%)

\subsection{Fragility curve}

Dynamic time-history analysis is used to evaluate the seismic response and to derive the fragility curve. This approach is the most tedious but it is also the more direct and accurate way to assess the fragility of Sichuan industrial buildings. The selected frame with confined 
masonry shear wall was subject to each group of the stochastic artificial records. Each group records were consisted of three stochastic ground motions.

There have some peak-displacement-history of original frame to be shown in Fig.22.
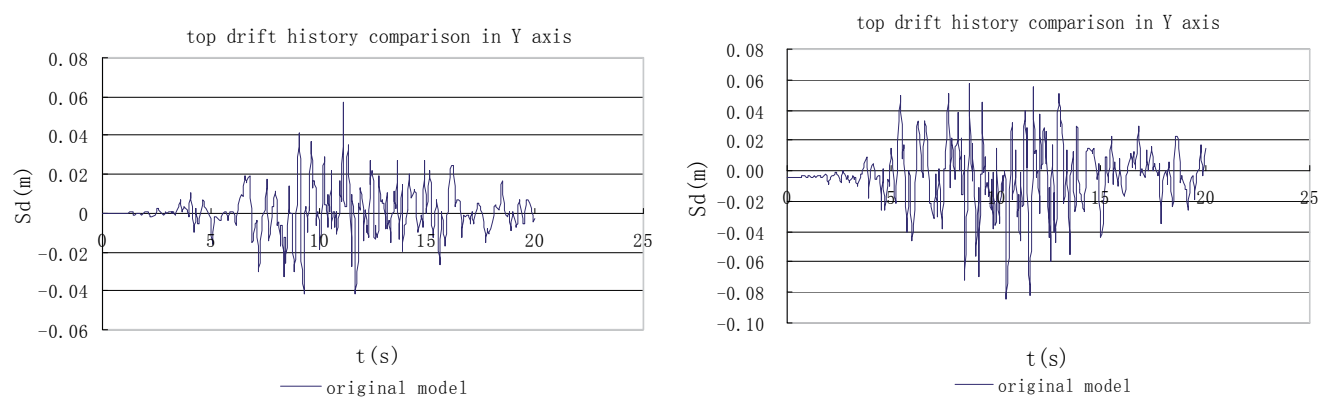

Fig. 22. Top drift time history with pga $=300$ gal in $X$ and $Y$ direction

The damage versus motion relationship is illustrated in Fig.23, The damage axis (Y-axis) described in terms of maximum inter storey drift ratio $\left(\mathrm{ISD}_{\max } \%\right)$ when the hazard axis $(\mathrm{X}$ axis) is described as spectral acceleration (Sa). Each vertical line of scattered data corresponds to an intensity level. From bottom to top, these are four limit states as light damage to complete damage respectively. Moreover the average value of each vertical data are connect by red line when \pm 0.3 variance have been considered as brown lines. The results also revealed that difference of seismic capacity of structure at two directions of industrial buildings, a standard deviation fit is assumed as 0.3.

The probability of exceedance of a certain limit state is obtained by calculating the area of the standard normal distribution over the horizontal line of that limit state.
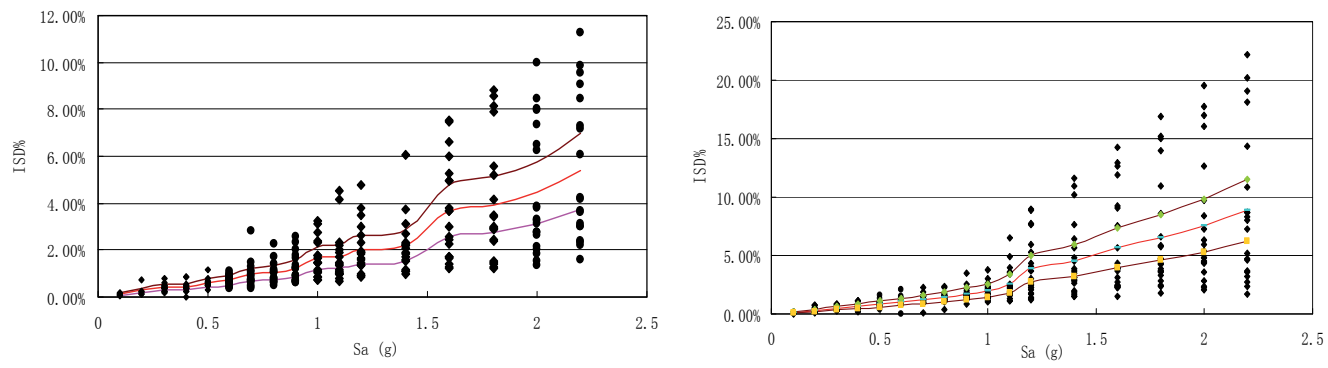

Fig. 23. Damage versus motion relationship in $X$ and $Y$ direction

After calculating the probability of exceedance of the limit state for each intensity level, the vulnerability curve can be constructed by plotting the calculated data versus Sa.

Fig.24 represents the fragility curves of typical industrial buildings in Western China with different spectral characteristic parameter. The curves become flatter as the nature of the statistical distribution of the response data. Vertical curves would represent deterministic response. 


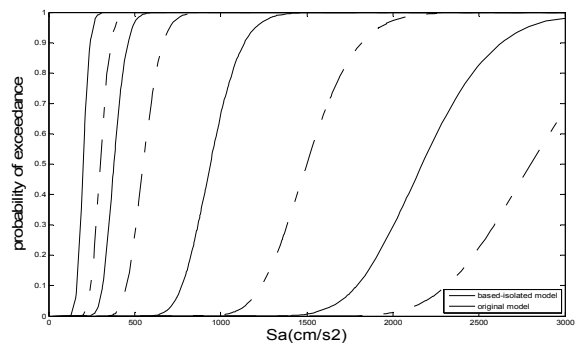

i

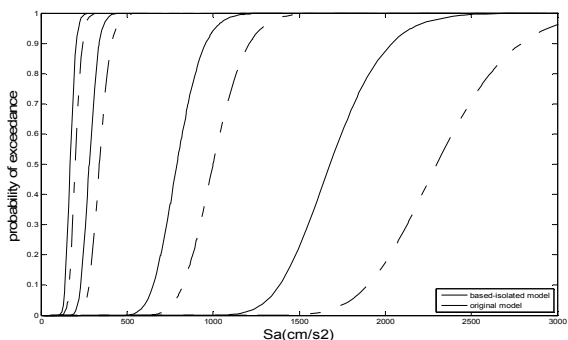

ii

Fig. 24. Vulnerability curve with spectral acceleration (i : X direction; ii: Y direction)

\section{Conclusion}

The following conclusions have been obtained.

1. The seismic vulnerability research is actual important part of perform based seismic design (PBSD), and the research work is inevitably lasting along perform based seismic design theory. The representative response spectrum of West China was been built and fit unsteady initial stochastic record in consideration of characteristic of frequency and random angle of phase position. The actual applied stochastic wave was been created after initial wave optimized with China code spectrum.

2. The representative small high-rise RC-shear wall buildings were been selected and seismic vulnerability analysis been made based dynamic time-history method, the analytical vulnerability curves were been obtained firstly in homeland. Probability of moderate damage was much seldom when PGA $<0.1 \mathrm{~g}$ under foundation stability precondition, in contrary probability of moderate damage was started to increasing when PGA $>0.3 \mathrm{~g}$. The seismic capability of longitudinal and transversal orientation of small high-rise RC-shear wall buildings was different, probability of slight damage was coherent under minor earthquake, vulnerability of longitudinal orientation ( $X$ axis) was less than transverse orientation ( $\mathrm{Y}$ axis) of frame under major earthquake obviously.

3. The typical MIRS in China were been modeled and their seismic fragility characteristic were studied on the base of dynamic nonlinear method. The results display better response features than general type of $\mathrm{RC}$ frames. The steep light damage curve reflects the roles of the infill brick panels that dominate the response in the vicinity of the light damage limit state. When the confined infill column are damaged and laminated rubber bearings began to play a key role in seicmic energy dissipation, the building reach interstory drift more flexible than before.

4. The seismic performance and vulnerability of industrial buildings were also been analyzed, seismic capacity and vulnerability of based-isolated models and original models were been compared. The seismic capability of two orientations was also different and longitudinal orientation of industrial buildings ( $\mathrm{X}$ axis) was more capacity in seismic resistance than transverse orientation ( $Y$ axis) of buildings. Based-isolated model minimized $30 \%-50 \%$ of $\mathrm{ISD}_{\max } \%$ comparing to original model under major earthquake, so the based-isolated industrial buildings are more high seismic resistance than original industrial buildings in major earthquake. 


\section{Acknowledgement}

The author was funded by the State key Program of National Natural Science Foundation of China (Grant No. 90815027), National Natural Science Foundation of China (Grant No. 51078097) and Natural Science Foundation of Ningxia Province (Grant No. NZ1156).

\section{References}

I. Takewaki (2011). Preliminary report of the 2011 off the Pacific coast of Tohoku Earthquake. Journal of Zhejiang Univ-Sci A (Appl Phys \& Eng), Vol.12, No.5, pp. (327334), ISSN: 1862-1775

Whitman, R.V.; Reed, J.W. and Hong, S.T. (1973). Earthquake Damage Probability Matrices, Proceedings of the Fifth World Conference on Earthquake Engineering, Rome, Italy, $12,1973$.

Braga, F.; Dolce, M. and Liberatore, D.A. (1982). Statistical Study on Damaged Buildings and an Ensuing Review of the MSK-76 Scale. Proceedings of the Seventh European Conference on Earthquake Engineering. Athens, Greece.1982.

Corsanego, A. \& Petrini, V. (1990). Seismic Vulnerability of Buildings - Work in Progress. Proceedings of the Workshop II on Seismic Risk Vulnerability and Risk Assessment, Trieste, Italy, 1990.

Di Pasquale, G.; Orsini, G. and Romeo, R.W. (2005). New Developments in Seismic Risk Assessment in Italy, Bulletin of Earthquake Engineering, Vol. 3, No. 1, (2005), pp. (101128), ISSN:1573-1456

Dolce, M.; Masi, A.; Marino, M. and Vona, M. (2003). Earthquake Damage Scenarios of the Building Stock of Potenza (Southern Italy) Including Site Effects, Bulletin of Earthquake Engineering, Vol. 1, No. 1, (2003), pp.(115-140), ISSN:1573-1456

Grünthal, G. (editor) (1998). Cahiers du Centre Européen de Géodynamique et de Séismologie: Volume 15 - European Macroseismic Scale 1998, European Center for Geodynamics and Seismology, ISBN : 2-87977-008-4,Luxembourg.

Yamazaki, F. \& Murao, O. (2000). Vulnerability functions for Japanese buildings based on damage data from the 1995 Kobe earthquake. Implication of recent earthquakes on seismic risk: Series on Innovation and Construction, Vol. 2, Imperial College Press, ISBN: 978-186094-233-4, London

Orsini, G. (1999). A Model for Buildings' Vulnerability Assessment Using the Parameterless Scale of Seismic Intensity (PSI). Earthquake Spectra, Vol. 15, No. 3, (1999), pp. (463483), ISSN: 8755-2930

M. A. Erberik \& Amr S. Elnashai. (2004). Fragilityanaly sis of flat-slab structures. Engineering Structures, Vol.26, pp. (937-948), ISSN: 0141-0296

T. Rossetto \& Amr Elnashai. (2005).A new analytical procedure for the derivation of displacement-based vulnerability curves for populations of RC structures. Engineer Structures, Vol.27, pp.(397-409), ISSN: 0141-0296

M. S. Kircil \& Z. Polat. (2006), Fragility analysis of mid-rise R/C frame buildings. Engineering Structures, Vol.28, pp.(1335-1345), ISSN: 0141-0296

Jun Ji ; Amr S. \& Elnashai. (2007). Daniel A. Kuchma, An analytical framework for seismic fragility analysis of RC high-rise buildings. Engineering Structures, Vol.29, pp.(31973209), ISSN: 0141-0296 
B. Borzi.; R. Pinho \& H. Crowley.(2008). Simplified pushover-based vulnerability analysis for large-scale assessment of RC buildings. Engineer Structures, Vol.30, pp.(804-820), ISSN: 0141-0296

Kaul, M.K. (1978). Stochastic Characterization of Earthquake through Their Response Spectrum. Earthquake Engineering and Structural Dynamics, Vol.6, No.5, pp.(497-509), ISSN: 0098-8847

Murphy JR \& O'Brien LJ. (1977).The correlation of peak ground acceleration amplitude with seismic intensity and other physical parameters. Bull Seismol Soc Am, Vol.67,pp.(877-915), ISSN: 0037-1106

Husid,R.L. (1969)Analisis de Terremotos: Analisis General, Revista de IDIEM. Santiago Chile, Vol.8, No.1,pp.(21-42), ISSN:0716-1832

Trifunacc,M.D \& Brady,A.G. (1975). On the Correlation of Peak Accelaration of Strong Motion with Earthquake Magnitude Epicentral Distance and Site Condition Proc.US Nat.Conf. Earthquake Engineering, pp.(43-52), ISSN: 1570-761X

Takizawa,H.et.al. (1980).Collapse of a Modal for Ductile Reinforced Concrete Frames under Extreme Earthquake Motion. EESD, Vol.8, No.2, ISSN: 1521-334X

Jennings PC. \& Housner GW. (1968). Tsai NC Simulated earthquake motions. Technical Report. Earthquake Engineering Research Laboratory, California Institute of Technology

Ministry of Construction P.R. China.(2002). Code for seismic design of buildings (GB500112001). Beijing: China Construction Press

China Association for Engineer construction standardization.(2001).Technical specification for seismic-isolation with laminated rubber beating isolators CECS126:2001. Beijing: China Construction Press.

Banon,H. \& Veneziano,D. (1982). Seismic Safety of Reinforced Concrete Members and Structures. Earthquake Engineering and Structural Dynamics, Vol.10, pp.(179-193), ISSN: 0098-8847

Park, Y.J. \& Ang, A.H.S. (1985). Mechanistic Seismic Damage Model for Reinforced Concrete. Journal of Structural Engineering, Vol. 111, No. 4, pp.(722-739), ISSN: 09700137

Colombo,A \& Negro,P. (2005). A damage index of generalised applicability. Engineer Structures, Vol.27, pp.(1164-1174), ISSN: 0141-0296

Madas, P. and Elnashai, A.S. (1992). A new passive confinement model for transient analysis of reinforced concrete structures. Earthquake Engineering and Structural Dynamics, Vol. 21, pp. (409-431), ISSN: 0098-8847

Martinez-Rueda, J.E. \& Elnashai, A.S. (1997). Confined concrete model under cyclic load. Materials and Structures, Vol. 30, No. 197, pp. (139-147), ISSN: 1359-5997

Menegotto, M., Pinto, P.E. (1973). Method of analysis for cyclically loaded RC plane frames including changes in geometry and non-elastic behaviour of elements under combined normal force and bending, Symposium on the Resistance and Ultimate Deformability of Structures Acted on by well defined Repeated Loads, International Association for Bridge and Structural Engineering, Zurich, Switzerland, pp. (15-22)

Filippou, F.C.\& Popov, E.P., Bertero, V.V. (1983) Modelling of R/C joints under cyclic excitations, ASCE Journal of Structural Engineering, Vol. 109, No. 11, pp. (2666-2684), ISSN: 0970-0137 
Monti, G. \& Nuti, C. (1992). Nonlinear cyclic behaviour of reinforcing bars including buckling, Journal of Structural Engineering, Vol. 118, No. 12, pp.(3268-3284), ISSN: 0970-0137

Prota, A., Cicco, F., Cosenza, E.(2009). Cyclic behavior of smooth steel reinforcing bars: experimental analysis and modeling issues, Journal of Earthquake Engineering, Vol. 13, No. 4, pp.(500-519), ISSN: 1363-2469

A. Ghobarah (1999). Response-based Damage Assessment of Structures, Earthquake Engineering and Structural Dynamics, Vol. 28, No. 1, pp.(79-104), ISSN: 1096-9845

Casarotti, C; Pinho, R \& Calvi, GM.(2005). Adaptive pushover-based methods for seismic assessment and design of bridge structures, ROSE Research Report 2005/06. Pavia, Italy: IUSS Press, ISBN: 0-646-00004-7

Ohsaki, Y.(1978). On the significance of Phase Content in Earthquake Ground Motions. Earthquake Engineering and Structural Dynamics, Vol. 7, No.5, ISSN: 0098-8847

Amin, M. \& Ang, A. S. (1968). Non-stationary Stochastic Model of Earthquake Motions. Journal of American Society of Civil Engineers, Vol.94, No.EM2, ISSN: 1090-0241

A.Moustafa (2011). Damage-Based Design Earthquake Loads for Single-Degree-Of -Freedom Inelastic Structures. Journal of structural engineering (ASCE), Vol.137, No.3, pp. (456467), ISSN: 0733-9445 


\title{
Seismic Behavior and Retrofit of Infilled Frames
}

\author{
Mohammad Reza Tabeshpour ${ }^{1}$, Amir Azad ${ }^{1}$ and Ali Akbar Golafshani² \\ ${ }^{1}$ Mechanical Engineering Department, \\ ${ }^{2}$ Civil Engineering Department \\ Sharif University of Technology, Tehran, \\ Iran
}

\section{Introduction}

The most important recent earthquakes showed the importance of seismic behavior of various types of structures such as infilled frames: Japan 2011 (Takewaki et al., 2011), Haiti 2010 (Eberhard et al.) and Newzealand 2010 (Ismail et al. 2011). There are many important questions in the field of infilled frames, such as:

1. What are the negative and positive effects of infill walls in various types of buildings (local and global)?

2. Where infills should be considered (in which type of structures, considering lateral resisting system)?

3. When it is better to have interaction between frame and infill? In what type of structures considering the number of stories?

4. When it is better to have a gap between frame and infill?

5. What is the state of the art in this field?

6. Comparing National and international codes, what is the state of the practice?

7. What are the suitable strategies to retrofit existing infilled frames?

The aim of this chapter is to answer some of these questions. A categorized discussion is presented here to classify the problems solved or to be solved.

An ideal model of structure (bare frame) is considered usually in order to analyze and design the structure, which undoubtedly has important differences with its actual model. The actual model has also some differences with the considered model such as effects of infill walls. Existence of the infill walls basically provides higher stiffness and strength for the frames, but their detrimental effects on the structure performance is ignored due to lack of adequate information about the behavior of frames and infill walls. Meanwhile, recent studies have shown that different arrangements of stiffness, mass and strength by each other can have significant effect on structure behavior and their response. One of the most common failure modes of structures in earthquakes is soft story failure which causes by discontinuity of lateral force resisting elements such as braces, shear walls or infill walls in the first story. In this case columns are imposed to large deformation and also plastic hinges are formed at top and bottom of the columns. This case usually is named as story mechanism. Due to eccentricity of the center of mass and stiffness, which causes by asymmetrical arrangement of infill walls, high torsional moment is produced. Other failure mode is short column that is a common mode in concrete structures. 
Tabeshpour (2009d) has presented a comparative study of several building codes about masonry infill wall for design purposes. Tabeshpour et al. (2011d) investigated the lateral drift of concrete infilled frames to answer the following question: When and how is it better to separate infill from frame?

\section{Masonry infill walls}

Regarding to the combined behavior from the infill wall and structural frame which observed in many earthquakes, researchers predicted these events with modeling the masonry infill walls based on Fig. 1 as a compression strut elements. The existence of infill walls can change the structural behavior from flexural action into axial action. The advantages in the conversion of flexural action to axial action are:

- Reduce contribution of frame in lateral resisting;

- $\quad$ Reducing the lateral deformations.

The disadvantages of converting the flexural action to axial action:

- Increase of the axial load in the column and foundation,

- Creation of the concentrated shears at top and bottom of the column,

- Creation concentrated shears at beginning and end of the beam,

- Creation of huge shears on the foundation.

The equivalent struts of infill wall may be modeled in 3 different types: beam-to-beam (Fig. 2), column-to-column (Fig. 3) and node-to-node (Fig. 4). If it take places-to-column model like Fig. 3 then some forces would be exchanged between walls and column. This part of column is known as short column.

Because of significant stiffness and strength of the infill walls, they may cause severe irregularities in stiffness and strength in the building's elevation and plan. Various effects of masonry infill walls are summarized in table 1.

\section{The history of modeling}

Finite element modeling of masonry infill wall is a very complex and unreliable task due to several parameters such as: mortar characteristics, brick specifications, the interaction between brick and mortar, the interaction between masonry infill wall and frame. Possible discussions on this issue would be:

1. Modeling of infill walls categorized in two methods:

- $\quad$ Detailed models (Micro)

- Simple models (Macro)

The former is offered based on the finite element of the masonry infill wall which has utilized common methods in theories of elasticity and plasticity. The behavior of macro models are based on physical behavior of infill walls that can be modeled by using one or some structural elements.

2. The second important issue is related to the capacity of model to cover some/all of the related nonlinear phenomena. For example, actual stiffness in some models are not considered in the elastic limit. Some other models take into account its stiffness and decrease carefully as well as the decline in strength. That is how the structural behavior can be studied before reaching the fracture.

3. Another issue is to study the effects of one directional and cyclic loading on behavior and characteristics of the system. 


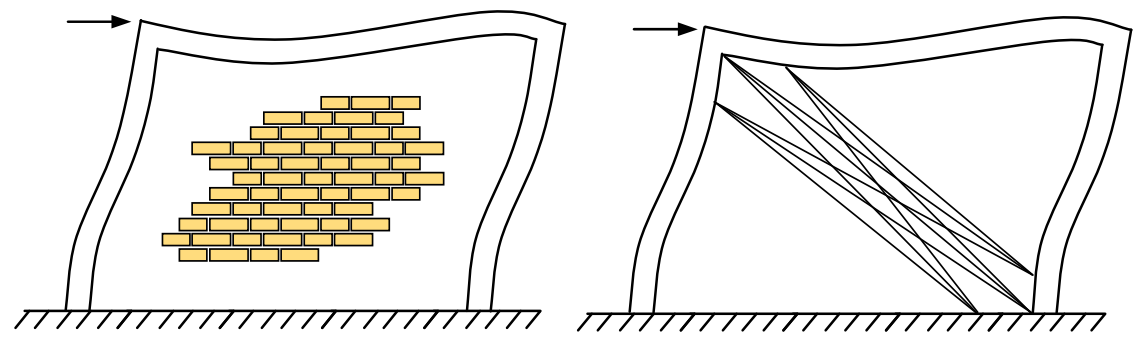

Fig. 1. Model with 9 struts

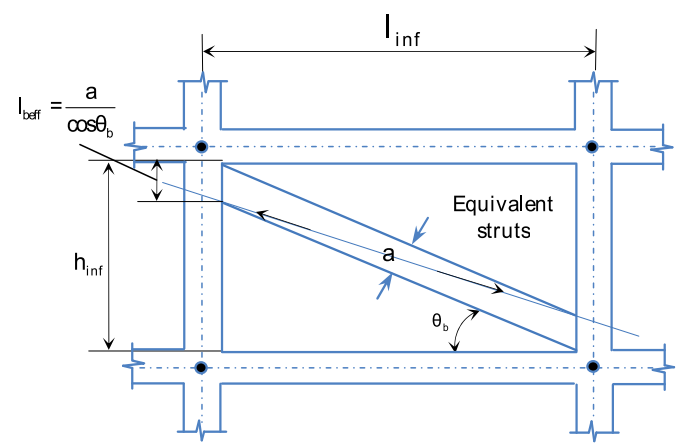

Fig. 2. Beam-to-beam model

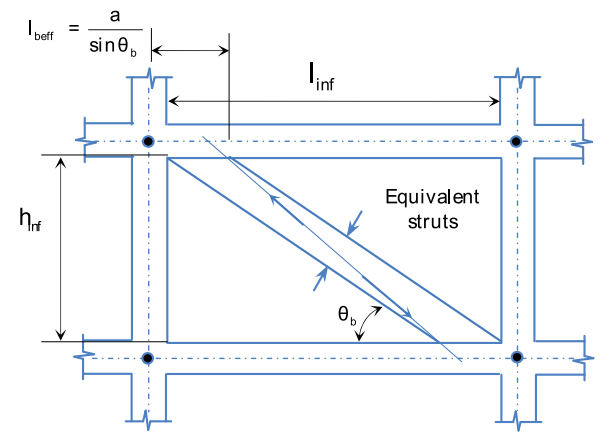

Fig. 3. Column-to-column model

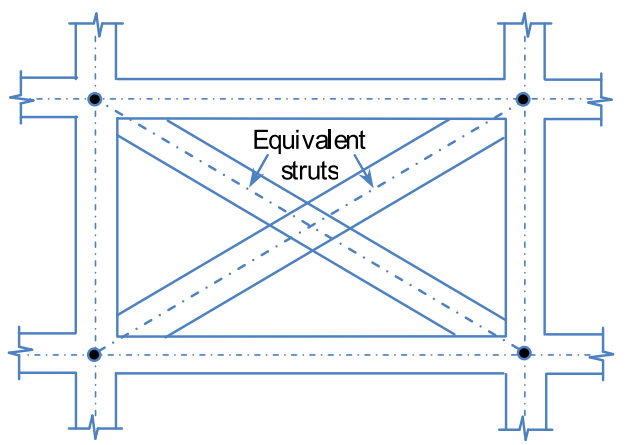

Fig. 4. Corner to corner model 


\begin{tabular}{|c|c|c|}
\hline No. & Advantages & Disadvantages \\
\hline 1 & $\begin{array}{c}\text { Higher stiffness and lower } \\
\text { displacement }\end{array}$ & Stiffness irregularity in height (soft story) \\
\hline 2 & Higher strength & Strength irregularity in height (weak story) \\
\hline 3 & Lower ductility requirements & Stiffness irregularity in plan (torsion) \\
\hline 4 & $\begin{array}{l}\text { Higher base level in special } \\
\text { conditions }\end{array}$ & $\begin{array}{l}\text { Improper distribution of force between columns } \\
\text { of a concrete frame }\end{array}$ \\
\hline 5 & $\begin{array}{l}\text { Ductile shear fracture in the steel } \\
\text { short column }\end{array}$ & $\begin{array}{l}\text { Improper distribution on force in plan (steel } \\
\text { short column) }\end{array}$ \\
\hline 6 & Frame design for small lateral loads & Increase in load design because of lower periods \\
\hline 7 & $\begin{array}{l}\text { Creation of couple system with } \\
\text { axial action of frame }\end{array}$ & $\begin{array}{l}\text { Increase on load design because of lower } \\
\text { behavior factor for joint system }\end{array}$ \\
\hline
\end{tabular}

Table 1. Advantages and disadvantages of masonry infill walls on steel or concrete frame

\subsection{Micro modeling}

All models discussed here are based on the finite element method which generally utilize 3 types of elements for providing the masonry infill wall, frame and the interaction between them. In most cases, special attention has been focused on the contacting elements between frame and masonry infill walls. Then, it has been clarified that numerical simulation of the infill wall is very important and their nonlinear phenamena must be modeled with great levels of accuracy. Research on this problem is closely related to develop elements used in masonry structures (Tabeshpour, 2009b).

Mallick and Severn (1967), have taken into account the contact of wall to frame particularly. The masonry infill walls were modeled as the rectangular elastic elements with 2 degree of freedom. The frame was also modeled using the elements without axial deformation. The slip of the frame against wall was also noticed along with the friction between them. They compared the results of analyses with experimental works and offered accurate presentation of the stiffness.

Goodman et al. (1968) developed an element in order to simulate the interaction between frame and wall. The rectangular element of the plain strain with 4 nodes and 2 transitional freedom degree in each node was modified to consider the contact condition properties. The shear strength of the element is dependent on adhesion and friction. This study has proposed a moderate mode between wall and frame with given length and zero initial width.

Researches from Malik and Garg (1971) modeled the effect of existence of shear slot between frame and masonry wall. They used the rectangular element of plain strain for the wall similar to the above mentioned technique. They also proposed that in the model of beam member for frame, the rotational degree of freedom must not be considered, which means that the frame must only be deformed under the shear and axial loads. This model was used to study 2 issues: the effect of openings available in the masonry infill wall in addition to the effect of shear slot available between frame and infill walls. Experimental work was also launched to evaluate the validity of the results.

Koset et al. (1974) observed that infill walls and frame cracks are occured and developed even in little lateral loads. This is attributed to the low tensile strength at the contact between infill walls and frame. Therefore, in order to simulate the system's response under 
the lateral load, opening/closing of gaps between masonry infill walls and frame must be considered.

King and Pandey (1978) used the element proposed by Goodman and his coworkers. Primary tests showed that the curves of shear stress were elasto-plastic in contact between the elements. Tangential stiffness characteristics $\left(K_{n}\right.$ and $\left.K_{s}\right)$ of the moderate elements were defined as functions of these elements. That was how the frictional slip of connection/separation between the elements was studied. They achieved acceptable results from Mallick and Severn (1967) models.

Liauw and Kwan (1984) advanced a plastic theory which allowed three different fracture modes. Based on the relative strength of columns, beams and masonry infill walls behaviour, three type of failure are mention below.

- Corner crashing of infill walls and fracture in columns

- Corner crashing of infill walls and fracture in beams

- Diagonal crack of masonry infill walls

Rivero and Walker (1984) developed a nonlinear model for simulating the response of the frame system which is Infilled by walls and is under stimulus of earthquake. 2 types of element were developed base on surface between frame and walls. Gap element and the connection element. The former was used aiming to show the distance between frame and infill walls in no tangential conditions, while the later was utilized to model the contact mode. The process of crack formation and development was studied carefully.

Shing et al. (2002) simulated the nonlinear behavior of the masonry wall elements using the plasticity theory. The cracks through bricks and mortar as well as the cracks between mortar joint and members of reinforced concrete were modeled and studied. In this model, overall behavior of the combined system before cracking was considered homogeneously and isotropically. The behavior of materials was presumed elasto-plastic based on the Von Mises yield criterion with tensions of Rankeen type. After calibrating the model by using experimental studies, the finite element model was capable to simulate the actual behavior of the system properties.

\subsection{Macro modeling}

The idea of using a simple member for simulating infill walls inside the frame has always been attractive, and has several advantages in the process of analysis and design. At the beginning, it was explained that a diagonal strut with appropriate mechanical properties can be a suitable candidate for walls. By using the diagonal strut model, it will be possible to enter the following items to the model:

- Shear stiffness of the infill wall,

- Small shear and tensile stress of column at the contact between wall and frame,

Although this simple model cannot notice the following complexities in the model:

- Decreasing the stiffness and strength under cyclic loads,

- Out of plane behavior for masonry infill walls when diagonal crack occurred,

- Shear slip along joints which occurs at the middle height of infill walls.

These problems were solved to some extent in the equivalent strut model. For example, Klinger and Bertero (1976) modeled masonry infill walls with two equivalent struts and noted the effects of stiffness dimming. Polyakov (1956) studied the normal and shear stresses at the middle of infill walls, using the variation calculation method and offered a numerical technique to estimate the load which cause diagonal crash. 
Holmz (1961) presented formula for a diagonal strut for the first time. He assumed that the width of equivalent strut is equal to one third of the diagonal length. After that, several studies were performed to define the width of the equivalent strut.

Stafford Smith (1968) observed that the equivalent diagonal strut has many simplifications and some modifications must be done on its equivalent width. He assumed that the distribution of the interactional forces between frame and infill walls is triangular. This idea has a very high accuracy and is still in use. Based on the interaction length between infill walls and frame, other proposals were introduced by Mainstone (1971) and Kadir (1971).

Klinger and Bertero (1976) provided the first diagonal member with cyclic behavior which was able to consider the stiffness dimming behavior through the modeling procedure.

Chrysostomou (1991) investigate the behavior of the frame and the infill wall system under the earthquake loading regarding the effects of decreasing of stiffness and wall strength. He modeled the wall in any diagonal direction with three bars based on Fig. 8 . The The aL length is equal to the plastic hinge in column or beam. These members act compressively.

The effective width of equivalent strut in the infill wall proposed by different researchers has severe variation from 10 to $35 \%$. Table 1 summarizes different relations for the effective width of equivalent brace in the masonry infill walls and Tabeshpour recommends some values for effective width in Hand book, part 18 (page 65) (2009). Modeling of infill wall using commercial softwares is needed for design purposes (Tabeshpour, 2009e).

\begin{tabular}{|c|c|c|c|c|}
\hline \multicolumn{2}{|c|}{ Researcher } & Effective Width $\left(b_{w}\right)$ & $\lambda h$ & \\
\hline \multicolumn{2}{|c|}{ Holmes (1961) } & $b_{w}=[0.33] d_{w}$ & - & $170(\max )$ \\
\hline \multicolumn{2}{|c|}{ Mainstone (1971) } & $b_{w}=0.16(\lambda h)^{-0.3} d_{w}$ & 5 & 50 \\
\hline \multicolumn{2}{|c|}{ Klingner and Bertero (1978) } & $b_{w}=0.175(\lambda . h)^{-0.4} d_{w}$ & 5 & 45 (min) \\
\hline \multicolumn{2}{|c|}{ Liauw and Kwan (1984) } & $b_{w}=0.95 h_{w} \cos \theta(\lambda h)^{-0.5}$ & 5 & 90 \\
\hline \multicolumn{2}{|c|}{ Paulay and Priestley (1992) } & $b_{w}=[0.25] d_{w}$ & - & 125 \\
\hline \multirow{2}{*}{ Recommended } & $\begin{array}{c}\text { Upper band, Negative } \\
\text { Effect }\end{array}$ & $b_{w}=[0.2] d_{w}$ & - & 100 \\
\hline & $\begin{array}{c}\text { Lower band, Positive } \\
\text { Effect }\end{array}$ & $b_{w}=[0.1] d_{w}$ & - & 50 \\
\hline
\end{tabular}

Table 2. Different formulae of equivalent masonry strut's effective width 


\subsection{Yung's modulus of masonry materials}

The important point about Young's modulus of masonry materials is the range of values obtained from the relations proposed by different researchers which is attributed to the nature of masonry materials. Table 2 lists some relations presented by some researchers as well as the value of Yung's modulus for $15 \mathrm{Kg} / \mathrm{cm}^{2}$ compressive stress (Tabeshpour Hand book, part 18, 2009). Shear strength of materials are usually demonstrated in codes by static friction relation as show in follow:

$$
\tau=\tau_{0}+\mu \sigma_{y}
$$

$\tau_{0}$ : Joint shear strength

$\mu \sigma_{y}$ : Frictional strength component

$\mu:$ Internal frictional factor

$\sigma_{y}:$ Normal stress component along the horizontal direction

\begin{tabular}{|c|c|c|}
\hline Researcher & Module of Elasticity & $\begin{array}{c}E_{m}\left(\mathrm{~kg} / \mathrm{cm}^{2}\right) \\
f_{m}=15 \mathrm{~kg} / \mathrm{cm}^{2}\end{array}$ \\
\hline Sahlin (1971) & $E_{m}=750 f_{m}$ & 1100 \\
\hline Paulay and Priestley (1992) & $E_{m}=750 f_{m}$ & 1100 \\
\hline Sanbartolome (1990) & $E_{m}=500 f_{m}$ & $7500(\mathrm{~min})$ \\
\hline Sinha\&Pedreschi (1983) & $E_{m}=1180 f_{m}$ & 16000 \\
\hline Hendry (1990) & $E_{m}=2116 f_{m}$ & $25000(\max )$ \\
\hline Some others & $E_{m}=1000 f_{m}$ & 15000 \\
\hline
\end{tabular}

Table 3. Module of elasticity for equivalent masonry struts

\begin{tabular}{cc} 
Researcher & Shear bond strengths, $\tau_{o}$ \\
\hline Hendry (1990) & 0.3 to $0.6 \mathrm{MPa}$ \\
Shrive (1991) & 0.1 to $0.7 \mathrm{MPa}$ \\
Paulay and Priestley (1992) & 0.1 to $1.5 \mathrm{MPa}$ \\
\hline
\end{tabular}

Table 4. Shear bond strength for equivalent masonry struts

\begin{tabular}{cc} 
Researcher & $\mu$ \\
\hline Sahlin (1971) & 0.1 to 1.2 \\
Stöckl and Hofmann (1988) & 0.1 to 1.2 \\
Atkinson et al. (1989) & 0.7 and 0.85 \\
Hendry (1990) & 0.1 to 1.2 \\
Paulay and Priestley (1992) & 0.3 for design purposes \\
\hline
\end{tabular}

Table 5. Ductility for equivalent masonry struts 


\section{Failure modes}

\subsection{Soft Story}

The base floors of the existing buildings are generally arranged as garages or offices. No walls are built in these floors due to its prescribed usage and comfort problems. But upper floors have walls separating rooms from each other for the residential usage. In these arrangements, the upper floors of most buildings are more rigid than their base floors. As a result, the seismic behaviors of the base and the upper floors are significantly different from each other. This phenomenon is called as the weak-story irregularity. Weak stories are subjected to larger lateral loads during earthquakes and under lateral loads their lateral deformations are greater than those of other floors so the design of structural members of weak stories is critical and it should be different from the upper floors.

Sattar and Liel (2000) shown in their results of pushover analysis that infill walls were increased the initial stiffness, strength, and energy dissipation of the infilled frame, compared to the bare frame, despite wall's brittle failure modes. Vulnerability and damage analysis of existing buildings using damage indices have been presented by Golafshani et al. (2005). Such studies can be used for quantitative investigation of existing buildings. A casestudy structure that collapsed because of a soft-story mechanism during the 2009 L'Aquila earthquake was studied with Verderame et al. (2009). Their study presented some peculiar details and results, but it could not be stated. It represents a common practice in the L'Aquila building stock.

Haque and Amanat (2009) shows that, when RC framed buildings having brick masonry infill on upper floor with soft ground floor is subjected to earthquake loading, base shear can be more than twice to that predicted by equivalent earthquake force method with or without infill or even by response spectrum method when no infill in the analysis model.

Tena-Colunga (2010) evaluated how the soft first story irregularity condition should be defined: (a) as a significant reduction of the lateral shear stiffness of all resisting frames within a given story, as established in the seismic provisions of Mexican building codes or, (b) as a substantial reduction of the lateral shear stiffness of one or more resisting frames within a given story, as proposed by the author.

Kirac et al. (2010) studied the seismic behavior of weak-story. Calculations were carried out for the building models which are consisting of various stories with different storey heights and spans. Some weak-story models were structural systems of existing buildings which were damaged during earthquakes. It was observed that negative effects of this irregularity could be reduced by some precautions during the construction stage. Also some recommendations were presented for the existing buildings with weak-story irregularity.

A conceptual and numerical analysis for investigating the effect of masonry infills on seismic behavior of concrete frames considering various type of infill arrangements was presented by Tabeshpour et al (2004). It was found that a large drift is concentrated in soft story (the story with no infill). Design of columns in soft stories is an important problem to have an acceptable mechanism in severe earthquakes. In order to avoid from soft story failure, columns should be designed for increased loads, Tabeshpour (2009f) has investigated increasing design load in specific columns and presented a simple formula for this purpose. 


\begin{tabular}{|c|c|c|c|c|c|}
\hline \multirow[b]{2}{*}{ Researcher } & \multirow{2}{*}{$\begin{array}{c}\delta_{c r}(\%) \\
\text { URM }\end{array}$} & \multicolumn{3}{|c|}{$\delta_{\max }(\%)$} & \multirow[b]{2}{*}{ Other note } \\
\hline & & URM & $\begin{array}{c}\text { Frame }+ \\
\text { Infill }\end{array}$ & $\begin{array}{l}\text { Bare } \\
\text { Frame }\end{array}$ & \\
\hline Fiorato (1970) & & & 1.1 & & \\
\hline Zarnic \& Tomazevic (1984) & 0.2 & & 1 & 3 & \\
\hline Govindan et al. (1986) & & & 3 & 1.5 & \\
\hline Valiasis \& Stylianidis (1989) & & & 0.6 & 1 & $\tau_{u} \approx 0.25-0.3 \mathrm{Mpa}$ \\
\hline Carydis et al. (1992) & & & & & $\begin{array}{c}\text { Good system behaviour up to } \\
0.14 \% \text { drift; steel frame with } \\
\text { infill }\end{array}$ \\
\hline Pires\&Carvalho (1992) & 0.1 & & 0.5 & & $\tau_{u} \approx 0.27-0.51 \mathrm{Mpa}$ \\
\hline Shing et al. (1992) & & & & & $\tau_{u} \approx 0.34 \mathrm{Mpa}$ \\
\hline Valiasis et al. (1993) & 0.2 to 0.3 & & & & \\
\hline Fardis \& Calvi (1995) & & & & & $\begin{array}{c}\delta_{\max } \text { for URM is } \\
0.1 \delta_{\max } \text { for frame }\end{array}$ \\
\hline Zarnic (1995) & 0.1 & 0.3 & 0.6 & 2 & \\
\hline Pires et al. (1995) & & & 0.3 & 2 & $V=0.8, V_{\max }$ at $6 \%$ drift \\
\hline Manos et al. (1995) & 0.15 & & 0.3 & 1 & $\begin{array}{c}\tau_{u} \approx 0.3 \mathrm{Mpa} V=0.8 \\
V_{\max } \text { at } 2 \% \text { drift for infill } \\
\text { frame }\end{array}$ \\
\hline Michailidis et al. (1995) & 0.1 & $0.25-0.35$ & & & \\
\hline Mehrabi et al. (1996) & 0.3 & & 0.6 & 3.1 & $\begin{array}{c}\tau_{u} \approx 0.5 \mathrm{Mpa}, V=0.8, \\
V_{\max } \text { at } 1.5 \% \text { drift for infilled } \\
\text { frame, } 6.8 \% \text { for bare frame }\end{array}$ \\
\hline Negro \& Verzeletti (1996) & $<0.3$ & & 1.1 & 2.4 & $\begin{array}{c}V_{\max }=0.4 W \text { for bare frame. } \\
V_{\max }=0.62 W \text { for infilled } \\
\text { frame }\end{array}$ \\
\hline Aguilar (1997) & & & 1.3 & & \\
\hline Marjani (1997) & 0.5 & & & & \\
\hline Zarnic \& Gostic (1997) & & 0.2 & 1 & $>1$ & \\
\hline Zarnic (1998) & & & 0.3 & & $\begin{array}{c}\qquad=V_{\max } \text { for } 0.3 \% \\
<\delta<2 \% \text { Used in } \\
\text { mathematical model of URM } \\
\text { infill }\end{array}$ \\
\hline Kappos et al. (1998) & 0.3 & & 3 & & \\
\hline Schneider et al. (1998) & 0.2 & & 2 & & \\
\hline Mosalam, White and Ayala (1998) & $\pm 0.1 \%$ and $\pm 0.2 \%$ & & 0.5 & & \\
\hline Mehrabi \& shing (1998) & $\begin{array}{l}0.013 \% \text { to } \\
0.037 \%\end{array}$ & & $\begin{array}{l}2 \% \text { to } \\
4.3 \% \\
\end{array}$ & & \\
\hline $\begin{array}{l}\text { Lili Anne Akin } \\
\text { (2004) }\end{array}$ & 0.3 & & 0.7 & & \\
\hline Al-Chaar et al. (2002) & $0.25 \%$ to $1 \%$ & & & & \\
\hline Santiago et al. (2008) & 1 & & & & $\begin{aligned} K_{\text {infill }} & =5 K_{\text {bare }} \\
f_{\text {infill }} & =2 f_{\text {bare }}\end{aligned}$ \\
\hline
\end{tabular}

Table 6. Deformation limitations 


\subsubsection{Conceptual discussion}

One of the main reasons of failure of structures due to earthquakes is discontinuity of lateral force resisting elements like bracing, shear wall or infill in the first story as show conceptually in Fig. 5. So first story act as soft story, in this case columns are imposed to large deformation and plastic hinges are formed at top and bottom of the element. Conceptual figure is obtained from actual earthquake observation as shown in fig. 6 . This phenomena is so-called story mechanism (severe drift of the story). Most of these buildings have collapsed. The upper stories have infills and consequently their stiffness is much more than the first story.

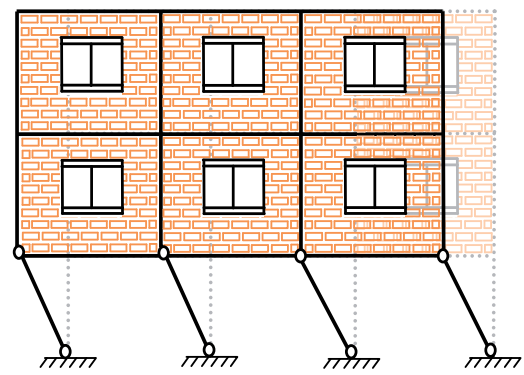

Fig. 5. Schematic view of soft story mechanism

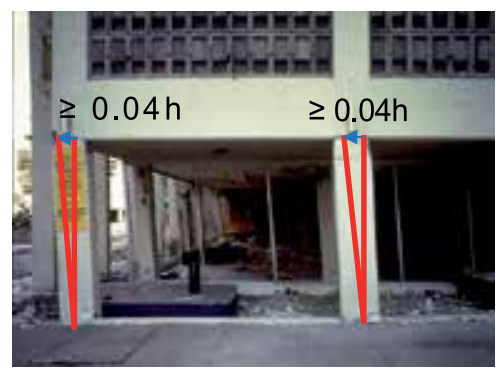

Fig. 6. Soft story failure in a building during earthquake (Italy 1976)

The performance of a building in earthquake is shown in Fig. 6. This building is RC structure and has parking in the first story; there is no infill in the parking story. Deformations are localized in the first story and the columns of this story undergo large deformation, passing collapsed limit ( $4 \%$ of height).

\subsection{Torsion}

The effects of masonry infill walls in the structures are significant and very important in seismic responses of structures due to the experiences of the previous earthquakes. Many existing buildings are irregular in plan or elevation because of asymmetric placement of masonry infills. This kind of torsion should be considered by engineers.

The inelastic seismic response of a class of one-way torsionally unbalanced structures is presented By Bozorgnia et al. (1986). Tso (1988) shown that much better correlation exists between inelastic torsional responses and strength eccentricity than the traditionally used stiffness eccentricity parameter. Tso and Ying (1990) used a single mass three-element 
model, a study was made on the effect of strength distribution among elements on the inelastic seismic responses of eccentric systems. Additional ductility demands on elements and additional edge displacements are taken as response parameters of interest in optimizing the strength distribution.

Yoon and Stafford Smith (1995) presented a method to predict the degree of translationaltorsional coupling of mixed-bent-type multistory building structures subject to dynamic loading.

Chopra and De la Llera (1996) focused on the description of two recently developed procedures to incorporate the effects of accidental and natural torsion in earthquake analysis and design of asymmetric buildings. Basu and Jain (2004) presented the definition of center of rigidity for rigid floor diaphragm buildings has been extended to unsymmetrical buildings with flexible floors.

Stefano et al. (2007) presents an overview of the progress in research regarding seismic response of plan and vertically irregular building structures. Dai Junw et al. (2009) shown some analytical results from 3D temporal characteristics of the responses of an RC frame building subjected to both a large aftershock and the main shock of Wenchuan $M_{\mathrm{s}}=8.0$ earthquake.

\subsubsection{Conceptual discussion}

Fig. 6 is a sample of previous earthquakes that shown the response of plan asymmetric structure. As a result, design codes incorporate procedures to account for such irregular plan-wise displacement distribution, leading to different stiffness's and capacities of resisting planes. Several researchers have carried out numerical and experimental investigations to understand these effects. The stiffness of masonry infill is a considerable value relating to that of the structure. Because of architectural and structural considerations, sometimes there is an eccentricity between center of mass and center of rigidity and the structure is irregular in plan called asymmetric building. The structure is also might be asymmetric as an irregular arrangement of infills in plan, which leads to unbalance distribution of stiffness. Produced torsion from eccentricity because of infill stiffness leads to extra forces and deformations in structural members and diaphragms. An appropriate alternative to solve this problem especially in existing buildings is using dampers.

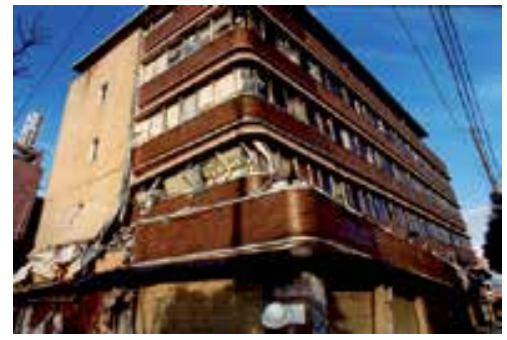

a) Tortional failure of structure (Kobe, 1995)

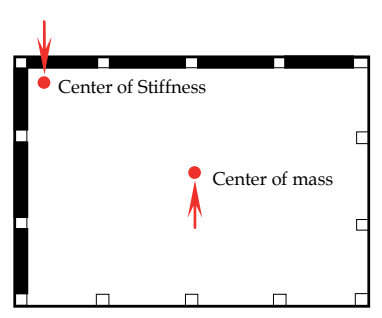

b) Intraction between infill walls and frame

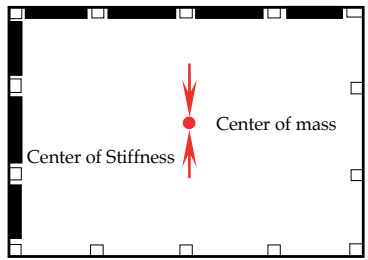

c) Intraction between infills wall and frame

Fig. 7. Torsion of building 


\subsection{Short column}

Shear failure is a critical kind of concrete column failure that occurs in short columns as repeatedly demonstrated during recent earthquake. Due to high brittle behavior and low ductility of these types of columns, it is important to investigate the behavior of short columns.

Moehle et al. (2000) examined loss of lateral and vertical load capacities by a study of columns tested in the laboratory. Correlations with geometric, materials, and loading characteristics were identified. They gathered test data to understand the effects of materials, geometry, and loading on failure mechanisms. Sezen and Moehle (2006) shown that columns with inadequate transverse reinforcement were vulnerable to damage including shear and axial load failure by earthquakes and laboratory experience. To study this behavior, they tested four full scale columns with light transfers reinforcement under unidirectional lateral load with either constant or varying axial load. Their tests shown that response of columns with nominally identical properties varied considerably with magnitude and historical and lateral loads. Kwon (2007) presented back-analysis result of a RC building in Ica, Peru which was severely damaged during the Pisco-Chincha Earthquake. Kwon confirmed in analysis results that shear force demand on columns with infill walls was significantly higher than those without infill walls. Turel Gur et al. (2007) made three surveys of damage to concrete structures following the 1999 Marmara and Düzce earthquakes. They observed that the sole severely damaged structure was damaged not by failure in the ground story, as all the other school buildings, but by failure of captive columns at basement level as a result of discontinuity of the foundation walls in height. The structural walls of the building, which were not damaged at all, prevented the collapse of the building by providing sufficient lateral strength and enhancing the gravity load capacity. Their observation was that the presence of structural walls improved the behavior of reinforced concrete systems drastically. Tabeshpour et al. (2005) presented numerical study of short column failure using IDARC. Non-ductile behavior of short columns was modeled for nonlinear damage analysis. Tabeshpour and Mousavy (2011b) presented plastic hinge properties for short column surveying because of masonry infill wall using nonlinear static analysis.

\subsubsection{Conceptual discussion}

\subsubsection{Local short column}

\subsection{Flexural failure}

Flexural failure in columns depends on the shear span ratio that is:

$$
a_{s}=M /(V H)
$$

Slender columns $\left(a_{s}>3.5\right)$ are characterized by a flexural type of failure. This type of damage consists of spalling of the concrete cover and then crushing of the compression zone, buckling of longitudinal bars and possible fracture of hoops due to the expansion of the core (Elnashai and Sarno, 2008).

\subsection{Shear failure}

Short columns $\left(a_{s}<2\right)$ are characterized by a shear failure presented a brittle failure. This type of failure occur when columns have conventional reinforcement (hoops and 
longitudinal bars) and high axial load, when subjected to cyclic loading results in crossinclined shear cracks. This behavior may be improved if cross-inclined reinforcement is utilized, and particularly if multiple cross-inclined reinforcement (forming a truss) is used. Some columns in RC frames may be considerably shorter in height than the other columns in the same story. Short columns are stiffer, and require a larger force to deform by the same amount than taller columns that are more flexible. Regarding to earthquake damage photos due to short column phenomena increased force generally incurs extensive damage on these columns. The upper portion of the column next to the window behaves as a short column due to the presence of the infill wall, which limits the movement of the lower portion of the column. In many cases, the heights of the columns in each story are the same, as there are no walls adjoining them. When the floor slab moves horizontally during an earthquake, the upper ends of all columns undergo the same displacement. Effective height of columns is shorter when masonry infills added during construction. Consequently these columns attract a larger force as compared to a regular column. The damage in these short columns as shown in fig. 7 is often in the form of X-shaped cracks, which is characteristic for shear failure. In new buildings, the short column effect should be avoided during the architectural design process.

\subsubsection{Global short column (very stiff non-ductile story)}

Earthquake observation indicates that, total collapse of the story occurs, due to shear failure of the columns in the story. If the negative effects of infill walls are not consider in design procedure, brittle failure in low drift ratio may occur and it makes detritions drop in strength.

\subsection{Interaction}

Interaction between infill walls and concrete columns cause the brittle failure as repeatedly demonstrated during recent earthquake. Existing of infill walls and adjacent to concrete frames is the most important and determinant effects in behavior of concrete structures during earthquake. During recent earthquake great damage occur because of interaction phenomena.

Smith \& Coull (1991) presented a design method for infilled frame based on diagonally braced frame criteria. They proposed a method that considered three possible failure modes of infill: shear along the masonry, diagonal crashing of infill walls and corner crushing of infill. Paulay \& Priestley (1992) proposed a theory about the seismic behavior of masonry infilled frame and a design method for infilled frames. They said that although masonry infill may increase the overall lateral load capacity, it can result in altering structural response and attracting forces to different or undesired part of structure with asymmetric arrangement. This means that masonry infill may affect on structural behavior in earthquake.

Bell and Davidson (2001) reported on the evaluation of a reinforced concrete frame building with brick infill walls. They used in their evaluation an equivalent strut approach for modeling the infill walls. Their results indicated that infill walls, where presented in a regular arrangement, had a significant beneficial influence on the behavior of RC buildings that contrasted with New Zealand guidelines which gave an impression that infill masonry walls had a detrimental influence on the behavior of buildings due to interaction effects. 
Naseer et al. (2007) overviewed buildings damaged during October 08, 2005 Kashmir earthquake. They understood that most of the buildings in the earthquake affected area were non-engineered. The following conclusions were drawn from the analysis of data collected in the post-earthquake damage assessment surveys that most of the buildings are either nonengineered or semi-engineered before October 08, 2005 earthquake. Mohyeddin-Kermani et al. (2009) focused specifically on observations made on concrete construction with masonry infill walls during the Sichuan earthquake with identification of damage and key of failure modes. This will be related to the damage and failure modes observed in past earthquakes because of interaction between masonry infill walls and concrete frame. Baran and Sevil (2010) studied on behavior of infilled frames under seismic loading. They considered hollow brick infills as "structural" members during the structural design process. They emphasized that since the behavior is nonlinear and closely related to the interaction conditions between frame and infill, analytical studies should be revised and supported by experimental data.

\subsubsection{Conceptual dissection}

Earthquake reports indicate the negative effect of infill wall as shown in fig. 7-9. Due to observation, damage of structure because of interaction effects categorize in two groups:

a. Interaction between masonry infill walls and concrete frame (Fig. 8)

b. Interaction effects in confined masonry structure (Fig. 9)

Design procedure of infilled frame structure in most codes is base on the bare frame. Earthquake observation indicate that infill frame have effective role in response of structure. Local damage of infill walls in recent earthquake indicate that the actual behavior of structure adjust to the material strength basis.

Confined masonry building is commonly used structure both in small cities and rural areas. This type of structure is very similar to infilled frames with two differences: masonry walls carry vertical loads and tie beams are not moment frames and don't carry vertical loads. Fig. 10 shows the interaction effect between tie beams and masonry wall. Because of concentrated shear force, the corner part of the beams is very vulnerable and shear cracks occur in earthquakes (Fig. 9).

In order to have a deep view on the structural behavior of infilled frames, simplified models of infills and frames are presented in table 7.

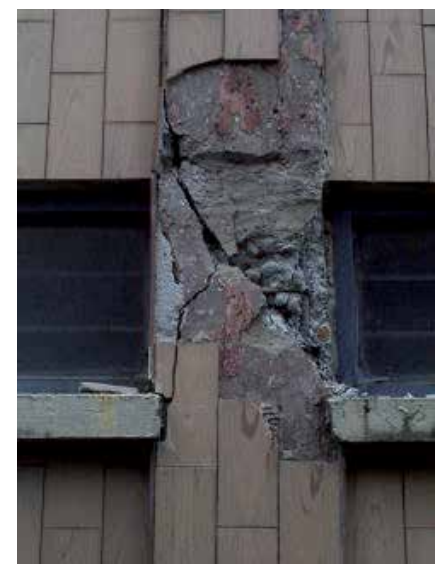

Fig. 8. shear failures in columns (California 1994) 


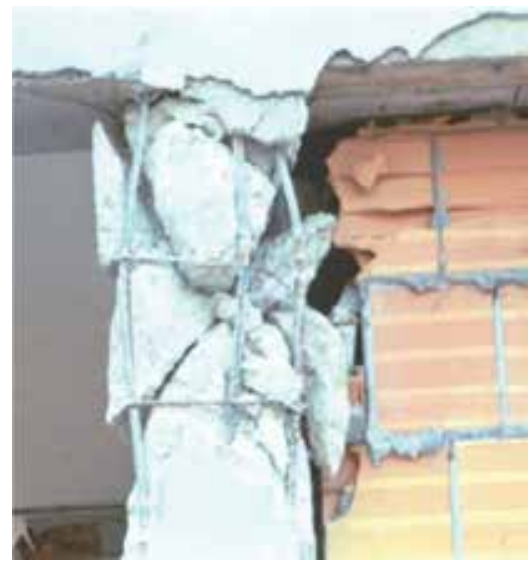

Fig. 9. Shear failure of column (Italia 2002)

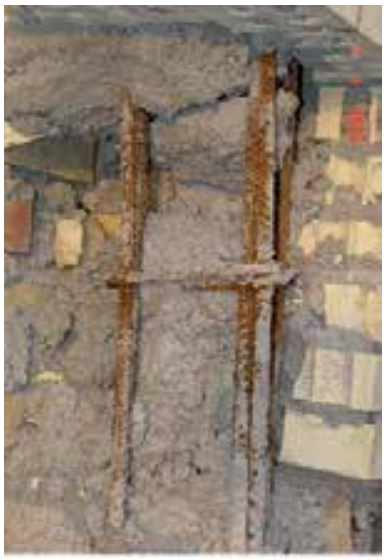

Fig. 10. interaction between infill wall and vertical tie beam
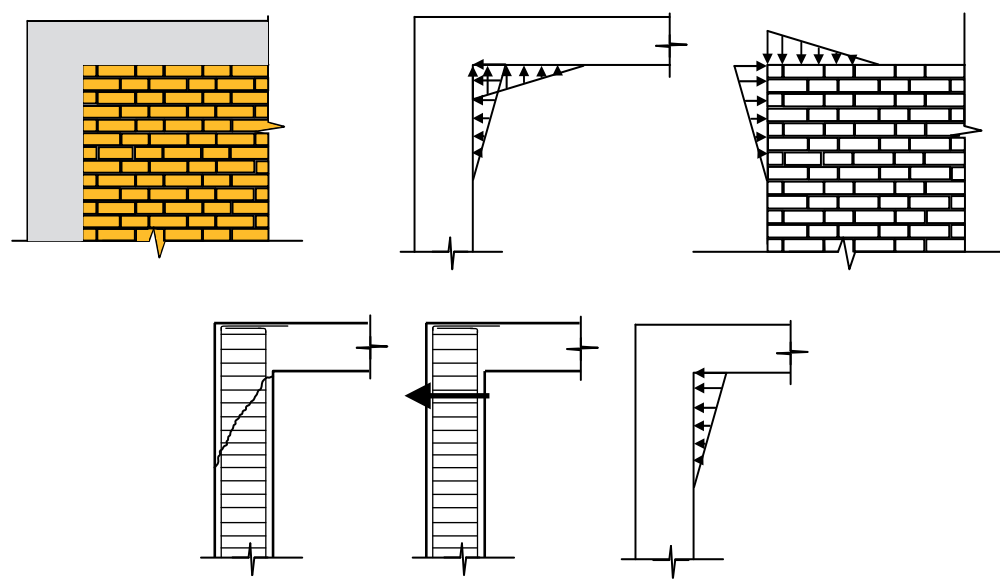

Fig. 11. Impose force from masonry wall into vertical tie beam 


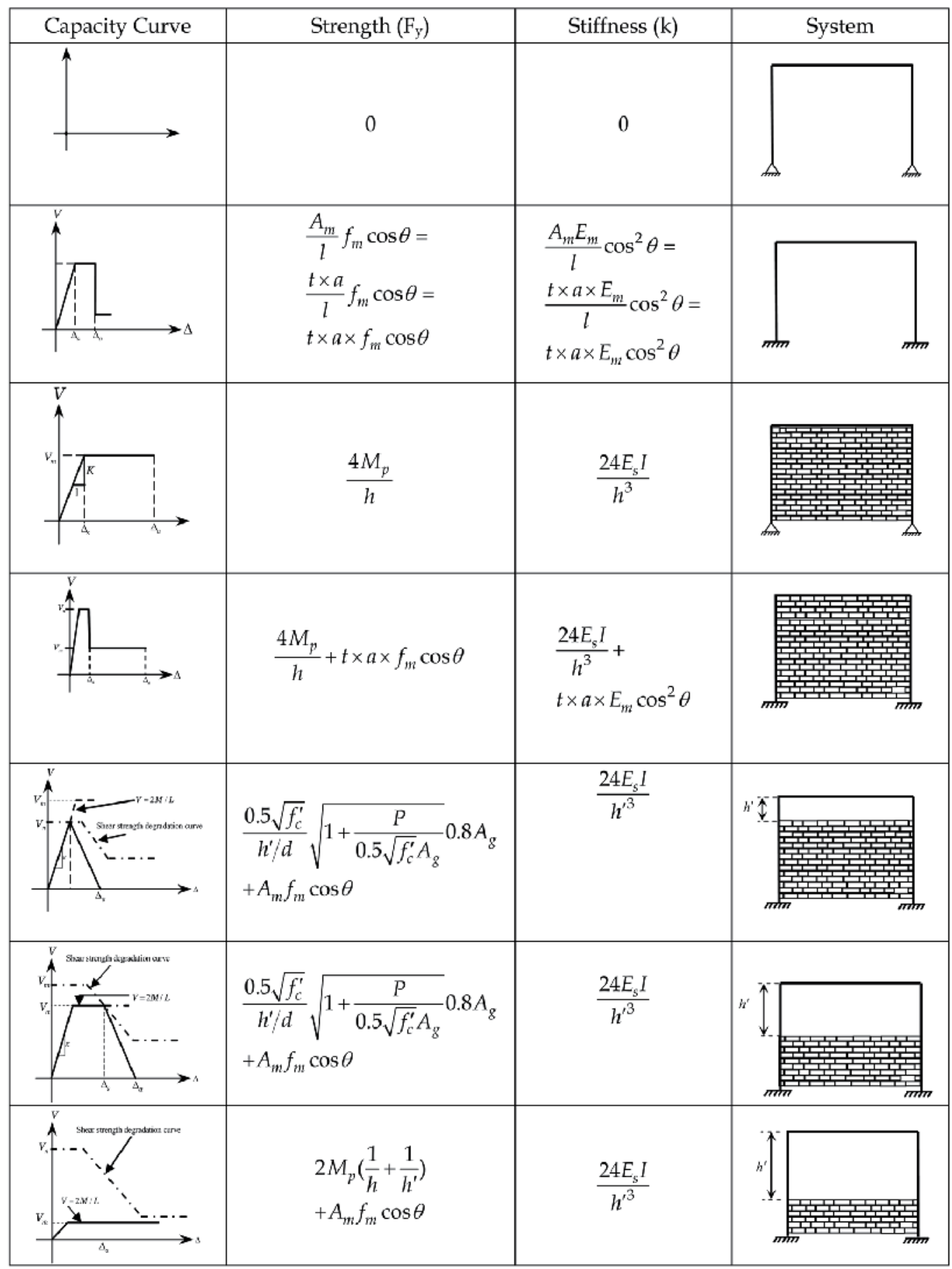

Table 7. Simplified models for capacity curves 


\section{Case study}

Tabeshpour et al. (2011c) showed that the infill walls can lead to severe torsion increase through the frame which can be solved by using friction damper device. We can say that in the irregularities and changes in structural properties because of infill walls will not considered, the structural design may be inefficient and the seismic response of the structures may not be acceptable. Tabeshpour and Ebrahimian, (2010) have presented design of friction/yielding damping devices. Considering the infill walls leads to determine the period of the structure in high accuracy and therefore, the seismic responses will be reliable and the design of a friction damper will be performing in a correct way. Many control devices have been developed to achieve the first purpose, and they have been applied to high-rise buildings and towers such as friction damper device (FDD) (Mualla and Belev, 2002). Friction damper is the simplest kind of dampers and easy to construct and install. The second purpose of vibration control is to prevent of imparting damage to the main elements of a structure during severe earthquakes. In seismic design of friction dampers, the structural stiffness and fundamental period directly affect the damper properties.

\subsection{Modeling of masonry infill walls}

From experimental observations, it is evident this type of structure exhibits a highly nonlinear inelastic behavior, even at low-level loading. The nonlinear effects mentioned above introduce analytical complexities, which require sophisticated computational techniques in order to be properly considered in the modeling. Due to the stiffness and strength degradation occurring under cyclic loading, the infilled frame structures cannot be modeled as elasto-plastic systems, while models that are more realistic should be used to obtain valid results, especially in the dynamic analysis of short period structures, such as infilled frames. The aim of this chapter is to introduce the modeling of a masonry infill walls, which will implement in the analysis in the following chapter. The elastic in-plane stiffness of a solid unreinforced masonry infill walls prior to cracking shall be represented with an equivalent diagonal compression strut of width, $a$, given by the following equation:

$$
\begin{gathered}
a=0.25\left(\lambda h_{\text {col }}\right)^{-0.4} r_{\text {inf }} \\
\lambda_{1}=\left(\frac{10 E_{m e} t_{\text {inf }} \sin 2 \theta}{E_{f e} I_{\text {col }} h_{\text {inf }}}\right)^{0.25}
\end{gathered}
$$

where:

$h_{\text {Col }}=$ Column height between centerlines of beams, $\mathrm{cm}$

$h_{\text {inf }}=$ Height of infill walls, $\mathrm{cm}$.

$E_{f e}=$ Expected modulus of elasticity of frame material, $\mathrm{kg} / \mathrm{cm}$

$E_{m e}=$ Expected modulus of elasticity of infill material, $\mathrm{kg} / \mathrm{cm}$

$I_{c o l}=$ Moment of inertia of column, $\mathrm{cm}$

$L_{\text {inf }}=$ Length of infill walls, $\mathrm{cm}$.

$r_{\text {inf }}=$ Diagonal length of walls panel, $\mathrm{cm}$. 
$t_{\text {inf }}=$ Thickness of infill walls and equivalent strut, $\mathrm{cm}$

$\theta=$ Angle (it's tangent is the infill height-to length, radians)

$\lambda_{1}=$ Coefficient used to determine equivalent width of infill strut

The equivalent strut shall have the same thickness and modulus of elasticity which is represented in fig. 4.

\begin{tabular}{cccc}
$E_{\text {me }}\left(\mathrm{kg} / \mathrm{cm}^{2}\right)$ & $t_{\text {inf }}(\mathrm{cm})$ & $\lambda_{1}$ & $a(\mathrm{~cm})$ \\
\hline 12000 & 20 & 0.009043 & 71.95 \\
\hline
\end{tabular}

Table 8. Summary of calculated masonry parameters

\subsection{Parameters of compression equivalent strut}

In this research equivalent compression strut used instead of masonry infill walls. This strut is in a diagonal and node-to-node manner whose length is equal to the diameter of the frame and its effective width is 0.2 of the diameter of the frame. The thickness of strut is same as the wall's thickness.

In order to obtain the masonry materials of strut, Australia's building code was used concerning the conventional compression bricks and mortars which produce stress-strain curves below relating to the mode of a $23 \mathrm{~cm}$ one. The equation of stress-strain of masonry materials (bricks) in compression is considered as a parabolic function up to the maximum stress $\left(f_{m 0}\right)$ based on Table 9. Then with increase in strain, the value of stress decreases linearly, therefore it remains constant. These values are described in details in appendix.

\begin{tabular}{ccccc} 
Parameter & Value & $23(\mathrm{Cm})$ \\
\hline Thickness & $4(\mathrm{Mpa})$ & \\
$f_{m o}$ & 0.0014 & \\
$\varepsilon_{m o}$ & $0.8(\mathrm{Mpa})$ & \\
$f_{m u}$ & 0.0028 & Parabola \\
$\varepsilon_{m u}$ & & $\varepsilon_{m u}$ \\
\hline
\end{tabular}

Table 9. Equivalent masonry strut's material properties

\subsection{Damper description and principle of action}

Friction dampers have often been employed as a component of these systems because they present high energy-dissipation potential at relatively low cost, easy to install and maintain. A friction damper is usually classified as one of the displacement-dependent energy dissipation devices, because its damper force is independent from the velocity and frequency-content of excitations. A friction damper is activated and starts to dissipate energy only if the friction force exerted on its friction interface exceeds the maximum friction force (slip force); otherwise, an inactivated damper is no different from a regular bracing. This devise used to dissipate the energy not only in the usual structure (building) but also it used in platforms and jackets (offshore structure) as well (Komachi et al., 2011). 
The damper main parts are the central (vertical) plate, two side (horizontal) plates and two circular friction pad discs placed in between the steel plates as shown in Fig. 11. The central plate has length $h$ and is attached to the girder mid span in a frame structure by a hinge. The hinge connection is meant to increase the amount of relative rotation between the central and side plates, which in turn enhances the energy dissipation in the system. The ends of the two side plates are connected to the members of inverted V-brace at a distance $r$ from the FDD center. The bracing makes use of pretension bars in order to avoid compression stresses and subsequent buckling. The bracing bars are pin-connected at both ends to the damper and to the column bases. The combination of two side plates and one central plate increases the frictional surface area and provides symmetry needed for obtaining plane action of the device. When a lateral force excites a frame structure, the girder tends to displace horizontally. The bracing system and the forces of friction developed at the interface of the steel plates and friction pads will resist the horizontal motion. Fig. 11 explain the functioning of the FDD under excitation. As is shown, the device is very simple in its components and can be arranged within different bracing configurations to obtain a complete damping system.

\subsection{Numerical study}

A 3-story frame with 3 bays has been investigated in this study. Fig. 12 show plan and elevation of the building. Frame $A$ has been filed by masonry walls with thickness of $23 \mathrm{~cm}$. Lateral force resisting system is intermediate steel moment frame and the type II of soil according to Iranian seismic code of practice (Standard No. 2800). Since investigating the effects of masonry infill walls is the main goal of this research, the considered frames are designed according to last version of Iranian building codes without considering infill walls. Dimensions of the elements have been shown in Table 10. Dead and live loads of stories are considered $600\left(\mathrm{~kg} / \mathrm{m}^{\wedge} 2\right)$ and $200\left(\mathrm{~kg} / \mathrm{m}^{\wedge} 2\right)$ respectively. These parameters are considered $550\left(\mathrm{~kg} / \mathrm{m}^{\wedge} 2\right)$ and $150\left(\mathrm{~kg} / \mathrm{m}^{\wedge} 2\right)$ respectively for roof story. Dead load is considered 133 $\left(\mathrm{kg} / \mathrm{m}^{\wedge} 2\right)$ for $23 \mathrm{~cm}$ thick walls respectively.
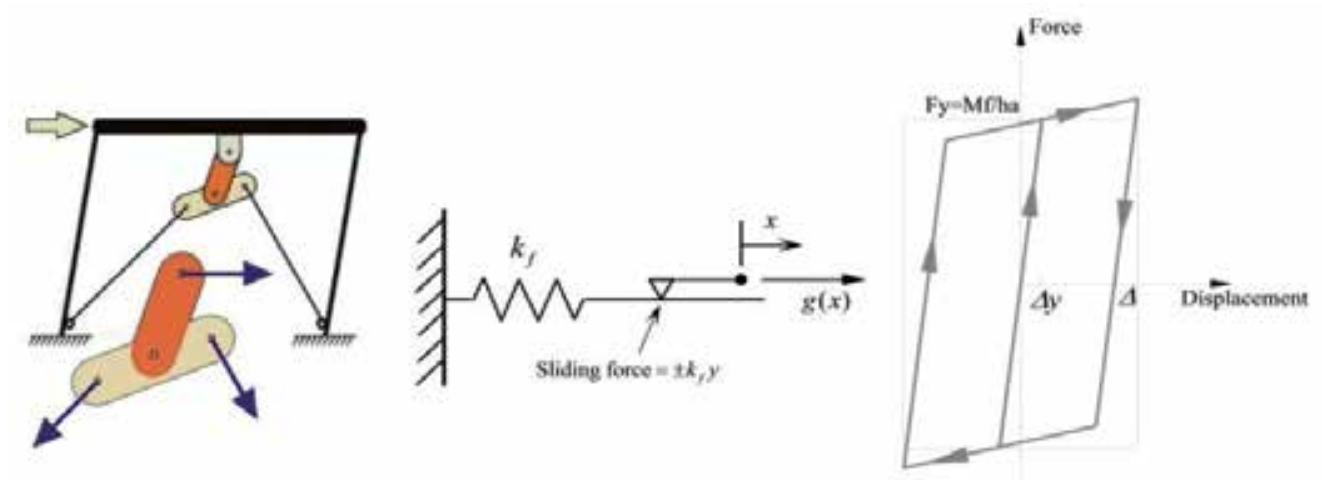

Fig. 12. Component of FDD

In order to compare the behavior of the original structure and equipped structure, the pushover curves of three cases are shown in Fig. 13. Infill walls lead to increase stiffness and strength of buildings compared to a building without considering infill walls. Changing the slope in pushover curves shows this phenomenon. In the push over curves with friction 
damper, stiffness and strength of buildings in the elastic part of analysis are increased. Since infill walls are brittle material and have a high stiffness, these walls absorb a large amount of lateral load until they fail. After failure of infill walls, we have a drop of stiffness (slope) and strength in curves. As it can be seen in the figure after failure of the infill walls, the slope of the curve will be the same as bare frame. The local interaction between frame A and infill walls is not considered. These results are achieved when the shear strength of columns are sufficient. This can be supposed in steel structures. In order to have a clear sense of the effect of infill and friction damper on the structural behavior, Fig.14 shows the scaled deformations of 3 cases named on Fig.13 as a, b and c.

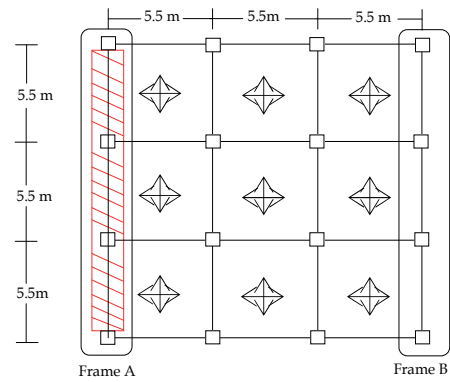

Plan view

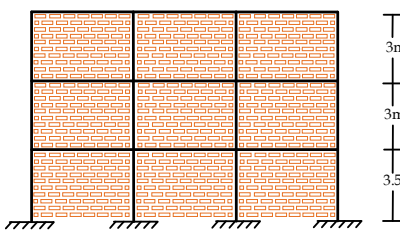

Frame A

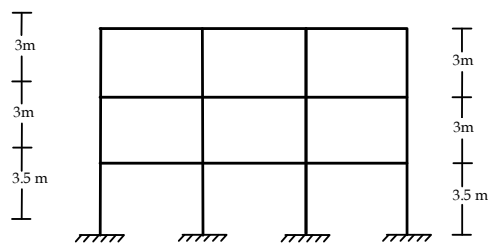

Frame B

Fig. 13. Building elevation

\begin{tabular}{|c|c|c|c|c|c|c|}
\hline \multirow{2}{*}{ Story } & \multicolumn{2}{|c|}{ Column } & \multicolumn{2}{c|}{ Exterior Beam } & \multicolumn{2}{c|}{ Interior Beam } \\
\cline { 2 - 7 } & $\begin{array}{c}\text { Dimension } \\
(\mathrm{cm})\end{array}$ & $\begin{array}{c}\text { Thickness } \\
(\mathrm{cm})\end{array}$ & $\begin{array}{c}\text { Web } \\
(\mathrm{cm})\end{array}$ & $\begin{array}{c}\text { Flange } \\
(\mathrm{cm})\end{array}$ & $\begin{array}{c}\text { Web } \\
(\mathrm{cm})\end{array}$ & $\begin{array}{c}\text { Flange } \\
(\mathrm{cm})\end{array}$ \\
\hline 1 & $25 \times 25$ & 1 & $30 \times 1$ & $12 \times 1$ & $30 \times 1$ & $15 \times 1$ \\
\hline 2 & $20 \times 20$ & 1 & $30 \times 1$ & $12 \times 1$ & $30 \times 1$ & $15 \times 1$ \\
\hline 3 & $20 \times 20$ & 1 & $30 \times 1$ & $12 \times 1$ & $30 \times 1$ & $15 \times 1$ \\
\hline
\end{tabular}

Table 10. Details of element sections

As shown in Fig. 14 by adding infill walls to the bare frame they lead to increase the stiffness of the system and the torsional problems occurs. This torsion leads to structural failure because of concentration of stress in one side and concentration of deformations in the other side. By using friction damper, eccentricity can be omitted and the distance between the center of mass and center of stiffness will be controlled to satisfy code requirement. In region I with increasing lateral deformation, rotation is increased both in infilled frame and equipped frame. However in this region the rotation at equipped frame is considerably less than infilled frame. This reduction of rotation is because of transforming asymmetric system (infilled frame) to a symmetric system (equipped frame). When infill walls start to fail, rotation starts to reduce Region II in the case of equipped frame. But for asymmetric infilled frame, the rotation increases with increasing lateral drift. In region III failing the infill walls cause to reduce the eccentricity and torsional rotation. Therefore the rotation decreases with 
increasing lateral displacement. In the case of equipped frame, failing the infill walls leads to increase rotation clockwise.

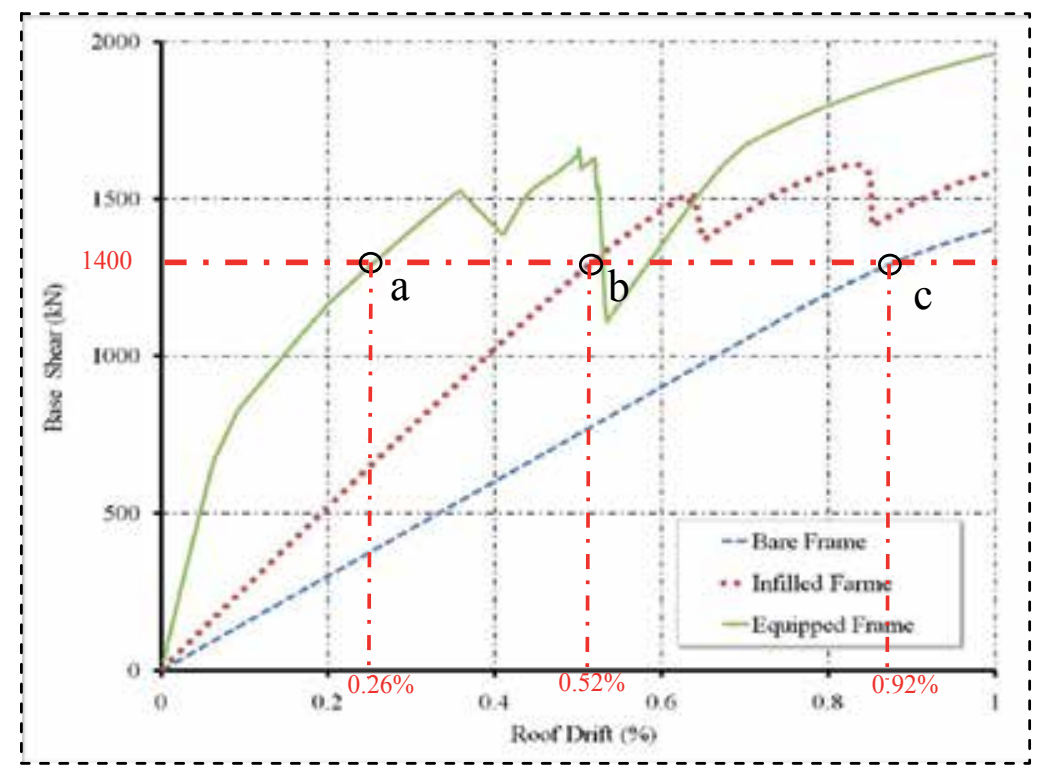

Fig. 14. Pushover curves

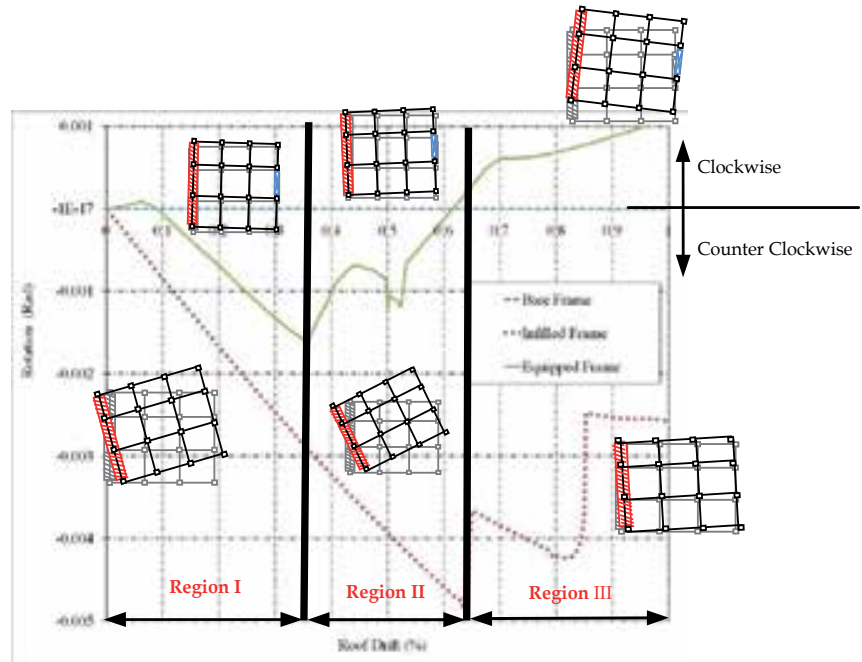

Fig. 15. Rotation curves for center of mass 
After record selection procedure the maximum response of the structure with this arrangement are selected. In this type of structure, in $Y$ direction infill wall is applied in one side and it changes the center of mass and stiffness in the structure. This eccentricity makes torsion in the plan. By applying the FDD in the perpendicular directions decreasing the eccentricity is possible.

Acceleration time history of Loma Perita earthquake has been shown in Fig. 15. Displacement time history response for bare and infilled frame under Loma Perita earthquake has been shown in Figs. 16 and 17 respectively. Comparing Figs. 17 and 18 it is seen a considerable reduction in response when it is equipped by FDD.

\begin{tabular}{|c|c|c|c|c|c|c|c|c|}
\hline Earthquake & Data & Site & $\begin{array}{l}\text { Station and } \\
\text { Component }\end{array}$ & $\mathrm{M}$ & $\mathrm{R}(\mathrm{km})$ & PGA (g) & $\begin{array}{c}\text { PGV } \\
(\mathrm{cm} / \mathrm{s})\end{array}$ & PGA/PGV (s) \\
\hline Loma perieta & 24.4.1989 & Soil & $\begin{array}{c}\text { Holister - } \\
\text { South \& Pine }\end{array}$ & 6.9 & 27.9 & 1.298 & 37.1 & 15.8 \\
\hline
\end{tabular}

Table 11. Details of records

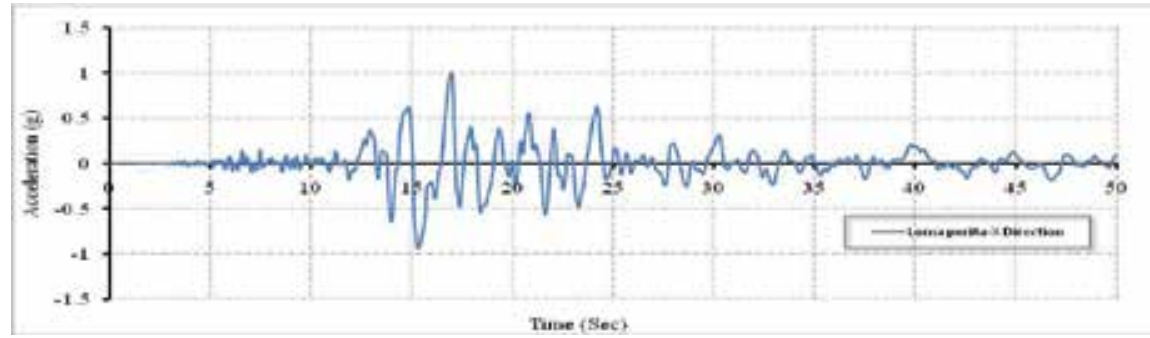

Fig. 16. Acceleration Time History of Loma Perita Earthquake

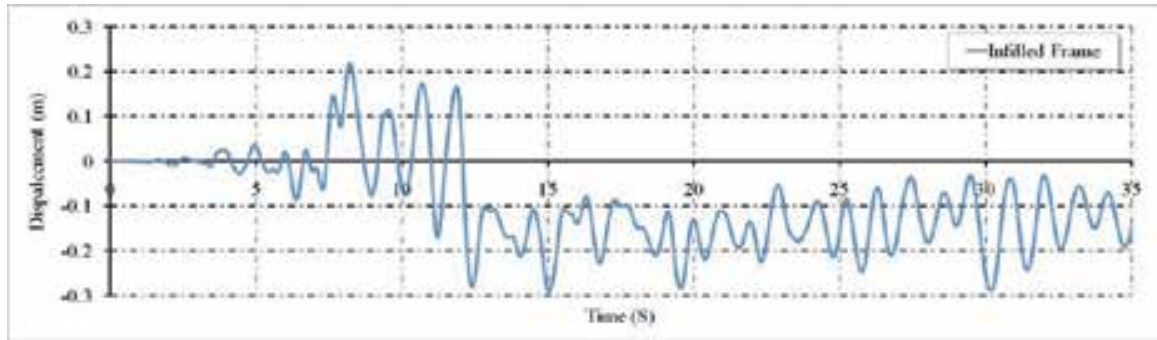

Fig. 17. Time history response for bare frame under Loma perita earthquake

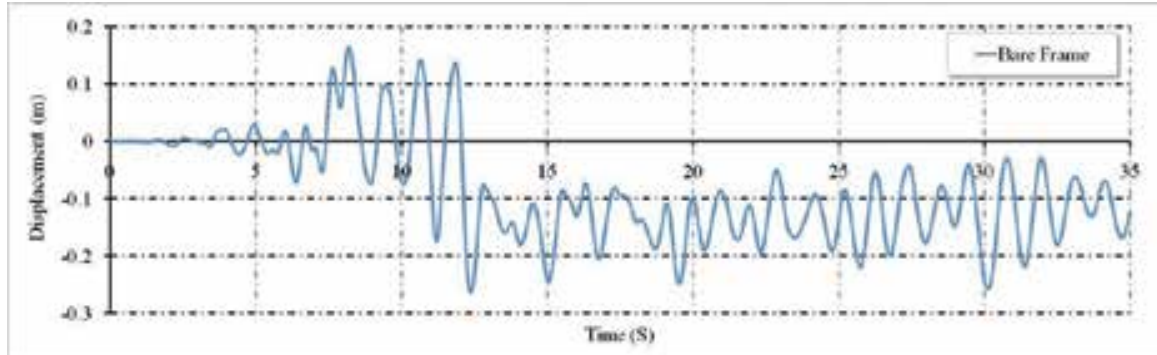

Fig. 18. Time history response for Infilled frame under Loma perita earthquake 


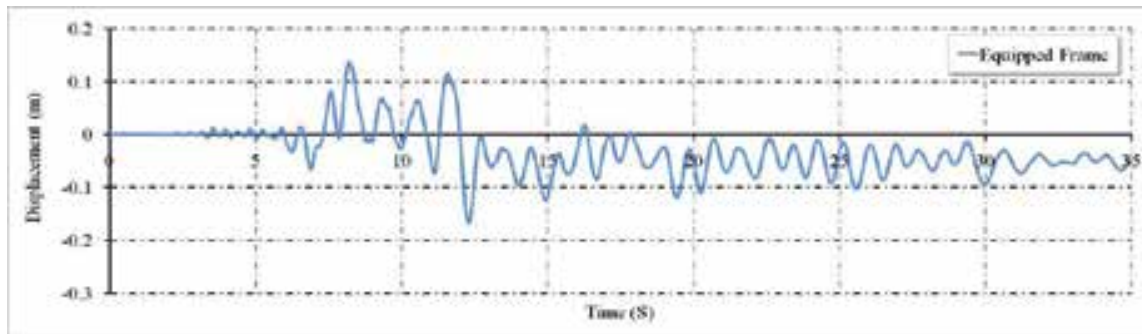

Fig. 19. Time history response for equipped frame under Loma perita earthquake

\subsection{Sumarry and conclusion}

Because of high stiffness of the infill walls, considering them as structural elements leads the initial stiffness of structures to increase. Such elements show high strength at the first step of seismic loading, but by reaching to the maximum strength, the infill walls fail and high loss of strength occurs in small drifts. This drop down of strength can be seen in push over curves of the structures. A relatively complete review on the positive and negative effects of masonry infills were presented in a categorized manner. As an example for numerical study torsion produced by infills were discussed as an engineering problem. Existing of these walls causes high differences between a center of mass and center of stiffness. Therefore by applying the lateral forces in center of mass, high torsional torque is generated in the diaphragm. For solving this problem, the FDD is used. Sensitivity analysis on effective variables on the FDD behavior shows that increasing sliding force causes decreasing the differences between the center of mass and center of stiffness, so the problem would be solved. It can be seen that, the FDD modifies structural torsion under earthquake excitation. By increasing PGA the positive effect of FDD in structural behavior is reduced, but equipped structure has better performance related to other structures without FDD. Seismic code requirements are considered. A detailed structural model has been produced using OpenSees. Both static and dynamic nonlinear analyses have been carried out. Because of sensitivity of the friction damper to pulse type excitation, near filed input motion has been considered as excitation force.

\section{Acknowledgement}

The authors gratefully acknowledge the contribution of Afsaneh Mousavi, graduated student of earthquake engineering, for helping in this research.

\section{References}

Aguilar, R. G. (1997). Efecto del Refuerzo Horizontal en el Comportamiento de Muros de Mampostería Confinada Ante Cargas Laterales. MSCE. Thesis, Universidad Nacional Autónoma de México.

Al-Chaar, G. Issa, M. \& Sweeney, S. (2002). Behavior of masonry-infilled nonductile reinforced concrete frames, J. Struct. Eng., ASCE 128, 1055-1063.

Arturo Tena-Colunga, (2010), Review of the Soft First Story Irregularity Condition of Buildings for Seismic Design, The Open Civil Engineering Journal, 4, 1-15. 
Atkinson, R.H., Amadei, B.P., Saeb, S. and Sture, S. (1989). Response of Masonry Bed Joints in Direct Shear, ASCE Journal of Structural Division, Vol. 115, No. 9, pp. 2276-2296

Australian Standard, AS 3700-2001, "Masonry Structures ", prepared by Committee BD004, published on 27 November 2001

Baran, M. \& Sevil, T. (2010), Analytical and experimental studies on infilled RC Frames, International Journal of the Physical Sciences Vol. 5(13), pp. 1981-1998, 18 October

Basu ,D. \& Jain, S. K. (2004), Seismic Analysis of Asymmetric Buildings with Flexible Floor Diaphragms, Journal of structural engineering, AUGUST

Bell, D.K. \& Davidson, B.J. (2001), Evaluation of Earthquake Risk Buildings with Masonry Infill Panels, NZSEE Conference, 2001

Bozorgnia1, Y., Tso, W. K. \& M. ASCE. (1986), Inelastic earthquake rresponse of asymmetric structures, Journal of Structural Engineering, Vol. 112, No. 2, February

Carydis, P.G. Mouzakis, H.P. Taflambas, J.M. and Vougioukas, E.A. (1992). Response of infilled frames with brickwalls to earthquake motions. Proceedings of the 10th World Conference on Earthquake Engineering, Madrid, A.A. Balkema, Rotterdam, Vol. 5, pp. 2829-2834.

Chrysostomou, C.Z. (1991). Effect of Degrading Infill Walls on the Nonlinear Seismic Response of Two-Dimensional Steel Frames, PhD dissertation, Cornell University, Ithaca, N.Y

Chopra, A.K.\& Juan C. De la Llera. (1996 ). Accidental and natural torsion in earthquake response and design of building, eleventh word conference on earthquake Engineering

Eberhard, M.O., Baldridge, S., Marshall, J. Mooney, W. \& Rix, G.J. (2010). The Mw7.0 Haiti earthquake of january 12, 2010: USGS/EERI Advance Reconnaissance Team

Elnashai, A. S. \& Sarno L. D. (2008), Fundamentals of Earthquake Engineering, John Wiley \& Sons, Ltd. ISBN: 978-0-470-02483-6

Fardis, M.N. and Calvi, M.C., 1995. Effects of infills on the global response o reinforced concrete frames, Proceedings of the 10th European Conference on Earthquake Engineering, Vol. 4, pp.2893-2898.A.A. Balkema, Rotterdam

Fiorato, A. E., Sozen, M. A. and Gamble, W. L.(1970), An Investigation of the Interaction of Reinforced Frames with Masonry Filler Walls, Structural Research Series, No. 370, November ,University of Illinois, Urbana, Illinois, Civil Engineering Studies.

Gerardo M. Verderame, Flavia De Luca, Paolo Ricci and Gaetano Manfredi. (2010), Preliminary analysis of a soft-storey mechanism after the 2009 L'Aquila earthquake, Earthquake Engng Struct. Dyn

Golafshani, A.A., Bakhshi, A. \& Tabeshpour, M.R. (2005). Vulnerability and damage analysis of existin buildings, Asian Journal of Civil Engineering, No. 1, 6, 85-100.

Goodman, R.E.; Taylor, R.L.; Brekke, T.L., (1968). A model for the mechanics of jointed rock. ASCE, Div.,94(3), pp. 637-659, USA

Govindan, P., Lakshmipathy, M., and Sanathkumar, A.R. (1986). Ductility of infilled frames. American Concrete Institute Journal, 83: 567 - 576.

Gur, T., Cihan Pay, A., Ramirez, J. A., Sozen, M. A., Johnson, A. M. \& Ayhan Irfanoglu, (2007), Performance of School Buildings in Turkey during the 1999 Düzce and the 2003 Bingöl Earthquakes, Submitted to Earthquake Spectra, EERI

Hendry, A.W. (1990) Structural Masonry, Macmillan Education Ltd, London, England.

Holmes M. (1963). Combined Loading on Infilled Frames, Proceeding Of The Institution Of Civil Engineers, Volume 25: 31-38 
Iranian Code of Practice for Seismic Design of Buildings, Standard No. 2800, $3^{\text {rd }}$ Edition, Building and housing research center, 2006

Ismail, N., Griffith, M.\& Ingham J.M. (2011) Performance of masonry buildings during the 2010 Darfield (New Zealand), 11 th NAMC

Junwu, D., Yanru ,W., Qingsong ,G., Xiaozhai, Q., Xueshan, Y., Mai, T. \& Lee, G. C. (2009), Three dimensional temporal characteristics of ground motions and building responses in Wenchuan earthquake, Earthq Eng \& Eng Vib, 8:287-299

Kadir M.R.A. (1974). The structural behavior of masonry infill panels framed structures, University of Edinburgh, PhD thesis

Kappos, A.J. Stylianidis, K.C. and Michailidis, C.N. (1998). Analytical models for brick masonry infilled r/c frames under lateral loading. Journal of Earthquake Engineering, Vol. 2(1),pp. 59-87

King G.J.W. \& Pandey P.C. (1978). The analysis of infilled frames using finite elements. Proc. Instn Civ. Engrs, Part 2, 65, 749-760

Klingner, R.E. Rubiano, N.R. Bashandy, T.R. \& Sweeney, S.C. (1996). Evaluation and Analytical Verification of Shaking Table Data from Infilled Frames, Part 2: Out-ofPlane Behavior, Proceedings of the 7th North American Masonry Conference, Notre Dame.

Klinger, R.E. \& Bertero, V.V. (1976). Infilled frames in earthquake resistant construction. Earthquake Engineering Research Center, University of California, Berkeley, CA, Rep. EERC 76-32, Dec

Komachi, Y. Tabeshpour, M.R. \& Mualla, I. \& Golafhshani, A.A. (2011). Retrofit of Ressalat Jacket Platform (Persian Gulf) using Friction Damper Device. Journal of Zhejiang University, in press

Koset, E.G. et al. (1974). Non-linear dynamic analysis of frames with filler panels, Am. Soc.Civ.Engrs, J.struct. Div.,100, 743-757

Kwon, O. (2008). Damaging Effects of the Pisco-Chincha (Peru) Earthquake on an Irregular RC Building, The 14th World Conference on Earthquake Engineering October 12-17, Beijing, China 2008

Lili Anne Akin. (2004). behavior of reinforced concrete frames with masonry infills in seismic regions. PHD Thesis in Purdue University

Liauw, T.C. \& Kawn, K.H. (1984). New Development in Research of Infilled Frames, Proc. 8th World Conf. on Earthq.Engng, San Francisko, 4, 623-630

Mainstone, R. J. (1971). On the stiffness and strengths of infilled frames, Proceedings, Institution of Civil Engineers, Supplement IV, 57-90

Mallick D.V. \& Severn R.T. (1967). The Behavior of Infilled Frames under Static Loading, The Institution of Civil Engineers, Proceedings, 39, 639-656

Mallick, D. V. \& Garg, R. P. (1971). Effect of openings on the lateral stiffness of infilled frames, Proceedings of the Institution of Civil Engineers 49, 193-209

Manos, G.C. Triamataki, M. \& Yasin, B. (1995). Experimental and numerical simulation of the influence of masonry infills on the seismic response of reinforced concrete framed structures.Proceedings of the 10th European Conference on Earthquake Engineering, A.A. Balkema, Rotterdam, Vol. 3, pp. 1513-1518.

Marjani, F. \& Ersoy, U. Behavior of Brick Infilled Reinforced Concrete Frames Under Reversed Cyclic Loading. 06/23/2004. 
Mehrabi, A. B., Shing, P. B., Schuller, M.P. and Noland, J.L. (1996). Experimental Evaluation of Masonry-Infilled RC Frames. ASCE Journal of Structural Engineering, Vol. 122, No. 3, pp. 228-237.

Mehrabi, A. B., and Shing, P. B. (1998). Seismic Resistance of Masonry- Infilled RC Frames. Proceedings of the 8th North American Masonry Conference, The Masonry Society, USA, pp. 465-476.

Michailidis, K., Sklavounos, S., and Plimer, I. (1995). Chromian dravite from the chromite ores of Vavdos area, Chalkidiki peninsula, Northern Greece. Neues Jahrbuch fur Mineralogie. Monatshefte, 1995, 513-528.

Moehle, J. P., Elwood, K. J. \& Sezen, H., Shear strength and axial load collapse of existing reinforced concrete columns

Moghadam, Hasan. (2001). Earthquake Engineering, Basic and Application Seismic design of buildings-2800 code. 3. S.1:Building and housing research center

Mohyeddin-Kermani, A. et al. (n.d).The Behaviour of RC Frames with Masonry Infill in Wenchuan Earthquake

Mosalam, K.M., White, R.N., \& Gergely, P. (1997). Static Response of Infilled Frames using Quasistatic Experimentation. ASCE Journal of Structural Engineering, Vol. 123, No. 11, pp. 1462-1469.

Mualla, IH, Belev B. Performance of steel frames with a new friction damper device under earthquake excitation, Engineering Structures, 24 (2002) 365-371

Naseer, A. et al. (2007). Building code of Pakistan: before and after the October o8, 2005 Kashmir earthquake, Sixth National Conference on Earthquake Engineering, 16-20 October, Istanbul, Turkey.

Negro P. and Verzeletti G. (1996). Effect of Infills on the Global Behaviour of R/C Frames: Energy Considerations from Pseudodynamic Tests. Earthquake Engineering and Structural Dynamics, 25, 753-773

Nevzat, Kirac. Mizam, Dogan, Hakan, Ozbasaran. (2010). Failure of weak-storey during earthquakes, Engineering Failure Analysis

Paulay, T. \& Priestley, M.J.N. (1992). Seismic Design of Reinforced Concrete and Masonry Buildings, John Wiley \& Sons, New York, United States

Paulay, T. \& Priestley, M. J. N. (1992). Seismic Design of Reinforced Concrete and Masonry Buildings", John Wiley \& Sons, Inc

Polyakov, S. V. (1956). Masonry in Framed Buildings (An investigation into the strength and stiffness of masonry infilling), Moscow.

Pires, F. \& Carvalho, E.C. (1992). The behaviour of infilled reinforced concrete frames under horizontal cyclic loading. Proceedings of the 10th World Conference on Earthquake Engineering, Madrid, A.A. Balkema, Rotterdam, Vol. 6, pp. 3419-3422.

Rivero C.E. \& Walker W.H. (1984), An Analytical Study of The Interaction of Frames and Infill Masonry Walls, Proc. 8th World Conf. on Earthq. Engng., San Francisko, 4, 591598.

Sahlin, S. (1971). Structural Masonry, Prentice-Hall Inc., New Jersey, United States.

San Bartolomé, A. (1990). Colleción del Ingeniero Civil, (in Spanish), Libro No. 4, Colegio de Ingenierios del Peru.E

Santiago, P. Amadeo, B.C. Mario, E. R. and Paul Smith-Pardo, J. masonry infill walls: an effective alternative for seismic strengthening of low-rise reinforced concrete 
building. The 14th World Conference on Earthquake Engineering October 12-17, 2008, Beijing, China

Schneider, S.P. Zagers, B.R. and Abrams, D.P. (1998). Lateral strength of steel frames with masonry infills having large openings. ASCE Journal of Structural Engineering, Vol. 124(8), pp. 896-904.

Sezen, H. Moehle, J. P, (2006), Seismic Test of Concrete Columns With Light Transverse Reinforcement, ACI Structural Journal, V.103, No.6, November-December

Sharany Haque \& Khan Mahmud Amanat. (2009), Strength and drift demand of columns of RC framed buildings with soft ground story, Journal of Civil Engineering (IEB), 37 (2) (2009) 99-110

Shing, P. B. \& Mehrabi, A. B. (2002). Behaviour and analysis of masonry-infilled frames, Progress in Structural Engineering and Materials, 4(3), 320-331.

Shrive, N.G. (1991). Materials and Material Properties, Reinforced and Prestressed Masonry, A.W.Hendry, Longman Scientific and Technical, London, England

Siamak Sattar \& Abbie B. Liel, (n.d), Seismic performance of reinforced concrete frame structures with and without masonry infill walls

Sinha, B.P. \& Pedreschi, R. (1983). Compressive Strength and Some Elastic Properties of Brickwork, International Journal of Masonry Construction, Vol. 3, No. 1, pp. 19-27.E

Smith, B. S. \& Coull, A. (1991), Tall Building Structures: Analysis and Design, John Wiley \& Sons, Inc

Stafford-Smith B.S. (1968). Model Test Results of Vertical and Horizontal Loading of Infilled Frames, ACI Journal, Volume 65, No. 8: 618-625

Stefano, Ma. D. \& Pintucchi, B. (2007), A review of research on seismic behaviour of irregular building structures since 2002, Springer Science+Business Media B.V. 2007.

Stöckl, S. \& Hofmann, P. (1988). Tests on the Shear Bond Behaviour in the Bed-joints of Masonry, Proceedings of the 8th International Brick and Block Masonry Conference, Dublin, Ireland.

Tabeshpour, M. R. Ghanad, M. A. Bakhshi, A. \& Golafshani, A. A. (2004). Effect of masonry infills on seismic behavior of concrete frames, 1th national conference of civil engineering, sheriff University, Tehran, Iran.

Tabeshpour, M. R. (2005). Numerical study of short column failure, Earthquake research letters, IISEE, Tehran, Iran.

Tabeshpour, M.R. (2009a). Masonry infills in structural frames (Handbook, Part 18) , FadakIsatis Publisher, Tehran, Iran.

Tabeshpour, M.R. (2009b). Seismic retrofit of infilled frames (Handbook, Part 19), Fadak Issatis Publisher, Tehran, Iran.

Tabeshpour, M. R. (2009c). Requirements of infills in Iranian seismic code of practice, Standard No. 2800 (Handbook, Part. 20), Fadak Issatis Publisher, Tehran, Iran.

Tabeshpour, M. R. (2009d) A comparative study of several codes about masonry infill wall, $4^{\text {th }}$ national conference of seismic design code of buildings (standard No. 2800), Tehran, Iran.

Tabeshpour, M. R. (2009e) Modeling of infill wall using commercial software, $4^{\text {th }}$ national conference of seismic design code of buildings (standard No. 2800), Tehran, Iran.

Tabeshpour, M. R. (2009f) Increasing design load in specific columns, $4^{\text {th }}$ national conference of seismic design code of buildings (standard No. 2800), Tehran, Iran. 
Tabeshpour, M.R. Ebrahimian, H. (2010). Seismic retrofit of existing structures using friction dampers, Asian Journal of Civil Engineering, No. 4, 11, 509-520.

Tabeshpour, M. R. Azad, A., \& Golafshani, A. A. (2011a). Nonlinear static analysis of steel frame with asymmetric arrangement of masonry infill wall, $3^{\text {rd }}$ national conference of urban management and retrofitting, Tehran, Iran.

Tabeshpour, M. R. Mousavy, A. (2011b). Short column surveying because of masonry infill wall existence using nonlinear static analysis, $6^{\text {th }}$ national conference of civil engineering, semnan, Iran.

Tabeshpour, M.R. Azad, A. Golafshani, A.A. (2011c). Seismic Retrofitting by using Friction damper in Horizontally Irregular Infilled Structures, $6^{\text {th }}$ National Congress on Civil Engineering, Semnan University, Semnan, Iran.

Tabeshpour, M. R. Kalatjari, V.R. \& Karimi, K. (2011d), Investigation of lateral drift of concrete infilled frames, $1^{\text {st }}$ National conference on earthquake and lifelines, Tehran, Iran.

Takewaki, I.; Murakami, S. \& Fujita, K. \& Yoshitomi, S. \& Tsuji, M. (2011). The 2011 off the Pacific coast of Tohoku earthquake and response of high-rise buildings under longperiod ground motions, Soil Dynamics and Earthquake Engineering, In Press

Tso, W.K. (1988), Strength eccentricity concept for inelastic analysis of asymmetrical structures, Eng. Struct. 1989, Vol. 11

Tso, W. K. \& Ying, H. (1992), Lateral strength distribution specification to limit additional inelastic deformation of torsionally unbalanced structures, Eng. Struct. Vol. 14, No 4 , July

Valiasis, T. Stylianidis, K. (1989). Masonry Infilled R/C Frames under Horizontal Loading experimental Results. European Earthquake Engineering, Vol. 3, No. 3, pp. 10-20

Valiasis, T.N. Stylianidis, K.C. and Penelis, G.G. (1993). Hysteresis model for weak brick masonry infills in $\mathrm{R} / \mathrm{C}$ frames under lateral reversals. European Earthquake Engineering, Vol. 7(1), pp. 3-9.

Yoon, Y. \& Stafford Smith, B. (1995), Assessment of translational-torsional coupling in asymmetric uniform wall-frame structures, Journal of structural engineering, October

Zarnic, R. (1994). Inelastic Model of R/C Frame with Masonry Infill - Analytical Approach. Engineering Modelling, 1-2, 47-54.

Zarnic, R. \& Tomazevic, M. (1985). Study of Behaviour of Masonry Infilled Reinforced Concrete Frames Subjected to Seismic Loading. Proc. 7th International Brick Masonry Conference, Brick Dev. Res. Inst. \& Dept. of Arch. And Bldg., University of Melbourne, 2, 1315-1325.

Zarnic, R. Gostic, S. (1998). Non Linear Modelling of Infilled Frames. 11 th European Conference Earthquake Engineering, Paris, Balkema: Rotterdam. 


\title{
Recent Advances in Seismic Response Analysis of Cylindrical Liquid Storage Tanks
}

\author{
Akira Maekawa \\ Institute of Nuclear Safety System, Inc. \\ Japan
}

\section{Introduction}

Japan is in a seismically active area and experiences many damaging earthquakes with loss of life. In 1995, the Hyogoken-Nanbu earthquake caused major destruction in Kobe City and in 2011 the Great Eastern Japan earthquake and tsunami caused major destruction in the Pacific coast areas of northeastern Japan. Additionally, recent relatively less destructive earthquakes include the 2003 Tokachi-Oki earthquake, the 2004 Niigataken Chuetsu earthquake, the 2005 Miyagiken-Oki earthquake, the 2007 Noto-Hanto earthquake, and the 2007 Niigataken Chuetsu-Oki earthquake.

In particular, the Great Eastern Japan, Noto-Hanto, Miyagiken-Oki, and NiigatakenChuetsu-Oki earthquakes occurred near nuclear power facilities and have been accompanied by enhanced public concern for seismic safety of nuclear plants. Within the Japanese national government, the Nuclear Safety Commission revised the Regulatory Guide for Reviewing Seismic Design of Nuclear Power Reactor Facilities (Nuclear Safety Commission of Japan, 2006) in 2006. This revised Regulatory Guide required seismic safety design of buildings, structures and equipment for larger seismic motions. In addition, seismic probabilistic safety assessment (seismic PSA) (American Nuclear Society, 2007; Atomic Energy Society of Japan, 2007) was urged, for which accurate evaluation techniques for seismic response of equipment installed in the nuclear power plants were needed.

Large cylindrical liquid storage tanks in nuclear power plants are classified as equipment requiring high seismic safety because many are containers storing cooling water used in normal plant operation and in accidents. Their seismic evaluation is done on the basis of the Technical Codes for Aseismic Design of Nuclear Power Plants (Japan Electric Association [JEA], 2008) published by the Japan Electric Association. The seismic evaluation methods used in the conventional seismic design of the tanks (Kanagawa Prefecture, 2002; High Pressure Gas Safety Institute of Japan [KHK], 2003; Architectural Institute of Japan [AIJ], 2010) such as the Technical Codes examine the bending vibration mode (beam-type vibration) which mainly affects the seismic resistance of the tanks, but they do not consider high order vibration modes (oval-type vibration) which are excited in the tank wall by large vibrations and cause oscillation patterns that look like petals of a flower. Therefore, it is necessary to reveal the influence of oval-type vibration on vibration characteristics and seismic safety and to consider the vibration in the seismic design of 
the tanks (Japan Society of Civil Engineers, 1989). However, research on oval-type vibration has only been of academic interest, including reports on fluid-structure interaction which causes oval-type vibration (Japan Society of Mechanical Engineers, 2003) and nonlinear behavior of oval-type vibration (Chiba, 1993). Though analysis techniques such as finite element methods are available as seismic evaluation methods at present, numerical seismic analysis of the tanks considering oval-type vibration has not been established because advanced techniques such as fluid-structure interaction analysis and nonlinear dynamic structure analysis are needed to simulate the oval-type vibration behavior.

In addition, capacity to resist buckling is an important evaluation item in seismic deign of cylindrical liquid storage tanks. Buckling is a dangerous mode for tanks which drastically lowers their structural strength (proof force) and collapses their geometries. In the ultimate buckled state and post-buckling, the cylindrical liquid storage tanks are deformed largely and display nonlinear inelastic behavior. Therefore, it is desirable to take into account the nonlinear inelastic dynamic behavior when evaluating seismic safety of the tanks. The conventional seismic design of tanks (Kanagawa Prefecture, 2002; KHK, 2003; JEA, 2008; AIJ, 2010) uses evaluation equations for static buckling derived from static buckling tests and the assumption of a linear response. However, the evaluation equations have not been validated sufficiently from the viewpoint of the dynamic liquid pressure effect in tanks subjected to seismic motions. Though a few dynamic experiments and development of numerical methods for buckling of cylindrical liquid storage tanks were done in the past, the developed numerical methods could simulate the experimental results only qualitatively (Fujita et al., 1992; Toyoda et al., 1997).

As described above, the conventional seismic design assumes linear behavior of the tanks and does not include nonlinear behavior in post-buckling. However, it is necessary to develop accurate seismic response analysis methods for the cylindrical liquid storage tanks to ensure seismic safety and conduct accurate seismic PSA for mega earthquakes. Therefore, an accurate dynamic analysis method to evaluate dynamic nonlinear behavior of the cylindrical liquid storage tanks subjected to seismic motions was proposed and validated by the dynamic experiment in this chapter. The research was done for the liquid storage tanks installed in nuclear power plants such as refueling water tanks and condensate water tanks. In this chapter, previous studies are overviewed and then sequential research findings on the dynamic analysis method are summarized.

First, the seismic damage modes of the cylindrical liquid storage tanks are explained briefly. Especially buckling modes caused by earthquakes are introduced. Secondly, the vibration behavior of the tanks is explained. Thirdly, previous studies are overviewed with regard to vibration characteristics and seismic evaluations. Special focus is given to the seismic response analysis and dynamic buckling evaluation. Fourthly, research studies concerned with oval-type vibration are summarized. Finally, the author's study regarding dynamic nonlinear analysis method for seismic response of the cylindrical liquid storage tanks is described and the method is shown to be suitable for actual tanks based on comparison with experimental results.

\section{Seismic damage modes of cylindrical liquid storage tanks}

Many typical examples of seismic damage modes of cylindrical storage tanks have been reported and many seismic damage analyses (see for example, (Fujii et al., 1969; Rahnama \& 
Morroe, 2000; Suzuki, 2008)) have been conducted. The damage modes of the tanks from the above analysis results are summarized as follows:

1. Buckling in the side walls

2. Failure of the tank roofs and their junctions

3. Sliding and lifting

4. Local fracture on the bases of the tanks and uneven settlement

5. Failure of anchor bolts

6. Cracking of annular parts of the base plate

The buckling modes of the side walls of tanks include shear buckling and bending buckling. The bending buckling includes diamond buckling and elephant foot bulges. These buckling modes are associated with geometry parameters of the tanks such as height to radius ratio and radius to thickness ratio. Figure 1 shows the relationship between the buckling modes and geometry parameters. Shear buckling occurs for small ratios of height to radius and bending buckling predominantly occurs for large ratios. Shear buckling is caused by shear force and brings about many large diagonal wrinkles in the center of a tank side wall. A typical example of diamond buckling is shown in Fig. 2. Diamond buckling is one of the bending buckling modes caused by the bending moment and it is generated on the base of a tank. When the buckling occurs, the cross section at the buckling region bends inward and has many wrinkles. Because the deformation is drastic, the structural strength (proof force) of the tanks decreases suddenly. Diamond buckling became widely know after it occurred in many wine storage tanks in the 1980 Greenville-Mt. Diablo earthquake. The elephant foot bulge is another bending buckling mode. This buckling mode was widely seen in the 1964 Alaska mega earthquake, the 1971 San Fernando earthquake and the 1994 Northridge earthquake and can cause spill incidents of liquid in the tanks through crack penetration. A typical example of the elephant foot bulge is shown in Fig. 3 (Ito et al., 2003). In the elephant foot bulge, the buckling cross section expands outward in a ring and the structural strength (proof force) decreases relatively gently through a gradual increase of the expansion. The occurrence condition of diamond buckling and the elephant foot bulge depends on the circumferential stress due to the internal pressure in the tanks, that is, hoop stress (Akiyama et al., 1989). The former occurs when the hoop stress is smaller and the latter occurs when the hoop stress is larger. In the 1995 Hyogoken-Nanbu earthquake, many observations of diamond buckling and elephant foot bulges were made in cylindrical liquid storage tanks.

The failure of tank roofs and their junctions is mainly caused by sloshing. This occurred in the 1964 earthquakes in Niigata and Alaska. More recently, the roofs and junctions of some petroleum tanks failed in the Kocaeli earthquake in Turkey and in the Chi-Chi earthquake in Taiwan, both in 1999. In Japan, a few petroleum tanks also failed in the 2003 Tokachi-Oki earthquake. In all three of these earthquakes, the floating roofs were damaged and fires broke out.

The sliding of tanks and lifting of base plates were observed in the 1964 Alaska earthquake. Local fracture on the bases of tanks, uneven settlement and failure of anchor bolts occurred in the 1995 Hyogoken-Nanbu earthquake. In the 1978 Miyagiken-Oki earthquake, cracking of annular parts of base plates occurred in petroleum tanks and stored petroleum leaked out. 


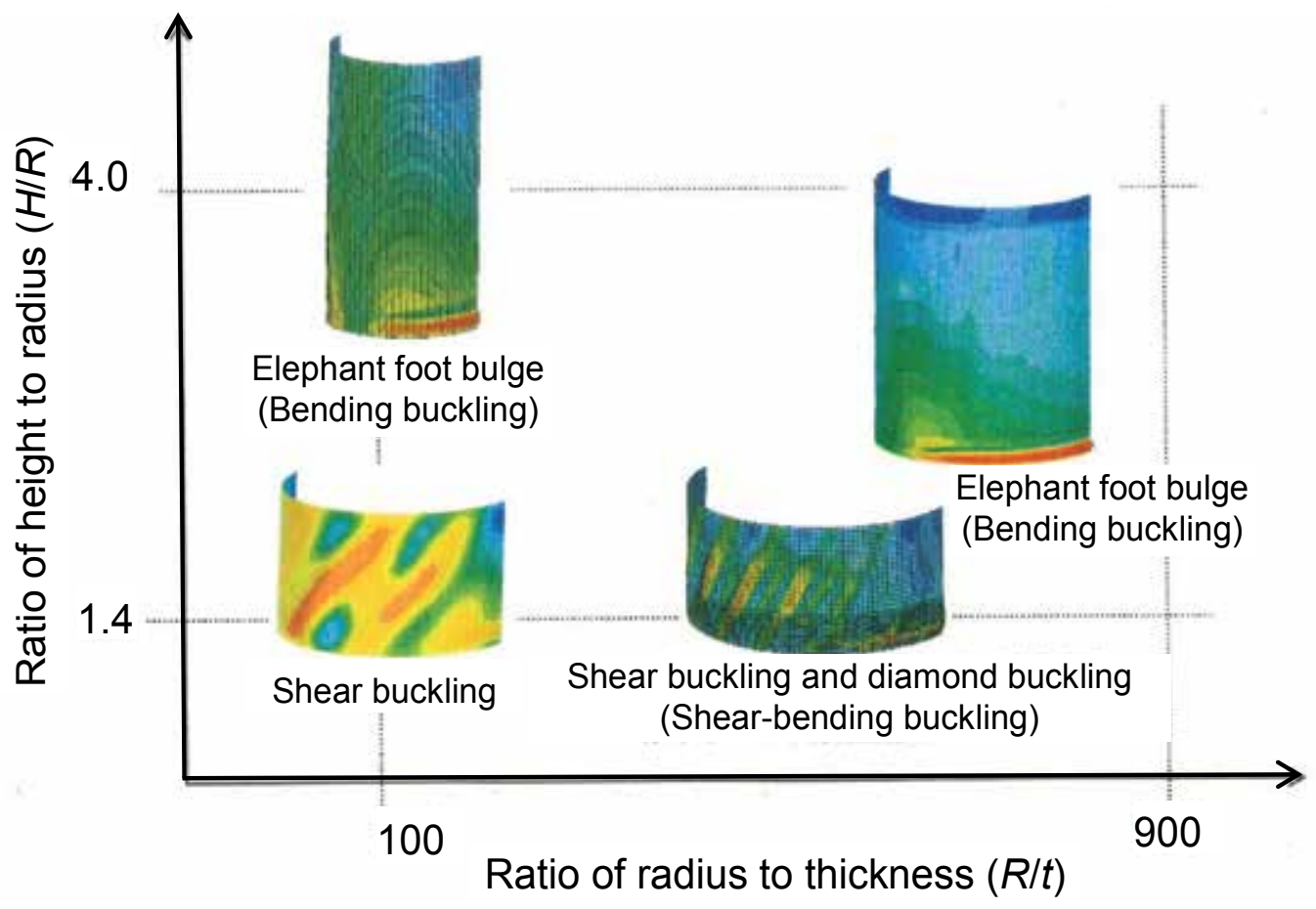

Fig. 1. Buckling modes of cylindrical tanks and geometry parameters.

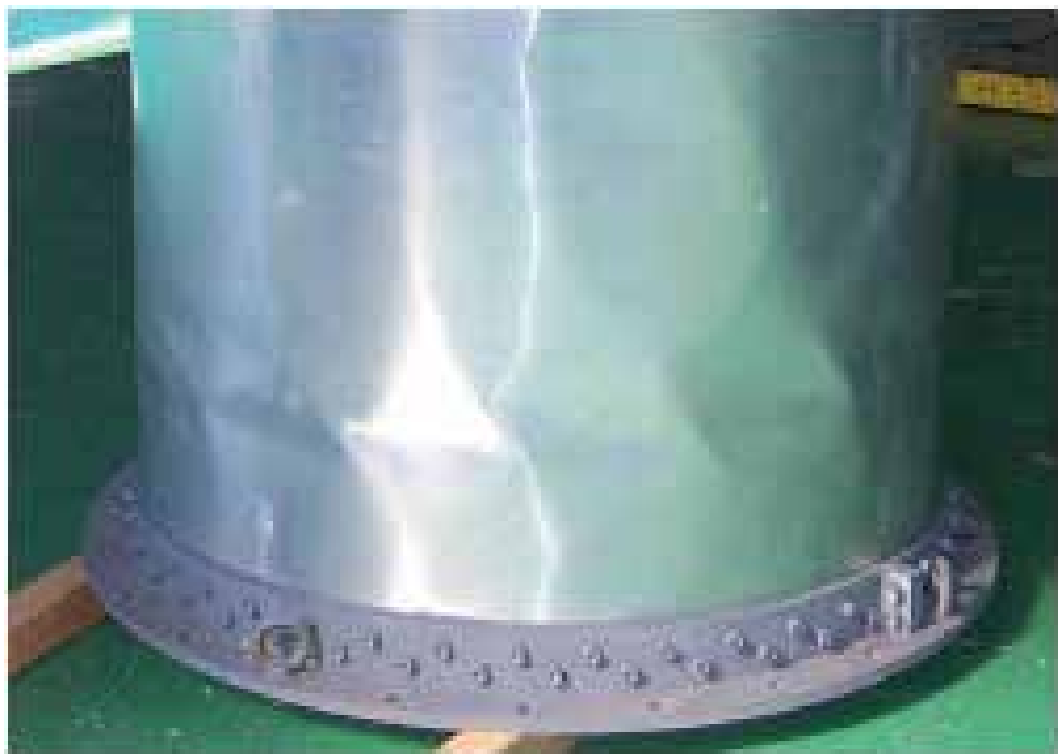

Fig. 2. Typical mode of diamond buckling. 


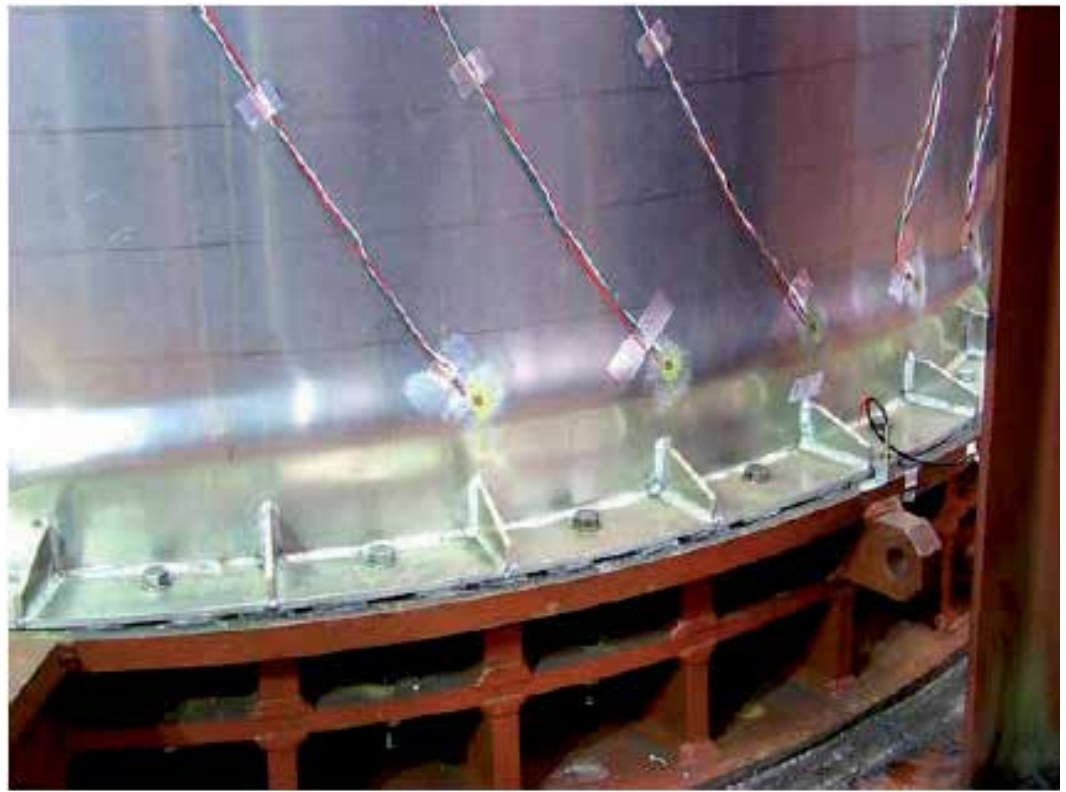

Fig. 3. Typical mode of elephant foot bulge. (Ito et al., 2003)

\section{Classification of vibration behaviour in cylindrical liquid storage tanks}

The vibration modes of the cylindrical liquid storage tanks are classified roughly into sloshing and bulging. The sloshing represents vibration of the free liquid surface and the bulging represents vibration of the tank structure. In large cylindrical tanks, shell vibration occurs because the side wall is relatively thinner compared to the radius length, which is regarded as a cylindrical shell. The modes of bulging including shell vibration are generally distinguished using the axial half wave number $m$ and circumferential wave number $n$. The modes with $m \geq 1$ and $n=1$ are the beam-type vibration and the modes with $m \geq 1$ and $\mathrm{n} \geq 2$ are the oval-type vibration. Typical examples of the vibration modes are shown in Fig. 4 (Fujita \& Saito, 2003). The figure shows the vibration modes of cylindrical tanks which are free on the top and rigid on the bottom; it is easy for readers to understand these modes of oval-type vibration. However, actual tanks have the vibration condition which is rigid on the top because of their fixed roofs.

In general linear analysis, vibration modes with $n \geq 2$ are not excited when a perfectly axisymmetric cylindrical shell such as a tank is vibrating. However, the oval-type vibration actually occurred in the vibration experiment using reduced models of cylindrical tanks (Kana, 1979; Fujita et al., 1984; Maekawa et al., 2010). Additionally it is not possible to say that the influence of oval-type vibration on seismic load of the tanks can be ignored (Clough et al., 1979). 


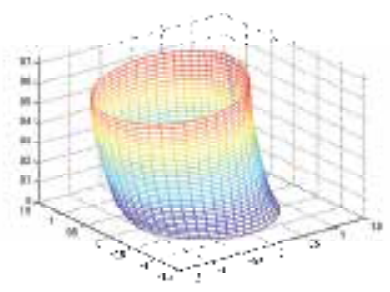

$m=1, n=1$

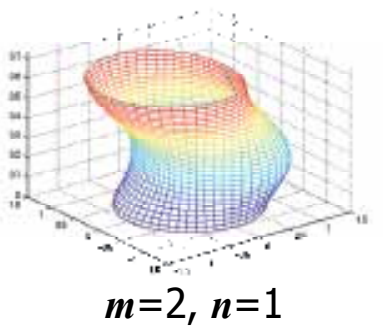

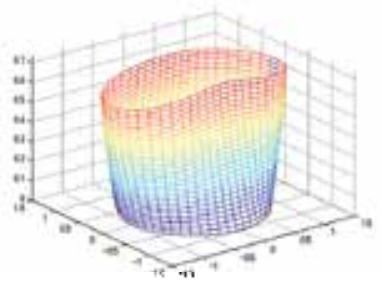

$m=1, n=2$

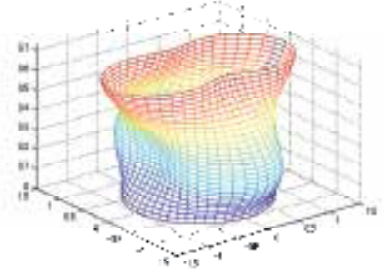

$m=2, n=2$

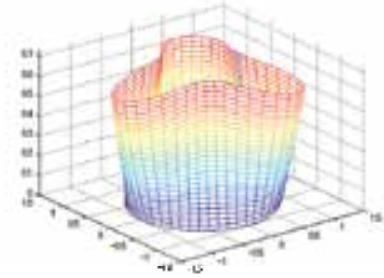

$m=1, n=3$

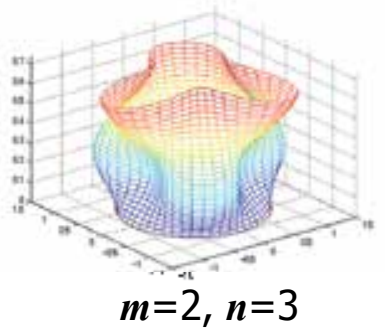

Fig. 4. Typical vibration modes of tanks: $m$, axial half wave number; $n$, circumferential wave number. (Fujita \& Saito, 2003)

\section{Overview of previous studies on vibration characteristics and seismic resistance of cylindrical liquid storage tanks}

In cylindrical liquid storage tanks, the liquid and the structure compose the coupled vibration system between fluid and structure and show complex vibration characteristics. Jacobsen (1949) and Werner and Sandquist (1949) were the first to study the influence of the contained liquid on dynamic behavior of cylindrical tanks. Their studies focused on only hydrodynamic behavior of the contained liquid assuming rigid containers. In the 1950s, the National Aeronautics and Space Administration (NASA) actively investigated the vibration behavior of rocket fuel tanks and then developed many empirical and analytical methods of sloshing and coupled vibration between fluid and structure (Abramson, 1966). The studies on sloshing developed as a specific field and their overview was done by Ibrahim et al. (2001). As for seismic evaluation of cylindrical liquid storage tanks, Housner (1957) proposed seismic response analysis method in 1957 which became known as Housner's theory and it has been adopted in some seismic design guidelines for cylindrical tanks. In this theory, the dynamic liquid pressure is calculated based on two separate pressures due to the horizontal inertia force of liquid and sloshing of the free liquid surface when cylindrical liquid storage tanks are subjected to seismic motions. However, tanks are assumed to be rigid bodies. Since Housner's proposal, many researchers have studied seismic evaluation methods. Veletsos and Yang (1976) and Fischer and Rammerstorfer (1982) proposed seismic response analysis methods of cylindrical liquid storage tanks assuming flexible structures for them by simplifying the vibration mode shape of the tanks. Moreover, Fujita (1981) also proposed seismic response analysis method in which tank structure was modeled by finite elements, the liquid pressure in tanks was expressed using velocity potential theory and series solution and coupled vibration between tank structure and contained liquid was considered. Ma et al. (1982) proposed an analysis method in which 
both tank structure and contained liquid were modeled by finite elements. The studies mentioned above focused on development of vibration response analysis methods of tanks subjected to seismic motions. Rammerstorfer et al. (1990) and Shimizu (1990) summarized research prior to 1990 on seismic evaluation methods of cylindrical liquid storage tanks. In their reviews, a few topics including bending vibration (beam-type vibration) and sloshing were covered, but not oval-type vibration. At present the behavior of oval-type vibration including the influence on seismic resistance of tanks remains unclarified.

Since the 1990s the issues of seismic study of cylindrical liquid storage tanks have shifted to dynamic buckling behavior. Though the buckling problem of cylindrical tanks was studied in the mid 1960s (NASA, 1968; Timoshenko et al., 1964), only the structural strength of an empty cylinder was investigated. The buckling problem of cylindrical liquid storage tanks subjected to seismic motions was in the limelight in the 1964 Alaska earthquake and in the 1971 San Fernando earthquake. In the 1980s Yamaki (1984) studied buckling behavior of cylindrical tanks filled with liquid systematically. In those studies, static buckling behavior was examined using small cylindrical tanks. Various dynamic buckling experiments were performed using different sizes of cylindrical tanks (Shin \& Babcock, 1980; Niwa \& Clough, 1982), and at the same time the occurrence mechanism of the buckling in the tanks was researched theoretically. In Japan, some dynamic buckling experiments were conducted using large scale models of cylindrical liquid storage tanks (Katayama et al., 1991; Tazuke et al., 2002). These experiments focused on proving tests of actual tanks, investigating the occurrence mechanism of the elephant foot bulge, and reflecting the experimental results into the seismic design. The experimental objects were vessels of fast breeder reactor and tanks for liquid natural gas. Toyoda and Masuko (1996), Fujita et al. (1990) and Akiyama (1997) studied the dynamic buckling of vessels and tanks vigorously and systematically, and Akiyama's proposal was adopted in a few guidelines for industrial tanks and vessels. However, the dynamic buckling behavior was not always clarified and Fujita et al. (1990) only examined the influence of oval-type vibration on seismic behavior of tanks. The numerical analysis methods, which could take into account fluid-structure interaction due to the contained liquid, were developed to simulate these experimental results but were not sufficient because of limited analysis conditions. Ito et al. (2003) conducted a dynamic buckling experiment on tall cylindrical liquid storage tanks such as the refueling water tanks installed in nuclear power plants. In their experiment, the ultimate state of the cylindrical liquid storage tanks was clarified but no numerical simulation development was discussed. Nowadays, seismic PSA should be performed as part of the seismic evaluation in nuclear power facilities. This requires that accurate failure modes and structure strength must be grasped for the ultimate state of equipment such as tanks. Therefore, it is urgent that the dynamic buckling simulation methods be established.

On the other hand, oval-type vibration has been studied from the standpoint of vibration engineering and the vibration behavior of a cylindrical shell, that is shell theory, has been investigated. Shell theory was studied from the 1920s and was systematically summarized by researchers such as Timoshenko and Woinowsky-Krieger (1959). The study on oval-type vibration of cylindrical liquid storage tanks was started in the 1960s as investigations of vibration and dynamic instability of a cylindrical shell partially filled with water. Chiba et al. $(1984,1986)$ conducted vibration tests using small cylindrical containers and undertook a theoretical study using Donnell's shell theory. More recently, Amabili (2003) investigated nonlinear response of oval-type vibration using Donnell's nonlinear thin shell theory 
vigorously. The contents of studies in the research field were reviewed by Amabili and Païdoussis (2003). However, these studies focused on nonlinear vibration characteristics and the instability region of oval-type vibration. Recently Maekawa et al. (2006) and Maekawa and Fujita (2007) clarified the influence of oval-type vibration on the resonance frequency and amplitude ratio of beam-type vibration in cylindrical tanks by vibration tests using a test tank. Their findings indicate that it is necessary to simulate oval-type vibration accurately in the seismic response analysis of cylindrical liquid storage tanks.

\section{Present status of dynamic analysis of cylindrical liquid storage tanks in Japan}

Cylindrical liquid storage tanks have a simple cylindrical geometry, but generate complex vibration behavior. In large tanks, the vibration behavior is a coupled system between the liquid and the tank structure because the tank walls are relatively thin and deformable. In the coupled system, various vibration modes simultaneously occur during earthquakes because the natural frequencies of most of the modes are in the exciting frequency range of the earthquakes. In the seismic design of tanks, the beam-type vibration which is the bending vibration mode is evaluated as the vibration mode which dominantly affects seismic response of the tanks. However, the vibration behavior of tanks is complex because oval-type vibration also actually occurs, which is a high order vibration mode and oscillation occurs with a petal-like pattern in the side wall. Highly advanced simulation techniques are needed to analyze such a complex vibration behavior. In conventional seismic evaluation methods, structural analysis is conducted assuming a hydrodynamic pressure distribution of liquid in the tanks as the load distribution with added mass based on velocity potential theory. However, the accuracy of time history analysis using conventional analysis methods is not good. Actually, the time history analysis of the hydrodynamic pressure distribution for successive fluid-structure interaction analysis is needed to simulate the behavior of beam-type vibration and oval-type vibration accurately. The conventional seismic evaluation of cylindrical liquid storage tanks (Kanagawa Prefecture, 2002; KHK, 2003; JEA, 2008; AIJ, 2010) is performed assuming the tank structure responses linearly and using evaluation equations for static buckling obtained on the basis of static buckling tests. However, an accurate buckling analysis method with dynamic effects and consideration of fluid-structure interaction has never been proposed. Though attempts were made to simulate dynamic buckling behaviors of cylindrical tanks (Fujita et al., 1992; Toyoda et al., 1997), accurate simulation methods were not realized.

Most recently Maekawa and Fujita (2009) proposed an accurate seismic response analysis and a dynamic buckling analysis for cylindrical liquid storage tanks. The proposed dynamic analysis method using explicit finite element analysis was validated by a dynamic seismic experiment which indicated that the analysis accuracy was good for evaluation of seismic response (vibration response and sloshing) and buckling behavior of cylindrical liquid storage tanks.

\section{Proposed dynamic analysis method}

The conventional seismic response analysis for cylindrical liquid storage tanks (Kanagawa Prefecture, 2002; KHK, 2003; JEA, 2008; AIJ, 2010) is appropriate as a conservative seismic design method but it is not appropriate for grasping actual phenomena during earthquakes. 
Maekawa and Fujita (2008) have proposed a nonlinear seismic response analysis method for cylindrical liquid storage tanks. In this method, the tank structure is three-dimensionally modeled using shell elements which can consider geometric nonlinearity (Belytschko et al., 1984), the contained liquid is modeled using solid elements which can calculate fluid behavior according to Euler's equation, and the fluid-structure interaction between the contained liquid and the tank structure is calculated by the arbitrary Lagrangian-Eulerian method (ALE method) (Hirt et al., 1974). Maekawa and Fujita (2008) compared analysis results obtained by their proposed method with experimental results and demonstrated that the nonlinear vibration behavior caused by the influence of oval-type vibration as well as the behavior of oval-type vibration could be simulated by the proposed method.

Maekawa and Fujita (2009) also applied their proposed method to dynamic buckling analysis. A nonlinear inelastic analysis and a large deformation analysis were conducted considering material nonlinearity to simulate plastic deformation and response in the postbuckling. The characteristics of the proposed method and the conventional dynamic buckling analysis method (Fujita et al., 1992; Toyoda et al., 1997) are compared in Table 1. As summarized there, the seismic response by the proposed method can be simulated more accurately and realistically.

Figure 5 shows an analysis model of a cylindrical tank used to validate the proposed method. This is a detailed finite element model of a test tank used for the dynamic buckling experiment presented in later sections. The numbers of nodes and elements are 424,460 and 410,482 , respectively. Figure 6 shows a photo of the test tank.

In the analysis model, the Lagrange elements are used for the structure part and the Euler elements are used for the fluid part in the model. The Belytschko-Lin-Tsay shell elements (Belytschko et al., 1984) based on Mindlin theory are used for modeling the tank structure. The shell elements can consider geometric nonlinearity and shear deformation. The shear deformation is formulated by rotating a cross section vertical to the center of a shell element surface and the nonlinear constitutive equations are expressed by only linear terms using an approach to remove the rigid body motion. For the present analysis case, a 200-kg weight on the tank top was assumed as an added mass rigidly fixed on the top of the model. The imperfection distribution from a perfect circle of the cylinder obtained by profile measurement was set in the model. The material constants used in the analysis are listed in Table 2. The stress-strain relationship of aluminum alloy used in the cylinder was obtained by a material test and was approximated by multiple lines for the elasto-plastic input condition. The material constants of steel and polycarbonate were nominal values.

The fluid part is modeled by solid elements which use Euler's equation as the fundamental equation. Using Euler elements, the fluid moves from one element to another element to reproduce the pressure change due to vibration. For the present analysis case, the properties of water were set for the $95 \%$ water level of the model and the remaining $5 \%$ part was assumed as empty because air and void elements were used. The ALE method is used for the fluid-structure interaction analysis.

In the analysis, static pressure of the liquid in the tank and the dead weight of the tank structure should be considered. Hence, the load balance in the static state was calculated by loading gravity force to the model before the dynamic analysis. The mass-proportional damping with $100 \%$ damping ratio was assumed to obtain the stationary solution quickly. Next, the analysis model was impacted using a triangular wave, the free vibration of the model was excited, and then the natural frequency of the primary beam-type vibration was 
obtained by frequency analysis of the response. Based on its natural frequency of $30.45 \mathrm{~Hz}$, $31 \mathrm{~Hz}$ was chosen as the excitation frequency. Finally, the dynamic buckling simulation was performed using sinusoidal waves with $31 \mathrm{~Hz}$ as input in which the liquid pressure change was calculated successively. The input acceleration $2.5 \mathrm{G}$ was chosen based on the experimental conditions described in the next section. The analysis conditions are summarized in Table 3. Time history analysis was conducted by an explicit integral method using the finite element analysis code LS-DYNA (Livermore Software Technology Corporation, 2003).

From the viewpoint of convergence, it is difficult to solve all problems when large deformation problems such as buckling are solved by an implicit method which often is used in the general finite element method. Therefore, the explicit method was applied to the seismic response analysis, which is a long time analysis, though the method has been used for only extremely short time analyses such as impact analysis until now. This was possible due to the rapid progress which has been made in computer calculation performance.

\begin{tabular}{|c|c|c|}
\hline Items & Proposed method & $\begin{array}{l}\text { Conventional method (Fujita et } \\
\text { al., 1992; Toyoda et al., 1997) }\end{array}$ \\
\hline $\begin{array}{l}\text { Behavior of water } \\
\text { contained in the } \\
\text { tank }\end{array}$ & $\begin{array}{l}\text { Contained water modeled by } \\
\text { solid elements; fluid-structure } \\
\text { interaction calculated } \\
\text { sequentially }\end{array}$ & $\begin{array}{l}\text { Contained water not modeled; } \\
\text { added mass considered. }\end{array}$ \\
\hline Oval-type vibration & All modes considered & $\begin{array}{l}\text { Higher order modes not } \\
\text { considered }\end{array}$ \\
\hline Sloshing & Analyzable & Non-analyzable \\
\hline 3D modeling & $\begin{array}{l}\text { 3D model for both } \\
\text { axisymmetric and non- } \\
\text { axisymmetric structures using } \\
\text { shell elements }\end{array}$ & $\begin{array}{l}\text { Axisymmetric model using } \\
\text { axisymmetric elements }\end{array}$ \\
\hline Solving method & Explicit method & $\begin{array}{l}\text { Newmark } \beta \text { method (Implicit } \\
\text { method) }\end{array}$ \\
\hline $\begin{array}{l}\text { Geometry } \\
\text { nonlinearity }\end{array}$ & $\begin{array}{l}\text { Rigid body motion removed to } \\
\text { allow use of a simple } \\
\text { constitutive equation with } \\
\text { linear terms for efficient } \\
\text { calculation and better analysis } \\
\text { accuracy }\end{array}$ & $\begin{array}{l}\text { Complicated constitutive } \\
\text { equation with high order } \\
\text { nonlinear terms used for better } \\
\text { analysis accuracy }\end{array}$ \\
\hline $\begin{array}{l}\text { Material } \\
\text { nonlinearity }\end{array}$ & $\begin{array}{l}\text { Elasto-plastic model using } \\
\text { multiple line approximation of } \\
\text { stress-strain relationship }\end{array}$ & $\begin{array}{l}\text { Elasto-plastic model using } \\
\text { multiple line approximation of } \\
\text { stress-strain relationship }\end{array}$ \\
\hline
\end{tabular}

Table 1. Comparison of the proposed and conventional dynamic buckling analysis methods 


\begin{tabular}{|c|c|c|c|c|}
\hline Materials & $\begin{array}{c}\text { Young's } \\
\text { modulus } \\
(\mathrm{MPa})\end{array}$ & $\begin{array}{l}\text { Poisson's } \\
\text { ratio }\end{array}$ & $\begin{array}{l}\text { Density } \\
\left(\mathrm{kg} / \mathrm{m}^{3}\right)\end{array}$ & $\begin{array}{c}\text { Bulk } \\
\text { modulus } \\
(\mathrm{MPa})\end{array}$ \\
\hline Aluminum alloy (A5052) (cylinder) & 69,420 & 0.33 & 2,680 & - \\
\hline Steel (flange and platform) & 203,000 & 0.3 & 7,800 & - \\
\hline Polycarbonate (top plate) & 1,960 & 0.3 & 11,900 & - \\
\hline Water (in the tank) & - & - & 1,000 & 2,200 \\
\hline
\end{tabular}

Table 2. Material constants for analysis

\begin{tabular}{|l|l|}
\hline \multicolumn{1}{|c|}{ Items } & \multicolumn{1}{c|}{ Analysis conditions } \\
\hline Structure & Model using nonlinear shell elements \\
\hline Water & Model using solid element with Euler's equation \\
\hline Air & Model using solid elements with voids \\
\hline Weight & Model using mass elements \\
\hline $\begin{array}{l}\text { Fluid-structure interaction } \\
\text { analysis }\end{array}$ & ALE method \\
\hline Time-history analysis & Explicit time integration method \\
\hline Damping & Rayleigh damping (3\% at resonance point) \\
\hline Excitation wave & Sinusoidal waves similar to those in the experiment \\
\hline Excitation acceleration & $2.5 \mathrm{G}$ \\
\hline Excitation frequency & $31 \mathrm{~Hz}$ \\
\hline Excitation direction & Horizontal $\left(0^{\circ}-180^{\circ}\right)$ \\
\hline
\end{tabular}

Table 3. Analysis conditions

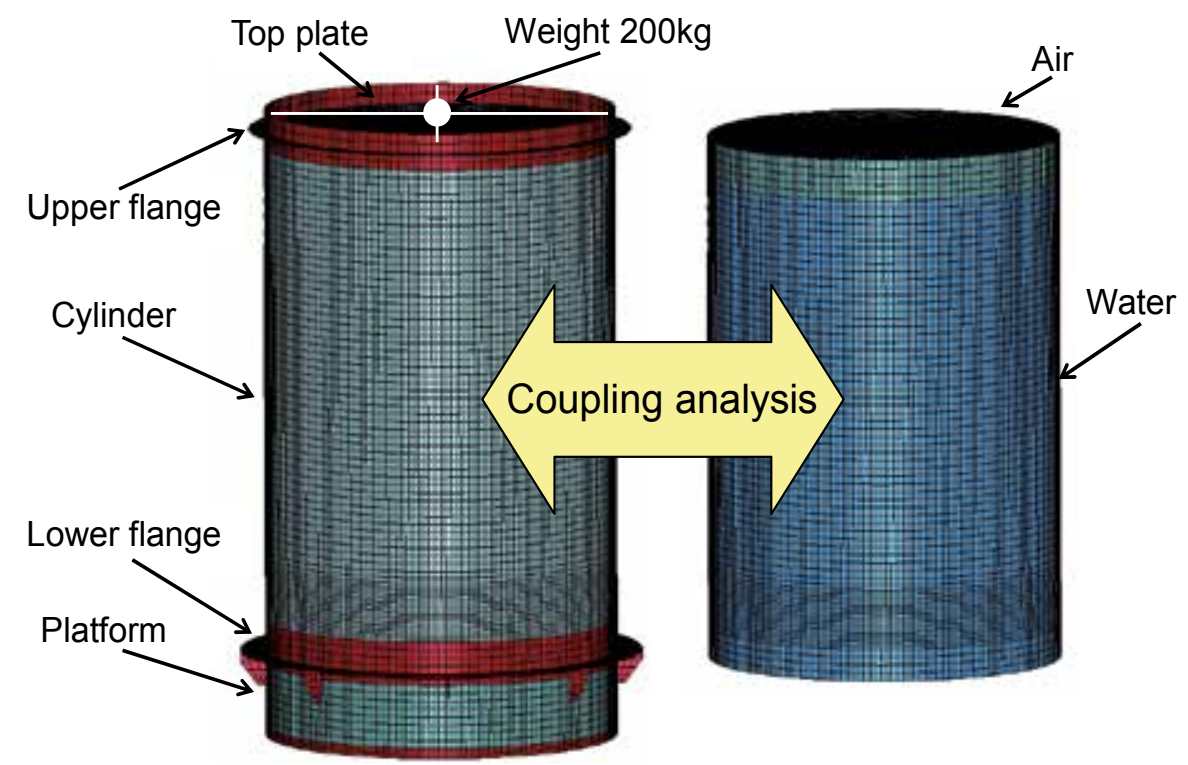

Structure part (shell elements) Fluid part (solid elements)

Fig. 5. Analysis model of $1 / 10$ reduced-scale cylindrical liquid storage tank. 


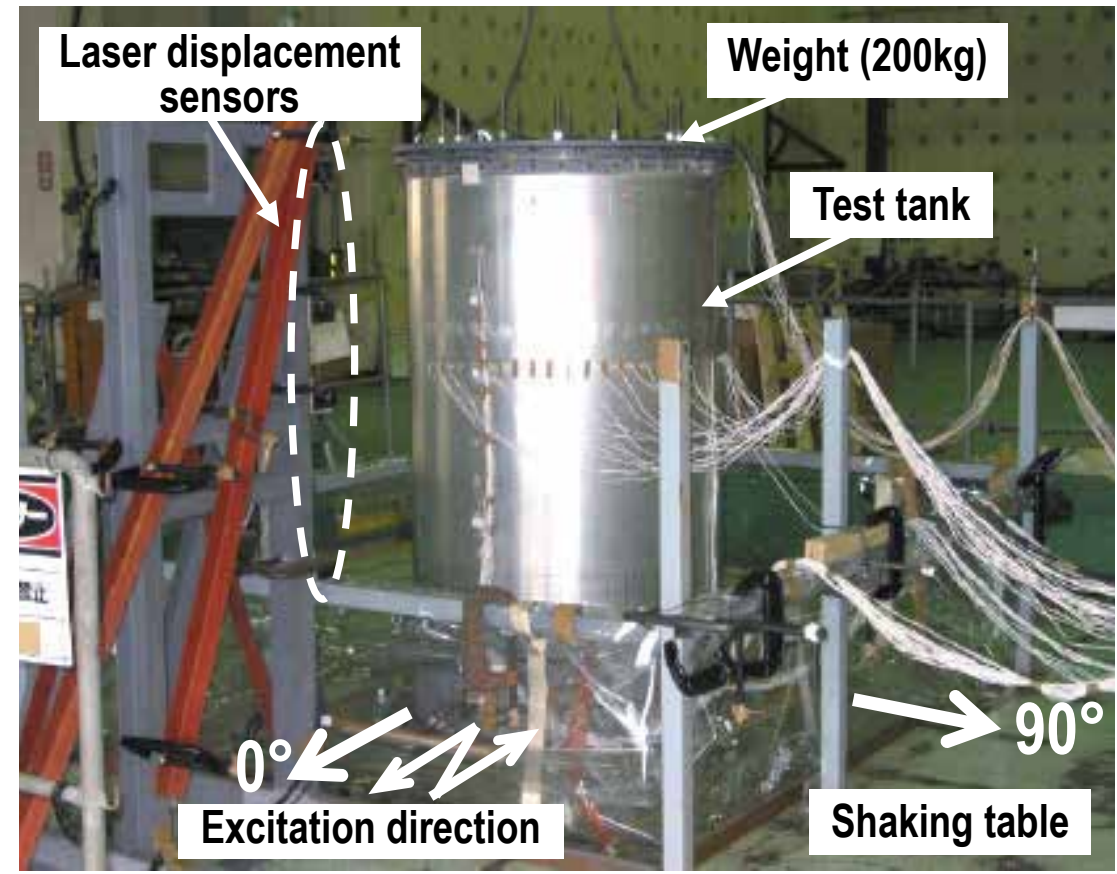

Fig. 6. Test tank of 1/10 reduced-scale cylindrical liquid storage tank.

\section{Dynamic buckling experiment}

The dynamic buckling experiment (Maekawa et al., 2007) of the test tank shown in Fig. 6 was conducted using a large shaking table setup to validate the proposed method. The test tank was a 1/10 reduced-scale model of the large cylindrical storage tanks installed in nuclear power plants such as condensate water tanks which are classified as equipment requiring the highest seismic safety. A $200-\mathrm{kg}$ doughnut-shaped weight was put on the top of the tank to amplify the response of the tank during shaking.

The dimensions and measurement locations of the test tank are shown in Fig. 7. The test tank was an aluminum alloy cylinder with a wall thickness of $1 \mathrm{~mm}$. The cylinder was fixed using steel flanges at both ends. A polycarbonate transparent board was used for the roof of the tank to look in the tank. The cylinder was made by shaping an aluminum alloy plate and using TIG welding. The shape imperfection from a perfect circle was measured by optical digital profilometry and the imperfection distribution was set in the analysis model. For the important geometry parameters in buckling, the height to radius ratio was 2.67 and radius to thickness ratio was 450 . The test tank was fixed on the shaking table using a steel platform with a 2-mm wall thickness. The shear force and bending moment of the whole tank were estimated using strain generated in the platform during excitation. The behavior of beam-type vibration was measured using accelerometers on the top of the tank. Laser displacement sensors were put in the $0^{\circ}$ direction at four heights of 290,680, 870 and 1200 $\mathrm{mm}$. The displacement changes of beam-type vibration and oval-type vibration were measured at the 1200-mm height and the other heights, respectively. In Fig. 7, many strain gauges were attached in the range from $-18^{\circ}$ to $138^{\circ}$ at the $700-\mathrm{mm}$ height. The mode shape of oval-type vibration was examined from the magnitude of strain in each position. 
Occurrence of the oval-type vibration was observed by a video camera at the $90^{\circ}$ position. The test tank was partially filled with water to the $95 \%$ level (to a height of $1140 \mathrm{~mm}$ ) and it was excited horizontally between $0^{\circ}$ and $180^{\circ}$ using sinusoidal waves. The acceleration amplitude of the sinusoidal waves gradually increased and decreased at the start and end, respectively because other frequency components, except the excitation frequency, were not included. The natural frequency of primary beam-type vibration of the tank was examined by a frequency sweep test. The natural frequency of $27 \mathrm{~Hz}$ was chosen as the excitation frequency. Three exciting tests were conducted while changing the magnitude of the input acceleration.

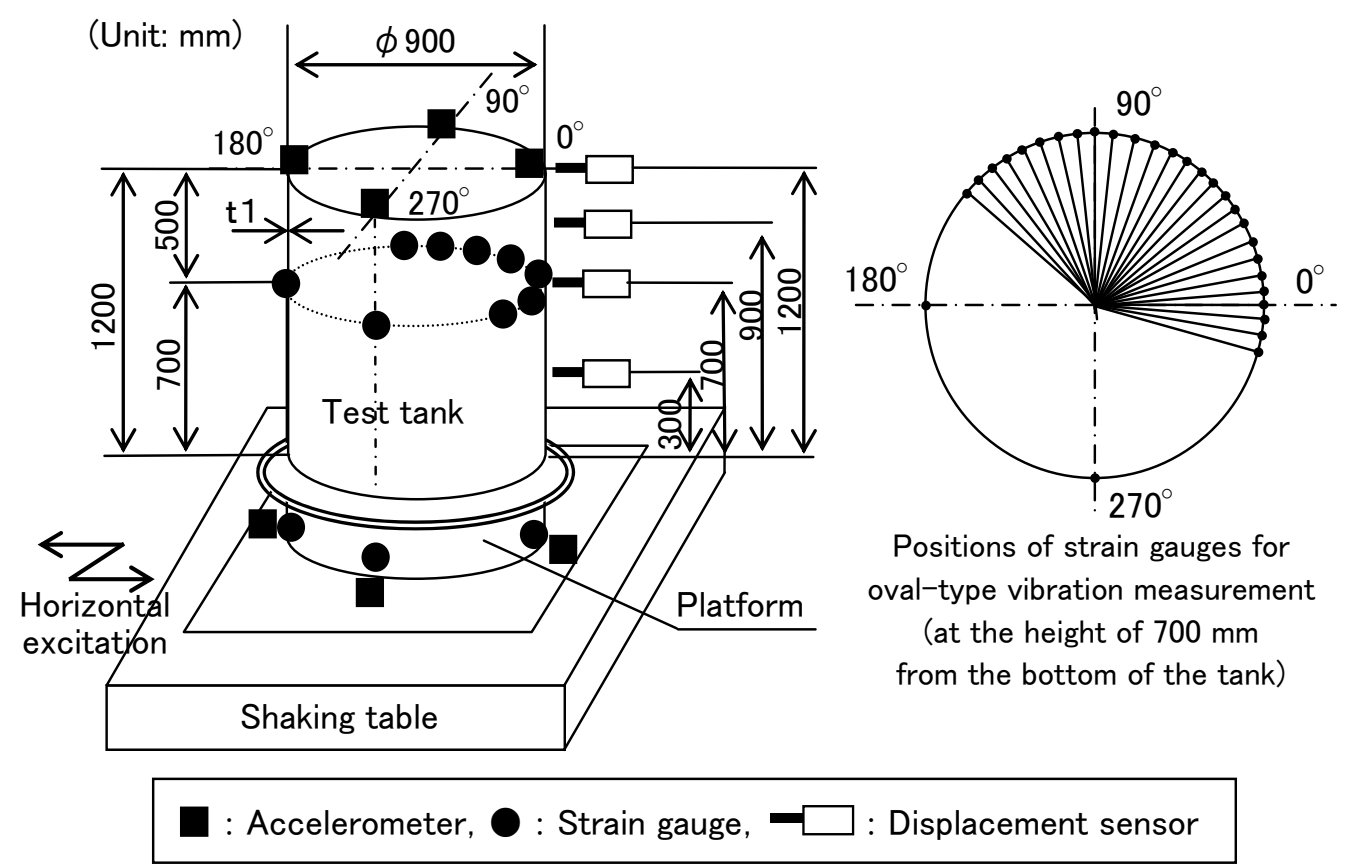

Fig. 7. Dimensions and measurement locations of test tank.

\section{Dynamic buckling experiment results}

Some distinct pictures expressing the dynamic behavior of the tank side wall are shown in Fig. 8. The vibration behavior of the wall is represented using lighted and shaded reflections from a spot light. The reflection of light in the center region of the cylinder was wide and narrow in the axis direction and indicated geometric change of the wall with time. Namely, oval-type vibration occurred and oscillation was large.

As shown in Fig. 9, plastic deformation was retained in the test tank after the experiment. The deformation in the side wall of the tank was probability caused by shear buckling and the deformed denting inward in the base was caused by bending buckling, specifically diamond buckling. Comparison of the maximum values of the shear stress and bending stress and the design allowance values indicated bending buckling occurred predominately because only the measured bending stress exceeded the design allowance value as shown in Table 4. 

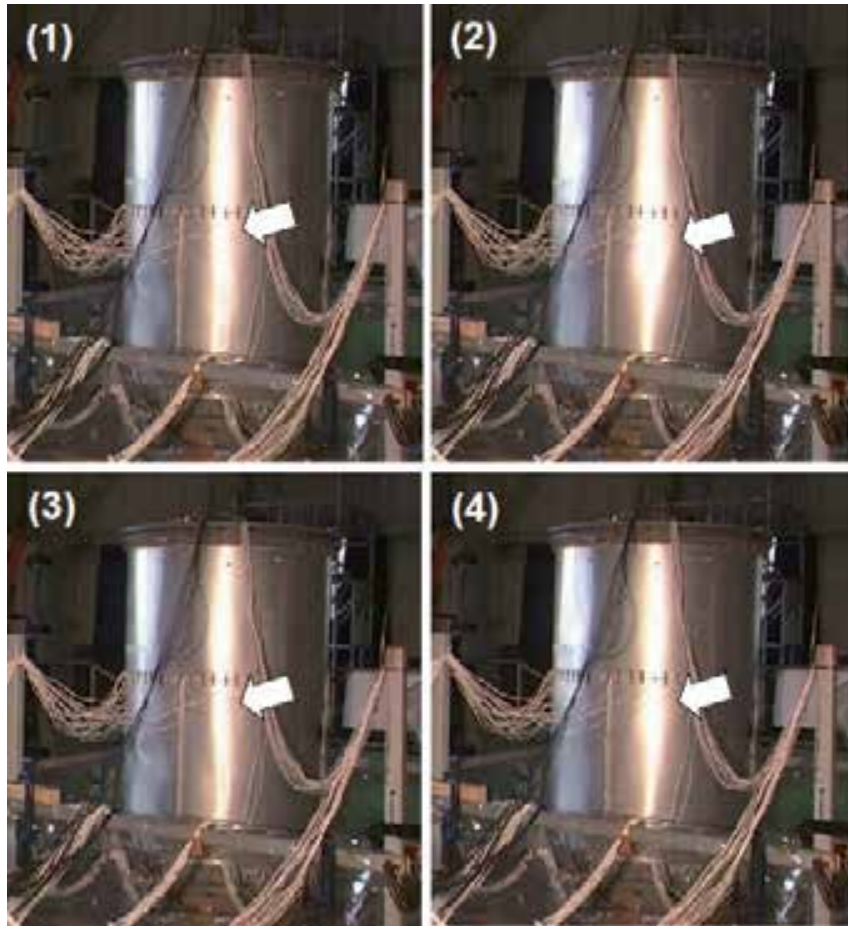

Fig. 8. Oval-type vibration occurring in the side wall of the test tank.

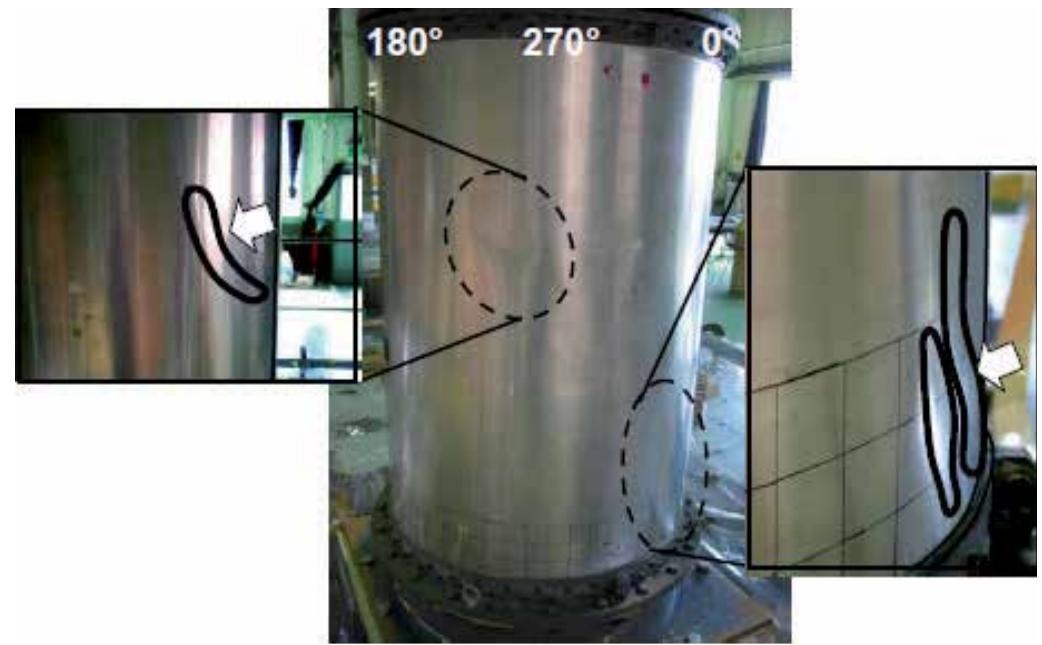

Fig. 9. Plastic deformation remaining in the tank wall after buckling.

\begin{tabular}{|l|c|c|}
\hline & Experimental values $\left(\mathrm{N} / \mathrm{mm}^{2}\right)$ & Design allowance values $\left(\mathrm{N} / \mathrm{mm}^{2}\right)$ \\
\hline Bending stress & 97.18 & 47.02 \\
\hline Shear stress & 33.45 & 54.65 \\
\hline
\end{tabular}

Table 4. Comparison of buckling stresses 


\section{Results of proposed dynamic analysis}

The time history analysis was conducted by the proposed method based on the analysis conditions in Table 3. Figure 10 shows distortion shape of the model after the analysis. The deformation factor was ten. Oval-type vibration occurred with large deformation of the wall. The bending buckling (diamond buckling) occurred with deformed denting inward in the base. These behaviors were similar to the experimental observation. Figure 11 shows the measured deformation shapes of a cross section of the test tank at a height of $700 \mathrm{~mm}$. These shapes represent modes of oval-type vibration excited in the experiment before and after buckling. These modes were different, indicating the influence of buckling. Figure 12 shows the deformation shapes of a cross section of the model obtained by the analysis. It was found that the analytical shapes were similar to those in Fig. 11. Namely, the proposed dynamic analysis method was demonstrated to simulate the experimental results accurately. Figures 13 and 14 show the pressure distributions of liquid in the tank model obtained by the analysis at the corresponding times to Fig. 12. Figure 13 shows liquid pressure distributions of the cross section at a height of $700 \mathrm{~mm}$ and Fig. 14 shows the vertical section between $0^{\circ}$ and $180^{\circ}$. The shape of the liquid pressure distributions in Fig. 13 corresponded to the mode shapes of oval-type vibration, for example, the regions are indicated by arrows. This indicates that the proposed method can simulate phenomena caused by the fluidstructure interaction in the tanks accurately. Consequently from the above results, the proposed dynamic analysis method is judged to be able to simulate oval-type vibration, liquid pressure behavior, buckling modes, and buckling response accurately.
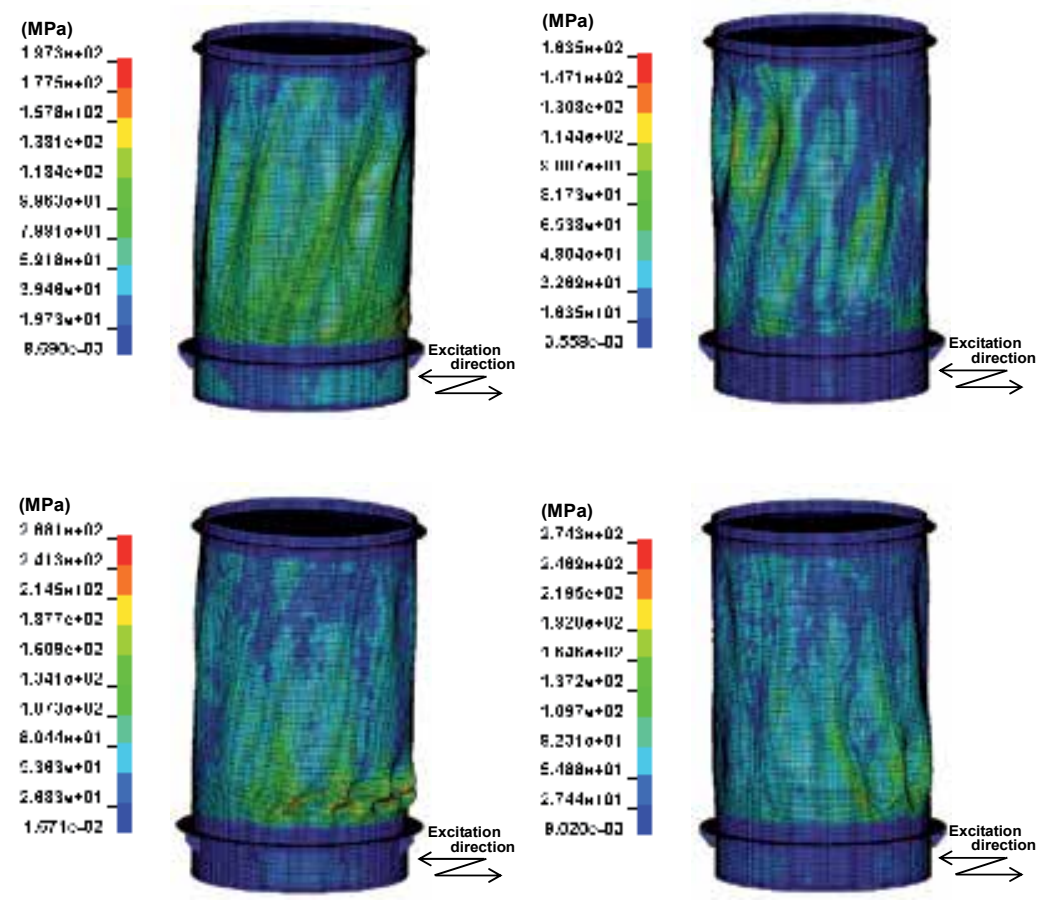

Fig. 10. Distortion shape and von Mises stress contour plots (displacement factor, 10) before (upper) and after (lower) buckling. 

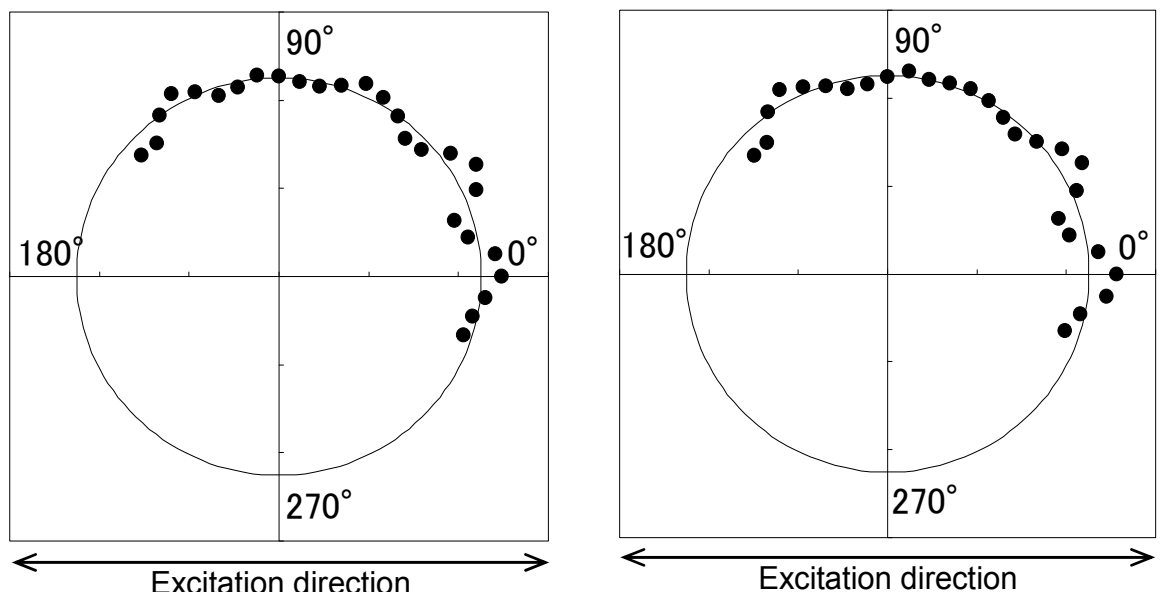

Fig. 11. Measured mode shapes of oval-type vibration in test tank at 700-mm height before (left) and after (right) buckling.

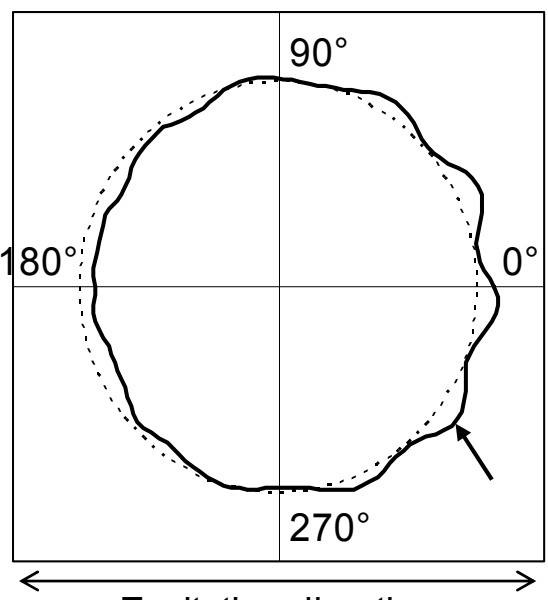

Excitation direction

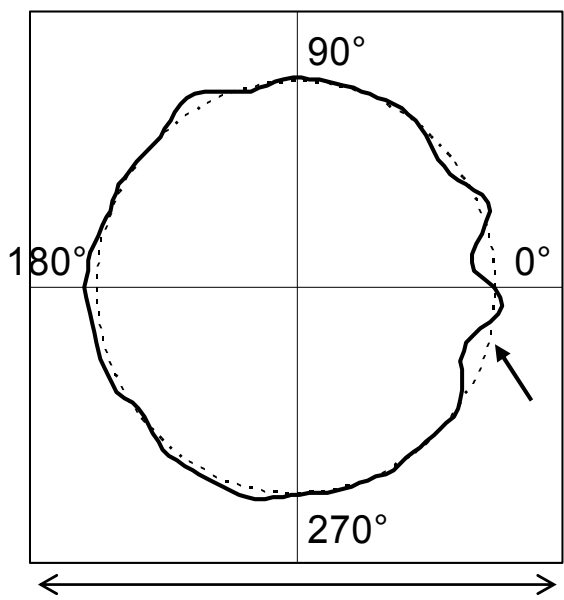

Excitation direction

Fig. 12. Calculated mode shapes of oval-type vibration in tank model at 700-mm height before (left) and after (right) buckling. 

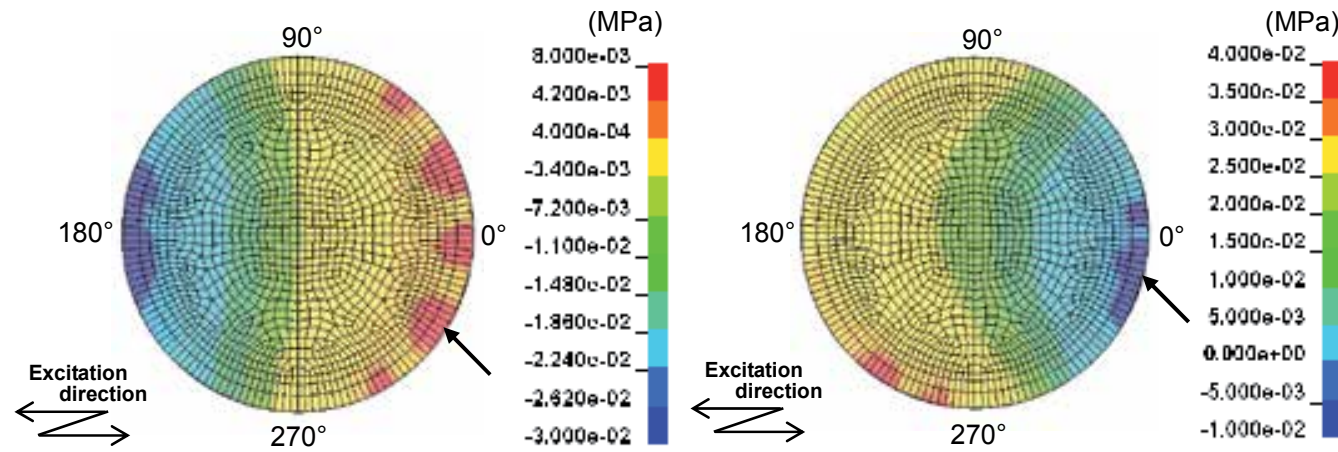

Fig. 13. Calculated liquid pressure distributions at 700-mm height before (left) and after (right) buckling.
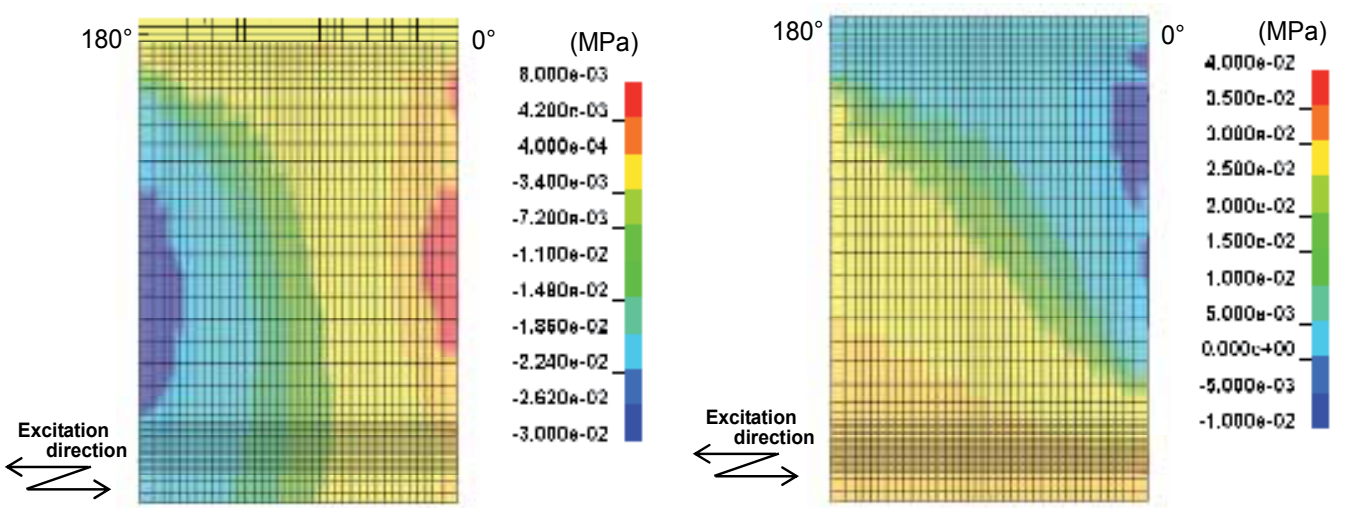

Fig. 14. Calculated liquid pressure distributions vertical section between $0^{\circ}$ and $180^{\circ}$ before (left) and after (right) buckling.

The mode shape of oval-type vibration excited was then discussed in detail using the proposed method. Figures 15 and 16 show the oval-type vibration behavior before and after buckling, respectively. The mode shape of oval-type vibration before buckling was specific and the circumferential wave number $n=12$ was dominant. On the other hand, the mode after buckling was unstable and non-uniform. This might be associated with the change of vibration characteristics of the tank model due to the development of buckling on the base of the tank model. As described above, the vibration behavior of cylindrical liquid storage tanks including oval-type vibration can be examined by numerical simulation using the proposed dynamic analysis method.

The dynamic behavior of the liquid pressure distribution also was discussed using the proposed method (see Figs. 13 and 14). In the liquid pressure distribution before buckling, a large dynamic liquid pressure occurred due to the large beam-type vibration. The change of liquid pressure due to oval-type vibration occurred locally. In the pressure distribution after buckling, the change of the dynamic liquid pressure due to beam-type vibration and oval-type vibration was not clear. The change of liquid pressure before and after buckling might also be attributed to the change of the vibration characteristics due to buckling. 

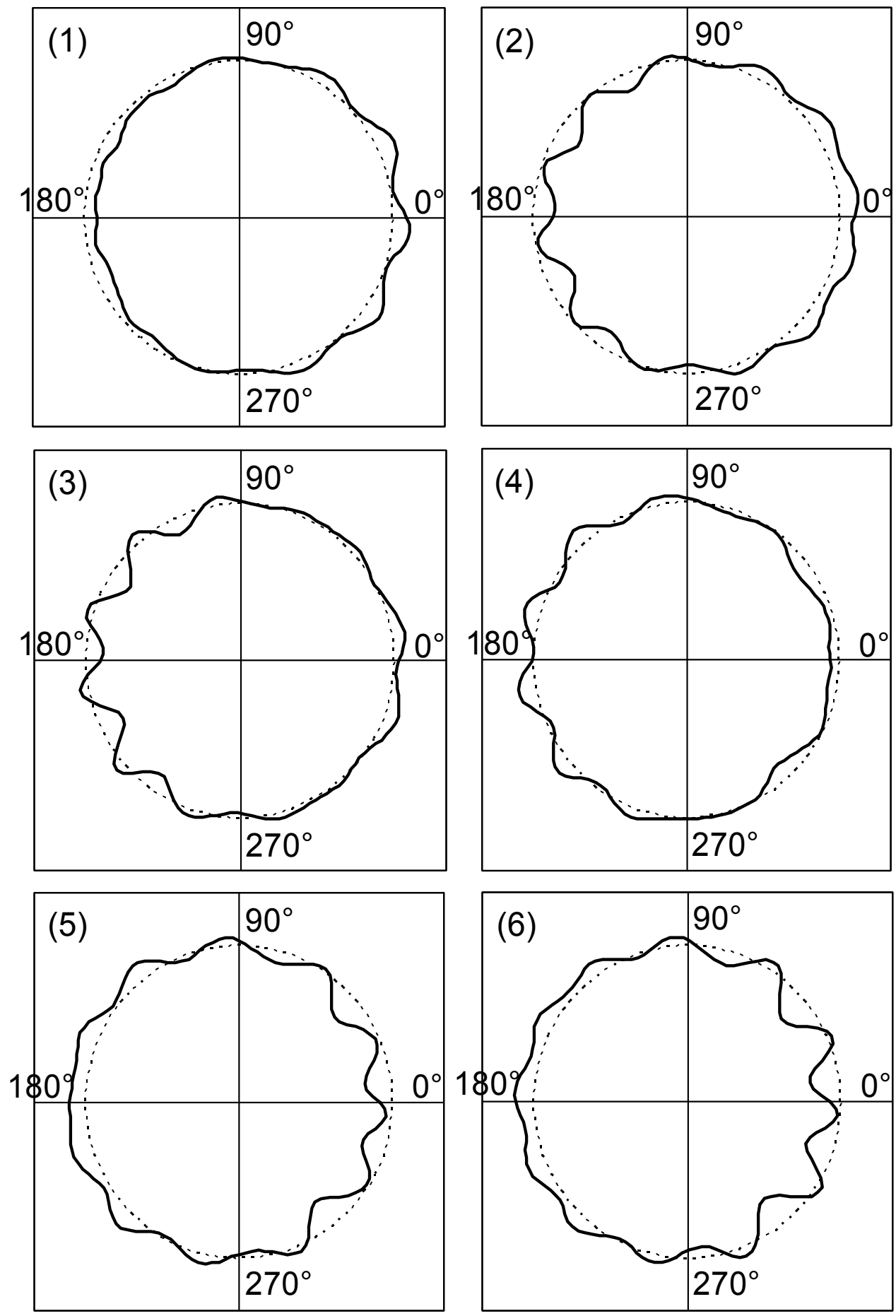

Fig. 15. Mode shape variation of oval-type vibration before buckling (time course: from (1) to (6)). 

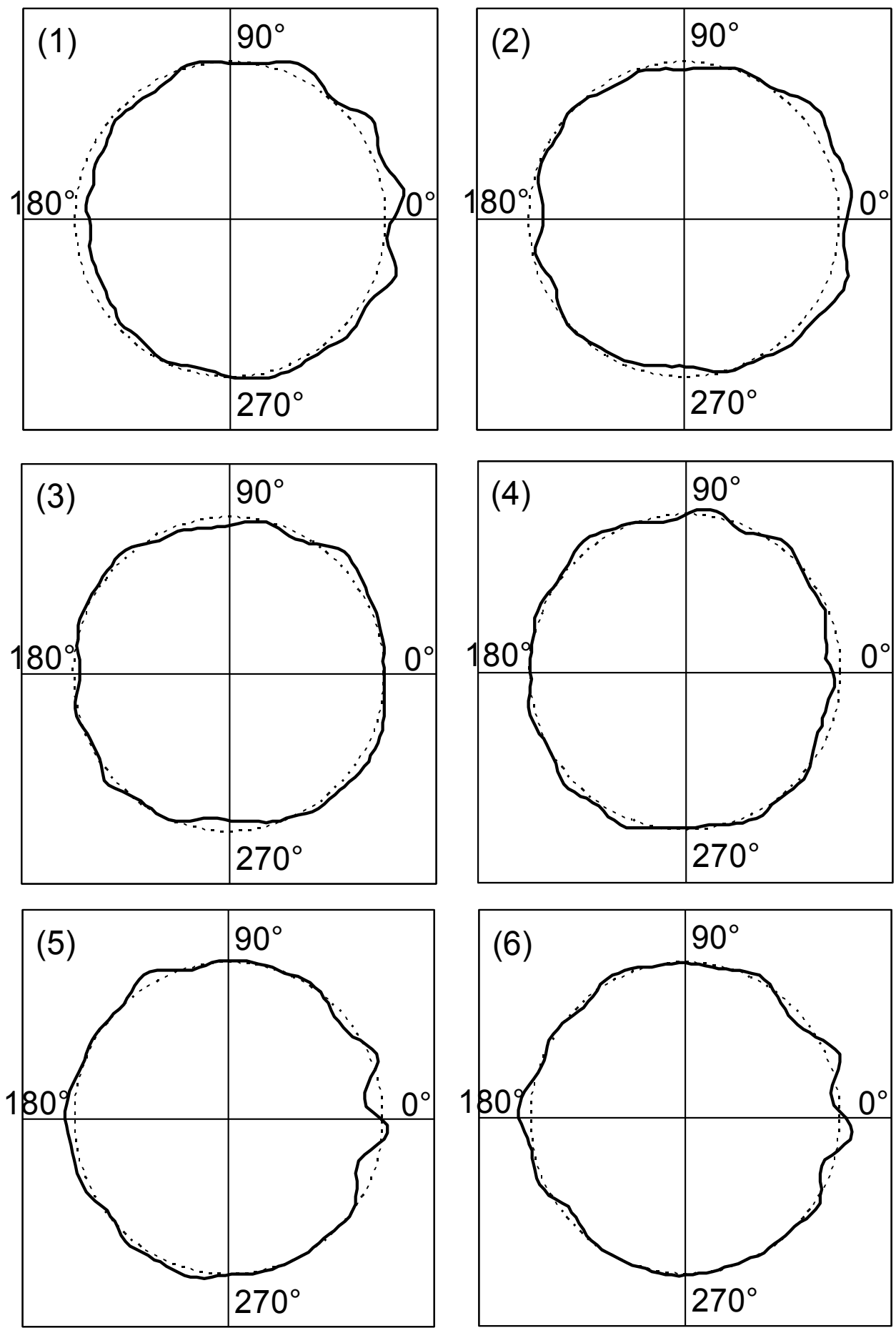

Fig. 16. Mode shape variation of oval-type vibration after buckling (time course: from (1) to (6)). 
The detailed analysis results of the dynamic liquid pressure distribution are shown in Figs. 17 and 18. The two figures show the vertical distribution of dynamic liquid pressure at the $0^{\circ}$ position before and after buckling, respectively. Here, a qualitative discussion was done because the lines of the graphs were ragged which could be attributed to the roughness of the mesh division and inherent properties of the explicit method. The two figures suggested that the dynamic liquid pressure distribution was caused by beam-type vibration and the distribution shape was typical of that in thin-walled and flexible cylindrical liquid storage tanks. The distribution shapes before and after buckling differed. The difference was thought to be associated with the change of vibration characteristics of the tank due to buckling.
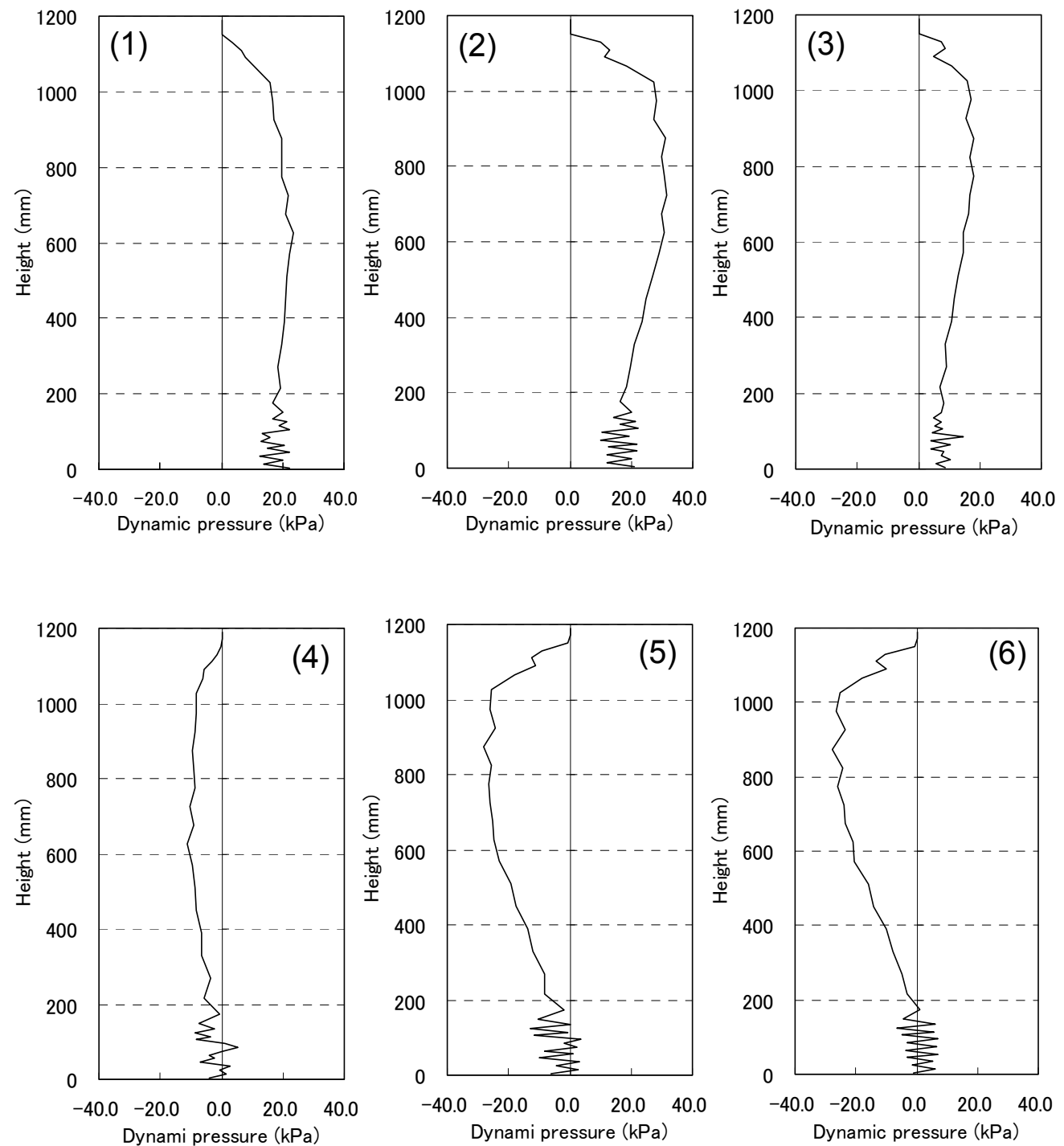

Fig. 17. Calculated vertical distributions change of dynamic liquid pressure at $0^{\circ}$ position before bucking (time course: from (1) to (6)). 

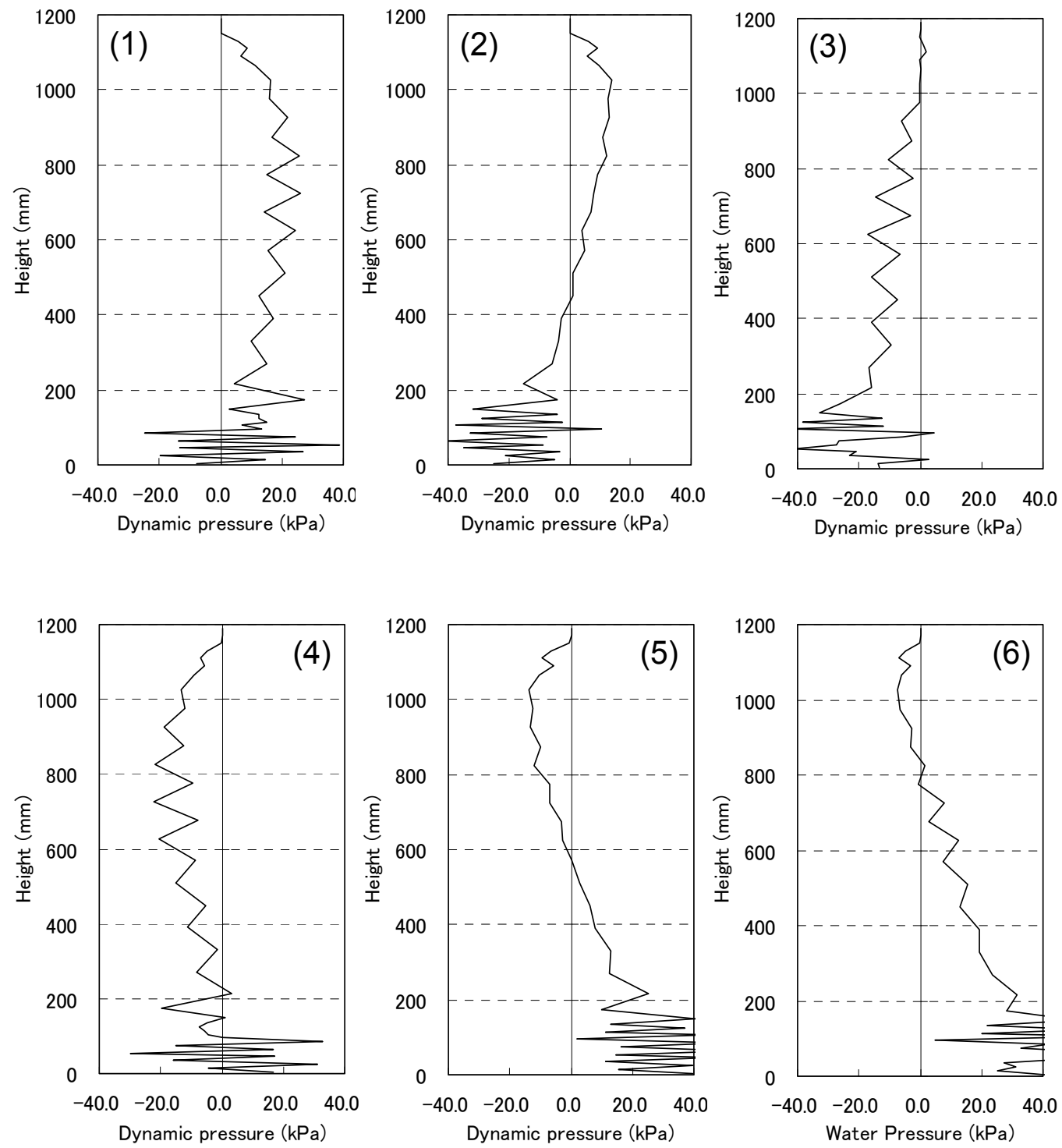

Fig. 18. Calculated vertical distributions change of dynamic liquid pressure at $0^{\circ}$ position after buckling (time course: from (1) to (6)).

The acceleration and buckling loads obtained from the dynamic experiment and the analysis are summarized in Table 5. The shear force and bending moment agreed well between them. The load that worked on the tank was well estimated by the analysis. Hence, the proposed dynamic analysis method can estimate dynamic behavior and buckling behavior of liquid storage tanks accurately even though the seismic response of the tanks was unknown. The input acceleration in the analysis agreed with that in the experiment. This indicates that the input magnitude to increase buckling, that is, the magnitude of seismic motion, is predicable by the proposed dynamic analysis method. 
In conclusion, it is demonstrated that the proposed dynamic analysis method can simulate the vibration response of cylindrical liquid storage tanks, the pressure behavior of the contained liquid and the buckling behavior accurately. Moreover, the buckling load and input magnitude to increase buckling can also be predicted accurately.

\begin{tabular}{|l|c|c|}
\hline & $\begin{array}{c}\text { Dynamic buckling } \\
\text { experiment }\end{array}$ & $\begin{array}{c}\text { Dynamic buckling } \\
\text { analysis }\end{array}$ \\
\hline Input acceleration $(\mathrm{G})$ & 2.37 & 2.61 \\
\hline Shear force $(\mathrm{kN})$ & 47.3 & 59.4 \\
\hline Bending moment $(\mathrm{MN} \mathrm{mm})$ & 61.8 & 67.7 \\
\hline
\end{tabular}

Table 5. Input excitation and associated base shear and base bending moment

\section{Applicability to actual tank}

The test tank and model described above are approximately a 1/10 reduced-scale model of an actual tank with uniform wall thickness. However, actual tanks are of many dimensions and shapes, and so research on larger tanks of different shapes would help assess the applicability of the proposed dynamic analysis method to actual tanks. Some dynamic buckling experiments using large-scale test tanks have been conducted. Ito et al. (2003) recently did a dynamic buckling experiment using a test tank similar to the refueling water storage tanks installed in nuclear power plants. The applicability of the proposed dynamic analysis method was assessed by using their experiment results as a benchmark (Maekawa \& Fujita, 2010).

A photograph of their tank is shown in Fig. 19 (Ito et al., 2003). It was a $1 / 5$ reduced-scale model. The tank, which had an internal diameter of $2200 \mathrm{~mm}$, cylinder height of $2650 \mathrm{~mm}$ and water level of $2526 \mathrm{~mm}$, had a non-uniform wall thickness with thickness decreasing in the height direction and was made of aluminum alloy. The height to radius ratio was 2.4 and the radius to average thickness ratio was 894 . The experiment results showed the occurrence of oval-type vibration in the wall and bending buckling (elephant foot bulge) in the base; the type of bending buckling was different from that of the $1 / 10$ reduced-scale test tank. Figure 20 shows the analysis model, which has 125,767 nodes and 131,712 elements. The Belytschko-Lin-Tsay shell elements (Belytschko et al., 1984) were used for the tank structure and solid elements with Euler's equation were used for the contained fluid. The coupling analysis between fluid and structure used the ALE method (Hirt et al., 1974) to estimate dynamic fluid-structure interaction. The free liquid surface was made by modeling the contained liquid and gas as solid elements in order to simulate sloshing. The same seismic wave as that used in the experiment was used as input. The magnitude of input acceleration was $2.7 \mathrm{Se}(1600 \mathrm{Gal}$ instantaneous maximum acceleration). In the experiment, buckling was caused and developed by the seismic wave of $2.7 \mathrm{Se}$.

The natural frequency of primary beam-type vibration of the analysis model was $16.6 \mathrm{~Hz}$ and that agreed with the experiment value of $16.8 \mathrm{~Hz}$, showing the appropriateness of the model. The analytical result for the seismic wave is shown in Fig. 21. The side wall of the model was severely deformed inward, representing the occurrence of oval-type vibration. The wall in the base of the model locally came outward, showing the occurrence of elephant foot bulge. These results confirm that the proposed method can simulate the occurrence of oval-type vibration and the elephant foot bulge. In addition, Fig. 22 shows the simulation of 
sloshing of the contained liquid. The boundary between the liquid and air was heaved up, representing sloshing of the free liquid surface. Finally, these results demonstrate that the proposed method can also accurately simulate the vibration characteristics and buckling behavior of tanks with various shapes.

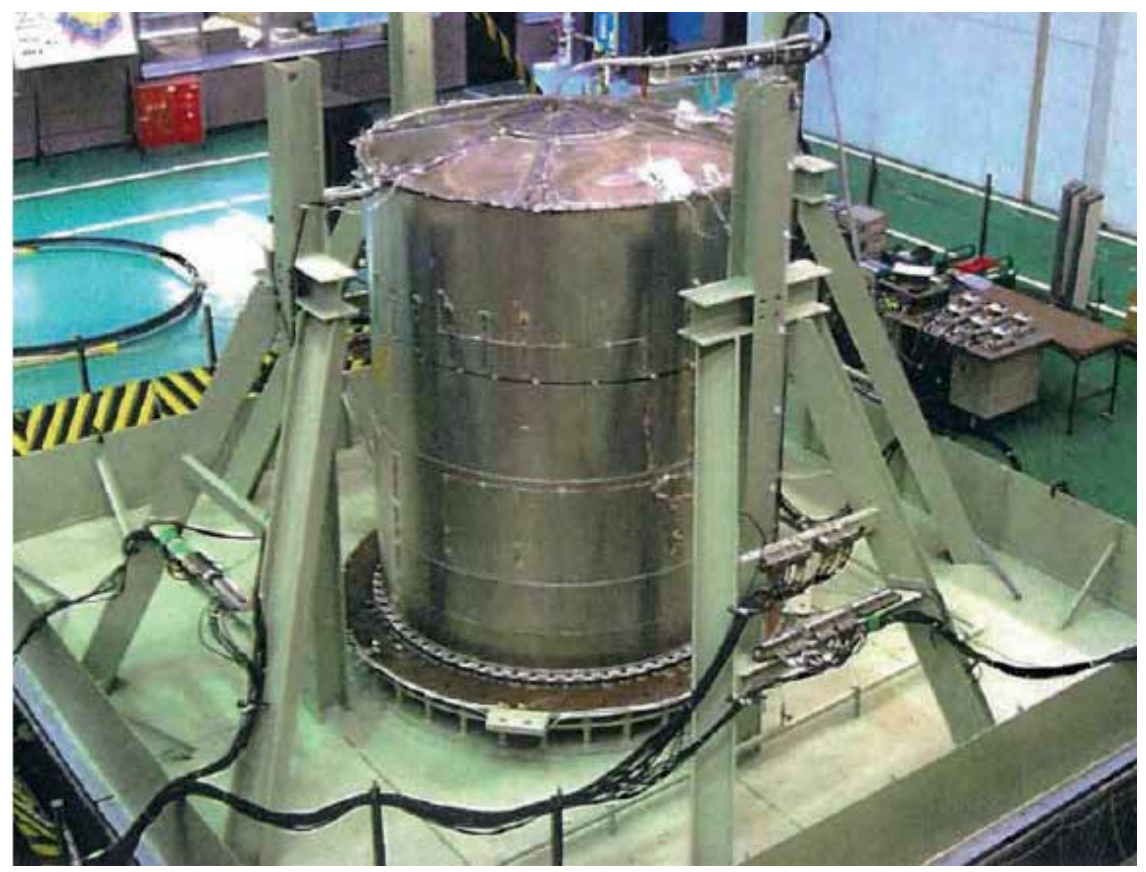

Fig. 19. Large test tank of $1 / 5$ reduced-scale cylindrical liquid storage tank. (Ito et al., 2003)

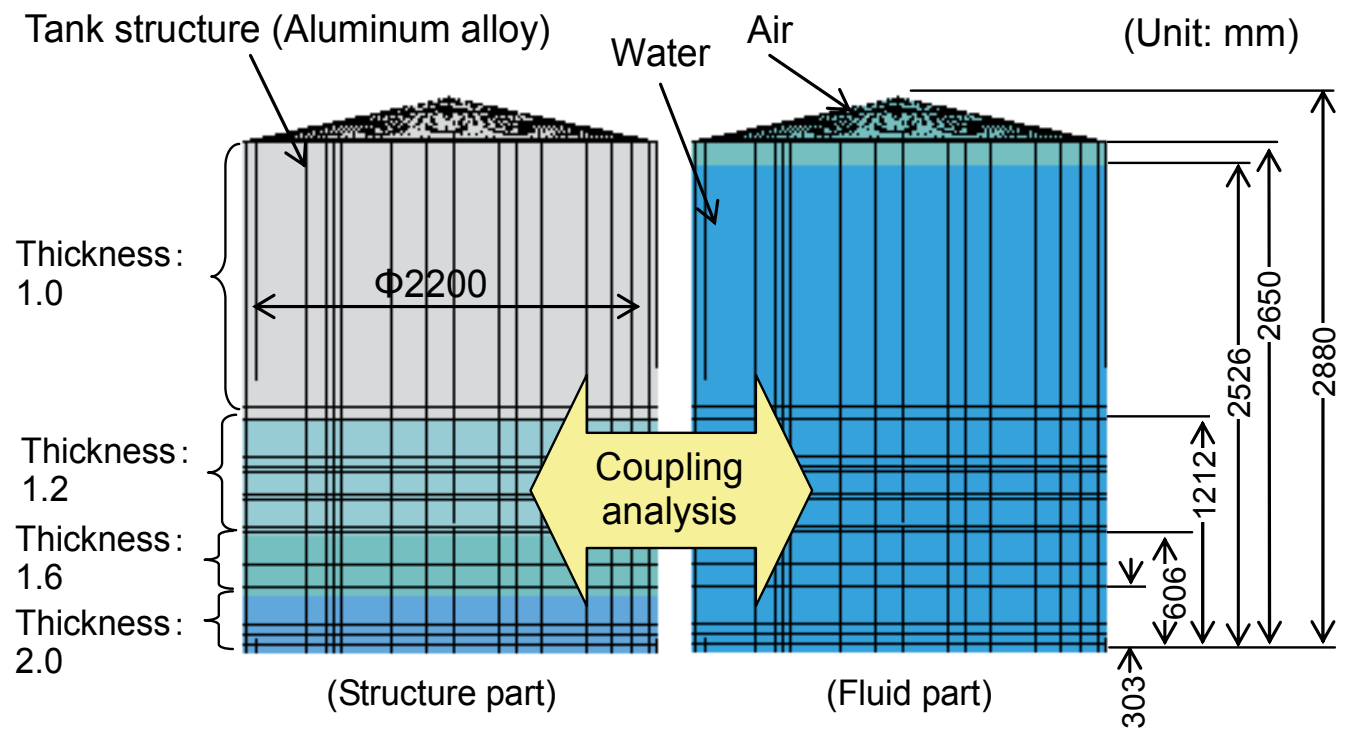

Fig. 20. Analysis model of large-scale tank. 


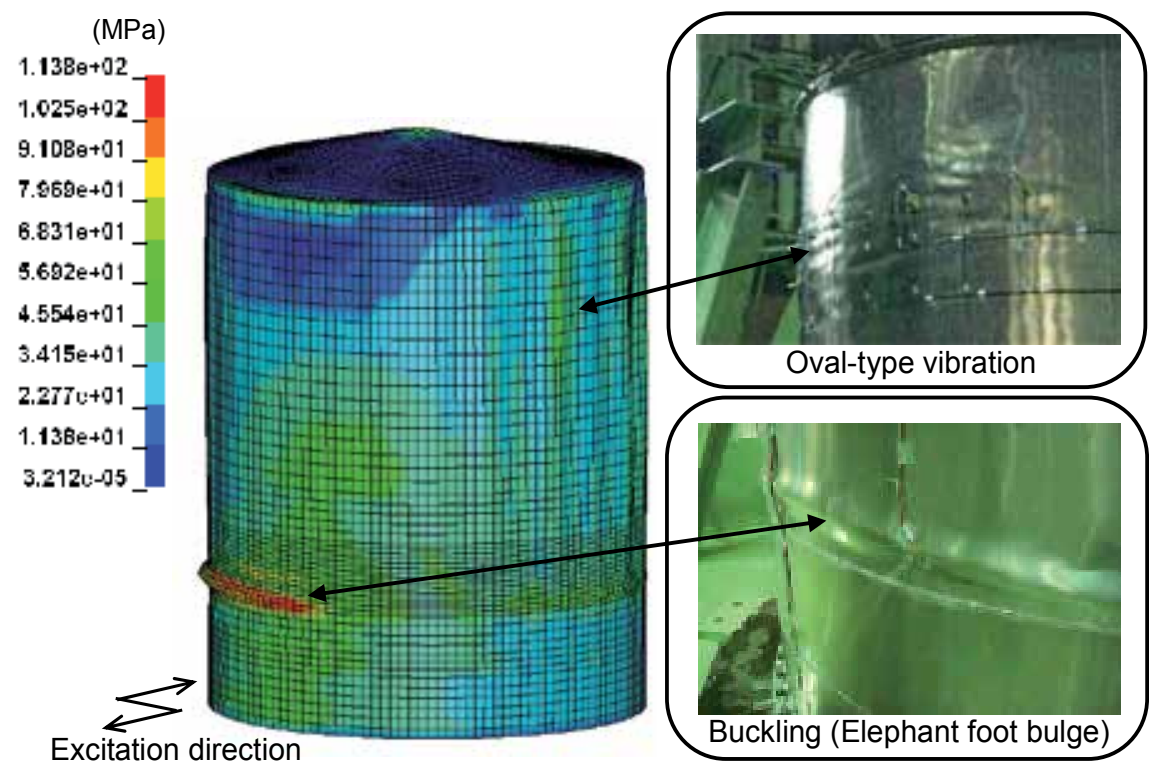

Fig. 21. Simulation of oval-type vibration and elephant foot bulge. 
(1)

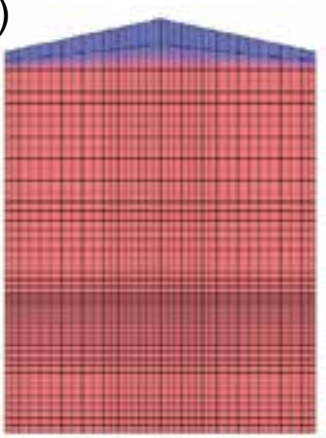

(4)

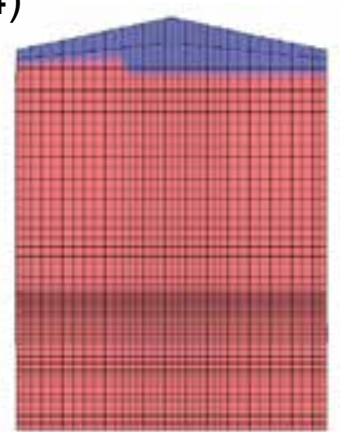

(2)

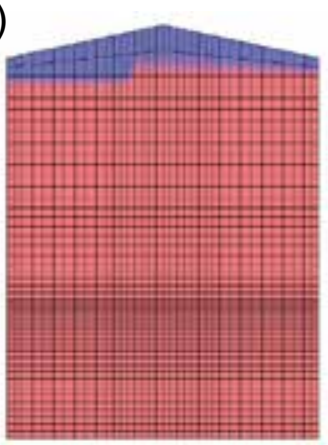

(5)

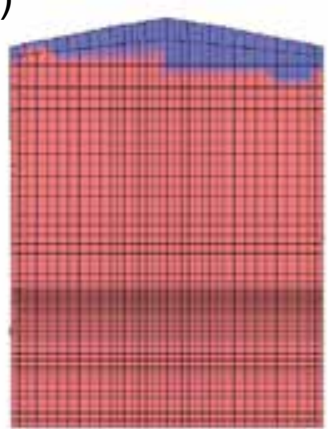

(3)

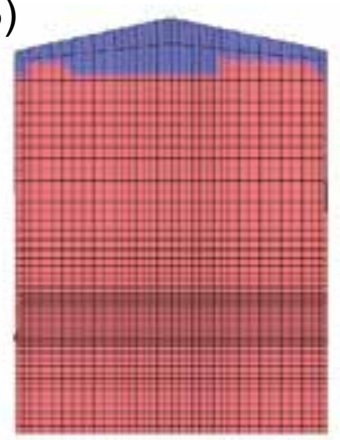

(6)

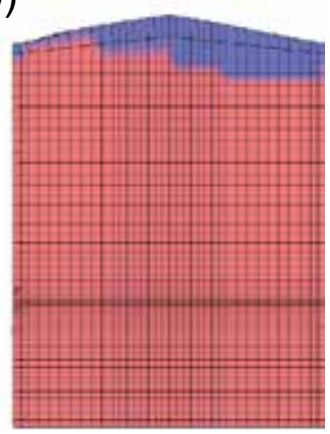

Fig. 22. Simulation of sloshing before (1) and during ((2) to (6)) excitation. The contained liquid is shown in red and the air in blue.

Comparison of analysis and experiment results for the $1 / 10$ and $1 / 5$ reduced-scale models show that the proposed method can simulate seismic response such as vibration response and sloshing and buckling behavior such as buckling mode and buckling load accurately. Consequently, the proposed dynamic analysis method is versatile and can be used to analyze many types of tanks. Additionally it is concluded that the method can adequately evaluate the seismic resistance of tanks such as their seismic safety margin (Maekawa et al., 2011).

\section{Conclusions}

The past and present research studies on seismic response analysis for cylindrical liquid storage tanks were reviewed. In addition, a new dynamic analysis method of seismic response for cylindrical liquid storage tanks which was developed by Maekawa and Fujita was introduced. The dynamic analysis method to evaluate seismic response (vibration and sloshing) and buckling was proposed and validated by the experimental results. The conclusions obtained are summarized as follows.

1. A new dynamic analysis method was proposed for evaluating seismic response and buckling behavior of cylindrical liquid storage tanks. In the method, the tank structure was three-dimensionally modeled by nonlinear shell elements which allowed geometric nonlinearity to be considered, the contained liquid was modeled by solid elements that calculated fluid behavior according to Euler's equation, and the fluid-structure 
interaction between contained liquid and tank structure was evaluated by the arbitrary Lagrangian-Eulerian method.

2. In the proposed method, the explicit method was applied to the seismic response analysis, which is a long time analysis, though the method had been used for only extremely short time analysis such as impact analysis until now. The time history analysis results using the explicit method could reproduce the vibration and buckling behavior accurately.

3. A dynamic buckling experiment was conducted by using the $1 / 10$ reduced-scale model of an actual tank. The experiment values were compared with the analysis results by the proposed method. The analysis and experiment results were in good agreement, especially with vibration response, buckling mode and buckling load. The results demonstrated that the proposed method could accurately simulate the seismic response of the tank.

4. The experiment and analysis values were compared for a 1/5 reduced-scale model. The proposed method was judged to be versatile and applicable to many types of tanks.

5. It was concluded that the proposed method could adequately evaluate the seismic resistance of tanks such as their seismic safety margin.

\section{Acknowledgment}

Some figures and photos were provided by Prof. Fujita of Osaka City University and Prof. Ito of Osaka Prefecture University, Japan. The experiment done by Prof. Ito and co-workers was conducted in a research project of the Kansai Electric Power Co., Kyushu Electric Power Co., Shikoku Electric Power Co., Hokkaido Electric Power Co., Japan Atomic Power Co. and Mitsubishi Heavy Industries, Ltd. The seismic wave used in the 1/5 reduced-scale tank was provided by staff members related to the project. The author sincerely thanks everyone for their cooperation.

\section{References}

Abramson, H.N. (1966). The Dynamic Behavior of Liquid in Moving Containers, NASA. SP-106, NASA, Washington, D.C., USA.

Akiyama, H.; Takahashi, M. \& Nomura, S. (1989). Buckling Tests of Steel Cylindrical Shells Subjected to Combined Bending, Shear and Internal Pressure, Journal of Structural and Construction Engineering, No. 400, pp. 113-122 (in Japanese).

Akiyama, H. (1997). Seismic Resistance of Fast Breeder Reactor Components Influenced by Buckling, Kajima Institute Publishing, Tokyo, Japan.

Amabili, M. (2003). Theory and Experiments for Large-Amplitude Vibrations of Empty and Fluid-Filled Circular Cylindrical Shells with Imperfections, Journal of Sound and Vibration Vol. 262, pp. 921-975.

Amabili, M. \& Païdoussis, M.P. (2003). Review of Studies on Geometrically Nonlinear Vibrations and Dynamics of Circular Cylindrical Shells and Panels, with and without Fluid-Structure Interaction, Applied Mechanics Review, Vol. 56, No. 4, pp. 349-381.

American Nuclear Society (ANS). (2007). American National Standard External Events in PRA Methodology, ANSI/ ANS-58.21-2007, ANS, La Grange Park, Illinois, USA. 
Architectural Institute of Japan (AIJ). (2010). Design Recommendation for Storage Tanks and Their Supports (Ver. 4), AIJ, Tokyo, Japan (in Japanese).

Atomic Energy Society of Japan (AESJ). (2007). Standard for Procedure of Seismic Probabilistic Safety Assessment for Nuclear Power Plants, AESJ-SC-P006:2007, AESJ, Tokyo, Japan (in Japanese).

Belytschko, T.; Lin, J. I. \& Tsay, C. S. (1984). Explicit Algorithms for the Nonlinear Dynamics of Shells, Computer Methods in Applied Mechanics and Engineering, No. 42, pp. 225251.

Chiba, M.; Yamaki, N. \& Tani, J. (1984). Free Vibration of a Clamped-Free Circular Cylindrical Shell Partially Filled with Liquid; Part I: Theoretical Analysis, ThinWalled Structures, Vol. 2, pp. 265-284.

Chiba, M.; Tani, J.; Hashimoto, H. \& Sudo, S. (1986). Dynamic Stability of Liquid-Filled Cylindrical Shells under Horizontal Excitation, Part I: Experiment, Journal of Sound and Vibration, Vol. 104, No. 2, pp. 301-319.

Chiba, M., (1993). Experimental Studies on a Nonlinear Hydroelastic Vibration of a Clamped Cylindrical Tank Partially Filled with Liquid, Journal of Pressure Vessel Technology, Vol. 115, pp. 381-388.

Clough, R. W.; Niwa, A. \& Clough, D. P. (1979). Experimental Seismic Study of Cylindrical Tanks, Journal of the Structural Division, Vol. 105, No. 12, pp. 2565-2590.

Fischer, D.F. \& Rammerstorfer, F.G. (1982). The Stability of Liquid-filled Cylindrical Shells under Dynamic Loading, In: Buckling of Shells, Ramm, E. (ed.), pp. 569-597, Springer-Verlag, Berlin.

Fujii, S.; Shibata, H.; Iguchi, M.; Sato, H. \& Akino, K. (1969). Observation of Damage of Industrial Firms in Niigata Earthquake, Proceedings of 4th World Conference on Earthquake Engineering, Vol. III, J2-1, pp. 1-19, Santiago, Chile.

Fujita, K. (1981). A Seismic Response Analysis of a Cylindrical Liquid Storage Tank, Bulletin of the JSME, Vol. 24, No. 192, pp. 1029-1036.

Fujita, K.; Ito, T.; Matsuo, T.; Shimomura, T. \& Morishita, M. (1984). Aseismic Study on the Reactor Vessel of a Fast Breeder Reactor, Nuclear Engineering and Design, Vol. 83, pp. 47-61.

Fujita, K.; Ito, T. \& Wada, H. (1990). Experimental Study on the Dynamic Buckling of Cylindrical Shell Due to Seismic Excitation, Proceedings of ASME Pressure Vessels and Piping Conference, Vol. 191, pp. 31-36, Nashville, Tennessee, USA.

Fujita, K.; Ito, T.; Baba, K.; Ochi, M. \& Nagata, K. (1992). Dynamic Buckling Analysis for Cylindrical Shell Due to Random Excitation, Proceedings of 10th World Conference on Earthquake Engineering, pp. 4981-4987, Madrid, Spain.

Fujita, K. \& Saito, A. (2003). Free Vibration and Seismic Response Analysis of a Liquid Storage Thin Cylindrical Shell with Unaxisymmetric Attached Mass and Stiffness, Proceedings of ASME Pressure Vessels and Piping Conference, Vol. 466, pp. 243-252.

High Pressure Gas Safety Institute of Japan (KHK). (2003). Seismic Design Standard for the High Pressure Gas Facilities, KHK E 012, KHK, Tokyo, Japan (in Japanese).

Hirt, C.W.; Amsden, A.A. \& Cook, J.L. (1974). An Arbitrary Lagrangian-Eulerian Computing Method for All Flow Speeds, Journal of Computational Physics, Vol. 4, pp. 227-253.

Housner, G.W. (1957). Dynamic Pressures on Accelerated Fluid Containers, Bulletin of the Seismological Society of America, Vol. 47, No. 1, pp. 15-35. 
Ibrahim, R.A.; Pilipchuk, V.N. \& Ikeda, T. (2001). Recent Advances in Liquid Sloshing Dynamics, Applied Mechanics Review, Vol. 54, No. 2, pp. 133-199.

Ito, T.; Morita, H.; Hamada, K.; Sugiyama, A.; Kawamoto, Y.; Ogo, H. \& Shirai, E. (2003). Investigation on Buckling Behavior of Cylindrical Liquid Storage Tanks under Seismic Excitation (1st Report; Investigation on Elephant Foot Bulge), Proceedings of ASME Pressure Vessels and Piping Conference, Vol. 466, pp. 193-201, Cleveland, Ohio, USA.

Jacobsen, L.S. (1949). Impulsive Hydrodynamics of Fluid inside a Cylindrical Tank and Fluid Surrounding a Cylindrical Pier, Bulletin of Seismological Society of America, pp. 189-204.

Japan Electric Association (JEA). (2008). Technical Codes for Aseismic Design of Nuclear Power Plants, JEAC4601-2008, JEA, Tokyo, Japan (in Japanese).

Japan Society of Civil Engineers (JSCE). (1989). Dynamic Analysis and Seismic Design -Energy Facilities-, pp. 190-191, Gihodo Shuppan Co. Ltd., Tokyo, Japan (in Japanese).

Japan Society of Mechanical Engineers (JSME). (2003). Handbook of Vibration and Buckling of Shells, pp. 243-293, Gihodo Shuppan Co. Ltd., Tokyo, Japan (in Japanese).

Kana, D.D. (1979). Seismic Response of Flexible Cylindrical Liquid Storage Tanks, Nuclear Engineering and Design, Vol. 52, pp. 185-199.

Kanagawa Prefecture. (2002). Seismic Design Standard for the High Pressure Gas Plants, Kanagawa Prefecture, Yokohama, Japan (in Japanese).

Katayama, H.; Kashima, Y.; Fujimoto, S; Kawamura, Y. \& Nakamura, H. (1991). Dynamic Buckling Test Using Scale Model for LMFBR Structure, Proceedings of 11th International Conference on Structural Mechanics in Reactor Technology (SMiRT 11), K15/5, pp. 415-420, Tokyo, Japan.

Livermore Software Technology Corporation. (2003). LS-DYNA Ver.970 Keyword User's Manual, Livermore Software Technology Corporation, Livermore, California, USA.

Ma, D.C.; Gvildys, J.; Chang, Y.W. \& Liu, W.K. (1982). Seismic Behavior of Liquid-Filled Shells, Nuclear Engineering and Design, Vol. 70, pp. 437-455.

Maekawa, A.; Suzuki, M. \& Fujita, K. (2006). Nonlinear Vibration Response of a Cylindrical Water Storage Tank Caused by Coupling Effect between Beam-Type Vibration and Oval-Type Vibration: Part 1 Vibration Experiment, Proceedings of ASME Pressure Vessels and Piping Conference, PVP2006-ICPVT-11-93261, Vancouver, Canada.

Maekawa, A. \& Fujita, K. (2007). Occurrence of Nonlinear Oval-Type Vibration under Large Sinusoidal Excitation: Experiment, Proceedings of ASME Pressure Vessels and Piping Conference, PVP2007-26461, San Antonio, Texas, USA.

Maekawa, A.; Fujita, K. \& Sasaki, T. (2007). Dynamic Buckling Test of Modified 1/10 Scale Model of Cylindrical Water Storage Tank, Proceedings of ASME Pressure Vessels and Piping Conference, PVP2007-26466, San Antonio, Texas, USA.

Maekawa, A. \& Fujita, K. (2008). Explicit Nonlinear Dynamic Analysis of Cylindrical Water Storage Tanks Concerning Coupled Vibration between Fluid and Structure, Proceedings of ASME Pressure Vessels and Piping Conference, PVP2008-61112, Chicago, Illinois, USA.

Maekawa, A. \& Fujita, K. (2009). Dynamic Buckling Analysis of Cylindrical Water Storage Tanks: A New Simulation Method Considering Coupled Vibration between Fluid and Structure, Proceedings of ASME Pressure Vessels and Piping Conference, PVP200977083, Prague, Czech Republic. 
Maekawa, A. \& Fujita, K. (2010). Dynamic Buckling Simulation of Cylindrical Liquid Storage Tanks Subjected to Seismic Motions, Proceedings of ASME Pressure Vessels and Piping Conference, PVP2010-25412, Bellevue, Washington, USA.

Maekawa, A.; Shimizu, Y.; Suzuki, M. \& Fujita, K. (2010). Vibration Test of a 1/10 Reduced Scale Model of Cylindrical Water Storage Tank, Journal of Pressure Vessel Technology, Vol. 132, No. 5, pp. 051801-1-051801-13.

Maekawa, A.; Takahashi, T. \& Fujita, K. (2011). Seismic Safety Margin of Cylindrical Liquid Storage Tanks in Nuclear Power Plants, Proceedings of ASME Pressure Vessels and Piping Conference, PVP2011-57241, Baltimore, Maryland, USA.

National Aeronautics and Space Administration (NASA). (1968). Buckling of Thin-Walled Circular Cylinders, NASA Space Vehicle Design Criteria, NASA SP-8007, NASA, Washington, D.C., USA.

Niwa, A. \& Clough, R.W. (1982). Buckling of Cylindrical Liquid-Storage Tanks under Earthquake Loading, Earthquake Engineering and Structural Dynamics, Vol. 10, pp. 107-122.

Nuclear Safety Commission of Japan (NSC). (2006). Regulatory Guide for Reviewing Seismic Design of Nuclear Facilities, NSC, Tokyo, Japan (in Japanese).

Rahnama, M. \& Morrow, G. (2000). Performance of Industrial Facilities in the August 17, 1999 Izmit Earthquake, Proceedings of 12th World Conference on Earthquake Engineering, 2851, Auckland, New Zealand.

Rammerstofer, F.G.; Scharf, K. \& Fischer, F.D. (1990). Storage Tanks under Earthquake Loading, Applied Mechanics Review, Vol. 43, No. 11, pp. 261-282.

Shimizu, N. (1990). Advances and Trends in Seismic Design of Cylindrical Liquid Storage Tanks, JSME International Journal, Series III, Vol. 33, No. 2, pp. 111-124.

Shin, C.F. \& Babcock, C.D. (1980). Scale Model Buckling Tests of a Fluid Filled Tank under Harmonic Excitation, Proceedings of ASME Pressure Vessels and Piping Conference, 80C2/PVP-66, pp. 1-7.

Suzuki, K. (2008). Earthquake Damage to Industrial Facilities and Development of Seismic and Vibration Control Technology, Journal of System Design and Dynamics, Vol. 2, No. 1, pp. 2-11.

Tazuke, H.; Yamaguchi, S.; Ishida, K.; Sakurai, T.; Akiyama, H. \& Chiba, T. (2002). Seismic Proving Test of Equipment and Structures in Thermal Conventional Power Plant, Journal of Pressure Vessel Technology, Vol. 124, pp. 133-143.

Timoshenko, S.P. \& Woinowsky-Krieger, S. (1959). Theory and Plate and Shells (2nd Edition), McGraw-Hill, New York, New York, USA.

Timoshenko, S.; Gere, J.M. \& Young, D. (1964). Theory of Elastic Stability (2nd Edition), McGraw-Hill, , New York, New York, USA.

Toyoda, Y. \& Masuko, Y. (1996). Dynamic Buckling Tests of Liquid-Filled Thin Cylindrical Tanks, Journal of Structural Engineering, Vol. 42A, pp. 179-188 (in Japanese).

Toyoda, Y.; Matsuura, S. \& Masuko, Y. (1997). Development of Computer Program for Dynamic Buckling Analysis of Liquid-Filled Thin Cylindrical Shell and Its Validation by Experiments, Journal of Structural Engineering, Vol. 43B, pp. 31-40 (in Japanese).

Veletsos, A.S. \& Yang, J.Y. (1976) Dynamic of Fixed-Based Liquid-Storage Tanks, Proceedings of U.S.-Japan Seminar for Earthquake Engineering Research with Emphasis on Lifeline Systems, pp. 317-341. 
Werner, P.W. \& Sandquist, K.J. (1949). On Hydrodynamic Earthquake Effects, Transactions of American Geophysical Union, Vol. 30, No. 5, pp. 636-657.

Yamaki, N. (1984). Elastic Stability of Circular Cylindrical Shells, North-Holland, Amsterdam, Netherlands. 


\title{
Seismic Reliability Analysis of Cable Stayed Bridges Against First Passage Failure
}

\author{
Rehan Ahmad Khan \\ Aligarh Muslim University \\ India
}

\section{Introduction}

In many structural applications, the ultimate purpose of using stochastic analysis is to determine the reliability of a structure, which has been designed to withstand the random excitations. The present study is concerned with one of the failures, which are the results of the dynamic response of the stable cable stayed bridges. If $\mathrm{Y}(\mathrm{t})$ is the dynamic response (either deflection, strain or stress) of the bridge at a critical point, the bridge may fail upon the occurrence of one of the following cases (Y.K. Lin, 1967):

i. $Y(t)$ reaches, for the first time, either an upper level A or lower level -B, where A and B are large positive numbers (Fig. 1(a)).

ii. Damage to the structure accumulated as $\mathrm{Y}(\mathrm{t})$ fluctuates at small or moderate excursion which are not large enough to cause a failure of the first type, and failure occurs when the accumulated damage reaches a fixed total.

iii. Failure may occur if a response spends too much of its time off range, i.e., it is. outside the set limits for more than a minimum fraction of its lifetime (Fig. 1(b))

The first case, which is called first passage failure (also called first excursion failure), is the objective of this chapter. According to definition, the first passage failure analysis means determining the probability that a prescribed threshold level (displacement, stress or other response level) will be exceeded, for the first time, during a fixed period of time. The first passage failure does not lead to the catastrophic failure of the bridge, but in view of serviceability consideration it is important. Therefore, the purpose of designing the structures against first passage failure is to reduce the probability of such failure, over the expected lifetime of the structure, to an acceptable level. In most random vibration problems, there is a probability close to unity that any given high response threshold level will be exceeded if the structure is excited for a long enough period of time.

Seismic response of cable stayed bridge due to the random ground motion is obtained in this chapter using frequency domain spectral analysis. The ground motion is assumed to be a partially correlated stationary random process. In the response analysis, the quasi-static response of the bridge deck produced due to the support motion is dully considered. The probability of first passage failure for the possibility of future earthquake is then presented for the analysis of various threshold levels (taken as fraction of yield stress) is considered. A parametric study is performed to show the effect of some important parameters such as threshold level, soil conditions, degree of correlation, angle of incidence of earthquake etc. on the reliability of cable stayed bridge against the first passage failure. 


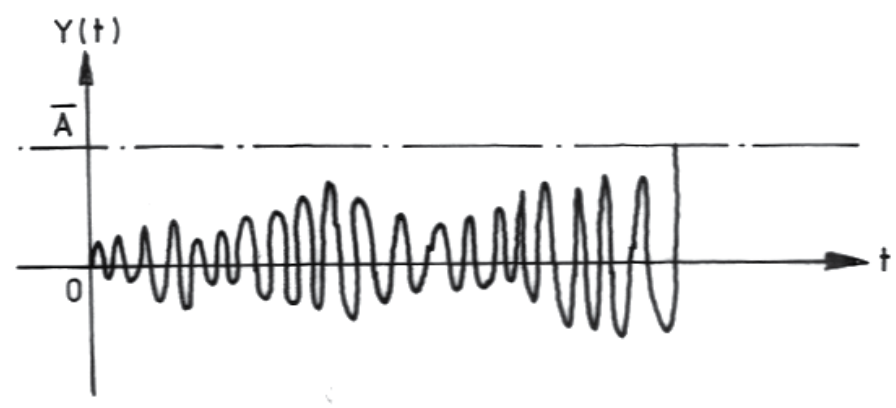

(a) Failure occurs when $\mathrm{Y}(\mathrm{t})$ first reaches the level $\mathrm{Y}(\mathrm{t})=\bar{A}$.

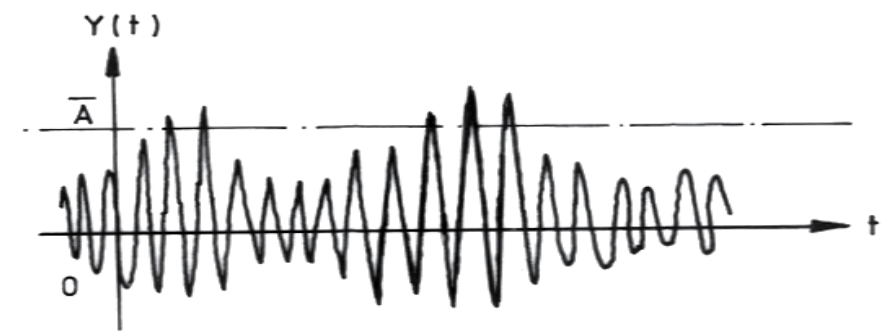

(b) Failure occurs if $Y(t)>\bar{A}$ for more than an acceptable fraction of the total elapse time.

Fig. 1. Possible modes of failure under random excitation.

\subsection{Brief review of earlier works}

A brief review of the earlier works on different aspects of reliability analysis of structures especially cable supported bridges subjected to seismic forces / dynamic excitations is presented below in order to highlight the need for the present work.

\subsubsection{Seismic response of cable supported bridges}

The vibration of cable stayed bridges under the earthquake excitation has been a topic of considerable research for many years. The dynamic behaviour of cable stayed bridges, subjected to earthquake ground motion, has been studied by several researchers.

Morris (1974) utilized the lumped mass approach for the linear and non-linear dynamic responses of two dimensional cable stayed bridges due to sinusoidal load applied at a node. It was concluded that four mode solutions were sufficient for both linear and nonlinear solutions. However, a time increment of $10 \%$ of the period corresponding to the highest mode gave satisfactory numerical results for all the dynamic analyses carried out.

Abdel Ghaffer and Nazmy (1987) investigated the effects of three dimensionality, multisupport excitation, travelling waves and non-linearity on the seismic behaviuor of cable stayed bridges. Their studies indicated that non-linearities become more important as the span of the bridge increases, and the effect of multiple support seismic excitation is more pronounced for a higher structural redundancy.

Nazmy and Abdel Ghaffar (1991), (1992) studied the effects of non-dispersive travelling seismic wave and ground motion spatial variability on the response of cable stayed bridges considering the cases of synchronous and non-synchronous support motions due to seismic excitations. The responses were obtained by time history analysis. They concluded that 
depending on the dynamic properties of the local soils at the supports, as well as the soils at the surrounding bridge site, the travelling seismic wave effect should be considered in the seismic analysis of these bridges. Also, there is multi-modal contribution from several modes of vibration to the total response of the bridge, for both displacement and member forces.

Hong Hao (1998) analyzed the effects of various bridge and ground motion parameters on the required seating lengths for bridge deck to prevent the pull-off and -drop collapse using random vibration method. He analyzed two span bridge model with different span lengths and vibration frequencies and subjected to various spatially varying ground excitations. Ground motions with different intensities, different cross correlations and different site conditions were considered in the analysis.

Soyluk and Dumanoglu (2000) carried out asynchronous and stochastic dynamic analysis of a cable stayed bridge (Jindo Bridge) using finite element method. In the asynchronous dynamic analysis, various wave velocities were used for the traveling ground motion. They found that response in the deck obtained from asynchronous dynamic analysis are much higher than the response obtained by the stochastic analysis. Further, shear wave velocity of ground motion greatly influences the response of the Jindo cable stayed bridge.

\subsubsection{Reliability analsysis of structures}

Lin (1967), Bolotin (1965) and Crandall, Mark (1963), Abbas and Manohar (2005a, b; 2007) discussed various models of structural failure under random dynamic loading and classified them into the following types: (i) failure due to the first excursion of the response beyond a safe level; (ii) failure due to the response remaining above a safe level for too long a duration and (iii) failure due to the accumulation of damage.

All these types of failure are associated with the reliability estimate of Structures subjected to dynamic loading like earthquake. A number of investigations are reported in the literature on the reliability analysis of Structures against the aforementioned types of failure. Relatively recent ones and a few important old ones are reviewed here.

Konishi (1969) studied the safety and reliability of suspension bridges under wind and earthquake actions. He treated the suspension safety of the structure under random ground motion from the standpoint of threshold crossing of a specified barrier.

Vanmarcke (1975) dealt with the problem of the probability of first-passage beyond a threshold value by a time dependent random process. Barrier was classified into three distinct categories: a single barrier, a double barrier, and a barrier defined for the envelope of the random process. The assumption that barrier crossings are independent, so that they constitute a Poisson process, is nearly true for high barrier levels. But for relatively low barrier levels, the Poisson process assumption is in error, as it does not account for the clustering effect in case of a narrow-band process while for a wide-band process, the actual time spent in the unsafe domain is not considered. Vanmarcke suggested improvements in the Poisson process assumption to allow for the above effects.

Chern (1976) dealt with the reliability of a bilinear hysteretic system, subjected to a random earthquake motion, considering first excursion beyond a specified barrier and low-cycle fatigue. Solomon and Spanos (1982) studied the structural reliability under a non-stationary seismic excitation, based on the first excursion beyond a specified barrier by the absolute value and envelope of the response process.

Schueller and stir (1987) reviewed various methods to calculate the failure probabilities of structural component or system in the light of their accuracy and computational efficiency. 
They analyzed that for problems of higher dimensions, approximation techniques utilizing linearization like FOSM introduced considerable error. In view of these difficulties, they provided an alternate method to calculate the failure probabilities, which combines the advantages of both the importance sampling technique and the design point calculation.

Mebarki et al. (1990) presented a new method for reliability assessment, called Hypercone method based on the principles of level-2 methods of reliability analysis. The main aim of the method was to evaluate reliability index 'beta' and to deduce the values of the probability of failure by considering the whole geometry of the failure domain. The restrictions and the practical application of the method were discussed. The results reported showed that the value of probability of failure deduced from the Hasofer-Lind index beta, assuming linearity of the limit state surface, and from Monte Carlo Simulations are in accordance with those deduce from the Hypercone method.

Zhu (1993) reviewed several methods for computing structural system reliability. A discretization or cell technique for determining the failure probabilities of structural system is proposed. The gaussian numerical integration method is introduced to improve its computational accuracy and can be applied to gaussian or non-gaussian variables with linear or non-linear safety margin. Harichandran et al. (1996) studied the stationary and transient response analyses of the Golden Gate suspension bridge, and the New River Gorge and Cold Spring Canyon deck arch bridges subjected to spatially varying earthquake ground motion (SVEGM). They found that transient lateral displacements of the suspension bridge center span significantly overshoot the corresponding stationary displacements for the filtered Kanai-Tajami excitation power spectrum; this spectrum may therefore be unsuitable for analyzing very flexible structures.

\section{Assumptions}

The following assumptions are made for the First Passage Failure analysis:

i. It is assumed that the response process is stationary Gaussian with zero mean value.

ii. As in spectral analysis method, the evaluated response is always positive, therefore the single barrier level (called type B barrier, according to Crandall et al.1966) is used.

iii. It is assumed that the threshold level crossing occur independently according to a Poisson process.

iv. The structure is assumed to be linear and lightly damped.

v. The bridge deck (girder) and the towers are assumed to be axially rigid.

vi. The bridge deck, assumed as continues beam, does not transmit any moment to the towers through the girder-tower connection.

vii. Cables are assumed to be straight under high initial tensions due to the dead load and well suited to support negative force increment during vibration without losing its straight configuration.

viii. An appropriate portion of the cable is included (in addition to deck mass) in the dynamic analysis of the bridge deck and is assumed to be uniformly distributed over the idealized deck.

ix. Beam-column effect, in the stiffness formulation of the beam is considered for the constant axial force in the beam and its fluctuating tension in the cable is ignored. Further, cable dynamics is ignored for the bridge deck vibration, i.e., the tension fluctuations in the cables are assumed to be quasi-static, and do not introduce any nonlinearity in the system. 


\section{Seismic excitation}

The seismic excitation is considered as a three component stationary random process. The earthquake ground motion is assumed as stationary random although in many cases it is assumed as a uniformly modulated non-stationary process. The response analysis remains the same for both cases. The response derived by assuming the process to be stationary can be multiplied by an envelope function to take care of the non-stationary. The components of the ground motion along an arbitrary set of orthogonal directions will be usually statistically correlated. However, as observed by Penzien and Watable (1975), the three components of ground motion along a set of principal axes are uncorrelated. These components, directed along the principal axes, are usually such that the major principal axis $(u)$ is directed towards the expected epicenter, the moderate principal axis $(w)$ is directed perpendicular to it (horizontally) and the minor principal axis (v) is directed vertically as shown in Fig.2. Nigam and Naranayan (1995) highlight the critical orientation of the alpha angle between the two sets of axes. Der Kiureghian (1996) developed the model for the coherency function describing spatial variability of earthquake ground motions. The model consists of three components characterizing three distinct effects of spatial variability, namely, the incoherence effect that arises from scattering of waves in the heterogeneous medium of the ground and their differential super positioning when arriving from an extended source, the wave-passage effect that arises from difference in the arrival times of waves at different stations, and the site-response effect that arises from difference in the local soil conditions at different stations. Abbas and Manohar (2002) developed a critical earthquake excitation models with emphasis on spatial variability characteristics of ground motion. In this study, the three components of the ground motion are assumed to be directed along the principal axes of the bridge $x, y, z$ or shifted with an angle a. Each component is assumed to be a stationary random and partially correlated process with zero mean characterized by a psdf. The psdf of the ground acceleration is defined by Clough and Penzien (1975) as

$$
S_{\ddot{f}_{g} \ddot{f}_{g}}=\left|H_{1}(i \omega)\right|^{2}\left|H_{2}(i \omega)\right|^{2} S_{o}
$$

in which $S_{o}$ is the spectrum of the white noise bedrock acceleration; $\left|H_{1}(i \omega)\right|^{2}$ and $\left|H_{2}(i \omega)\right|^{2}$ are the transfer functions of the first and the second filters representing the dynamic characteristic of the soil layers above the bedrock, where

$$
\begin{aligned}
\left|H_{1}(i \omega)\right|^{2} & =\frac{1+\left(2 \xi_{g} \omega / \omega_{g}\right)^{2}}{\left[1-\left(\omega / \omega_{g}\right)^{2}\right]^{2}+\left(2 \xi_{g} \omega / \omega_{g}\right)^{2}} \\
\left|H_{2}(i \omega)\right|^{2} & =\frac{\left(\omega / \omega_{f}\right)^{4}}{\left[1-\left(\omega / \omega_{g}\right)^{2}\right]^{2}+\left(2 \xi_{g} \omega / \omega_{g}\right)^{2}}
\end{aligned}
$$

in which $\omega_{g}, \xi_{g}$ are the resonant frequency and damping ratio of the first filter, and $\omega_{f}, \xi_{f}$ are those of the second filter. The clough and Penzien is a double filter for spectral density of 
the ground acceleration for which the corresponding displacement psdf does not become unrealistic. In case of Kanai-Tajimi spectrum, although the psdf of acceleration is simpler but it has problem that the corresponding displacement psdf become undefined at zero frequency.

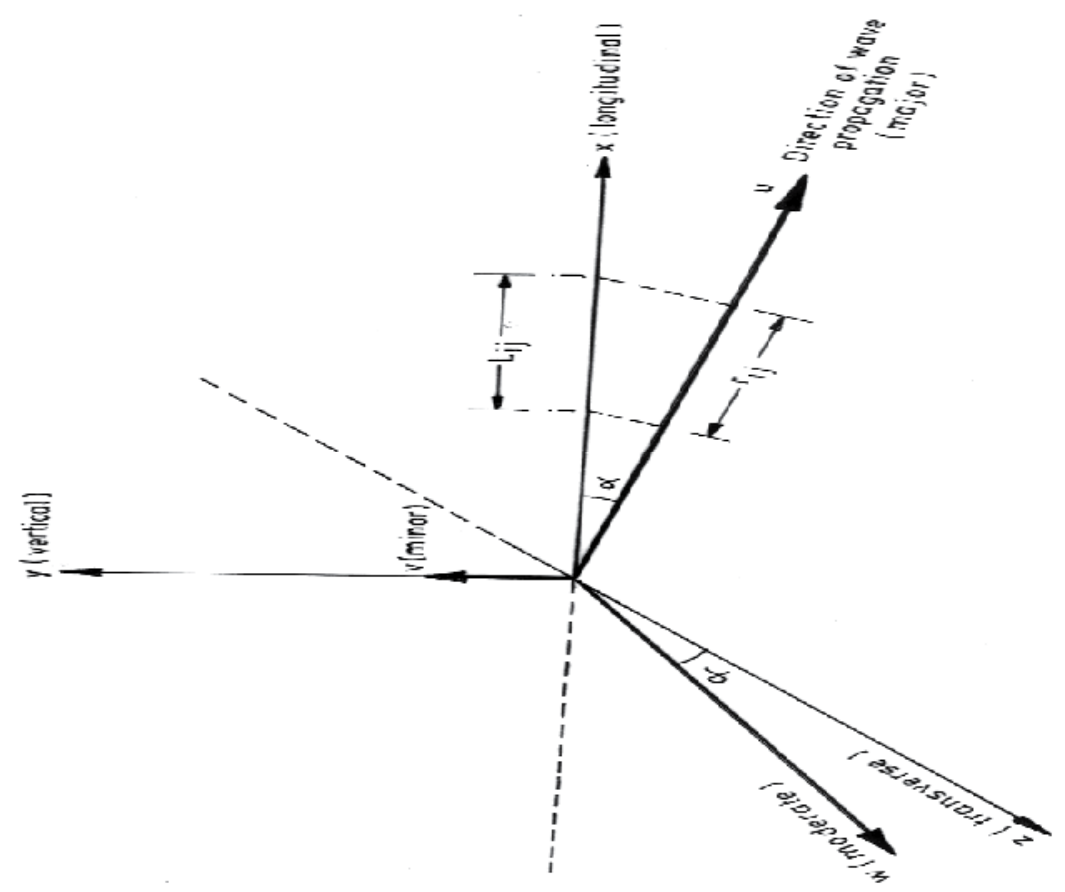

Fig. 2. Principal directions of the bridge $(x, y, z)$ and the ground motion $(u, v, w)$

The cross spectrum between the random ground motions $\ddot{f}_{g_{i}}$ and $\ddot{f}_{g_{j}}$ at two stations $\mathrm{i}$ and $\mathrm{j}$ is described by that given by Hindy and Novak (1980) as

$$
S_{\ddot{f}_{g i} \ddot{f}_{g h}}\left(r_{i j}, \omega\right)=S_{\ddot{f}_{g} \ddot{f}_{g}}(\omega) \rho_{i j}(\omega)
$$

in which $S_{\ddot{f}_{f}} \ddot{f}_{\mathrm{f}}(\omega)$ local spectrum of ground acceleration as given in Eqn.(1) which is assumed to be the same for all supports and $\rho_{i j}(\omega)$ is the cross correlation function (coherence function) of the ground motion between two excitation points $i$, $j$ and is represented by

$$
\rho_{i j}(\omega)=\exp \left[-c\left(\frac{r_{i j} \omega}{2 \pi V_{s}}\right)\right]
$$

in which $r_{i j}$ is the separation distance between stations $\mathrm{i}$ and $\mathrm{j}$ measured in the direction of wave propagation; $c$ is a constant depending upon the distance from the epicenter and the inhomogeneity of the medium; $V_{s}$ is the shear wave velocity of the soil; and $\omega$ is the frequency ( $\mathrm{rad} / \mathrm{sec})$ of the ground motion. 
For one sided spectrum it is well known that

$$
\sigma_{\ddot{f}_{g}}{ }^{2}=S_{o}\left[\int_{0}^{\alpha}\left|H_{1}(i \omega)\right|^{2}\left|H_{2}(i \omega)\right|^{2} d \omega\right]
$$

$\sigma_{\ddot{f}_{g}}{ }^{2}$ is the variance of ground acceleration. The Modified Mercalli intensity of an earthquake Is is a measures of the strength of the shaking at a particular location and will vary with distance, substrate conditions and other factors. The empirical relation between the standard deviation of peak ground acceleration and earthquake intensity Is is given as

$$
\sigma_{\ddot{f}_{g}}^{2}=10^{\left(I_{s} / 3-0.5\right)} / K^{*}
$$

where $\mathrm{K}^{*}$ is a peak factor given as

$$
K^{*}=K^{\prime}+\frac{0.5772}{K^{\prime}} ; K^{\prime}=\sqrt{2 \ln \left(N_{0} T\right)}
$$

The empirical relation between the magnitude of earthquake and intensity of earthquake is given as

$$
\mathrm{M}=1.3+0.6 \mathrm{I}
$$

In Eqn.(9), $\mathrm{N}_{0}$ is the mean rate of zero crossing and is given by

$$
N_{0}=\frac{1}{2 \pi} \sqrt{\int_{0}^{\alpha} \omega^{2} S_{\ddot{f}_{g}}(\omega) d \omega / \int_{0}^{\alpha} S_{\ddot{f}_{g}}(\omega) d \omega}
$$

By defining the filter characteristics $\omega_{\mathrm{g}}, \xi_{\mathrm{g}}, \omega_{\mathrm{f}}, \xi_{\mathrm{f}}$ and specifying a standard deviation of the ground acceleration $\sigma_{\ddot{f}_{\mathrm{g}}}$, the psdf of the ground acceleration can be completely defined. The psdfs $S_{f_{g} f_{g}}(\omega)$ and $S_{\dot{f}_{g} \dot{f}_{g}}(\omega)$ of the ground displacement and velocity respectively are related to $S_{\ddot{f}_{g} \ddot{f}_{g}}(\omega)$ by

$$
\begin{aligned}
& S_{f_{g} f_{g}}(\omega)=S_{\ddot{f}_{g} \ddot{f}_{g}}(\omega) / \omega^{4} \\
& S_{\dot{f}_{g} \dot{f}_{g}}(\omega)=S_{\ddot{f}_{g} \ddot{f}_{g}}(\omega) / \omega^{2}
\end{aligned}
$$

The ground motion is represented along the three principal directions $(\mathrm{u}, \mathrm{v}, \mathrm{w})$ by defining ratio $R_{u}, R_{v}$ and $R_{w}$ along them such that

$$
\ddot{u}_{g}(t)=R_{u} \ddot{f}_{g}(t) ; \ddot{\mathrm{v}}_{\mathrm{g}}(t)=R_{v} \ddot{f}_{g}(t) ; \ddot{\mathrm{w}}_{\mathrm{g}}(t)=R_{w} \ddot{f}_{g}(t)
$$

and the psdfs of the ground acceleration in the principal directions of the ground motion $(\mathrm{u}, \mathrm{v}, \mathrm{w})$ can be defined as

$$
S_{\ddot{u}_{g} \ddot{u}_{g}}(\omega)=R_{u}{ }^{2} S_{\ddot{f}_{g} \ddot{f}_{g}}(\omega) ; S_{\ddot{v}_{g} \ddot{v}_{g}}(\omega)=R_{v}{ }^{2} S_{\ddot{f}_{g} \ddot{f}_{g}}(\omega) \quad ; S_{\ddot{w}_{g} \ddot{w}_{g}}(\omega)=R_{w}{ }^{2} S_{\ddot{f}_{g} \ddot{f}_{g}}(\omega)
$$


so that

$$
{\sigma_{\ddot{u}_{g}}}^{2}=R_{u}{ }^{2} \sigma_{\ddot{f}_{g}}{ }^{2} ; \sigma_{\ddot{v}_{g}}{ }^{2}=R_{v}{ }^{2} \sigma_{\ddot{f}_{g}}{ }^{2} ; \sigma_{\ddot{w}_{g}}{ }^{2}=R_{w}{ }^{2} \sigma_{\ddot{f}_{g}}{ }^{2}
$$

When the angle of incidence of the ground motion with respect to the principal direction of the bridge is defined as $a$, the ground motions along the principal directions of the bridge $(\mathrm{x}, \mathrm{y}, \mathrm{z})$ are defined as

$$
\begin{aligned}
& \ddot{x}_{g}(t)=\ddot{u}_{g}(t) \cos \alpha-\ddot{w}_{g}(t) \sin \alpha \\
& \ddot{z}_{g}(t)=\ddot{u}_{g}(t) \sin \alpha-\ddot{w}_{g}(t) \cos \alpha \\
& \ddot{y}_{g}(t)=\ddot{v}_{g}(t)
\end{aligned}
$$

The psdfs of the ground accelerations along $x, y, z$ can be written as

$$
\begin{aligned}
& S_{\ddot{x}_{g} \ddot{x}_{g}}=\cos ^{2} \alpha S_{\ddot{u}_{g} \ddot{u}_{g}}+\sin ^{2} \alpha S_{\ddot{w}_{g} \ddot{w}_{g}}=R^{2} S_{\ddot{f}_{g}} \ddot{f}_{g} \\
& S_{\ddot{z}_{g} \ddot{z}_{g}}=\sin ^{2} \alpha S_{\ddot{u}_{g} \ddot{u}_{g}}+\cos ^{2} \alpha S_{\ddot{w}_{g} \ddot{w}_{g}}=R_{z}^{2} S_{\ddot{f}_{g}} \ddot{f}_{g} \\
& S_{\ddot{y}_{g} \ddot{y}_{g}}=S_{\ddot{v}_{g} \ddot{v}_{g}}=R_{y}{ }^{2} S_{\ddot{f}_{g}} \ddot{f}_{g}
\end{aligned}
$$

where $R_{x}, R_{y}$, and $R_{z}$ are the ratios of the ground motion along the principal axes of the bridge and given as

$$
\begin{aligned}
& R_{x}{ }^{2}=R_{u}{ }^{2} \cos ^{2} \alpha+R_{w}{ }^{2} \sin ^{2} \alpha \\
& R_{z}{ }^{2}=R_{u}{ }^{2} \sin ^{2} \alpha+R_{w}{ }^{2} \cos ^{2} \alpha \\
& R_{y}{ }^{2}=R_{v}{ }^{2}
\end{aligned}
$$

and $R_{u}, R_{v}$, and $R_{w}$ are ratios of the ground motion along the principal directions of the ground motion $(\mathrm{u}, \mathrm{v}, \mathrm{w})$ as shown in Fig.2.

\section{Distribution function of magnitude of earthquake}

Two types of distribution functions of the magnitude of earthquake are considered in the study.

\subsection{Exponential distribution}

This type of probability distribution function of the magnitude of earthquake is based on the Gutenberg - Ritcher Recurrence law (Kagan, 2002a)

$$
\begin{gathered}
\log \lambda_{m}=a-b m \\
\lambda_{m}=10 a-b m=\exp (\alpha-\beta m)
\end{gathered}
$$

where $\lambda_{\mathrm{m}}$ is the mean annual rate of exceedence of magnitude $\mathrm{m} ; 10^{\mathrm{a}}$ is the mean yearly number of earthquakes greater than or equal to zero,; and $\mathrm{b}$ describes the relative 
likelihood of large or small earthquakes. Eqn.(18b) implies that the magnitudes are exponentially distributed. Based on Eqn. (18b), the probability density function (PDF) is given by

$$
\mathrm{P}_{\mathrm{M}}(\mathrm{m})=\beta \mathrm{e}^{-\beta\left(m-\mathrm{m}_{0}\right)}
$$

where, $\beta=2.303 \mathrm{~b}$, and $\mathrm{m}_{0}$ is the lower threshold magnitude of earthquake, earthquakes smaller than which are eliminated, and $\mathrm{m}$ is the magnitude of earthquake.

The cumulative distribution function (CDF) of magnitude of earthquake for exponential distribution is given by the following expression

$$
\mathrm{F}_{\mathrm{M}}(\mathrm{m})=\left\{1-\exp \left(-\beta\left(\mathrm{m}-\mathrm{m}_{0}\right)\right)\right\}
$$

\subsection{Gumbel type-I distribution}

The cumulative distribution function of magnitude of earthquake for Gumbel type-I distribution is given by the following expression

$$
\mathrm{F}(\mathrm{m})=\operatorname{Exp}(-\exp -\alpha(\mathrm{m}-\mathrm{u}))
$$

where $\alpha$ and $u$ are the parameters for Gumbel Type- 1 distribution given by

$$
\begin{gathered}
\bar{M}=\mathrm{u}+0.5772 / \alpha \\
\sigma_{\mathrm{m}}^{2}=\pi^{2} / 6 \alpha^{2}
\end{gathered}
$$

in which $\bar{M}$ and $\sigma_{\mathrm{m}}$ are the mean and standard deviation of the magnitudes of earthquake respectively.

\section{Theoretical analysis}

\subsection{Free vibration analysis of cable stayed bridge deck (girder)}

The bridge deck, as shown in Figs.3(a) is idealized as a continuous beam over the outer abutments and the interior towers, and the effect of cable is taken as vertical springs at the points of intersections between the cables and the bridge deck shown in Fig.3(b). Further, the effect of the spring stiffness is taken as an additional vertical stiffness to the flexural stiffness of the bridge.

Referring to Fig.3(c) and Fig.3(d), the fluctuations of tension in the cable at any instant of time $(t)$ can be written as

$$
h_{i}(t)=K_{i} V\left(x_{i}, t\right) \sin \psi_{i}+K_{i} \Delta_{j}(t) \cos \psi_{i}
$$

where, $K_{i}=E_{c} A_{i} / L_{i}$ is the stiffness of the $\mathrm{i}^{\text {th }}$ cable; $\mathrm{V}\left(\mathrm{x}_{\mathrm{i}}, \mathrm{t}\right)$ is the displacement of the girder at time $t$ at the joint of the $i^{\text {th }}$ cable with the girder; $\Delta_{j}(t)$ is the horizontal sway of the tower at the $i^{\text {th }}$ tower cable joint connecting the $i^{\text {th }}$ cable; $\Psi_{\mathrm{i}}$ is the angle of inclination of the $i^{\text {th }}$ cable to the horizontal (measured clockwise from the cable to the horizontal line as shown in Fig.3.3(c); $A_{i}, L_{i}$ are the cross-sectional area and the length of the $i^{\text {th }}$ cable respectively and $E_{c}$ is the equivalent modulus of elasticity of the straight cable under dead loads. 


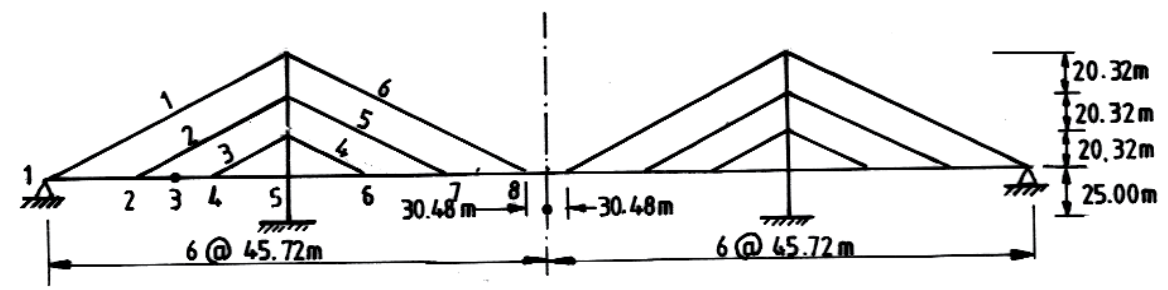

(a) Harp type cable stayed bridge considered for parametric study(Bridge-I)

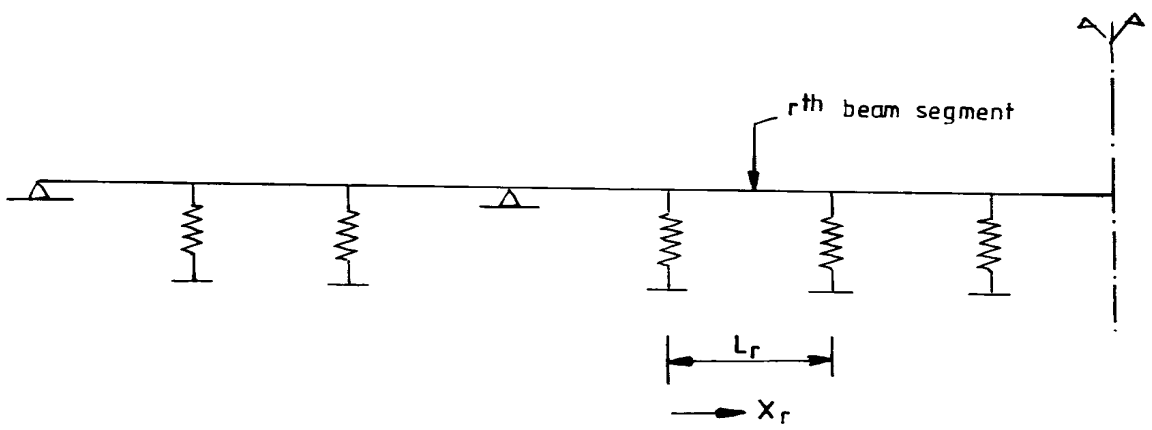

(b) Idealization of the bridge deck

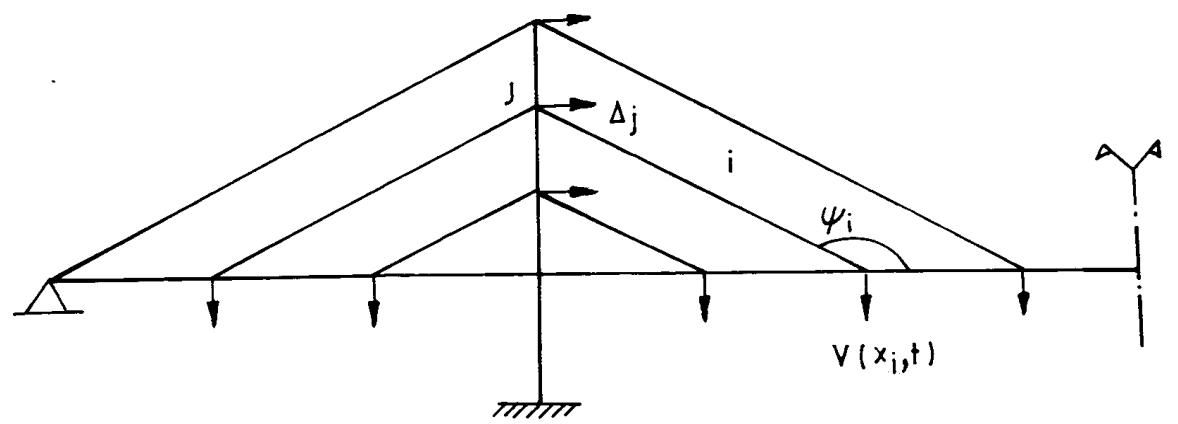

(c)Displacement due to the fluctuation of the $i^{\text {th }}$ cable

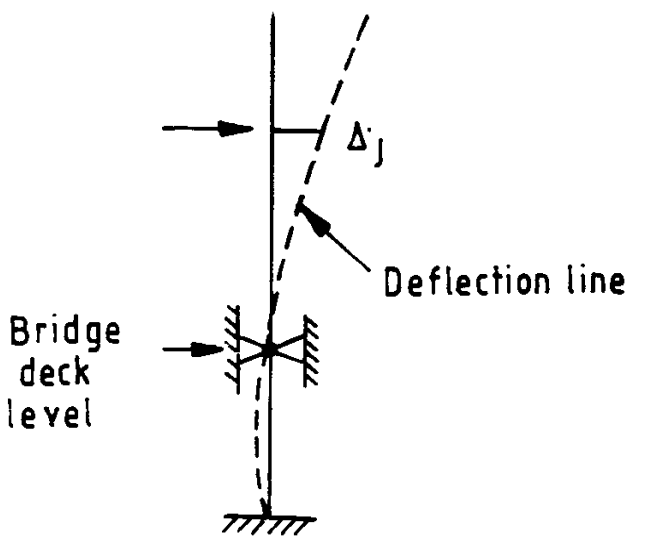

(d)Main system and the displacement of the tower

Fig. 3. (a, b, c, d) Problem identification 
Following Eqn.(23), the changes in tensions in the array of cables can be put in the following matrix form

$$
\{\mathrm{h}\}_{\mathrm{Ncx} 1}=(\mathbf{A})_{\mathrm{NcxNd}}\{\mathrm{V}\}_{\mathrm{Ndxl}}+(\mathbf{B})_{\mathrm{Ncx} 1}\{\Delta\}_{\mathrm{Nt} x 1}
$$

Where Nc is the number of cable (or pair of cable in case of two-phase cable stayed bridge); $\mathrm{Nd}$ is the number of unrestrained vertical degrees of freedom of the girder at the cablegirder joints; $\mathrm{Nt}$ is the number of horizontal tower degrees of freedom at the cable-tower joints; $\{\mathrm{V}\}$ and $\{\Delta\}$ are the girder and the tower displacement vectors; $\{\mathrm{h}\}$ is the vector of incremental cable tensions; (A) and (B) are the matrices which are formed by proper positioning of the elements $\mathrm{K}_{\mathrm{i}} \sin \psi_{\mathrm{i}}$ and $\mathrm{K}_{\mathrm{i}} \cos \psi_{\mathrm{i}}$ respectively.

The deflections of the tower at the cable joints can be obtained by assuming that the tower behaves like a vertical beam fixed at the bottom end and constrained horizontally at the level of the bridge deck and subjected to the transverse forces $h_{i}(t) \cos \psi_{i}(i=1, N c)$ at the cable tower joints as shown in Fig.3(b) and are given by

$$
\{\Delta\}=(C)\{h\}
$$

where the elements of the matrix $(\mathbf{C})$ can be easily obtained from the deflection equation of the tower (vertical beam) subjected to concentrated load, as mentioned above, using standard structural analysis procedure. Eliminating $\{\Delta\}$ from Eqns.(24) and (25), the relation between the vectors of incremental cable tensions and girder deflections may be written as

$$
\{\mathrm{h}\}=((\mathrm{l})-(\mathrm{B})(\mathrm{C}))^{-1}(\mathrm{~A})\{\mathrm{V}\}
$$

where (I) is the unit matrix of order Nc.

Premultiplying both sides of Eqn. (26) by a diagonal matrix (D) of order Nc, where the diagonals consists of the terms of $\sin \psi_{\mathrm{i}}(\mathrm{i}=1$ to $\mathrm{Nc}$ ), Eqn. (26) can be written as

$$
\left\{\mathrm{h}_{\mathrm{v} \cdot \mathrm{b}}\right\}=\left(\mathbf{K}_{\mathrm{c}, \mathrm{v}}\right)\{\mathrm{V}\}
$$

where $\left\{h_{v, b}\right\}$ is the vector containing the vertical components of incremental cable tensions, and $\left(K_{c, v}\right)=((D)(I)-(B)(C))^{-1}(A)$ is the stiffness matrix of the bridge contributed by the cables in vertical vibration.

The equation of motion for the relative vertical vibration $Y\left(x_{r}, t\right)$ of the beam segment $r$ of the idealized deck with constant axial force $\mathrm{N}_{\mathrm{r}}$, neglecting the shear deformation and rotary moment of inertia is given by

$$
E_{d} I_{r} \frac{\partial^{4} Y}{\partial X_{r}^{4}}+N_{r} \frac{\partial^{2} Y\left(x_{r}, t\right)}{\partial X_{r}{ }^{2}}+C_{r} \frac{\partial Y\left(x_{r}, t\right)}{\partial t}+\frac{\bar{W}_{r}}{g} \frac{\partial^{2} Y\left(x_{r}, t\right)}{\partial t^{2}}=P\left(x_{r}, t\right) \text { for } \mathrm{r}=1,2,3-\cdots .-\mathrm{N}_{\mathrm{b}}
$$

in which $E_{d}$ and $I_{r}$ are the modulus of elasticity of the bridge deck and vertical moment of inertia of the beam segment $r$ of the deck respectively.

$\mathrm{P}\left(\mathrm{x}_{\mathrm{r}}, \mathrm{t}\right)$ is defined as the load induced due to seismic excitations at different support degree of freedom and is given by

$$
P\left(X_{r}, t\right)=-\frac{\bar{W}_{r}}{g} \sum_{j=1}^{8} g_{j r}\left(x_{r}\right) \ddot{f}_{j}(t)
$$


where $\ddot{f}_{j}(t), \quad j=1,2,--------, 8$ are the accelerations at the different support degrees of freedom and $g_{j r}\left(x_{r}\right)$ is the vertical displacement of the $\mathrm{r}^{\text {th }}$ segment of the bridge deck due to unit displacement at the $\mathrm{j}^{\text {th }}$ degree of the supports. In Eqns. (28) and (29), it is assumed that for lightly damped system, the effect of damping term associated with quasi-static movement of the supports is negligible (Clough and Penzien, 1993).

The expression for $\mathrm{n}^{\text {th }}$ mode shape (undamped) for vertical vibration of the $\mathrm{r}^{\text {th }}$ segment of the bridge deck is given by (Chatterjee, 1992)

$$
\phi_{n}\left(x_{r}\right)=A_{n r} \cos \beta_{n r} x_{r}+B_{n r} \sin \beta_{n r} x_{r}+C_{n r} \cosh \gamma_{n r} x_{r}+D_{n r} \sinh \gamma_{n r} x_{r}
$$

where $A_{n r}, B_{n r}, C_{n r}$ and $D_{n r}$ are the integration constants expressed in terms of $n^{\text {th }}$ natural frequency of vertical vibration $\omega_{\text {bn }}$ and

$$
\beta_{n r}=\sqrt{\frac{N_{r}\left(Z_{n r}+1\right)}{2 E_{d} I_{r}}} ; \gamma_{\mathrm{nr}}=\sqrt{\frac{N_{r}\left(Z_{n r}-1\right)}{2 E_{d} I_{r}}}
$$

where

$$
Z_{n r}=\sqrt{1+\frac{4 E_{d} I_{r} \bar{W}_{r} / g \omega_{b n}{ }^{2}}{N_{r}^{2}}}
$$

in which the suffix $r$ is used to mean the $r^{\text {th }}$ segment of the beam. The origin for the $r^{\text {th }}$ segment is fixed at the left end as shown in Fig.3.3(c).Utilizing Eqn.(30), a relation between end displacements (vertical deflection and slope) and end forces(shear forces and bending moments) for the $\mathrm{r}^{\text {th }}$ segment may be written as

$$
\{\mathrm{F}\}_{\mathrm{r}}=(\mathrm{K})_{\mathrm{r}}\left\{\mathrm{x}_{\mathrm{r}}\right\}
$$

where $\{F\}_{r}$ and $\left\{x_{r}\right\}$ are the end forces and end displacement vectors and $(K)_{r}$ is the flexural dynamic stiffness matrix of the $r^{\text {th }}$ beam segment. The integration constants $A_{n r}, B_{n r}$, etc. are related to the end displacements as

$$
\{C\}_{\mathrm{r}}=(\mathrm{T})_{\mathrm{r}}\left\{\mathrm{x}_{\mathrm{r}}\right\}
$$

where $\{C\}_{r}$ is the vector of integration constants containing $A_{n r}$ etc., and $(T)_{r}$ is the matrix integration constants. The sign conventions used in the dynamic stiffness formulation are shown in Fig.4. The explicit expressions for the elements of $(\mathrm{K})_{\mathrm{r}}$ and $(\mathrm{T})_{\mathrm{r}}$ are given by Chatterjee (1992). Assembling the stiffness $(\mathbf{K})_{\mathrm{r}}$ for each element $(\mathrm{r})$ and adding the vertical stiffness due to cables $\left(\mathrm{K}_{\mathrm{C}, \mathrm{V}}\right)$, the overall stiffness of the bridge $(\mathbf{K})$ is obtained. The condition for the free vibration of the bridge deck may then be written as

$$
\text { (K) }\{\mathrm{U}\}=\{0\}
$$

where $\{U\}$ is the unknown end displacement vector for the beam corresponding to the dynamic degrees of freedom Fig.4. Using Eqn.(33) leads to

$$
\operatorname{det}(\mathrm{K})=0
$$




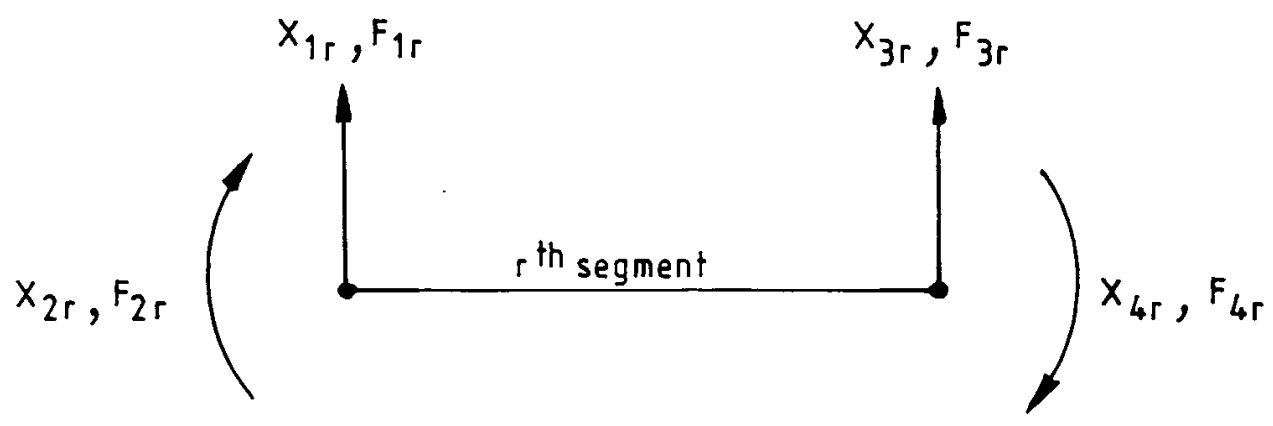

Fig. 4. Sign conventions used in the dynamic stiffness formulation.

Using Regula Falsi approach, the natural frequencies for the system are determined from the solution of Eqn.(33). Once the natural frequencies are obtained, mode shapes can be known through the use of Eqns.(32 \& 33).

\subsection{Modal transformation}

The modal transformation of the relative vertical displacement $y\left(x_{r}, t\right)$ for any point in the $r^{\text {th }}$ deck segment is given as

$$
y\left(x_{r}, t\right)=\sum_{n=1}^{\alpha} \varphi_{n}\left(x_{r}\right) q_{n}(t) \mathrm{r}=1,2,----, \mathrm{N}_{\mathrm{b}}
$$

in which $\Phi_{n}\left(x_{r}\right)$ is the $n^{\text {th }}$ mode shape of the $\mathrm{r}^{\text {th }}$ beam segment of the bridge deck and $q_{n}(t)$ is the $\mathrm{n}^{\text {th }}$ generalized coordinate. Substituting Eqn.(35) into Eqn.(28), multiplying by $\Phi_{\mathrm{m}}\left(\mathrm{x}_{\mathrm{r}}\right)$, integrating w.r.t. $\mathrm{L}_{\mathrm{r}}$ and using the orthogonality of the mode shapes leads to

$$
\ddot{q_{n}}(t)+2 \xi_{n} \omega_{n} \dot{q}_{n}(t)+\omega_{n}{ }^{2} q_{n}(t)=\bar{P}_{n}(t) \mathrm{n}=1,---, \mathrm{M}
$$

in which $\zeta_{n}$ and $\omega_{n}$ are the damping ratio and the natural frequency of the $\mathrm{n}^{\text {th }}$ vertical mode; $\mathrm{M}$ is the number of modes considered and $\vec{P}_{n}(t)$ is the generalized force given as

$$
\bar{P}_{n}(t)=\sum_{j=1}^{8} R_{j n}\left(x_{r}\right) \ddot{f}_{j}(t)
$$

where $R_{\text {jn }}$ is the modal participation factor given by

$$
R_{j n}=-\frac{\sum_{r=1}^{N_{b}} \frac{\bar{W}_{r}}{g} \int_{0}^{L_{r}} g_{j r}\left(x_{r}\right) \phi_{n}\left(x_{r}\right) d x_{r}}{\sum_{r=1}^{N_{b}} \frac{\bar{W}_{r}}{g} \int_{0}^{L_{r}} \phi_{n}^{2}\left(x_{r}\right) d x_{r}}
$$

in which $g_{j r}\left(x_{r}\right)$, the quasi static function, is the vertical displacement of the $\mathrm{r}^{\text {th }}$ beam segment of the bridge deck due to unit displacement given in the $j^{\text {th }}$ direction of support movement. 
Eqn.(37) can be put in the following matrix form

$$
\bar{P}_{n}(t)=\left[G_{n}\right]\{f\}
$$

in which

$$
\left[G_{n}\right]=\left\{G_{1 n}--------G_{8 n}\right\} \text { and }
$$$$
\left.\{f\}^{T}=\left\{f_{1}(t)------f_{8} \ddot{(} t\right)\right\}
$$

where $\left[G_{n}\right]$ is the generalized force coefficients for the $n^{\text {th }}$ mode and can be obtained by Eqns.(36 \& 37) i.e. $G_{1 n}=R_{1 n},----, G_{8 n}=R_{8 n}$.

\subsection{Spectral analysis}

\subsubsection{Evaluation of the relative displacement}

Applying the principles of modal analysis, the $\mathrm{n}^{\text {th }}$ generalized coordinate in frequency domain can be written as

$$
q_{n}(\omega)=H_{n}(\omega) \bar{P}_{n}(\omega)
$$

in which $H_{n}(\omega)$ is the $\mathrm{n}^{\text {th }}$ modal frequency response function given by

$$
H_{n}(\omega)=\left[\left(\omega_{n}^{2}-\omega^{2}\right)+i\left(2 \xi_{n} \omega_{n} \omega\right)\right]^{-1}
$$

Similarly, the $\mathrm{m}^{\text {th }}$ generalized coordinate can be written as

$$
q_{m}(\omega)=H_{m}(\omega) \bar{P}_{m}(\omega)
$$

The cross power spectral density function between the two generalized coordinate $q_{n}(\omega)$ and $q_{m}(\omega)$ is given by

$$
S_{q_{n} q_{m}}(\omega)=H_{n}(\omega) H_{m}(\omega) S_{\bar{P}_{n} \bar{P}_{m}}
$$

$H_{n}(\omega)$ denotes the complex conjugate of the $H_{n}(\omega)$ and $S_{\bar{P}_{n} \bar{P}_{m}}$ can be written in the matrix form as

$$
S_{\bar{P}_{n} \bar{P}_{m}}=\left[G_{n}\right]\left[S_{f f}\right]\left[G_{m}\right]^{T}
$$

$\left[\mathrm{S}_{\mathrm{ff}}\right]$ is the psdf matrix for the ground motion inputs (of size $8 \times 8$ ) which are the support accelerations i.e. $\ddot{f}_{1}(t), \ddot{f}_{2}(t), \ddot{f}_{3}, \ddot{f}_{4}, \ddot{f}_{5}, \ddot{f}_{6}, \ddot{f}_{7}, \ddot{f}_{8}$.

Any element of the matrix $\left[\mathrm{S}_{\mathrm{ff}}\right]$ may be written in terms of the psdf of ground acceleration $S_{\ddot{f}_{g} \ddot{f}_{g}}(\omega)$ by using the coherence function Eqn.(5), the ratio between the three components of the ground motion $\left(\mathrm{R}_{\mathrm{u}}: \mathrm{R}_{\mathrm{v}}: \mathrm{R}_{\mathrm{w}}\right)$ and Eqns.(16 \& 17) as explained earlier. For example $(1,4)$ and $(5,8)$ can be written in the form 


$$
\begin{aligned}
& S_{\ddot{f}_{1} \ddot{f}_{4}}=\rho_{14}(\omega) R_{y}{ }^{2} S_{\ddot{f}_{g} \ddot{f}_{g}} \\
& S_{\ddot{f}_{5} \ddot{f}_{8}}=\rho_{58}(\omega) R_{x}{ }^{2} S_{\ddot{f}_{g} \ddot{f}_{g}}
\end{aligned}
$$

Using the expression given in Eqn.(42), the elements of the matrix $\left[S_{\mathrm{qq}}\right]$ may be formed which has the dimension of $M \times M$. Since the relative displacement $y\left(x_{r}, t\right)$ is given by

$$
y\left(x_{r}, t\right)=\sum_{n=1}^{M} \phi_{n}\left(x_{r}\right) q_{n}(t)=\left[\phi\left(x_{r}\right)\right]_{(1 \times m)}\{q\}_{(1 \times m)}
$$

The psdf of the response $y\left(x_{r}, t\right)$ is given by

$$
S_{y y}\left(x_{r}, \omega\right)=\left[\phi\left(x_{r}\right)\right]\left[S_{q q}\right]\left[\phi\left(x_{r}\right)\right]^{T}
$$

\subsubsection{Evaluation of the quasi-static displacement}

The quasi-static component of the vertical displacement at any point in the $\mathrm{r}^{\text {th }}$ deck segment at time $(\mathrm{t})$ is given as

$$
g\left(x_{r}, t\right)=\sum_{j=1}^{8} g_{j r}\left(x_{r}\right) f_{j}(t)=[Q]\{\bar{f}\}
$$

where

$$
[Q]=\left\{g_{1 r}\left(x_{r}\right) g_{2 r}\left(x_{r}\right)---g_{8 r}\left(x_{r}\right)\right\} ;\left\{f^{-}\right\}^{T}=\left\{f_{1}(t) f_{2}(t)\right.
$$

$\mathrm{g}_{\mathrm{jr}}\left(\mathrm{x}_{\mathrm{r}}\right)$ is the vertical displacement at any point in the $\mathrm{r}^{\text {th }}$ beam segment of the bridge deck due to unit movement of the $\mathrm{j}^{\text {th }}$ support d.o.f. The psdf of the quasi-static displacement at any point in the $\mathrm{r}^{\text {th }}$ deck segment is given by

$$
S_{g g}\left(x_{r}, \omega\right)=[Q]\left[S_{\bar{f} \overline{\mathrm{f}}}\right][Q]^{T}
$$

where $\left[S_{\bar{f} \overline{\mathrm{f}}}\right]$ is the psdf matrix for the ground displacements at the support d.o.f.s and can be obtained in terms of the psdf of ground acceleration $S_{\ddot{f}_{o} \ddot{f}_{g}}$ with the help of both the coherence function, the ratio between the three components of ground motion and Eqns.(11, $16 \& 17)$.

\subsubsection{Evaluation of the total displacement}

The total displacement at any point of the $\mathrm{r}^{\text {th }}$ beam segment of the bridge deck at any time (t) can be written as

$$
Y\left(x_{r}, t\right)=y\left(x_{r}, t\right)+g\left(x_{r}, t\right)
$$

The psdf of the total vertical displacement can be expressed as 


$$
S_{Y Y}\left(x_{r}, \omega\right)=S_{y y}\left(x_{r}, \omega\right)+S_{g g}\left(x_{r}, \omega\right)+S_{y g}\left(x_{r}, \omega\right)+S_{g y}\left(x_{r}, \omega\right)
$$

$S_{y g}\left(x_{r}, \omega\right)$ and $S_{g y}\left(x_{r}, \omega\right)$ are the cross power spectral density functions between relative and the quasi-static displacements. Using Eqns.(-------), the expression for $S_{y g}\left(x_{r}, \omega\right)$ can be obtained as

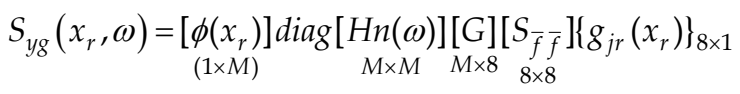

$$
\begin{aligned}
& \mathrm{n}=1,2,----, \mathrm{M} ; \mathrm{j}=1,2,-----, 8 ; \quad \mathrm{r}=1,2,------, \mathrm{N}_{\mathrm{b}}
\end{aligned}
$$

where $\left[S_{\bar{f} \bar{f}}\right]$ is the cross power spectral density matrix of the random vectors $\{\ddot{f}\}$ and $\left\{f_{f}^{-}\right\}$ i.e., the support accelerations and displacements. $S_{g y}\left(x_{r}, \omega\right)$ is the complex conjugate of $S_{y g}\left(x_{r}, \omega\right)$.

\subsubsection{Evaluation of the bending moment}

Using Eqns.(45 \& 47) and differentiating the expression for $Y\left(x_{r}, t\right)$ twice with respect to $x$, the following expression for the bending moment can be obtained as

$$
E_{d} l_{r} \frac{\partial^{2} y}{\partial x^{2}}=\sum_{n=1}^{M} E_{d} l_{r} \frac{d^{2} \phi\left(x_{r}\right)}{d x^{2}} q_{n}(t)+\sum_{j=1}^{8} E_{d} l_{r} \frac{d^{2} g_{j r}\left(x_{r}\right)}{d x^{2}} f_{j}(t)
$$

Similar expressions can be obtained for the psdf of the bending moment at any point in the $\mathrm{r}^{\text {th }}$ beam segment of the bridge deck as those derived for the total displacement by replacing $\phi\left(x_{r}\right)$ and $g_{\mathrm{jr}}\left(\mathrm{x}_{\mathrm{r}}\right)$ by $E_{d} I_{r} d^{2} \phi\left(x_{r}\right) / d x^{2} \quad$ and $E_{d} I_{r} d^{2} g_{j r}\left(x_{r}\right) / d x^{2} \quad$ respectively. $E_{d} I_{r} d^{2} g_{j r}\left(x_{r}\right) / d x^{2}$ is obtained from the quasi-static analysis of the entire bridge using the stiffness approach as mentioned before.

\subsection{Statistical parameters of response}

For studying the statistical properties of the response process, the first few moments of the response power spectral density function are needed. The $\mathrm{j}^{\text {th }}$ moment of the PSDF function may be defined as follows:

$$
\lambda_{j}=\int_{0}^{\alpha} w^{j} S_{Y Y}(w) d w \quad \text { where } \mathrm{j}=0,1,2,---
$$

The zeroth and second moments may be recognized as the variances of the response and the first time derivative of the response respectively.

$$
\begin{gathered}
\lambda_{0}=\int_{0}^{\alpha} S_{Y Y}(w) d w=\sigma_{Y Y}{ }^{2} \\
\lambda_{2}=\int_{0}^{\alpha} w^{2} S_{Y Y}(w) d w=\sigma_{\dot{Y} \dot{Y}}{ }^{2}
\end{gathered}
$$


The mean rate of zero crossing at positive slopes is by

$$
v_{0}=\frac{1}{2 \pi} \sqrt{\frac{\lambda_{2}}{\lambda_{0}}}
$$

Another quantity of interest is the dispersion parameter q given by

$$
q=\sqrt{1-\frac{\lambda_{1}^{2}}{\lambda_{0} \lambda_{2}}}
$$

The value of $q$ lies in the range [0,1]. It can shown that $q$ is small for a narrow band process and relatively large for a wide band process. The mean rate of crossing a specified level A at a positive slope by a stationary zero mean Gaussian random process $\mathrm{z}(\mathrm{t})$ can be expressed by Lin (1967) as

$$
v_{a}=v_{0} e^{\frac{-\psi^{2}}{2}}
$$

where

$$
\psi=\frac{A}{\sigma_{Y Y}}
$$

It has been confirmed by theoretical as well as simulation studies that the probability of a stationary response process remaining below a specified barrier level decays approximately exponentially with time as given by the relationship (Coleman,1959 and Crandal et.al., 1966)

$$
L(T)=L_{0} e^{-\alpha T}
$$

Where, $L_{0}$ is the probability of starting below the threshold, $\mathrm{a}$ is the decay rate, and $\mathrm{T}$ is the duration of the response process.

At high barrier levels, $L_{o}$ is practically equal to one, and the decay rate is given by the following expressions for processes with double barrier and one sided barrier respectively (Vanmarcke, 1975; Lin, 1967)

$$
\begin{gathered}
\alpha_{D}=2 v_{a} \\
\alpha_{S}=v_{a}
\end{gathered}
$$

In case of relatively low threshold levels, an improved value can be obtained by using the expressions for the probability of starting below the threshold and the decay rate (Vanmarcke, 1975)

$$
\begin{gathered}
L_{0}=1-e^{\frac{-\psi^{2}}{2}} \\
\alpha_{D}^{*}=\alpha_{D} \frac{1-e^{-\sqrt{\frac{\pi}{2}}(\mathrm{q} \psi)}}{1-\mathrm{e}^{-\psi^{2} / 2}}
\end{gathered}
$$




$$
\alpha_{S}^{*}=\alpha_{S} \frac{1-e^{-\sqrt{2 \pi}(\mathrm{q} \psi)}}{1-\mathrm{e}^{-\psi^{2} / 2}}
$$

\subsection{Reliability estimation against first passage failure}

For an earthquake with given magnitude M, the probability of First Passage Failure, i.e., the probability that the response is larger than a threshold level $\mathrm{A}$, can be determined from the following relationship

$$
p\left[z>A \mid M_{r}\right]=1-L(T)
$$

where $\mathrm{T}$ is the duration of the response.

If $f_{M}(M)$ is the probability density functions of earthquake magnitude, the probability of First Passage Failure, provided that an earthquake occurs, can be calculated from (Ang and Tang, 1975)

$$
p_{E}=p[z>A]=\int_{M=4}^{9} p\left[z>A \mid M_{r}\right] f_{M}(M) d M
$$

If the rate of earthquake occurrence for the seismo - tectonic region considered in the study is a constant and $\mathrm{n}$ is the average number of earthquakes per year in the magnitude range of interest for the source region, the probability of atleast one failure due to earthquake in " $\mathrm{m}$ " years can be expressed as

$$
P_{F}=1-\left(1-p_{E}\right)^{m \cdot n}
$$

\section{Numerical study}

A double plane symmetrical harp type cable stayed bridges, (Morris, 1974 ) used as illustrative example is shown in Fig.3.3(a). The structural data of the bridge is shown in Table-1.

In addition, the following data are assumed for the analysis of the problem, $E_{c}=E_{d} ; \xi=0.02$ for all modes; and the tower - deck inertia ratio, the ratio between three components of the ground motion $\left(R_{\mathrm{u}}: \mathrm{R}_{\mathrm{v}}: \mathrm{R}_{\mathrm{w}}\right)$, $\alpha$, duration of earthquake, $\beta$ values for exponential distribution of magnitude of earthquake are taken $4,(1.0: 1.0: 1.0), 0.0^{0}, 15 \mathrm{sec}$ and $(1.5,2.303,2.703)$ respectively unless mentioned otherwise. Also, the ground motion is assumed to be partially correlated in firm soil unless mentioned otherwise.

The random ground motion is assumed to be homogeneous stochastic process which is represented by Clough and Penzien double filter psdf given by two sets of filter coefficients representing the soft and firm soils respectively. For the soft soil, the coefficients are $\omega_{\mathrm{g}}=$ $6.2832 \mathrm{rad} / \mathrm{sec} ; \omega_{\mathrm{f}}=0.62832 \mathrm{rad} / \mathrm{sec} ; \xi_{\mathrm{g}}=\xi_{\mathrm{f}}=0.4$, while those for the firm soils are $\omega_{\mathrm{g}}=$ $15.708 \mathrm{rad} / \mathrm{sec} ; \omega_{\mathrm{f}}=1.5708 \mathrm{rad} / \mathrm{sec} ; \xi_{\mathrm{g}}=\xi_{\mathrm{f}}=0.6$. The two psdfs corresponding to the two sets of filter coefficients are shown in Fig.5. The spatial correlation function used in the parametric study is given by Eqn. 5 in which the value of $\mathrm{c}=2.0, \mathrm{Vs}=70 \mathrm{~m} / \mathrm{sec}$ and $\mathrm{Vs}=330$ $\mathrm{m} / \mathrm{sec}$ for the first and second psdfs respectively. The r.m.s (root mean square) ground acceleration is related to intensity of earthquake by empirical equation given by Eqn.7. Intensity of earthquake $I_{S}$ in turn is related to magnitude of earthquake given by Eqn.9. The 
input for excitation is thus the intensity of earthquake for which $\sigma_{\ddot{u}_{g}}$ value can be calculated using Eqn.7. From $\sigma_{\ddot{u}_{g}}$ the value of $\mathrm{S}_{0}$ defining the ordinates of the double filter psdf can be obtained using equation Eqn.1.

\begin{tabular}{|c|c|c|}
\hline Parameter & Centre Span & Side Span \\
\hline $\begin{array}{l}\text { Deck length } \\
\text { Deck Area } \\
\text { Deck Depth } \\
\text { Modulus of Elasticity of deck } \\
\text { Moment of Inertia of deck } \\
\end{array}$ & $\begin{array}{c}\mathrm{L}_{2}=335.28 \mathrm{~m} \\
\mathrm{~A}_{2}=0.32 \mathrm{~m}^{2} \\
\mathrm{D}_{2}=4.0 \mathrm{~m} \\
\mathrm{E}_{2}=2.0683 \times 10^{11} \mathrm{~N} / \mathrm{m}^{2} \\
\mathrm{I}_{2}=1.131 \mathrm{~m}^{4}\end{array}$ & $\begin{array}{c}\mathrm{L}_{1==} \mathrm{L}_{3}=137.16 \mathrm{~m} \\
\mathrm{~A}_{1}=\mathrm{A}_{3}=0.32 \mathrm{~m}^{2} \\
\mathrm{D}_{1}=\mathrm{D}_{3}=4.0 \\
\mathrm{E}_{1}=\mathrm{E}_{3}=2.0683 \times 10^{11} \mathrm{~N} / \mathrm{m}^{2} \\
\mathrm{I}_{1}=\mathrm{I}_{3}=1.131 \mathrm{~m}^{4}\end{array}$ \\
\hline Tower Properties & $\mathrm{L}_{\mathrm{t}}=85.96 \mathrm{~m} ; \quad \mathrm{E}_{\mathrm{t}}=2.0683$ & $10^{11} \mathrm{~N} / \mathrm{m}^{2} ; \quad \mathrm{A}_{\mathrm{t}}=0.236 \mathrm{~m}^{2}$ \\
\hline Cable Properties & $\begin{array}{r}\text { Area of the cables ( } 1 \text { to } 6) \\
\text { and } \\
\text { Tension in cables }(1 \text { to } 6) \\
4.3 \times 10^{6}, 5.9 \times 10 \\
\text { Modulus of cables }\end{array}$ & $\begin{array}{l}.04,0.016,0.016,0.016,0.016 \\
.04 \mathrm{~m}^{2} \\
5.5 \times 10^{6}, 5.9 \times 10^{6}, 5.9 \times 10^{6} \\
\text { and } 15.5 \times 10^{6} \mathrm{~N} / \mathrm{m}^{2} \\
=2.0683 \times 10^{11} \mathrm{~N} / \mathrm{m}^{2}\end{array}$ \\
\hline \multicolumn{2}{|c|}{ Distributed mass of the bridge over half width deck } & $9.016 \times 10^{3} \mathrm{Kg} / \mathrm{m}$ \\
\hline $\begin{array}{l}\text { Properties of flexible } \\
\text { foundation }\end{array}$ & \multicolumn{2}{|c|}{$\begin{array}{c}\text { Radius of circular foundation }=3 \mathrm{~m} \\
\text { Poission's ratio }=0.33 \\
\text { Density of the soil }=12 \mathrm{Kn} / \mathrm{m}^{3}\end{array}$} \\
\hline
\end{tabular}

Table 1. Structural data of the Harp Type Cable Stayed Bridge

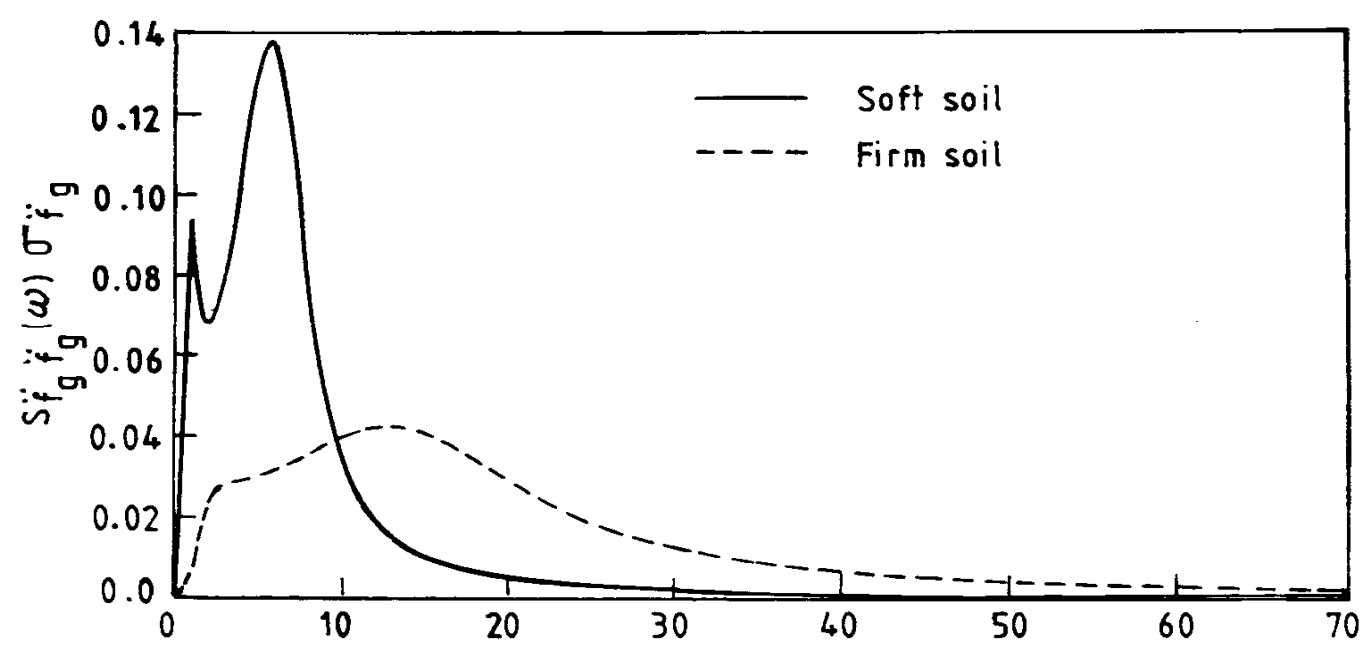

Fig. 5. Normalized PSDF of ground acceleration

Fig. 6 shows the first five modes of the bridge corresponding to $I_{t} / I_{d}=4.0$. The first five frequencies and the corresponding nature of the mode shapes for the bridge is given in Table-2 for different $I_{t} / I_{d}$ ratios. 
356

Earthquake-Resistant Structures - Design, Assessment and Rehabilitation
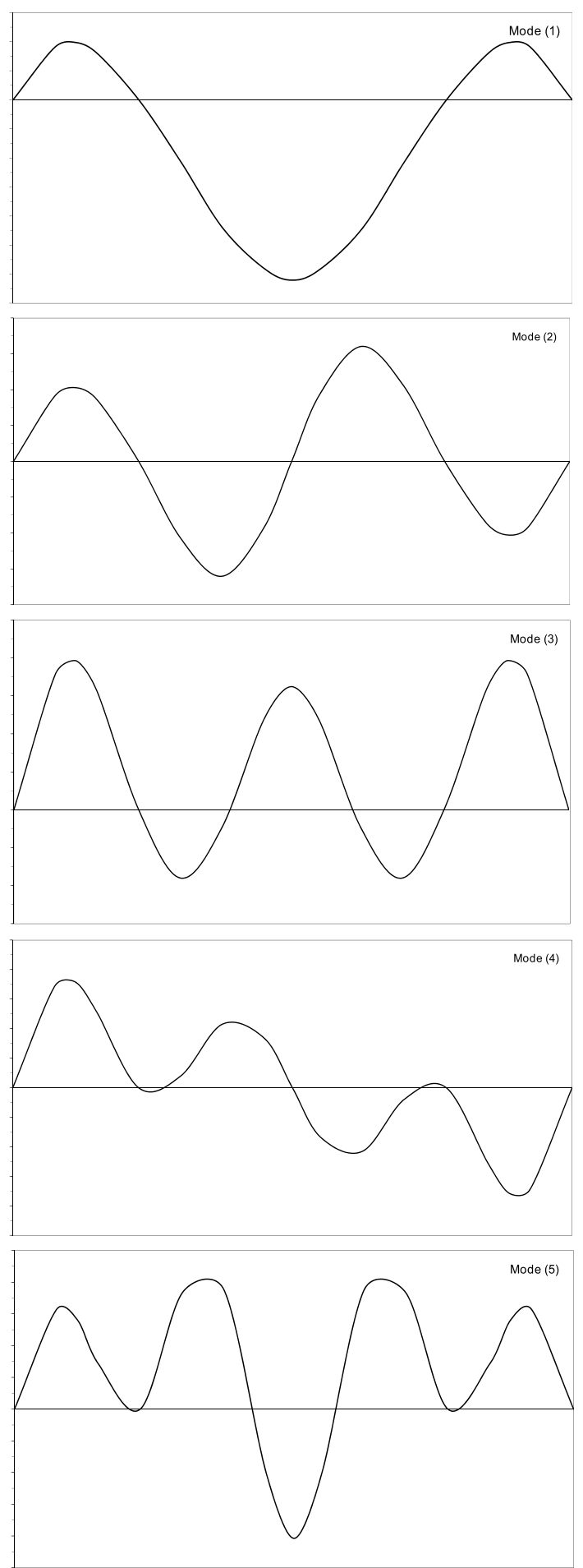

Fig. 6. First 5 mode shapes 


\begin{tabular}{|c|c|c|c|c|}
\hline \multirow{2}{*}{ Mode No. } & \multicolumn{3}{|c|}{ Fundamental Frequencies (rad/sec) } & \multirow{2}{*}{ Nature } \\
\cline { 2 - 4 } & $\mathbf{I}_{\mathbf{t}} / \mathbf{I}_{\mathbf{d}} \mathbf{= 2 . 0}$ & $\mathbf{I}_{\mathbf{t}} / \mathbf{I}_{\mathbf{d}}=\mathbf{4 . 0}$ & $\mathbf{I}_{\mathbf{t}} / \mathbf{I}_{\mathbf{d}}=\mathbf{6 . 0}$ & \\
\hline 1 & 2.838 & 2.983 & 3.072 & Symmetric \\
\hline 2 & 3.247 & 3.551 & 3.716 & Anti-symmetric \\
\hline 3 & 4.443 & 4.706 & 4.885 & Symmetric \\
\hline 4 & 5.319 & 5.374 & 5.409 & Anti-symmetric \\
\hline 5 & 6.429 & 6.450 & 6.467 & Symmetric \\
\hline
\end{tabular}

Table 2. Fundamental frequencies of the bridge deck for different tower - deck inertia ratio.

\subsection{Effect of the tower - Deck inertia ratio $\left(I_{t} / I_{d}\right)$}

The first 5 fundamental frequencies are obtained for different ratios of the tower - deck inertias i.e. 2, 4 and 6 respectively as shown in Tables-2. It is seen from the tables that with the increase of the tower - deck inertia ratio, the frequencies of the bridge deck increase. This is due to the fact that the increase in the tower stiffness increases the component of the vertical stiffness of the bridge provided by the cables.

\subsection{Effect of the quasi-static component on the response}

Total and relative displacement of the bridge decks obtained for the firm soil is shown in Tables-3. It is seen from the Tables that the contribution of the quasi-static component to the total response is significant for displacement where as it is small for bending moment.

\begin{tabular}{|c|c|c|c|c|}
\hline \multirow{2}{*}{ Point No. } & \multicolumn{2}{|c|}{ Relative } & \multicolumn{2}{c|}{ Total } \\
\cline { 2 - 5 } & $\begin{array}{c}\text { Displacement } \\
(\mathbf{m})\end{array}$ & $\begin{array}{c}\text { Moment } \\
\mathbf{( t - m})\end{array}$ & $\begin{array}{c}\text { Displacement } \\
(\mathbf{m})\end{array}$ & $\begin{array}{c}\text { Moment } \\
(\mathbf{t}-\mathbf{m})\end{array}$ \\
\hline 1 & 0.0 & 0.0 & 0.0509 & 0.0 \\
\hline 2 & 0.0961 & 1424.0 & 0.1108 & 1429.0 \\
\hline 3 & 0.1040 & 1432.0 & 0.1184 & 1435.0 \\
\hline 4 & 0.0831 & 727.0 & 0.0995 & 740.0 \\
\hline 5 & 0.0 & 996.0 & 0.0509 & 1009 \\
\hline 6 & 0.0906 & 715.0 & 0.1099 & 726 \\
\hline 7 & 0.1621 & 974.0 & 0.1808 & 976.0 \\
\hline 8 & 0.1886 & 702.0 & 0.2109 & 699 \\
\hline 9 & 0.1957 & 1160.0 & 0.2164 & 1160.0 \\
\hline
\end{tabular}

Table 3. Effect of the quasi - static part of the response on the r.m.s responses

\subsection{Effect of barrier level on the reliability}

Effect of the barrier level on the reliability of the bridge is shown in Figs. 7. The barrier level are taken as $15 \%, 20 \%, 25 \%, 30 \%, 33 \%, 40 \%, 50 \%$ and $70 \%$ of the yield stress assuming that the barrier level is the difference between yield stress and the pre-stress in the girder (deck). It is seen from the figures that the reliability increases as the barrier levels increases as it would be expected. However, the variation is not linear; it tends to follow an S shaped curve. For certain condition, the variation of reliability with barrier level may be very steep in the lower range of barrier levels. The same figures also compare between the reliabilities for firm and soft soils conditions. It is seen from the Fig.7 that the reliability for a particular barrier level is higher for firm soil. The difference between the two is considerably more at the lower end of the barrier level. 


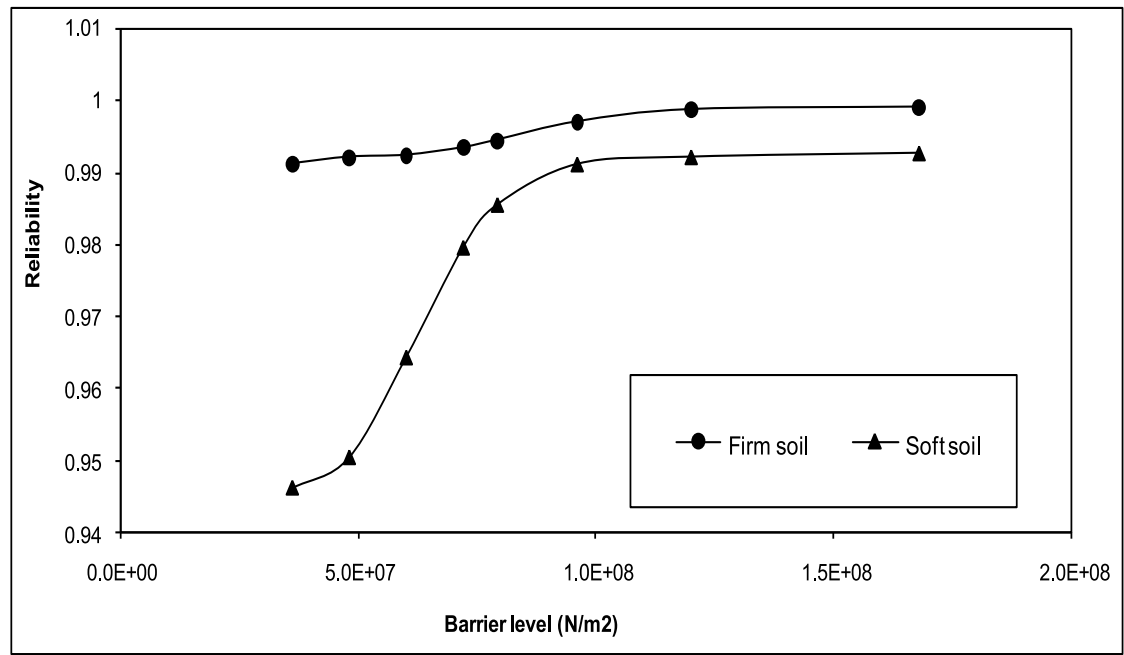

Fig. 7. Variation of Reliability with Barrier level

\subsection{Effect of magnitude of earthquake on the reliability}

The effect of the distribution of magnitude of earthquake on the reliability is shown in Fig.8. The figure shows the variation of reliability with barrier level for different distributions of the magnitude of earthquake obtained by exponential and gumbel distribution. It is seen from the figures that the reliability increases with the increase in beta values for exponential distribution. The difference between reliabilities obtained for two beta values considerably is more for lower values of barrier level. Above a certain value of beta, the reliability nearly approaches unity for all barrier levels. Further, Gumbel distribution provides much higher value of reliability as compared to Exponential distribution (for beta $=1.5$ ). For soft soil condition (Fig.9), the effect of the distribution of the magnitude of earthquake is more produced.

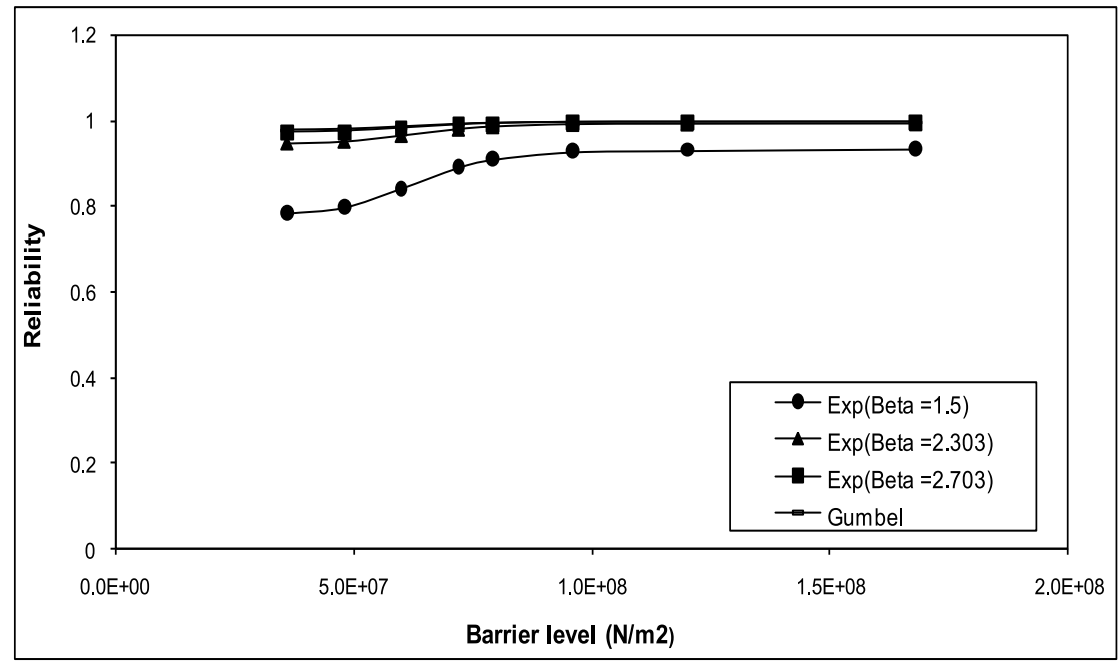

Fig. 8. Variation of Reliability with Barrier level for different distributions of magnitude of earthquake (Hard soil) 


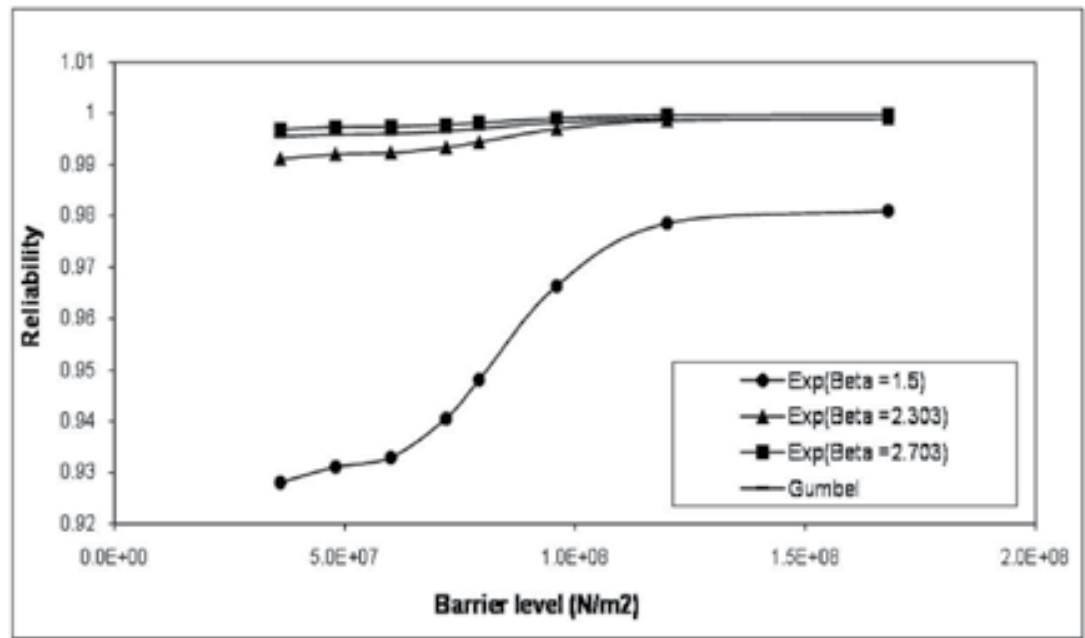

Fig. 9. Variation of Reliability with Barrier level for different distributions of magnitude of earthquake (Soft soil)

\subsection{Effect of ratio between the three components of earthquake (Ru:Rv:Rw) on the reliability}

The effect of this ratio on the variation of reliability with the barrier level is shown in Figs.10 and 11 for two angle of incidences of earthquake $\left(\alpha=0^{0}\right.$ and $\left.\alpha=45^{\circ}\right)$. It is seen from the figures that the ratio has significant effect on this variation, especially at the lower end of the barrier level.

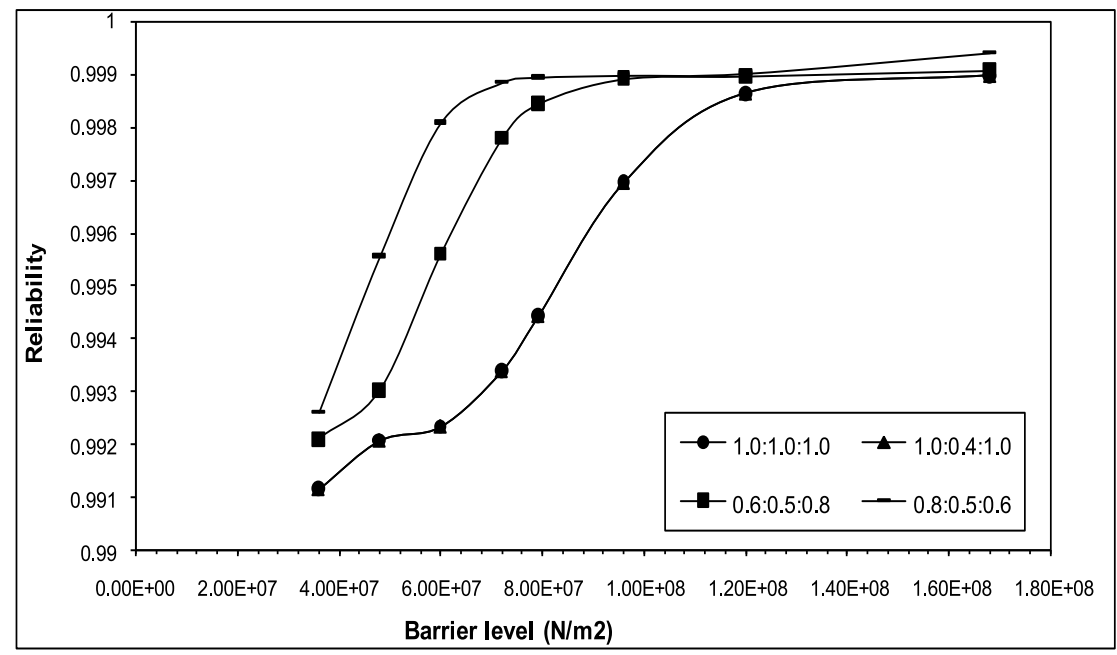

Fig. 10. Variation of Reliability for different ratios of Earthquake components (Alpha $=0.0$ degree) 


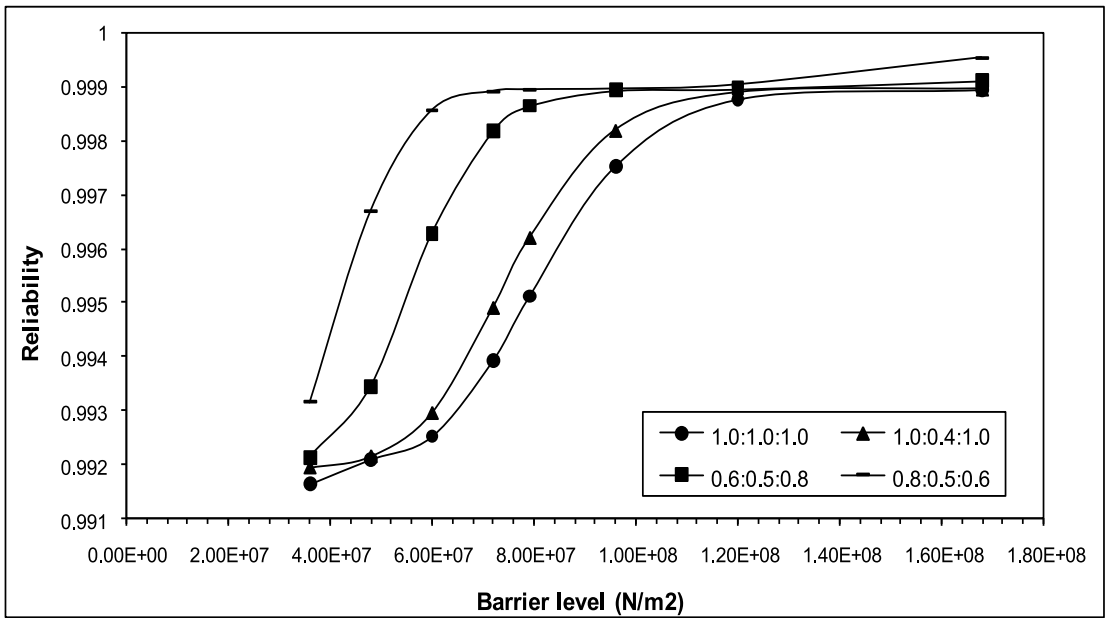

Fig. 11. Variation of Reliability for different ratios of Earthquake components (Alpha $=45$ degree)

\subsection{Effect of angle of incidence on the reliability}

Fig.12 show the effect of angle of incidence on the variation of reliability with barrier level. Three values of angle of incidence are considered namely, $0^{0}$ i.e. major direction of earthquake is along the longitudinal axis of the bridge and the other two cases are having $30^{\circ}$ and $70^{\circ}$ angle of incidence with the longitudinal axis of the bridge. It is seen from the figures that $0^{0}$ angle of incidence provides minimum reliability while $70^{\circ}$ angle of incidence provides maximum reliability. This is expected because $0^{0}$ angle of incidence produces maximum stress in the bridge.

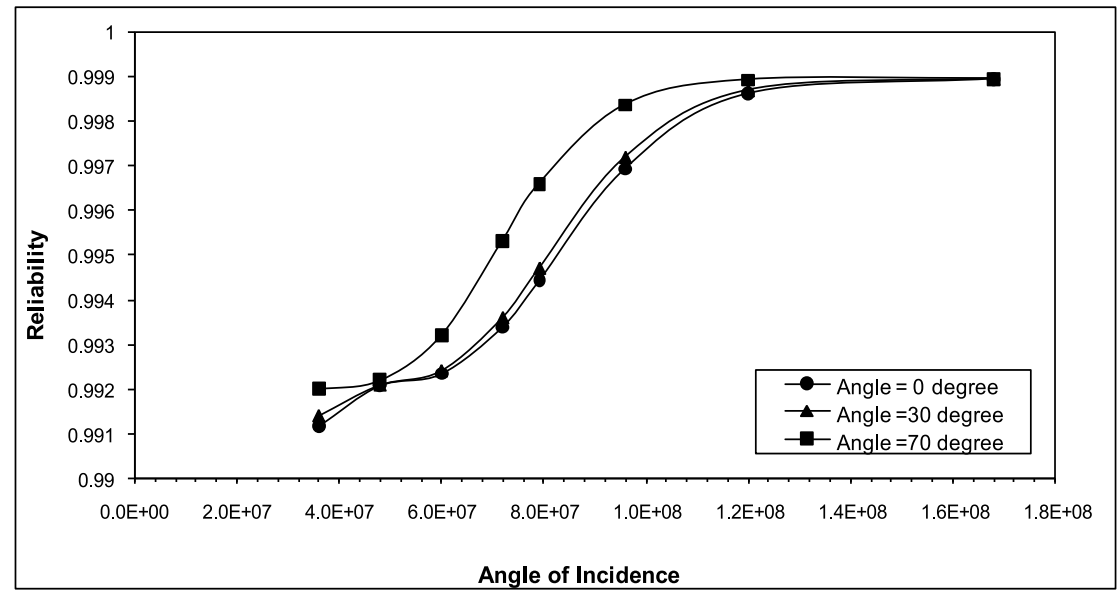

Fig. 12. Variation of Reliability for different Angles of Incidence 


\subsection{Effect of correlation of ground motion on the reliability}

Fig.13 shows the variation of reliability with barrier level for three cases of ground motion that is fully correlated, partially correlated and uncorrelated. It is seen from the figures that fully correlated ground motion provides the highest reliability while uncorrelated ground motion gives the lowest value. The partially correlated ground motion gives reliability in between the two. This is the case because uncorrelated / partially correlated ground motion induces additional bending moment in the deck due to the phase lag of ground motion between different supports. The difference between the reliabilities for the three cases is nor very significant for the hard soil. However, the difference between them is significant for the soft soil (Fig.14). Further, the difference between the reliabilities are considerably reduced at the higher end of the barrier level.

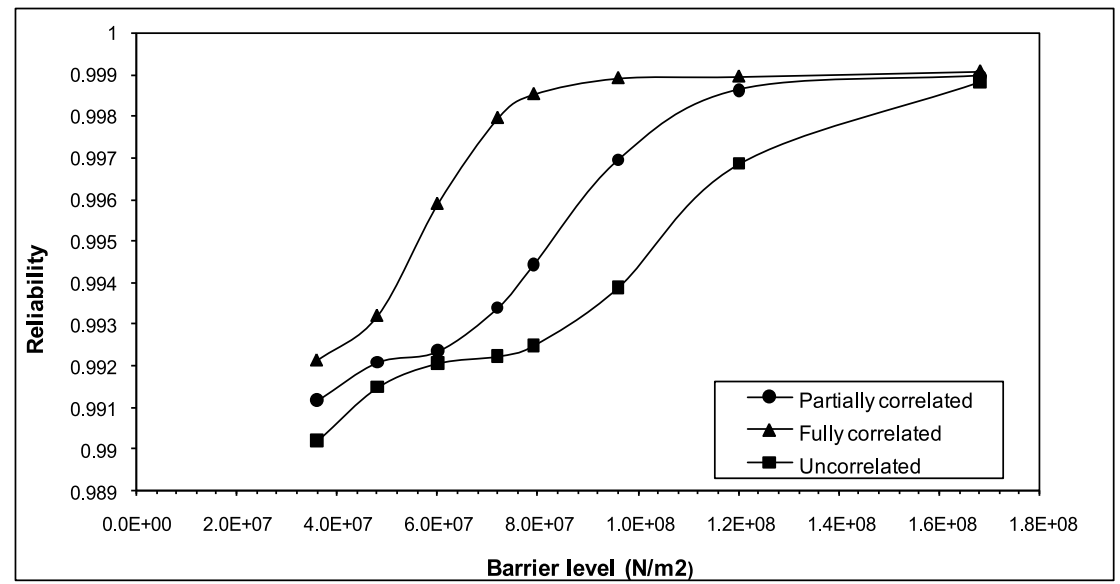

Fig. 13. Variation of Reliability with Barrier level for different degrees of correlation of ground motion (Hard soil)

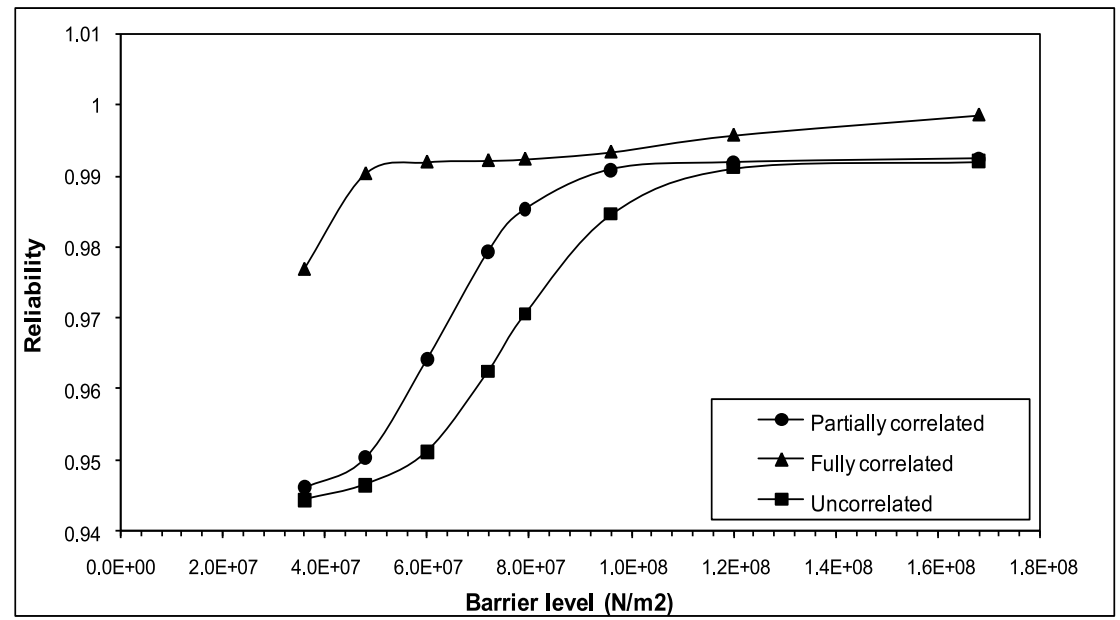

Fig. 14. Variation of Reliability with Barrier level for different degrees of correlation of ground motion (Soft soil) 


\subsection{Effect of duration of earthquake on the reliability}

Fig.15 shows the variation of reliability with duration of earthquake for a barrier level of $33 \%$. It is seen from the figures that reliability decreases mildly with the increase of duration of earthquake for both soft and firm soil. Thus, the duration of earthquake does not have significant influence on the reliability.

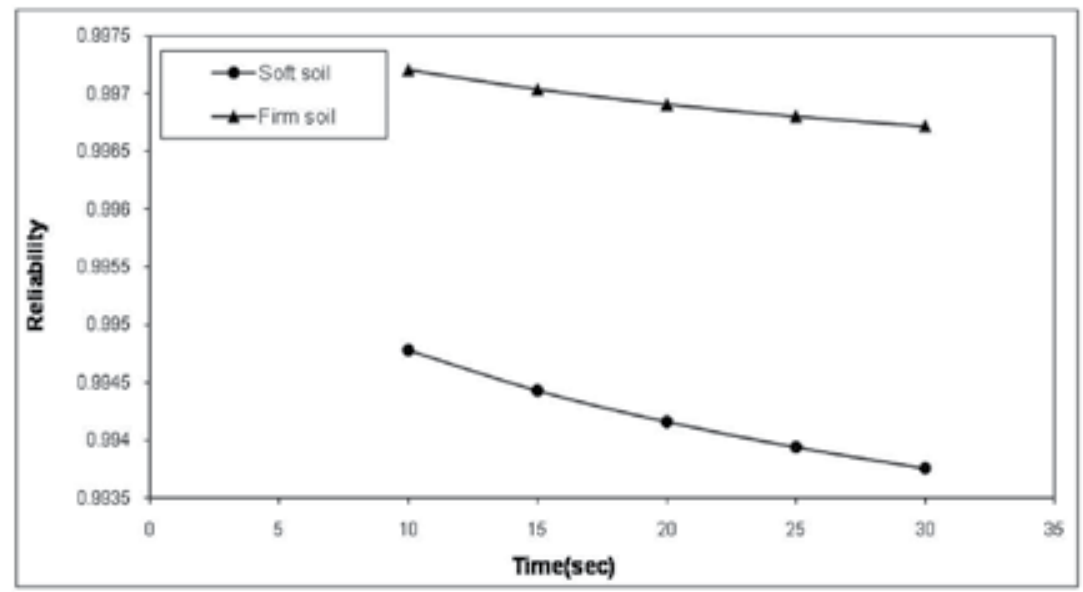

Fig. 15. Variation of Reliability with duration of Earthquakes (for a barrier level of 33\%)

\section{Conclusions}

Reliability against first passage failure of cable stayed bridges under earthquake excitation is presented. The responses of cable stayed bridges are obtained for random ground motion which is modeled as stationary random process represented by double filter power spectral density function (psdf) and a correlation function. The responses are obtained by frequency domain spectral analysis. Conditional probability of first crossing the threshold level for a given RMS ground acceleration is obtained by using the moments of the psdf of the response. The RMS value of the ground acceleration is related to the magnitude of earthquake by an empirical equation, and the probability density function of the earthquake is integrated with the conditional probability of failure to find the probability of first passage failure. Using the above method of analysis, two cable stayed bridges are analyzed and probabilities of first passage failure are obtained for a number of parametric variations. The results of the numerical study lead to the following conclusions:

i. The reliability against first passage failure increases sharply with the increase in barrier level in the lower range of its values.

ii. For the soft soil condition, the reliability is considerably less as compared to the firm soil condition.

iii. Gumbel distribution of the magnitude of the earthquake provides a very high estimate of reliability and gives values close to those obtained by exponential distribution with high values of the parameter $\beta$.

iv. Uncorrelated ground motion provides lower estimates of the reliability as compared to the fully correlated ground motion. The difference significantly more for the soft soil. 
v. The ratios between the components of ground motion have considerable influence on the reliability estimates. For soft soil condition, the difference between the reliability estimates, especially in the lower range of barrier level.

vi. In the lower range of barrier level, considerable difference between reliabilities is observed for $0^{0}$ and $70^{\circ}$ angle of incidence with respect to the longitudinal axis of the bridge. For higher value of the barrier level, there is practically no difference between the two reliabilities.

vii. The duration of ground motion does not have significant influence on the reliability estimates.

viii. It is found that reliability decreases with the increase of average number of earthquake per year. The variation is nonlinear and more steep for the soft soil condition. For an average number of earthquake 0.5 per year, the reliability could be as 0.3 .

\section{References}

[1] Abbas, A.M., and Manohar, C.S. (2007)."Reliability-based vector non-stationary random critical earthquake excitations for parametrically excited systems", Structural Safety, Vol.29(1), pp.32-48.

[2] Abbas, A.M., and Manohar, C.S. (2005)."Reliability-based critical earthquake load models. Part1:Linear Structures", Journal of Sound and Vibration, Vol.287, pp.865882.

[3] Abbas, A.M., and Manohar, C.S. (2002)."Critical spatially-varying earthquake load models for extended structures", Journal of Structural Engineering, ASCE, Vol.29, pp.39-52.

[4] Ang, A.H.S., and Tang, W.H. (1975). “ Probability concept in engineering planning and design basic principles", Vol. 1, John Wiley and Sons, New York.

[5] Abdel Ghaffar, A.M. and Nazmy, A.S. (1991). "3-D Nonlinear Seismic Behaviour of Cable Stayed Bridge" Journal of Structural Engineering, ASCE, Vol. 117, No. 11, pp. 3456-3476.

[6] Abdel Ghaffar, A.M., and Nazmy, A.S. (1987). "Effect of Three Dimensionality and NonLinearity on the Dynamic and Seismic Behaviour of Cable Stayed Bridges", Bridges and Transmission Line Structures, Proceedings of Structures Congress, ASCE, Orlando, Florida, USA.

[7] Bolotin, V.V. (1965). "Statistical Methods in Structural Mechanics”, STROIIDAT, Moscow (Translated by M.D. Friedman, Lockheed Missiles and Space Company).

[8] Crandall, S.H., and Mark, W.D. (1963). "Random Vibrations in Mechanical Systems", Academic Press, New York.

[9] Chern, C.H. (1976). "Reliability of Structure under Random Earthquake Motion”, M.Eng. Thesis No. 952, Asian Institute of Technology, Bangkok.

[10] Clough, R.W., and Penzien, J. (1975). "Dynamics of Structures" , McGraw Hill, New York.

[11] Clough, R.W., and Penzien, J. (1993). “Dynamics of Structures" , McGraw Hill, New York.

[12] Harichandran, R.S., Hawwari, A. and Sweidan, B.N.(1996)."Response of Long-Span Bridges to Spatially Varying Ground motion", Journal of Structural Engineering, Vo.122(5), pp/476-484. 
[13] Hong Hao (1998). “A Parametric Study of the Required Seating Length for Bridge Decks During Earthquake", Earthquake Engineering and Structural Dynamics, Vol. 27, pp. $91-103$.

[14] Hindy, A., and Novak, M. (1980). "Earthquake Response of Buried Insulated Pipes", Proc. ASCE, J. Engg. Mech. Div., Vol.106, pp.1135-1149.

[15] Kangan, Y.Y. (2002a)."Seismic moment distribution revisited: I. Statistical Result", Int. Journal of Geo-physical, Vol.148, pp.520-541.

[16] Kiureghian, A.D.(1996)."A coherency model for spatially varying ground motions", Earthquake Engineering \& Structural Dynamics, Vol.29, pp.99-111.

[17] Konishi, I. (1969). "Safety and Reliability of Suspension Bridges", Proc. .First Int. Conf. on Structural Safety and Reliability (ICOSSAR), Ed. A.M. Freudenthal, Washington D.C.

[18] Lin, Y.K. (1967). "Probabilistic Methods in Structural Dynamics", McGraw-Hill, New York.

[19] Morris, N.F. (1974). "Dynamics Analysis of Cable -Stiffened Structures", Journal of the Structural Division, ASCE, Vol.100, No.ST5, pp.971-981.

[20] Mebarki, A., Lorrain, M., and Bertin, J. (1990). “ Structural Reliability Analysis by a New Level - 2 Method: The Hypercone Method", Structural Safety, Vol. 9, No.1, pp. 91 103.

[21] Nigam, N.C. and Narayanan, S.(1994). "Applications of Random Vibrations",Narosa Publishing House, New Delhi.

[22] Penzien, J., and Watable, M. (1975). “ Characteristics of 3-Dimensional Earthquake Ground Motions", Earthquake Engineering and Structural Dynamics, Vol. 3, pp. 365-373.

[23] Vanmarcke , E.H. (1975). "On the Distribution of the First -Passage Time for Normal Stationary Random Processes", Journal of the Applied Mechanics, Transactions of ASME, Vol.42, Paper No. 75-APMW-12, pp.215-220.

[24] Schueller, G.I. (1987) "Structures under Wind and Earthquakes Loading -Design and Safety Considerations" , A Short Course taught at Civil Engineering Department, Chulalongkorn University, Bangkok.

[25] Solomos ,G.P., and Spanos, P.T.D. (1982), "Structural Reliability under Evolutionary Seismic Excitation", Proc. First Int. Conf. on Soil Dynamics and Earthquake Engineering,U.K.(Published in Soil Dynamics and Earthquake Engineering „,Vol.2, No.2).

[26] Soyluk, K., and Dumanoglu, A.A. (2000). "Comparison of Asynchronous and Stochastic Dynamic Response of a Cable Stayed Bridge", Engineering Structures, Vol. 22, pp. $435-445$.

[27] Zhu, T. L. (1993). "A cell Technique for Computing the Failure Probability of Structural Systems", Computers and Structures, Vol. 46, No. 6, pp. 1001 - 1005. 


\title{
Compound Stochastic Seismic Vulnerability Analysis and Seismic Risk Evaluation for Super Large Cable-Stayed Bridge
}

\author{
Feng Qing-Hai ${ }^{1}{ }^{2}$, Yuan Wan-Cheng ${ }^{1}$ and Chih-Chen Chang ${ }^{3}$ \\ ${ }^{1}$ State Key Laboratory of Disaster Reduction in Civil Engineering, \\ Tongji University, Shanghai \\ ${ }^{2}$ CCCC Highway Consultants CO., Ltd. Beijing \\ ${ }^{3}$ Department of Civil and Environmental Engineering, Hong Kong University of \\ Science and Technology, Clear Water Bay, Kowloon, Hong Kong,
}

China

\section{Introduction}

Previous seismic disasters indicate bridge structures are the most vulnerable component of the road transportation system in seism, such as the Haiti earthquake and Tohoku earthquake. Bitter lesson of bridge damage leads to the development of seismic analysis theories for bridge such as seismic vulnerability analysis.

Generally, seismic vulnerability is the probability of different damage in different seismic levels, which combines the intensity measure of seism with damage index for bridge structure. Methods of seismic vulnerability analysis include expert vulnerability analysis, experience vulnerability analysis and theory vulnerability analysis. Nevertheless, only seismic randomness has been taken into account in most seismic vulnerability analysis (e.g. Shinozuka et al. 2000; Kiremidjian et al. 1997; Basoz et al. 1997; Yamazaki et al. 2000). Apart from seismic randomness, bridge structure parameters are also stochastic, such as material properties, bridge geometry, boundary condition and so on, which cause the randomness of structure seismic response. Therefore, seismic vulnerability for bridge structure should be determined by both the randomness of seism and that of structure parameters. Due to the complexity of bridge structure system, it is very difficult to gain the analytic solution of seismic response for stochastic structure. Since the stochastic numerical simulation is time consuming and inefficient, this method is suspended at the threshold of thought.

Now, based on traditional seismic vulnerability analysis method, Artificial Neural Network (ANN), Monte Carlo (MC) technologies combining with Incremental Dynamic Analysis (IDA) and PUSHOVER method, Compound Stochastic Seismic Vulnerability Analysis (CSSVA) method is developed to take both randomness of material and that of seism into account from the point of total probability, which not only gives full play to the ANN, MC, IDA and PUSHOVER,but also increases the the efficiency of analysis greatly (Feng Qing-hai 2009).

Generally, the damage of bridge might lead to more serious results and secondary damage than that of road, and the seismic risk level of bridge determines that of the whole road transportation system. Due to the above mentioned reasons, more and more scholars pay attention to the bridge seismic risk evaluation. 
Safety is relative, risk is absolute. So far, bridge structure risk evaluation is only limited to that of transportation, maintenance, management and so on. Since paper or reports for seismic risk evaluation for bridge structure performance is few, the only essential aspect for bridge seismic risk evaluation by those paper or reports is the seismic risk according to the damage probability of a determined bridge structure (e.g. Furuta et al. 2006; Hays et al. 1998; Padgett et al. 2007). However, that is unilateral. The truth is the randomness of material and that of seism exist at the same time. Moreover, the bridge damage probability according to the seismic vulnerability is gained within seism happening. In order to overcome this shortage, a method of seismic risk evaluation based on IDA and MC is presented, which has taken the difference of site type, randomness of time, space and intensity into consideration. It has indeed reflects the seismic risk situation within any years.

So, by the aforementioned method for seismic vulnerability and risk assessment, more real performance state and seismic risk lever are gained, which are good for design, maintenance and earthquake insurance of long span bridge.

\section{Method of compound stochastic seismic vulnerability analysis}

\subsection{Basic theory}

The Damage Index(DI) of bridge is affected by many uncertainties such as randomness of parameters of structure, seism and so on, which makes it a complicated process in gaining the seismic vulnerability curve. The capability of bridge structure could be expressed in the form of $R(M, G, C, \ldots .), M, G,$.$C stand for variety of material, size and calculation methods,$ respectively. At the same time, the seismic response could be expressed as $P(I M)$. So, DI is the systemic combination of $R(M, G, C, \ldots .$.$) and P(I M)$, which is shown as following.

$$
D I=f(R(M, G, C, \ldots \ldots .), P(I M))
$$

Clearly, DI is also variable.

Easy to see, the calculation of DI for bridge structure is divided into several parts, the first is the statistic for capability of bridge structure itself, the second is the statistic for seismic response of bridge structure, and the final is the combination of them. The flow chart is shown in Figure 1.

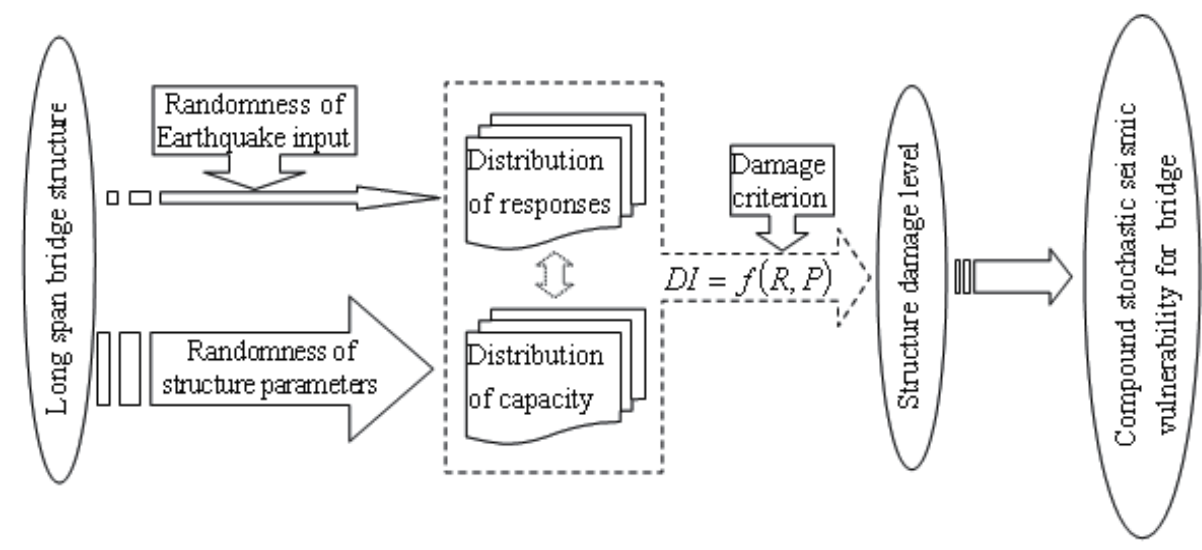

Fig. 1. Flow Chart for Compound Stochastic Seismic Vulnerability Analysis 


\subsection{Statistic for capability of bridge structure}

Statistic for capability of bridge structure is analyzed based on method of PUSHOVER combining ANN and MC. The analysis process is shown in the following. Radial Basic Function Neural Network (RBFNN) is adopted in this method according to the conclusion of reference ( Feng Qing-hai 2007).

1. Main parameters which affect the capability of bridge structure most are analyzed. Distributions for each parameter are determined, too.

2. By the method of orthogonal design, $A+B$ groups of finite element models of bridge structures are built.

3. The response of capability is derived by the method of PUSHOVER.

4. RBFNN is built, and trained by responses of A groups and checked by that of B groups.

5. Go on if the result of Step 4 is successful, or rebuild RBFNN from Step 4.

6. Plenty of responses of capability are simulated by inputting large number of structure model parameters generated by the method of MC and RBFNN.

7. The characteristic of capability of bridge structure is gained by statistics on all those responses of capability.

The analysis flow chart is shown in Figure 2.

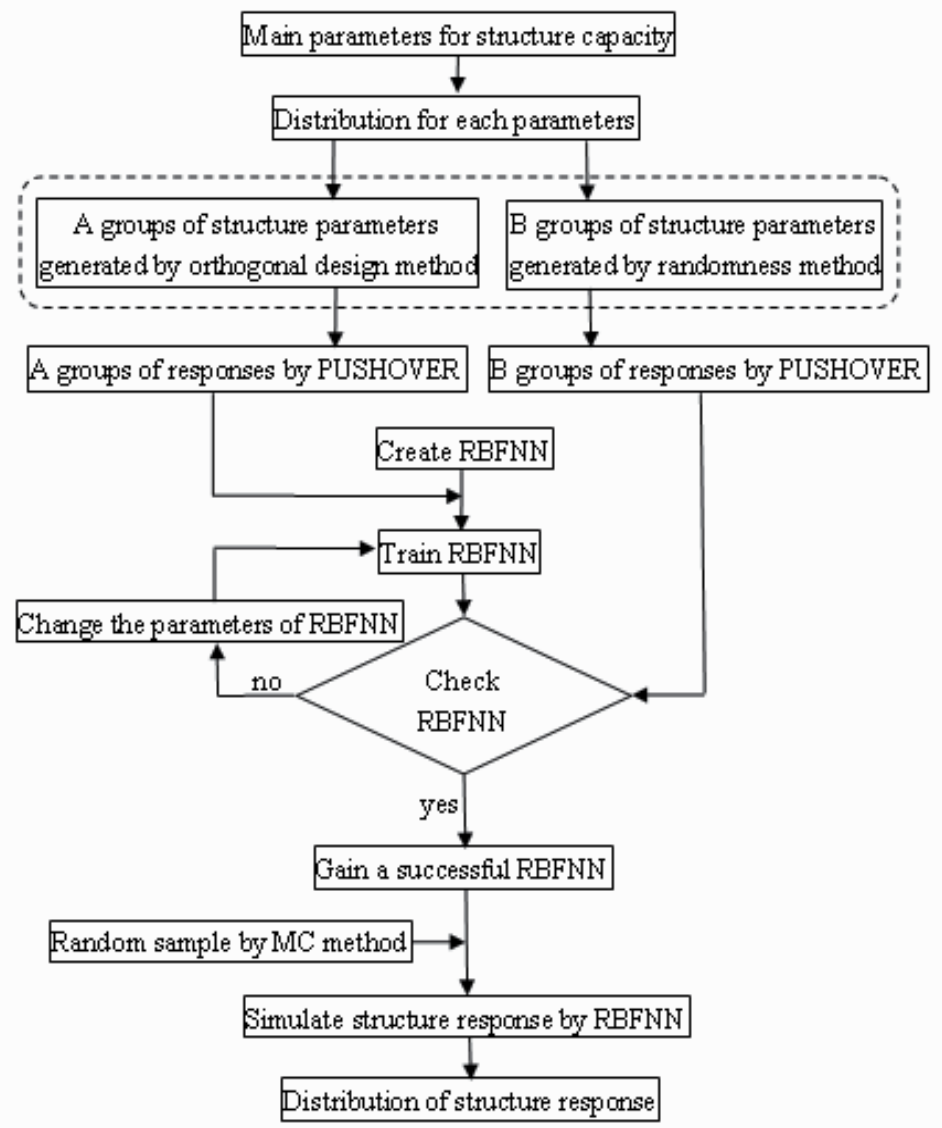

Fig. 2. Flow Chart for Stochastic Structure Capacity analysis 


\subsection{Statistic for seismic response of bridge structure}

Seism is a ground motion of great randomness which might happen at anytime and anywhere. Therefore, it is very necessary to analyze the seismic response from the view of probability. While, the variability of bridge structure itself has a small effect on the seismic response when the randomness of both structure and ground motion are considered ( $\mathrm{Hu} \mathrm{Bo}$ 2000). In order to simplify the calculation method and reduce calculation time, only seismic randomness is taken into account in the statistic for seismic response of bridge structure in this paper.

Statistic analysis of seismic response is performed based on the method of IDA under multiearthquake waves. The analysis process is shown in the following.

1. The finite element model of bridge structure is built.

2. Multi-earthquake waves are selected, and scaled to different intensities by scale factors (SF).

3. IDA is performed. Plenty of seismic responses are gained.

4. The characteristic of seismic responses of bridge structure is gained by statistic analysis on all those seismic responses.

The analysis flow chart is shown in Figure 3.

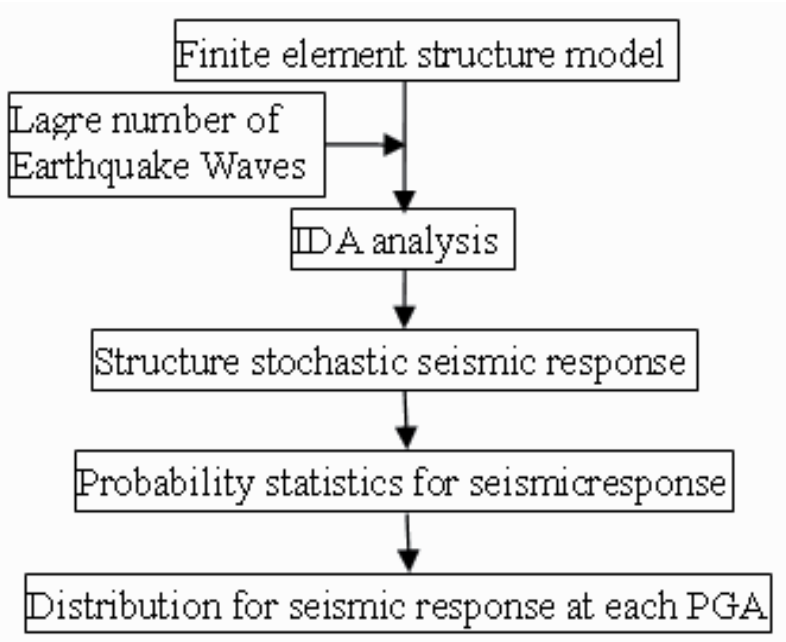

Fig. 3. Flow Chart for Stochastic Seismic Response Analysis 


\subsection{Methodology of CSSVA for bridge structure}

Based on the theories mentioned in above sections, the distribution characteristic of capability and seismic response of bridge structure, CSSVA is performed combining with ANN-MC technology. The analysis process is shown in the following.

1. Get the distributions of capability and seismic responses of bridge structure, respectively.

2. Generate adequate numbers of capability and seismic responses by the method of MC and orthogonal design based on the distribution characteristic got in Step 1.

3. Two RBFNN are built, one for capability and the other for seismic response, then trained and checked by the data gained in Step 2.

4. Go on if the result of check is successful, or rebuild RBFNN from Step 3.

5. A large number of DI are gained according to Equation 1.

6. Select the damage criterion.

7. Compare DI with the damage criterion. The probability of damage is calculated at each IM.

8. The seismic vulnerability curves of bridge structure are drawn.

The analysis flow chart is shown in Figure 4.

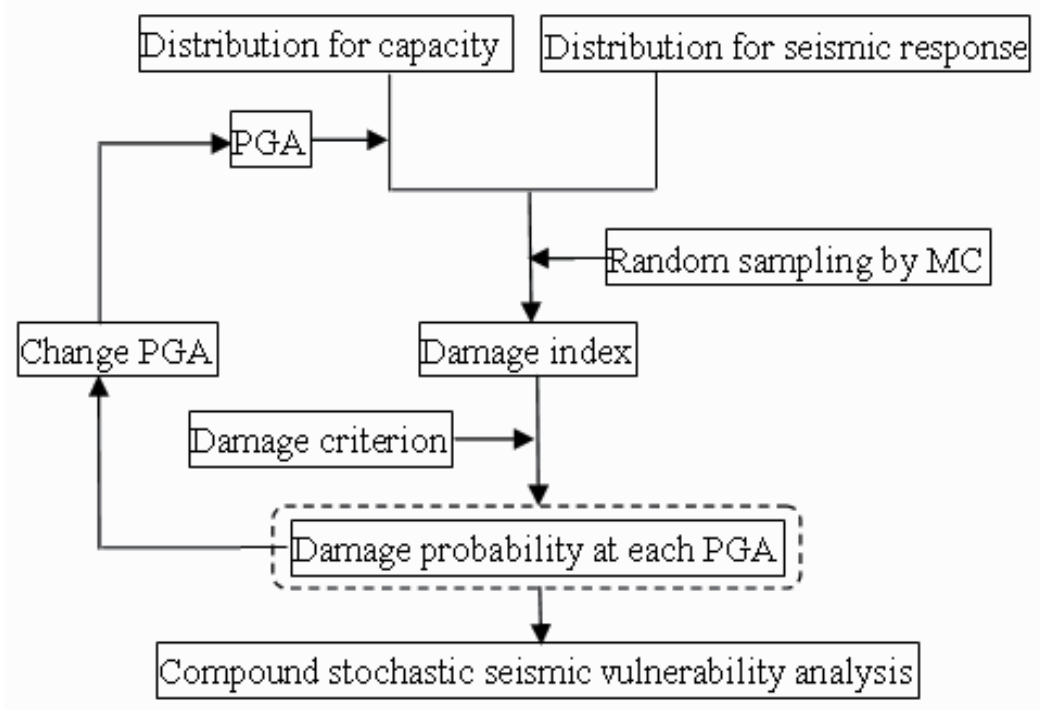

Fig. 4. Flow Chart for Compound Stochastic Seismic Vulnerability Analysis 


\subsection{Effect on seismic vulnerability by compound stochastic}

Based on CSSVA, some examples are performed. For the limited space of paper, only part of results is drawn to display the effect on seismic vulnerability by compound stochastic. Seismic vulnerability curve of determination structure and that of compound stochastic are shown in Figure 5.

Easy to see, when structure stochastic are neglected, seismic vulnerability curves, which are gained by the method of two order spline curve fittings, are folded. Meanwhile, the curve deviate to the vulnerability point distinctly. By comparison, vulnerability curves of compound stochastic are smooth, and are getting through every vulnerability point. For the two curves, the PGA for beginning damage is the same. However, when damage probability is less than $80 \%$, vulnerability value of determination structure is bigger than that of compound stochastic. When damage probability ranges from $80 \%$ to $95 \%$, the contrary is the case.

In all, vulnerability curve of determination structure spreads around that of compound stochastic. Besides, the structure stochastic indeed affects the seismic vulnerability, although not too enormous. Compound stochastic reflects the seismic vulnerability of bridge structure better.
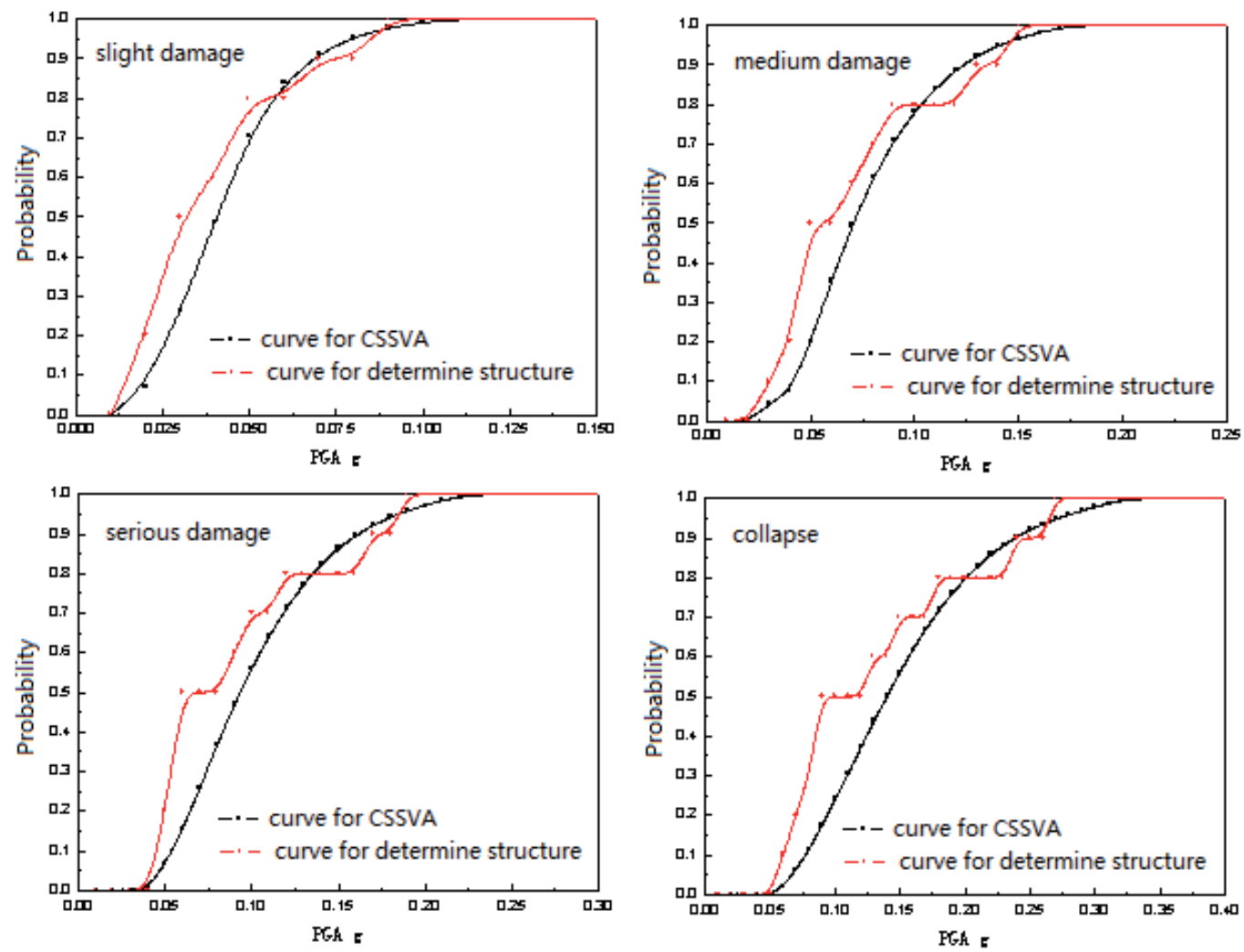

Fig. 5. Effect on Seismic Vulnerability by Compound Stochastic 


\section{Seismic risk probability evaluation for long span bridge}

\subsection{Concept for probability evaluation of seismic risk}

Bridge seismic risk analysis involves two aspects, namely the probability of event occurrence and that of event consequence. The consequence mainly describes the damage severity and has no relationship with structure analysis. Risk analysis, usually said, is the probability of event occurrence without considering the consequence.

Seismic risk probability evaluation for bridge is one of the basis of risk analysis, which also involves two aspects, namely seismic risk and structure seismic vulnerability. Seismic risk probability evaluation for bridge could be expressed as following: the probability of different damage states with consideration of earthquake dangerous. It indicates that the reliability of bridge is threatened by both seism and structure seismic vulnerability during the same determined period.

Seism is regarded as a risk event, the sign is $H$. According to structure reliability thought, limit state function could be expressed as following (Alfredo H-S. ANG \& Wilson H.Tang, 2007).

$$
Z=R-S
$$

in which, $Z$ is the performance function, $R$ is the comprehensive resistance, $S$ is comprehensive response on structure induced by risk events. Then, the damage probability is expressed by expression as following.

$$
P=P(R<S)=\int_{R}^{\infty} f(S) d S
$$

in which, $f(S)$ is the probability density function of comprehensive effect induced by risk events. Clearly, $S$ is closely related to $H$. The $f(S)$ should be expressed in the form of $f(S, H)$, namely the joint density function.

$$
f(S, H)=f(S \mid H) f(H)
$$

in which, $f(S \mid H)$ is the conditional probability density function for different damage states under given risk event $H . f(H)$ is the probability density function of risk event $H$. So, $f(S)$ is expressed as following.

$$
f(S)=\int_{-\infty}^{+\infty} f(S \mid H) f(H) d H
$$

then, the expression combining Equation 5 and Equation 3 is as following.

$$
\begin{gathered}
P=p(R<S)=\int_{R}^{+\infty}\left[\int_{-\infty}^{+\infty} f(S \mid H) f(H) d H\right] d S=\int_{0}^{+\infty}\left[\int_{R}^{+\infty} f(S \mid H) d S\right] f(H) d H \\
P=\int_{0}^{+\infty} F_{S}(H) f(H) d H
\end{gathered}
$$

briefly,

$$
P=\int_{0}^{+\infty} F_{S}(H) f(H) d H
$$


in which, $F_{S}(H)$ stands for $\int_{R}^{+\infty} f(S \mid H) d S$.

\subsection{Method of seismic risk probability evaluation for bridge based on IDA-MC}

In order to avoid complicated calculation, IDA is applied in association with MC. The specific procedure is interpreted at the following sections.

\subsubsection{Probability distribution of seismic intensity and according PGA}

Seismic dangerous analysis is the basis of seismic risk probability evaluation, which reflects the probable maximum effect of seismic damage for a bridge in a district within determined coming period. The effect could be depicted in many ways. Since bridges designed in China are based on the design criterion of seismic intensity, probability distribution of seismic intensity introduced in reference (Gao Xiao-wang et al. 1986) is adopted as the seismic dangerous, namely:

$$
F_{I I I}(x)=\exp \left(-\left(\frac{\omega-x}{\omega-\varepsilon}\right)^{K}\right)
$$

in which, $\omega$ is the upper limited value of intensity, usually equals to 12 . $\varepsilon$ usually satisfied with the equation of $1-e^{-1}=0.632$. $K$ is form parameter. From the point of engineering application, $K$ equals to the value of intensity according to the seismic probability of $10 \%$.

If the probability distribution of seismic intensity for 50 years is determined, then the probability distribution in any limited period could be expressed as the following.

$$
F_{i}(i)=\left[F_{T}(I)\right]^{t / T}=\left[\exp \left(-\left(\frac{\omega-i}{\omega-\varepsilon}\right)^{K}\right)\right]^{t / T}=\exp \left(-\frac{t}{T}\left(\frac{\omega-i}{\omega-\varepsilon}\right)^{K}\right)
$$

According to the existing seismic records, PGA according to a determined intensity are of big discreteness. In order to be convenient for calculation, $A$ has the relationship to $I$ as following.

$$
A=10^{(I \cdot \log 2-0.01)}
$$

in which, $A$ and $I$ is the value of PGA and seismic intensity, respectively. The unit for $A$ is $\mathrm{gal}$. Here, it is very necessary to illuminate that, $A$ is a continuous value only for the necessary of statistic.

\subsubsection{Method and steps}

1. To build a finite element analysis model of bridge structure, and choose enough seismic waves.

2. IDA is performed. Seismic response according to every wave is recorded to form IDA curve.

3. Determine design reference period (prior to a small one) and the probability distribution.

4. According to MC, a great lot of $I$ are generated.

5. According to Equation 11, the same number of $A$ are gained based on Step 4 . 
6. Based on $A$, corresponding seismic responses are gained from the IDA curve.

7. Structure damage probability is gained by statistics based on damage criterion.

The analysis procedures are shown in Figure 6.

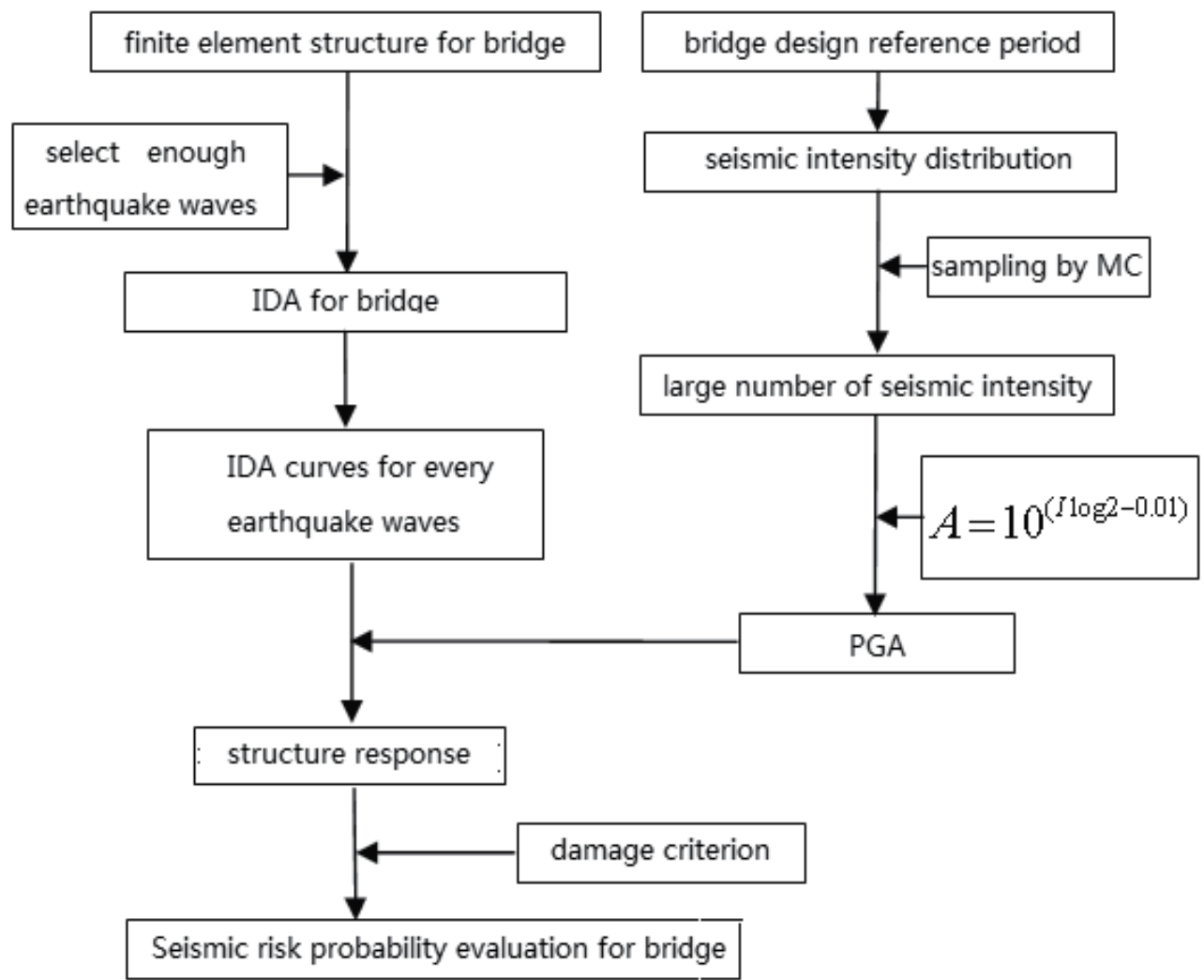

Fig. 6. Seismic risk probability evaluation for bridge

\subsection{Example analysis}

\subsubsection{Analysis model for floating system cable-stayed bridge}

According to reference (Yan Hai-quan and Wang Jun-jie 2007), floating cable-stayed bridge could be simplified to a main tower with a lumped mass on the top of main tower, and the tolerance of results are acceptable. In this section, simplified models are adopted and only longitudinal cases are analyzed. The refined finite element model for main tower is built by OpenSees as shown in Figure 7, main tower is divided into 3 parts, namely upper tower, middle tower and low tower. In consideration of the strengthening at the upper tower by steel pile casting, the upper tower is regarded elastic. Fiber element is adopted in middle tower and low tower.

Main tower is $300 \mathrm{~m}$ in height, $90 \mathrm{~m}$ for upper tower, $150 \mathrm{~m}$ for middle tower and $60 \mathrm{~m}$ for low tower, respectively. Tower is divided into 30 elements of 10 meters in vertical direction, and every element and joint is numbered from 1 to 30 and 1 to 31 , respectively. The bridge site belongs to $3^{\text {rd }}$ type, the seismic intensity is 8 and design period is 50 years. 


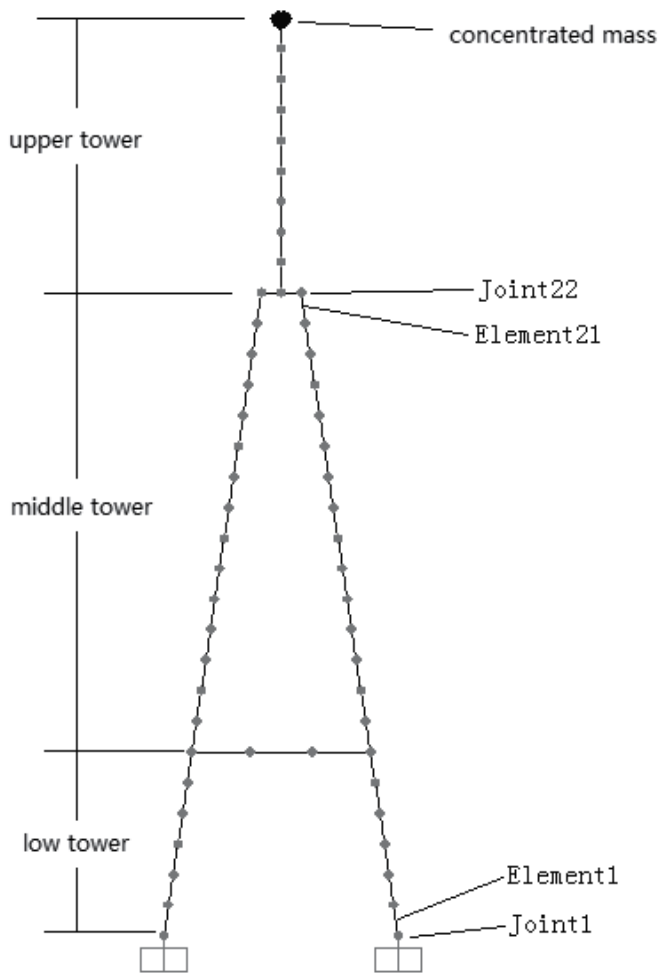

Fig. 7. Finite element model for main tower

\subsubsection{Damage criterion}

Double control damage criterion put forward by Park and Ang are adopted, which is shown as following:

$$
D I=f\left(\delta_{m}, \delta_{u}, Q_{y}, \int d E_{h}, \beta\right)=\frac{\delta_{m}}{\delta_{u}}+\frac{\beta}{\delta_{u} Q_{y}} \int d E_{h}
$$

in which, the signal and suggestion value are illuminated particularly in reference (Fan Lichu and Zhuo Wei-dong 2001). The relationship between damage levels, damage state and DI described by Park, Ang and Wen (H. Hwang et al. 2001) is shown in Table 1.

\begin{tabular}{lll}
\hline Damage level & Damage character & Park-Ang DI \\
\hline 1 no damage & Some slight fracture at local part & $D I<0.1$ \\
\hline 2 slight damage & Slight fracture distributed widely & $0.1 \leq D I<0.25$ \\
\hline 3 medium damage & Serious fracture or spall partly & $0.25 \leq D I<0.4$ \\
\hline 4 serious damage & Concrete crushed or steel break & $0.4 \leq D I<0.8$ \\
\hline 5 collapse & collapse & $D I \geq 0.8$ \\
\hline
\end{tabular}

Table 1. Damage Character and Damage Index at Each Level 


\subsubsection{IDA for main tower}

Only longitudinal case is studied. IDA is performed with PGA being scaled from $0.1 \mathrm{~g}$ to $1.0 \mathrm{~g}$ with step of $0.1 \mathrm{~g}$. Seismic responses such as moment, curvature, hysteretic energy are recorded. Representative response distributions along main tower are described in Figure 8, 9,10 , respectively..

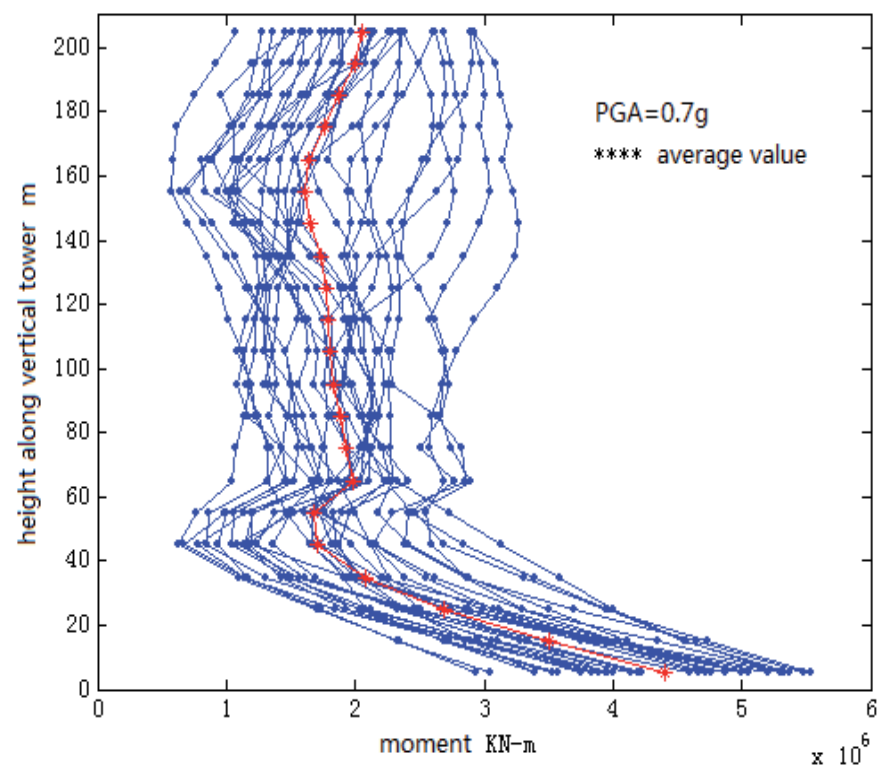

Fig. 8. Moment distribution along vertical tower

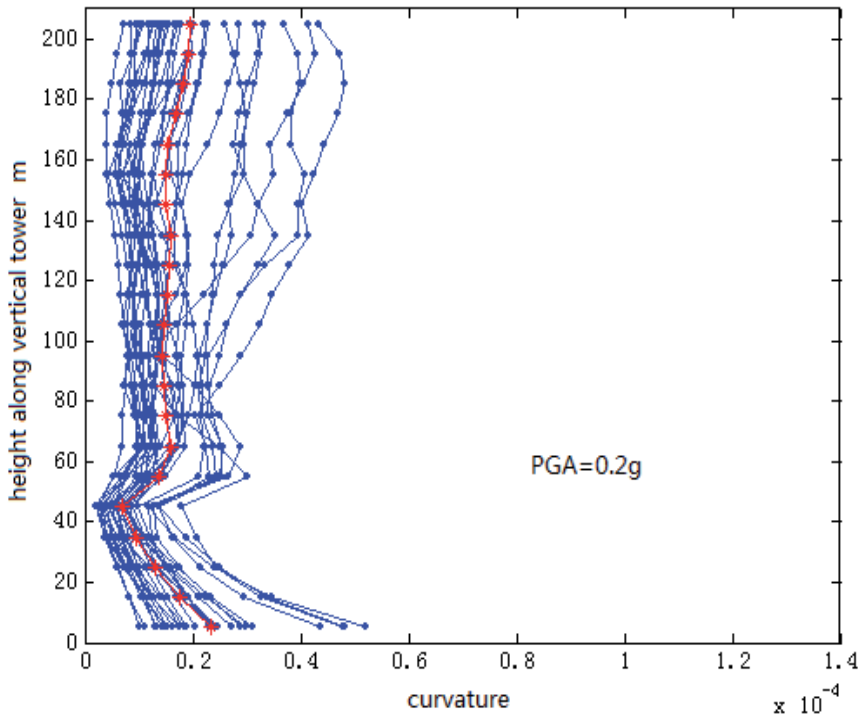

Fig. 9. Curvature distribution along vertical tower 


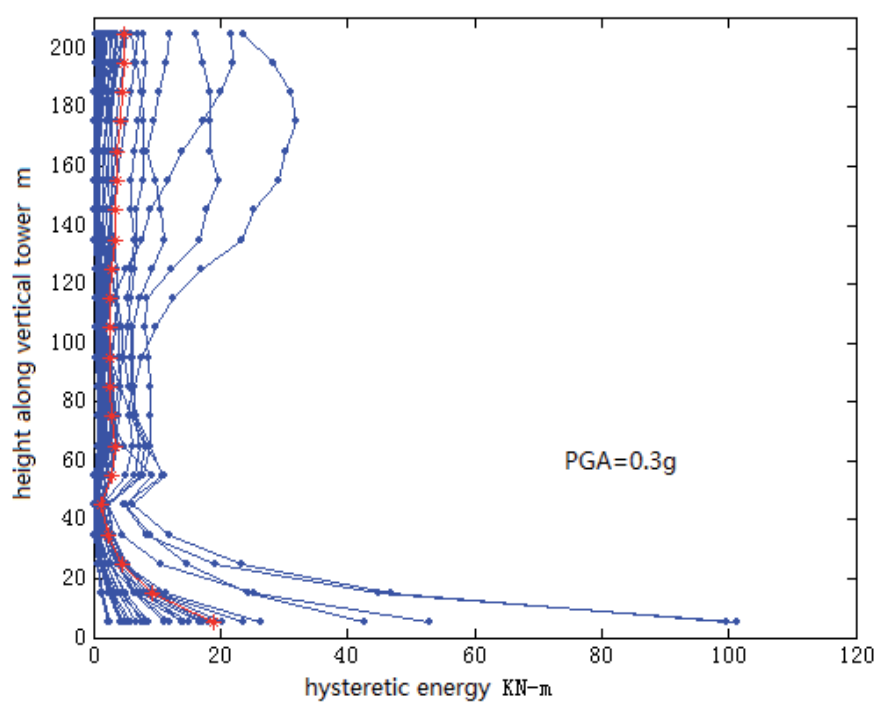

Fig. 10. Hysteretic energy distribution along vertical towe

\subsubsection{Stochastic sampling for PGA}

According to reference (Ye Ai-jun 2002) combining the site type, design period and seismic intensity, $K$ is determined as following:

$$
1-0.1=\exp \left(-\left(\frac{12-8}{12-6.45}\right)^{K}\right)
$$

According to Equation 13 , the $K=6.87$. Then, the seismic intensity probability distribution within $t$ years is

$$
F_{t}(i)=\exp \left(-\frac{t}{50}\left(\frac{12-i}{5.55}\right)^{6.87}\right)
$$

When $\mathrm{t}=1$, and according to the method of $\mathrm{MC}, 50000$ random intensity value are generated, numbered Rand(1) to Rand(50000). Then, the according PGA numbered from RandPGA(1) to RandPGA(50000) which reflect the seismic random from time, space and intensity are gained as following.

$$
\operatorname{RandPGA}(i)=10^{(\operatorname{Rand}(i) \log (2)-0.01)}
$$

\subsubsection{Bridge structure seismic risk probability evaluation within 1 year}

Based on the stochastic PGA and IDA curve, responses for every element in any stochastic PGA are gained, the total number is 560000 . Based on the damage criterion, the damage time for every element in different damage levels are accounted. For example, the damage number for slight damage at the bottom of main tower is 7649 . Then the damage probability is

$$
\frac{7649}{560000} \times 100 \%=1.37 \%
$$


Damage probability distribution along main tower in different damage level is shown in Figure 11(a). Easy to see, in different damage levels, the damage probability is different, especially damage probability of the bottom is larger than that of the others, there are two main reasons for this phenomenon as following:

Firstly, the main tower is the most important component of large long span bridge, any damage could cause devastating damage for the bridge. So in the design, main tower is conservatively designed. Not only stiffness but also strength is strengthened.

Secondly, when the bottom element begins to be damaged, the upper elements are protected.

Based on the thought of structural reliability, the main tower is a series of structure, so the total damage probability is the biggest one in different damage levels.

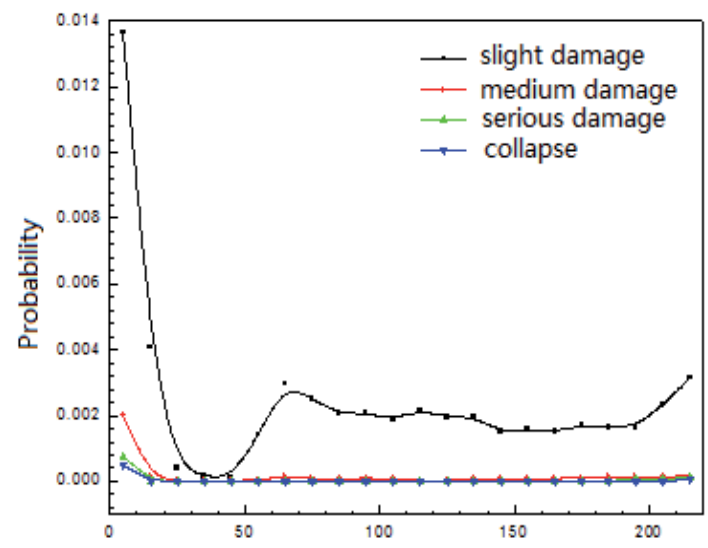

height along vertical main tower $\mathrm{m}$

(a) Damage probability distribution along vertical tower at different states

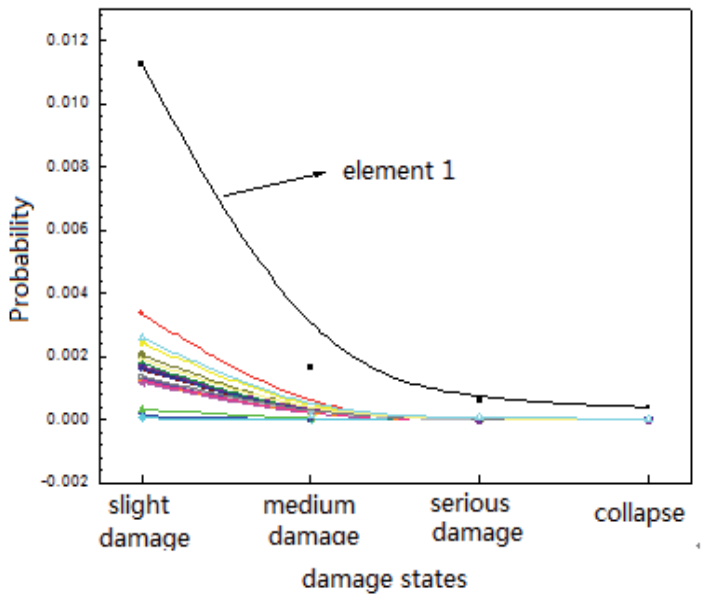

(b) Element damage probability at different damage states

Fig. 11. Element damage probability at different damage states 


\subsubsection{Bridge structure seismic risk probability evaluation within any years}

With different years, the seismic risk is different, and so does the seismic intensity distribution. According to the method as in Section 3.3.5, seismic risk probability from 10 to 100 years with interval of 10 years are calculated, as shown in Figure 11(a).

In order to get a reasonable cognition, risk levels are shown in Table 2. Combining figure 11(a) with Table 2(Basoz, Nesrin, Kiremidjian \& Anne S, 1997), the log curve for main tower seismic risk is shown in Figure 11(b).

\begin{tabular}{cccc}
\hline Grade & Risk Description & Probability Range & Intermediate Value \\
\hline 1 & Very unlikely & $<0.0003$ & 0.0001 \\
\hline 2 & Impossible & $0.0003-0.003$ & 0.001 \\
\hline 3 & Occasional & $0.003-0.03$ & 0.01 \\
\hline 4 & Possible & $0.03-0.3$ & 0.1 \\
\hline 5 & Very likely & $>0.3$ & 1 \\
\hline
\end{tabular}

Table 2. Risk Probability Description

Easy to see from Figure 11(b), for main tower, within 1 year, slight damage and medium damage is impossible, and serious damage will never happen. Within other years, especially over 30 years, slight damage is possible, medium damage and serious damage will be occasional, and collapse will never happen.

\section{Conclusion}

Compound stochastic seismic vulnerability analysis for bridge structure is presented, in which double damage criterion is adopted. The effect of compound stochastic on seismic vulnerability are analyzed, which proves that, compound stochastic indeed has little effect on seismic vulnerability. The results show that compound stochastic seismic vulnerability curve reflects the actual situation.

Based on method of MC and results of seismic vulnerability analysis for large long span cable-stayed bridge, seismic risk analysis evaluation for bridges is performed, which has taken difference of site type, randomness of time, space and intensity into consideration. Seismic risk situation within any years for bridge structure is measured by using the experience of situation description for bridge risk evaluation.

\section{Acknowledgements}

This research is supported by Ministry of Science and Technology of China under Grant No. SLDRCE 09-B-08, Kwang-Hua Fund for College of Civil Engineering, Tongji University and the National Science Foundation of China under Grant No.50978194 and No.90915011, and Science and technology project of Ministry of Transport, China. 


\section{References}

Alfredo H-S. Ang \& Wilson H.Tang.(2007). Probability concepts in engineering..

Shinozuka M., Feng, M.Q., Lee, J. \& Naganuma, T. (2000). Statistical Analysis of Fragility Curves[J]. Journal of Engineering Mechanics, ASCE, Vol. 126, No.12, (December, 2000), pp. 1224-1231

Kiremidjian A. S. \& Bosöz, N. (1997). Evaluation of Bridge Damage Data from Recent Earthquakes. NCEER Bulletin, Vol. 11, No. 2, pp. 1-7

Basoz, Nesrin, Kiremidjian \& Anne S. (1997). Risk Assessment of Bridges and Highway Systems from the Northridge Earthquake. Proceedings of the National Seismic Conference on Bridges and Highways: "Progress in Research and Practice", pp. 65-79, Sacramento, California, USA

Yamazaki, F., Motomura, H. \& Hamada, T. (2000). Damage Assessment of Expressway Networks in Japan based on Seismic Monitoring. Proceeding of the 12th World Conference on Earthquake Engineering, pp. 0551, Upper Hutt, New Zealand

Feng Qing-hai (2009). Study on Seismic Vulnerability and Risk Probability Analysis of Super-Large Bridge[D]. Tongji University, Shanghai, China

Furuta H., Katayama H. \& Dogaki M. (2006) • Effects of Seismic Risk on Life-cycle Cost Analysis for Bridge Maintenance. Proceedings of the 4th International Conference on Current and Future Trends in Bridge Design, Construction and Maintenance, pp. 22-33

Hays W.W - (1998). Reduction of Earthquake Risk in the United States : Bridging the Gap between Research and Practice - IEEE Transactions on Engineering Management, Vol.45, No.2, (May 1998), pp. 176-180

Padgett, Jamie E, DesRoches \& Reginald (2007). Bridge Functionality Relationships for Improved Seismic Risk Assessment of Transportation Networks. Earthquake Spectra, Vol.23, No.1, (February 2007), pp. 115-130

Feng Qing-hai, Yuan Wan-cheng (2007). Comparative Study on BP Neural Network and RBF Neural Network in Performance Evaluation of Seismic Resistance for Pier Columns[J]. Structrual Engineers, Vol.23, No.5, pp. 41-47

$\mathrm{Hu}$ Bo (2000) - Study on the Probabilistic Seismic Design Method for Bridges[D] - Tongji University, Shanghai, China

Gao Xiao-wang, Bao Ai-bin (1986). Determination of Anti-Seismic Level by Probabilistic Method[J]. Journal of Building Structures. Vol.3, No.2, pp. 55-63.

Yan Hai-quan; Wang Jun-jie (2007). A Tower Model for Seismic Response Prediction of Floating Cable-stayed Bridge in Longitudinal Direction[J]. Journal of Earthquake Engineering and Engineering Vibration, , Vol.27, No.4, (February 2007), pp. 80-86.

Fan Li-chu, Zhuo Wei-dong (2001). Seismic Design for Ductility of Bridge[M]. Beijing: China Communications Press, 2001

California Office of Emergency Services, Vision 2000: Performance Based Seismic Engineering of Buildings, Structural Engineers Association of California, Sacramento, CA, 1995 
H. Hwang, J.B.Liu \& Y.H.Chiu (2001). Seismic Fragility Analysis of Highway Bridges, MidAmeirica Earthquake Center Technical Report, MAEC-RR-4 Project, 2001

Ye Ai-jun (2002). Bridge Seismic[M]. Beijing: China Communication Press, 2002 


\title{
Assessment and Rehabilitation of Jacket Platforms
}

\author{
Mohammad Reza Tabeshpour ${ }^{1}$, Younes Komachi ${ }^{3}$ and Ali Akbar Golafshani ${ }^{2}$ \\ ${ }^{1}$ Mechanical Engineering Department, \\ ${ }^{2}$ Civil Engineering Department \\ Sharif University of Technology, Tehran \\ ${ }^{3}$ Department of Civil Eng., Pardis Branch, Islamic Azad University, Pardis \\ Iran
}

\section{Introduction}

The most important recent earthquake showed the importance of seismic assessment of both onshore and offshore structures (Takewaki et al., 2011). Strong earthquake can cause damages to engineering structures. Many strong earthquakes normally take place in offshore such as 2011 Japan earthquake (Moustafa, 2011 and Takewaki et al., 2011) can cause severe damage to offshore structures. The steel jacket structure is a kind of fixed offshore platform that is suitable for construction in water depth from a few meters to more than 100 $\mathrm{m}$. Compared to regular structures, a jacket offshore platform is a complicated system and is composed of many parts include structural and nonstructural elements. Structural modeling includes two major division; structure and pile foundation. Fig. 1 shows parts of jacket offshore platform system and some of related researches.

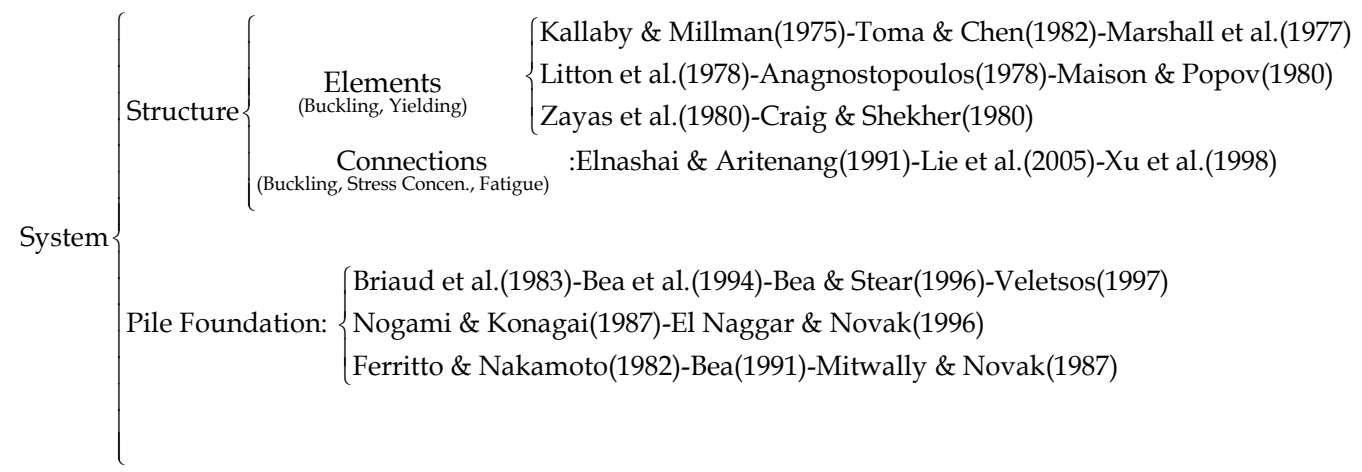

Fig. 1. Parts of jacket offshore platform system.

The major structural components of such an offshore platform are jacket, piles, and deck. A jacket structure which serves as bracing for the piles against lateral loads is fixed by piles driven through the inside of the legs of the jacket structure and into soil many tens of meters deep. The deck structure is fixed upon the jacket structure. Oceans in which offshore 
platforms are built present a set of complicated and harsh environmental conditions. Dynamic loads including wind, wave, current, and earthquakes dominate the design of offshore structures. Venkataramana (1998) presented a time domain analysis of the dynamic response of a simplified offshore structure to simultaneous loadings by random sea waves and random earthquake ground motions. Kawano and Venkataramana (1999) investigate the dynamic response and reliability analysis of offshore structure under the action of sea waves, currents and earthquakes. The dynamic loads affect not only the routine operation of an offshore platform such as drilling and production activities, but also the safety and serviceability of the structure. Approximately 100 template-type offshore platforms have been installed in seismically active regions of the world's oceans. New regions with the potential for significant seismic activity are now beginning to be developed. Older platforms in seismic regions may have three areas of deficiency:

1. Inadequate ground motions for original design.

2. Structural framing which is not arranged or detailed for ductile behavior.

3. Reduced capacity resulting from damage, corrosion or fatigue.

Many of these platforms are now beyond their original design life (20-25 years), as well. From the economic point of view the continued use of an existing installation will in many cases be preferable compared to a new installation. The assessment of existing platforms under environmental (wave, wind, current etc.) loads and probable future loads (earthquake) (Moustafa, 2011) is a relatively new process and has not yet been standardized as design is. This lack of standardization creates some difficulty in establishing performance requirements which must be developed depending upon the risks (i.e., hazards, exposures and consequences) associated with the future operations of the platform. The present criteria of the offshore structure standards for seismic assessment can be improved using building pre-standards.

Assessment of jacket platforms has rarely been studied. Krieger et al. (1994) describe the process of assessment of existing platforms. Petrauskas et al. (1994) illustrate assessment of structural members and foundation of jacket platforms against metocean loads. Craig et al. explain assessment criteria for various loading conditions. Ersdal (2005) evaluates the possible life extension of offshore installations and procedures of standards in this matter, with a focus on ultimate limit state analysis and fatigue analysis. Gebara et al. (2000) assess the performance of the jacket platform under subsidence and perform ultimate strength and reliability analyses for four levels of sea floor subsidence. The assessment process of building prestandards was studied also. Bardakis and Dritsos (2007) compared the criteria of FEMA-356 and GRECO (Based on the EC-8). Hueste and Bai (2007) described the assessment and rehabilitation of an existing concrete building based on the FEMA-356 procedure and criteria. Golafshani et al. (2009) suggested this idea that API procedures for seismic assessment of jacket platforms can be evaluated and improved with respect to building documents for the first time at 2006.

Fig. 2 shows some standards for offshore structures that include detailed procedures for the assessment of existing structures. Petroleum and natural gas industries Offshore Structures Part 1: General Requirements,' ISO (2002) is one of the most general accepted standards. A detailed assessment procedure for existing structures is found in ISO 19902. The Norwegian regulations (PSA 2004) refer to ISO 19900 (ISO 2002) for the assessment of existing structures. Other standards, like API RP2A-WSD (API 2000) and ISO/DIS 13822 (ISO 2000), also include detailed procedures for the assessment of existing structures. 
API RP2A is one of the most useful standards for the design and assessment of offshore structures. Section 17 of this standard has recommendations for the assessment of offshore structures. The assessment criteria of this standard are based on the objective of collapse prevention of the structure under extreme earthquake conditions. The results of an assessment with API give information about the total structure's condition. This standard describes the rehabilitation objective globally and does not present a routine methodology for rehabilitation.

In the last decade, several building documents such as FEMA- 356 and ATC-40 were developed for the assessment and rehabilitation of these structures. In the FEMA-356 document seismic deficiencies identified using an evaluation methodology considering building performance at a certain seismic hazard. The FEMA-356 document developed an extensive assessment and rehabilitation procedure. This document not only has numerical criteria for assessment but also presents design procedures for rehabilitation.

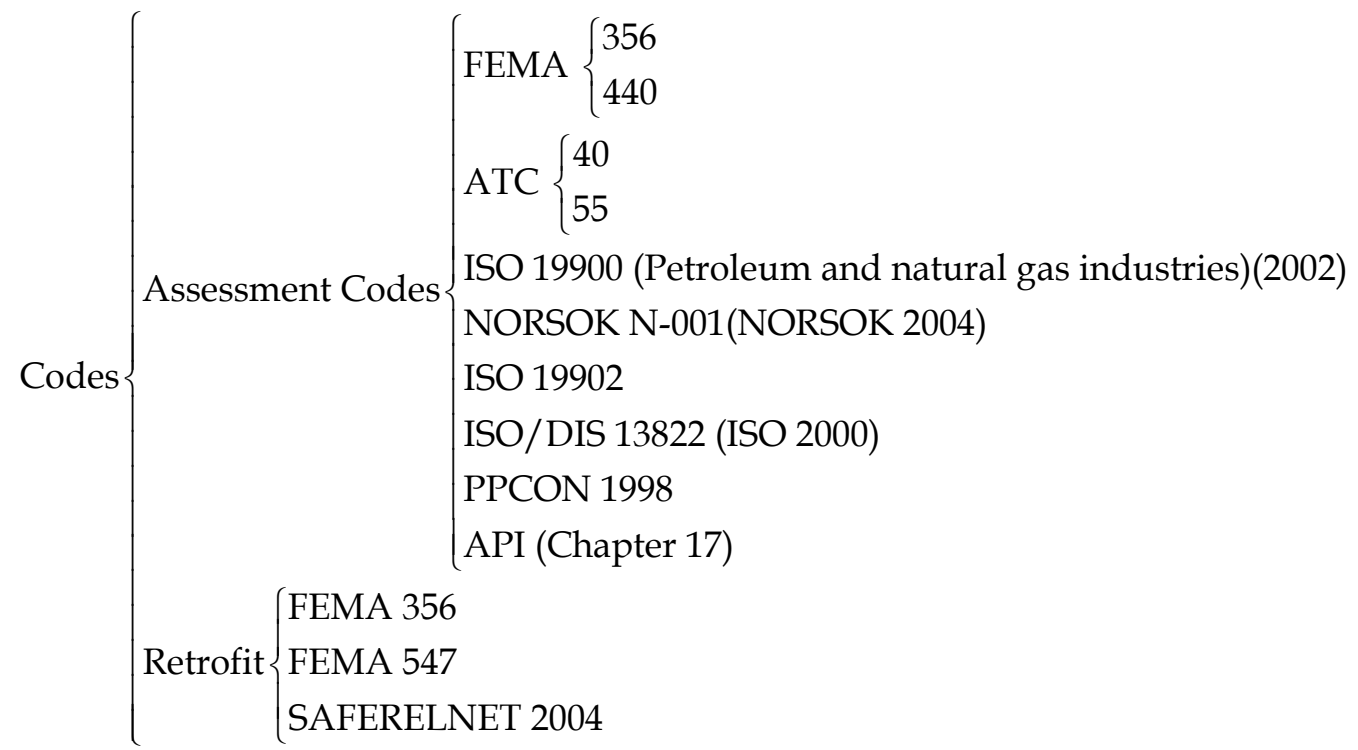

Fig. 2. Some standards for assessment and rehabilitation of jacket offshore platforms

The API approach is based on simple collapse mechanism investigation without describing the methodology in detail. However FEMA-356 consists of detailed processes for both seismic assessment and rehabilitation of building structures. However there are advices in API documents (API RP 2A) in order to seismic assessment of jacket platforms, but because of brief existing comments in this field, it is necessary to use more appropriate pre-standards for seismic assessment of these structures. For example Golafshani et al., (2009) compares FEMA-356 and API approach for assessment of jacket offshore platform structures. Komachi et al., (2009a) presented the performance based assessment of jacket platforms for seismic vulnerability.

Current methods for seismic upgrading of existing structures can be classified into two major groups: traditional and modern. Traditional methods aim to increase the strength and/or ductility of the structure by repairing/upgrading members. Nowadays there are 
some new technologies (for example seismic isolation and energy dissipation) for seismic protection of the structures. The passive control approach is of current concern to many researchers and there are several attempts exploring its application to offshore structures. Recently, although, there have been several studies for the effectiveness of active and passive control mechanisms in controlling the response of offshore platforms under wave loading.

Incorporation of energy dissipation systems in a traditional earthquake-resistant structure has been recognized as an effective strategy for seismic protection of structures (Soong and Dargush, 1997). New vibration control technologies have been applied to offshore structures in the following cases. Vandiver and Mitome (1979) used storage tanks as Tuned Liquid Damper (TLD) on a fixed platform to mitigate the vibration of the structure subjected to random wave forces. Kawano and Venkataraman (1992) and Kawano (1993) studied the application of an active tuned mass damper to reduce the response of platforms due to wave loading. Abdel-Rohman (1996) studied the dynamic response of a steel jacket platform with certain active and passive control due to wave-induced loading. Lee (1997) used stochastic analysis and demonstrated the efficiency of mechanical dampers for an offshore platform. Suneja and Datta (1998) demonstrated the efficiency of an active control system for articulated leg platforms under wave loading. Vincenzo and Roger (1999) developed an Active Mass Damper for suppression of vortex- induced vibrations of offshore structures. Chen et al. (1999) studied the response of a jacket platform installed with TLD due to earthquake loading. Ou et al. (1999) studied the response reduction of jacket platforms with a viscoelastic damper with respect to ice loads. Terro et al. (1999) developed a multi-loop feedback-control design as applied to an offshore steel jacket platform. Suhardjo and Kareem (2001) used both passive and active control systems for the control of offshore platforms. Ding (2001) studied the response reduction of jacket platforms with a viscous damper due to ice loads. Qu et al. (2001) presented a rational analytical method for determining the dynamic response of large truss towers equipped with friction dampers under wind-excitation and investigated the efficiency of friction dampers. Wang (2002) used Magnetorheological dampers for vibration control of offshore platforms for wave-excited response. Mahadik and Jangid (2003) studied the response of offshore jacket platforms with an active tuned mass damper under wave loading. Patil and Janjid (2005) studied the behavior of a platform with viscoelastic, viscous and friction damper for wave loads. Lee et al. (2006) studied the effectiveness of a Tuned Liquid Column Damper (TLCD), which dissipates energy by water flow between two water columns, for offshore structures and also, Ou et al. (2006) studied the application of damping isolation systems for response mitigation of offshore platform structures. Jin et al. (2007) studied the effect of Tuned Liquid Dampers (TLD) and found that the larger the ratio of water-mass to platform-mass, the higher the reduction of responses. Komachi et al., (2009) presented Friction Damper Devices (FDD) as a control system to rehabilitation existing jacket offshore platforms. Golafshani and Gholizad (2009) studied the performance of friction dampers for mitigating of waveinduced vibrations and used mathematical formulation to evaluate the response of the model. Yoe et al. (2009) used Tuned Mass Damper (TMD) for mitigation of dynamic ice loads.

The service life of an offshore structure can be doubled if the dynamic stress amplitude reduces by $15 \%$. Few studies have reported on the effectiveness of the passive control 
systems using dampers in controlling the response of offshore platforms under a parametric variation studying the influence of important system parameters and comparative performance of dampers. In order to reduce possible damage to jacket offshore platforms in harsh marine environments, the necessity of carrying out further studies on developing efficient and practical vibration control strategies for the suppression of dynamic responses of existing offshore structures should be emphasized. In this chapter rough and global comments of API are compared with detailed method of FEMA. As an example seismic assessment of the existing 4 legged Service platform placed in the Persian Gulf is presented. A very useful method for rehabilitation of existing jacket platforms is damper. In this study, a Friction Damper Device (FDD) proposed by Mualla (2002) is used to mitigate the vibration of a typical fixed jacket offshore platform in Persian Gulf. The contents of this study mainly include the investigation of the influence of the damping system parameters on vibration control of offshore platforms under the actions of earthquake excitations. This chapter shows that FDD improves the structural behavior and performance of jacket platforms.

\section{Assessment}

\subsection{API recommendations}

The API is currently developing recommendations for the assessment of existing platforms including requirements for platforms subjected to hurricanes, storms, earthquakes and ice loading. These recommendations will likely focus on a demonstration of adequate ductility for platforms located in earthquake dominated regions. The focus towards ductility, or demonstrated survivability, under extreme earthquake conditions is based on the objective of prevention of loss of life and pollution. The performance criterion for assessment is essentially identical to that of the Design Level Earthquake (DLE) requirement for new designs.

The structures need to meet one of two sets of global structural performance criteria, depending on the platform's exposure category. In addition, local structural performance requirements for topside equipment and appurtenances must be met, independent of the platform's exposure category classification. In the case of high exposure platforms, they must be shown by rational analysis (Pushover or Nonlinear Time history) to remain globally stable under median ground motions representative of an earthquake with an associated return period of 1000 years. For lower exposure platforms located in areas with high seismic activity, a return period of 500 years must be selected.

\subsection{FEMA recommendations}

Performance based design (PBD) has been fully described in the guidelines published by FEMA and ATC. These documents do not have the force of codes but provide details of best practice for the evaluation and strengthening of existing buildings. These are continuing to be expanded as PBD becomes more widespread. In these standards a criterion such as drift is applied indirectly when the elements are assessed.

Four levels of building performance consist of Operational (O), Immediate Occupancy $(\mathrm{IO})$, Life Safety (LS), and Collapse Prevention (CP) in increasing levels of damage is considered in FEMA. The Immediate Occupancy (IO) performance level requires that the building remain essentially functional during and immediately after the earthquake. The 
last performance level, Collapse Prevention (CP) will result in a building on the point of collapse and probably economically irreparable. The Life Safety level (LS) is the level usually implicit in codes and may also result in a building which is not economic to repair. The rehabilitation objectives are formed of combinations of earthquake hazard and building performance (consisting of structural and nonstructural performance levels).

FEMA sets a desirable goal for rehabilitation, a Basic Safety Objective (BSO) which comprises two targets:

1. Life Safety building performance at Basic Safety Earthquake 1 (BSE-1), the 475 year earthquake.

2. Collapse Prevention performance at BSE-2, the 2500 year earthquake.

Depending on the function of the structure, other objectives may be set. For example, an enhanced objective may set higher building performance levels at BSE-1 and BSE-2 for critical facilities such as hospitals. Component actions are classified as deformation or force-controlled. Table 1 shows some of the action types. A component acceptance criterion for force-controlled actions is based on the force and is independent of the performance level and components shall have lower-bound strengths not less than the maximum design forces. For deformation-controlled actions the criterion is based on the target performance level and in these components shall have expected deformation capacities not less than maximum deformation demands calculated at the target displacement.

Action

Force-Control

Deformation-Control

Braces in Tension and Compression

\begin{tabular}{ccc}
\hline Columns - Compression & $\checkmark$ \\
\hline & $P<0.5 P_{c r}$ & $\checkmark$ \\
\cline { 2 - 3 } Columns-flexure & $\checkmark>0.5 P_{c r}$ & $\checkmark$ \\
\hline
\end{tabular}

Table 1. Type of action for some elements

FEMA provides modeling parameters and numerical acceptance criteria for beams, columns, and braces as a function of parameters such as diameter to thickness ratio. The leg and brace in the jacket act like a column and brace in the steel braced frame, respectively. Therefore, for assessment of these structures using FEMA, criteria of chapter five of this document will be used.

\subsection{Comparison of API RP 2A and FEMA-356}

In the Table 2 procedures of FEMA and API for assessment are compared to each other. In contrast to API that assesses structures globally, FEMA evaluates each member of the structure for assessment. This matter has some advantages in several manners: 


\begin{tabular}{lcc}
\hline \hline Structure & API RP 2A & FEMA-356 \\
\hline Criteria for & $\begin{array}{c}\text { Jacket } \\
\text { Platform }\end{array}$ & Building \\
\hline Return Period of extreme earthquake & Total structure & $\begin{array}{c}\text { Members and } \\
\text { structure }\end{array}$ \\
\hline $\begin{array}{l}\text { Modeling: } \\
\text { Soil-pile-Structure Interaction } \\
\begin{array}{l}\text { Modeling parameters and Criteria for } \\
\text { members }\end{array}\end{array}$ & 1000 years & 2500 years \\
\hline Simplified procedure for assessment & By details & Global \\
\hline Rehabilitation method & Don't have & Have \\
\hline
\end{tabular}

Table 2. Comparison of API RP 2A and FEMA-356

1. Comparing with buildings, the redundancy of the jacket platform structure is low, and failure of some members can affect not only the routine operation of structure such as production activities, but also the safety and serviceability of the structure. Then the results of FEMA are more reliable and economical than API.

2. Specific criteria can be taken into account for the evaluation of each member with respect to its condition. This matter is more important for a jacket platform in that strength degradation of members due to fatigue and corrosion and etc. is feasible.

3. Rehabilitation of the structure can be performed better knowing the behavior of members.

The return period adopted for collapse requirement ground motions in API RP 2A is lower than that of FEMA-356. Earthquake return periods of FEMA and API are compared to each other in the Table 4. From a point of comparison with FEMA-356, there are four principal reasons why the earthquake return period of API is low:

1. Importance of offshore structures is higher than buildings.

2. Seismic loads imposed on a structure are highly dependent on the stiffness and energy dissipation characteristics of the structural system, including the piling and supporting soils, and so higher uncertainties in soil properties result directly in higher uncertainties in loads.

3. Uncertainties in the estimation of ground motions for offshore structures are higher than those for buildings.

4. Because of lower redundancy, the sensitivity to increase in return period is greater for offshore structures than buildings. This item is an important difference between jacket platforms and buildings.

The FEMA document represents simplified procedures such as a coefficient method that can be used for assessment and rehabilitation of buildings for different loading conditions and this document consists of useful procedures for the rehabilitation of buildings.

\subsection{Response determination using nonlinear pushover analysis}

Nonlinear time-history analysis can be used for assessment and rehabilitation of all types of structures. This procedure is complicated and time consuming. Nowadays nonlinear static procedures are widely used for the assessment and rehabilitation of structures. These procedures can be used to estimate the response of structures under seismic 
loading. The target displacement for each level of load is calculated. The target displacement is intended to represent the maximum displacement likely to be experienced during the design earthquake. The stresses and deformations in each component are then evaluated at this displacement level. FEMA-356 utilizes the Coefficient Method in which several empirically derived factors are used to modify the response of a single-degree-offreedom (SDOF) model of the structure assuming that it remains elastic. The CapacitySpectrum Method of ATC-40 uses empirically derived relationships for the effective period and damping as a function of ductility to estimate the response of an equivalent linear SDOF oscillator. Recently these methods evaluated and improved in the FEMA-440 document [16].

\subsubsection{Capacity-spectrum method (ATC-40)}

In the Capacity-Spectrum Method, the base shear versus roof displacement relationship (capacity) and seismic ground motion (demand) are plotted in Acceleration-Displacement Response Spectrum (ADRS) format. The performance point (maximum inelastic displacement) can be obtained from the intersection point of demand and capacity. This procedure is presented in Fig. 3. In this figure $S_{a}$ and $S_{d}$ are spectral acceleration and spectral displacement respectively.

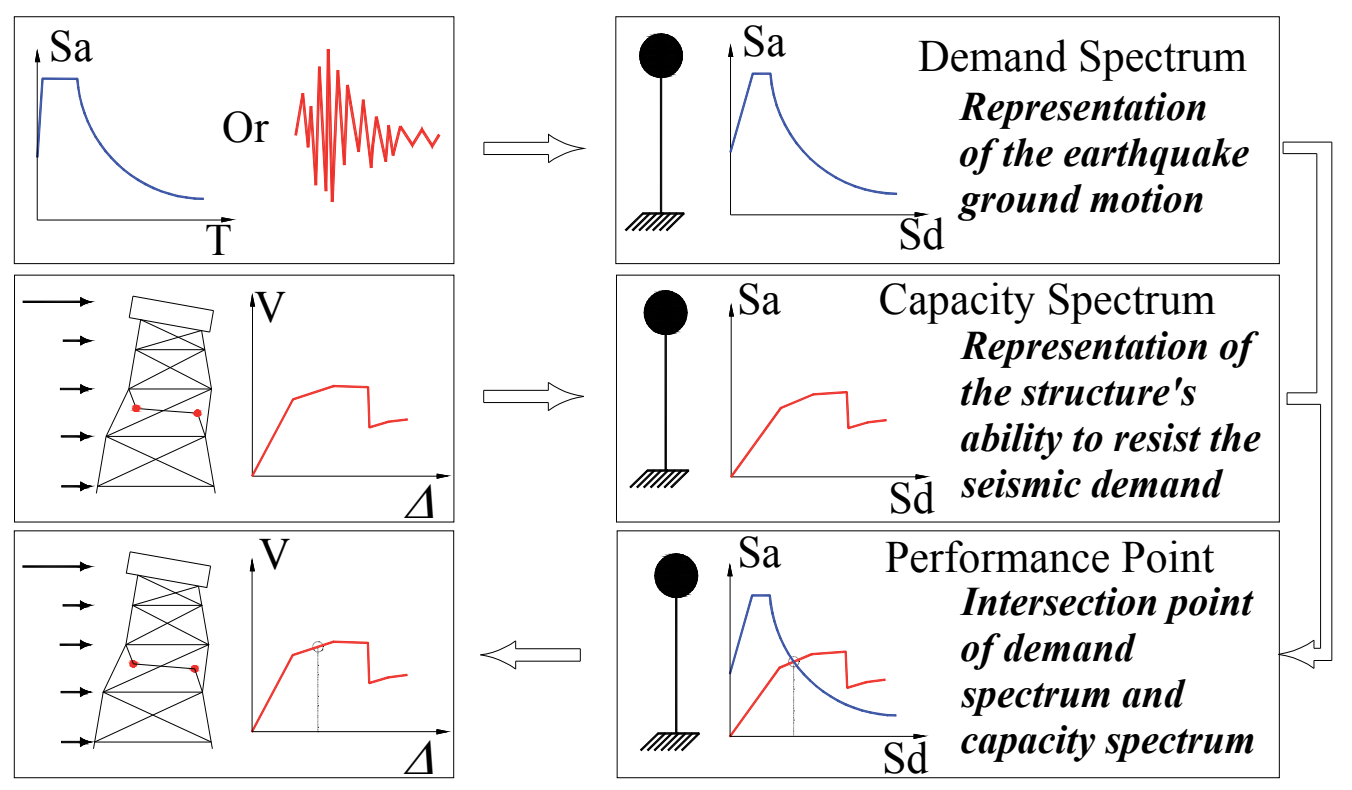

Fig. 3. Capacity-spectrum method.

\subsubsection{The coefficient method (FEMA-356)}

In the Coefficient Method, the maximum inelastic displacement (Target Displacement) is obtained from multiplying the linear elastic response by a series of coefficients $C_{0}$ through $C_{3}$. These coefficients are derived from statistical studies of the nonlinear time-history analyses of SDOF oscillators. 


\subsubsection{FEMA-440 recommendations}

The FEMA-440 document evaluates and improves the abovementioned simplified inelastic analysis procedures. Proposed modifications to the Coefficient Method of FEMA-356 relate primarily to the coefficients themselves. For coefficients $C_{1}$ and $C_{2}$ new relationships are proposed. It is also proposed that instead of coefficient $C_{3}$ a limitation on minimum strength be used. The improved procedure for the Capacity-Spectrum Method consists of new estimates of equivalent period and damping. This Linearization Method is calibrated for certain hysteretic loops with different calibration equations for the nondegrading and degrading cases.

\subsection{Case study}

\subsubsection{Description of the jacket platform}

The existing offshore complex consists of a drilling platform, a production platform, a service platform and a flare tripod in the field. The field was originally developed and put in production in 1968. There has been some damage imposed during Iran/Iraq war and some other extended damage due to adverse climate conditions afterwards. The service platform consists of a four leg battered jacket and topside located in $67.40 \mathrm{~m}$ water depth which is connected to production platform by means of one existing bridge. The service life of the platform is 25 years.

\subsubsection{Load cases}

For time history analysis of the platform, a 'best fit' set of scaled, natural time histories is used provided the velocity spectrum values have been properly modulated to equal or exceed the standard spectrum velocity values at specified periods $(0.2 \mathrm{~T}$ to $1.5 \mathrm{~T})$ as mentioned in International Building Code (IBC).

\subsubsection{Numerical model}

Analytical models were created using the open source finite element platform, OpenSees. This program is useful for modeling of jacket platform structures because of its capability of modelling of the post-buckling behavior of tubular members, soil-pilestructure interaction and etc. A two-dimensional model of a single frame is developed for the structure. A force-based nonlinear beam-column element (utilizing a layered fiber section) is used to model all components of the frame. Steel material is modeled using a bilinear stress-strain curve with $0.3 \%$ post-yield hardening. Initial imperfections in the struts are accounted for, with a value of $0: 001 \mathrm{~L}$ where $\mathrm{L}$ is the length of the member. This idea is useful for modeling the post-buckling behavior of the strut members, respectively.

The mathematical model of the pile-soil-structure system consists of the following sets of elements (Fig. 4):

1. Pile elements, modeled by a number of nonlinear beam-column elements.

2. Far-field soil model representing the free-field motion of the soil column, vertically and horizontally that is unaffected by the pile motions. The soil is modeled using elastic quad elements. The nodes that are at the same depth are constrained.

3. Near-field elements that connect the piles to the soil, vertically and horizontally. The strength and stiffness of these elements depends on the state of the far-field soil and the relative motion of the pile and far-field soil. The interface between the pile and 
surrounding soil is modeled using $\mathrm{p}-\mathrm{y}, \mathrm{t}-\mathrm{z}, \mathrm{q}-\mathrm{z}$ nonlinear spring elements. Hysteretic and radiation damping are considered using these elements. The group effects are not considered. The input motion is applied to the fixed nodes at the bottom of the soil column. The seismic record at bedrock is found from the input motion at the surface. Hydrodynamic effects are considered in terms of hydrodynamic damping from drag forces and added masses.

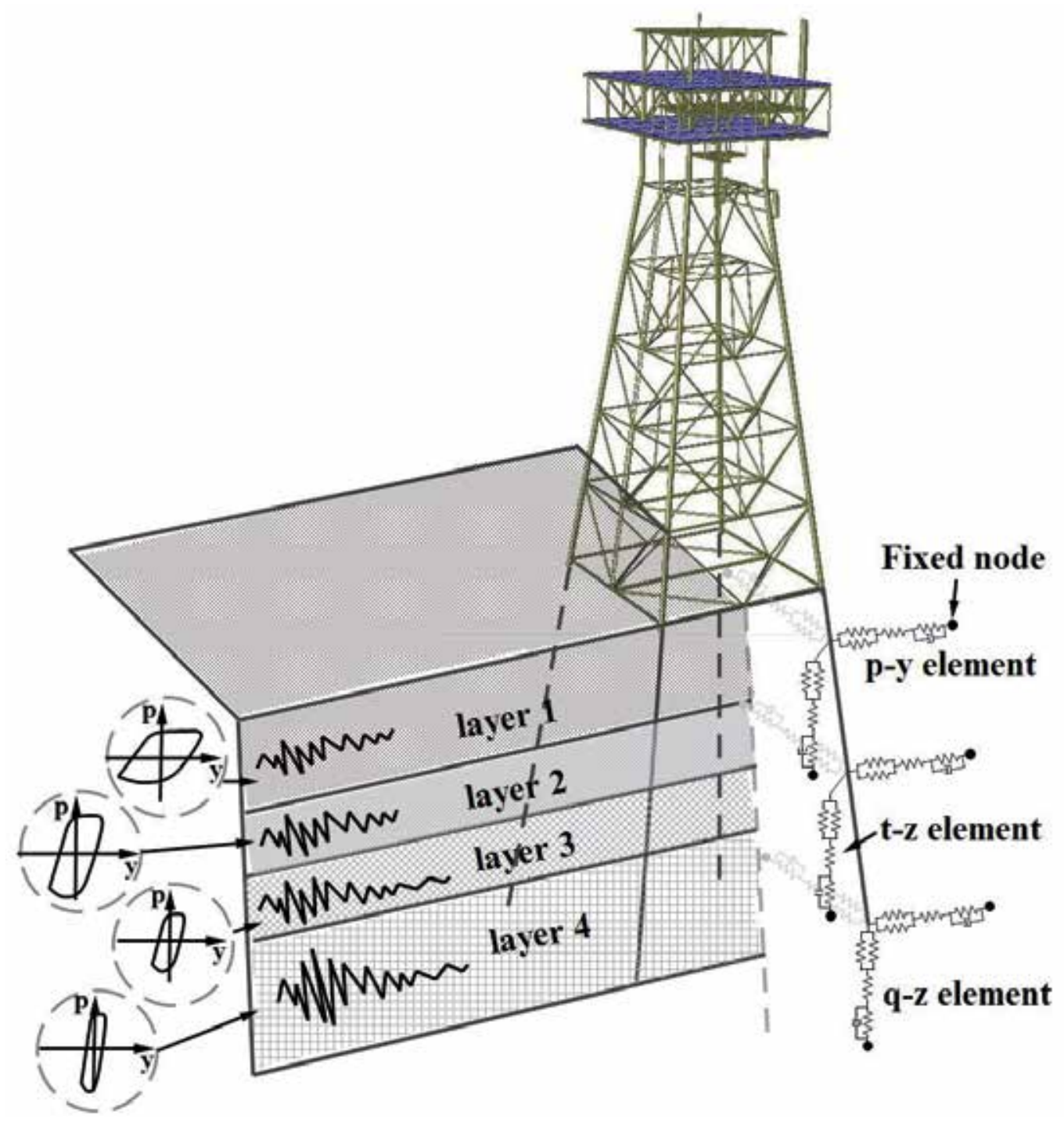

Fig. 4. Modeling of soil-pile-structure interaction. 


\subsubsection{Numerical study}

A uniform distribution of load is applied for the pushover analysis. The backbone curve of the deformation-control action of members is needed for an assessment of the structure based on the FEMA procedure. Fig. 5 shows the backbone curve of the axial action for strut-2 of the platform and FEMA criteria. Table 6 shows dimensions and assessment parameters of jacket struts. In this Table $\Delta_{C}$ and $\Delta_{T}$ are the axial deformations at the expected buckling load and at the expected tensile yielding load, respectively. Fig. 6 shows the pushover curve of the structure. This Figure shows an instantaneous loss of strength at a deck displacement equal to $0.29 \mathrm{~m}$. It can be seen that after the point with a deck displacement of $1.15 \mathrm{~m}$ the load-deformation curve has a negative slope. This figure also shows that for a deck displacement equal to $2.0 \mathrm{~m}$, the 1st and 2nd platform levels remain elastic.

The typical platform in Persian Gulf was assessed using responses obtained from a series of nonlinear time history analyses using three best fit records for each hazard level.

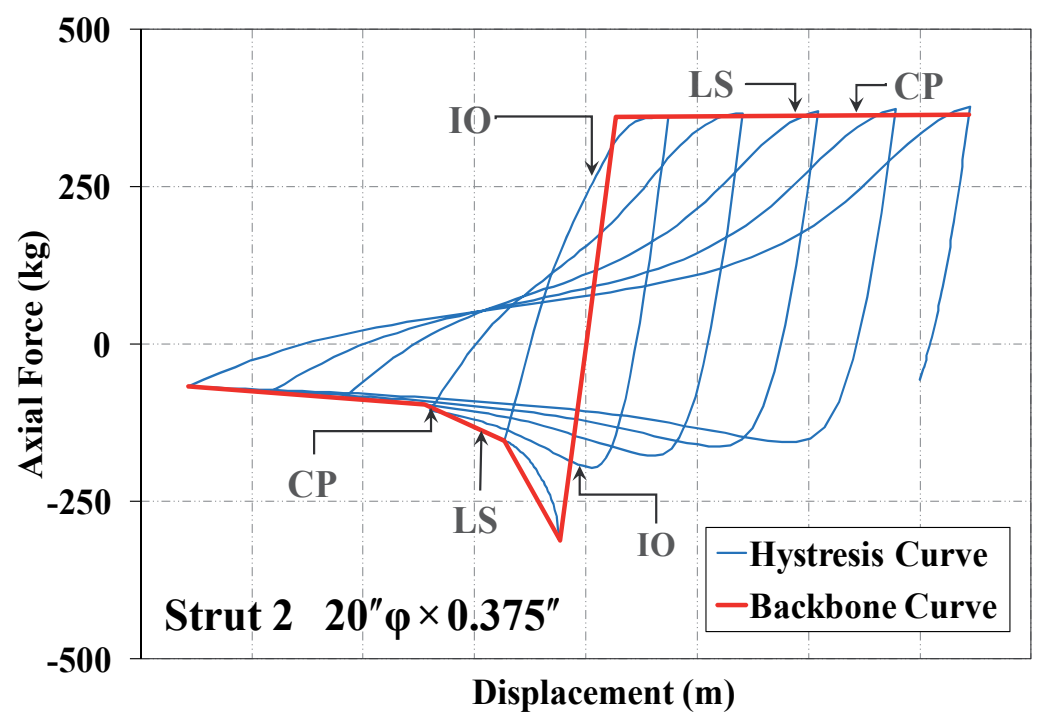

Fig. 5. Hysteretic behavior of Strut-2 and its Backbone curve.

\subsubsection{Structural assessment}

This section provides processes of assessment of the typical jacket platform based on the API and FEMA-356. The demands are obtained from mean values of the time history analysis.

\subsubsection{API standard}

The collapse of the structure is defined by its lack of ability to withstand to the load. The collapse load is defined as the maximum load the structure can withstand, before the loaddisplacement curve starts a negative trend. The deck displacement at the collapse point of the jacket is $1.15 \mathrm{~m}$.

\subsubsection{FEMA-356 document}

Leg behavior. Compression action in leg elements is a force control action, but the action type for flexure in these elements depends on the axial force of legs. 
Brace behavior. Braces are members with deformation controlled action and mainly determine the performance level of jackets. Fig. 6 shows the performance of the jacket for each hazard level. This figure shows that the jacket platform is highly sensitive to input motion.

\subsubsection{Directivity effects}

One of the primary factors affecting motion in the near-fault region is the directivity in which rupture progresses from the hypocenter along the zone of rupture. "Directivity" refers to the direction of rupture propagation as opposed to the direction of ground displacement (Abrahamson, 1998). A site may be classified after an earthquake as demonstrating forward, reverse, or neutral directivity effects. If the rupture propagates toward the site and the angle between the fault and the direction from the hypocenter to the site is reasonably small, the site is likely to demonstrate forward directivity. If rupture propagates away from the site, it will likely demonstrate reverse directivity (Abrahamson, 1998). If the site is more or less perpendicular to the fault from the hypocenter it will likely demonstrate neutral directivity. The phrase "directivity effects" usually refers to "forward directivity effects", as these case results in ground motions that are more critical to engineered structures.

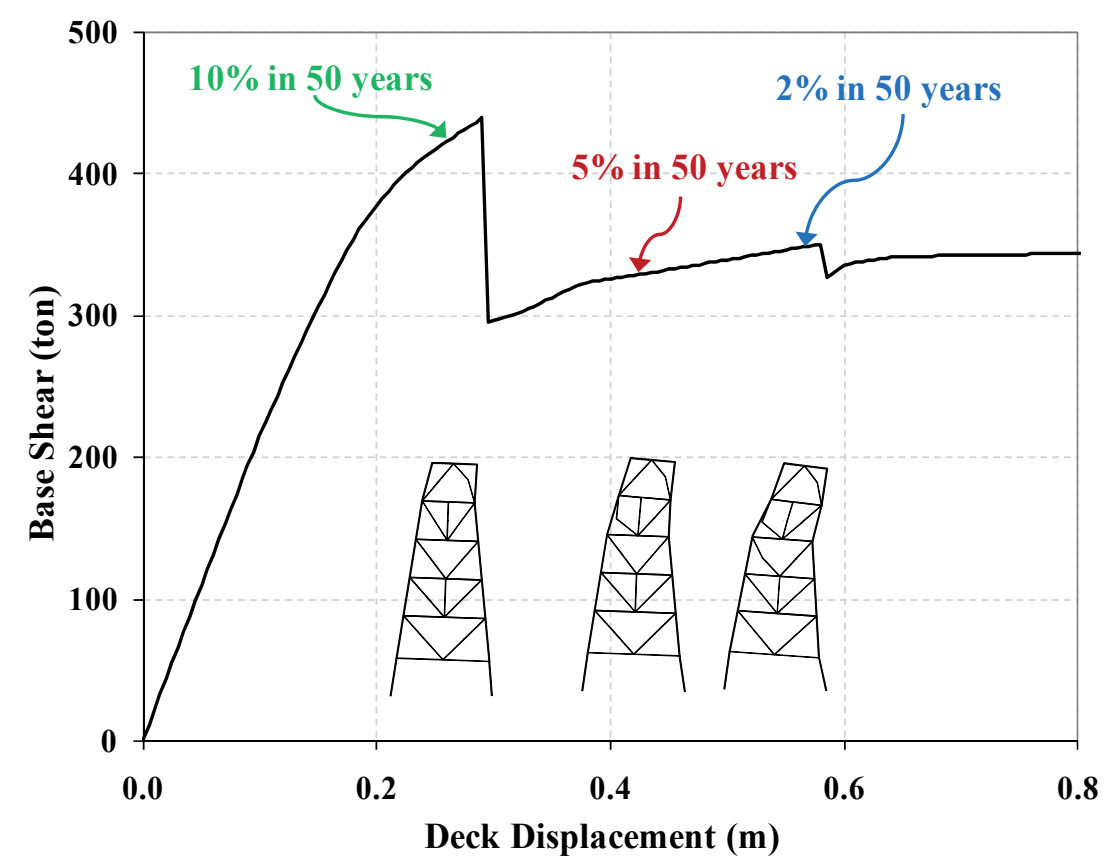

Fig. 6. Performance of the jacket for various levels of hazard.

A large velocity directivity pulse occurs when the conditions of forward directivity are met. These conditions include:

1. The earthquake is sufficiently large (moment magnitude greater than 6);

2. The site is located sufficiently close to the fault rupture (within $10 \mathrm{~km}$ ); and

3. The rupture propagates toward the site. 
This large velocity directivity pulse will be evident in the fault-normal direction. It is typically located toward the front of the time history and consists of, on average, one cycle of motion. Peak velocities usually are between $30 \mathrm{~cm} / \mathrm{sec}$ to $200 \mathrm{~cm} / \mathrm{s}$ with a mean value about $100 \mathrm{~cm} / \mathrm{sec}$. The period of the pulse can range from $0.5 \mathrm{sec}$ to $5 \mathrm{sec}$ with a mean value about $2.5 \mathrm{sec}$. Tabeshpour presented a conceptual discussion on the effect of near field earthquakes on various structures (2009).

\subsubsection{Pulse-type excitation}

For consideration of directivity effects, the analytical velocity pulse model proposed by $\mathrm{He}$ (2003) is expressed as:

$$
\dot{u}_{p}=C t^{n} e^{-a t} \sin \omega_{p} t
$$

Where $\mathrm{C}$ is the amplitude scaling factor, $a$ is the decay factor, $\mathrm{n}$ is the shape parameter of the envelope, and $\omega_{p}$ is the pulse frequency in $\mathrm{rad} / \mathrm{s}$. Differentiating above equation, the acceleration $\ddot{u}_{p}$ of the pulse can be obtained as:

$$
\ddot{u}_{p}=C t^{n} e^{-a t}\left[\left(\frac{n}{t}-a\right) \sin \omega_{p} t+\omega_{p} \cos \omega_{p} t\right]
$$

The acceleration $\ddot{u}_{p}$ in above Equation is considered as ground acceleration for numerical simulations in this chapter. To illustrate the performance of structure during the near-fault excitations, the parameter $\beta=T_{p} / T$ is used, where $T$ and $T_{p}$ are the fundamental period of jacket platform and pulse period, respectively. Fig. 7 shows time-history plots of acceleration for a velocity pulse with parameters $a=2.51, C=7.17, n=1$ for three $T_{p}$.

\subsubsection{Results and discussions}

Base shear of the structure vs. deck displacement (hysteresis loops) is presented in Fig. 8. A global view of the nonlinear behavior of the structure is seen clearly at the first pulse of excitation. In is seen that all energy dissipation by hysteretic behavior of the elements is occurred just in the one loop. Maximum displacements are shown by points A, B and C in horizontal axis.

\section{Rehabilitation}

Fig. 9 provides rehabilitation process of jacket offshore platforms. Rehabilitation of existing jacket consists of two phases of assessment and rehabilitation. Many researches have been carried out in this matter that is shown in this figure. With respect to type of loads, many types of control systems can be used for rehabilitation of jacket offshore platforms. Fig. 10 shows types of control systems usable for jacket offshore platforms.

\subsection{Tuned mass damper (TMD), wind and wave protection}

TMD is suitable passive control device for narrow band loads such as wind and wave loads. The efficiency of this device should be investigated for environmental loads. Fig. 11 shows jacket platform equipped with TMD and equal single degree of freedom. Fig. 12 shows the effect of TMD on the displacement response of jacket platform under the harmonic wave load with wave period equal to the fundamental period of the structure $(\mathrm{T})$ and wave height $\mathrm{H}=0.212 \mathrm{~m}$. A clearly decrease is observed in structural response. 


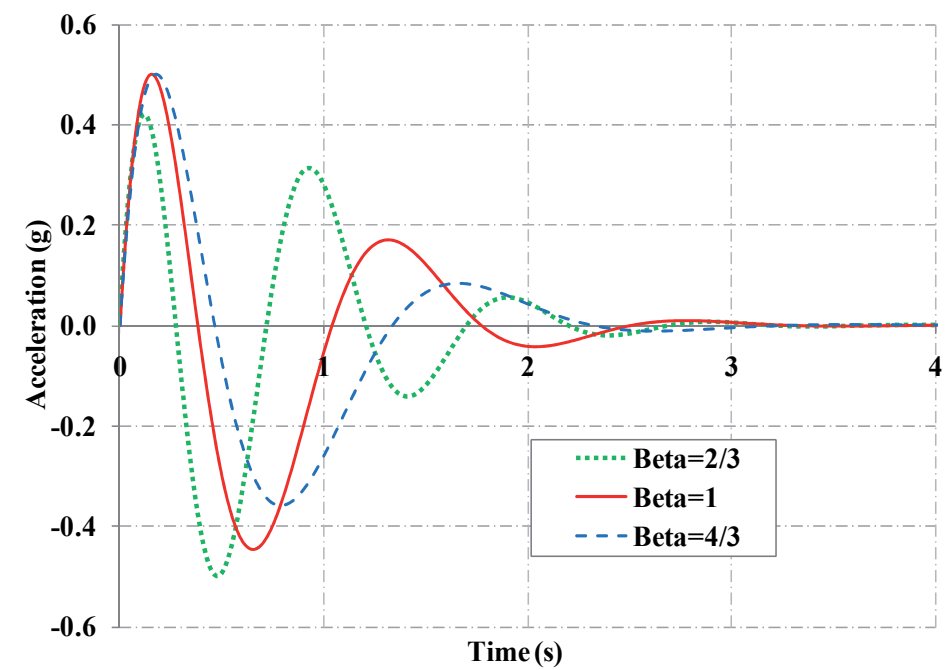

Fig. 7. Time-history plots of acceleration for $\beta=2 / 3,1,4 / 3$.

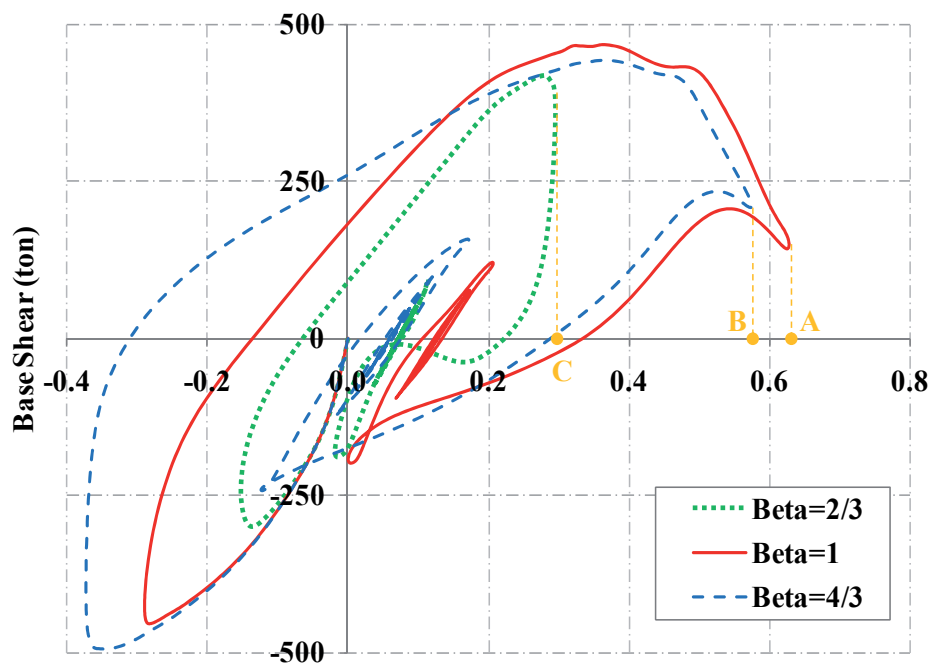

Deck Disp. (m)

Fig. 8. Hysteresis loops of the structure for $\beta=2 / 3,1,4 / 3$. 


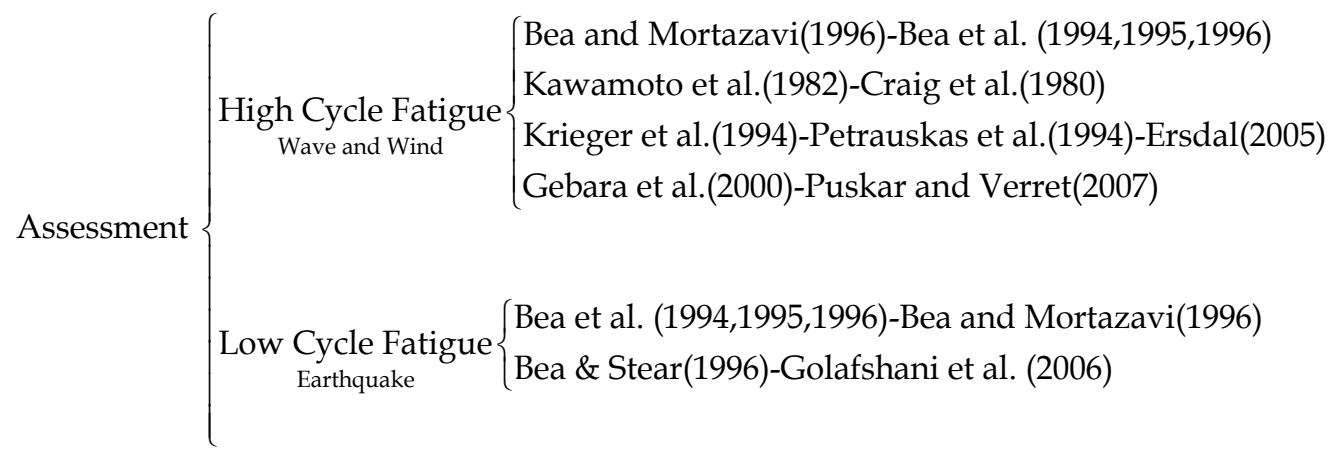

Fig. 9. Rehabilitation studies process for jacket offshore platforms.

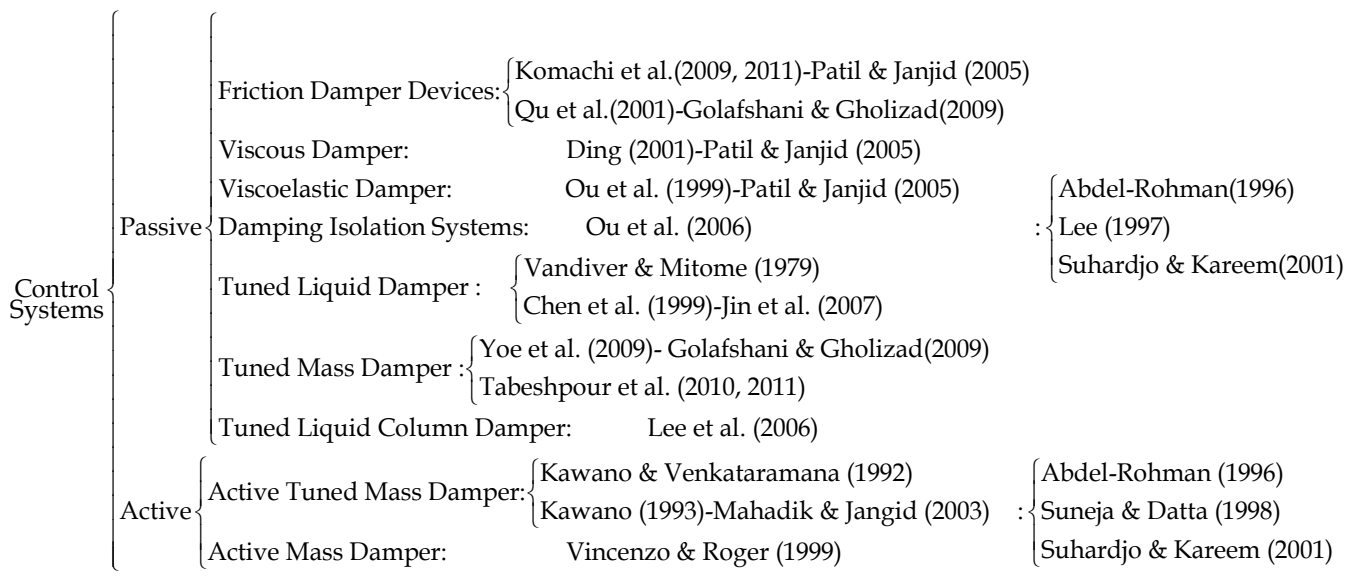

Fig. 10. Types of control systems used for jacket offshore platforms.
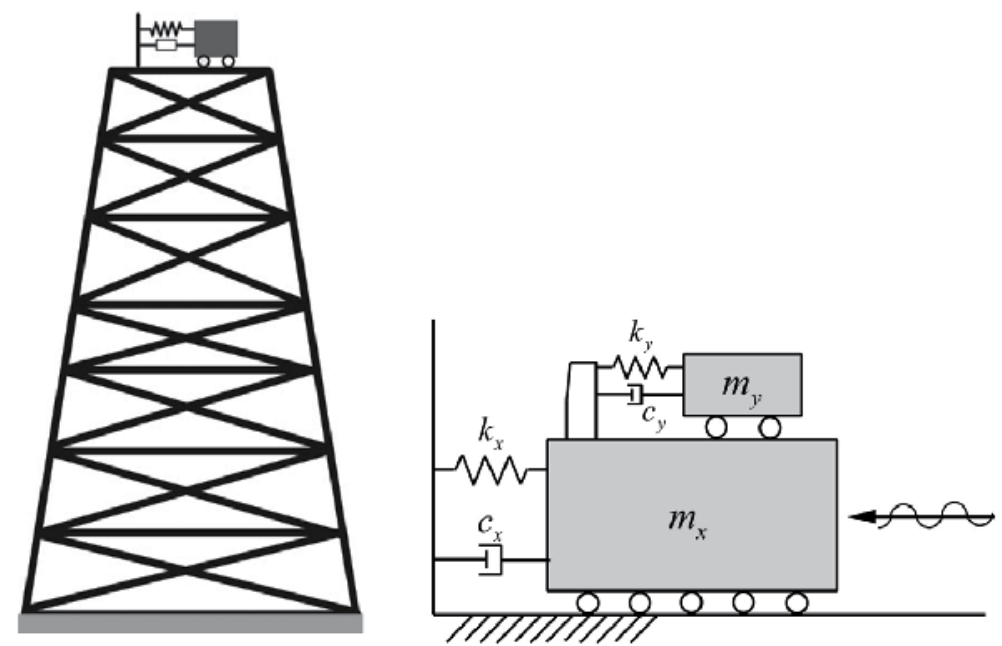

Fig. 11. Steel jacket platform utilized with a TMD and its equivalent SDOF system. 


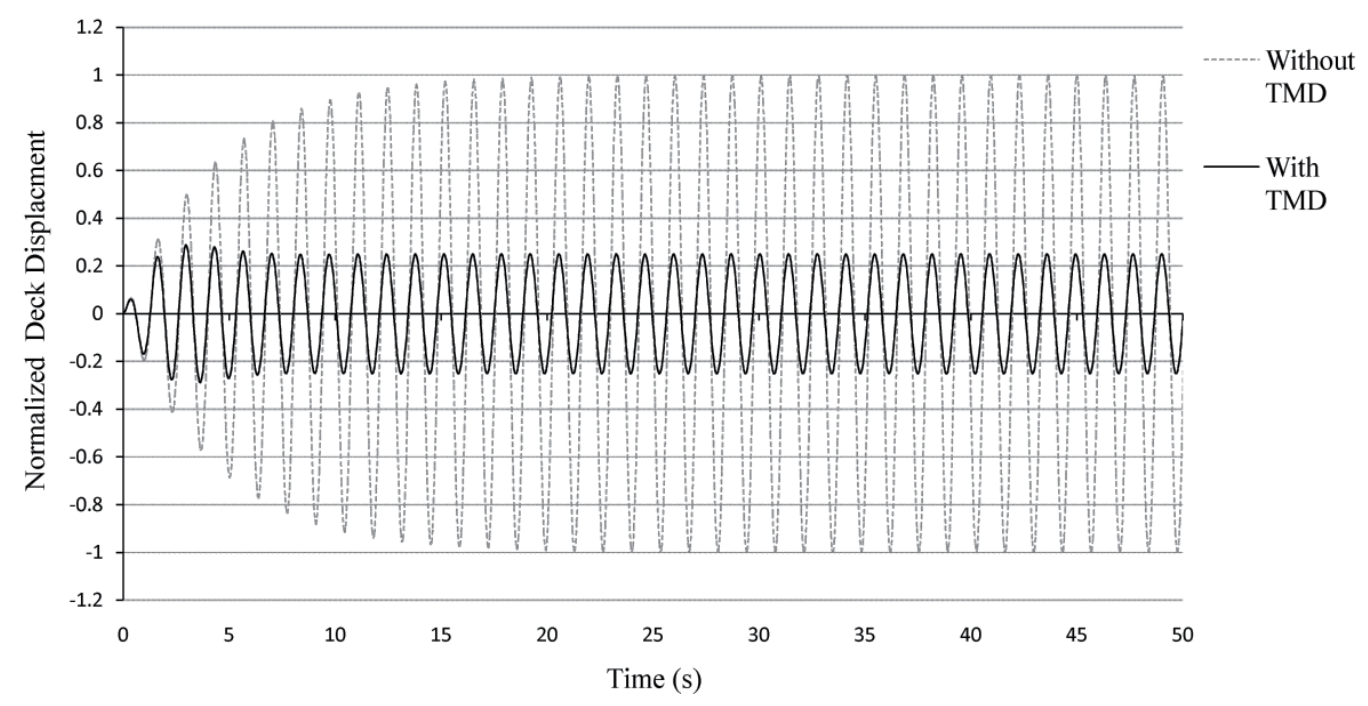

Fig. 12. Time history of deck displacement for harmonic load; W/O \& W TMD ( $\mathrm{H}=0.212 \mathrm{~m}, \mathrm{~T})$.

Fig. 13 also shows a clearly decrease in structural response for the harmonic wave load with wave period equal to three times of the fundamental period of the structure (3T) and wave height $\mathrm{H}=1.91 \mathrm{~m}$. Time history of deck acceleration for this loading has been shown in Fig. 14. It can be seen that acceleration reduces highly.

Fig. 15 shows the effect of TMD on the response of structure opposed to wind load. Mean velocity of wind with return period of 50 years at $10 \mathrm{~m}$ height from sea surface has been assumed to be equal to $22.5 \mathrm{~m} / \mathrm{s}$. This figure shows that TMD is effective for wind load cases as well.

\subsection{Friction damper devices (FDD), seismic protection}

In this research, a novel friction damper device (FDD), Mualla and Belev (2002), which is economical, can be easily manufactured and quickly installed, is used. The damper main parts are the central (vertical) plate, two side (horizontal) plates and two circular friction pad discs placed in between the steel (Fig. 16). The hinge connection is meant to increase the amount of relative rotation between the central and side plates, which in turn enhances the energy dissipation in the system. The ends of the two side plates are connected to the members of inverted V-brace at a distance $r$ from the FDD centre. The bracing makes use of pretensioned bars in order to avoid compression stresses and subsequent buckling. The bracing bars are pin-connected at both ends to the damper and to the column bases. The combination of two side plates and one central plate increases the frictional surface area and provides symmetry needed for obtaining plane action of the device. Zero-length element of program used for modeling of the frictional hinge. In order to verify modeling assumptions, model related to Mualla article evaluated. Tabeshpour and Ebrahimian presented a simple procedure for design of friction damper $(2007,2009)$. Komachi et al. investigated the efficiency of FDD for rehabilitation of jacket platforms (2011). 


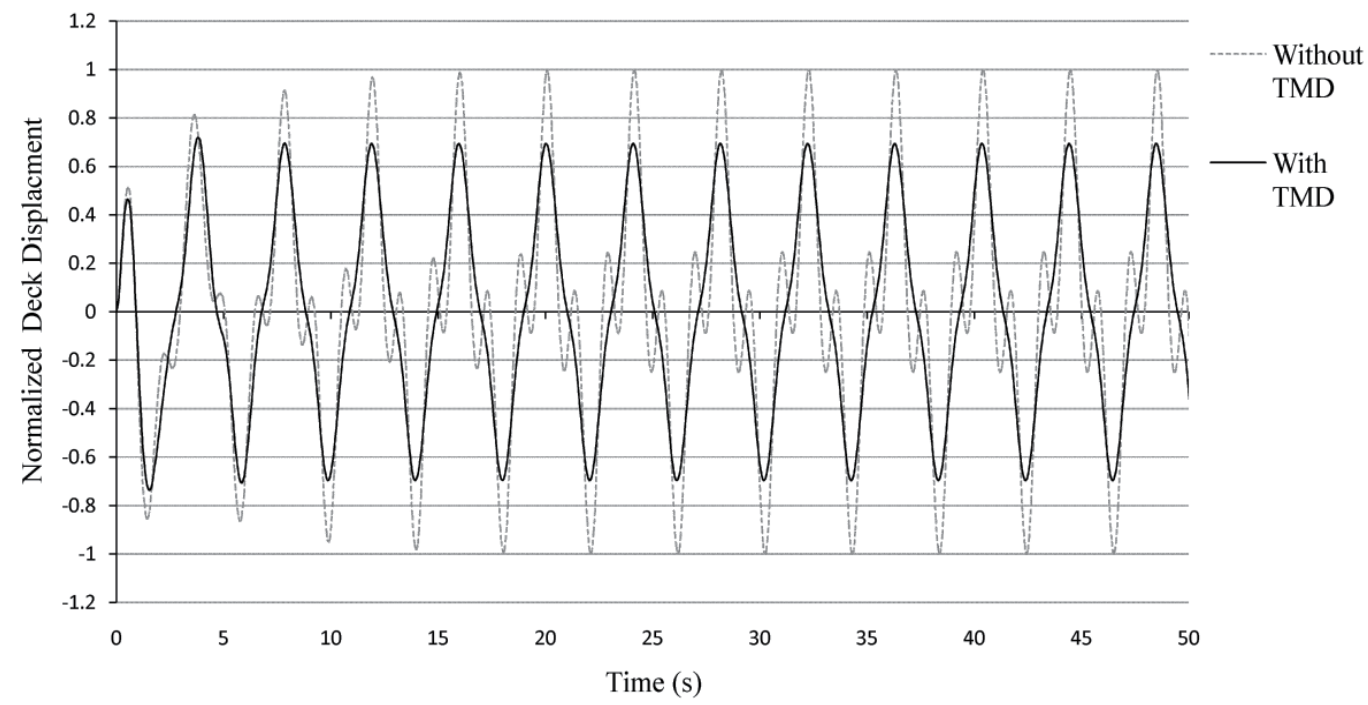

Fig. 13. Time history of deck displacement for harmonic load; W/O \& W TMD (H=1.91 m, 3T).

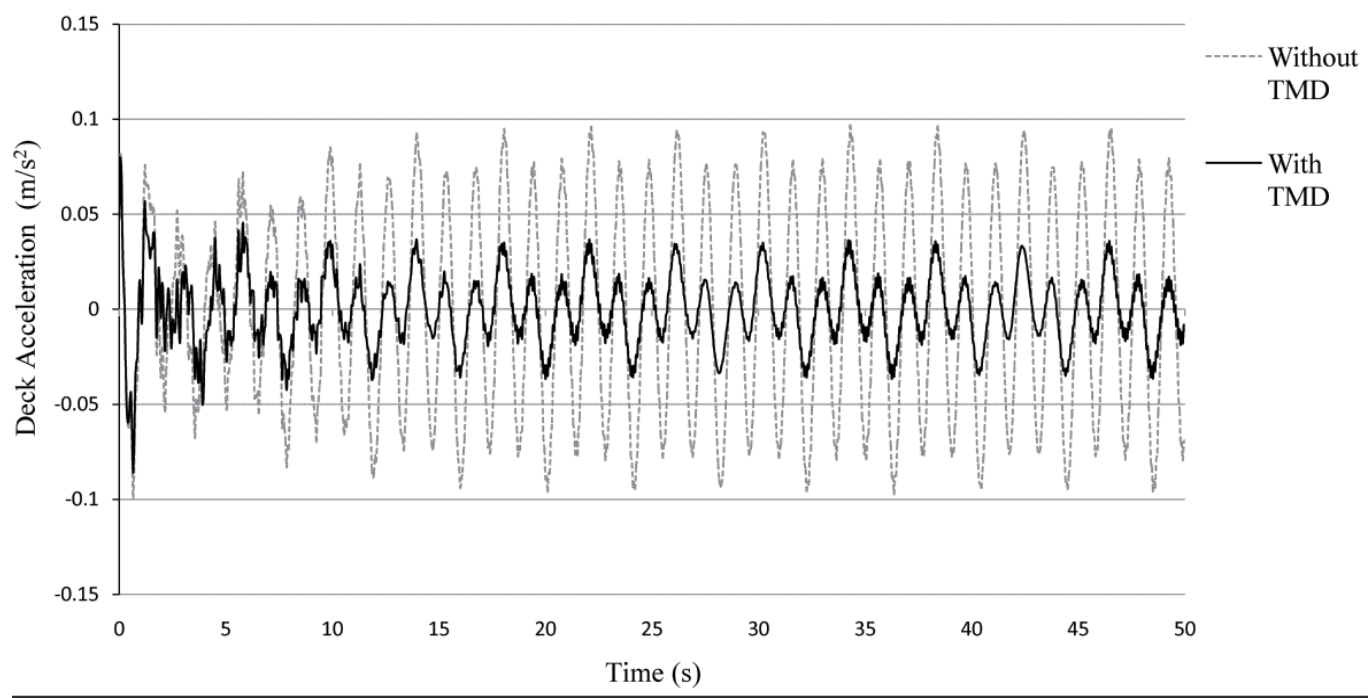

Fig. 14. Time history of deck acceleration for harmonic load; W/O \& W TMD (H=1.91 m). 


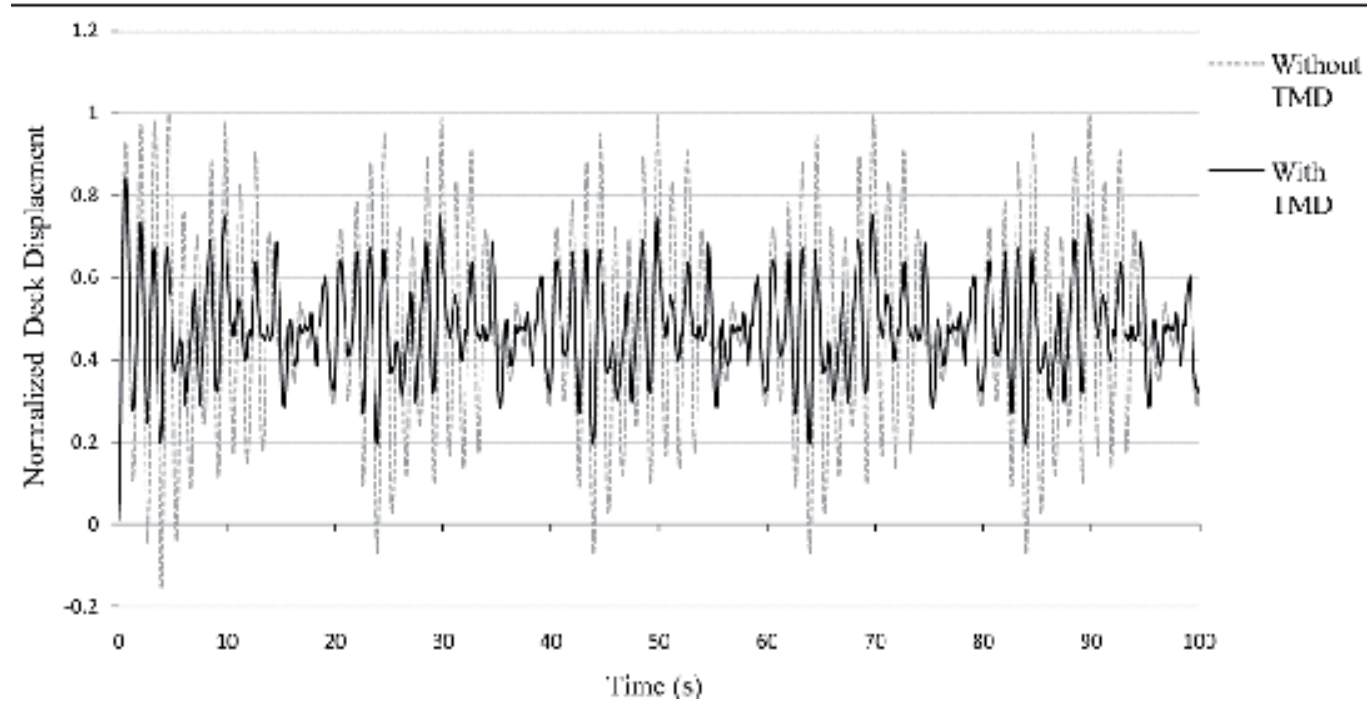

Fig. 15. Time history of deck displacement for wind load; W/O \& W TMD.

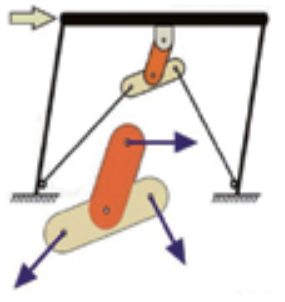

a

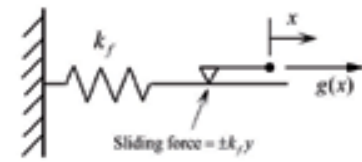

b

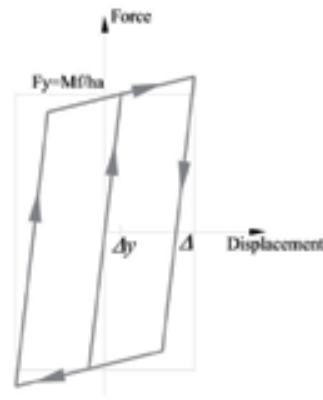

C

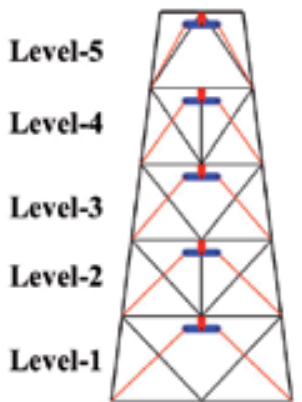

d

Fig. 16. a) Configuration of friction damper, b) Mathematical model, c)Hystresis behavior, d) Arrangement of FDD.

The equivalent viscous damping of this system is obtained by:

$$
\beta_{\text {eff }}=\frac{2}{\pi} \frac{F R(S R-F R)}{\left(S R+F R^{2}\right)}, \quad \frac{F R}{S R} \prec 1
$$

That FR and SR are the damper properties in terms of the structure properties are defined as follows:

$$
\left\{\begin{array}{l}
F R=\frac{F_{y}}{F_{e}}: \text { the ratio of damper stiffness to total structure stiffness } \\
S R=\frac{K_{b d}}{K_{e}}: \text { the ratio of damper yield force to total structure force }
\end{array}\right.
$$


Fig. 17 shows the comparison of pushover curves of the jacket with and without the damper. It can be seen that the damper improves the performance of the jacket especially at the nonlinear region. Base shear of the structure vs. deck displacement (hysteresis loops) for CHY101W record is presented in Fig. 18 for cases of with and without FDD.

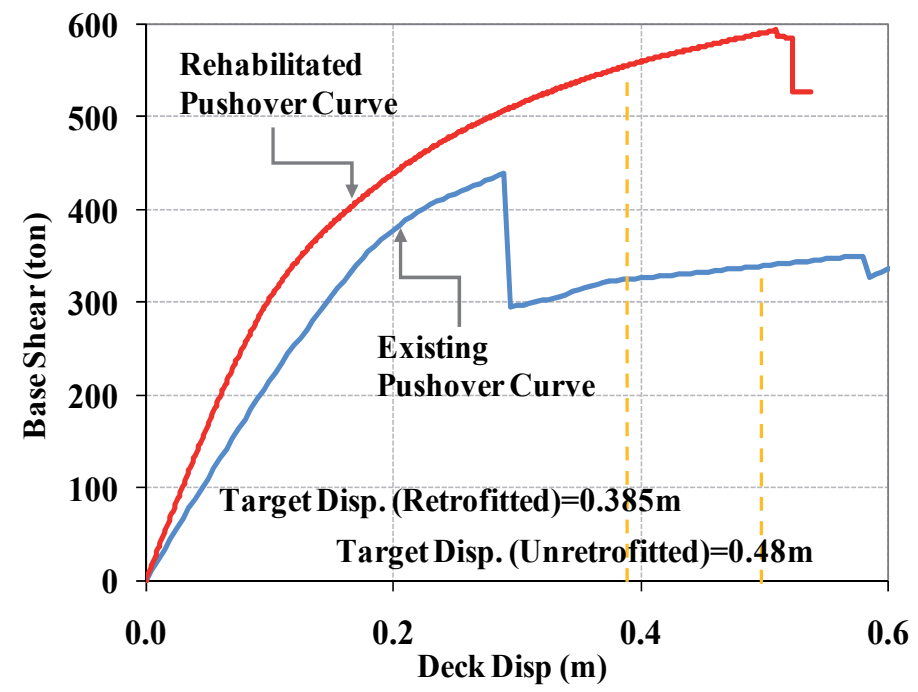

Fig. 17. Pushover curve of the jacket for rehabilitated and existing cases.

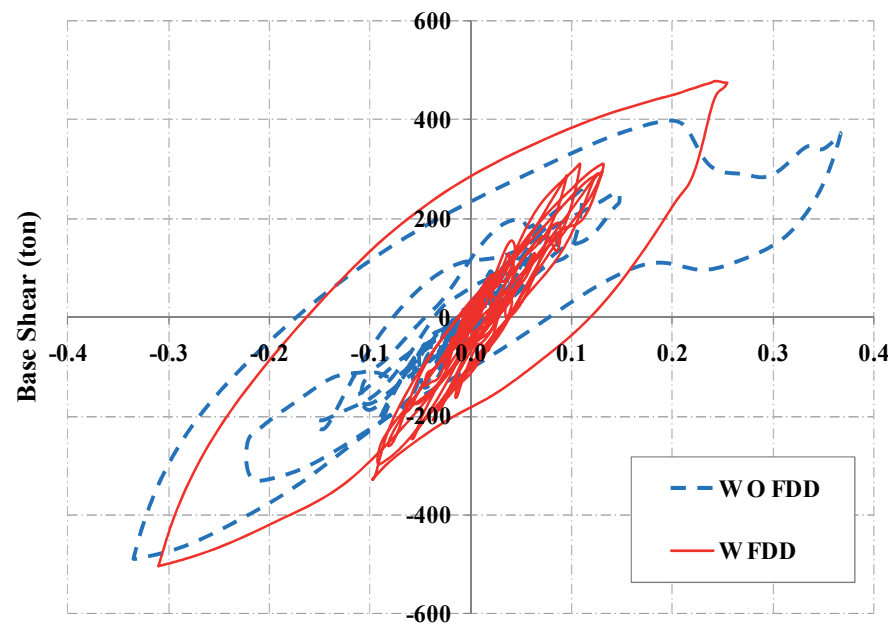

Deck Disp. (m)

Fig. 18. Hysteresis loops of jacket for CHY101W record.

Fig. 19 shows time history of frictional hinge rotation of dampers at various levels of jacket for CHY101W record. Fig. 20 shows the time history of deck displacement for CHY101W record. This figure shows that deck displacement reduces highly (about 60\%) and base shear of structure reduce about $10 \%$ too. 


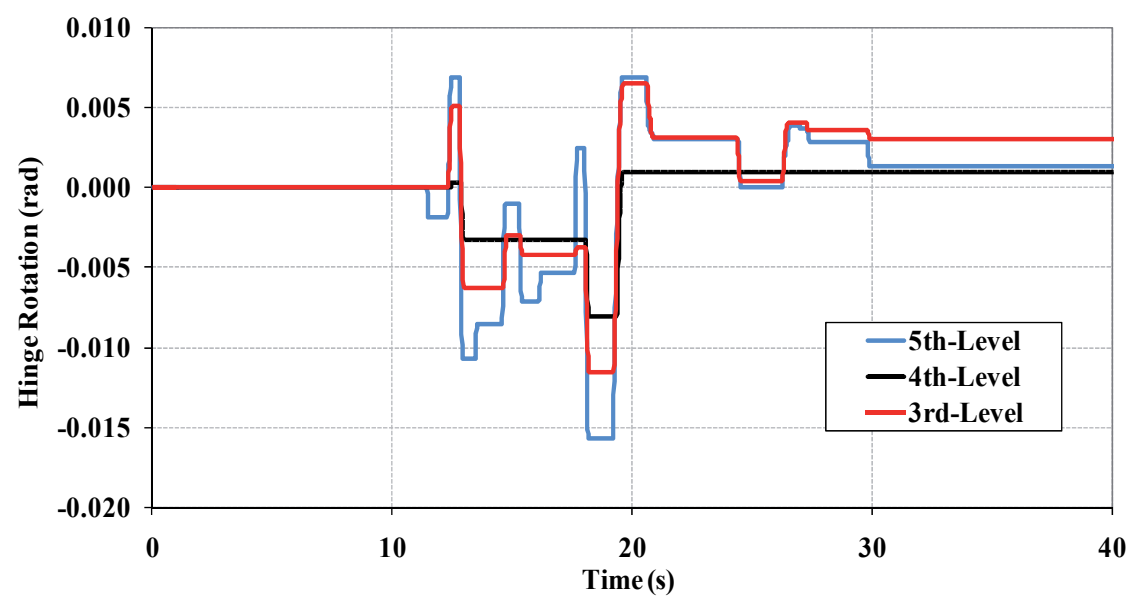

Fig. 19. Time history of friction hinge rotation for CHY101W record.

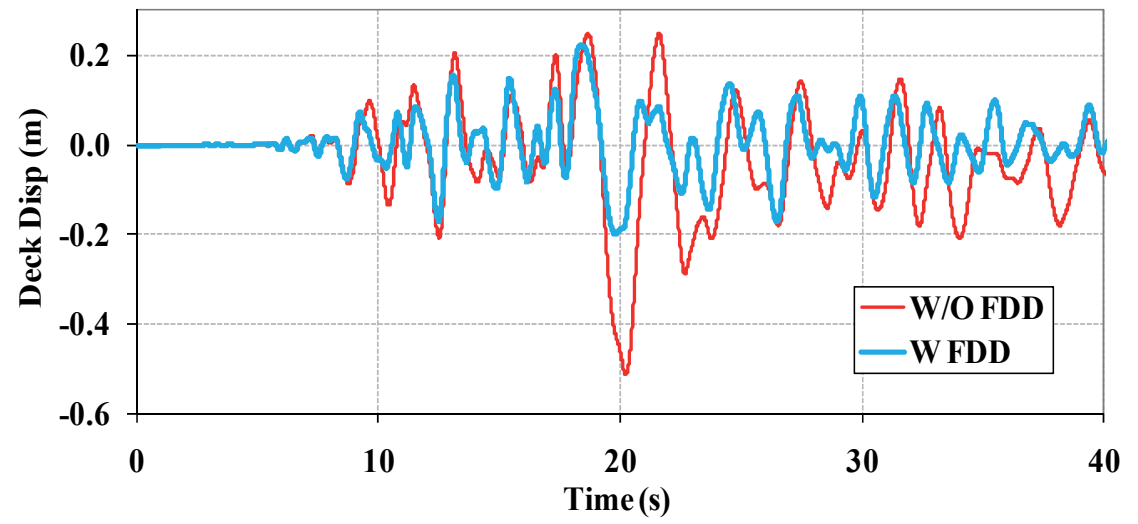

Fig. 20. Time history of deck displacement of structure for cases with and without damper for CHY101W record.

\section{Conclusion}

This chapter presented process of assessment and rehabilitation of jacket platform offshore structures. A seismic assessment of API standard and FEMA-356 documents was compared to each other. As an example an existing 4 legged service platform was assessed with API and FEMA-356 with respect to various earthquake hazard levels. Nonlinear static and dynamic analyses were used to determine the response of the structure. This study shows that:

1. Comparative building redundancy of a jacket platform structure is low and failure of one member in these structures can lead to immediate reduction of strength and afterward collapse of the structure.

2. However global criteria for seismic assessment of jacket platforms are presented in the API standard but there is no numerical or specific criterion in order to assess the structure. It is observed that building documents can be used to develop numerical and 
applicable criteria for seismic assessment of such structures. Assessment with respect to member's criteria has some advantages such as better decision making for rehabilitation and detecting the full capacity of members.

3. Return periods related to collapse prevention in API and FEMA are 1000 and 2500 years respectively. The return period of API should probably be reviewed because the expected mean life time of the jacket is greater than design mean life time. However the approach and methodology presented in building structure documents (such as FEMA356) is very appropriate and efficient in the seismic assessment of jacket platform structures.

The effects of near-fault earthquakes on the behavior of steel jacket platforms has been presented. Pulse type excitation has been used for investigation of structural behavior. It is shown that the maximum response of structure occurs when $T=T_{p}$ and also that input pulse with $\beta>1.0$ gives a higher amplitude rather than $\beta<1.0$, that denotes importance of ratio of period of directivity pulse to structure period. The increasing in dynamic amplitude can be more than two times than that of both static and far field responses.

Rehabilitation process using TMD and FDD for these structures presented. Effect of TMD on the response of jacket offshore platform under the wave and wind loads presented. It was shown that TMD is very effective for reduction of jacket responses under the these loads.

FDD was used on a steel jacket platform located in Iranian waters of the Persian Gulf. Results were shown that responses of jacket reduce dramatically. A numerical study was performed using pushover and nonlinear dynamic analysis. Pushover analysis results were shown that use of FDD system reduce target displacement of the structure and also was shown that a sudden decrease of jacket strength does not occur when this system is installed on the structure. Due to the low redundancy of jacket platform structures, the strength of these structures can decrease suddenly and the use of FDD systems can be extremely useful. Analysis results were shown that friction damper greatly reduces deck displacement. It was observed that for large record accelerations structure behavior becomes highly nonlinear and the performance of the friction damper for response reduction increases (for example up to $65 \%$ deck displacement reductions). Numerical studies clearly exhibit that these control systems represent a practical alternative for rehabilitation of existing jacket platforms.

\section{References}

Abdel-Rohman, M. (1996). Structural control of steel jacket platform. Structural Engineering and Mechanics, 4, 25-38

American petroleum institute recommended practice for planning, design and constructing fixed offshore platforms. API RP 2A, 21th ed. Washington (DC): American Petroleum Institute; 2000

Anagnostopoulos, S. (October 1978). Post-yield Flexural Properties of Tubular Members. Preprint 3302, ASCE Convention and Exposition, Chicago

Applied Technology Council (ATC). Seismic evaluation and retrofit of concrete buildings. vol. 1 and 2. Report no. ATC-40. Redwood City (CA): 1996 
Bea, R.G.; Loch, K. J. \& Young, P. L. (October 2, 1994). Screening Methodologies for Use in Platform Assessments and Requalifications. Department of Civil Engineering, University of California at Berkeley

Bea, R.G.; et. al. (May 1-4, 1995). Verification of a Simplified Methods to Evaluate the Capacities of Template-Type Platforms, 27th Annual Offshore Technology Conference, Houston, Texas

Bea, R. G.; Mortazavi, M. (May 26-31, 1996). A Simplified Structural Reliability Analysis Procedure for Use in Assessments and Requalifications of Template-Type Offshore Platforms, 6th International Offshore and Polar Engineering Conference, Los Angeles, California

Bea, R.G.; Stear, J.D. (March 1996). Using Static Pushover Analysis to Determine the Ultimate Limit States of Gulf of Mexico Steel Template-Type Platforms Subjected to Hurricane Wind and Wave Loads. Marine Technology and Management Group Project, Department of Civil Engineering, University of California at Berkeley

Bea, R.; Stear, J. (March 1999). Report 3: Benchmark Platform and Site Studies, Department of Civil \& Engineering, University of California at Berkeley

Briaud, J. L.; Tucker, L. M. \& Lytton, R. L. \& Coyle, H. M. (September 1983). Behavior of Piles and Pile Groups in Cohesionless Soils, Texas A\&M University, College Station, Texas

Briaud, J. L.; Tucker, L. M. (October 1983). Piles in Sand: A Design Method Including Residual Stresses, Texas A\&M University, College Station, Texas

Briaud, J. L.; Tucker, L. M. (May 17, 1984). Coefficient of Variation of In-Situ Tests in Sand, ASCE Symposium 'Probabilistic Characterization of Soil Properties: Bridge Between Theory and Practice, Atlanta, Georgia

Bardakis, V.G.; Dritsos, S.E. (2007). Evaluating assumptions for seismic assessment of existing buildings. Soil Dyn Earthq Eng, 27(3), 223_33

Chen, X.; Wang, L.Y. \& Xu, J. (1999). TLD technique for reducing ice-induced vibration on platforms. J Cold Reg. Eng, 13(3), 139-52

Cornell, C. A. (August 1987). Offshore Structural Systems Reliability, C. Allin Cornell, Inc., Portola Valley, California

Craig, M.J.K.; Shekher, V. (1980). Inelastic Earthquake Analysis of an Offshore California Platform, Proceedings, Offshore Technology Conference, OTC 3822, Houston, Texas, $259-267$

Craig, M.J.K.; \& Digre, K.A. (1994). Assessment of high-consequence platforms; Issues and applications, Proceedings of offshore technology conference, OTC 7485, Houston (TX)

Ding, J.H. (2001). Theoretical and experimental study on structural vibration repressed system using viscous fluid dampers, Ph.D. dissertation, Harbin Institute of Technology, [in Chinese]

El Naggar, M.H.; Novak, M. (1996). Influence of foundation nonlinearity on offshore towers response. J Geotech Engng, ASCE, 1996, 122(9), 717-24

Elnashai, A. S.; Aritenang, W. (1991). Nonlinear modelling of weld-beaded composite tubular connections. Eng. Struct, 1991, Vol 13 34-42

Ettema, R.; Stern, F. \& Lazaro, J. (April 1987). Dynamics of Continuous-Mode Icebreaking by a Polar-Class Icebreaking Hull. IIHR Report, Iowa Institute of Hydraulic Research, The University of Iowa, Iowa City, Iowa 
Ersdal, G. (2005). G. Assessment of existing offshore structures for life extension, Ph.D. dissertation, University of Stavanger

FEMA-356 (2000). Prestandard and commentary for the seismic rehabilitation of buildings. Report FEMA-356. Washington (DC): Federal Emergency Management Agency

FEMA-440 (2005). Improvement of nonlinear static seismic analysis procedures. Report FEMA-356. Washington (DC): Federal Emergency Management Agency

Ferritto, J. M.; Nakamoto, R. T. (September 1982). The Effective Stress Soil Model, Technical Memorandum 51-82-07, Naval Civil Engineering Laboratory, Port Hueneme, California

Filiatrault, A.; Cherry, S. (1989). Parameters influencing the design of friction damped structures. Department of civil eng., University of British Columbia, Vancouver, B.C., Canada V6T 1W5, 753-766.

Gebara, J.; Dolan, D. \& Pawsey, S. \& Jeanjean, P. \& Dahl-Stamnes, K. (2000). Assessment of offshore platforms under subsidence Part I: Approach. ASME J Offshore Mech Arct Eng, 122, 260-6

Golafhshani, A.A.; Tabeshpure, M.R. \& Komachi, Y. (2006). Assessment and retrofit of existing jacket platform using friction damper devices, 8th international conference of ocean industries, Bushehr (Iran), Bushehr University, [in Persian]

Golafhshani, A.A.; Gholizad, A. (2009). Friction damper for vibration control in offshore steel jacket platforms. Journal of Constructional Steel Research, 65(1), 180-187

Golafhshani, A.A.; Tabeshpure, M.R. \& Komachi, Y. (2009). FEMA approaches in seismic assessment of jacket platforms, (case study: Ressalat jacket of Persian gulf). Journal of Constructional Steel Research, 65(10), 1979-1986

Grilli, S.T.; Fake, T. \& Spaulding, M.L. (May 26, 2000). Numerical Modeling of Oil Containment by a Boom/Barrier System: Phase III Final Report. University of Rhode Island

Hueste, M.D.; Bai, J.W. (2007). Seismic retrofit of a reinforced concrete flat-slab structure: Part I seismic performance evaluation. Eng Struct, 29(6), 1165-77

IBC, 2000. International Building Code. International, Code Council. Falls Church (Virginia).

ISO (2000). ISO/DIS 13822 Bases for design of structures_Assessment of existing structures. International Standardisation Organisation

ISO (2002). ISO 19900 Petroleum and natural gas industries_Offshore structures_Part 1: General requirements. International Standardisation Organisation

ISO (2004). ISO/DIS 19902 Design of fixed steel jackets. DIS Draft. International Standardisation Organisation

Jin, Q.; Li, X. \& Sun, N. \& Zhou, J. \& Guan, J. (2007). Experimental and numerical study on tuned liquid dampers for controlling earthquake response of jacket offshore platform. Marine Structures, 20(4), 238-254

Kallaby, J.; Millman, D.N. (1975). Inelastic Analysis of Fixed Offshore Platforms for Earthquake Loading, Proceedings, Offshore Technology Conference, Houston, Texas

Kawamoto, J.; Sunder, S. and Connor, J. (1982). An assessment of uncertainties in fatigue analyses of steel jacket offshore platforms. Applied Ocean Research, Vol. 4, No.1, 9-16

Kawano, K.; Venkataramana, K. (1992). Seismic response of offshore platform with TMD, Proc. of 10th World Conference on Earthquake Engineering, 4, 241-246

Kawano, K. (1993).Active control effects on dynamic response of offshore structures, Proc. of 3rd ISOPE Conference, 3, 494-498 
Kawano, K.; Venkataramana, K. (January 1999). Dynamic response and reliability analysis of large offshore structures, Computer Methods in Applied Mechanics and Engineering, Volume 168, Issues 1-4, 6, 255-272.

Komachi, Y.; Tabeshpour, M.R. \& Golafhshani, A.A. (2009a).Assessment of seismic vulnerability of jacket platforms based on the performance, 11th international conference of ocean industries, Kish, Iran, (in Persian)

Komachi, Y.; Tabeshpour, M.R. \& Golafhshani, A.A. (2009b).Use of energy damping systems for rehabilitation of jacket platforms, 11th international conference of ocean industries, Kish, Iran, [in Persian]

Komachi, Y.; Tabeshpour, M.R. \& Golafhshani, A.A. (2010). Special considerations for finite element modeling of jacket platforms, 12th international conference of ocean industries, Zibakenar, Iran, [in Persian]

Komachi, Y.; Tabeshpour, M.R. \& Mualla, I. \& Golafhshani, A.A. (2011). Retrofit of Ressalat Jacket Platform (Persian Gulf) using Friction Damper Device. Journal of Zhejiang University, Sci A (Appl Phys \& Eng) 2011 12(9):680-691

Krieger, W.F.; Banon, H. \& Lloyd, J.R. \& De, R.S. \& Digre, K.A. \& Nair, D. et al. (1994). Process for assessment of existing platforms to determine their fitness for purpose, Proceedings of offshore technology conference, OTC 7482, Houston (TX)

Lee, H.H. (1997). Stochastic analysis for offshore structures with added mechanical dampers. Ocean Engineering, 24, 817-834

Lee, H.N.; Wong, S.H. \& Lee, R.S. (2006). Response mitigation on the offshore floating platform system with tuned liquid column damper. Ocean Eng., 33(8-9), 1118-42

Lie, S.T.; Lee, C.K. \& Chiew, S.P. \& Shao, Y.B. (2005). Validation of surface crack stress intensity factors of a tubular K-joint. International Journal of Pressure Vessels and Piping, 82, 610-617

Litton, R.W.; Pawsey, S.F. \& Stock, D. \& Wilson, B.M. (1978). Efficient Numerical Procedures for Nonlinear Seismic Response Analysis of Braced Tubular Structures. Preprint 3302, ASCE Convention and Exposition, Chicago, October

Mahadik, A.S.; Jangid, R.S. (2003). Active control of offshore jacket platforms. International Shipbuilding Progress, 50, 277-295

Maison, B.; Popov, E.P. (1980). Cyclic response prediction for braced steel frames. J. Struct., Div., ASCE, 106, 1401-1416

Marshall, P.W.; Gates, W.E. \& Anagnostopoulos, S. (1977). Inelastic Dynamic Analysis of Tubular Offshore Structures, Proceedings, Offshore Technology Conference, OTC 2908, Houston, Texas, 235-246

Mitwally, H.; Novak, M. (1987). Response of offshore towers with pile interaction. J Geotech Engng, ASCE, 1987, 113(7), 1065-84

Moustafa, A. (March, 2011). Damage-Based Design Earthquake Loads for Single-Degree-OfFreedom Inelastic Structures, Journal of structural engineering, Vol. 137, No. 3, 456467

Mualla, I.H.; Belev, B. (2002). Performance of steel frames with a new friction damper device under earthquake excitation. Engineering Structures, 24, 365-371

Mualla, I.H.; Nielsen, L.O. (2002). A Friction Damping System Low order behavior and design. Department of Civil Engineering, DTU-bygning, 118 
Nath, J. H.; Hsu, M. K. \& Hudspeth, R.T. \& Dummen, J. (September 11-13, 1984). Laboratory Wave Forces on Vertical Cylinders, Proceedings Ocean Structural Dynamics Symposium, Oregon State University, Corvallis, Oregon, pp. 312-330

Nogami, T.; Konagai, K. (February 1987). Dynamic Response of Vertically Loaded Nonlinear Pile Foundations. Paper No. 21252, ASCE Journal of Geotechnical Engineering, Volume 113, No. 2

Nogami, T.; Leung, M. B. (March 1-6, 1987). Dynamic Soil-Structure Analysis of Offshore Gravity Platforms Using Simplified Model, Proceedings of the Sixth International Offshore Mechanics and Arctic Symposium, Houston, Texas

OpenSees 2005. Open system for earthquake engineering simulation. Available online http://opensees.berkeley.edu.

Ou, J.P.; Duan, Z.D. \& Xiao, Y.Q. (1999). Ice-induced vibration analysis of JZ20-2MUQ offshore platform using in-situ ice force histories. The Ocean Engineering, 17(2), 7080

Ou, J.P.; Xiao, Y.Q. \& Duan, Z.D. \& Zou, X.Y. \& Wu, B. \& Wei, J.S. (2000). Ice-induced vibration control of JZ20-2MUQ platform structure with viscoelastic energy dissipators. The Ocean Engineering, 18(3), 9-14

Ou, J.; Long, X. \& Li, Q.S. \& Xiao, Y.Q. (2006). Vibration control of steel jacket offshore platform structures with damping isolation systems. Engineering Structure, 29(7), $1525-1538$

Patil, K.C.; Jangid, R.S. (2005). Passive control of offshore jacket platforms. Ocean Engineering, 32, 1933-1949

Petrauskas, C.; Finnigan, T.D. \& Heideman, J.C. \& Vogel, M. \& Santala, M. \& Berek, G.P. (1994). Metocean criteria/loads for use in assessment of existing offshore platforms, Proceedings of offshore technology conference, OTC 7484, Houston (TX)

PSA (2004). Regulation relating to management in the petroleum activities. Petroleum Safety Authority Norway. 2004

Puskar, F.J.; Verret, S.M. and Roberts, C. (2007). Fixed platform performance during recent hurricanes: comparison to design standards. OTC 18989, Presented at the 2007 Offshore Technology Conference. Houston, TX

Qu, W.L.; Chen, Z.H. \& Xu, Y.L. (2001). Dynamic analysis of wind-excited truss tower with friction dampers. Computers \& Structures, 79(32), 2817-2831

Ressalat (R1). Offshore complex renovation and reconstruction project. Production platform in place analysis report. Rsl-P1-St-Cn-1001-C2. Amid Engineering \& Development Company

Soong, T.T.; Dargush, G.F. (1997). Passive energy dissipation systems in structural engineering. Wiley, London

Suhardjo, J.; Kareem, A. (2001). Feedback-feedforward control of offshore platforms under random waves. Earthquake Engineering and Structural Dynamics, 30(2), 213-235

Suneja, B.P.; Datta, T.K. (1998). Active control of ALP with improved performance functions. Ocean Engineering, 25, 817-835

Suneja, B.P.; Datta, T.K. (1999). Nonlinear open-close loop active control of articulated leg platform. International Journal of Offshore and Polar Engineering, 9, 141-148

Tabeshpour, M.R.; Ebrahimian, H. (2007). Application of friction dampers in seismic retrofit of existing structures, 5th international conference on seismology and earthquake engineering, SEE5, Tehran, Iran 
Tabeshpour, M.R. (2009). Effect of near field motions on seismic behavior of long period structures. Bana Journal, Vol. 11, No.1 [In Persian]

Tabeshpour, M.R.; Ebrahimian, H. (2010). Seismic Retrofit of Existing Structures using Friction. Asia Journal of Civil Engineering, 11(4), 509-520

Tabeshpour, M.R.; Dehkharghanian, V. \& Dovlatshahi, M. (2010). Effect tuned mass damper on vertical vibration of tension leg platform, MIC2010, Zibakenar, Iran, [In Persian]

Tabeshpour, M.R.; Rezaie, E. \& Arafati, N. (2011). Response mitigation of jacket platforms using tuned mass damper, 1st National conference on steel structures, Tehran, Iran

Takewaki, I.; Murakami, S. \& Fujita, K. \& Yoshitomi, S. \& Tsuji, M. (2011). The 2011 off the Pacific coast of Tohoku earthquake and response of high-rise buildings under longperiod ground motions, Soil Dynamics and Earthquake Engineering, In Press

Terro, M.J.; Mahmoud, M.S. \& Abdel-Rohman M. (1999). Multi-loop feedback control of offshore steel jacket platforms. Computers \& Structures, 70(2), 185-202

Toma, S.; Chen, W.F. (1982). Inelastic cyclic analysis of pin-ended tubes. I Struct Div., ASCE, 108, 2279-2294

Vandiver, J.K.; Mitome, S. (1979). Effect of liquid storage tanks on the dynamics response of offshore platform. Applied Ocean Research, 1, 67-74

Veletsos, A.S. (1977). Dynamics of Structure-Foundation Systems. Structural and Geotechnical Mechanics: A Volume Honoring Nathan M. Newmark, editor: W. J. Hall, PrenticeHall, Englewood Cliffs, NJ

Venkataramana, K.; Kawano, K. \& Yoshihara, S. (May 24-29, 1998). Time-domain dynamic analysis of offshore structures under combined wave and earthquake loadings, proceeding of the eighth international offshore and polar engineering conference, Montreal, Canada

Vincenzo, G.; Roger, G. (1999). Adaptive control of flow-induced oscillation including vortex effects. International Journal of Non-Linear Mechanics, 34, 853-68

Wang, S. (2002). Semi-active control of wave-induced vibration for offshore platforms by use of MR damper, International Conference on Offshore Mechanics and Artic Engineering, Oslo, Norway, 23-28

Xu, T.; Bea, R. (January 1998). Reassessment of the Tubular Joint Capacity: Uncertainty and Reliability. Screening Methodologies Project Phase IV, Report to Joint Industry Project Sponsors, Marine Technology and Management Group, Department of Civil Engineering, University of California at Berkeley

Yue, Q.; Zhang, L. \& Zhang, W. \& Kärnä, T. (2009). Mitigating ice-induced jacket platform vibrations utilizing a TMD system. Cold Regions Science and Technology, 56(2-3), 8489

Zayas, V.A.; Popov, E.P. \& Mahin, S.A. (1980). Cyclic inelastic buckling of tubular steel braces. Report UCB/EERC-80/16, Earthquake Engineering Research Center, Berkeley (CA), University of California 


\title{
Seismic Strengthening of Reinforced Concrete Buildings
}

\author{
Hasan Kaplan and Salih Y1lmaz \\ Pamukkale University, Department of Civil Engineering \\ Turkey
}

\section{Introduction}

Many buildings have either collapsed or experienced different levels of damage during past earthquakes. Several investigations have been carried out on buildings that were damaged by earthquakes. Low-quality concrete, poor confinement of the end regions, weak column-strong beam behavior, short column behavior, inadequate splice lengths and improper hooks of the stirrups were some of the important structural deficiencies (Yakut et al., 2005). Most of those buildings were constructed before the introduction of modern building codes. They usually cannot provide the required ductility, lateral stiffness and strength, which are definitely lower than the limits imposed by the modern building codes (Kaplan et al., 2011). Due to low lateral stiffness and strength, vulnerable structures are subjected to large displacement demands, which cannot be met adequately as they have low ductility.

One of the first known examples of strengthening is the strengthening of Hagia Sophia by Sinan the Architect in 1573. With an insight casting light on the modern era, Sinan built buttress type shear walls around the mosque in order to reduce horizontal displacements of the building. When looked at the scientific studies that were carried out on this field, it is seen a research process started in 1950s (Whitney et al., 1955). In those years, infill wall tests which had started to be performed on single storey reinforced concrete frames continued with several structural types and strengthening methods.

Deficiencies that emerge in reinforced concrete buildings in terms of stiffness, strength, ductility and redundancy led to studies intended to strengthen buildings against earthquakes. The strengthening methods used today are intended to improve one or some of the behavioral characteristics of buildings listed above. Methods for the strengthening of buildings may basically be categorized into two main groups: System based strengthening and member based strengthening (Moehle, 2000). In the system based strengthening methods, a structural system is modified by adding members such as reinforced concrete shear walls, mainly improving the strength and stiffness characteristics of the system (Jirsa \& Kreger, 1989; Albanesi et al., 2006). As to the member based strengthening methods, it is aimed at ensuring an improvement in the ductility of a system by means of enhancements made to those members with inadequate capacity or ductility. In these methods, it can be considered that there are no significant changes occurring in strength and stiffness characteristics of the load-bearing system. 


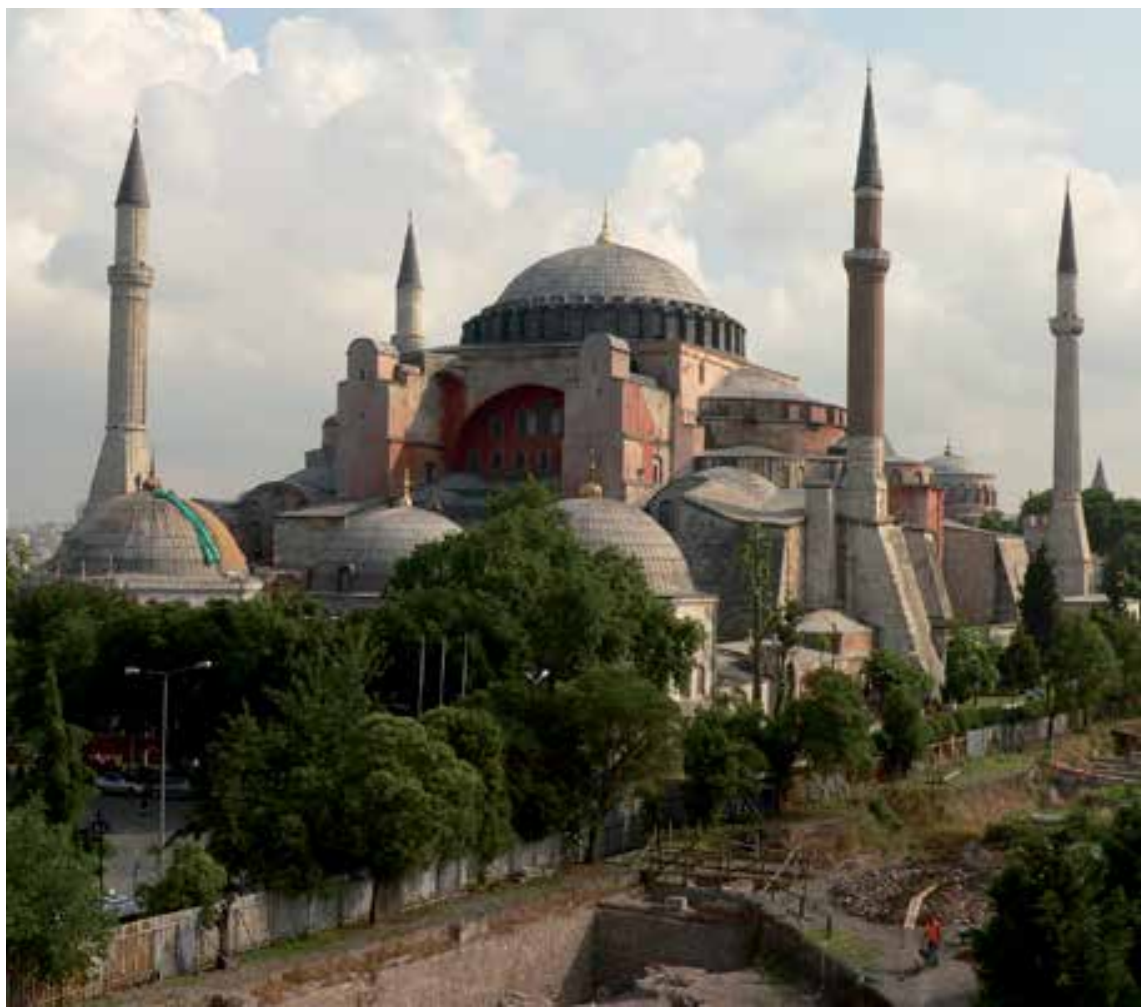

Fig. 1. Hagia Sophia is strengthened with external buttress type walls by Sinan in 1573.

\section{Design philosophy for strengthening}

During earthquake motions, deformations take place across the elements of the load-bearing system as a result of the response of buildings to the ground motion. As a consequence of these deformations, internal forces develop across the elements of the load-bearing system and a displacement behavior appears across the building. The resultant displacement demand varies depending on the stiffness and mass of the building. In general, buildings with higher stiffness and lower mass have smaller horizontal displacements demands. On the contrary, displacement demands are to increase. On the other hand, each building has a specific displacement capacity. In other words, the amount of horizontal displacement that a building can afford without collapsing is limited. The purpose of strengthening methods is to ensure that the displacement demand of a building is to be kept below its displacement capacity (Yllmaz et al., 2011). This can mainly be achieved by reducing expected displacement demand of the structure during the strong motion or improving the displacement capacity of the structure.

In the system strengthening, new elements are added to a building to enhance its global stiffness. With an increase in the stiffness, the natural period of vibration of the building is to decrease. This, in turn, will result in a decrease in the amount of horizontal displacement that must be achieved by the building to resist earthquakes. When the building has enough stiffness, it will no longer able to achieve the amount of displacement which would cause it to collapse. Moreover, addition of new members to the building shall mostly increase the horizontal load 
capacity of the building as well (Canbay et al., 2003). The increased capacity will therefore require greater ground motions to allow the building to develop a yielding behavior. Thus, it can be said that the system strengthening does not only prevent collapsing but also delays structural damages. More clearly, the damage level of a building which is expected to sustain significant damages during a medium intensity earthquake may be reduced to minimum levels when it is strengthened by system strengthening techniques.

The element strengthening is a method based on the reinforcement of inadequate elements triggering the loss of stability due to the sustained damages without undertaking major changes in the load-bearing system of the building. In this method, negligible changes take place in the characteristics of a building such as stiffness and mass since no significant changes are made to the load-bearing system. Therefore, it must be considered that there will be no significant changes in the displacement demand after the member strengthening.

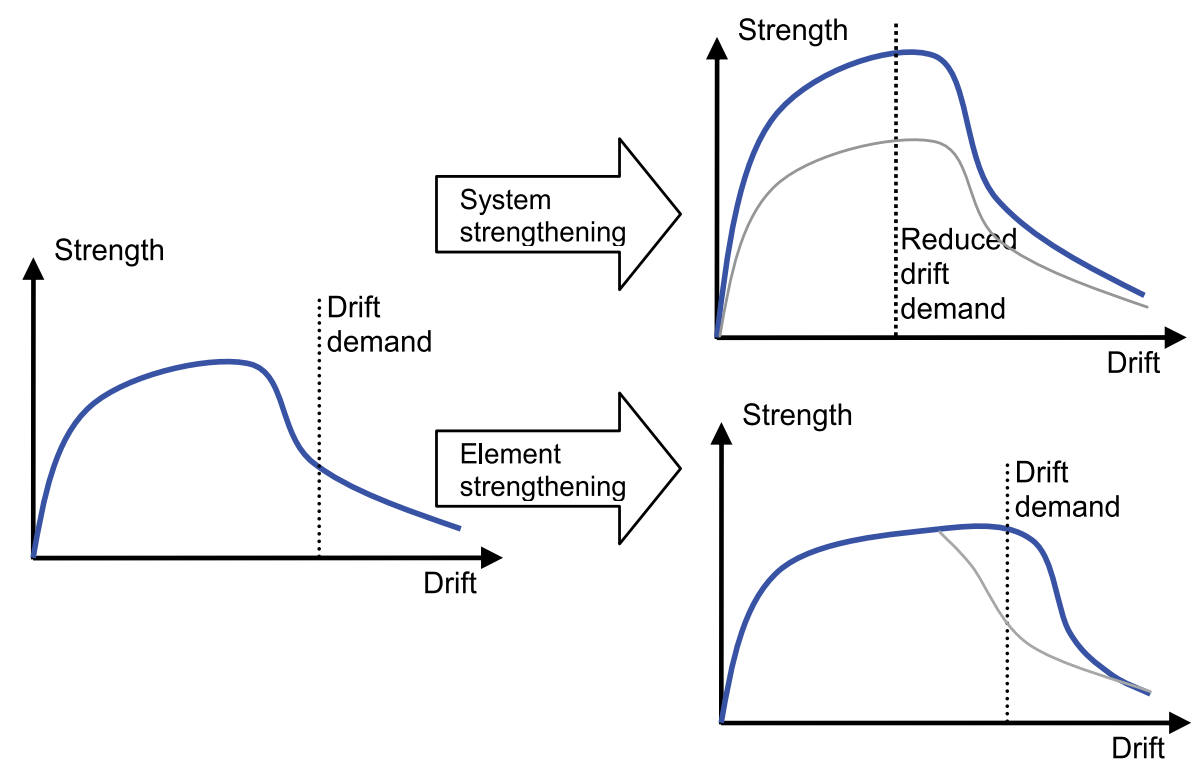

Fig. 2. Change in the displacement demand and capacity of the structure after strengthening

\section{System based strengthening techniques}

Most of the strengthening strategies have recently been based on global strengthening schemes as per which the structure is usually strengthened for limiting lateral displacements in order to compensate the low ductility (Sonuvar et al., 2004). In these methods causing a change in the global behavior of a building, as explained above, a behavioral change takes place when new members are added to the building. For a building which is currently used, it is important that the new members which are to be added to the structure are few in number and they are designed to ensure a significant increase in the load capacity and stiffness of the structure. It is well known by construction engineers that this target may most easily and economically be achieved through reinforced concrete and steel shear walls. Shear walls that are to be added to the building can be designed in the form of infill shear wall (Higashi et al., 1982) or external shear wall (Kaplan et al., 2009). Below are presented some of the most frequently applied system based strengthening methods. 


\subsection{Infill shear walls}

Among the global strengthening methods, addition of RC infill is the most popular one. Many researchers have focused on the addition of infill RC walls and found that the installation of RC infill walls greatly improves lateral load capacity and stiffness of the structure. Even in cases of application to damaged buildings, the infill method can yield satisfactory results (Canbay et al., 2003; Sonuvar et al., 2004).

In the strengthening method with infill walls, the existing partition walls in the building are removed and high strength reinforced concrete shear walls are built instead (Figure 3). In such a strengthening application, the shear walls bear majority of the earthquake loads and limits the displacement behavior of the building while the frame system resists very low amounts of the earthquake loads. Reinforced concrete infill walls can also be used as partial walls and wing walls (Higashi et al., 1982; Bush et al., 1992). Door and window openings may also be provided in these walls to allow the building to deliver its architectural functions although they reduce stiffness and strength of the wall. In such applications preventing the construction of infill walls in the form of a full-fill wall, many experimental studies indicated that these walls develop more brittle damages compared to full-fill walls.

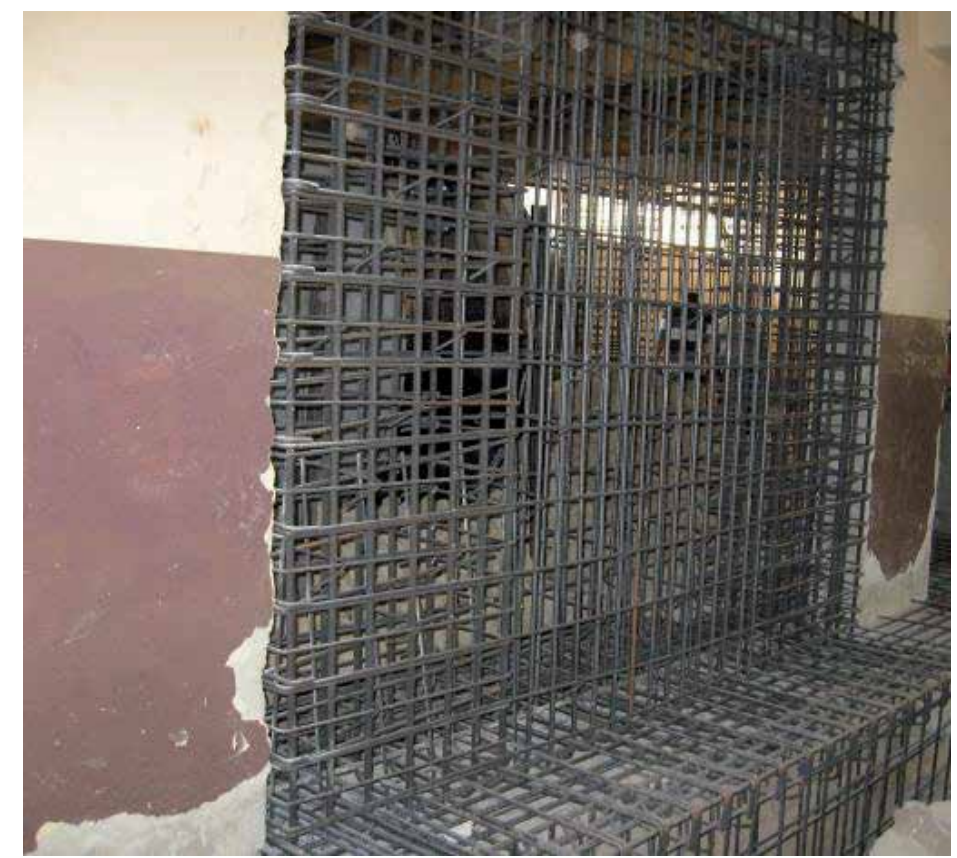

Fig. 3. Infill wall application in an RC building

As seen in Figure 4, columns of the existing building constitute the end regions of infill walls. Well confined end regions of reinforced concrete walls are considerably important in terms of the ductility of the wall. However, at the end regions of the strengthening walls, there generally exist inadequately confined columns with lower concrete strength. In that case, the end region must be confined in order to enhance ductility of the wall. Figure 4 presents various alternatives for confining end zones. Confined end region can be both made by jacketing of the existing column and can also be formed in the infill wall. If confinement and material characteristics of the existing columns are relatively good, the 
confined end region may be provided in the infill wall (TEC, 2007). In case the characteristics of end column are poor, it should be preferred to jacket the existing column.
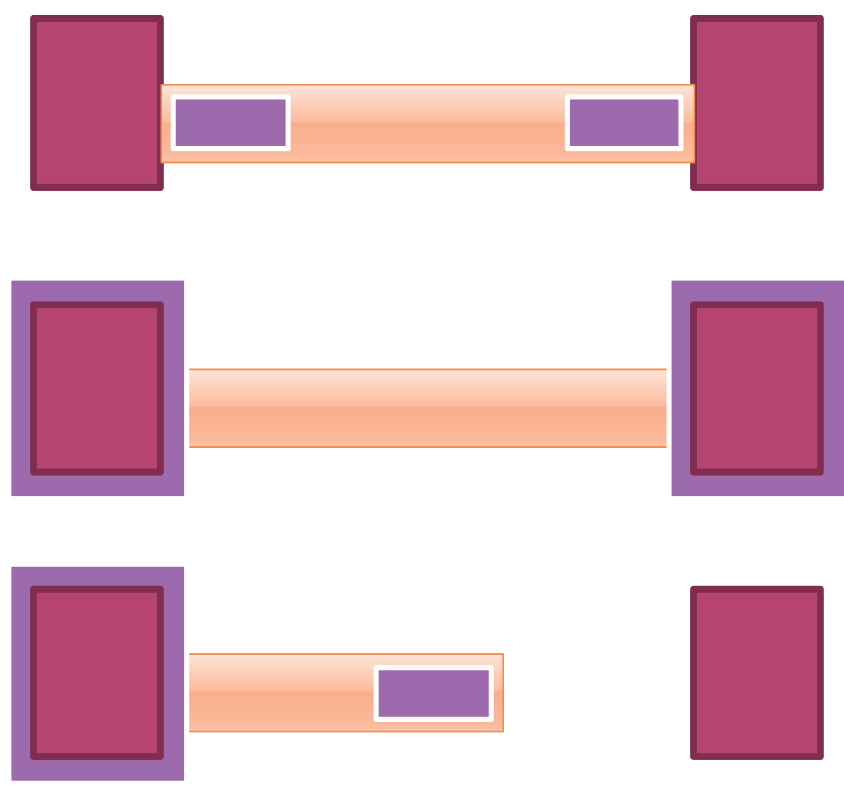

Fig. 4. Formation of the end zones in full or partial shear walls

Minimum reinforcement and anchor requirements for infill walls are given in Figure 5. It is also considerably important to ensure the continuity of longitudinal reinforcement in a manner that allows the wall to achieve an adequate moment capacity. Reinforcement continuity can be maintained by means of continuous anchors which are installed across beams and extended to both storeys. Anchors that are both installed across the foundation and the beams and fastened to the longitudinal reinforcements must have an enough lap splice length and embedment depth. If beam height is big, it would be difficult, or even impossible to drill the beam along its height and to install anchors. In such cases, wall can be constructed by breaking the beam or the longitudinal reinforcements can be placed on the sides of the beam by making the infill walls wider than the beam. In that case, anchored crossties must be installed on the lateral faces of the beam in order to restrict the buckling length of the reinforcement by fastening to the lateral face of the beam. In Figure 6, it is seen that with such anchors, there is a decrease in the buckling length of the longitudinal reinforcement extending along the sides of a beam. Anchored crossties can either be installed on both sides of a beam or be installed on each face separately.

Reinforced concrete walls to be added to the structure must be placed in a manner that it is not caused torsional effects on the structure and irregularities in the structure are eliminated, as observed in the design of new buildings. Some appropriate and inappropriate shear wall layouts are presented in Figure 7. The added walls must be regularly distributed across the plan. Unlike the design of existing building, a special example for strengthening is seen in Figure 7, at the bottommost. Although new walls are seemingly added in a symmetric and regular way to a building which have 2 irregular walls made from S220 
grade steel, this layout is actually not appropriate. Since stiffness of the walls are close to each other, it is obvious that, in this building which is initially displaced without buckling, old walls will first reach the yield moment. There will be no enhancements in the new walls and the building will undergo twisting until the old walls lose their bearing capacity since there is no force balancing the earthquake loads that impact on the center of mass after these shear walls are failed. As it can be seen, in this seemingly correct design, the old walls are collapsed without any yielding in the new high capacity walls. However, the alternative of this seemingly appropriate incorrect design has a more regular behavior even though it exhibits a more irregular layout. Although some buckling is expected to occur across the elastic zone, the total capacities of those walls placed on the left- and right-hand sides of the structure are close to each other and will approximately yield at the same time. Any resultant accidental buckling is compensated through a shear wall placed in the middle zone.

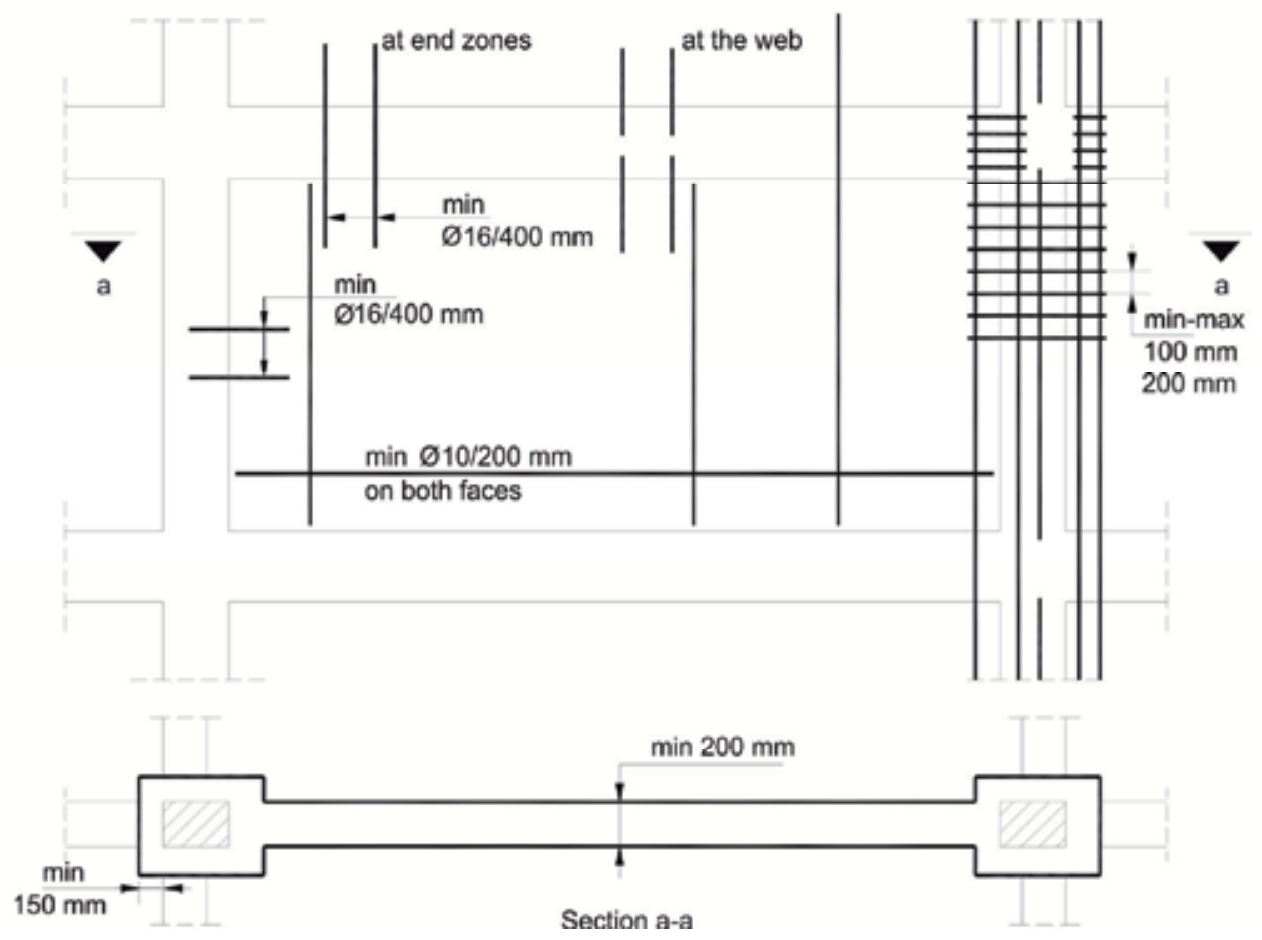

Fig. 5. Minimum reinforcement and anchor requirements for infill walls

\subsection{External shear walls}

Although the use of shear walls becomes widespread due to the fact that they are effective strengthening elements, they are also known to result in some difficulties hence they require a great deal of demolition and construction works in the existing structure. Application of external shear walls is an approach introduced to diminish such difficulties (Sucuoglu, 2006). In this approach, shear walls are applied to the external facade of a building without demolishing the existing infill walls. In that case, the shear wall can be placed in parallel with or perpendicular to the existing frame members. 

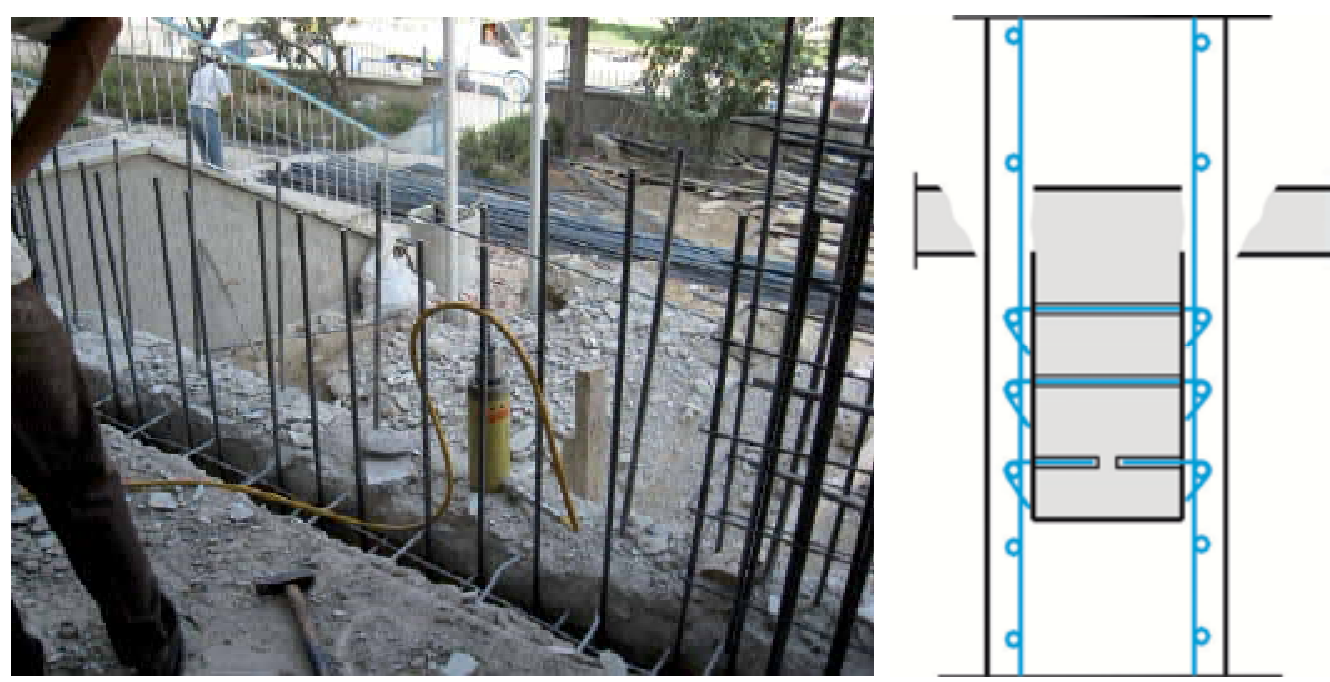

Fig. 6. Wide infill walls and anchored crossties confining the longitudinal reinforcements

In case the shear walls are located perpendicular to the building façade (Kaltakci et al., 2008), large openings are needed in the building facade. The shear walls installed function like a buttress. In cases where pile foundation is not applied for the shear wall foundation, such shear walls are effective in only one direction. In order to create a positive effect on earthquake resistance of the building, they must be installed at opposite facades. This increases the required amount of shear walls and costs. For all these reasons, external shear walls that located perpendicular to the external facade are not preferred in the application.

The more preferred form of the external shear walls is the application where they are installed in parallel with the building façade (Y1lmaz et al., 2010c, 2011). An example of the application of a shear wall located in parallel with the building facade is seen in Figure 8 . Design and application of external shear walls are easier compared to that of infill walls. End zones of shear walls can be detailed in itself. These shear walls may also be manufactured as prefabricated panels and assembled in place (Y1lmaz et al., 2010a, 2011; Kaplan et al., 2009).

Contrary to various advantages of external shear walls, there is a significant difference between external shear walls and infill shear walls. External shear walls shall not make a positive contribution to the frame strength when anchors connecting the external shear walls to the existing frame are damaged. However, even if infill shear walls are designed inadequately or applied incorrectly, it is apparent that they will have a bracing effect on the frame (Ohmura et al., 2006). In reinforced concrete design of external shear walls, it should be conformed to those guidelines that must be considered when designing reinforced concrete shear walls. What has importance is to correctly connect the external shear wall to the existing frame (Yilmaz, et al., 2010a, 2011). Although there is no methods for this provided in the applicable codes, from the experimental studies carried out by the authors, it was found that anchors can exhibit adequate performance when the design anchor shear force is determined in accordance with the capacity design principles. 

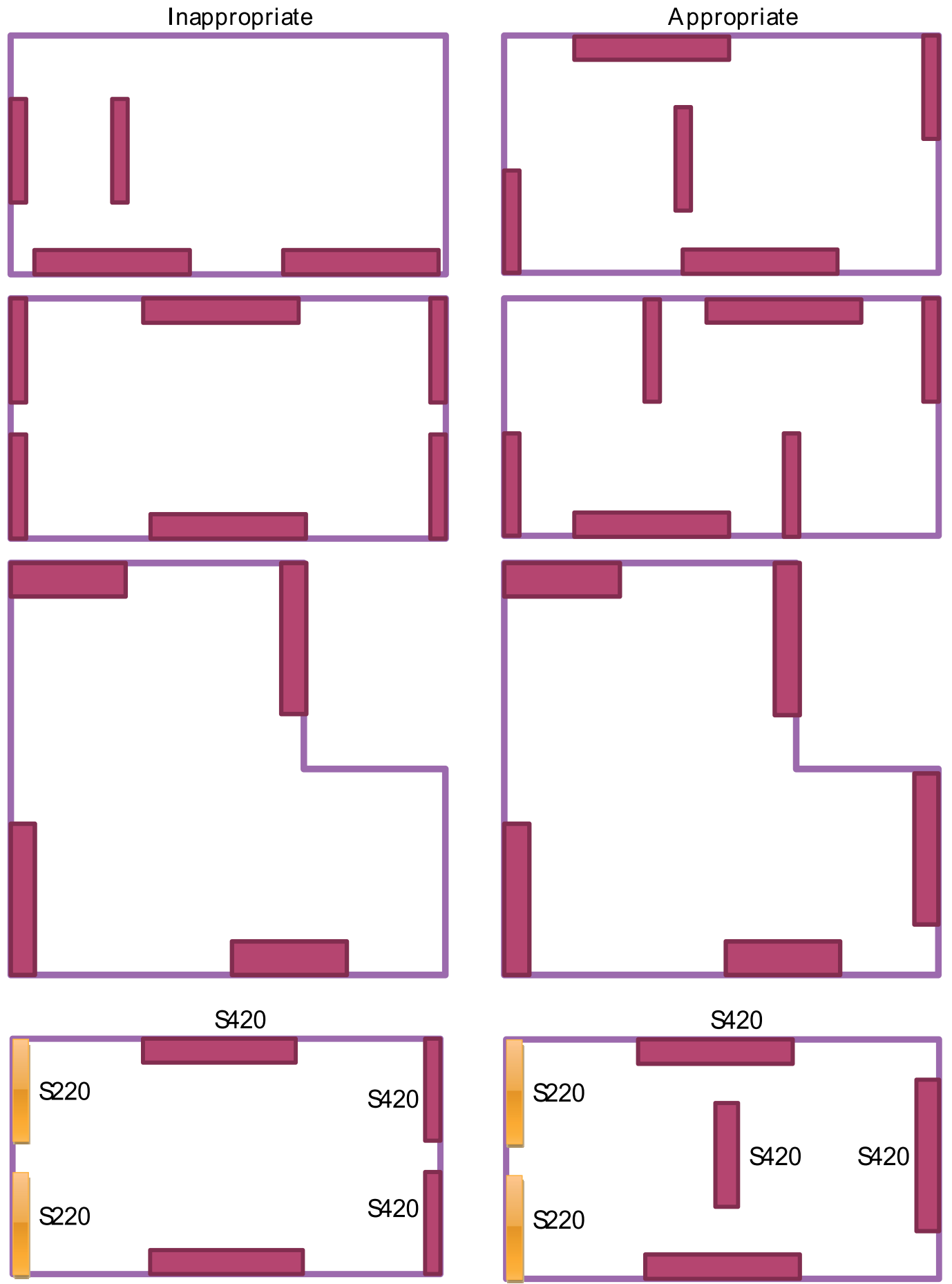

S420

S420

Fig. 7. Inappropriate and appropriate shear wall layouts 


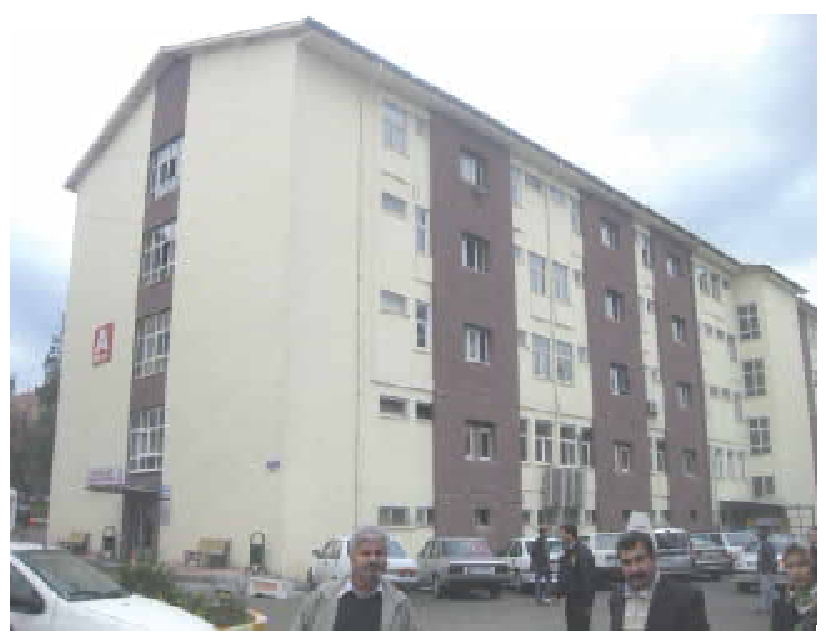

Fig. 8. An external shear wall strengthened building

The load transfer the external shear wall and the existing structure by the external shear wall anchors is schematically presented in Figure 9. To design anchors of the external shear wall, it is first determined the shear wall's ultimate moment capacity $\left(M_{p}\right)$. The design shear force of the anchors applied to beams at the storey level, $V_{a b}$ is calculated for $\mathrm{i}^{\text {th }}$ storey by the equation 1 given below. For calculations made for the last storey, it is taken that $V_{d, i+1}=0$. This force will give the anchor force required to transfer the necessary shear force to the shear wall at the relevant storey to allow the shear wall to reach its capacity at its bottom.

$$
V_{a b, i}=\left(V_{d, i}-V_{d, i+1}\right) \cdot \frac{M_{p}}{M_{d}}
$$

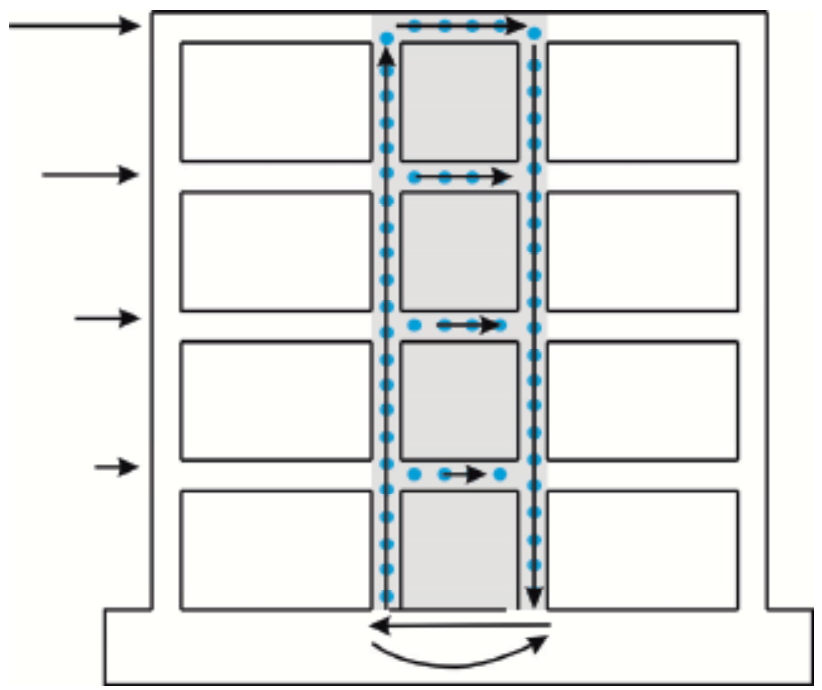

Fig. 9. Load transfer between the structure and external shear walls by anchors 
After the storey anchor design obtained, contribution of the anchors to the shear wall moment capacity is established. When $M_{a b}$ moment created by $V_{a b}$ forces at the bottom of shear wall is subtracted from the ultimate bearing capacity of the shear wall, the residual moment $\left(M_{a c}\right)$ obtained is expected to be carried by a force couple which is to be created by the anchors installed in the columns (Equation 3).

$$
\begin{aligned}
& M_{a b}=\sum_{i=1}^{n} V_{a b, i} \cdot H_{i} \\
& M_{a c}=M_{p}-M_{a b}
\end{aligned}
$$

Experimental studies carried out indicated that an increase in the design anchor shear force for the last storey by 25 to $30 \%$ has positive effects in terms of behavior (Y1lmaz, et al., 2010a). In general, anchor damage which is to be created at the top storey subjected to the maximum anchor shear force results in a significant loss in the bearing capacity of the shear wall and causes the anchors at the lower storeys to be damaged or leads to serious damages at the top storey level (Figure 10).

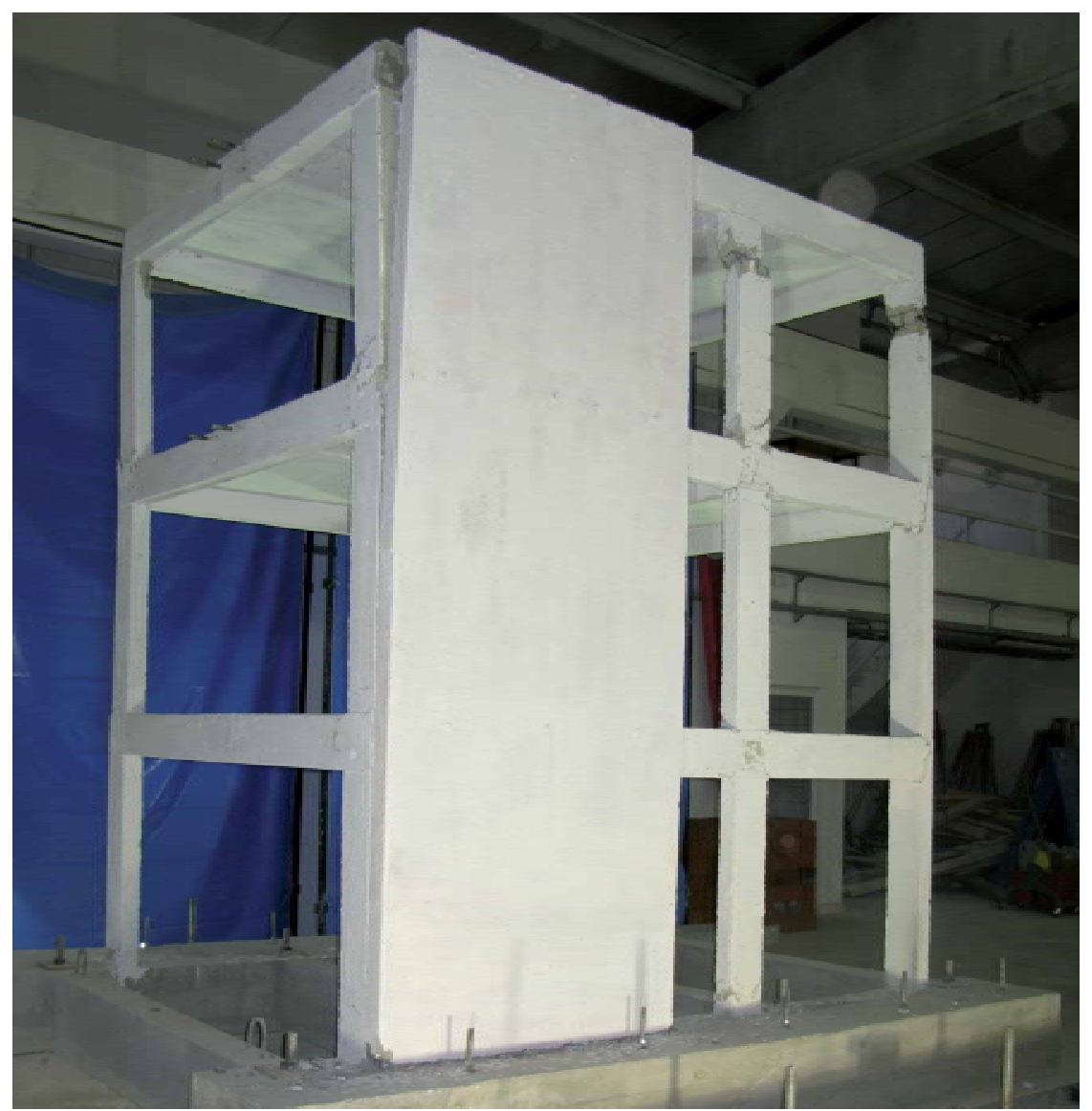

Fig. 10. A damaged upper storey due to poor anchor design 


\subsection{Steel bracing}

Steel bracing for RC frames has also been used to reduce drift demands. Bracing can either be implemented inside the frame (Masri \& Goel, 1996) or applied from outside of the system (Bush et al., 1991) like RC walls. Post-tensioning can also be applied to bracing elements (Gilmore et al., 1996). In either case, steel bracing offers more suitable solutions in aesthetical terms for numerous applications. Although its application inside the building is not easy for those buildings with small openings, it particularly allows easy installation across the axes on external facades (Gorgulu et al., 2011). Research on various types of bracing styles is available in the literature (Perera et al., 2004; Ohmura et al., 2006). Architectural characteristics and functionality can be less disturbed by using an appropriate bracing style. Figure 11 presents the use of buttress type steel shear wall constructed on the building's external facade as a different example.

Design of steel elements must be made in conformity with the details specified in steel standards and codes. For the connection between the existing structure and steel members, the anchor design principles given in the subsequent section may be utilized. Another mode of damage that must especially be considered in the buttress type steel shear walls is to be the out-of-plane buckling of the compression elements of the buttress type shear wall placed. If possible, it is recommended to install lateral supports at storey levels to prevent lateral buckling. Use of such elements may also economize design of shear walls to a certain extent.

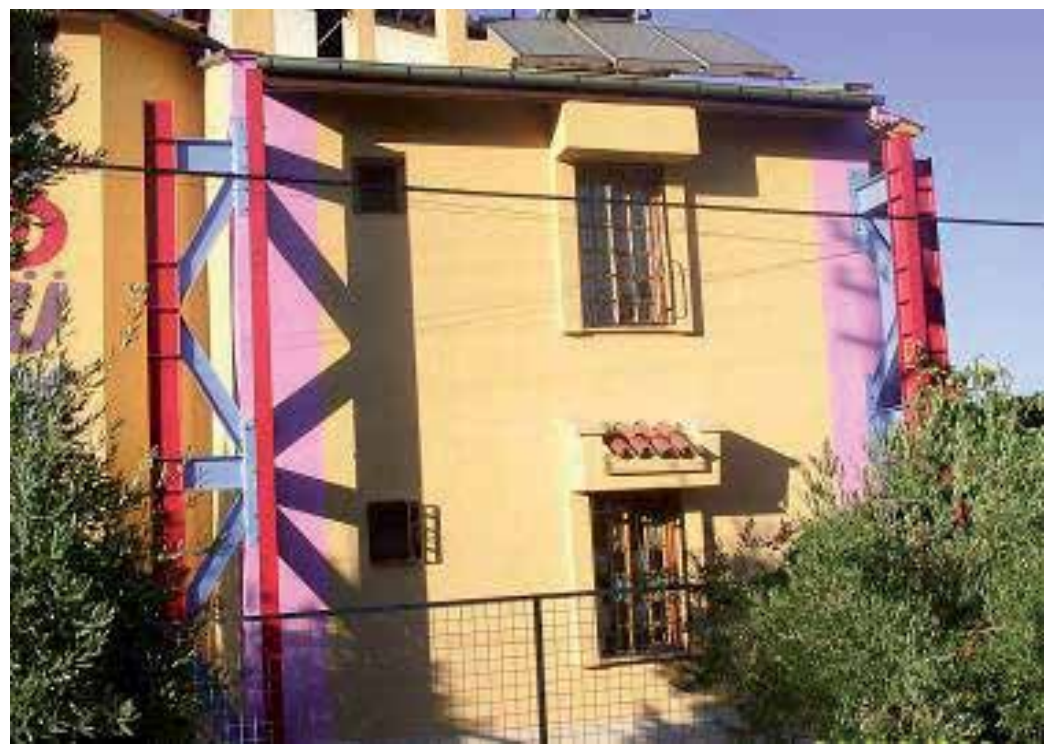

Fig. 11. Buttress type external steel shear wall (Photo by Dr. Yavuz Selim Tama)

\subsection{Infill strengthening}

Another method that can be used apart from adding new ductile shear walls to a building with an inadequate earthquake resistance is to improve both capacities and ductility of the existing brittle partition walls which are constructed between columns and beams in the form of fills. Those walls of which effects on the behavior are not considered during structural design can generally produce a certain bracing effect and make a positive 
contribution to the behavior. There are too many uncertainties regarding the behavior of these members and they are not likely to produce a desired bracing effect at all times. Partition walls may also lead to brittle damages across the surrounding columns by exhibiting many different behaviors. In addition, these walls are the members that are first damaged and lose their bearing capacity in a building under earthquake loads. A wide range of methods were developed to enhance capacity and ductility of these walls. Some of them include strengthening with mesh reinforcement, strengthening with precast panel and strengthening with FRP materials. The infill walls which are strengthened with those methods provide a bracing effect to the reinforced concrete frame for a longer period of time, restrict the building's displacement and produce a similar effect like the shear walls (Baran, 2005; Frosch et al., 1996). However, decrease in force is more brittle since the strengthened infill walls cannot display a bending behavior while the strengthening shear walls exhibits a bending behavior and reach their capacity in a ductile manner. Design engineer is expected to consider that these methods bring out a more brittle solution compared to those structures strengthened with reinforced concrete or steel shear walls.

\section{Element-based strengthening}

Element-based strengthening approach is the modification of deficient elements to increase ductility so that the deficient elements will reach their limit states in a ductile manner when subjected to design events. However, this strategy is more expensive and harder to implement in cases of many deficient elements which is the reason that the global strengthening methods have been more popular than element strengthening. Effective results can be obtained by using such methods in buildings with a limited number of deficient elements along with the global strengthening methods.

\subsection{RC jackets}

One of the most frequently used methods for strengthening of the reinforced concrete columns is the reinforced concrete jacketing (Figure 12). Jacketing which can be defined as the confinement of the column with new and higher quality reinforced concrete elements may be implemented for various purposes based on the type of deficiencies that the structural member has. Columns subjected to brittle damages can be jacketed in order to enhance resistance against shear and/or axial loads. In that case, although the purpose of jacketing is only to increase axial load or shear strength, some changes will also occur in the bending stiffness and moment capacity of the member after the jacketing application. By considering these changes during the jacketing design, the jacketed section is ensured to achieve adequate shear and axial load strength.

Except for such brittle damages, the jacketing is applied for elements with inadequate bending capacity or ductility. By this way, strength of the columns displaying a splice failure as a result of bending can also be improved. Jacketing of the columns is to produce the best result if it is implemented at 4 sides of the column. Where necessary, confinement at 3 sides can also provide adequate performance. However, it is not generally recommended to implement the jacketing at 1 or 2 sides. Because, with such jacketing applications, no significant changes take place in the confinement characteristics of the member. 


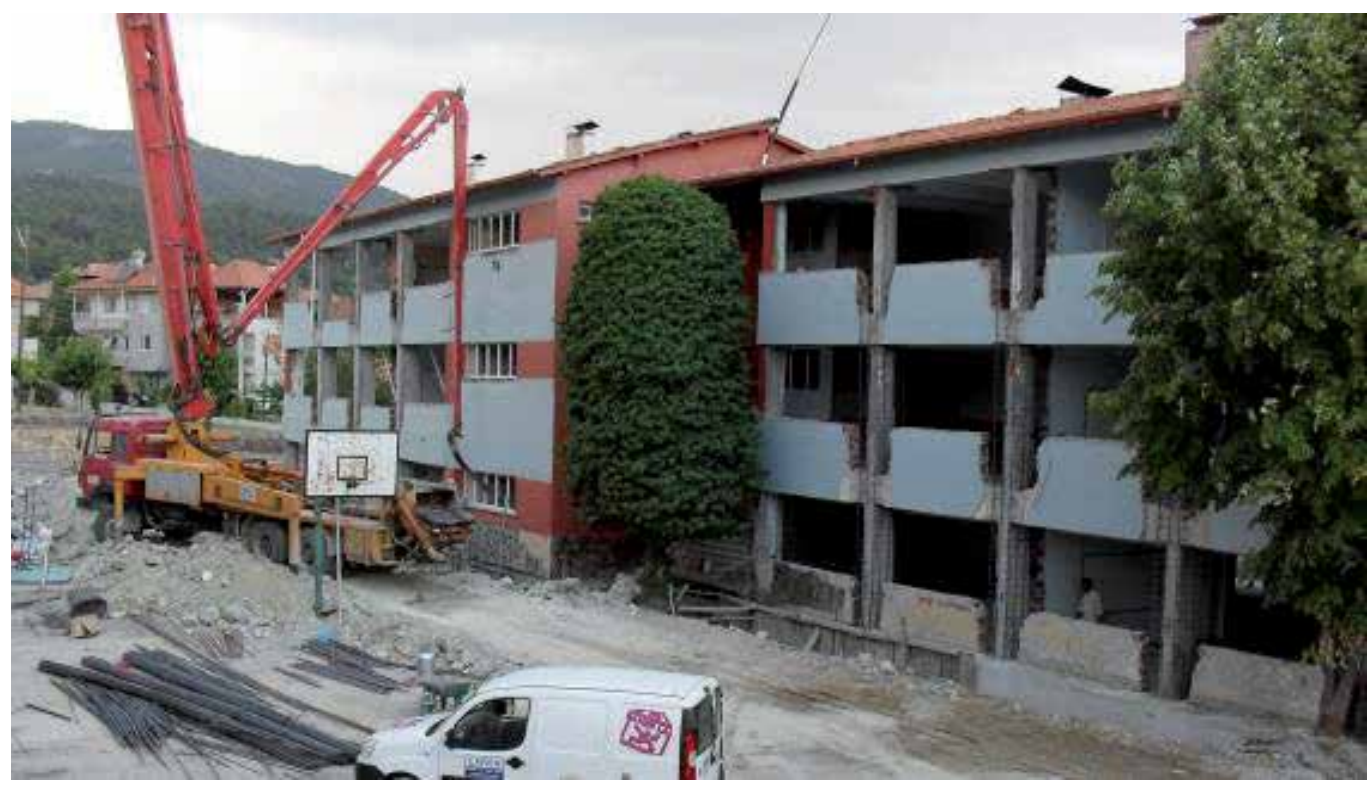

Fig. 12. An RC jacketing application (Photo by Ahmet Sarışın)

Although the reinforced concrete jacketing can technically be applied for all the structural members, a jacketing application which will increase the beam's bending capacity is not advised since it may cause the strong beam-weak column formation resulted by an increase in the capacity of beam members.

\subsection{Steel jackets}

Jacketing with steel elements is a practical method used frequently for various applications. A typical steel jacketing application is presented in Figure 13. Steel jacketing can readily be used to especially enhance the shear strength of reinforced concrete elements. Located at the corners of an element, L-profiles are coupled by means of steel plates and confined. With the maintenance of continuity between storeys, steel jacketing can also be used to increase the bending strength. Also, the maintenance of adequate strength between the steel element and reinforced concrete element is inevitable for the improvement of bending capacity.

\subsection{Fiber reinforced polymers}

In recent years, use of Fiber Reinforced Polymer has considerably become widespread in strengthening applications. Fiber polymer fabrics that can be used to improve bending, shear and axial capacities of the columns and beams may be manufactured from various materials such as carbon, glass and aramidWithout an increase in the volume of the strengthened member, significant improvements can be achieved in the capacity and ductility characteristics of the element. In Figure 14, beam strenghthening for a parking structure is shown. Due to space restrictions in this structure, it was not feasible to utilize classical element strengthening techniques. These materials may practically be used for numerous purposes such as enhancement of the flexural capacity of floor slabs and improvement of shear capacity of beams, columns or shear walls. 


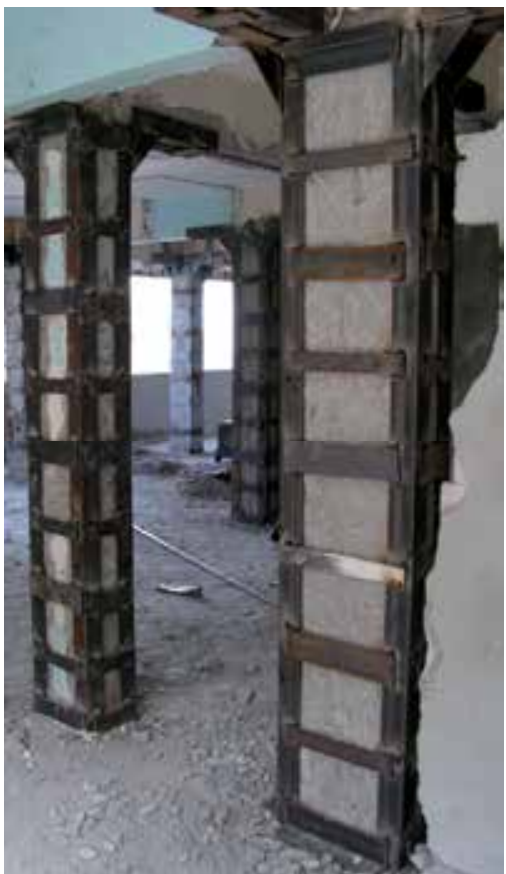

Fig. 13. Steel jacketing applied to RC columns (Photo by Dr. Ali Haydar Kayhan)

In case FRP material is used like a longitudinal reinforcement, the additional flexural capacity produced can easily be found by a simple calculation of cross-section. Calculations for determining its contribution to the shear capacity is not very different from the conventional reinforced concrete calculations. To measure enhancement in the axial capacity and change in the ductility of the member, more complex calculations beyond the limits of basic reinforced concrete knowledge are needed.

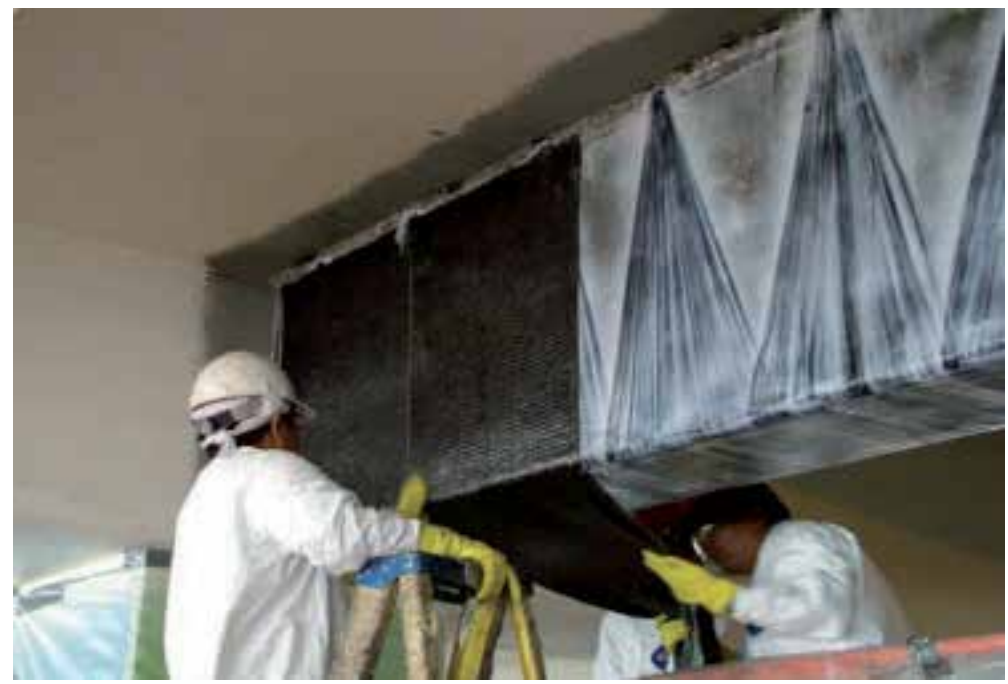

Fig. 14. FRP confinement of a beam (reprinted with the permission of FYFE Europe) 


\section{Connections between new and existing elements: Anchors}

\subsection{Design}

Anchor design should primarily be made by considering different modes of behavior that the anchor members can exhibit. The most general approach to determine the anchor strength is the capacity approach given in ACI318 Appendix D (2005). Although the approach suggested in ACI318 is recommended for cast-in-place and mechanical anchors, it was found from the experimental studies that it can also be used for most of the chemical anchors. Both in shear or tensile loads, the ultimate capacity could be calculated for the different collapse modes in the ACI318 method and the ultimate capacity would be determined taking the collapse mode with the lowest strength into consideration.

Possible failure modes that the anchor elements are likely to sustain under tensile forces are shown in Figure 15 (Yilmaz \& Özen, 2010a). In the ACI method, capacity values for the failure modes indicated below are calculated separately or determined empirically. After the relevant capacity values are established for the steel failure $\left(N_{s a}\right)$, debonding failure $\left(N_{p}\right)$, cone failure $\left(N_{c b}\right)$ conditions, the tensile capacity of the anchor element is considered to be the lowest one of these values. While possibility of the occurrence of a cone failure in shallow anchors which are implemented for those elements with lower concrete strengths increases, increased diameter of the steel also raises that possibility. Debonding failure is affected by concrete strength and diameter of the steel as well as numerous unknown factors related to the application and is definitely recommended to be determined experimentally.

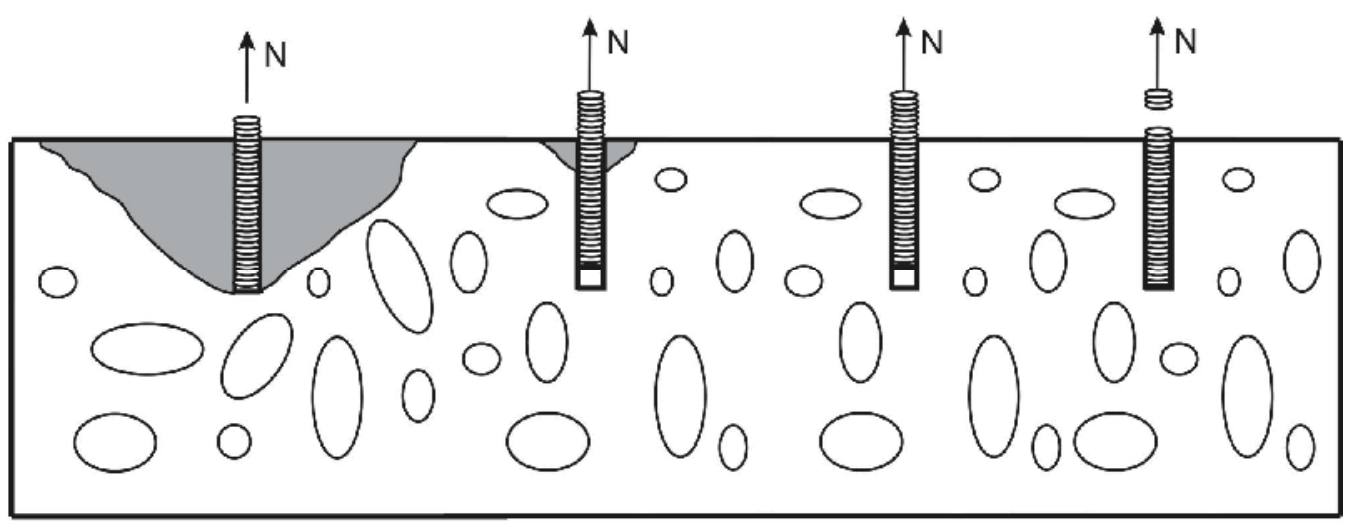

Fig. 15. Failure modes of post-installed anchors under tensile forces (concrete cone, small cone with debonding, debonding, steel failures)

Similar to tensile failure modes, in ACI318 Appendix D, three different failure modes were defined upon reaching the anchor shear capacity: steel failure $\left(V_{s a}\right)$, concrete pryout failure $\left(V_{c p}\right)$ and concrete breakout failure $\left(V_{c b}\right)$ of near edge anchors. These modes of collapse are shown in Figure 16. In situations where the anchor bars is closer to the edge, the ultimate concrete cone capacity generally governs the anchor capacity and where it is further away from the edge, the shear capacity of the anchor bar is the main determining factor. For shallow or high diameter anchors, concrete pryout failure may also govern the behavior (Çalışkan et al., 2010). 
Tensile or shear capacity determined with the abovementioned approach should be reduced by a strength reduction coefficient for design purposes. Various coefficients are listed in ACI318 depending on the environmental conditions and workmanship quality. These reduction coefficients vary between 0.45 and 0.75 depending on the loading type, failure mode and reliability of the application. Details of the formulations are to be studied from related codes or standards.

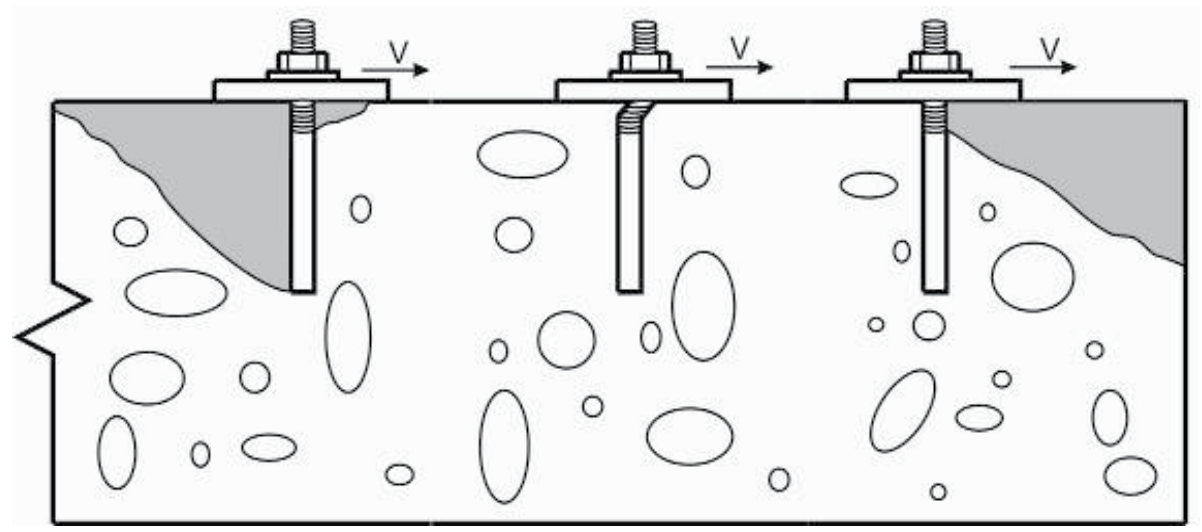

Fig. 16. Failure modes of post-installed anchors under shear forces

In some studies carried out recently on low strength concretes, it is suggested to use a reduction coefficient depending on the diameter of reinforcement when the anchor capacity is governed by steel failure mode per ACI318 formulations. Accordingly, the strength of reinforcements with larger diameters (bar diameters greater than $16 \mathrm{~mm}$ ) should be reduced, as seen in Figure 17. Research on this area is not completed yet. Designers are recommended to use high diameter bars with concern.

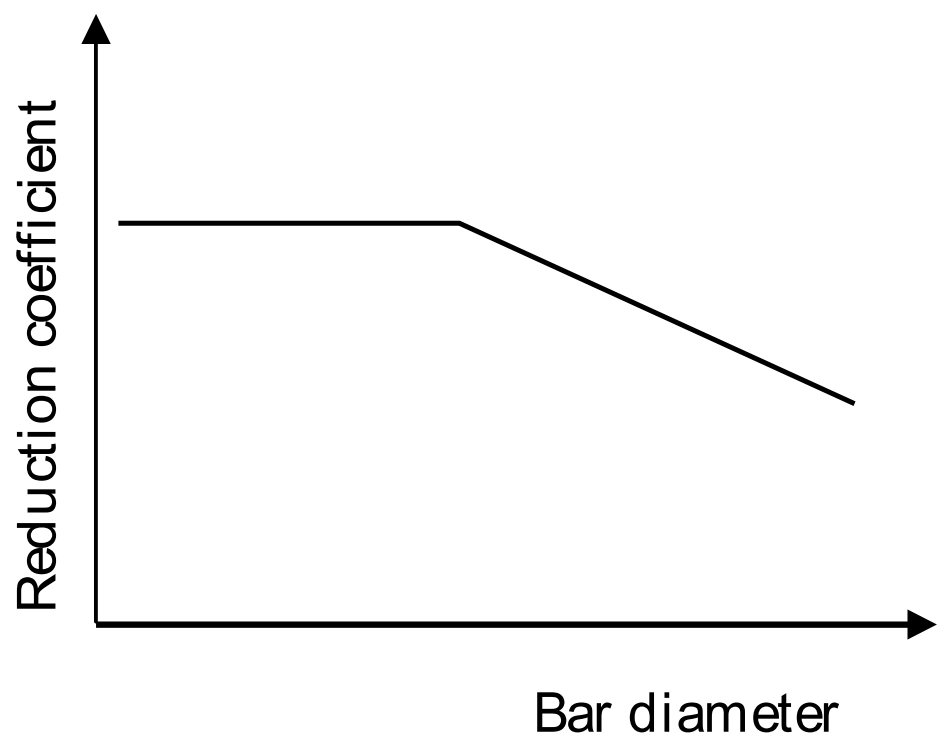

Fig. 17. Strength reduction for steel failure mode depending on bar diameter 
An important question for the design engineer is which failure modes will be allowed or not allowed. That is to say, it must become apparent that anchor capacity would allow failure modes such as cone or pryout which are of brittle failure modes or always a ductile failure arising from anchor bar should take place. The answer to this question varies depending on the usage mode of the anchor. For example, let us consider anchors which are overlapped to the longitudinal reinforcements at the bottom of shear wall. Since the shear wall shall achieve bending capacity due to tensile forces developed in these anchors, the shear wall cannot have a ductile bending behavior as expected if collapse takes place in a brittle failure mode in these anchors. For ductile behavior of the shear wall, these anchors must exhibit a yield behavior without formation of debonding or concrete cone. In case that an anchor with a diameter larger than the longitudinal reinforcement of shear wall is installed, the anchor capacity can be determined by brittle failure modes, but in that case, the anchor strength must not be lower than that of the longitudinal reinforcement connected so that the longitudinal reinforcement can display a ductile tensile yielding. When the shear wall reaches the bending capacity, capacity of other anchors that are found not to develop a yield mechanism can also be allowed to be designed according to brittle failure modes. In that case, brittle anchor failures will never occur since the anchor cannot reach its capacity.

\subsection{Application}

Performance of the elements connecting the old and new members mostly has an effect on the performance of the strengthened system. Thus, connection elements used in the strengthening must carefully be designed and implemented. Since majority of the application is comprised of anchor elements, this section is focused on some topics related to such elements. The most frequently used anchor type in strengthening applications is chemical anchors. Being readily applied in field, these anchors are also substantially cost-effective.

However, many reinforced concrete elements to which anchors are applied for the strengthening works have a very poor concrete quality and some of these elements have been seriously damaged during the service life of the structure. However, it is crucial to consider failure conditions of these elements during anchor installation. It is also important that holes to be used for anchor installation must be bored so as to cause the lowest level of damage to these elements. Figure 18 presents some incorrect applications made on low strength concretes.

One of the most important defects that can occur during the installation of chemical anchors is to carry out installation by applying chemical material around the steel bar only without adequately filling the holes with adhesive. Tensile tested specimen of an anchor bar installed in this way is presented in Figure 19. It is seen that epoxy on the surface of reinforcement is not in contact with a vast section of the hole. It is obvious that such an anchor will prevent the strengthening element from reaching its capacity.

Hole must be bored in a manner that the hole diameter is at least $4 \sim 5 \mathrm{~mm}$ higher than the bar diameter and between $120 \%$ and $150 \%$ of the bar diameter. Before the hole boring process, a reinforcement detection device must be used, otherwise lots of trials may be needed to bore holes and this results in unnecessary damages (Figure 20). In addition, where applicable, anchor bars must not be installed in defective zones. It should be considered that anchors that are near the edge of reinforced concrete element will have a decreased efficiency. Some anchors implemented across inappropriate locations are shown in Figure 20. 

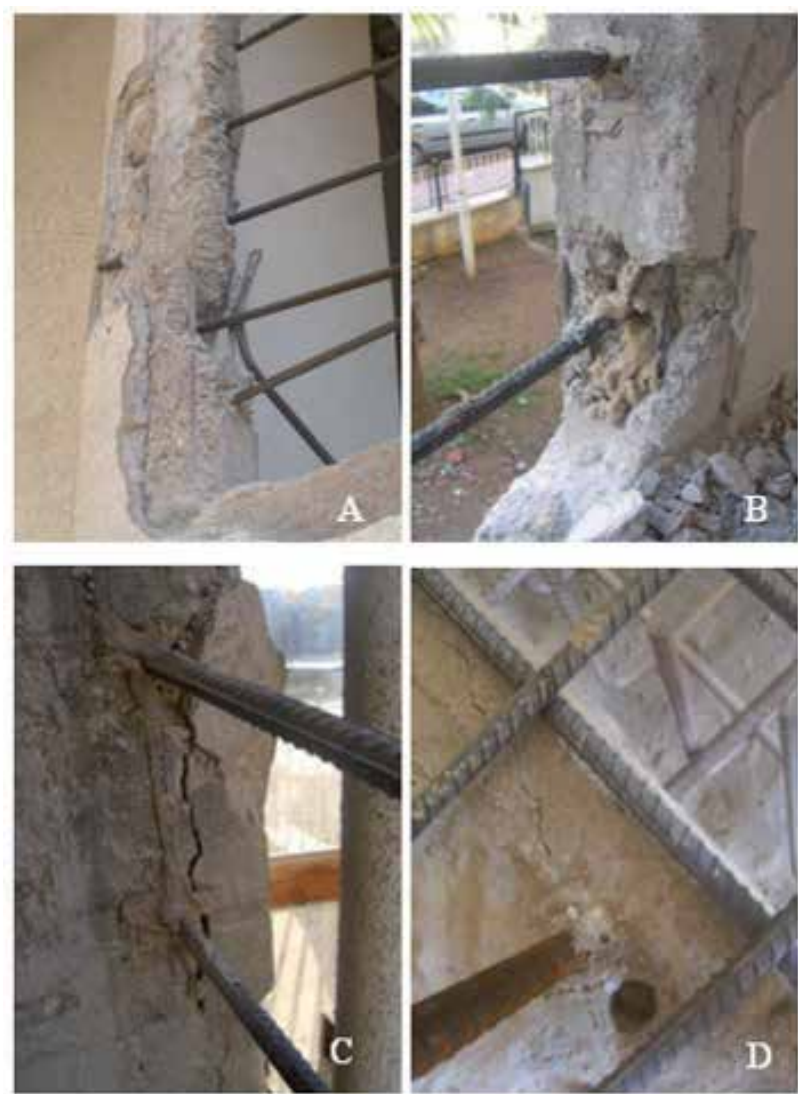

Fig. 18. Poorly implemented anchor examples (A, B: anchors implemented on defective surfaces; C: Anchor implemented near a crack; D: Anchor fracturing the concrete during the tensile test)

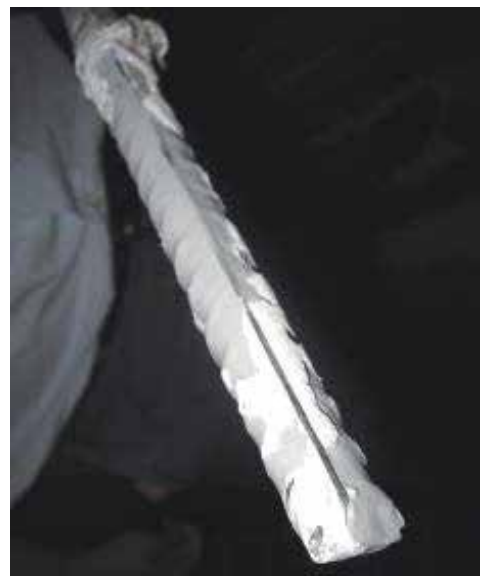

Fig. 19. Interlocking surface in an anchor installed into a hole which is not filled with epoxy (Photo by Dr. Halil Nohutcu) 

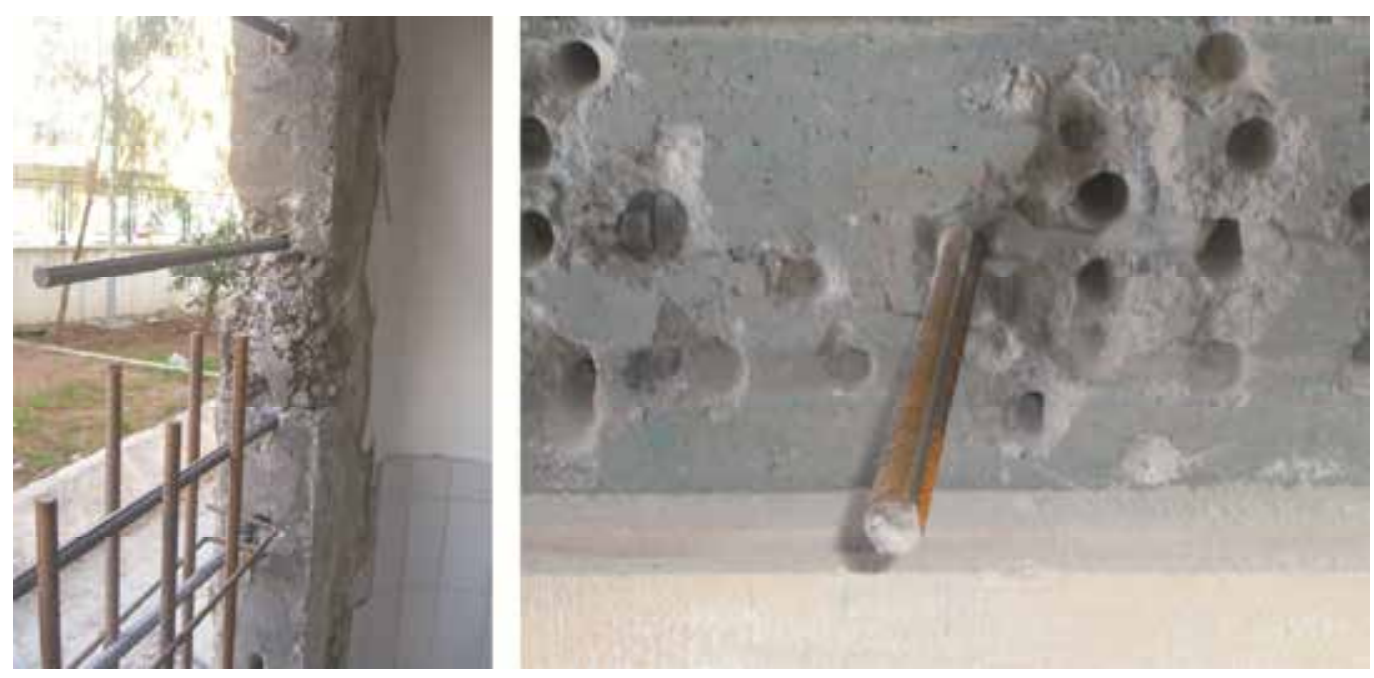

Fig. 20. Inappropriate anchor layouts 


\section{Conclusion}

A brief summary of the available literature on seismic strengthening methods is presented in this chapter. In general, the structural engineer has alternatives of element based and system based methods. Element based techniques are more economical solutions when local problems are the main reason of the strengthening decision. If the problems related with the global ductility, stiffness or strength of the structure are the main concern, global strengthening techniques are more advantageous. Both approaches can also be utilized if needed.

On the other hand, performance of the elements connecting the old and new members mostly has a vital effect on the performance of the strengthened system. Thus, connection elements used in the strengthening must carefully be designed and implemented. Since majority of the application is comprised of anchor elements, this section is focused on some topics related to anchors.

\section{Acknowledgment}

Authors greatly acknowledges Dr. Halil Nohutcu, Dr. Nihat Cetinkaya, Dr. Özlem Çalışkan, Ahmet Sarisin, and Prof. Dr. Ergin Atimtay for their efforts and contributions in experimental program on external shear walls. Dr. Yavuz Selim Tama, Dr. Ali Haydar Kayhan are also acknowledged for providing some photos on strengthening. Authors supervises several strengthening projects and carried out many research projects. Funding institutions (TUBITAK, DPT and Pamukkale University) and organizations (Denizli, Mugla and Kütahya Governorships) are also acknowledged for their valuable support.

\section{References}

ACI Committee 318. Building code requirements for structural concrete and commentary (ACI 318M-05). American Concrete Institute, 2005.

Albanesi T., Biondi S., Candigliota E. and Nuti C. Experimental analysis on a regular full scale infilled frame. Proceedings of the First European Conference on Earthquake Engineering and Seismology, Geneva, 2006, Paper No. 1608.

Baran M. Precast Concrete Panel Infill Walls for Seismic Strengthening of Reinforced Concrete Framed Strcutures. PhD thesis, Middle East Technical University, Ankara, 2005.

Bush T. D., Wyllie L. A. and Jirsa, J. O. Observations on two seismic strengthening schemes for concrete frames. Earthquake Spectra, 1991, 7, No.4, 511-527.

Çalışkan, Ö., Yilmaz, S., Kaplan, H., "Shear Capacity of Post-Installed Anchors According to ACI318 and TS500" 9th International Congress on Advances in Civil Engineering, 2010, Trabzon, Turkey.

Canbay E., Ersoy U. and Ozcebe G. Contribution of reinforced concrete infills to seismic behavior of structural systems. ACI Structural Journal, 2003, 100, No.5, 637-643.

Frosch R. J., Wanzhi L., Jirsa J. O. and Kreger M. E. Retrofit of non-ductile momentresisting frames using precast infill wall panels. Earthquake Spectra, 1996, 12, No.4, 741-760. 
Gilmore A. T., Bertero V. V. and Youssef N. F. G. Seismic rehabilitation of infilled nonductile frame buildings using post-tensioned steel braces. Earthquake Spectra, 1996, 12, No.4, 863-882.

Gorgulu, T., Tama, Y.S., Yilmaz, S., Kaplan, H., Ay, Z., Strengthening of Reinforced Concrete Structures With External Steel Shear Walls, Journal of Constructional Steel Research, 2011, doi:10.1016/j.jcsr.2011.08.010.

Higashi Y., Endo T. and Shimizu Y. Effects on behaviors of reinforced concrete frames by adding shear walls. Proceedings of the Third Seminar on Repair and Retrofit of Structures, Michigan, 1982, pp. 265-290.

Jirsa J. and Kreger M. Recent research on repair and strengthening of reinforced concrete structures. Proceedings of the ASCE Structures Congress, California, 1989, 1, 679688.

Kaltakci, M.Y., Arslan, M. H., Yilmaz, U. S. and Arslan, H. D. A new approach on the strengthening of primary school buildings in Turkey: An application of external shear wall, Building and Environment, 2008, 43, No.6, 983-990.

Kaplan, H., Yılmaz, S., Cetinkaya, N., Atımtay, E., Seismic strengthening of RC structures with exterior shear walls, Sadhana - Academy Proceedings in Engineering Science, 2011, 36(1), 17-34.

Kaplan, H., Yılmaz, S., Cetinkaya, N., Nohutcu, H. and Atımtay, E. Gönen, H. A New Method for Strengthening of Precast Industrial Structures. Journal of The Faculty of Engineering and Architecture of Gazi University, 2009, 24 No.4, 659-665, (in Turkish).

Masri A. and Goel S. Seismic design and testing of an RC slab-column frame strengthened with steel bracing. Earthquake Spectra, 1996, 12, No.4, 645-666.

TEC-2007, Ministry of Public Works and Settlement, Turkish Earthquake Code-2007: Specifications for buildings to be built in seismic areas. Ankara, Turkey (in Turkish), 2007.

Moehle J. P. State of research on seismic retrofit of concrete building structures in the US. Proceeding of US-Japan Symposium and Workshop on Seismic Retrofit of Concrete Structures - State of Research and Practice, USA, 2000.

Ohmura, T., Hayashi, S., Kanata, K. and Fujimura, T., Seismic retrofit of reinforced concrete frames by steel braces using no anchors. Proceedings of the 8th National Conference on Earthquake Engineering. California, 2006.

Perera, R., Gómez, S. and Alarcón, E. Experimental and analytical study of masonry infill reinforced concrete frames retrofitted with steel braces. ASCE Journal of Structural Engineering, 2004, 130, No.12, 2032-2039.

Sonuvar M. O., Ozcebe G. and Ersoy, U. Rehabilitation of reinforced concrete frames with reinforced concrete infills. ACI Structural Journal, 2004, 101, No.4, 494-500.

Sucuoglu H., Jury R., Ozmen A., Hopkins D. and Ozcebe G. Developing retrofit solutions for the residential building stocks in Istanbul. Proceedings of 100th Anniversary Earthquake Conference, California, 2006

Whitney, C.S., Anderson, B.G. ve Cohen, E., Design of Blast Resistant Construction for Atomic Explosions, ACI Structural Journal, 1955, 26(7):589-683.

Yakut A., Gülkan P., Bakır B.S., Yılmaz M.T., Re-examination of damage distribution in Adapazarı structural considerations. Engineering Structures, 2005, 27, No.7, 9901001. 
Yılmaz, S., Cetinkaya, N., Nohutcu, H., Caliskan, O., Çırak, İ.F., Experimental Investigation on Anchor Applications for External Shear Walls, Technical Report, 192 p, 2010a, Denizli.

Yılmaz, S., Kaplan, H., Çalışkan, Ö., Kıraç, N., “Cyclic Shear Resistance of Epoxy Anchors Bonded to Low Strength Concrete" 14th European Conference on Earthquake Engineering, Paper no. 510, 2010b, Ohrid, Macedonia.

Yilmaz, S., Özen M.A., "Tensile Strength of Chemical Anchors Embedded to Low and Normal Strength Concrete" Pamukkale University, Report no: 2009FBE025, 131 p. 2010a, Denizli (in Turkish).

Yilmaz, S., Özen, M.A., "Strength of Chemical Anchors Embedded to Low Strength Concrete" 14th European Conference on Earthquake Engineering, Paper no. 513, 2010b, Ohrid, Macedonia.

Yılmaz, S., Tama, Y.S., Kaplan, H., “Design and Construction of External Precast Shear Walls for Seismic Retrofit" 14th European Conference on Earthquake Engineering, Paper no. 1238, 2010c, Ohrid, Macedonia.

Yılmaz, S., Kaplan, H., Tama, Y.S., Çalışkan, Ö., Solak, A., “Experimental program on design and application of external retrofit walls for low ductility RC frames" 4th International Conference on Advances in Experimental Structural Engineering, 2011, Ispra, Italy. 


\title{
Bond-Based Earthquake-Proof of RC Bridge Columns Reinforced with Steel Rebars and SFCBs
}

\author{
Mohamed F.M. Fahmy ${ }^{1}$ and Zhishen $\mathrm{Wu}^{2}$ \\ ${ }^{1}$ Assiut University \\ 2Ibaraki University, \\ ${ }^{1}$ Egypt \\ 2Japan
}

\section{Introduction}

Bond between reinforcement and the adjoining concrete has been extensively studied, and it is confirmed that the use of deformed bars is essential for composite behavior of reinforced concrete (RC) structures. But since bond between the longitudinal bars and concrete results in concentration of damage at a specific localized interval of longitudinal bars where the local buckling occurs, Takiguchi et al. (1976) suggested mitigating this concentration of damage through unbonding of the longitudinal bars from concrete at plastic hinge zone. Kawashima et al. (2001) conducted an experimental study on RC columns reinforced with different lengths of unbonded bars at the plastic hinge zone. It was noticed that the failure of concrete was much less in the unbonded column than standard column, and strain on unbonded bar was less than that on the reinforcement of standard column. Recently, to improve the seismic performance of RC members, it is highlighted in the study of Pandey and Mutsuyoshi (2005) that reducing bond strength between the longitudinal bars and concrete has a favored effect on the failure mode, shear capacity and ductility of RC bridge piers: failure mode at ultimate state is changed from shear to flexural and shear strength and ductility are increased.

In the performance-based design approach, the design is primarily focused on meeting a performance objective, which is in line with a desired level of service (Floren et al., 2001, Priestley et al., 2007). For instance, new seismic design philosophies for bridges recommend that important bridges subject to massive earthquakes should be able to sustain the expected maximum lateral force in the inelastic stage with limited damages. To achieve this aim, structure should realize the existence of post-yield stiffness, damage level should be limited, and its permanent deformations (residual deformations) should be smaller than a specified limit; and all these indices are essentially dependent on the composite behavior of RC structures. On the other hand, the studies of Kawashima et al. (2001) \& Pandey and Mutsuyoshi (2005) revealed the importance of reducing concrete-to-steel bond to mitigate the concentrated damage in the plastic hinge zone.

In the last two decades, civil engineers and designers have attempted to develop and adopt new forms of materials that would assist in the building of stronger, larger, more longer 
lasting, and aesthetic structures.Because the advantages of advanced composite materials, i.e., fiber reinforced polymers (FRPs), include: light weight, high strength or stiffness-toweight ratios, corrosion resistance, and, in particular, the elastic performance, steel bars hybridized in the longitudinal direction with FRPs were recommended in the study of Fahmy et al. (2010) as an innovative reinforcement method for recoverable structures, where strain-hardening behavior of the innovative bars can be controlled based on both the amount and type of fibers used. Also, the use of the innovative rebars, i.e., steel fiber composite bars (SFCBs), will increase the life spans of structures because the inner steel bar is protected against corrosion (Fig 1). Uniaxial and cyclic tensile behavior of the innovative rebars were experimentally tested by $\mathrm{Wu}$ et al. (2010).
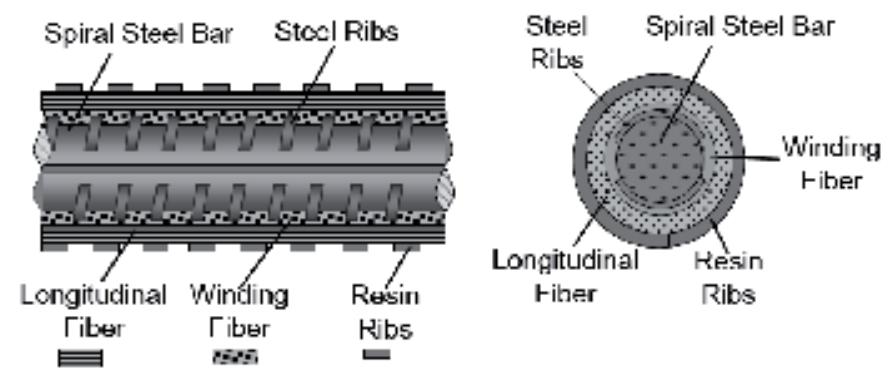

Fig. 1. Details of steel fiber composite bar (SFCB)

Due to the limited studies on bond-controlled structures which are reinforced with the ordinary steel rebars and because of the successful performance of the innovative SFCBs, it is essential to identify a suitable bond-based damage-controllable system, which guarantees limitation of the damage and mitigation of the permanent deformations. In addition, application of this system should not affect the structure load carrying capacity in the inelastic stage: structure should continue able to carrying load in the inelastic stage to withstand strong earthquakes. Hence, in the following, analytical studies using a computer program (Open System for Earthquake Engineering Simulation (Open SEES) [Mazzoni et al.]) are conducted, where effects of concrete-to-steel bond properties on the performance of RC bridge columns reinforced with rebars having different strainhardening levels are determined. Effects of different bond conditions on column elastic and post-yield stiffnesses, residual deformations, and damaged zone are addressed. Validity of the analytical findings is established based on the experimental results of columns reinforced with unbonded deformed steel bars (DSBs) in the plastic hinge zone by Kawashima et al. (2001), columns with bond-controlled reinforcements by Pandey and Mutsuyoshi (2005), and two columns reinforced with rebars having a controlled strain-hardening behavior, i.e. steel fiber composite bars. Ultimately, the influence of concrete-to-SFCBs bond on the recoverability of RC bridge columns is analytically studied.

\section{Idealized load-deformation model of damage-controllable RC structures}

Fig. 2 shows a mechanical model of damage-controllable RC structures located in high seismicity zones. The proposed model exhibits the required performance from newly constructed structures under the effect of different levels of seismic load, where the 
lateral response proceeds along O-A-B-C-D-E-F-G. The behavior of a general RC flexural structure whose lateral response is along $O-A^{\prime}-B^{\prime}-C^{\prime}-D^{\prime}-F^{\prime}$ is also given for comparison. Prior to the yielding of steel reinforcement, lines $\mathrm{OAB}$ and $\mathrm{OA}^{\prime} \mathrm{B}^{\prime}$ corresponding to both types of structures share similar stiffnesses, $K_{1}$. The most remarkable difference occurs after the yielding of the steel reinforcement: after point $C$ and $C^{\prime}$. For the general $R C$ structure, the deformation increases dramatically almost without any significant increase in load carrying capability: along line $C^{\prime} \mathrm{D}^{\prime}$ almost zero post-yield stiffness is demonstrated. However, with the proposed approach, the structure can still carry the load even after the steel reinforcement yields and hardening behavior has been exhibited along line $\mathrm{CD}$. Based on the codes requirements for ductile structures to withstand strong earthquakes, the proposed structure is characterized by the part DEF after the hardening zone, where favorable ductility is demonstrated. The ultimate drift $\left(\delta_{u}\right)$ corresponding to point $\mathrm{F}$ or $\mathrm{F}^{\prime}$ for the proposed structure and the general RC structure, respectively, is defined for both structures to be at 20 percent strength decay, (Park \& Paulay, 1975).

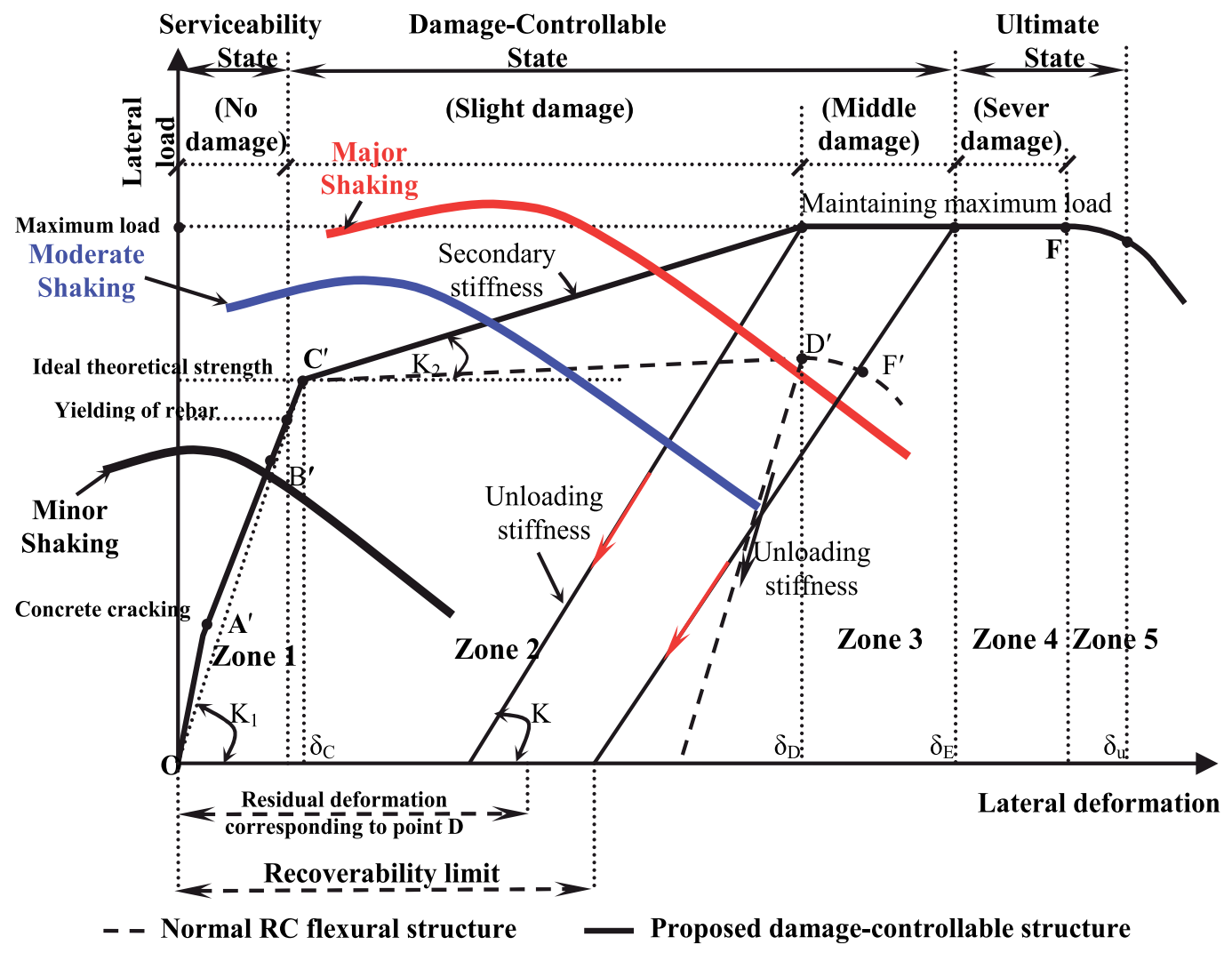

Fig. 2. Idealized load-deformation behavior of proposed damage-controlled structures

According to the mechanical behavior shown in Fig.2, the load-deformation of the proposed structure can be divided into five main zones; Zone 1: from point $\mathrm{O}$ to $\mathrm{B}$; Zone 2: from point $\mathrm{B}$ to $\mathrm{D}$; Zone 3: from point $\mathrm{D}$ to $\mathrm{E}$; Zone 4: from point $\mathrm{E}$ to point $\mathrm{F}$; and Zone 5: after point $\mathrm{F}$. Zone 1 corresponds to a stage of no damage or concrete cracking. Under a 
small earthquake, the mechanical behavior should be controlled in this zone, and the original function of the structure can be maintained without any repair and displacement of elements. Zone 2 corresponds to the hardening behavior after the yielding of steel reinforcements, where a distinct secondary stiffness is demonstrated and the dramatic deformation can be effectively controlled. Under a medium or strong earthquake, the mechanical behavior of the proposed structure should be within zone 2 . Thus, damage can be effectively controlled by the secondary stiffness. The original function of the structures can be quickly recovered through repairs after a medium or large earthquake. Zone 3 corresponds to ductile behavior after hardening, where favorable ductility is demonstrated under a large earthquake but with middle level of damage. Furthermore, within this zone structure should be recoverable, where residual deformation does not exceed recoverability limit, i.e. residual deformations should be less than $1 \%$ of structure height for quick recovery of original functions of structure after an earthquake. Zone 4 corresponds to additional ductile behavior, where the proposed structure can be kept in place without collapse during a large earthquake, though severe damage may occur. The original function of the structures may be recovered through the replacement of some elements. During a severe earthquake, the mechanical behavior may enter zone 5 with collapse.

It is clear that two indices should be applied to measure the recoverability of RC structures: secondary stiffness and residual deformation. However, to have a complete description of structure performance, damage level should be considered in the evaluation of the recoverability of structures after seismic excitation. In the study of Mostafa (2011) several damage measures based on a single response parameter are summarized.

\section{Analytical investigation}

Of crucial importance in the anti-seismic design of $\mathrm{RC}$ bridges is to ensure the gradual increase of strength after yielding (existence of positive post-yield stiffness) and to minimize the permanent deformation due to a massive earthquake so that the damage can be easily repaired. That is quickly recoverable bridges after sever seismic actions. In this study mechanical properties of steel reinforcement used and its bond conditions to the surrounding concrete are key parameters to investigate how could be the required recoverability of RC bridges controlled? Thus, in the following section, analytical studies are carried out to detect their effects on the performance of RC columns: elastic and post-yield stiffnesses, residual deformations, and damage level.

\subsection{Fiber-based model of RC columns with zero-length section element}

Cyclic loading analysis is conducted using a computer program (Open SEES) [Mazzoni et al.]. This program has a variety of predefined material models for multiple applications that can be manipulated to fit specific criteria and properties. Since the fiber analysis remains the most economic and accurate means to capture seismic behavior of concrete structures (Spacone et al. (1996) and Saiidi et al. (2009)), the fiber model was used, Fig. 3. The confined concrete properties were based on Kent-Scott-Park's model. The widely used GiuffréMenegotto-Pinto model is employed in this study to represent the hysteretic stress-strain behavior of longitudinal steel reinforcement. The model includes the yielding, strain hardening, and Bauschinger effect of the steel bar. 


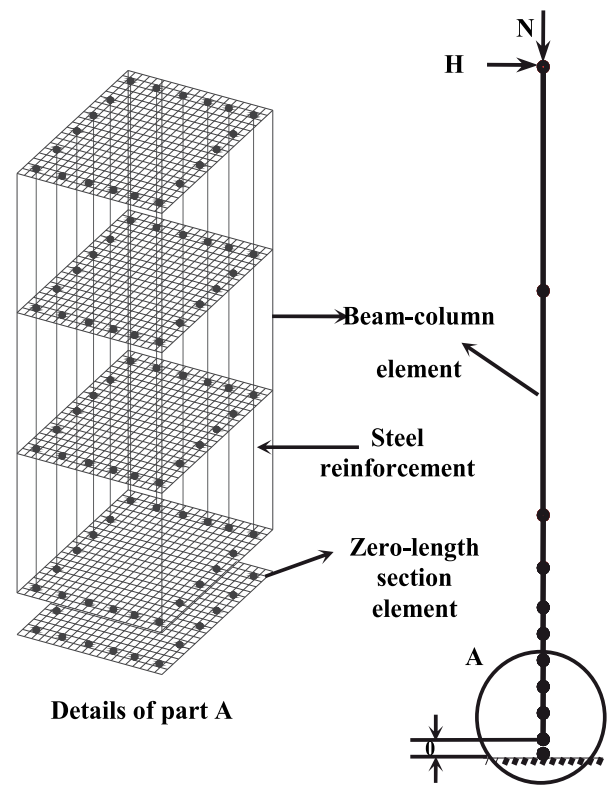

Fig. 3. Fiber-based modeling of the studied columns

Zhao \& Sritharan (2007) have developed fiber-based analysis of concrete structure through the incorporation of a zero-length section element to reflect the effect of the fixed-end rotation that arises at the column-foundation or column-beam interface on the performance of structures. The conducted analyses by Zhao \& Sritharan (2007) on cyclic responses of cantilever columns and a bridge tee-joint system, satisfactorily captured deflections, force versus displacement hysteresis responses, and strains in the longitudinal reinforcing bars. The developed constitutive model by Zhao \& Sritharan (2007) for the steel fibers of the zerolength section element expresses the bar stresses $(\sigma)$ versus loaded-end slip $(S)$ response. The main parameters of this model are the loaded-end slips $S_{y}$ and $S_{u}$ and their corresponding bar stresses $\sigma_{y}$ and $\sigma_{u}$, respectively. Incase the bar has a sufficient anchorage length, $f_{y}$ and $f_{u}$ are the stresses corresponding to $S_{y}$ and $S_{u}$, respectively, where $f_{y}$ and $\mathrm{f}_{\mathrm{u}}$ are the yield and ultimate strengths of the steel reinforcing bar, respectively, Fig. 4(a). Zhao \& Sritharan (2007) defined that the sufficient anchorage length is not less than $\left(\left(d_{b} / 7\right)\left(f_{y} /\left(f_{c o}^{\prime}\right)^{0.5}\right)\right.$, where $d_{b}$ is the bar diameter $(\mathrm{mm})$ and $f^{\prime}{ }_{c o}$ is the concrete compressive strength in MPa. To determine the suitable value of $S_{y}$, Zhao \& Sritharan (2007) proposed the following experimentally-based equation:

$$
S_{y}=2.54\left[\frac{d_{b}}{8437} \frac{f_{y}}{\sqrt{f_{c o}^{\prime}}}(2 \alpha+1)\right]^{1 / \alpha}+0.34
$$

where $a$ was taken as 0.4 . Definition of $S_{u}$ value is based on the determined value of $S_{y}: S_{u}=$ 30 40 $S_{y}$. This model is employed for capturing the slip effect in flexural members subjected to reversed cyclic loading, and hysteretic rules were established, Fig. 4(b). Consequently, this model is adopted here to find out the impact of different bond behaviors on the performance of cyclically loaded RC columns. 


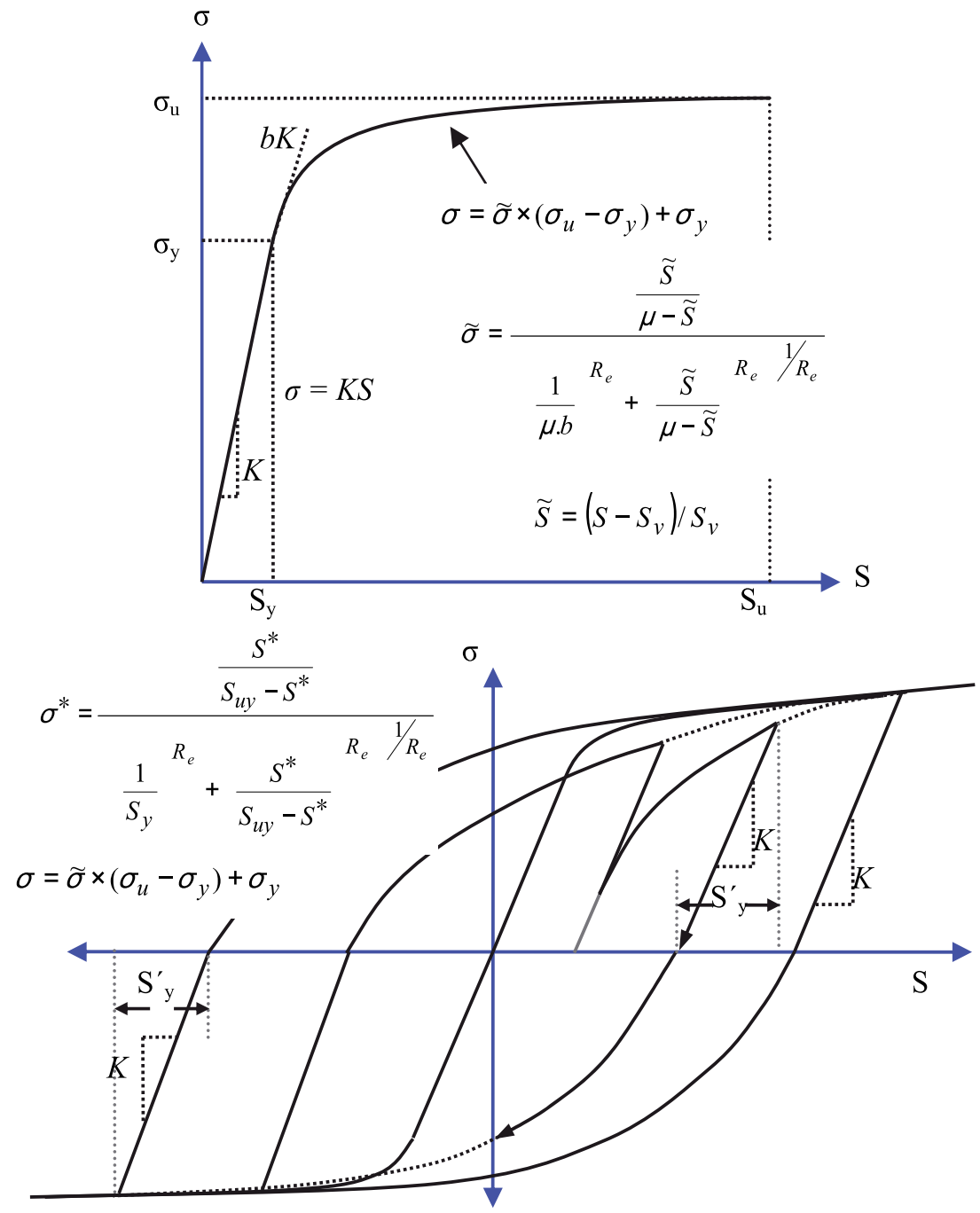

Fig. 4. (a) Envelop curve and (b) Hysteretic model for steel bar stress versus loaded-end slip relationship (Zhao \& Sritharan, 2007).

\subsection{Effect of bond parameters and rebar mechanical properties on the performance of RC column}

Kawashima et al. (2001) conducted an experimental study on four RC columns (A, B, C, and D). Specimen A was tested to simulate the performance of conventionally reinforced column but the others were reinforced with bars unbonded at the plastic hinge zone. Here, hysteretic response of Specimen A is considered as a control sample to find out the effects of different bond conditions between column reinforcement and the surrounding concrete on its hysteretic performance (in particular the required recoverability). The tested specimen had square crosssection of 400x400mm and 1450-mm-height. It was reinforced with twelve longitudinal rebars of 13-mm-diameter of $367 \mathrm{MPa}$ yield strength, lateral reinforcement consisted of 6-mm-diameter ordinary rebars with yield strength of $376 \mathrm{MPa}$ and spacing of $50 \mathrm{~mm}$. The tested compressive 
strength of the concrete cylinder was 20.6 MPa. The column was tested under constant axial load $(160 \mathrm{kN})$ and reversed cyclic lateral load. Using the four parameters of Zhao and Sritharan model $\left(\left(S_{y}\right.\right.$ and $\left.\sigma_{y}\right)$ and $\left(S_{u}\right.$ and $\left.\left.\sigma_{u}\right)\right)$, ten different bond conditions are studied (Table 1$)$.

\begin{tabular}{|c|c|c|c|c|c|c|c|c|c|}
\hline \multirow{2}{*}{$\begin{array}{l}\frac{3}{3} \\
\frac{0}{0}\end{array}$} & \multirow[b]{2}{*}{ 芯 } & \multirow[b]{2}{*}{ Column } & \multicolumn{3}{|c|}{ Steel mechanical properties } & \multicolumn{4}{|c|}{ Bond properties } \\
\hline & & & $\begin{array}{c}f_{y} \\
(\mathrm{MPa})\end{array}$ & $\begin{array}{c}f_{u} \\
(\mathrm{MPa}) \\
\end{array}$ & $E_{2} / E_{1}$ & $\begin{array}{c}S_{y} \\
(\mathrm{~mm})\end{array}$ & $\begin{array}{c}\sigma_{y} \\
(\mathrm{MPa}) \\
\end{array}$ & $\begin{array}{c}S_{u} \\
(\mathrm{~mm})\end{array}$ & $\begin{array}{c}\sigma_{u} \\
(\mathrm{MPa}) \\
\end{array}$ \\
\hline \multirow{3}{*}{$I$} & 1 & $0.01-S_{y}-0.8 f_{y}$ & 367 & $1.5 f_{y}$ & 0.01 & $S_{y}$ & $f_{y}$ & $S_{u}$ & $0.8 f_{y}$ \\
\hline & 2 & $0.01-2 S_{y}-0.8 f_{y}$ & 367 & $1.5 f_{y}$ & 0.01 & $2 S_{y}$ & $f_{y}$ & $2 S_{u}$ & $0.8 f_{y}$ \\
\hline & 3 & $0.01-3 S_{y}-0.8 f_{y}$ & 367 & $1.5 f_{y}$ & 0.01 & $3 S_{y}$ & $f_{y}$ & $3 S_{u}$ & $0.8 f_{y}$ \\
\hline \multirow{2}{*}{ II } & 4 & $0.01-S_{y}-f_{y}$ & 367 & $1.5 f_{y}$ & 0.01 & $S_{y}$ & $f_{y}$ & $S_{u}$ & $f_{y}$ \\
\hline & 5 & $0.01-2 S_{y}-f_{y}$ & 367 & $1.5 f_{y}$ & 0.01 & $2 S_{y}$ & $f_{y}$ & $2 S_{u}$ & $f_{y}$ \\
\hline \multirow[t]{2}{*}{ III } & 6 & $0.01-S_{y}-1.5 f_{y}$ & 367 & $1.5 f_{y}$ & 0.01 & $S_{y}$ & $f_{y}$ & $S_{u}$ & $1.5 f_{y}$ \\
\hline & 7 & $0.02-S_{y}-2 f_{y}$ & 367 & $2.0 f_{y}$ & 0.02 & $S_{y}$ & $f_{y}$ & $S_{u}$ & $2 f_{y}$ \\
\hline \multirow[t]{2}{*}{$I V$} & 8 & $0.04-S_{y}-2 f_{y}$ & 367 & $2.0 f_{y}$ & 0.04 & $S_{y}$ & $f_{y}$ & $S_{u}$ & $2 f_{y}$ \\
\hline & 9 & $0.08-S_{y}-2 f_{y}$ & 367 & $2.0 f_{y}$ & 0.08 & $S_{y}$ & $f_{y}$ & $S_{u}$ & $2 f_{y}$ \\
\hline$V$ & 10 & $0.12-3 S_{y}-1.5 f_{y}$ & 367 & $2.5 f_{y}$ & 0.12 & $3 S_{y}$ & $f_{y}$ & $3 S_{u}$ & $1.5 f_{y}$ \\
\hline
\end{tabular}

Note: $E_{1}=200 \mathrm{GPa}$

Table 1. Reinforcement mechanical properties and corresponding bond properties

To evaluate the effect of the strain-hardening behavior of steel reinforcement used on the performance of bond-controlled structures, five values of the bilinear ratio factor $\left(r=E_{2} / E_{1}\right)$, where $E_{2}$ and $E_{1}$ are the steel elastic and post-yielding stiffnesses, are examined here, i.e. $r=0.01$, $0.02,0.04,0.08$, and 0.12 . Of course bond between reinforcement and the adjoining concrete is an essential factor in the definition of both $\sigma_{y}$ and $\sigma_{u}$ and the corresponding slip values. Therefore, five groups (I, II, III, IV, and V) are studied here, Table 1. The groups I, II \&III consider the effects of bond parameters on the performance of columns reinforced with ordinary rebars (bilinear ratio factor $=0.01$ ). In case no. 6 of group III, bond parameters are those proposed by Zhao \& Sritharan (2007) to include the effect of strain penetration of longitudinal bars into foundation on the lateral response of conventionally RC columns. Meanwhile, the aim in the groups I and II is to characterize the effect of weak bond strength between ordinary rebars and concrete, so, it is assumed that rebars may not approach the ultimate strength, e.g. $\sigma_{u} \leq f_{y}$, and also $S_{y}$ would increase to two or three times of its value evaluated based on Eq. 1. Effect of higher bilinear ratios of steel reinforcement is examined in the last two groups (IV and V). In group IV, the ultimate strength of rebars used is kept constant $\left(2 f_{y}\right)$ while different bilinear ratios $(r=0.02,0.04$ and 0.08$)$ are studied. In the last case study (group $(\mathrm{V}))$, column is reinforced with rebars having bilinear ratio factor of 0.12 but it is assumed that, through controlling the bond conditions, rebars may not approach more than $60 \%$ of their ultimate strength along with the effect of additional slippage. It is noteworthy that the studied cases of RC columns are typified based on the bilinear ratio between post-yield and elastic stiffnesses of steel reinforcing bar, rebar loaded-end slip at $\sigma_{y}$, and maximum achieved strength of reinforcing steel at the ultimate slip $\left(S_{u}\right)$ of rebars. For instance, $\left(0.01-2 S_{y}-0.8 f_{y}\right)$ is the designation of case two of group I, where 0.01 is the bilinear ratio factor of reinforcing steel; $2 S_{y}$ is double of rebar slip value calculated by Eq. 1 and $\sigma_{y}$ is the corresponding strength; and $0.8 f_{y}$ is $80 \%$ of rebar yield strength, which is considered the maximum achieved strength $\left(\sigma_{u}\right)$.

Hysteretic responses of the studied cases are depicted in Fig. 5, wherein the measured forcedrift relationship of Specimen A is superimposed for comparison. 

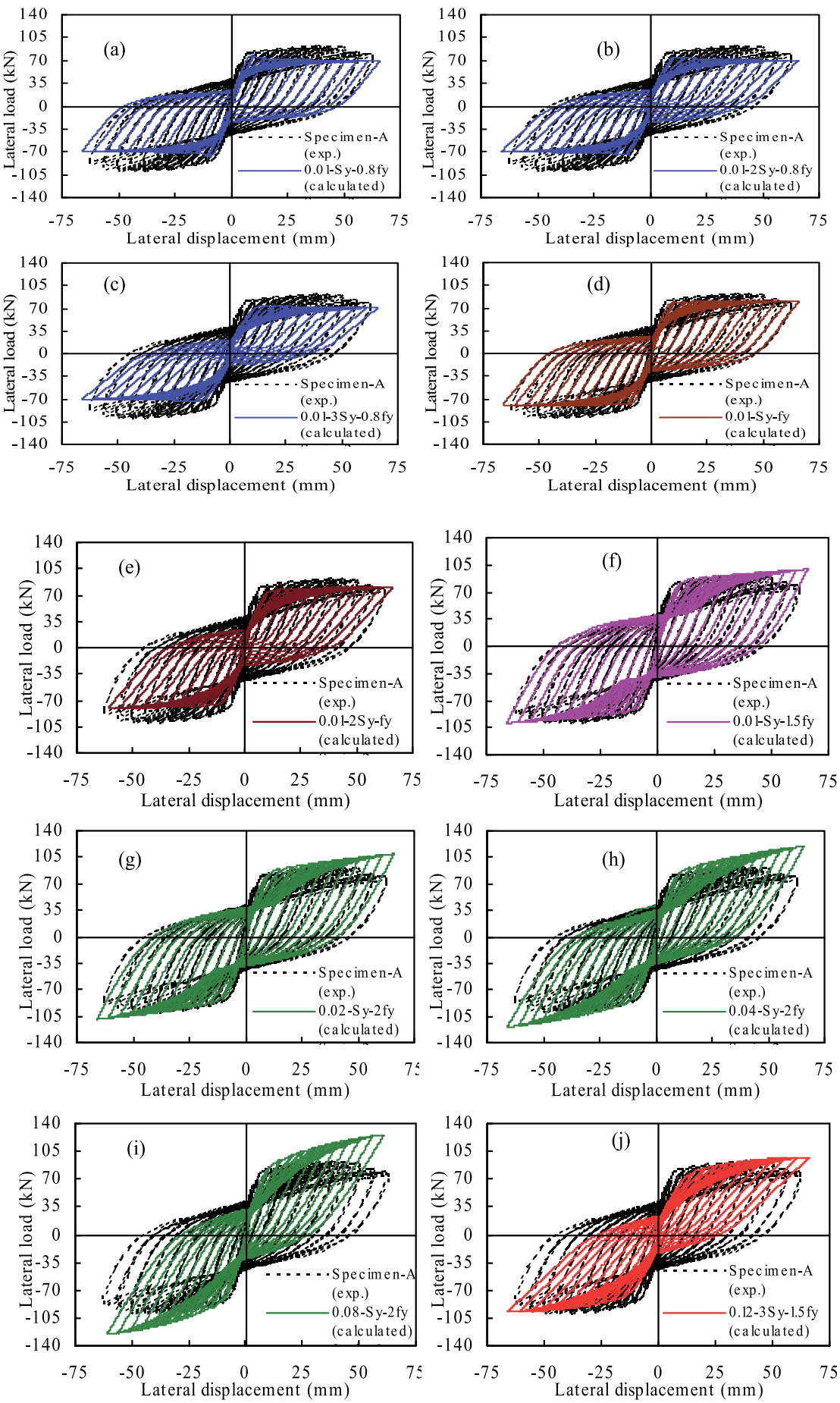

Fig. 5. Effect of bond properties on the hysteretic responses of RC bridge columns reinforced with rebars of different levels of strain-hardening 


\subsubsection{Detailed discussion on bond-based performance of RC columns}

In the light of the aforementioned analytical results, this section presents a detailed discussion about the effects of altering bond conditions and steel type on the performance of RC column in terms of its post-yield stiffness, residual deformation, and damage level.

\subsubsection{Post-yield stiffness}

The post-yield stiffness is defined as the gradual increase of column strength after fulfilling its theoretical moment capacity $M_{i}$, and the end point of the post-yield stiffness is that corresponding to the maximum lateral capacity. The theoretical moment capacity is calculated using ACI rectangular stress block for concrete in compression, which has a mean stress of $0.85 f^{\prime}{ }_{c o}$, the measured concrete compression strength $f^{\prime}{ }_{c o}$ and steel yield strength $f_{y}$, and an ultimate concrete compression strain of 0.003 [ACI 318-08]. Based on the specified details, dimensions, and material properties of the tested specimen $\mathrm{A}$, theoretical ideal strength $\left(P_{i}=M_{i} / L\right.$, where $L$ is column height) is defined and superimposed in Figs. $6(\mathrm{a}-\mathrm{d})$. Figs. 6 (a-d) show the skeleton curves of the hysteretic responses of the analytical studied cases.

The experimental results of the conventionally tested column (Specimen A) showed that column was able to carry the load even after achieving the theoretical strength $(84.4 \mathrm{kN})$ and hardening behavior has been exhibited. The envelope of the measured force-drift relationship of Specimen A is superimposed on the results of the studied cases, as shown in Figs. 6 (a-d). The curves are the average of the envelopes for the push-and-pull loadings. It was reported by Pettinga et al. (2006) that systems exhibiting post-yield stiffness ratios greater than $5 \%$ will have significantly reduced permanent displacements. The ratio between column post-yield stiffness $\left(k_{2}\right)$ and elastic stiffness $\left(k_{1}\right)$ of Specimen A is $2.35 \%$. Accordingly, to achieve the aim of quickly recoverable bridges after massive earthquakes, an enhancement of the performance of conventionally RC column is still necessary.

When weak bond strength between ordinary rebars $(r=0.01)$ and the surrounding concrete is considered, the analytical results show that the column performance in the inelastic stage is function of reinforcement stresses at the ultimate slippage, Figs. 6 (a \& b). For instance, in the studied cases of group I, where reinforcement stresses would not exceed $80 \%$ of the yield strength, column deformation increases dramatically with a decrease in the load carrying capability after yielding (negative post-yield stiffness), Fig. 6 (a); but, In group II, zero postyield stiffness is the column performance in the inelastic stage when reinforcement stresses would approach the yield strength at the ultimate slippage, Fig. 6 (b). It is noteworthy that, in both groups, columns could not reach up to the theoretical strength of Specimen A.

As is clear from Fig. 6 (c), replacement of ordinary rebars with others, having higher strainhardening levels and perfect bond to the surrounding concrete, would enhance column performance in the inelastic stage. Where the increase in $r$ value leads to an increase in column post-yield stiffness, e.g. the ratios between column post-yield and elastic stiffnesses $\left(k_{2} / k_{1}\right)$ are $2.88 \%, 4.05 \%$, and $5.2 \%$ when steel bilinear ratio are $0.02,0.04$, and 0.08 , respectively. It should be noted that the first stiffness was the same among the studied cases and Specimen A, as shown in Fig. 6 (c).

In the last case study, where $r$ is 0.12 , although merely $60 \%$ of rebar ultimate strength could be achieved along with an increase in rebars slippage, Fig. 6 (d) shows that columns could realize the existence of post-yield stiffness $\left(k_{2} / k_{1}=3.18 \%\right)$ and reach up to the same lateral strength of specimen A. Moreover, in a distinction from the other studied cases, a third zone with zero post-yield stiffness characterizes the inelastic stage of this column. 
It is noteworthy that the slope of column elastic stiffness would slightly affect by the increase in rebar slippage at yielding when it is double of the value calculated from Eq. 1, Figs. 6 (a \& b); however, it decreases to two-third of the elastic stiffness of conventionally RC column when rebar slippage increases to $3 S_{y}$, regardless of whether the bilinear ratio is small or high, Figs. 6 (a \& d). The lower stiffness of the columns would lead to longer vibration period for the columns and would generally reduce earthquake forces (Saiidi et al. (2009)).
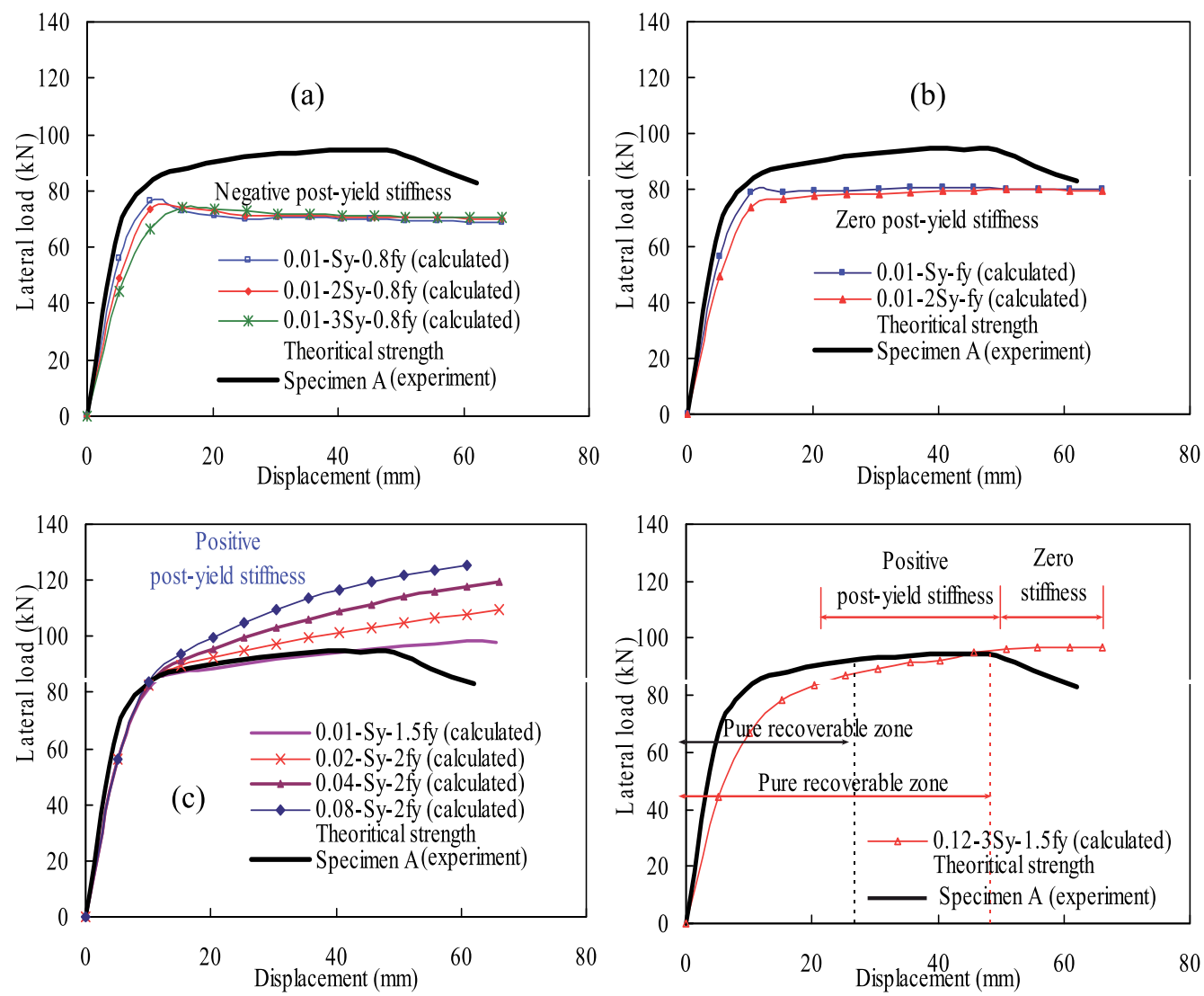

Fig. 6. Effect of bond properties on the post-yield stiffness of RC bridge columns reinforced with rebars of different levels of strain-hardening

\subsubsection{Residual deformations}

Using the test results of specimen A and analytical results of the studied cases (Fig. 5), the drift ratios versus columns residual drift ratios are plotted in Fig. 7. The residual deformation, which is defined as the displacement of zero-crossing at unloading on the hysteresis loops, should not exceed $1 \%$ of the column height for a quick recovery after an earthquake (JSCE, (2000)). For specimen A, the drift ratios versus residual drift ratios are superimposed on Figs. 7 (a-d) for comparison. While specimen A was able to reach up to $4.6 \%$ lateral drift ratio with almost $12.1 \%$ strength decay, the end of the recoverable stat is corresponding to $1.83 \%$ lateral drift, Fig 5 (d) \& Fig. 7. After lateral column drift of $1.83 \%$, 
regardless of whether damage level is light, medium, or sever, column can not continue to function following major earthquakes and demolishing may be required. Consequently, mitigation of column residual deformations is critical to have ductile-recoverable bridges.

Figs. $7(\mathrm{a} \& \mathrm{~b})$ present the residual drift ratios verses lateral drift ratios of columns with different levels of bond capacity between ordinary rebars and the surrounding concrete. As is clear, mitigation of column residual deformation is in direct relationship with rebar slippage, where the increase in rebar slippage to two and three times of the value defined by Zhao \& Sritharan model shifts the recoverable zone by almost $0.5 \%$ and $1 \%$ lateral drift, respectively.

The effects of using reinforcement with high bilinear ratio $(r)$ on column residual deformations are shown in Fig. 7 (c), where a favorable effect could be noticed. For example, $2.16 \%$ lateral drift is the end of the recoverable state of column reinforced with rebars of 0.02 bilinear ratio factor; and when the bilinear ratio increases to 0.04 and 0.08 , the end of the recoverable stat is shifted to $2.38 \%$ and $2.67 \%$, respectively. Furthermore, Fig. 7 (d) exhibits the effect of using rebars with a higher bilinear ratio $(r=0.12)$ coupled with the effects of weak bond strength to the adjoining concrete. The simulation results indicate that column performance is ductile-recoverable, where column could be recoverable until lateral drift ratio of $3.34 \%$ with the existence of post-yield stiffness, as shown in Fig. 6 (d).
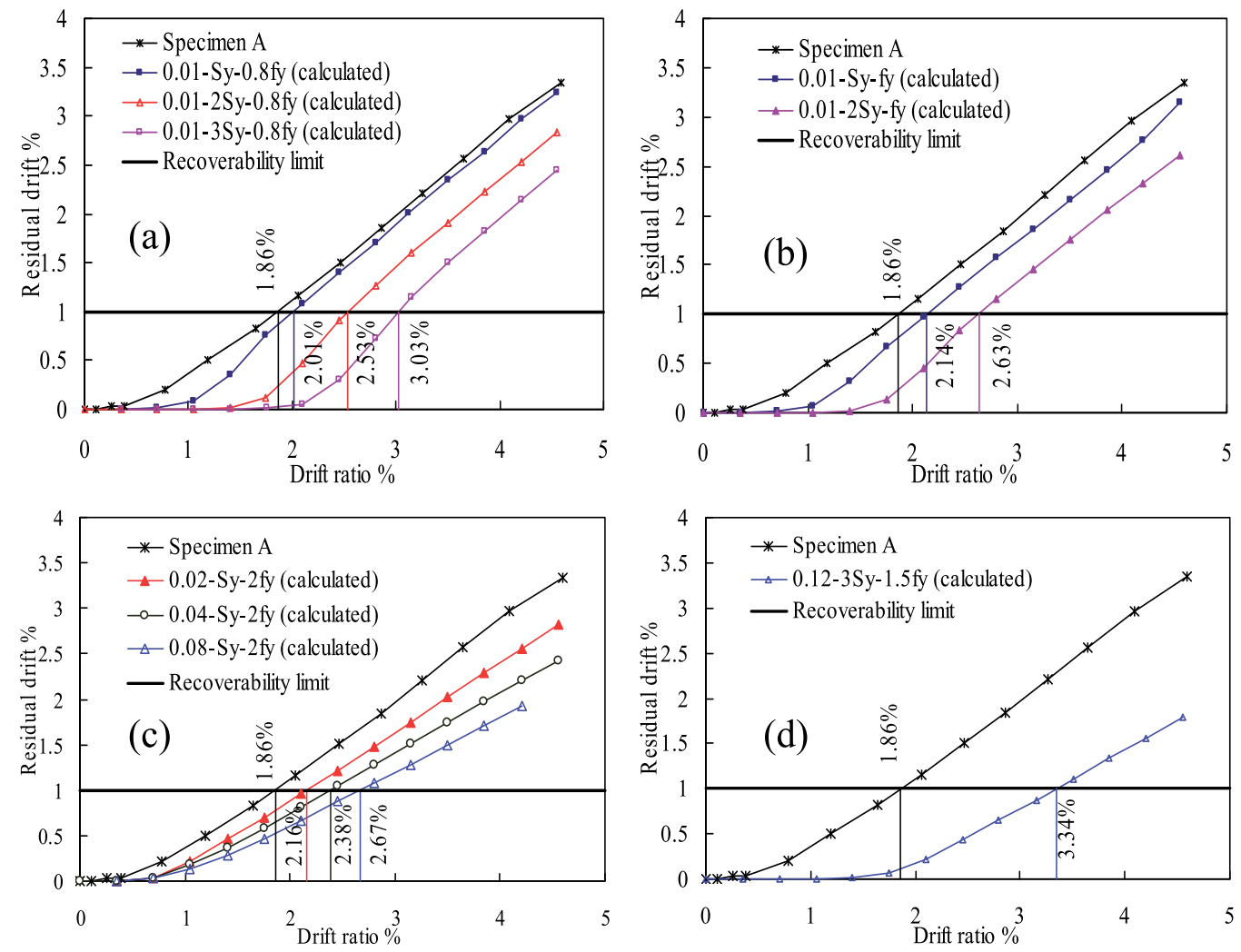

Fig. 7. Effect of bond properties on the residual deformations of RC bridge columns reinforced with rebars of different levels of strain-hardening 


\subsubsection{Damage level}

The main conclusion taken from the aforementioned analytical results presented in Figs. 6 and 7 is that both steel strain-hardening behavior and concrete-to-rebar bond capacity are key parameters, which can be employed to achieve the required performance from structures during and after seismic actions. Even so, to define a suitable system guaranteeing the aim of damage-free recoverable structures, investigating the effects of the studied parameters (Table 1) on column damage level is indispensable. The literature on damage measures of structures under ground motions is vast (e.g. Cosenza et al. 1993; Ghobarah et al. 1999; Abbas 2011). Damage indices are based on either a single or combination of structural response parameters.

To have a structure capable of deforming in a ductile manner when subjected to several cycles of lateral loading, deformations concentrate in the region of the primary lateral force resisting mechanism (plastic deformation zone), wherein the demand of high deformability (curvature capacity) increases with the increase in the ductility demand. Consequently, length of this zone, which is one of the crucial aspects in the performancebased design, along with the distribution of curvature ductility $\left(\varphi / \varphi_{y}\right)$ would be useful to find out the impact of the studied parameters on the damage level, because the curvature $(\varphi)$ is dependent on steel strains. $\varphi$ is calculated using $\left(\varepsilon_{s t}-\varepsilon_{s c}\right) /\left(d-d^{\prime}\right)$, where $\varepsilon_{s t}$ and $\varepsilon_{s c}$ are the steel strains in the tension and compression sides of the loaded column, respectively (tensile strains are taken as positive and compression strains as negative), $d$ - $d$ ' is the distance between the tension and compression steel pieces, and $\varphi_{y}$ is the curvature at first yield.

\subsection{Depth of yielding zone (Plastic deformation zone)}

At the ultimate achieved lateral drift of Specimen A, which is almost $4.2 \%$, length of the plastic deformation zone is defined for each of the studied cases in Table 1 based on the calculated distribution of curvature ductility through the column height. Fig. 8 shows the depth of the yielding zone as a ratio of the column height for all the studied cases. It is clear from the figure that depth of the yielding zone is in direct proportion to the value of $r$ of reinforcement used when it is in perfect bond to the surrounding concrete, where the normalized depth of the yielding zone could be changed from 0.41 to 0.54 by the increase of $r$ from 0.02 to 0.08 . In addition, it is evident that depth of the yielding zone is significantly affected by bond conditions, where the smallest depth of the yielding zone would be for columns with weak bond strength between ordinary rebars and the adjoining concrete, and this depth may possibly not change by the increase in the slippage of rebars.

\subsection{Curvature distribution}

While the yielding zone may locate in a small region at column base or extend beyond the normal range of the yielding zone based on the reinforcement type and its bond condition to the surrounding concrete, the worst case, i.e. with high damage level, still can not be defined. For example, the column typified $\left(0.08-S_{y}-2 f_{y}\right)$ has the greatest normalized depth of the yielding zone $(0.54>0.36$ of conventionally reinforced column) among the studied cases, however damage level would not be the worst based on the curvature distribution of both columns. Hence, distribution of curvature ductility is determined for all of the studied cases. 


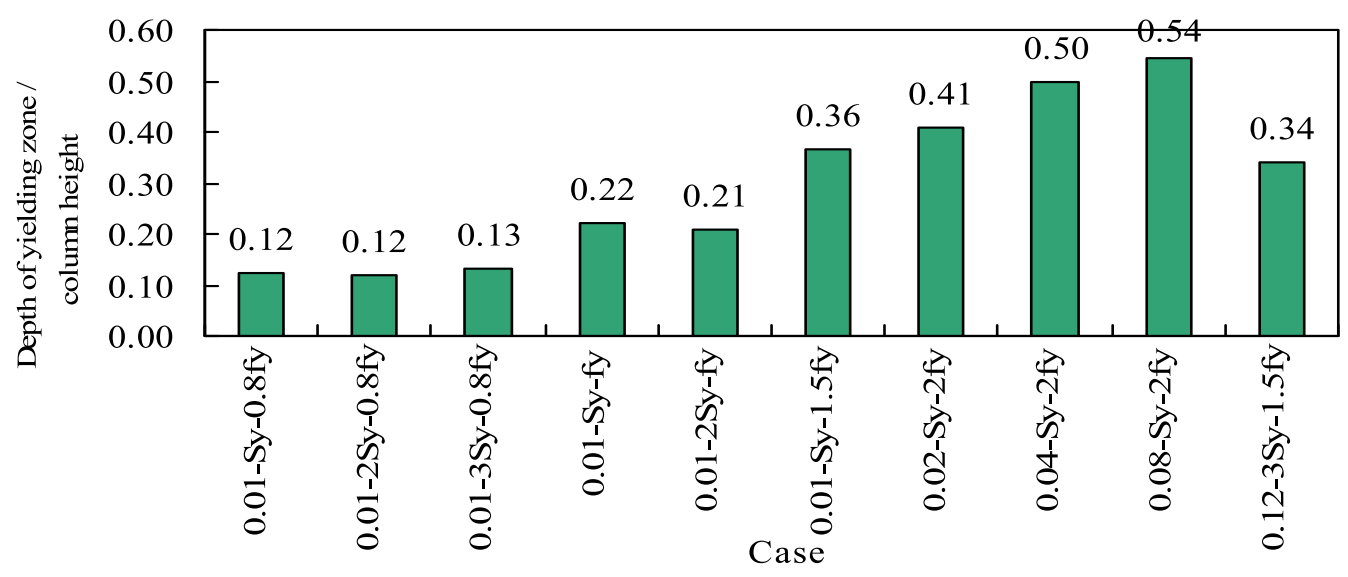

Fig. 8. Depth of the yielding zone of the studied cases at drift of $4.2 \%$ of column height

When bond strength is sufficient to assure the composite behavior between concrete and rebars, steel greatly contributes to the required deformability in the plastic zone. This is clear from Fig. 9 (a), where conventionally reinforced columns (case no. 6) exhibits high concentration of curvature ductility within the first $250 \mathrm{~mm}$ of the column height, i.e. curvature ductility is 32.3 at column base and reduces to 5.1 at $250 \mathrm{~mm}$. On the other hand, steel contribution to the required curvature ductility reduces when concrete-to-steel bond is weak. This can be seen from Fig. 9 (b) where curvature ductility reduces to $14.6 \%$ and $4 \%$ of that achieved by the conventionally reinforced column at its base when steel stresses at the ultimate slippage are $f_{y}$ and $0.8 f_{y}$, respectively, regardless of rebar ultimate slippage value. However, those columns can achieve the same level of the lateral drift of Specimen A as shown in Figs. $5 \& 6$, and this could be attributed to the increase in the contribution of column fixed-end rotation to lateral column deformation as shown in Fig. 10 (cases 1 to 6, listed in Table 1). In other words, when bond strength is weak, the reduction in steel contribution to lateral column deformation is compensated through the contribution of the fixed-end rotation due to rebars slippage, and this in turn reveals mitigation of damage in the plastic deformation zone.

Fig. 11 (a) details the distribution of curvature ductility of columns reinforced with rebars having bilinear ratio factors higher than that of ordinary rebars, e.g. rebars with $r=0.02$, 0.04 , and 0.08 , where perfect bond with the adjoining concrete are assumed for the three cases. It is clear that curvature ductility at column base and up until a height of $250 \mathrm{~mm}$ reduces by the increase in the value of $r$; nevertheless, out of this region the increase in $r$ adversely affects the distribution of curvature ductility: at $400 \mathrm{~mm}$ of column height, the change in $r$ from 0.02 to 0.04 and 0.08 increases curvature ductility from 1.8 to 4.1 and 3.8, respectively, and at $600 \mathrm{~mm}$ it increases from 0.97 to 1.16 and 1.3 , respectively. Furthermore, in these three cases the curvature ductility demands within 100-mm from the column base are smaller than those of the conventionally reinforced column (Fig. 9 (a)). In conclusion, using rebars with high level of strain-hardening may contribute to the mitigation of damage in the plastic hinge zone as a result of the redistribution of curvature ductility through the column height, and this propagation of yielding to a higher depth would compensate the demand of high deformability at column base to achieve a certain level of lateral drift. 

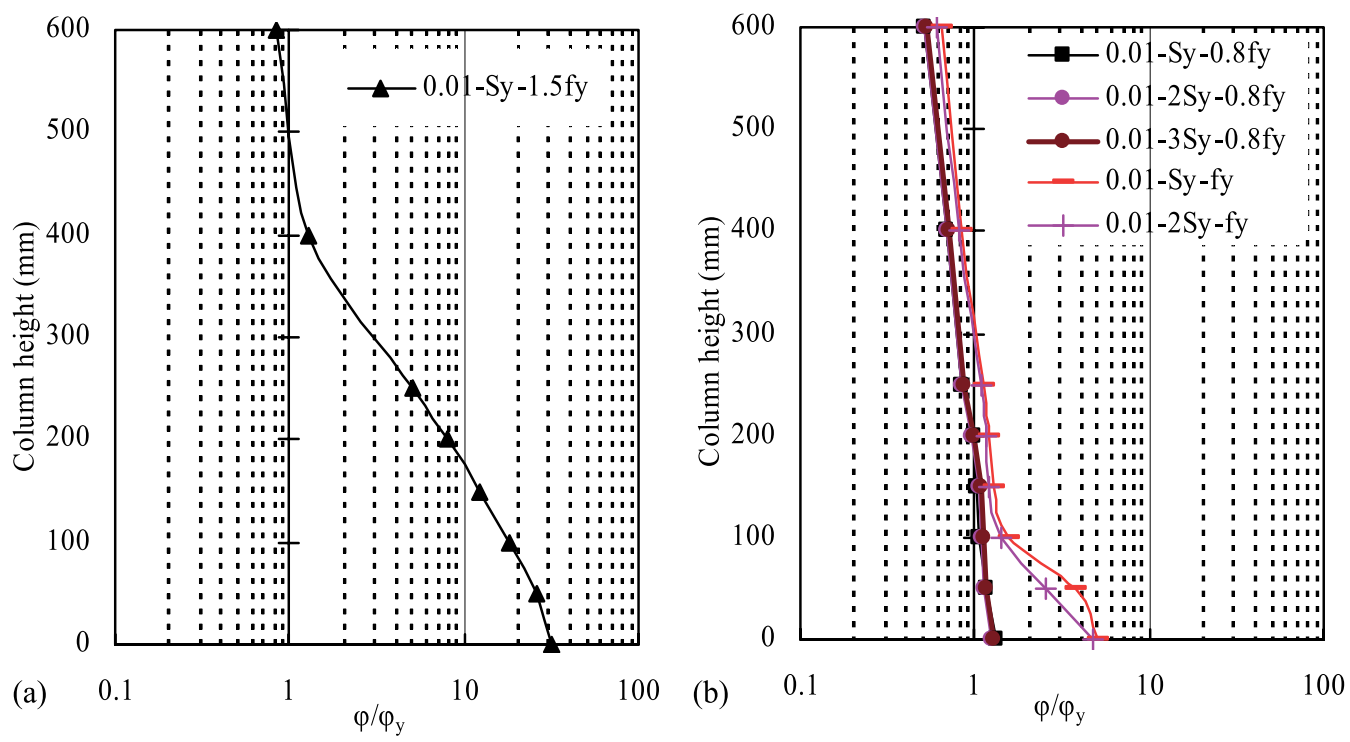

Fig. 9. The effect of bond properties between ordinary rebars $(r=0.01)$ and concrete on distribution of curvature ductility through column height at lateral drift of $4.2 \%$
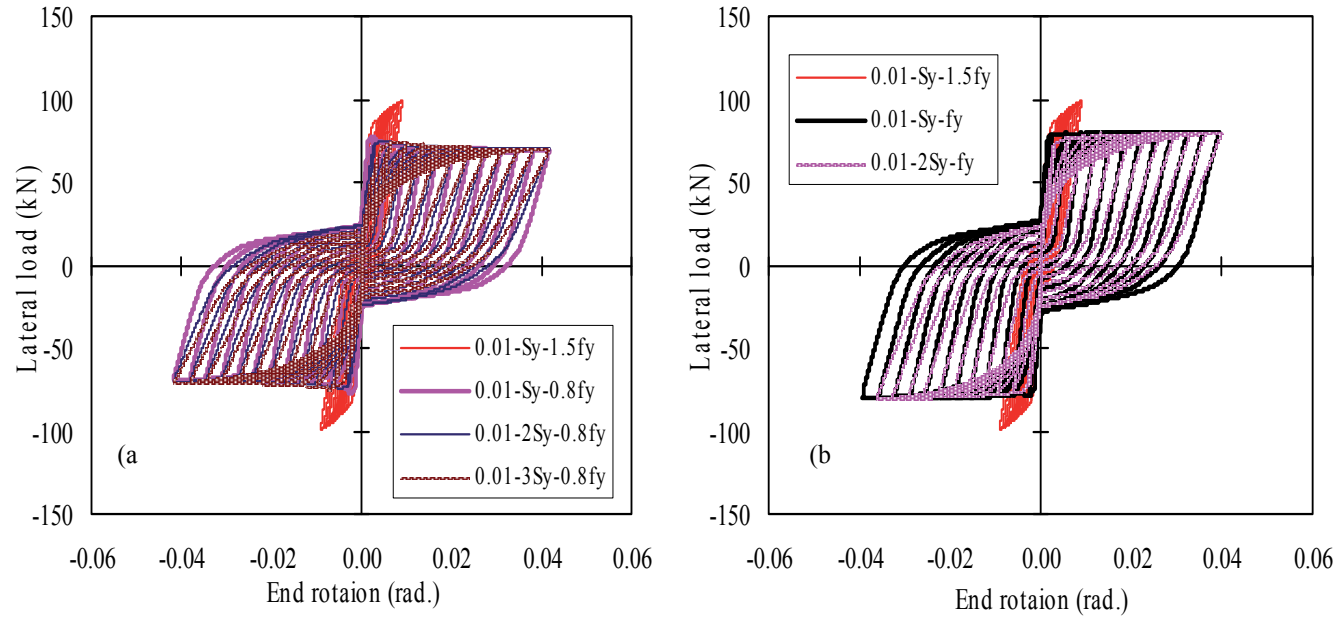

Fig. 10. Calculated fixed end-rotation versus lateral load 

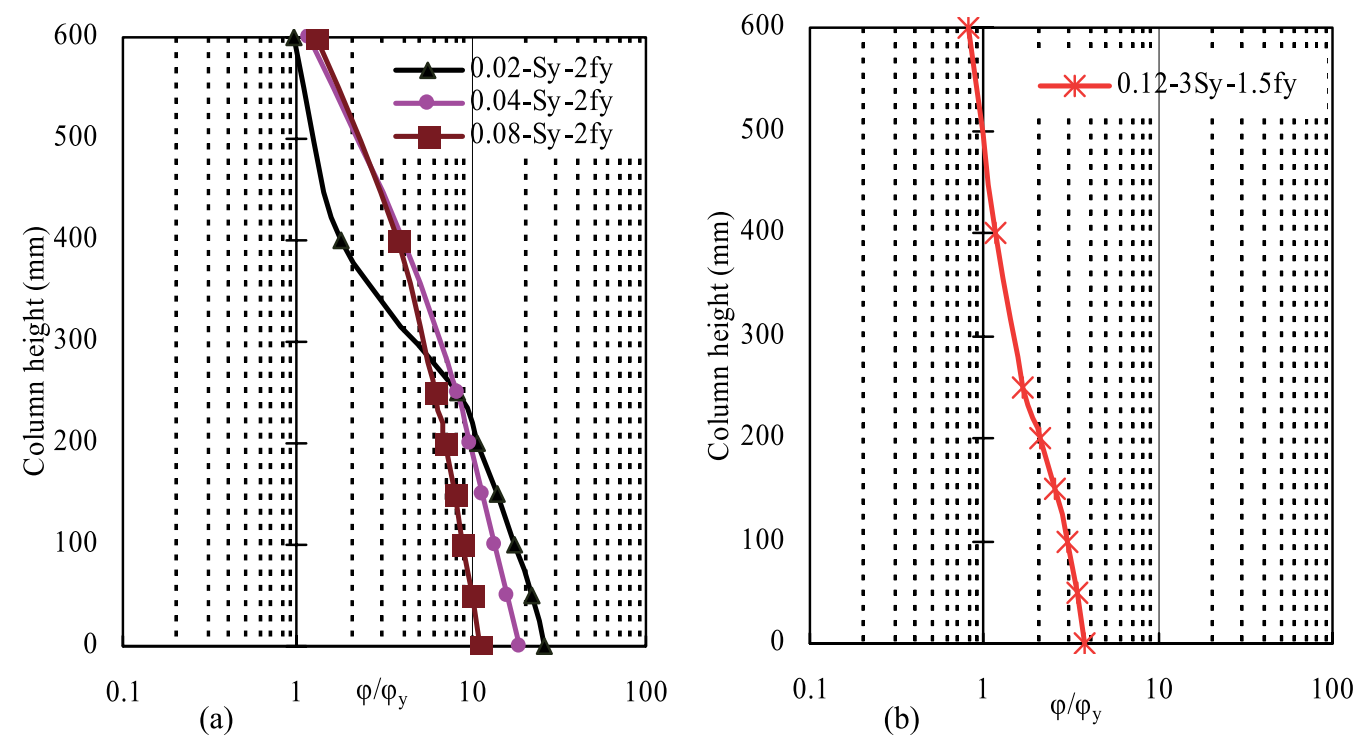

Fig. 11. The effect of bond properties between rebars $(r>0.01)$ and concrete on distribution of curvature ductility through column height at lateral drift of $4.2 \%$

The combined effect of both high bilinear ratio and bond capacity on the distribution of curvature ductility can be evaluated from Fig. 11 (b), where a well distribution of column curvature could be achieved. Curvature ductility is almost 3.78 at the interface section, is reduced to 2.12 and 1.17 at $200 \mathrm{~mm}$ and $400 \mathrm{~mm}$ from the column base, respectively, and ends at 0.8 at $600 \mathrm{~mm}$. Curvature distribution in this case also reveals the importance of using rebars with high strain-hardening performance, because when ordinary rebars loss their abilities of carrying load after yielding (cases number $4 \& 5$ in Table 1) due to the weak bond strength, concentration of damage very close to column base is highly probable, see Fig. 9 (b).

Based on the aforementioned analytical results, contribution of steel deformations to lateral deformation of RC columns should be reduced to mitigate damage at the zone of plastic deformations and thus contribution of the fixed-end rotation, due to rebars slippage, is critical to achieve the desired drift demand. For conventionally reinforced structures, contribution of column fixed-end rotation could only guarantee mitigation of the concentrated damage in plastic deformation zones, namely, structures reinforced with rebars having elastic perfectlyplastic behavior or small bilinear factor $(r \leq 0.01)$, this contribution would be accompanied by a negative or zero post-yield stiffness behavior, Fig.6 (a\&b). However, reinforcing structures with bond-controlled rebars having high bilinear ratio factor would be a reasonable system to have a damage-free recoverable structure. Because structure might be able to continue carrying load in the inelastic stage and both damage level and residual deformations could be controlled.

\section{Experimental evaluation of recoverability of RC bridge columns reinforced with bond-controlled rebars and SFCBs}

In order to verify the analytical findings, experimental results of columns reinforced with unbonded deformed bars in plastic hinge zone (Kawashima et al., 2001) and those with bond-controlled reinforcements (Pandey \& Mutsuyoshi, 2005) are restudied here from the 
view point of the required recoverability. Furthermore, two columns reinforced with SBFCBs and SCFCBs were tested by Fahmy et al. (2010) to examine the effect of high strainhardening behavior of reinforcement used on the required recoverability. Reinforcements of both specimens were in normal bond conditions to the surrounding concrete.

\subsection{Bridge columns reinforced with unbonded deformed bars}

To enhance ductility of RC bridge columns, Kawashima et al. (2001) conducted an experimental study on scale-model RC columns reinforced with ordinary deformed bars, which were unbonded at the plastic hinge zone. Different unbonding lengths were considered in their study, e.g. $200 \mathrm{~mm}, 400 \mathrm{~mm}$, and $600 \mathrm{~mm}$ were the unbonded lengths in Specimens B, C, and D, (Fig. 12, and Table 2), respectively. The tested specimens had square cross-section of $400 \times 400 \mathrm{~mm}$ and $1450-\mathrm{mm}$-height. In view of the results of Kawashima et al. (2001), the required recoverability of unbonded RC columns are examined in this study.

The envelopes of the measured force-drift relationships of the three unbonded specimens are shown in Fig. 13. The curves are the average of the envelopes for the push-and-pull loadings. Additionally, for comparison, the measured force-drift relationship of Specimen A is superimposed on the same figure. It is evident from Fig. 13 that the increase of the unbonded length is accompanied by a decrease in the elastic stiffness, reduction of the achieved ultimate strength, and diminishing of post-yield stiffness.
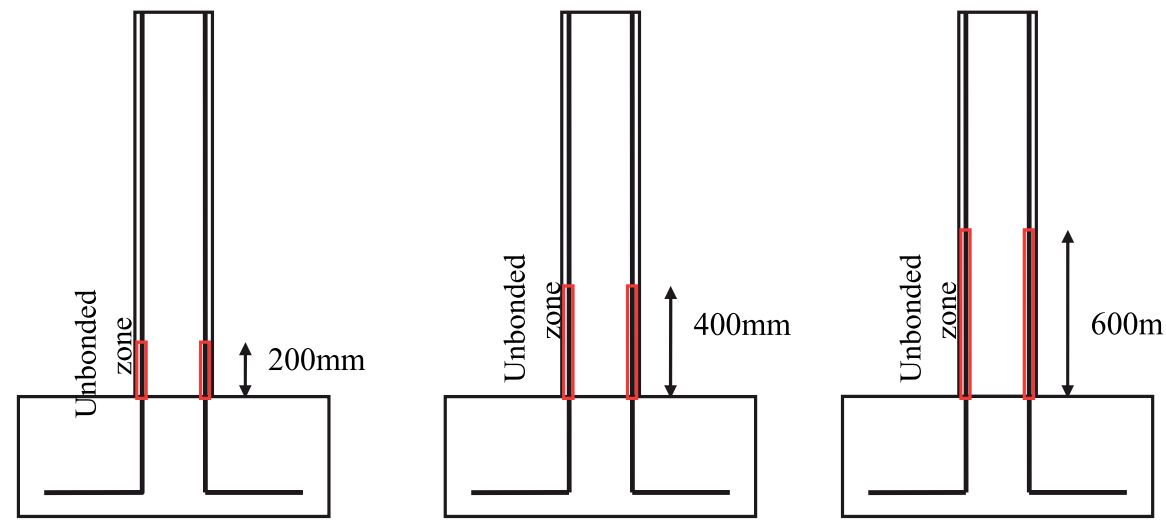

Fig. 12. Schematic details of the unbonded length of RC bridge columns

\begin{tabular}{|c|c|c|c|c|c|c|c|}
\hline Author & Sample & 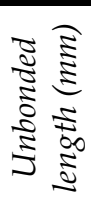 & $\begin{array}{l}\text { Axial } \\
\text { Load, } \\
\text { ratio } \\
(\%)^{a}\end{array}$ & $\begin{array}{c}\text { Main } \\
\text { steel }\end{array}$ & $v^{8} \sum_{\Sigma}^{\overparen{\varepsilon}}$ & $\begin{array}{c}\text { Yield } \\
\text { stress of } \\
\text { main } \\
\text { steel, } \\
(\mathrm{MPa})\end{array}$ & $\begin{array}{c}\text { Yield } \\
\text { stress of } \\
\text { lateral } \\
\text { steel, } \\
(\mathrm{MPa})\end{array}$ \\
\hline \multirow{3}{*}{$\begin{array}{l}\text { Kawashima et al. } \\
(2001)\end{array}$} & spe & 200 & 4.2 & $12 \mathrm{D} 13$ & 23.6 & 367 & 376 \\
\hline & Specimen C & 400 & 4.1 & 12 D13 & 24.6 & 367 & 376 \\
\hline & Specimen D & 600 & 4.2 & 12 D13 & 23.5 & 367 & 376 \\
\hline
\end{tabular}

a axial load ratio $=\left((\right.$ axial load $\left.) /\left(A_{g} \cdot f_{c o}^{\prime}\right)\right)$, where $A_{g}$ is the gross section area.

D13 = deformed bar with diameter of $13 \mathrm{~mm}$

Table 2. Details, dimensions, and material properties of unbonded RC columns 
Residual drift ratios verses column lateral drift ratios are plotted in Fig. 14 for the three unbonded specimens and the control one. This figure shows that there is a close similarity in the relation between the column drifts and the corresponding residual deformations for the four columns, which reveals that unbonding of deformed rebars at the plastic hinge zone with any length could not assure the alleviation of column residual deformations.

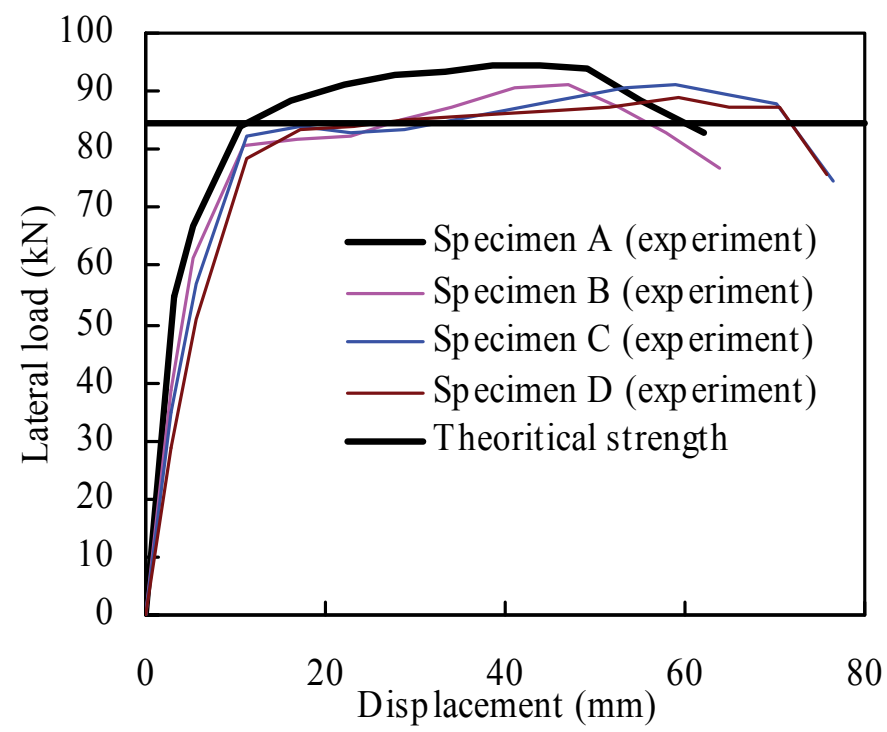

Fig. 13. Skeleton curves of the hysteretic responses of columns reinforced with bonded and unbonded deformed bars

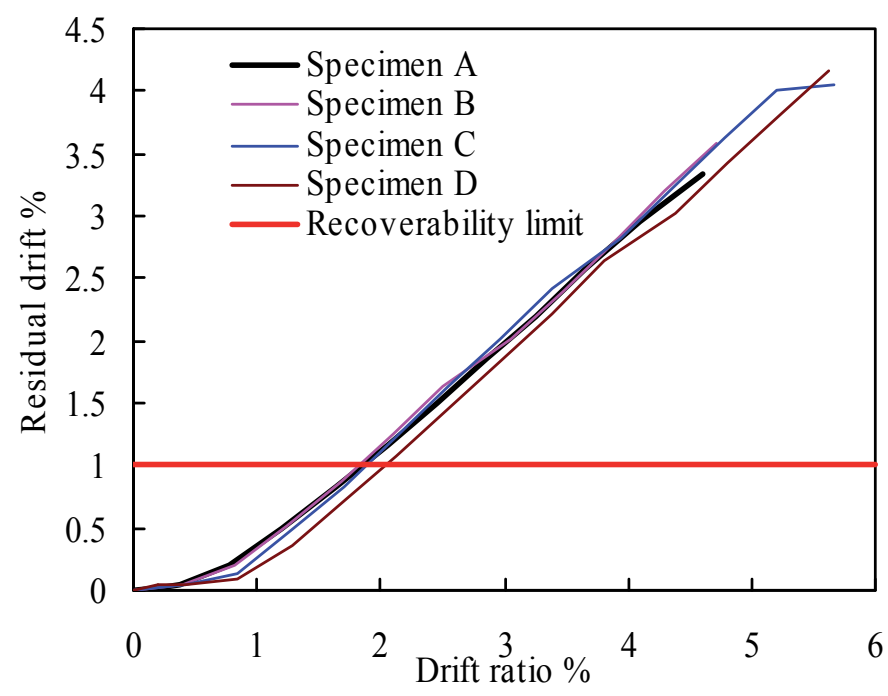

Fig. 14. Residual deformations of columns reinforced with bonded and unbonded deformed bars 


\subsection{Bridge columns reinforced with bond-controlled rebars}

An experimental investigation was carried out by Pandey and Mutsuyoshi (2005) to examine how controlling the bond of the longitudinal reinforcements can improve seismic performance factors, such as shear strength and ductility, of RC structures. Results of three of the tested columns (C-1, C-6, and C-7) are examined here to find the effects of surface characteristics of rebars used and also bond conditions of steel-to-concrete on the required recoverability: columns C-1 and C-6 were reinforced with DSBs and RSBs (round steel bars), respectively, but column C-7 was reinforced with RSBs, which had been coated with grease to further reduce bond strength through $800 \mathrm{~mm}$ length. Details of those columns are given in Table 3.

\begin{tabular}{|c|c|c|c|c|c|c|c|c|c|}
\hline Author & Sample & 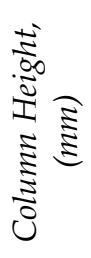 & 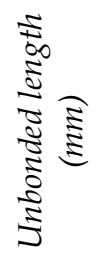 & $\begin{array}{l}\text { Axial } \\
\text { Load, } \\
\text { ratio } \\
(\%)^{a}\end{array}$ & $\begin{array}{c}\text { Main } \\
\text { steel }\end{array}$ & $\begin{array}{c}\text { Transverse } \\
\text { steel } \\
\text { (Size and } \\
\text { spacing, } m m \text { ) }\end{array}$ & $4^{8} \sum^{\overparen{E}}$ & $\begin{array}{c}\text { Yield } \\
\text { stress } \\
\text { of main } \\
\text { steel, } \\
(\mathrm{MPa})\end{array}$ & $\begin{array}{c}\text { Yield } \\
\text { stress } \\
\text { of } \\
\text { lateral } \\
\text { steel, } \\
(\mathrm{MPa})\end{array}$ \\
\hline \multirow{3}{*}{$\begin{array}{l}\text { Pandey and } \\
\text { Mutsuyoshi } \\
\text { (2005) }\end{array}$} & C-1 & 1050 & ---- & 2.7 & 12 D16 & D6 at 150 & 36.4 & 396.4 & 426.7 \\
\hline & C-6 & 1050 & ---- & 2.6 & $12 \Phi 16$ & D6 at 150 & 39.21 & 298.6 & 426.7 \\
\hline & $C-7$ & 1050 & 800 & 2.4 & $12 \Phi 16$ & D6 at 150 & 42.52 & 298.6 & 426.7 \\
\hline
\end{tabular}

a axial load ratio $=\left((\right.$ axial load $\left.) /\left(A_{g} . f_{c o}^{\prime}\right)\right)$, where $A_{g}$ is the gross section area.

D16 = deformed bar with diameter of $16 \mathrm{~mm}$; and $\Phi 16=$ round bar with diameter of $16 \mathrm{~mm}$.

Table 3. Details, dimensions, and material properties of bond-controlled RC columns

Because the lateral response of any of the investigated columns differs from that of others, the effect of the studied parameters on the achieved post-yield stiffness was evaluated as shown in Fig. 15, where average drifts of pull-and-push excursions of loading are plotted in relation to the corresponding column lateral strengths $(P)$ divided by the theoretical strength $\left(P_{i}\right)$. As a result of coating RSBs with grease, elastic stiffness of column C-7 was the smallest among the examined columns, Fig. 15. On the other hand, replacement of DSBs with RSBs slightly affected the elastic stiffness of column C-6, and it could not alter the shear failure mode of column C-1 to the ductile failure mode of column C-7, which was due to crushing and spalling of the concrete cover, followed by yielding of the longitudinal bars at the column-footing joint. In all cases shown in Fig. 15, negative post-yield stiffness is the performance in the inelastic stage, where the greatest deterioration of strength appeared in column C-1.

Relationship between column drifts and residual drifts is depicted in Fig. 16 for the columns C-1, C-6, and C-7. As is seen from Fig. 16, residual deformations of columns C-1 and C-6 are the same at any lateral drift, and their drifts at the recoverability limit are almost identical. This means that the use of RSBs in place of DSBs has no impact on column residual deformations. On the other hand, a favorable mitigation of column residual deformations was for column C-7 reinforced with unbonded RSBs. Column C-7 was capable of staying recoverable until lateral drift of $3.05 \%$, which is almost 1.5 times the drift of column C-6 at the recoverability limit. 
Although unbonding of DSBs at plastic hinge zone had no effect on column residual deformations, Fig. 14 and 16, it is interesting to stress on this finding which would help in realizing how could be the residual deformations controlled through the bond conditions between reinforcement and the surrounding concrete. When DSBs or RSBs are unbonded to the surrounding concrete, rigid body rotation due to rebar slippage would contribute to column lateral deformation and thus contribution of steel greatly reduces, and this was acknowledged in the study of Kawashima et al. (2001) through the measurements of steel strain at the plastic hinge zone, where strains of unbonded rebars were much lower than the counterpart in conventional RC columns. Despite the reduction of steel deformations, column residual deformations did not significantly change, particularly, when longitudinal reinforcement used was DSBs, and this can be attributed to partial unbonding of the DSBs, i.e. length of the unbonded zone, and characteristics of rebar surface. That is, owing to the interlocking of DSBs ribs of the bonded regions with the surrounding concrete, rebar cannot return to its former place after slippage. Therefore, unbonding of RSBs with grease coating for a length of $\sim 0.76$ of column height (column C7) increased the opportunity of limiting the residual fixed-end rotation and in turn the permanent deformations. In the study of Pandey and Mutsuyoshi (2005), it was reported that unbonding of most column height is an appropriate technique to avoid initiation of flexural cracks from the bonded regions. Consequently, mitigation of residual deformations of bond-controlled RC columns is dependent on the geometry of rebar surface as well as the length of the bonded regions.

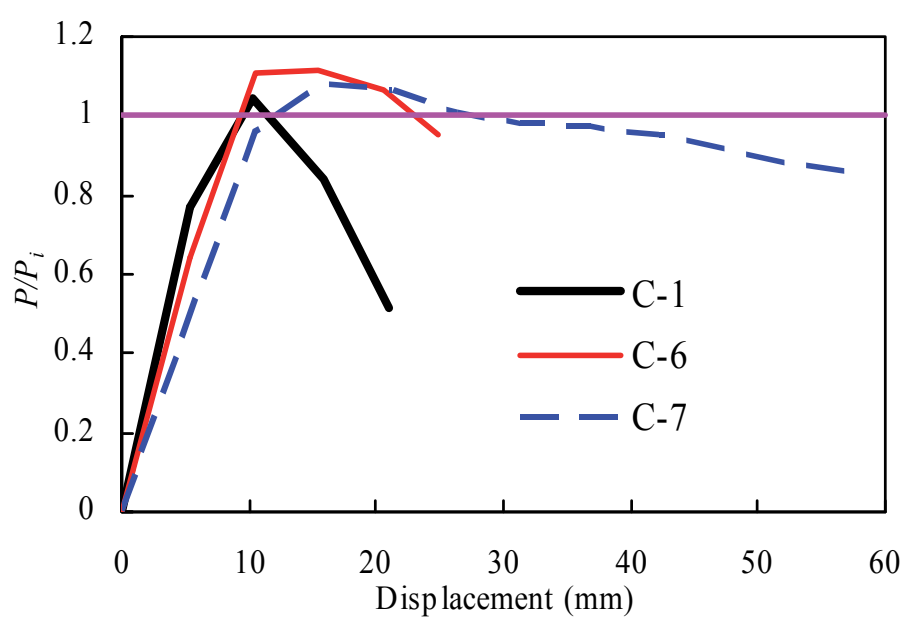

Fig. 15. Skeleton curves of the hysteretic responses of columns reinforced with bonded deformed bars and bonded and unbonded round bars 


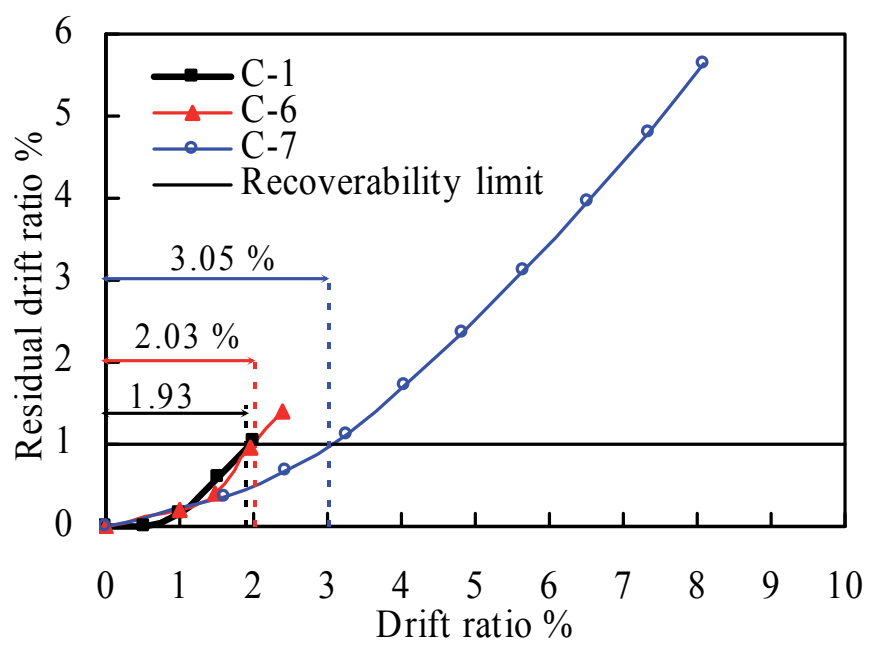

Fig. 16. Residual deformations of columns reinforced with bonded deformed bars and bonded and unbonded round bars

\subsection{Bridge columns reinforced with steel fiber composite bars (SFCBs)}

The results of this study are treated in a longer length in the study of Fahmy et al. (2010). Here, the hysteretic responses of the tested three columns are given to verify the possibility of enhancing recoverability of RC bridges using rebars with high level of strain-hardening as longitudinal reinforcement in place of ordinary rebars. Fig. 17 shows the results of three tested columns (CS14, CS10-C40 and CS10-B30), where the first column was reinforced with twelve deformed rebars with elastic perfectly-plastic behavior and the other columns were reinforced with steel fiber composite bars ( $r$ is over 10\%). It is evident from Fig. 17 that conventionally reinforced column was able to achieve lateral drift until $30 \mathrm{~mm}$ without any significant lose in the lateral capacity. However, after $\sim 21 \mathrm{~mm}$ later displacement $(1.91 \%$ lateral drift ratio) the column fell in the irrecoverable stat. on the other hand, the results of the other two columns (CS10-C40 and CS10-B30) showed recoverable performance till lateral drifts of $3.06 \%$ and $3.16 \%$, which correspond to $10.2 \%$ and $5.9 \%$ drop in the achieved ultimate strengths, respectively. However, due to the good bond between SFCBs and the adjoining concrete, Fig. 18 reveals extension of the plastic zone of column CS10-B30 beyond the counterpart in the conventionally reinforced column: depth of the damaged zone was almost 25\% of the column height for the columns CS14 and 31\% for CS10-B30, respectively.

Overall, the agreement found between all the addressed experimental results and the theoretical findings is acceptable. For instance, the decrease in the slope of both column elastic and post-yield stiffnesses due to poor bond qualities between ordinary rebars and the surrounding concrete is evident in the experimental results of Kawashima et al. (2001) and Pandey \& Mutsuyoshi (2005), Figs. 13 and 15. Moreover, the enhancement in the inelastic stage with the existence of post-yield stiffness was successfully achieved by columns reinforced with SFCBs. The analytical results showed that column residual deformations could be reduced when rebars slippage at yielding increases or rebars used have high level of the strain-hardening, and both effects are validated by the results of column C-7 reinforced with RSBs coated with grease (Fig. 16) and the results of the columns CS10-C40 and CS10-B30 reinforced with SFCBs, respectively, see Fig. 17. 

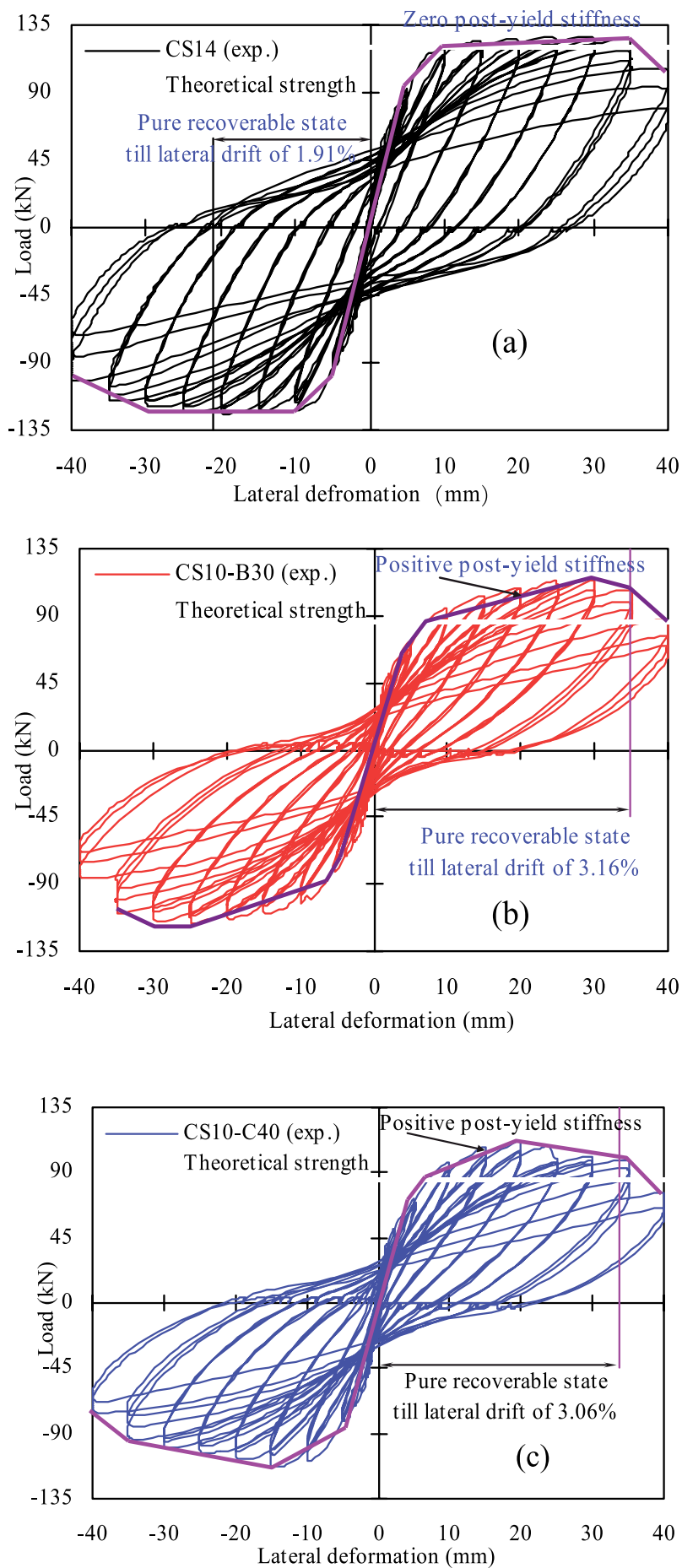

Fig. 17. Measured hysteretic lateral responses of the columns (a) CS14, (b) CS10-B30, and (c) CS10-C40 


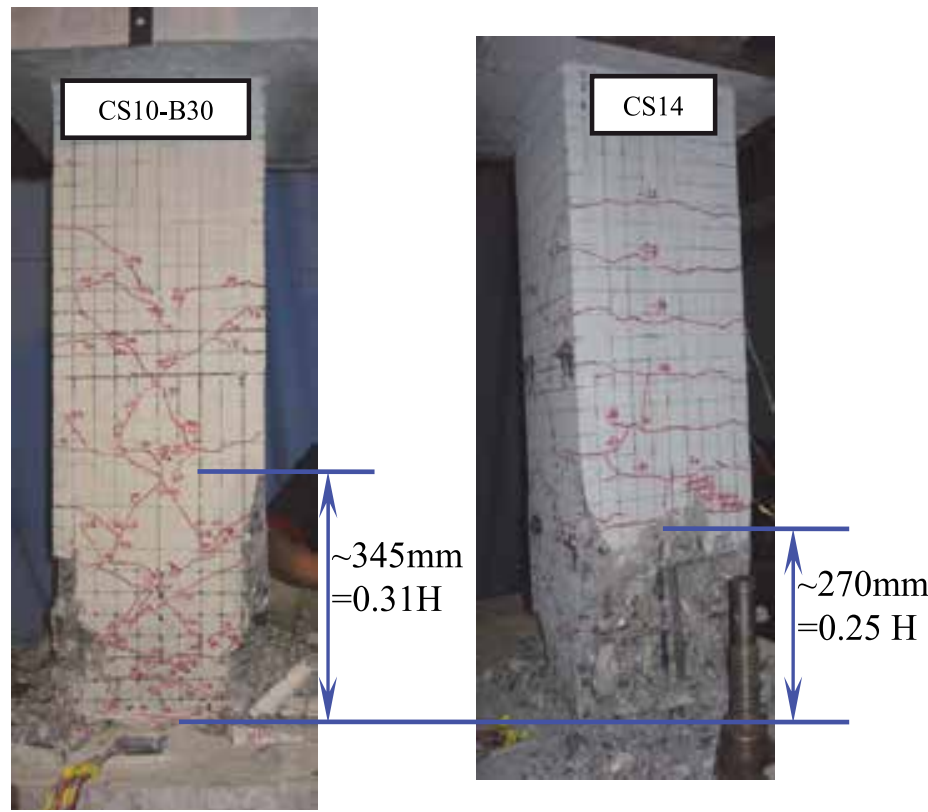

Fig. 18. Depth of damaged zone of the columns (a) CS10-B30 and (b) CS14

In conclusion, recoverable performance of RC columns lying in high seismicity regions can not be guaranteed with bond-controlled techniques using DSBs or RSBs. While using unbonded DSBs or RSBs would mitigate the concentration of damage at plastic hinge regions, bonded regions limit the probability of residual deformation mitigation. Moreover, unbonded columns hardly can achieve the theoretical strength with a negative or zero postyield stiffness performance in the inelastic stage. On the other hand, due to the promising performance of the innovative rebars (SFCBs), it is highly desirable to further study the effect of bond conditions on the recoverability of columns reinforced with SFCBs.

\section{Analytical study on RC columns reinforced with bond-controlled SFCBs}

Fig. 1 shows that the inner core ribbed rebar is first looped around with bundles of continuous fibers roving to fill up the space between its ribs, such that bond strength is guaranteed with the outer longitudinal fibers. This figure also shows that there is a spiral distribution of resin ribs along the SFCB. The main function of those ribs is to ensure the bond performance between a SFCB and the surrounding material, such as concrete, allowing SFCBs to achieve the designed level of strength. For instance, ribs with adequately designed spacing and height would lead SFCBs to approach their ultimate strength; however, in case ribs spacing is wide and the height is undersized, rebars may fail to continue to carry additional load until approaching the designed strength level. Besides, slippages of SFCBs at both yielding and maximum achieved strength depend on the applied bond technique. Consequently, five cases of RC columns reinforced with SFCBs are studied (Table 4), where four cases examine the effect of the achieved strength of SBFCBs on the performance of RC bridge columns. The last case (case no. 5) considers the effect of additional slippage of SFCBs when the ultimate strength is greatly affected by bond conditions, i.e. merely $70 \%$ of the ultimate strength could be achieved. 


\begin{tabular}{ccccc}
\hline & \multicolumn{5}{c}{ Bond properties } \\
\cline { 2 - 5 } Case & $\begin{array}{c}S_{y} \\
(\mathrm{~mm})\end{array}$ & $\begin{array}{c}\sigma_{y} \\
(\mathrm{MPa})\end{array}$ & $\begin{array}{c}S_{u} \\
(\mathrm{~mm})\end{array}$ & $\begin{array}{c}\sigma_{u} \\
(\mathrm{MPa})\end{array}$ \\
\hline 1 & $S_{y}$ & $f_{y}$ & $S_{u}$ & $f_{u}$ \\
2 & $S_{y}$ & $f_{y}$ & $S_{u}$ & $0.9 f_{u}$ \\
3 & $S_{y}$ & $f_{y}$ & $S_{u}$ & $0.8 f_{u}$ \\
4 & $S_{y}$ & $f_{y}$ & $S_{u}$ & $0.7 f_{u}$ \\
5 & $2 S_{y}$ & $f_{y}$ & $2 S_{u}$ & $0.7 f_{u}$ \\
\hline
\end{tabular}

Table 4. Studied bond properties of column reinforced with SFCBs

The studied column has square cross-section of $300 \times 300 \mathrm{~mm}$ and $1300-\mathrm{mm}$-tall. It is typified CS10-B30 and reinforced with twelve SBFCB, Table 5. The concrete compressive strength is $\sim 38 \mathrm{MPa}$. The columns are analyzed under the effect of constant axial load (12\% of column axial strength) and reversed cyclic lateral load. Under normal bond condition, a column with these given details was experimentally tested by Fahmy et al. (2010) with another column typified CS10-C40, where its longitudinal reinforcement was twelve SCFCB, Table 5.

\begin{tabular}{ccccccc}
\hline Type & $\begin{array}{c}\text { Diameter } \\
(\mathrm{mm})\end{array}$ & $\begin{array}{c}\text { Elastic } \\
\text { modulus } \\
\left(E_{1}\right)(\mathrm{GPa})\end{array}$ & $\begin{array}{c}\text { Yield } \\
\text { strength } \\
(\mathrm{MPa})\end{array}$ & $\begin{array}{c}\text { Post-yield } \\
\text { stiffness } \\
\left(E_{2}\right),(\mathrm{GPa})\end{array}$ & $\begin{array}{c}\text { Ultimate } \\
\text { strength } \\
(\mathrm{MPa})\end{array}$ & $E_{2} / E_{1}(\%)$ \\
\hline$\Phi 10+30 \mathrm{~B}$ & 13.2 & 142 & 312.4 & 16.6 & 691.3 & 11.7 \\
$\begin{array}{c}\text { (SBFCB }) \\
\text { Ф10+40C }\end{array}$ & 12.9 & 155.5 & 342.2 & 30.2 & 641.8 & 19.7 \\
$\begin{array}{c}(\mathrm{SCFCB}) \\
\Phi 10\end{array}$ & 10 & 200 & 420 & - & 480 & - \\
\hline
\end{tabular}

Table 5. Properties of SFCB and ordinary rebars

Figure 19 shows the effect of bond properties on both column post-yield stiffness (its slop and end point) and residual deformations. For instance, with the assumption of extremely sufficient bond strength between SFCBs and the adjoining concrete, Figure 19 (a) shows the highest achieved inelastic stiffness which ends at lateral drift of $40-\mathrm{mm}$ (due to rupture of the outer basalt fibers) with the minimum residual deformations. But, when the composite behavior between SBFCBs and surrounding concrete can not assure the development of the ultimate strength of SFCBs, the inelastic hysteretic response after column yielding would be divided to two parts: the first shows that the column can still carry the load after the SBFCBs yield and hardening behavior has been exhibited; and the second part demonstrates a zero post-yield stiffness, which starts by the end of the hardening zone. As is clear from Figs. 19 (a-e), the slop of the post-yield stiffness and its end point is dependent on the bond conditions. In addition, the controlled increase in slippage of SFCBs would assist in further mitigation of residual deformations, Figs. 19 (d \& e). 

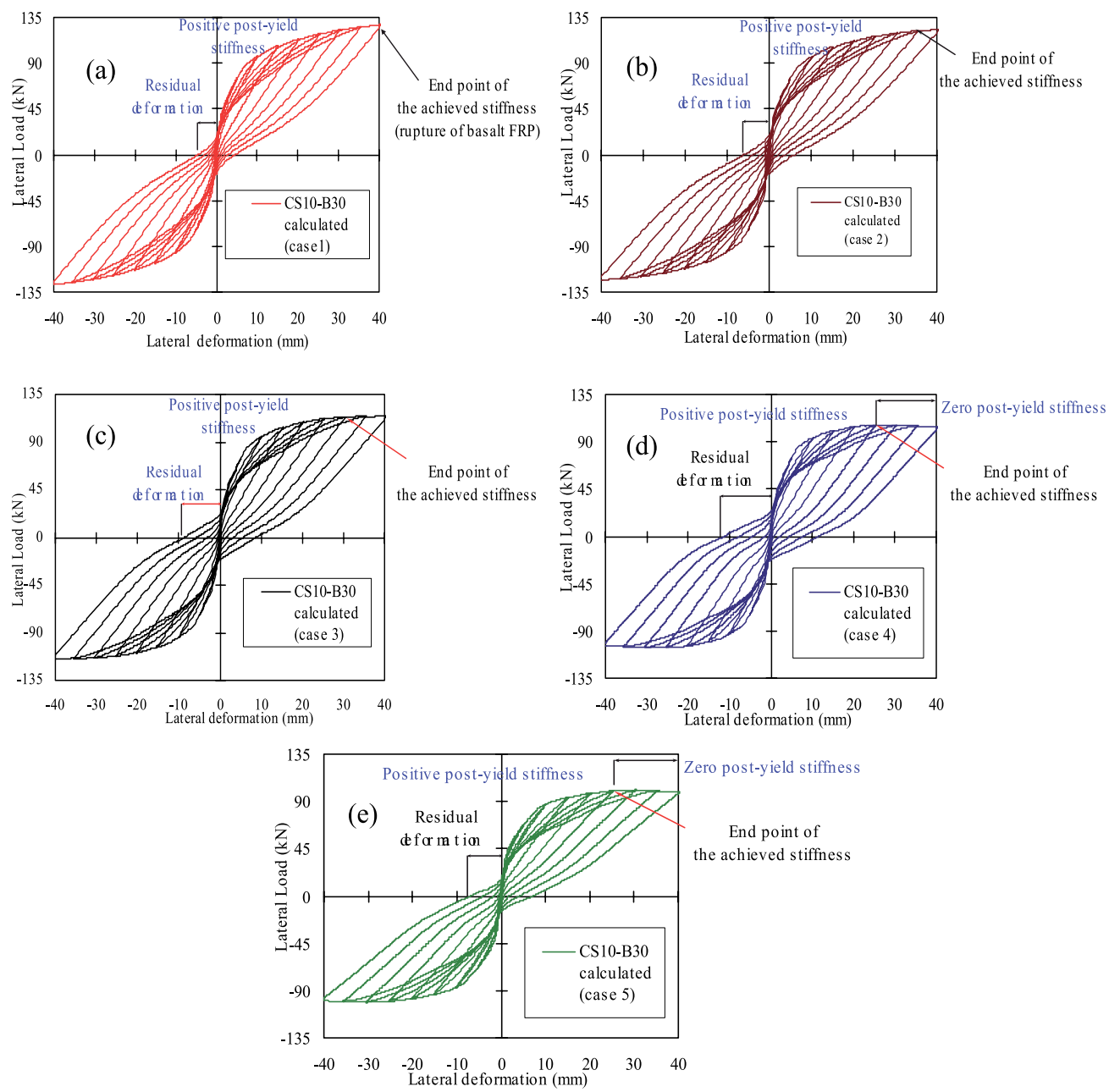

Fig. 19. Bond effect on performance of RC column reinforced with SBFCBs

\section{Summary and conclusions}

The demand for efficient and effective damage-controllable systems has received strong attention in the last decade, where the main goal is the limitation of the damage at plastic hinge zones along with substantial mitigation of the static residual deformations. Since bond between longitudinal reinforcement and concrete is a key factor controlling structural performance of reinforced concrete (RC) structures, here a study was conducted to examine the effect of alternating the bond conditions between longitudinal reinforcement and concrete on the performance of RC columns. Effects of different conditions of concrete-tosteel bond on the elastic and post-yield stiffnesses, residual deformations, and damage level of RC bridge columns reinforced with rebars having different strain-hardening levels were determined. For ordinary steel bars RSBs and DSBs, the study showed that damage of plastic hinge zone is mitigated provided that reinforcement is completely unbonded from the surrounding concrete. Also, the study revealed that residual deformation of columns 
reinforced with RSBs can be mitigated only if the unbonded zone represents most of the column height. Despite these encouraging findings, unbonded columns could barely achieve the theoretical strength with zero or negative post-yield stiffness in the inelastic stage. That is, quick recovery of of RC columns reinforced with unbonded DSBs or RSBs can not be guaranteed, which limits the practical application of this technique in RC bridge columns lying in high seismicity zones. Since RC column reinforced with SBFCBs distinctly outperforms its RC counterpart, bond-based damage-controllable system using SBFCBs could be applied for structures reinforced with the innovative rebars, where slope and end point of the achieved post-yield stiffness can be controlled. In addition, both residual deformations and damage level at plastic hinge zone could be limited.

\section{References}

ACI Committee 318. Building code requirements for structural concrete (ACI 318-08) and commentary (318R-08). American Concrete Institute (ACI) 2008, Farmington Hills, Mich., 430 pp.

Cosenza, C.; Manfredi, G. \& Ramasco, R. (1993). The use of damage functionals in earthquake engineering: a comparison between different methods. Earthquake Eng. Struct. Dyn., Vol. 22, pp. 855-868.

Fahmy, M.F.M.; Wu, Z.S., Wu, G. \& Sun, Z.Y. (2010). Post-yield stiffnesses and residual deformations of $\mathrm{RC}$ bridge columns reinforced with ordinary rebars and steel fiber composite bars. Journal of Engineering Structures, Vol.32, pp. 2969-2983.

Ghobara, A.; Abou-Elfath, H. \& Biddah, A. (1999). Response-based damage assessment of structures. Earthquake Eng. Struct. Dyn., Vol. 28, pp. 79-104.

JSCE Earthquake Engineering Committee (2000). Earthquake resistant design codes in Japan. Japan Society of Civil Engineers (JSCE), Tokyo, Japan, 150 pp.

Kawashima, K.; Hosoiri, K.; Shoji, G., \& Sakai, J. (2001). Effects of unbonding of main reinforcements at plastic hinge region on enhanced ductility of reinforced concrete bridge columns. Structural and Earthquake Engineering, Proc. JSCE, 689/I-57, pp. 4564.

Mazzoni, S; McKenne, F; Scott, MH; Fenves, GL, et al. Open System for Earthquake Engineering Simulation User Manual version 2.1.0. Pacific Earthquake Engineering Center, University of California, Berkeley, CA, http://opensees.berkeley.edu/OpenSees/manuals/usermanual/

Moustafa, A. (2011). Damage-based design earthquake loads for single-degree-of-freedom inelastic structures. Journal of Structural Engineering (ASCE), Vol. 137, No.3, pp. 456467.

Pandey, GR \& Mustsuyoshi, H. (2005). Seismic performance of reinforced concrete piers with bond-controlled reinforcements. ACI Structural Journal, Vol.102, No.2, pp. 295304.

Park, R. \& Paulay, T. (1975). Reinforced concrete structures. John Wiley and Sons, 769pp.

Pettinga, D. ; Pampanin, S. ; Christopoulos, C. \& Priestley, N. (2006). Accounting for residual deformations and simple approaches to their mitigation. First European Conference on Earthquake Engineering and Seismology, Geneva, Switzerland.

Priestley, M.J.N.; Calvi, G.M. \& Kowalsky. (2007). Displacement-based seismic design of structures. IUSS Press, Pavia, 721pp. 
Saiidi, M.S. ; O’Brein, M. \& Sadrossadat-Zadeh, M. (2009). Cyclic response of concrete bridge columns using superelastic nitinol and bendable concrete. ACI Structural Journal; Vol.106, No.1, pp. 69-77.

Spacone, E. ; Filippou, F. \& Taucer, F. (1996). Fiber beam-column model for nonlinear analysis of R/C frames-part II: applications. Journal of Earthquake Engineering and Structural Dynamics, Vol. 25, pp. 727-742.

Takiguchi, K. ; Okada, K. \& Sakai, M. (1976). Ductility capacity of bonded and unbonded reinforced concrete members. Proc. Architectural Institute of Japan. 249, pp. 1-11.

Wu, G. ; Wu, ZS. ; Luo, YB. ; Sun, ZY. \& Hu, XQ (2010). Mechanical properties of steel fiber composite bar (SFCB) under uniaxial and cyclic tensile loads. Journal of Materials in Civil Engineering, Vol. 22, No. 10, pp. 1056-1066.

Zhao, J. and Sritharan, S. (2007). Modeling of strain penetration effects in fiber-based analysis of reinforced concrete structures. ACI Structural Journal, Vol. 104, No.2, pp. 133-141. 


\title{
Electromagnetic Sensing Techniques for Non-Destructive Diagnosis of Civil Engineering Structures
}

\author{
Massimo Bavusi et al. ${ }^{*}$ \\ CNR-IMAA, \\ Italy
}

\section{Introduction}

Health Assessment Methods (HAM) and Structural Health Monitoring (SHM) aim to improve the standard of knowledge regarding the safety and maintenance of structures and infrastructure acquiring information about geometrical, mechanical and dynamical characteristics of structures. In earthquake-prone areas, this activity has the double aim of assessing the buildings structural integrity and extracting information regarding their response during a seismic event in order to define appropriate activities for risk mitigation.

A number of factors afflict buildings and infrastructure safety in seismic areas:

- Outdated codes of practice: a significant number of highly urbanized areas are present globally, where a high percentage of structures have been designed and erected considering only gravity loading.

- The age of the structures and the real in-situ performance of construction material significantly affect their overall behaviour.

- Structural deficiencies such as poor material qualities and/or degradation of structural materials (rust, spalling etc.), inadequate construction detailing, low levels of ductility, brittle collapse mechanisms.

The seismic assessment of structures is performed in terms of the estimation of the earthquake intensity that would lead to a certain damage condition and/or collapse. The assessment of the seismic vulnerability of existing buildings is generally based on the knowledge of building characteristics and through a complex analysis of the possible collapse mechanisms in order to identify the most probable failure for the given structure (as example: Ansari, 2005; Douglas, 2007; Moustafa et al., 2010).

The methodological approach for the evaluation of a structure resistance is represented in Figure 1 where structural knowledge obtained through a series of test assessments is needed in order to define vulnerability and thus design suitable retrofit strategies.

\footnotetext{
${ }^{*}$ Romeo Bernini2 ${ }^{2}$, Vincenzo Lapenna ${ }^{1}$, Antonio Loperte ${ }^{1}$, Francesco Soldovieri² ${ }^{2}$ Felice Carlo Ponzo ${ }^{3}$, Antonio Di Cesare ${ }^{3}$ and Rocco Ditommaso ${ }^{3}$

${ }^{1}$ CNR-IMAA, Italy

${ }^{2} \mathrm{CNR}$-IREA, Italy

${ }^{3}$ Basilicata University/DiSGG, Italy
} 
Since the level of reliability of the assessment method is related to the adequacy of the model and to the completeness of the information, all useful available data have to be collected in order to define the original structural characteristics such as: geometry of structural elements, characteristics and behaviour of the construction materials, presence of degradation, arrangement of longitudinal and transversal reinforcement.

The knowledge of an existing structure is never complete and the level and accuracy of construction details obviously corresponds proportionally with the available original design documentation, the time and funds available for in situ investigations and experimental tests on the structural elements.

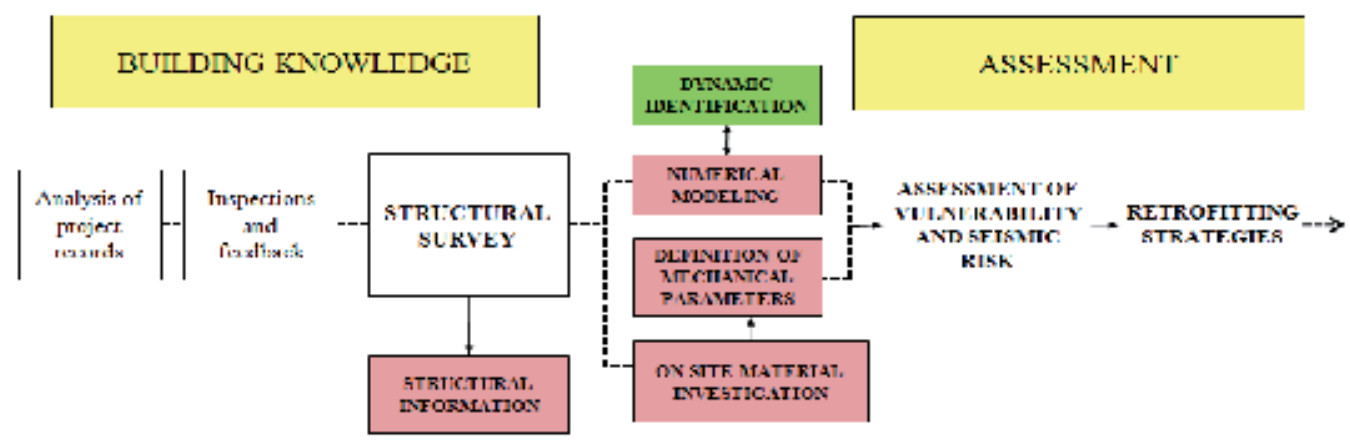

Fig. 1. Methodological approach.

A reliable assessment of the vulnerability of buildings is also strictly connected to the evaluation of the mechanical characteristics of the constitutive materials. This can be particularly complex for concrete, due to the high variability of its resistance that depends on intrinsic factors such as the composition as well as the environmental and maturing conditions, and other factors attributable to the collection technique and reworking of the concrete sample and the test conditions in general (Barlet, 1994).

A number of tests and methods have been developed for evaluating the resistance of construction materials ranging from completely non-destructive tests (NDT), where there is no damage to the structural element, using methods where the concrete surface is only slightly damaged, to partially destructive or destructive tests (DT), where the structural element has to be repaired afterwards.

The classical NDT methods, generally used for Reinforced concrete (R/C) structures, are the surface hardness method coupled with the ultrasonic method. As these methods are influenced in different and/or opposing ways by some fundamental parameters, their combined use allows outputs with minimal dispersion to be obtained. It is generally not advisable to use a single non-destructive test to estimate the strength in situ of concrete.

The range of properties that can be assessed using the range of NDT methods is significant and includes fundamental parameters such as density, elastic modulus as well as surface hardness, reinforcement location and depth of cover concrete. In some cases it is also possible to check the quality of workmanship and structural integrity through the ability to detect voids, cracking and delamination.

Preliminary tests can be performed with a covermeter according to the procedures described in the British Standard 188:204. With this technique it is possible to determine the presence and size of reinforcing bars, laps, transverse steel and depth and position of reinforcement. 
The identification of the position of the reinforcement bars is also used as a preliminary to the other NDT (such as Ultrasonic Pulse Velocity, Schmidt Rebound Hammer, Pull-out UNI EN 12504-3, 2005) and also the DT (Core Extraction and Compression Test). In addition the partially destructive method of removing the cover concrete in some areas and measuring directly the diameter and type of the reinforcement can be performed.

The Schmidt rebound hammer test is principally a surface hardness tester and is carried out according to UNI EN 12504-2 (2001). The system works on the principle that the rebound of an elastic mass depends on the hardness of the surface against which the mass impinges. There is little apparent theoretical relationship between the strength of concrete and the rebound number of the hammer, however within limits, experimental correlations are established between strength properties and the rebound number. All of this cannot be generalized and should be calibrated for each type of existing concrete, for example using the results of compression tests. In the some cases the results of the hammer tests, taken as a rebound average (Ir) is used individually to assess the homogeneity of the concrete.

The Ultrasonic test is carried out in compliance with UNI EN 12504-4 (2005) and is aimed at determining the propagation velocity of a mechanical vibration pulse in concrete. By measuring the pulse crossing time and the distance between the two probes, the apparent propagation velocity can be calculated. This value can differ from the real value when the elastic waves undergo deviations from the path identified by the conjunction line between the two probes (RILEM 1972). The factors that affect the ultrasonic test the most are linked to the concrete composition, environmental and test conditions.

When interpreting the NDT results, special attention is needed regarding the presence of possible anomalies which can negatively influence the experimental assessment of the in situ concrete mechanical characteristics. Such anomalies are generally characterized through an evident correlation of the experimental datum with either a physical parameter of reference (usually compressive strength from DT) or with respect to the trend shown by the data acquired in the same context of structural homogeneity. These anomalies usually arise from improper execution of the test or from the fact that the test is carried out in non-ideal conditions.

Direct measure of the compressive strength of concrete in a structure is provided by the Concrete Core Extraction and Compression method (DT). The process of obtaining core specimens and interpreting the strength test results is often affected by various factors that influence either the in-place strength of the concrete or the measured strength of the test specimen (UNI EN 12504-1, 2002). In spite of such disturbance factors, values measured in this way are certainly the most reliable possible. Furthermore errors can be reduced using the A.C.I. 214.4R-03 guidelines which summarize current practices for obtaining cores and interpreting core compressive strength test results.

Immediately after extraction, the core concrete is tested for carbonation (also called depassivation). Carbonation penetrates below the exposed surface of concrete extremely slowly. The significance of carbonation is that the usual protection of the reinforcing steel generally present in the concrete due to the alkaline conditions caused by the hydrated cement paste is neutralized. Thus, if the entirety of the cover concrete is carbonated, corrosion of the steel will occur if moisture and oxygen can infiltrate the section.

The necessary destruction of the test object usually makes DT methods more expensive, and these testing methods can also be inappropriate in many circumstances. Therefore the use of NDT plays a crucial role in ensuring an economical operation. A general proportion of 1 
core to 4 non-destructive investigations is recommended. Both the results from the DT and NDT are then combined in order to estimate the in situ concrete strength using the SonReb method. This method is the principal combination of Schmidt Rebound Hammer with Ultrasonic Pulse Velocity used for quality control and strength estimation of in situ concrete (Braga, 1992).

Another group of NDT methods are the Dynamic identification tests which can be used in order to assess fundamental dynamic properties (frequencies and/or modal shapes) of the structure and indirectly estimate the Young's modulus of the material (Ponzo et al., 2010). All dynamic characteristics can be estimated using two different approaches: classical methodologies based on Fourier analysis (Ditommaso et al., 2010a) or innovative methodologies based on time-frequency and interferometric analyses (Ditommaso et al., 2010b; Picozzi et al., 2011). These latter analyses are also useful to detect possible structural damage occurred after an earthquake (Ditommaso et al., 2011).

The results of the above testing methods (both NDT and DT) are used to calibrate numerical models. These models can then be compared to the likely seismic loading thus providing the overall vulnerability of the structure being considered (Ponzo et al. 2011).

Even if the methods described above can ensure (when correctly applied) a high level of structural knowledge a number of issues remain to be addressed. In order to do this innovative technologies and new methods must be developed: reduce uncertainty regarding core extraction points, improve the detection of deflection and deficiencies, detect water infiltration, improve reinforcement information, improve the depth under investigation, and reduce time and cost.

Due to rapid and flexible execution, high spatial resolution and deep investigation depth, electromagnetic sensing techniques are a group of NDT methods which can achieve these objectives. They can direct the use of classical NDT and DT methods and reduce uncertainties, coring number and the survey cost. Furthermore, their contribution to the structural knowledge allows the adoption of lower safety coefficients (through the increase in available information thus minimising spread) and thus higher calculation resistances. This in turn reduces the extent and cost of the actions required for the improvement or seismic retrofit of structures, if needed.

The building and infrastructure diagnostics can be take advantage from the use of new NDT techniques enabling larger investigation depths, spatial resolution, void and defect detection capacity, low cost and fastness. Electromagnetic sensing techniques can be an useful tool in order to achieve these objectives. They are based on injection of a form of electromagnetic energy (electrical current, radiowave, microwave, light, etc.) into the surveyed object and gathering of returned signal in order to measure electromagnetic properties (resistivity, electrical permittivity), reconstruct the inner structure, detect embedded defects.

In table 1 an overview of the advantages and disadvantages of several techniques (both classic and innovative) is presented, in terms of cost, speed of procedure, non intrusivity, accuracy of data obtained and degree of correlation with actual values.

Nevertheless, applying electromagnetic sensing techniques to man made structures, some adaptation needs to fit stringent requirements in terms of exploration depth, spatial resolution and signal/noise ratio. In fact, commonly used building materials pose challenging issues in terms of electrode impedance, coupling antennas, survey modalities, tomographic reconstruction, sensor size. 
Electromagnetic sensing techniques suitable for civil infrastructures and building diagnostic such as Ground Penetrating Radar (GPR) and Electrical Resistivity Tomography (ERT) are presented in this chapter. Concerning the GPR we focus the attention on the possibility to improve the imaging at low and radio/microwave frequencies by using novel inversion approaches such as the Microwave Tomography (MT).

Then, a novel distributed fiber optic sensor technology able to monitor strain and temperature variations, is described. Finally, we discuss about the real contribution provided by electromagnetic sensing techniques in the building and infrastructure monitoring.

\begin{tabular}{|l|c|c|c|c|c|}
\hline \multicolumn{1}{|c|}{ Test Method } & Cost & $\begin{array}{c}\text { Speed of } \\
\text { procedure }\end{array}$ & $\begin{array}{c}\text { Damage } \\
\text { effected }\end{array}$ & $\begin{array}{c}\text { Accuracy } \\
\text { of data }\end{array}$ & $\begin{array}{c}\text { Degree of } \\
\text { correlation }\end{array}$ \\
\hline Core samples & High & Slow & Moderate & Moderate & High \\
\hline Rebound hammer & Very low & Fast & None & $\begin{array}{c}\text { Only } \\
\text { surface } \\
\text { info }\end{array}$ & Low \\
\hline Ultrasonic waves & Low & Fast & None & $\begin{array}{c}\text { Complete } \\
\text { penetration }\end{array}$ & Moderate \\
\hline Covermeter & Low & Fast & None & Moderate & - \\
\hline $\begin{array}{l}\text { Electromagnetic } \\
\text { sensing }\end{array}$ & High & Moderate & None & Good & $\begin{array}{c}\text { Low/ } \\
\text { Moderate }\end{array}$ \\
\hline Dynamic Test & Low & Moderate & None & Good & None/Low \\
\hline
\end{tabular}

Table 1. Advantages and disadvantages of different health assessment techniques.

\section{Electromagnetic sensing techniques}

Electromagnetic sensing techniques use is now rather diffuse in several earth science fields such as geology, hydrogeology, seismology, glaciology, stratigraphy of urban areas study, polluted areas study, landslides characterization, etc. Few years ago, non intrusiveness and quickness of these techniques suggested their use for investigating buildings and civil engineering structures.

The migration of these techniques towards the engineering can be identified with the Microgeophysics where specific issues are the sensor miniaturizing, signal/noise ratio improvement, exploitation of all available free surfaces for energizing and acquiring signals (Cosentino et al., 2011).

Electromagnetic sensing techniques are an useful tool for the diagnostics of civil infrastructures, such as transport ones, in the framework of their static and dynamic behavior before, during and after a crisis event such as an earthquake.

In fact, they ensure a fast and not-intrusive diagnosis useful in the pre-event stage since a precautionary diagnosis of strategic buildings and transport infrastructure can be a critical issue in the seismic risk prevention.

Moreover, they can represent, during the crisis, a valid tool for the rapid damage mapping of the civil buildings and infrastructures (bridges, roads, dams, assessment) in order to have preliminary estimations of those safe for rescue forces. Then, a rapid damage assessment for private buildings enable a correct estimate of the damaged houses and resources to be 
committed. Finally, in the post-event stage, restoration interventions can be driven by the electromagnetic sensing techniques in order to minimize the costs and maximize the results. The electromagnetic sensing techniques provides information about investigated materials in terms of amplitude and phase of the gathered signals, in turn function of the electromagnetic properties of the materials.

Amongst the requirements of the infrastructure diagnostics there is certainly the determination of the structural element thickness, rebar diameters, fractures and defects detection, water content or moisture (as indicators of chemical reactions occurrence), strain. Therefore, the information obtained by the electromagnetic sensing techniques have to be converted in order to provide information directly usable by the engineers.

Not all electromagnetic sensing techniques are suitable in becoming NDT techniques. Sensing technique selection have to keep into account a certain degree of electromagnetic noise immunity, high spatial resolution and a suitable sensor size. Other aspects to be kept into account are:

- $\quad$ proper survey design;

- $\quad$ useful spatial resolution and investigation depth;

- suitable electrical contact, electromagnetic and/or mechanical coupling, precise positioning, boundary problems minimization;

- $\quad$ efficient data processing, including image processing and inversion techniques;

- $\quad$ easy data interpretation;

- possibility of data integration.

Ground Penetrating Radar (GPR) and Electrical Resistivity Tomography (ERT) have these qualities since they are active techniques providing the control on the injected signal, an adjustable spatial resolution and an useful sensor size. Moreover a number of processing codes and inversion routines are available allowing well interpretable images, although personnel with certain degree of experience is required in the data processing and interpretation.

Another class of sensors is the distributed ones, ensuring the availability of measurements along the entire envelop of the sensor. Among these, Fiber Optic Distributed Sensors based on Brillouin scattering phenomenon is a promising experimental technique able to provide field of temperature and strain along the fiber which can be a standard low cost telecommunication fiber. Unlike other fiber optic sensors, this technique permits the remote and spatially continuous monitoring of the structure in terms of temperature and strain with the resolution of some tens of centimetres.

In the following paragraphs we describe those three techniques providing examples of application and highlighting their strengths and limitations.

\subsection{Ground penetrating radar}

Ground Penetrating Radar is an electromagnetic sensing technique based on the same operating principles of classical radars (Daniels, 2004). In fact, it works by emitting an electromagnetic signal (generally modulated pulses or continuous harmonic waves) into the ground or another natural or manmade object; the electromagnetic wave propagates through the opaque medium and when it impinges on a non-homogeneity of the electromagnetic properties, in terms of dielectric permittivity and electrical conductivity, a backscattered electromagnetic field arises. Such a backscattered field is then collected by the receiving antenna located at the air/opaque medium interface and undergoes a subsequent processing and visualization, usually as a 2D image (Figure 2). 

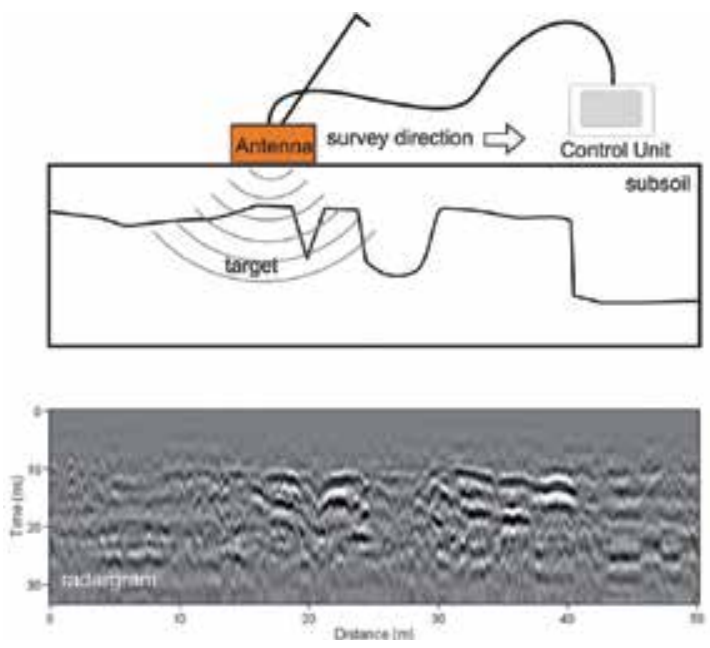

Fig. 2. GPR survey design (upper panel) and radargram (lower panel).

Spatial resolution and investigation depth of GPR method are strictly dependent by the central frequency of the used antenna. In fact, antennas with low and mid frequency $(40 \mathrm{MHz}-750 \mathrm{MHz})$ provided high investigation depth $(10 \mathrm{~m}-3 \mathrm{~m})$ associated to a relative low spatial resolution $(2 \mathrm{~m}-10 \mathrm{~cm})$. On the contrary, high central frequency antennas $(900$ $\mathrm{MHz}-2.5 \mathrm{GHz})$ provide low investigation depths $(1 \mathrm{~m}-10 \mathrm{~cm})$ and high spatial resolution $(5 \mathrm{~cm}-0.5 \mathrm{~cm})$. Since the physical size of the antennas decreases as the frequency increases, the requirement of miniaturizing the sensors is naturally achieved for the GPR.

As a consequence, the GPR technique is useful for the study of bedrock stratigraphy and cavity detection (Lazzari et al. 2006), groundwater and pollution (Chianese et al. 2006), metal and plastic pipelines such as cables in urban areas, archaeological finds (Bavusi et al. 2009) when low central frequency antennas are used.

On the contrary, when high central frequency antennas are used, the GPR, more properly named in this case Surface Penetrating Radar (SPR), can be considered a NDT technique (McCann and Forde 2001) providing precious information about the presence of "embedded" objects such as, reinforced rebars (Shaw et al., 2005; Che et al., 2009), but also embedded "defects" such as voids and, by using special antennas (Huston et al, 2000; Forest and Utsi, 2004; Utsi et al., 2008), fractures. Moreover, the GPR technique can contribute to determine the concrete moisture content (Shaari et al., 2004; Hugenschmidt and Loser, 2008;

GPR survey design is a crucial issue since it determines not only the possibility to detect the target (rebar, defect, water infiltration, ecc.), but also the format output in terms or 2D (cross section, time-slice, depth slice) or 3D data volume, kind of processing and difficult of interpretation.

A proper GPR survey design have to keep into account:

- orientation of searched target;

- possibility of exploit one or more suitable free surfaces;

- desired spatial resolution and investigation depth;

- mispositioning error minimization;

- format of the output;

- processing and interpretation effort minimization. 
When rebars are searched, the most used method of acquisition requires a regular orthogonal survey grid with a proper spacing (a few centimeters) in order to have a suitable spatial resolution. Figure 3 shows a the survey design performed in order to check the continuity of longitudinal and transversal rebars and check the degree of success of the concrete restoration intervention based on epoxy resin injection (Bavusi et al., 2010a).
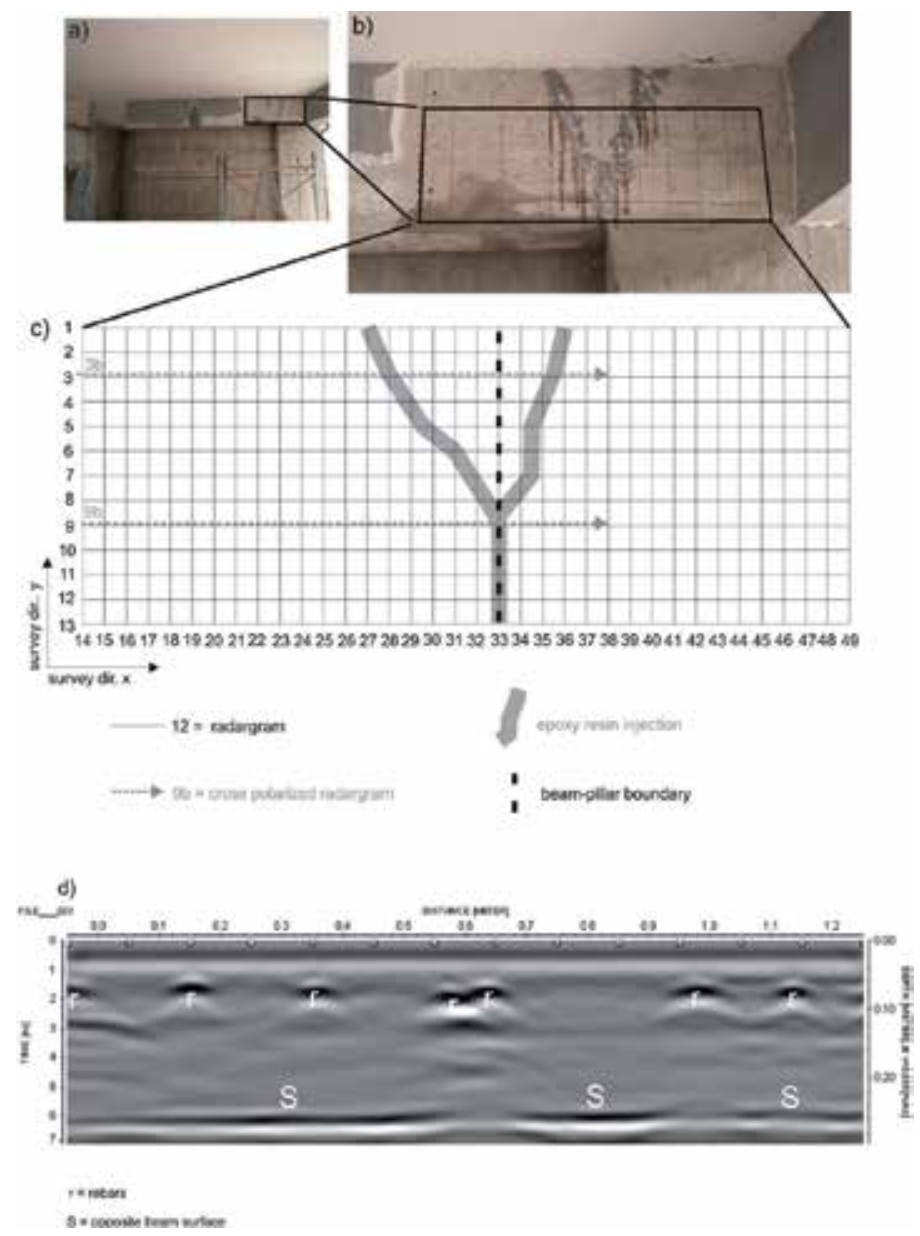

Fig. 3. GPR survey design and results on a beam of a school of L'Aquila damaged by the Abruzzo earthquake of 6th April 2009. a) beam; b) detail of damaged area with gridding; c) regular $4 \mathrm{~cm}$ square grid drawn on the beam; d) longitudinal processed radargram n.3.

A radargram has been gathered along each longitudinal and transversal survey line by using a $1500 \mathrm{MHz}$ antenna provided by survey wheel. This survey design allows to select proper cross-sections and built a data volume. In fact, transversal radargrams offer a view of longitudinal rebars, while longitudinal radargrams are useful for visualizing transversal rebars. Then, the interpolation of all radargrams allows to built a data volume and selects more significant time-slices or depth-slices in order to have a plan view of all rebars (Figure 4). 


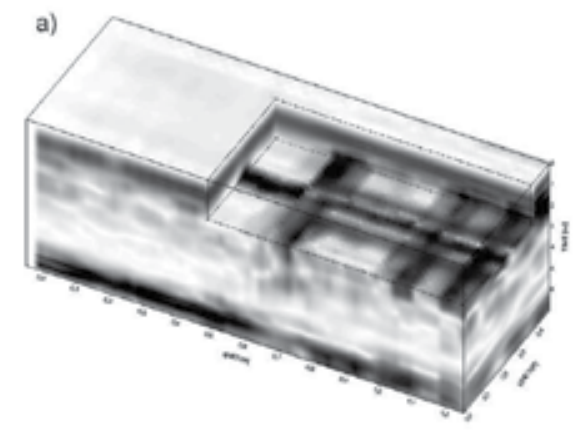

b)

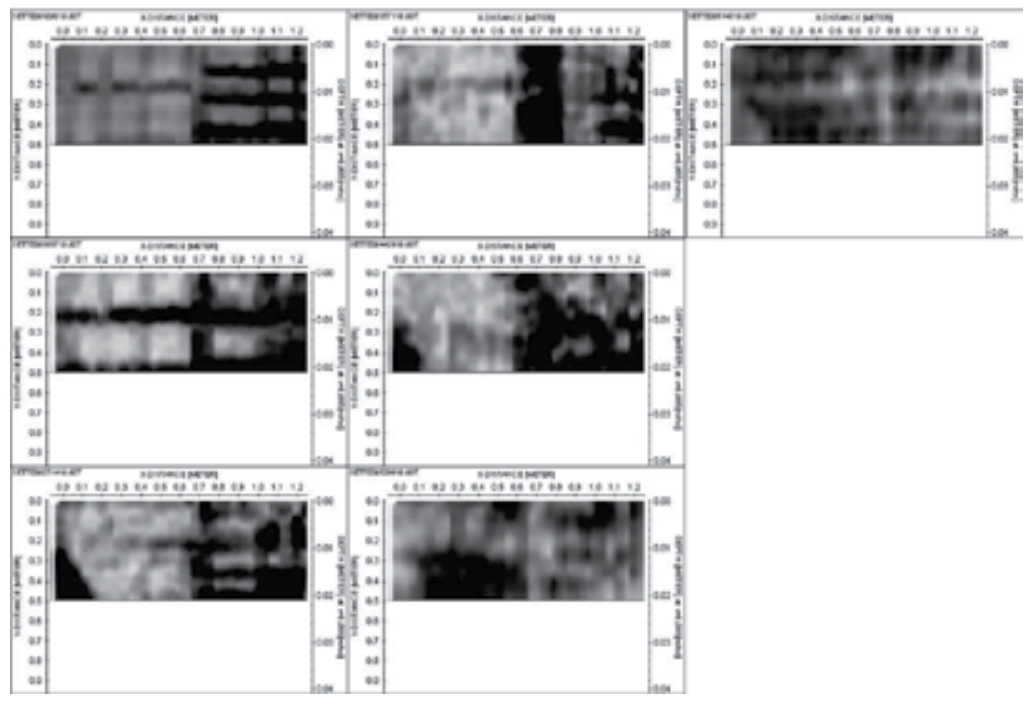

Fig. 4. a) data volume built by interpolating all radargrams gathered along the survey lines of figure $3 \mathrm{c} . \mathrm{b}$ ) slices extracted at several depths.

Then, GPR method provides very impressive and effective images of the inner of a reinforced concrete structure. However, the main limitation is that deeper rebar layer is not well detected due to scattering phenomena and attenuation losses producing in turn a loss in spatial definition in depth. Moreover the upper layer of rebars produces a strong disturbance on the rest of radargram.

In order to overcome this drawback, several strategies can be applied:

1. Repeating the measurements on the opposite surface of the structural element, if possible, in order to focus the other rebar layer;

2. Acquiring in cross-polarization mode: this method enables to focus the attention on the concrete matrix and not on the rebars;

3. Applying more robust inversion routines such as the Microwave Tomography.

First strategy can be effective, but increases the time consuming. The second one exploits the property of cross-polarized radargrams which are less sensitive to the rebars normal to the survey direction and more sensitive to the rebars parallel to the survey direction (Figure 5). 
a)

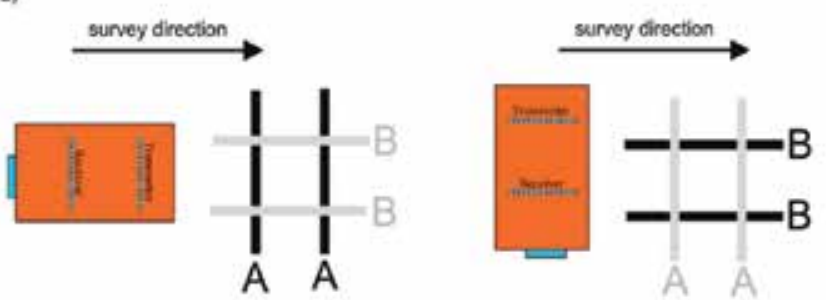

b)

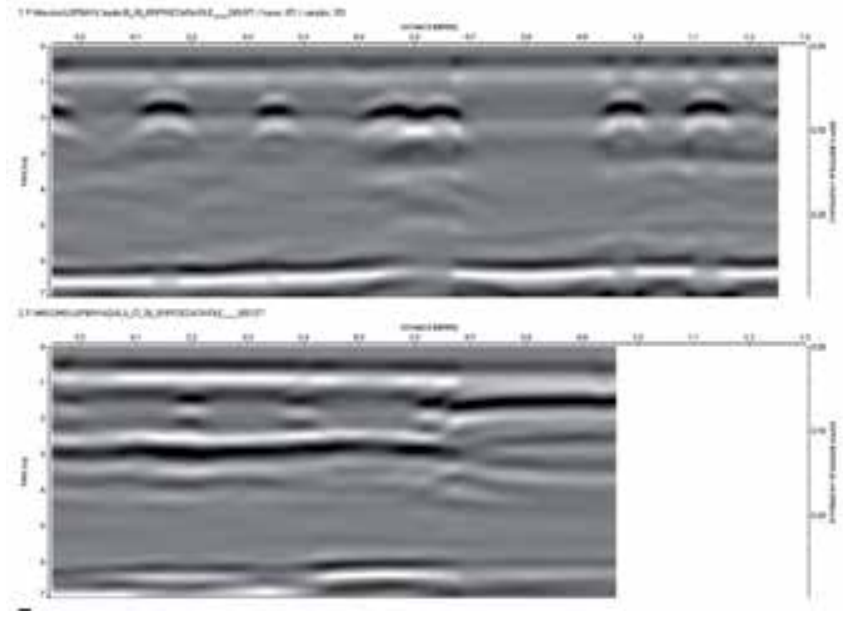

Fig. 5. a) Effect of the antenna polarization on the rebar reflection intensity. b) comparison between the normal-polarized and cross-polarized radargram gathered on the longitudinal survey lines n.9 of figure 2c.

Despite of the above mentioned advantages of GPR, one of the obstacles to its use regards the "low interpretability" of the radargram; therefore a an understandable "interpretation and visualization" of the investigated scene entails a high level operator's expertise and often a-priori information is required. This difficulty of the interpretation is further on affected in the case that no a priori information is available as, for example, it often happens in the case of historical heritage (Masini et al., 2010) where a lack of knowledge about the constructive modalities and materials of the structure can arise.

Therefore, a GPR data processing is often necessary to achieve more easily interpretable and reliable reconstructions of the scene, i.e. images that be easily understandable even by a not expert operator.

The usual radaristic approaches are based on migration procedures that essentially aim at reconstructing buried scattering objects from measurements collected above or just at the air/soil interface. These approaches were first based on graphical methods (Hagendoorn, 1954) based on high frequency assumption of the electromagnetic propagation and scattering; afterward this approach found a more consistent mathematical background based on the wave equation of the electromagnetic scattering (Stolt, 1978).

The absence of reflection in the concrete corresponding to the restored fracture indicates the success of the epoxy injection which filled all possible voids. Finally, a retrofit reinforcing intervention can be designed on the bases of the existent rebar arrangement. 
Recently, new data processing based on the inverse scattering problem have been developed and implemented also in realistic situations for infrastructure monitoring (Catapano et al., 2006; Soldovieri and Orlando, 2009, Bavusi et al., 2011). In particular, the microwave tomography approaches have arose as the most suitable ones for the on field exploitation (Soldovieri and Solimene, 2010; Persico et al., 2005).

Such a class of approaches is based on the modeling of the electromagnetic scattering phenomena. According to this modelization, the imaging problem is cast as an inverse scattering problem where one attempts to infer the electromagnetic properties of the scattering object starting from the scattered field measured somewhere outside it.

The statement of the problem is then the following: given an incident field, $E_{\text {inc }}$, which is the electromagnetic field existing in the whole space (the background medium) in absence of the scattering object and is generated by a transmitting antenna, by the interaction of the incident field with the embedded objects the scattered field $E_{S}$ arises; from the knowledge of the scattered field $E_{S}$ properties about the scattering targets, either geometrical and/or structural, have to be retrieved. The mathematical equations subtending the scattering phenomena to solve the above stated problem are in order.

To this end, we refer to a two-dimensional and scalar geometry. We consider a cylindrical dielectric object (i.e. invariant along the axis out-coming from the sheet) enclosed within the domain $\mathrm{D}$ illuminated by an incident field linearly polarized along the axis of invariance. The scattered field is observed over the domain $\Sigma$ (not necessarily rectilinear). Moreover, we denote by $\varepsilon(\underline{r})$ e by $\varepsilon_{\mathrm{b}}(\underline{\mathrm{r}})$ the permittivity profile of the unknown object and of the background medium, respectively. In particular, the latter is not necessarily constant (i.e., a non-homogeneous background medium is allowed too) but has to be known. The magnetic permeability is assumed equal to that of the free space $\mu_{0}$ everywhere. The geometry of the problem is detailed in Figure 6.

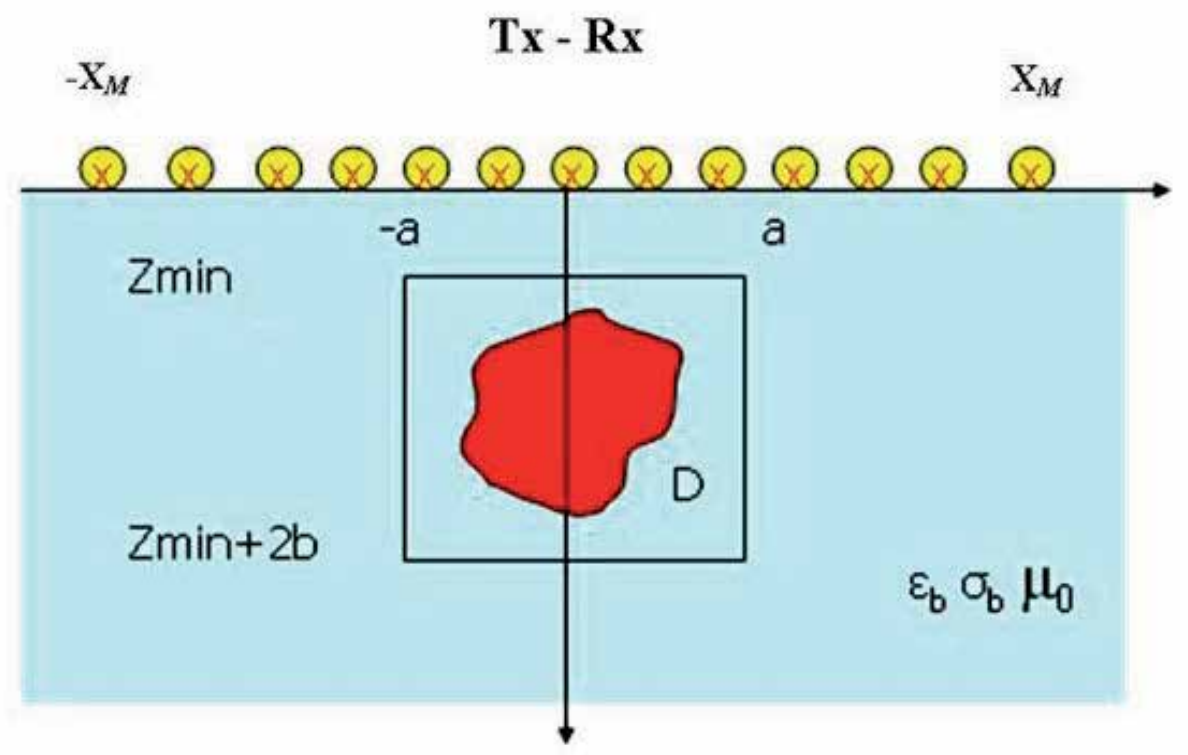

Fig. 6. Geometry of the subsurface prospecting problem 
The problem, thus, amounts to retrieving the dielectric permittivity profile $\varepsilon(\underline{\mathrm{r}})$ of the unknown object(s) from the knowledge of the scattered field $E_{S}$. The physical phenomenon is governed by the two equations (Chew, 1995)

$$
\begin{array}{ll}
\mathrm{E}\left(\underline{\mathrm{r}}, \underline{\mathrm{r}}_{\mathrm{S}} ; \mathrm{k}_{\mathrm{b}}\right)=\mathrm{E}_{\mathrm{inc}}\left(\underline{\mathrm{r}}^{\prime} \underline{\mathrm{r}}_{\mathrm{S}} ; \mathrm{k}_{\mathrm{b}}\right)+\mathrm{k}_{\mathrm{b}}^{2} \int_{\mathrm{D}} \mathrm{G}\left(\underline{\mathrm{r}}^{\prime} \underline{\mathrm{r}}^{\prime} ; \mathrm{k}_{\mathrm{b}}\right) \mathrm{E}\left(\underline{\mathrm{r}}^{\prime}, \underline{\mathrm{r}}_{\mathrm{S}} ; \mathrm{k}_{\mathrm{b}}\right) \chi\left(\underline{\mathrm{r}}^{\prime}\right) \mathrm{d} \underline{\mathrm{r}}^{\prime} & \underline{\mathrm{r}} \in \mathrm{D} \\
\mathrm{E}_{\mathrm{S}}\left(\underline{\mathrm{r}}_{\mathrm{O}}, \underline{\mathrm{r}}_{\mathrm{S}} ; \mathrm{k}_{\mathrm{b}}\right)=\mathrm{k}_{\mathrm{b}}^{2} \int_{\mathrm{D}} \mathrm{G}\left(\underline{\mathrm{r}}_{\mathrm{O}}, \underline{\mathrm{r}} ; \mathrm{k}_{\mathrm{b}}\right) \mathrm{E}\left(\underline{r}^{\prime}, \underline{\mathrm{r}}_{\mathrm{S}} ; \mathrm{k}_{\mathrm{b}}\right) \chi(\underline{\mathrm{r}}) \mathrm{d} \underline{\mathrm{r}} & \underline{\mathrm{r}}_{\mathrm{O}} \in \Sigma
\end{array}
$$

where $\mathrm{E}=\mathrm{E}_{\mathrm{inc}}+\mathrm{E}_{\mathrm{S}}$ is the total field, $\mathrm{k}_{\mathrm{b}}$ is the subsurface (background) wave-number and $\chi(\underline{\mathrm{r}})=\varepsilon(\underline{\mathrm{r}}) / \varepsilon_{\mathrm{b}}-1$ is the dimensionless contrast function. $\mathrm{G}(\bullet, \bullet)$ is the pertinent Green's function (Leone and Soldovieri, 2003), $\underline{r}_{O}$ is the observation point and $\underline{r}_{S}$ is the position of the source.

In accordance to the volumetric equivalence theorem (Harrington, 1961), the above integral formulation permits to interpret the scattered field as being radiated by secondary sources (the "polarization currents") which are just located within the space occupied by the targets. The reconstruction problem thus consists of inverting the "system of equations (1)" versus the contrast function. However, since (from the first of the equations 1) the field inside the buried targets depends on the unknown contrast function, the relationship between the contrast function and the scattered field is nonlinear. However, the problem can be cast as a linear one if the first line equation is arrested at the first term of its Neumann expansion. After doing this $\mathrm{E} \cong \mathrm{E}_{\text {inc }}$ is assumed within the targets and the so-called Born linear model is obtained (Chew, 1995). Accordingly, the scattering model becomes

$$
\mathrm{E}_{\mathrm{S}}\left(\underline{\mathrm{r}}_{\mathrm{O}}, \underline{\mathrm{r}}_{\mathrm{S}} ; \mathrm{k}_{\mathrm{b}}\right)=\mathrm{k}_{\mathrm{b}}^{2} \int_{\mathrm{D}}^{\mathrm{G}}\left(\underline{\mathrm{r}}_{\mathrm{O}}, \underline{\mathrm{r}} ; \mathrm{k}_{\mathrm{b}}\right) \mathrm{E}_{\mathrm{inc}}\left(\underline{\mathrm{r}}^{\prime} \underline{\mathrm{r}}_{\mathrm{S}} ; \mathrm{k}_{\mathrm{b}}\right) \chi(\underline{\mathrm{r}}) \mathrm{dr} \underline{\mathrm{r}} \quad \underline{\mathrm{r}}_{\mathrm{O}} \in \Sigma
$$

Let us just remark that, within the linear approximation, the internal field does not depend on the dielectric profile, which is the same as to say that mutual interactions between different parts of any object or between different objects are neglected. In other words, this means to consider each part of the object as an elementary scatterer that does not depend on the presence of the other scatterers.

Consequently, at this point the problem can be cast as the inversion of the linear integral equation (2) and the numerical implementation of the solution algorithm requires the discretization of eq. (2). This task is pursued by resorting to the method of moments (MoM) (Harrington, 1961).

One of the main feature of a GPR data is its ability to provide images of the inner structure of a building or infrastructure at all useful observation scales by exploiting antennas with several central frequencies. Concerning this, the bridge inspection, which is normally carried out by using classical DT and NDT methods, can derive benefit from the GPR technique (Scott et al., 2003; Hugenschmidt and Mastrangelo, 2006). Structural particulars of interest are inner rebars, tendons, boundary conditions, anchors, saddles and other internal elements. On the other hand, the observation can involve the entire deck of a bridge. Such observations became crucial when all original project documentations are partially or completely lost.

Figure 7 shows the survey design drown for the deck survey of the deck of Musmeci bridge in Potenza (Basilicata Region, Southern Italy) (Bavusi et al., 2011). 

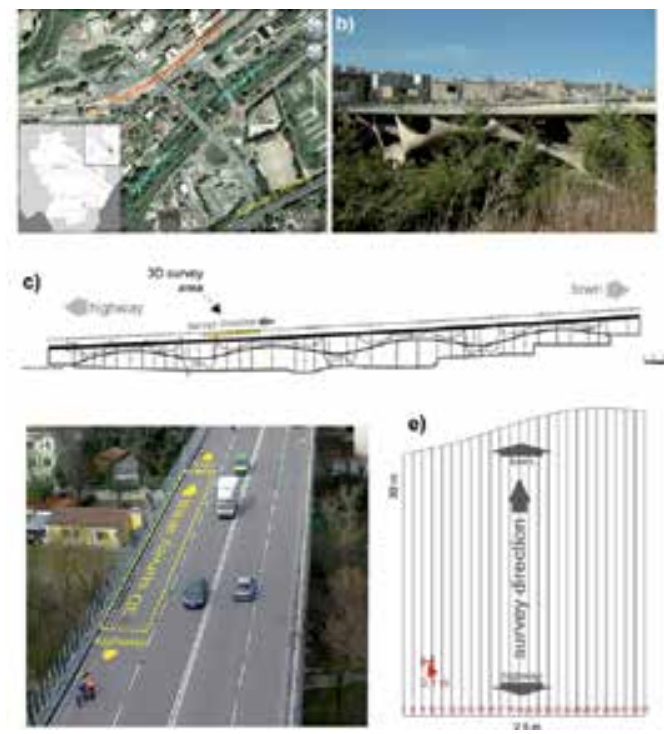

Fig. 7. a) location of the Musmeci bridge in Potenza, Basilicata Region, Italy. b) location of the survey area respect to the bridge; d) surveyed lane; e) survey grid. From Bavusi M., Soldovieri F, Di Napoli R., Loperte A., Di Cesare A., Ponzo F.C. and Lapenna V (2010).Ground Penetrating Radar and Microwave Tomography 3D applications for the deck evaluation of the Musmeci bridge (Potenza, Italy). J. Geophys. Eng. 8 (2011) 1-14. Courtesy of IOP Publishing Ltd.

A such survey design is able to provide several depth-slices in order to observe the asphalt layer, the sects layer and the lower reinforced concrete plate of the deck. Figure 8 shows more significant depth-slices showing the deck structure at several depths.
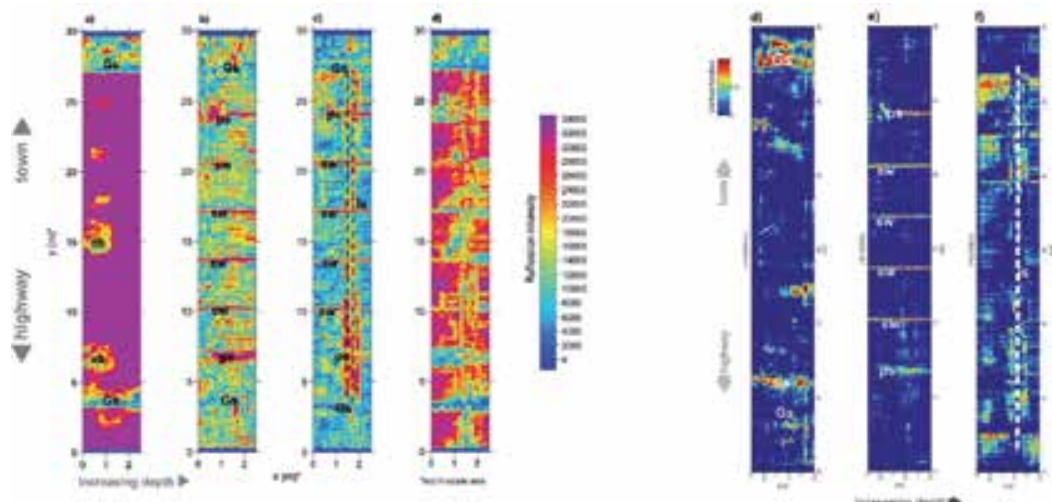

Fig. 8. a-d) depth-slices built at $6 \mathrm{~cm}$ (a), $20 \mathrm{~cm}$ (b), $40 \mathrm{~cm}$ (c) and $50 \mathrm{~cm}$ (d) by interpolating all radargrams gathered on the survey lines of figure $5 \mathrm{e}$; $\mathrm{d}$-f) depth-slices built at $6 \mathrm{~cm}(\mathrm{~d}), 20$ $\mathrm{cm}(\mathrm{e})$ and $40 \mathrm{~cm}(\mathrm{f})$, by interpolating the same radargrams inverted by means of the Microwave Tomography. ab: absorption zone, Gs: Gerber saddle, ps: pillar support, sw: stiffening wall, ls: longitudinal stiffening wall. From Bavusi M., Soldovieri F, Di Napoli R., Loperte A., Di Cesare A., Ponzo F.C. and Lapenna V (2010).Ground Penetrating Radar and Microwave Tomography 3D applications for the deck evaluation of the Musmeci bridge (Potenza, Italy). J. Geophys. Eng. 8 (2011) 1-14. Courtesy of IOP Publishing Ltd. 
In particular the asphalt layer shows concentrated absorptive zones that can be related to water infiltration zones. In this case the precise positioning of traces is a critical issue since it can produce a staggering effect when rectilinear features are detected. The use of a survey wheel is mandatory such as a certain degree of care in the cart dragging in order to limit mispositioning errors. In this way a residual error can be subsequently reduced by using proper algorithms.

Among possible defects afflicting buildings and infrastructures, fractures are a very warring problem. In fact, fractures can be due to several causes: temperature $(3 \mathrm{~mm})$, dry up $(0.4 \mathrm{~mm})$, load $(0.4 \mathrm{~mm})$. They can involve loss of mechanical strength and represent a preferential way for the water infiltration which in turn can favour the developin $g$ of chemical reactions (expanding salt crystallization, oxidation, carbonation, etc.).

Crack detection is an important issue in the field of non-destructive testing. Several techniques can be employed in order to check, localize and characterize fractures in manmade buildings: ultrasonic shear waves (De La Haza et al. 2008), elastic waves (Ohtsu et al. 2008), GPR (Utsi et al. 2008).

Due to their small size and variable orientation, fractures detection represents a very challenge for the GPR technique. In fact, the crack detection requires the exploitation of all spatial resolution available in the frequency range used. Moreover it requires fracture to be surveyed is filled by air, water or a material different from the host medium in order to produce a backscattered field (Grandjean and Gourry 1996). In addition, the geometry of the fracture with respect to the survey line plays a fundamental role (Tsoflias et al. 2004). For a vertically oriented fracture, a reflection hyperbola arises due to the bottom of the fracture and to each change in the direction of the fracture with respect to the vertical path (Forest and Utsi 2004). By exploiting this property, it is theoretically possible to detect a fracture by using a common GPR dipole antenna, even the use of specifically designed high vertical resolution antennas is very helpful (Forest and Utsi 2004; Utsi et al. 2008). Moreover, data processing plays a fundamental role to improve the 'imaging' and the focusing of the buried reflectors (Grandjean and Gourry 1996; Leucci et al. 2007).

Figure 9 shows a $1500 \mathrm{MHz}$ GPR survey carried out on a fracture in the floor of the Prefecture of Chania (Crete Island, Greece) (Bavusi et al., 2010b).

Fracture zone, located at the middle point of the radargram, is detected by using a classical processing approach, but best performances in terms of spatial resolution can be obtained by using the Microwave Tomography.

\subsection{Electrical resistivity tomography}

Electrical resistivity tomography (ERT) is an electromagnetic sensing technique used to obtain 2D and 3D images in terms of electrical resistivity of areas of complex geology (Griffiths and Baker 1993), landslides, watertable, basins, faults.

Technically, during an electrical resistivity measurement, the electric current is injected into the ground via two $30-40 \mathrm{~cm} \times 1.5 \mathrm{~cm}$ steel electrodes and the resulting electrical voltage is measured between two other electrodes in line with current electrodes (Sharma, 1997). ERT can be carried out by using different electrode configurations such as dipole-dipole, Wenner, Schulumberger, pole-dipole, etc. (Figure 10).

At present, such configurations can be carried out by using multi-electrode systems enabling the automatic switch of all available electrodes previously fixed into the ground. The system manages the current injection and simultaneous potential measurements which can occur simultaneously at more potential electrodes in case of multichannel systems. 

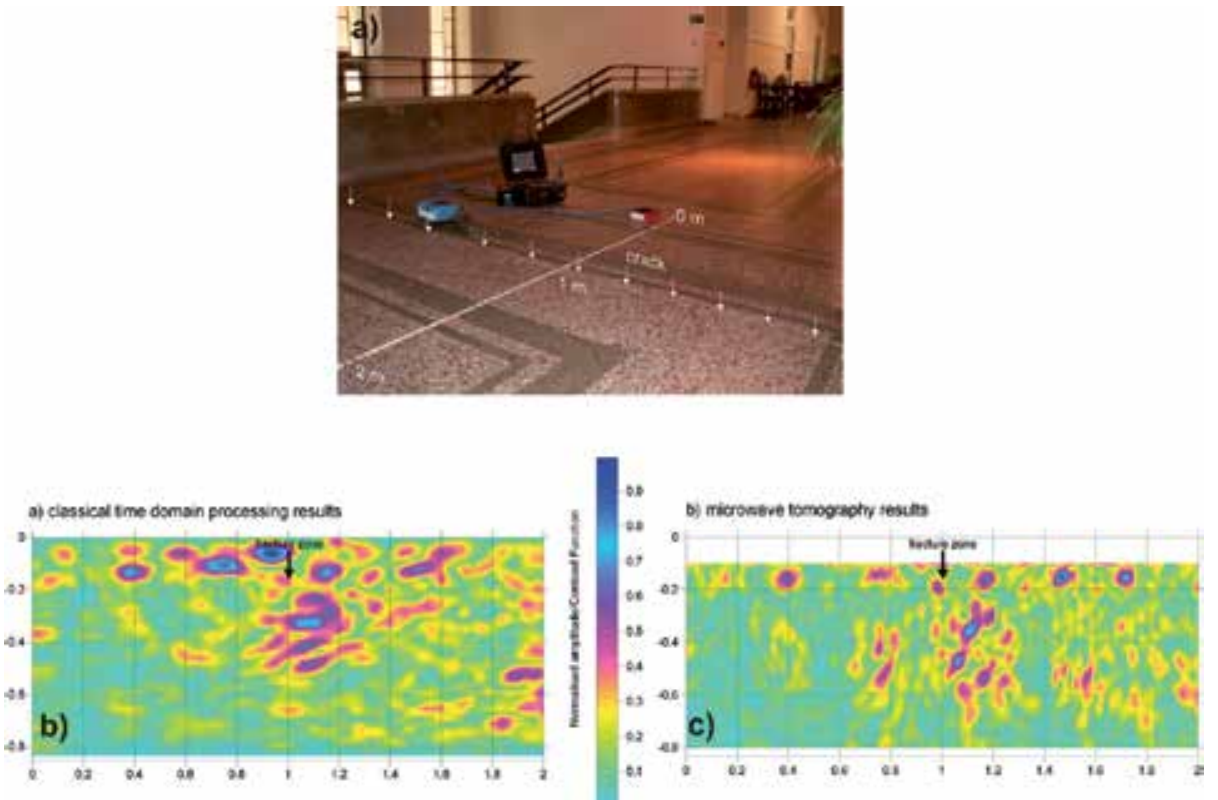

Fig. 9. a) GPR survey design carried out at the Prefecture of Chania (Crete, Greece) on a fracture in the floor. Fracture zone is at the middle point of the radargrams. b) processed radargram; c) Microwave Tomography From Bavusi M., Soldovieri F., Piscitelli S., Loperte A., Vallianatos F. and Soupios P. (2010). Ground-penetrating radar and microwave tomography to evaluate the crack and joint geometry in historical buildings: some examples from Chania, Crete, Greece. Near Surface Geophysics, Vol.8, No. 5, pp. 377-387. Courtesy of EAGE Publications BV..

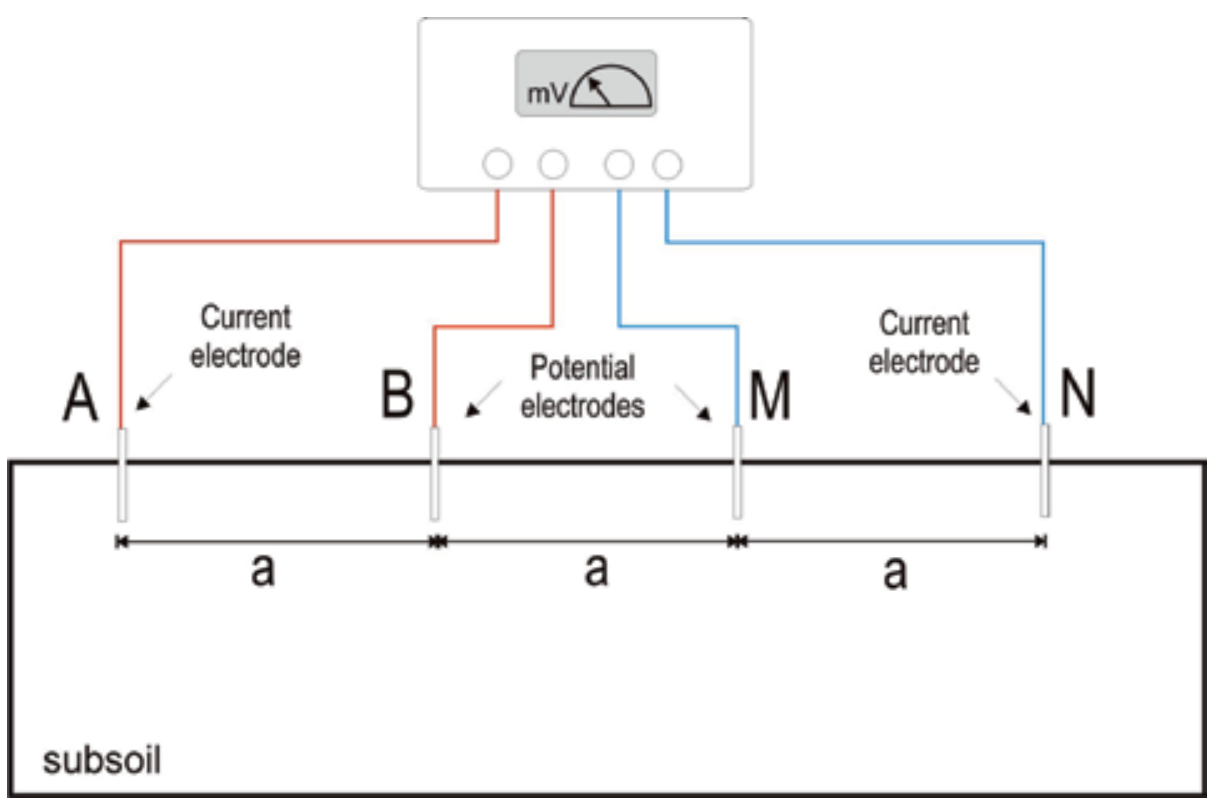

Fig. 10. a) Dipole-dipole array for the acquisition of a measure of electrical resistivity. 
The result of an ERT survey is a distribution 2D or 3D of apparent resistivity where each data point is defined by two coordinates $(x$ and $z$ ) depending on the position of the quadrupole (couple of current and potential dipoles) used and a value of apparent resistivity. Then, in order to reconstruct real resistivity distribution, an inversion routine is required. A number of algorithms are available in order to perform this reconstruction such as Res2DInv (Loke and Barker, 1996) for the automatic 2D inversion of apparent resistivity data was used. The inversion routine is based on the smoothness constrained least-squares inversion (Sasaki, 1992) implemented by a quasi-Newton optimization technique.

ERT surveys have been successfully applied in geology for stratigraphy and cavity detection (Lazzari et al., 2010), fault characterization (Caputo et al., 2007), landslide studies (Lapenna et al., 2005), in hydrogeology, in environmental problems for contaminant plume detection and waste dump characterization (Bavusi et al., 2006), for hydrogeology and coastal salt water intrusion detection (Satriani et al., 2011a), in agricultural for the root-zone characterization (Al Hagrey, 2007; Satriani et al, 2011b), in archaeology and cultural heritage studies (Bavusi et al., 2009). Figure 11 shows an example of ERT carried out on a piling in an area subjected to landslides. It is well visible the effect of the structure on the water distribution.

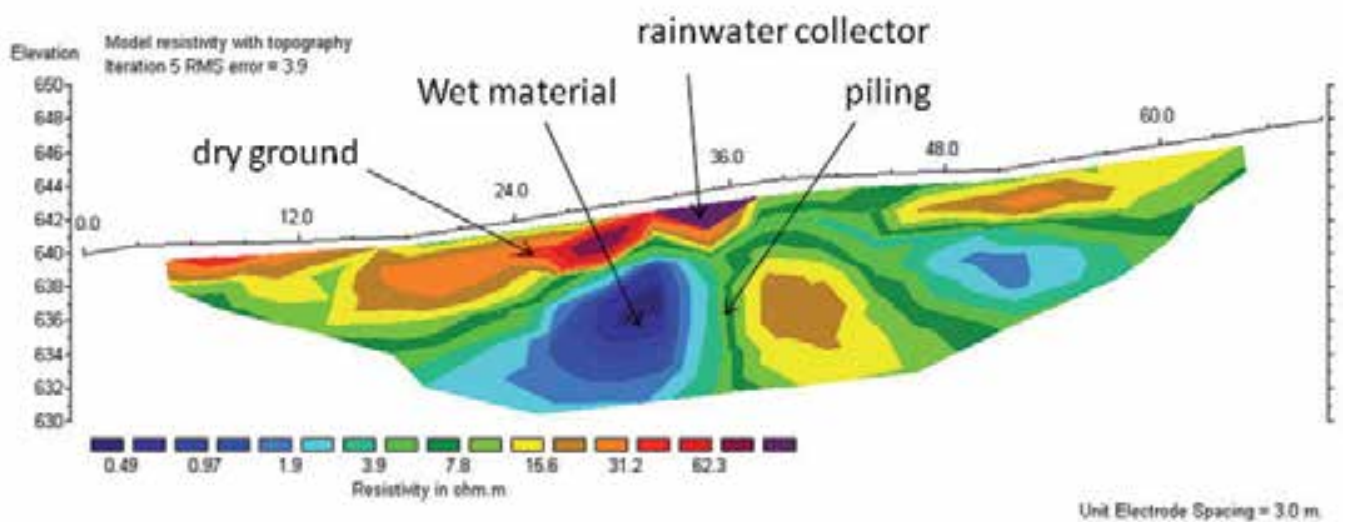

Fig. 11. Example of ERT carried out on a piling in an area subjected to landslides.

The ERT exhibits significant potentialities in terms of high resolution and flexibility of the investigation depth that can be varied in a simple way, by varying the electrode spacing. A large electrode spacing provides a high investigation dept and a low spatial resolution. On the contrary, a small electrode spacing allows to achieve a great spatial resolution but a low investigation depth.

This characteristic makes the ERT a candidate for the structure and infrastructure monitoring, even though some problems have to be enfaced. First, a structure or infrastructure survey requires an electrode spacing ranging between one centimeter and some decimeter, then sensors have to miniaturized in order to respect the assumption of geophysics stating transducers have to be punctual (i.e. small respect to dimension of the investigated volume) and reduce modeling errors (Cosentino et al. 2011; Athanasiou et al., 2007). Then, a low contact resistance have to be ensured in order to put an adequate current injection (Cosentino et al. 2011). 
In order to meet these requirements, several devices can be used such as $\mathrm{Cu}$ flat-base electrodes with conductive gel (Athanasiou et al., 2007), $\mathrm{Ag} / \mathrm{AgCl}$ medical electrodes and nails (Cosentino et al 2011), Cu-CuSO electrodes (Seppänen et al., 2009). Main limitation of these devices is the difficulty of put them on a vertical or steeply slope surface, even worse under a ceiling. Moreover, medical electrodes are not stable in time (Cosentino et al 2011), while flat-base electrodes are not suitable for the asphalt, where the only possibility to apply the ERT is making holes in order to put the electrode in the substratum.

In spite of these limitations, the ERT has been successfully applied on masonry, floors, artifacts in order to detect fractures, voids, previous restoration works, structural particulars, moisture. The application to reinforced concrete is possible in order to detect those targets and rebars, but experiments demonstrates target detection capacity in simple geometrical configurations (Seppänen et al., 2009; Karhunen et al., 2009).

In presence of complex rebar configurations such as reinforcement cages, the potential field produced by the injected current suffers a warping due to the circuit represented by the cage not easily modelable. A new class of inversion routines is then required in order to solve this problem. This technique appears still not adequate to be applied to structures and infrastructures, but technological development can provide technical solutions able to mitigate and overcame described limitations.

\subsection{Distributed fiber optic sensors}

Typically standard NDT systems are based on the use of point sensors, however the Structural Health Monitoring (SHM) of large civil infrastructures like bridges, dams could require a large number of sensors. For these applications, there is an increasing interest towards the use of distributed optical fiber sensors. In these sensors is the optical fiber itself that acts as a sensor providing measurements all along the fiber. This approach permits to monitoring the whole structure by use of a single optical fiber avoiding the need of a huge amount of measurement points and lead to the comprehension of the real static behavior of the structure rather than a limited number of sensors. Furthermore, distributed sensors could play a fundamental role in civil engineering because no other tools allow the detection of local phenomena whose location is impossible to be predicted "a priori" like, for instance, for crack detection. Distributed fiber optic sensors are substantially different from other fiber optic sensors technologies being based on optical scattering mechanism (Raleigh, Raman, Brillouin) occurring during light propagation in common telecom optical fibres.

Spatial resolution is typically achieved by using the optical time domain reflectometry (OTDR) (Barnoski 1976), in which optical pulses are launched into an optical fiber and consequent variations in backscattering intensity is detected as a function of time. Alternative detection techniques such as frequency-domain approaches have been also demonstrated. Raleigh scattering based sensors were first developed, in order to locate fiber breaks or bad splices along a fiber link. However Rayleigh backscatter in standard fibers gives information only about optical attenuation, and it can not be related to other parameters such as temperature or strain. Distributed temperature sensing was first demonstrated by Hartog and Payne (1982), who used temperature-induced variation of the Rayleigh scattering coefficient along the length of liquid-core, but the low reliability of liquid-core fibers may restrict their use. A recent, very interesting approach makes use of the very high spatial resolution allowed by sweptwavelength interferometry, in order to correlate temperature and strain of the fiber with the spectrum of the Rayleigh backscatter spatial fluctuations (Measures 2001). This approach 
requires standard telecommunication fibers and very high spatial resolution (a few millimeters) has been demonstrated. On the other hand, the main disadvantages are the equipment cost (a tunable laser source is needed for the measurements), and the limited sensing length $(70 \mathrm{~m})$. Dakin et al. (1985) demonstrated temperature profiles measurement using the variations in the Raman backscattering coefficients of anti-Stokes and Stokes light. The Raman approaches are very practical because conventional silica-based optical fibers can be used as the sensor. The anti-Stokes-Raman-backscattered light is about $30 \mathrm{~dB}$ weaker than the Rayleigh-backscattered light. However, its sensitivity to temperature is great. Therefore, systems based on Raman scattering have been commercialized by several manufacturers. Nevertheless, Raman scattering based systems do not allow performing deformation measurements. In 1989, it was reported that the frequency shift of the Stokes-Brillouinbackscattered light (the so-called Brillouin frequency shift) greatly varies with strain and temperature along the fiber (Horiguchi 1989, Culverhouse 1989). Since then, considerable attention has been paid to exploiting Brillouin scattering for distributed sensing. This is for the following reasons. First, strain is a very important parameter in the monitoring of the integrity of civil structures. Secondly, unlike the Raman technique, Brillouin frequency shift measurement does not require calibration of the optical-fiber loss. Furthermore, a very attractive feature of Brillouin-based sensors stems from the use of a standard telecommunications-grade optical fiber as the sensor head. The low-cost and low-loss nature of the sensor make possible to perform distributed measurements over distances of many kilometers. Finally, the tremendous developments in the optical telecommunications market have reduced considerably the cost and increased the performances of optical fibers and their associated optical components.

Distributed optical fiber sensors based on stimulated Brillouin scattering (SBS) rely on the interaction between two lightwaves and an acoustic wave in the optical fiber. The measurement principle is based on the characteristic that the Brillouin frequency of the optical fiber is shifted when strain as well as temperature changes occur. Spatial information along the length of the fiber can be obtained through Brillouin optical time domain analysis (BOTDA) by measuring propagation times for light pulses travelling in the fiber. This allows for continuous distributions of the parameter to be monitored.

The research in the beginning of distributed fiber optic strain sensing was mostly based on laboratory applications (Bernini 2005, Bernini 2008) only in last years in-field demonstration by a fully distributed sensor have been previously reported (Komatsu 2002).

About bridge structures recently the applications and the validation of distributed strain sensor during load test has been demonstrated (Matta 2008, Minardo 2011). Other examples are the installation of a distributed fiber optic strain sensing cable into the inspection gallery of a dam (Inaudi and Glisic 2005, Glisic and Inaudi, 2007) or the monitoring of extra-long tunnel, running over $150 \mathrm{~km}$ of seafloor geologic body with complicated topographic and geologic units (Shi, 2003).

However, the use of distributed fiber optic sensors for crack detection in concrete are rare. This is mainly due to the fact that the instrumentations available for in-field application have a limited spatial resolution (1m) (Deif 2010). In fact, distributed sensors measure the with average strain at each measurement point, where the strain is averaged over the length called spatial resolution. Today, new methods for distributed fiber optic strain sensing with sub-meter spatial resolution are being developed in order to increase the opportunities in NDT of civil structures especially for crack detection (Hotate 2002, Zou 2005, Bernini 2007). 
As an example, we report the results obtained in a load test on a road-bridge (Minardo 2011). In particular, the tests were performed by an stimulate Brilouin Scattering portable sensor prototype with $3 \mathrm{~m}$-spatial resolution. The fiber employed for the measurements was a PVC-coated, single-mode, standard telecom optical fiber. The fiber was bonded along the lower flange of a 44-m-long, double-T steel beam, by use of a epoxy adhesive. Strain measurements were performed while loading the bridge with an increasing weight by use of gravel-loaded trucks (Figure 12a). During the loading test, data were also collected by other instruments for a cross-correlation. In particular, two vibrating wire (VW) strain gauges were previously spot-welded to the surface of the steel beam, so as to provide the strain at the quarter and the middle section of the loaded beam. Figure 10b depicts the results of the optical fiber measurements, for different load conditions. In particular, the solid lines refer to the bridge loaded by two, four, and five gravel trucks, respectively. Each truck had a weight of approximately 47 tons. The same figure also reports the results of a finite-element-method (FEM)-based numerical analysis (circles). Numerical data were obtained by modelling each gravel truck as three concentrated loads applied in correspondence of the three truck axles. A good agreement exists between the experimental and numerical data. The standard deviation was always less than $20 \mu \varepsilon$, corresponding to the nominal accuracy of the instrument. Moreover, the maximum strain provided by the optical fiber sensor $(\approx 350 \mu \varepsilon)$, is in good agreement with the value provided by the strain gauge placed at the middle beam section $(\approx 330 \mu \varepsilon)$. Another interesting feature is that the optical fiber sensor was able to reveal the right-shift of the center of gravity (CG), when loading the beam with five trucks. Actually, the fifth truck was not disposed symmetrically with respect to the middle of the bridge; rather it was closer to the right side. As a consequence, the section at which maximum strain occurs is shifted to the right in the final load test. Finally, in figure $12 \mathrm{~b}$ are also reported, for comparison, the data obtained by the strain gauges (squares). As can be observed a good agreement between the two different observations is achieved.
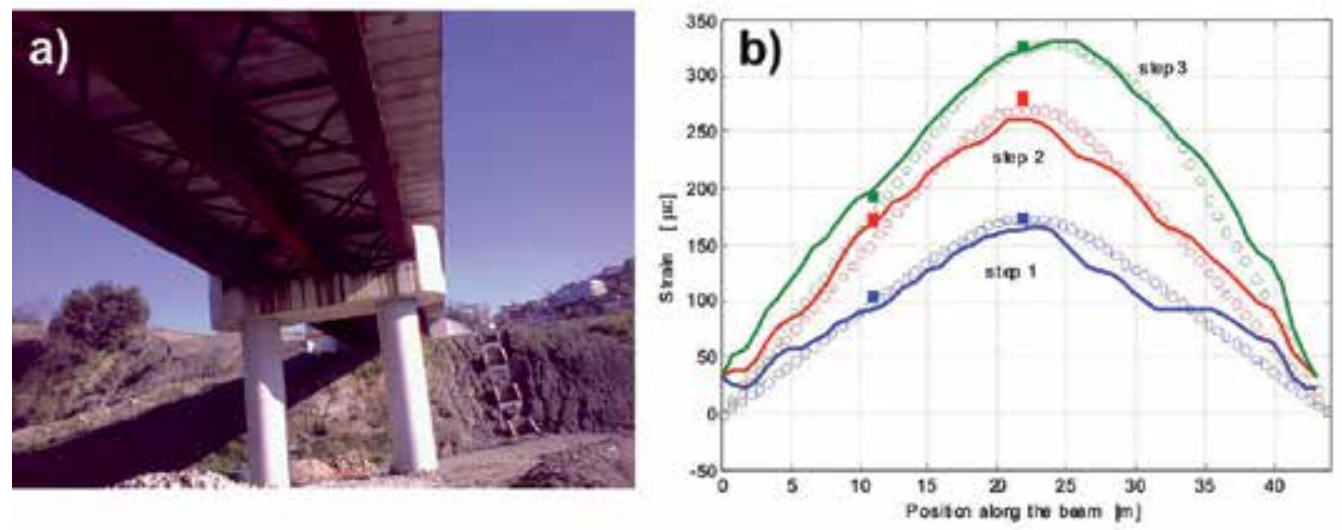

Fig. 12. a) bridge used for the load test. b) Distributed strain measurement along the girder, for different load conditions (solid lines). FEM simulations (open circles). VW strain gauges (squares). 


\section{Conclusion}

Health monitoring of civil engineering structures is devoted to assess the structural integrity and dynamic behaviour during a seismic event in order to define appropriate activities for risk mitigation. This need is more stringent for aged infrastructures built following outdated codes of practice and where the chemical degradation of concrete and irons acted for more time.

The design of suitable retrofit strategies requires a series of test assessments in order to define the vulnerability. A number of destructive (DT) and non destructive tests (NTD) and methods has been developed and applied around the world in order to achieve all necessary information about construction material. An appropriate survey have to include a suitable proportion of DT and NDT methods in order to reduce possible damage to investigated structure, achieve a balanced combination of punctual and distributed information and reduce the global cost of the survey.

Then, the requirement of new non invasive technique is a stringent need that can be satisfied by the electromagnetic sensing techniques, this class of geophysical techniques which can be easily adapted to the specific requirements of civil infrastructures. This is the case of the Ground Penetrating Radar which at high frequencies provides the needed spatial resolution and a convenient small size of the antenna. Moreover, this technique can benefit on a new kind of processing based on the Microwave Tomography (MT) inversion. In this way the technique can focus small defects such as fractures and voids, detect rebars with great precision even if deep. The combination of GPR and MT will provide in future a new class of devices able to supply a not focused image ready for use by non expert users.

The Electrical Resistivity Tomography can be another suitable technique useful to depict embedded structural particulars and defects, but its systematic application requires to solve two problems. First is the design of appropriate non intrusive electrodes, stable in time and easy to install in any position. A solution can be provided by medical industries that have experience in the design of electrodes for human body applications. The latter is the lack of inversion routines able to model the effect of a rebar cage on the potential field and properly reconstruct the inner of a reinforced concrete structure. Anyway the ERT is successfully applied on floors, pavements, masonry and reinforced concrete structures having a simple inner arrangement. Moreover, the industry will provide in future new sensors, equipments and inversion routines able to mitigate or solve described problems.

Another class of sensors which will change the way of monitor a civil infrastructure is the distributed fiber optic sensor able to provide temperature and strain information along the fiber. This sensor is based on standard telecom fiber optic which is inexpensive and allows to design sensor sized on the infrastructure to be surveyed or on a particular. It allows to achieve in real time information by several points of a distributed civil infrastructure such as a railway, aqueduct, gas or oil pipeline without using transmission devices. In future optic fibers will be embedded in construction materials allowing a for life monitoring of an infrastructure. Moreover, a possible technological improvement of this technique can allow in future its application to the ambient vibration monitoring (Wenzel and Pichler, 2005). 


\section{Acknowledgment}

The research leading to these results concerning the Musmeci bridge has received funding from the European Community's Seventh Framework Programme (FP7/2007-2013) under Grant Agreement n 225663 Joint Call FP7-ICT-SEC-2007-1.

Moreover, the authors would like to thank the "Soprintendenza per i Beni Architettonici e Paesaggistici della Basilicata" and the "Direzione Regionale per i beni Culturali e Paesaggistici della Basilicata" that have partially funded the activities. Finally, the authors would like to thank the owner of the Musmeci Bridge, ASI Consortium, for their interest to these research activities and the Municipality of Potenza for the granted authorization to access and work on the structure. Furthermore, the authors would like to thank EAGE Publications BV and IOP Publishing Ltd for copyright permissions granted.

Finally, the authors would like to thank TeRN Consortium for supporting this work.

\section{References}

A.C.I. 214.4R (2003). Guide for Obtaining Cores and Interpreting Compressive Strength Results.

Al Hagrey, S.A., (2007). Geophysical imaging of root-zone, trunk, and moisture heterogeneity, J. Exp. Bot., Vol. 58, pp. 839-854.

Ansari F.(2005). Sensing issues in civil structural health monitoring. Spinger.

Athanasiou E.N. , Tsourlos P.I., Vargemezis G.N., Papazachos C.B. and

Barlet, F.M. \& MacGregor, J. (1994). Effect of Core Diameter on Concrete Core Strengths. ACI Materials Journal, Vol. 91, No. 5, September-October.

Barnoski J. K., Jensen S. M., (1976). Fiber waveguides: A novel technique for investigation attenuation characteristics. Appl. Opt., Vol. 15, pp. 2112 - 2115.

Bavusi M., Rizzo E., Lapenna V. (2006). Electromagnetic methods to characterize the Savoia di Lucania waste dump (Southern Italy). Environmental Geology, Vol. 51, No. 2, pp. 301-308. DOI 10.1007/s00254-006-0327-9

Bavusi M., Rizzo E., Giocoli A., Lapenna V., (2009). Geophysical characterisation of Carlo's V Castle (Crotone, Italy). Journal of Applied Geophysics, Vol. 67, No. 4, ,pp. 386-401.

Bavusi, M., Loperte, A.; Lapenna, V., Soldovieri, F., (2010a). Rebars and defects detection by a GPR survey at a L'Aquila school damaged by the earthquake of April 2009., 2010 13th International Conference on Ground Penetrating Radar (GPR), 21-25 June 2010.

Bavusi M., Soldovieri F., Piscitelli S., Loperte A., Vallianatos F.and Soupios P. (2010b). Ground-penetrating radar and microwave tomography to evaluate the crack and joint geometry in historical buildings: some examples from Chania, Crete, Greece. Near Surface Geophysics, Vol.8, No. 5, pp. 377-387.

Bavusi M., Soldovieri F., Di Napoli R., Loperte A., Di Cesare A., Ponzo F. C. and Lapenna V. (2011). Ground Penetrating Radar and Microwave Tomography 3D applications for the deck evaluation of the Musmeci bridge (Potenza, Italy). Journal of Goph. and Eng, No. 8, pp. 1-14. 
Bernini R., Fraldi M., Minardo A., Minutolo V., Carannante F., Nunziante L, Zeni L. (2005). Damage detection in bending beams through Brillouin distributed optic-fibre sensor. Bridge Struct. Vol. 1, 355-363.

Bernini R., Minardo A., Zeni L., (2007). Accurate high-resolution fiber-optic distributed strain measurements for structural health monitoring. Sensors and Actuators A, Vol. 134, pp. 389-395.

Bernini R., Minardo A., Zeni L. (2008). Vectorial dislocation monitoring of pipelines by use of Brillouin-based fiber-optics sensors. Smart Mater. Struct., Vol. 17, pp. 015006,

Braga, F., Dolce, M., Masi, A., Nigro, D. (1992). Valutazione delle caratteristiche meccaniche dei calcestruzzi di bassa resistenza mediante prove non distruttive. L'Industria Italiana del Cemento, Vol. 3, pp. 201-208.

Caputo R., Salviulo L., Piscitelli S., Loperte A., (2007). Late Quaternary activity along the Scorciabuoi Fault (Southern Italy) as inferred from electrical resistivity tomographies. Annals of Geophysics, Vol. 50, No., 2, pp.213-224.

Catapano I., Crocco L., Persico R., Pieraccini M., Soldovieri F. (2006). Linear and Nonlinear Microwave Tomography Approaches for Subsurface Prospecting: Validation on Real Data. Antennas and Wireless Propagation Letters, Vol. 5, pp. 49-53.

Che W. C., Chen H. L. and Hung S. L. (2009). Measurement radius of reinforcing steel bar in concrete using digital image GPR. Constr. Build. Mater., Vol. 23, pp. 10571063.

Chew C. W.(1995). Waves and Fields in inhomogeneous media. Piscataway,NJ: IEEE Press.

Chianese D., D’Emilio M., Bavusi M., Lapenna V., Macchiato M. (2006). Magnetic and ground probing radar measurements for soil pollution mappingin the industrial area of Val Basento (Basilicata Region, Southern Italy): a case study. Environ Geol , Vol. 49, pp. 389-404.

Cosentino P.L., , Capizzi P., Martorana R., Messina P. and Schiavone S. (2011). From Geophysics to Microgeophysics for Engineering and Cultural Heritage. International journal of Geophysics, Vol. 2011, pp. 1-8. DOI: 10.1155/2011/428412.

Culverhouse D., Fahari F., Pannell C. N., Jackson D. A.(1989). Potential of stimulated Brillouin scattering as sensing mechanism for distributed temperature sensors. Electron. Lett., Vol. 25, No. 14, pp. 913-915.

Dakin J. P., Pratt D.J, Bibby G. W., Ross J. N., (1985), Distributed optical fiber Raman temperature sensor using a semiconductor light source and detector, Electron. Lett., Vol. 21, No. 13, pp. 569-570.

Daniels D.J. (2006). Ground Penetrating Radar. 2nd edition. Institution of Engineering and Technology, pp.634. DOI: 978-0-86341-360-5; electronic DOI: 978-1-59124-893-4

De La Haza A.O., Petersen C. G. and Samokrutov A. (2008). Three dimesional imaging of concrete structures using ultrasonic shear waves. Structural Faults $\mathcal{E}$ Repair-2008, 12th International Conference, Edinburgh, UK: 10th -12th June 2008.

Deif A., Martın-Perez B., Cousin B., Zhang C., Bao X., Li W. (2010). Detection of cracks in a reinforced concrete beam using distributed Brillouin fibre sensors. Smart Mater. Struct., Vol. 19, 055014. 
Ditommaso R., Parolai S., Mucciarelli M., Eggert S., Sobiesiak M. and Zschau J. (2010a). Monitoring the response and the back-radiated energy of a building subjected to ambient vibration and impulsive action: the Falkenhof Tower (Potsdam, Germany). Bulletin of Earthquake Engineering. Vol..8, No. 3. DOI: 10.1007/s10518009-9151-4.

Ditommaso R., Mucciarelli M., Ponzo F. C. (2010b). S-Transform based filter applied to the analysis of non-linear dynamic behaviour of soil and buildings. 14th European Conference on Earthquake Engineering. Ohrid, Republic of Macedonia, August 30 September 03.

Ditommaso R, Mucciarelli M., Ponzo F. C. (2011). Analysis of non-stationary structural systems by using a band-variable filter. Submitted to Bulletin of Earthquake Engineering.

Douglas A. (2007). Health Monitoring of Structural Materials and Components: Methods with Applications. Copyright (C) 2007 John Wiley \& Sons, Ltd.

Forest R. and Utsi V. (2004). Non destructive crack depth measurements with ground penetrating radar. 10th Int. Conf. on Ground Penetrating Radar,Delft, The Netherlands, 21-24 June 2004.

Glisic B. and Inaudi D. (2007). Fibre Optic Methods for Structural Health Monitoring. John Wiley \& Sons. ISBN: 0470061421.

Grandjean C. and Gourry J.C. (1996). GPR data processing for 3D fracture mapping in a marble quarry (Thassos, Greece). Journal of Applied Geophysics, Vol. 36, pp.19-30.

Griffiths, D.H. and R.D. Baker (1993): Two-dimensional resistivity imaging and modelling in areas of complex geology. J. Appl. Geophys., Vol. 29, pp. 211-226.

Hagedoorn, J. G. (1954). A Process of Seismic Reflection Interpretation. Geophys. Prospect. Vol. 2, pp 85-127.

Harrington, R.F. (1961). Time-Harmonic Electromagnetic Fields, Mc Graw Hill.

Hartog A. H., and D. N. Payne (1982). Remote measurement of temperature distribution using an optical fiber. Proc. ECOC '82, Cannes, France, pp. 215-220, 1982.

Horiguchi T, Kurashima T., Tateda M., (1989). Tensile strain dependence of Brillouin frequency shift in silica optical fibers. IEEE Photonics Tech. Lett., Vol. 1, No. 5, pp. 107-108.

Hotate K., Tanaka M., (2002). Distributed fiber Brillouin strain sensing with 1-cm spatial resolution by correlation-based continuous-wave technique. IEEE Phot. Technol. Let. Vol. 14, 179-181.

Hugenschmidt J. and Mastrangelo R. (2006). GPR inspection of concrete bridges Cem. Concr. Compos., Vol. 28, pp. 384-92.

Hugenschmidt J. and Loser R. (2008). Detection of chlorides and moisture in concrete structures with ground penetrating radar. Mater. Struct. Vol. 41, pp. 785-92

Huston D., Hu J. Q., Maser. K., Weedon W. and Adam C. (2000). GIMAground penetrating radar system for monitoring concrete bridge decks. J. Appl. Geophys., Vol. 43, pp. $139-46$.

Inaudi D., Glisic B., (2005). Application of distributed Fiber Optic Sensory for SHM", 2nd International Conference on Structural Health Monitoring of Intelligent Infrastructure (SHMII-2'2005), Shenzhen, China, November 16-18. 
Karhunen K., Seppänen A., Lehikoinen A.\&. Kaipio J.P,(2009). Locating reinforcing bars in concrete with Electrical Resistance Tomography. Concrete Repair, Rehabilitation and Retrofitting II - Alexander et al (eds). (C) 2009 Taylor \& Francis Group, London, ISBN 978-0-415-46850-3

Komatsu, K., Fujihashi, K. \& Okutsu, M., (2002). Application of optical sensing technology to the civil engineering field with optical fiber strain measurement device (BOTDR). Proc. SPIE, Vol. 4920: 352-361.

Lapenna V., Lorenzo P., Perrone A., Piscitelli P., Rizzo E. Sdao F., (2005). 2D electrical resistivity imaging of some complex landslides in the Lucanian Apennine chain, southern Italy. Geophysics, Vol. 70, No. 3; pp. B11-B18. DOI: $10.1190 / 1.1926571$

Lazzari M., Loperte A., and Perrone A. (2006). Near surface geophysics techniques and geomorphological approach to reconstruct the hazard cave map in historical and urban areas. Adv. Geosci., Vol. 24, pp. 35-44.

Leucci G., Persico R. and Soldovieri F. (2007) Detection of fractures from GPR data: the case history of the Cathedral of Otranto. J. Geophys. Eng., Vol. 4, pp. 452-461.

Lazzari M., Loperte A., and Perrone A. (2010). Near surface geophysics techniques and geomorphological approach. Adv. Geosci., Vol. 24, pp. 35-44.

to reconstruct the hazard cave map in historical and urban areas

Leone G. and Soldovieri F. (2003). Analysis of the distorted Born approximation for subsurface reconstruction: truncation and uncertainties effect", IEEE Trans. Geoscience and Remote Sensing, vol. 41, no. 1, pp. 66-74, Jan.

Loke, M.H and Baker R.D., (1996). Rapid least-squares inversion of apparent resistivity pseudosections by quasi-Newton method. Geophys. Prospect., Vol. 44, pp.131-152.

Masini L, Persico R., Rizzo E, Calia A, Giannotta m. T., Quarta G., Pagliuca A. (2010). Integrated Techniques for Analysis and Monitoring of Historical Monuments: the case of S.Giovanni al Sepolcro in Brindisi (Southern Italy). Accepted for publication on Near Surface Geophysics.

McCann D. M., Forde M. C. (2001). Review of NDT methods in the assessment of concrete and masonry structures. NDTEE International, Vol. 34, pp. 71-84

Matta F., Bastianini F., Galati N., Casadei P., Nanni A. (2008). Distributed Strain Measurement in Steel Bridge with Fiber Optic Sensors: Validation through Diagnostic Load Test. ASCE Journal of Performance of Constructed Facilities, Vol. 22, pp. 264-273.

Measures R. M., Structural monitoring with fiber optic technology. Academic Press, 2001

Minardo A., Bernini R., Amato L., Zeni L., (2011). Bridge monitoring using Brillouin fiberoptic sensors. IEEE Sensors Journal, Vol. 99, pp.

Moustafa A., Mahadevan S., Daigle M., Biswas G. (2010). Structural and sensor damage identification using the bond graph approach. Structural Control and Health Monitoring, 17: 178-197.

Ohtsu M., Tokay M., Ohno K. and Isoda T., (2008). Elastic-wave methods for crack detection and damage evaluation in concrete. Structural Faults $\mathcal{E}$ Repair-2008, 12th International Conference, Edinburgh, UK: 10th -12th June 2008. 
R. Persico, R. Bernini, Soldovieri F. (2005). The role of the measurement configuration in inverse scattering from buried objects under the Born approximation. IEEE Trans. Antennas and Propagation, Vol. 53, No.6, pp. 1875-1887.

Picozzi M., S. Parolai, M. Mucciarelli, C. Milkereit, D. Bindi, R. Ditommaso, M. Vona, M.R. Gallipoli, and J. Zschau. (2011). Interferometric Analysis of Strong Ground Motion for Structural Health Monitoring: The Example of the L'Aquila, Italy, Seismic Sequence of 2009. Bulletin of the Seismological Society of America, Vol. 101, No. 2, pp. 635-651, April 2011, DOI: 10.1785/0120100070.

Ponzo, F., Ditommaso, R., Auletta, G., Mossucca, A. (2010). A fast method for a structural health monitoring of Italian reinforced concrete strategic buildings. Bulletin of Earthquake Engineering. DOI: 10.1007/s10518-010-9194-6. Vol. 8, No. 6, pp. 14211434.

Ponzo F. C., Mossucca A., Di Cesare A., Nigro D., Dolce M., Moroni C. (2011). Seismic assessment of the R/C buildings: the case study of Di.Coma.C Centre after the L'Aquila (Italy) 2009 seismic sequence. 9th Pacific Conference on Earthquake Engineering. Building an Earthquake-Resilient Society. 14-16 April, 2011, Auckland, New Zealand.

RILEM Recommendation NDT 1, Testing of Concrete by the Ultrasonic Pulse Method, Paris, December 1972.

Sasaky, Y. (1992). Resolution of resistivity tomography inferred from numerical simulation, Geophys. Prospect., Vol. 54, pp. 453-464.

A. Satriani, A. Loperte, M. Proto, and M. Bavusi (2011a). Building damage caused by tree roots: laboratory experiments of GPR and ERT survey. Adv. Geosci., Vol. 24, pp. 133-137.

Satriani A., A. Loperte, M. Proto (2011b). Electrical resistivity tomography for coastal salt water intrusion characterization along the Ionian coast of Basilicata Region (Southern Italy). Fifteenth International Water Technology Conference, IWTC-15 2011, Alexandria, Egypt, 28-30 May.

Scott M,. Rezaizadeha A., Delahazab A., Santosc C. G., Moored M., Graybeale B. and Washerf G. (2003). A comparison of nondestructive evaluation methods for bridge deck assessment. NDTEE Int., Vol., 36, pp. 245-55.

Seppänen A., Karhunen K., Lehikoinen A. \& Kaipio J.P., (2009). Electrical resistance tomography imaging of concrete. Concrete Repair, Rehabilitation and Retrofitting II pp. 571-577. Alexander et al (eds). (c) 2009 Taylor \& Francis Group, London, ISBN 978-0-415-46850-3

Shaari A., Millard S. G. and Bungeyb J. H. (2004). Modelling the propagation of a radar signal through concrete as a low-pass filter. NDTEE Int., Vol. 37, pp. 237-42.

Sharma, P.S. (1997). Enviromental and Engineering Geophysics. Cambridge University Press.

Shi B., Xu H., Chen B., Zhang D., Ding,Y., Cui H., Gao J.,(2003). Feasibility study on the application of fiber-optic distributed sensors for strain measurementin the Taiwan Strait Tunnel project. Marine Georesources and Geotechnology, Vol. 21, pp. 333-343. 
Shaw M. R., Millard S. G. Molyneauxc T. C. K., Taylord M. J., Bungeyb J. H. (2005). Location of steel reinforcement in concrete using ground penetrating radar and neural networks. NDTEE International, Vol. 38, pp. 203-212.

Soldovieri F. and Orlando L.(2009). Novel tomographic based approach and processing strategies for multi-frequency antennas GPR measurements using multi-frequency antennas. Journal of Cultural Heritage, Vol. 10, pp. e83-e92.

Soldovieri F., Solimene R., (2010). Ground Penetrating Radar Subsurface Imaging of Buried Objects, in Radar Technology, IN-TECH, Vienna Austria, ISBN 978-953-307-029-2, Edited by: Guy Kouemou, January 2010.

Stolt, R. H. (1978) Migration by Fourier Transform. Geophysics, Vol. 43 pp. 23-48.

Tsoflias G.P., Van Gestelz J.P., Blankenship D.D. and Sen M., (2004). Vertical fracture detection by ex-ploiting the polarization properties of ground-penetrating radar signals. Geophysics, Vol. 69, pp. 803-810.

UNI EN 12504-1 (2002) Prove sul calcestruzzo nelle strutture - Carote - Prelievo, esame e prova di compressione

UNI EN 12504-2 (2001) Prove sul calcestruzzo nelle strutture - Prove non distruttive Determinazione dell'indice sclerometrico

UNI EN 12504-3 (2005) Prove sul calcestruzzo nelle strutture - Parte 3: Determinazione della forza di estrazione

UNI EN 12504-4 (2005) Prove sul calcestruzzo nelle strutture - Parte 4: Determinazione della velocità di propagazione degli impulsi ultrasonici

Utsi V., Birtwisle A. and Coock J. (2008). Detection of subsurface reflective cracking using GPR. Structural Faults \& Repair-2008: 12th Int. Conf., Edinburgh, UK, 10-12 June 2008.

Wenzel H. and Pichler D. (2005). Ambient Vibration Monitoring. Wilhey.

Zou L., Bao X., Wan Y., Chen L., (2005). Coherent probe-pump-based Brillouin sensor for centimetre-crack detection. Opt. Let., Vol. 15, pp. 370-372. 


\title{
Steel Building Assessment in Post-Earthquake Fire Environments with Fiber Optical Sensors
}

\author{
Genda Chen, Ying Huang and Hai Xiao \\ Missouri University of Science and Technology \\ Untied States of America
}

\section{Introduction}

This chapter is aimed to develop and calibrate a quasi-distributed optical sensor network of long period fiber gratings and extrinsic Fabry-Perot interferometers for simultaneous high temperature and large strain measurements, and to validate it for the post-earthquake assessment of a steel frame in high temperature environments. The steel frame represents typical steel buildings that are susceptible to the high temperature effect of earthquakeinduced fires such as those observed at multiple locations during the March 11, 2011, Japan earthquake.

Critical buildings such as hospitals and police stations must remain functional for postearthquake responses and evacuation immediately following a major earthquake event. However, they often experience large strains due to shaking effects as observed during recent earthquakes, causing permanent inelastic deformation. The post-earthquake fires associated with the earthquake-induced short fuse of electrical systems and leakage of gas devices can further strain the already damaged structures during the earthquakes, potentially leading to a progressive collapse of buildings. In a matter of seconds to an hour, tenants can be injured and trapped in the collapsed buildings, desperately waiting for rescue in helpless situations. Therefore, real time monitoring and assessment of the structural condition of critical buildings is of paramount importance to post-earthquake responses and evacuation in earthquake-prone regions. An accurate assessment of the buildings in these harsh conditions can assist fire fighters in their rescue efforts and save earthquake victims.

For structural condition assessment in fire environments, the most widely used, commercial sensing tools are based on electrical principles, including electrical resistance gauges for strain measurements and thermocouples for temperature measurements. Electrical resistance gauges were initially proposed in 1856 by Lord Kelvin (Thomson 1857). A strain gauge is basically a conductive metal foil that is printed on a non-conductive insulating flexible backing. It can be applied to the surface of a solid structure with suitable adhesives, such as cyanoacrylate. When perfectly attached, the foil and then the backing are deformed together with the structure to be monitored, causing a change in electrical resistance of the alloy. Due to electromechanical properties of the alloy, the foil and the adhesives, the maximum strain that a strain gauge can measure prior to its failure is typically limited to $1 \%$. The readings from a strain gauge also vary with the temperature during measurements. They become significant in high temperature applications and must be compensated for temperature effects. 
For strain measurements in high temperature environments, significant improvements on the temperature properties of strain gauges were made by Easerling (1963) and Gregory et al. (2007). Commercial products introduced by Vichy Micro-Measurement can be applied to measure a strain of $5,000 \mu \varepsilon$ at $1,000^{\circ} \mathrm{F}$. However, wired strain gauges would likely lose their signals due to power outage during and immediately following a strong earthquake when structures being monitored are subjected to large strains and post-earthquake fire environments. For the same reason, conductive textiles that are operated with their electromechanical properties through a special design of "sensing string" will likely be malfunctional even though the sensors can be used for large strain measurements (Zhang 2006).

For temperature measurements, thermocouples were initially proposed in 1822 by Fourier and Oersted, and based on the thermoelectricity principle discovered by Thomas in 1821 (ASTM 1981). Since then, thermocouples have been widely used for high temperature measurements. Today, a variety of thermocouples are commercially available for various temperature ranges, including Type $\mathrm{K}, \mathrm{E}, \mathrm{J}, \mathrm{N}, \mathrm{B}, \mathrm{R}, \mathrm{S}, \mathrm{T}, \mathrm{C}$, and $\mathrm{M}$. A thermocouple is a device consisting of two different conductors (usually metal alloys) that produce a voltage proportional to a temperature difference between either ends of the pair of conductors. The change in voltage corresponds to the temperature to be measured. Like strain gauges, the measured voltage change and then the converted temperature may not be able to be transferred to a central data acquisition in post-earthquake fire environments.

The above review clearly indicates that a conventional monitoring system with strain gauges and thermocouples is inadequate for structural condition assessment in harsh environments, such as extremely high temperature in the event of a post-earthquake fire. On the other hand, an optical fiber monitoring system can provide a viable means for this application. In addition to its high temperature tolerance, optical fibers are compact, immune to electromagnetic interference, durable in acid environment, and capable of being integrated into various types of structures and materials. Once developed for large strain measurement capabilities, optical fiber sensors can be multiplexed and integrated into a quasi-distributed, multi-parameter sensing network in harsh environments.

Various fiber optical sensors have been developed mainly based on the intensity changes, gratings, and interferometers with the last two most widely investigated. Grating based optical sensors are led by the fiber Bragg grating (FBG) and the long period fiber grating (LPFG) technologies. A FBG sensor couples two light beams in their respective forwardand backward-propagating core-guided modes near a resonant wavelength, functioning like a wavelength-selective mirror (Othonos 1999). A LPFG sensor with a periodic refractive index perturbation of its fiber core has a period of the hundreds of micrometer and couples the core mode (guided light inside the core of the fiber) into the cladding modes at certain discrete wavelengths (known as resonance wavelengths) (Vengsarkar et al. 1996). For strain measurements at high temperatures, FBG sensors were applied by Wnuk et al. (2005) and Mateus et al. (2007); LPFG sensors were investigated by Huang et al. (2010a). In addition, extrinsic Fabry-Perot interferometer (EFPI) sensors were developed both for a small range of strain measurement (Xiao et al. 1997) and for a large range of strain measurement (Huang et al. 2010b, 2011). However, fiber optical sensors for large strain measurements at high temperature are yet to be studied. Furthermore, their applications for structural behaviour monitoring in post-earthquake fire environments have not been investigated. 
In this chapter, an EFPI-based sensor is first introduced and characterized for large strain measurements with high resolution. In particular, the operational principle, signal processing algorithm, and experimental validation of the EFPI sensor are investigated in detail. The EFPI sensor developed for large strain measurements is then combined with a LPFG-based temperature sensor for simultaneous large strain and high temperature measurements. A straightforward signal decomposition technique is introduced for the evaluation of temperature and strain from the readings of an EFPI-LPFG sensor. Lastly, the developed fiber optical sensors are multiplexed in an optical fiber sensing network and validated in their application to a steel frame under a simulated post-earthquake fire environment for structural behaviour monitoring and assessment.

\section{An EFPI based large strain sensor with high resolution}

An EFPI sensor can be made by first inserting two cleaved optical fibers into a capillary tube and then bonding them to the tube with epoxy or in thermo fusion. This way of packaging improves the sensor's robustness in applications, but limits the sensor's dynamic range to the corresponding maximum deformation of the capillary tube. On the other hand, if the two cleaved ends are left unattached to the tube, the packaged device is essentially a displacement sensor. By dividing the measured displacement by the initial distance between the two cleaved ends, the device can be implemented as a large strain sensor. For example, if one or two ends of the fibers are adhered to a substructure to be monitored, the packaged device will experience an applied strain that can be determined from the measured displacement by the EFPI as the substructure deforms under an external load. In this case, the technological challenge remains in achieving high resolution during a large strain measurement. The concept of movable EFPI sensors was developed by Habel et al. (1996) and applied for strain measurements during the first few hours of hydration reaction in concrete (Habel et al. 1996, 1997, 2008).

Up to date, most applications of movable EFPI sensors deal with strain measurements in small range. The resolution of the sensors for large strain measurement has not been analysed systematically. Over the past twenty years, several data processing methods have been investigated to analyse data series from EFPI sensors in structural health monitoring applications (Liu et al. 2001 and Qi et al. 2003). Due to a small range of strain measurement, the so-called phase tracking method with relatively high resolution has been widely applied. Qi et al. (2003) developed a hybrid data processing method by combining several methods using a white light interferometer. Although only tested for a small dynamic range in strain measurements, the data processing technique appears to be a promising concept that can be extended to achieve an increasing dynamic range and resolution with EFPI sensors.

\subsection{Operational principle}

\subsubsection{Sensor structure and measurement system}

Fig. 1 shows the schematic of an EFPI sensor head and its associated measurement system. As illustrated by the enlarged view of the sensor head in Fig. 1(a), the EFPI is formed by two perpendicularly cleaved end faces of a single-mode optical fiber (Corning SMF-28). The left side of the fiber serves as a lead-in fiber and the right side serves as a low reflective mirror. 


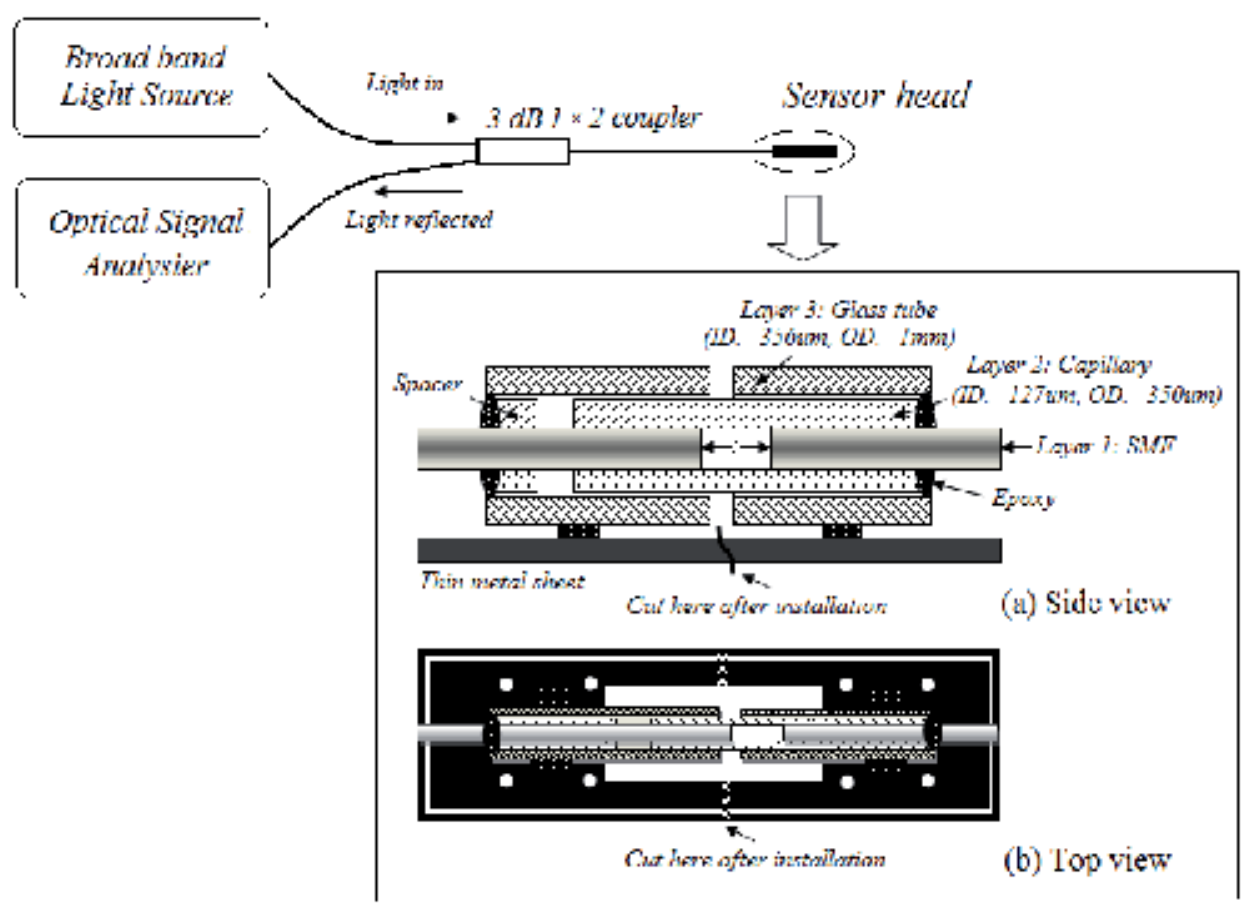

Fig. 1. Schematic of an EFPI sensor: sensor structure and measurement system

In theory, a Fabry-Perot cavity with a freely movable end face can be constructed by inserting two cleaved fiber ends into the two ends of a glass tube and gluing one side of the bare fiber to the tube. However, the freely movable bare fiber end, when not glued to the tube, is easy to break in applications since optical fibers are susceptible to any shear action. To address this issue, a three layer structure is used to package an EFPI strain sensor, including an inner, intermediate and outer layer. The inner layer or Layer 1 in Fig. 1(a) is an optical glass fiber of $125 \mu \mathrm{m}$ in diameter. The intermediate layer or Layer 2 in Fig. 1(a) is a capillary glass tube with an inside diameter of $127 \mu \mathrm{m}$ and an outside diameter of $350 \mu \mathrm{m}$. The capillary tube is designed to guide the cleaved fiber to ensure that its two end faces can move in parallel. The outer layer or Layer 3 in Fig. 1(a) is a glass tube with an inside diameter of $356 \mu \mathrm{m}$ and an outside diameter of 1,000 $\mu \mathrm{m}$, which is designed to enhance the overall stability of the packaged sensor. On one side (right) of the interferometer, all three layers are bonded together with epoxy as illustrated in Fig. 1(a). On the other side (left), the fiber is bonded to the third layer through an inserted spacer while the intermediate layer is unbounded to allow for free movement of the fiber end faces within the capillary tube.

The three-layer structure transfers a shear force from the bare fiber to the intermediate layer (spacer) during operation. As its diameter increases, the intermediate layer is less susceptible to the applied shear force so that the proposed structure can operate stably without breakage. The two pieces of the outer glass tube are bonded to a thin metal sheet both at the lead-in side and mirror side of the fiber sensor. The metal sheet has two perforated side strips and a pre-cut rectangular hole in the middle section. It can be bolted to a steel substructure in applications as illustrated in Fig. 1(b). The sensor installation is completed by cutting the two side strips across the perforation of the thin metal sheet. The 
separation of the attachment sheet ensures that the sensor actually measures the elongation of the steel substructure between the two sides of the cut section as clearly illustrated in the top view of Fig. 1(b). The distance between the two bolts closest to the cut section is defined as the gauge length of the sensor, which is $L=2 \mathrm{~mm}$ in this study. The EFPI cavity length is designated as $l$. Based on the proposed sensor structure, a sensor prototype was fabricated as shown in Fig. 2(a) and the micro-view of its sensor head can be seen in Fig. 2(b).

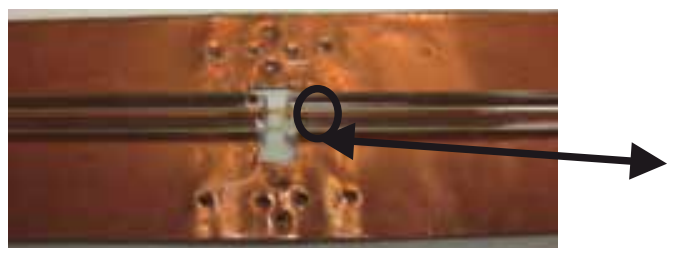

(a) Top view of a sensor prototype: sensor head and metal attachment sheet

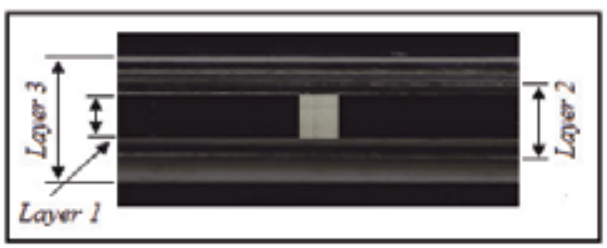

(b) Side view of the sensor head taken with an optical microscope

Fig. 2. Sensor prototype

As shown in Fig. 1, the measurement system of an EFPI sensor uses a broadband light source (BBS) of wavelength from $1520 \mathrm{~nm}$ to $1620 \mathrm{~nm}$, which is generated by multiplexing a C-band (BBS 1550A-TS) and an L-band (HWT-BS-L-P-FC/UPC-B) Erbium Doped fiber amplified spontaneous emission (ASE). The light propagates into the EFPI sensor through a $3 \mathrm{~dB}$ coupler. As the light travels through the lead-in fiber, part of the light is reflected at both cleaved end faces of the EFPI sensor, producing a backward travelling interference signal. The reflected interference spectrum coupled back by the coupler is detected by an optical spectrum analyzer (OSA, HP 70952B). A personal computer is used to record and process the interference spectra. Finally, the characteristic wavelength on the spectrum can be related to the cavity of the EFPI or the strain applied to the substructure in various resolutions with the signal processing algorithms presented in Section 2.1.2.

\subsubsection{Signal processing algorithms}

To simultaneously achieve a large dynamic range and high resolution in strain measurements, three data processing methods are introduced and studied to characterize their performance. These methods include 1) interference frequency tracking of the Fourier transform of a spectral interferogram, 2) period tracking and 3) phase tracking of the spectral interferogram.

Interference Frequency Tracking Method A low finesse EFPI can generally be modelled by a two-beam interference theory (Taylor 2008). The spectral interferogram of an EFPI typically represents a harmonic function of wavenumber with a dominant frequency known as the interference frequency. By taking the Fourier transform of such an interferogram, an approximate delta function of cavity length corresponding to the interference frequency is obtained (Liu et al. 2000). The cavity length of the EFPI, $l$, can then be calculated by Equation 1:

$$
l=\frac{n \pi}{\left(v_{E}-v_{S}\right)}
$$


in which $v_{S}$ and $v_{E}$ are the wavenumbers of the start and end points of an observation bandwidth, respectively, and $\mathrm{n}$ is an integer representing the Fourier series index.

It can easily be observed from Equation 1 that the minimum detectable cavity length change of an EFPI large strain sensor is $\pi /\left(v_{E}-v_{S}\right)$ when $n=1$. For a light source with a spectrum width of $100 \mathrm{~nm}$, the detectable cavity length change is approximately $12 \mu \mathrm{m}$. This corresponds to strain resolution of approximately $6,000 \mu \varepsilon$ when a gauge length of $2 \mathrm{~mm}$ is used. As indicated by Equation 1, the strain resolution is inversely proportional to the bandwidth of the light source. Higher resolution in strain measurement thus requires an optical source with a broader bandwidth, which can only be provided by a limited selection of equipment available in the market.

Period Tracking Method Due to the interrelation between period and interference frequency, the change in period of the spectral interferogram can also be used to determine the cavity length of an EFPI (Taylor 2008). The period of a spectral interferogram is defined as the distance between two consecutive valleys on the spectral interferogram. By introducing a wavenumber-wavelength relation $(v=2 \pi / \lambda)$, the cavity length can be evaluated by Equation 2:

$$
l=\frac{\lambda_{1} \lambda_{2}}{2\left(\lambda_{2}-\lambda_{1}\right)}
$$

where $\lambda_{1}$ and $\lambda_{2}\left(\lambda_{2}>\lambda_{1}\right)$ represent the first and second wavelengths of two consecutive valleys on the spectral interferogram that can be directly taken from OSA measurements.

Let $S_{l}$ be the resolution of a strain sensor, which is defined as the minimum detectable change in cavity length when using the period tracing method. Therefore, when $\lambda_{1}$ and $\lambda_{2}$ are assumed to be two independent random variables, $S_{l}$ can be derived from Equation 2 and expressed into Equation 3:

$$
S_{l}=\sqrt{\left(\frac{\partial l}{\partial \lambda_{1}}\right)^{2}\left(S_{\lambda_{1}}\right)^{2}+\left(\frac{\partial l}{\partial \lambda_{2}}\right)^{2}\left(S_{\lambda_{2}}\right)^{2}}=\sqrt{\frac{\lambda_{2}{ }^{4}}{4\left(\lambda_{2}-\lambda_{1}\right)^{4}}\left(S_{\lambda_{1}}\right)^{2}+\frac{\lambda_{1}{ }^{4}}{4\left(\lambda_{2}-\lambda_{1}\right)^{4}}\left(S_{\lambda 2}\right)^{2}}
$$

in which $S_{\lambda 1}$ and $S_{\lambda 2}$ represent the OSA measurement resolutions of the two consecutive valleys, respectively. Determined from the performance specifications of a particular OSA instrument, $S_{\lambda 1}$ and $S_{\lambda 2}$ are equal $\left(S_{\lambda 1}=S_{\lambda 2}=S_{\lambda}\right)$ since the instrument has a consistent measurement resolution of wavelength within the specified observation bandwidth. In addition, within a relatively small observation spectrum range, both $\lambda_{1}$ and $\lambda_{2}$ can be approximated by the center wavelength of the range, $\lambda_{0}$. As a result, Equation 3 can be simplified into:

$$
S_{l} \approx S_{\lambda} \frac{\lambda_{0}^{2}}{\sqrt{2}(\Delta \lambda)^{2}}
$$

where $\Delta \lambda$ is the wavelength difference between the two consecutive valleys. For the estimation of measurement errors, $\Delta \lambda$ at a given cavity length can be considered to be a constant within the wavelength bandwidth of observation, though $\Delta \lambda$ slightly increases with wavelength. Equations 2 and 4 indicate that the minimum detectable cavity length decreases in a quadratic manner as $\Delta \mathcal{\lambda}$ increases or the cavity length decreases. In other words, as the cavity length increases, the resolution of cavity length or strain measurement becomes lower. 
Phase Tracking Method Based on the two beam interference theory (Taylor 2008), the spectral interferogram reaches its minimum when the phase difference between the two beams satisfies the following condition:

$$
\frac{4 \pi l}{\lambda_{v}}=(2 m+1) \pi
$$

where $m$ is an integer that can be estimated following the procedure as specified in Qi et al. (2003), and $\lambda_{v}$ is the center wavelength of a specific interference valley. Taking the derivative of the cavity length with respect to $\lambda_{v}$ yields

$$
\frac{d l}{d \lambda_{v}}=\frac{2 m+1}{4}
$$

Therefore, the change in cavity length can be estimated from Equations 5 and 6 as follows:

$$
\Delta l=\frac{\Delta \lambda_{v}}{\lambda_{v}} l
$$

where $\Delta \lambda_{v}$ is the change in center wavelength of the specific interference valley and $\Delta l$ is the change in cavity length. As Equation 7 indicates, the cavity length change is directly proportional to the wavelength shift of the interferogram and to the cavity length of the EFPI. Since the minimum $\Delta \mathcal{\lambda}_{v}$ is represented by the instrument measurement resolution or $S_{\lambda 1}$ and $S_{\lambda 2}$, the resolution of the phase tracking method decreases linearly as the EFPI cavity length increases.

Comparison among Three Processing Methods Fig. 3 compares the theoretical strain measurement resolution of three data processing methods when $L=2 \mathrm{~mm}$. To cover a range of wavelength measurement resolution from various commercial OSAs, let $S_{\lambda}=\Delta \lambda_{v}$ be equal to $0.001 \mathrm{~nm}, 0.01 \mathrm{~nm}$, and $0.1 \mathrm{~nm}$. It can be clearly observed from Fig. 3 that the interference frequency tracking method has constant resolution of approximately $6,000 \mu \varepsilon$. The resolution of the period tracking method decreases in a quadratic manner as the EFPI cavity length increases. The resolution of the period tracking method is strongly influenced by the resolution of a particular OSA system. If $S_{\lambda}=\Delta \lambda_{v}=0.01 \mathrm{~nm}$, the strain resolution of period tracking method is $600 \mu \varepsilon$. If $S_{\lambda}=\Delta \lambda_{v}=0.1 \mathrm{~nm}$, the period tracking method has higher resolution than the interference frequency tracking method for $l<320$ $\mu \mathrm{m}$. In addition, the resolution of the phase tracking method decreases linearly as the EFPI cavity length increases. Among the three methods, the phase tracking method has the highest resolution since it represents the local (most detailed information) change of phase. When $l=320 \mu \mathrm{m}$ and the given OSA resolution is $0.1 \mathrm{~nm}$, the strain resolution of the phase tracking method is $10 \mu \varepsilon$ in comparison with $6,000 \mu \varepsilon$ for the other two methods. However, the phase tracking method can only measure a relatively small change of the cavity length within a $2 \pi$ phase range to avoid ambiguity. Therefore, its operation range is limited to a change of approximately $0.75 \mu \mathrm{m}$ in cavity length or a change of $375 \mu \varepsilon$ in strain. On the other hand, the other two methods can be used to measure a large change of cavity length. 


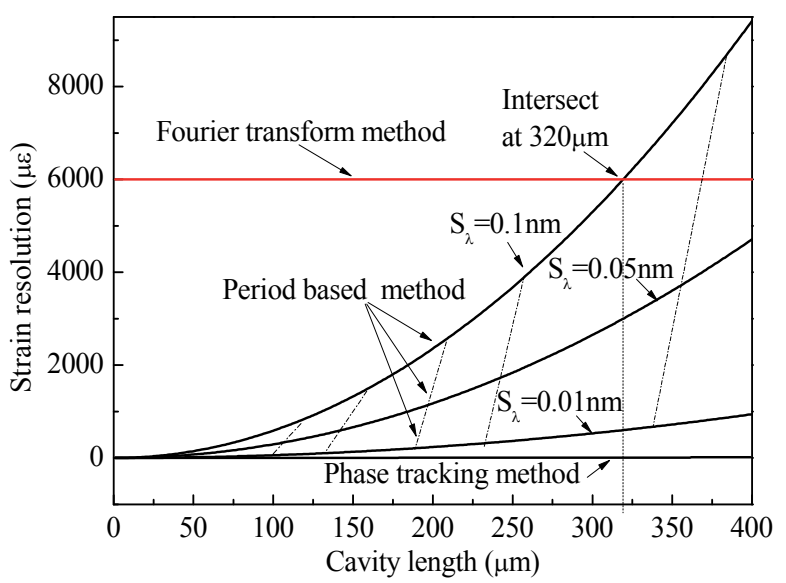

Fig. 3. Resolution as a function of cavity length

\subsection{Experiment and discussions}

To evaluate the performance of the proposed sensor for large strain measurements, an EFPIbased prototype sensor was constructed with transparent glass tubes so that any change in cavity length can be observed in the laboratory by using an optical microscope as shown in Fig. 2(b). The lead-in side of the fiber sensor was fixed on an aluminum block and the fiber mirror side of the sensor was attached to a computer-controlled precision stage so that the cavity length can be controlled precisely. The gauge length of the strain sensor was set to 2 $\mathrm{mm}$. The reference strain, which will be further discussed in Fig. 5, was determined by dividing the change in cavity length, directly measured by stage movement, by the gauge length. The strain detected by the EFPI sensor is obtained by dividing the cavity length calculated from an EFPI signal to the gauge length.

Fig. 4(a) presents two interferograms of the EFPI sensor prototype with a cavity length of 65 $\mu m$ and $175 \mu m$, respectively. It can be observed from Fig. 4(a) that the interference frequency increases as the EFPI cavity length increases or as more fringes are condensed into a given observation spectrum range. However, the range of interference signal intensities decreases as the EFPI cavity length increases. The signal range is often quantified by a fringe visibility $(\mathrm{V})$ as defined by Equation 8 :

$$
V=\frac{I_{\max }-I_{\min }}{I_{\max }+I_{\min }}
$$

where $I_{\max }$ and $I_{\min }$ represent the maximum and minimum intensities of an interference signal, respectively. The fringe visibility determined from Equation 8 is plotted in Fig. 4(b) as a function of cavity length. The experiment stopped when the fringe visibility dropped below $20 \%$, corresponding to a maximum cavity length of approximately $265 \mu \mathrm{m}$ of the prototype sensor. The drop in fringe visibility as a function of cavity length was mainly caused by the divergence of the output beam from the lead-in fiber, which was governed by the numerical aperture (NA) of the fiber (Han et al. 2004). Other potential factors such as misalignment are negligible in this study since the glass tubes of the three-layer sensor prototype were assembled with a tight tolerance. 


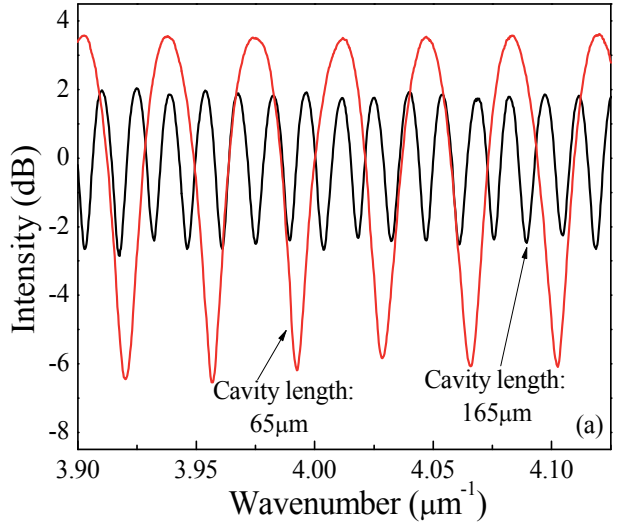

(a) Interferograms with a cavity length of 65 $\mu \mathrm{m}$ and $175 \mu \mathrm{m}$

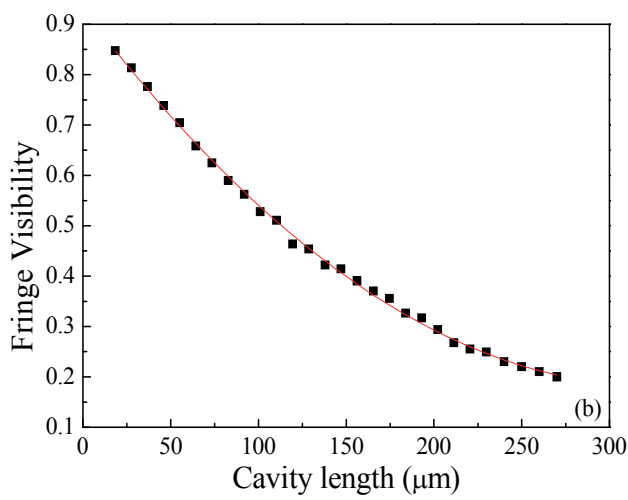

(b) Fringe visibility as a function of cavity length

Fig. 4. Interferogram and fringe visibility

To investigate the measurement resolution of the interference frequency tracking method, large strain measurement experiments were designed and executed. During various tests, the cavity length of the sensor prototype ranged from $15 \mu \mathrm{m}$ to $265 \mu \mathrm{m}$ at $10 \mu \mathrm{m}$ intervals. The maximum change of cavity length was approximately $250 \mu \mathrm{m}$, corresponding to a dynamic strain range of $12.5 \%$. Fig. 5 relates the reference strain measured by the change in stage movement to the strain measured by the change in cavity length of the EFPI sensor. The theoretic values were directly calculated based on the stage movement; they follow a straight line with a slope of 1:1 as represented by the solid line in Fig. 5. The experimental data points demonstrated only slight fluctuations around the theoretic line. To compare the measurement resolution of the interference frequency tracking method and the period tracking method, refined experiments were conducted within a strain range of $11,000 \mu \varepsilon$ to $21,000 \mu \varepsilon$. In this case, the precision stage was moved at $2 \mu \mathrm{m}$ intervals, giving rise to a strain change of 1,000 $\mu \varepsilon$ between two consecutive measurements. The results from the refined experiments processed with both the interference frequency tracking and period tracking methods are presented as an inset in Fig. 5. It can be seen from Fig. 5 that the theoretical prediction strongly agrees with the test data points that were processed with the period tracking method and the measured strains processed with the interference frequency tracking method follow a zig-zag trend with respect to the theoretic prediction. This comparison indicates that the interference frequency tracking method is unable to resolve a strain difference within an interval of $12 \mu \mathrm{m}$ in cavity length. This length resolution corresponds to a strain measurement of approximately $6,000 \mu \varepsilon$, which agrees with the calculated strain resolution that is limited by the light source bandwidth of $100 \mathrm{~nm}$.

The relative accuracy between the interference frequency method and period tracking method is supported by Fig. 3 since the cavity length observed during the refined experiments was significantly less than $320 \mu \mathrm{m}$ when the two methods had the same resolution. The interference frequency tracking method is advantageous over the other two methods in terms of computational efficiency and constant resolution over the entire dynamic range. In addition, it is immune to localized spectrum distortions that could potentially result in large errors when waveform based signal processing methods are used. 


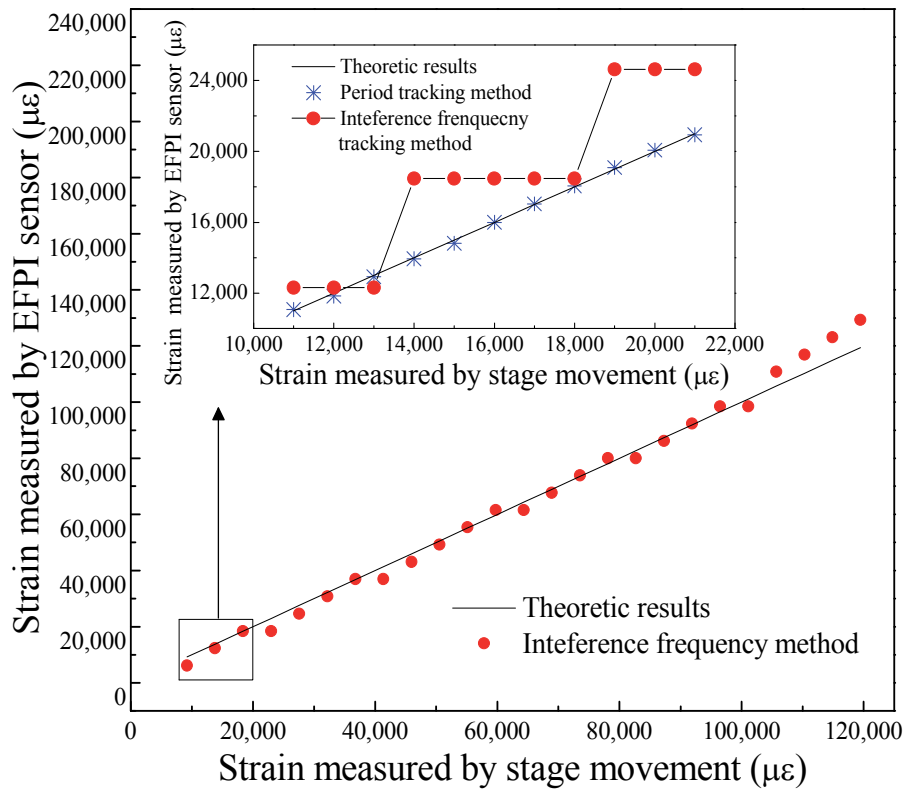

Fig. 5. Measured stains processed with the interference frequency tracking method (inset: comparison between the frequency and period tracking methods)

To verify the accuracy of the phase tracking method, more refined experiments were performed with a smaller stage movement interval of $0.1 \mu \mathrm{m}$. The cavity length of the EFPI sensor was set to range from $15 \mu \mathrm{m}$ to $30 \mu \mathrm{m}$, which corresponds to a strain of 7,500 $\mu \varepsilon$. Fig. 6(a) shows two representative spectral interferograms of the EFPI sensor at two consecutive stage positions with a cavity length difference of $0.1 \mu \mathrm{m}$. Fig. 6(b) compares the measured strains processed with the phase tracking and the period tracking methods. It can be observed from Fig. 6(b) that the theoretically predicted strain is in agreement with the strain data points processed with the phase tracking method and that of the period tracking method shows a notable deviation from the theoretic prediction based on the reference strains. This comparison indicates that refined resolution can be achieved with the use of the phase tracking method. The maximum deviation of the period tracking method was estimated to be $50 \mu \varepsilon$ at an EFPI cavity length of $30 \mu \mathrm{m}$, which is consistent with the theoretic prediction given in Fig. 3. The deviation is expected to further increase as the EFPI cavity length increases. However, it is worth repeating that the period tracking method can measure a large range of strain while the phase tracking method is limited to a strain measurement range of approximately $375 \mu \varepsilon$, which corresponds to a phase shift of $2 \pi$. 


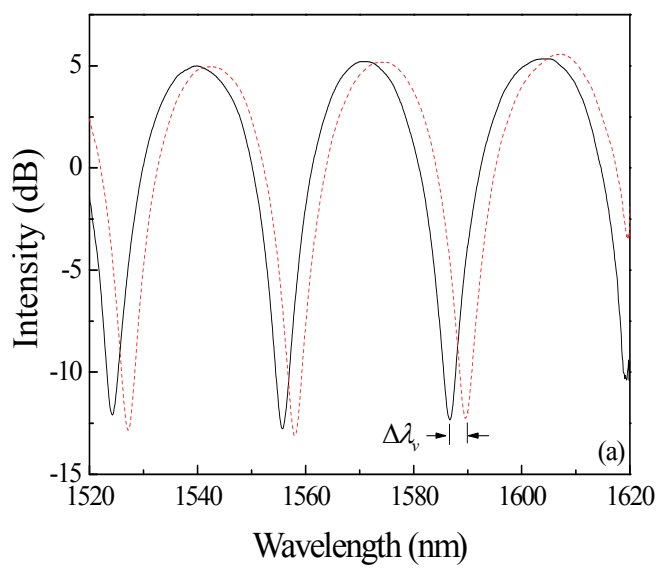

(a) Phase tracking method

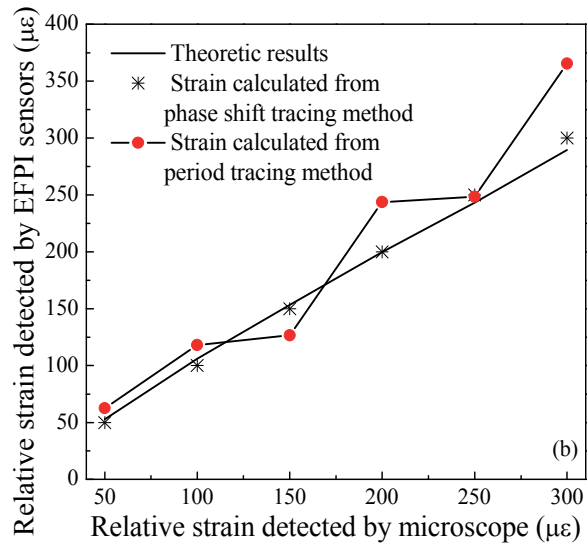

(b) Period versus phase tracking methods

Fig. 6. Typical spectral Interferograms and strain measurements

\section{A quasi-distributed optical fiber sensing system for large strain and high temperature measurements}

A hybrid LPFG/EFPI sensor was designed and fabricated by linking a $\mathrm{CO}_{2}$ laser induced LPFG with a movable EFPI in series. On one hand, LPFG is at least two orders (approximately 100 times) more sensitive to temperature than strain, and thus regarded as a temperature sensing component of the hybrid sensor. On the other hand, packaged with a glass tube only, EFPI has a weak cross effect of temperature on strain measurements in the order of $0.5 \mu \varepsilon{ }^{\circ} \mathrm{C}$, depending upon thermal coefficients of the optical fiber and the glass tube. Therefore, EFPI mainly works as a strain sensing component of the hybrid sensor (Huang et al. 2010).

\subsection{Sensor structure and measurement system}

Built upon Fig. 1(a), Fig. 7 shows the schematic of a hybrid fiber optical LPFG/EFPI sensor structure. The EFPI was formed by two perpendicularly cleaved end faces of a single-mode optical fiber (Corning SMF-28). The left part of the fiber with written gratings (LPFG) serves as a lead-in fiber and the right cleaved end face functions like a low reflective mirror. Both the cleaved end faces were placed inside a capillary tube with an inner diameter of $127 \mu \mathrm{m}$ and an outer diameter of $250 \mu \mathrm{m}$ to ensure the two end faces move in parallel. One end of the fiber was bonded on the capillary tube and the other end was not. The sensor installation was completed by bonding the capillary tube and the right fiber to a host structure over a gauge length of $L$ between the two bonding points. In this study, $L=2 \mathrm{~mm}$. The EFPI cavity length was designated as $l$. The bonding between all the components was completed after high temperature tolerable adhesives had been applied (Huang et al. 2008).

Fig. 7 also illustrates the expected main mechanism of light reflection and transmission around a hybrid sensor. As light at the light-in end of the fiber transmits through the LPFG, one part of the light is reflected by the left cleaved end face and the other part is reflected by the right cleaved end face. Since the LPFG is very close to the EFPI, typically less than $5 \mathrm{~cm}$ apart, both the EFPI-reflected light beams will be further reflected by the LPFG mirror towards the EFPI. At the light-out end of the fiber, the two branches of secondly reflected lights by the LPFG mirror thus form an interferometer at the OSA output spectrum together 
with the spectrum of the LPFG itself. The spatial frequency of the interferometer is only a function of the EFPI cavity length. The refractive index change of the interferometer does not affect the transmission signal of the LPFG itself as a sensor component.

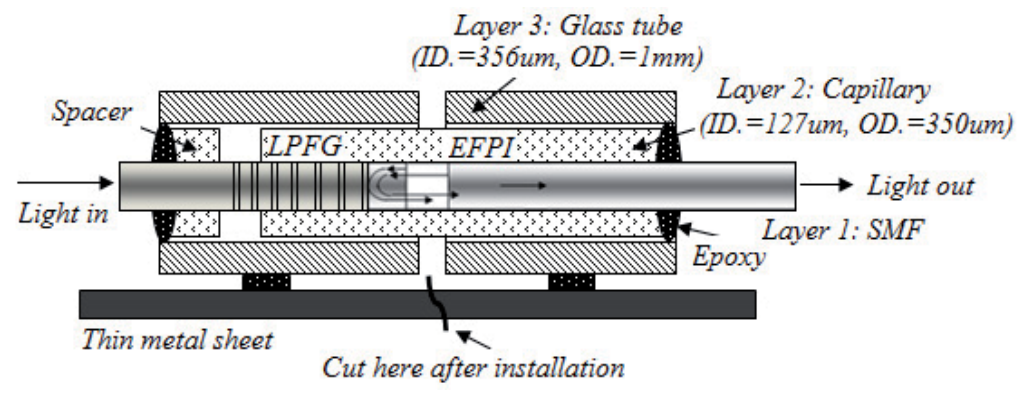

Fig. 7. Sensor structure and operational principle of a hybrid EFPI/LPFG sensor

Fig. 8 shows an optical sensing system of multiple hybrid LPFG/EFPI sensors. Light from a broadband source was channelled through each sensor head by two N-channel D configuration Input/output optic switchers, and received by the OSA. The OSA was then connected to a personal computer for real time data processing. A typical transmission spectrum seen on the OSA is illustrated in Fig. 8 as well. With the two N-channel D configuration optic switchers, $\mathrm{N}$ strategic locations of a critical structure can be monitored for simultaneous large strain and high temperature measurements.

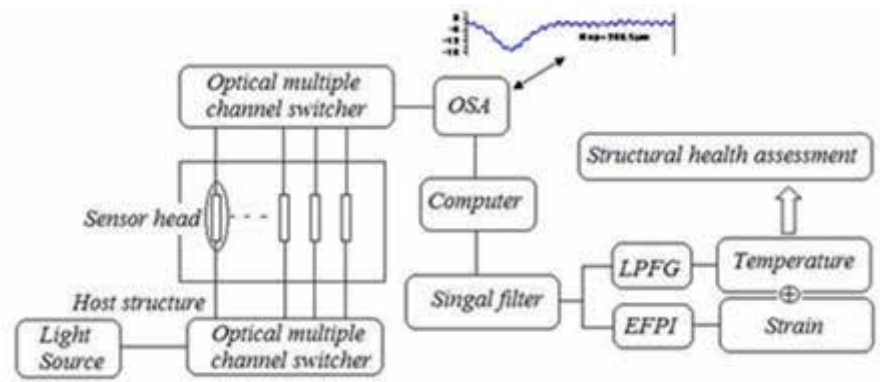

Fig. 8. An optical sensing system of hybrid EFPI/LPFG sensors

\subsection{Decomposition of the signal from a hybrid LPFG/EFPI sensor}

To enable the decomposition of a recorded signal into components from each LPFG sensor and each EFPI sensor in the hybrid sensing system, LPFG sensors must be designed with different wavelengths for multiplexing and EFPI sensors are given different optical paths. The signal processing algorithm for the signal decomposition is discussed below.

To measure temperature and strain at a specific location of a structure, the distance between the LPFG and EFPI components of a hybrid sensing system must be short, i.e., within $5 \mathrm{~cm}$. With such a short distance between the two sensor components, the transmission signal of a hybrid sensor approximately represents a combined effect of individual LPFG and EFPI components. Fig. 9(a) illustrates a typical spectral interferogram of the hybrid LPFG/EFPI sensor as detailed in Fig. 7 with a cavity length of over $20 \mu m$, and Fig. 9(b) shows the phase and amplitude of the Fast Fourier Transform (FFT) of the interferogram. It can be seen from Fig. 9(b) that the spatial frequency of the LPFG component is low and that of the EFPI 


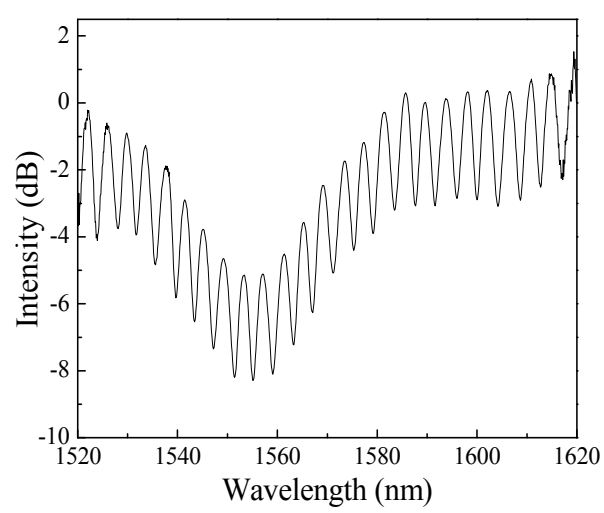

(a) Spectral interferogram of a hybrid sensor

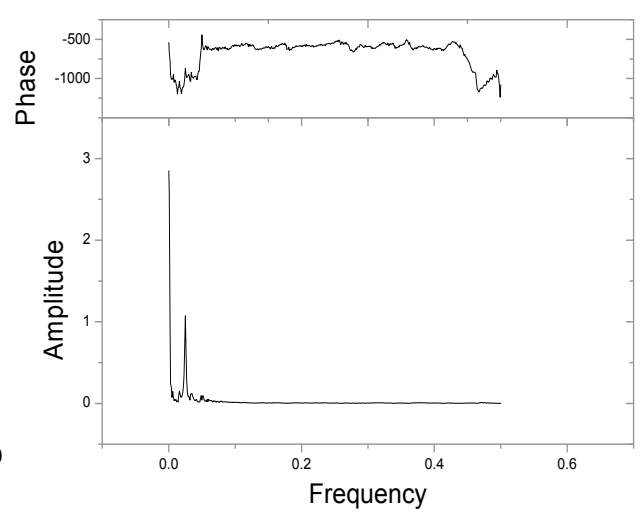

(b) FFT of the hybrid sensor's spectral interferogram

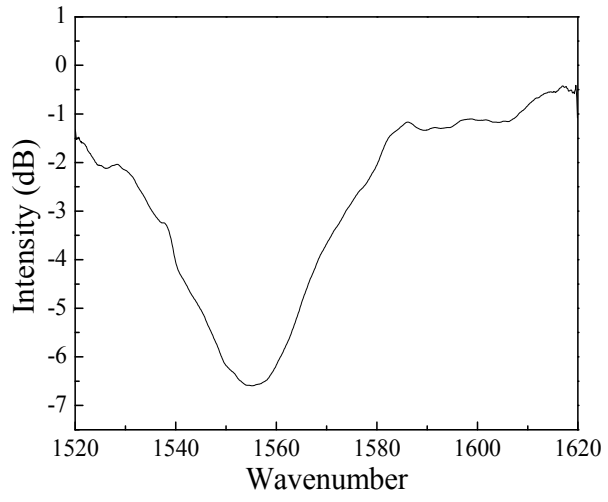

(c) Low-pass spectrum of the LPFG

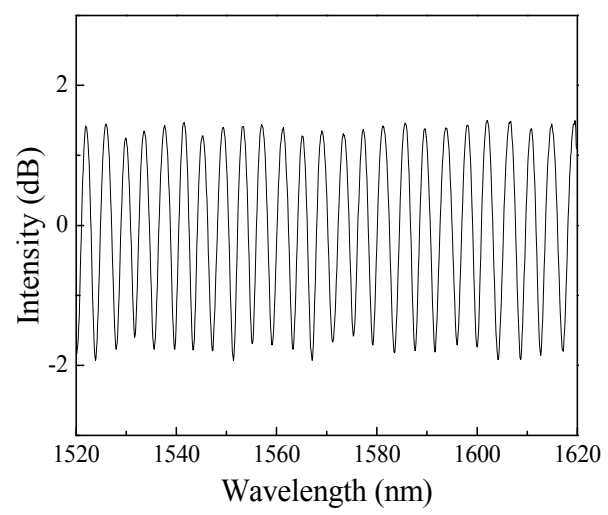

(e) High-pass interferogram of the EFPI

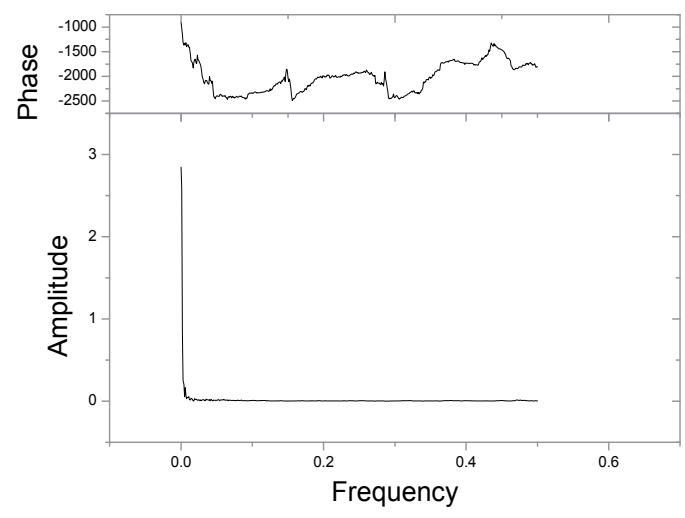

(d) FFT of the LPFG's spectrum

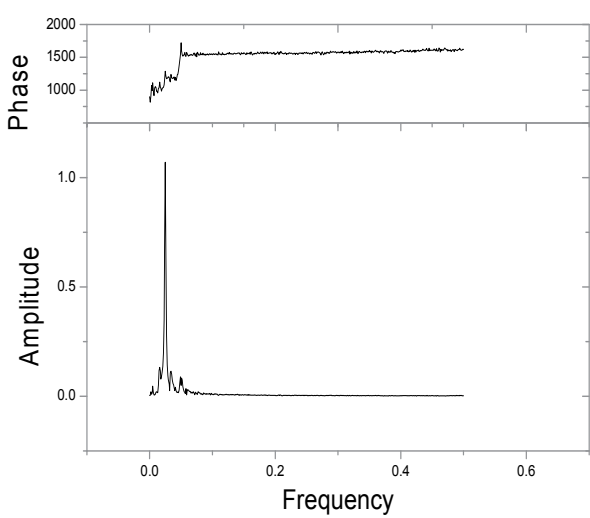

(f) FFT from the EFPI

Fig. 9. Transmission spectra and their FFTs of various sensors with low-pass and high-pass filters 
component is significantly higher. Therefore, to decompose the signal into two parts from the LPFG and EFPI components, respectively, a low-pass filter was applied. Figs. 9(c, d) present the spectrum of the LPFG, a low-passed signal from Fig. 9(a), and its Fourier transform. As such, the interferogram of the EFPI was then obtained by subtracting Fig. 9(c) from Fig. 9(a), as shown in Fig. 9(e). Its Fourier transform is presented in Fig. 9(f). The cavity length of the EFPI can be calculated from the decomposed signal based on the spectral frequency tracking method as discussed in Section 2.1.2.

\subsection{Experiments and discussions}

As indicated in Fig. 5, the EFPI component only of a hybrid LPFG/EFPI sensor can measure a gap distance change of up to $260 \mu \mathrm{m}$, corresponding to a strain of $12 \%$ for a gauge length of $2 \mathrm{~mm}$, and temperature as high as $1292^{\circ} \mathrm{F}\left(700^{\circ} \mathrm{C}\right)$. In this section, a hybrid LPFG/EFPI sensor was tested in the laboratory for simultaneous strain and temperature measurements. The hybrid sensor was installed on two steel channels of $2 \mathrm{~mm}$ apart, which were fixed on two computer controlled precise stages. The steel channels together with the hybrid sensor were further put into the high temperature furnace (made by Thermo Electron Corporation). Figs. 10(a, b) illustrate the measured temperature and strain. The hybrid LPFG/EFPI worked well up to $1292^{\circ} \mathrm{F}\left(700^{\circ} \mathrm{C}\right)$ and the difference between the strain calculated from the interference frequency method and that from the movable stage was within $5 \%$, which is acceptable for large strain measurement. Fig. 10(b) also illustrates that the influence of temperature on the EFPI signal was small and insignificant.

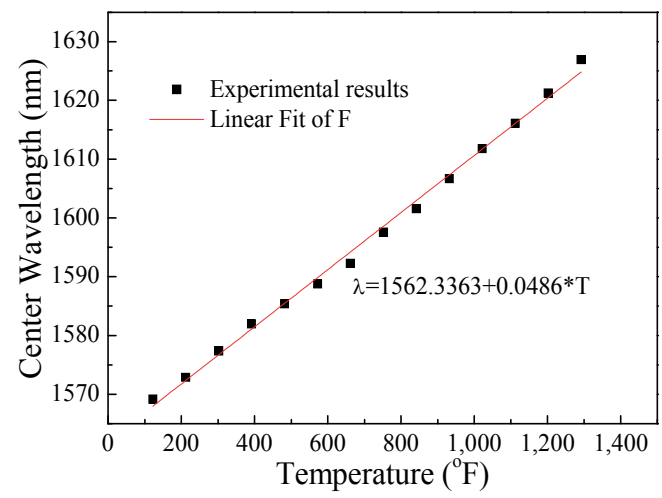

(a) Wavelength change with temperature

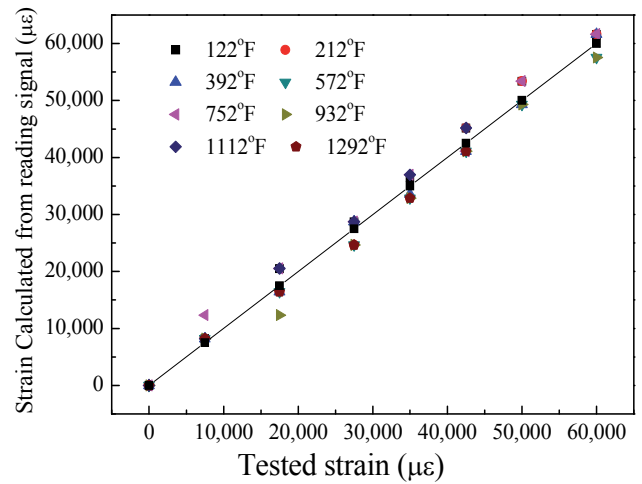

(b) Strain accuracy and temperature effect

Fig. 10. Temperature sensitivity, cross temperature-strain effect, and strain measurement accuracy of a LPFG/EFPI sensor

\section{Post-earthquake assessment of steel buildings under simulated earthquake and fire loadings with an optical fiber sensor network}

\subsection{Design of an idealized steel frame}

A single-bay rigid frame of one top beam and two columns is considered in this study. The frame was made of A36 steel and built with hot-rolled S-shapes as shown in Fig. 11. To illustrate a potential switch of failure modes from one column to another under earthquake and post-earthquake fire loadings, respectively, a substructure of the frame consisting of one 
column (\#1 in Fig. 11) and the top beam was tested under a static lateral load to simulate earthquake effects and the entire frame with two identical columns was tested with the other column (\#2 in Fig. 11) placed in a high temperature environment to represent postearthquake fire effects. The former is referred to as an L-shaped frame and the latter is referred to as a two-column frame for clarity in the following discussions.

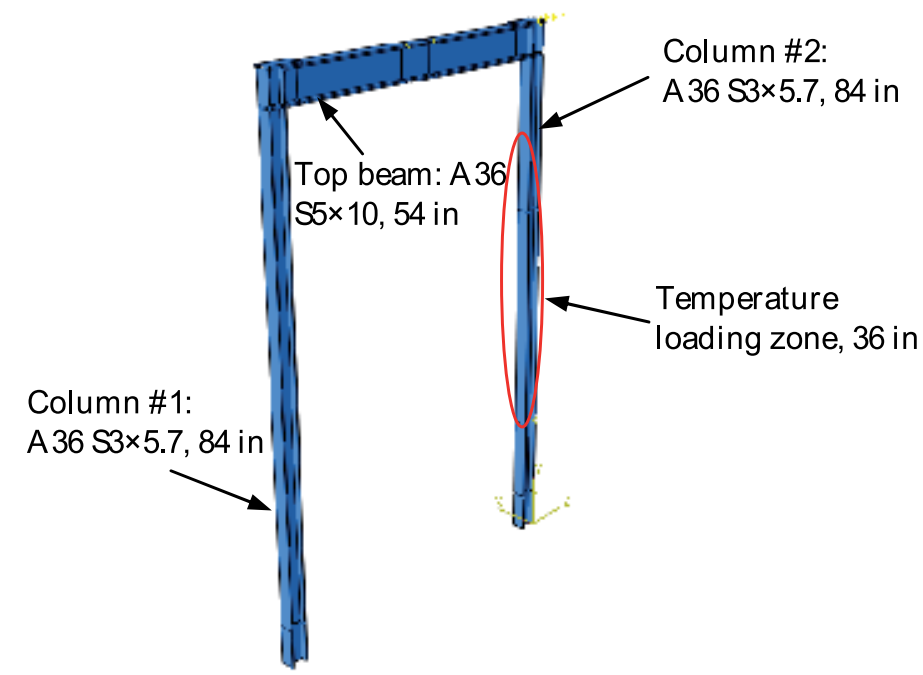

Fig. 11. Rendering of the steel frame

The dimensions of the steel columns were determined based on the size of an electric furnace (Lindberg/Blue M Tube Furnaces) used to simulate the high temperature effect of post-earthquake fires on the progressive collapse of the frame structure. The overall dimension of the furnace used for testing is $22 \times 54 \times 16 \mathrm{in} .(55.9 \times 137.2 \times 66 \mathrm{~cm})$ with an actual heating zone of $36 \mathrm{in}$. $(91.4 \mathrm{~cm})$ in length and an inner diameter of $6 \mathrm{in}$. $(15.24 \mathrm{~cm})$. Considering additional spaces required for the assembling (welding of stiffeners, beamcolumn joint, and column-tube connection) of the two-column frame after column \#2 has been placed through the round furnace, the length of the columns was selected to be $84 \mathrm{in}$. $(213.4 \mathrm{~cm})$. To provide a sufficient space for frame deformation at high temperature, the columns of the steel frame were selected as $S 3 \times 5.7$, which has a flange width of $3 \mathrm{in}$. (7.62 $\mathrm{cm})$ and a height of $4 \mathrm{in}$. $(10.16 \mathrm{~cm})$. To design a rigid beam, the stiffness of the top beam was set at least 5 times that of each column. As such, a hot-rolled $S 5 \times 10$ beam was selected. Since the anchors on the strong floor in the structures laboratory are spaced $36 \mathrm{in}$. $(91.44 \mathrm{~cm})$ apart, the length of the top beam was selected to be $56 \mathrm{in}$. $(142.2 \mathrm{~cm})$, which is equal to the anchor spacing plus the width of one bolted plate on the floor and twice the width of an angle stiffener. To ensure a rigid beam-column connection, three stiffeners were provided on each column: a $3 \times 12 \times 0.5 \mathrm{in} .(7.62 \times 30.48 \times 1.27 \mathrm{~cm})$ stiffener placed on the top cross section of the column, and two $1.4 \times 3.9 \times 0.5 \mathrm{in}$. $(3.556 \times 9.9 \times 1.27 \mathrm{~cm})$ stiffeners placed between the two flanges of the column on two sides of the column web, extending the bottom flange of the beam. Stiffeners were welded to the steel frame at the beam-column connection. The overall design of the steel frame is shown in Fig. 11. The column subjected to earthquake effects only is referred to as Column \#1 while the other column directly subjected to earthquakeinduced fire effects is referred to as Column \#2. 


\subsection{L-shaped steel frame and earthquake-induced damage \\ 4.2.1 Test setup and instrumentation under lateral loading}

To simulate earthquake damage of the steel frame (Column \#1 only), Column \#1 and the top beam was placed on the strong floor and subjected to cyclic loading. Fig. 12 shows the test setup of the L-shaped frame and its prototype in the inset. The L-shaped frame was welded on a steel tube of $6 \times 6 \times 132 \mathrm{in} .(15.24 \times 15.24 \times 335 \mathrm{~cm})$ with $1 / 2 \mathrm{in} .(1.27 \mathrm{~cm})$ wall thickness. In addition, two triangle brackets were individually welded to the two flanges of the column and the square tube to ensure a rigid connection between the column and the tube. The square tube was anchored into the strong floor at four anchor locations. To prevent it from experiencing large deformation, the square tube was stiffened near the base of the column by three stiffener plates of $12 \times 5.5 \times 0.5 \mathrm{in} .(30.48 \times 13.94 \times 1.27 \mathrm{~cm})$. The stiffeners were welded on the three sides of the square tube: column base face and two side faces. To approximately represent the twocolumn frame behavior, the free end of the top beam was transversely restrained by a rollertype support. A cyclic load was applied to the top of the column or the top beam by means of a hydraulic actuator. The applied load was measured by a 5-kip load cell installed between the actuator and the frame. To monitor the structural behavior under the cyclic load, 15 conventional strain gauges were deployed and distributed along the column and the beam as located in Fig. 12. They are designated with a prefix of SG\#. For example, SG\#1 means the strain gauge \#1 that was deployed to monitor the strain in the column-to-tube connection. Similarly, SG\#12 was used to assess the beam-column joint condition. In addition, two linear variable differential transformers (LVDTs) were respectively deployed $8 \mathrm{in}$. (203.2 $\mathrm{mm}$ ) above the column base and $14 \mathrm{in}$. $(355.6 \mathrm{~mm}$ ) below the bottom flange of the top beam. LVDT\#1 was deployed at the bottom of the column to ensure that the column is not displaced during testing. LVDT\#2 was deployed at this location for convenience.

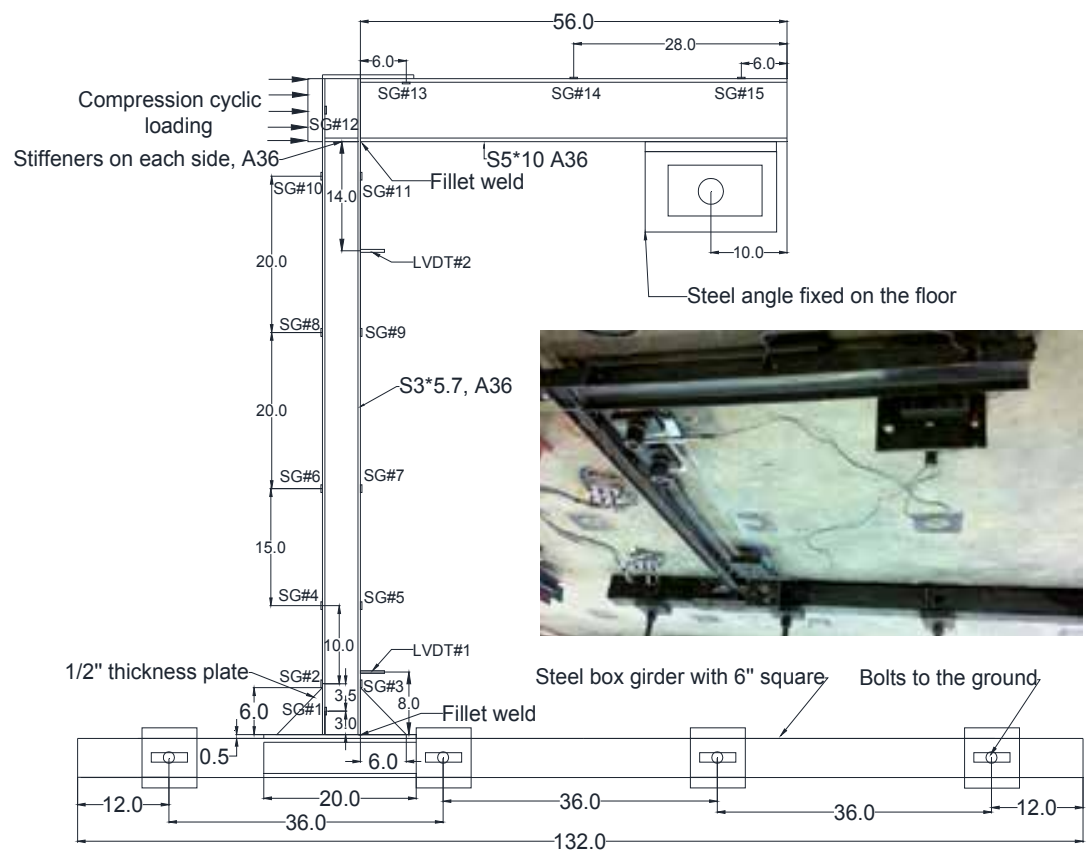

Fig. 12. Test setup and instrumentation of the L-shaped steel frame with a prototype inset (unit: in.) 


\subsubsection{Loading protocol and simulated earthquake damage}

Fig. 13 presents the cyclic loading protocol, measured strains and displacements of the Lshaped frame structure. As shown in Fig. 13(a), five cycles of loading were applied to the frame following a sawteeth pattern. The first four cycles of loading reached 3.4 kips $(15.1 \mathrm{kN})$ at which the column expects to experience initial yielding, and the last cycle reached 3.8 kips $(16.9 \mathrm{kN})$ to ensure that the column is subject to inelastic deformation. For all cycles, the frame structure was loaded and then unloaded at a rate of $-5.46 \mathrm{lb} / \mathrm{sec}(-24.29 \mathrm{~N} / \mathrm{sec})$ and $10.92 \mathrm{lb} / \mathrm{sec}(-48.57 \mathrm{~N} / \mathrm{sec})$, respectively.

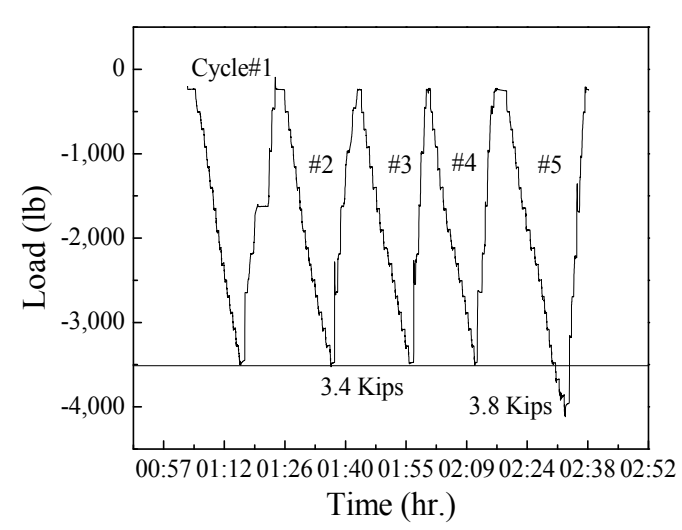

(a) Loading protocol

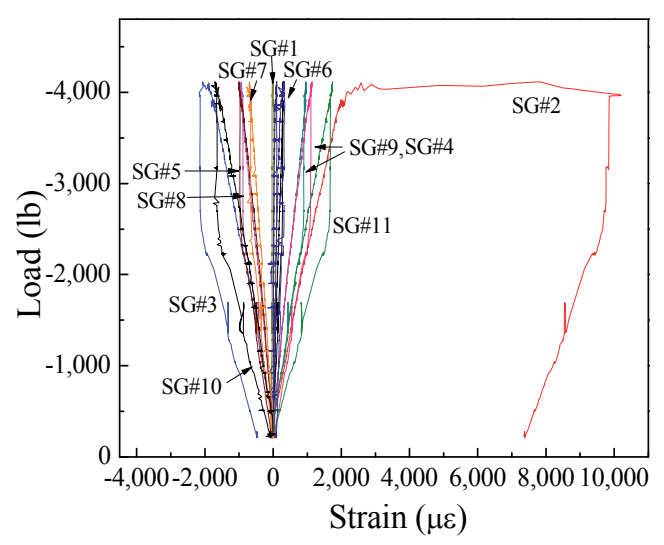

(c) Load-strain relations for cycle \#5

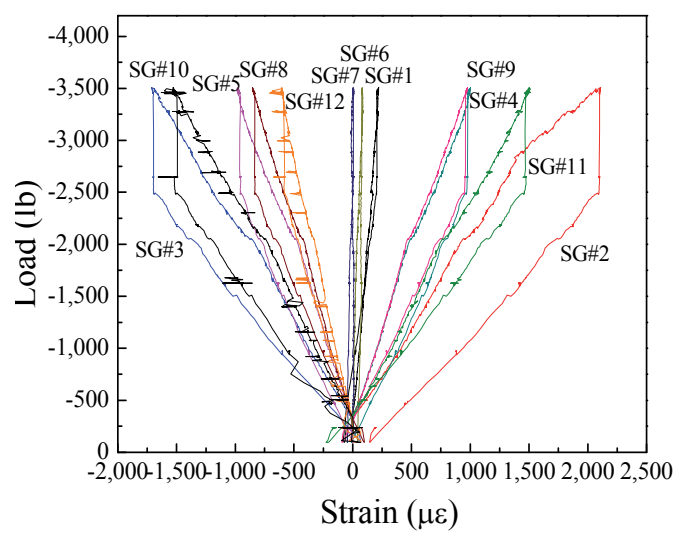

(b) Load-strain relations for cycle \#1 to \#4

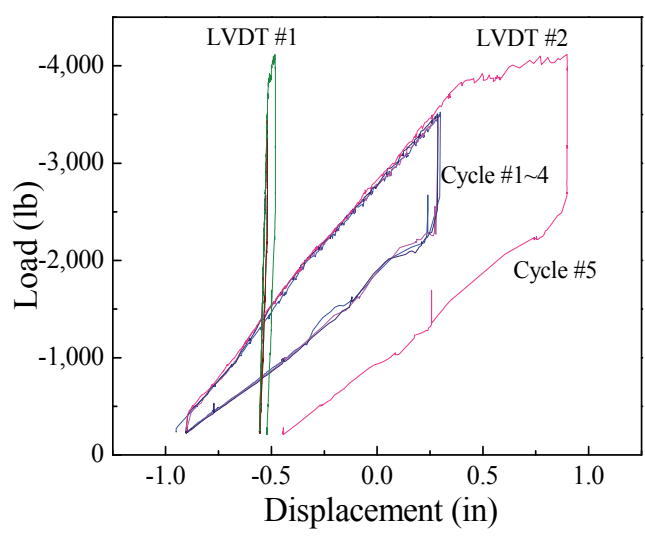

(d) Load-displacement relation

Fig. 13. Cyclic loading, measured strains and displacements

The measured strains (raw data) of the column are presented as a function of the applied load in Fig. 13(b) during the first four cycles of loading and in Fig. 13(c) during the last cycle. For the first four loading cycles, the maximum strain at the bottom of the column (SG\#2) was approximately $0.2 \%$, indicating initial yielding of the test frame. For the fifth cycle, the strain reached $1 \%$ as the load was held at 3.8 kips $(16.9 \mathrm{kN})$ for a few seconds. After unloading, a permanent strain of $0.75 \%$ remained at the column base (SG\#2). Throughout the tests, the maximum strains in the beam-column and column-tube connection areas are 
both insignificant due to their significantly stronger designs than that of the column member. The maximum strains at locations slightly away from the connection areas are the highest as shown in Fig. 14 for strain distributions along the height of the column (outside face) during the first four cycles and the fifth cycle of loading. It can be clearly observed from Fig. 14 that Column \#1 was subject to double curvatures with a zero strain around 20 in. $(50.8 \mathrm{~cm})$ above the column base. The extent of inelastic deformation was mainly limited to the bottom portion of the column during the fifth cycle.

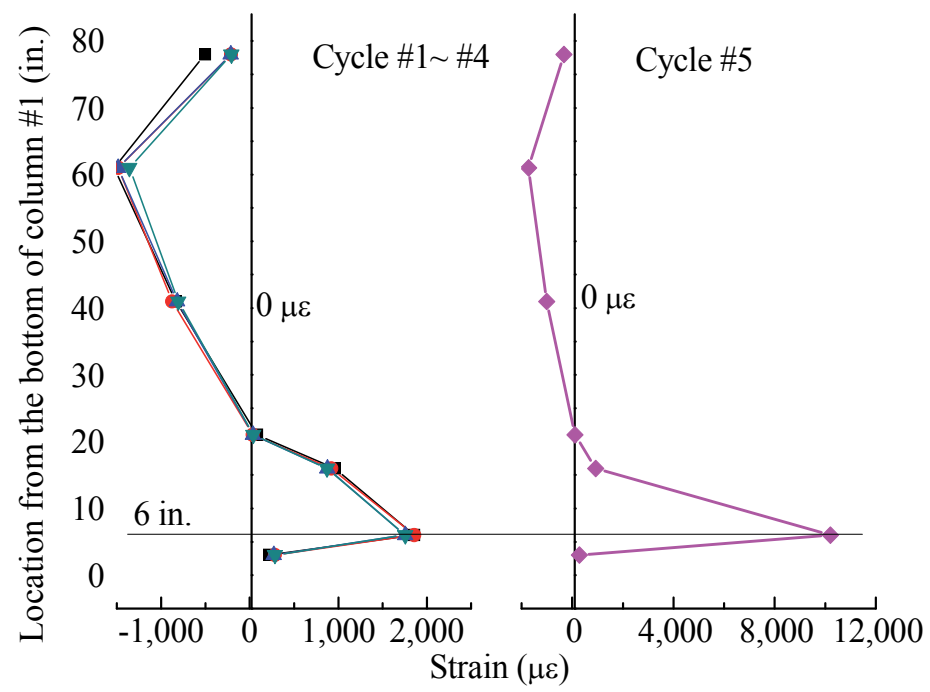

Fig. 14. Distribution of the maximum strains along the column height

The displacement change with load is presented in Fig. 13(d) in various loading cycles. It can be observed from Fig. 13(d) that the displacement change from LVDT\#1 is negligible, indicating that the frame base was basically fixed to the strong floor. The largest displacement of $1.8 \mathrm{in}$. $(4.572 \mathrm{~cm})$ was observed at the top of the column (location of LVDT\#2), leaving behind $0.5 \mathrm{in} .(1.27 \mathrm{~cm})$ permanent deformation in the column when unloaded. The permanent plastic strain and deformation introduced by the cyclic loading represented a large strain condition that can be induced by an earthquake event.

\subsection{Two-column steel frame and fire induced damage 4.3.1 Test setup and instrumentation under fire loading}

Fig. 15 shows the overall test setup of the two-column frame and various data acquisition systems used during tests as well as side views of the frame (shown in the inset). The frame was fixed to the strong floor at the base of both columns through the same square tube as used for cyclic tests. To simulate the gravity effect, the frame is subjected to a vertical load applied in displacement control by a hydraulic actuator against a rigid reaction beam. One column (\#2) passed through a high temperature furnace before it was welded to the remaining L-shaped frame. A wood frame was built as a lateral support for instrumentation. Commercial sensors included 10 K-type thermocouples (TM), 5 high temperature strain gauges (HSG), and 15 strain gauges deployed during the earthquake test. Fiber optical sensors consisted of 1 FBG temperature sensor, 2 LPFG high temperature sensors, 5 EFPI 
large strain sensors, and 2 hybrid EFPI/LPFG sensors. As shown in Fig. 16, to protect them from potential damage, the fiber optical sensors on Column \#2 were attached to the inside face of its flanges. The FBG and 1 LPFG were placed immediately above the furnace to monitor the temperature and strain at this transition area. The other LPFG sensor was located at the base of Column \#1 to ensure a good estimate on the boundary condition. Three out of the five EFPI sensors were placed at the end of the heating zone (36" total height) and two were located at the third and two-third points as illustrated in Fig. 16. One hybrid EFPI/LPFG sensor was placed at the bottom of the furnace and the other was at the $2 / 3$ length from the bottom of the furnace together with one EFPI sensor.

To closely monitor the structural behavior of the entire steel frame, other than fiber optical sensors, a comprehensive sensing network of commercial sensors was also developed and applied to the frame structure as illustrated in Fig. 17. As illustrated in an inset in Fig. 17, ceramic adhesives that can endure high temperature up to $2012{ }^{\circ} \mathrm{F}$ were used to attach optical sensors on the inside surface of the column flanges.

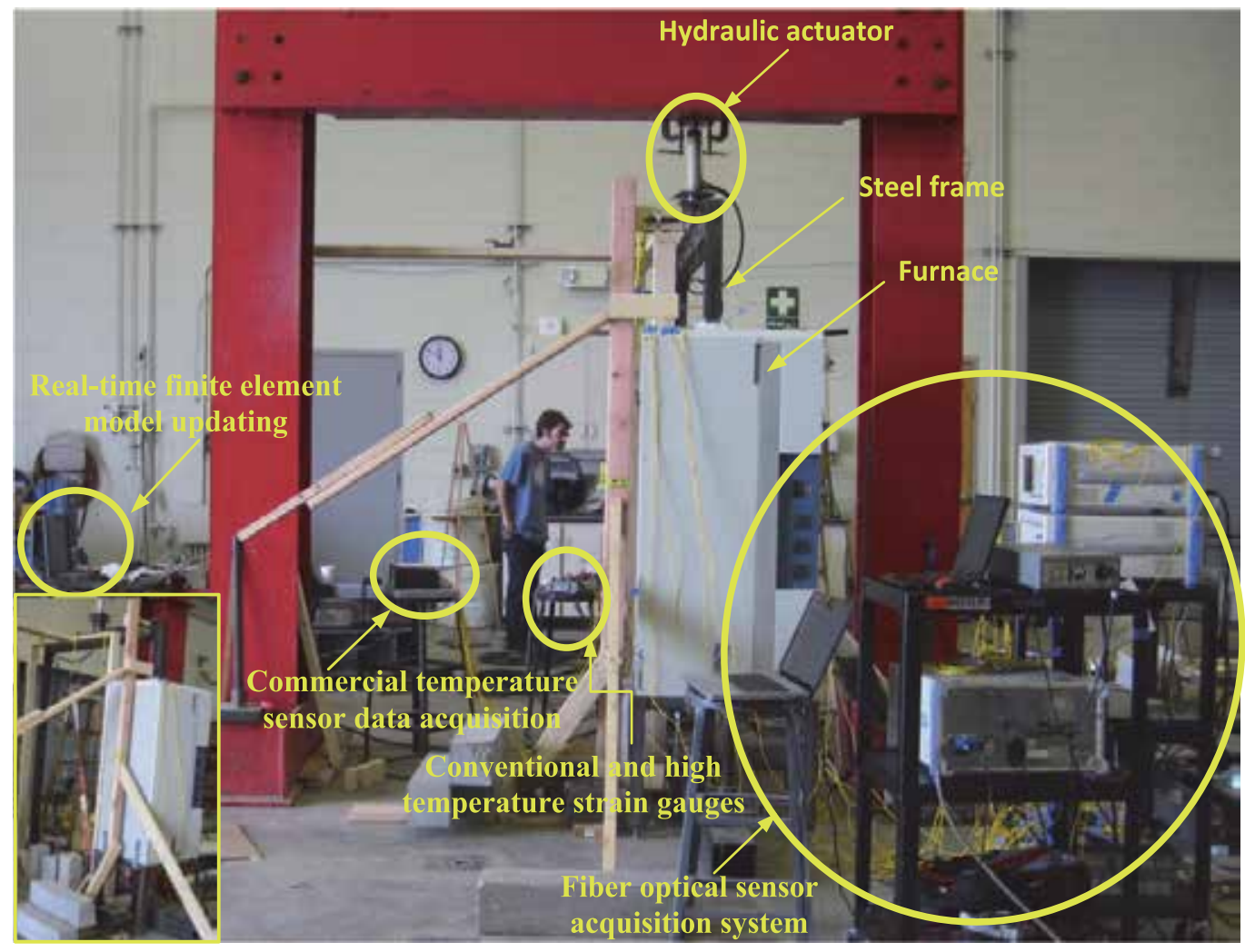

Fig. 15. Overview of the two-column frame test setup and data acquisition systems 


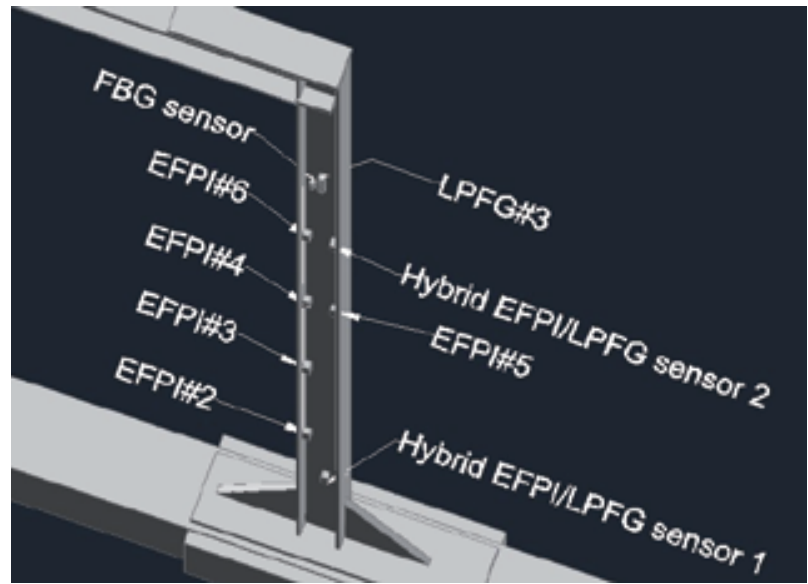

Fig. 16. Specific locations of fiber optical sensors in three-dimensional view

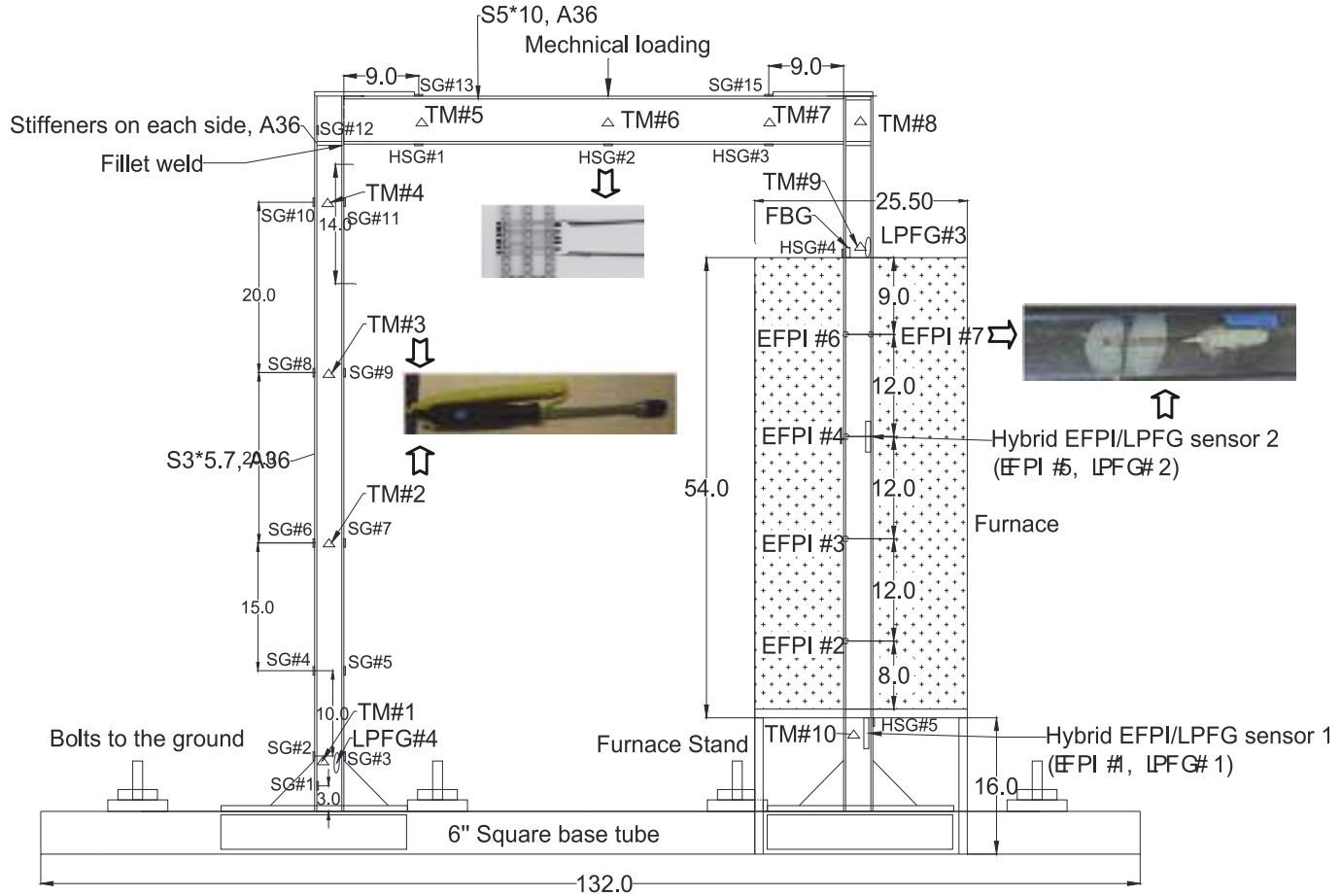

Fig. 17. Instrumentation for simulated post-earthquake fire tests with photos showing the EFPI and hybrid EFPI/LPFG sensors, high temperature strain gauges, and thermocouples

\subsubsection{Loading protocol and simulated fire damage}

A Lindberg/Blue $M$ tube furnace made by Thermo Scientific was used to provide a high temperature environment that simulates the high temperature effect of a post-earthquake fire. It had three temperature zones that can be programmed and operated independently. In this study, the three temperature zones were programmed to have the same temperature 
increase profile. As shown in Fig. 18(a), temperature was increased at a rate of $18{ }^{\circ} \mathrm{F} / \mathrm{min}$ from $72{ }^{\circ} \mathrm{F}$ (room temperature, $22^{\circ} \mathrm{C}$ ) to $1472^{\circ} \mathrm{F}\left(800^{\circ} \mathrm{C}\right.$ ) by an interval of $180^{\circ} \mathrm{F}\left(100^{\circ} \mathrm{C}\right)$ or $90^{\circ} \mathrm{F}\left(50^{\circ} \mathrm{C}\right)$. At each temperature level, the test paused for 10 minutes to ensure that the temperature distribution is stable both inside and outside the furnace.

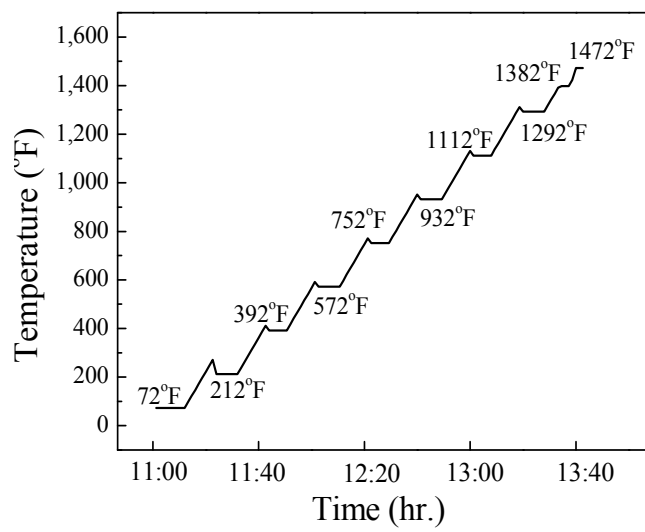

(a) Temperature loading profile

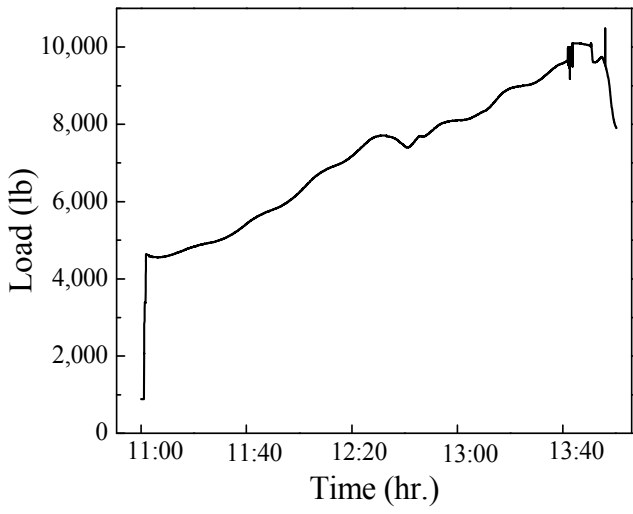

(b) Vertical load history

Fig. 18. Loading condition during post-earthquake fire tests

As temperature increased, Column \#2 was elongated, increasing the stroke of the hydraulic actuator. As a result, the vertical load applied on the top beam by the displacement-controlled actuator was increased significantly. Corresponding to the increasing of furnace temperature, Fig. 18(b) shows that the frame was subjected to a vertical load of $4.6 \mathrm{kips}(20.46 \mathrm{kN})$ to $10 \mathrm{kips}(44.48 \mathrm{kN})$. Since the applied load was introduced to mainly simulate the gravity effect on the frame structure, an alternate scheme to load the frame needs to be developed in the future to maintain a nearly constant gravity effect.

Fig. 19(a) shows the measured temperatures from 4 LPFG sensors. LPFG\#2, which was placed inside the furnace, measured the furnace temperature profile as shown in Fig. 18(a). The temperature on the top of the furnace increased from room temperature to 550 ${ }^{\circ} \mathrm{F}$ as indicated by the measurement of LPFG\#3. Fig. 19(b) shows the measured strains from 7 EFPI sensors after temperature compensation, including those as part of the two hybrid sensors. Note that EFPI\#6 and EFPI\#7 were malfunctional after strains exceeded $11 \%$. This is because all EFPI sensors were designed to have the maximum strain of approximately $11 \%$ with an initial cavity of 50 60 $\mu \mathrm{m}$. At the top of the heating zone, the steel column is subject to a strain of $8 \%$ at $752{ }^{\circ} \mathrm{F}$ and would have been strained for over $11 \%$ at $927^{\circ} \mathrm{F}$ based on the strain increasing trend over the time. Other locations with maximum strains of less than $11 \%$ were continuously monitored by the EFPI sensors till the end of high temperature tests. It is also observed from Fig. 19(b) that the strains measured by EFPI\#4 and EFPI\#5 are similar, both slightly larger than that of EFPT\#3 for most part. These three sensors were all located inside the furnace. However, the strain measurements by EFPI\#2 (bottom of the furnace) were substantially smaller than those from EFPI\#6 and EFPI\#7 (top of the furnace) even though they were both located at the end of the furnace. This comparison indicated the effect of gravity on the temperature distribution at the boundary of the furnace. 


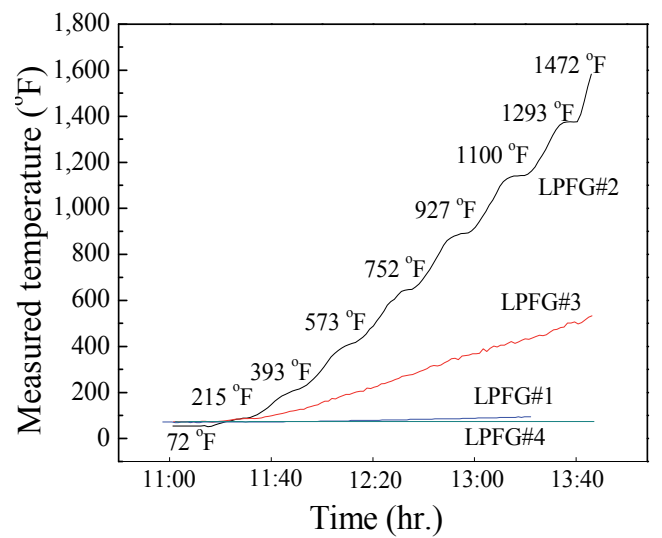

(a) Measured temperature by LPFG sensors

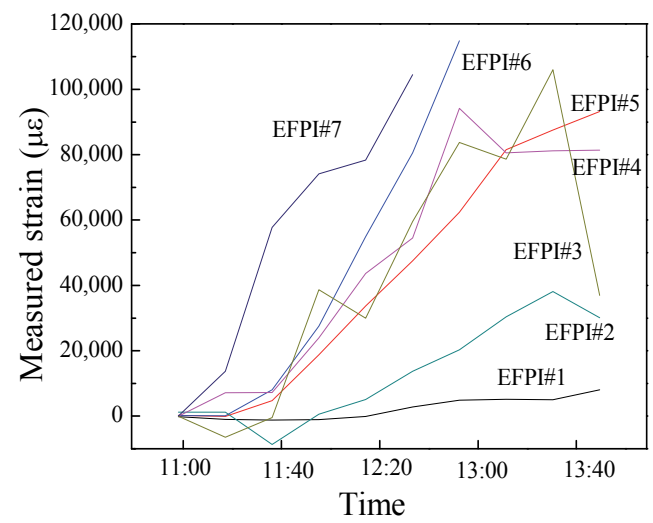

(b) Measured strains by EFPI sensors

Fig. 19. Measurements from LPFG and EFPI sensors

By comparing Fig. 13(c) with Fig. 19(b), relative effects of simulated earthquakes and earthquake-induced fires on column stability of the test frame can be discussed. During the cyclic load tests simulating earthquake effects, Column \#1 was subjected to approximately $1 \%$ strain or ductility of 8 for A36 steel. This level of strain likely represents the effect of a moderate earthquake. In a post-earthquake fire environment, Column \#2 was subjected to over $10 \%$ strain or ductility of over 80 for A36 steel. At $1472{ }^{\circ} \mathrm{F}$, Column \#2 became unstable due to extensive strain and deformation, resulting in a progressive collapse of the steel frame. This was confirmed by a visual inspection of the tested frame towards the completion of the experiment. It can thus be concluded that the fire-induced inelastic deformation of steel structures can far exceed the earthquake-induced deformation under a moderate earthquake event.

To understand how temperature affects the structural condition of the frame outside the furnace, Fig. 20 presents the strain measurements from strain gauges and high temperature strain gauges as the furnace temperature increased. Without direct temperature loading on Column \#1, the permanent plastic strain of $0.75 \%$ induced by the simulated earthquake (SG\#2) remained nearly constant during the simulated post-earthquake fire condition. The strains at other locations varied little with temperature as well.

\subsubsection{Validation of the optical fiber sensing network against conventional sensors}

The strains measured by EFPI\#1 and HSG\#5 are compared in Fig. 21(a) near the bottom of Column \#2 immediately below the furnace. The two measurements showed a similar trend with a correlation coefficient of 0.963 . This comparison verified the viability of using fiber optical sensors for strain measurements. Similarly, Fig. 21(b) compares various temperature measurements by TM\#9, LPFG\#3, and FBG sensors near the top of Column \#2 immediately above the furnace. Overall they are in good agreement even though the LPFG sensor appeared to give a better comparison with the thermocouple in two temperature ranges as seen in Fig. 21(b). 


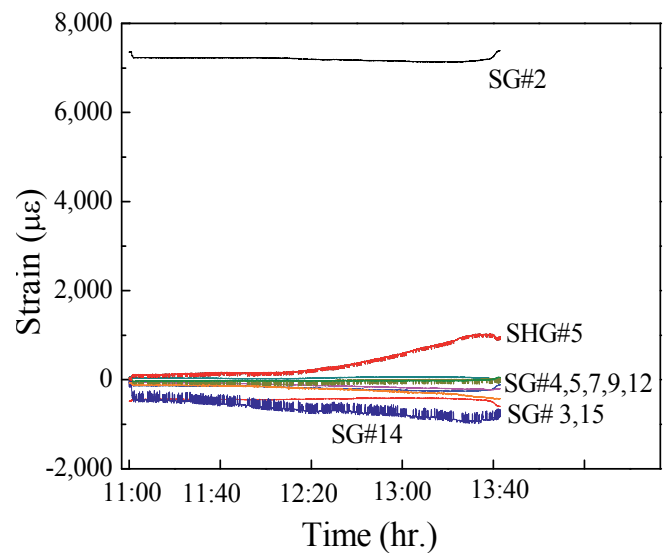

Fig. 20. Change of strains in the frame outside the furnace

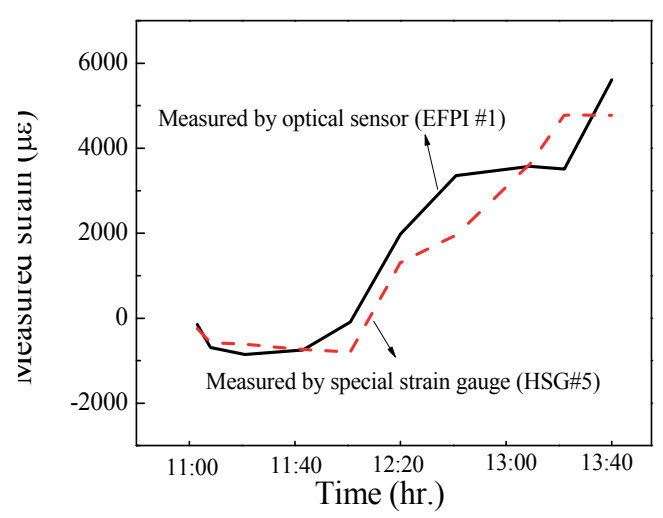

(a) Strains near the bottom of column \#2

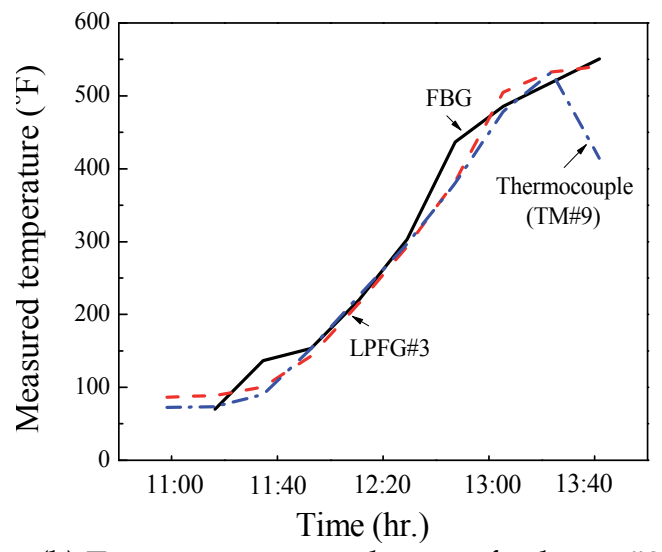

(b) Temperature near the top of column \#2

Fig. 21. Comparison between fiber optical sensors and commercial sensors

\section{Conclusions}

In this chapter, an optical fiber sensing network of extrinsic Fabry-Perot interferometers and long-period fiber gratings sensors has been developed and validated with laboratory experiments for large strain, high resolution measurements in high temperature environments. The operational principle of the hybrid sensors and three signal processing algorithms were presented, including interference frequency tracking, period tracking, and phase tracking methods. A prototype of the hybrid sensors has achieved strain resolution of $10 \mu \varepsilon$ within a $12 \%$ dynamic range at temperature up to $1472^{\circ} \mathrm{F}\left(800^{\circ} \mathrm{C}\right)$. Through extensive tests on a steel frame in a high temperature environment, the developed fiber optical sensors were validated against commercial temperature and strain sensors in their limited measurement ranges; the optical fiber sensing network was proven effective to monitor the structural condition of the frame in real time. On one hand, one column of the two-column frame structure was subjected to significant inelastic deformation during an earthquake event. On the other hand, the other column of the steel structure may experience extensive 
strains under post-earthquake fires, resulting in a progressive collapse of the structure. Overall, the developed optical sensing network and fiber optical sensors have been demonstrated to be viable devices for the monitoring and behaviour assessment of steel structures under extreme loads such as earthquakes and earthquake-induced fires. They can be employed in practical applications under harsh conditions.

\section{Acknowledgments}

Financial support to complete this study was provided in part by the U.S. National Science Foundation under Award No.CMMI-0825942 and by the Mid-America Transportation Centre under Award No. 0018358. The findings and opinions expressed in this chapter are those of the authors only and do not necessarily reflect the views of the sponsors.

\section{References}

ASTM Committee E20. (1981). Manual on the Use of Thermocouples in Temperature Measurement (STP-470B), pp.28-36, ISBN: 0-8031-0502-9, West Conshohocken: ASTM International, 1981

Bhatia, V. \& Vengsarkar, A. (1996). Optical fibre long period grating sensors, Opt. Lett., Vol. 21, No. 9, (March 1996), pp.692-694.

Corte, G. D.; Landolfo, R. \& Mazzolania, F. M. (2003). Post-earthquake fire resistance of moment resisting steel frames, Fire Safety Journal, Vol. 38, No.7, (November 2003), pp. 593-612.

Easerling, K. E. (1963). High temperature resistance strain gauges, Brit. J. Appl. Phys., Vol. 14,No. 2, (February 1963), pp.79-84.

Gregory, O. J. \& Chen, X. M. (2007). A low TCR nano-composite strain gage for high temperature aerospace applications, IEEE Sensors 2007 Conference, pp.624-627, ISSN 1930-0395, Atlanta, GA, USA, Oct. 28-31 2007.

Habel, W. R.; Hofmann, D.; Hillemeier, B. \& Basedau, F. (1996). Fibre sensors for damage detection on large structures and for assessment if deformation behavior of cementitious materials, Proc. of 11th Engineering Mechanics ASCE-Conference, pp.355358, Fort Lauderdale, FL, USA., 19-22 May, 1996.

Habel, W. R. \& Hillemeier, B. (1998). Non-reactive measurement of mortar deformation at very early ages by means of embedded compliant fibre-optic micro strain sensors, Proc. of 12th Engineering Mechanics ASCE-Conference, pp.799-802, La Jolla, CA, USA., 17-20 May 1998.

Habel, W. R.; Hofmann, D. \& Hillemeier, B. (1997). Deformation measurement of mortars at early ages and of large concrete components on site by means of embedded fibreoptic microstrain sensors, Cement and Concrete Composites, Vol.19, No.1, (May 1998), pp.81-102.

Han, M. \& Wang, A. (2004). Exact analysis of low-finesse multimode fibre extrinsic Fabry-Perot interferometers, Applied Optics, Vol.43, No. 24, (August 2004), pp.4659-4666. 
Huang, Y.; Chen, G.; Xiao, H.; Zhang, Y.; \& Zhou, Z. (2011). A quasi-distributed optical fibre sensor network for large strain and high-temperature measurements of structures, Proc. SPIE, Vol. 7983, (March 2011), pp.17-27.

Huang, Y.; Wei, T.; Zhou, Z.; Zhang, Y.; Chen, G. \& Xiao, H. (2010b). An extrinsic FabryPerot interferometer-based large strain sensor with high resolution, Meas. Sci. Technol., Vol. 21, No. 10, (Sep. 2010), pp.105308.1-8.

Huang, Y.; Zhou, Z.; Zhang, Y.; Chen, G. \& Xiao, H. (2010a). A temperature selfcompensated LPFG sensor for large strain measurements at high temperature, IEEE Trans. Instru. \& Meas., Vol. 50, No.11, (Nov. 2010), pp. 2997 - 3004.

Li, Y. J.; Wei, T.; Montoya, J. A.; Saini, S. V.; Lan, X. W.; Tang, X. L.; Dong, J. H. \& Xiao, H. (2008). Measurement of $\mathrm{CO}_{2}$-laser-irradiation-induced refractive index modulation in single-mode fibre toward long- period fibre grating design and fabrication, Applied Opt., Vol. 47, No.29, (Oct. 2008), pp. 5296-5304.

Liu, T. \& Fernando, G. F. (2000). A frequency division multiplexed low-finesse fibre optic Fabry-Perot sensor system for strain and displacement measurements, Review of Scientific Instruments, Vol.71, No.3, (Nov. 1999), pp.1275-1278.

Mateus, C. F. R. \& Barbosa, C. L. (2007). Harsh environment temperature and strain sensor using tunable VCSEL and multiple fibre Bragg gratings, 2007 SBMO/IEEE MTT-S International Microwave \& Optoelectronics Conference, pp.496-498, ISBN: 978-1-42440661-6, Brazil, Oct. 29 -Nov. 12007

Othonos, A. \& Kalli, K. (1999). Fibre Bragg Gratings: Fundamentals and Applications in Telecommunications and Sensing, ISBN-13: 978-0890063446, Boston: Artech House, June 1999

Qi, B.; Pickrell, G. R.; Xu, J.; Zhang, P.; Duan, Y.; Peng, W.; Huang, Z.; Huo, W.; Xiao, H.; May, G. R. \& Wang, A. (2003). Novel data processing techniques for dispersive white light interferometer, Opt. Engrg., Vol.42, No.11, (April, 2003), pp. 3165-3171.

Schuler, S.; Habel, W. \& Hillemeier, B. (2008). Embedded fibre optic micro strain sensors for assessment of shrinkage at very early ages, International Conference on Microdurability 2008, pp.1377-1387, ISBN: 978-2-35158-065-3, Nanjing, China, 13-15 October 2008

Taylor, H. F. (2008). Fibre Optic Sensor (second edition), pp. 35-64, CRC Press, ISBN: 1420053655, Boca Raton, 2008

Thomson, W. (1857). On the electro-dynamic qualities of metals, Proc. R. Soc., Vol. 8, pp. 54650.

Udd, E. (1991). An Introduction for Scientists and Engineers, Fibre Optic Sensors, New York: John Wiley and Sons, 1991

Vengsarkar, A. M.; Perdrazzani, J. R.; Judkins, J. B.; Lemaire, P. J.; Bergano, N. S. \& Davidson, C. R. (1996). Long-period fibre gratings based gain equalizers, Opt. Lett., Vol. 21, No. 5, (March 1996), pp. 336-338.

Wnuk, V. P.; Méndez, A.; Ferguson, S. \& Graver, T. (2005). Process for mounting and packaging of fibre Bragg grating strain sensors for use in harsh environment applications, Proc. of SPIE, Vol. 5758, (March 2005), pp. 46-54.

Xiao, H.; Zhao, W.; Lockhard, R. \& Wang, A. (1997). Absolute Sapphire optical fibre sensor for high temperature applications, Proc. SPIE., Vol. 3201, (April 1997), pp. $36-42$. 
Zhang, B. \& Kahrizi, M. (2007). High-temperature resistance fibre Bragg grating temperature sensor fabrication, IEEE Sensors Journal, (April, 2007), Vol. 7, No.4, pp. $586-591$.

Zhang, H.; Tao, X. M.; Yu, T. X.; Wang, S. Y. \& Cheng, X. Y. (2006). A novel sensate 'string' for large-strain measurement at high temperature, Meas. Sci. Technol., (February 2006) Vol. 17, No. 2, pp.450-458. 


\title{
Response of Underground Pipes to Blast Loads
}

\author{
A.J. Olarewaju, R.N.S.V. Kameswara and M.A. Mannan \\ Universiti Malaysia Sabah \\ Malaysia
}

\section{Introduction}

Underground structures are divided into two major categories, fully buried structures and partially buried structures regardless of the shape of the structure. Underground cylindrical structures like pipes, shafts, tunnels, tanks, etc. are used for services such as water supply, sewage, drainage, etc. Most structures have now become targets of terrorist attack in recent years. Examples are 1995 Paris subway in France, 2004 Moscow subway is Russia (Dix, 2004; Huabei, 2009), 1995 Alfred Murrah Federal Building in Oklahoma City. The main sources of blast are: terrorist attacks, war, accidental explosion from military formations, etc. The constituents of blast comprises of: 1) rock media, 2) soils, 3) structure, 4) thin-layer elements surrounding the structure; blast loads, and 5) procedure for the analysis of interaction and responses of these constituents. In order to synchronize the interaction and responses of these variables, relevant data is required which could be obtained from field tests, laboratory tests, theoretical studies, work done in related fields and extension of work done in related fields (Ngo et al., 2007; Greg, 2008; Bibiana \& Ricardo, 2008; Olarewaju at al. 2010a).

There are lots of methods available to determine the responses of underground structures to blast loads. These are: i) the analytical methods, and ii) the numerical methods using numerical tools (Ngo et al., 2007; Peter \& Andrew, 2009). The problem of analytical method is that the solution allows only a small elastic response or limited plastic response and does not allow for large deflection and may lead to unstable responses. To overcome these problems, the finite element analysis paves the way towards a more rational blast resistance design. Though the drawback is the time and expertise required in pre- and post-processing for a given structural system. In structural design, the methods of structural analysis and design are broadly divided into three categories, namely, theoretical methods which can be used to carry out analysis and the use of design codes, by testing the full size structure or a scaled model using experimental methods, and by making use of model studies (Ganesan, 2000). There are different types of static and dynamic loads acting on underground pipes. In the case of static loads, there surcharge load on the ground surface due various engineering activities. In the case of dynamic loads, these are cyclic load, earthquake, blast, etc. Blast being one of the dynamic load acting on underground pipes either from surface blast, underground blast, open trench blast or internal explosion is a short discontinuous event.

\section{Background study}

Under blast loading, though typically adopted constitutive relations of soils are elastic, elasto-plastic, or visco-plastic, the initial response is the most important (Huabei, 2009). It 
involves some plastic deformation that takes place within the vicinity of the explosion and as a result of this one could model the soil as an elasto-plastic material. Beyond this region, the soil can be taken as an elastic material at certain distance from the explosion. Viscoelastic soils exhibit elastic behavior upon loading followed by a slow and continuous increase of strain at a decreasing rate (Duhee et al., 2009). In this study, the soil and pipes are considered as linear elastic, homogeneous, isotropic materials (Boh et al., 2007; Greg, 2008). For such materials, Kameswara (1998) has shown that only two elastic constants are needed to study the mechanics/behavior. These can be the usual elastic constants (the Young's modulus, $E$ and Poisson's ratio, $v$ ) or the Lame's constants $(\lambda$ and $\mu)$.

When explosion occurs, surface waves and body waves are generated. Consequent upon these are the isotropic component and deviatory component of the stress pulse (Robert, 2002). Transient stress pulse due to isotropic components causes compression and dilation of soil or rock with particle motion which is known as compression or P-waves. The deviatory component causes shearing of stress with particle velocity perpendicular in the direction to the wave propagation and these are known as shear or S-waves. On the surface of the ground, the particles adopt ellipse motion known as Rayleigh waves or R-waves (Kameswara Rao, 1998; Robert 2002). Energy impulse from explosion decreases for two reasons: (i) due to geometric effect, and (ii) due to energy dissipation as a result of work done in plastically deforming the soil matrix (Dimitiri \& Jerosen, 1999; Huabei, 2009; Omang et al., 2009).

The categories of blast in this study that are applicable to underground pipes are; (i) underground blast, (ii) blast in open trench, (iii) internal explosion inside the pipes as well as (iv) surface blast (Olarewaju et al. 2010b). Blasts can create sufficient tremors to damage substructures over a wide area (Eric Talmadge and Shino Yuasa, 2011). With regards to the severity of destruction of explosion as a result of blast, it has been reported by James (2008) that typical residence structure will collapse by an overpressure of $35 \mathrm{kPa}$ while a blast wave of $83 \mathrm{kPa}$ will convert most large office buildings into rubbles. Accordingly, blast could be thought of as an artificial earthquake. Consequently, there is need to study the relationships and consequences of blasts in underground structures specifically in pipes. This is with a view to designing protective underground structures specifically pipes to resist the effects of blast and to suggest possible mitigation measures.

A lot of works have been done on dynamic soil-structure interaction majorly for linear, homogeneous, and semi-infinite half space soil media. This is contained in Olarewaju et al. (2010a). In this work, observations were limited to displacements at the crown and springline of pipe buried in a soil layer. Effect of slip between the soil and pipe was not considered. Huabei (2009) recently obtained the responses of subway structures under blast loading using the Abaqus finite element numerical software. This study is limited to the determination of the responses of empty underground pipes under blast loads. The material properties are limited to linear, elastic, homogeneous and isotropic materials. It is assumed that blast takes place far away from the vicinity of the underground pipes.

\section{Blast load characteristics and determination}

Explosive has to detonate in order to produce explosive effect. The term detonation as explained in the Unified Facilities Criteria (2008) refers to a very rapid and stable chemical reaction that proceeds through the explosive material at a speed termed the detonation velocity. This velocity ranges from $6705.6 \mathrm{~m} / \mathrm{s}$ to $8534.4 \mathrm{~m} / \mathrm{s}$ for high explosives. The detonation waves rapidly convert the explosive into a very hot, dense, high-pressure gas. 
The volume of the gas of this explosive material generates strong blast waves in air. The pressures behind the detonation front range from $18619 \mathrm{MPa}$ to $33785 \mathrm{MPa}$. Only about onethird of the total energy generated in most high explosives is released in the detonation process. The remaining two-thirds of the energy is released in air more slowly during explosions as the detonation products mix with air and burn.

According to the same source, the blast effects of an explosion are in the form of shock waves composed of high-intensity shocks which expand outward from the surface of the explosive into the surrounding air. As the shock wave expand, they decay in strength, lengthen in duration, and decrease in velocity (Longinow \& Mniszewski, 1996; Remennikov, 2003; Unified Facilities Criteria, 2008). According to the Unified Facilities Criteria (2008), blast loads on structures can be categorized into two main headings; i) unconfined explosions (i. e. free air burst, air burst and surface), ii) confined explosions (i. e. fully vented, partially confined and fully confined).

According to the same source, the violent release of energy from a detonation converts the explosive material into a very high pressure gas at very high temperatures. This is followed by pressure front associated with the high pressure gas which propagates radially into the surrounding atmosphere as a strong shock wave, driven and supported by the hot gases. The shock front, term the blast wave is characterized by an almost instantaneous rise from atmospheric pressure to a peak incident pressure Pso. Over pressure, Pso is the rise in blast pressure above the atmospheric pressure. This pressure increases or the shock front travels radially from the point of explosion with a diminishing shock velocity $U$ which is always in excess of the sonic velocity of the medium. The shock front arrives at a given location at time $t_{A}(\mathrm{~ms})$. After the rise to the peak value of over pressure Pso, the incident pressure decays to the atmospheric value in time $t_{\mathrm{o}}$ (ms - millisecond) which is the positive duration (Olarewaju et al. 2011n).

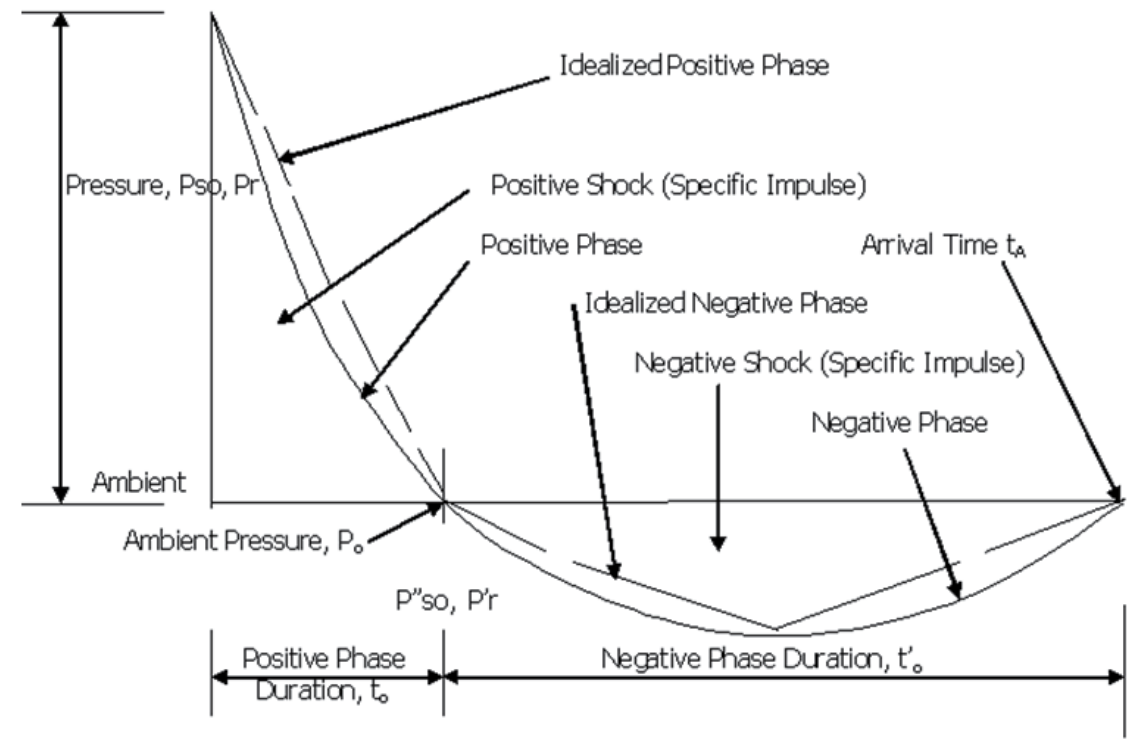

Time after Explosion

Fig. 1. Pressure Time Variation (Unified Facilities Criteria, 2008; Olarewaju et al.2011 and 2011n) 
The negative phase with duration $\mathrm{t}^{-}$( $\mathrm{ms}$ ) is usually longer than the positive phase. It is characterized by a negative pressure (usually below atmospheric pressure) having a maximum value of negative overpressure $\mathrm{Pso}^{-}$as well as reversal of the particle flow. The negative phase is usually less important in design than the positive phase because it is very small and is usually ignored. The incident pulse density (i. e., specific impulse) associated with the blast wave is the integrated area under the pressure-time curve and is denoted by $i_{s}$ for the positive phase and by $i_{s}-$ for the negative phase as illustrated in Fig. 1. An additional parameter of the blast wave, the wave length, is sometimes required in the analysis of structures. The positive wave length $\mathrm{LW}_{W}$ is the length at a given distance from the detonation which, at a particular instance of time, is experiencing positive pressure (Longinow \& Mniszewski, 1996; Remennikov, 2003; Unified Facilities Criteria, 2008). Unified Facilities Criteria (2008) allows for an increase of $20 \%$.

In case of underground blast, most of the energy is spent in fracturing, heating, melting, and vaporizing the surrounding soils and rocks (Johnson \& Sammis, 2001) with only a very small amount being converted to seismic energy. The fraction of the small amount of total energy that goes into seismic energy is a measure of the seismic efficiency of underground explosions. There are three methods available for predicting blast loads on structures. These are: empirical, semi-empirical and numerical methods. Details could be found in Peter and Andrew (2009), Olarewaju (2010), Olarewaju et al. (2010i), (2010j) and (2011p).
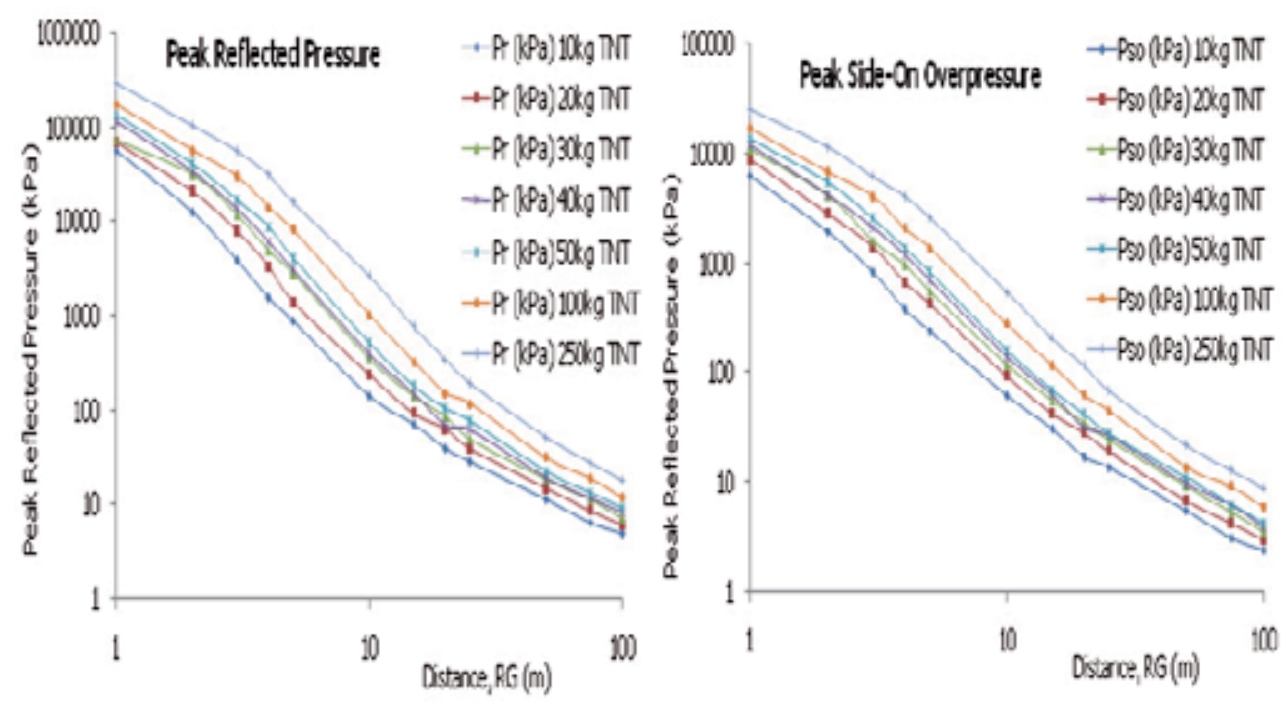

Fig. 2. Peak Reflected Pressure and Peak Side-On Overpressure for Surface Blast (Olarewaju et al. 2010c, 2010e, 2010i) 

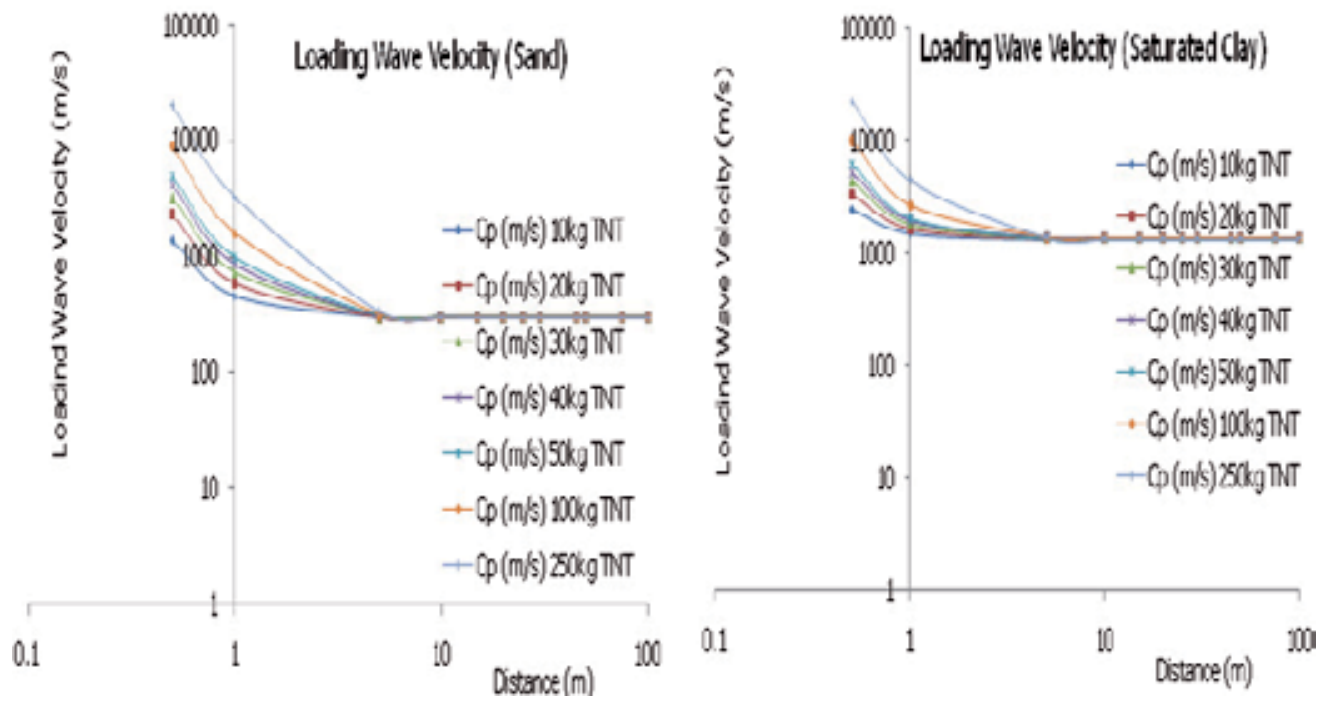

Fig. 3. Loading Wave Velocity for Sand and Saturated Clay for Underground Blast (Olarewaju, et al. 2010c, 2010e, 1020f, 2010i)

Mitigation techniques are meant to reduce the impact of blast and seismic related issues on underground structures. These techniques include: soil stabilization using mechanical and/or additive, grout, ground improvement using i) prefabricated vertical drains, placing soil surcharge and maintaining it for the required time, vacuum consolidation, stone column; ii) chemical modification (with deep soil mixing, jet grouting, etc); iii) densification (using vibro compaction dynamic compaction, compaction grouting, etc), reinforcement (using stone columns, geo-synthetic reinforcement) (Olarewaju, 2004a; 2008b; Raju, 2010; Kameswara, 1998; Olarewaju et al. 2011). Tire-chip backfill has also been used by Towhata \& Sim (2010) to reduce the bending stress and moment caused by displacement of underground pipes. If the thickness of the tire-chip backfilling is increased, it can resist larger displacement caused by blast and thereby reduces the bending stress and the moment caused by large displacements. Similarly, trenchless technique can also be used to rehabilitate damaged underground pipes due to blast, aging, etc. (Randall, 1999) especially in congested and built-up areas.

\section{Methodology}

The existing model studied by Ronanki (1997) was validated using the Abaqus numerical package and the results are compared well. From the results, the crown displacement at H/D=1 is 1.31 times that of crown displacement at $\mathrm{H} / \mathrm{D}=2$. The maximum horizontal sprig-line response in terms of pressure, displacement, maximum principal strain and mises for $H / D=1$ is 1.24 times that of maximum horizontal spring-line response for $H / D=2$. This is in line with the submissions of Roanaki (1997) that "Embedment depth has significant effect on both the crown and spring-line response (deflection). With increase of depth of embedment of pipes, the response (deflection) decreases. The maximum crown response for $H / D=1$ is about 1.3 times that of the maximum crown response (deflection) of $\mathrm{H} / \mathrm{D}=2$. In case of spring-line response (deflection), the maximum horizontal spring-line deflection for $\mathrm{H} / \mathrm{D}=1$ is about 1.2 times that of maximum horizontal spring-line deflection of $\mathrm{H} / \mathrm{D}=2$ ". These results is also in agreement with those reported by Ramakrishan (1979) though no numerical data are presented. 


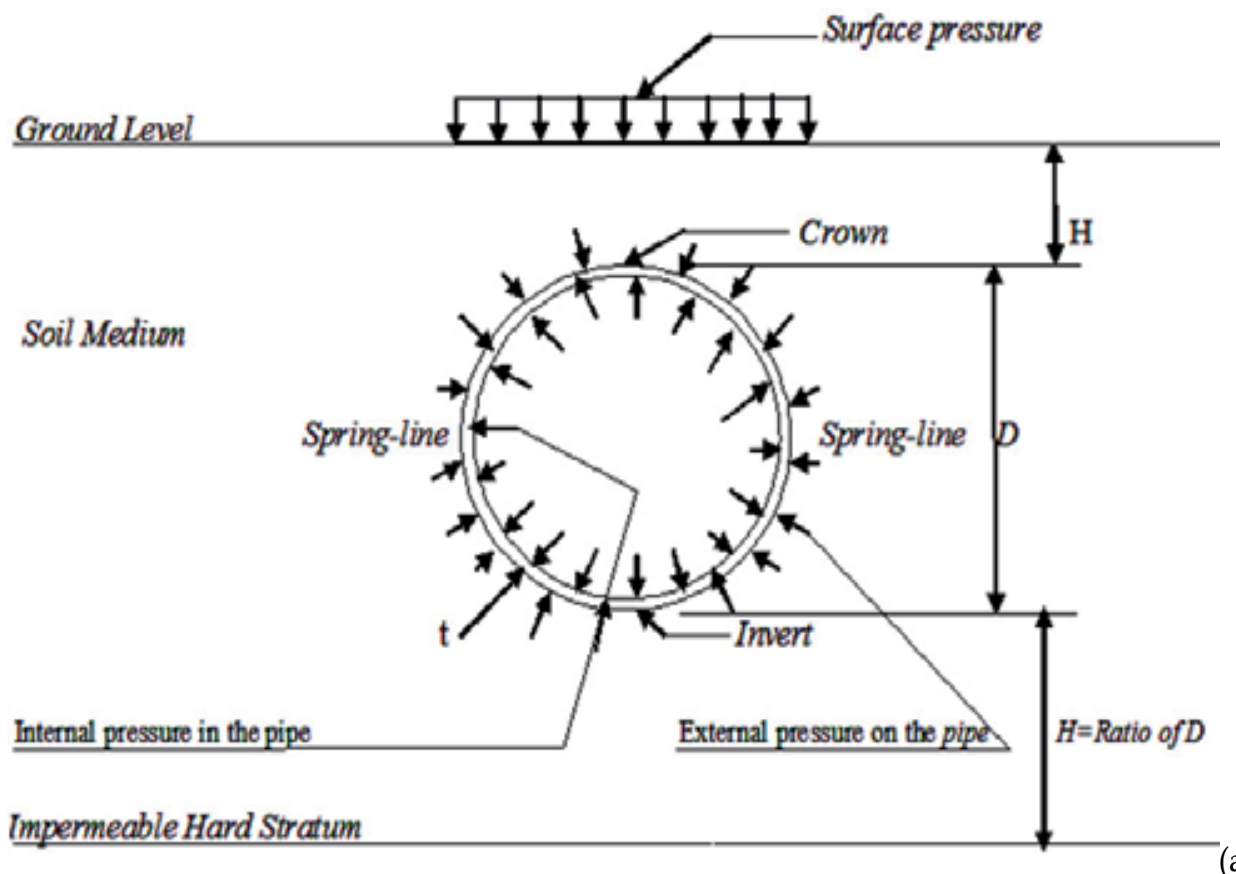

(a)

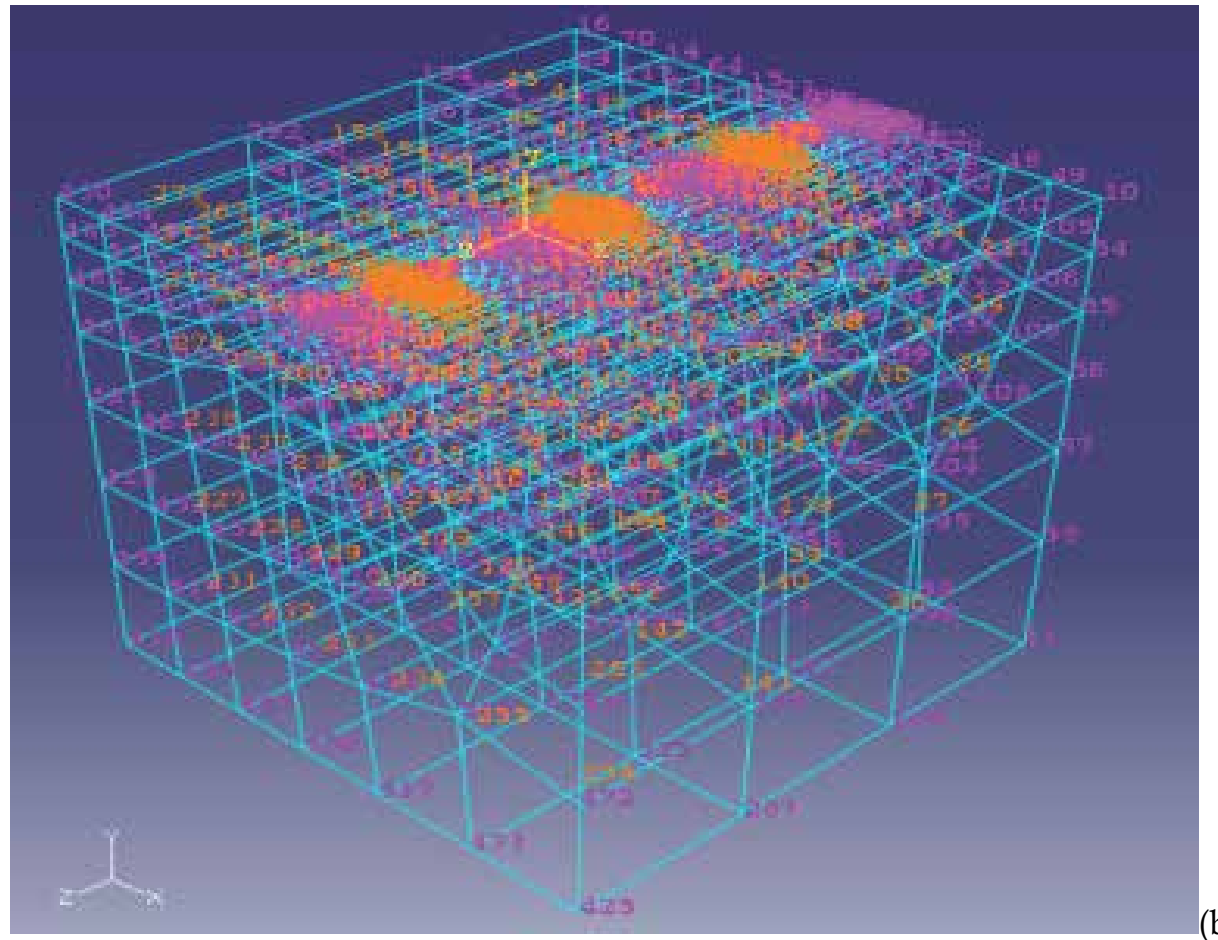

(b)

Fig. 4. (a) Cross-section of underground pipe (Olarewaju et al. 2011n); (b) Finite element model of underground pipe using Abaqus 


\begin{tabular}{|c|c|c|c|}
\hline Material & $\begin{array}{c}\text { Density, } \rho \\
\left(\mathrm{kg} / \mathrm{m}^{3}\right)\end{array}$ & $\begin{array}{c}\text { Young's Modulus, } \\
\mathrm{E}(\mathrm{kPa})\end{array}$ & $\begin{array}{c}\text { Poisson's } \\
\text { Ratio, } \mathrm{v}\end{array}$ \\
\hline Loose sand & 1800 & 18500 & 0.3 \\
Dense sand & 1840 & 51500 & 0.375 \\
Undrained Clay & 2060 & 6000 & 0.5 \\
Intervening medium & 1800 & 18500 & 0.3 \\
Steel pipe & 7950 & $200 \times 10^{6}$ & 0.2 \\
Concrete pipe & 2500 & $20 \times 10^{6}$ & 0.175 \\
\hline
\end{tabular}

Table 1. Material properties for the study

The ground media considered in this study are loose sand, dense sand and undrained clay. The geotechnical properties shown in Table 1 as revealed by several researchers (Das, 1994; FLAC, 2000; Coduto, 2001; Duncan, 2001; Unified Facilities Criteria, 2008; Kameswara, 1998; etc) were used to study the response of underground pipes due to blast loads. Since the two elastic constants are enough to study the mechanics of an elastic body, the material properties used are the modulus of elasticity, E, Poisson's ratio and density of soil and pipe materials. The largest possible value of Poisson's ratio is 0.5 and is normally attained during plastic flow and this signifies constancy of volume (Chen, 1995). Huabei (2009) pointed out that undrained behavior is relevant for saturated soft soils especially clay that is subjected to rapid blast loading since the movement of pore water is negligible under such circumstance. For $10 \mathrm{~kg}, 20 \mathrm{~kg}, 30 \mathrm{~kg}, 40 \mathrm{~kg}, 50 \mathrm{~kg}, 100 \mathrm{~kg}$ and $250 \mathrm{~kg}$ explosives, Unified Facilities Criteria (2008) was used to predict positive phase of blast loads at various stand-off point for surface blast and results are presented in Figs 2. Analytical method was used to predict the blast load for underground blast at various stand-off points and results presented in Figs. 3 . According to Huabei (2009), it is not likely for terrorists to use very large amount of explosive in an attack targeting underground pipes. Soil model in the problem definition shown in Figs. $4(\mathrm{a}, \mathrm{b})$ of $100 \mathrm{~m}$ by $100 \mathrm{~m}$ by $100 \mathrm{~m}$ depth consist of buried pipe $100 \mathrm{~m}$ long and $1 \mathrm{~m}$ diameter buried at various embedment ratios were study for the various categories of blast applicable to underground pipes. Parametric studies were carried out for various blasts. Blast load duration was verified and it was observed that, for response to take place in underground pipe, most especially pipes buried in loose sand, duration of blast should be greater than 0.02s (Olarewaju, et al 2011n).

\section{Method of analysis}

Abaqus package was used to solve the equations of motion of the system:

$$
[\mathrm{m}][\ddot{U}]+[\mathrm{c}][\dot{U}]+[\mathrm{k}][\mathrm{U}]=[\mathrm{P}]
$$

with the initial conditions:

$$
\mathrm{U}(\mathrm{t}=0)=\mathrm{U}_{\mathrm{o}} \text { and } \dot{U}(\mathrm{t}=0)=\dot{U}_{\mathrm{o}}=\mathrm{v}_{\mathrm{o}}
$$

where $\mathrm{m}, \mathrm{c}$, and $\mathrm{k}$ are the global mass, damping and stiffness matrices of the pipes system and $t$ is the time. $U$ and $P$ are displacement and load vectors while dot indicate their time derivatives. The time duration for the numerical solution (Abaqus Analysis User's Manual, 2009) was divided into intervals of time $\Delta t=h$, where $h$ is the time increment. Finite difference in Abaqus/Explicit was used to calculate the response (Abaqus Analysis User's 
Manual, 2009). Stability limit is the largest time increment that can be taken without the method generating large rapid growing errors (Abaqus Analysis User's Manual, 2009; Abaqus/Explicit: Advanced Topics, 2009). The difficulty is that the accuracy of the sensitivities can depend on the number of elements. This dependency is not seen with either analytical sensitivity analysis or with the overall finite difference method (explicit). Sensitivity analysis is not required in Finite difference of Abaqus/Explicit because. According to Abaqus Analysis User's Manual (2009), the default value of perturbation has been proved to provide the required accuracy in Abaqus /Standard.

Boundary condition of the model was defined with respect to global Cartesian axes in order to account for the infinite soil medium (Geoetchnical Modeling and Analysis with Abaqus, 2009; Ramakrishan, 1979; Ronanki, 1997). Contrary to our usual engineering intuition, introducing damping to the solution reduces the stable time increment. However, a small amount of numerical damping is introduced in the form of bulk viscosity to control high frequency oscillations (Abaqus Analysis User's Manual, 2009; Geoetchnical Modeling and Analysis with Abaqus, 2009). Estimation of blast load parameters could be done by empirical method, semi-empirical methods and numerical methods. The method to be adopted depends on the numerical tool available for the study of response of underground structures to blast loads. In this study, empirical method using Unified Facilities Criteria (2008) was used. The blast load parameters to be determined using this method depend on the available numerical tool. According to Unified facilities Criteria (2008), pressure is the governing factor in design and the study of the response of underground structures. Load due to surface blast was represented by pressure load with short duration (in millisecond, $\mathrm{ms}$ ) while load due to underground blast was represented by loading wave velocity with short load duration (in millisecond, ms).

\section{Results and discussion}

\subsection{Response of underground pipes to surface blast}

The blast load was represented by pressure load (Figures 1 and 2) whose centre coincide with the centre of the explosive. The pressure load reduces to zero at $0.025 \mathrm{~s}$. At low pressure load due surface blast, there was no response observed on underground pipe. Due to surface blast, it was observed that crown, invert and spring-line displacement reduces as embedment ratios increases in loose sand, dense sand and undrained clay. This is shown shown in Figs. 5. Crown, invert and spring-line pressures, stresses and strains increase at embedment ratios of 2 and 3 after which it reduces as the embedment ratios increases.

For steel pipe at $\mathrm{H} / \mathrm{D}=1$, crown and invert displacement in loose sand is the highest and least in undrained clay. This is in agreement with the findings of Huabei (2009), that increasing the burial depth enhances the confinement of underground pipe, hence reduces the maximum lining stress under internal blast loading (Huabei, 2009). The results indicate that it is necessary to evaluate the blast-resistance of underground structures with small burial depth. Materials yield easily and more at lower depth of burial (Huabei, 2009).

With small burial depth, due to low confinement from ground, displacement, pressure, stress and strain could be significantly large and underground structures like pipes could be severely damaged even with moderate surface blast, underground blast and open trench blast (Olarewaju et al 2010c). According to James (2009), the effect of varying the depth of burial of structures below the ground level is an important phenomenon to study. The depth of soil cover above the increases the over burden stresses on it, which can help in stabilizing 
it with respect to its response to an externally applied impulsive action. This can help in reduction of the vibrations which occur in response to an explosive blast action.

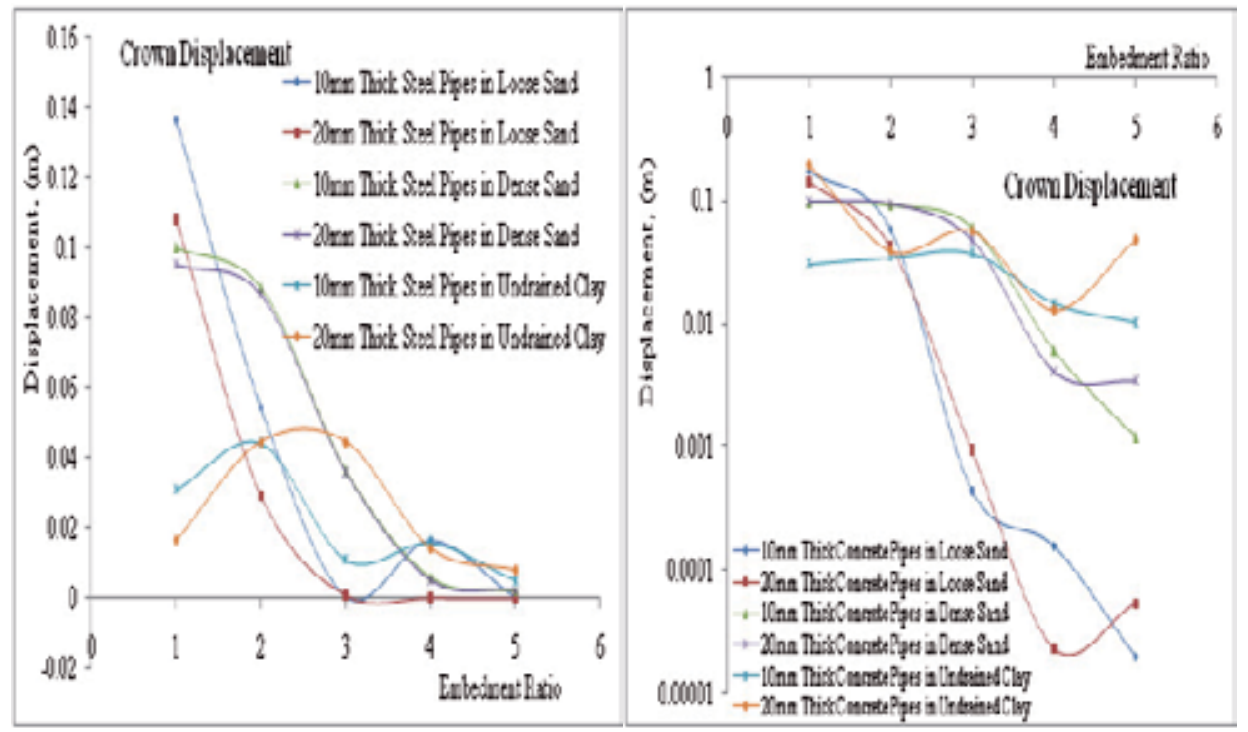

(a) Crown displacement (steel pipe)

(b) Crown Displacement (concrete pipe)

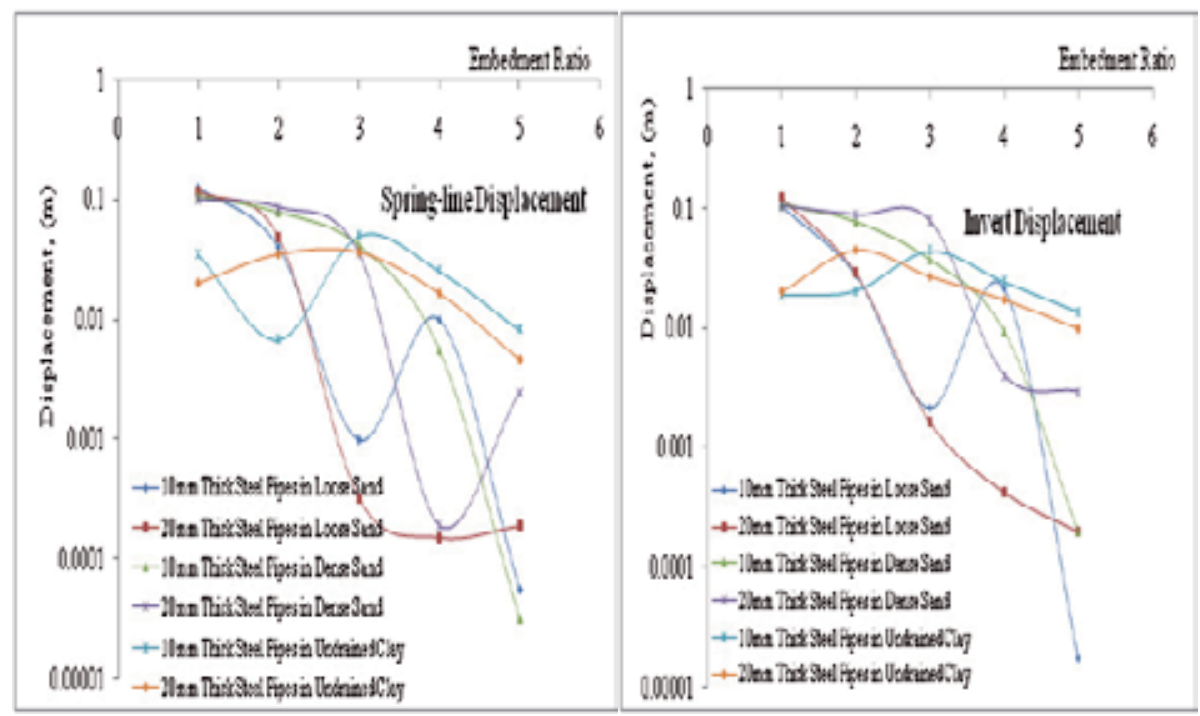

(c) Spring-line displacement

(d) Invert displacement

Fig. 5. Displacement in underground pipes due to surface blast 


\subsection{Response of underground pipes to underground blast}

The blast load was represented by loading wave velocity (Figures 1 and 3) which reduces to zero at $0.025 \mathrm{~s}$. For a given loading wave velocity, crown, invert and spring-line displacements in pipes is almost constant at all the embedment ratios considered irrespective of the material properties. This is higher compared to that obtained in open trench blast. This is because, as the peak particle velocity due to underground blast travels within the soil medium, it transmits the load bodily to the buried pipes along the direction of travel. As a result of this, displacement is bound to be higher compared to open trench blast where the wave energy only impeaches on the side of the trench.

Reduction in pressure, stress and strain is noticeable at embedded ratios of 3 to 5 . This is in agreement with the submission of Ronanki (1997) on the effect of seismic/loading wave velocity that, the spring-line horizontal displacement remains almost constant with increasing mode shape number. The vertical crown displacement increases with mode shape number up to a value 15, beyond that the displacement tends to be constant (Ronanki, 1997). Finally, crown, invert and spring-line pressures, stresses and strains in pipes showed wide variation as the embedment ratio increases in all the soil media considered. Though there is reduction in all these parameters as the embedment ratio increases (Olarewaju et al. 2010f).

\subsection{Response of underground pipes to open trench blast}

The blast load was represented by pressure load (Figures 1 and 2) which reduces to zero at 0.025s. Displacement (Figs. 6) in pipes due to open trench blast is lower compared to that obtained in underground blast. In addition, virtually all the parameters observed i. e. displacement, pressure, stress and strain at the crown, invert and spring-line of pipes reduces at embedment ratios of 3 beyond which no significant changes occurred. Finally, crown, invert and spring-line displacements, pressures, stresses and strains reduce as the embedment ratio increases with a sharp increase at embedment ratio of 2 in all the ground media considered (Olarewaju et al. 2010e). Increasing the burial depth of underground pipe enhances the confinement on the underground pipe, hence reduces the maximum displacement, pressure, stress and strain under blast loading (Huabei, 2009). Details could be found in Olarewaju et al $(2010 b)$

\subsection{Response of underground pipes to internal explosion}

The blast load was represented by pressure load (Figures 1 and 2) whose centre coincide with the centre of the explosive. The pressure load reduces to zero at $0.025 \mathrm{~s}$. The result shows that as the diameter of pipes increases, blast load parameters generated inside the pipe increases. As the thickness of pipes reduces, time history as a result of internal explosion increases in the same proportion. In addition to this, depth of burial of pipes showed no significant changes in the time history of external work and energies generated due to internal explosion (Olarewaju et al 2010d and 2010l). Furthermore, stress components on the ground surface reduced as the depth of embedment of pipes increases. Equivalent earthquake parameters on the surface of the ground due to $50 \mathrm{~kg}$ TNT explosion in pipe are higher than that recorded in San Fernando earthquake of 1971 (Robert, 2002). Finally, pressure changes from negative to positive within the soil medium due to dilations and compressions caused by the transient stress pulse of compression wave while velocity, displacement and stresses reduce as it approaches the ground surface. This reduction is more in loose sand than undrained clay due to arching effect (Craig, 1994). Details could be found in Olarewaju et al. (2010d). 


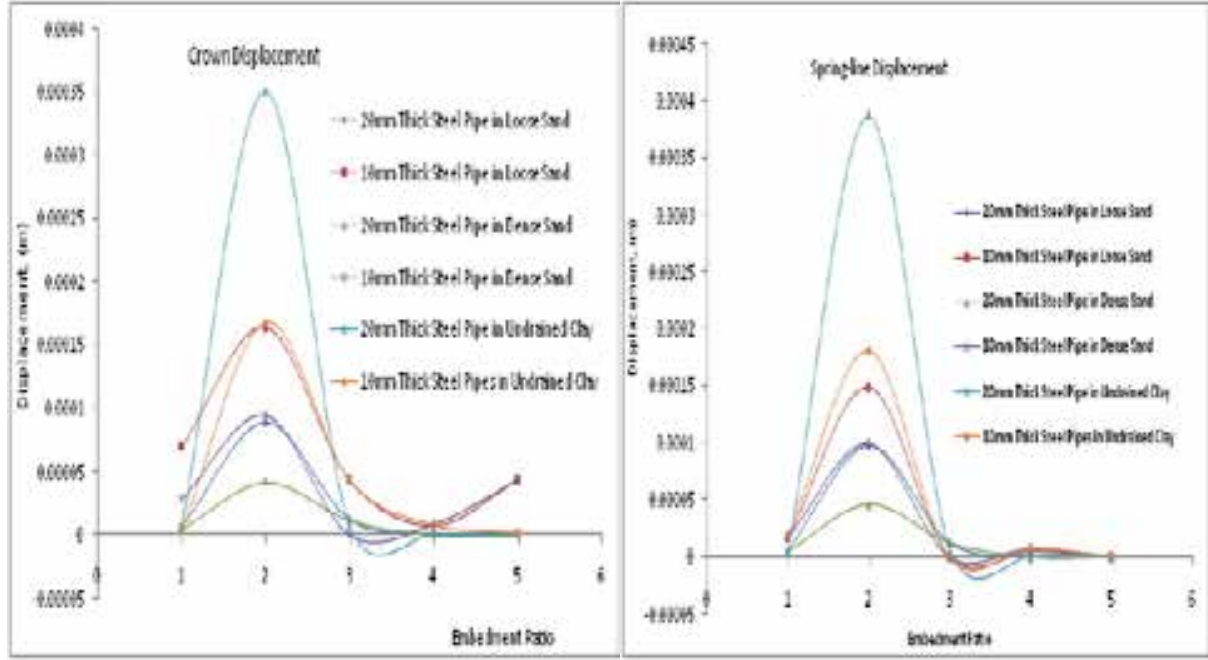

(a) Crown displacement

(b) Spring-line displacement

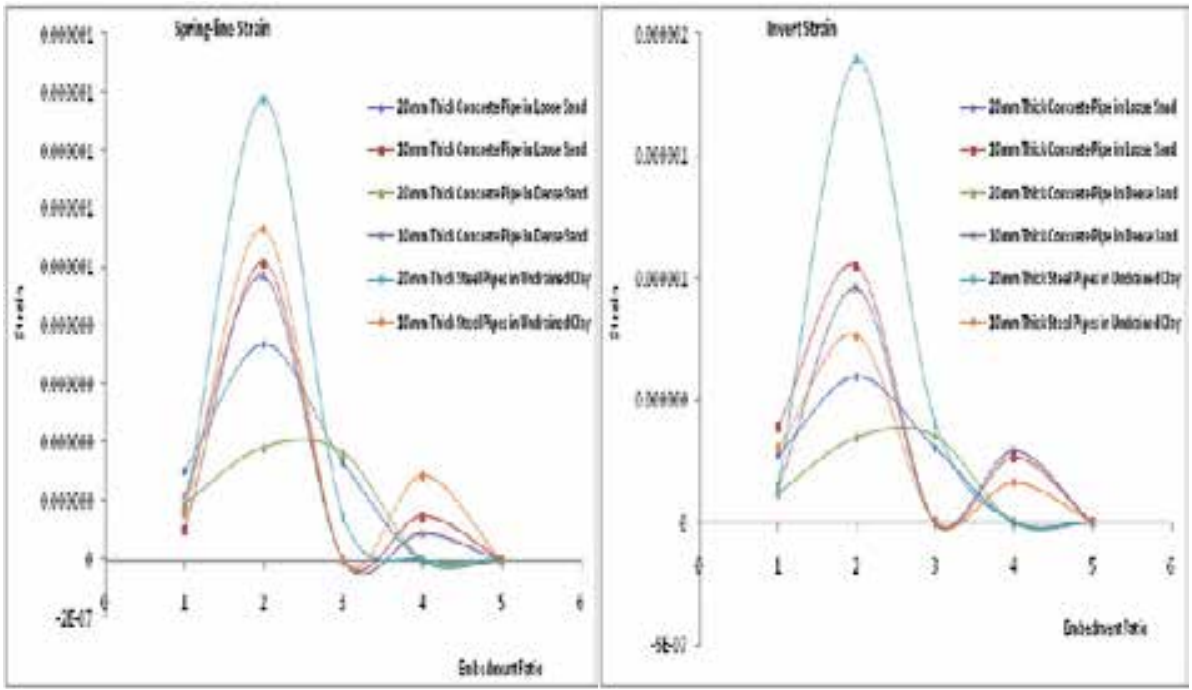

(c) Spring-line strain

(d) Invert strain

Fig. 6. Displacement and Strain in pipes in open trench blast 


\section{Parametric studies}

\subsection{Effects of coefficient of friction}

Due to surface blast, displacement at the crown reduces at coefficient of friction of 0.2 to 0.4 and above in dense sand. The reverse is the case in loose sand where displacement increases as the coefficient of friction increases. Invert displacement reduces as the coefficient of friction increases. Spring-line displacement increases as the coefficient of friction increases. Due to the dynamic nature of surface blast loads, there is wide variation in the results; there is reduction in the values of crown, invert and spring-line pressures, stresses and strains for coefficient of friction of 0.2 to 0.4 . This is also noticeable for the increased values of peak reflected pressure. Liang-Chaun (1978) pointed out that in cases when test data are not available, the following friction coefficient can be used: Silt $=0.3$; Sand $=0.4$; Gavel $=0.5 \mathrm{~m}$ and added that the above coefficients are the lower bond values equivalent to the sliding friction. The static and dynamic coefficient of friction can be as much as $70 \%$ higher.

\subsection{Effects of young's modulus of soil}

Effects of liquefaction as observed in the varying Young's modulus for soil for surface blast and underground blast is similar to the varying Young's modulus for intervening medium. Varying the Young's modulus, E of soil, displacement became higher at E of $1 \times 10^{6} \mathrm{~Pa}$. Between Young's modulus, E of $10000 \mathrm{~Pa}$ and $3000000 \mathrm{~Pa}$, pressure, stress and strain get to the peak value with maximum value at $\mathrm{E}$ of $1000000 \mathrm{~Pa}$. Crown has the maximum values of stress and strain while invert has the maximum pressure. With the value of Young's modulus, E soil ranging from $0 \mathrm{~Pa}$ to $10000 \mathrm{~Pa}$, displacement, pressure, stress and strain (Figs 7) reduce with no substantial increase. This is in agreement with the submission of Susana \& Rafael (2006). From the result of the work by Huabei (2009), it showed that as Young's modulus of soil is increasing, mises stress is reducing. For the constant value of stress with increasing value of Young's modulus, E of soil, it shows that the soil has yielded.

\subsection{Effects of young's modulus of pipes}

Displacement is high at the crown but low at the invert and spring-line of pipes having low value of Young's modulus. At higher Young's modulus, the displacement at the crown, invert and spring-line became the same. Pressure and stress is low at low Young's modulus but increases as the Young's modulus increases. Large strain is observed between the values of $100 \mathrm{~Pa}$ and $10000 \mathrm{~Pa}$ beyond which the value of strain reduces. Low stiffness pipes are pvc pipes, clay pipes, etc while high stiffness pipes are steel pipes, reinforced concrete pipes, etc. It is evident that as the Young's modulus E of pipes increases, strain reduced due to increased stiffness but the pressure and stress increases from E of $1 \times 10^{7} \mathrm{~Pa}$. This shows that pipes of lower value of $\mathrm{E}$ have lower displacement, pressure, stress and strain induced in them due to surface blast compared to pipes with higher stiffness like steel and reinforced concrete pipes.

The result presented by Frans (2001) clearly shows that the low stiffness pipes suffer less from subsidence than the one with the higher stiffness. At the same time a higher deflection is observed when using low stiffness pipes. This proves that rigid pipes transfer load, and flexible pipes deform and the load is transferred by the soil. When the bed is firm, hardly any subsidence takes place hence the stiffness of the pipe has no effect either. However, when the bed is loose or soft, subsidence becomes a real issue and also the effect of pipe stiffness is significant. 


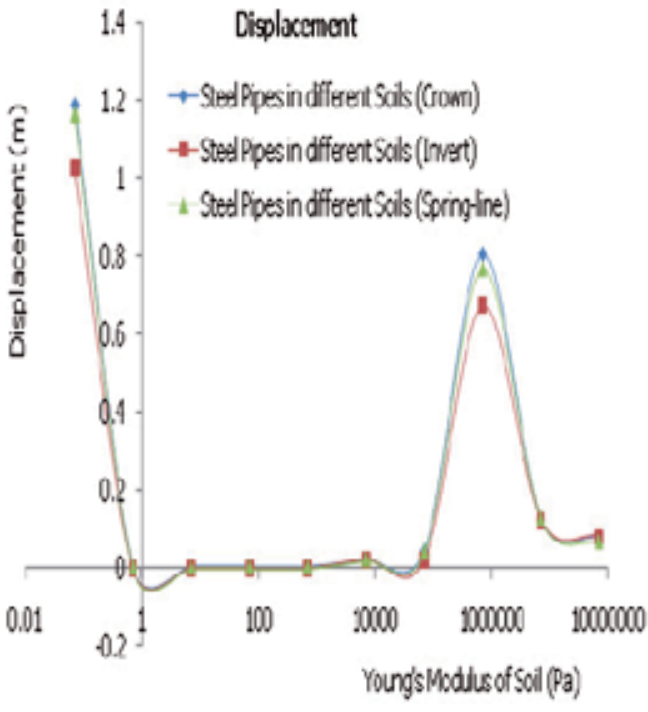

(a) Displacement

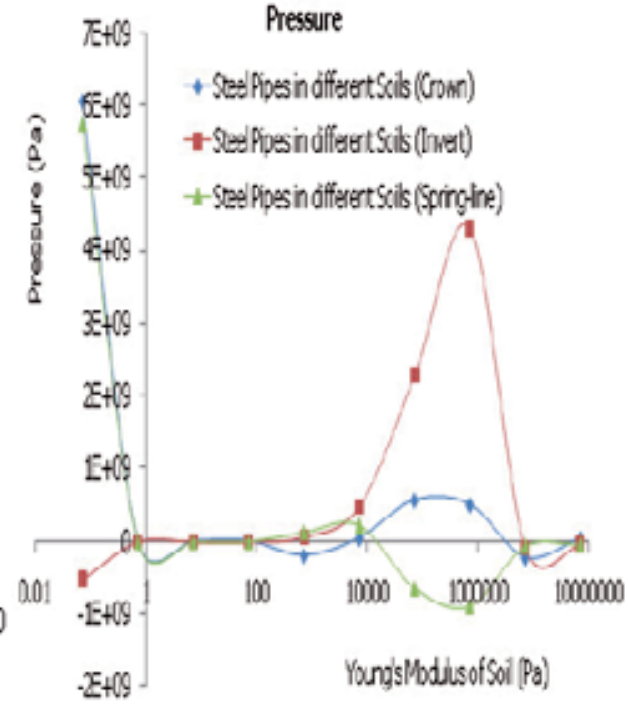

(d) Pressure

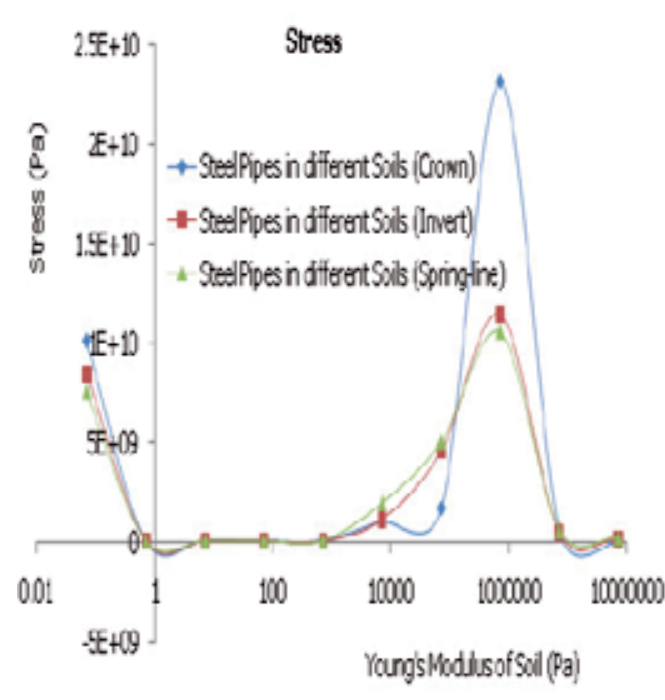

(a) Stress

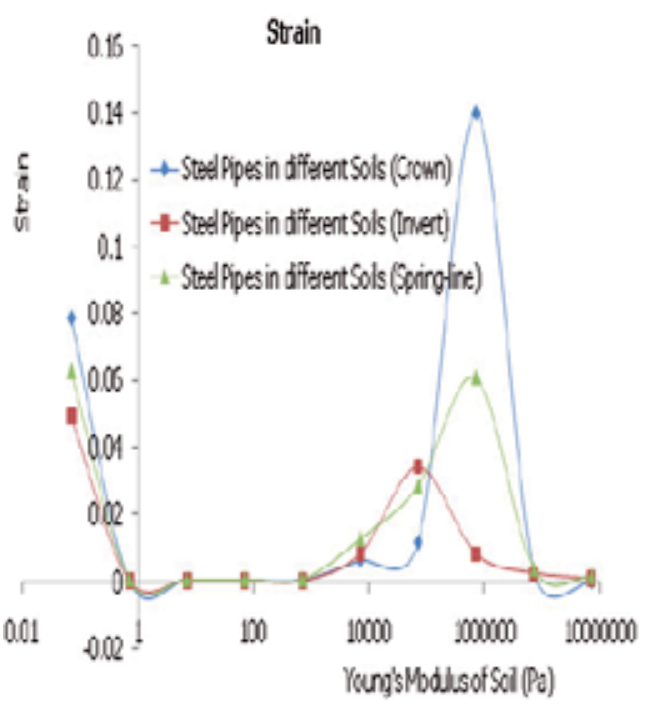

(d) Strain

Fig. 7. Displacement, Pressure, Stress and Strain in buried pipes for varying Young's modulus of soil for surface blast 


\subsection{Effects of young's modulus of pipes}

Displacement is high at the crown but low at the invert and spring-line of pipes having low value of Young's modulus. At higher Young's modulus, the displacement at the crown, invert and spring-line became the same. Pressure and stress is low at low Young's modulus but increases as the Young's modulus increases. Large strain is observed between the values of $100 \mathrm{~Pa}$ and $10000 \mathrm{~Pa}$ beyond which the value of strain reduces. Low stiffness pipes are pvc pipes, clay pipes, etc while high stiffness pipes are steel pipes, reinforced concrete pipes, etc. It is evident that as the Young's modulus E of pipes increases, strain reduced due to increased stiffness but the pressure and stress increases from $\mathrm{E}$ of $1 \times 10^{7} \mathrm{~Pa}$. This shows that pipes of lower value of $E$ have lower displacement, pressure, stress and strain induced in them due to surface blast compared to pipes with higher stiffness like steel and reinforced concrete pipes.

The result presented by Frans (2001) clearly shows that the low stiffness pipes suffer less from subsidence than the one with the higher stiffness. At the same time a higher deflection is observed when using low stiffness pipes. This proves that rigid pipes transfer load, and flexible pipes deform and the load is transferred by the soil. When the bed is firm, hardly any subsidence takes place hence the stiffness of the pipe has no effect either. However, when the bed is loose or soft, subsidence becomes a real issue and also the effect of pipe stiffness is significant.

\subsection{Effects of pipe thickness}

The result indicates that steel and concrete pipes show similar characteristics and behavior in thickness. In other words, as the thickness of pipes increases, displacement, pressure, stress and strain reduces. At low pipe thickness, displacement, stress and strain in steel and concrete pipes buried in undrained clay, is low at the invert but remain constant at the crown, invert and spring-line as the thickness increases. According to James (2009), the size and thickness of the structure under consideration is a major factor which can potentially influence the stresses generated on it. The reason could be attributed to the fact that smaller size structure has lower mass, making it easier to displace under blast loadings.

Higher displacements in the structure can result in larger strain deformations, causing the corresponding stresses to be lower due to energy dissipation in deforming the structure. According to Zhengwen (1997), rigid structures experience higher pressure and less displacement during the first half-wave of response, when compared with more flexible counterparts. In that case, underground pipes with smaller thickness are considered as flexible while those with increased thickness are considered as rigid structures.

\section{Conclusions}

Blast is a short discontinuous event whose duration is very small compared to earthquake. Considering the various constituent of blast, ground pipes and intervening media can be modeled. It must be remembered that soil exists as semi-infinite medium. Numerical tool to be used must incorporate the notion of infinite in the formulation. To account for the infinity of soil medium, in this study, in the absence of infinite element, Global Cartesian axis in Abaqus software was used. In other words, it shows that soil is a continuous media. To account for material damping, small numerical damping in the form of bulk viscosity was introduced. Blast and/or blast parameters can be represented or modeled using software (i. e. BLASTXW, SPLIT-X, BLAPAN, SPIDS, etc) or by using Eulerian numerical techniques 
developed using finite volume and finite difference solver (i. e. SHAMRC, ANSYS, AUTODYN 2D AND 3D, etc) (Olarewaju et al., 2010i).

To represent blast load parameters, it can to be determined by empirical method using available code like Technical Manual 1990, Unified Facilities Criteria 2008, etc (Unified Facilities Criteria 2008 supersede other available technical manual). In this study, blast load parameters were estimated using empirical method, (i. e. Unified facilities Criteria (2008)) and represented in the model. Other blast load parameters applicable to the design and study of response underground pipes to blast loads that can be estimated by empirical method are: peak reflected pressure, side-on overpressure, specific impulse, horizontal and vertical acceleration, horizontal and vertical displacement, shock front velocity, horizontal and vertical velocity, duration, arrival time, etc (Olarewaju et al. 2010a; 2010i). To capture the short duration of blast load, time integration technique in Abaqus/Explicit was used in this study.

Conclusively, this study has shown the various responses of underground pipes due to various blasts scenarios. Results of parametric studies were also presented and discussed. Finally, possible mitigation measures were also suggested. Consequently, the parameters thus obtained will help in designing underground pipes to resist effects of various blast loads.

\section{Acknowledgment}

The financial supports provided by Ministry of Science Technology and Innovation, MOSTI, Malaysia under Universiti Malaysia Sabah (UMS) e-Science Grant no. 03-01-10-SF0042 is gratefully appreciated.

\section{References}

Abaqus Inc. (2009). Abaqus Analysis User's Manuals - Documentation, Dassault Systemes Simulia, Providence, Rhode Island, USA

Abaqus Inc. (2009). Geotechnical Modeling and Analysis with Abaqus, Dassault Systemes Simulia, Providence, Rhode Island, USA

Bibiana, M. Luccioni, and D. Ricardo Ambrosini, (2008). Evaluating the effect of underground explosions on structures, Asociacion Argentina de Mecanica Computacional, Mecanica Computacional XXVII, Noviembre, pp 1999-2019

Boh, J. W., Louca, L. A. and Choo, Y. S. (2007). Finite Element Analysis of Blast Resistance Structures in the Oil and Gas Industry, Singapore and UK, ABAQUS User's Conference, pp 1-15

Chen, W. F. (1995). The Civil Engineering Handbook, CRC Press, London. 1386 Coduto, D. P. (2001). Foundation Design: Principles and Practices (2nd edition), Prentice Hall, Inc., New Jersey, 883

Craig, R. F. (1994). Soil Mechanics, (5th edition), Chapman and Hall, Great Britain Das, B. M. (1994). Principles of Geotechnical Engineering (3rd edition), PWS Publishing, Co., Boston, Massachusetts, 672

Dix, A. (2004). Terrorism - the new challenge for old tools. Tunnels and Tunneling International 36 (10): pp 41-43

Duncan, J. M. (2001). CEE 5564: Seepage and Earth Structure, Course Notes, Spring 2001, Virginia Tech, Blacksburg, Virginia 
Dimitri Komatitsch and Jerosen Tromp (1999). Introduction to the spectral element method for three dimensional seismic wave propagation, Geophys. J. Int., 139, pp 806-822

Duhee P., Myung S., Dong-Yeop K. and Chang-Gyun J., (2009). Simulation of tunnel response under spatially varying ground motion, International Journal Soil Dynamics and Earthquake Engineering, Science Direct Ltd Eric Talmadge and Shino Yuasa, 2011. Stricken Japan nuclear plant rocked by 2nd blast.

Fukushima Dai-Ichi Nuclear Plant Plagued By Cooling Issues The Associated Press, 14 March. http://www.news4jax.com/nationalnews/27184574/detail.html

FLAC User's Manual (2000). Version 4.0. Itasca Consulting Group, Inc., Minneapolis, Minnesota

Frans Alferink. (2001). Soil-Pipe Interaction: A next step in understanding and suggestions for improvements for design methods, Waving $M \& T$, The Netherlands, Plastic Pipes XI, Munich, 3rd-6th September

Ganesan, T. P. (2000). Model of Structures (1 $1^{\text {st }}$ edition), University Press Ltd., India, ISBN: $817371123-2$

Greg B. C. (2008). Modeling Blast Loading on Reinforced Concrete Structures with Zapotec,Sandia National Laboratories, Albuquerque, ABAQUS User's Conference

Huabei Liu, (20090. Dynamic Analysis of Subways Structures under Blast Loading,University Transportation Research Center, New York, USA

James A. Marusek, (2008). Personal Shelters, Abaqus User's Conference, US Department ofthe Navy

Johnson, L. R. and Sammis, C. G. (2001). Effects of rock damaged on seismic wavesgenerated by explosions. International Journal of Pure Applied Geophysics, 158, pp 1869-1908

Kameswara Rao, N. S. V. (1998). Vibration Analysis and Foundation Dynamics (1 ${ }^{\text {st }}$ edition),Wheeler Publishing Co. Ltd., New Delhi, India, ISBN: 81-7544-001-5

Liang-Chaun Peng. (1978). Soil-pipe interaction - Stress analysis methods for undergroundpipelines, AAA Technology and Specialties Co., Inc., Houston, Pipeline Industry, May, 67-76

Longinow, A. and Mniszewski, R. K. (1996). Protecting buildings against vehicle bombattacks, Practice Periodical on Structural Design and Construction, ASCE, New York, pp 51-54

Mosley, W. H. and Bungey, J. H. (1990). Reinforced Concrete Design (4th edition), ELBS, withMacmillan, Hampshire

Neil, J. and Ravindra, K. D. (1996). Civil Engineering Materials, (5 $5^{\text {th }}$ edition), MacmillanPress Ltd., London

Ngo, T. J., Mendis, J., Gupta, A. and Ramsay, J. (2007). Blast Loading and Blast Effects onStructures - An Overview, University of Melbourne, Australia, International Journal of Structural Engineering, EJSE, International Special Issue: Loading on Structures, pp 76-91

Olarewaju, A. J., Kameswara Rao, N.S.V and Mannan, M.A., (2010a). Response ofUnderground Pipes due to Blast Loads by Simulation - An Overview, Journal of Geotechnical Engineering, EJGE, (15/G), June, pp 831-852, ISSN 1089-3032

Olarewaju, A. J., Kameswara Rao, N.S.V and Mannan, M.A., (2010b). Guidelines for theDesign of Buried Pipes to Resist Effects of Internal Explosion, Open Trench and Underground Blasts, Journal of Geotechnical Engineering, EJGE, (15/J), July, pp 959-971, ISSN 1089-3032

Olarewaju, A. J., Kameswara Rao, N.S.V and Mannan, M.A., (2010c). Blast Effects onUnderground Pipes, Journal of Geotechnical Engineering, EJGE, (15/F), May, pp 645-658, ISSN 1089-3032 
Olarewaju, A. J., Kameswara Rao, N.S.V and Mannan, M.A., (2010d), Behaviors of BuriedPipes due to Internal Explosion, Malaysia Construction Research Journal, MCRJ, September, (in press)

Olarewaju, A. J., Kameswara Rao, N.S.V and Mannan, M.A., (2010e), Design Hints forBuried Pipes to Resist Effects of Blast, Proceedings of Indian Geotechnical Conference (IGC), ISBN 13: 978-0230-33211-9, ISBN 10: 0230-33207-2, Indian Institute of Technology, Powai, Mumbai, India, Macmillan Publishers India Ltd., December $16^{\text {th }}-18^{\text {th }}$, Published in 2011, pp 881-884

Olarewaju, A. J., Kameswara Rao, N.S.V and Mannan, M.A., (2010f), Response ofUnderground Pipes Due to Underground Blast, Proceedings of the International Agricultural Engineering Conference on Innovation, Cooperation and Sharing, Chinese Academy of Agricultural Mechanization Sciences and Shanghai Society for Agricultural Machinery, Shanghai, China, September 17th-20th, pp (I) 321-329

Olarewaju, A. J., Kameswara Rao, N.S.V and Mannan, M.A., (2010g), Behavior of BuriedPipes Due to Surface Blast Using Finite Element Method, Proceedings of $1^{\text {st }}$ Graduate Student Research International Conference, Brunei, on Contributions Towards Environment, Bio-diversity and Sustainable Development, Universiti Brunei Darussalam, December $13^{\text {th }}-15^{\text {th }}$, pp 17 (1-6)

Olarewaju, A. J., Kameswara Rao, N.S.V and Mannan, M.A., (2011h), Response ofUnderground Pipes Due to Surface Blast Using Finite Element Method, Proceedings of International Soil Tillage Research Organization (ISTRO) - Nigeria Symposium on Tillage for Agricultural Productivity and Environmental Sustainability, University of Ilorin, Ilorin, Nigeria, February 21st $-24^{\text {th }}, 241-251$

Olarewaju, A. J., Kameswara Rao, N.S.V and Mannan, M.A., (2010i). Blast Prediction andCharacteristics for Simulating the Response of Underground Structures, Proceedings of the $3^{\text {rd }}$ International Conference of Southeast Asian on Natural Resources and Environmental Management (3-SANREM), ISBN: 978-983-2641-59-9, August $3^{\text {rd }} 5^{\text {th }}$, Universiti Malaysia Sabah, Malaysia, pp $384-391$

Olarewaju, A. J. (2010j). Blast Effects on Underground Pipes, SKTM (School of Engineeringand Information Technology) PG Newsletter, Universiti Malaysia, Sabah, Special Ed. (1/1), July, pp 5, ISSN 2180-0537

Olarewaju, A. J., Kameswara Rao, N.S.V and Mannan, M.A., (2011k), Blast Effects onUnderground Pipes Using Finite Element Method, Proceedings of $12^{\text {th }}$ International Conference on Quality in Research, ISSN: 114-1284, Faculty of Engineering, University of Indonesia, Bali, Indonesia, July $4^{\text {th }}-7^{\text {th }}$, (in press)

Olarewaju, A. J., Kameswara Rao, N.S.V and Mannan, M.A., (20101), Response ofUnderground Pipes due to Blast Load, Proceedings of the $3^{\text {rd }}$ International Earthquake Symposium Bangladesh, ISBN: 978-984-8725-01-6, Bangladesh University of Engineering Technology, Dhaka, $4^{\text {th }}-6^{\text {th }}$ March, pp 165-172

Olarewaju, A. J., Kameswara Rao, N.S.V and Mannan, M.A., (2011m). Dimensionless Response of Underground Pipes Due to Blast Loads Using Finite Element Method, Journal of Geotechnical Engineering, EJGE, (16/E), March, ISSN 1089-3032, pp 563-574

Olarewaju, A. J., Kameswara Rao, N.S.V and Mannan, M.A., (2011n). Simulation and Verification of Blast Load Duration for Studying the Response of Underground Horizontal and Vertical Pipes Using Finite Element Method, Journal of Geotechnical Engineering, EJGE, (16/G), April, ISSN 1089-3032 pp 785-796

Olarewaju, A. J. (2008a). Engineering Properties of Recycled Concrete and Used Steel Reinforcement Bars. M. Eng Thesis, Civil Engineering Department, Federal University of Technology, Alure, Ondo State, Nigeria, July 
Olarewaju, A. J., (2004b). Soil Stabilization, M.ENG Seminar Paper on Course Title: Advanced Soil Mechanics (CVE 821), Civil Engineering Department, Federal University of Technology, Akure, Ondo State, Nigeria, June

Olarewaju, A. J., Balogun, M. O. and Akinlolu, S. O. (2011). Suitability of Eggshell Stabilized Lateritic Soil as Subgrade Material for Road Construction. Electronic Journal of Geotechnical Engineering (EJGE), ISSN 1089-3032, (16/H), April, pp 899-908

Omang, M. Borve, S. and Trulsen, J. (2009). Numerical Simulations of Blast Wave Propagation in Underground Facilities, Norwegian Defense Research Establishment, Norway

Peter, D. S. and Andrew, T. (2009). Blast Load Assessment by Simplified and Advanced Methods, Defence College of Management and Technology, Defence Academy of the United Kingdom, Cranfield University, UK. http:/ / www.civ.uth.gr/

Prakash, S. and Puri, V. K. (2010). Past and Future of Liquefaction, Proceedings of Indian Geotechnical Conference, ISBN: 978-0230332089, ISBN: 0230-332080, GEOtrendz, Macmillan Publisher India Ltd., Vol. III, 63-72

Raju, V. R. (2010). Ground Improvement - Application and Quality Control, ISBN: 978 0230332089, ISBN: 0230-332080, Proceedings of Indian Geotechnical Conference, GEOtrendz, Macmillan Publisher India Ltd., Vol. III, 121-131

Ramakrishan, K. (1979). Finite element analysis of pipes buried in linearly and non linear elastic media. M.Tech thesis, Civil Engineering Department, Indian Institute of Technology, Kanpur, India

Randall C. Conner, (1999). Pipeline and Rehabilitation, Proceedings of the group of ASCE Technical Sessions at the 1999 American Public Works Association International Public Works Congress and exposition, September, 19-22, Denver Colorado

Remennikov, A. M. (2003). A Review of Methods for Predicting Bomb Blast Effects on Buildings, University of Wollongong, International Journal of Battlefield Technology, 6(3): pp 5-10

Robert, W. D. (2002). Geotechnical Earthquake Engineering Handbook, McGraw Hill, New York, ISBN: 0-07-137782-4

Ronanki, S. S. (1997). Response Analysis of Buried Circular Pipes under 3 Dimensional Seismic Loading, M.Tech thesis, Civil Engineering Department, Indian Institute of Technology, Kanpur, India

Susana Lopex Querol and Rafael Blazquez, (2006). Identification of failure mechanisms of road embankments due to liquefaction: optimal corrective measures at seismic sites. Canadian Geotechnic Journal, NRC Canada, 43: pp 889-0-902

Towhata, I. and Sim, W. W. (2010). Model Tests on Embedded Pipeline Crossing a Seismic Fault. ISBN: 978-984-8725-01-6, Proceedings of the 3rd International Earthquake Symposium, Bangladesh University of Engineering and Technology, Dhaka, 1-12

Unified Facilities Criteria. (2008). Structures to Resist the Effects of Accidental Explosions, UFC 3 340-02, Department of Defense, US Army Corps of Engineers, Naval Facilities Engineering Command, Air Force Civil Engineer Support Agency, United States of America

Zhenweng Yang. (1997). Finite element simulation of response of buried shelters to blast loadings, National University of Singapore, Republic of Singapore, International Journal of Finite Element in Analysis and Design, 24: pp 113-132 

